Cover. Photograph showing the Balkhab River looking upstream at Sari Puhl in northern Afghanistan. (Photograph by Thomas J. Mack, U.S. Geological Survey) 


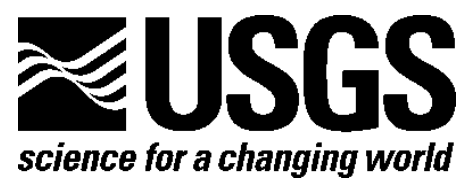

Prepared under the auspices of the U.S. Task Force for Business and Stability Operations

\section{Streamflow Characteristics at Streamgages in Northern Afghanistan and Selected Locations}

By Scott A. Olson and Tara Williams-Sether

Data Series 529

U.S. Department of the Interior

U.S. Geological Survey

USGS Afghanistan Project Product Number 179 


\section{U.S. Department of the Interior \\ KEN SALAZAR, Secretary}

\section{U.S. Geological Survey \\ Marcia K. McNutt, Director}

U.S. Geological Survey, Reston, Virginia: 2010

For product and ordering information:

World Wide Web: http://www.usgs.gov/pubprod

Telephone: 1-888-ASK-USGS

For more information on the USGS-the Federal source for science about the Earth, its natural and living resources, natural hazards, and the environment:

World Wide Web: http://www.usgs.gov

Telephone: 1-888-ASK-USGS

Suggested citation:

Olson, S.A., and Williams-Sether, T., 2010, Streamflow characteristics at streamgages in northern Afghanistan and selected locations: U.S. Geological Survey Data Series 529, 512 p., at http://pubs.usgs.gov/ds/529/.

Any use of trade, product, or firm names is for descriptive purposes only and does not imply endorsement by the U.S. Government.

Although this report is in the public domain, permission must be secured from the individual copyright owners to reproduce any copyrighted material contained within this report. 


\section{Contents}

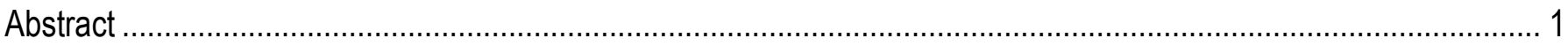

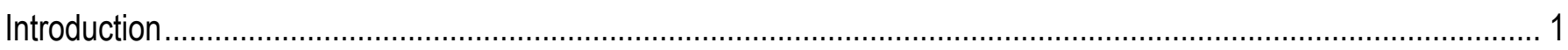

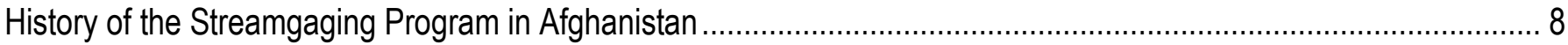

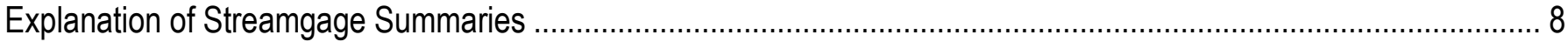

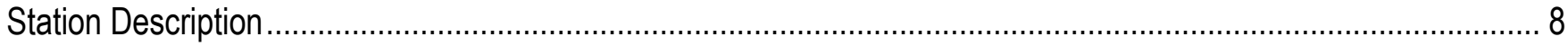

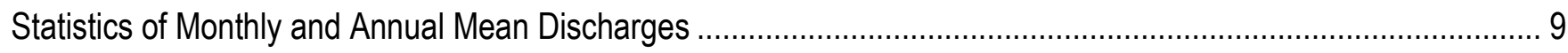

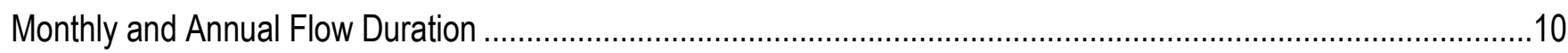

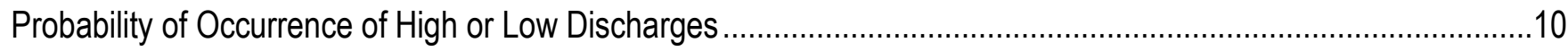

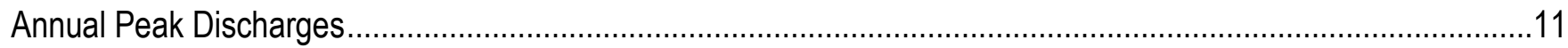

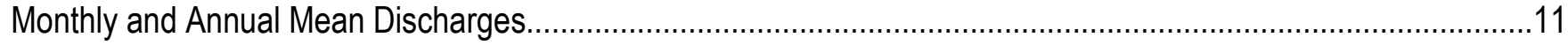

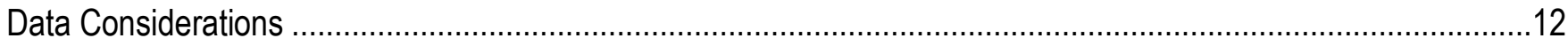

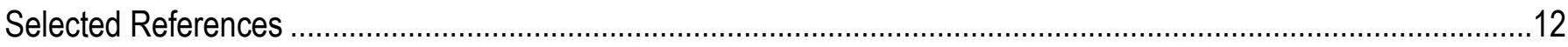

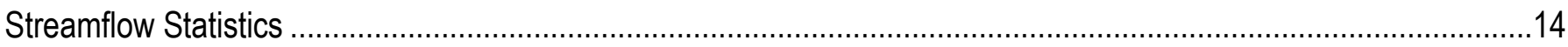

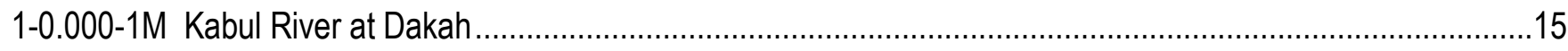

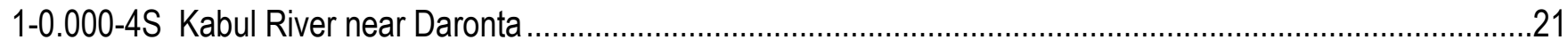

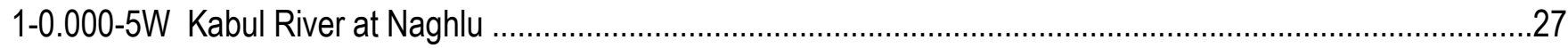

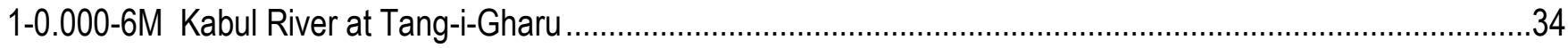

1-2.R00-7T Hazarnaw River at Sabay ...............................................................................................

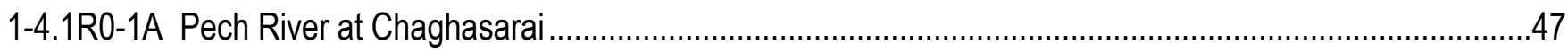

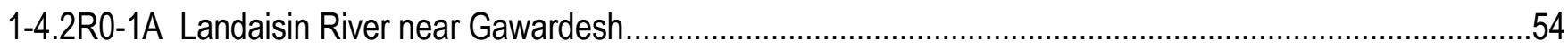

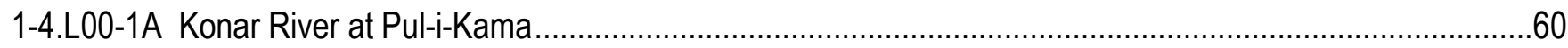

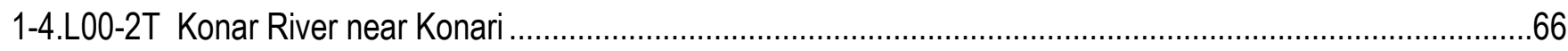

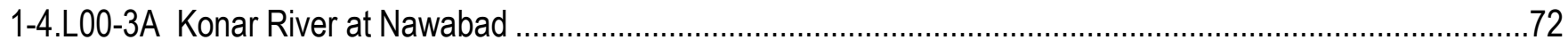

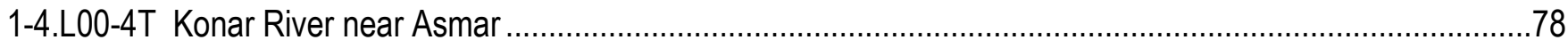

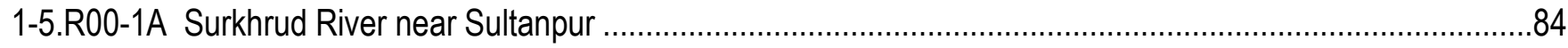

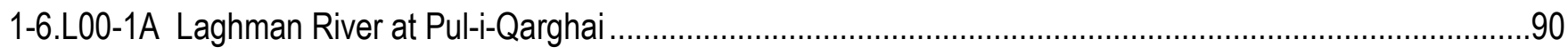

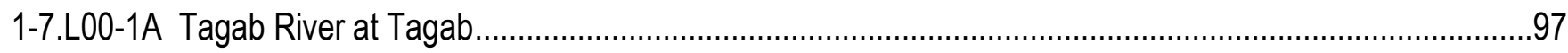

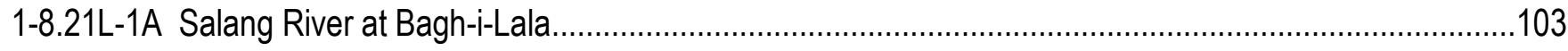

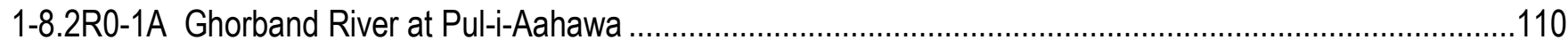

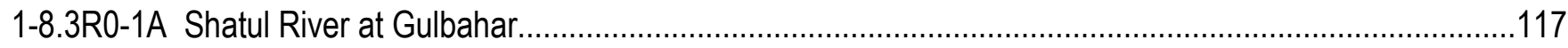

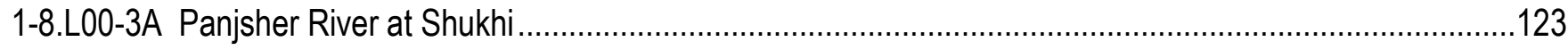

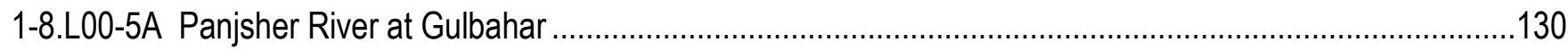

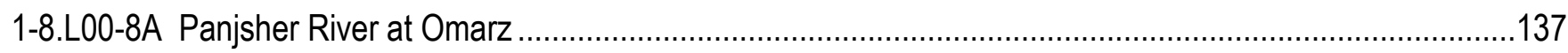

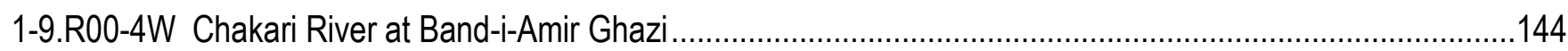

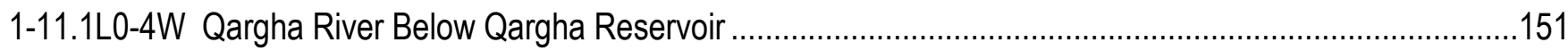




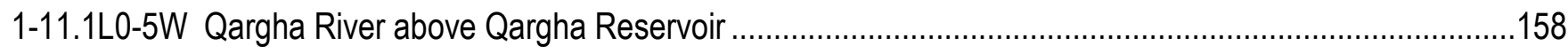

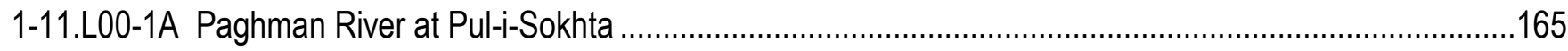

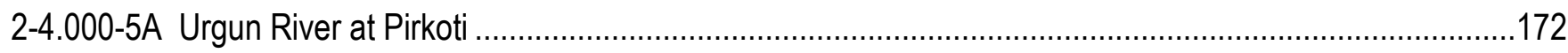

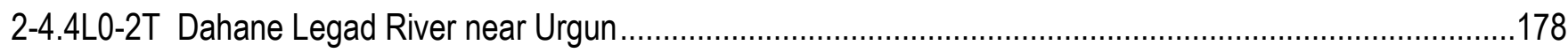

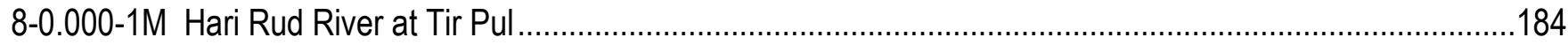

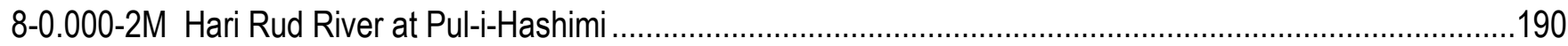

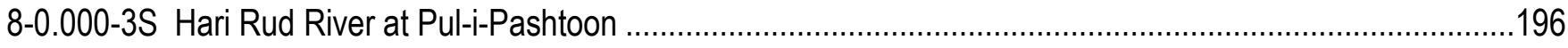

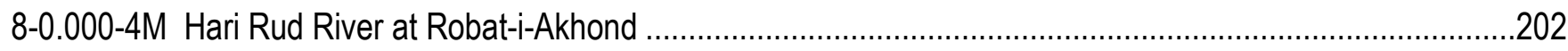

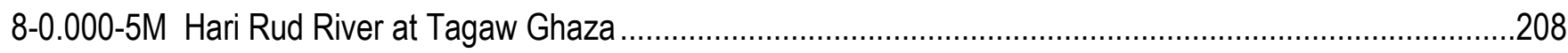

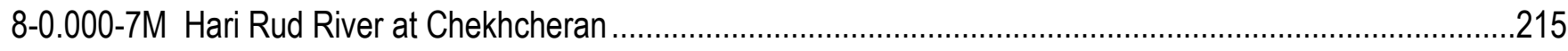

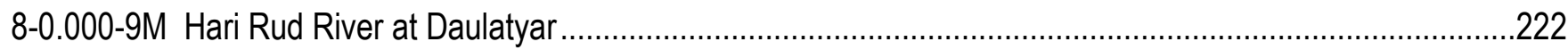

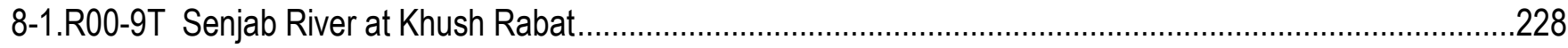

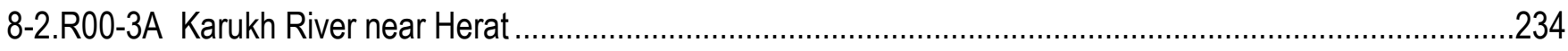

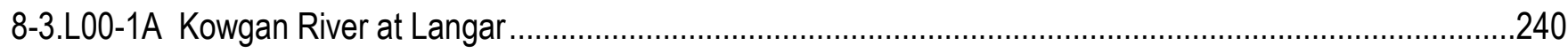

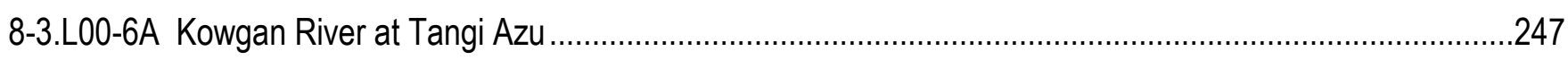

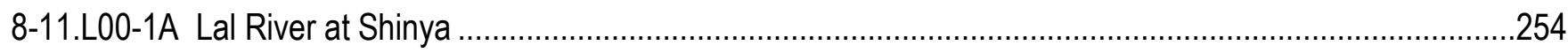

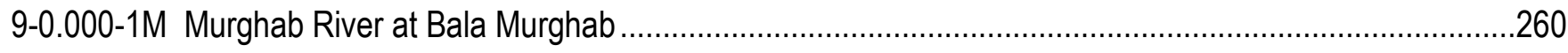

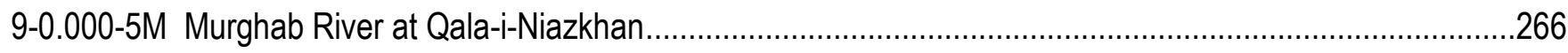

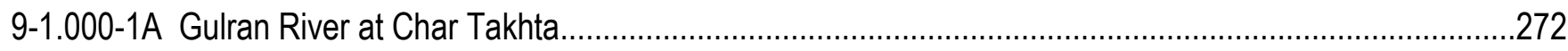

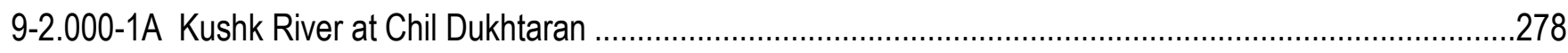

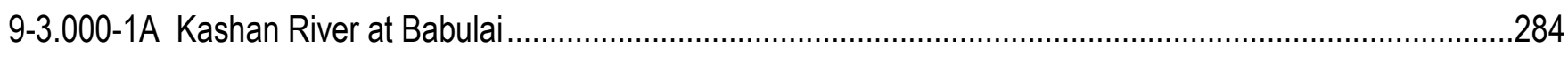

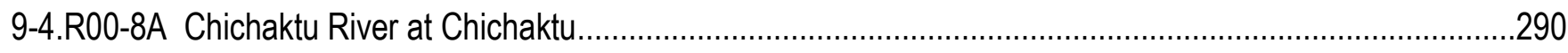

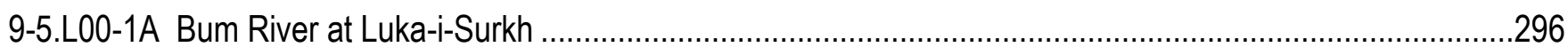

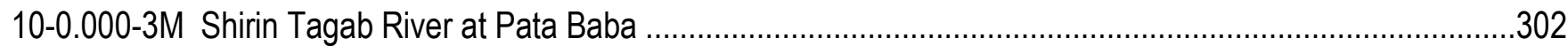

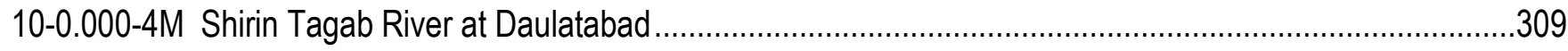

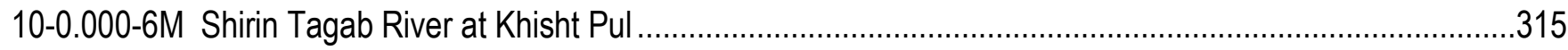

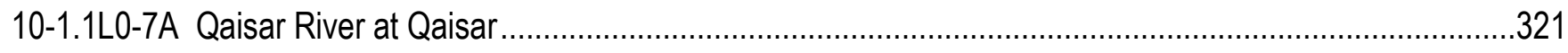

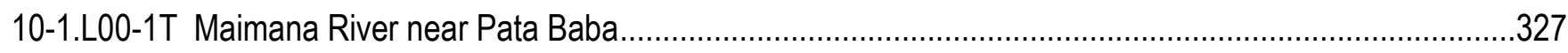

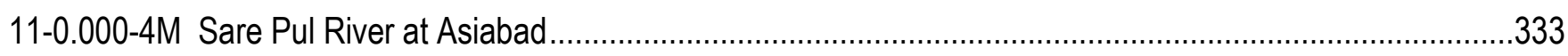

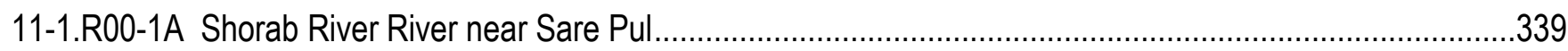

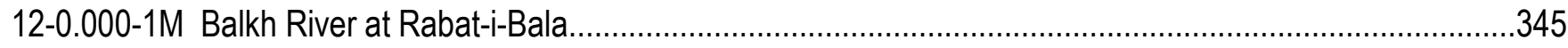

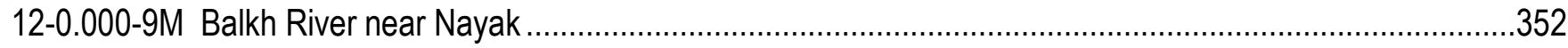

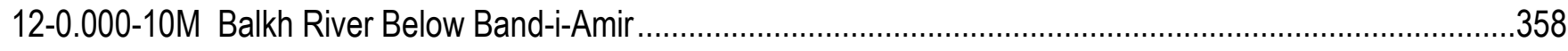

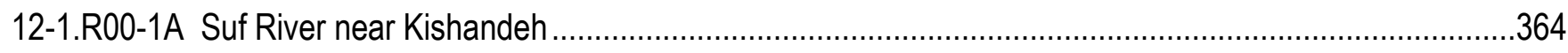

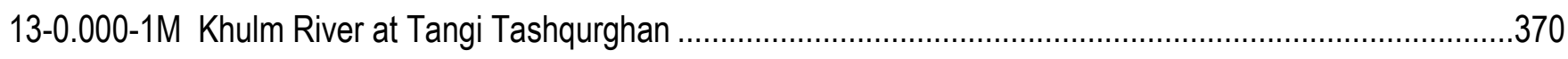

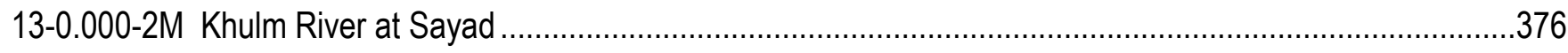

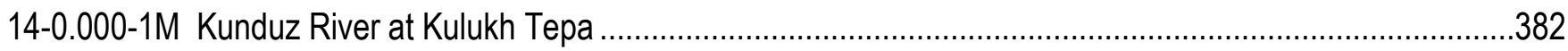




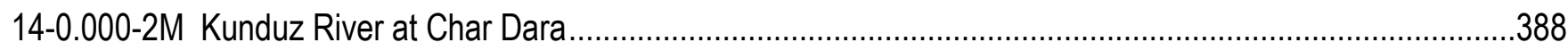

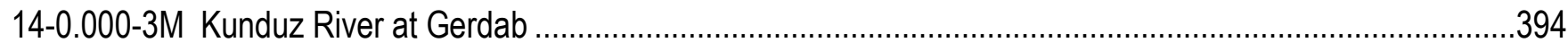

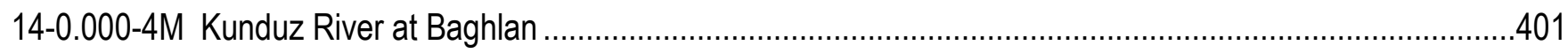

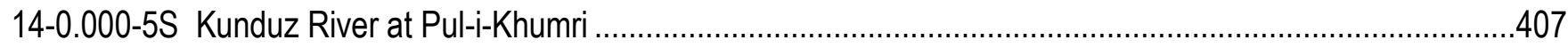

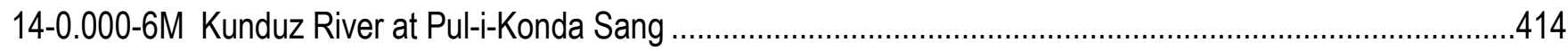

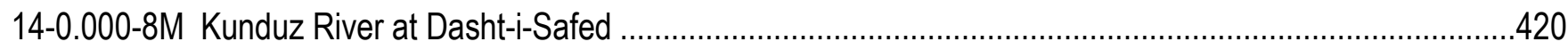

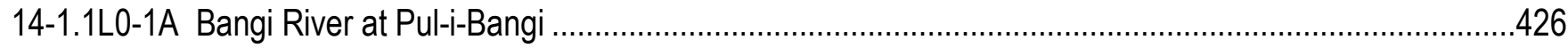

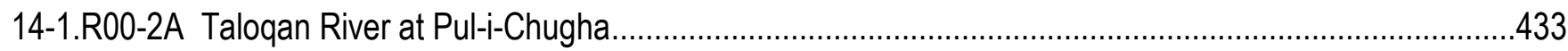

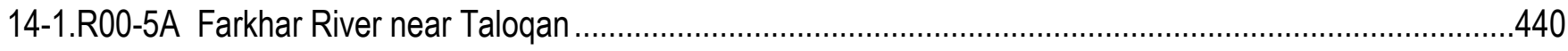

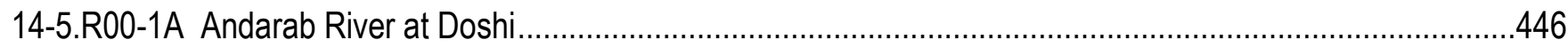

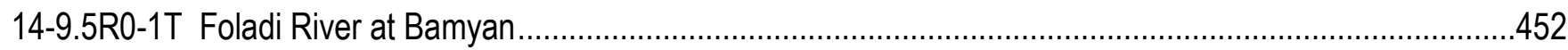

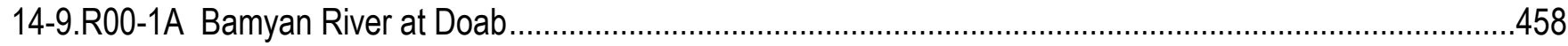

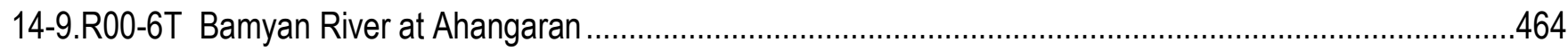

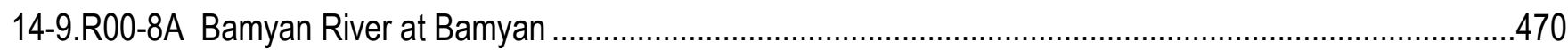

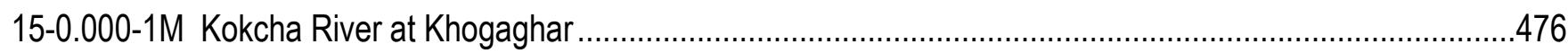

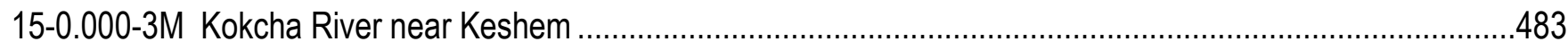

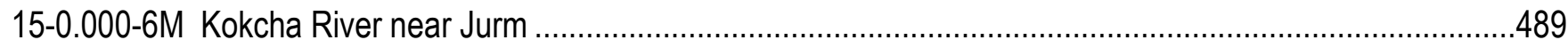

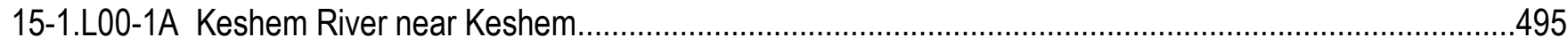

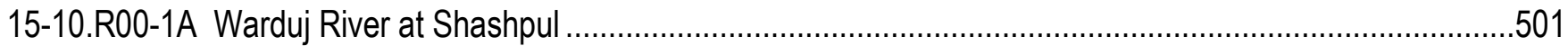

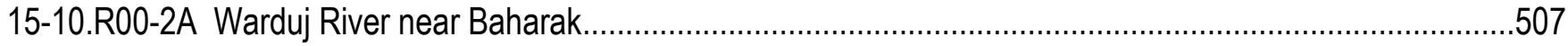




\section{Figures}

Figure 1. Map showing location of streamgages within the Kabul and Indus Basins for which streamflow statistics are published in this report 2

Figure 2. Map showing location of streamgages within the Amu Darya Basin for which streamflow statistics are published in this report 3

Figure 3. Map showing locations of streamgages within the Northern Basins for which streamflow statistics are published in this report

Figure 4. Map showing locations of streamgages within the Hari Rud and Murghab Basins for which streamflow statistics are published in this report

\section{Table}

Table 1. List of streamgages in Afghanistan for which streamflow statistics are published in this report 6 


\section{Conversion Factors}

\begin{tabular}{|c|c|c|}
\hline Multiply & By & To obtain \\
\hline \multicolumn{3}{|c|}{ Length } \\
\hline meter $(\mathrm{m})$ & 3.281 & foot $(\mathrm{ft})$ \\
\hline kilometer (km) & 0.6214 & mile (mi) \\
\hline \multicolumn{3}{|c|}{ Area } \\
\hline square meter $\left(\mathrm{m}^{2}\right)$ & 0.0002471 & acre \\
\hline hectare (ha) & 2.471 & acre \\
\hline square kilometer $\left(\mathrm{km}^{2}\right)$ & 247.1 & acre \\
\hline square meter $\left(\mathrm{m}^{2}\right)$ & 10.76 & square foot $\left(\mathrm{ft}^{2}\right)$ \\
\hline square hectometer $\left(\mathrm{hm}^{2}\right)$ & 0.003861 & section (640 acres or 1 square mile) \\
\hline hectare (ha) & 0.003861 & square mile $\left(\mathrm{mi}^{2}\right)$ \\
\hline square kilometer $\left(\mathrm{km}^{2}\right)$ & 0.3861 & square mile $\left(\mathrm{mi}^{2}\right)$ \\
\hline \multicolumn{3}{|c|}{ Volume } \\
\hline cubic meter $\left(\mathrm{m}^{3}\right)$ & 264.2 & gallon (gal) \\
\hline cubic meter $\left(\mathrm{m}^{3}\right)$ & 0.0002642 & million gallons (Mgal) \\
\hline cubic meter $\left(\mathrm{m}^{3}\right)$ & 35.31 & cubic foot $\left(\mathrm{ft}^{3}\right)$ \\
\hline cubic meter $\left(\mathrm{m}^{3}\right)$ & 1.308 & cubic yard $\left(\mathrm{yd}^{3}\right)$ \\
\hline cubic meter $\left(\mathrm{m}^{3}\right)$ & 0.0008107 & acre-foot (acre-ft) \\
\hline \multicolumn{3}{|c|}{ Flow rate } \\
\hline cubic meter per second $\left(\mathrm{m}^{3} / \mathrm{s}\right)$ & 70.07 & acre-foot per day (acre-ft/d) \\
\hline cubic meter per second $\left(\mathrm{m}^{3} / \mathrm{s}\right)$ & 35.31 & cubic foot per second $\left(\mathrm{ft}^{3} / \mathrm{s}\right)$ \\
\hline $\begin{array}{l}\text { cubic meter per second per square } \\
\text { kilometer }\left[\left(\mathrm{m}^{3} / \mathrm{s}\right) / \mathrm{km}^{2}\right]\end{array}$ & 91.49 & $\begin{array}{l}\text { cubic foot per second per square mile } \\
{\left[\left(\mathrm{ft}^{3} / \mathrm{s}\right) / \mathrm{mi}^{2}\right]}\end{array}$ \\
\hline cubic meter per second $\left(\mathrm{m}^{3} / \mathrm{s}\right)$ & 22.83 & million gallons per day (Mgal/d) \\
\hline
\end{tabular}

Altitude, as used in this report, refers to distance above mean sea level at an unknown vertical datum. 


\title{
Streamflow Characteristics at Streamgages in Northern Afghanistan and Selected Locations
}

\author{
By Scott A. Olson and Tara Williams-Sether
}

\begin{abstract}
Statistical summaries of streamflow data for 79 historical streamgages in Northern Afghanistan and other selected historical streamgages are presented in this report. The summaries for each streamgage include (1) station description, (2) graph of the annual mean discharge for the period of record, (3) statistics of monthly and annual mean discharges, (4) monthly and annual flow duration, (5) probability of occurrence of annual high discharges, (6) probability of occurrence of annual low discharges, (7) probability of occurrence of seasonal low discharges, (8) annual peak discharges for the period of record, and (9) monthly and annual mean discharges for the period of record.

\section{Introduction}

Preliminary hydrologic evaluations are needed to determine the water-resource priorities in all areas of Afghanistan for agricultural, industrial, and residential water uses. This report focuses on providing streamflow characteristics of 79 streamgages in the northern half of Afghanistan, primarily in the Kabul, Amu Darya, Northern, Hari Rud and Murghab Basins (figs. 1-4). This report also includes some streamgages in other parts of Afghanistan that were not included in previous reports (WilliamsSether, 2008; Vining, 2010). The basins represented in the report are in arid and mountainous environments, often fed by melting snow from the high mountains and infrequent storms.

Because there can be great fluctuations in streamflow, from flood to drought, in Afghanistan, knowledge of the magnitude and time distribution of streamflow is essential for all aspects of water management and environmental planning. To provide the Afghan managers with necessary streamflow information, the U.S. Geological Survey, in cooperation with the U.S. Task Force for Business and Stability Operations, computed streamflow statistics for data collected at historical streamgages in northern Afghanistan and selected locations in southeastern Afghanistan. Agencies responsible for the development and management of the surface-water resources of Afghanistan can use the data provided in this report to assist in making safe, economical, and environmentally sound water-resource planning decisions. The historical streamgages used are listed in table 1 and their locations are shown in figures $1-4$.
\end{abstract}




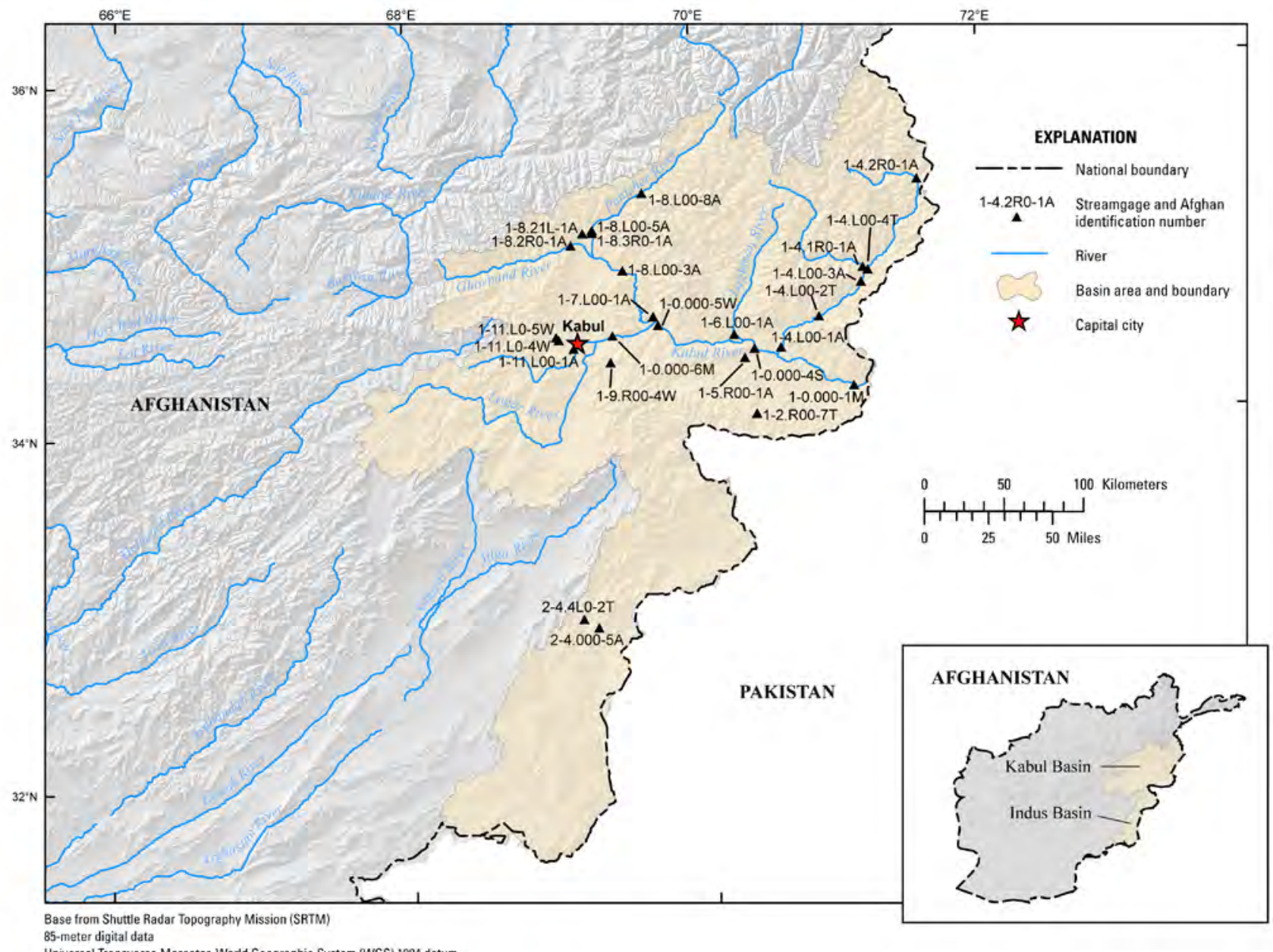

Universal Transverse Mercator, World Geographic System (WGS) 1984 datum

Figure 1. Location of streamgages within the Kabul and Indus Basins for which streamflow statistics are published in this report. Streamgage identification data shown in table 1. 


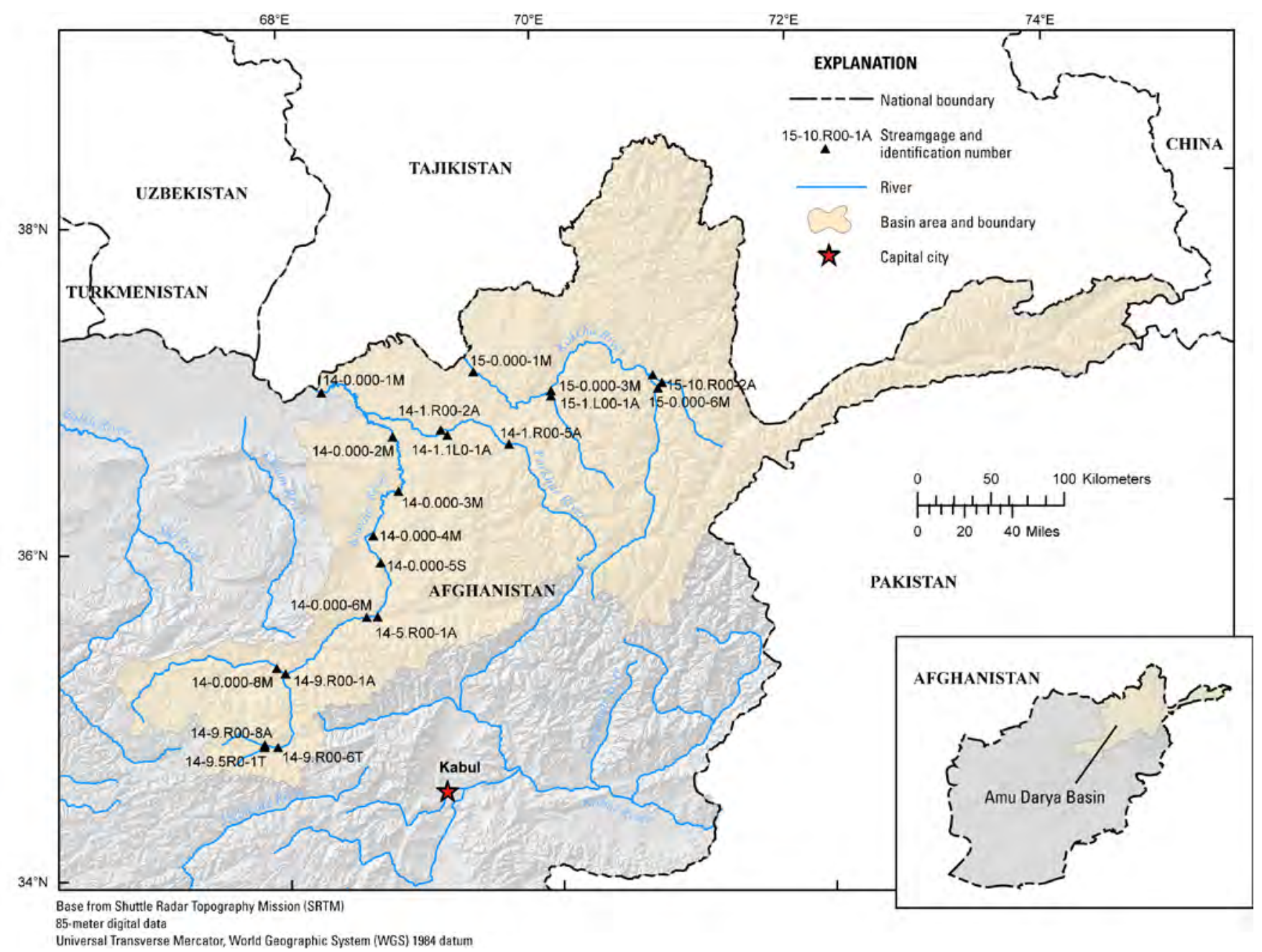

Figure 2. Location of streamgages within the Amu Darya Basin for which streamflow statistics are published in this report. Streamgage identification data shown in table 1. 


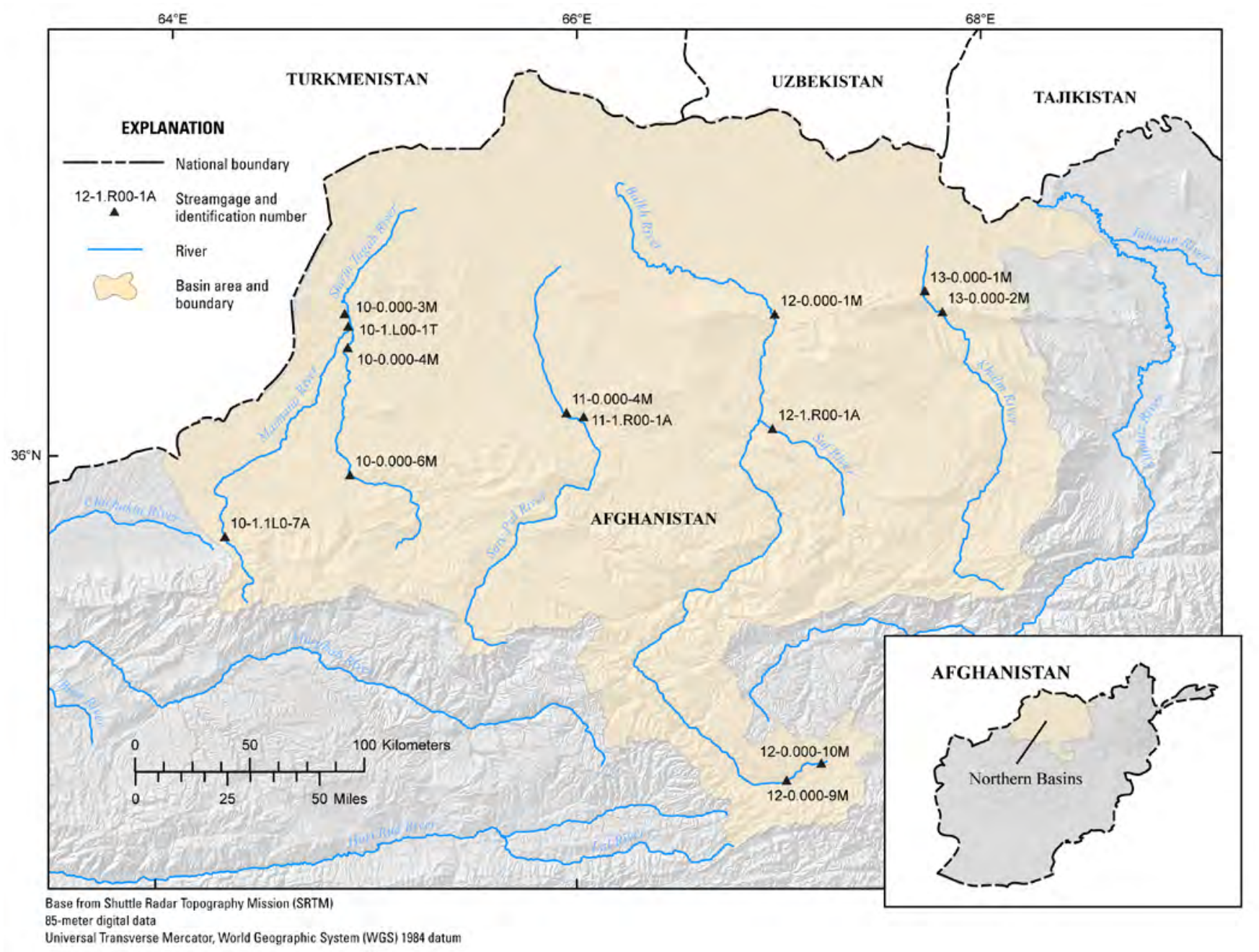

Figure 3. Locations of streamgages within the Northern Basins for which streamflow statistics are published in this report. Streamgage identification data shown in table 1. 


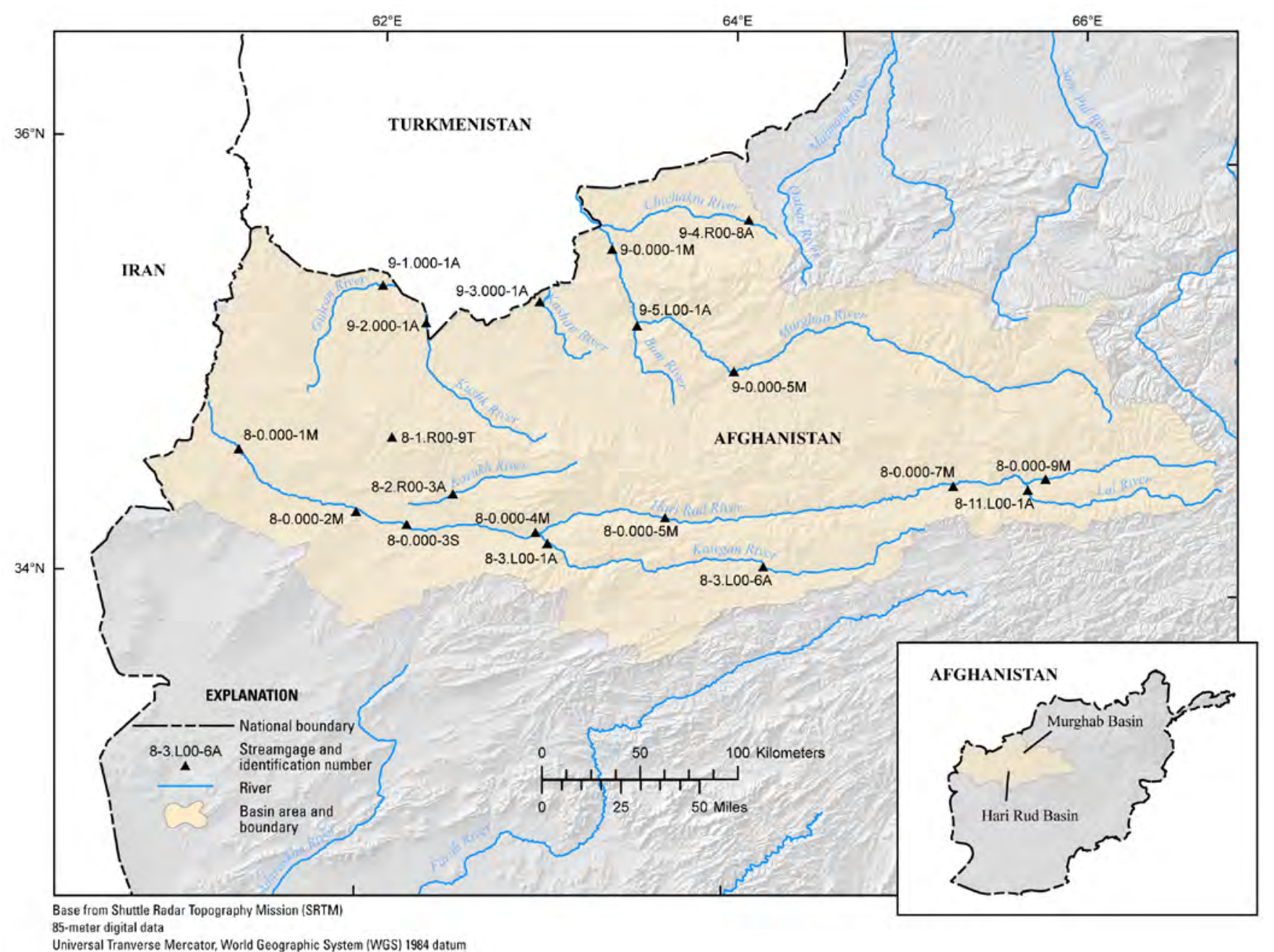

Figure 4. Locations of streamgages within the Hari Rud and Murghab Basins for which streamflow statistics are published in this report. Streamgage identification data shown in table 1. 
Table 1. List of streamgages in Afghanistan for which streamflow statistics are published in this report.

\begin{tabular}{|c|c|c|}
\hline $\begin{array}{c}\text { Afghan } \\
\text { identification } \\
\text { number }\end{array}$ & $\begin{array}{c}\text { U.S. Geological } \\
\text { Survey identification } \\
\text { number }\end{array}$ & Station name \\
\hline $1-0.000-1 \mathrm{M}$ & 341400071020000 & Kabul River at Dakah \\
\hline $1-0.000-4 S$ & 342800070220000 & Kabul River near Daronta \\
\hline $1-0.000-5 \mathrm{~W}$ & 343700069430000 & Kabul River at Naghlu \\
\hline $1-0.000-6 \mathrm{M}$ & 343400069240000 & Kabul River at Tang-i-Gharu \\
\hline 1-2.R00-7T & 340600070220000 & Hazarnaw River at Sabay \\
\hline $1-4.1 \mathrm{R} 0-1 \mathrm{~A}$ & 345400071080000 & Pech River at Chaghasarai \\
\hline $1-4.2 \mathrm{R} 0-1 \mathrm{~A}$ & 352300071320000 & Landaisin River near Gawardesh \\
\hline 1-4.L00-1A & 342800070330000 & Konar River at Pul-i-Kama \\
\hline 1-4.L00-2T & 343800070490000 & Konar River near Konari \\
\hline 1-4.L00-3A & 344900071070000 & Konar River at Nawabad \\
\hline 1-4.L00-4T & 345300071100000 & Konar River near Asmar \\
\hline $1-5 . R 00-1 \mathrm{~A}$ & 342500070180000 & Surkhrud River near Sultanpur \\
\hline 1-6.L00-1A & 343300070140000 & Laghman River at Pul-i-Qarghai \\
\hline 1-7.L00-1A & 344000069410000 & Tagab River at Tagab \\
\hline $1-8.21 \mathrm{~L}-1 \mathrm{~A}$ & 350900069130000 & Salang River at Bagh-i-Lala \\
\hline $1-8.2 \mathrm{R} 0-1 \mathrm{~A}$ & 350500069080000 & Ghorband River at Pul-i-Ashawa \\
\hline $1-8.3 \mathrm{R} 0-1 \mathrm{~A}$ & 350900069170000 & Shatul River at Gulbahar \\
\hline 1-8.L00-3A & 345600069290000 & Panjsher River at Shukhi \\
\hline 1-8.L00-5A & 351000069170000 & Panjsher River at Gulbahar \\
\hline 1-8.L00-8A & 352200069380000 & Panjsher River at Omarz \\
\hline 1-9.R00-4W & 342500069230000 & Chakari River at Band-i-Amir Ghazi \\
\hline $1-11.1 \mathrm{~L} 0-4 \mathrm{~W}$ & 343300069020000 & Qargha River below Qargha Reservior \\
\hline $1-11.1 \mathrm{~L} 0-5 \mathrm{~W}$ & 343400069010000 & Qargha River above Qargha Reservoir \\
\hline 1-11.L00-1A & 343000069080000 & Paghman River at Pul-i-Sokhta \\
\hline $2-4.000-5 \mathrm{~A}$ & 325500069150000 & Urgun River at Pirkoti \\
\hline $2-4.4 \mathrm{~L} 0-2 \mathrm{~T}$ & 325800069090000 & Dahane Legad River near Urgun \\
\hline $8-0.000-1 \mathrm{M}$ & 343600061160000 & Hari Rud River at Tir Pul \\
\hline $8-0.000-2 \mathrm{M}$ & 342000061560000 & Hari Rud River at Pul-i-Hashimi \\
\hline $8-0.000-3 \mathrm{~S}$ & 341700062130000 & Hari Rud River at Pul-i-Pashtoon \\
\hline $8-0.000-4 \mathrm{M}$ & 341600062560000 & Hari Rud River at Robat-I-Akhond \\
\hline $8-0.000-5 \mathrm{M}$ & 342100063390000 & Hari Rud River at Tagaw Ghaza \\
\hline $8-0.000-7 \mathrm{M}$ & 343100065150000 & Hari Rud River at Chekhcheran \\
\hline $8-0.000-9 \mathrm{M}$ & 343300065460000 & Hari Rud River at Daulatyar \\
\hline 8-1.R00-9T & 344100062070000 & Senjab River at Khush Rabat \\
\hline 8-2.R00-3A & 342600062280000 & Karukh River near Herat \\
\hline 8-3.L00-1A & 341300063000000 & Kowgan River at Langar \\
\hline 8-3.L00-6A & 340800064120000 & Kowgan River at Tangi Azu \\
\hline 8-11.L00-1A & 343000065400000 & Lal River at Shinya \\
\hline $9-0.000-1 \mathrm{M}$ & 353500063190000 & Murghab River at Bala Murghab \\
\hline $9-0.000-5 \mathrm{M}$ & 350200064010000 & Murghab River at Qala-i-Niazkhan \\
\hline $9-1.000-1 \mathrm{~A}$ & 352300062020000 & Gulran River at Char Takhta \\
\hline
\end{tabular}


Table 1. List of streamgages in Afghanistan for which streamflow statistics are published in this report.

-Continued

\begin{tabular}{|c|c|c|}
\hline $\begin{array}{c}\text { Afghan } \\
\text { identification } \\
\text { number }\end{array}$ & $\begin{array}{c}\text { U.S. Geological } \\
\text { Survey identification } \\
\text { number }\end{array}$ & Station name \\
\hline $9-2.000-1 \mathrm{~A}$ & 351300062170000 & Kushk River at Chil Dukhtaran \\
\hline $9-3.000-1 \mathrm{~A}$ & 352000062550000 & Kashan River at Babulai \\
\hline 9-4.R00-8A & 354400064050000 & Chichaktu River at Chichaktu \\
\hline 9-5.L00-1A & 351400063280000 & Bum River at Luka-i-Surkh \\
\hline $10-0.000-3 \mathrm{M}$ & 363500064520000 & Shirin Tagab River at Pata Baba \\
\hline $10-0.000-4 \mathrm{M}$ & 362700064530000 & Shirin Tagab River at Daulatabad \\
\hline $10-0.000-6 \mathrm{M}$ & 355700064540000 & Shirin Tagab River at Khisht Pul \\
\hline 10-1.1L0-7A & 354200064180000 & Qaisar River at Qaisar \\
\hline 10-1.L00-1T & 363200064530000 & Maimana River near Pata Baba \\
\hline $11-0.000-4 \mathrm{M}$ & 361200065570000 & Sare Pul River at Asiabad \\
\hline 11-1.R00-1A & 361100066020000 & Shorab River near Sare Pul \\
\hline $12-0.000-1 \mathrm{M}$ & 363500066580000 & Balkh River at Rabat-i-Bala \\
\hline $12-0.000-9 \mathrm{M}$ & 344500067000000 & Balkh River near Nayak \\
\hline $12-0.000-10 \mathrm{M}$ & 344900067100000 & Balkh River below Band-i-Amir \\
\hline 12-1.R00-1A & 360800066570000 & Suf River near Kishandeh \\
\hline $13-0.000-1 \mathrm{M}$ & 364000067420000 & Khulm River at Tangi Tashqurghan \\
\hline $13-0.000-2 \mathrm{M}$ & 363500067470000 & Khulm River at Sayad \\
\hline $14-0.000-1 \mathrm{M}$ & 365900068180000 & Kunduz River at Kulukh Tepa \\
\hline $14-0.000-2 \mathrm{M}$ & 364200068500000 & Kunduz River at Char Dara \\
\hline $14-0.000-3 \mathrm{M}$ & 362200068520000 & Kunduz River at Gerdab \\
\hline $14-0.000-4 \mathrm{M}$ & 360600068400000 & Kunduz River at Baghlan \\
\hline $14-0.000-5 \mathrm{~S}$ & 355600068430000 & Kunduz River at Pul-i-Khumri \\
\hline $14-0.000-6 \mathrm{M}$ & 353600068360000 & Kunduz River at Pul-i-Konda Sang \\
\hline $14-0.000-8 \mathrm{M}$ & 351800067550000 & Kunduz River at Dasht-i-Safed \\
\hline $14-1.1 \mathrm{~L} 0-1 \mathrm{~A}$ & 364200069150000 & Bangi River at Pul-i-Bangi \\
\hline 14-1.R00-2A & 364400069120000 & Taloqan River at Pul-i-Chugha \\
\hline 14-1.R00-5A & 363800069430000 & Farkhar River near Taloqan \\
\hline 14-5.R00-1A & 353600068410000 & Andarab River at Doshi \\
\hline $14-9.5 \mathrm{R} 0-1 \mathrm{~T}$ & 344900067490000 & Foladi River at Bamyan \\
\hline 14-9.R00-1A & 351600067590000 & Bamyan River at Doab \\
\hline 14-9.R00-6T & 344900067550000 & Bamyan River at Ahangaran \\
\hline 14-9.R00-8A & 345000067490000 & Bamyan River at Bamyan \\
\hline $15-0.000-1 \mathrm{M}$ & 370500069280000 & Kokcha River at Khojaghar \\
\hline $15-0.000-3 \mathrm{M}$ & 365700070030000 & Kokcha River near Keshem \\
\hline $15-0.000-6 \mathrm{M}$ & 365600070520000 & Kokcha River near Jurm \\
\hline 15-1.L00-1A & 365500070030000 & Keshem River near Keshem \\
\hline 15-10.R00-1A & 370100070500000 & Warduj River at Shashpul \\
\hline $15-10 \cdot \mathrm{R} 00-2 \mathrm{~A}$ & 365800070540000 & Warduj River near Baharak \\
\hline
\end{tabular}




\section{History of the Streamgaging Program in Afghanistan}

Streamgaging began in Afghanistan in the mid-1940s at a few sites. The number of sites increased over the years until the late 1970s. Streamgaging was discontinued soon after the Soviet invasion of Afghanistan in 1979. Until 1978, Afghanistan had a network of approximately 160 streamgages. No streamflow data were collected after September 1980 until recently. In 2005, three historical streamgages were re-established and currently (2010) much of the historic network has been re-established and is being operated by the Afghanistan Ministry of Energy and Water.

\section{Explanation of Streamgage Summaries}

Streamgage summaries are presented so that each station description and tables of streamflow statistics are presented in the same order and format for each streamgage, including the same relative placement on the pages. Because the statistical information listed in the tables was created by computer "data retrievals" or from statistical program results, significant figures were not always rounded to U.S. Geological Survey standards. The order of presentation is as follows:

1. Station description,

2. Graph of the annual mean discharge for the period of record,

3. Table of statistics of monthly and annual mean discharges,

4. Table of monthly and annual flow duration,

5. Table of probability of occurrence of annual high discharges,

6. Table of probability of occurrence of annual low discharges,

7. Table of probability of occurrence of seasonal low discharges,

8. Table of annual peak discharge and corresponding gage height for the period of record, and

9. Table of monthly and annual mean discharges for the period of record.

\section{Station Description}

The location, drainage area, period of record, and other available information about each streamgage included in this report are presented in the station description. This information is compiled from records originally published by the Afghanistan Ministry of Energy and Water. The following comments clarify information presented under the various headings of the station description.

LOCATION: Information on streamgage location is limited to latitude and longitude.

DRAINAGE AREA: Drainage area is as reported by the Afghanistan Ministry of Energy and Water from Survey of India maps. The accuracy of the drainage areas is unknown.

ALTITUDE: The datum of the streamgage referenced to mean sea level.

PERIOD OF RECORD: The period of record is the period for which there are published records for the streamgage or for an equivalent streamgage. An equivalent streamgage is a streamgage that was in operation prior to or after the subject streamgage, and whose location is such that records from it can reasonably be considered equivalent with records from the subject streamgage. This situation arises 
when a streamgage is relocated upstream or downstream and given a new streamgage number or name, but the changes in drainage area and other basin characteristics are not significantly different.

GAGE: The type of streamgage or recorder that is or was used to collect data. A condensed history of the types, locations, and datums of previous streamgages are given under this heading when available.

\section{Statistics of Monthly and Annual Mean Discharges}

Statistics of monthly and annual mean discharges presented for each streamgage include (1) the maximum, minimum, and mean monthly discharges and (2) the maximum, minimum, and mean annual discharges. The water years (October 1 through September 30) in which the maximum and minimum discharges occurred are listed with the respective values, and the standard deviation and coefficient of variation of the monthly and annual mean discharges are listed with the respective values. Also, the percentage of the annual discharge that is comprised by each monthly mean discharge is listed in the table. The statistics of monthly and annual means discharges were computed using statistical applications built into the U.S. Geological Survey Automated Data Processing System (ADAPS) Version 4.9 (U.S. Geological Survey, 2009).

Each of the statistics is explained in the following paragraphs. As an aid to the readers' understanding of how the monthly mean and annual mean discharges are determined, data for the streamgage Kokcha River at Khojaghar (15-0.000-1M) are used as an example. Each monthly mean is the mean of the daily values for the month. Months or years for which all daily values are not available are not included in the compilation of statistics.

The maximum monthly mean discharge is the maximum value of all the monthly mean values for a given month during the period of record. The maximum mean value for October is $142 \mathrm{~m}^{3} / \mathrm{s}$, which occurred during water year 1968. Similarly, the minimum monthly mean discharge is the minimum value of all the monthly mean values for a specific month over the period of record. The minimum mean value for October is $82.2 \mathrm{~m}^{3} / \mathrm{s}$, which occurred during water year 1972 . The maximum and minimum monthly mean values can be found in the statistics of monthly and annual mean discharges table or by searching the columns of the monthly and annual mean discharges table.

The mean monthly discharge is the mean of all the monthly mean discharges for a given month for the period of record, and the standard deviation is a measure of the variability of the values. The mean monthly discharge for October is $110 \mathrm{~m}^{3} / \mathrm{s}$, and the standard deviation is $19.9 \mathrm{~m}^{3} / \mathrm{s}$. The mean monthly discharge for October is the same as the mean of all October daily values for the period of record used. However, the standard deviation obtained using monthly values is smaller than the standard deviation obtained using all daily values. The standard deviation is smaller because the monthly values have less variability than the daily values.

The coefficient of variation is the ratio of the standard deviation to the mean. The coefficient of variation is dimensionless. Because monthly mean discharges are generally much greater in spring than in winter, the standard deviations also are generally much greater in spring than in winter. However, dividing the standard deviation by the mean monthly discharge tends to normalize the measures for all months so a more meaningful comparison among months can be made.

The percentage of annual discharge is the percentage of the annual discharge occurring each month. It is calculated by dividing the mean monthly discharge for a given month by the total of the 
12 mean monthly discharges and multiplying by 100 . Because of rounding of the monthly percentage, the sum of the 12 percentages may not always equal 100 percent.

The maximum, minimum, and mean annual discharges are selected or computed from the annual mean discharges for the period of record. The water years of occurrence of the maximum and minimum values are listed with the respective values, and the standard deviation of the annual mean discharge is listed with the mean discharge. For the Kokcha River at Khojaghar streamgage, the maximum annual mean discharge of $262 \mathrm{~m}^{3} / \mathrm{s}$ occurred in 1969, and the minimum annual mean discharge of $144 \mathrm{~m}^{3} / \mathrm{s}$ occurred in 1972. The mean annual discharge for the period of record is $198 \mathrm{~m}^{3} / \mathrm{s}$.

\section{Monthly and Annual Flow Duration}

The monthly and annual flow-duration table is a magnitude and frequency analysis of daily discharge values. It is computed by tabulating the number of daily discharge values that fall within preselected class limits, computing the percentage of values within each class, and interpolating discharge values for the percentages shown in the table. Monthly values are calculated from daily values for all complete months of record, and annual values are calculated for all complete water years. The monthly and annual flow durations were computed using statistical applications built into U.S. Geological Survey ADAPS Version 4.9 (U.S. Geological Survey, 2009).

For example, at the Kokcha River at Khojaghar (15-0.000-1M) streamgage, the 90-percent flowduration value for October is $77.3 \mathrm{~m}^{3} / \mathrm{s}$. This indicates that 90 percent of all October daily discharge values for the period of record were equal to or greater than $77.3 \mathrm{~m} / \mathrm{s}$.

\section{Probability of Occurrence of High or Low Discharges}

The probabilities of occurrence of annual high discharges, annual low discharges, and seasonal low discharges are presented in three tables for each streamgage. Probability of occurrence is an estimate of the likelihood that a particular discharge in a stream will be equaled or exceeded in 1 year or, in the case of low flows, the likelihood that the discharge will not be equaled or exceeded during the year or season. The probability of occurrence of a high flow is called the exceedance probability, and the probability of occurrence of low flow is called the nonexceedance probability. For example, if the instantaneous discharge for the 0.20 exceedance probability is listed as $1,050 \mathrm{~m}^{3} / \mathrm{s}$, then a 20 -percent chance exists that a discharge equal to or greater than $1,050 \mathrm{~m}^{3} / \mathrm{s}$ will occur once during the year.

Recurrence interval is another way of expressing annual probability, and it is the reciprocal of the probability of occurrence. The recurrence interval for an exceedance probability of 0.20 is 5 years ( 1 divided by 0.20 ). For a long discharge record, the annual maximum discharge can be expected to equal or exceed $1,050 \mathrm{~m}^{3} / \mathrm{s}$ on average once every 5 years.

The table of probability of annual high discharges for each streamgage lists the maximum instantaneous discharge and the maximum mean discharge for 3, 7, 15, and 30 consecutive-day periods for selected exceedance probabilities and equivalent recurrence intervals. Values for the maximum instantaneous discharge are computed from the streamflow record according to the guidelines established by the U.S. Interagency Advisory Committee on Water Data (1982). No adjustments are made for length of record, and a station skew instead of a regional skew was used in the computations. The U.S. Geological Survey PeakFQ software (Flynn and others, 2006) was used to compute the maximum instantaneous discharges for the selected exceedance probabilities. 
Values for the maximum daily mean discharges for 3, 7, 15, and 30 consecutive-day periods are computed from the annual high mean values of the corresponding periods. The computations are based on the log-Pearson Type III distribution (Helsel and Hirsch, 1992) using values obtained for the water year.

The table of probability of annual low discharges for each streamgage lists the minimum daily mean discharge for $1,3,7,14,30,60,90,120$, and 183 consecutive-day periods for selected nonexceedance probabilities and equivalent recurrence intervals. Values for the minimum mean discharges are computed from the annual low discharge values of the corresponding periods using the log-Pearson Type III distribution. Probabilities of annual low discharges are computed using values obtained for the climatic year (April 1 through March 31).

The table of probability of seasonal low discharges for each streamgage lists the minimum daily mean discharge for 1, 7, 14, and 30 consecutive-day periods for selected probabilities and equivalent recurrence intervals. These values are computed from the seasonal low mean values of the corresponding periods using the log-Pearson Type III distribution. The seasonal periods provided are December through February, March through May, June through August, and September through November.

The probabilities of the maximum mean discharge and the annual and seasonal minimum mean discharge were computed using SWSTAT (Lumb and others, U.S. Geological Survey, written commun., 2002). SWSTAT is a U.S. Geological Survey computer program for interactive computation of surfacewater statistics.

The annual low discharge and the seasonal low discharges that occur in any given year are sensitive to natural-channel processes, such as evapotranspiration, and human-induced hydrologic modifications, such as the operation of many small water-storage reservoirs; the effects of surface-water withdrawal for agricultural, municipal, and industrial use; and the effects of return flow to the river. Evaluations of such processes and conditions should be made when using the statistics provided in this report.

\section{Annual Peak Discharges}

The annual peak discharges for each station are provided by water year. These peak discharges were used in the computation of the instantaneous maximum discharge exceedance probabilities for each streamgage. The data for the peaks discharges were taken directly from the Afghanistan Hydrologic Data Reports published by the Afghanistan Ministry of Water and Power. Peaks from incomplete water years were checked using hydrograph comparison with nearby streamgages to insure that the discharge was the annual peak discharge.

\section{Monthly and Annual Mean Discharges}

The monthly and annual mean discharges are found in the final table for each streamgage. The monthly and annual means discharges were computed using statistical applications built into U.S. Geological Survey ADAPS Version 4.9 (U.S. Geological Survey, 2009). 


\section{Data Considerations}

The reliability of statistical data is related to the length of streamgage record. The Hydrology Subcommittee of the Interagency Advisory Committee on Water Data (1982) recommends that at least 10 years of record be used for computing flood-frequency estimates. However, the record length for each streamgage in Afghanistan can vary substantially. The 10-year requirement was relaxed in this report so statistics could be computed for all streamgages. Subsequently, extreme high or low flows may be included in the streamflow record of one streamgage and not in another, resulting in inconsistencies in the streamflow statistics when comparing streamgage data. In some instances, there was insufficient data to compute a statistic and the statistic is reported as "not given."

Statistics provided in the report are presented as computed by software with little evaluation. In addition, any differences in statistical values for pre- and post-regulation periods were not addressed in this report. It is the user's responsibility to interpret the data and understand the limitations of the statistics computed with limited record length.

\section{Selected References}

Democratic Republic of Afghanistan, Ministry of Irrigation and Water Resources, 1985, Hydrological yearbook 1979-1980, Part I and II, Rivers of Indus and Helmand Basin (Kabul, Khuram, Helmand and Ghazni): Institute of Water Resources Development, Afghanistan Hydrologic Data Report RO 211, $131 \mathrm{p}$.

Democratic Republic of Afghanistan, Ministry of Water and Power, 1976, Hydrological yearbook 1962-1975, Part III-8, Hari Rud river basin: Water and Soil Survey Department, Afghanistan Hydrologic Data Report RO 214, 118 p.

Democratic Republic of Afghanistan, Ministry of Water and Power, 1977, Hydrological yearbook 1965-1975, Part I-1A, Lower Kabul river basin up to Naghlu reservoir: Water and Soil Survey Department, Afghanistan Hydrologic Data Report RO 213, 186 p.

Democratic Republic of Afghanistan, Ministry of Water and Power, 1979a, Hydrological yearbook 1960-1975, Part V-14 and 15, Kunduz and Kokcha river basin: Water and Soil Survey Department, Afghanistan Hydrologic Data Report RO 222, 212 p.

Democratic Republic of Afghanistan, Ministry of Water and Power, 1979b, Hydrological yearbook 1976-1978, Part V, Rivers of Amu basin (Kunduz and Kokcha): Water and Soil Survey Department, Afghanistan Hydrologic Data Report RO 220, 69 p.

Democratic Republic of Afghanistan, Ministry of Water and Power, 1981, Hydrological yearbook 1976-1978, Part I, Rivers of Indus basin (Kabul, Khurran and Shamal): Water and Soil Survey Department, Afghanistan Hydrologic Data Report RO 218, 83 p.

Democratic Republic of Afghanistan, Ministry of Water and Power, date unknown, Hydrological yearbook 1963-1975, Part I-2, Khurran river basin: Water and Soil Survey Department, Afghanistan Hydrologic Data Report RO 216, 110 p. 
Democratic Republic of Afghanistan, Ministry of Water and Power, date unknown, Hydrological yearbook 1964-1975, Part IV-9 to 13, Murghab, Shirintagab, Serepul, Balkh, and Khulm river basins: Water and Soil Survey Department, Afghanistan Hydrologic Data Report RO 208, 190 p.

Democratic Republic of Afghanistan, Ministry of Water and Power, date unknown, Hydrological yearbook 1976-1978, Part III, West flowing rivers (Khash, Farah, Adraskan and Harirud): Water and Soil Survey Department, Afghanistan Hydrologic Data Report RO 219, 77 p.

Democratic Republic of Afghanistan, Ministry of Water and Power, date unknown, Hydrological yearbook 1976-1978, Part IV, North flowing rivers (Murghab, Shirin Tagab, Sarepul, Balkh and Khulm): Water and Soil Survey Department, Afghanistan Hydrologic Data Report RO 209, 75 p.

Flynn, K.M., Kirby, W.H., and Hummel, P.R., 2006, User's manual for program PeakFQ, Annual floodfrequency analysis using bulletin 17B guidelines: U.S. Geological Survey Techniques and Methods, book 4, chap. B4, 42 p.

Helsel, D.R., and Hirsch, R.M., 1992, Statistical methods in water resources: New York, Elsevier Science Publishing Co., 522 p.

Lumb, A.M., Thomas, W.O., Jr., and Flynn, K.M., 2002, User's manual for SWSTAT, a computer program for interactive computation of surface-water statistics: U.S. Geological Survey website accessed April 5, 2010, at http://water.usgs.gov/software/SWSTAT/.

U.S. Interagency Advisory Committee on Water Data, 1982, Guidelines for determining flood flow frequency, Bulletin 17-B of the Hydrology Subcommittee: Reston, Va., U.S. Geological Survey, Office of Water Coordination, $183 \mathrm{p}$.

U.S. Geological Survey, 2009, User's manual for the national water information system of the U.S. Geological Survey_Automated data processing system (ADAPS) Version 4.9: U.S. Geological Survey website accessed April 5, 2010, at http://nwis.usgs.gov/currentdocs/adaps/adaps.book.html.

Vining, K.C., 2010, Streamflow characteristics of streams in southeastern Afghanistan: U.S. Geological Survey Data Series 508, 104 p.

Williams-Sether, Tara, 2008, Streamflow characteristics of streams in the Helmand Basin, Afghanistan: U.S. Geological Survey Data Series 333, 341 p. 
STEAMFLOW STATISTICS 


\section{1-0.000-1M KABUL RIVER AT DAKAH}

\section{(U.S. Geological Survey identification number: 341400071020000)}

LOCATION: Lat $34^{\circ} 14^{\prime} \mathrm{N}$., long $71^{\circ} 02^{\prime} \mathrm{E}$.

DRAINAGE AREA: $67,370 \mathrm{~km}^{2}$.

ELEVATION: 420 meters above mean sea level.

PERIOD OF RECORD: February 21, 1968 to July 22, 1980.

GAGE: Water-stage recorder.

Annual mean discharge

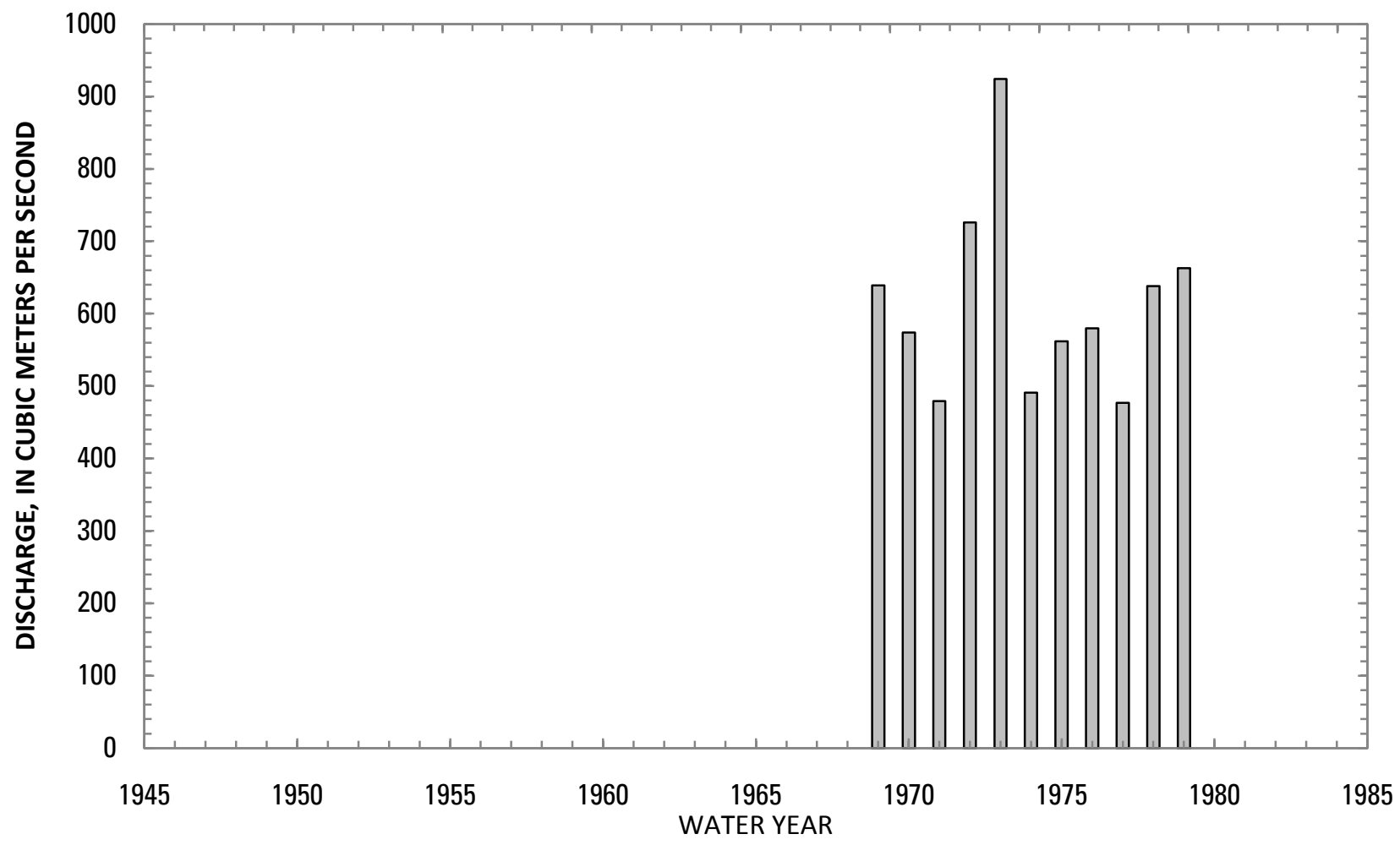




\section{1-0.000-1M KABUL RIVER AT DAKAH, Continued}

Statistics of monthly and annual mean discharges $\left[\mathrm{m}^{3} / \mathrm{s}\right.$, cubic meters per second]

\begin{tabular}{|c|c|c|c|c|c|c|c|c|}
\hline \multirow[b]{2}{*}{ Month } & \multicolumn{2}{|c|}{ Maximum } & \multicolumn{2}{|c|}{ Minimum } & \multicolumn{4}{|c|}{ Mean } \\
\hline & $\begin{array}{c}\text { Discharge } \\
\left(\mathrm{m}^{3} / \mathrm{s}\right)\end{array}$ & $\begin{array}{c}\text { Water year } \\
\text { of } \\
\text { occurrence }\end{array}$ & $\begin{array}{c}\text { Discharge } \\
\left(\mathrm{m}^{3} / \mathrm{s}\right)\end{array}$ & $\begin{array}{c}\text { Water year } \\
\text { of } \\
\text { occurrence }\end{array}$ & $\begin{array}{c}\text { Discharge } \\
\left(\mathrm{m}^{3} / \mathrm{s}\right)\end{array}$ & $\begin{array}{c}\text { Standard } \\
\text { deviation } \\
\left(\mathrm{m}^{3} / \mathrm{s}\right)\end{array}$ & $\begin{array}{c}\text { Coefficient } \\
\text { of } \\
\text { variation }\end{array}$ & $\begin{array}{c}\text { Percentage } \\
\text { of annual } \\
\text { discharge }\end{array}$ \\
\hline October & 310 & 1974 & 209 & 1975 & 269 & 35.3 & 0.13 & 3.56 \\
\hline November & 268 & 1970 & 132 & 1975 & 217 & 37.2 & 0.17 & 2.88 \\
\hline December & 248 & 1970 & 105 & 1975 & 187 & 37.1 & 0.2 & 2.47 \\
\hline January & 252 & 1970 & 89.1 & 1975 & 164 & 43.8 & 0.27 & 2.17 \\
\hline February & 267 & 1980 & 81.2 & 1975 & 165 & 50.5 & 0.31 & 2.19 \\
\hline March & 377 & 1968 & 104 & 1975 & 234 & 89.3 & 0.38 & 3.10 \\
\hline April & 1,290 & 1973 & 369 & 1971 & 651 & 245 & 0.38 & 8.61 \\
\hline May & 1,880 & 1973 & 593 & 1977 & 1,020 & 327 & 0.32 & 13.4 \\
\hline June & 2,230 & 1972 & 1,150 & 1970 & 1,570 & 404 & 0.26 & 20.8 \\
\hline July & 2,080 & 1973 & 999 & 1971 & 1,480 & 355 & 0.24 & 19.6 \\
\hline August & 1,430 & 1973 & 817 & 1974 & 1,080 & 188 & 0.17 & 14.3 \\
\hline September & 763 & 1973 & 380 & 1974 & 529 & 118 & 0.22 & 7.00 \\
\hline Annual & 924 & 1973 & 477 & 1977 & 614 & 130 & 0.21 & 100 \\
\hline
\end{tabular}




\section{1-0.000-1M KABUL RIVER AT DAKAH, Continued}

Monthly and annual flow duration, in cubic meters per second

[ng, not given]

\begin{tabular}{|c|c|c|c|c|c|c|c|c|c|c|c|c|c|}
\hline \multirow{2}{*}{$\begin{array}{l}\text { Percentage } \\
\text { of days } \\
\text { discharge } \\
\text { equaled or } \\
\text { exceeded }\end{array}$} & \multicolumn{12}{|c|}{ Month } & \multirow{2}{*}{ Annua } \\
\hline & October & November & December & January & February & March & April & May & June & July & August & September & \\
\hline 95 & 198 & 140 & 109 & 91.1 & 84.5 & 102 & 254 & 447 & 862 & 862 & 679 & 295 & 122 \\
\hline 90 & 217 & 166 & 140 & 113 & 112 & 117 & 297 & 518 & 998 & 940 & 710 & 314 & 140 \\
\hline 85 & 223 & 175 & 148 & 127 & 121 & 125 & 329 & 569 & 1,080 & 1,030 & 743 & 342 & 157 \\
\hline 80 & 227 & 185 & 163 & 131 & 127 & 135 & 360 & 631 & 1,160 & 1,220 & 800 & 359 & 173 \\
\hline 75 & 233 & 197 & 168 & 136 & 133 & 146 & 388 & 692 & 1,200 & 1,180 & 852 & 388 & 192 \\
\hline 70 & 239 & 205 & 170 & 138 & 137 & 153 & 410 & 759 & 1,290 & 1,230 & 893 & 418 & 210 \\
\hline 65 & 244 & 212 & 172 & 141 & 140 & 165 & 429 & 815 & 1,340 & 1,290 & 936 & 448 & 226 \\
\hline 60 & 251 & 215 & 176 & 145 & 145 & 182 & 454 & 876 & 1,380 & 1,350 & 979 & 479 & 246 \\
\hline 55 & 258 & 217 & 180 & 152 & 149 & 195 & 486 & 933 & 1,450 & 1,400 & 1,020 & 497 & 277 \\
\hline 50 & 263 & 220 & 185 & 157 & 152 & 206 & 529 & 1,000 & 1,500 & 1,450 & 1,070 & 513 & 322 \\
\hline 45 & 268 & 224 & 189 & 161 & 156 & 216 & 583 & 1,050 & 1,550 & 1,500 & 1,100 & 529 & 417 \\
\hline 40 & 278 & 229 & 193 & 165 & 161 & 224 & 635 & 1,090 & 1,610 & 1,540 & 1,150 & 543 & 534 \\
\hline 35 & 284 & 232 & 201 & 171 & 170 & 236 & 676 & 1,140 & 1,670 & 1,590 & 1,190 & 564 & 679 \\
\hline 30 & 290 & 236 & 206 & 180 & 179 & 258 & 735 & 1,180 & 1,740 & 1,650 & 1,240 & 594 & 823 \\
\hline 25 & 298 & 241 & 214 & 192 & 193 & 280 & 845 & 1,240 & 1,830 & 1,740 & 1,280 & 638 & 983 \\
\hline 20 & 309 & 247 & 223 & 207 & 210 & 306 & 939 & 1,320 & 1,970 & 1,850 & 1,340 & 679 & 1,140 \\
\hline 15 & 320 & 255 & 231 & 219 & 229 & 358 & 1,060 & 1,400 & 2,150 & 1,910 & 1,410 & 710 & 1,280 \\
\hline 10 & 335 & 266 & 240 & 234 & 248 & 412 & 1,220 & 1,540 & 2,360 & 2,020 & 1,500 & 754 & 1,460 \\
\hline 5 & 353 & 278 & 256 & 252 & 281 & 481 & 1,400 & 1,830 & 2,570 & 2,230 & 1,560 & 827 & 1,740 \\
\hline
\end{tabular}




\section{1-0.000-1M KABUL RIVER AT DAKAH, Continued}

Probability of occurrence of annual high discharges

[ $\mathrm{m}^{3} / \mathrm{s}$, cubic meters per second; ng, not given]

\begin{tabular}{|c|c|c|c|c|c|c|}
\hline \multirow{2}{*}{$\begin{array}{c}\text { Exceedance } \\
\text { probability }\end{array}$} & \multirow{2}{*}{$\begin{array}{c}\text { Recurrence } \\
\text { interval } \\
\text { (years) }\end{array}$} & \multirow{2}{*}{$\begin{array}{c}\text { Maximum } \\
\text { instantaneous } \\
\text { discharge } \\
\left(\mathrm{m}^{3} / \mathrm{s}\right)\end{array}$} & \multicolumn{4}{|c|}{ Maximum daily mean discharge $\left(\mathrm{m}^{3} / \mathrm{s}\right)$} \\
\hline & & & $\begin{array}{l}\text { 3-day } \\
\text { period }\end{array}$ & $\begin{array}{l}\text { 7-day } \\
\text { period }\end{array}$ & $\begin{array}{l}\text { 15-day } \\
\text { period }\end{array}$ & $\begin{array}{l}\text { 30-day } \\
\text { period }\end{array}$ \\
\hline 0.99 & 1.01 & 1,230 & 1,170 & 1,100 & 1,060 & 966 \\
\hline 0.95 & 1.05 & 1,460 & 1,350 & 1,270 & 1,200 & 1,100 \\
\hline 0.90 & 1.11 & 1,590 & 1,470 & 1,380 & 1,290 & 1,180 \\
\hline 0.80 & 1.25 & 1,760 & 1,620 & 1,530 & 1,420 & 1,300 \\
\hline 0.50 & 2 & 2,140 & 1,970 & 1,860 & 1,730 & 1,570 \\
\hline 0.20 & 5 & 2,600 & 2,430 & 2,290 & 2,150 & 1,940 \\
\hline 0.10 & 10 & 2,860 & 2,720 & 2,570 & 2,430 & 2,180 \\
\hline 0.04 & 25 & 3,170 & 3,080 & 2,900 & 2,790 & 2,480 \\
\hline 0.02 & 50 & 3,390 & 3,340 & 3,140 & 3,060 & 2,710 \\
\hline 0.01 & 100 & 3,590 & 3,610 & 3,380 & 3,340 & 2,930 \\
\hline 0.005 & 200 & 3,790 & 3,870 & ${ }^{1} 3,620$ & 3,630 & 3,160 \\
\hline 0.002 & 500 & 4,040 & $\mathrm{ng}$ & $\mathrm{ng}$ & $\mathrm{ng}$ & $\mathrm{ng}$ \\
\hline
\end{tabular}

'Data does not fit log-Pearson Type III curve, use with caution.

\section{1-0.000-1M KABUL RIVER AT DAKAH, Continued}

Probability of occurrence of annual low discharges $\left[\mathrm{m}^{3} / \mathrm{s}\right.$, meters per second]

\begin{tabular}{|c|c|c|c|c|c|c|c|c|c|c|}
\hline \multirow{3}{*}{$\begin{array}{c}\text { Nonexceedance } \\
\text { probability }\end{array}$} & \multirow{3}{*}{$\begin{array}{c}\text { Recurrence } \\
\text { interval } \\
\text { (years) }\end{array}$} & \multicolumn{9}{|c|}{ Minimum daily mean discharge $\left(\mathrm{m}^{3} / \mathrm{s}\right)$} \\
\hline & & \multicolumn{9}{|c|}{ Number of consecutive days } \\
\hline & & 1 & 3 & 7 & 14 & 30 & 60 & 90 & 120 & 183 \\
\hline 0.05 & 20 & 73.4 & 74.6 & 77.0 & 81.3 & 87.6 & 92.9 & 98.7 & 107 & 135 \\
\hline 0.10 & 10 & 85.9 & 86.5 & 88.9 & 93.2 & 99.3 & 106 & 112 & 122 & 151 \\
\hline 0.20 & 5 & 101 & 102 & 104 & 109 & 114 & 122 & 128 & 140 & 171 \\
\hline 0.50 & 2 & 128 & 132 & 136 & 140 & 146 & 156 & 163 & 175 & 207 \\
\hline
\end{tabular}




\section{1-0.000-1M KABUL RIVER AT DAKAH, Continued}

Probability of occurrence of seasonal low discharges

$\left[\mathrm{m}^{3} / \mathrm{s}\right.$, meters per second]

\begin{tabular}{|c|c|c|c|c|c|c|c|c|c|}
\hline \multirow{3}{*}{$\begin{array}{c}\text { Nonexceedance } \\
\text { probability }\end{array}$} & \multirow{3}{*}{$\begin{array}{c}\text { Recurrence } \\
\text { interval } \\
\text { (years) }\end{array}$} & \multicolumn{8}{|c|}{ Minimum daily mean discharge $\left(\mathrm{m}^{3} / \mathrm{s}\right)$} \\
\hline & & \multicolumn{8}{|c|}{ Number of consecutive days } \\
\hline & & 1 & 7 & 14 & 30 & 1 & 7 & 14 & 30 \\
\hline & & \multicolumn{4}{|c|}{ December-January-February } & \multicolumn{4}{|c|}{ March-April-May } \\
\hline 0.05 & 20 & 74.8 & 80.3 & 87.0 & 91.9 & 76.2 & 82.4 & 92.4 & 113 \\
\hline 0.10 & 10 & 88.1 & 92.0 & 97.9 & 103 & 87.5 & 95.2 & 105 & 131 \\
\hline 0.20 & 5 & 104 & 107 & 112 & 118 & 103 & 113 & 124 & 156 \\
\hline \multirow[t]{2}{*}{0.50} & 2 & 131 & 139 & 143 & 150 & 140 & 154 & 167 & 218 \\
\hline & & \multicolumn{4}{|c|}{ June-July-August } & \multicolumn{4}{|c|}{ September-October-November } \\
\hline 0.05 & 20 & 551 & 644 & 688 & 777 & 121 & 132 & 137 & 148 \\
\hline 0.10 & 10 & 564 & 653 & 723 & 831 & 139 & 150 & 156 & 166 \\
\hline 0.20 & 5 & 586 & 669 & 770 & 901 & 161 & 171 & 179 & 188 \\
\hline 0.50 & 2 & 654 & 730 & 877 & 1,050 & 198 & 208 & 216 & 224 \\
\hline
\end{tabular}

\section{1-0.000-1M KABUL RIVER AT DAKAH, Continued}

Annual peak discharges

$\left[\mathrm{m}^{3} / \mathrm{s}\right.$, meters per second]

\begin{tabular}{|c|c|c|c|c|c|}
\hline \multicolumn{3}{|c|}{$\begin{array}{c}\text { Annual peak discharge, } \\
\text { by year }\end{array}$} & \multicolumn{3}{|c|}{$\begin{array}{l}\text { Annual peak discharge, } \\
\text { from highest to lowest }\end{array}$} \\
\hline $\begin{array}{l}\text { Water } \\
\text { year }\end{array}$ & Date & $\begin{array}{c}\text { Peak } \\
\text { discharge } \\
\left(\mathrm{m}^{3} / \mathrm{s}\right)\end{array}$ & $\begin{array}{l}\text { Water } \\
\text { year }\end{array}$ & Date & $\begin{array}{c}\text { Peak } \\
\text { discharge } \\
\left(\mathrm{m}^{3} / \mathrm{s}\right)\end{array}$ \\
\hline 1968 & June 11, 1968 & 2,655 & 1978 & July 8, 1978 & 3,016 \\
\hline 1969 & June 20, 1969 & 2,091 & 1973 & June 13, 1973 & 2,870 \\
\hline 1970 & June 29, 1970 & 1,600 & 1972 & June 16, 1972 & 2,750 \\
\hline 1971 & June 1, 1971 & 1,410 & 1968 & June 11, 1968 & 2,655 \\
\hline 1972 & June 16, 1972 & 2,750 & 1979 & June 28, 1979 & 2,218 \\
\hline 1973 & June 13, 1973 & 2,870 & 1975 & May 17, 1975 & 2,180 \\
\hline 1974 & July 17, 1974 & 1,790 & 1969 & June 20, 1969 & 2,091 \\
\hline 1975 & May 17, 1975 & 2,180 & 1976 & July 10, 1976 & 2,050 \\
\hline 1976 & July 10, 1976 & 2,050 & 1980 & June 26, 1980 & 1,962 \\
\hline 1977 & June 26, 1977 & 1,860 & 1977 & June 26, 1977 & 1,860 \\
\hline 1978 & July 8, 1978 & 3,016 & 1974 & July 17,1974 & 1,790 \\
\hline 1979 & June 28, 1979 & 2,218 & 1970 & June 29, 1970 & 1,600 \\
\hline 1980 & June 26,1980 & 1,962 & 1971 & June 1, 1971 & 1,410 \\
\hline
\end{tabular}




\section{1-0.000-1M KABUL RIVER AT DAKAH, Continued}

Monthly and annual mean discharges, in cubic meters per second

[Data may not be rounded in accordance with U.S. Geological Survey publication standards; --, no data]

\begin{tabular}{|c|c|c|c|c|c|c|c|c|c|c|c|c|c|}
\hline \multirow{2}{*}{$\begin{array}{c}\text { Water } \\
\text { year }\end{array}$} & \multicolumn{12}{|c|}{ Monthly mean discharge } & \multirow{2}{*}{$\begin{array}{l}\text { Annual } \\
\text { discharge }\end{array}$} \\
\hline & October & November & December & January & February & March & April & May & June & July & August & September & \\
\hline 1968 & -- & -- & -- & -- & -- & 377 & 861 & 1,190 & 2,220 & 1,870 & 1,230 & 500 & -- \\
\hline 1969 & 308 & 255 & 231 & 197 & 162 & 345 & 655 & 789 & 1,580 & 1,460 & 1,120 & 540 & 639 \\
\hline 1970 & 309 & 268 & 248 & 252 & 227 & 222 & 507 & 858 & 1,150 & 1,030 & 1,120 & 676 & 574 \\
\hline 1971 & 249 & 186 & 183 & 153 & 137 & 146 & 369 & 848 & 1,160 & 999 & 871 & 427 & 479 \\
\hline 1972 & 224 & 218 & 176 & 126 & 131 & 196 & 569 & 1,260 & 2,230 & 1,710 & 1,210 & 650 & 726 \\
\hline 1973 & 269 & 210 & 178 & 168 & 221 & 342 & 1,290 & 1,880 & 2,210 & 2,080 & 1,430 & 763 & 924 \\
\hline 1974 & 310 & 220 & 168 & 139 & 135 & 172 & 417 & 679 & 1,240 & 1,190 & 817 & 380 & 491 \\
\hline 1975 & 209 & 132 & 105 & 89.1 & 81.2 & 104 & 576 & 975 & 1,490 & 1,280 & 1,130 & 532 & 562 \\
\hline 1976 & 251 & 199 & 180 & 164 & 175 & 185 & 659 & 899 & 1,300 & 1,550 & 891 & 496 & 580 \\
\hline 1977 & 283 & 223 & 191 & 165 & 148 & 168 & 427 & 593 & 1,180 & 1,110 & 839 & 380 & 477 \\
\hline 1978 & 237 & 197 & 158 & 132 & 150 & 228 & 563 & 1,030 & 1,470 & 1,740 & 1,160 & 543 & 638 \\
\hline 1979 & 304 & 254 & 208 & 158 & 150 & 209 & 735 & 994 & 1,570 & 1,710 & 1,160 & 458 & 663 \\
\hline 1980 & 278 & 246 & 216 & 222 & 267 & 351 & 834 & 1,200 & 1,590 & -- & -- & -- & -- \\
\hline
\end{tabular}




\section{1-0.000-4S KABUL RIVER NEAR DARONTA}

\section{(U.S. Geological Survey identification number: 342800070220000)}

LOCATION: Lat $34^{\circ} 28^{\prime} \mathrm{N}$., long $70^{\circ} 22^{\prime} \mathrm{E}$.

DRAINAGE AREA: $34,375 \mathrm{~km}^{2}$.

ELEVATION: 585 meters above mean sea level.

PERIOD OF RECORD: October 1, 1959 to September 30, 1964.

GAGE: Water-stage recorder.

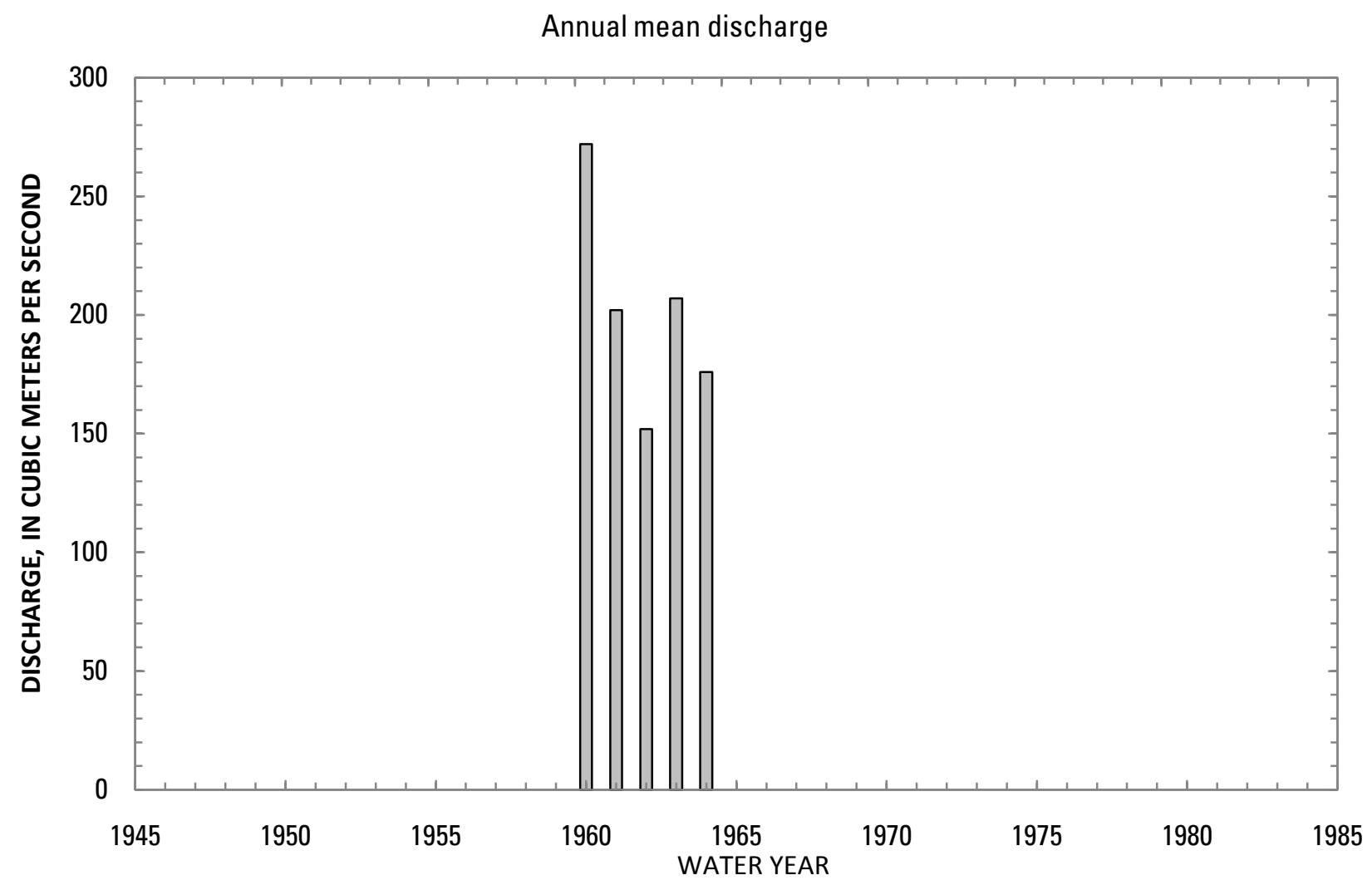




\section{1-0.000-4S KABUL RIVER NEAR DARONTA, Continued}

Statistics of monthly and annual mean discharges

[ $\mathrm{m}^{3} / \mathrm{s}$, cubic meters per second]

\begin{tabular}{|c|c|c|c|c|c|c|c|c|}
\hline \multirow[b]{2}{*}{ Month } & \multicolumn{2}{|c|}{ Maximum } & \multicolumn{2}{|c|}{ Minimum } & \multicolumn{4}{|c|}{ Mean } \\
\hline & $\begin{array}{c}\text { Discharge } \\
\left(\mathrm{m}^{3} / \mathrm{s}\right)\end{array}$ & $\begin{array}{c}\text { Water year } \\
\text { of } \\
\text { occurrence }\end{array}$ & $\begin{array}{c}\text { Discharge } \\
\left(\mathrm{m}^{3} / \mathrm{s}\right)\end{array}$ & $\begin{array}{c}\text { Water year } \\
\text { of } \\
\text { occurrence }\end{array}$ & $\begin{array}{c}\text { Discharge } \\
\left(\mathrm{m}^{3} / \mathrm{s}\right)\end{array}$ & $\begin{array}{c}\text { Standard } \\
\text { deviation } \\
\left(\mathrm{m}^{3} / \mathrm{s}\right)\end{array}$ & $\begin{array}{c}\text { Coefficient } \\
\text { of } \\
\text { variation }\end{array}$ & $\begin{array}{c}\text { Percentage } \\
\text { of annual } \\
\text { discharge }\end{array}$ \\
\hline October & 67.7 & 1961 & 50.2 & 1960 & 58.5 & 6.98 & 0.12 & 2.42 \\
\hline November & 88.3 & 1960 & 64.9 & 1963 & 76.6 & 9.34 & 0.12 & 3.16 \\
\hline December & 88.6 & 1961 & 83.6 & 1962 & 85.7 & 1.82 & 0.02 & 3.54 \\
\hline January & 104 & 1964 & 80.2 & 1960 & 90.0 & 9.71 & 0.11 & 3.72 \\
\hline February & 94.4 & 1964 & 73.8 & 1962 & 81.2 & 8.13 & 0.10 & 3.36 \\
\hline March & 183 & 1960 & 70.5 & 1962 & 116 & 45.3 & 0.39 & 4.81 \\
\hline April & 476 & 1960 & 164 & 1963 & 310 & 136 & 0.44 & 12.8 \\
\hline May & 623 & 1960 & 202 & 1962 & 414 & 161 & 0.39 & 17.1 \\
\hline June & 723 & 1963 & 415 & 1964 & 582 & 120 & 0.21 & 24.1 \\
\hline July & 588 & 1960 & 296 & 1964 & 399 & 124 & 0.31 & 16.5 \\
\hline August & 256 & 1960 & 94.1 & 1964 & 142 & 68.4 & 0.48 & 5.88 \\
\hline September & 82.1 & 1960 & 51.9 & 1962 & 62.8 & 11.5 & 0.18 & 2.60 \\
\hline Annual & 272 & 1960 & 152 & 1962 & 202 & 45.0 & 0.22 & 100 \\
\hline
\end{tabular}


1-0.000-4S KABUL RIVER NEAR DARONTA, Continued

Monthly and annual flow duration, in cubic meters per second

[ng, not given]

\begin{tabular}{|c|c|c|c|c|c|c|c|c|c|c|c|c|c|}
\hline \multirow{2}{*}{$\begin{array}{l}\text { Percentage } \\
\text { of days } \\
\text { discharge } \\
\text { equaled or } \\
\text { exceeded }\end{array}$} & \multicolumn{12}{|c|}{ Month } & \multirow{2}{*}{ Annual } \\
\hline & October & November & December & January & February & March & April & May & June & July & August & September & \\
\hline 95 & 46.5 & 58.5 & 77.2 & 76.2 & 68.4 & 64.8 & 118 & 165 & 271 & 169 & 69.1 & 45.3 & 54.9 \\
\hline 90 & 49.7 & 59.4 & 78.8 & 77.6 & 70.9 & 67.2 & 127 & 178 & 355 & 197 & 72.5 & 49.0 & 60.1 \\
\hline 85 & 50.8 & 60.7 & 80.2 & 78.3 & 72.0 & 69.6 & 138 & 207 & 460 & 208 & 76.4 & 54.9 & 65.0 \\
\hline 80 & 51.5 & 63.0 & 80.9 & 80.5 & 72.9 & 71.4 & 167 & 228 & 478 & 239 & 82.0 & 55.6 & 69.7 \\
\hline 75 & 53.7 & 67.0 & 82.3 & 82.2 & 74.2 & 73.2 & 187 & 263 & 491 & 287 & 86.0 & 56.3 & 74.3 \\
\hline 70 & 54.9 & 68.9 & 83.1 & 83.3 & 75.3 & 78.6 & 199 & 306 & 511 & 321 & 90.1 & 56.9 & 78.1 \\
\hline 65 & 56.6 & 70.5 & 83.6 & 83.9 & 76.1 & 81.4 & 212 & 370 & 521 & 337 & 93.1 & 57.5 & 81.9 \\
\hline 60 & 58.5 & 72.9 & 83.9 & 85.2 & 76.9 & 84.6 & 230 & 385 & 531 & 361 & 96.6 & 58.1 & 85.1 \\
\hline 55 & 58.8 & 74.1 & 85.0 & 87.4 & 77.9 & 88.2 & 249 & 411 & 603 & 379 & 101 & 58.6 & 88.3 \\
\hline 50 & 59.2 & 75.0 & 87.0 & 88.8 & 79.1 & 95.0 & 263 & 430 & 624 & 397 & 107 & 59.1 & 91.7 \\
\hline 45 & 59.7 & 76.6 & 87.3 & 90.2 & 79.8 & 107 & 299 & 452 & 644 & 417 & 115 & 59.6 & 101 \\
\hline 40 & 61.2 & 79.9 & 87.6 & 91.4 & 80.6 & 111 & 325 & 465 & 660 & 439 & 128 & 60.1 & 118 \\
\hline 35 & 61.8 & 82.3 & 88.1 & 92.6 & 81.5 & 115 & 360 & 485 & 676 & 464 & 134 & 62.6 & 165 \\
\hline 30 & 62.4 & 84.0 & 89.8 & 93.9 & 82.9 & 128 & 375 & 508 & 689 & 485 & 151 & 64.1 & 212 \\
\hline 25 & 63.6 & 85.4 & 90.1 & 96.1 & 86.7 & 149 & 399 & 525 & 702 & 500 & 170 & 68.0 & 280 \\
\hline 20 & 65.7 & 86.8 & 90.3 & 97.3 & 90.1 & 166 & 426 & 544 & 715 & 517 & 204 & 69.4 & 379 \\
\hline 15 & 66.8 & 89.2 & 90.5 & 99.9 & 93.8 & 186 & 458 & 592 & 729 & 548 & 243 & 72.0 & 455 \\
\hline 10 & 67.9 & 91.2 & 91.5 & 105 & 98.4 & 202 & 506 & 632 & 750 & 620 & 279 & 74.6 & 518 \\
\hline 5 & $\mathrm{ng}$ & 100 & 93.2 & 110 & 105 & 228 & 594 & 678 & 780 & 693 & 316 & 86.3 & 655 \\
\hline
\end{tabular}




\section{1-0.000-4S KABUL RIVER NEAR DARONTA, Continued}

Probability of occurrence of annual high discharges

[ $\mathrm{m}^{3} / \mathrm{s}$, cubic meters per second; $\mathrm{ng}$, not given]

\begin{tabular}{|c|c|c|c|c|c|c|}
\hline \multirow{2}{*}{$\begin{array}{c}\text { Exceedance } \\
\text { probability }\end{array}$} & \multirow{2}{*}{$\begin{array}{c}\text { Recurrence } \\
\text { interval } \\
\text { (years) }\end{array}$} & \multirow{2}{*}{$\begin{array}{l}\text { Maximum } \\
\text { instantaneous } \\
\text { discharge } \\
\left(\mathrm{m}^{3} / \mathrm{s}\right)\end{array}$} & \multicolumn{4}{|c|}{ Maximum daily mean discharge $\left(\mathrm{m}^{3} / \mathrm{s}\right)$} \\
\hline & & & $\begin{array}{l}\text { 3-day } \\
\text { period }\end{array}$ & $\begin{array}{l}\text { 7-day } \\
\text { period }\end{array}$ & $\begin{array}{l}\text { 15-day } \\
\text { period }\end{array}$ & $\begin{array}{l}\text { 30-day } \\
\text { period }\end{array}$ \\
\hline 0.99 & 1.01 & 423 & 416 & 395 & ${ }^{2} 378$ & 394 \\
\hline 0.95 & 1.05 & 492 & 491 & 480 & 464 & 448 \\
\hline 0.90 & 1.11 & 541 & 539 & 528 & 510 & 479 \\
\hline 0.80 & 1.25 & 614 & 603 & 588 & 564 & 519 \\
\hline 0.50 & 2 & 821 & 754 & 705 & 656 & 600 \\
\hline 0.20 & 5 & 1,170 & 951 & 822 & 728 & 690 \\
\hline 0.10 & 10 & 1,440 & 1,080 & 881 & 756 & 741 \\
\hline 0.04 & 25 & 1,850 & 1,230 & 941 & ${ }^{2} 779$ & 797 \\
\hline 0.02 & 50 & 2,190 & 1,350 & 978 & ${ }^{2} 790$ & 835 \\
\hline 0.01 & 100 & 2,570 & 1,460 & 1,010 & ${ }^{2} 799$ & 869 \\
\hline 0.005 & 200 & 3,000 & 1,580 & 1,040 & ${ }^{2} 805$ & 902 \\
\hline 0.002 & 500 & 3,650 & $\mathrm{ng}$ & $\mathrm{ng}$ & $\mathrm{ng}$ & $\mathrm{ng}$ \\
\hline
\end{tabular}

'Less than 10 years of data used.

${ }^{2}$ Data does not fit log-Pearson Type III curve, use with caution.

\section{1-0.000-4S KABUL RIVER NEAR DARONTA, Continued}

Probability of occurrence of annual low discharges [m³ $/ \mathrm{s}$, meters per second; ng, not given]

\begin{tabular}{|c|c|c|c|c|c|c|c|c|c|c|}
\hline \multirow{3}{*}{$\begin{array}{c}\text { Nonexceedance } \\
\text { probability }\end{array}$} & \multirow{3}{*}{$\begin{array}{c}\text { Recurrence } \\
\text { interval } \\
\text { (years) }\end{array}$} & \multicolumn{9}{|c|}{ Minimum daily mean discharge $\left(\mathrm{m}^{3} / \mathrm{s}\right)$} \\
\hline & & \multicolumn{9}{|c|}{ Number of consecutive days } \\
\hline & & 1 & 3 & 7 & 14 & 30 & 60 & 90 & 120 & 183 \\
\hline 0.05 & 20 & ng & ng & ng & ng & ng & ng & $\mathrm{ng}$ & ng & ng \\
\hline 0.10 & 10 & $\mathrm{ng}$ & ng & ng & ng & ng & ng & ng & ng & ng \\
\hline 0.20 & 5 & ng & ng & $\mathrm{ng}$ & ng & ng & ng & $\mathrm{ng}$ & ng & ng \\
\hline 0.50 & 2 & $\mathrm{ng}$ & ng & $\mathrm{ng}$ & ng & ng & ng & $\mathrm{ng}$ & ng & ng \\
\hline
\end{tabular}




\section{1-0.000-4S KABUL RIVER NEAR DARONTA, Continued}

Probability of occurrence of seasonal low discharges [m³/s, meters per second; ng, not given]

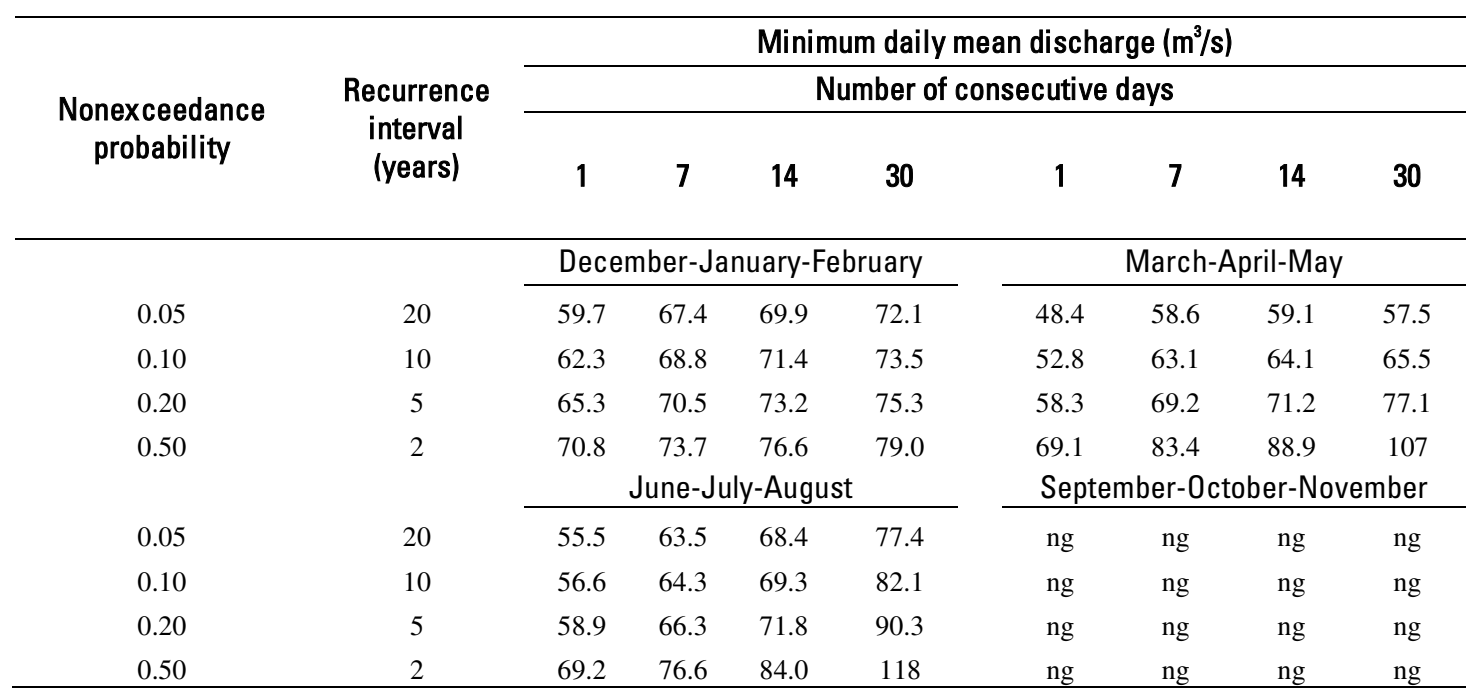

\section{1-0.000-4S KABUL RIVER NEAR DARONTA, Continued}

\begin{tabular}{|c|c|c|c|c|c|}
\hline \multicolumn{6}{|c|}{$\begin{array}{l}\text { Annual peak discharges } \\
\text { [m³/s, meters per second] }\end{array}$} \\
\hline \multicolumn{3}{|c|}{$\begin{array}{c}\text { Annual peak discharge, } \\
\text { by year }\end{array}$} & \multicolumn{3}{|c|}{$\begin{array}{l}\text { Annual peak discharge, } \\
\text { from highest to lowest }\end{array}$} \\
\hline $\begin{array}{l}\text { Water } \\
\text { year }\end{array}$ & Date & $\begin{array}{c}\text { Peak } \\
\text { discharge } \\
\left(\mathrm{m}^{3} / \mathrm{s}\right)\end{array}$ & $\begin{array}{l}\text { Water } \\
\text { year }\end{array}$ & Date & $\begin{array}{c}\text { Peak } \\
\text { discharge } \\
\left(\mathrm{m}^{3} / \mathrm{s}\right)\end{array}$ \\
\hline 1960 & April 17, 1960 & 1,560 & 1960 & April 17, 1960 & 1,560 \\
\hline 1961 & June 5, 1961 & 790 & 1962 & June 12, 1962 & 900 \\
\hline 1962 & June 12, 1962 & 900 & 1963 & June, 1963 & 795 \\
\hline 1963 & June, 1963 & 795 & 1961 & June 5, 1961 & 790 \\
\hline 1964 & June 11, 1964 & 528 & 1964 & June 11, 1964 & 528 \\
\hline
\end{tabular}




\section{1-0.000-4S KABUL RIVER NEAR DARONTA, Continued}

Monthly and annual mean discharges, in cubic meters per second $[--$, no data]

\begin{tabular}{|c|c|c|c|c|c|c|c|c|c|c|c|c|c|}
\hline \multirow{2}{*}{$\begin{array}{l}\text { Water } \\
\text { year }\end{array}$} & \multicolumn{12}{|c|}{ Monthly mean discharge } & \multirow{2}{*}{$\begin{array}{l}\text { Annual } \\
\text { discharge }\end{array}$} \\
\hline & October & November & December & January & February & March & April & May & June & July & August & September & \\
\hline 1960 & 50.2 & 88.3 & 85.8 & 80.2 & 78.9 & 183 & 476 & 623 & 670 & 588 & 256 & 82.1 & 272 \\
\hline 1961 & 67.7 & 82.0 & 88.6 & 92.2 & 83.0 & 110 & 353 & 472 & 568 & 342 & 101 & 62.7 & 202 \\
\hline 1962 & 61.4 & 77.8 & 83.6 & 81.3 & 73.8 & 70.5 & 176 & 202 & 536 & 310 & 103 & 51.9 & 152 \\
\hline 1963 & 53.0 & 64.9 & 85.1 & 92.1 & 76.0 & 81.9 & 164 & 460 & 723 & 461 & 157 & 60.1 & 207 \\
\hline 1964 & 60.2 & 69.9 & 85.5 & 104 & 94.4 & 137 & 382 & 315 & 415 & 296 & 94.1 & 57.2 & 176 \\
\hline
\end{tabular}




\section{1-0.000-5W KABUL RIVER AT NAGHLU \\ (U.S. Geological Survey identification number: 343700069430000)}

LOCATION: Lat $34^{\circ} 37^{\prime} \mathrm{N}$., long $69^{\circ} 43^{\prime} \mathrm{E}$.

DRAINAGE AREA: $26,046 \mathrm{~km}^{2}$.

ELEVATION: 985 meters above mean sea level.

PERIOD OF RECORD: October 1, 1959 to September 30, 1980.

GAGE: Water-stage recorder.

Annual mean discharge

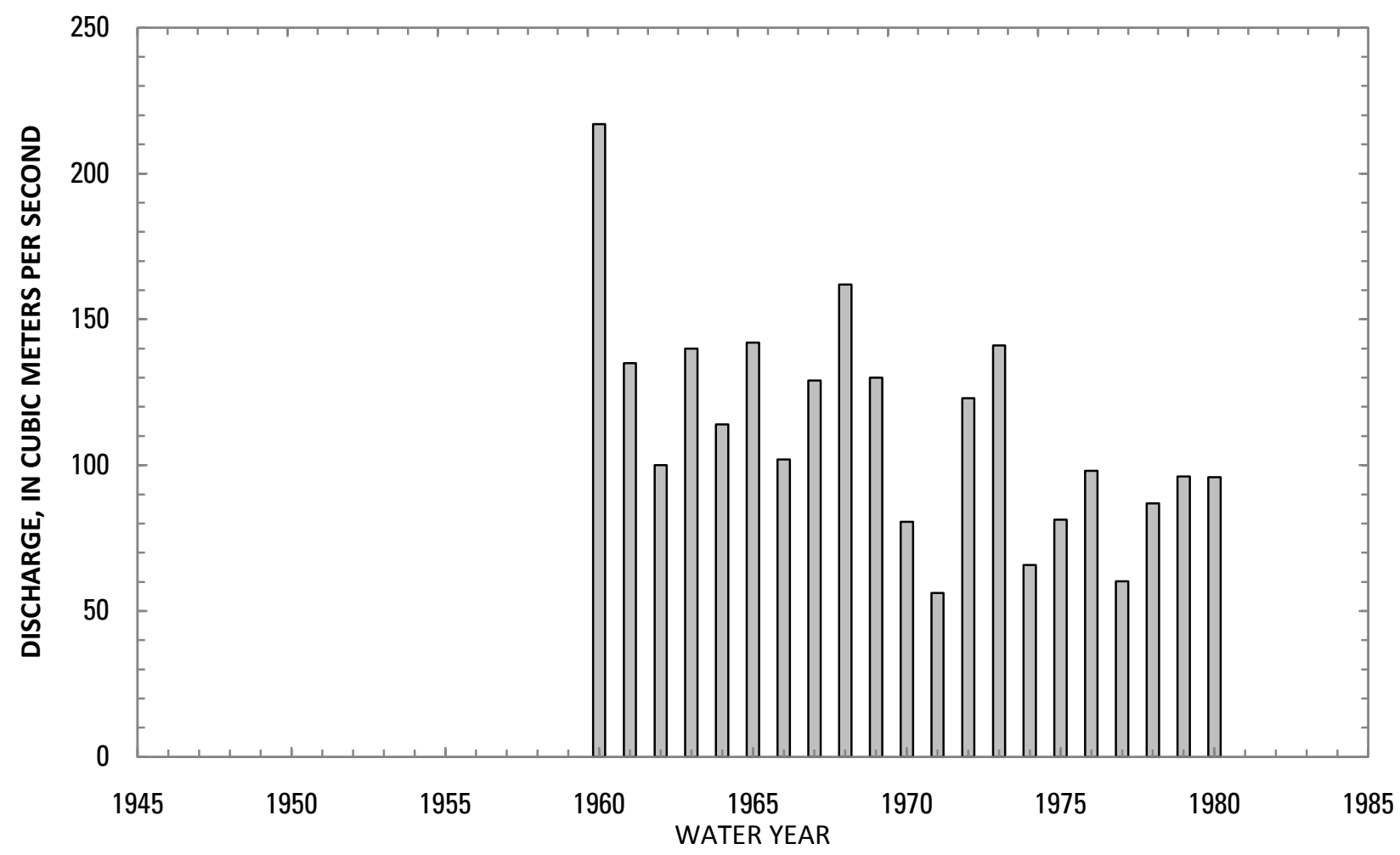




\section{1-0.000-5W KABUL RIVER AT NAGHLU, Continued}

Statistics of monthly and annual mean discharges $\left[\mathrm{m}^{3} / \mathrm{s}\right.$, cubic meters per second]

\begin{tabular}{|c|c|c|c|c|c|c|c|c|}
\hline \multirow[b]{2}{*}{ Month } & \multicolumn{2}{|c|}{ Maximum } & \multicolumn{2}{|c|}{ Minimum } & \multicolumn{4}{|c|}{ Mean } \\
\hline & $\begin{array}{c}\text { Discharge } \\
\left(\mathrm{m}^{3} / \mathrm{s}\right)\end{array}$ & $\begin{array}{c}\text { Water year } \\
\text { of } \\
\text { occurrence }\end{array}$ & $\begin{array}{c}\text { Discharge } \\
\left(\mathrm{m}^{3} / \mathrm{s}\right)\end{array}$ & $\begin{array}{c}\text { Water year } \\
\text { of } \\
\text { occurrence }\end{array}$ & $\begin{array}{c}\text { Discharge } \\
\left(\mathrm{m}^{3} / \mathrm{s}\right)\end{array}$ & $\begin{array}{c}\text { Standard } \\
\text { deviation } \\
\left(\mathrm{m}^{3} / \mathrm{s}\right)\end{array}$ & $\begin{array}{c}\text { Coefficient } \\
\text { of } \\
\text { variation }\end{array}$ & $\begin{array}{c}\text { Percentage } \\
\text { of annual } \\
\text { discharge }\end{array}$ \\
\hline October & 60.2 & 1974 & 33.7 & 1967 & 44.8 & 8.01 & 0.18 & 3.32 \\
\hline November & 70.0 & 1960 & 39.1 & 1976 & 54.2 & 10.2 & 0.19 & 4.03 \\
\hline December & 85.6 & 1968 & 34.1 & 1978 & 55.0 & 15.2 & 0.28 & 4.09 \\
\hline January & 102 & 1968 & 34.9 & 1972 & 59.9 & 16.8 & 0.28 & 4.45 \\
\hline February & 89.2 & 1973 & 41.0 & 1979 & 57.5 & 12.4 & 0.22 & 4.27 \\
\hline March & 159 & 1968 & 31.7 & 1967 & 67.1 & 32.8 & 0.49 & 4.98 \\
\hline April & 362 & 1960 & 45.9 & 1971 & 171 & 86.7 & 0.51 & 12.7 \\
\hline May & 475 & 1960 & 64.0 & 1977 & 228 & 95.9 & 0.42 & 16.9 \\
\hline June & 563 & 1960 & 117 & 1971 & 313 & 131 & 0.42 & 23.3 \\
\hline July & 524 & 1960 & 34.4 & 1971 & 185 & 112 & 0.60 & 13.8 \\
\hline August & 178 & 1960 & 29.2 & 1971 & 67.3 & 32.5 & 0.48 & 5.00 \\
\hline September & 62.0 & 1960 & 31.1 & 1971 & 42.8 & 7.68 & 0.18 & 3.18 \\
\hline Annual & 217 & 1960 & 56.2 & 1971 & 112 & 37.9 & 0.34 & 100 \\
\hline
\end{tabular}


1-0.000-5W KABUL RIVER AT NAGHLU, Continued

Monthly and annual flow duration, in cubic meters per second

\begin{tabular}{|c|c|c|c|c|c|c|c|c|c|c|c|c|c|}
\hline \multirow{2}{*}{$\begin{array}{l}\text { Percentage } \\
\text { of days } \\
\text { discharge } \\
\text { equaled or } \\
\text { exceeded }\end{array}$} & \multicolumn{12}{|c|}{ Month } & \multirow{2}{*}{ Annual } \\
\hline & October & November & December & January & February & March & April & May & June & July & August & September & \\
\hline 95 & 32.2 & 33.6 & 29.3 & 31.1 & 34.0 & 26.9 & 30.7 & 50.5 & 70.1 & 38.0 & 30.6 & 29.9 & 31.6 \\
\hline 90 & 34.2 & 38.8 & 32.3 & 34.7 & 37.8 & 30.8 & 43.3 & 72.4 & 133 & 49.7 & 34.8 & 32.8 & 35.8 \\
\hline 85 & 35.4 & 40.4 & 34.8 & 37.5 & 40.7 & 35.1 & 48.5 & 98.4 & 151 & 57.5 & 37.8 & 34.9 & 38.5 \\
\hline 80 & 36.4 & 42.1 & 37.0 & 40.7 & 43.2 & 38.4 & 55.1 & 123 & 170 & 69.5 & 41.1 & 36.0 & 41.4 \\
\hline 75 & 37.6 & 43.9 & 39.5 & 44.0 & 46.5 & 41.7 & 73.1 & 143 & 197 & 85.1 & 43.7 & 36.9 & 44.4 \\
\hline 70 & 38.7 & 45.9 & 42.7 & 47.8 & 48.7 & 46.3 & 88.2 & 159 & 220 & 98.2 & 45.5 & 37.6 & 47.4 \\
\hline 65 & 39.7 & 47.6 & 46.8 & 51.1 & 51.6 & 50.3 & 102 & 175 & 240 & 110 & 47.4 & 38.3 & 50.4 \\
\hline 60 & 40.6 & 49.1 & 49.9 & 53.7 & 53.4 & 52.0 & 114 & 191 & 262 & 123 & 49.2 & 39.2 & 53.6 \\
\hline 55 & 41.5 & 50.5 & 51.8 & 57.0 & 55.1 & 53.7 & 129 & 203 & 290 & 139 & 51.1 & 40.2 & 56.9 \\
\hline 50 & 42.6 & 52.6 & 53.7 & 59.8 & 56.7 & 55.4 & 144 & 214 & 307 & 156 & 53.0 & 41.1 & 60.5 \\
\hline 45 & 43.9 & 54.8 & 55.9 & 61.8 & 58.2 & 58.9 & 161 & 230 & 321 & 174 & 54.9 & 42.2 & 65.3 \\
\hline 40 & 45.4 & 57.5 & 58.0 & 63.8 & 59.8 & 62.8 & 178 & 247 & 340 & 193 & 58.9 & 43.5 & 70.8 \\
\hline 35 & 47.3 & 60.4 & 60.4 & 67.2 & 61.8 & 67.3 & 195 & 265 & 358 & 212 & 62.8 & 44.7 & 77.2 \\
\hline 30 & 49.2 & 62.6 & 63.0 & 70.5 & 64.4 & 72.4 & 214 & 284 & 376 & 234 & 66.8 & 46.6 & 93.2 \\
\hline 25 & 51.6 & 64.3 & 67.1 & 72.6 & 67.4 & 77.2 & 240 & 303 & 407 & 258 & 71.9 & 48.5 & 131 \\
\hline 20 & 53.9 & 65.9 & 71.6 & 74.8 & 69.9 & 81.7 & 268 & 328 & 450 & 283 & 80.3 & 50.6 & 174 \\
\hline 15 & 56.1 & 68.4 & 73.9 & 77.1 & 73.2 & 93.1 & 298 & 356 & 500 & 309 & 95.1 & 53.4 & 225 \\
\hline 10 & 58.5 & 71.3 & 76.3 & 79.4 & 78.0 & 118 & 338 & 385 & 542 & 350 & 120 & 55.8 & 287 \\
\hline 5 & 62.2 & 74.7 & 81.5 & 81.7 & 83.8 & 158 & 391 & 449 & 597 & 416 & 167 & 58.1 & 368 \\
\hline
\end{tabular}




\section{1-0.000-5W KABUL RIVER AT NAGHLU, Continued}

Probability of occurrence of annual high discharges [ $\mathrm{m}^{3} / \mathrm{s}$, cubic meters per second; ng, not given]

\begin{tabular}{|c|c|c|c|c|c|c|}
\hline \multirow{2}{*}{$\begin{array}{l}\text { Exceedance } \\
\text { probability }\end{array}$} & \multirow{2}{*}{$\begin{array}{c}\text { Recurrence } \\
\text { interval } \\
\text { (years) }\end{array}$} & \multirow{2}{*}{$\begin{array}{l}\text { Maximum } \\
\text { instantaneous } \\
\text { discharge } \\
\left(\mathrm{m}^{3} / \mathrm{s}\right)\end{array}$} & \multicolumn{4}{|c|}{ Maximum daily mean discharge $\left(\mathrm{m}^{3} / \mathrm{s}\right)$} \\
\hline & & & $\begin{array}{l}\text { 3-day } \\
\text { period }\end{array}$ & $\begin{array}{l}\text { 7-day } \\
\text { period }\end{array}$ & $\begin{array}{l}\text { 15-day } \\
\text { period }\end{array}$ & $\begin{array}{l}\text { 30-day } \\
\text { period }\end{array}$ \\
\hline 0.99 & 1.01 & 188 & 178 & 157 & 143 & 124 \\
\hline 0.95 & 1.05 & 251 & 235 & 208 & 188 & 166 \\
\hline 0.90 & 1.11 & 294 & 271 & 241 & 217 & 193 \\
\hline 0.80 & 1.25 & 354 & 320 & 286 & 257 & 231 \\
\hline 0.50 & 2 & 506 & 435 & 392 & 354 & 320 \\
\hline 0.20 & 5 & 721 & 582 & 525 & 481 & 436 \\
\hline 0.10 & 10 & 867 & 672 & 607 & 563 & 509 \\
\hline 0.04 & 25 & 1,050 & 779 & 704 & 663 & 597 \\
\hline 0.02 & 50 & 1,200 & 855 & 773 & 736 & 659 \\
\hline 0.01 & 100 & 1,340 & 928 & 839 & 807 & 720 \\
\hline 0.005 & 200 & 1,490 & 999 & 902 & 878 & 779 \\
\hline 0.002 & 500 & 1,680 & ng & $\mathrm{ng}$ & $\mathrm{ng}$ & $\mathrm{ng}$ \\
\hline
\end{tabular}

\section{1-0.000-5W KABUL RIVER AT NAGHLU, Continued}

Probability of occurrence of annual low discharges $\left[\mathrm{m}^{3} / \mathrm{s}\right.$, meters per second]

\begin{tabular}{|c|c|c|c|c|c|c|c|c|c|c|}
\hline \multirow{3}{*}{$\begin{array}{c}\text { Nonexceedance } \\
\text { probability }\end{array}$} & \multirow{3}{*}{$\begin{array}{c}\text { Recurrence } \\
\text { interval } \\
\text { (years) }\end{array}$} & \multicolumn{9}{|c|}{ Minimum daily mean discharge $\left(\mathrm{m}^{3} / \mathrm{s}\right)$} \\
\hline & & \multicolumn{9}{|c|}{ Number of consecutive days } \\
\hline & & 1 & 3 & 7 & 14 & 30 & 60 & 90 & 120 & 183 \\
\hline 0.05 & 20 & 10.4 & 14.7 & 18.7 & 24.2 & 30.5 & 32.8 & 34.0 & 36.7 & 38.7 \\
\hline 0.10 & 10 & 12.1 & 16.6 & 20.6 & 25.4 & 31.2 & 33.8 & 35.5 & 38.2 & 40.9 \\
\hline 0.20 & 5 & 14.7 & 19.2 & 23.2 & 27.2 & 32.3 & 35.3 & 37.5 & 40.2 & 43.8 \\
\hline 0.50 & 2 & 21.5 & 25.6 & 29.2 & 31.8 & 35.5 & 39.0 & 42.1 & 45.0 & 49.9 \\
\hline
\end{tabular}




\section{1-0.000-5W KABUL RIVER AT NAGHLU, Continued}

\section{Probability of occurrence of seasonal low discharges}

$\left[\mathrm{m}^{3} / \mathrm{s}\right.$, meters per second]

\begin{tabular}{|c|c|c|c|c|c|c|c|c|c|}
\hline \multirow{3}{*}{$\begin{array}{c}\text { Nonexceedance } \\
\text { probability }\end{array}$} & \multirow{3}{*}{$\begin{array}{c}\text { Recurrence } \\
\text { interval } \\
\text { (years) }\end{array}$} & \multicolumn{8}{|c|}{ Minimum daily mean discharge $\left(\mathrm{m}^{3} / \mathrm{s}\right)$} \\
\hline & & \multicolumn{8}{|c|}{ Number of consecutive days } \\
\hline & & 1 & 7 & 14 & 30 & 1 & 7 & 14 & 30 \\
\hline & & \multicolumn{4}{|c|}{ December-January-February } & \multicolumn{4}{|c|}{ March-April-May } \\
\hline 0.05 & 20 & 14.4 & 22.6 & 26.2 & 30.8 & 11.2 & 18.9 & 23.1 & 27.5 \\
\hline 0.10 & 10 & 17.5 & 25.9 & 29.2 & 33.8 & 14.7 & 23.3 & 27.2 & 31.8 \\
\hline 0.20 & 5 & 22.0 & 30.5 & 33.5 & 38.0 & 20.0 & 29.4 & 33.1 & 38.2 \\
\hline \multirow[t]{2}{*}{0.50} & 2 & 33.3 & 41.0 & 43.4 & 47.6 & 33.6 & 43.9 & 47.5 & 55.8 \\
\hline & & \multicolumn{4}{|c|}{ June-July-August } & \multicolumn{4}{|c|}{ September-October-November } \\
\hline 0.05 & 20 & 24.3 & 29.5 & 33.8 & 34.2 & 20.7 & 27.6 & 29.9 & 31.9 \\
\hline 0.10 & 10 & 26.1 & 31.2 & 35.4 & 37.8 & 22.3 & 28.8 & 31.0 & 33.1 \\
\hline 0.20 & 5 & 28.7 & 33.9 & 37.9 & 43.2 & 24.5 & 30.5 & 32.6 & 34.7 \\
\hline 0.50 & 2 & 36.1 & 41.5 & 45.8 & 58.2 & 29.8 & 34.9 & 36.6 & 38.7 \\
\hline
\end{tabular}




\section{1-0.000-5W KABUL RIVER AT NAGHLU, Continued}

\begin{tabular}{|c|c|c|c|c|c|}
\hline \multicolumn{6}{|c|}{$\begin{array}{l}\text { Annual peak discharges } \\
{\left[\mathrm{m}^{3} / \mathrm{s} \text {, meters per second] }\right.}\end{array}$} \\
\hline \multicolumn{3}{|c|}{$\begin{array}{c}\text { Annual peak discharge, } \\
\text { by year }\end{array}$} & \multicolumn{3}{|c|}{$\begin{array}{l}\text { Annual peak discharge, } \\
\text { from highest to lowest }\end{array}$} \\
\hline $\begin{array}{l}\text { Water } \\
\text { year }\end{array}$ & Date & $\begin{array}{c}\text { Peak } \\
\text { discharge } \\
\left(\mathrm{m}^{3} / \mathrm{s}\right)\end{array}$ & $\begin{array}{l}\text { Water } \\
\text { year }\end{array}$ & Date & $\begin{array}{c}\text { Peak } \\
\text { discharge } \\
\left(\mathrm{m}^{3} / \mathrm{s}\right)\end{array}$ \\
\hline 1960 & April 17, 1960 & 1,260 & 1960 & April 17, 1960 & 1,260 \\
\hline 1961 & June 5, 1961 & 780 & 1967 & April 28, 1967 & 863 \\
\hline 1962 & June 12, 1962 & 626 & 1961 & June 5, 1961 & 780 \\
\hline 1963 & June 28, 1963 & 706 & 1968 & June 11, 1968 & 733 \\
\hline 1964 & June 12, 1964 & 534 & 1963 & June 28, 1963 & 706 \\
\hline 1965 & April 23, 1965 & 482 & 1978 & July 7, 1978 & 698 \\
\hline 1966 & June 19, 1966 & 358 & 1962 & June 12, 1962 & 626 \\
\hline 1967 & April 28, 1967 & 863 & 1972 & April 28, 1972 & 580 \\
\hline 1968 & June 11, 1968 & 733 & 1964 & June 12, 1964 & 534 \\
\hline 1969 & June 20, 1969 & 490 & 1973 & June 13, 1973 & 531 \\
\hline 1970 & May 23, 1970 & 353 & 1980 & May 15, 1980 & 500 \\
\hline 1971 & June 7, 1971 & 224 & 1969 & June 20, 1969 & 490 \\
\hline 1972 & April 28, 1972 & 580 & 1965 & April 23, 1965 & 482 \\
\hline 1973 & June 13, 1973 & 531 & 1976 & April 25, 1976 & 474 \\
\hline 1974 & June 18, 1974 & 258 & 1979 & June 24, 1979 & 427 \\
\hline 1975 & June 18,1975 & 384 & 1975 & June 18,1975 & 384 \\
\hline 1976 & April 25, 1976 & 474 & 1966 & June 19, 1966 & 358 \\
\hline 1977 & June 25, 1977 & 277 & 1970 & May 23, 1970 & 353 \\
\hline 1978 & July 7, 1978 & 698 & 1977 & June 25, 1977 & 277 \\
\hline 1979 & June 24, 1979 & 427 & 1974 & June 18, 1974 & 258 \\
\hline
\end{tabular}




\section{1-0.000-5W KABUL RIVER AT NAGHLU, Continued}

Monthly and annual mean discharges, in cubic meters per second

[Data may not be rounded in accordance with U.S. Geological Survey publication standards]

\begin{tabular}{|c|c|c|c|c|c|c|c|c|c|c|c|c|c|}
\hline \multirow{2}{*}{$\begin{array}{l}\text { Water } \\
\text { year }\end{array}$} & \multicolumn{12}{|c|}{ Monthly mean discharge } & \multirow{2}{*}{$\begin{array}{c}\text { Annual } \\
\text { discharge }\end{array}$} \\
\hline & October & November & December & January & February & March & April & May & June & July & August & September & \\
\hline 1960 & 39.4 & 70.0 & 75.6 & 74.3 & 65.9 & 113 & 362 & 475 & 563 & 524 & 178 & 62.0 & 217 \\
\hline 1961 & 41.8 & 58.9 & 60.5 & 62.0 & 58.3 & 60.5 & 279 & 305 & 394 & 196 & 62.9 & 38.5 & 135 \\
\hline 1962 & 38.5 & 57.4 & 67.0 & 72.0 & 62.8 & 54.8 & 132 & 143 & 314 & 167 & 57.6 & 38.4 & 100 \\
\hline 1963 & 35.1 & 42.4 & 54.7 & 59.2 & 49.0 & 52.8 & 104 & 310 & 525 & 307 & 100 & 39.5 & 140 \\
\hline 1964 & 39.4 & 45.5 & 55.2 & 67.0 & 60.9 & 87.8 & 246 & 199 & 275 & 191 & 60.7 & 37.9 & 114 \\
\hline 1965 & 36.1 & 44.6 & 56.0 & 65.2 & 58.0 & 76.4 & 251 & 325 & 343 & 286 & 100 & 56.7 & 142 \\
\hline 1966 & 57.5 & 67.3 & 75.7 & 75.4 & 67.2 & 83.1 & 165 & 171 & 253 & 103 & 66.2 & 40.6 & 102 \\
\hline 1967 & 33.7 & 47.7 & 60.6 & 73.4 & 64.8 & 31.7 & 102 & 292 & 425 & 296 & 87.8 & 35.0 & 129 \\
\hline 1968 & 46.7 & 63.2 & 85.6 & 102 & 52.3 & 159 & 238 & 247 & 535 & 302 & 83.4 & 36.1 & 162 \\
\hline 1969 & 45.8 & 62.7 & 73.3 & 74.5 & 74.7 & 111 & 211 & 170 & 368 & 230 & 90.1 & 45.6 & 130 \\
\hline 1970 & 46.7 & 60.5 & 70.5 & 76.5 & 74.7 & 73.9 & 72.4 & 142 & 175 & 80.0 & 49.9 & 45.2 & 80.6 \\
\hline 1971 & 36.9 & 59.1 & 41.1 & 50.9 & 43.8 & 36.8 & 45.9 & 148 & 117 & 34.4 & 29.2 & 31.1 & 56.2 \\
\hline 1972 & 56.4 & 44.5 & 36.4 & 34.9 & 52.0 & 33.2 & 198 & 318 & 407 & 185 & 61.2 & 46.2 & 123 \\
\hline 1973 & 55.4 & 61.5 & 44.3 & 47.0 & 89.2 & 103 & 307 & 313 & 406 & 166 & 51.1 & 48.6 & 141 \\
\hline 1974 & 60.2 & 44.0 & 36.3 & 44.5 & 51.2 & 52.0 & 87.5 & 90.8 & 140 & 82.8 & 49.4 & 51.9 & 65.8 \\
\hline 1975 & 42.0 & 51.9 & 39.2 & 36.8 & 43.1 & 43.5 & 104 & 157 & 234 & 135 & 47.8 & 39.9 & 81.3 \\
\hline 1976 & 42.1 & 39.1 & 43.8 & 50.3 & 54.5 & 48.0 & 208 & 246 & 211 & 149 & 45.2 & 39.8 & 98.1 \\
\hline 1977 & 43.8 & 39.1 & 42.1 & 59.2 & 44.9 & 41.7 & 85.7 & 64.0 & 157 & 73.8 & 36.2 & 36.4 & 60.2 \\
\hline 1978 & 38.3 & 46.3 & 34.1 & 43.7 & 42.8 & 48.7 & 92.4 & 228 & 231 & 147 & 41.7 & 47.6 & 86.9 \\
\hline 1979 & 53.6 & 64.5 & 46.7 & 38.7 & 41.0 & 35.2 & 123 & 196 & 270 & 166 & 69.3 & 47.1 & 96.1 \\
\hline 1980 & 50.3 & 68.1 & 56.9 & 50.1 & 56.9 & 62.2 & 170 & 245 & 240 & 71.9 & 45.0 & 35.4 & 95.9 \\
\hline
\end{tabular}




\section{1-0.000-6M KABUL RIVER AT TANG-I-GHARU}

(U.S. Geological Survey identification number: 343400069240000 )

LOCATION: Lat $34^{\circ} 34^{\prime} \mathrm{N}$., long $69^{\circ} 24^{\prime} \mathrm{E}$.

DRAINAGE AREA: $12,850 \mathrm{~km}^{2}$.

ELEVATION: 1,770 meters above mean sea level.

PERIOD OF RECORD: October 1, 1959 to September 30, 1980.

GAGE: Water-stage recorder.

Annual mean discharge

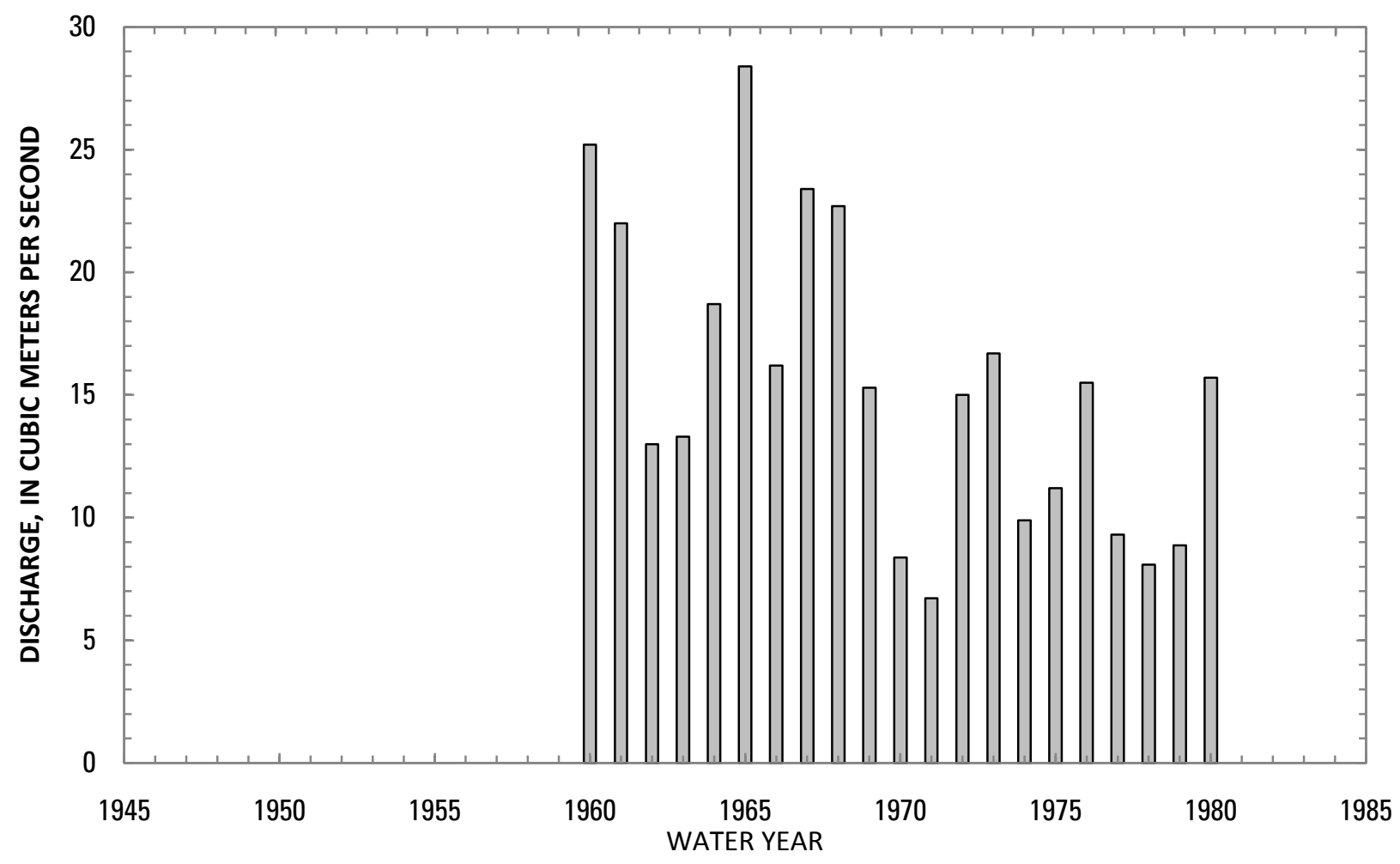




\section{1-0.000-6M KABUL RIVER AT TANG-I-GHARU, Continued}

Statistics of monthly and annual mean discharges [ $\mathrm{m}^{3} / \mathrm{s}$, cubic meters per second]

\begin{tabular}{|c|c|c|c|c|c|c|c|c|}
\hline \multirow[b]{2}{*}{ Month } & \multicolumn{2}{|c|}{ Maximum } & \multicolumn{2}{|c|}{ Minimum } & \multicolumn{4}{|c|}{ Mean } \\
\hline & $\begin{array}{c}\text { Discharge } \\
\left(\mathrm{m}^{3} / \mathrm{s}\right)\end{array}$ & $\begin{array}{c}\text { Water year } \\
\text { of } \\
\text { occurrence }\end{array}$ & $\begin{array}{c}\text { Discharge } \\
\left(\mathrm{m}^{3} / \mathrm{s}\right)\end{array}$ & $\begin{array}{c}\text { Water year } \\
\text { of } \\
\text { occurrence }\end{array}$ & $\begin{array}{c}\text { Discharge } \\
\left(\mathrm{m}^{3} / \mathrm{s}\right)\end{array}$ & $\begin{array}{c}\text { Standard } \\
\text { deviation } \\
\left(\mathrm{m}^{3} / \mathrm{s}\right)\end{array}$ & $\begin{array}{c}\text { Coefficient } \\
\text { of } \\
\text { variation }\end{array}$ & $\begin{array}{c}\text { Percentage } \\
\text { of annual } \\
\text { discharge }\end{array}$ \\
\hline October & 9.85 & 1961 & 0.06 & 1971 & 2.93 & 3.45 & 1.18 & 1.58 \\
\hline November & 24.4 & 1961 & 0.56 & 1972 & 12.0 & 7.47 & 0.62 & 6.49 \\
\hline December & 26.6 & 1966 & 6.77 & 1972 & 17.7 & 5.85 & 0.33 & 9.52 \\
\hline January & 27.9 & 1968 & 11.5 & 1972 & 19.9 & 4.71 & 0.24 & 10.7 \\
\hline February & 28.1 & 1969 & 13.5 & 1979 & 20.5 & 3.97 & 0.19 & 11.1 \\
\hline March & 45.5 & 1968 & 14.4 & 1979 & 25.8 & 7.67 & 0.3 & 13.9 \\
\hline April & 96.2 & 1964 & 6.17 & 1970 & 48.0 & 28.4 & 0.59 & 25.9 \\
\hline May & 93.1 & 1965 & 0.44 & 1970 & 28.3 & 27.8 & 0.98 & 15.3 \\
\hline June & 31.1 & 1965 & 0.15 & 1970 & 5.86 & 8.51 & 1.45 & 3.16 \\
\hline July & 13.6 & 1965 & 0.09 & 1979 & 2.60 & 3.71 & 1.43 & 1.40 \\
\hline August & 5.64 & 1978 & 0.01 & 1974 & 1.14 & 1.49 & 1.30 & 0.61 \\
\hline September & 3.76 & 1965 & 0.04 & 1970 & 0.72 & 0.91 & 1.26 & 0.39 \\
\hline Annual & 28.4 & 1965 & 6.71 & 1971 & 15.4 & 6.15 & 0.40 & 100 \\
\hline
\end{tabular}


1-0.000-6M KABUL RIVER AT TANG-I-GHARU, Continued

Monthly and annual flow duration, in cubic meters per second

\begin{tabular}{|c|c|c|c|c|c|c|c|c|c|c|c|c|c|}
\hline \multirow{2}{*}{$\begin{array}{l}\text { Percentage } \\
\text { of days } \\
\text { discharge } \\
\text { equaled or } \\
\text { exceeded }\end{array}$} & \multicolumn{12}{|c|}{ Month } & \multirow{2}{*}{ Annual } \\
\hline & October & November & December & January & February & March & April & May & June & July & August & September & \\
\hline 95 & 0.07 & 0.22 & 8.65 & 12.2 & 13.6 & 13.8 & 4.93 & 0.29 & 0.12 & 0.08 & 0.03 & 0.04 & 0.12 \\
\hline 90 & 0.12 & 0.47 & 10.0 & 13.8 & 15.6 & 15.6 & 8.67 & 0.46 & 0.18 & 0.09 & 0.08 & 0.08 & 0.19 \\
\hline 85 & 0.15 & 3.03 & 10.9 & 14.8 & 16.2 & 16.8 & 13.5 & 0.67 & 0.25 & 0.13 & 0.11 & 0.13 & 0.27 \\
\hline 80 & 0.19 & 4.51 & 11.5 & 15.8 & 16.8 & 18.4 & 17.1 & 1.05 & 0.33 & 0.16 & 0.14 & 0.14 & 0.38 \\
\hline 75 & 0.21 & 5.64 & 12.6 & 16.2 & 17.3 & 19.5 & 21.0 & 2.25 & 0.42 & 0.19 & 0.15 & 0.16 & 0.64 \\
\hline 70 & 0.22 & 6.62 & 13.8 & 16.8 & 17.8 & 20.4 & 27.3 & 5.21 & 0.52 & 0.22 & 0.17 & 0.18 & 1.04 \\
\hline 65 & 0.24 & 7.35 & 15.2 & 18.3 & 18.3 & 21.2 & 31.4 & 7.00 & 0.67 & 0.27 & 0.20 & 0.20 & 2.30 \\
\hline 60 & 0.31 & 8.12 & 16.8 & 18.9 & 18.8 & 21.8 & 34.3 & 9.99 & 0.78 & 0.34 & 0.24 & 0.23 & 4.65 \\
\hline 55 & 0.47 & 9.34 & 17.3 & 19.4 & 19.4 & 22.4 & 37.3 & 13.3 & 0.94 & 0.40 & 0.30 & 0.26 & 7.78 \\
\hline 50 & 0.70 & 11.2 & 17.8 & 19.9 & 20.5 & 23.3 & 40.3 & 16.9 & 1.27 & 0.47 & 0.34 & 0.28 & 11.5 \\
\hline 45 & 0.91 & 13.2 & 18.6 & 20.4 & 21.2 & 24.3 & 44.4 & 20.8 & 1.94 & 0.59 & 0.40 & 0.31 & 14.3 \\
\hline 40 & 1.79 & 14.9 & 19.3 & 20.8 & 21.9 & 25.5 & 48.9 & 24.5 & 2.49 & 0.78 & 0.50 & 0.37 & 16.9 \\
\hline 35 & 2.76 & 16.4 & 20.1 & 21.6 & 22.5 & 26.8 & 54.4 & 30.0 & 3.14 & 1.00 & 0.65 & 0.51 & 18.6 \\
\hline 30 & 3.62 & 17.7 & 21.1 & 22.2 & 23.1 & 28.1 & 59.6 & 36.6 & 4.43 & 1.29 & 0.81 & 0.65 & 20.2 \\
\hline 25 & 4.60 & 19.0 & 22.3 & 23.2 & 23.5 & 30.1 & 64.6 & 43.1 & 6.70 & 2.20 & 0.98 & 0.77 & 21.9 \\
\hline 20 & 5.69 & 20.5 & 23.3 & 24.1 & 24.5 & 32.1 & 72.7 & 52.4 & 10.4 & 3.07 & 1.22 & 0.91 & 24.6 \\
\hline 15 & 6.82 & 22.0 & 24.4 & 25.8 & 24.9 & 34.4 & 83.8 & 65.5 & 14.7 & 5.17 & 1.53 & 1.21 & 27.8 \\
\hline 10 & 8.54 & 23.7 & 25.5 & 26.6 & 25.9 & 38.0 & 95.2 & 78.3 & 20.5 & 8.28 & 2.48 & 2.08 & 32.9 \\
\hline 5 & 12.9 & 25.7 & 26.8 & 27.8 & 26.8 & 48.1 & 122 & 102 & 27.5 & 12.3 & 4.57 & 3.50 & 52.8 \\
\hline
\end{tabular}




\section{1-0.000-6M KABUL RIVER AT TANG-I-GHARU, Continued}

Probability of occurrence of annual high discharges

[ $\mathrm{m}^{3} / \mathrm{s}$, cubic meters per second; $\mathrm{ng}$, not given]

\begin{tabular}{|c|c|c|c|c|c|c|}
\hline \multirow{2}{*}{$\begin{array}{c}\text { Exceedance } \\
\text { probability }\end{array}$} & \multirow{2}{*}{$\begin{array}{c}\text { Recurrence } \\
\text { interval } \\
\text { (years) }\end{array}$} & \multirow{2}{*}{$\begin{array}{c}\text { Maximum } \\
\text { instantaneous } \\
\text { discharge } \\
\left(\mathrm{m}^{3} / \mathrm{s}\right)\end{array}$} & \multicolumn{4}{|c|}{ Maximum daily mean discharge $\left(\mathrm{m}^{3} / \mathrm{s}\right)$} \\
\hline & & & $\begin{array}{l}\text { 3-day } \\
\text { period }\end{array}$ & $\begin{array}{l}\text { 7-day } \\
\text { period }\end{array}$ & $\begin{array}{l}\text { 15-day } \\
\text { period }\end{array}$ & $\begin{array}{l}\text { 30-day } \\
\text { period }\end{array}$ \\
\hline 0.99 & 1.01 & 27.4 & 16.2 & 15.0 & 12.6 & 11.6 \\
\hline 0.95 & 1.05 & 41.5 & 26.4 & 23.8 & 20.4 & 18.2 \\
\hline 0.90 & 1.11 & 51.0 & 33.8 & 30.3 & 26.1 & 22.9 \\
\hline 0.80 & 1.25 & 64.8 & 45.1 & 40.3 & 35.0 & 30.1 \\
\hline 0.50 & 2 & 98.3 & 75.5 & 67.9 & 59.6 & 50.1 \\
\hline 0.20 & 5 & 142 & 121 & 111 & 97.9 & 81.6 \\
\hline 0.10 & 10 & 169 & 152 & 142 & 125 & 104 \\
\hline 0.04 & 25 & 201 & 192 & 183 & 161 & 135 \\
\hline 0.02 & 50 & 223 & 221 & 215 & 189 & 159 \\
\hline 0.01 & 100 & 244 & 250 & 247 & 217 & 183 \\
\hline 0.005 & 200 & 263 & ${ }^{1} 279$ & 281 & 245 & 208 \\
\hline 0.002 & 500 & 288 & ng & ng & ng & ng \\
\hline
\end{tabular}

'Data does not fit log-Pearson Type III curve, use with caution.

\section{1-0.000-6M KABUL RIVER AT TANG-I-GHARU, Continued}

Probability of occurrence of annual low discharges

$\left[\mathrm{m}^{3} / \mathrm{s}\right.$, meters per second]

\begin{tabular}{|c|c|c|c|c|c|c|c|c|c|c|}
\hline \multirow{3}{*}{$\begin{array}{c}\text { Nonexceedance } \\
\text { probability }\end{array}$} & \multirow{3}{*}{$\begin{array}{c}\text { Recurrence } \\
\text { interval } \\
\text { (years) }\end{array}$} & \multicolumn{9}{|c|}{ Minimum daily mean discharge $\left(\mathrm{m}^{3} / \mathrm{s}\right)$} \\
\hline & & \multicolumn{9}{|c|}{ Number of consecutive days } \\
\hline & & 1 & 3 & 7 & 14 & 30 & 60 & 90 & 120 & 183 \\
\hline 0.05 & 20 & 0 & 0 & 0.01 & 0.02 & 0.02 & 0.05 & 0.08 & 0.11 & 0.28 \\
\hline 0.10 & 10 & 0.01 & 0.01 & 0.02 & 0.03 & 0.04 & 0.07 & 0.12 & 0.16 & 0.46 \\
\hline 0.20 & 5 & 0.03 & 0.03 & 0.04 & 0.06 & 0.07 & 0.12 & 0.19 & 0.26 & 0.82 \\
\hline 0.50 & 2 & 0.11 & 0.13 & 0.14 & 0.18 & 0.22 & 0.32 & 0.51 & 0.70 & 2.31 \\
\hline
\end{tabular}




\section{1-0.000-6M KABUL RIVER AT TANG-I-GHARU, Continued}

Probability of occurrence of seasonal low discharges

[ $\mathrm{m}^{3} / \mathrm{s}$, meters per second]

\begin{tabular}{|c|c|c|c|c|c|c|c|c|c|}
\hline \multirow{3}{*}{$\begin{array}{c}\text { Nonexceedance } \\
\text { probability }\end{array}$} & \multirow{3}{*}{$\begin{array}{c}\text { Recurrence } \\
\text { interval } \\
\text { (years) }\end{array}$} & \multicolumn{8}{|c|}{ Minimum daily mean discharge $\left(\mathrm{m}^{3} / \mathrm{s}\right)$} \\
\hline & & \multicolumn{8}{|c|}{ Number of consecutive days } \\
\hline & & 1 & 7 & 14 & 30 & 1 & 7 & 14 & 30 \\
\hline & & \multicolumn{4}{|c|}{ December-January-February } & \multicolumn{4}{|c|}{ March-April-May } \\
\hline 0.05 & 20 & 4.95 & 6.06 & 6.93 & 8.42 & 0.04 & 0.12 & 0.18 & 0.56 \\
\hline 0.10 & 10 & 6.79 & 7.90 & 8.72 & 10.1 & 0.14 & 0.29 & 0.44 & 1.28 \\
\hline 0.20 & 5 & 9.44 & 10.5 & 11.2 & 12.3 & 0.50 & 0.80 & 1.19 & 3.06 \\
\hline \multirow[t]{2}{*}{0.50} & 2 & 15.3 & 16.0 & 16.5 & 17.1 & 3.65 & 4.24 & 5.99 & 11.7 \\
\hline & & \multicolumn{4}{|c|}{ June-July-August } & \multicolumn{4}{|c|}{ September-October-November } \\
\hline 0.05 & 20 & 0 & 0.01 & 0.02 & 0.03 & 0.01 & 0.02 & 0.03 & 0.05 \\
\hline 0.10 & 10 & 0.01 & 0.03 & 0.04 & 0.05 & 0.03 & 0.03 & 0.05 & 0.07 \\
\hline 0.20 & 5 & 0.03 & 0.05 & 0.07 & 0.09 & 0.05 & 0.06 & 0.08 & 0.12 \\
\hline 0.50 & 2 & 0.14 & 0.17 & 0.22 & 0.29 & 0.16 & 0.20 & 0.23 & 0.33 \\
\hline
\end{tabular}




\section{1-0.000-6M KABUL RIVER AT TANG-I-GHARU, Continued}

\begin{tabular}{|c|c|c|c|c|c|}
\hline \multicolumn{6}{|c|}{ 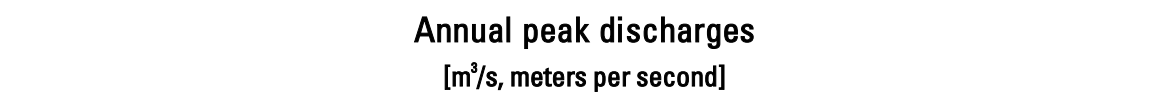 } \\
\hline \multicolumn{3}{|c|}{$\begin{array}{c}\text { Annual peak discharge, } \\
\text { by year }\end{array}$} & \multicolumn{3}{|c|}{$\begin{array}{l}\text { Annual peak discharge, } \\
\text { from highest to lowest }\end{array}$} \\
\hline $\begin{array}{l}\text { Water } \\
\text { year }\end{array}$ & Date & $\begin{array}{c}\text { Peak } \\
\text { discharge } \\
\left(\mathrm{m}^{3} / \mathrm{s}\right)\end{array}$ & $\begin{array}{l}\text { Water } \\
\text { year }\end{array}$ & Date & $\begin{array}{c}\text { Peak } \\
\text { discharge } \\
\left(\mathrm{m}^{3} / \mathrm{s}\right)\end{array}$ \\
\hline 1960 & May 2,1960 & 175 & 1967 & April 28, 1967 & 192 \\
\hline 1961 & April 18, 1961 & 98.0 & 1960 & May 2, 1960 & 175 \\
\hline 1962 & November 15, 1961 & 39.5 & 1963 & May 19, 1963 & 165 \\
\hline 1963 & May 19, 1963 & 165 & 1964 & April 10, 1964 & 158 \\
\hline 1964 & April 10, 1964 & 158 & 1965 & April 25, 1965 & 156 \\
\hline 1965 & April 25, 1965 & 156 & 1976 & April 25, 1976 & 128 \\
\hline 1966 & April 26, 1966 & 79.7 & 1968 & April 30, 1968 & 115 \\
\hline 1967 & April 28, 1967 & 192 & 1978 & July 7, 1978 & 109 \\
\hline 1968 & April 30, 1968 & 115 & 1972 & April 28, 1972 & 107 \\
\hline 1969 & April 19, 1969 & 85.5 & 1973 & April 11, 1973 & 107 \\
\hline 1970 & July 7, 1970 & 101 & 1980 & April 19, 1980 & 107 \\
\hline 1971 & March 26, 1971 & 43.6 & 1970 & July 7,1970 & 101 \\
\hline 1972 & April 28, 1972 & 107 & 1961 & April 18, 1961 & 98.0 \\
\hline 1973 & April 11, 1973 & 107 & 1969 & April 19, 1969 & 85.5 \\
\hline 1974 & April 7, 1974 & 48.2 & 1966 & April 26, 1966 & 79.7 \\
\hline 1975 & April 4, 1975 & 68.8 & 1979 & April 14, 1979 & 69.5 \\
\hline 1976 & April 25, 1976 & 128 & 1975 & April 4, 1975 & 68.8 \\
\hline 1977 & April 18, 1977 & 47.4 & 1974 & April 7, 1974 & 48.2 \\
\hline 1978 & July 7, 1978 & 109 & 1977 & April 18, 1977 & 47.4 \\
\hline 1979 & April 14, 1979 & 69.5 & 1971 & March 26, 1971 & 43.6 \\
\hline 1980 & April 19, 1980 & 107 & 1962 & November 15,1961 & 39.5 \\
\hline
\end{tabular}


1-0.000-6M KABUL RIVER AT TANG-I-GHARU, Continued

Monthly and annual mean discharges, in cubic meters per second

[Data may not be rounded in accordance with U.S. Geological Survey publication standards]

\begin{tabular}{|c|c|c|c|c|c|c|c|c|c|c|c|c|c|}
\hline \multirow{2}{*}{$\begin{array}{c}\text { Water } \\
\text { year }\end{array}$} & \multicolumn{12}{|c|}{ Monthly mean discharge } & \multirow{2}{*}{$\begin{array}{c}\text { Annual } \\
\text { discharge }\end{array}$} \\
\hline & October & November & December & January & February & March & April & May & June & July & August & September & \\
\hline 1960 & 3.65 & 19.8 & 22.3 & 20.9 & 21.8 & 26.6 & 83.1 & 75.4 & 18.4 & 7.22 & 1.75 & 1.49 & 25.2 \\
\hline 1961 & 9.85 & 24.4 & 24.6 & 26.1 & 25.6 & 28.2 & 69.4 & 41.3 & 6.89 & 5.51 & 1.47 & 1.07 & 22.0 \\
\hline 1962 & 6.88 & 22.2 & 22.5 & 23.6 & 23.5 & 21.7 & 28.8 & 6.22 & 0.593 & 0.621 & 0.337 & 0.287 & 13.0 \\
\hline 1963 & 2.33 & 10.2 & 19.8 & 20.4 & 18.7 & 17.4 & 13.5 & 50.5 & 4.43 & 0.386 & 0.85 & 0.293 & 13.3 \\
\hline 1964 & 0.67 & 11.2 & 17.6 & 19.4 & 19.5 & 34.8 & 96.2 & 21.5 & 2.80 & 1.00 & 0.432 & 0.351 & 18.7 \\
\hline 1965 & 1.81 & 15.3 & 19.8 & 18.7 & 21.9 & 28.4 & 89.5 & 93.1 & 31.1 & 13.6 & 3.86 & 3.76 & 28.4 \\
\hline 1966 & 9.62 & 22.2 & 26.6 & 25.4 & 24.3 & 26.8 & 42.2 & 12.3 & 2.08 & 1.15 & 0.851 & 1.73 & 16.2 \\
\hline 1967 & 5.32 & 16.0 & 20.2 & 22.5 & 23.6 & 23.7 & 60.0 & 74.3 & 22.8 & 7.75 & 2.62 & 2.04 & 23.4 \\
\hline 1968 & 9.06 & 21.7 & 25.8 & 27.9 & 27.0 & 45.5 & 56.7 & 41.8 & 13.8 & 1.42 & 0.494 & 1.05 & 22.7 \\
\hline 1969 & 6.51 & 18.1 & 26.6 & 27.9 & 28.1 & 32.9 & 30.9 & 10.6 & 2.25 & 0.335 & 0.306 & 0.321 & 15.3 \\
\hline 1970 & 2.08 & 17.3 & 17.4 & 19.7 & 18.3 & 18.0 & 6.17 & 0.443 & 0.154 & 1.34 & 0.172 & 0.035 & 8.37 \\
\hline 1971 & 0.06 & 4.26 & 12.8 & 15.3 & 16.7 & 19.0 & 11.6 & 0.697 & 0.438 & 0.134 & 0.13 & 0.164 & 6.71 \\
\hline 1972 & 0.21 & 0.56 & 6.77 & 11.5 & 14.6 & 29.2 & 53.5 & 53.8 & 6.80 & 1.84 & 0.409 & 0.759 & 15.0 \\
\hline 1973 & 0.79 & 8.90 & 16.5 & 19.1 & 18.8 & 39.1 & 78.9 & 17.2 & 1.29 & 0.743 & 0.392 & 0.235 & 16.7 \\
\hline 1974 & 1.13 & 8.43 & 17.8 & 21.4 & 21.3 & 22.8 & 22.2 & 2.09 & 2.11 & 0.124 & 0.013 & 0.098 & 9.89 \\
\hline 1975 & 0.20 & 4.41 & 10.5 & 16.9 & 18.4 & 24.4 & 43.6 & 14.0 & 1.71 & 0.253 & 0.601 & 0.149 & 11.2 \\
\hline 1976 & 0.12 & 5.23 & 13.5 & 16.6 & 18.1 & 24.8 & 67.1 & 35.5 & 3.16 & 1.73 & 0.237 & 0.197 & 15.5 \\
\hline 1977 & 0.64 & 9.83 & 16.8 & 21.8 & 22.8 & 18.3 & 20.6 & 1.26 & 0.222 & 0.142 & 0.149 & 0.13 & 9.30 \\
\hline 1978 & 0.18 & 4.23 & 10.9 & 13.7 & 16.0 & 18.5 & 16.5 & 2.02 & 0.239 & 8.71 & 5.64 & 0.571 & 8.08 \\
\hline 1979 & 0.23 & 4.27 & 10.2 & 13.2 & 13.5 & 14.4 & 34.6 & 12.9 & 0.289 & 0.086 & 3.12 & 0.212 & 8.87 \\
\hline 1980 & 0.22 & 4.19 & 11.7 & 15.2 & 18.0 & 27.7 & 82.8 & 26.7 & 1.46 & 0.42 & 0.101 & 0.151 & 15.7 \\
\hline
\end{tabular}




\section{1-2.R00-7T HAZARNAW RIVER AT SABAY}

(U.S. Geological Survey identification number: 340600070220000)

LOCATION: Lat $34^{\circ} 06^{\prime} \mathrm{N}$., long $70^{\circ} 22^{\prime} \mathrm{E}$.

DRAINAGE AREA: $120 \mathrm{~km}^{2}$.

ELEVATION: 1,400 meters above mean sea level.

PERIOD OF RECORD: December 26, 1975 to September 30, 1979.

GAGE: Staff gage.

Annual mean discharge

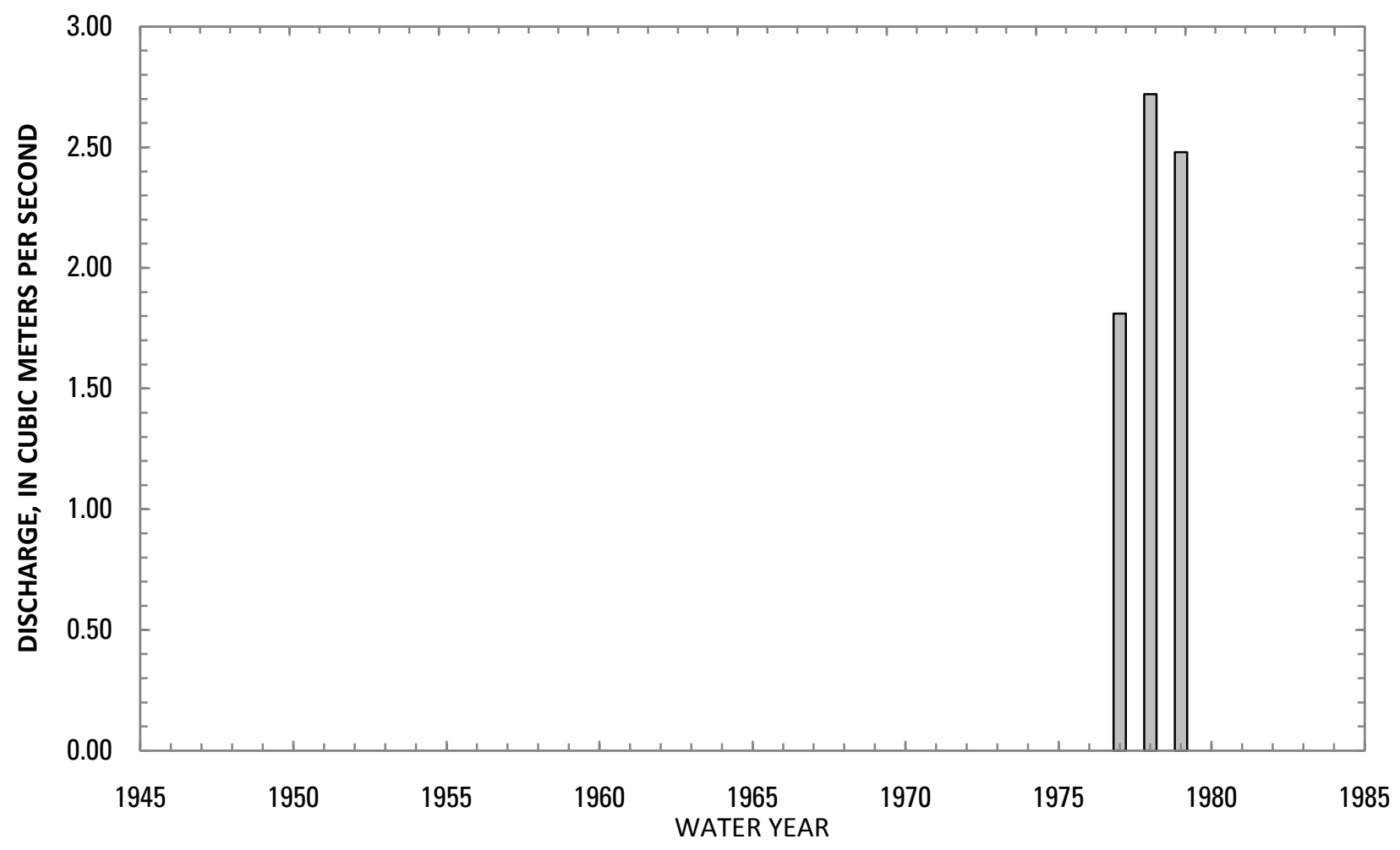




\section{1-2.R00-7T HAZARNAW RIVER AT SABAY, Continued}

Statistics of monthly and annual mean discharges [ $\mathrm{m}^{3} / \mathrm{s}$, cubic meters per second]

\begin{tabular}{|c|c|c|c|c|c|c|c|c|}
\hline \multirow[b]{2}{*}{ Month } & \multicolumn{2}{|c|}{ Maximum } & \multicolumn{2}{|c|}{ Minimum } & \multicolumn{4}{|c|}{ Mean } \\
\hline & $\begin{array}{c}\text { Discharge } \\
\left(\mathrm{m}^{3} / \mathrm{s}\right)\end{array}$ & $\begin{array}{l}\text { Water year } \\
\text { of } \\
\text { occurrence }\end{array}$ & $\begin{array}{c}\text { Discharge } \\
\left(\mathrm{m}^{3} / \mathrm{s}\right)\end{array}$ & $\begin{array}{c}\text { Water year } \\
\text { of } \\
\text { occurrence }\end{array}$ & $\begin{array}{c}\text { Discharge } \\
\left(\mathrm{m}^{3} / \mathrm{s}\right)\end{array}$ & $\begin{array}{c}\text { Standard } \\
\text { deviation } \\
\left(\mathrm{m}^{3} / \mathrm{s}\right)\end{array}$ & $\begin{array}{c}\text { Coefficient } \\
\text { of } \\
\text { variation }\end{array}$ & $\begin{array}{c}\text { Percentage } \\
\text { of annual } \\
\text { discharge }\end{array}$ \\
\hline October & 0.53 & 1977 & 0.30 & 1978 & 0.43 & 0.12 & 0.27 & 1.58 \\
\hline November & 0.50 & 1977 & 0.31 & 1978 & 0.39 & 0.10 & 0.25 & 1.44 \\
\hline December & 0.41 & 1977 & 0.28 & 1979 & 0.34 & 0.07 & 0.20 & 1.24 \\
\hline January & 0.30 & 1979 & 0.25 & 1978 & 0.28 & 0.02 & 0.09 & 1.03 \\
\hline February & 0.35 & 1977 & 0.17 & 1978 & 0.28 & 0.08 & 0.28 & 1.03 \\
\hline March & 0.54 & 1977 & 0.36 & 1976 & 0.42 & 0.08 & 0.20 & 1.55 \\
\hline April & 4.32 & 1978 & 2.76 & 1976 & 3.58 & 0.76 & 0.21 & 13.2 \\
\hline May & 8.17 & 1978 & 4.95 & 1979 & 6.38 & 1.44 & 0.23 & 23.5 \\
\hline June & 9.45 & 1978 & 4.93 & 1977 & 7.26 & 2.17 & 0.30 & 26.8 \\
\hline July & 5.77 & 1978 & 1.88 & 1977 & 3.91 & 1.80 & 0.46 & 14.4 \\
\hline August & 3.22 & 1979 & 0.76 & 1977 & 2.30 & 1.12 & 0.49 & 8.49 \\
\hline September & 2.89 & 1977 & 0.91 & 1978 & 1.58 & 0.93 & 0.59 & 5.80 \\
\hline Annual & 2.72 & 1978 & 1.81 & 1977 & 2.34 & 0.47 & 0.20 & 100 \\
\hline
\end{tabular}




\section{1-2.R00-7T HAZARNAW RIVER AT SABAY, Continued}

Monthly and annual flow duration, in cubic meters per second

[ng, not given]

\begin{tabular}{|c|c|c|c|c|c|c|c|c|c|c|c|c|c|}
\hline \multirow{2}{*}{$\begin{array}{l}\text { Percentage } \\
\text { of days } \\
\text { discharge } \\
\text { equaled or } \\
\text { exceeded }\end{array}$} & \multicolumn{12}{|c|}{ Month } & \multirow{2}{*}{ Annua } \\
\hline & October & November & December & January & February & March & April & May & June & July & August & September & \\
\hline 95 & 0.27 & 0.29 & 0.26 & 0.20 & 0.15 & 0.26 & 0.67 & 2.53 & 3.35 & 1.24 & 0.54 & 0.45 & 0.26 \\
\hline 90 & 0.29 & 0.29 & 0.27 & 0.23 & 0.16 & 0.27 & 0.82 & 3.06 & 3.77 & 1.53 & 0.60 & 0.53 & 0.27 \\
\hline 85 & 0.29 & 0.29 & 0.29 & 0.25 & 0.19 & 0.29 & 0.98 & 3.50 & 4.13 & 1.85 & 0.71 & 0.55 & 0.29 \\
\hline 80 & 0.30 & 0.30 & 0.29 & 0.26 & 0.20 & 0.29 & 1.27 & 3.81 & 4.28 & 2.07 & 0.83 & 0.56 & 0.30 \\
\hline 75 & 0.30 & 0.30 & 0.29 & 0.26 & 0.27 & 0.30 & 1.96 & 4.31 & 4.55 & 2.49 & 1.06 & 0.58 & 0.32 \\
\hline 70 & 0.30 & 0.30 & 0.29 & 0.27 & 0.27 & 0.33 & 2.22 & 4.48 & 4.94 & 2.64 & 1.20 & 0.61 & 0.37 \\
\hline 65 & 0.43 & 0.30 & 0.30 & 0.27 & 0.28 & 0.34 & 2.49 & 4.66 & 5.29 & 2.80 & 1.42 & 0.67 & 0.42 \\
\hline 60 & 0.43 & 0.33 & 0.30 & 0.27 & 0.28 & 0.36 & 2.67 & 4.92 & 6.29 & 3.00 & 1.66 & 0.75 & 0.46 \\
\hline 55 & 0.44 & 0.34 & 0.30 & 0.28 & 0.29 & 0.38 & 2.82 & 5.13 & 6.64 & 3.24 & 1.80 & 0.84 & 0.50 \\
\hline 50 & 0.44 & 0.37 & 0.30 & 0.29 & 0.29 & 0.38 & 3.24 & 5.33 & 7.29 & 3.50 & 1.91 & 0.90 & 0.56 \\
\hline 45 & 0.44 & 0.38 & 0.30 & 0.29 & 0.30 & 0.39 & 3.50 & 5.52 & 7.65 & 3.67 & 2.01 & 0.96 & 0.80 \\
\hline 40 & 0.49 & 0.43 & 0.36 & 0.30 & 0.31 & 0.42 & 3.77 & 5.71 & 7.89 & 3.83 & 2.13 & 1.22 & 1.41 \\
\hline 35 & 0.53 & 0.48 & 0.37 & ng & 0.32 & 0.43 & 4.40 & 6.16 & 8.06 & 4.05 & 2.25 & 1.38 & 1.97 \\
\hline 30 & 0.53 & 0.48 & 0.38 & ng & 0.32 & 0.44 & 4.95 & 6.64 & 8.23 & 4.26 & 2.41 & 1.43 & 2.61 \\
\hline 25 & 0.53 & 0.49 & 0.39 & ng & 0.33 & 0.45 & 5.33 & 7.48 & 8.48 & 4.45 & 2.60 & 1.48 & 3.49 \\
\hline 20 & 0.54 & 0.49 & ng & ng & 0.34 & 0.49 & 5.61 & 8.46 & 8.81 & 4.81 & 2.82 & 1.53 & 4.34 \\
\hline 15 & 0.54 & 0.53 & ng & ng & ng & 0.61 & 5.92 & 9.35 & 10.00 & 5.65 & 3.16 & 1.63 & 5.25 \\
\hline 10 & ng & 0.53 & ng & ng & ng & 0.66 & 6.25 & 10.10 & 11.60 & 6.76 & 4.05 & 1.83 & 6.46 \\
\hline 5 & ng & 0.54 & ng & ng & ng & 0.81 & 7.21 & 11.80 & 13.90 & 8.23 & 4.63 & 4.54 & 8.67 \\
\hline
\end{tabular}




\section{1-2.R00-7T HAZARNAW RIVER AT SABAY, Continued}

Probability of occurrence of annual high discharges

[ $\mathrm{m}^{3} / \mathrm{s}$, cubic meters per second; $\mathrm{ng}$, not given]

\begin{tabular}{|c|c|c|c|c|c|c|}
\hline \multirow{2}{*}{$\begin{array}{l}\text { Exceedance } \\
\text { probability }\end{array}$} & \multirow{2}{*}{$\begin{array}{c}\text { Recurrence } \\
\text { interval } \\
\text { (years) }\end{array}$} & \multirow{2}{*}{$\begin{array}{l}\text { Maximum } \\
\text { instantaneous } \\
\text { discharge } \\
\left(\mathrm{m}^{3} / \mathrm{s}\right)\end{array}$} & \multicolumn{4}{|c|}{ Maximum daily mean discharge $\left(\mathrm{m}^{3} / \mathrm{s}\right)$} \\
\hline & & & $\begin{array}{l}\text { 3-day } \\
\text { period }\end{array}$ & $\begin{array}{l}\text { 7-day } \\
\text { period }\end{array}$ & $\begin{array}{l}\text { 15-day } \\
\text { period }\end{array}$ & $\begin{array}{l}\text { 30-day } \\
\text { period }\end{array}$ \\
\hline 0.99 & 1.01 & ng & ng & ng & $\mathrm{ng}$ & ng \\
\hline 0.95 & 1.05 & ng & ng & ng & $\mathrm{ng}$ & ng \\
\hline 0.90 & 1.11 & ng & ng & ng & $\mathrm{ng}$ & ng \\
\hline 0.80 & 1.25 & ng & ng & ng & $\mathrm{ng}$ & $\mathrm{ng}$ \\
\hline 0.50 & 2 & ng & $\mathrm{ng}$ & ng & ng & ng \\
\hline 0.20 & 5 & ng & $\mathrm{ng}$ & ng & ng & ng \\
\hline 0.10 & 10 & ng & $\mathrm{ng}$ & ng & $\mathrm{ng}$ & $\mathrm{ng}$ \\
\hline 0.04 & 25 & ng & $\mathrm{ng}$ & ng & $\mathrm{ng}$ & ng \\
\hline 0.02 & 50 & ng & $\mathrm{ng}$ & ng & $\mathrm{ng}$ & $\mathrm{ng}$ \\
\hline 0.01 & 100 & ng & $\mathrm{ng}$ & $\mathrm{ng}$ & $\mathrm{ng}$ & $\mathrm{ng}$ \\
\hline 0.005 & 200 & ng & ng & ng & ng & $\mathrm{ng}$ \\
\hline 0.002 & 500 & $\mathrm{ng}$ & ng & $\mathrm{ng}$ & $\mathrm{ng}$ & $\mathrm{ng}$ \\
\hline
\end{tabular}

\section{1-2.R00-7T HAZARNAW RIVER AT SABAY, Continued}

\section{Probability of occurrence of annual low discharges}

[ $\mathrm{m}^{3} / \mathrm{s}$, meters per second; $\mathrm{ng}$, not given]

\begin{tabular}{|c|c|c|c|c|c|c|c|c|c|c|}
\hline \multirow{3}{*}{$\begin{array}{c}\text { Nonexceedance } \\
\text { probability }\end{array}$} & \multirow{3}{*}{$\begin{array}{c}\text { Recurrence } \\
\text { interval } \\
\text { (years) }\end{array}$} & \multicolumn{9}{|c|}{ Minimum daily mean discharge $\left(\mathrm{m}^{3} / \mathrm{s}\right)$} \\
\hline & & \multicolumn{9}{|c|}{ Number of consecutive days } \\
\hline & & 1 & 3 & 7 & 14 & 30 & 60 & 90 & 120 & 183 \\
\hline 0.05 & 20 & ng & ng & $\mathrm{ng}$ & ng & ng & ng & ng & ng & ng \\
\hline 0.10 & 10 & ng & ng & $\mathrm{ng}$ & $\mathrm{ng}$ & $\mathrm{ng}$ & ng & ng & ng & ng \\
\hline 0.20 & 5 & ng & ng & ng & ng & ng & ng & ng & ng & ng \\
\hline 0.50 & 2 & ng & ng & ng & ng & ng & ng & ng & ng & ng \\
\hline
\end{tabular}




\section{1-2.R00-7T HAZARNAW RIVER AT SABAY, Continued}

Probability of occurrence of seasonal low discharges [ $\mathrm{m}^{3} / \mathrm{s}$, meters per second; $\mathrm{ng}$, not given]

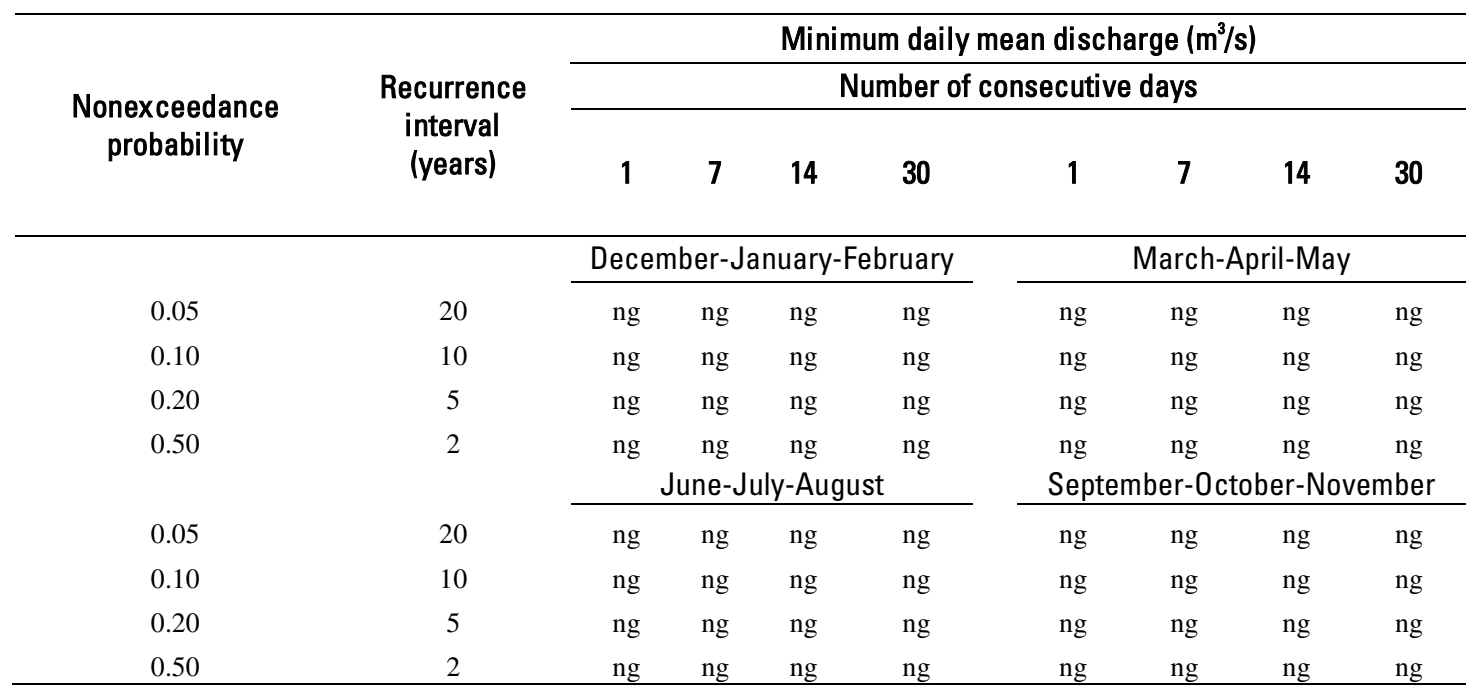

\section{1-2.R00-7T HAZARNAW RIVER AT SABAY, Continued}

Annual peak discharges

[ $\mathrm{m}^{3} / \mathrm{s}$, meters per second]

\begin{tabular}{|c|c|c|c|c|c|}
\hline \multicolumn{3}{|c|}{$\begin{array}{c}\text { Annual peak discharge, } \\
\text { by year }\end{array}$} & \multicolumn{3}{|c|}{$\begin{array}{l}\text { Annual peak discharge, } \\
\text { from highest to lowest }\end{array}$} \\
\hline $\begin{array}{l}\text { Water } \\
\text { year }\end{array}$ & Date & $\begin{array}{c}\text { Peak } \\
\text { discharge } \\
\left(\mathrm{m}^{3} / \mathrm{s}\right)\end{array}$ & $\begin{array}{l}\text { Water } \\
\text { year }\end{array}$ & Date & $\begin{array}{c}\text { Peak } \\
\text { discharge } \\
\left(\mathrm{m}^{3} / \mathrm{s}\right)\end{array}$ \\
\hline 1976 & July 21, 1976 & 49.4 & 1977 & $\begin{array}{c}\text { September 4, } \\
1977\end{array}$ & 110 \\
\hline 1977 & September 4, 1977 & 110 & 1976 & July 21, 1976 & 49.4 \\
\hline 1978 & July 7, 1978 & 18.6 & 1979 & August 7, 1979 & 38.5 \\
\hline 1979 & August 7, 1979 & 38.5 & 1978 & July 7,1978 & 18.6 \\
\hline
\end{tabular}




\section{1-2.R00-7T HAZARNAW RIVER AT SABAY, Continued}

Monthly and annual mean discharges, in cubic meters per second

[Data may not be rounded in accordance with U.S. Geological Survey publication standards; --, no data]

\begin{tabular}{|c|c|c|c|c|c|c|c|c|c|c|c|c|c|}
\hline \multirow{2}{*}{$\begin{array}{l}\text { Water } \\
\text { year }\end{array}$} & \multicolumn{12}{|c|}{ Monthly mean discharge } & \multirow{2}{*}{$\begin{array}{c}\text { Annual } \\
\text { discharge }\end{array}$} \\
\hline & October & November & December & January & February & March & April & May & June & July & August & September & \\
\hline 1976 & -- & -- & -- & 0.29 & 0.28 & 0.36 & 2.76 & 6.88 & 5.95 & 2.95 & 3.05 & 0.91 & -- \\
\hline 1977 & 0.53 & 0.50 & 0.41 & 0.28 & 0.35 & 0.54 & 3.12 & 5.53 & 4.93 & 1.88 & 0.76 & 2.89 & 1.81 \\
\hline 1978 & 0.30 & 0.31 & 0.32 & 0.25 & 0.17 & 0.38 & 4.32 & 8.17 & 9.45 & 5.77 & 2.19 & 0.91 & 2.72 \\
\hline 1979 & 0.47 & 0.37 & 0.28 & 0.30 & 0.32 & 0.41 & 4.12 & 4.95 & 8.72 & 5.03 & 3.22 & 1.59 & 2.48 \\
\hline
\end{tabular}




\section{1-4.1R0-1A PECH RIVER AT CHAGHASARAI \\ (U.S. Geological Survey identification number: 345400071080000)}

LOCATION: Lat $34^{\circ} 54^{\prime} \mathrm{N}$., long $71^{\circ} 08^{\prime} \mathrm{E}$.

DRAINAGE AREA: $3,855 \mathrm{~km}^{2}$.

ELEVATION: 790 meters above mean sea level.

PERIOD OF RECORD: February 23, 1960 to February 28, 1979.

GAGE: Water-stage recorder.

Annual mean discharge

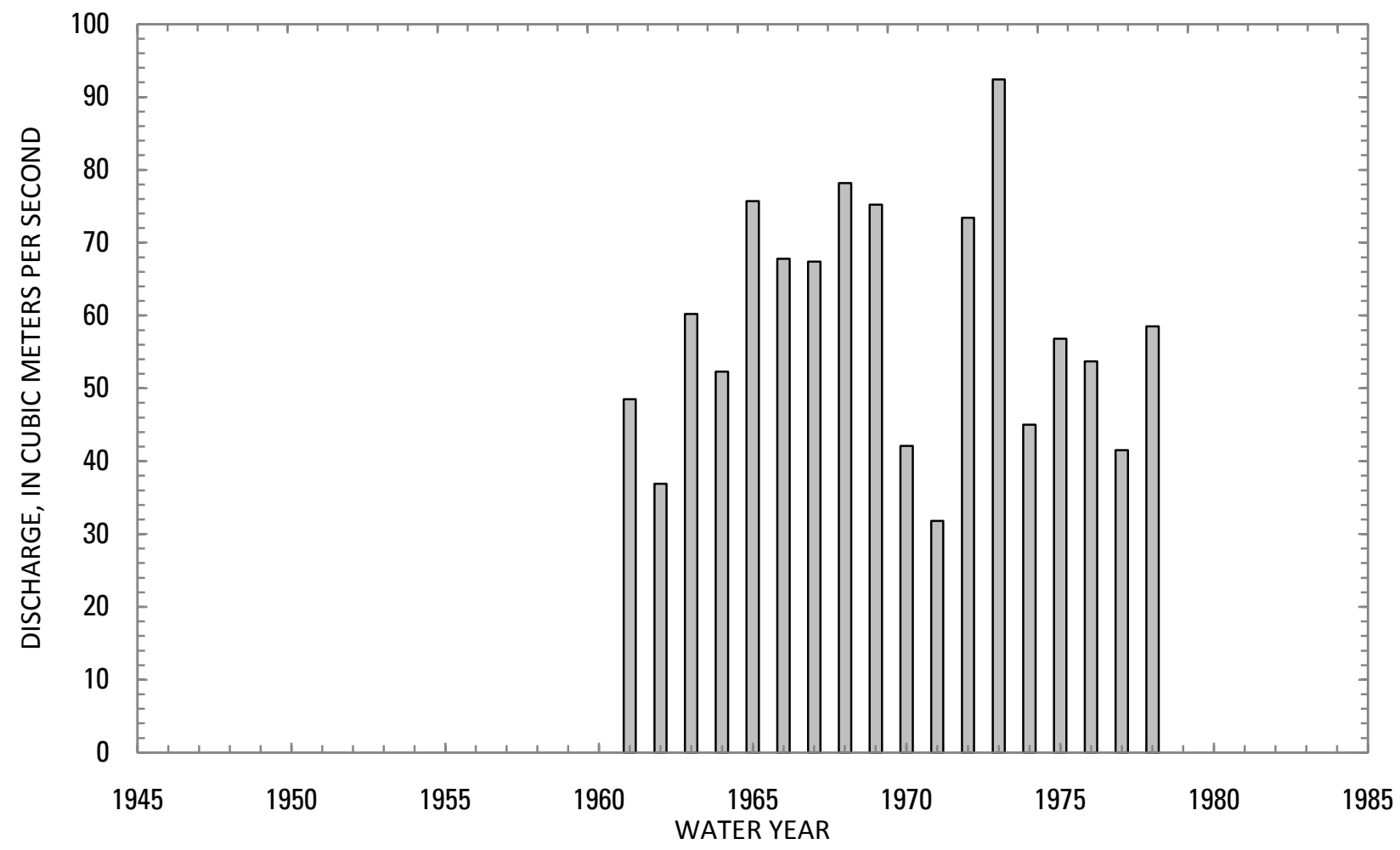




\section{1-4.1R0-1A PECH RIVER AT CHAGHASARAI, Continued}

Statistics of monthly and annual mean discharges $\left[\mathrm{m}^{3} / \mathrm{s}\right.$, cubic meters per second]

\begin{tabular}{|c|c|c|c|c|c|c|c|c|}
\hline \multirow[b]{2}{*}{ Month } & \multicolumn{2}{|c|}{ Maximum } & \multicolumn{2}{|c|}{ Minimum } & \multicolumn{4}{|c|}{ Mean } \\
\hline & $\begin{array}{c}\text { Discharge } \\
\left(\mathrm{m}^{3} / \mathrm{s}\right)\end{array}$ & $\begin{array}{c}\text { Water year } \\
\text { of } \\
\text { occurrence }\end{array}$ & $\begin{array}{c}\text { Discharge } \\
\left(\mathrm{m}^{3} / \mathrm{s}\right)\end{array}$ & $\begin{array}{c}\text { Water year } \\
\text { of } \\
\text { occurrence }\end{array}$ & $\begin{array}{c}\text { Discharge } \\
\left(\mathrm{m}^{3} / \mathrm{s}\right)\end{array}$ & $\begin{array}{c}\text { Standard } \\
\text { deviation } \\
\left(\mathrm{m}^{3} / \mathrm{s}\right)\end{array}$ & $\begin{array}{c}\text { Coefficient } \\
\text { of } \\
\text { variation }\end{array}$ & $\begin{array}{c}\text { Percentage } \\
\text { of annual } \\
\text { discharge }\end{array}$ \\
\hline October & 30.1 & 1970 & 6.74 & 1972 & 14.4 & 6.30 & 0.44 & 2.05 \\
\hline November & 23.1 & 1970 & 5.94 & 1972 & 11.3 & 4.21 & 0.37 & 1.62 \\
\hline December & 13.1 & 1973 & 4.01 & 1972 & 8.59 & 2.39 & 0.28 & 1.22 \\
\hline January & 13.2 & 1973 & 3.50 & 1971 & 7.68 & 2.19 & 0.28 & 1.09 \\
\hline February & 16.9 & 1973 & 4.24 & 1971 & 9.78 & 3.30 & 0.34 & 1.39 \\
\hline March & 54.7 & 1969 & 11.4 & 1975 & 24.9 & 13.0 & 0.52 & 3.55 \\
\hline April & 174 & 1973 & 42.7 & 1977 & 82.7 & 29.3 & 0.35 & 11.8 \\
\hline May & 278 & 1973 & 75.8 & 1962 & 150 & 42.3 & 0.28 & 21.3 \\
\hline June & 331 & 1972 & 96.4 & 1971 & 206 & 64.5 & 0.31 & 29.4 \\
\hline July & 219 & 1965 & 33.5 & 1970 & 118 & 51.5 & 0.44 & 16.9 \\
\hline August & 84.5 & 1968 & 17.0 & 1974 & 46.5 & 17.6 & 0.38 & 6.62 \\
\hline September & 33.8 & 1967 & 9.17 & 1971 & 21.8 & 6.65 & 0.31 & 3.10 \\
\hline Annual & 92.4 & 1973 & 31.8 & 1971 & 58.7 & 16.4 & 0.28 & 100 \\
\hline
\end{tabular}


1-4.1R0-1A PECH RIVER AT CHAGHASARAI, Continued

Monthly and annual flow duration, in cubic meters per second

\begin{tabular}{|c|c|c|c|c|c|c|c|c|c|c|c|c|c|}
\hline \multirow{2}{*}{$\begin{array}{l}\text { Percentage } \\
\text { of days } \\
\text { discharge } \\
\text { equaled or } \\
\text { exceeded }\end{array}$} & \multicolumn{12}{|c|}{ Month } & \multirow{2}{*}{ Annual } \\
\hline & October & November & December & January & February & March & April & May & June & July & August & September & \\
\hline 95 & 6.99 & 5.86 & 4.16 & 3.14 & 5.26 & 8.39 & 32.4 & 64.8 & 91.4 & 28.6 & 16.3 & 8.93 & 6.11 \\
\hline 90 & 7.81 & 6.61 & 4.92 & 4.52 & 5.89 & 9.26 & 36.5 & 77.6 & 119 & 36.0 & 22.5 & 10.6 & 7.11 \\
\hline 85 & 8.51 & 6.95 & 5.67 & 5.50 & 6.39 & 10.3 & 40.2 & 88.3 & 132 & 41.9 & 25.2 & 12.3 & 7.97 \\
\hline 80 & 9.05 & 7.63 & 6.21 & 5.98 & 6.89 & 11.2 & 44.9 & 98.8 & 142 & 50.1 & 28.4 & 14.0 & 8.76 \\
\hline 75 & 9.58 & 8.04 & 6.77 & 6.22 & 7.26 & 12.5 & 48.7 & 106 & 151 & 59.5 & 30.1 & 16.1 & 9.67 \\
\hline 70 & 10.1 & 8.64 & 7.10 & 6.38 & 7.58 & 13.4 & 51.8 & 113 & 162 & 71.3 & 32.9 & 17.7 & 10.9 \\
\hline 65 & 10.6 & 8.98 & 7.33 & 6.62 & 7.81 & 15.0 & 54.9 & 119 & 170 & 79.5 & 34.9 & 18.7 & 12.6 \\
\hline 60 & 11.0 & 9.24 & 7.60 & 6.88 & 8.03 & 16.3 & 58.1 & 125 & 179 & 89.1 & 37.2 & 19.4 & 14.6 \\
\hline 55 & 11.5 & 9.55 & 7.90 & 7.14 & 8.35 & 18.2 & 62.2 & 132 & 188 & 99.7 & 39.3 & 20.3 & 17.9 \\
\hline 50 & 12.3 & 9.94 & 8.18 & 7.34 & 8.73 & 20.1 & 67.9 & 141 & 198 & 112 & 41.3 & 21.5 & 21.9 \\
\hline 45 & 12.8 & 10.5 & 8.54 & 7.57 & 9.10 & 21.5 & 76.1 & 150 & 214 & 120 & 43.3 & 22.4 & 27.3 \\
\hline 40 & 13.3 & 11.2 & 8.94 & 7.84 & 9.55 & 23.4 & 82.5 & 157 & 226 & 131 & 45.9 & 23.3 & 34.7 \\
\hline 35 & 14.2 & 11.9 & 9.15 & 8.22 & 10.1 & 25.8 & 90.2 & 165 & 237 & 140 & 49.0 & 24.2 & 43.3 \\
\hline 30 & 15.9 & 12.5 & 9.37 & 8.76 & 10.7 & 29.2 & 97.3 & 175 & 252 & 149 & 53.1 & 25.3 & 58.1 \\
\hline 25 & 17.7 & 13.3 & 9.80 & 9.18 & 12.0 & 32.8 & 106 & 186 & 263 & 160 & 57.9 & 26.6 & 81.2 \\
\hline 20 & 19.3 & 14.4 & 10.8 & 9.58 & 13.2 & 35.4 & 115 & 198 & 276 & 172 & 63.0 & 28.3 & 111 \\
\hline 15 & 21.2 & 15.3 & 12.1 & 9.96 & 14.1 & 41.0 & 126 & 213 & 292 & 189 & 69.5 & 31.2 & 140 \\
\hline 10 & 24.8 & 17.3 & 12.9 & 10.8 & 15.6 & 46.3 & 147 & 237 & 309 & 219 & 78.7 & 33.3 & 173 \\
\hline 5 & 29.0 & 21.6 & 13.8 & 13.0 & 17.7 & 55.8 & 184 & 263 & 332 & 258 & 94.8 & 36.0 & 232 \\
\hline
\end{tabular}




\section{1-4.1R0-1A PECH RIVER AT CHAGHASARAI, Continued}

Probability of occurrence of annual high discharges

[m $\mathrm{m}^{3} / \mathrm{s}$, cubic meters per second; ng, not given]

\begin{tabular}{|c|c|c|c|c|c|c|}
\hline \multirow{2}{*}{$\begin{array}{c}\text { Exceedance } \\
\text { probability }\end{array}$} & \multirow{2}{*}{$\begin{array}{c}\text { Recurrence } \\
\text { interval } \\
\text { (years) }\end{array}$} & \multirow{2}{*}{$\begin{array}{l}\text { Maximum } \\
\text { instantaneous } \\
\text { discharge } \\
\left(\mathrm{m}^{3} / \mathrm{s}\right)\end{array}$} & \multicolumn{4}{|c|}{ Maximum daily mean discharge $\left(\mathrm{m}^{3} / \mathrm{s}\right)$} \\
\hline & & & $\begin{array}{l}\text { 3-day } \\
\text { period }\end{array}$ & $\begin{array}{l}\text { 7-day } \\
\text { period }\end{array}$ & $\begin{array}{l}\text { 15-day } \\
\text { period }\end{array}$ & $\begin{array}{l}\text { 30-day } \\
\text { period }\end{array}$ \\
\hline 0.99 & 1.01 & 181 & 139 & 135 & 129 & 117 \\
\hline 0.95 & 1.05 & 228 & 176 & 167 & 154 & 140 \\
\hline 0.90 & 1.11 & 256 & 198 & 186 & 170 & 154 \\
\hline 0.80 & 1.25 & 293 & 225 & 209 & 190 & 172 \\
\hline 0.50 & 2 & 373 & 279 & 256 & 234 & 215 \\
\hline 0.20 & 5 & 465 & 332 & 305 & 285 & 267 \\
\hline 0.10 & 10 & 517 & 359 & 331 & 316 & 300 \\
\hline 0.04 & 25 & 576 & 385 & 357 & 351 & 339 \\
\hline 0.02 & 50 & 616 & 401 & ${ }^{1} 374$ & 375 & 367 \\
\hline 0.01 & 100 & 652 & 415 & ${ }^{1} 388$ & 398 & 394 \\
\hline 0.005 & 200 & 686 & 427 & ${ }^{1} 401$ & ${ }^{1} 420$ & 421 \\
\hline 0.002 & 500 & 728 & ng & ng & $\mathrm{ng}$ & ng \\
\hline
\end{tabular}

'Data does not fit log-Pearson Type III curve, use with caution.

\section{1-4.1R0-1A PECH RIVER AT CHAGHASARAI, Continued}

\section{Probability of occurrence of annual low discharges} $\left[\mathrm{m}^{3} / \mathrm{s}\right.$, meters per second]

\begin{tabular}{|c|c|c|c|c|c|c|c|c|c|c|}
\hline \multirow{3}{*}{$\begin{array}{c}\text { Nonexceedance } \\
\text { probability }\end{array}$} & \multirow{3}{*}{$\begin{array}{c}\text { Recurrence } \\
\text { interval } \\
\text { (years) }\end{array}$} & \multicolumn{9}{|c|}{ Minimum daily mean discharge $\left(\mathrm{m}^{3} / \mathrm{s}\right)$} \\
\hline & & \multicolumn{9}{|c|}{ Number of consecutive days } \\
\hline & & 1 & 3 & 7 & 14 & 30 & 60 & 90 & 120 & 183 \\
\hline 0.05 & 20 & 2.76 & 3.01 & 3.10 & 3.23 & 3.59 & 4.24 & 4.77 & 5.30 & 6.84 \\
\hline 0.10 & 10 & 3.28 & 3.53 & 3.62 & 3.80 & 4.22 & 4.92 & 5.43 & 6.01 & 7.61 \\
\hline 0.20 & 5 & 4.01 & 4.24 & 4.34 & 4.58 & 5.07 & 5.80 & 6.28 & 6.94 & 8.65 \\
\hline 0.50 & 2 & 5.69 & 5.90 & 6.02 & 6.36 & 6.94 & 7.67 & 8.09 & 8.87 & 11.0 \\
\hline
\end{tabular}




\section{1-4.1R0-1A PECH RIVER AT CHAGHASARAI, Continued}

Probability of occurrence of seasonal low discharges [ $\mathrm{m}^{3} / \mathrm{s}$, meters per second]

\begin{tabular}{|c|c|c|c|c|c|c|c|c|c|}
\hline \multirow{3}{*}{$\begin{array}{c}\text { Nonexceedance } \\
\text { probability }\end{array}$} & \multirow{3}{*}{$\begin{array}{c}\text { Recurrence } \\
\text { interval } \\
\text { (years) }\end{array}$} & \multicolumn{8}{|c|}{ Minimum daily mean discharge $\left(\mathrm{m}^{3} / \mathrm{s}\right)$} \\
\hline & & \multicolumn{8}{|c|}{ Number of consecutive days } \\
\hline & & 1 & 7 & 14 & 30 & 1 & 7 & 14 & 30 \\
\hline & & \multicolumn{4}{|c|}{ December-January-February } & \multicolumn{4}{|c|}{ March-April-May } \\
\hline 0.05 & 20 & 2.81 & 3.15 & 3.28 & 3.64 & 5.19 & 6.11 & 7.32 & 10.3 \\
\hline 0.10 & 10 & 3.31 & 3.67 & 3.84 & 4.26 & 5.91 & 7.04 & 8.40 & 11.9 \\
\hline 0.20 & 5 & 4.02 & 4.40 & 4.61 & 5.10 & 7.01 & 8.45 & 10.0 & 14.2 \\
\hline \multirow[t]{2}{*}{0.50} & 2 & 5.69 & 6.06 & 6.35 & 6.92 & 10.1 & 12.4 & 14.7 & 20.9 \\
\hline & & \multicolumn{4}{|c|}{ June-July-August } & \multicolumn{4}{|c|}{ September-October-November } \\
\hline 0.05 & 20 & 13.9 & 16.4 & 17.0 & 19.1 & 4.40 & 5.17 & 5.58 & 6.13 \\
\hline 0.10 & 10 & 16.9 & 19.5 & 20.8 & 23.6 & 4.99 & 5.66 & 6.02 & 6.72 \\
\hline 0.20 & 5 & 20.9 & 23.6 & 25.9 & 30.0 & 5.80 & 6.37 & 6.70 & 7.59 \\
\hline 0.50 & 2 & 29.0 & 31.9 & 36.2 & 44.5 & 7.70 & 8.24 & 8.61 & 9.92 \\
\hline
\end{tabular}




\section{1-4.1R0-1A PECH RIVER AT CHAGHASARAI, Continued}

\section{Annual peak discharges}

$\left[\mathrm{m}^{3} / \mathrm{s}\right.$, meters per second]

\begin{tabular}{|c|c|c|c|c|c|}
\hline \multicolumn{3}{|c|}{$\begin{array}{c}\text { Annual peak discharge, } \\
\text { by year }\end{array}$} & \multicolumn{3}{|c|}{$\begin{array}{l}\text { Annual peak discharge, } \\
\text { from highest to lowest }\end{array}$} \\
\hline $\begin{array}{l}\text { Water } \\
\text { year }\end{array}$ & Date & $\begin{array}{c}\text { Peak } \\
\text { discharge } \\
\left(\mathrm{m}^{3} / \mathrm{s}\right)\end{array}$ & $\begin{array}{c}\text { Water } \\
\text { year }\end{array}$ & Date & $\begin{array}{c}\text { Peak } \\
\text { discharge } \\
\left(\mathrm{m}^{3} / \mathrm{s}\right)\end{array}$ \\
\hline 1961 & June 5, 1961 & 375 & 1972 & June 25, 1972 & 540 \\
\hline 1962 & June 12, 1962 & 240 & 1978 & July 6, 1978 & 530 \\
\hline 1963 & May 13, 1963 & 515 & 1965 & April 18, 1965 & 525 \\
\hline 1964 & July 3, 1964 & 274 & 1963 & May 13, 1963 & 515 \\
\hline 1965 & April 18, 1965 & 525 & 1967 & June 12, 1967 & 456 \\
\hline 1966 & June 20, 1966 & 406 & 1969 & June 18, 1969 & 445 \\
\hline 1967 & June 12, 1967 & 456 & 1973 & June 12,1973 & 435 \\
\hline 1968 & July 8, 1968 & 366 & 1966 & June 20, 1966 & 406 \\
\hline 1969 & June 18, 1969 & 445 & 1975 & June 16,1975 & 387 \\
\hline 1970 & May 21, 1970 & 254 & 1961 & June 5, 1961 & 375 \\
\hline 1971 & May 23, 1971 & 352 & 1968 & July 8, 1968 & 366 \\
\hline 1972 & June 25, 1972 & 540 & 1974 & June 5, 1974 & 362 \\
\hline 1973 & June 12, 1973 & 435 & 1971 & May 23, 1971 & 352 \\
\hline 1974 & June 5,1974 & 362 & 1979 & June 23, 1979 & 326 \\
\hline 1975 & June 16, 1975 & 387 & 1977 & May 25, 1977 & 315 \\
\hline 1976 & May 23, 1976 & 291 & 1976 & May 23, 1976 & 291 \\
\hline 1977 & May 25, 1977 & 315 & 1964 & July 3, 1964 & 274 \\
\hline 1978 & July 6, 1978 & 530 & 1970 & May 21, 1970 & 254 \\
\hline 1979 & June 23, 1979 & 326 & 1962 & June 12,1962 & 240 \\
\hline 1980 & June 11, 1980 & 213 & 1980 & June 11,1980 & 213 \\
\hline
\end{tabular}


1-4.1R0-1A PECH RIVER AT CHAGHASARAI, Continued

Monthly and annual mean discharges, in cubic meters per second $[--$, no data]

\begin{tabular}{|c|c|c|c|c|c|c|c|c|c|c|c|c|c|}
\hline \multirow{2}{*}{$\begin{array}{l}\text { Water } \\
\text { year }\end{array}$} & \multicolumn{12}{|c|}{ Monthly mean discharge } & \multirow{2}{*}{$\begin{array}{c}\text { Annual } \\
\text { discharge }\end{array}$} \\
\hline & October & November & December & January & February & March & April & May & June & July & August & September & \\
\hline 1960 & -- & -- & -- & -- & -- & 33.3 & 85.8 & 92.3 & 166 & 168 & 64.5 & 18.3 & -- \\
\hline 1961 & 10.5 & 13.5 & 8.18 & 6.46 & 8.49 & 15.4 & 58.4 & 124 & 179 & 85.5 & 41.5 & 30.7 & 48.5 \\
\hline 1962 & 10.0 & 12.3 & 9.74 & 8.03 & 8.96 & 12.4 & 55.9 & 75.8 & 129 & 68.2 & 32.5 & 19.0 & 36.9 \\
\hline 1963 & 22.1 & 15.1 & 7.97 & 6.58 & 7.86 & 27.0 & 74.9 & 145 & 216 & 124 & 53.3 & 20.5 & 60.2 \\
\hline 1964 & 11.8 & 9.30 & 11.8 & 7.79 & 8.94 & 18.7 & 71.6 & 129 & 175 & 112 & 52.4 & 18.6 & 52.3 \\
\hline 1965 & 10.2 & 8.47 & 9.03 & 8.26 & 9.18 & 21.7 & 93.0 & 166 & 272 & 219 & 61.2 & 26.9 & 75.7 \\
\hline 1966 & 13.0 & 9.24 & 9.02 & 9.26 & 14.1 & 37.8 & 105 & 153 & 223 & 142 & 63.8 & 32.5 & 67.8 \\
\hline 1967 & 24.6 & 15.9 & 8.37 & 6.17 & 7.89 & 18.4 & 77.9 & 149 & 246 & 161 & 56.1 & 33.8 & 67.4 \\
\hline 1968 & 21.3 & 16.0 & 10.5 & 9.64 & 15.3 & 39.6 & 100 & 153 & 259 & 204 & 84.5 & 25.1 & 78.2 \\
\hline 1969 & 19.6 & 13.8 & 12.6 & 10.2 & 13.5 & 54.7 & 102 & 163 & 290 & 145 & 52.1 & 24.3 & 75.2 \\
\hline 1970 & 30.1 & 23.1 & 10.4 & 9.49 & 8.73 & 17.4 & 63.7 & 137 & 120 & 33.5 & 31.4 & 18.9 & 42.1 \\
\hline 1971 & 9.32 & 6.44 & 4.73 & 3.50 & 4.24 & 14.5 & 46.1 & 127 & 96.4 & 38.2 & 19.4 & 9.17 & 31.8 \\
\hline 1972 & 6.74 & 5.94 & 4.01 & 4.57 & 12.7 & 39.5 & 105 & 196 & 331 & 123 & 32.6 & 20.7 & 73.4 \\
\hline 1973 & 15.1 & 13.2 & 13.1 & 13.2 & 16.9 & 46.1 & 174 & 278 & 306 & 151 & 60.1 & 20.1 & 92.4 \\
\hline 1974 & 13.1 & 8.78 & 7.71 & 6.24 & 8.39 & 19.7 & 64.6 & 142 & 174 & 67.5 & 17.0 & 10.3 & 45.0 \\
\hline 1975 & 8.60 & 7.85 & 6.84 & 6.85 & 6.15 & 11.4 & 87.2 & 138 & 213 & 112 & 55.4 & 27.7 & 56.8 \\
\hline 1976 & 16.2 & 9.46 & 7.71 & 6.56 & 10.5 & 15.0 & 73.8 & 159 & 165 & 116 & 43.1 & 21.4 & 53.7 \\
\hline 1977 & 10.4 & 8.24 & 6.60 & 7.59 & 7.60 & 18.3 & 42.7 & 125 & 171 & 57.9 & 25.0 & 15.6 & 41.5 \\
\hline 1978 & 10.4 & 9.23 & 7.98 & 9.02 & 6.44 & 12.4 & 89.1 & 191 & 188 & 119 & 37.4 & 19.7 & 58.5 \\
\hline 1979 & 10.8 & 9.54 & 7.00 & 6.60 & 9.90 & -- & -- & -- & -- & -- & -- & -- & -- \\
\hline
\end{tabular}




\section{1-4.2R0-1A LANDAISIN RIVER NEAR GAWARDESH}

\section{(U.S. Geological Survey identification number: 352300071320000)}

LOCATION: Lat $35^{\circ} 23^{\prime} \mathrm{N}$., long $71^{\circ} 32^{\prime} \mathrm{E}$.

DRAINAGE AREA: $3,130 \mathrm{~km}^{2}$.

ELEVATION: 1,135 meters above mean sea level.

PERIOD OF RECORD: May 1, 1975 to July 22, 1978.

GAGE: Water-stage recorder. Staff gage at same site and datum prior to 1976 water year.

Annual mean discharge

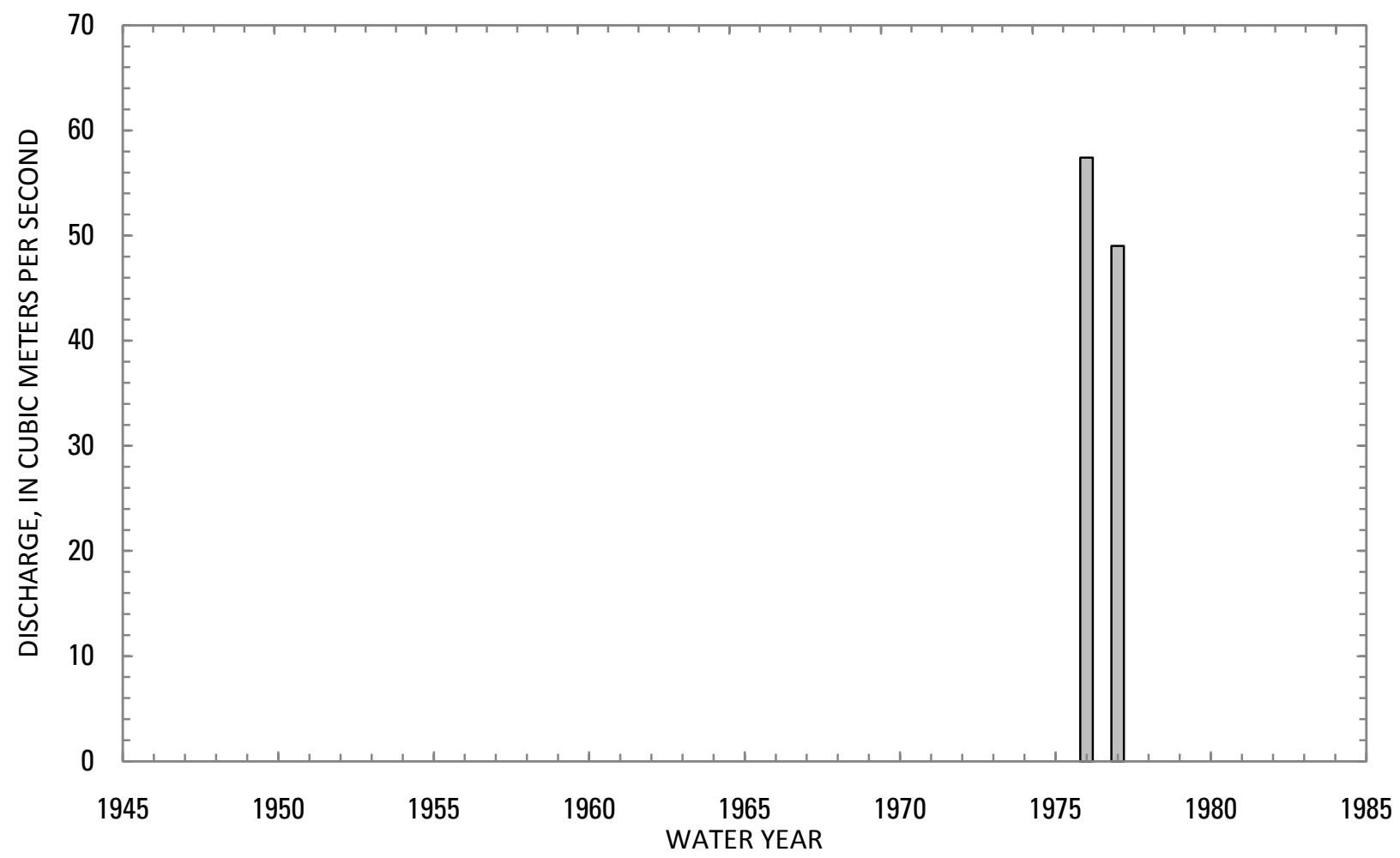




\section{1-4.2R0-1A LANDAISIN RIVER NEAR GAWARDESH, Continued}

Statistics of monthly and annual mean discharges $\left[\mathrm{m}^{3} / \mathrm{s}\right.$, cubic meters per second]

\begin{tabular}{|c|c|c|c|c|c|c|c|c|}
\hline \multirow[b]{2}{*}{ Month } & \multicolumn{2}{|c|}{ Maximum } & \multicolumn{2}{|c|}{ Minimum } & \multicolumn{4}{|c|}{ Mean } \\
\hline & $\begin{array}{c}\text { Discharge } \\
\left(\mathrm{m}^{3} / \mathrm{s}\right)\end{array}$ & $\begin{array}{c}\text { Water year } \\
\text { of } \\
\text { occurrence }\end{array}$ & $\begin{array}{c}\text { Discharge } \\
\left(\mathrm{m}^{3} / \mathrm{s}\right)\end{array}$ & $\begin{array}{c}\text { Water year } \\
\text { of } \\
\text { occurrence }\end{array}$ & $\begin{array}{c}\text { Discharge } \\
\left(\mathrm{m}^{3} / \mathrm{s}\right)\end{array}$ & $\begin{array}{c}\text { Standard } \\
\text { deviation } \\
\left(\mathrm{m}^{3} / \mathrm{s}\right)\end{array}$ & $\begin{array}{c}\text { Coefficient } \\
\text { of } \\
\text { variation }\end{array}$ & $\begin{array}{c}\text { Percentage } \\
\text { of annual } \\
\text { discharge }\end{array}$ \\
\hline October & 23.6 & 1976 & 21.8 & 1978 & 23.0 & 1.01 & 0.04 & 3.38 \\
\hline November & 16.4 & 1977 & 15.6 & 1978 & 16.1 & 0.44 & 0.03 & 2.37 \\
\hline December & 13.0 & 1977 & 11.4 & 1978 & 12.4 & 0.87 & 0.07 & 1.83 \\
\hline January & 12.1 & 1976 & 9.7 & 1978 & 11.0 & 1.21 & 0.11 & 1.61 \\
\hline February & 11.9 & 1976 & 10.3 & 1978 & 10.9 & 0.90 & 0.08 & 1.60 \\
\hline March & 19.7 & 1977 & 14.1 & 1976 & 16.4 & 2.93 & 0.18 & 2.41 \\
\hline April & 53.4 & 1976 & 44.4 & 1977 & 49.8 & 4.76 & 0.10 & 7.33 \\
\hline May & 169 & 1975 & 80.0 & 1977 & 124 & 36.4 & 0.29 & 18.2 \\
\hline June & 175 & 1978 & 150 & 1977 & 162 & 11.9 & 0.07 & 23.9 \\
\hline July & 144 & 1976 & 112 & 1977 & 129 & 16.0 & 0.12 & 19.0 \\
\hline August & 99.0 & 1975 & 69.9 & 1977 & 82.5 & 15.0 & 0.18 & 12.1 \\
\hline September & 47.2 & 1975 & 35.5 & 1977 & 43.0 & 6.49 & 0.15 & 6.33 \\
\hline Annual & 57.4 & 1976 & 49.0 & 1977 & 53.2 & 5.94 & 0.11 & 100 \\
\hline
\end{tabular}


1-4.2R0-1A LANDAISIN RIVER NEAR GAWARDESH, Continued

Monthly and annual flow duration, in cubic meters per second

[ng, not given]

\begin{tabular}{|c|c|c|c|c|c|c|c|c|c|c|c|c|c|}
\hline \multirow{2}{*}{$\begin{array}{l}\text { Percentage } \\
\text { of days } \\
\text { discharge } \\
\text { equaled or } \\
\text { exceeded }\end{array}$} & \multicolumn{12}{|c|}{ Month } & \multirow{2}{*}{ Annua } \\
\hline & October & November & December & January & February & March & April & May & June & July & August & September & \\
\hline 95 & 18.6 & 13.5 & 10.5 & 9.33 & 9.33 & 11.7 & 16.6 & 51.2 & 121 & 82.5 & 55.3 & 29.6 & 11.1 \\
\hline 90 & 19.3 & 14.2 & 11.1 & 9.36 & 9.37 & 12.0 & 19.1 & 62.0 & 130 & 90.8 & 62.5 & 30.3 & 11.8 \\
\hline 85 & 19.5 & 14.3 & 11.2 & 9.39 & 9.84 & 12.7 & 23.6 & 68.6 & 136 & 93.3 & 67.2 & 31.1 & 12.4 \\
\hline 80 & 20.0 & 14.9 & 11.6 & 9.90 & 9.86 & 13.2 & 33.4 & 75.7 & 141 & 99.0 & 68.2 & 31.8 & 12.9 \\
\hline 75 & 20.6 & 15.0 & 11.7 & 9.94 & 9.89 & 13.4 & 34.8 & 88.1 & 147 & 109 & 69.2 & 32.5 & 13.4 \\
\hline 70 & 20.9 & 15.1 & 12.1 & 10.5 & 9.91 & 14.1 & 36.9 & 104 & 152 & 114 & 71.3 & 34.0 & 14.4 \\
\hline 65 & 21.7 & 15.5 & 12.1 & 10.6 & 10.5 & 14.3 & 39.5 & 113 & 156 & 118 & 73.9 & 35.1 & 16.1 \\
\hline 60 & 21.8 & 15.6 & 12.2 & 10.6 & 10.6 & 14.5 & 40.5 & 120 & 161 & 122 & 76.5 & 36.1 & 18.6 \\
\hline 55 & 22.3 & 15.7 & 12.2 & 11.0 & 10.6 & 14.7 & 41.3 & 125 & 163 & 127 & 78.8 & 38.6 & 23.0 \\
\hline 50 & 22.5 & 15.7 & 12.7 & 11.1 & 11.0 & 14.9 & 42.1 & 129 & 167 & 133 & 81.2 & 41.1 & 28.6 \\
\hline 45 & 23.4 & 16.0 & 12.8 & 11.1 & 11.1 & 15.6 & 47.1 & 133 & 169 & 137 & 82.3 & 44.8 & 34.9 \\
\hline 40 & 23.6 & 16.5 & 12.8 & 11.7 & 11.1 & 15.8 & 51.8 & 138 & 170 & 140 & 83.5 & 46.9 & 43.5 \\
\hline 35 & 24.1 & 17.0 & 12.8 & 11.7 & 11.6 & 16.0 & 53.9 & 142 & 172 & 143 & 84.7 & 47.5 & 55.6 \\
\hline 30 & 24.3 & 17.2 & 12.9 & ng & 11.6 & 16.2 & 57.5 & 145 & 176 & 147 & 86.2 & 48.3 & 69.5 \\
\hline 25 & 24.9 & 17.6 & 12.9 & $\mathrm{ng}$ & 11.7 & 16.5 & 62.3 & 150 & 179 & 151 & 89.9 & 50.4 & 80.8 \\
\hline 20 & 25.1 & 17.8 & 13.5 & ng & 11.7 & 17.9 & 67.1 & 155 & 183 & 155 & 91.3 & 52.3 & 98.2 \\
\hline 15 & 25.9 & 17.9 & 13.5 & ng & 12.2 & 19.5 & 79.4 & 160 & 190 & 158 & 98.2 & 56.0 & 125 \\
\hline 10 & 27.5 & 18.4 & ng & ng & 12.3 & 21.8 & 88.4 & 166 & 194 & 162 & 106 & 61.5 & 141 \\
\hline 5 & 29.1 & 18.5 & ng & ng & $\mathrm{ng}$ & 29.3 & 98.9 & 175 & 198 & 173 & 130 & 63.8 & 159 \\
\hline
\end{tabular}




\section{1-4.2R0-1A LANDAISIN RIVER NEAR GAWARDESH, Continued}

Probability of occurrence of annual high discharges

[ $\mathrm{m}^{3} / \mathrm{s}$, cubic meters per second; $\mathrm{ng}$, not given]

\begin{tabular}{|c|c|c|c|c|c|c|}
\hline \multirow{2}{*}{$\begin{array}{l}\text { Exceedance } \\
\text { probability }\end{array}$} & \multirow{2}{*}{$\begin{array}{c}\text { Recurrence } \\
\text { interval } \\
\text { (years) }\end{array}$} & \multirow{2}{*}{$\begin{array}{l}\text { Maximum } \\
\text { instantaneous } \\
\text { discharge } \\
\left(\mathrm{m}^{3} / \mathrm{s}\right)\end{array}$} & \multicolumn{4}{|c|}{ Maximum daily mean discharge $\left(\mathrm{m}^{3} / \mathrm{s}\right)$} \\
\hline & & & $\begin{array}{l}\text { 3-day } \\
\text { period }\end{array}$ & $\begin{array}{l}\text { 7-day } \\
\text { period }\end{array}$ & $\begin{array}{l}\text { 15-day } \\
\text { period }\end{array}$ & $\begin{array}{l}\text { 30-day } \\
\text { period }\end{array}$ \\
\hline 0.99 & 1.01 & ng & ng & ng & $\mathrm{ng}$ & ng \\
\hline 0.95 & 1.05 & ng & ng & ng & $\mathrm{ng}$ & ng \\
\hline 0.90 & 1.11 & ng & ng & ng & $\mathrm{ng}$ & ng \\
\hline 0.80 & 1.25 & ng & ng & ng & $\mathrm{ng}$ & $\mathrm{ng}$ \\
\hline 0.50 & 2 & ng & $\mathrm{ng}$ & ng & ng & ng \\
\hline 0.20 & 5 & ng & $\mathrm{ng}$ & ng & ng & ng \\
\hline 0.10 & 10 & ng & $\mathrm{ng}$ & ng & $\mathrm{ng}$ & $\mathrm{ng}$ \\
\hline 0.04 & 25 & ng & $\mathrm{ng}$ & ng & $\mathrm{ng}$ & ng \\
\hline 0.02 & 50 & ng & $\mathrm{ng}$ & ng & $\mathrm{ng}$ & $\mathrm{ng}$ \\
\hline 0.01 & 100 & ng & $\mathrm{ng}$ & $\mathrm{ng}$ & $\mathrm{ng}$ & $\mathrm{ng}$ \\
\hline 0.005 & 200 & ng & ng & ng & ng & $\mathrm{ng}$ \\
\hline 0.002 & 500 & $\mathrm{ng}$ & ng & $\mathrm{ng}$ & $\mathrm{ng}$ & $\mathrm{ng}$ \\
\hline
\end{tabular}

\section{1-4.2R0-1A LANDAISIN RIVER NEAR GAWARDESH, Continued}

Probability of occurrence of annual low discharges

[ $\mathrm{m}^{3} / \mathrm{s}$, meters per second; $\mathrm{ng}$, not given]

\begin{tabular}{|c|c|c|c|c|c|c|c|c|c|c|}
\hline \multirow{3}{*}{$\begin{array}{c}\text { Nonexceedance } \\
\text { probability }\end{array}$} & \multirow{3}{*}{$\begin{array}{c}\text { Recurrence } \\
\text { interval } \\
\text { (years) }\end{array}$} & \multicolumn{9}{|c|}{ Minimum daily mean discharge $\left(\mathrm{m}^{3} / \mathrm{s}\right)$} \\
\hline & & \multicolumn{9}{|c|}{ Number of consecutive days } \\
\hline & & 1 & 3 & 7 & 14 & 30 & 60 & 90 & 120 & 183 \\
\hline 0.05 & 20 & ng & ng & ng & ng & ng & ng & ng & ng & ng \\
\hline 0.10 & 10 & ng & ng & $\mathrm{ng}$ & ng & ng & ng & ng & ng & ng \\
\hline 0.20 & 5 & ng & ng & ng & ng & ng & ng & ng & $\mathrm{ng}$ & ng \\
\hline 0.50 & 2 & $\mathrm{ng}$ & $\mathrm{ng}$ & $\mathrm{ng}$ & $\mathrm{ng}$ & $\mathrm{ng}$ & $\mathrm{ng}$ & $\mathrm{ng}$ & ng & $\mathrm{ng}$ \\
\hline
\end{tabular}




\section{1-4.2R0-1A LANDAISIN RIVER NEAR GAWARDESH, Continued}

Probability of occurrence of seasonal low discharges [m³/s, meters per second; ng, not given]

\begin{tabular}{|c|c|c|c|c|c|c|c|c|c|}
\hline \multirow{3}{*}{$\begin{array}{c}\text { Nonexceedance } \\
\text { probability }\end{array}$} & \multirow{3}{*}{$\begin{array}{c}\text { Recurrence } \\
\text { interval } \\
\text { (years) }\end{array}$} & \multicolumn{8}{|c|}{ Minimum daily mean discharge $\left(\mathrm{m}^{3} / \mathrm{s}\right)$} \\
\hline & & \multicolumn{8}{|c|}{ Number of consecutive days } \\
\hline & & 1 & 7 & 14 & 30 & 1 & 7 & 14 & 30 \\
\hline & & \multicolumn{4}{|c|}{ December-January-February } & \multicolumn{4}{|c|}{ March-April-May } \\
\hline 0.05 & 20 & ng & ng & ng & ng & ng & $\mathrm{ng}$ & ng & ng \\
\hline 0.10 & 10 & ng & ng & ng & ng & ng & ng & ng & ng \\
\hline 0.20 & 5 & ng & ng & ng & ng & ng & ng & ng & ng \\
\hline \multirow[t]{2}{*}{0.50} & 2 & ng & ng & ng & ng & ng & ng & ng & ng \\
\hline & & \multicolumn{4}{|c|}{ June-July-August } & \multicolumn{4}{|c|}{ September-October-November } \\
\hline 0.05 & 20 & $\mathrm{ng}$ & ng & ng & ng & ng & ng & ng & ng \\
\hline 0.10 & 10 & ng & ng & ng & ng & ng & ng & ng & ng \\
\hline 0.20 & 5 & $\mathrm{ng}$ & ng & ng & ng & ng & ng & $\mathrm{ng}$ & ng \\
\hline 0.50 & 2 & $\mathrm{ng}$ & $\mathrm{ng}$ & $\mathrm{ng}$ & $\mathrm{ng}$ & $\mathrm{ng}$ & $\mathrm{ng}$ & $\mathrm{ng}$ & ng \\
\hline
\end{tabular}

\section{1-4.2R0-1A LANDAISIN RIVER NEAR GAWARDESH, Continued}

\begin{tabular}{|c|c|c|c|c|c|}
\hline \multicolumn{6}{|c|}{$\begin{array}{c}\text { Annual peak discharges } \\
\text { [m³/s, meters per second] }\end{array}$} \\
\hline \multicolumn{3}{|c|}{$\begin{array}{c}\text { Annual peak discharge, } \\
\text { by year }\end{array}$} & \multicolumn{3}{|c|}{$\begin{array}{l}\text { Annual peak discharge, } \\
\text { from highest to lowest }\end{array}$} \\
\hline $\begin{array}{l}\text { Water } \\
\text { year }\end{array}$ & Date & $\begin{array}{c}\text { Peak } \\
\text { discharge } \\
\left(\mathrm{m}^{3} / \mathrm{s}\right)\end{array}$ & $\begin{array}{l}\text { Water } \\
\text { year }\end{array}$ & Date & $\begin{array}{c}\text { Peak } \\
\text { discharge } \\
\left(\mathrm{m}^{3} / \mathrm{s}\right)\end{array}$ \\
\hline 1975 & May 15, 1975 & 342 & 1975 & May 15,1975 & 342 \\
\hline 1976 & June 4, 1976 & 222 & 1977 & June 22, 1977 & 272 \\
\hline 1977 & June 22, 1977 & 272 & 1978 & June 6, 1978 & 236 \\
\hline 1978 & June 6,1978 & 236 & 1976 & June 4, 1976 & 222 \\
\hline
\end{tabular}


1-4.2R0-1A LANDAISIN RIVER NEAR GAWARDESH, Continued

Monthly and annual mean discharges, in cubic meters per second

[Data may not be rounded in accordance with U.S. Geological Survey publication standards; --, no data]

\begin{tabular}{|c|c|c|c|c|c|c|c|c|c|c|c|c|c|}
\hline \multirow{2}{*}{$\begin{array}{c}\text { Water } \\
\text { year }\end{array}$} & \multicolumn{12}{|c|}{ Monthly mean discharge } & \multirow{2}{*}{$\begin{array}{c}\text { Annual } \\
\text { discharge }\end{array}$} \\
\hline & October & November & December & January & February & March & April & May & June & July & August & September & \\
\hline 1975 & -- & -- & -- & -- & -- & -- & -- & 169 & 169 & 130 & 99.0 & 47.2 & -- \\
\hline 1976 & 23.6 & 16.3 & 12.8 & 12.1 & 11.9 & 14.1 & 53.4 & 120 & 154 & 144 & 78.5 & 46.2 & 57.4 \\
\hline 1977 & 23.5 & 16.4 & 13.0 & 11.1 & 10.4 & 19.7 & 44.4 & 80.0 & 150 & 112 & 69.9 & 35.5 & 49.0 \\
\hline 1978 & 21.8 & 15.6 & 11.4 & 9.69 & 10.3 & 15.4 & 51.6 & 125 & 175 & -- & -- & -- & -- \\
\hline
\end{tabular}




\section{1-4.L00-1A KONAR RIVER AT PUL-I-KAMA}

\section{(U.S. Geological Survey identification number: 342800070330000)}

LOCATION: Lat $34^{\circ} 28^{\prime} \mathrm{N}$., long $70^{\circ} 33^{\prime} \mathrm{E}$.

DRAINAGE AREA: $26,005 \mathrm{~km}^{2}$.

ELEVATION: 555 meters above mean sea level.

PERIOD OF RECORD: December 28, 1966 to September 30, 1979.

GAGE: Water-stage recorder.

Annual mean discharge

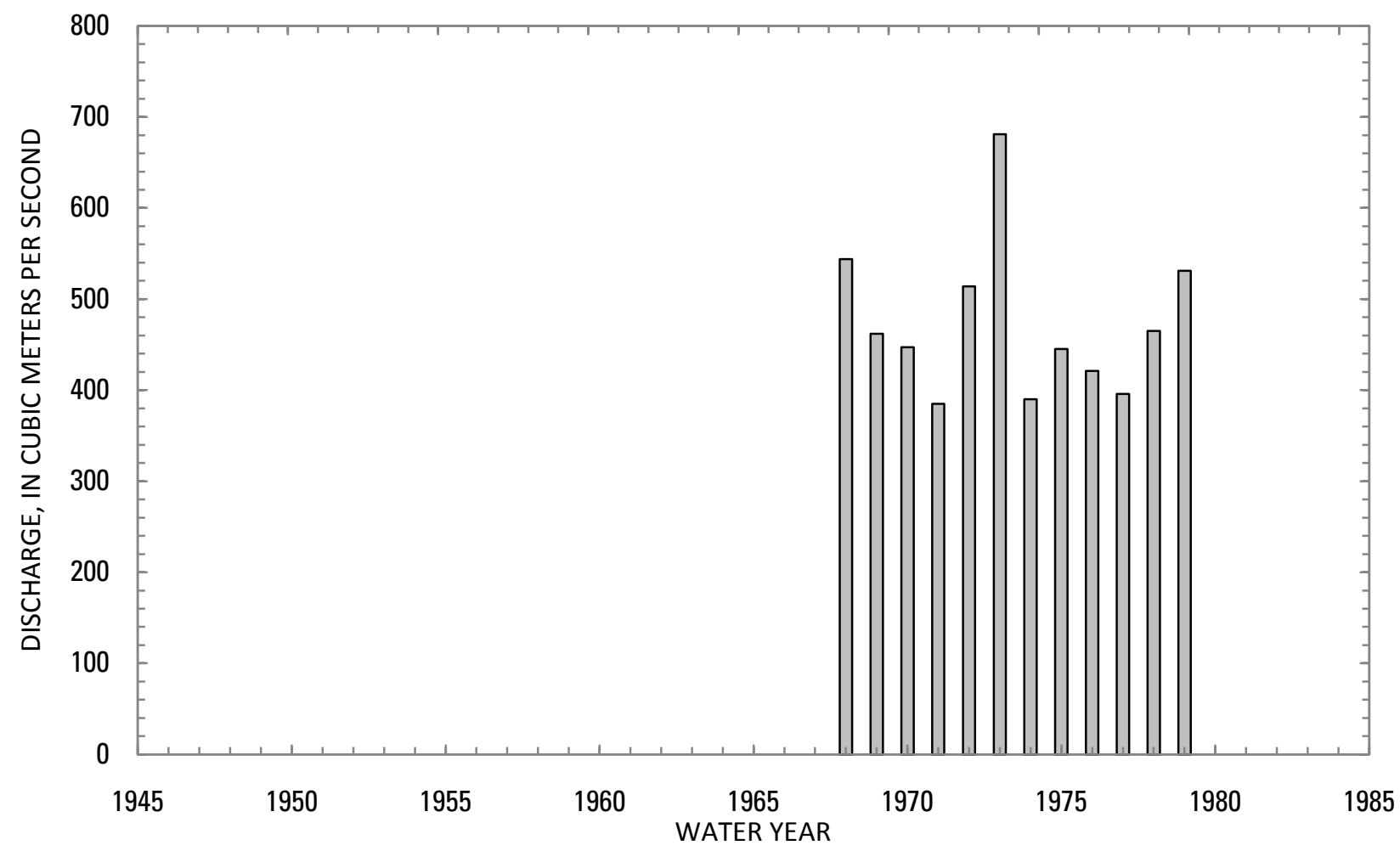




\section{1-4.L00-1A KONAR RIVER AT PUL-I-KAMA, Continued}

Statistics of monthly and annual mean discharges [ $\mathrm{m}^{3} / \mathrm{s}$, cubic meters per second]

\begin{tabular}{|c|c|c|c|c|c|c|c|c|}
\hline \multirow[b]{2}{*}{ Month } & \multicolumn{2}{|c|}{ Maximum } & \multicolumn{2}{|c|}{ Minimum } & \multicolumn{4}{|c|}{ Mean } \\
\hline & $\begin{array}{c}\text { Discharge } \\
\left(\mathrm{m}^{3} / \mathrm{s}\right)\end{array}$ & $\begin{array}{l}\text { Water year } \\
\text { of } \\
\text { occurrence }\end{array}$ & $\begin{array}{c}\text { Discharge } \\
\left(\mathrm{m}^{3} / \mathrm{s}\right)\end{array}$ & $\begin{array}{c}\text { Water year } \\
\text { of } \\
\text { occurrence }\end{array}$ & $\begin{array}{c}\text { Discharge } \\
\left(\mathrm{m}^{3} / \mathrm{s}\right)\end{array}$ & $\begin{array}{c}\text { Standard } \\
\text { deviation } \\
\left(\mathrm{m}^{3} / \mathrm{s}\right)\end{array}$ & $\begin{array}{c}\text { Coefficient } \\
\text { of } \\
\text { variation }\end{array}$ & $\begin{array}{c}\text { Percentage } \\
\text { of annual } \\
\text { discharge }\end{array}$ \\
\hline October & 249 & 1979 & 177 & 1975 & 223 & 22.3 & 0.10 & 3.92 \\
\hline November & 195 & 1972 & 113 & 1975 & 164 & 26.77 & 0.16 & 2.88 \\
\hline December & 173 & 1970 & 88.2 & 1975 & 131 & 22.3 & 0.17 & 2.30 \\
\hline January & 147 & 1970 & 74.8 & 1975 & 109 & 16.4 & 0.15 & 1.92 \\
\hline February & 131 & 1970 & 60.2 & 1975 & 103 & 18.1 & 0.18 & 1.81 \\
\hline March & 205 & 1969 & 65.0 & 1975 & 136 & 38.6 & 0.28 & 2.40 \\
\hline April & 776 & 1973 & 223 & 1971 & 378 & 146 & 0.39 & 6.64 \\
\hline May & 1,380 & 1973 & 408 & 1977 & 657 & 236 & 0.36 & 11.6 \\
\hline June & 1,360 & 1968 & 800 & 1970 & 1,055 & 204 & 0.19 & 18.5 \\
\hline July & 1,690 & 1973 & 883 & 1970 & 1,243 & 246 & 0.20 & 21.8 \\
\hline August & 1,280 & 1973 & 786 & 1974 & 1,010 & 143 & 0.14 & 17.7 \\
\hline September & 690 & 1973 & 335 & 1977 & 481 & 111 & 0.23 & 8.46 \\
\hline Annual & 681 & 1973 & 385 & 1971 & 473 & 84.4 & 0.18 & 100 \\
\hline
\end{tabular}


1-4.L00-1A KONAR RIVER AT PUL-I-KAMA, Continued

Monthly and annual flow duration, in cubic meters per second

\begin{tabular}{|c|c|c|c|c|c|c|c|c|c|c|c|c|c|}
\hline \multirow{2}{*}{$\begin{array}{l}\text { Percentage } \\
\text { of days } \\
\text { discharge } \\
\text { equaled or } \\
\text { exceeded }\end{array}$} & \multicolumn{12}{|c|}{ Month } & \multirow{2}{*}{ Annua } \\
\hline & October & November & December & January & February & March & April & May & June & July & August & September & \\
\hline 95 & 158 & 115 & 90.3 & 76.1 & 65.9 & 70.1 & 151 & 295 & 627 & 767 & 628 & 257 & 89.6 \\
\hline 90 & 176 & 123 & 105 & 88.4 & 82.0 & 82.2 & 171 & 355 & 681 & 898 & 659 & 278 & 102 \\
\hline 85 & 186 & 130 & 109 & 89.8 & 90.5 & 92.4 & 180 & 398 & 719 & 967 & 701 & 302 & 110 \\
\hline 80 & 193 & 137 & 110 & 99.2 & 92.0 & 101 & 193 & 433 & 786 & 1,020 & 755 & 321 & 119 \\
\hline 75 & 198 & 143 & 112 & 100 & 93.5 & 105 & 212 & 464 & 833 & 1,060 & 814 & 346 & 130 \\
\hline 70 & 202 & 149 & 115 & 101 & 95.6 & 109 & 232 & 489 & 874 & 1,100 & 870 & 375 & 144 \\
\hline 65 & 205 & 153 & 120 & 106 & 97.6 & 112 & 245 & 515 & 902 & 1,120 & 918 & 400 & 161 \\
\hline 60 & 209 & 158 & 124 & 107 & 98.6 & 116 & 258 & 547 & 929 & 1,150 & 966 & 418 & 184 \\
\hline 55 & 213 & 160 & 129 & 108 & 100 & 119 & 280 & 574 & 978 & 1,170 & 1,010 & 439 & 209 \\
\hline 50 & 218 & 166 & 132 & 109 & 101 & 123 & 308 & 604 & 1,010 & 1,200 & 1,030 & 458 & 243 \\
\hline 45 & 222 & 172 & 134 & 110 & 103 & 128 & 329 & 637 & 1,050 & 1,230 & 1,060 & 472 & 304 \\
\hline 40 & 226 & 175 & 137 & 111 & 106 & 133 & 360 & 667 & 1,090 & 1,260 & 1,080 & 488 & 413 \\
\hline 35 & 230 & 181 & 139 & 114 & 108 & 138 & 391 & 696 & 1,130 & 1,310 & 1,100 & 507 & 517 \\
\hline 30 & 237 & 185 & 141 & 116 & 110 & 143 & 426 & 726 & 1,180 & 1,350 & 1,120 & 536 & 643 \\
\hline 25 & 244 & 190 & 148 & 119 & 113 & 150 & 477 & 759 & 1,240 & 1,410 & 1,160 & 570 & 753 \\
\hline 20 & 251 & 193 & 151 & 122 & 117 & 160 & 535 & 805 & 1,340 & 1,470 & 1,200 & 638 & 907 \\
\hline 15 & 262 & 197 & 156 & 128 & 126 & 174 & 622 & 891 & 1,420 & 1,590 & 1,270 & 686 & 1,040 \\
\hline 10 & 279 & 202 & 163 & 132 & 131 & 206 & 734 & 1,010 & 1,520 & 1,670 & 1,330 & 730 & 1,170 \\
\hline 5 & 307 & 207 & 178 & 146 & 135 & 246 & 867 & 1,380 & 1,660 & 1,800 & 1,460 & 789 & 1,360 \\
\hline
\end{tabular}




\section{1-4.L00-1A KONAR RIVER AT PUL-I-KAMA, Continued}

Probability of occurrence of annual high discharges

[ $\mathrm{m}^{3} / \mathrm{s}$, cubic meters per second; $\mathrm{ng}$, not given]

\begin{tabular}{|c|c|c|c|c|c|c|}
\hline \multirow{2}{*}{$\begin{array}{c}\text { Exceedance } \\
\text { probability }\end{array}$} & \multirow{2}{*}{$\begin{array}{l}\text { Recurrence } \\
\text { interval } \\
\text { (years) }\end{array}$} & \multirow{2}{*}{$\begin{array}{l}\text { Maximum } \\
\text { instantaneous } \\
\text { discharge } \\
\left(\mathrm{m}^{3} / \mathrm{s}\right)\end{array}$} & \multicolumn{4}{|c|}{ Maximum daily mean discharge $\left(\mathrm{m}^{3} / \mathrm{s}\right)$} \\
\hline & & & $\begin{array}{l}\text { 3-day } \\
\text { period }\end{array}$ & $\begin{array}{l}\text { 7-day } \\
\text { period }\end{array}$ & $\begin{array}{l}\text { 15-day } \\
\text { period }\end{array}$ & $\begin{array}{l}\text { 30-day } \\
\text { period }\end{array}$ \\
\hline 0.99 & 1.01 & 1,110 & 1,000 & 984 & 949 & 919 \\
\hline 0.95 & 1.05 & 1,240 & 1,110 & 1,080 & 1,030 & 982 \\
\hline 0.90 & 1.11 & 1,320 & 1,180 & 1,140 & 1,080 & 1,020 \\
\hline 0.80 & 1.25 & 1,430 & 1,270 & 1,230 & 1,150 & 1,090 \\
\hline 0.50 & 2 & 1,680 & 1,500 & 1,430 & 1,330 & 1,240 \\
\hline 0.20 & 5 & 2,020 & 1,820 & 1,720 & 1,590 & 1,470 \\
\hline 0.10 & 10 & 2,240 & 2,040 & 1,910 & 1,760 & 1,630 \\
\hline 0.04 & 25 & 2,510 & 2,310 & 2,160 & 1,990 & 1,830 \\
\hline 0.02 & 50 & 2,710 & 2,520 & 2,350 & 2,160 & 1,990 \\
\hline 0.01 & 100 & 2,910 & 2,730 & 2,540 & 2,340 & 2,160 \\
\hline 0.005 & 200 & 3,110 & 2,950 & 2,730 & 2,520 & 2,330 \\
\hline 0.002 & 500 & 3,380 & $\mathrm{ng}$ & $\mathrm{ng}$ & ng & $\mathrm{ng}$ \\
\hline
\end{tabular}

\section{1-4.L00-1A KONAR RIVER AT PUL-I-KAMA, Continued}

Probability of occurrence of annual low discharges

[m $\mathrm{m}^{3} / \mathrm{s}$, meters per second]

\begin{tabular}{|c|c|c|c|c|c|c|c|c|c|c|}
\hline \multirow{3}{*}{$\begin{array}{c}\text { Nonexceedance } \\
\text { probability }\end{array}$} & \multirow{3}{*}{$\begin{array}{c}\text { Recurrence } \\
\text { interval } \\
\text { (years) }\end{array}$} & \multicolumn{9}{|c|}{ Minimum daily mean discharge $\left(\mathrm{m}^{3} / \mathrm{s}\right)$} \\
\hline & & \multicolumn{9}{|c|}{ Number of consecutive days } \\
\hline & & 1 & 3 & 7 & 14 & 30 & 60 & 90 & 120 & 183 \\
\hline 0.05 & 20 & 51.8 & 52.0 & 55.6 & 58.4 & 63.9 & 68.8 & 74.6 & 80.6 & 106 \\
\hline 0.10 & 10 & 59.6 & 60.5 & 64.6 & 67.3 & 72.7 & 77.7 & 83.4 & 89.9 & 117 \\
\hline 0.20 & 5 & 69.3 & 70.9 & 75.4 & 78.1 & 83.2 & 88.3 & 93.8 & 101 & 129 \\
\hline 0.50 & 2 & 87.4 & 90.3 & 94.4 & 97.0 & 101 & 106 & 111 & 119 & 149 \\
\hline
\end{tabular}




\section{1-4.L00-1A KONAR RIVER AT PUL-I-KAMA, Continued}

Probability of occurrence of seasonal low discharges [ $\mathrm{m}^{3} / \mathrm{s}$, meters per second]

\begin{tabular}{|c|c|c|c|c|c|c|c|c|c|}
\hline \multirow{3}{*}{$\begin{array}{c}\text { Nonexceedance } \\
\text { probability }\end{array}$} & \multirow{3}{*}{$\begin{array}{c}\text { Recurrence } \\
\text { interval } \\
\text { (years) }\end{array}$} & \multicolumn{8}{|c|}{ Minimum daily mean discharge $\left(\mathrm{m}^{3} / \mathrm{s}\right)$} \\
\hline & & \multicolumn{8}{|c|}{ Number of consecutive days } \\
\hline & & 1 & 7 & 14 & 30 & 1 & 7 & 14 & 30 \\
\hline & & \multicolumn{4}{|c|}{ December-January-February } & \multicolumn{4}{|c|}{ March-April-May } \\
\hline 0.05 & 20 & 51.7 & 59.0 & 63.6 & 68.6 & 58.3 & 60.3 & 65.5 & 75.5 \\
\hline 0.10 & 10 & 60.0 & 67.7 & 72.2 & 76.6 & 67.9 & 70.9 & 76.2 & 87.3 \\
\hline 0.20 & 5 & 70.3 & 78.1 & 82.3 & 86.1 & 79.4 & 83.5 & 89.2 & 103 \\
\hline \multirow[t]{2}{*}{0.50} & 2 & 89.5 & 95.9 & 99.4 & 102 & 99.3 & 105 & 112 & 134 \\
\hline & & \multicolumn{4}{|c|}{ June-July-August } & \multicolumn{4}{|c|}{ September-October-November } \\
\hline 0.05 & 20 & 465 & 515 & 606 & 741 & 93.7 & 102 & 106 & 117 \\
\hline 0.10 & 10 & 491 & 549 & 640 & 786 & 102 & 113 & 117 & 128 \\
\hline 0.20 & 5 & 523 & 591 & 684 & 844 & 114 & 126 & 131 & 141 \\
\hline 0.50 & 2 & 589 & 672 & 781 & 963 & 136 & 150 & 155 & 166 \\
\hline
\end{tabular}

\section{1-4.L00-1A KONAR RIVER AT PUL-I-KAMA, Continued}

Annual peak discharges

$\left[\mathrm{m}^{3} / \mathrm{s}\right.$, meters per second]

\begin{tabular}{|c|c|c|c|c|c|}
\hline \multicolumn{3}{|c|}{$\begin{array}{c}\text { Annual peak discharge, } \\
\text { by year }\end{array}$} & \multicolumn{3}{|c|}{$\begin{array}{l}\text { Annual peak discharge, } \\
\text { from highest to lowest }\end{array}$} \\
\hline $\begin{array}{l}\text { Water } \\
\text { year }\end{array}$ & Date & $\begin{array}{c}\text { Peak } \\
\text { discharge } \\
\left(\mathrm{m}^{3} / \mathrm{s}\right)\end{array}$ & $\begin{array}{l}\text { Water } \\
\text { year }\end{array}$ & Date & $\begin{array}{c}\text { Peak } \\
\text { discharge } \\
\left(\mathrm{m}^{3} / \mathrm{s}\right)\end{array}$ \\
\hline 1967 & July 24, 1967 & 1,706 & 1973 & July 18,1973 & 2,350 \\
\hline 1968 & July 9, 1968 & 1,800 & 1975 & May 16, 1975 & 2,342 \\
\hline 1969 & July 24, 1969 & 1,325 & 1978 & July 8, 1978 & 2,200 \\
\hline 1970 & July 17, 1970 & 1,490 & 1979 & July 7, 1979 & 1,900 \\
\hline 1971 & July 29, 1971 & 1,312 & 1968 & July 9, 1968 & 1,800 \\
\hline 1972 & June 19, 1972 & 1,700 & 1967 & July 24, 1967 & 1,706 \\
\hline 1973 & July 18,1973 & 2,350 & 1972 & June 19,1972 & 1,700 \\
\hline 1974 & July 17,1974 & 1,680 & 1974 & July 17, 1974 & 1,680 \\
\hline 1975 & May 16,1975 & 2,342 & 1970 & July 17, 1970 & 1,490 \\
\hline 1976 & July 10, 1976 & 1,382 & 1977 & June 25, 1977 & 1,414 \\
\hline 1977 & June 25, 1977 & 1,414 & 1976 & July 10, 1976 & 1,382 \\
\hline 1978 & July 8, 1978 & 2,200 & 1969 & July 24, 1969 & 1,325 \\
\hline 1979 & July 7, 1979 & 1,900 & 1971 & July 29, 1971 & 1,312 \\
\hline
\end{tabular}




\section{1-4.L00-1A KONAR RIVER AT PUL-I-KAMA, Continued}

Monthly and annual mean discharges, in cubic meters per second

[Data may not be rounded in accordance with U.S. Geological Survey publication standards; --, no data]

\begin{tabular}{|c|c|c|c|c|c|c|c|c|c|c|c|c|c|}
\hline \multirow{2}{*}{$\begin{array}{l}\text { Water } \\
\text { year }\end{array}$} & \multicolumn{12}{|c|}{ Monthly mean discharge } & \multirow{2}{*}{$\begin{array}{c}\text { Annual } \\
\text { discharge }\end{array}$} \\
\hline & October & November & December & January & February & March & April & May & June & July & August & September & \\
\hline 1967 & -- & -- & -- & 111 & 109 & 134 & 351 & 587 & 1,170 & 1,460 & 1,100 & 603 & -- \\
\hline 1968 & 233 & 189 & 140 & 115 & 127 & 176 & 493 & 745 & 1,360 & 1,380 & 1,090 & 457 & 544 \\
\hline 1969 & 235 & 164 & 131 & 102 & 93.8 & 205 & 408 & 535 & 1,030 & 1,140 & 980 & 494 & 462 \\
\hline 1970 & 246 & 189 & 173 & 147 & 131 & 150 & 345 & 611 & 800 & 883 & 1,070 & 588 & 447 \\
\hline 1971 & 203 & 123 & 110 & 99.7 & 93.8 & 115 & 223 & 539 & 930 & 923 & 839 & 402 & 385 \\
\hline 1972 & 213 & 195 & 147 & 108 & 96.9 & 129 & 247 & 630 & 1,340 & 1,320 & 1,110 & 615 & 514 \\
\hline 1973 & 241 & 158 & 138 & 114 & 116 & 198 & 776 & 1,380 & 1,350 & 1,690 & 1,280 & 690 & 681 \\
\hline 1974 & 244 & 167 & 127 & 95.2 & 88.6 & 104 & 248 & 471 & 894 & 1,090 & 786 & 337 & 390 \\
\hline 1975 & 177 & 113 & 88.2 & 74.8 & 60.2 & 65 & 343 & 717 & 1,120 & 1,080 & 1,050 & 424 & 445 \\
\hline 1976 & 219 & 183 & 142 & 118 & 112 & 119 & 357 & 632 & 829 & 1,110 & 794 & 420 & 421 \\
\hline 1977 & 217 & 151 & 120 & 109 & 100 & 123 & 265 & 408 & 835 & 1,160 & 908 & 335 & 396 \\
\hline 1978 & 199 & 147 & 109 & 106 & 104 & 115 & 377 & 612 & 953 & 1,310 & 1,040 & 472 & 465 \\
\hline 1979 & 249 & 187 & 148 & 121 & 106 & 141 & 481 & 679 & 1,100 & 1,610 & 1,080 & 422 & 531 \\
\hline
\end{tabular}




\section{1-4.LOO-2T KONAR RIVER NEAR KONARI \\ (U.S. Geological Survey identification number: 343800070490000 )}

LOCATION: Lat $34^{\circ} 38^{\prime} \mathrm{N}$., long $70^{\circ} 49^{\prime} \mathrm{E}$.

DRAINAGE AREA: $24,895 \mathrm{~km}^{2}$.

ELEVATION: 665 meters above mean sea level.

PERIOD OF RECORD: October 1, 1959 to September 30, 1967.

GAGE: Water-stage recorder.

Annual mean discharge

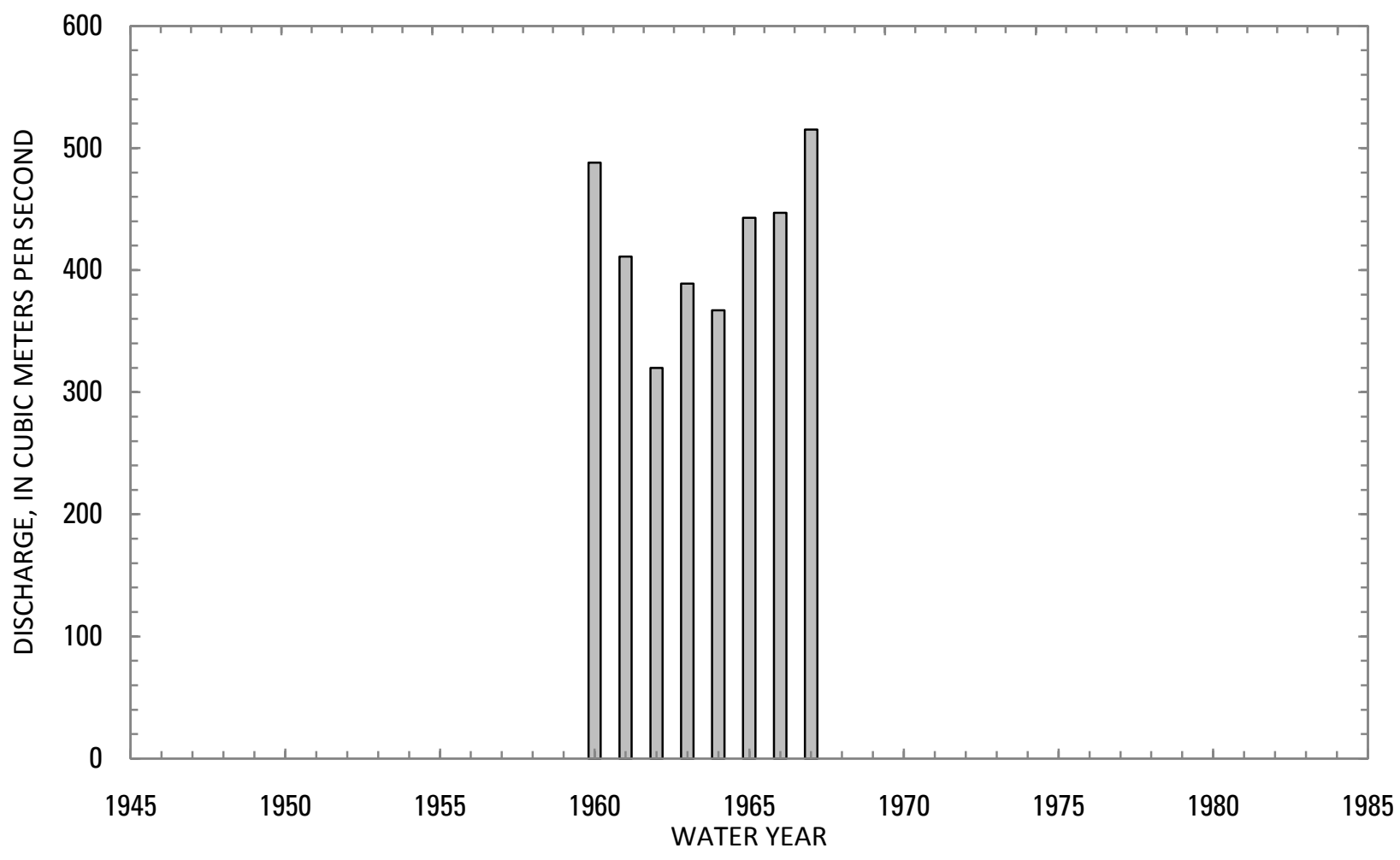




\section{1-4.LO0-2T KONAR RIVER NEAR KONARI, Continued}

Statistics of monthly and annual mean discharges [ $\mathrm{m}^{3} / \mathrm{s}$, cubic meters per second]

\begin{tabular}{|c|c|c|c|c|c|c|c|c|}
\hline \multirow[b]{2}{*}{ Month } & \multicolumn{2}{|c|}{ Maximum } & \multicolumn{2}{|c|}{ Minimum } & \multicolumn{4}{|c|}{ Mean } \\
\hline & $\begin{array}{c}\text { Discharge } \\
\left(\mathrm{m}^{3} / \mathrm{s}\right)\end{array}$ & $\begin{array}{c}\text { Water year } \\
\text { of } \\
\text { occurrence }\end{array}$ & $\begin{array}{c}\text { Discharge } \\
\left(\mathrm{m}^{3} / \mathrm{s}\right)\end{array}$ & $\begin{array}{c}\text { Water year } \\
\text { of } \\
\text { occurrence }\end{array}$ & $\begin{array}{c}\text { Discharge } \\
\left(\mathrm{m}^{3} / \mathrm{s}\right)\end{array}$ & $\begin{array}{c}\text { Standard } \\
\text { deviation } \\
\left(\mathrm{m}^{3} / \mathrm{s}\right)\end{array}$ & $\begin{array}{c}\text { Coefficient } \\
\text { of } \\
\text { variation }\end{array}$ & $\begin{array}{c}\text { Percentage } \\
\text { of annual } \\
\text { discharge }\end{array}$ \\
\hline October & 293 & 1960 & 175 & 1966 & 222 & 44.6 & 0.20 & 4.39 \\
\hline November & 227 & 1960 & 133 & 1965 & 159 & 32.1 & 0.20 & 3.15 \\
\hline December & 203 & 1960 & 116 & 1966 & 139 & 27.8 & 0.20 & 2.75 \\
\hline January & 181 & 1960 & 103 & 1966 & 128 & 23.8 & 0.19 & 2.53 \\
\hline February & 185 & 1960 & 100 & 1966 & 126 & 25.6 & 0.20 & 2.50 \\
\hline March & 227 & 1960 & 122 & 1962 & 153 & 35.7 & 0.23 & 3.04 \\
\hline April & 440 & 1965 & 197 & 1962 & 331 & 81.4 & 0.25 & 6.54 \\
\hline May & 783 & 1965 & 289 & 1962 & 547 & 139 & 0.25 & 10.8 \\
\hline June & 1,270 & 1967 & 597 & 1962 & 918 & 219 & 0.24 & 18.2 \\
\hline July & 1,400 & 1967 & 745 & 1962 & 1,030 & 203 & 0.20 & 20.5 \\
\hline August & 1,090 & 1967 & 672 & 1961 & 822 & 147 & 0.18 & 16.3 \\
\hline September & 589 & 1961 & 393 & 1965 & 472 & 74.1 & 0.16 & 9.35 \\
\hline Annual & 515 & 1967 & 320 & 1962 & 423 & 64.0 & 0.15 & 100 \\
\hline
\end{tabular}


1-4.L00-2T KONAR RIVER NEAR KONARI, Continued

Monthly and annual flow duration, in cubic meters per second

\begin{tabular}{|c|c|c|c|c|c|c|c|c|c|c|c|c|c|}
\hline \multirow{2}{*}{$\begin{array}{l}\text { Percentage } \\
\text { of days } \\
\text { discharge } \\
\text { equaled or } \\
\text { exceeded }\end{array}$} & \multicolumn{12}{|c|}{ Month } & \multirow{2}{*}{ Annua } \\
\hline & October & November & December & January & February & March & April & May & June & July & August & September & \\
\hline 95 & 152 & 130 & 114 & 101 & 98.2 & 116 & 154 & 251 & 499 & 696 & 563 & 278 & 115 \\
\hline 90 & 157 & 131 & 118 & 109 & 102 & 117 & 162 & 334 & 607 & 760 & 613 & 296 & 119 \\
\hline 85 & 161 & 133 & 121 & 113 & 112 & 118 & 174 & 403 & 677 & 795 & 628 & 321 & 124 \\
\hline 80 & 166 & 134 & 124 & 116 & 114 & 120 & 191 & 428 & 708 & 840 & 646 & 350 & 130 \\
\hline 75 & 172 & 136 & 125 & 117 & 116 & 122 & 215 & 450 & 732 & 887 & 666 & 363 & 136 \\
\hline 70 & 181 & 138 & 126 & 118 & 117 & 125 & 238 & 471 & 757 & 916 & 697 & 377 & 145 \\
\hline 65 & 191 & 140 & 127 & 119 & 118 & 129 & 254 & 492 & 788 & 949 & 721 & 395 & 159 \\
\hline 60 & 198 & 142 & 128 & 120 & 119 & 132 & 264 & 508 & 830 & 974 & 738 & 423 & 184 \\
\hline 55 & 204 & 145 & 129 & 121 & 120 & 135 & 283 & 521 & 869 & 996 & 769 & 444 & 209 \\
\hline 50 & 209 & 148 & 130 & 122 & 120 & 137 & 294 & 533 & 901 & 1,020 & 789 & 462 & 246 \\
\hline 45 & 214 & 151 & 131 & 122 & 121 & 141 & 310 & 553 & 929 & 1,040 & 806 & 488 & 303 \\
\hline 40 & 223 & 153 & 133 & 123 & 121 & 144 & 330 & 572 & 958 & 1,070 & 836 & 509 & 398 \\
\hline 35 & 229 & 158 & 135 & 124 & 122 & 146 & 351 & 591 & 990 & 1,100 & 866 & 525 & 494 \\
\hline 30 & 237 & 164 & 138 & 126 & 123 & 151 & 372 & 612 & 1,020 & 1,140 & 900 & 538 & 571 \\
\hline 25 & 251 & 174 & 143 & 129 & 130 & 158 & 406 & 639 & 1,050 & 1,180 & 944 & 562 & 659 \\
\hline 20 & 264 & 186 & 150 & 138 & 133 & 185 & 447 & 682 & 1,100 & 1,230 & 990 & 603 & 748 \\
\hline 15 & 277 & 194 & 157 & 142 & 136 & 219 & 493 & 723 & 1,150 & 1,300 & 1060 & 632 & 850 \\
\hline 10 & 300 & 213 & 198 & 175 & 180 & 228 & 544 & 768 & 1,370 & 1,330 & 1,120 & 668 & 978 \\
\hline 5 & 364 & 229 & 206 & 184 & 188 & 250 & 621 & 823 & 1,490 & 1,420 & 1,190 & 722 & 1,130 \\
\hline
\end{tabular}




\section{1-4.L00-2T KONAR RIVER NEAR KONARI, Continued}

Probability of occurrence of annual high discharges [ $\mathrm{m}^{3} / \mathrm{s}$, cubic meters per second; ng, not given]

\begin{tabular}{|c|c|c|c|c|c|c|}
\hline \multirow{2}{*}{$\begin{array}{c}\text { Exceedance } \\
\text { probability }\end{array}$} & \multirow{2}{*}{$\begin{array}{c}\text { Recurrence } \\
\text { interval } \\
\text { (years) }\end{array}$} & \multirow{2}{*}{$\begin{array}{l}\text { Maximum } \\
\text { instantaneous } \\
\text { discharge } \\
\left(\mathrm{m}^{3} / \mathrm{s}\right)\end{array}$} & \multicolumn{4}{|c|}{ Maximum daily mean discharge $\left(\mathrm{m}^{3} / \mathrm{s}\right)$} \\
\hline & & & $\begin{array}{l}\text { 3-day } \\
\text { period }\end{array}$ & $\begin{array}{l}\text { 7-day } \\
\text { period }\end{array}$ & $\begin{array}{l}\text { 15-day } \\
\text { period }\end{array}$ & $\begin{array}{l}\text { 30-day } \\
\text { period }\end{array}$ \\
\hline 0.99 & 1.01 & 836 & 679 & 677 & ${ }^{2} 657$ & 672 \\
\hline 0.95 & 1.05 & 975 & 822 & 805 & 783 & 772 \\
\hline 0.90 & 1.11 & 1,060 & 905 & 880 & 855 & 830 \\
\hline 0.80 & 1.25 & 1,160 & 1,010 & 976 & 948 & 905 \\
\hline 0.50 & 2 & 1,380 & 1,230 & 1,180 & 1,140 & 1,070 \\
\hline 0.20 & 5 & 1,620 & 1,460 & 1,400 & 1,350 & 1,250 \\
\hline 0.10 & 10 & 1,760 & 1,580 & 1,520 & 1,460 & 1,350 \\
\hline 0.04 & 25 & 1,910 & 1,710 & 1,660 & 1,580 & 1,480 \\
\hline 0.02 & 50 & 2,010 & 1,800 & 1,750 & 1,660 & 1,560 \\
\hline 0.01 & 100 & 2,110 & 1,880 & 1,840 & 1,740 & 1,640 \\
\hline 0.005 & 200 & 2,200 & 1,950 & 1,910 & 1,810 & 1,710 \\
\hline 0.002 & 500 & 2,310 & ng & ng & ng & ng \\
\hline
\end{tabular}

\section{1-4.L00-2T KONAR RIVER NEAR KONARI, Continued}

Probability of occurrence of annual low discharges

$\left[\mathrm{m}^{3} / \mathrm{s}\right.$, meters per second]

\begin{tabular}{|c|c|c|c|c|c|c|c|c|c|c|}
\hline \multirow{3}{*}{$\begin{array}{c}\text { Nonexceedance } \\
\text { probability }\end{array}$} & \multirow{3}{*}{$\begin{array}{c}\text { Recurrence } \\
\text { interval } \\
\text { (years) }\end{array}$} & \multicolumn{9}{|c|}{ Minimum daily mean discharge $\left(\mathrm{m}^{3} / \mathrm{s}\right)$} \\
\hline & & \multicolumn{9}{|c|}{ Number of consecutive days } \\
\hline & & 1 & 3 & 7 & 14 & 30 & 60 & 90 & 120 & 183 \\
\hline 0.05 & 20 & 95.4 & 96 & 97.3 & 97.9 & 99.2 & 101 & 106 & 111 & 131 \\
\hline 0.10 & 10 & 101 & 101 & 102 & 102 & 104 & 105 & 109 & 114 & 133 \\
\hline 0.20 & 5 & 106 & 107 & 107 & 108 & 109 & 110 & 113 & 117 & 136 \\
\hline 0.50 & 2 & 116 & 117 & 117 & 117 & 118 & 119 & 120 & 123 & 144 \\
\hline
\end{tabular}




\section{1-4.L00-2T KONAR RIVER NEAR KONARI, Continued}

Probability of occurrence of seasonal low discharges [ $\mathrm{m}^{3} / \mathrm{s}$, meters per second]

\begin{tabular}{|c|c|c|c|c|c|c|c|c|c|}
\hline \multirow{3}{*}{$\begin{array}{c}\text { Nonexceedance } \\
\text { probability }\end{array}$} & \multirow{3}{*}{$\begin{array}{c}\text { Recurrence } \\
\text { interval } \\
\text { (years) }\end{array}$} & \multicolumn{8}{|c|}{ Minimum daily mean discharge $\left(\mathrm{m}^{3} / \mathrm{s}\right)$} \\
\hline & & \multicolumn{8}{|c|}{ Number of consecutive days } \\
\hline & & 1 & 7 & 14 & 30 & 1 & 7 & 14 & 30 \\
\hline & & \multicolumn{4}{|c|}{ December-January-February } & \multicolumn{4}{|c|}{ March-April-May } \\
\hline 0.05 & 20 & 97.8 & 99.6 & 100 & 101 & 104 & 111 & 113 & 115 \\
\hline 0.10 & 10 & 100 & 102 & 102 & 103 & 105 & 111 & 114 & 119 \\
\hline 0.20 & 5 & 105 & 106 & 106 & 107 & 108 & 113 & 115 & 124 \\
\hline \multirow[t]{2}{*}{0.50} & 2 & 117 & 117 & 118 & 118 & 117 & 121 & 124 & 142 \\
\hline & & \multicolumn{4}{|c|}{ June-July-August } & \multicolumn{4}{|c|}{ September-0ctober-November } \\
\hline 0.05 & 20 & 304 & 361 & 482 & 585 & 102 & 124 & 125 & 129 \\
\hline 0.10 & 10 & 361 & 410 & 515 & 617 & 110 & 125 & 127 & 131 \\
\hline 0.20 & 5 & 430 & 472 & 560 & 662 & 119 & 128 & 130 & 135 \\
\hline 0.50 & 2 & 549 & 595 & 664 & 767 & 136 & 136 & 138 & 145 \\
\hline
\end{tabular}

\section{1-4.LO0-2T KONAR RIVER NEAR KONARI, Continued}

Annual peak discharges

$\left[\mathrm{m}^{3} / \mathrm{s}\right.$, meters per second]

\begin{tabular}{|c|c|c|c|c|c|}
\hline \multicolumn{3}{|c|}{$\begin{array}{c}\text { Annual peak discharge, } \\
\text { by year }\end{array}$} & \multicolumn{3}{|c|}{$\begin{array}{l}\text { Annual peak discharge, } \\
\text { from highest to lowest }\end{array}$} \\
\hline $\begin{array}{l}\text { Water } \\
\text { year }\end{array}$ & Date & $\begin{array}{c}\text { Peak } \\
\text { discharge } \\
\left(\mathrm{m}^{3} / \mathrm{s}\right)\end{array}$ & $\begin{array}{l}\text { Water } \\
\text { year }\end{array}$ & Date & $\begin{array}{c}\text { Peak } \\
\text { discharge } \\
\left(\mathrm{m}^{3} / \mathrm{s}\right)\end{array}$ \\
\hline 1960 & July 11,1960 & 1,490 & 1967 & June 12, 1967 & 1,820 \\
\hline 1961 & July 16, 1961 & 1,410 & 1966 & June 20, 1966 & 1,670 \\
\hline 1962 & July 22, 1962 & 998 & 1960 & July 11, 1960 & 1,490 \\
\hline 1963 & June 28, 1963 & 1,110 & 1961 & July 16, 1961 & 1,410 \\
\hline 1964 & July 29, 1964 & 1,280 & 1965 & May 22, 1965 & 1,348 \\
\hline 1965 & May 22, 1965 & 1,348 & 1964 & July 29, 1964 & 1,280 \\
\hline 1966 & June 20, 1966 & 1,670 & 1963 & June 28, 1963 & 1,110 \\
\hline 1967 & June 12, 1967 & 1,820 & 1962 & July 22, 1962 & 998 \\
\hline
\end{tabular}


1-4.L00-2T KONAR RIVER NEAR KONARI, Continued

Monthly and annual mean discharges, in cubic meters per second

\begin{tabular}{|c|c|c|c|c|c|c|c|c|c|c|c|c|c|}
\hline \multirow{2}{*}{$\begin{array}{l}\text { Water } \\
\text { year }\end{array}$} & \multicolumn{12}{|c|}{ Monthly mean discharge } & \multirow{2}{*}{$\begin{array}{l}\text { Annual } \\
\text { discharge }\end{array}$} \\
\hline & October & November & December & January & February & March & April & May & June & July & August & September & \\
\hline 1960 & 293 & 227 & 203 & 181 & 185 & 227 & 368 & 618 & 878 & 1,180 & 969 & 506 & 488 \\
\hline 1961 & 256 & 183 & 150 & 139 & 134 & 143 & 292 & 518 & 824 & 1,010 & 672 & 589 & 411 \\
\hline 1962 & 259 & 140 & 127 & 122 & 119 & 122 & 197 & 289 & 597 & 745 & 708 & 400 & 320 \\
\hline 1963 & 189 & 151 & 132 & 124 & 121 & 143 & 274 & 547 & 868 & 890 & 805 & 405 & 389 \\
\hline 1964 & 188 & 139 & 123 & 114 & 113 & 130 & 300 & 469 & 729 & 884 & 740 & 464 & 367 \\
\hline 1965 & 180 & 133 & 126 & 119 & 119 & 136 & 440 & 783 & 1,070 & 1,090 & 713 & 393 & 443 \\
\hline 1966 & 175 & 137 & 116 & 103 & 100 & 188 & 427 & 576 & 1,110 & 1,070 & 882 & 463 & 447 \\
\hline 1967 & 233 & 163 & 133 & 120 & 118 & 138 & 346 & 578 & 1,270 & 1,400 & 1,090 & 559 & 515 \\
\hline
\end{tabular}




\section{1-4.L00-3A KONAR RIVER AT NAWABAD}

\section{(U.S. Geological Survey identification number: 344900071070000)}

LOCATION: Lat $34^{\circ} 49^{\prime} \mathrm{N}$., long $71^{\circ} 07^{\prime} \mathrm{E}$.

DRAINAGE AREA: $23,960 \mathrm{~km}^{2}$.

ELEVATION: 798 meters above mean sea level.

PERIOD OF RECORD: April 1, 1976 to September 30, 1979.

GAGE: Water-stage recorder.

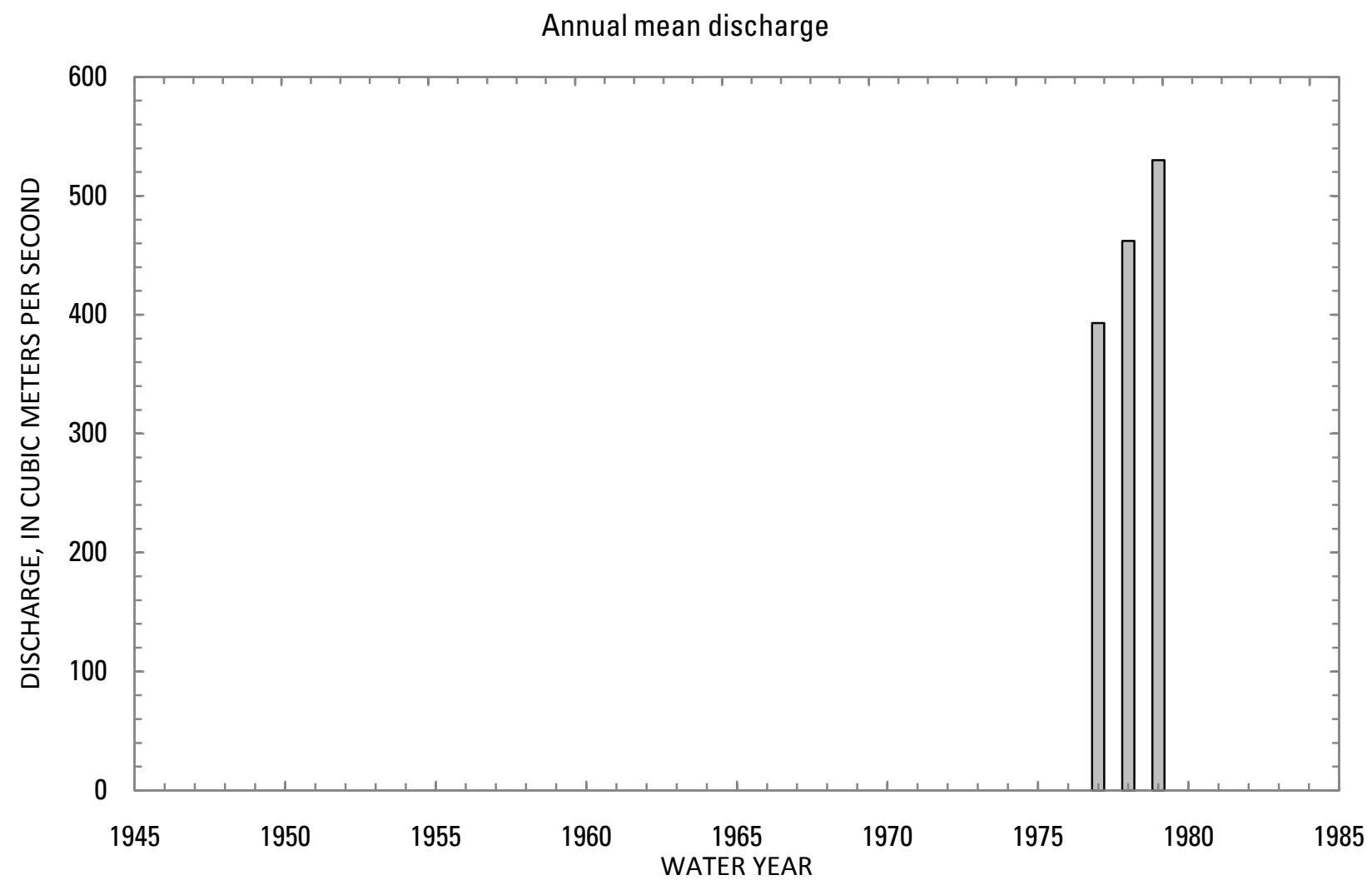




\section{1-4.L00-3A KONAR RIVER AT NAWABAD, Continued}

Statistics of monthly and annual mean discharges [ $\mathrm{m}^{3} / \mathrm{s}$, cubic meters per second]

\begin{tabular}{|c|c|c|c|c|c|c|c|c|}
\hline \multirow[b]{2}{*}{ Month } & \multicolumn{2}{|c|}{ Maximum } & \multicolumn{2}{|c|}{ Minimum } & \multicolumn{4}{|c|}{ Mean } \\
\hline & $\begin{array}{c}\text { Discharge } \\
\left(\mathrm{m}^{3} / \mathrm{s}\right)\end{array}$ & $\begin{array}{c}\text { Water year } \\
\text { of } \\
\text { occurrence }\end{array}$ & $\begin{array}{c}\text { Discharge } \\
\left(\mathrm{m}^{3} / \mathrm{s}\right)\end{array}$ & $\begin{array}{c}\text { Water year } \\
\text { of } \\
\text { occurrence }\end{array}$ & $\begin{array}{c}\text { Discharge } \\
\left(\mathrm{m}^{3} / \mathrm{s}\right)\end{array}$ & $\begin{array}{c}\text { Standard } \\
\text { deviation } \\
\left(\mathrm{m}^{3} / \mathrm{s}\right)\end{array}$ & $\begin{array}{c}\text { Coefficient } \\
\text { of } \\
\text { variation }\end{array}$ & $\begin{array}{c}\text { Percentage } \\
\text { of annual } \\
\text { discharge }\end{array}$ \\
\hline October & 256 & 1979 & 203 & 1978 & 226 & 27.3 & 0.12 & 4.23 \\
\hline November & 185 & 1979 & 142 & 1978 & 159 & 22.7 & 0.14 & 2.98 \\
\hline December & 144 & 1979 & 109 & 1978 & 124 & 18.0 & 0.15 & 2.32 \\
\hline January & 114 & 1979 & 87.0 & 1978 & 103 & 14.2 & 0.14 & 1.93 \\
\hline February & 102 & 1979 & 90.0 & 1978 & 94.2 & 6.76 & 0.07 & 1.76 \\
\hline March & 137 & 1979 & 123 & 1977 & 131 & 7.21 & 0.06 & 2.45 \\
\hline April & 468 & 1979 & 236 & 1977 & 349 & 100 & 0.29 & 6.53 \\
\hline May & 674 & 1979 & 423 & 1977 & 593 & 115 & 0.19 & 11.1 \\
\hline June & 1,100 & 1979 & 785 & 1976 & 927 & 135 & 0.15 & 17.4 \\
\hline July & 1,620 & 1979 & 1,050 & 1976 & 1,280 & 260 & 0.20 & 23.9 \\
\hline August & 1,090 & 1979 & 746 & 1976 & 935 & 159 & 0.17 & 17.5 \\
\hline September & 489 & 1978 & 382 & 1977 & 426 & 46.2 & 0.11 & 7.98 \\
\hline Annual & 530 & 1979 & 393 & 1977 & 462 & 68.5 & 0.15 & 100 \\
\hline
\end{tabular}




\section{1-4.LO0-3A KONAR RIVER AT NAWABAD, Continued}

Monthly and annual flow duration, in cubic meters per second

[ng, not given]

\begin{tabular}{|c|c|c|c|c|c|c|c|c|c|c|c|c|c|}
\hline \multirow{2}{*}{$\begin{array}{l}\text { Percentage } \\
\text { of days } \\
\text { discharge } \\
\text { equaled or } \\
\text { exceeded }\end{array}$} & \multicolumn{12}{|c|}{ Month } & \multirow{2}{*}{ Annual } \\
\hline & October & November & December & January & February & March & April & May & June & July & August & September & \\
\hline 95 & 154 & 130 & 96.6 & 84.1 & 83.6 & 91.3 & 158 & 253 & 497 & 912 & 582 & 287 & 89.3 \\
\hline 90 & 166 & 136 & 99.2 & 85.3 & 85.3 & 101 & 182 & 326 & 610 & 984 & 627 & 297 & 96.4 \\
\hline 85 & 172 & 138 & 107 & 86.0 & 86.7 & 106 & 214 & 365 & 647 & 1,020 & 663 & 308 & 107 \\
\hline 80 & 176 & 139 & 110 & 86.9 & 87.7 & 110 & 219 & 450 & 673 & 1,070 & 726 & 318 & 117 \\
\hline 75 & 179 & 142 & 113 & 89.2 & 88.5 & 115 & 225 & 465 & 700 & 1,100 & 765 & 327 & 127 \\
\hline 70 & 187 & 144 & 115 & 91.0 & 89.1 & 117 & 235 & 493 & 733 & 1,110 & 803 & 353 & 138 \\
\hline 65 & 192 & 146 & 116 & 94.7 & 89.5 & 120 & 242 & 528 & 784 & 1,130 & 837 & 366 & 153 \\
\hline 60 & 198 & 151 & 117 & 96.5 & 89.9 & 123 & 252 & 558 & 814 & 1,140 & 858 & 387 & 173 \\
\hline 55 & 201 & 158 & 119 & 100 & 90.2 & 124 & 268 & 575 & 833 & 1,160 & 880 & 403 & 196 \\
\hline 50 & 205 & 159 & 121 & 101 & 90.6 & 126 & 288 & 599 & 852 & 1,180 & 912 & 412 & 237 \\
\hline 45 & 214 & 161 & 125 & 103 & 91.6 & 130 & 296 & 631 & 873 & 1,200 & 942 & 442 & 296 \\
\hline 40 & 226 & 167 & 129 & 107 & 92.8 & 132 & 303 & 663 & 910 & 1,220 & 967 & 462 & 376 \\
\hline 35 & 235 & 172 & 131 & 109 & 93.6 & 134 & 340 & 686 & 965 & 1,250 & 1,020 & 481 & 482 \\
\hline 30 & 244 & 174 & 136 & 111 & 94.3 & 137 & 382 & 700 & 1,020 & 1,330 & 1,060 & 493 & 582 \\
\hline 25 & 254 & 179 & 140 & 116 & 95.6 & 141 & 468 & 714 & 1,100 & 1,460 & 1,090 & 504 & 695 \\
\hline 20 & 266 & 181 & 141 & 120 & 97.8 & 145 & 518 & 738 & 1,160 & 1,610 & 1,120 & 515 & 853 \\
\hline 15 & 287 & 189 & 145 & 121 & 103 & 153 & 579 & 763 & 1,220 & 1,690 & 1,170 & 534 & 1,030 \\
\hline 10 & 293 & 191 & 147 & 122 & 106 & 159 & 628 & 785 & 1,360 & 1,760 & 1,230 & 561 & 1,150 \\
\hline 5 & 388 & 193 & $\mathrm{ng}$ & 126 & 111 & 191 & 732 & 875 & 1,690 & 1,890 & 1,430 & 612 & 1,390 \\
\hline
\end{tabular}




\section{1-4.L00-3A KONAR RIVER AT NAWABAD, Continued}

Probability of occurrence of annual high discharges

[m $\mathrm{m}^{3} / \mathrm{s}$, cubic meters per second; ng, not given]

\begin{tabular}{|c|c|c|c|c|c|c|}
\hline \multirow{2}{*}{$\begin{array}{c}\text { Exceedance } \\
\text { probability }\end{array}$} & \multirow{2}{*}{$\begin{array}{c}\text { Recurrence } \\
\text { interval } \\
\text { (years) }\end{array}$} & \multirow{2}{*}{$\begin{array}{l}\text { Maximum } \\
\text { instantaneous } \\
\text { discharge } \\
\left(\mathrm{m}^{3} / \mathrm{s}\right)\end{array}$} & \multicolumn{4}{|c|}{ Maximum daily mean discharge $\left(\mathrm{m}^{3} / \mathrm{s}\right)$} \\
\hline & & & $\begin{array}{l}\text { 3-day } \\
\text { period }\end{array}$ & $\begin{array}{l}\text { 7-day } \\
\text { period }\end{array}$ & $\begin{array}{l}\text { 15-day } \\
\text { period }\end{array}$ & $\begin{array}{l}\text { 30-day } \\
\text { period }\end{array}$ \\
\hline 0.99 & 1.01 & ng & ng & ng & ng & ng \\
\hline 0.95 & 1.05 & ng & ng & ng & ng & $\mathrm{ng}$ \\
\hline 0.90 & 1.11 & ng & ng & ng & ng & $\mathrm{ng}$ \\
\hline 0.80 & 1.25 & ng & ng & ng & ng & ng \\
\hline 0.50 & 2 & ng & ng & ng & ng & ng \\
\hline 0.20 & 5 & ng & ng & ng & ng & ng \\
\hline 0.10 & 10 & ng & ng & ng & ng & ng \\
\hline 0.04 & 25 & ng & ng & ng & ng & ng \\
\hline 0.02 & 50 & ng & ng & ng & $\mathrm{ng}$ & $\mathrm{ng}$ \\
\hline 0.01 & 100 & ng & ng & ng & $\mathrm{ng}$ & ng \\
\hline 0.005 & 200 & ng & ng & ng & $\mathrm{ng}$ & ng \\
\hline 0.002 & 500 & $\mathrm{ng}$ & $\mathrm{ng}$ & ng & $\mathrm{ng}$ & $\mathrm{ng}$ \\
\hline
\end{tabular}

\section{1-4.L00-3A KONAR RIVER AT NAWABAD, Continued}

Probability of occurrence of annual low discharges

[ $\mathrm{m}^{3} / \mathrm{s}$, meters per second; ng, not given]

\begin{tabular}{|c|c|c|c|c|c|c|c|c|c|c|}
\hline \multirow{3}{*}{$\begin{array}{c}\text { Nonexceedance } \\
\text { probability }\end{array}$} & \multirow{3}{*}{$\begin{array}{c}\text { Recurrence } \\
\text { interval } \\
\text { (years) }\end{array}$} & \multicolumn{9}{|c|}{ Minimum daily mean discharge $\left(\mathrm{m}^{3} / \mathrm{s}\right)$} \\
\hline & & \multicolumn{9}{|c|}{ Number of consecutive days } \\
\hline & & 1 & 3 & 7 & 14 & 30 & 60 & 90 & 120 & 183 \\
\hline 0.05 & 20 & ng & ng & $\mathrm{ng}$ & $\mathrm{ng}$ & $\mathrm{ng}$ & $\mathrm{ng}$ & ng & ng & ng \\
\hline 0.10 & 10 & $\mathrm{ng}$ & ng & $\mathrm{ng}$ & ng & $\mathrm{ng}$ & $\mathrm{ng}$ & ng & ng & ng \\
\hline 0.20 & 5 & ng & ng & ng & ng & ng & ng & ng & ng & ng \\
\hline 0.50 & 2 & ng & ng & ng & ng & ng & ng & ng & ng & ng \\
\hline
\end{tabular}




\section{1-4.L00-3A KONAR RIVER AT NAWABAD, Continued}

Probability of occurrence of seasonal low discharges [ $\mathrm{m}^{3} / \mathrm{s}$, meters per second; $\mathrm{ng}$, not given]

\begin{tabular}{|c|c|c|c|c|c|c|c|c|c|}
\hline \multirow{3}{*}{$\begin{array}{c}\text { Nonexceedance } \\
\text { probability }\end{array}$} & \multirow{3}{*}{$\begin{array}{c}\text { Recurrence } \\
\text { interval } \\
\text { (years) }\end{array}$} & \multicolumn{8}{|c|}{ Minimum daily mean discharge $\left(\mathrm{m}^{3} / \mathrm{s}\right)$} \\
\hline & & \multicolumn{8}{|c|}{ Number of consecutive days } \\
\hline & & 1 & 7 & 14 & 30 & 1 & 7 & 14 & 30 \\
\hline & & \multicolumn{4}{|c|}{ December-January-February } & \multicolumn{4}{|c|}{ March-April-May } \\
\hline 0.05 & 20 & ng & ng & $\mathrm{ng}$ & ng & ng & ng & ng & ng \\
\hline 0.10 & 10 & ng & ng & ng & ng & ng & ng & ng & ng \\
\hline 0.20 & 5 & ng & ng & ng & ng & ng & ng & ng & ng \\
\hline \multirow[t]{2}{*}{0.50} & 2 & ng & ng & $\mathrm{ng}$ & ng & ng & ng & ng & ng \\
\hline & & \multicolumn{4}{|c|}{ June-July-August } & \multicolumn{4}{|c|}{ September-October-November } \\
\hline 0.05 & 20 & ng & ng & ng & ng & ng & ng & ng & ng \\
\hline 0.10 & 10 & ng & $\mathrm{ng}$ & $\mathrm{ng}$ & ng & ng & ng & ng & ng \\
\hline 0.20 & 5 & ng & ng & ng & ng & ng & $\mathrm{ng}$ & $\mathrm{ng}$ & ng \\
\hline 0.50 & 2 & $\mathrm{ng}$ & $\mathrm{ng}$ & $\mathrm{ng}$ & $\mathrm{ng}$ & $\mathrm{ng}$ & $\mathrm{ng}$ & $\mathrm{ng}$ & ng \\
\hline
\end{tabular}

\section{1-4.L00-3A KONAR RIVER AT NAWABAD, Continued}

Annual peak discharges

$\left[\mathrm{m}^{3} / \mathrm{s}\right.$, meters per second]

\begin{tabular}{|c|c|c|c|c|c|}
\hline \multicolumn{3}{|c|}{$\begin{array}{c}\text { Annual peak discharge, } \\
\text { by year }\end{array}$} & \multicolumn{3}{|c|}{$\begin{array}{l}\text { Annual peak discharge, } \\
\text { from highest to lowest }\end{array}$} \\
\hline $\begin{array}{l}\text { Water } \\
\text { year }\end{array}$ & Date & $\begin{array}{c}\text { Peak } \\
\text { discharge } \\
\left(\mathrm{m}^{3} / \mathrm{s}\right)\end{array}$ & $\begin{array}{l}\text { Water } \\
\text { year }\end{array}$ & Date & $\begin{array}{c}\text { Peak } \\
\text { discharge } \\
\left(\mathrm{m}^{3} / \mathrm{s}\right)\end{array}$ \\
\hline 1976 & July 21, 1976 & 1,190 & 1978 & July 8, 1978 & 2,000 \\
\hline 1977 & June 24, 1977 & 1,394 & 1979 & July 8, 1979 & 1,900 \\
\hline 1978 & July 8, 1978 & 2,000 & 1977 & June 24, 1977 & 1,394 \\
\hline 1979 & July 8, 1979 & 1,900 & 1976 & July 21,1976 & 1,190 \\
\hline
\end{tabular}




\section{1-4.LO0-3A KONAR RIVER AT NAWABAD, Continued}

Monthly and annual mean discharges, in cubic meters per second

[Data may not be rounded in accordance with U.S. Geological Survey publication standards; --, no data]

\begin{tabular}{|c|c|c|c|c|c|c|c|c|c|c|c|c|c|}
\hline \multirow{2}{*}{$\begin{array}{l}\text { Water } \\
\text { year }\end{array}$} & \multicolumn{12}{|c|}{ Monthly mean discharge } & \multirow{2}{*}{$\begin{array}{c}\text { Annual } \\
\text { discharge }\end{array}$} \\
\hline & October & November & December & January & February & March & April & May & June & July & August & September & \\
\hline 1976 & -- & -- & -- & -- & -- & -- & 386 & 654 & 785 & 1,050 & 746 & 404 & -- \\
\hline 1977 & 218 & 151 & 119 & 108 & 90.6 & 123 & 236 & 423 & 867 & 1,100 & 863 & 382 & 393 \\
\hline 1978 & 203 & 142 & 109 & 87.0 & 90.0 & 133 & 306 & 619 & 954 & 1,330 & 1,040 & 489 & 462 \\
\hline 1979 & 256 & 185 & 144 & 114 & 102 & 137 & 468 & 674 & 1,100 & 1,620 & 1,090 & 430 & 530 \\
\hline
\end{tabular}




\section{1-4.L00-4T KONAR RIVER NEAR ASMAR}

\section{(U.S. Geological Survey identification number: 345300071100000)}

LOCATION: Lat $34^{\circ} 53^{\prime} \mathrm{N}$., long $71^{\circ} 10^{\prime} \mathrm{E}$.

DRAINAGE AREA: $19,960 \mathrm{~km}^{2}$.

ELEVATION: 795 meters above mean sea level.

PERIOD OF RECORD: February 23, 1960 to September 30, 1971.

GAGE: Water-stage recorder.

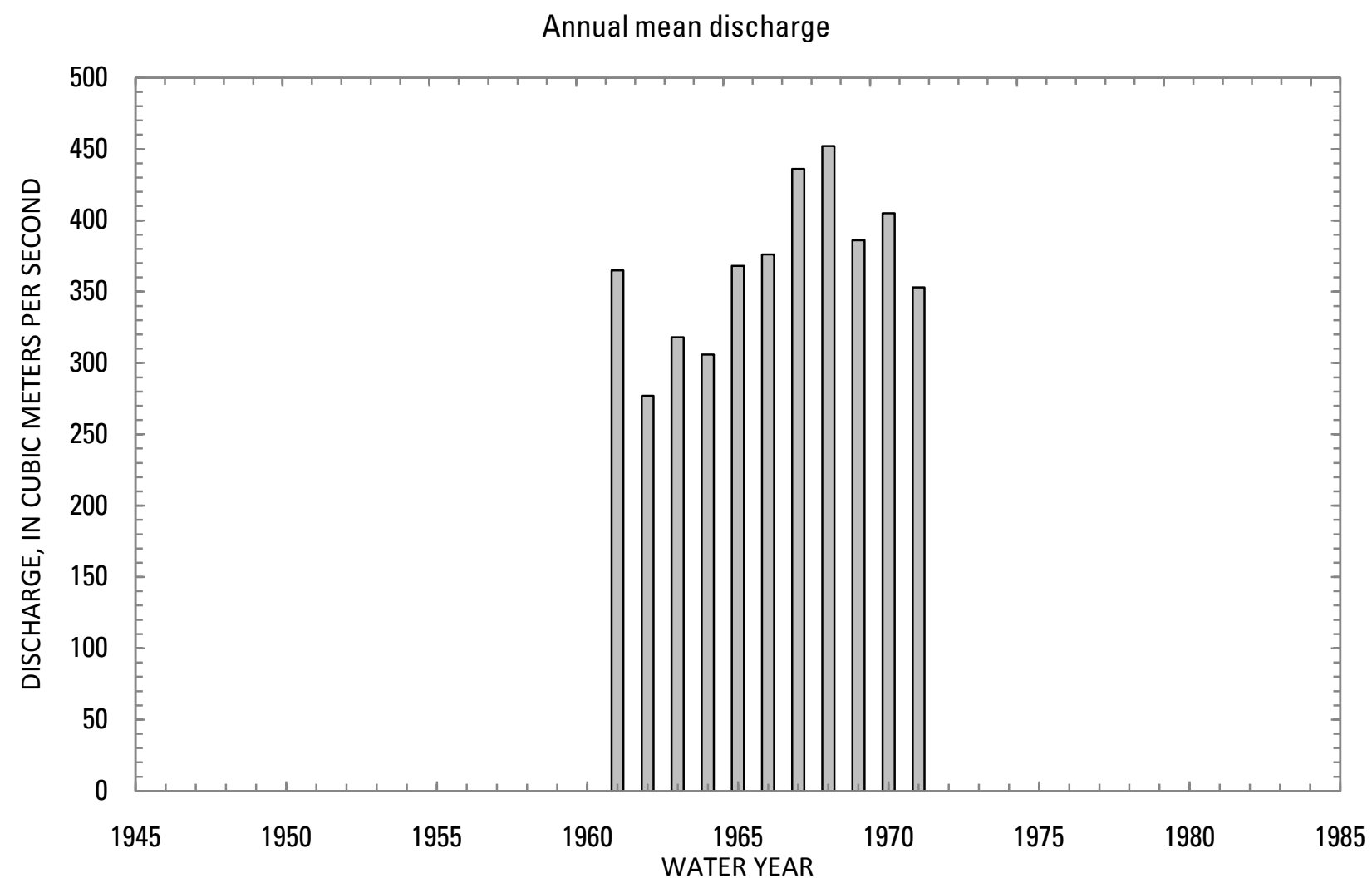




\section{1-4.L00-4T KONAR RIVER NEAR ASMAR, Continued}

Statistics of monthly and annual mean discharges [ $\mathrm{m}^{3} / \mathrm{s}$, cubic meters per second]

\begin{tabular}{|c|c|c|c|c|c|c|c|c|}
\hline \multirow[b]{2}{*}{ Month } & \multicolumn{2}{|c|}{ Maximum } & \multicolumn{2}{|c|}{ Minimum } & \multicolumn{4}{|c|}{ Mean } \\
\hline & $\begin{array}{c}\text { Discharge } \\
\left(\mathrm{m}^{3} / \mathrm{s}\right)\end{array}$ & $\begin{array}{c}\text { Water year } \\
\text { of } \\
\text { occurrence }\end{array}$ & $\begin{array}{c}\text { Discharge } \\
\left(\mathrm{m}^{3} / \mathrm{s}\right)\end{array}$ & $\begin{array}{c}\text { Water year } \\
\text { of } \\
\text { occurrence }\end{array}$ & $\begin{array}{l}\text { Discharge } \\
\left(\mathrm{m}^{3} / \mathrm{s}\right)\end{array}$ & $\begin{array}{c}\text { Standard } \\
\text { deviation } \\
\left(\mathrm{m}^{3} / \mathrm{s}\right)\end{array}$ & $\begin{array}{c}\text { Coefficient } \\
\text { of } \\
\text { variation }\end{array}$ & $\begin{array}{c}\text { Percentage } \\
\text { of annual } \\
\text { discharge }\end{array}$ \\
\hline October & 251 & 1961 & 144 & 1963 & 194 & 35.2 & 0.18 & 4.38 \\
\hline November & 176 & 1970 & 107 & 1964 & 135 & 26.9 & 0.20 & 3.06 \\
\hline December & 161 & 1970 & 88.9 & 1964 & 112 & 21.2 & 0.19 & 2.53 \\
\hline January & 137 & 1970 & 75.4 & 1966 & 97.7 & 16.2 & 0.17 & 2.21 \\
\hline February & 123 & 1970 & 75.6 & 1966 & 89.7 & 13.0 & 0.14 & 2.03 \\
\hline March & 167 & 1960 & 95.3 & 1963 & 121 & 22.2 & 0.18 & 2.73 \\
\hline April & 373 & 1968 & 157 & 1962 & 263 & 65.7 & 0.25 & 5.96 \\
\hline May & 593 & 1965 & 236 & 1962 & 437 & 94.4 & 0.22 & 9.88 \\
\hline June & 1070 & 1968 & 503 & 1962 & 760 & 158 & 0.21 & 17.2 \\
\hline July & 1200 & 1967 & 674 & 1962 & 925 & 155 & 0.17 & 20.9 \\
\hline August & 1040 & 1970 & 644 & 1962 & 831 & 144 & 0.17 & 18.8 \\
\hline September & 583 & 1961 & 361 & 1963 & 454 & 79.4 & 0.18 & 10.3 \\
\hline Annual & 452 & 1968 & 277 & 1962 & 367 & 53.2 & 0.14 & 100 \\
\hline
\end{tabular}


1-4.L00-4T KONAR RIVER NEAR ASMAR, Continued

Monthly and annual flow duration, in cubic meters per second

\begin{tabular}{|c|c|c|c|c|c|c|c|c|c|c|c|c|c|}
\hline \multirow{2}{*}{$\begin{array}{l}\text { Percentage } \\
\text { of days } \\
\text { discharge } \\
\text { equaled or } \\
\text { exceeded }\end{array}$} & \multicolumn{12}{|c|}{ Month } & \multirow{2}{*}{ Annual } \\
\hline & October & November & December & January & February & March & April & May & June & July & August & September & \\
\hline 95 & 120 & 103 & 85.9 & 76.7 & 71.5 & 82.4 & 114 & 204 & 448 & 629 & 579 & 245 & 84.9 \\
\hline 90 & 129 & 108 & 88.4 & 82.5 & 76.4 & 85.2 & 133 & 259 & 523 & 684 & 621 & 264 & 90.0 \\
\hline 85 & 138 & 110 & 90.8 & 84.3 & 80.4 & 89.2 & 143 & 304 & 568 & 709 & 639 & 292 & 95.1 \\
\hline 80 & 145 & 111 & 93.1 & 85.2 & 81.2 & 91.5 & 149 & 327 & 599 & 758 & 665 & 314 & 102 \\
\hline 75 & 155 & 113 & 97.0 & 86.2 & 82.1 & 93.4 & 165 & 347 & 623 & 798 & 685 & 341 & 109 \\
\hline 70 & 165 & 114 & 100 & 87.3 & 82.9 & 95.3 & 183 & 368 & 644 & 825 & 701 & 357 & 118 \\
\hline 65 & 170 & 116 & 102 & 88.7 & 83.7 & 97.7 & 195 & 384 & 669 & 852 & 719 & 376 & 131 \\
\hline 60 & 178 & 117 & 104 & 90.6 & 84.5 & 100 & 206 & 398 & 695 & 874 & 739 & 399 & 148 \\
\hline 55 & 184 & 119 & 104 & 93.3 & 85.6 & 103 & 221 & 409 & 712 & 894 & 759 & 420 & 174 \\
\hline 50 & 190 & 127 & 105 & 95.2 & 86.8 & 105 & 236 & 422 & 732 & 913 & 782 & 438 & 208 \\
\hline 45 & 199 & 133 & 109 & 96.1 & 87.9 & 113 & 250 & 444 & 756 & 941 & 806 & 462 & 253 \\
\hline 40 & 205 & 138 & 114 & 97.1 & 89.0 & 123 & 268 & 470 & 784 & 966 & 835 & 479 & 326 \\
\hline 35 & 209 & 143 & 117 & 98.5 & 90.7 & 128 & 280 & 489 & 817 & 990 & 883 & 492 & 407 \\
\hline 30 & 214 & 151 & 118 & 101 & 92.3 & 133 & 294 & 507 & 854 & 1,020 & 938 & 509 & 502 \\
\hline 25 & 222 & 158 & 123 & 106 & 94.1 & 138 & 312 & 534 & 885 & 1,050 & 990 & 527 & 598 \\
\hline 20 & 229 & 166 & 125 & 113 & 96.8 & 147 & 359 & 551 & 916 & 1,090 & 1,040 & 551 & 679 \\
\hline 15 & 239 & 179 & 130 & 114 & 99.9 & 157 & 397 & 577 & 972 & 1,120 & 1,080 & 614 & 761 \\
\hline 10 & 267 & 182 & 140 & 119 & 104 & 173 & 470 & 616 & 1,090 & 1,180 & 1,120 & 677 & 881 \\
\hline 5 & 297 & 187 & 160 & 0.05 & 119 & 205 & 500 & 677 & 1,180 & 1,270 & 1,170 & 739 & 1,050 \\
\hline
\end{tabular}




\section{1-4.L00-4T KONAR RIVER NEAR ASMAR, Continued}

Probability of occurrence of annual high discharges [ $\mathrm{m}^{3} / \mathrm{s}$, cubic meters per second; $\mathrm{ng}$, not given]

\begin{tabular}{|c|c|c|c|c|c|c|}
\hline \multirow{2}{*}{$\begin{array}{c}\text { Exceedance } \\
\text { probability }\end{array}$} & \multirow{2}{*}{$\begin{array}{c}\text { Recurrence } \\
\text { interval } \\
\text { (years) }\end{array}$} & \multirow{2}{*}{$\begin{array}{l}\text { Maximum } \\
\text { instantaneous } \\
\text { discharge } \\
\left(\mathrm{m}^{3} / \mathrm{s}\right)\end{array}$} & \multicolumn{4}{|c|}{ Maximum daily mean discharge $\left(\mathrm{m}^{3} / \mathrm{s}\right)$} \\
\hline & & & $\begin{array}{l}\text { 3-day } \\
\text { period }\end{array}$ & $\begin{array}{l}\text { 7-day } \\
\text { period }\end{array}$ & $\begin{array}{l}\text { 15-day } \\
\text { period }\end{array}$ & $\begin{array}{l}\text { 30-day } \\
\text { period }\end{array}$ \\
\hline 0.99 & 1.01 & 766 & 650 & 642 & 638 & 620 \\
\hline 0.95 & 1.05 & 876 & 771 & 755 & 742 & 712 \\
\hline 0.90 & 1.11 & 939 & 841 & 820 & 801 & 764 \\
\hline 0.80 & 1.25 & 1,020 & 930 & 903 & 875 & 831 \\
\hline 0.50 & 2 & 1,180 & 1,110 & 1,080 & 1,030 & 967 \\
\hline 0.20 & 5 & 1,350 & 1,310 & 1,260 & 1,190 & 1,110 \\
\hline 0.10 & 10 & 1,450 & 1,410 & 1,370 & 1,280 & 1,200 \\
\hline 0.04 & 25 & 1,550 & 1,530 & 1,480 & 1,370 & 1,290 \\
\hline 0.02 & 50 & 1,620 & 1,600 & 1,560 & 1,430 & 1,350 \\
\hline 0.01 & 100 & 1,680 & 1,670 & 1,630 & 1,490 & 1,400 \\
\hline 0.005 & 200 & 1,740 & 1,730 & 1,700 & 1,540 & 1,450 \\
\hline 0.002 & 500 & 1,820 & $\mathrm{ng}$ & $\mathrm{ng}$ & $\mathrm{ng}$ & $\mathrm{ng}$ \\
\hline
\end{tabular}

\section{1-4.L00-4T KONAR RIVER NEAR ASMAR, Continued}

Probability of occurrence of annual low discharges $\left[\mathrm{m}^{3} / \mathrm{s}\right.$, meters per second]

\begin{tabular}{|c|c|c|c|c|c|c|c|c|c|c|}
\hline \multirow{3}{*}{$\begin{array}{c}\text { Nonexceedance } \\
\text { probability }\end{array}$} & \multirow{3}{*}{$\begin{array}{c}\text { Recurrence } \\
\text { interval } \\
\text { (years) }\end{array}$} & \multicolumn{9}{|c|}{ Minimum daily mean discharge $\left(\mathrm{m}^{3} / \mathrm{s}\right)$} \\
\hline & & \multicolumn{9}{|c|}{ Number of consecutive days } \\
\hline & & 1 & 3 & 7 & 14 & 30 & 60 & 90 & 120 & 183 \\
\hline 0.05 & 20 & 32.1 & 61.5 & 66.9 & 69.1 & 74.6 & 77.9 & 81.6 & 85.4 & 99.8 \\
\hline 0.10 & 10 & 43.1 & 65.0 & 69.4 & 71.3 & 75.8 & 79.1 & 83.2 & 87.2 & 104 \\
\hline 0.20 & 5 & 57.8 & 69.9 & 73.0 & 74.5 & 77.8 & 81.1 & 85.6 & 90.0 & 110 \\
\hline 0.50 & 2 & 84.4 & 81.1 & 82.1 & 82.9 & 84.4 & 87.8 & 92.9 & 98.1 & 123 \\
\hline
\end{tabular}




\section{1-4.L00-4T KONAR RIVER NEAR ASMAR, Continued}

Probability of occurrence of seasonal low discharges [ $\mathrm{m}^{3} / \mathrm{s}$, meters per second]

\begin{tabular}{|c|c|c|c|c|c|c|c|c|c|}
\hline \multirow{3}{*}{$\begin{array}{c}\text { Nonexceedance } \\
\text { probability }\end{array}$} & \multirow{3}{*}{$\begin{array}{c}\text { Recurrence } \\
\text { interval } \\
\text { (years) }\end{array}$} & \multicolumn{8}{|c|}{ Minimum daily mean discharge $\left(\mathrm{m}^{3} / \mathrm{s}\right)$} \\
\hline & & \multicolumn{8}{|c|}{ Number of consecutive days } \\
\hline & & 1 & 7 & 14 & 30 & 1 & 7 & 14 & 30 \\
\hline & & \multicolumn{4}{|c|}{ December-January-February } & \multicolumn{4}{|c|}{ March-April-May } \\
\hline 0.05 & 20 & 32.6 & 68.3 & 71.4 & 76.0 & 63.7 & 71.8 & 84.0 & 88.8 \\
\hline 0.10 & 10 & 44.1 & 70.5 & 73.1 & 77.2 & 68.5 & 74.6 & 85.1 & 93.7 \\
\hline 0.20 & 5 & 59.2 & 73.7 & 75.9 & 79.2 & 74.8 & 78.9 & 87.3 & 100 \\
\hline \multirow[t]{2}{*}{0.50} & 2 & 85.7 & 82.2 & 83.5 & 85.6 & 88.4 & 91.0 & 95.5 & 116 \\
\hline & & \multicolumn{4}{|c|}{ June-July-August } & \multicolumn{4}{|c|}{ September-October-November } \\
\hline 0.05 & 20 & 275 & 322 & 413 & 516 & 92.0 & 92.8 & 95.8 & 98.8 \\
\hline 0.10 & 10 & 329 & 377 & 460 & 558 & 96.8 & 97.8 & 100 & 105 \\
\hline 0.20 & 5 & 394 & 446 & 518 & 613 & 103 & 104 & 107 & 113 \\
\hline 0.50 & 2 & 504 & 567 & 632 & 726 & 116 & 119 & 123 & 131 \\
\hline
\end{tabular}

\section{1-4.L00-4T KONAR RIVER NEAR ASMAR, Continued}

Annual peak discharges

[ $\mathrm{m}^{3} / \mathrm{s}$, meters per second]

\begin{tabular}{|c|c|c|c|c|c|}
\hline \multicolumn{3}{|c|}{$\begin{array}{c}\text { Annual peak discharge, } \\
\text { by year }\end{array}$} & \multicolumn{3}{|c|}{$\begin{array}{l}\text { Annual peak discharge, } \\
\text { from highest to lowest }\end{array}$} \\
\hline $\begin{array}{l}\text { Water } \\
\text { year }\end{array}$ & Date & $\begin{array}{c}\text { Peak } \\
\text { discharge } \\
\left(\mathrm{m}^{3} / \mathrm{s}\right)\end{array}$ & $\begin{array}{l}\text { Water } \\
\text { year }\end{array}$ & Date & $\begin{array}{c}\text { Peak } \\
\text { discharge } \\
\left(\mathrm{m}^{3} / \mathrm{s}\right)\end{array}$ \\
\hline 1960 & July 11,1960 & 1,230 & 1967 & July 24,1967 & 1,530 \\
\hline 1961 & July 16, 1961 & 1,180 & 1968 & July 10, 1968 & 1,472 \\
\hline 1962 & July 26, 1962 & 876 & 1970 & July 3, 1970 & 1,290 \\
\hline 1963 & July 12,1963 & 926 & 1966 & June 20, 1966 & 1,263 \\
\hline 1964 & July 13,1964 & 976 & 1960 & July 11,1960 & 1,230 \\
\hline 1965 & July 27, 1965 & 1,165 & 1969 & August 12, 1969 & 1,196 \\
\hline 1966 & June 20, 1966 & 1,263 & 1961 & July 16,1961 & 1,180 \\
\hline 1967 & July 24,1967 & 1,530 & 1965 & July 27,1965 & 1,165 \\
\hline 1968 & July 10,1968 & 1,472 & 1971 & July 14,1971 & 1,130 \\
\hline 1969 & August 12, 1969 & 1,196 & 1964 & July 13,1964 & 976 \\
\hline 1970 & July 3, 1970 & 1,290 & 1963 & July 12,1963 & 926 \\
\hline 1971 & July 14,1971 & 1,130 & 1962 & July 26, 1962 & 876 \\
\hline
\end{tabular}




\section{1-4.L00-4T KONAR RIVER NEAR ASMAR, Continued}

Monthly and annual mean discharges, in cubic meters per second $[--$, no data]

\begin{tabular}{|c|c|c|c|c|c|c|c|c|c|c|c|c|c|}
\hline \multirow{2}{*}{$\begin{array}{l}\text { Water } \\
\text { year }\end{array}$} & \multicolumn{12}{|c|}{ Monthly mean discharge } & \multirow{2}{*}{$\begin{array}{c}\text { Annual } \\
\text { discharge }\end{array}$} \\
\hline & October & November & December & January & February & March & April & May & June & July & August & September & \\
\hline 1960 & -- & -- & -- & -- & -- & 167 & 338 & 528 & 739 & 1,010 & 858 & 473 & -- \\
\hline 1961 & 251 & 158 & 117 & 101 & 100 & 117 & 245 & 418 & 654 & 885 & 736 & 583 & 365 \\
\hline 1962 & 217 & 116 & 102 & 95.7 & 92.9 & 95.9 & 157 & 236 & 503 & 674 & 644 & 369 & 277 \\
\hline 1963 & 144 & 109 & 93.2 & 86.3 & 86.0 & 95.3 & 186 & 424 & 710 & 798 & 700 & 361 & 318 \\
\hline 1964 & 144 & 107 & 88.9 & 84.2 & 84.0 & 123 & 244 & 347 & 544 & 767 & 699 & 432 & 306 \\
\hline 1965 & 153 & 110 & 104 & 94.0 & 85.1 & 99.1 & 312 & 593 & 850 & 937 & 681 & 372 & 368 \\
\hline 1966 & 175 & 122 & 88.9 & 75.4 & 75.6 & 147 & 295 & 442 & 883 & 929 & 827 & 430 & 376 \\
\hline 1967 & 208 & 146 & 119 & 113 & 91.1 & 110 & 270 & 462 & 887 & 1,200 & 1,030 & 569 & 436 \\
\hline 1968 & 208 & 175 & 130 & 97.5 & 81.1 & 124 & 373 & 535 & 1,070 & 1,180 & 1,010 & 434 & 452 \\
\hline 1969 & 220 & 153 & 120 & 94.2 & 79.0 & 133 & 285 & 373 & 767 & 988 & 932 & 461 & 386 \\
\hline 1970 & 216 & 176 & 161 & 137 & 123 & 136 & 279 & 473 & 682 & 849 & 1,040 & 562 & 405 \\
\hline 1971 & 193 & 116 & 105 & 96.3 & 88.8 & 102 & 175 & 410 & 836 & 885 & 816 & 396 & 353 \\
\hline
\end{tabular}




\section{1-5.R00-1A SURKHRUD RIVER AT SULTANPUR}

(U.S. Geological Survey identification number: 342500070180000)

LOCATION: Lat $34^{\circ} 25^{\prime} \mathrm{N}$., long $70^{\circ} 18^{\prime} \mathrm{E}$.

DRAINAGE AREA: $2,590 \mathrm{~km}^{2}$.

ELEVATION: 700 meters above mean sea level.

PERIOD OF RECORD: March 8, 1968 to September 30, 1975 and January 15, 1976 to March 31, 1980.

GAGE: Water-stage recorder.

Annual mean discharge

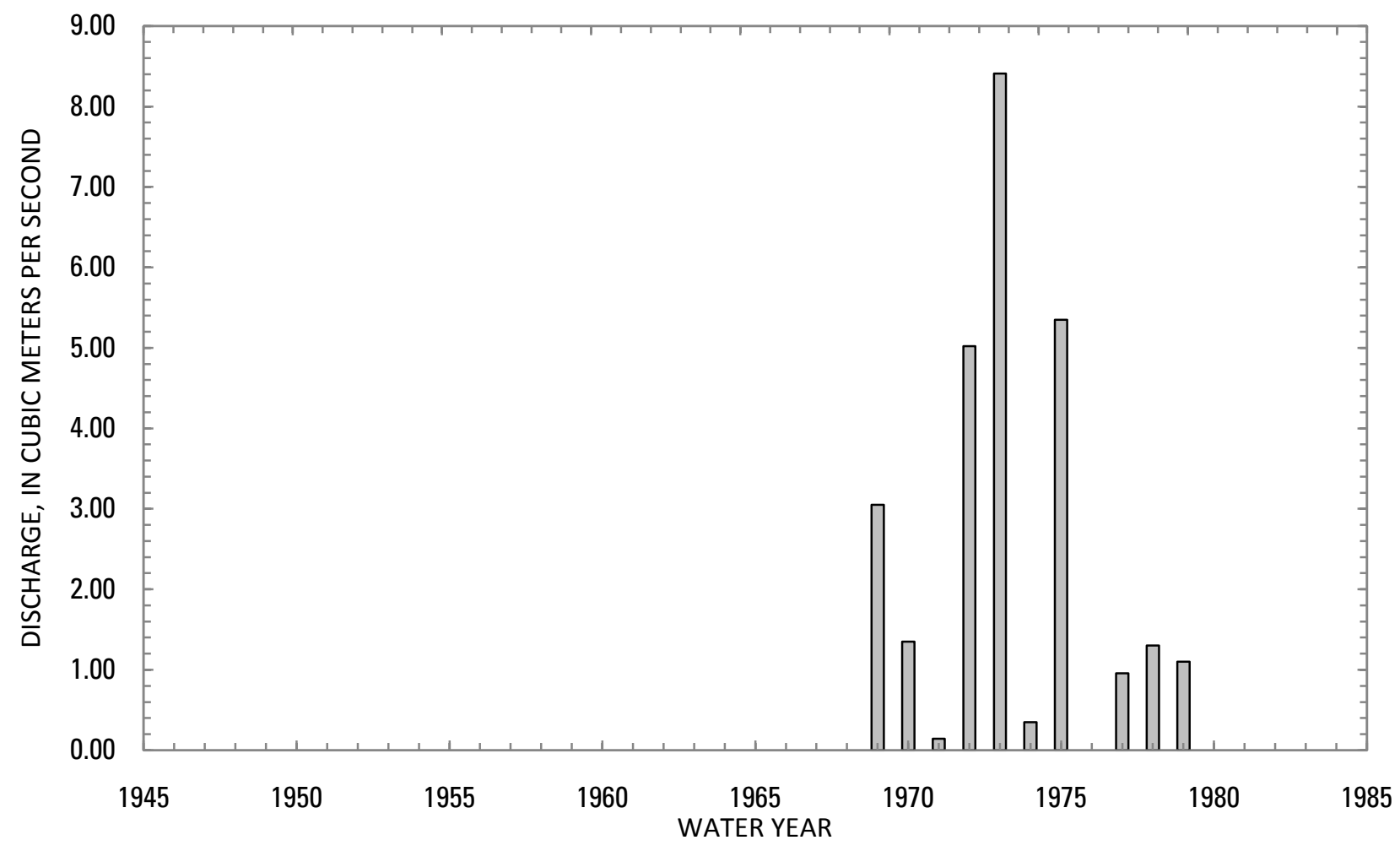




\section{1-5.R00-1A SURKHRUD RIVER NEAR SULTANPUR, Continued}

Statistics of monthly and annual mean discharges

[ $\mathrm{m}^{3} / \mathrm{s}$, cubic meters per second; ng, not given]

\begin{tabular}{|c|c|c|c|c|c|c|c|c|}
\hline \multirow[b]{2}{*}{ Month } & \multicolumn{2}{|c|}{ Maximum } & \multicolumn{2}{|c|}{ Minimum } & \multicolumn{4}{|c|}{ Mean } \\
\hline & $\begin{array}{c}\text { Discharge } \\
\left(\mathrm{m}^{3} / \mathrm{s}\right)\end{array}$ & $\begin{array}{c}\text { Water year } \\
\text { of } \\
\text { occurrence }\end{array}$ & $\begin{array}{c}\text { Discharge } \\
\left(\mathrm{m}^{3} / \mathrm{s}\right)\end{array}$ & $\begin{array}{c}\text { Water year } \\
\text { of } \\
\text { occurrence }\end{array}$ & $\begin{array}{c}\text { Discharge } \\
\left(\mathrm{m}^{3} / \mathrm{s}\right)\end{array}$ & $\begin{array}{c}\text { Standard } \\
\text { deviation } \\
\left(\mathrm{m}^{3} / \mathrm{s}\right)\end{array}$ & $\begin{array}{c}\text { Coefficient } \\
\text { of } \\
\text { variation }\end{array}$ & $\begin{array}{c}\text { Percentage } \\
\text { of annual } \\
\text { discharge }\end{array}$ \\
\hline October & 1.33 & 1969 & 0.01 & 1972 & 0.49 & 0.39 & 0.79 & 1.40 \\
\hline November & 2.71 & 1970 & 0.07 & 1972 & 0.82 & 0.9 & 1.09 & 2.36 \\
\hline December & 3.13 & 1969 & 0.09 & 1972 & 1.09 & 1.04 & 0.95 & 3.12 \\
\hline January & 2.01 & 1973 & 0.18 & 1971 & 0.89 & 0.65 & 0.74 & 2.54 \\
\hline February & 2.47 & 1979 & 0.15 & 1974 & 1.18 & 0.87 & 0.74 & 3.38 \\
\hline March & 8.16 & 1973 & 0.18 & 1971 & 1.92 & 2.25 & 1.17 & 5.51 \\
\hline April & 22.6 & 1973 & 0.35 & 1974 & 7.23 & 6.62 & 0.92 & 20.7 \\
\hline May & 27.5 & 1972 & 0.14 & 1971 & 11.0 & 11.4 & 1.04 & 31.6 \\
\hline June & 24.1 & 1973 & 0 & 1971 & 6.63 & 8.38 & 1.27 & 19.0 \\
\hline July & 8.98 & 1973 & 0 & ng & 1.83 & 2.57 & 1.41 & 5.24 \\
\hline August & 5.50 & 1975 & 0 & ng & 1.33 & 1.88 & 1.41 & 3.81 \\
\hline September & 1.35 & 1972 & 0 & ng & 0.48 & 0.47 & 0.98 & 1.38 \\
\hline Annual & 8.41 & 1973 & 0.15 & 1971 & 2.70 & 2.72 & 1.01 & 100 \\
\hline
\end{tabular}


1-5.R00-1A SURKHRUD RIVER NEAR SULTANPUR, Continued

Monthly and annual flow duration, in cubic meters per second

\begin{tabular}{|c|c|c|c|c|c|c|c|c|c|c|c|c|c|}
\hline \multirow{2}{*}{$\begin{array}{l}\text { Percentage } \\
\text { of days } \\
\text { discharge } \\
\text { equaled or } \\
\text { exceeded }\end{array}$} & \multicolumn{12}{|c|}{ Month } & \multirow{2}{*}{ Annual } \\
\hline & October & November & December & January & February & March & April & May & June & July & August & September & \\
\hline 95 & 0 & 0.09 & 0.09 & 0.09 & 0.13 & 0.14 & 0.20 & 0 & 0 & 0 & 0 & 0 & 0 \\
\hline 90 & 0.02 & 0.12 & 0.16 & 0.18 & 0.18 & 0.18 & 0.26 & 0.12 & 0.01 & 0 & 0 & 0 & 0 \\
\hline 85 & 0.10 & 0.16 & 0.18 & 0.19 & 0.20 & 0.19 & 0.34 & 0.17 & 0.07 & 0 & 0 & 0 & 0.05 \\
\hline 80 & 0.13 & 0.18 & 0.18 & 0.21 & 0.25 & 0.21 & 0.39 & 0.21 & 0.11 & 0 & 0 & 0 & 0.11 \\
\hline 75 & 0.18 & 0.19 & 0.23 & 0.26 & 0.30 & 0.25 & 0.65 & 0.41 & 0.16 & 0 & 0 & 0 & 0.18 \\
\hline 70 & 0.21 & 0.20 & 0.35 & 0.32 & 0.33 & 0.31 & 1.01 & 0.89 & 0.29 & 0 & 0 & 0 & 0.20 \\
\hline 65 & 0.22 & 0.21 & 0.42 & 0.37 & 0.36 & 0.35 & 1.35 & 1.49 & 0.43 & 0 & 0 & 0.01 & 0.22 \\
\hline 60 & 0.23 & 0.29 & 0.53 & 0.41 & 0.41 & 0.41 & 1.76 & 2.07 & 0.57 & 0.13 & 0.08 & 0.09 & 0.29 \\
\hline 55 & 0.25 & 0.39 & 0.61 & 0.47 & 0.55 & 0.58 & 2.29 & 3.11 & 0.78 & 0.42 & 0.15 & 0.17 & 0.37 \\
\hline 50 & 0.28 & 0.47 & 0.71 & 0.58 & 0.81 & 0.71 & 3.30 & 5.18 & 1.61 & 0.52 & 0.18 & 0.19 & 0.45 \\
\hline 45 & 0.31 & 0.62 & 0.85 & 0.74 & 1.02 & 0.89 & 4.95 & 9.63 & 2.63 & 0.66 & 0.20 & 0.22 & 0.56 \\
\hline 40 & 0.36 & 0.68 & 0.96 & 0.90 & 1.23 & 1.23 & 6.01 & 13.0 & 5.20 & 0.84 & 0.22 & 0.27 & 0.73 \\
\hline 35 & 0.51 & 0.74 & 1.04 & 1.00 & 1.38 & 1.64 & 6.98 & 15.7 & 7.63 & 0.94 & 0.31 & 0.31 & 0.94 \\
\hline 30 & 0.65 & 0.82 & 1.26 & 1.22 & 1.51 & 1.95 & 7.83 & 18.0 & 9.21 & 1.11 & 0.36 & 0.38 & 1.19 \\
\hline 25 & 0.74 & 1.01 & 1.44 & 1.45 & 1.67 & 2.13 & 9.83 & 20.2 & 11.2 & 1.53 & 0.44 & 0.53 & 1.65 \\
\hline 20 & 0.87 & 1.27 & 2.08 & 1.60 & 1.83 & 2.37 & 13.1 & 23.3 & 14.2 & 1.91 & 0.58 & 0.60 & 2.21 \\
\hline 15 & 0.93 & 1.91 & 2.47 & 1.94 & 2.07 & 2.66 & 17.8 & 26.9 & 18.0 & 3.35 & 0.91 & 0.72 & 3.16 \\
\hline 10 & 0.99 & 2.37 & 3.03 & 2.18 & 2.34 & 4.17 & 21.3 & 29.7 & 22.2 & 5.27 & 1.28 & 0.86 & 7.56 \\
\hline 5 & 1.35 & 3.05 & 3.37 & 2.46 & 2.64 & 9.03 & 25.6 & 32.5 & 25.3 & 9.21 & 3.55 & 1.08 & 18.9 \\
\hline
\end{tabular}




\section{1-5.R00-1A SURKHRUD RIVER NEAR SULTANPUR, Continued}

Probability of occurrence of annual high discharges

[ $\mathrm{m}^{3} / \mathrm{s}$, cubic meters per second; ng, not given]

\begin{tabular}{|c|c|c|c|c|c|c|}
\hline \multirow{2}{*}{$\begin{array}{l}\text { Exceedance } \\
\text { probability }\end{array}$} & \multirow{2}{*}{$\begin{array}{c}\text { Recurrence } \\
\text { interval } \\
\text { (years) }\end{array}$} & \multirow{2}{*}{$\begin{array}{l}\text { Maximum } \\
\text { instantaneous } \\
\text { discharge } \\
\left(\mathrm{m}^{3} / \mathrm{s}\right)\end{array}$} & \multicolumn{4}{|c|}{ Maximum daily mean discharge $\left(\mathrm{m}^{3} / \mathrm{s}\right)$} \\
\hline & & & $\begin{array}{l}\text { 3-day } \\
\text { period }\end{array}$ & $\begin{array}{l}\text { 7-day } \\
\text { period }\end{array}$ & $\begin{array}{l}\text { 15-day } \\
\text { period }\end{array}$ & $\begin{array}{l}\text { 30-day } \\
\text { period }\end{array}$ \\
\hline 0.99 & 1.01 & 1.40 & 0.35 & 0.35 & 0.25 & 0.17 \\
\hline 0.95 & 1.05 & 7.80 & 1.45 & 1.21 & 0.84 & 0.58 \\
\hline 0.90 & 1.11 & 16.3 & 2.81 & 2.18 & 1.53 & 1.06 \\
\hline 0.80 & 1.25 & 33.9 & 5.70 & 4.20 & 3.00 & 2.10 \\
\hline 0.50 & 2 & 88.7 & 17.1 & 12.3 & 9.33 & 6.99 \\
\hline 0.20 & 5 & 144 & 37.4 & 28.6 & 24.1 & 20.2 \\
\hline 0.10 & 10 & 162 & 50.6 & 41.0 & 37.0 & 33.3 \\
\hline 0.04 & 25 & 173 & 65.0 & 57.0 & 55.4 & 54.5 \\
\hline 0.02 & 50 & 176 & 73.8 & ${ }^{1} 68.4$ & ${ }^{1} 70.2$ & 73.4 \\
\hline 0.01 & 100 & 178 & ' 81.2 & ${ }^{1} 79.2$ & ${ }^{1} 85.4$ & 94.6 \\
\hline 0.005 & 200 & 179 & ${ }^{1} 87.2$ & ${ }^{1} 89.2$ & ${ }^{1} 101$ & 118 \\
\hline 0.002 & 500 & 179 & $\mathrm{ng}$ & $\mathrm{ng}$ & $\mathrm{ng}$ & $\mathrm{ng}$ \\
\hline
\end{tabular}

'Data does not fit log-Pearson Type III curve, use with caution.

\section{1-5.R00-1A SURKHRUD RIVER NEAR SULTANPUR, Continued}

Probability of occurrence of annual low discharges

$\left[\mathrm{m}^{3} / \mathrm{s}\right.$, meters per second]

\begin{tabular}{|c|c|c|c|c|c|c|c|c|c|c|}
\hline \multirow{3}{*}{$\begin{array}{c}\text { Nonexceedance } \\
\text { probability }\end{array}$} & \multirow{3}{*}{$\begin{array}{c}\text { Recurrence } \\
\text { interval } \\
\text { (years) }\end{array}$} & \multicolumn{9}{|c|}{ Minimum daily mean discharge $\left(\mathrm{m}^{3} / \mathrm{s}\right)$} \\
\hline & & \multicolumn{9}{|c|}{ Number of consecutive days } \\
\hline & & 1 & 3 & 7 & 14 & 30 & 60 & 90 & 120 & 183 \\
\hline 0.05 & 20 & 0 & 0 & 0 & 0 & 0 & 0 & 0 & 0 & 0.01 \\
\hline 0.10 & 10 & 0 & 0 & 0 & 0 & 0 & 0 & 0 & 0 & 0.03 \\
\hline 0.20 & 5 & 0 & 0 & 0 & 0 & 0 & 0 & 0.01 & 0.05 & 0.10 \\
\hline 0.50 & 2 & 0 & 0 & 0 & 0.07 & 0.10 & 0.15 & 0.20 & 0.30 & 0.47 \\
\hline
\end{tabular}




\section{1-5.R00-1A SURKHRUD RIVER NEAR SULTANPUR, Continued}

Probability of occurrence of seasonal low discharges

[ $\mathrm{m}^{3} / \mathrm{s}$, meters per second]

\begin{tabular}{|c|c|c|c|c|c|c|c|c|c|}
\hline \multirow{3}{*}{$\begin{array}{c}\text { Nonexceedance } \\
\text { probability }\end{array}$} & \multirow{3}{*}{$\begin{array}{c}\text { Recurrence } \\
\text { interval } \\
\text { (years) }\end{array}$} & \multicolumn{8}{|c|}{ Minimum daily mean discharge $\left(\mathrm{m}^{3} / \mathrm{s}\right)$} \\
\hline & & \multicolumn{8}{|c|}{ Number of consecutive days } \\
\hline & & 1 & 7 & 14 & 30 & 1 & 7 & 14 & 30 \\
\hline & & \multicolumn{4}{|c|}{ December-January-February } & \multicolumn{4}{|c|}{ March-April-May } \\
\hline 0.05 & 20 & 0.02 & 0.05 & 0.06 & 0.07 & 0 & 0 & 0 & 0.09 \\
\hline 0.10 & 10 & 0.04 & 0.07 & 0.08 & 0.11 & 0.02 & 0.05 & 0.06 & 0.15 \\
\hline 0.20 & 5 & 0.06 & 0.10 & 0.13 & 0.17 & 0.05 & 0.11 & 0.14 & 0.28 \\
\hline \multirow[t]{2}{*}{0.50} & 2 & 0.16 & 0.23 & 0.29 & 0.40 & 0.15 & 0.29 & 0.38 & 0.86 \\
\hline & & \multicolumn{4}{|c|}{ June-July-August } & \multicolumn{4}{|c|}{ September-0ctober-November } \\
\hline 0.05 & 20 & 0 & 0 & 0 & 0 & 0 & 0 & 0 & 0 \\
\hline 0.10 & 10 & 0 & 0 & 0 & 0 & 0 & 0 & 0 & 0 \\
\hline 0.20 & 5 & 0 & 0 & 0 & 0 & 0 & 0 & 0 & 0.01 \\
\hline 0.50 & 2 & 0 & 0.01 & 0.10 & 0.24 & 0 & 0.04 & 0.10 & 0.18 \\
\hline
\end{tabular}

\section{1-5.R00-1A SURKHRUD RIVER NEAR SULTANPUR, Continued}

Annual peak discharges

[ $\mathrm{m}^{3} / \mathrm{s}$, meters per second]

\begin{tabular}{|c|c|c|c|c|c|}
\hline \multicolumn{3}{|c|}{$\begin{array}{c}\text { Annual peak discharge, } \\
\text { by year }\end{array}$} & \multicolumn{3}{|c|}{$\begin{array}{l}\text { Annual peak discharge, } \\
\text { from highest to lowest }\end{array}$} \\
\hline $\begin{array}{l}\text { Water } \\
\text { year }\end{array}$ & Date & $\begin{array}{c}\text { Peak } \\
\text { discharge } \\
\left(\mathrm{m}^{3} / \mathrm{s}\right)\end{array}$ & $\begin{array}{l}\text { Water } \\
\text { year }\end{array}$ & Date & $\begin{array}{c}\text { Peak } \\
\text { discharge } \\
\left(\mathrm{m}^{3} / \mathrm{s}\right)\end{array}$ \\
\hline 1968 & March 10, 1968 & 62.8 & 1975 & August 13, 1975 & 281 \\
\hline 1969 & May 22, 1969 & 41.8 & 1978 & August 16, 1978 & 117 \\
\hline 1970 & April 16, 1970 & 105 & 1971 & April 27, 1971 & 115 \\
\hline 1971 & April 27, 1971 & 115 & 1970 & $\begin{array}{l}\text { April 16, } 1970 \\
\text { September } 18,\end{array}$ & 105 \\
\hline 1972 & September 18, 1972 & 80.0 & 1972 & 1972 & 80.0 \\
\hline 1973 & April 25, 1973 & 47.0 & 1979 & February 18, 1979 & 74.8 \\
\hline 1974 & April 8, 1974 & 3.36 & 1977 & April 4, 1977 & 73.2 \\
\hline 1975 & August 13, 1975 & 281 & 1976 & August 15, 1976 & 64.1 \\
\hline 1976 & August 15, 1976 & 64.1 & 1968 & March 10, 1968 & 62.8 \\
\hline 1977 & April 4, 1977 & 73.2 & 1973 & April 25, 1973 & 47.0 \\
\hline 1978 & August 16, 1978 & 117 & 1969 & May 22, 1969 & 41.8 \\
\hline 1979 & February 18,1979 & 74.8 & 1974 & April 8, 1974 & 3.36 \\
\hline
\end{tabular}


1-5.R00-1A SURKHRUD RIVER NEAR SULTANPUR, Continued

Monthly and annual mean discharges, in cubic meters per second

[Data may not be rounded in accordance with U.S. Geological Survey publication standards; --, no data]

\begin{tabular}{|c|c|c|c|c|c|c|c|c|c|c|c|c|c|}
\hline \multirow{2}{*}{$\begin{array}{l}\text { Water } \\
\text { year }\end{array}$} & \multicolumn{12}{|c|}{ Monthly mean discharge } & \multirow{2}{*}{$\begin{array}{c}\text { Annual } \\
\text { discharge }\end{array}$} \\
\hline & October & November & December & January & February & March & April & May & June & July & August & September & \\
\hline 1968 & -- & -- & -- & -- & -- & -- & 8.20 & 18.4 & 14.1 & 4.28 & 0.74 & 0.43 & -- \\
\hline 1969 & 1.33 & 2.35 & 3.13 & 2.00 & 1.92 & 1.14 & 6.91 & 10.4 & 5.87 & 1.32 & 0.24 & 0 & 3.05 \\
\hline 1970 & 0.39 & 2.71 & 1.95 & 0.92 & 0.97 & 0.84 & 5.75 & 2.49 & 0.19 & 0 & 0 & 0.08 & 1.35 \\
\hline 1971 & 0.20 & 0.20 & 0.18 & 0.18 & 0.18 & 0.18 & 0.49 & 0.14 & 0 & 0 & 0 & 0 & 0.15 \\
\hline 1972 & 0.01 & 0.07 & 0.09 & 0.40 & 1.44 & 2.03 & 6.11 & 27.5 & 19.6 & 1.45 & 0.27 & 1.35 & 5.02 \\
\hline 1973 & 1.00 & 1.05 & 2.61 & 2.01 & 2.22 & 8.16 & 22.6 & 27.5 & 24.1 & 8.98 & 0.28 & 0.25 & 8.41 \\
\hline 1974 & 0.62 & 0.79 & 1.11 & 0.54 & 0.15 & 0.20 & 0.35 & 0.17 & 0.21 & 0 & 0 & 0.01 & 0.35 \\
\hline 1975 & 0.15 & 0.23 & 0.36 & 0.33 & 0.36 & 3.84 & 15.5 & 27.3 & 8.25 & 1.25 & 5.50 & 0.72 & 5.35 \\
\hline 1976 & -- & -- & -- & -- & 2.11 & 1.83 & 11.9 & 13.0 & 5.75 & 0.98 & 1.47 & 1.28 & -- \\
\hline 1977 & 0.50 & 0.50 & 0.72 & 1.23 & 0.49 & 0.27 & 2.49 & 0.93 & 0.65 & 0.96 & 2.36 & 0.34 & 0.96 \\
\hline 1978 & 0.55 & 0.34 & 0.54 & 0.44 & 0.35 & 0.45 & 1.92 & 2.30 & 0.68 & 2.57 & 4.60 & 0.65 & 1.30 \\
\hline 1979 & 0.29 & 0.18 & 0.22 & 0.48 & 2.47 & 1.66 & 4.53 & 2.09 & 0.13 & 0.15 & 0.51 & 0.68 & 1.10 \\
\hline 1980 & 0.36 & 0.66 & 1.08 & 1.23 & 1.51 & 2.48 & -- & -- & -- & -- & -- & -- & -- \\
\hline
\end{tabular}




\section{1-6.L00-1A LAGHMAN RIVER AT PUL-I-0ARGHAI}

(U.S. Geological Survey identification number: 343300070140000)

LOCATION: Lat $34^{\circ} 33^{\prime} \mathrm{N}$., long $70^{\circ} 14^{\prime} \mathrm{E}$.

DRAINAGE AREA: $6,155 \mathrm{~km}^{2}$.

ELEVATION: 640 meters above mean sea level.

PERIOD OF RECORD: October 1, 1960 to September 30, 1979.

GAGE: Water-stage recorder.

Annual mean discharge

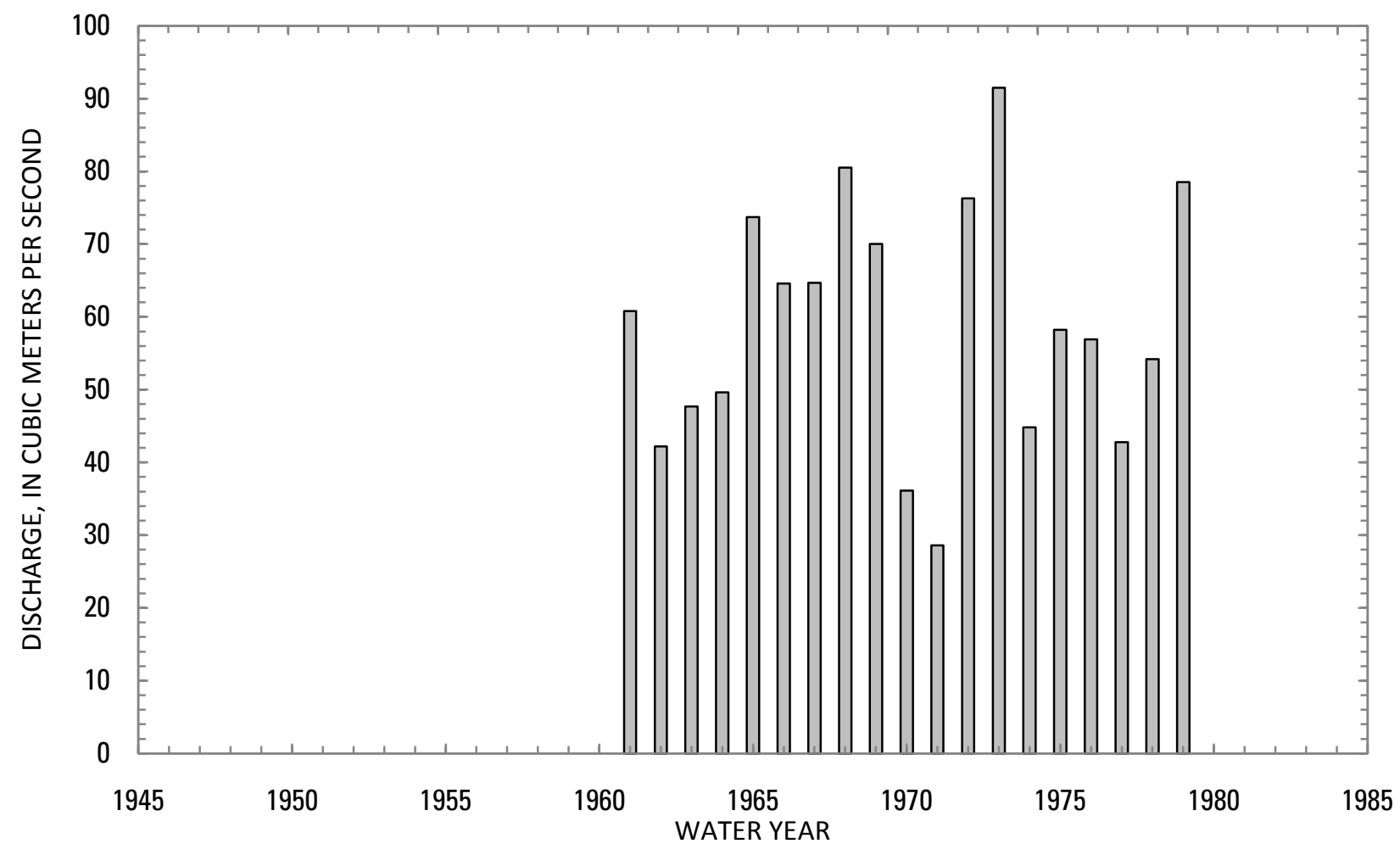




\section{1-6.L00-1A LAGHMAN RIVER AT PUL-I-QARGHAI, Continued}

Statistics of monthly and annual mean discharges

$\left[\mathrm{m}^{3} / \mathrm{s}\right.$, cubic meters per second]

\begin{tabular}{|c|c|c|c|c|c|c|c|c|}
\hline \multirow[b]{2}{*}{ Month } & \multicolumn{2}{|c|}{ Maximum } & \multicolumn{2}{|c|}{ Minimum } & \multicolumn{4}{|c|}{ Mean } \\
\hline & $\begin{array}{c}\text { Discharge } \\
\left(\mathrm{m}^{3} / \mathrm{s}\right)\end{array}$ & $\begin{array}{c}\text { Water year } \\
\text { of } \\
\text { occurrence }\end{array}$ & $\begin{array}{c}\text { Discharge } \\
\left(\mathrm{m}^{3} / \mathrm{s}\right)\end{array}$ & $\begin{array}{c}\text { Water year } \\
\text { of } \\
\text { occurrence }\end{array}$ & $\begin{array}{c}\text { Discharge } \\
\left(\mathrm{m}^{3} / \mathrm{s}\right)\end{array}$ & $\begin{array}{c}\text { Standard } \\
\text { deviation } \\
\left(\mathrm{m}^{3} / \mathrm{s}\right)\end{array}$ & $\begin{array}{c}\text { Coefficient } \\
\text { of } \\
\text { variation }\end{array}$ & $\begin{array}{c}\text { Percentage } \\
\text { of annual } \\
\text { discharge }\end{array}$ \\
\hline October & 18.6 & 1962 & 1.50 & 1972 & 6.37 & 4.33 & 0.68 & 0.90 \\
\hline November & 15.2 & 1962 & 1.23 & 1972 & 5.41 & 3.10 & 0.57 & 0.76 \\
\hline December & 14.0 & 1963 & 0.95 & 1972 & 5.09 & 3.43 & 0.67 & 0.72 \\
\hline January & 12.5 & 1962 & 1.23 & 1971 & 4.67 & 2.69 & 0.58 & 0.66 \\
\hline February & 23.1 & 1972 & 1.79 & 1971 & 6.38 & 5.08 & 0.80 & 0.90 \\
\hline March & 58.2 & 1969 & 4.88 & 1970 & 24.4 & 15.5 & 0.63 & 3.45 \\
\hline April & 203 & 1973 & 46.6 & 1962 & 106 & 35.7 & 0.34 & 15.0 \\
\hline May & 282 & 1973 & 55.9 & 1962 & 169 & 47.8 & 0.28 & 23.9 \\
\hline June & 358 & 1972 & 81.5 & 1971 & 225 & 70.3 & 0.31 & 31.8 \\
\hline July & 196 & 1965 & 13.8 & 1971 & 112 & 51.00 & 0.46 & 15.8 \\
\hline August & 67.5 & 1979 & 9.02 & 1971 & 33.0 & 19.0 & 0.58 & 4.67 \\
\hline September & 27.6 & 1961 & 2.79 & 1962 & 10.1 & 6.17 & 0.61 & 1.43 \\
\hline Annual & 91.5 & 1973 & 28.6 & 1971 & 59.0 & 16.7 & 0.28 & 100 \\
\hline
\end{tabular}


1-6.L00-1A LAGHMAN RIVER AT PUL-I-QARGHAI, Continued

Monthly and annual flow duration, in cubic meters per second

\begin{tabular}{|c|c|c|c|c|c|c|c|c|c|c|c|c|c|}
\hline \multirow{2}{*}{$\begin{array}{l}\text { Percentage } \\
\text { of days } \\
\text { discharge } \\
\text { equaled or } \\
\text { exceeded }\end{array}$} & \multicolumn{12}{|c|}{ Month } & \multirow{2}{*}{ Annual } \\
\hline & October & November & December & January & February & March & April & May & June & July & August & September & \\
\hline 95 & 1.67 & 1.30 & 1.06 & 1.03 & 1.89 & 2.92 & 30.1 & 64.7 & 88.9 & 14.4 & 7.23 & 2.05 & 2.11 \\
\hline 90 & 1.92 & 2.20 & 2.04 & 1.90 & 2.11 & 3.99 & 39.3 & 90.3 & 125 & 22.0 & 8.29 & 2.98 & 2.89 \\
\hline 85 & 2.01 & 2.86 & 2.10 & 2.00 & 2.65 & 4.93 & 50.2 & 106 & 150 & 32.3 & 10.2 & 4.18 & 3.48 \\
\hline 80 & 2.78 & 3.01 & 2.82 & 2.60 & 2.96 & 5.62 & 57.9 & 114 & 167 & 48.0 & 11.4 & 4.78 & 4.28 \\
\hline 75 & 3.46 & 3.20 & 2.96 & 2.84 & 3.13 & 6.83 & 65.0 & 121 & 176 & 57.9 & 12.9 & 5.31 & 4.80 \\
\hline 70 & 4.07 & 3.50 & 3.15 & 3.04 & 3.32 & 8.31 & 71.4 & 130 & 184 & 67.2 & 15.5 & 5.90 & 5.40 \\
\hline 65 & 4.37 & 4.14 & 3.44 & 3.27 & 3.61 & 10.5 & 77.6 & 140 & 193 & 77.5 & 18.8 & 6.41 & 6.21 \\
\hline 60 & 4.59 & 4.42 & 3.74 & 3.50 & 3.99 & 12.8 & 83.8 & 152 & 202 & 89.1 & 21.0 & 6.95 & 7.49 \\
\hline 55 & 4.89 & 4.61 & 4.33 & 3.82 & 4.60 & 15.3 & 90.0 & 164 & 209 & 97.3 & 23.6 & 7.55 & 9.32 \\
\hline 50 & 5.23 & 4.81 & 4.59 & 4.14 & 4.79 & 18.3 & 96.2 & 173 & 216 & 105 & 26.3 & 8.27 & 12.5 \\
\hline 45 & 5.52 & 5.01 & 4.79 & 4.42 & 4.97 & 20.0 & 103 & 181 & 226 & 113 & 30.0 & 9.50 & 17.7 \\
\hline 40 & 5.87 & 5.23 & 4.93 & 4.67 & 5.45 & 21.8 & 111 & 190 & 237 & 126 & 32.7 & 10.3 & 25.4 \\
\hline 35 & 6.39 & 5.46 & 5.07 & 4.82 & 5.90 & 23.7 & 118 & 198 & 251 & 136 & 36.1 & 10.8 & 40.8 \\
\hline 30 & 7.10 & 5.79 & 5.27 & 4.97 & 6.41 & 26.9 & 126 & 203 & 266 & 146 & 39.8 & 11.6 & 64.4 \\
\hline 25 & 7.62 & 6.23 & 5.57 & 5.12 & 7.34 & 30.8 & 135 & 207 & 284 & 157 & 44.8 & 13.0 & 92.4 \\
\hline 20 & 8.12 & 6.67 & 5.94 & 5.55 & 8.90 & 40.7 & 144 & 214 & 300 & 168 & 53.5 & 14.8 & 124 \\
\hline 15 & 9.65 & 7.05 & 6.94 & 5.96 & 10.6 & 48.7 & 161 & 225 & 316 & 186 & 60.9 & 16.3 & 159 \\
\hline 10 & 12.2 & 9.11 & 11.1 & 7.97 & 12.7 & 56.4 & 185 & 247 & 343 & 209 & 71.3 & 18.6 & 195 \\
\hline 5 & 17.8 & 12.9 & 14.0 & 12.1 & 16.3 & 70.1 & 224 & 278 & 372 & 238 & 81.5 & 25.6 & 239 \\
\hline
\end{tabular}




\section{1-6.L00-1A LAGHMAN RIVER AT PUL-I-QARGHAI, Continued}

Probability of occurrence of annual high discharges

[m $\mathrm{m}^{3} / \mathrm{s}$, cubic meters per second; ng, not given]

\begin{tabular}{|c|c|c|c|c|c|c|}
\hline \multirow{2}{*}{$\begin{array}{l}\text { Exceedance } \\
\text { probability }\end{array}$} & \multirow{2}{*}{$\begin{array}{c}\text { Recurrence } \\
\text { interval } \\
\text { (years) }\end{array}$} & \multirow{2}{*}{$\begin{array}{c}\text { Maximum } \\
\text { instantaneous } \\
\text { discharge } \\
\left(\mathrm{m}^{3} / \mathrm{s}\right)\end{array}$} & \multicolumn{4}{|c|}{ Maximum daily mean discharge $\left(\mathrm{m}^{3} / \mathrm{s}\right)$} \\
\hline & & & $\begin{array}{l}\text { 3-day } \\
\text { period }\end{array}$ & $\begin{array}{l}\text { 7-day } \\
\text { period }\end{array}$ & $\begin{array}{l}\text { 15-day } \\
\text { period }\end{array}$ & $\begin{array}{l}\text { 30-day } \\
\text { period }\end{array}$ \\
\hline 0.99 & 1.01 & 222 & 177 & 161 & 155 & 148 \\
\hline 0.95 & 1.05 & 263 & 202 & 184 & 175 & 165 \\
\hline 0.90 & 1.11 & 288 & 218 & 199 & 188 & 176 \\
\hline 0.80 & 1.25 & 320 & 239 & 219 & 206 & 192 \\
\hline 0.50 & 2 & 389 & 288 & 266 & 248 & 229 \\
\hline 0.20 & 5 & 470 & 349 & 327 & 304 & 280 \\
\hline 0.10 & 10 & 517 & 388 & 367 & 342 & 315 \\
\hline 0.04 & 25 & 571 & 436 & 417 & 390 & 359 \\
\hline 0.02 & 50 & 609 & 471 & 454 & 426 & 393 \\
\hline 0.01 & 100 & 644 & 505 & 491 & 462 & 427 \\
\hline 0.005 & 200 & 677 & 539 & 528 & 499 & 462 \\
\hline 0.002 & 500 & 720 & $\mathrm{ng}$ & $\mathrm{ng}$ & ng & $\mathrm{ng}$ \\
\hline
\end{tabular}

\section{1-6.L00-1A LAGHMAN RIVER AT PUL-I-QARGHAI, Continued}

Probability of occurrence of annual low discharges

[ $\mathrm{m}^{3} / \mathrm{s}$, meters per second]

\begin{tabular}{|c|c|c|c|c|c|c|c|c|c|c|}
\hline \multirow{3}{*}{$\begin{array}{c}\text { Nonexceedance } \\
\text { probability }\end{array}$} & \multirow{3}{*}{$\begin{array}{c}\text { Recurrence } \\
\text { interval } \\
\text { (years) }\end{array}$} & \multicolumn{9}{|c|}{ Minimum daily mean discharge $\left(\mathrm{m}^{3} / \mathrm{s}\right)$} \\
\hline & & \multicolumn{9}{|c|}{ Number of consecutive days } \\
\hline & & 1 & 3 & 7 & 14 & 30 & 60 & 90 & 120 & 183 \\
\hline 0.05 & 20 & 1.01 & 1.01 & 1.03 & 1.05 & 1.07 & 1.24 & 1.41 & 1.58 & 2.49 \\
\hline 0.10 & 10 & 1.31 & 1.32 & 1.34 & 1.37 & 1.40 & 1.60 & 1.79 & 2.01 & 2.93 \\
\hline 0.20 & 5 & 1.75 & 1.76 & 1.80 & 1.84 & 1.91 & 2.13 & 2.36 & 2.64 & 3.56 \\
\hline 0.50 & 2 & 2.85 & 2.89 & 2.95 & 3.02 & 3.18 & 3.47 & 3.77 & 4.19 & 5.19 \\
\hline
\end{tabular}




\section{1-6.L00-1A LAGHMAN RIVER AT PUL-I-QARGHAI, Continued}

Probability of occurrence of seasonal low discharges

[ $\mathrm{m}^{3} / \mathrm{s}$, meters per second]

\begin{tabular}{|c|c|c|c|c|c|c|c|c|c|}
\hline \multirow{3}{*}{$\begin{array}{c}\text { Nonexceedance } \\
\text { probability }\end{array}$} & \multirow{3}{*}{$\begin{array}{c}\text { Recurrence } \\
\text { interval } \\
\text { (years) }\end{array}$} & \multicolumn{8}{|c|}{ Minimum daily mean discharge $\left(\mathrm{m}^{3} / \mathrm{s}\right)$} \\
\hline & & \multicolumn{8}{|c|}{ Number of consecutive days } \\
\hline & & 1 & 7 & 14 & 30 & 1 & 7 & 14 & 30 \\
\hline & & \multicolumn{4}{|c|}{ December-January-February } & \multicolumn{4}{|c|}{ March-April-May } \\
\hline 0.05 & 20 & 1.08 & 1.10 & 1.10 & 1.12 & 1.86 & 2.46 & 2.75 & 5.85 \\
\hline 0.10 & 10 & 1.40 & 1.44 & 1.44 & 1.47 & 2.30 & 3.05 & 3.60 & 7.75 \\
\hline 0.20 & 5 & 1.88 & 1.95 & 1.96 & 2.01 & 3.04 & 4.02 & 5.01 & 10.8 \\
\hline \multirow[t]{2}{*}{0.50} & 2 & 3.01 & 3.19 & 3.25 & 3.40 & 5.55 & 7.26 & 9.60 & 19.7 \\
\hline & & \multicolumn{4}{|c|}{ June-July-August } & \multicolumn{4}{|c|}{ September-0ctober-November } \\
\hline 0.05 & 20 & 4.73 & 5.51 & 6.52 & 8.10 & 1.31 & 1.35 & 1.42 & 1.49 \\
\hline 0.10 & 10 & 5.88 & 6.92 & 8.32 & 10.9 & 1.68 & 1.71 & 1.80 & 1.89 \\
\hline 0.20 & 5 & 7.64 & 9.10 & 11.1 & 15.3 & 2.24 & 2.25 & 2.39 & 2.53 \\
\hline 0.50 & 2 & 12.6 & 15.3 & 18.9 & 27.9 & 3.70 & 3.76 & 3.99 & 4.34 \\
\hline
\end{tabular}




\section{1-6.L00-1A LAGHMAN RIVER AT PUL-I-QARGHAI, Continued}

Annual peak discharges

$\left[\mathrm{m}^{3} / \mathrm{s}\right.$, meters per second]

\begin{tabular}{|c|c|c|c|c|c|}
\hline \multicolumn{3}{|c|}{$\begin{array}{c}\text { Annual peak discharge, } \\
\text { by year }\end{array}$} & \multicolumn{3}{|c|}{$\begin{array}{l}\text { Annual peak discharge, } \\
\text { from highest to lowest }\end{array}$} \\
\hline $\begin{array}{l}\text { Water } \\
\text { year }\end{array}$ & Date & $\begin{array}{c}\text { Peak } \\
\text { discharge } \\
\left(\mathrm{m}^{3} / \mathrm{s}\right)\end{array}$ & $\begin{array}{l}\text { Water } \\
\text { year }\end{array}$ & Date & $\begin{array}{c}\text { Peak } \\
\text { discharge } \\
\left(\mathrm{m}^{3} / \mathrm{s}\right)\end{array}$ \\
\hline 1961 & May 6, 1961 & 250 & 1968 & April 30, 1968 & 592 \\
\hline 1962 & June 12, 1962 & 421 & 1972 & June 16, 1972 & 519 \\
\hline 1963 & May 13, 1963 & 359 & 1967 & June 12, 1967 & 506 \\
\hline 1964 & July 3, 1964 & 450 & 1979 & June 11, 1979 & 465 \\
\hline 1965 & April 18, 1965 & 442 & 1964 & July 3, 1964 & 450 \\
\hline 1966 & June 19, 1966 & 410 & 1973 & June 3, 1973 & 444 \\
\hline 1967 & June 12, 1967 & 506 & 1965 & April 18, 1965 & 442 \\
\hline 1968 & April 30, 1968 & 592 & 1962 & June 12, 1962 & 421 \\
\hline 1969 & June 16, 1969 & 417 & 1969 & June 16, 1969 & 417 \\
\hline 1970 & May 22, 1970 & 332 & 1966 & June 19, 1966 & 410 \\
\hline 1971 & May 20, 1971 & 336 & 1963 & May 13, 1963 & 359 \\
\hline 1972 & June 16, 1972 & 519 & 1978 & July 6, 1978 & 353 \\
\hline 1973 & June 3, 1973 & 444 & 1975 & June 17, 1975 & 339 \\
\hline 1974 & May 31, 1974 & 270 & 1971 & May 20, 1971 & 336 \\
\hline 1975 & June 17, 1975 & 339 & 1976 & June 4, 1976 & 334 \\
\hline 1976 & June 4, 1976 & 334 & 1970 & May 22, 1970 & 332 \\
\hline 1977 & June 23, 1977 & 292 & 1977 & June 23, 1977 & 292 \\
\hline 1978 & July 6,1978 & 353 & 1974 & May 31, 1974 & 270 \\
\hline 1979 & June 11, 1979 & 465 & 1961 & May 6, 1961 & 250 \\
\hline
\end{tabular}


1-6.L00-1A LAGHMAN RIVER AT PUL-I-QARGHAI, Continued

Monthly and annual mean discharges, in cubic meters per second

\begin{tabular}{|c|c|c|c|c|c|c|c|c|c|c|c|c|c|}
\hline \multirow{2}{*}{$\begin{array}{l}\text { Water } \\
\text { year }\end{array}$} & \multicolumn{12}{|c|}{ Monthly mean discharge } & \multirow{2}{*}{$\begin{array}{c}\text { Annual } \\
\text { discharge }\end{array}$} \\
\hline & October & November & December & January & February & March & April & May & June & July & August & September & \\
\hline 1961 & 4.37 & 4.13 & 6.1 & 5.29 & 5.06 & 25.6 & 110 & 205 & 184 & 106 & 43.3 & 27.6 & 60.8 \\
\hline 1962 & 18.6 & 15.2 & 13.5 & 12.5 & 9.21 & 8.09 & 46.6 & 55.9 & 210 & 93.6 & 20.7 & 2.79 & 42.2 \\
\hline 1963 & 2.07 & 5.95 & 14.0 & 8.92 & 1.94 & 20.1 & 72.3 & 132 & 182 & 103 & 22.9 & 5.49 & 47.7 \\
\hline 1964 & 4.45 & 4.57 & 4.65 & 4.83 & 5.10 & 14.3 & 88.8 & 134 & 178 & 117 & 32.7 & 6.80 & 49.6 \\
\hline 1965 & 5.29 & 4.91 & 4.81 & 5.32 & 6.61 & 17.5 & 107 & 194 & 265 & 196 & 60.2 & 14.6 & 73.7 \\
\hline 1966 & 8.55 & 6.36 & 5.50 & 5.36 & 10.0 & 42.6 & 120 & 168 & 243 & 104 & 40.6 & 19.2 & 64.6 \\
\hline 1967 & 12.9 & 8.15 & 3.58 & 4.18 & 5.13 & 12.9 & 82 & 155 & 276 & 166 & 39.6 & 8.85 & 64.7 \\
\hline 1968 & 5.70 & 5.68 & 5.79 & 4.11 & 6.10 & 42.2 & 131 & 184 & 324 & 190 & 55.9 & 11.6 & 80.5 \\
\hline 1969 & 6.65 & 4.23 & 5.02 & 4.91 & 5.85 & 58.2 & 98.5 & 149 & 262 & 173 & 54.4 & 14.5 & 70.0 \\
\hline 1970 & 12.2 & 9.33 & 5.31 & 3.24 & 3.00 & 4.88 & 73.2 & 143 & 126 & 27.9 & 12.5 & 11.2 & 36.1 \\
\hline 1971 & 3.58 & 2.77 & 2.00 & 1.23 & 1.79 & 10.4 & 59.0 & 154 & 81.5 & 13.8 & 9.02 & 3.24 & 28.6 \\
\hline 1972 & 1.50 & 1.23 & 0.95 & 2.42 & 23.1 & 50.2 & 115 & 213 & 358 & 119 & 21.4 & 12.6 & 76.3 \\
\hline 1973 & 8.65 & 7.00 & 6.84 & 7.93 & 14.2 & 43.1 & 203 & 282 & 327 & 156 & 31.5 & 8.00 & 91.5 \\
\hline 1974 & 5.16 & 4.66 & 2.44 & 2.23 & 2.93 & 23.5 & 87.5 & 143 & 184 & 65.1 & 11.5 & 3.98 & 44.8 \\
\hline 1975 & 2.00 & 2.21 & 2.02 & 1.96 & 2.24 & 8.01 & 114 & 162 & 224 & 113 & 54.2 & 11.4 & 58.2 \\
\hline 1976 & 7.25 & 5.14 & 4.25 & 3.81 & 6.28 & 19.5 & 142 & 172 & 187 & 97.0 & 28.7 & 9.98 & 56.9 \\
\hline 1977 & 4.74 & 2.94 & 3.07 & 4.36 & 3.71 & 19.1 & 95.3 & 131 & 181 & 54.8 & 10.4 & 3.75 & 42.8 \\
\hline 1978 & 2.35 & 3.11 & 3.34 & 2.77 & 3.36 & 19.1 & 125 & 192 & 202 & 81.2 & 9.91 & 5.03 & 54.2 \\
\hline 1979 & 5.00 & 5.17 & 3.44 & 3.34 & 5.64 & 24.7 & 145 & 238 & 282 & 147 & 67.5 & 12.0 & 78.5 \\
\hline
\end{tabular}




\section{1-7.L00-1A TAGAB RIVER AT TAGAB}

\section{(U.S. Geological Survey identification number: 344000069410000)}

LOCATION: Lat $34^{\circ} 40^{\prime} \mathrm{N}$., long $69^{\circ} 41^{\prime} \mathrm{E}$.

DRAINAGE AREA: $800 \mathrm{~km}^{2}$.

ELEVATION: 1,060 meters above mean sea level.

PERIOD OF RECORD: August 7, 1968 to April 30, 1979.

GAGE: Water-stage recorder.

Annual mean discharge

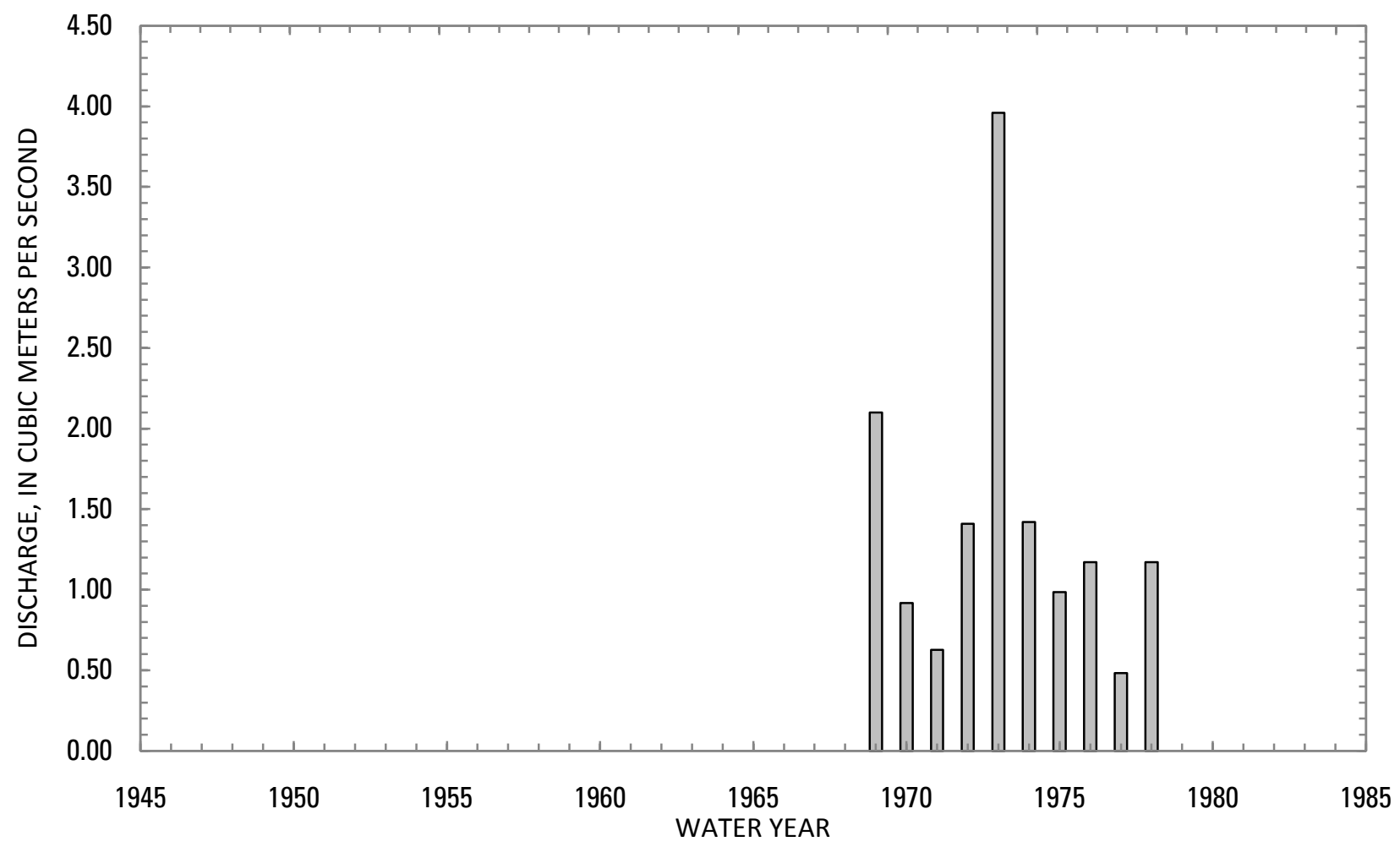




\section{1-7.L00-1A TAGAB RIVER AT TAGAB, Continued}

Statistics of monthly and annual mean discharges $\left[\mathrm{m}^{3} / \mathrm{s}\right.$, cubic meters per second]

\begin{tabular}{|c|c|c|c|c|c|c|c|c|}
\hline \multirow[b]{2}{*}{ Month } & \multicolumn{2}{|c|}{ Maximum } & \multicolumn{2}{|c|}{ Minimum } & \multicolumn{4}{|c|}{ Mean } \\
\hline & $\begin{array}{c}\text { Discharge } \\
\left(\mathrm{m}^{3} / \mathrm{s}\right)\end{array}$ & $\begin{array}{c}\text { Water year } \\
\text { of } \\
\text { occurrence }\end{array}$ & $\begin{array}{c}\text { Discharge } \\
\left(\mathrm{m}^{3} / \mathrm{s}\right)\end{array}$ & $\begin{array}{c}\text { Water year } \\
\text { of } \\
\text { occurrence }\end{array}$ & $\begin{array}{c}\text { Discharge } \\
\left(\mathrm{m}^{3} / \mathrm{s}\right)\end{array}$ & $\begin{array}{c}\text { Standard } \\
\text { deviation } \\
\left(\mathrm{m}^{3} / \mathrm{s}\right)\end{array}$ & $\begin{array}{c}\text { Coefficient } \\
\text { of } \\
\text { variation }\end{array}$ & $\begin{array}{c}\text { Percentage } \\
\text { of annual } \\
\text { discharge }\end{array}$ \\
\hline October & 1.18 & 1974 & 0.06 & 1979 & 0.46 & 0.37 & 0.80 & 2.76 \\
\hline November & 1.64 & 1970 & 0.20 & 1976 & 0.70 & 0.52 & 0.75 & 4.15 \\
\hline December & 2.32 & 1973 & 0.43 & 1978 & 1.06 & 0.62 & 0.59 & 6.30 \\
\hline January & 1.90 & 1973 & 0.47 & 1978 & 1.16 & 0.51 & 0.44 & 6.89 \\
\hline February & 2.35 & 1973 & 0.56 & 1978 & 1.28 & 0.57 & 0.44 & 7.63 \\
\hline March & 4.69 & 1973 & 0.41 & 1977 & 1.48 & 1.16 & 0.78 & 8.83 \\
\hline April & 18.4 & 1973 & 0.27 & 1977 & 5.37 & 4.79 & 0.89 & 32.0 \\
\hline May & 10.8 & 1973 & 0.07 & 1977 & 3.84 & 3.64 & 0.95 & 22.9 \\
\hline June & 2.29 & 1973 & 0.07 & 1975 & 0.70 & 0.86 & 1.23 & 4.19 \\
\hline July & 2.16 & 1978 & 0.01 & 1977 & 0.45 & 0.77 & 1.69 & 2.69 \\
\hline August & 0.26 & 1973 & 0.01 & 1976 & 0.09 & 0.10 & 1.11 & 0.53 \\
\hline September & 0.80 & 1973 & 0.01 & 1977 & 0.20 & 0.24 & 1.15 & 1.21 \\
\hline Annual & 3.96 & 1973 & 0.48 & 1977 & 1.42 & 1.00 & 0.70 & 100 \\
\hline
\end{tabular}


1-7.L00-1A TAGAB RIVER AT TAGAB, Continued

Monthly and annual flow duration, in cubic meters per second

\begin{tabular}{|c|c|c|c|c|c|c|c|c|c|c|c|c|c|}
\hline \multirow{2}{*}{$\begin{array}{l}\text { Percentage } \\
\text { of days } \\
\text { discharge } \\
\text { equaled or } \\
\text { exceeded }\end{array}$} & \multicolumn{12}{|c|}{ Month } & \multirow{2}{*}{ Annual } \\
\hline & October & November & December & January & February & March & April & May & June & July & August & September & \\
\hline 95 & 0.05 & 0.20 & 0.41 & 0.52 & 0.55 & 0.23 & 0.22 & 0.04 & 0.07 & 0 & 0.01 & 0.01 & 0.02 \\
\hline 90 & 0.10 & 0.23 & 0.45 & 0.58 & 0.63 & 0.51 & 0.45 & 0.11 & 0.10 & 0.01 & 0.01 & 0.01 & 0.03 \\
\hline 85 & 0.12 & 0.25 & 0.47 & 0.60 & 0.66 & 0.65 & 0.87 & 0.13 & 0.11 & 0.01 & 0.01 & 0.02 & 0.09 \\
\hline 80 & 0.15 & 0.27 & 0.48 & 0.62 & 0.70 & 0.72 & 1.16 & 0.27 & 0.12 & 0.02 & 0.01 & 0.02 & 0.12 \\
\hline 75 & 0.19 & 0.30 & 0.52 & 0.68 & 0.79 & 0.83 & 1.43 & 0.41 & 0.13 & 0.02 & 0.01 & 0.02 & 0.17 \\
\hline 70 & 0.22 & 0.32 & 0.55 & 0.73 & 0.89 & 0.87 & 1.74 & 0.52 & 0.13 & 0.02 & 0.02 & 0.03 & 0.23 \\
\hline 65 & 0.24 & 0.34 & 0.62 & 0.90 & 0.97 & 0.91 & 2.02 & 0.77 & 0.14 & 0.03 & 0.02 & 0.05 & 0.29 \\
\hline 60 & 0.26 & 0.36 & 0.69 & 1.00 & 1.02 & 0.95 & 2.57 & 1.04 & 0.16 & 0.06 & 0.02 & 0.08 & 0.37 \\
\hline 55 & 0.27 & 0.38 & 0.77 & 1.06 & 1.06 & 1.02 & 2.79 & 2.02 & 0.17 & 0.10 & 0.02 & 0.09 & 0.47 \\
\hline 50 & 0.30 & 0.40 & 0.86 & 1.14 & 1.16 & 1.12 & 3.11 & 2.29 & 0.19 & 0.10 & 0.05 & 0.10 & 0.59 \\
\hline 45 & 0.33 & 0.47 & 0.95 & 1.20 & 1.26 & 1.21 & 3.75 & 2.90 & 0.21 & 0.11 & 0.06 & 0.10 & 0.73 \\
\hline 40 & 0.39 & 0.56 & 1.18 & 1.25 & 1.43 & 1.28 & 4.67 & 3.37 & 0.26 & 0.12 & 0.09 & 0.11 & 0.88 \\
\hline 35 & 0.46 & 0.83 & 1.34 & 1.31 & 1.54 & 1.37 & 5.77 & 4.59 & 0.50 & 0.13 & 0.10 & 0.17 & 1.03 \\
\hline 30 & 0.58 & 1.04 & 1.43 & 1.50 & 1.67 & 1.48 & 6.77 & 5.65 & 0.63 & 0.17 & 0.11 & 0.19 & 1.21 \\
\hline 25 & 0.77 & 1.13 & 1.51 & 1.58 & 1.75 & 1.62 & 7.70 & 6.56 & 0.70 & 0.22 & 0.12 & 0.25 & 1.43 \\
\hline 20 & 0.86 & 1.21 & 1.60 & 1.69 & 1.80 & 1.78 & 8.55 & 7.56 & 0.79 & 0.31 & 0.16 & 0.29 & 1.70 \\
\hline 15 & 0.93 & 1.31 & 1.73 & 1.78 & 1.85 & 2.17 & 9.90 & 8.78 & 0.94 & 0.43 & 0.24 & 0.37 & 2.06 \\
\hline 10 & 1.03 & 1.48 & 1.99 & 1.88 & 1.90 & 2.63 & 12.1 & 9.90 & 2.12 & 0.60 & 0.26 & 0.44 & 2.95 \\
\hline 5 & 1.27 & 1.85 & 2.31 & 1.99 & 2.04 & 3.39 & 18.2 & 11.0 & 3.89 & 0.93 & 0.29 & 0.67 & 7.04 \\
\hline
\end{tabular}




\section{1-7.L00-1A TAGAB RIVER AT TAGAB, Continued}

Probability of occurrence of annual high discharges

[m $\mathrm{m}^{3} / \mathrm{s}$, cubic meters per second; ng, not given]

\begin{tabular}{|c|c|c|c|c|c|c|}
\hline \multirow{2}{*}{$\begin{array}{l}\text { Exceedance } \\
\text { probability }\end{array}$} & \multirow{2}{*}{$\begin{array}{c}\text { Recurrence } \\
\text { interval } \\
\text { (years) }\end{array}$} & \multirow{2}{*}{$\begin{array}{l}\text { Maximum } \\
\text { instantaneous } \\
\text { discharge } \\
\left(\mathrm{m}^{3} / \mathrm{s}\right)\end{array}$} & \multicolumn{4}{|c|}{ Maximum daily mean discharge $\left(\mathrm{m}^{3} / \mathrm{s}\right)$} \\
\hline & & & $\begin{array}{l}\text { 3-day } \\
\text { period }\end{array}$ & $\begin{array}{l}\text { 7-day } \\
\text { period }\end{array}$ & $\begin{array}{l}\text { 15-day } \\
\text { period }\end{array}$ & $\begin{array}{l}\text { 30-day } \\
\text { period }\end{array}$ \\
\hline 0.99 & 1.01 & 3.00 & 1.30 & 1.16 & 1.06 & 0.97 \\
\hline 0.95 & 1.05 & 6.00 & 2.64 & 2.34 & 2.04 & 1.65 \\
\hline 0.90 & 1.11 & 8.50 & 3.73 & 3.28 & 2.81 & 2.17 \\
\hline 0.80 & 1.25 & 12.8 & 5.49 & 4.79 & 4.03 & 3.02 \\
\hline 0.50 & 2 & 25.6 & 10.6 & 8.97 & 7.45 & 5.60 \\
\hline 0.20 & 5 & 46.8 & 18.2 & 14.9 & 12.6 & 10.1 \\
\hline 0.10 & 10 & 62.0 & 23.1 & 18.6 & 15.9 & 13.7 \\
\hline 0.04 & 25 & 81.7 & 29.1 & 22.8 & 20.0 & 18.9 \\
\hline 0.02 & 50 & 96.4 & 33.2 & 25.6 & ${ }^{1} 22.9$ & 23.1 \\
\hline 0.01 & 100 & 111 & 37.1 & 28.1 & ${ }^{1} 25.7$ & 27.6 \\
\hline 0.005 & 200 & 125 & 40.7 & ${ }^{1} 30.4$ & ${ }^{1} 28.3$ & 32.4 \\
\hline 0.002 & 500 & 144 & ng & $\mathrm{ng}$ & $\mathrm{ng}$ & $\mathrm{ng}$ \\
\hline
\end{tabular}

'Data does not fit log-Pearson Type III curve, use with caution.

\section{1-7.L00-1A TAGAB RIVER AT TAGAB, Continued}

\section{Probability of occurrence of annual low discharges}

$\left[\mathrm{m}^{3} / \mathrm{s}\right.$, meters per second]

\begin{tabular}{|c|c|c|c|c|c|c|c|c|c|c|}
\hline \multirow{3}{*}{$\begin{array}{c}\text { Nonexceedance } \\
\text { probability }\end{array}$} & \multirow{3}{*}{$\begin{array}{l}\text { Recurrence } \\
\text { interval } \\
\text { (years) }\end{array}$} & \multicolumn{9}{|c|}{ Minimum daily mean discharge $\left(\mathrm{m}^{3} / \mathrm{s}\right)$} \\
\hline & & \multicolumn{9}{|c|}{ Number of consecutive days } \\
\hline & & 1 & 3 & 7 & 14 & 30 & 60 & 90 & 120 & 183 \\
\hline 0.05 & 20 & 0 & 0 & 0 & 0 & 0 & 0 & 0.01 & 0.03 & 0.06 \\
\hline 0.10 & 10 & 0 & 0 & 0 & 0 & 0 & 0.01 & 0.01 & 0.04 & 0.08 \\
\hline 0.20 & 5 & 0.01 & 0.01 & 0.01 & 0.01 & 0.01 & 0.01 & 0.02 & 0.05 & 0.11 \\
\hline 0.50 & 2 & 0.02 & 0.03 & 0.03 & 0.03 & 0.03 & 0.04 & 0.06 & 0.11 & 0.23 \\
\hline
\end{tabular}




\section{1-7.L00-1A TAGAB RIVER AT TAGAB, Continued}

\section{Probability of occurrence of seasonal low discharges}

[ $\mathrm{m}^{3} / \mathrm{s}$, meters per second]

\begin{tabular}{|c|c|c|c|c|c|c|c|c|c|}
\hline \multirow{3}{*}{$\begin{array}{c}\text { Nonexceedance } \\
\text { probability }\end{array}$} & \multirow{3}{*}{$\begin{array}{c}\text { Recurrence } \\
\text { interval } \\
\text { (years) }\end{array}$} & \multicolumn{8}{|c|}{ Minimum daily mean discharge $\left(\mathrm{m}^{3} / \mathrm{s}\right)$} \\
\hline & & \multicolumn{8}{|c|}{ Number of consecutive days } \\
\hline & & 1 & 7 & 14 & 30 & 1 & 7 & 14 & 30 \\
\hline & & \multicolumn{4}{|c|}{ December-January-February } & \multicolumn{4}{|c|}{ March-April-May } \\
\hline 0.05 & 20 & 0.26 & 0.29 & 0.30 & 0.34 & 0.03 & 0.03 & 0.04 & 0.10 \\
\hline 0.10 & 10 & 0.31 & 0.35 & 0.37 & 0.42 & 0.04 & 0.06 & 0.07 & 0.19 \\
\hline 0.20 & 5 & 0.40 & 0.44 & 0.47 & 0.53 & 0.08 & 0.12 & 0.14 & 0.39 \\
\hline \multirow[t]{2}{*}{0.50} & 2 & 0.65 & 0.70 & 0.75 & 0.83 & 0.25 & 0.38 & 0.47 & 1.09 \\
\hline & & \multicolumn{4}{|c|}{ June-July-August } & \multicolumn{4}{|c|}{ September-0ctober-November } \\
\hline 0.05 & 20 & 0 & 0 & 0 & 0 & 0.01 & 0.01 & 0.01 & 0.01 \\
\hline 0.10 & 10 & 0 & 0 & 0 & 0 & 0.01 & 0.01 & 0.01 & 0.01 \\
\hline 0.20 & 5 & 0.01 & 0.01 & 0.01 & 0.01 & 0.01 & 0.01 & 0.02 & 0.03 \\
\hline 0.50 & 2 & 0.02 & 0.03 & 0.03 & 0.04 & 0.03 & 0.04 & 0.05 & 0.10 \\
\hline
\end{tabular}

\section{1-7.L00-1A TAGAB RIVER AT TAGAB, Continued}

Annual peak discharges

[ $\mathrm{m}^{3} / \mathrm{s}$, meters per second]

\begin{tabular}{|c|c|c|c|c|c|}
\hline \multicolumn{3}{|c|}{$\begin{array}{c}\text { Annual peak discharge, } \\
\text { by year }\end{array}$} & \multicolumn{3}{|c|}{$\begin{array}{l}\text { Annual peak discharge, } \\
\text { from highest to lowest }\end{array}$} \\
\hline $\begin{array}{l}\text { Water } \\
\text { year }\end{array}$ & Date & $\begin{array}{c}\text { Peak } \\
\text { discharge } \\
\left(\mathrm{m}^{3} / \mathrm{s}\right)\end{array}$ & $\begin{array}{l}\text { Water } \\
\text { year }\end{array}$ & Date & $\begin{array}{c}\text { Peak } \\
\text { discharge } \\
\left(\mathrm{m}^{3} / \mathrm{s}\right)\end{array}$ \\
\hline 1969 & April 20, 1969 & 21.4 & 1978 & July 6, 1978 & 60.0 \\
\hline 1970 & April 15, 1970 & 41.5 & 1973 & April 4, 1973 & 56.3 \\
\hline 1971 & March 26, 1971 & 15.0 & 1976 & April 24, 1976 & 50.0 \\
\hline 1972 & April 28, 1972 & 25.8 & 1970 & April 15, 1970 & 41.5 \\
\hline 1973 & April 4, 1973 & 56.3 & 1972 & April 28, 1972 & 25.8 \\
\hline 1974 & April 8, 1974 & 10.1 & 1969 & April 20, 1969 & 21.4 \\
\hline 1975 & April 5, 1975 & 18.5 & 1975 & April 5, 1975 & 18.5 \\
\hline 1976 & April 24, 1976 & 50.0 & 1971 & March 26, 1971 & 15.0 \\
\hline 1977 & January 10, 1977 & 5.80 & 1974 & $\begin{array}{c}\text { April 8, } 1974 \\
\text { January 10, }\end{array}$ & 10.1 \\
\hline 1978 & July 6,1978 & 60.0 & 1977 & 1977 & 5.80 \\
\hline
\end{tabular}




\section{1-7.L00-1A TAGAB RIVER AT TAGAB, Continued}

Monthly and annual mean discharges, in cubic meters per second

[Data may not be rounded in accordance with U.S. Geological Survey publication standards; --, no data]

\begin{tabular}{|c|c|c|c|c|c|c|c|c|c|c|c|c|c|}
\hline \multirow{2}{*}{$\begin{array}{l}\text { Water } \\
\text { year }\end{array}$} & \multicolumn{12}{|c|}{ Monthly mean discharge } & \multirow{2}{*}{$\begin{array}{c}\text { Annual } \\
\text { discharge }\end{array}$} \\
\hline & October & November & December & January & February & March & April & May & June & July & August & September & \\
\hline 1968 & -- & -- & -- & -- & -- & -- & -- & -- & -- & -- & -- & 0.21 & -- \\
\hline 1969 & 0.76 & 1.24 & 1.58 & 1.55 & 1.71 & 2.20 & 5.68 & 7.37 & 2.09 & 0.37 & 0.26 & 0.42 & 2.10 \\
\hline 1970 & 0.92 & 1.64 & 1.51 & 1.20 & 1.06 & 1.28 & 2.59 & 0.39 & 0.16 & 0.13 & 0.05 & 0.12 & 0.92 \\
\hline 1971 & 0.23 & 0.30 & 0.62 & 0.60 & 0.65 & 0.72 & 2.37 & 1.53 & 0.21 & 0.10 & 0.10 & 0.10 & 0.63 \\
\hline 1972 & 0.27 & 0.34 & 0.47 & 0.64 & 0.75 & 1.30 & 3.45 & 7.79 & 1.29 & 0.13 & 0.13 & 0.31 & 1.41 \\
\hline 1973 & 0.76 & 1.36 & 2.32 & 1.90 & 2.35 & 4.69 & 18.4 & 10.8 & 2.29 & 1.58 & 0.26 & 0.80 & 3.96 \\
\hline 1974 & 1.18 & 1.08 & 1.66 & 1.73 & 1.87 & 1.35 & 6.51 & 1.55 & 0.16 & 0.03 & 0.01 & 0.10 & 1.42 \\
\hline 1975 & 0.27 & 0.31 & 0.62 & 1.04 & 1.02 & 0.98 & 4.55 & 2.86 & 0.07 & 0.02 & 0.01 & 0.13 & 0.99 \\
\hline 1976 & 0.18 & 0.20 & 0.54 & 0.76 & 1.02 & 0.85 & 5.57 & 4.44 & 0.54 & 0.01 & 0.01 & 0.04 & 1.17 \\
\hline 1977 & 0.35 & 0.40 & 1.05 & 1.71 & 1.42 & 0.41 & 0.27 & 0.07 & 0.12 & 0.01 & 0.01 & 0.01 & 0.48 \\
\hline 1978 & 0.14 & 0.35 & 0.43 & 0.47 & 0.56 & 0.97 & 7.16 & 1.60 & 0.12 & 2.16 & 0.06 & 0.01 & 1.17 \\
\hline 1979 & 0.06 & 0.45 & 0.87 & 1.15 & 1.69 & 1.57 & 2.54 & -- & -- & -- & -- & -- & -- \\
\hline
\end{tabular}




\section{1-8.21L-1A SALANG RIVER AT BAGH-I-LALA}

(U.S. Geological Survey identification number: 350900069130000)

LOCATION: Lat $35^{\circ} 09^{\prime} \mathrm{N}$., long $69^{\circ} 13^{\prime} \mathrm{E}$.

DRAINAGE AREA: $485 \mathrm{~km}^{2}$.

ELEVATION: 1,660 meters above mean sea level.

PERIOD OF RECORD: October 1, 1961 to March 23, 1964 and December 2, 1964 to February 29, 1980.

GAGE: Water-stage recorder. Station was destroyed by flooding on March 23, 1964.

Annual mean discharge

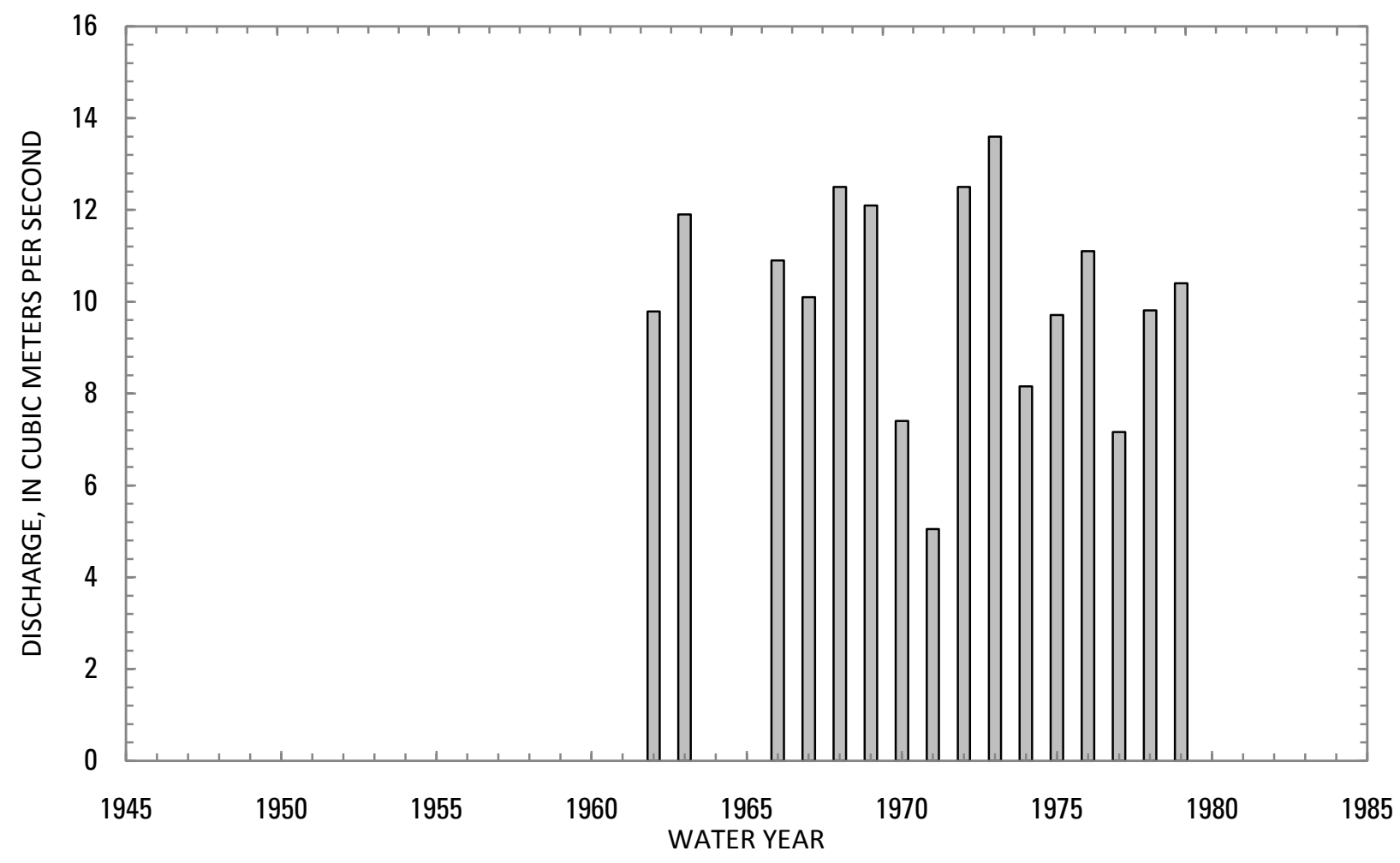




\section{1-8.21L-1A SALANG RIVER AT BAGH-I-LALA, Continued}

Statistics of monthly and annual mean discharges $\left[\mathrm{m}^{3} / \mathrm{s}\right.$, cubic meters per second]

\begin{tabular}{|c|c|c|c|c|c|c|c|c|}
\hline \multirow[b]{2}{*}{ Month } & \multicolumn{2}{|c|}{ Maximum } & \multicolumn{2}{|c|}{ Minimum } & \multicolumn{4}{|c|}{ Mean } \\
\hline & $\begin{array}{c}\text { Discharge } \\
\left(\mathrm{m}^{3} / \mathrm{s}\right)\end{array}$ & $\begin{array}{c}\text { Water year } \\
\text { of } \\
\text { occurrence }\end{array}$ & $\begin{array}{c}\text { Discharge } \\
\left(\mathrm{m}^{3} / \mathrm{s}\right)\end{array}$ & $\begin{array}{l}\text { Water year } \\
\quad \text { of } \\
\text { occurrence }\end{array}$ & $\begin{array}{c}\text { Discharge } \\
\left(\mathrm{m}^{3} / \mathrm{s}\right)\end{array}$ & $\begin{array}{c}\text { Standard } \\
\text { deviation } \\
\left(\mathrm{m}^{3} / \mathrm{s}\right)\end{array}$ & $\begin{array}{c}\text { Coefficient } \\
\text { of } \\
\text { variation }\end{array}$ & $\begin{array}{c}\text { Percentage } \\
\text { of annual } \\
\text { discharge }\end{array}$ \\
\hline October & 5.08 & 1962 & 2.39 & 1979 & 3.47 & 0.69 & 0.20 & 2.78 \\
\hline November & 4.59 & 1962 & 2.23 & 1975 & 3.12 & 0.69 & 0.22 & 2.50 \\
\hline December & 4.37 & 1962 & 1.92 & 1975 & 2.90 & 0.57 & 0.20 & 2.33 \\
\hline January & 4.30 & 1962 & 1.97 & 1964 & 2.73 & 0.60 & 0.22 & 2.19 \\
\hline February & 4.81 & 1980 & 1.71 & 1971 & 3.17 & 0.84 & 0.26 & 2.54 \\
\hline March & 15.3 & 1969 & 4.03 & 1975 & 7.80 & 3.18 & 0.41 & 6.25 \\
\hline April & 31.7 & 1973 & 10.3 & 1971 & 22.4 & 5.68 & 0.25 & 18.0 \\
\hline May & 43.9 & 1973 & 11.1 & 1971 & 27.4 & 8.68 & 0.32 & 22.0 \\
\hline June & 45.4 & 1968 & 9.55 & 1971 & 27.1 & 9.24 & 0.34 & 21.7 \\
\hline July & 30.8 & 1965 & 6.47 & 1971 & 14.1 & 5.79 & 0.41 & 11.3 \\
\hline August & 11.7 & 1965 & 3.90 & 1971 & 6.48 & 1.88 & 0.29 & 5.19 \\
\hline September & 5.51 & 1962 & 3.24 & 1975 & 4.11 & 0.73 & 0.18 & 3.30 \\
\hline Annual & 13.6 & 1973 & 5.05 & 1971 & 10.14 & 2.28 & 0.23 & 100 \\
\hline
\end{tabular}


1-8.21L-1A SALANG RIVER AT BAGH-I-LALA, Continued

Monthly and annual flow duration, in cubic meters per second

\begin{tabular}{|c|c|c|c|c|c|c|c|c|c|c|c|c|c|}
\hline \multirow{2}{*}{$\begin{array}{l}\text { Percentage } \\
\text { of days } \\
\text { discharge } \\
\text { equaled or } \\
\text { exceeded }\end{array}$} & \multicolumn{12}{|c|}{ Month } & \multirow{2}{*}{ Annual } \\
\hline & October & November & December & January & February & March & April & May & June & July & August & September & \\
\hline 95 & 2.44 & 2.12 & 2.01 & 1.90 & 1.78 & 3.01 & 8.77 & 12.0 & 11.2 & 6.07 & 3.63 & 2.94 & 2.24 \\
\hline 90 & 2.65 & 2.29 & 2.25 & 2.03 & 2.13 & 3.20 & 10.7 & 14.8 & 13.5 & 6.87 & 3.99 & 3.06 & 2.53 \\
\hline 85 & 2.73 & 2.43 & 2.44 & 2.11 & 2.22 & 3.60 & 12.2 & 16.9 & 16.5 & 7.39 & 4.30 & 3.26 & 2.69 \\
\hline 80 & 2.84 & 2.58 & 2.48 & 2.23 & 2.34 & 4.16 & 13.4 & 19.4 & 17.9 & 8.61 & 4.67 & 3.36 & 2.88 \\
\hline 75 & 2.98 & 2.62 & 2.59 & 2.27 & 2.42 & 4.53 & 14.7 & 21.1 & 19.3 & 9.39 & 5.04 & 3.46 & 3.09 \\
\hline 70 & 3.08 & 2.67 & 2.62 & 2.40 & 2.55 & 4.87 & 15.9 & 22.0 & 20.5 & 10.1 & 5.21 & 3.51 & 3.30 \\
\hline 65 & 3.13 & 2.81 & 2.66 & 2.46 & 2.72 & 5.18 & 17.5 & 22.9 & 21.8 & 10.5 & 5.42 & 3.56 & 3.51 \\
\hline 60 & 3.21 & 2.88 & 2.72 & 2.51 & 2.86 & 5.50 & 18.3 & 23.8 & 23.7 & 10.9 & 5.63 & 3.72 & 3.78 \\
\hline 55 & 3.40 & 2.94 & 2.76 & 2.59 & 2.96 & 5.94 & 19.3 & 24.7 & 25.1 & 11.5 & 5.86 & 3.78 & 4.10 \\
\hline 50 & 3.47 & 3.06 & 2.80 & 2.66 & 3.06 & 6.24 & 20.6 & 25.6 & 26.2 & 12.1 & 6.04 & 3.95 & 4.62 \\
\hline 45 & 3.55 & 3.11 & 2.88 & 2.72 & 3.22 & 6.67 & 22.0 & 26.6 & 27.9 & 13.3 & 6.23 & 4.01 & 5.30 \\
\hline 40 & 3.63 & 3.16 & 2.92 & 2.76 & 3.32 & 7.26 & 23.6 & 27.8 & 29.1 & 14.2 & 6.64 & 4.20 & 6.49 \\
\hline 35 & 3.67 & 3.21 & 2.96 & 2.81 & 3.44 & 7.86 & 25.8 & 29.4 & 30.4 & 15.2 & 6.88 & 4.41 & 8.40 \\
\hline 30 & 3.71 & 3.25 & 3.09 & 2.90 & 3.59 & 8.74 & 27.5 & 31.4 & 31.6 & 16.2 & 7.17 & 4.56 & 10.9 \\
\hline 25 & 3.74 & 3.44 & 3.18 & 3.09 & 3.71 & 9.56 & 28.7 & 34.1 & 33.3 & 17.4 & 7.62 & 4.78 & 14.6 \\
\hline 20 & 3.94 & 3.60 & 3.31 & 3.21 & 3.90 & 10.5 & 30.1 & 36.1 & 37.3 & 18.9 & 8.11 & 4.92 & 18.5 \\
\hline 15 & 4.20 & 3.91 & 3.57 & 3.49 & 4.09 & 12.0 & 32.1 & 38.9 & 39.5 & 20.8 & 8.57 & 5.02 & 22.3 \\
\hline 10 & 4.50 & 4.37 & 3.68 & 3.57 & 4.30 & 15.3 & 35.2 & 42.7 & 41.9 & 22.9 & 9.30 & 5.28 & 26.8 \\
\hline 5 & 4.88 & 4.61 & 4.17 & 3.98 & 4.73 & 18.8 & 40.1 & 45.0 & 45.2 & 29.3 & 10.2 & 5.69 & 32.8 \\
\hline
\end{tabular}




\section{1-8.21L-1A SALANG RIVER AT BAGH-I-LALA, Continued}

Probability of occurrence of annual high discharges

[m $\mathrm{m}^{3} / \mathrm{s}$, cubic meters per second; ng, not given]

\begin{tabular}{|c|c|c|c|c|c|c|}
\hline \multirow{2}{*}{$\begin{array}{c}\text { Exceedance } \\
\text { probability }\end{array}$} & \multirow{2}{*}{$\begin{array}{c}\text { Recurrence } \\
\text { interval } \\
\text { (years) }\end{array}$} & \multirow{2}{*}{$\begin{array}{c}\text { Maximum } \\
\text { instantaneous } \\
\text { discharge } \\
\left(\mathrm{m}^{3} / \mathrm{s}\right)\end{array}$} & \multicolumn{4}{|c|}{ Maximum daily mean discharge $\left(\mathrm{m}^{3} / \mathrm{s}\right)$} \\
\hline & & & $\begin{array}{l}\text { 3-day } \\
\text { period }\end{array}$ & $\begin{array}{l}\text { 7-day } \\
\text { period }\end{array}$ & $\begin{array}{l}\text { 15-day } \\
\text { period }\end{array}$ & $\begin{array}{l}\text { 30-day } \\
\text { period }\end{array}$ \\
\hline 0.99 & 1.01 & 18.9 & 11.9 & 10.7 & 10.1 & 9.83 \\
\hline 0.95 & 1.05 & 26.8 & 17.7 & 16.7 & 16.0 & 15.1 \\
\hline 0.90 & 1.11 & 32.0 & 21.6 & 20.6 & 19.7 & 18.5 \\
\hline 0.80 & 1.25 & 39.4 & 27.1 & 26.0 & 24.7 & 22.9 \\
\hline 0.50 & 2 & 57.2 & 40.1 & 37.7 & 34.8 & 31.8 \\
\hline 0.20 & 5 & 80.6 & 56.2 & 50.3 & 44.4 & 40.2 \\
\hline 0.10 & 10 & 95.2 & 65.8 & 56.7 & 48.6 & 44.0 \\
\hline 0.04 & 25 & 113 & 76.7 & 63.1 & 52.4 & 47.3 \\
\hline 0.02 & 50 & 125 & 84.0 & 66.9 & 54.3 & 49.1 \\
\hline 0.01 & 100 & 137 & 90.8 & 70.1 & 55.8 & 50.5 \\
\hline 0.005 & 200 & 149 & 97.0 & 72.7 & 57.0 & 51.5 \\
\hline 0.002 & 500 & 164 & $\mathrm{ng}$ & $\mathrm{ng}$ & ng & $\mathrm{ng}$ \\
\hline
\end{tabular}

1-8.21L-1A SALANG RIVER AT BAGH-I-LALA, Continued

Probability of occurrence of annual low discharges $\left[\mathrm{m}^{3} / \mathrm{s}\right.$, meters per second]

\begin{tabular}{|c|c|c|c|c|c|c|c|c|c|c|}
\hline \multirow{3}{*}{$\begin{array}{c}\text { Nonexceedance } \\
\text { probability }\end{array}$} & \multirow{3}{*}{$\begin{array}{c}\text { Recurrence } \\
\text { interval } \\
\text { (years) }\end{array}$} & \multicolumn{9}{|c|}{ Minimum daily mean discharge $\left(\mathrm{m}^{3} / \mathrm{s}\right)$} \\
\hline & & \multicolumn{9}{|c|}{ Number of consecutive days } \\
\hline & & 1 & 3 & 7 & 14 & 30 & 60 & 90 & 120 & 183 \\
\hline 0.05 & 20 & 1.28 & 1.54 & 1.58 & 1.63 & 1.73 & 1.86 & 2.04 & 2.14 & 2.44 \\
\hline 0.10 & 10 & 1.41 & 1.66 & 1.70 & 1.75 & 1.86 & 1.99 & 2.16 & 2.26 & 2.56 \\
\hline 0.20 & 5 & 1.59 & 1.81 & 1.87 & 1.92 & 2.03 & 2.17 & 2.32 & 2.42 & 2.72 \\
\hline 0.50 & 2 & 2.00 & 2.17 & 2.25 & 2.30 & 2.41 & 2.55 & 2.67 & 2.77 & 3.09 \\
\hline
\end{tabular}




\section{1-8.21L-1A SALANG RIVER AT BAGH-I-LALA, Continued}

Probability of occurrence of seasonal low discharges

[ $\mathrm{m}^{3} / \mathrm{s}$, meters per second]

\begin{tabular}{|c|c|c|c|c|c|c|c|c|c|}
\hline \multirow{3}{*}{$\begin{array}{c}\text { Nonexceedance } \\
\text { probability }\end{array}$} & \multirow{3}{*}{$\begin{array}{c}\text { Recurrence } \\
\text { interval } \\
\text { (years) }\end{array}$} & \multicolumn{8}{|c|}{ Minimum daily mean discharge $\left(\mathrm{m}^{3} / \mathrm{s}\right)$} \\
\hline & & \multicolumn{8}{|c|}{ Number of consecutive days } \\
\hline & & 1 & 7 & 14 & 30 & 1 & 7 & 14 & 30 \\
\hline & & \multicolumn{4}{|c|}{ December-January-February } & \multicolumn{4}{|c|}{ March-April-May } \\
\hline 0.05 & 20 & 1.18 & 1.47 & 1.63 & 1.76 & 2.15 & 2.24 & 2.56 & 4.00 \\
\hline 0.10 & 10 & 1.34 & 1.63 & 1.76 & 1.88 & 2.45 & 2.63 & 2.98 & 4.46 \\
\hline 0.20 & 5 & 1.56 & 1.85 & 1.94 & 2.05 & 2.83 & 3.14 & 3.56 & 5.14 \\
\hline \multirow[t]{2}{*}{0.50} & 2 & 2.07 & 2.34 & 2.37 & 2.45 & 3.63 & 4.19 & 4.88 & 6.93 \\
\hline & & \multicolumn{4}{|c|}{ June-July-August } & \multicolumn{4}{|c|}{ September-October-November } \\
\hline 0.05 & 20 & 3.00 & 3.16 & 3.45 & 3.95 & 1.79 & 1.83 & 1.91 & 2.10 \\
\hline 0.10 & 10 & 3.35 & 3.51 & 3.79 & 4.34 & 1.97 & 2.01 & 2.09 & 2.25 \\
\hline 0.20 & 5 & 3.80 & 3.97 & 4.25 & 4.88 & 2.19 & 2.25 & 2.32 & 2.46 \\
\hline 0.50 & 2 & 4.70 & 4.92 & 5.24 & 6.13 & 2.65 & 2.72 & 2.79 & 2.90 \\
\hline
\end{tabular}


1-8.21L-1A SALANG RIVER AT BAGH-I-LALA, Continued

\begin{tabular}{|c|c|c|c|c|c|}
\hline \multicolumn{6}{|c|}{$\begin{array}{l}\text { Annual peak discharges } \\
\text { [m³/s, meters per second] }\end{array}$} \\
\hline \multicolumn{3}{|c|}{$\begin{array}{c}\text { Annual peak discharge, } \\
\text { by year }\end{array}$} & \multicolumn{3}{|c|}{$\begin{array}{l}\text { Annual peak discharge, } \\
\text { from highest to lowest }\end{array}$} \\
\hline $\begin{array}{l}\text { Water } \\
\text { year }\end{array}$ & Date & $\begin{array}{c}\text { Peak } \\
\text { discharge } \\
\left(\mathrm{m}^{3} / \mathrm{s}\right)\end{array}$ & $\begin{array}{l}\text { Water } \\
\text { year }\end{array}$ & Date & $\begin{array}{c}\text { Peak } \\
\text { discharge } \\
\left(\mathrm{m}^{3} / \mathrm{s}\right)\end{array}$ \\
\hline 1962 & April 25, 1962 & 43.8 & 1967 & April 27, 1967 & 124 \\
\hline 1963 & May 14,1963 & 92.8 & 1963 & May 14,1963 & 92.8 \\
\hline 1965 & June 13, 1965 & 50.0 & 1969 & April 14, 1969 & 91.0 \\
\hline 1966 & April 25, 1966 & 60.6 & 1972 & May 13, 1972 & 80.8 \\
\hline 1967 & April 27, 1967 & 124 & 1968 & April 29, 1968 & 76.1 \\
\hline 1968 & April 29, 1968 & 76.1 & 1966 & April 25, 1966 & 60.6 \\
\hline 1969 & April 14, 1969 & 91.0 & 1975 & May 15,1975 & 60.0 \\
\hline 1970 & April 15, 1970 & 58.5 & 1970 & April 15, 1970 & 58.5 \\
\hline 1971 & May 19, 1971 & 22.4 & 1973 & June 3, 1973 & 56.7 \\
\hline 1972 & May 13, 1972 & 80.8 & 1976 & April 23, 1976 & 53.1 \\
\hline 1973 & June 3, 1973 & 56.7 & 1965 & June 13, 1965 & 50.0 \\
\hline 1974 & May 1, 1974 & 45.5 & 1979 & April 30, 1979 & 48.9 \\
\hline 1975 & May 15, 1975 & 60.0 & 1974 & May 1, 1974 & 45.5 \\
\hline 1976 & April 23, 1976 & 53.1 & 1962 & April 25, 1962 & 43.8 \\
\hline 1977 & May 27, 1977 & 27.9 & 1978 & May 4, 1978 & 41.6 \\
\hline 1978 & May 4, 1978 & 41.6 & 1977 & May 27, 1977 & 27.9 \\
\hline 1979 & April 30, 1979 & 48.9 & 1971 & May 19,1971 & 22.4 \\
\hline
\end{tabular}


1-8.21L-1A SALANG RIVER AT BAGH-I-LALA, Continued

Monthly and annual mean discharges, in cubic meters per second

[Data may not be rounded in accordance with U.S. Geological Survey publication standards; --, no data]

\begin{tabular}{|c|c|c|c|c|c|c|c|c|c|c|c|c|c|}
\hline \multirow{2}{*}{$\begin{array}{l}\text { Water } \\
\text { year }\end{array}$} & \multicolumn{12}{|c|}{ Monthly mean discharge } & \multirow{2}{*}{$\begin{array}{c}\text { Annual } \\
\text { discharge }\end{array}$} \\
\hline & October & November & December & January & February & March & April & May & June & July & August & September & \\
\hline 1962 & 5.08 & 4.59 & 4.37 & 4.30 & 4.39 & 5.59 & 19.8 & 23.2 & 20.6 & 12.6 & 7.33 & 5.51 & 9.79 \\
\hline 1963 & 4.51 & 4.12 & 3.55 & 3.52 & 3.87 & 7.73 & 17.5 & 39.6 & 34.1 & 14.2 & 6.36 & 3.35 & 11.9 \\
\hline 1964 & 2.86 & 2.57 & 2.21 & 1.97 & 3.25 & -- & -- & -- & -- & -- & -- & -- & -- \\
\hline 1965 & -- & -- & -- & 3.33 & 4.06 & 8.93 & 25.7 & 38.1 & 40.5 & 30.8 & 11.7 & 5.25 & -- \\
\hline 1966 & 3.72 & 3.26 & 2.86 & 2.84 & 3.46 & 9.05 & 26.2 & 25.1 & 32.3 & 11.8 & 6.25 & 4.56 & 10.9 \\
\hline 1967 & 3.62 & 3.10 & 2.75 & 2.45 & 2.37 & 5.35 & 28.2 & 23.0 & 25.6 & 12.8 & 6.63 & 5.02 & 10.1 \\
\hline 1968 & 3.60 & 2.28 & 2.54 & 2.38 & 2.76 & 9.03 & 23.5 & 26.8 & 45.4 & 19.5 & 7.26 & 4.66 & 12.5 \\
\hline 1969 & 3.74 & 3.27 & 3.79 & 2.75 & 3.68 & 15.3 & 29.1 & 21.4 & 30.7 & 18.0 & 8.18 & 4.54 & 12.1 \\
\hline 1970 & 3.60 & 3.82 & 2.82 & 2.26 & 2.79 & 5.62 & 16.6 & 21.1 & 15.0 & 7.28 & 4.42 & 3.34 & 7.40 \\
\hline 1971 & 2.66 & 2.52 & 2.72 & 1.97 & 1.71 & 4.29 & 10.3 & 11.1 & 9.55 & 6.47 & 3.90 & 3.28 & 5.05 \\
\hline 1972 & 2.70 & 2.62 & 2.53 & 2.82 & 3.12 & 13.7 & 24.2 & 36.8 & 32.0 & 18.0 & 7.43 & 4.37 & 12.5 \\
\hline 1973 & 4.13 & 4.22 & 3.33 & 3.42 & 4.24 & 11.4 & 31.7 & 43.9 & 33.5 & 13.5 & 6.40 & 3.70 & 13.6 \\
\hline 1974 & 3.75 & 3.05 & 2.55 & 2.06 & 2.28 & 5.81 & 18.6 & 23.2 & 19.4 & 8.72 & 4.60 & 3.66 & 8.16 \\
\hline 1975 & 3.09 & 2.23 & 1.92 & 2.19 & 2.18 & 4.03 & 23.2 & 28.0 & 26.9 & 14.0 & 5.30 & 3.24 & 9.71 \\
\hline 1976 & 3.18 & 2.91 & 2.96 & 2.51 & 2.72 & 5.50 & 23.4 & 33.1 & 28.8 & 16.9 & 7.08 & 4.37 & 11.1 \\
\hline 1977 & 3.94 & 3.27 & 2.91 & 2.79 & 2.84 & 7.09 & 13.6 & 15.9 & 17.1 & 8.75 & 4.21 & 3.34 & 7.16 \\
\hline 1978 & 3.02 & 3.06 & 2.93 & 3.04 & 3.23 & 7.74 & 23.5 & 30.6 & 21.0 & 10.1 & 5.36 & 3.94 & 9.81 \\
\hline 1979 & 2.39 & 2.23 & 2.80 & 2.61 & 2.45 & 6.48 & 26.3 & 25.0 & 27.6 & 15.6 & 7.71 & 3.77 & 10.4 \\
\hline 1980 & 2.90 & 3.01 & 2.67 & 2.67 & 4.81 & -- & -- & -- & -- & -- & -- & -- & -- \\
\hline
\end{tabular}




\section{1-8.2R0-1A GHORBAND RIVER AT PUL-I-ASHAWA}

(U.S. Geological Survey identification number: 350500069080000)

LOCATION: Lat $35^{\circ} 05^{\prime} \mathrm{N}$., long $69^{\circ} 08^{\prime} \mathrm{E}$.

DRAINAGE AREA: $4,020 \mathrm{~km}^{2}$.

ELEVATION: 1,610 meters above mean sea level.

PERIOD OF RECORD: October 1, 1959 to February 4, 1980.

GAGE: Water-stage recorder.

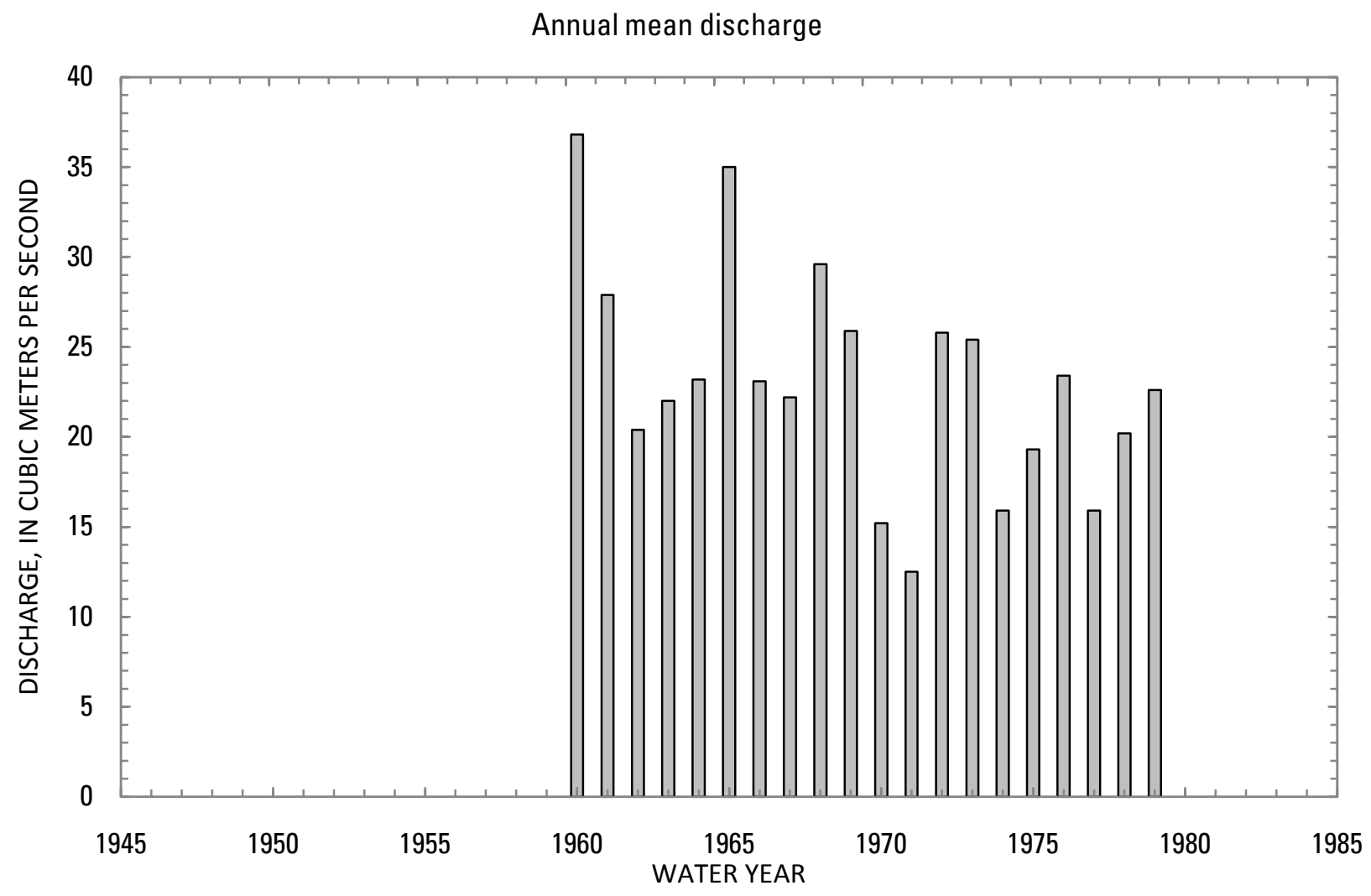




\section{1-8.2R0-1A GHORBAND RIVER AT PUL-I-ASHAWA, Continued}

Statistics of monthly and annual mean discharges

[ $\mathrm{m}^{3} / \mathrm{s}$, cubic meters per second]

\begin{tabular}{|c|c|c|c|c|c|c|c|c|}
\hline \multirow[b]{2}{*}{ Month } & \multicolumn{2}{|c|}{ Maximum } & \multicolumn{2}{|c|}{ Minimum } & \multicolumn{4}{|c|}{ Mean } \\
\hline & $\begin{array}{c}\text { Discharge } \\
\left(\mathrm{m}^{3} / \mathrm{s}\right)\end{array}$ & $\begin{array}{c}\text { Water year } \\
\text { of } \\
\text { occurrence }\end{array}$ & $\begin{array}{c}\text { Discharge } \\
\left(\mathrm{m}^{3} / \mathrm{s}\right)\end{array}$ & $\begin{array}{c}\text { Water year } \\
\text { of } \\
\text { occurrence }\end{array}$ & $\begin{array}{c}\text { Discharge } \\
\left(\mathrm{m}^{3} / \mathrm{s}\right)\end{array}$ & $\begin{array}{c}\text { Standard } \\
\text { deviation } \\
\left(\mathrm{m}^{3} / \mathrm{s}\right)\end{array}$ & $\begin{array}{c}\text { Coefficient } \\
\text { of } \\
\text { variation }\end{array}$ & $\begin{array}{c}\text { Percentage } \\
\text { of annual } \\
\text { discharge }\end{array}$ \\
\hline October & 15.4 & 1961 & 5.08 & 1972 & 9.08 & 2.49 & 0.27 & 3.28 \\
\hline November & 16.2 & 1961 & 6.76 & 1960 & 10.3 & 2.33 & 0.23 & 3.73 \\
\hline December & 14.4 & 1961 & 6.68 & 1960 & 9.74 & 2.11 & 0.22 & 3.52 \\
\hline January & 13.0 & 1961 & 5.72 & 1972 & 8.80 & 1.97 & 0.22 & 3.18 \\
\hline February & 16.0 & 1960 & 4.63 & 1971 & 8.82 & 2.78 & 0.32 & 3.19 \\
\hline March & 35.0 & 1960 & 8.45 & 1970 & 15.5 & 6.11 & 0.40 & 5.58 \\
\hline April & 54.1 & 1965 & 21.7 & 1970 & 36.5 & 10.5 & 0.29 & 13.2 \\
\hline May & 94.9 & 1960 & 33.2 & 1977 & 57.6 & 16.7 & 0.29 & 20.8 \\
\hline June & 107 & 1968 & 22.5 & 1971 & 66.8 & 22.7 & 0.34 & 24.1 \\
\hline July & 75.0 & 1965 & 7.68 & 1971 & 32.7 & 17.1 & 0.52 & 11.8 \\
\hline August & 26.8 & 1960 & 3.40 & 1971 & 12.5 & 6.11 & 0.49 & 4.50 \\
\hline September & 16.4 & 1960 & 3.41 & 1971 & 8.56 & 3.49 & 0.41 & 3.09 \\
\hline Annual & 36.8 & 1960 & 12.5 & 1971 & 23.1 & 6.18 & 0.27 & 100 \\
\hline
\end{tabular}


1-8.2R0-1A GHORBAND RIVER AT PUL-I-ASHAWA, Continued

Monthly and annual flow duration, in cubic meters per second

\begin{tabular}{|c|c|c|c|c|c|c|c|c|c|c|c|c|c|}
\hline \multirow{2}{*}{$\begin{array}{l}\text { Percentage } \\
\text { of days } \\
\text { discharge } \\
\text { equaled or } \\
\text { exceeded }\end{array}$} & \multicolumn{12}{|c|}{ Month } & \multirow{2}{*}{ Annual } \\
\hline & October & November & December & January & February & March & April & May & June & July & August & September & \\
\hline 95 & 5.63 & 6.81 & 6.82 & 5.61 & 4.92 & 7.19 & 15.9 & 27.2 & 26.5 & 9.06 & 3.88 & 3.40 & 5.73 \\
\hline 90 & 6.22 & 7.82 & 7.19 & 6.18 & 5.55 & 7.87 & 18.9 & 33.1 & 33.5 & 12.0 & 5.26 & 4.27 & 6.76 \\
\hline 85 & 6.70 & 8.16 & 8.05 & 6.61 & 6.09 & 8.54 & 20.7 & 36.8 & 39.8 & 13.8 & 5.82 & 4.91 & 7.43 \\
\hline 80 & 7.11 & 8.55 & 8.37 & 7.06 & 6.39 & 9.06 & 22.1 & 39.9 & 46.1 & 15.7 & 6.60 & 5.61 & 8.04 \\
\hline 75 & 7.43 & 8.91 & 8.59 & 7.39 & 6.76 & 9.87 & 23.6 & 43.5 & 49.9 & 17.4 & 7.74 & 6.08 & 8.63 \\
\hline 70 & 7.68 & 9.10 & 8.69 & 7.74 & 7.06 & 10.9 & 25.1 & 46.3 & 53.5 & 19.7 & 8.74 & 6.55 & 9.11 \\
\hline 65 & 7.89 & 9.26 & 8.79 & 8.10 & 7.28 & 11.7 & 26.8 & 48.4 & 56.2 & 21.7 & 9.53 & 6.96 & 9.58 \\
\hline 60 & 8.10 & 9.41 & 8.91 & 8.29 & 7.64 & 12.4 & 28.4 & 50.6 & 59.1 & 23.4 & 10.2 & 7.16 & 10.1 \\
\hline 55 & 8.30 & 9.58 & 9.03 & 8.49 & 8.05 & 13.0 & 30.5 & 53.1 & 61.8 & 25.2 & 10.9 & 7.36 & 11.0 \\
\hline 50 & 8.58 & 9.82 & 9.23 & 8.75 & 8.41 & 13.5 & 33.0 & 55.6 & 64.4 & 28.1 & 11.6 & 7.72 & 11.9 \\
\hline 45 & 8.83 & 9.98 & 9.38 & 8.95 & 8.72 & 14.1 & 35.4 & 58.3 & 67.4 & 30.3 & 12.2 & 8.20 & 12.9 \\
\hline 40 & 9.03 & 10.2 & 9.51 & 9.17 & 9.11 & 14.6 & 37.6 & 61.0 & 70.9 & 32.9 & 12.7 & 8.95 & 14.6 \\
\hline 35 & 9.38 & 10.4 & 9.88 & 9.42 & 9.59 & 15.5 & 39.6 & 63.5 & 75.3 & 36.3 & 13.3 & 9.66 & 17.2 \\
\hline 30 & 10.1 & 10.7 & 10.1 & 9.64 & 10.0 & 17.1 & 41.6 & 66.2 & 80.3 & 39.6 & 14.2 & 10.3 & 22.6 \\
\hline 25 & 10.7 & 11.9 & 10.2 & 9.87 & 10.5 & 18.4 & 44.2 & 70.4 & 84.7 & 43.3 & 15.5 & 10.9 & 29.4 \\
\hline 20 & 11.1 & 12.4 & 11.0 & 10.1 & 11.3 & 20.4 & 48.4 & 75.2 & 90.1 & 47.3 & 16.7 & 11.8 & 38.6 \\
\hline 15 & 12.3 & 12.8 & 12.4 & 10.7 & 12.0 & 23.3 & 53.1 & 79.8 & 96.1 & 52.3 & 18.3 & 12.5 & 48.5 \\
\hline 10 & 13.0 & 13.6 & 13.7 & 11.9 & 12.5 & 26.4 & 59.7 & 85.0 & 103 & 59.9 & 20.5 & 13.7 & 59.6 \\
\hline 5 & 14.8 & 15.6 & 14.4 & 12.9 & 13.0 & 29.9 & 74.3 & 93.4 & 111 & 76.5 & 24.8 & 15.3 & 76.7 \\
\hline
\end{tabular}




\section{1-8.2R0-1A GHORBAND RIVER AT PUL-I-ASHAWA, Continued}

Probability of occurrence of annual high discharges

[m $\mathrm{m}^{3} / \mathrm{s}$, cubic meters per second; ng, not given]

\begin{tabular}{|c|c|c|c|c|c|c|}
\hline \multirow{2}{*}{$\begin{array}{c}\text { Exceedance } \\
\text { probability }\end{array}$} & \multirow{2}{*}{$\begin{array}{c}\text { Recurrence } \\
\text { interval } \\
\text { (years) }\end{array}$} & \multirow{2}{*}{$\begin{array}{l}\text { Maximum } \\
\text { instantaneous } \\
\text { discharge } \\
\left(\mathrm{m}^{3} / \mathrm{s}\right)\end{array}$} & \multicolumn{4}{|c|}{ Maximum daily mean discharge $\left(\mathrm{m}^{3} / \mathrm{s}\right)$} \\
\hline & & & $\begin{array}{l}\text { 3-day } \\
\text { period }\end{array}$ & $\begin{array}{l}\text { 7-day } \\
\text { period }\end{array}$ & $\begin{array}{l}\text { 15-day } \\
\text { period }\end{array}$ & $\begin{array}{l}\text { 30-day } \\
\text { period }\end{array}$ \\
\hline 0.99 & 1.01 & 55.0 & 48.0 & 44.4 & 41.5 & 38.2 \\
\hline 0.95 & 1.05 & 67.0 & 57.1 & 53.0 & 49.6 & 45.7 \\
\hline 0.90 & 1.11 & 74.2 & 62.6 & 58.3 & 54.6 & 50.3 \\
\hline 0.80 & 1.25 & 83.7 & 70.0 & 65.5 & 61.2 & 56.4 \\
\hline 0.50 & 2 & 104 & 86.8 & 81.8 & 76.3 & 70.3 \\
\hline 0.20 & 5 & 129 & 107 & 102 & 94.9 & 87.7 \\
\hline 0.10 & 10 & 143 & 120 & 115 & 106 & 98.4 \\
\hline 0.04 & 25 & 159 & 135 & 130 & 120 & 111 \\
\hline 0.02 & 50 & 170 & 146 & 142 & 130 & 121 \\
\hline 0.01 & 100 & 181 & 157 & 152 & 139 & 130 \\
\hline 0.005 & 200 & 190 & 167 & 163 & 148 & 138 \\
\hline 0.002 & 500 & 203 & $\mathrm{ng}$ & $\mathrm{ng}$ & $\mathrm{ng}$ & $\mathrm{ng}$ \\
\hline
\end{tabular}

1-8.2RO-1A GHORBAND RIVER AT PUL-I-ASHAWA, Continued

Probability of occurrence of annual low discharges

$\left[\mathrm{m}^{3} / \mathrm{s}\right.$, meters per second]

\begin{tabular}{|c|c|c|c|c|c|c|c|c|c|c|}
\hline \multirow{3}{*}{$\begin{array}{c}\text { Nonexceedance } \\
\text { probability }\end{array}$} & \multirow{3}{*}{$\begin{array}{c}\text { Recurrence } \\
\text { interval } \\
\text { (years) }\end{array}$} & \multicolumn{9}{|c|}{ Minimum daily mean discharge $\left(\mathrm{m}^{3} / \mathrm{s}\right)$} \\
\hline & & \multicolumn{9}{|c|}{ Number of consecutive days } \\
\hline & & 1 & 3 & 7 & 14 & 30 & 60 & 90 & 120 & 183 \\
\hline 0.05 & 20 & 3.27 & 3.34 & 3.43 & 3.57 & 3.77 & 4.12 & 4.65 & 5.31 & 6.00 \\
\hline 0.10 & 10 & 3.81 & 3.89 & 3.99 & 4.15 & 4.39 & 4.79 & 5.37 & 5.98 & 6.56 \\
\hline 0.20 & 5 & 4.54 & 4.66 & 4.77 & 4.95 & 5.23 & 5.69 & 6.31 & 6.85 & 7.31 \\
\hline 0.50 & 2 & 6.26 & 6.45 & 6.58 & 6.78 & 7.11 & 7.62 & 8.25 & 8.67 & 8.95 \\
\hline
\end{tabular}




\section{1-8.2R0-1A GHORBAND RIVER AT PUL-I-ASHAWA, Continued}

Probability of occurrence of seasonal low discharges

[m $\mathrm{m}^{3} / \mathrm{s}$, meters per second]

\begin{tabular}{|c|c|c|c|c|c|c|c|c|c|}
\hline \multirow{3}{*}{$\begin{array}{c}\text { Nonexceedance } \\
\text { probability }\end{array}$} & \multirow{3}{*}{$\begin{array}{c}\text { Recurrence } \\
\text { interval } \\
\text { (years) }\end{array}$} & \multicolumn{8}{|c|}{ Minimum daily mean discharge $\left(\mathrm{m}^{3} / \mathrm{s}\right)$} \\
\hline & & \multicolumn{8}{|c|}{ Number of consecutive days } \\
\hline & & 1 & 7 & 14 & 30 & 1 & 7 & 14 & 30 \\
\hline & & \multicolumn{4}{|c|}{ December-January-February } & \multicolumn{4}{|c|}{ March-April-May } \\
\hline 0.05 & 20 & 4.22 & 4.44 & 4.70 & 5.09 & 5.74 & 6.52 & 7.07 & 8.81 \\
\hline 0.10 & 10 & 4.72 & 4.99 & 5.24 & 5.64 & 6.15 & 6.92 & 7.57 & 9.53 \\
\hline 0.20 & 5 & 5.40 & 5.73 & 5.96 & 6.36 & 6.79 & 7.56 & 8.35 & 10.6 \\
\hline \multirow[t]{2}{*}{0.50} & 2 & 6.95 & 7.37 & 7.56 & 7.96 & 8.66 & 9.46 & 10.7 & 13.7 \\
\hline & & \multicolumn{4}{|c|}{ June-July-August } & \multicolumn{4}{|c|}{ September-October-November } \\
\hline 0.05 & 20 & 3.44 & 3.70 & 3.92 & 4.45 & 2.40 & 2.90 & 3.50 & 3.95 \\
\hline 0.10 & 10 & 4.26 & 4.56 & 4.85 & 5.57 & 3.20 & 3.61 & 4.13 & 4.57 \\
\hline 0.20 & 5 & 5.46 & 5.82 & 6.20 & 7.21 & 4.35 & 4.63 & 5.01 & 5.45 \\
\hline 0.50 & 2 & 8.50 & 8.97 & 9.57 & 11.3 & 7.04 & 7.05 & 7.14 & 7.52 \\
\hline
\end{tabular}


1-8.2R0-1A GHORBAND RIVER AT PUL-I-ASHAWA, Continued

\begin{tabular}{|c|c|c|c|c|c|}
\hline \multicolumn{6}{|c|}{$\begin{array}{l}\text { Annual peak discharges } \\
\text { [m³/s, meters per second] }\end{array}$} \\
\hline \multicolumn{3}{|c|}{$\begin{array}{c}\text { Annual peak discharge, } \\
\text { by year }\end{array}$} & \multicolumn{3}{|c|}{$\begin{array}{l}\text { Annual peak discharge, } \\
\text { from highest to lowest }\end{array}$} \\
\hline $\begin{array}{l}\text { Water } \\
\text { year }\end{array}$ & Date & $\begin{array}{c}\text { Peak } \\
\text { discharge } \\
\left(\mathrm{m}^{3} / \mathrm{s}\right)\end{array}$ & $\begin{array}{l}\text { Water } \\
\text { year }\end{array}$ & Date & $\begin{array}{c}\text { Peak } \\
\text { discharge } \\
\left(\mathrm{m}^{3} / \mathrm{s}\right)\end{array}$ \\
\hline 1960 & April 18, 1960 & 146 & 1967 & April 27, 1967 & 161 \\
\hline 1961 & June 5, 1961 & 139 & 1960 & April 18, 1960 & 146 \\
\hline 1962 & June 11, 1962 & 84.4 & 1961 & June 5, 1961 & 139 \\
\hline 1963 & May 15,1963 & 96.2 & 1968 & June 10, 1968 & 131 \\
\hline 1964 & April 11, 1964 & 93.0 & 1976 & June 4, 1976 & 130 \\
\hline 1965 & June 13, 1965 & 118 & 1972 & June 13, 1972 & 123 \\
\hline 1966 & June 18, 1966 & 108 & 1975 & May 16, 1975 & 121 \\
\hline 1967 & April 27, 1967 & 161 & 1965 & June 13,1965 & 118 \\
\hline 1968 & June 10, 1968 & 131 & 1973 & June 4, 1973 & 111 \\
\hline 1969 & June 17, 1969 & 97.5 & 1966 & June 18,1966 & 108 \\
\hline 1970 & May 21, 1970 & 73.2 & 1969 & June 17, 1969 & 97.5 \\
\hline 1971 & May 20, 1971 & 69.7 & 1979 & June 22, 1979 & 96.8 \\
\hline 1972 & June 13, 1972 & 123 & 1963 & May 15,1963 & 96.2 \\
\hline 1973 & June 4, 1973 & 111 & 1964 & April 11, 1964 & 93.0 \\
\hline 1974 & June 1, 1974 & 63.2 & 1977 & May 27, 1977 & 86.4 \\
\hline 1975 & May 16, 1975 & 121 & 1978 & April 17, 1978 & 85.6 \\
\hline 1976 & June 4, 1976 & 130 & 1962 & June 11, 1962 & 84.4 \\
\hline 1977 & May 27, 1977 & 86.4 & 1970 & May 21, 1970 & 73.2 \\
\hline 1978 & April 17, 1978 & 85.6 & 1971 & May 20, 1971 & 69.7 \\
\hline 1979 & June 22, 1979 & 96.8 & 1974 & June 1,1974 & 63.2 \\
\hline
\end{tabular}


1-8.2R0-1A GHORBAND RIVER AT PUL-I-ASHAWA, Continued

Monthly and annual mean discharges, in cubic meters per second

[Data may not be rounded in accordance with U.S. Geological Survey publication standards; --, no data]

\begin{tabular}{|c|c|c|c|c|c|c|c|c|c|c|c|c|c|}
\hline \multirow{2}{*}{$\begin{array}{l}\text { Water } \\
\text { year }\end{array}$} & \multicolumn{12}{|c|}{ Monthly mean discharge } & \multirow{2}{*}{$\begin{array}{c}\text { Annual } \\
\text { discharge }\end{array}$} \\
\hline & October & November & December & January & February & March & April & May & June & July & August & September & \\
\hline 1960 & 6.21 & 6.76 & 6.68 & 7.48 & 16.0 & 35.0 & 54.0 & 94.9 & 102 & 68.4 & 26.8 & 16.4 & 36.8 \\
\hline 1961 & 15.4 & 16.2 & 14.4 & 13.0 & 12.1 & 13.6 & 39.3 & 68.3 & 77.7 & 37.1 & 15.8 & 11.7 & 27.9 \\
\hline 1962 & 11.1 & 12.8 & 13.7 & 12.9 & 12.6 & 14.0 & 29.1 & 40.5 & 48.2 & 23.7 & 12.3 & 14.1 & 20.4 \\
\hline 1963 & 8.40 & 9.63 & 8.95 & 8.01 & 7.00 & 10.3 & 22.2 & 61.1 & 72.8 & 35.9 & 10.9 & 7.58 & 22.0 \\
\hline 1964 & 7.61 & 9.66 & 9.53 & 9.26 & 9.02 & 16.0 & 46.5 & 59.0 & 62.1 & 31.3 & 11.3 & 6.85 & 23.2 \\
\hline 1965 & 7.66 & 9.07 & 8.65 & 8.44 & 7.99 & 18.6 & 54.1 & 89.5 & 100 & 75.0 & 25.7 & 13.4 & 35.0 \\
\hline 1966 & 12.7 & 12.9 & 12.0 & 10.3 & 10.5 & 14.9 & 34.5 & 53.0 & 72.0 & 25.7 & 10.5 & 8.51 & 23.1 \\
\hline 1967 & 9.18 & 9.49 & 8.43 & 7.24 & 6.77 & 10.5 & 33.8 & 48.1 & 70.6 & 35.7 & 15.2 & 11.1 & 22.2 \\
\hline 1968 & 9.45 & 9.88 & 10.2 & 9.23 & 9.32 & 19.3 & 42.9 & 59.1 & 107 & 51.9 & 17.0 & 10.1 & 29.6 \\
\hline 1969 & 11.8 & 12.3 & 13.9 & 10.5 & 11.5 & 23.2 & 39.3 & 50.0 & 74.7 & 38.1 & 15.0 & 10.5 & 25.9 \\
\hline 1970 & 12.4 & 14.4 & 9.43 & 8.29 & 7.38 & 8.45 & 21.7 & 40.8 & 34.0 & 13.4 & 6.21 & 5.85 & 15.2 \\
\hline 1971 & 8.57 & 9.28 & 8.10 & 6.12 & 4.63 & 10.9 & 22.8 & 42.6 & 22.5 & 7.68 & 3.40 & 3.41 & 12.5 \\
\hline 1972 & 5.08 & 6.91 & 6.84 & 5.72 & 5.26 & 19.7 & 36.7 & 74.0 & 91.1 & 36.9 & 13.2 & 7.98 & 25.8 \\
\hline 1973 & 10.4 & 10.8 & 10.1 & 10.4 & 9.73 & 19.8 & 45.1 & 73.1 & 67.3 & 25.4 & 12.5 & 9.24 & 25.4 \\
\hline 1974 & 9.47 & 10.5 & 9.95 & 9.82 & 8.57 & 11.1 & 23.1 & 39.5 & 42.6 & 14.9 & 5.60 & 5.86 & 15.9 \\
\hline 1975 & 8.30 & 8.96 & 8.48 & 6.99 & 6.32 & 11.2 & 29.2 & 49.2 & 61.1 & 29.0 & 8.48 & 4.43 & 19.3 \\
\hline 1976 & 6.85 & 8.18 & 9.29 & 8.63 & 6.24 & 11.7 & 41.5 & 71.3 & 69.8 & 31.9 & 8.51 & 7.27 & 23.4 \\
\hline 1977 & 8.20 & 8.73 & 9.50 & 9.45 & 10.0 & 15.2 & 24.7 & 33.2 & 47.9 & 13.6 & 5.63 & 4.55 & 15.9 \\
\hline 1978 & 6.60 & 8.89 & 8.91 & 9.03 & 8.77 & 15.6 & 47.5 & 57.3 & 42.8 & 20.7 & 8.85 & 7.22 & 20.2 \\
\hline 1979 & 8.21 & 10.1 & 9.27 & 8.05 & 6.71 & 10.2 & 42.3 & 47.5 & 70.5 & 37.1 & 16.2 & 5.21 & 22.6 \\
\hline 1980 & 7.12 & 11.2 & 8.27 & 6.03 & -- & -- & -- & -- & -- & -- & -- & -- & -- \\
\hline
\end{tabular}




\section{1-8.3R0-1A SHATUL RIVER AT GULBAHAR}

(U.S. Geological Survey identification number: 350900069170000)

LOCATION: Lat $35^{\circ} 09^{\prime} \mathrm{N}$., long $69^{\circ} 17^{\prime} \mathrm{E}$.

DRAINAGE AREA: $205 \mathrm{~km}^{2}$.

ELEVATION: 1,590 meters above mean sea level.

PERIOD OF RECORD: May 30, 1967 to March 6, 1980.

GAGE: Water-stage recorder.

Annual mean discharge

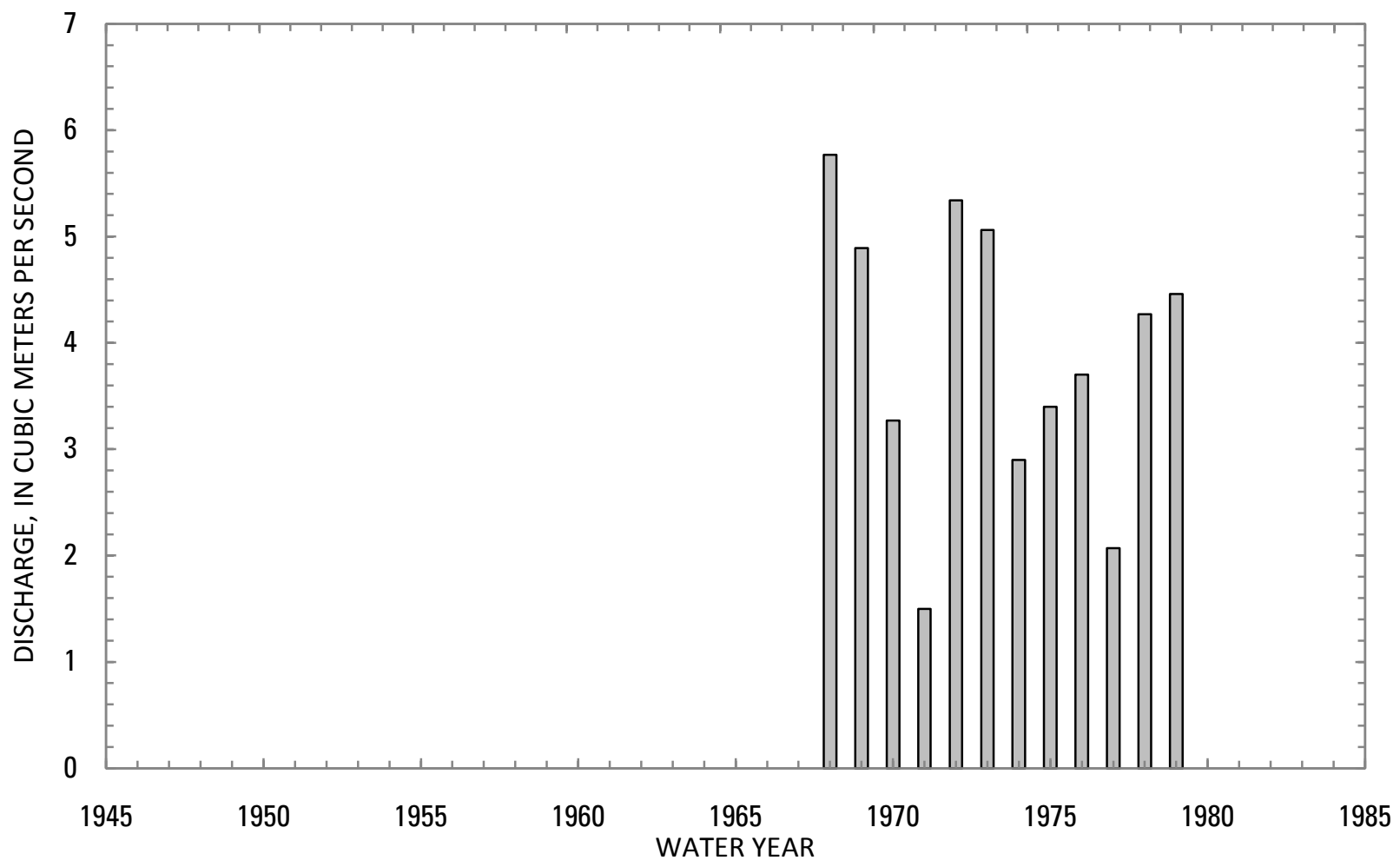




\section{1-8.3R0-1A SHATUL RIVER AT GULBAHAR, Continued}

Statistics of monthly and annual mean discharges [m $\mathrm{m}^{3} / \mathrm{s}$, cubic meters per second]

\begin{tabular}{|c|c|c|c|c|c|c|c|c|}
\hline \multirow[b]{2}{*}{ Month } & \multicolumn{2}{|c|}{ Maximum } & \multicolumn{2}{|c|}{ Minimum } & \multicolumn{4}{|c|}{ Mean } \\
\hline & $\begin{array}{c}\text { Discharge } \\
\left(\mathrm{m}^{3} / \mathrm{s}\right)\end{array}$ & $\begin{array}{l}\text { Water year } \\
\text { of } \\
\text { occurrence }\end{array}$ & $\begin{array}{c}\text { Discharge } \\
\left(\mathrm{m}^{3} / \mathrm{s}\right)\end{array}$ & $\begin{array}{c}\text { Water year } \\
\text { of } \\
\text { occurrence }\end{array}$ & $\begin{array}{c}\text { Discharge } \\
\left(\mathrm{m}^{3} / \mathrm{s}\right)\end{array}$ & $\begin{array}{c}\text { Standard } \\
\text { deviation } \\
\left(\mathrm{m}^{3} / \mathrm{s}\right)\end{array}$ & $\begin{array}{c}\text { Coefficient } \\
\text { of } \\
\text { variation }\end{array}$ & $\begin{array}{c}\text { Percentage } \\
\text { of annual } \\
\text { discharge }\end{array}$ \\
\hline October & 1.03 & 1970 & 0.03 & 1971 & 0.25 & 0.27 & 1.09 & 0.53 \\
\hline November & 0.59 & 1970 & 0.03 & 1979 & 0.14 & 0.15 & 1.13 & 0.29 \\
\hline December & 0.27 & 1970 & 0.03 & 1979 & 0.12 & 0.10 & 0.82 & 0.25 \\
\hline January & 0.62 & 1973 & 0.02 & 1979 & 0.19 & 0.17 & 0.90 & 0.39 \\
\hline February & 0.58 & 1976 & 0.01 & 1971 & 0.29 & 0.17 & 0.60 & 0.62 \\
\hline March & 3.51 & 1969 & 0.36 & 1971 & 1.77 & 0.91 & 0.51 & 3.73 \\
\hline April & 11.0 & 1978 & 2.20 & 1971 & 7.05 & 2.47 & 0.35 & 14.9 \\
\hline May & 17.5 & 1978 & 5.23 & 1977 & 10.7 & 3.71 & 0.35 & 22.5 \\
\hline June & 25.3 & 1972 & 6.13 & 1971 & 15.6 & 5.53 & 0.36 & 32.9 \\
\hline July & 15.7 & 1968 & 1.27 & 1971 & 8.38 & 4.55 & 0.54 & 17.7 \\
\hline August & 6.16 & 1968 & 0.75 & 1974 & 2.44 & 1.89 & 0.77 & 5.16 \\
\hline September & 1.58 & 1969 & 0.03 & 1979 & 0.53 & 0.50 & 0.96 & 1.11 \\
\hline Annual & 5.77 & 1968 & 1.50 & 1971 & 3.89 & 1.32 & 0.34 & 100 \\
\hline
\end{tabular}


1-8.3R0-1A SHATUL RIVER AT GULBAHAR, Continued

Monthly and annual flow duration, in cubic meters per second

\begin{tabular}{|c|c|c|c|c|c|c|c|c|c|c|c|c|c|}
\hline \multirow{2}{*}{$\begin{array}{l}\text { Percentage } \\
\text { of days } \\
\text { discharge } \\
\text { equaled or } \\
\text { exceeded }\end{array}$} & \multicolumn{12}{|c|}{ Month } & \multirow{2}{*}{ Annual } \\
\hline & October & November & December & January & February & March & April & May & June & July & August & September & \\
\hline 95 & 0.04 & 0.02 & 0.02 & 0.02 & 0.02 & 0.07 & 2.09 & 3.64 & 6.37 & 1.03 & 0.22 & 0.02 & 0.03 \\
\hline 90 & 0.05 & 0.03 & 0.03 & 0.03 & 0.06 & 0.25 & 2.90 & 4.61 & 8.14 & 1.51 & 0.37 & 0.03 & 0.04 \\
\hline 85 & 0.06 & 0.03 & 0.03 & 0.03 & 0.07 & 0.34 & 3.45 & 5.54 & 9.15 & 1.88 & 0.49 & 0.06 & 0.06 \\
\hline 80 & 0.07 & 0.04 & 0.04 & 0.04 & 0.09 & 0.39 & 3.78 & 6.32 & 10.1 & 2.72 & 0.64 & 0.07 & 0.08 \\
\hline 75 & 0.08 & 0.04 & 0.04 & 0.04 & 0.10 & 0.48 & 4.13 & 6.97 & 11.0 & 3.57 & 0.78 & 0.08 & 0.12 \\
\hline 70 & 0.10 & 0.05 & 0.04 & 0.05 & 0.12 & 0.66 & 4.45 & 7.80 & 11.8 & 4.66 & 0.93 & 0.12 & 0.17 \\
\hline 65 & 0.11 & 0.05 & 0.05 & 0.06 & 0.15 & 0.75 & 4.93 & 8.37 & 12.7 & 5.22 & 1.10 & 0.17 & 0.23 \\
\hline 60 & 0.12 & 0.05 & 0.06 & 0.06 & 0.17 & 0.88 & 5.43 & 8.90 & 13.5 & 6.39 & 1.33 & 0.20 & 0.32 \\
\hline 55 & 0.13 & 0.06 & 0.06 & 0.07 & 0.22 & 1.05 & 6.11 & 9.58 & 14.2 & 7.02 & 1.46 & 0.23 & 0.44 \\
\hline 50 & 0.15 & 0.06 & 0.07 & 0.09 & 0.24 & 1.35 & 6.53 & 10.3 & 15.0 & 7.84 & 1.65 & 0.27 & 0.69 \\
\hline 45 & 0.17 & 0.08 & 0.07 & 0.12 & 0.28 & 1.61 & 7.04 & 11.0 & 16.1 & 8.73 & 1.95 & 0.33 & 1.01 \\
\hline 40 & 0.19 & 0.10 & 0.09 & 0.16 & 0.33 & 1.82 & 7.49 & 11.5 & 17.3 & 9.50 & 2.21 & 0.40 & 1.68 \\
\hline 35 & 0.21 & 0.13 & 0.10 & 0.19 & 0.35 & 2.03 & 8.14 & 12.2 & 18.3 & 10.3 & 2.74 & 0.49 & 2.84 \\
\hline 30 & 0.28 & 0.15 & 0.13 & 0.24 & 0.37 & 2.37 & 8.92 & 13.2 & 19.2 & 11.5 & 3.02 & 0.72 & 4.13 \\
\hline 25 & 0.36 & 0.18 & 0.16 & 0.30 & 0.40 & 2.96 & 9.61 & 14.6 & 20.1 & 12.7 & 3.49 & 0.85 & 6.08 \\
\hline 20 & 0.42 & 0.22 & 0.20 & 0.34 & 0.43 & 3.28 & 10.3 & 15.5 & 21.2 & 13.7 & 4.00 & 0.99 & 8.15 \\
\hline 15 & 0.46 & 0.25 & 0.27 & 0.38 & 0.50 & 3.58 & 11.4 & 16.2 & 22.6 & 14.6 & 4.82 & 1.19 & 10.4 \\
\hline 10 & 0.52 & 0.31 & 0.29 & 0.43 & 0.64 & 4.02 & 12.4 & 17.2 & 24.4 & 15.5 & 5.99 & 1.47 & 13.1 \\
\hline 5 & 0.93 & 0.52 & 0.36 & 0.59 & 0.82 & 4.71 & 13.6 & 18.7 & 26.7 & 18.1 & 7.39 & 1.76 & 17.1 \\
\hline
\end{tabular}




\section{1-8.3R0-1A SHATUL RIVER AT GULBAHAR, Continued}

Probability of occurrence of annual high discharges

[ $\mathrm{m}^{3} / \mathrm{s}$, cubic meters per second; $\mathrm{ng}$, not given]

\begin{tabular}{|c|c|c|c|c|c|c|}
\hline \multirow{2}{*}{$\begin{array}{c}\text { Exceedance } \\
\text { probability }\end{array}$} & \multirow{2}{*}{$\begin{array}{c}\text { Recurrence } \\
\text { interval } \\
\text { (years) }\end{array}$} & \multirow{2}{*}{$\begin{array}{l}\text { Maximum } \\
\text { instantaneous } \\
\text { discharge } \\
\left(\mathrm{m}^{3} / \mathrm{s}\right)\end{array}$} & \multicolumn{4}{|c|}{ Maximum daily mean discharge $\left(\mathrm{m}^{3} / \mathrm{s}\right)$} \\
\hline & & & $\begin{array}{l}\text { 3-day } \\
\text { period }\end{array}$ & $\begin{array}{l}\text { 7-day } \\
\text { period }\end{array}$ & $\begin{array}{l}\text { 15-day } \\
\text { period }\end{array}$ & $\begin{array}{l}\text { 30-day } \\
\text { period }\end{array}$ \\
\hline 0.99 & 1.01 & 16.4 & 9.49 & 8.84 & 8.19 & 7.05 \\
\hline 0.95 & 1.05 & 19.2 & 11.9 & 11.2 & 10.3 & 9.10 \\
\hline 0.90 & 1.11 & 20.9 & 13.4 & 12.6 & 11.7 & 10.4 \\
\hline 0.80 & 1.25 & 23.2 & 15.4 & 14.5 & 13.5 & 12.1 \\
\hline 0.50 & 2 & 28.5 & 19.8 & 18.6 & 17.5 & 15.9 \\
\hline 0.20 & 5 & 35.2 & 25.0 & 23.7 & 22.4 & 20.6 \\
\hline 0.10 & 10 & 39.5 & 28.0 & 26.6 & 25.4 & 23.4 \\
\hline 0.04 & 25 & 44.6 & 31.5 & 30.0 & 28.9 & 26.7 \\
\hline 0.02 & 50 & 48.4 & 33.9 & 32.4 & 31.3 & 28.9 \\
\hline 0.01 & 100 & 52.1 & 36.1 & 34.6 & 33.6 & 31.0 \\
\hline 0.005 & 200 & 55.7 & 38.2 & 36.7 & 35.9 & 33.0 \\
\hline 0.002 & 500 & 60.6 & $\mathrm{ng}$ & $\mathrm{ng}$ & $\mathrm{ng}$ & ng \\
\hline
\end{tabular}

\section{1-8.3R0-1A SHATUL RIVER AT GULBAHAR, Continued}

Probability of occurrence of annual low discharges

$\left[\mathrm{m}^{3} / \mathrm{s}\right.$, meters per second]

\begin{tabular}{|c|c|c|c|c|c|c|c|c|c|c|}
\hline \multirow{3}{*}{$\begin{array}{c}\text { Nonexceedance } \\
\text { probability }\end{array}$} & \multirow{3}{*}{$\begin{array}{c}\text { Recurrence } \\
\text { interval } \\
\text { (years) }\end{array}$} & \multicolumn{9}{|c|}{ Minimum daily mean discharge $\left(\mathrm{m}^{3} / \mathrm{s}\right)$} \\
\hline & & \multicolumn{9}{|c|}{ Number of consecutive days } \\
\hline & & 1 & 3 & 7 & 14 & 30 & 60 & 90 & 120 & 183 \\
\hline 0.05 & 20 & 0.01 & 0.01 & 0.01 & 0.01 & 0.01 & 0.02 & 0.02 & 0.02 & 0.03 \\
\hline 0.10 & 10 & 0.01 & 0.01 & 0.01 & 0.01 & 0.01 & 0.02 & 0.02 & 0.03 & 0.05 \\
\hline 0.20 & 5 & 0.01 & 0.01 & 0.01 & 0.02 & 0.02 & 0.03 & 0.03 & 0.04 & 0.07 \\
\hline 0.50 & 2 & 0.02 & 0.02 & 0.02 & 0.03 & 0.04 & 0.05 & 0.07 & 0.08 & 0.16 \\
\hline
\end{tabular}




\section{1-8.3R0-1A SHATUL RIVER AT GULBAHAR, Continued}

Probability of occurrence of seasonal low discharges [ $\mathrm{m}^{3} / \mathrm{s}$, meters per second]

\begin{tabular}{|c|c|c|c|c|c|c|c|c|c|}
\hline \multirow{3}{*}{$\begin{array}{c}\text { Nonexceedance } \\
\text { probability }\end{array}$} & \multirow{3}{*}{$\begin{array}{c}\text { Recurrence } \\
\text { interval } \\
\text { (years) }\end{array}$} & \multicolumn{8}{|c|}{ Minimum daily mean discharge $\left(\mathrm{m}^{3} / \mathrm{s}\right)$} \\
\hline & & \multicolumn{8}{|c|}{ Number of consecutive days } \\
\hline & & 1 & 7 & 14 & 30 & 1 & 7 & 14 & 30 \\
\hline & & \multicolumn{4}{|c|}{ December-January-February } & \multicolumn{4}{|c|}{ March-April-May } \\
\hline 0.05 & 20 & 0.01 & 0.01 & 0.01 & 0.01 & 0.02 & 0.03 & 0.04 & 0.40 \\
\hline 0.10 & 10 & 0.01 & 0.01 & 0.02 & 0.02 & 0.05 & 0.07 & 0.11 & 0.59 \\
\hline 0.20 & 5 & 0.01 & 0.02 & 0.02 & 0.03 & 0.11 & 0.17 & 0.26 & 0.88 \\
\hline \multirow[t]{2}{*}{0.50} & 2 & 0.03 & 0.03 & 0.05 & 0.06 & 0.34 & 0.50 & 0.81 & 1.64 \\
\hline & & \multicolumn{4}{|c|}{ June-July-August } & \multicolumn{4}{|c|}{ September-0ctober-November } \\
\hline 0.05 & 20 & 0.05 & 0.10 & 0.18 & 0.54 & 0.01 & 0.01 & 0.01 & 0.01 \\
\hline 0.10 & 10 & 0.09 & 0.16 & 0.26 & 0.69 & 0.01 & 0.01 & 0.01 & 0.02 \\
\hline 0.20 & 5 & 0.17 & 0.26 & 0.40 & 0.94 & 0.01 & 0.02 & 0.02 & 0.03 \\
\hline 0.50 & 2 & 0.55 & 0.70 & 0.93 & 1.74 & 0.03 & 0.04 & 0.05 & 0.07 \\
\hline
\end{tabular}

\section{1-8.3R0-1A SHATUL RIVER AT GULBAHAR, Continued}

Annual peak discharges

[ $\mathrm{m}^{3} / \mathrm{s}$, meters per second]

\begin{tabular}{|c|c|c|c|c|c|}
\hline \multicolumn{3}{|c|}{$\begin{array}{c}\text { Annual peak discharge, } \\
\text { by year }\end{array}$} & \multicolumn{3}{|c|}{$\begin{array}{l}\text { Annual peak discharge, } \\
\text { from highest to lowest }\end{array}$} \\
\hline $\begin{array}{l}\text { Water } \\
\text { year }\end{array}$ & Date & $\begin{array}{c}\text { Peak } \\
\text { discharge } \\
\left(\mathrm{m}^{3} / \mathrm{s}\right)\end{array}$ & $\begin{array}{l}\text { Water } \\
\text { year }\end{array}$ & Date & $\begin{array}{c}\text { Peak } \\
\text { discharge } \\
\left(\mathrm{m}^{3} / \mathrm{s}\right)\end{array}$ \\
\hline 1968 & June 25, 1968 & 41.8 & 1968 & June 25, 1968 & 41.8 \\
\hline 1969 & June 16, 1969 & 28.2 & 1972 & June 12,1972 & 40.6 \\
\hline 1970 & May 21, 1970 & 27.6 & 1973 & June 11,1973 & 35.1 \\
\hline 1971 & May 19, 1971 & 20.6 & 1979 & June 13, 1979 & 35.0 \\
\hline 1972 & June 12, 1972 & 40.6 & 1975 & June 17,1975 & 30.9 \\
\hline 1973 & June 11, 1973 & 35.1 & 1969 & June 16, 1969 & 28.2 \\
\hline 1974 & June 4, 1974 & 21.6 & 1970 & May 21, 1970 & 27.6 \\
\hline 1975 & June 17,1975 & 30.9 & 1978 & June 5, 1978 & 27.0 \\
\hline 1976 & July 6, 1976 & 24.5 & 1976 & July 6, 1976 & 24.5 \\
\hline 1977 & June 22, 1977 & 20.7 & 1974 & June 4, 1974 & 21.6 \\
\hline 1978 & June 5, 1978 & 27.0 & 1977 & June 22, 1977 & 20.7 \\
\hline 1979 & June 13, 1979 & 35.0 & 1971 & May 19,1971 & 20.6 \\
\hline
\end{tabular}




\section{1-8.3R0-1A SHATUL RIVER AT GULBAHAR, Continued}

Monthly and annual mean discharges, in cubic meters per second

[Data may not be rounded in accordance with U.S. Geological Survey publication standards; --, no data]

\begin{tabular}{|c|c|c|c|c|c|c|c|c|c|c|c|c|c|}
\hline \multirow{2}{*}{$\begin{array}{l}\text { Water } \\
\text { year }\end{array}$} & \multicolumn{12}{|c|}{ Monthly mean discharge } & \multirow{2}{*}{$\begin{array}{c}\text { Annual } \\
\text { discharge }\end{array}$} \\
\hline & October & November & December & January & February & March & April & May & June & July & August & September & \\
\hline 1967 & -- & -- & -- & -- & -- & -- & -- & -- & 19.1 & 12.5 & 4.03 & 0.92 & -- \\
\hline 1968 & 0.45 & 0.23 & 0.19 & 0.37 & 0.35 & 2.54 & 8.24 & 11.3 & 22.5 & 15.7 & 6.16 & 1.21 & 5.77 \\
\hline 1969 & 0.42 & 0.23 & 0.26 & 0.22 & 0.39 & 3.51 & 8.36 & 9.03 & 16.1 & 12.5 & 5.85 & 1.58 & 4.89 \\
\hline 1970 & 1.03 & 0.59 & 0.27 & 0.30 & 0.35 & 1.67 & 6.47 & 12.3 & 12.2 & 2.92 & 0.99 & 0.04 & 3.27 \\
\hline 1971 & 0.03 & 0.04 & 0.03 & 0.03 & 0.01 & 0.36 & 2.20 & 6.89 & 6.13 & 1.27 & 0.75 & 0.17 & 1.50 \\
\hline 1972 & 0.18 & 0.10 & 0.06 & 0.06 & 0.08 & 2.37 & 6.42 & 13.5 & 25.3 & 11.8 & 3.53 & 0.78 & 5.34 \\
\hline 1973 & 0.37 & 0.06 & 0.21 & 0.62 & 0.44 & 2.86 & 9.84 & 16.3 & 19.8 & 8.57 & 1.28 & 0.24 & 5.06 \\
\hline 1974 & 0.17 & 0.10 & 0.08 & 0.08 & 0.37 & 1.56 & 6.82 & 8.91 & 10.7 & 5.08 & 0.75 & 0.13 & 2.90 \\
\hline 1975 & 0.09 & 0.03 & 0.03 & 0.05 & 0.16 & 0.67 & 6.14 & 7.47 & 14.4 & 9.38 & 1.98 & 0.33 & 3.40 \\
\hline 1976 & 0.19 & 0.08 & 0.07 & 0.13 & 0.58 & 1.71 & 6.30 & 9.28 & 12.2 & 11.0 & 2.40 & 0.44 & 3.70 \\
\hline 1977 & 0.09 & 0.04 & 0.04 & 0.15 & 0.33 & 1.34 & 3.76 & 5.23 & 9.55 & 3.49 & 0.75 & 0.07 & 2.07 \\
\hline 1978 & 0.09 & 0.05 & 0.04 & 0.18 & 0.13 & 1.29 & 11.0 & 17.5 & 14.5 & 3.91 & 1.47 & 0.90 & 4.27 \\
\hline 1979 & 0.06 & 0.03 & 0.03 & 0.02 & 0.12 & 1.31 & 9.05 & 10.1 & 20.0 & 10.8 & 1.84 & 0.03 & 4.46 \\
\hline 1980 & 0.10 & 0.19 & 0.23 & 0.22 & 0.49 & -- & -- & -- & -- & -- & -- & -- & -- \\
\hline
\end{tabular}




\section{1-8.L00-3A PANJSHER RIVER AT SHUKHI}

\section{(U.S. Geological Survey identification number: 345600069290000)}

LOCATION: Lat $34^{\circ} 56^{\prime} \mathrm{N}$., long $69^{\circ} 29^{\prime} \mathrm{E}$.

DRAINAGE AREA: $10,850 \mathrm{~km}^{2}$.

ELEVATION: 1,400 meters above mean sea level.

PERIOD OF RECORD: October 1, 1966 to September 30, 1980.

GAGE: Water-stage recorder.

\section{Annual mean discharge}

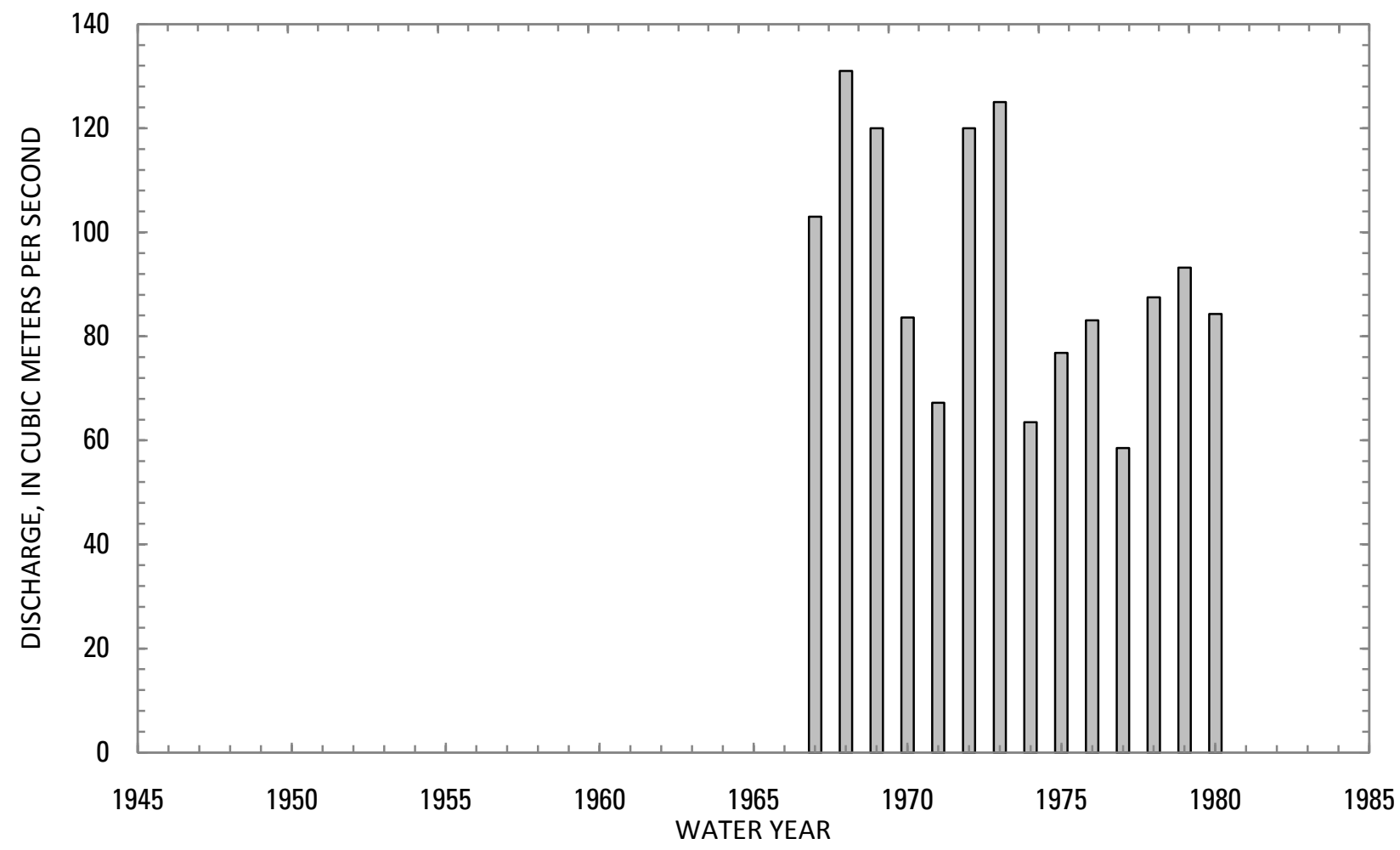




\section{1-8.L00-3A PANJSHER RIVER AT SHUKHI, Continued}

Statistics of monthly and annual mean discharges $\left[\mathrm{m}^{3} / \mathrm{s}\right.$, cubic meters per second]

\begin{tabular}{|c|c|c|c|c|c|c|c|c|}
\hline \multirow[b]{2}{*}{ Month } & \multicolumn{2}{|c|}{ Maximum } & \multicolumn{2}{|c|}{ Minimum } & \multicolumn{4}{|c|}{ Mean } \\
\hline & $\begin{array}{c}\text { Discharge } \\
\left(\mathrm{m}^{3} / \mathrm{s}\right)\end{array}$ & $\begin{array}{c}\text { Water year } \\
\text { of } \\
\text { occurrence }\end{array}$ & $\begin{array}{c}\text { Discharge } \\
\left(\mathrm{m}^{3} / \mathrm{s}\right)\end{array}$ & $\begin{array}{c}\text { Water year } \\
\text { of } \\
\text { occurrence }\end{array}$ & $\begin{array}{c}\text { Discharge } \\
\left(\mathrm{m}^{3} / \mathrm{s}\right)\end{array}$ & $\begin{array}{c}\text { Standard } \\
\text { deviation } \\
\left(\mathrm{m}^{3} / \mathrm{s}\right)\end{array}$ & $\begin{array}{c}\text { Coefficient } \\
\text { of } \\
\text { variation }\end{array}$ & $\begin{array}{c}\text { Percentage } \\
\text { of annual } \\
\text { discharge }\end{array}$ \\
\hline October & 32.2 & 1973 & 23.6 & 1970 & 27.7 & 2.52 & 0.09 & 2.49 \\
\hline November & 32.9 & 1970 & 22.6 & 1972 & 29.4 & 3.24 & 0.11 & 2.64 \\
\hline December & 45.0 & 1969 & 23.9 & 1972 & 32.8 & 5.25 & 0.16 & 2.95 \\
\hline January & 44.1 & 1969 & 26.0 & 1972 & 34.9 & 5.20 & 0.15 & 3.13 \\
\hline February & 37.2 & 1969 & 24.7 & 1972 & 31.3 & 4.20 & 0.13 & 2.81 \\
\hline March & 67.2 & 1969 & 29.0 & 1974 & 41.3 & 11.5 & 0.28 & 3.71 \\
\hline April & 207 & 1973 & 60.2 & 1977 & 123 & 38.3 & 0.31 & 11.1 \\
\hline May & 345 & 1973 & 95.5 & 1977 & 203 & 64.6 & 0.32 & 18.2 \\
\hline June & 493 & 1968 & 190 & 1971 & 326 & 102 & 0.31 & 29.3 \\
\hline July & 362 & 1968 & 45.2 & 1971 & 177 & 93.0 & 0.53 & 15.9 \\
\hline August & 112 & 1968 & 25.9 & 1980 & 55.9 & 26.9 & 0.48 & 5.02 \\
\hline September & 38.9 & 1970 & 22.9 & 1980 & 30.6 & 4.13 & 0.13 & 2.75 \\
\hline Annual & 131 & 1968 & 58.5 & 1977 & 92.6 & 23.7 & 0.26 & 100 \\
\hline
\end{tabular}


1-8.LO0-3A PANJSHER RIVER AT SHUKHI, Continued

Monthly and annual flow duration, in cubic meters per second

\begin{tabular}{|c|c|c|c|c|c|c|c|c|c|c|c|c|c|}
\hline \multirow{2}{*}{$\begin{array}{l}\text { Percentage } \\
\text { of days } \\
\text { discharge } \\
\text { equaled or } \\
\text { exceeded }\end{array}$} & \multicolumn{12}{|c|}{ Month } & \multirow{2}{*}{ Annual } \\
\hline & October & November & December & January & February & March & April & May & June & July & August & September & \\
\hline 95 & 22.7 & 22.9 & 25.0 & 25.9 & 24.1 & 24.5 & 44.5 & 70.0 & 133 & 37.7 & 25.6 & 22.7 & 24.6 \\
\hline 90 & 24.2 & 23.9 & 26.0 & 28.5 & 25.1 & 25.6 & 50.2 & 98.5 & 175 & 47.6 & 27.2 & 23.1 & 26.3 \\
\hline 85 & 24.4 & 24.6 & 28.1 & 29.5 & 26.0 & 27.0 & 57.5 & 111 & 197 & 57.9 & 28.5 & 25.9 & 27.8 \\
\hline 80 & 25.2 & 27.1 & 28.9 & 30.0 & 27.2 & 27.8 & 64.1 & 124 & 216 & 70.3 & 30.5 & 27.0 & 28.6 \\
\hline 75 & 25.5 & 27.6 & 29.5 & 30.4 & 27.7 & 28.6 & 72.7 & 134 & 230 & 81.1 & 31.7 & 27.7 & 29.4 \\
\hline 70 & 26.2 & 28.1 & 30.2 & 30.7 & 28.6 & 29.3 & 79.7 & 141 & 250 & 94.1 & 34.3 & 28.3 & 30.3 \\
\hline 65 & 26.9 & 28.5 & 30.7 & 31.3 & 28.8 & 29.9 & 87.2 & 153 & 262 & 109 & 36.0 & 29.1 & 31.4 \\
\hline 60 & 27.2 & 28.8 & 31.1 & 32.6 & 29.0 & 31.9 & 96.9 & 165 & 284 & 120 & 37.9 & 29.6 & 32.8 \\
\hline 55 & 27.5 & 29.1 & 31.7 & 34.2 & 29.5 & 33.1 & 105 & 179 & 302 & 135 & 40.1 & 29.9 & 34.3 \\
\hline 50 & 27.7 & 29.4 & 32.2 & 34.7 & 30.1 & 34.3 & 112 & 194 & 316 & 155 & 43.0 & 30.3 & 36.4 \\
\hline 45 & 28.0 & 29.7 & 32.5 & 35.4 & 30.7 & 35.4 & 118 & 204 & 336 & 174 & 48.4 & 30.7 & 39.0 \\
\hline 40 & 28.4 & 30.1 & 33.0 & 36.4 & 31.9 & 36.7 & 127 & 214 & 360 & 192 & 52.3 & 31.2 & 43.8 \\
\hline 35 & 28.8 & 30.9 & 33.7 & 37.3 & 32.6 & 38.1 & 135 & 226 & 378 & 205 & 56.3 & 31.6 & 56.3 \\
\hline 30 & 29.2 & 31.3 & 34.5 & 37.9 & 33.7 & 40.3 & 144 & 243 & 392 & 224 & 60.2 & 32.7 & 81.2 \\
\hline 25 & 29.6 & 31.9 & 35.3 & 39.0 & 34.8 & 43.6 & 160 & 261 & 412 & 239 & 66.5 & 33.2 & 114 \\
\hline 20 & 29.9 & 32.5 & 36.1 & 39.9 & 35.8 & 49.4 & 175 & 290 & 437 & 265 & 73.0 & 33.9 & 152 \\
\hline 15 & 30.2 & 33.3 & 37.8 & 41.5 & 36.6 & 58.3 & 193 & 312 & 471 & 298 & 83.0 & 35.0 & 201 \\
\hline 10 & 30.6 & 34.2 & 39.1 & 42.2 & 38.2 & 67.9 & 213 & 341 & 508 & 349 & 108 & 36.1 & 255 \\
\hline 5 & 32.0 & 35.4 & 44.3 & 44.1 & 40.8 & 90.9 & 248 & 376 & 543 & 422 & 139 & 40.1 & 353 \\
\hline
\end{tabular}




\section{1-8.L00-3A PANJSHER RIVER AT SHUKHI, Continued}

Probability of occurrence of annual high discharges

[m $\mathrm{m}^{3} / \mathrm{s}$, cubic meters per second; ng, not given]

\begin{tabular}{|c|c|c|c|c|c|c|}
\hline \multirow{2}{*}{$\begin{array}{c}\text { Exceedance } \\
\text { probability }\end{array}$} & \multirow{2}{*}{$\begin{array}{c}\text { Recurrence } \\
\text { interval } \\
\text { (years) }\end{array}$} & \multirow{2}{*}{$\begin{array}{l}\text { Maximum } \\
\text { instantaneous } \\
\text { discharge } \\
\left(\mathrm{m}^{3} / \mathrm{s}\right)\end{array}$} & \multicolumn{4}{|c|}{ Maximum daily mean discharge $\left(\mathrm{m}^{3} / \mathrm{s}\right)$} \\
\hline & & & $\begin{array}{l}\text { 3-day } \\
\text { period }\end{array}$ & $\begin{array}{l}\text { 7-day } \\
\text { period }\end{array}$ & $\begin{array}{l}\text { 15-day } \\
\text { period }\end{array}$ & $\begin{array}{l}\text { 30-day } \\
\text { period }\end{array}$ \\
\hline 0.99 & 1.01 & 318 & 248 & 227 & 200 & 178 \\
\hline 0.95 & 1.05 & 375 & 296 & 273 & 242 & 215 \\
\hline 0.90 & 1.11 & 409 & 325 & 300 & 267 & 238 \\
\hline 0.80 & 1.25 & 453 & 362 & 336 & 300 & 268 \\
\hline 0.50 & 2 & 548 & 440 & 412 & 374 & 338 \\
\hline 0.20 & 5 & 659 & 528 & 499 & 461 & 424 \\
\hline 0.10 & 10 & 724 & 577 & 549 & 514 & 477 \\
\hline 0.04 & 25 & 798 & 633 & 605 & 575 & 541 \\
\hline 0.02 & 50 & 849 & 670 & 644 & 617 & 586 \\
\hline 0.01 & 100 & 897 & 705 & 679 & 658 & 630 \\
\hline 0.005 & 200 & 943 & 737 & 713 & 696 & 673 \\
\hline 0.002 & 500 & 1,000 & ng & $\mathrm{ng}$ & $\mathrm{ng}$ & $\mathrm{ng}$ \\
\hline
\end{tabular}

\section{1-8.L00-3A PANJSHER RIVER AT SHUKHI, Continued}

\section{Probability of occurrence of annual low discharges}

$\left[\mathrm{m}^{3} / \mathrm{s}\right.$, meters per second]

\begin{tabular}{|c|c|c|c|c|c|c|c|c|c|c|}
\hline \multirow{3}{*}{$\begin{array}{c}\text { Nonexceedance } \\
\text { probability }\end{array}$} & \multirow{3}{*}{$\begin{array}{c}\text { Recurrence } \\
\text { interval } \\
\text { (years) }\end{array}$} & \multicolumn{9}{|c|}{ Minimum daily mean discharge $\left(\mathrm{m}^{3} / \mathrm{s}\right)$} \\
\hline & & \multicolumn{9}{|c|}{ Number of consecutive days } \\
\hline & & 1 & 3 & 7 & 14 & 30 & 60 & 90 & 120 & 183 \\
\hline 0.05 & 20 & 20.7 & 20.8 & 21.2 & 21.5 & 21.9 & 23.0 & 24.3 & 24.9 & 26.0 \\
\hline 0.10 & 10 & 21.6 & 21.7 & 22.0 & 22.4 & 22.8 & 24.0 & 25.3 & 25.9 & 27.0 \\
\hline 0.20 & 5 & 22.7 & 22.8 & 23.1 & 23.4 & 24.0 & 25.2 & 26.5 & 27.2 & 28.2 \\
\hline 0.50 & 2 & 24.8 & 24.9 & 25.1 & 25.4 & 26.2 & 27.6 & 28.6 & 29.6 & 30.6 \\
\hline
\end{tabular}




\section{1-8.L00-3A PANJSHER RIVER AT SHUKHI, Continued}

Probability of occurrence of seasonal low discharges [m $\mathrm{m}^{3} / \mathrm{s}$, meters per second]

\begin{tabular}{|c|c|c|c|c|c|c|c|c|c|}
\hline \multirow{3}{*}{$\begin{array}{c}\text { Nonexceedance } \\
\text { probability }\end{array}$} & \multirow{3}{*}{$\begin{array}{c}\text { Recurrence } \\
\text { interval } \\
\text { (years) }\end{array}$} & \multicolumn{8}{|c|}{ Minimum daily mean discharge $\left(\mathrm{m}^{3} / \mathrm{s}\right)$} \\
\hline & & \multicolumn{8}{|c|}{ Number of consecutive days } \\
\hline & & 1 & 7 & 14 & 30 & 1 & 7 & 14 & 30 \\
\hline & & \multicolumn{4}{|c|}{ December-January-February } & \multicolumn{4}{|c|}{ March-April-May } \\
\hline 0.05 & 20 & 22.9 & 23.0 & 23.5 & 24.5 & 22.7 & 23.0 & 24.0 & 27.3 \\
\hline 0.10 & 10 & 23.9 & 24.1 & 24.6 & 25.6 & 23.7 & 23.9 & 25.1 & 29.0 \\
\hline 0.20 & 5 & 25.0 & 25.4 & 25.9 & 27.1 & 24.9 & 25.1 & 26.7 & 31.5 \\
\hline \multirow[t]{2}{*}{0.50} & 2 & 27.1 & 27.8 & 28.5 & 30.2 & 27.3 & 27.9 & 30.6 & 38.2 \\
\hline & & \multicolumn{4}{|c|}{ June-July-August } & \multicolumn{4}{|c|}{ September-October-November } \\
\hline 0.05 & 20 & 23.1 & 23.3 & 23.3 & 23.7 & 20.6 & 21.1 & 21.5 & 21.8 \\
\hline 0.10 & 10 & 25.1 & 25.6 & 26.2 & 27.7 & 21.7 & 22.1 & 22.5 & 22.9 \\
\hline 0.20 & 5 & 27.8 & 28.6 & 30.1 & 33.6 & 23.0 & 23.4 & 23.8 & 24.2 \\
\hline 0.50 & 2 & 33.7 & 35.6 & 39.4 & 49.2 & 25.5 & 25.8 & 26.0 & 26.7 \\
\hline
\end{tabular}




\section{1-8.L00-3A PANJSHER RIVER AT SHUKHI, Continued}

\begin{tabular}{|c|c|c|c|c|c|}
\hline \multicolumn{6}{|c|}{ 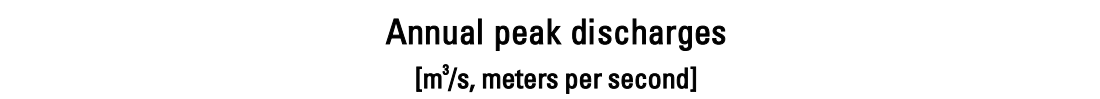 } \\
\hline \multicolumn{3}{|c|}{$\begin{array}{c}\text { Annual peak discharge, } \\
\text { by year }\end{array}$} & \multicolumn{3}{|c|}{$\begin{array}{l}\text { Annual peak discharge, } \\
\text { from highest to lowest }\end{array}$} \\
\hline $\begin{array}{l}\text { Water } \\
\text { year }\end{array}$ & Date & $\begin{array}{c}\text { Peak } \\
\text { discharge } \\
\left(\mathrm{m}^{3} / \mathrm{s}\right)\end{array}$ & $\begin{array}{l}\text { Water } \\
\text { year }\end{array}$ & Date & $\begin{array}{c}\text { Peak } \\
\text { discharge } \\
\left(\mathrm{m}^{3} / \mathrm{s}\right)\end{array}$ \\
\hline 1967 & June 12, 1967 & 750 & 1967 & June 12, 1967 & 750 \\
\hline 1968 & June 25, 1968 & 680 & 1972 & June 28, 1972 & 719 \\
\hline 1969 & June 20, 1969 & 626 & 1973 & June 13, 1973 & 700 \\
\hline 1970 & June 3, 1970 & 509 & 1968 & June 25, 1968 & 680 \\
\hline 1971 & May 28, 1971 & 522 & 1969 & June 20, 1969 & 626 \\
\hline 1972 & June 28, 1972 & 719 & 1978 & July 7, 1978 & 590 \\
\hline 1973 & June 13, 1973 & 700 & 1979 & June 22, 1979 & 558 \\
\hline 1974 & June 18,1974 & 380 & 1971 & May 28, 1971 & 522 \\
\hline 1975 & June 17, 1975 & 451 & 1970 & June 3, 1970 & 509 \\
\hline 1976 & June 4, 1976 & 482 & 1976 & June 4, 1976 & 482 \\
\hline 1977 & June 23, 1977 & 392 & 1980 & June 9, 1980 & 452 \\
\hline 1978 & July 7, 1978 & 590 & 1975 & June 17, 1975 & 451 \\
\hline 1979 & June 22, 1979 & 558 & 1977 & June 23, 1977 & 392 \\
\hline 1980 & June 9, 1980 & 452 & 1974 & June 18, 1974 & 380 \\
\hline
\end{tabular}


1-8.L00-3A PANJSHER RIVER AT SHUKHI, Continued

Monthly and annual mean discharges, in cubic meters per second

[Data may not be rounded in accordance with U.S. Geological Survey publication standards]

\begin{tabular}{|c|c|c|c|c|c|c|c|c|c|c|c|c|c|}
\hline \multirow{2}{*}{$\begin{array}{l}\text { Water } \\
\text { year }\end{array}$} & \multicolumn{12}{|c|}{ Monthly mean discharge } & \multirow{2}{*}{$\begin{array}{c}\text { Annual } \\
\text { discharge }\end{array}$} \\
\hline & October & November & December & January & February & March & April & May & June & July & August & September & \\
\hline 1967 & 27.8 & 28.3 & 33.8 & 36.1 & 25.8 & 29.6 & 122 & 176 & 390 & 268 & 70.0 & 32.5 & 103 \\
\hline 1968 & 29.5 & 30.2 & 37.2 & 42.3 & 36.0 & 56.1 & 143 & 201 & 493 & 362 & 112 & 34.4 & 131 \\
\hline 1969 & 29.4 & 32.1 & 45.0 & 44.1 & 37.2 & 67.2 & 164 & 167 & 407 & 312 & 104 & 33.5 & 120 \\
\hline 1970 & 23.6 & 32.9 & 32.7 & 37.7 & 28.6 & 34.3 & 98.3 & 216 & 270 & 125 & 64.5 & 38.9 & 83.6 \\
\hline 1971 & 30.1 & 28.1 & 30.2 & 30.2 & 28.5 & 36.8 & 86.7 & 241 & 190 & 45.2 & 29.1 & 29.1 & 67.2 \\
\hline 1972 & 27.7 & 22.6 & 23.9 & 26.0 & 24.7 & 45.0 & 143 & 298 & 492 & 244 & 65.1 & 32.9 & 120 \\
\hline 1973 & 32.2 & 32.8 & 38.2 & 40.6 & 36.3 & 55.3 & 207 & 345 & 439 & 194 & 51.1 & 31.9 & 125 \\
\hline 1974 & 29.7 & 29.0 & 31.6 & 32.8 & 31.5 & 29.0 & 82.2 & 136 & 217 & 87.9 & 28.0 & 27.8 & 63.5 \\
\hline 1975 & 27.8 & 27.7 & 28.8 & 30.5 & 30.2 & 31.9 & 91.2 & 145 & 267 & 157 & 53.3 & 30.1 & 76.8 \\
\hline 1976 & 25.5 & 23.8 & 26.6 & 28.8 & 27.8 & 37.9 & 123 & 198 & 261 & 173 & 40.7 & 30.8 & 83.1 \\
\hline 1977 & 25.9 & 28.2 & 31.2 & 37.0 & 35.1 & 31.1 & 60.2 & 95.5 & 207 & 88.7 & 32.6 & 29.5 & 58.5 \\
\hline 1978 & 29.3 & 31.7 & 31.3 & 33.9 & 30.8 & 35.1 & 120 & 230 & 294 & 143 & 39.5 & 29.9 & 87.5 \\
\hline 1979 & 26.3 & 32.5 & 36.3 & 33.1 & 28.8 & 44.1 & 138 & 170 & 324 & 193 & 66.7 & 23.7 & 93.2 \\
\hline 1980 & 23.6 & 31.6 & 32.0 & 35.1 & 36.2 & 45.1 & 150 & 217 & 315 & 80.2 & 25.9 & 22.9 & 84.3 \\
\hline
\end{tabular}




\section{1-8.L00-5A PANJSHER RIVER AT GULBAHAR}

\section{(U.S. Geological Survey identification number: 351000069170000)}

LOCATION: Lat $35^{\circ} 10^{\prime} \mathrm{N}$., long $69^{\circ} 17^{\prime} \mathrm{E}$.

DRAINAGE AREA: $3,565 \mathrm{~km}^{2}$.

ELEVATION: 1,605 meters above mean sea level.

PERIOD OF RECORD: October 1, 1959 to September 30, 1980.

GAGE: Water-stage recorder.

Annual mean discharge

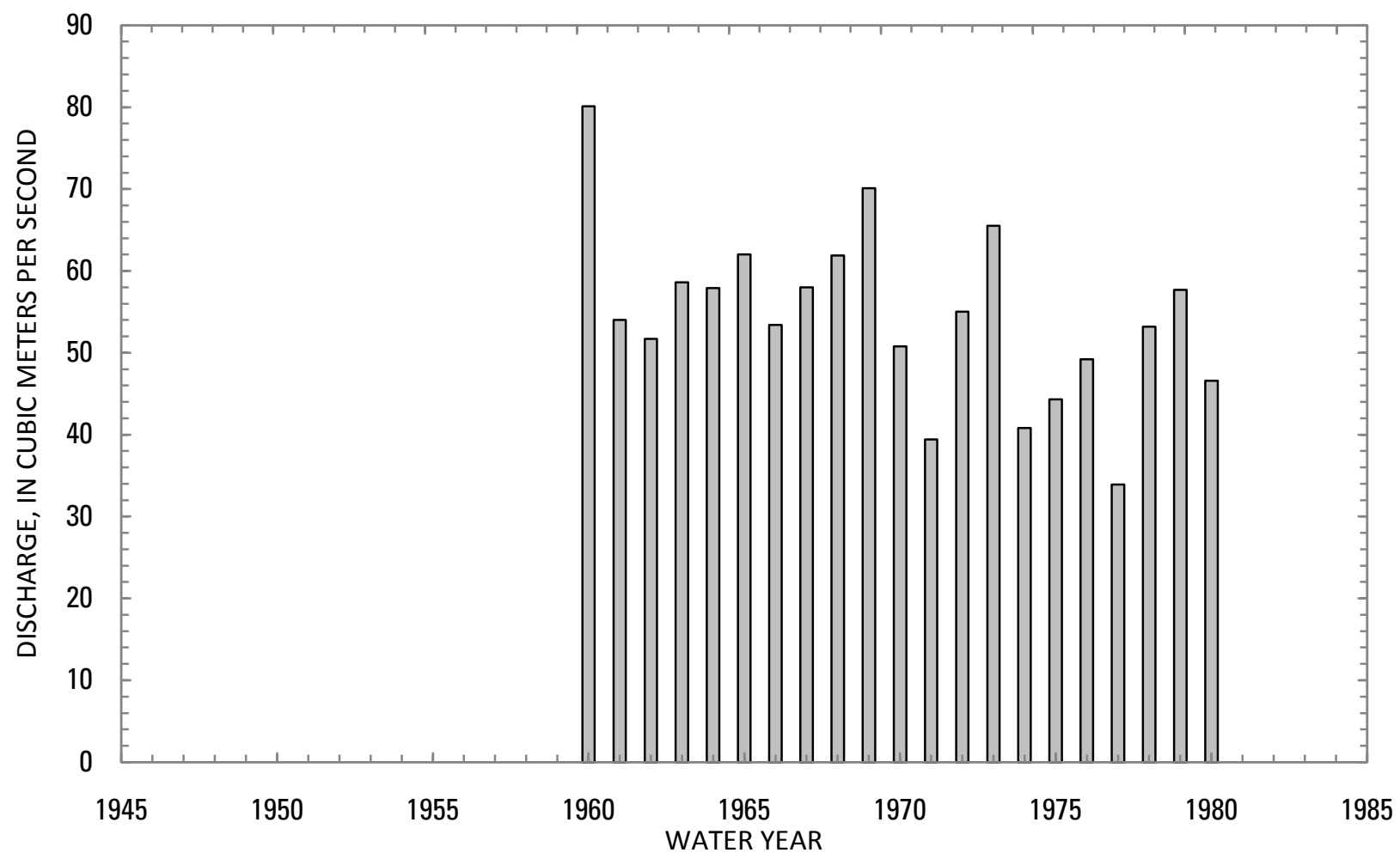




\section{1-8.L00-5A PANJSHER RIVER AT GULBAHAR, Continued}

Statistics of monthly and annual mean discharges [m $\mathrm{m}^{3} / \mathrm{s}$, cubic meters per second]

\begin{tabular}{|c|c|c|c|c|c|c|c|c|}
\hline \multirow[b]{2}{*}{ Month } & \multicolumn{2}{|c|}{ Maximum } & \multicolumn{2}{|c|}{ Minimum } & \multicolumn{4}{|c|}{ Mean } \\
\hline & $\begin{array}{c}\text { Discharge } \\
\left(\mathrm{m}^{3} / \mathrm{s}\right)\end{array}$ & $\begin{array}{c}\text { Water year } \\
\text { of } \\
\text { occurrence }\end{array}$ & $\begin{array}{c}\text { Discharge } \\
\left(\mathrm{m}^{3} / \mathrm{s}\right)\end{array}$ & $\begin{array}{c}\text { Water year } \\
\text { of } \\
\text { occurrence }\end{array}$ & $\begin{array}{c}\text { Discharge } \\
\left(\mathrm{m}^{3} / \mathrm{s}\right)\end{array}$ & $\begin{array}{c}\text { Standard } \\
\text { deviation } \\
\left(\mathrm{m}^{3} / \mathrm{s}\right)\end{array}$ & $\begin{array}{c}\text { Coefficient } \\
\text { of } \\
\text { variation }\end{array}$ & $\begin{array}{c}\text { Percentage } \\
\text { of annual } \\
\text { discharge }\end{array}$ \\
\hline October & 27.1 & 1970 & 12.3 & 1964 & 17.2 & 3.40 & 0.20 & 2.64 \\
\hline November & 24.3 & 1970 & 10.5 & 1964 & 14.4 & 3.10 & 0.22 & 2.20 \\
\hline December & 19.3 & 1970 & 8.59 & 1971 & 12.2 & 2.48 & 0.20 & 1.87 \\
\hline January & 17.2 & 1970 & 7.24 & 1971 & 10.6 & 2.40 & 0.23 & 1.62 \\
\hline February & 14.1 & 1970 & 7.18 & 1971 & 10.4 & 2.01 & 0.19 & 1.59 \\
\hline March & 23.4 & 1968 & 8.48 & 1962 & 13.9 & 3.55 & 0.26 & 2.12 \\
\hline April & 62.9 & 1973 & 19.1 & 1977 & 36.2 & 10.0 & 0.28 & 5.54 \\
\hline May & 148 & 1971 & 49.8 & 1977 & 92.8 & 27.7 & 0.30 & 14.2 \\
\hline June & 316 & 1963 & 131 & 1971 & 219 & 51.5 & 0.24 & 33.5 \\
\hline July & 298 & 1960 & 47.8 & 1971 & 148 & 60.6 & 0.41 & 22.7 \\
\hline August & 99.4 & 1969 & 30.3 & 1971 & 53.9 & 19.0 & 0.35 & 8.26 \\
\hline September & 36.3 & 1969 & 13.7 & 1963 & 24.4 & 5.61 & 0.23 & 3.74 \\
\hline Annual & 80.1 & 1960 & 33.9 & 1977 & 54.5 & 10.6 & 0.19 & 100 \\
\hline
\end{tabular}


1-8.L00-5A PANJSHER RIVER AT GULBAHAR, Continued

Monthly and annual flow duration, in cubic meters per second

\begin{tabular}{|c|c|c|c|c|c|c|c|c|c|c|c|c|c|}
\hline \multirow{2}{*}{$\begin{array}{l}\text { Percentage } \\
\text { of days } \\
\text { discharge } \\
\text { equaled or } \\
\text { exceeded }\end{array}$} & \multicolumn{12}{|c|}{ Month } & \multirow{2}{*}{ Annual } \\
\hline & October & November & December & January & February & March & April & May & June & July & August & September & \\
\hline 95 & 12.5 & 10.9 & 8.85 & 7.16 & 7.06 & 7.81 & 14.9 & 36.5 & 96.2 & 48.2 & 25.3 & 14.4 & 8.63 \\
\hline 90 & 13.0 & 11.1 & 9.42 & 7.54 & 7.49 & 8.84 & 16.2 & 42.6 & 119 & 58.1 & 27.9 & 16.3 & 10.0 \\
\hline 85 & 13.3 & 11.4 & 9.93 & 8.03 & 7.96 & 9.72 & 18.1 & 50.1 & 137 & 69.5 & 30.5 & 17.7 & 10.9 \\
\hline 80 & 13.9 & 11.8 & 10.2 & 8.70 & 8.24 & 10.5 & 19.7 & 55.7 & 152 & 78.9 & 32.8 & 18.5 & 11.6 \\
\hline 75 & 14.5 & 12.0 & 10.5 & 9.00 & 8.91 & 10.9 & 21.0 & 61.4 & 167 & 89.1 & 35.0 & 19.5 & 12.4 \\
\hline 70 & 15.4 & 12.2 & 10.7 & 9.21 & 9.12 & 11.4 & 23.0 & 66.3 & 177 & 96.0 & 36.9 & 20.1 & 13.3 \\
\hline 65 & 16.0 & 12.4 & 10.9 & 9.43 & 9.77 & 11.9 & 25.0 & 70.1 & 186 & 102 & 39.8 & 21.1 & 14.4 \\
\hline 60 & 16.3 & 12.9 & 11.1 & 9.78 & 10.0 & 12.2 & 27.0 & 74.0 & 197 & 111 & 42.2 & 22.1 & 15.8 \\
\hline 55 & 16.6 & 13.2 & 11.6 & 10.0 & 10.2 & 12.6 & 29.0 & 78.1 & 208 & 121 & 45.5 & 22.8 & 17.2 \\
\hline 50 & 16.9 & 13.6 & 11.8 & 10.1 & 10.5 & 13.1 & 31.3 & 83.1 & 216 & 130 & 48.7 & 23.6 & 19.3 \\
\hline 45 & 17.2 & 14.2 & 12.1 & 10.2 & 10.7 & 13.4 & 34.2 & 88.8 & 224 & 142 & 51.3 & 24.6 & 22.9 \\
\hline 40 & 17.5 & 14.9 & 12.6 & 10.7 & 11.0 & 13.7 & 37.3 & 95.3 & 235 & 154 & 53.9 & 25.4 & 28.0 \\
\hline 35 & 17.8 & 15.3 & 12.9 & 11.0 & 11.2 & 14.3 & 39.9 & 102 & 249 & 167 & 57.4 & 26.2 & 35.6 \\
\hline 30 & 18.3 & 15.7 & 13.1 & 11.3 & 11.6 & 15.0 & 42.8 & 110 & 261 & 184 & 60.8 & 27.5 & 47.5 \\
\hline 25 & 18.9 & 16.0 & 13.6 & 11.7 & 11.9 & 15.5 & 47.0 & 118 & 272 & 198 & 67.2 & 28.8 & 63.3 \\
\hline 20 & 19.7 & 16.4 & 14.1 & 12.5 & 12.2 & 16.2 & 50.4 & 129 & 284 & 219 & 71.5 & 30.1 & 83.3 \\
\hline 15 & 20.5 & 17.0 & 14.9 & 13.1 & 12.6 & 17.6 & 54.1 & 142 & 303 & 236 & 78.4 & 31.6 & 116 \\
\hline 10 & 21.8 & 18.0 & 15.6 & 14.0 & 13.0 & 19.4 & 61.2 & 158 & 326 & 252 & 86.5 & 33.2 & 164 \\
\hline 5 & 25.6 & 21.1 & 17.0 & 15.2 & 13.8 & 22.7 & 71.2 & 185 & 354 & 277 & 109 & 37.0 & 230 \\
\hline
\end{tabular}




\section{1-8.L00-5A PANJSHER RIVER AT GULBAHAR, Continued}

Probability of occurrence of annual high discharges

[m $\mathrm{m}^{3} / \mathrm{s}$, cubic meters per second; ng, not given]

\begin{tabular}{ccccccc}
\hline & & \multicolumn{3}{c}{ Maximum daily mean discharge $\left(\mathbf{m}^{3} / \mathbf{s}\right)$} \\
$\begin{array}{c}\text { Exceedance } \\
\text { probability }\end{array}$ & $\begin{array}{c}\text { Recurrence } \\
\text { interval } \\
\text { (years) }\end{array}$ & $\begin{array}{c}\text { Maximum } \\
\text { instantaneous } \\
\text { discharge } \\
\text { (m } \mathbf{3} / \mathbf{s})\end{array}$ & $\begin{array}{c}\text { 3-day } \\
\text { period }\end{array}$ & $\begin{array}{c}\text { 7-day } \\
\text { period }\end{array}$ & $\begin{array}{c}\text { 15-day } \\
\text { period }\end{array}$ & $\begin{array}{c}\text { 30-day } \\
\text { period }\end{array}$ \\
\hline 0.99 & 1.01 & 209 & 179 & 166 & 147 & 125 \\
0.95 & 1.05 & 255 & 209 & 193 & 172 & 152 \\
0.90 & 1.11 & 282 & 227 & 210 & 188 & 168 \\
0.80 & 1.25 & 318 & 250 & 232 & 209 & 189 \\
0.50 & 2 & 394 & 300 & 281 & 257 & 234 \\
0.20 & 5 & 483 & 360 & 343 & 317 & 286 \\
0.10 & 10 & 534 & 395 & 381 & 353 & 316 \\
0.04 & 25 & 592 & 436 & 427 & 397 & 349 \\
0.02 & 50 & 631 & 465 & 460 & 429 & 372 \\
0.01 & 100 & 668 & 492 & 492 & 460 & 393 \\
0.005 & 200 & 702 & 518 & 523 & 490 & 413 \\
0.002 & 500 & 746 & ng & ng & ng & ng \\
\hline
\end{tabular}

'Data does not fit log-Pearson Type III curve, use with caution.

\section{1-8.L00-5A PANJSHER RIVER AT GULBAHAR, Continued}

\section{Probability of occurrence of annual low discharges}

[ $\mathrm{m}^{3} / \mathrm{s}$, meters per second]

\begin{tabular}{|c|c|c|c|c|c|c|c|c|c|c|}
\hline \multirow{3}{*}{$\begin{array}{c}\text { Nonexceedance } \\
\text { probability }\end{array}$} & \multirow{3}{*}{$\begin{array}{l}\text { Recurrence } \\
\text { interval } \\
\text { (years) }\end{array}$} & \multicolumn{9}{|c|}{ Minimum daily mean discharge $\left(\mathrm{m}^{3} / \mathrm{s}\right)$} \\
\hline & & \multicolumn{9}{|c|}{ Number of consecutive days } \\
\hline & & 1 & 3 & 7 & 14 & 30 & 60 & 90 & 120 & 183 \\
\hline 0.05 & 20 & 6.39 & 6.47 & 6.59 & 6.72 & 6.85 & 7.20 & 7.60 & 8.31 & 10.0 \\
\hline 0.10 & 10 & 6.89 & 7.00 & 7.14 & 7.28 & 7.44 & 7.78 & 8.18 & 8.83 & 10.5 \\
\hline 0.20 & 5 & 7.53 & 7.69 & 7.86 & 8.01 & 8.19 & 8.53 & 8.94 & 9.52 & 11.1 \\
\hline 0.50 & 2 & 8.91 & 9.14 & 9.34 & 9.52 & 9.74 & 10.1 & 10.6 & 11.1 & 12.7 \\
\hline
\end{tabular}




\section{1-8.L00-5A PANJSHER RIVER AT GULBAHAR, Continued}

Probability of occurrence of seasonal low discharges

[ $\mathrm{m}^{3} / \mathrm{s}$, meters per second]

\begin{tabular}{|c|c|c|c|c|c|c|c|c|c|}
\hline \multirow{3}{*}{$\begin{array}{c}\text { Nonexceedance } \\
\text { probability }\end{array}$} & \multirow{3}{*}{$\begin{array}{c}\text { Recurrence } \\
\text { interval } \\
\text { (years) }\end{array}$} & \multicolumn{8}{|c|}{ Minimum daily mean discharge $\left(\mathrm{m}^{3} / \mathrm{s}\right)$} \\
\hline & & \multicolumn{8}{|c|}{ Number of consecutive days } \\
\hline & & 1 & 7 & 14 & 30 & 1 & 7 & 14 & 30 \\
\hline & & \multicolumn{4}{|c|}{ December-January-February } & \multicolumn{4}{|c|}{ March-April-May } \\
\hline 0.05 & 20 & 6.43 & 6.66 & 6.77 & 6.93 & 7.04 & 7.30 & 7.68 & 9.15 \\
\hline 0.10 & 10 & 6.89 & 7.20 & 7.32 & 7.49 & 7.76 & 8.11 & 8.44 & 9.85 \\
\hline 0.20 & 5 & 7.49 & 7.88 & 8.02 & 8.21 & 8.67 & 9.13 & 9.45 & 10.8 \\
\hline \multirow[t]{2}{*}{0.50} & 2 & 8.84 & 9.33 & 9.50 & 9.74 & 10.4 & 11.2 & 11.6 & 13.1 \\
\hline & & \multicolumn{4}{|c|}{ June-July-August } & \multicolumn{4}{|c|}{ September-October-November } \\
\hline 0.05 & 20 & 17.5 & 19.1 & 22.8 & 29.2 & 9.85 & 10.2 & 10.4 & 10.8 \\
\hline 0.10 & 10 & 20.5 & 22.2 & 25.6 & 32.7 & 10.2 & 10.5 & 10.7 & 11.2 \\
\hline 0.20 & 5 & 24.3 & 26.3 & 29.4 & 37.7 & 10.7 & 11.1 & 11.3 & 11.8 \\
\hline 0.50 & 2 & 32.3 & 34.8 & 38.3 & 49.7 & 12.2 & 12.6 & 12.9 & 13.5 \\
\hline
\end{tabular}




\section{1-8.L00-5A PANJSHER RIVER AT GULBAHAR, Continued}

\begin{tabular}{|c|c|c|c|c|c|}
\hline \multicolumn{6}{|c|}{$\begin{array}{c}\text { Annual peak discharges } \\
{\left[\mathrm{m}^{3} / \mathrm{s} \text {, meters per second] }\right.}\end{array}$} \\
\hline \multicolumn{3}{|c|}{$\begin{array}{c}\text { Annual peak discharge, } \\
\text { by year }\end{array}$} & \multicolumn{3}{|c|}{$\begin{array}{l}\text { Annual peak discharge, } \\
\text { from highest to lowest }\end{array}$} \\
\hline $\begin{array}{l}\text { Water } \\
\text { year }\end{array}$ & Date & $\begin{array}{c}\text { Peak } \\
\text { discharge } \\
\left(\mathrm{m}^{3} / \mathrm{s}\right)\end{array}$ & $\begin{array}{l}\text { Water } \\
\text { year }\end{array}$ & Date & $\begin{array}{c}\text { Peak } \\
\text { discharge } \\
\left(\mathrm{m}^{3} / \mathrm{s}\right)\end{array}$ \\
\hline 1960 & July 10,1960 & 584 & 1960 & July 10, 1960 & 584 \\
\hline 1961 & June 5, 1961 & 515 & 1964 & July 4, 1964 & 569 \\
\hline 1962 & June 10, 1962 & 488 & 1963 & June 20, 1963 & 520 \\
\hline 1963 & June 20, 1963 & 520 & 1961 & June 5, 1961 & 515 \\
\hline 1964 & July 4, 1964 & 569 & 1962 & June 10, 1962 & 488 \\
\hline 1965 & July 15,1965 & 300 & 1979 & June 23, 1979 & 477 \\
\hline 1966 & June 19, 1966 & 335 & 1973 & June 13, 1973 & 473 \\
\hline 1967 & June 12, 1967 & 358 & 1969 & June 20, 1969 & 424 \\
\hline 1968 & July 8, 1968 & 412 & 1978 & June 5, 1978 & 418 \\
\hline 1969 & June 20, 1969 & 424 & 1968 & July 8, 1968 & 412 \\
\hline 1970 & June 3, 1970 & 350 & 1972 & June 25, 1972 & 385 \\
\hline 1971 & June 9, 1971 & 321 & 1967 & June 12, 1967 & 358 \\
\hline 1972 & June 25, 1972 & 385 & 1970 & June 3,1970 & 350 \\
\hline 1973 & June 13, 1973 & 473 & 1977 & June 23, 1977 & 338 \\
\hline 1974 & June 16, 1974 & 292 & 1966 & June 19, 1966 & 335 \\
\hline 1975 & June 17, 1975 & 335 & 1975 & June 17, 1975 & 335 \\
\hline 1976 & June 4, 1976 & 327 & 1976 & June 4, 1976 & 327 \\
\hline 1977 & June 23, 1977 & 338 & 1971 & June 9, 1971 & 321 \\
\hline 1978 & June 5, 1978 & 418 & 1965 & July 15, 1965 & 300 \\
\hline 1979 & June 23, 1979 & 477 & 1974 & June 16, 1974 & 292 \\
\hline 1980 & June 9, 1980 & 220 & 1980 & June 9, 1980 & 220 \\
\hline
\end{tabular}




\section{1-8.L00-5A PANJSHER RIVER AT GULBAHAR, Continued}

Monthly and annual mean discharges, in cubic meters per second

[Data may not be rounded in accordance with U.S. Geological Survey publication standards]

\begin{tabular}{|c|c|c|c|c|c|c|c|c|c|c|c|c|c|}
\hline \multirow{2}{*}{$\begin{array}{l}\text { Water } \\
\text { year }\end{array}$} & \multicolumn{12}{|c|}{ Monthly mean discharge } & \multirow{2}{*}{$\begin{array}{c}\text { Annual } \\
\text { discharge }\end{array}$} \\
\hline & October & November & December & January & February & March & April & May & June & July & August & September & \\
\hline 1960 & 17.3 & 15.4 & 12.2 & 9.58 & 10.1 & 16.2 & 37.8 & 110 & 306 & 298 & 93.5 & 33.5 & 80.1 \\
\hline 1961 & 20.3 & 16.1 & 12.4 & 9.64 & 8.07 & 9.15 & 25.4 & 92.4 & 235 & 138 & 52.8 & 27.7 & 54.0 \\
\hline 1962 & 17.7 & 14.7 & 10.5 & 8.82 & 7.84 & 8.48 & 24.3 & 57.8 & 238 & 153 & 48.6 & 29.5 & 51.7 \\
\hline 1963 & 17.9 & 13.4 & 9.70 & 7.44 & 7.57 & 11.7 & 27.4 & 75.6 & 316 & 162 & 41.2 & 13.7 & 58.6 \\
\hline 1964 & 12.3 & 10.5 & 9.74 & 7.78 & 7.46 & 12.6 & 40.8 & 99.7 & 226 & 191 & 54.1 & 21.6 & 57.9 \\
\hline 1965 & 15.4 & 13.4 & 12.9 & 11.9 & 11.7 & 15.2 & 38.4 & 90.6 & 202 & 231 & 68.5 & 28.2 & 62.0 \\
\hline 1966 & 22.4 & 18.1 & 14.8 & 10.7 & 10.6 & 14.4 & 39.0 & 80.7 & 228 & 120 & 56.0 & 25.1 & 53.4 \\
\hline 1967 & 16.7 & 13.0 & 10.7 & 9.22 & 12.9 & 14.1 & 35.5 & 77.1 & 219 & 184 & 72.3 & 30.5 & 58.0 \\
\hline 1968 & 17.2 & 12.9 & 13.6 & 13.3 & 12.2 & 23.4 & 37.1 & 64.2 & 246 & 203 & 73.9 & 26.3 & 61.9 \\
\hline 1969 & 20.0 & 16.8 & 15.3 & 13.4 & 11.7 & 19.1 & 37.8 & 88.9 & 257 & 222 & 99.4 & 36.3 & 70.1 \\
\hline 1970 & 27.1 & 24.3 & 19.3 & 17.2 & 14.1 & 14.5 & 41.9 & 121 & 177 & 74.6 & 48.9 & 27.8 & 50.8 \\
\hline 1971 & 15.0 & 12.0 & 8.59 & 7.24 & 7.18 & 11.1 & 34.5 & 148 & 131 & 47.8 & 30.3 & 17.4 & 39.4 \\
\hline 1972 & 13.5 & 12.0 & 11.3 & 8.84 & 10.0 & 12.5 & 21.1 & 70.9 & 271 & 155 & 50.0 & 25.0 & 55.0 \\
\hline 1973 & 17.4 & 15.5 & 13.5 & 11.7 & 12.5 & 20.3 & 62.9 & 146 & 280 & 138 & 44.5 & 22.4 & 65.5 \\
\hline 1974 & 19.3 & 15.2 & 12.2 & 10.2 & 10.0 & 12.7 & 33.1 & 82.0 & 152 & 91.2 & 31.5 & 18.2 & 40.8 \\
\hline 1975 & 13.7 & 11.5 & 9.98 & 9.32 & 9.20 & 10.7 & 31.4 & 66.4 & 175 & 119 & 52.2 & 22.6 & 44.3 \\
\hline 1976 & 16.7 & 14.8 & 12.0 & 11.3 & 10.9 & 12.9 & 38.6 & 99.2 & 164 & 139 & 46.5 & 23.7 & 49.2 \\
\hline 1977 & 15.7 & 11.8 & 10.6 & 10.8 & 11.2 & 12.2 & 19.1 & 49.8 & 138 & 80.2 & 30.6 & 16.5 & 33.9 \\
\hline 1978 & 13.2 & 11.7 & 11.1 & 9.55 & 9.18 & 12.6 & 41.4 & 138 & 221 & 103 & 44.4 & 22.0 & 53.2 \\
\hline 1979 & 14.6 & 11.6 & 11.0 & 10.0 & 10.5 & 13.3 & 53.6 & 81.4 & 223 & 175 & 62.0 & 23.6 & 57.7 \\
\hline 1980 & 18.0 & 16.7 & 15.5 & 13.9 & 13.0 & 13.7 & 38.4 & 108 & 189 & 82.8 & 31.1 & 20.5 & 46.6 \\
\hline
\end{tabular}




\section{1-8.L00-8A PANJSHER RIVER AT OMARZ}

\section{(U.S. Geological Survey identification number: 352200069380000)}

LOCATION: Lat $35^{\circ} 22^{\prime} \mathrm{N}$., long $69^{\circ} 38^{\prime} \mathrm{E}$.

DRAINAGE AREA: $2,240 \mathrm{~km}^{2}$.

ELEVATION: 2,010 meters above mean sea level.

PERIOD OF RECORD: October 1, 1962 to February 20, 1963 and June 25, 1963 to September 30, 1980.

GAGE: Water-stage recorder.

Annual mean discharge

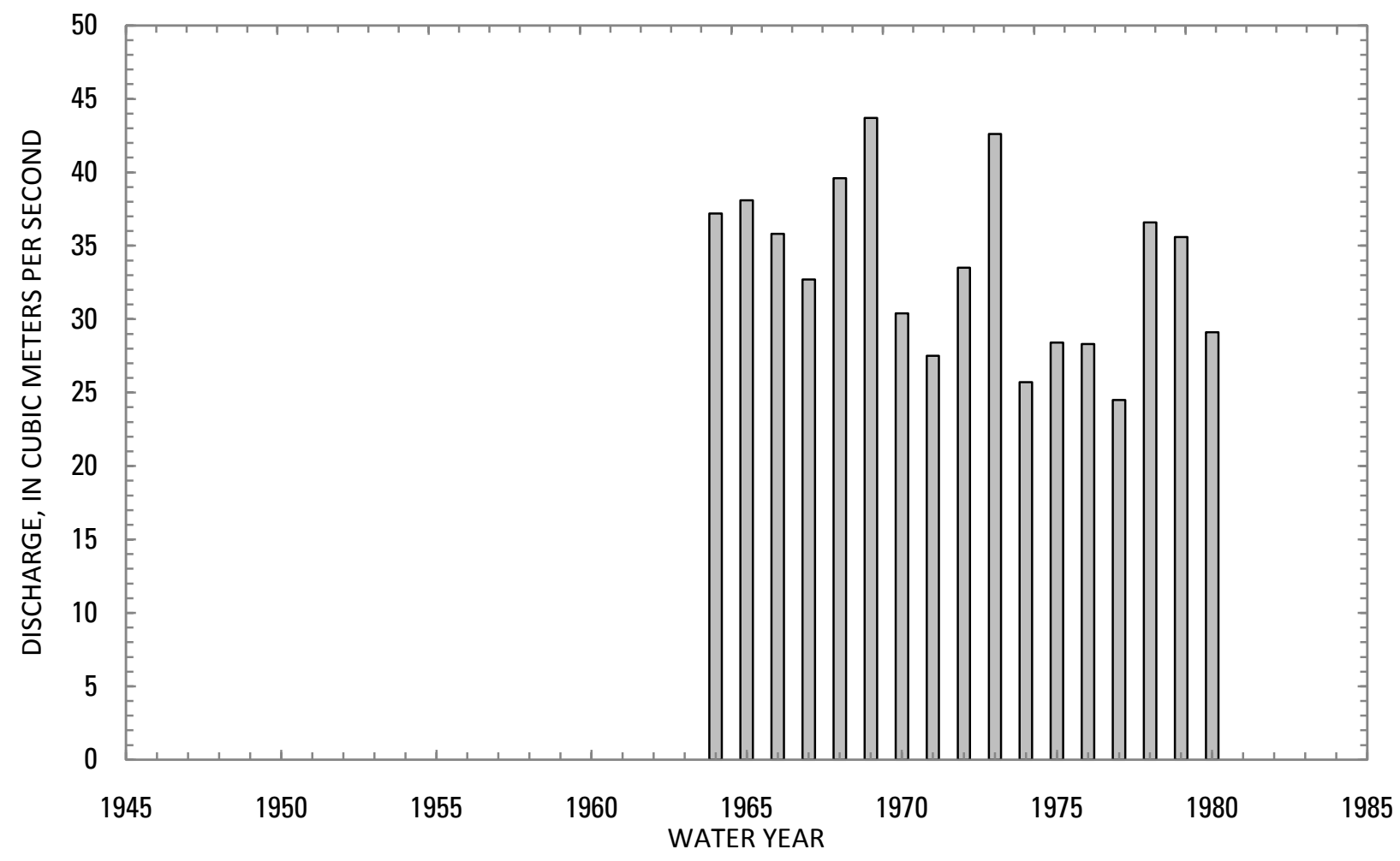




\section{1-8.L00-8A PANJSHER RIVER AT OMARZ, Continued}

Statistics of monthly and annual mean discharges $\left[\mathrm{m}^{3} / \mathrm{s}\right.$, cubic meters per second]

\begin{tabular}{|c|c|c|c|c|c|c|c|c|}
\hline \multirow[b]{2}{*}{ Month } & \multicolumn{2}{|c|}{ Maximum } & \multicolumn{2}{|c|}{ Minimum } & \multicolumn{4}{|c|}{ Mean } \\
\hline & $\begin{array}{c}\text { Discharge } \\
\left(\mathrm{m}^{3} / \mathrm{s}\right)\end{array}$ & $\begin{array}{c}\text { Water year } \\
\text { of } \\
\text { occurrence }\end{array}$ & $\begin{array}{c}\text { Discharge } \\
\left(\mathrm{m}^{3} / \mathrm{s}\right)\end{array}$ & $\begin{array}{c}\text { Water year } \\
\text { of } \\
\text { occurrence }\end{array}$ & $\begin{array}{c}\text { Discharge } \\
\left(\mathrm{m}^{3} / \mathrm{s}\right)\end{array}$ & $\begin{array}{c}\text { Standard } \\
\text { deviation } \\
\left(\mathrm{m}^{3} / \mathrm{s}\right)\end{array}$ & $\begin{array}{c}\text { Coefficient } \\
\text { of } \\
\text { variation }\end{array}$ & $\begin{array}{c}\text { Percentage } \\
\text { of annual } \\
\text { discharge }\end{array}$ \\
\hline October & 15.8 & 1970 & 7.90 & 1972 & 11.9 & 2.05 & 0.17 & 2.95 \\
\hline November & 12.4 & 1973 & 7.51 & 1972 & 9.50 & 1.55 & 0.16 & 2.36 \\
\hline December & 10.7 & 1973 & 6.04 & 1975 & 7.96 & 1.35 & 0.17 & 1.98 \\
\hline January & 9.11 & 1970 & 5.27 & 1979 & 6.85 & 1.18 & 0.17 & 1.71 \\
\hline February & 8.72 & 1970 & 5.05 & 1975 & 6.46 & 0.92 & 0.14 & 1.61 \\
\hline March & 10.0 & 1971 & 5.55 & 1975 & 7.45 & 1.32 & 0.18 & 1.86 \\
\hline April & 23.7 & 1971 & 10.5 & 1977 & 16.3 & 4.12 & 0.25 & 4.05 \\
\hline May & 92.4 & 1971 & 32.1 & 1977 & 50.1 & 18.1 & 0.36 & 12.5 \\
\hline June & 190 & 1973 & 89.2 & 1971 & 131 & 28.5 & 0.22 & 32.5 \\
\hline July & 153 & 1969 & 34.7 & 1971 & 95.8 & 33.3 & 0.35 & 23.9 \\
\hline August & 75.5 & 1969 & 24.0 & 1974 & 40.3 & 13.2 & 0.33 & 10.0 \\
\hline September & 27.6 & 1969 & 11.9 & 1971 & 18.5 & 3.72 & 0.20 & 4.60 \\
\hline Annual & 43.7 & 1969 & 24.5 & 1977 & 33.5 & 5.80 & 0.17 & 100 \\
\hline
\end{tabular}


1-8.L00-8A PANJSHER RIVER AT OMARZ, Continued

Monthly and annual flow duration, in cubic meters per second

\begin{tabular}{|c|c|c|c|c|c|c|c|c|c|c|c|c|c|}
\hline \multirow{2}{*}{$\begin{array}{l}\text { Percentage } \\
\text { of days } \\
\text { discharge } \\
\text { equaled or } \\
\text { exceeded }\end{array}$} & \multicolumn{12}{|c|}{ Month } & \multirow{2}{*}{ Annual } \\
\hline & October & November & December & January & February & March & April & May & June & July & August & September & \\
\hline 95 & 8.49 & 7.02 & 5.88 & 5.12 & 4.96 & 5.52 & 7.15 & 17.3 & 57.5 & 35.9 & 20.2 & 11.7 & 5.78 \\
\hline 90 & 9.04 & 7.43 & 6.19 & 5.42 & 5.40 & 5.67 & 8.45 & 20.1 & 68.9 & 41.2 & 22.7 & 12.7 & 6.26 \\
\hline 85 & 9.55 & 7.78 & 6.30 & 5.51 & 5.67 & 5.97 & 9.04 & 22.5 & 79.0 & 46.5 & 23.9 & 14.2 & 6.73 \\
\hline 80 & 9.79 & 8.10 & 6.62 & 5.74 & 5.77 & 6.09 & 9.63 & 25.4 & 86.8 & 55.1 & 25.1 & 14.8 & 7.18 \\
\hline 75 & 10.0 & 8.22 & 6.94 & 6.00 & 5.85 & 6.19 & 10.0 & 29.1 & 94.9 & 62.7 & 26.8 & 15.4 & 7.74 \\
\hline 70 & 10.5 & 8.37 & 7.05 & 6.09 & 5.92 & 6.28 & 10.6 & 31.9 & 101 & 66.8 & 28.7 & 15.7 & 8.38 \\
\hline 65 & 11.0 & 8.57 & 7.20 & 6.19 & 5.99 & 6.58 & 11.4 & 34.3 & 108 & 70.8 & 31.4 & 16.1 & 9.03 \\
\hline 60 & 11.2 & 8.76 & 7.51 & 6.29 & 6.13 & 6.69 & 12.1 & 36.8 & 114 & 75.0 & 33.2 & 16.7 & 9.81 \\
\hline 55 & 11.4 & 8.92 & 7.72 & 6.62 & 6.22 & 6.83 & 13.3 & 39.9 & 123 & 81.4 & 34.8 & 17.4 & 11.0 \\
\hline 50 & 11.7 & 9.07 & 8.00 & 6.82 & 6.29 & 6.99 & 14.2 & 43.9 & 130 & 88.8 & 36.4 & 17.8 & 12.3 \\
\hline 45 & 12.0 & 9.48 & 8.13 & 6.95 & 6.53 & 7.27 & 15.2 & 48.0 & 137 & 95.6 & 38.5 & 18.1 & 14.8 \\
\hline 40 & 12.4 & 9.77 & 8.24 & 7.21 & 6.63 & 7.44 & 16.7 & 51.0 & 145 & 102 & 40.7 & 19.2 & 18.2 \\
\hline 35 & 12.8 & 10.0 & 8.38 & 7.3 & 6.91 & 7.62 & 17.8 & 54.2 & 154 & 108 & 42.7 & 20.0 & 22.0 \\
\hline 30 & 13.0 & 10.4 & 8.55 & 7.46 & 6.99 & 7.84 & 19.2 & 58.1 & 161 & 115 & 45.0 & 20.7 & 28.6 \\
\hline 25 & 13.4 & 10.6 & 9.00 & 7.73 & 7.08 & 8.36 & 20.0 & 63.2 & 170 & 123 & 49.0 & 21.4 & 37.9 \\
\hline 20 & 13.8 & 11.1 & 9.18 & 7.87 & 7.18 & 9.02 & 21.7 & 69.3 & 177 & 139 & 53.7 & 22.1 & 52.1 \\
\hline 15 & 14.4 & 11.7 & 9.35 & 8.08 & 7.34 & 9.34 & 23.3 & 76.9 & 185 & 153 & 58.3 & 22.9 & 70.2 \\
\hline 10 & 14.9 & 12.1 & 9.89 & 8.76 & 7.65 & 9.74 & 26.1 & 91.2 & 194 & 163 & 62.6 & 24.9 & 100 \\
\hline 5 & 15.7 & 12.7 & 10.8 & 8.95 & ng & 10.8 & 31.8 & 109 & 204 & 180 & 71.9 & 28.3 & 144 \\
\hline
\end{tabular}




\section{1-8.L00-8A PANJSHER RIVER AT OMARZ, Continued}

Probability of occurrence of annual high discharges [m $\mathrm{m}^{3} / \mathrm{s}$, cubic meters per second; ng, not given]

\begin{tabular}{|c|c|c|c|c|c|c|}
\hline \multirow{2}{*}{$\begin{array}{l}\text { Exceedance } \\
\text { probability }\end{array}$} & \multirow{2}{*}{$\begin{array}{l}\text { Recurrence } \\
\text { interval } \\
\text { (years) }\end{array}$} & \multirow{2}{*}{$\begin{array}{l}\text { Maximum } \\
\text { instantaneous } \\
\text { discharge } \\
\left(\mathrm{m}^{3} / \mathrm{s}\right)\end{array}$} & \multicolumn{4}{|c|}{ Maximum daily mean discharge $\left(\mathrm{m}^{3} / \mathrm{s}\right)$} \\
\hline & & & $\begin{array}{l}\text { 3-day } \\
\text { period }\end{array}$ & $\begin{array}{l}\text { 7-day } \\
\text { period }\end{array}$ & $\begin{array}{l}\text { 15-day } \\
\text { period }\end{array}$ & $\begin{array}{l}\text { 30-day } \\
\text { period }\end{array}$ \\
\hline 0.99 & 1.01 & 181 & 125 & 113 & 97.8 & 82.1 \\
\hline 0.95 & 1.05 & 202 & 142 & 130 & 114 & 98.0 \\
\hline 0.90 & 1.11 & 214 & 152 & 139 & 124 & 107 \\
\hline 0.80 & 1.25 & 230 & 163 & 151 & 135 & 119 \\
\hline 0.50 & 2 & 264 & 187 & 174 & 160 & 144 \\
\hline 0.20 & 5 & 303 & 213 & 200 & 185 & 172 \\
\hline 0.10 & 10 & 326 & 227 & 214 & 199 & 187 \\
\hline 0.04 & 25 & 353 & 242 & 229 & 214 & 204 \\
\hline 0.02 & 50 & 371 & 252 & 239 & 224 & 216 \\
\hline 0.01 & 100 & 389 & 261 & 248 & 233 & 226 \\
\hline 0.005 & 200 & 405 & 270 & 257 & 241 & 236 \\
\hline 0.002 & 500 & 427 & $\mathrm{ng}$ & $\mathrm{ng}$ & $\mathrm{ng}$ & $\mathrm{ng}$ \\
\hline
\end{tabular}

\section{1-8.L00-8A PANJSHER RIVER AT OMARZ, Continued}

Probability of occurrence of annual low discharges $\left[\mathrm{m}^{3} / \mathrm{s}\right.$, meters per second]

\begin{tabular}{|c|c|c|c|c|c|c|c|c|c|c|}
\hline \multirow{3}{*}{$\begin{array}{c}\text { Nonexceedance } \\
\text { probability }\end{array}$} & \multirow{3}{*}{$\begin{array}{c}\text { Recurrence } \\
\text { interval } \\
\text { (years) }\end{array}$} & \multicolumn{9}{|c|}{ Minimum daily mean discharge $\left(\mathrm{m}^{3} / \mathrm{s}\right)$} \\
\hline & & \multicolumn{9}{|c|}{ Number of consecutive days } \\
\hline & & 1 & 3 & 7 & 14 & 30 & 60 & 90 & 120 & 183 \\
\hline 0.05 & 20 & 3.83 & 4.27 & 4.63 & 4.77 & 4.99 & 5.15 & 5.28 & 5.54 & 6.55 \\
\hline 0.10 & 10 & 4.10 & 4.52 & 4.83 & 4.98 & 5.17 & 5.35 & 5.52 & 5.80 & 6.85 \\
\hline 0.20 & 5 & 4.47 & 4.84 & 5.11 & 5.27 & 5.44 & 5.62 & 5.83 & 6.14 & 7.24 \\
\hline 0.50 & 2 & 5.26 & 5.56 & 5.76 & 5.93 & 6.06 & 6.26 & 6.54 & 6.91 & 8.13 \\
\hline
\end{tabular}




\section{1-8.L00-8A PANJSHER RIVER AT OMARZ, Continued}

Probability of occurrence of seasonal low discharges $\left[\mathrm{m}^{3} / \mathrm{s}\right.$, meters per second]

\begin{tabular}{|c|c|c|c|c|c|c|c|c|c|}
\hline \multirow{3}{*}{$\begin{array}{c}\text { Nonexceedance } \\
\text { probability }\end{array}$} & \multirow{3}{*}{$\begin{array}{c}\text { Recurrence } \\
\text { interval } \\
\text { (years) }\end{array}$} & \multicolumn{8}{|c|}{ Minimum daily mean discharge $\left(\mathrm{m}^{3} / \mathrm{s}\right)$} \\
\hline & & \multicolumn{8}{|c|}{ Number of consecutive days } \\
\hline & & 1 & 7 & 14 & 30 & 1 & 7 & 14 & 30 \\
\hline & & \multicolumn{4}{|c|}{ December-January-February } & \multicolumn{4}{|c|}{ March-April-May } \\
\hline 0.05 & 20 & 3.81 & 4.6 & 4.76 & 4.95 & 5.06 & 5.31 & 5.34 & 5.55 \\
\hline 0.10 & 10 & 4.14 & 4.86 & 5.02 & 5.20 & 5.25 & 5.53 & 5.59 & 5.87 \\
\hline 0.20 & 5 & 4.57 & 5.20 & 5.35 & 5.54 & 5.52 & 5.82 & 5.92 & 6.31 \\
\hline \multirow[t]{2}{*}{0.50} & 2 & 5.47 & 5.94 & 6.09 & 6.27 & 6.14 & 6.47 & 6.66 & 7.26 \\
\hline & & \multicolumn{4}{|c|}{ June-July-August } & \multicolumn{4}{|c|}{ September-October-November } \\
\hline 0.05 & 20 & 17.7 & 18.6 & 18.7 & 22.5 & 6.49 & 6.68 & 6.76 & 7.23 \\
\hline 0.10 & 10 & 18.8 & 20.0 & 20.8 & 25.1 & 6.79 & 6.98 & 7.10 & 7.6 \\
\hline 0.20 & 5 & 20.2 & 21.9 & 23.6 & 28.6 & 7.20 & 7.39 & 7.55 & 8.10 \\
\hline 0.50 & 2 & 23.8 & 26.3 & 29.9 & 37.4 & 8.16 & 8.38 & 8.61 & 9.24 \\
\hline
\end{tabular}




\section{1-8.L00-8A PANJSHER RIVER AT OMARZ, Continued}

\begin{tabular}{|c|c|c|c|c|c|}
\hline \multicolumn{6}{|c|}{$\begin{array}{c}\text { Annual peak discharges } \\
{\left[\mathrm{m}^{3} / \mathrm{s} \text {, meters per second] }\right.}\end{array}$} \\
\hline \multicolumn{3}{|c|}{$\begin{array}{c}\text { Annual peak discharge, } \\
\text { by year }\end{array}$} & \multicolumn{3}{|c|}{$\begin{array}{l}\text { Annual peak discharge, } \\
\text { from highest to lowest }\end{array}$} \\
\hline $\begin{array}{l}\text { Water } \\
\text { year }\end{array}$ & Date & $\begin{array}{c}\text { Peak } \\
\text { discharge } \\
\left(\mathrm{m}^{3} / \mathrm{s}\right)\end{array}$ & $\begin{array}{l}\text { Water } \\
\text { year }\end{array}$ & Date & $\begin{array}{c}\text { Peak } \\
\text { discharge } \\
\left(\mathrm{m}^{3} / \mathrm{s}\right)\end{array}$ \\
\hline 1964 & July 2, 1964 & 272 & 1973 & June 12, 1973 & 342 \\
\hline 1965 & July 14, 1965 & 224 & 1979 & June 23, 1979 & 326 \\
\hline 1966 & June 19, 1966 & 300 & 1968 & July 18,1968 & 318 \\
\hline 1967 & June 28, 1967 & 237 & 1972 & June 21, 1972 & 306 \\
\hline 1968 & July 18,1968 & 318 & 1978 & July 6, 1978 & 305 \\
\hline 1969 & June 20, 1969 & 279 & 1966 & June 19, 1966 & 300 \\
\hline 1970 & June 3, 1970 & 226 & 1977 & June 22, 1977 & 284 \\
\hline 1971 & May 27, 1971 & 252 & 1969 & June 20, 1969 & 279 \\
\hline 1972 & June 21, 1972 & 306 & 1964 & July 2, 1964 & 272 \\
\hline 1973 & June 12, 1973 & 342 & 1971 & May 27, 1971 & 252 \\
\hline 1974 & June 16, 1974 & 219 & 1967 & June 28, 1967 & 237 \\
\hline 1975 & June 15,1975 & 228 & 1975 & June 15, 1975 & 228 \\
\hline 1976 & July 8, 1976 & 210 & 1970 & June 3, 1970 & 226 \\
\hline 1977 & June 22, 1977 & 284 & 1965 & July 14, 1965 & 224 \\
\hline 1978 & July 6, 1978 & 305 & 1974 & June 16, 1974 & 219 \\
\hline 1979 & June 23, 1979 & 326 & 1980 & June 11, 1980 & 213 \\
\hline 1980 & June 11,1980 & 213 & 1976 & July 8, 1976 & 210 \\
\hline
\end{tabular}




\section{1-8.L00-8A PANJSHER RIVER AT OMARZ, Continued}

Monthly and annual mean discharges, in cubic meters per second $[--$, no data]

\begin{tabular}{|c|c|c|c|c|c|c|c|c|c|c|c|c|c|}
\hline \multirow{2}{*}{$\begin{array}{l}\text { Water } \\
\text { year }\end{array}$} & \multicolumn{12}{|c|}{ Monthly mean discharge } & \multirow{2}{*}{$\begin{array}{c}\text { Annual } \\
\text { discharge }\end{array}$} \\
\hline & October & November & December & January & February & March & April & May & June & July & August & September & \\
\hline 1963 & 12.1 & 10.4 & 8.71 & 7.61 & -- & -- & -- & -- & -- & 105 & 41.1 & 19.4 & -- \\
\hline 1964 & 14.8 & 10.9 & 8.32 & 7.43 & 7.58 & 8.45 & 14.5 & 45.3 & 130 & 126 & 52.7 & 20.2 & 37.2 \\
\hline 1965 & 11.0 & 7.64 & 6.21 & 6.20 & 6.02 & 8.53 & 12.6 & 42.1 & 139 & 140 & 53.0 & 22.6 & 38.1 \\
\hline 1966 & 12.9 & 11.4 & 9.67 & 8.39 & 6.67 & 6.82 & 13.8 & 54.1 & 143 & 93.8 & 45.7 & 21.7 & 35.8 \\
\hline 1967 & 11.9 & 8.68 & 6.79 & 5.79 & 5.97 & 6.07 & 11.5 & 33.3 & 126 & 113 & 42.3 & 19.5 & 32.7 \\
\hline 1968 & 11.2 & 8.19 & 7.19 & 6.72 & 6.42 & 7.65 & 17.2 & 34.4 & 161 & 142 & 54.2 & 19.0 & 39.6 \\
\hline 1969 & 13.8 & 10.2 & 9.25 & 7.26 & 7.08 & 9.56 & 20.5 & 38.4 & 149 & 153 & 75.5 & 27.6 & 43.7 \\
\hline 1970 & 15.8 & 12.2 & 9.73 & 9.11 & 8.72 & 9.27 & 19.4 & 63.9 & 103 & 53.3 & 37.3 & 21.8 & 30.4 \\
\hline 1971 & 11.8 & 9.52 & 7.77 & 6.09 & 6.47 & 10.0 & 23.7 & 92.4 & 89.2 & 34.7 & 25.1 & 11.9 & 27.5 \\
\hline 1972 & 7.90 & 7.51 & 6.08 & 5.34 & 5.67 & 7.28 & 12.3 & 34.3 & 151 & 104 & 40.9 & 19.6 & 33.5 \\
\hline 1973 & 14.7 & 12.4 & 10.7 & 8.71 & 7.19 & 7.15 & 21.1 & 79.1 & 190 & 106 & 36.4 & 17.4 & 42.6 \\
\hline 1974 & 10.4 & 8.16 & 7.40 & 6.00 & 6.03 & 7.56 & 14.8 & 41.7 & 101 & 67.5 & 24.0 & 13.2 & 25.7 \\
\hline 1975 & 9.50 & 7.63 & 6.04 & 5.51 & 5.05 & 5.55 & 12.5 & 35.8 & 113 & 86.0 & 37.6 & 16.0 & 28.4 \\
\hline 1976 & 9.71 & 8.05 & 7.08 & 5.90 & 5.63 & 5.79 & 13.0 & 45.3 & 95.0 & 92.6 & 32.1 & 18.1 & 28.3 \\
\hline 1977 & 12.5 & 10.1 & 8.84 & 7.82 & 6.84 & 7.10 & 10.5 & 32.1 & 98.8 & 57.5 & 25.8 & 15.2 & 24.5 \\
\hline 1978 & 9.74 & 8.78 & 8.05 & 7.26 & 6.93 & 7.32 & 19.4 & 73.4 & 168 & 80.6 & 31.5 & 17.6 & 36.6 \\
\hline 1979 & 12.1 & 9.56 & 7.18 & 5.27 & 5.08 & 6.23 & 21.9 & 41.4 & 142 & 113 & 45.2 & 17.5 & 35.6 \\
\hline 1980 & 11.6 & 9.68 & 8.21 & 6.95 & 6.51 & 6.42 & 17.6 & 63.8 & 123 & 56.8 & 24.2 & 14.5 & 29.1 \\
\hline
\end{tabular}




\section{1-9.R00-4W CHAKARI RIVER AT BAND-I-AMIR GHAZI}

\section{(U.S. Geological Survey identification number: 342500069230000)}

LOCATION: Lat $34^{\circ} 25^{\prime} \mathrm{N}$., long $69^{\circ} 23^{\prime} \mathrm{E}$.

DRAINAGE AREA: $395 \mathrm{~km}^{2}$.

ELEVATION: 2,050 meters above mean sea level.

PERIOD OF RECORD: May 26, 1965 to September 30, 1980.

GAGE: Staff gage.

Annual mean discharge

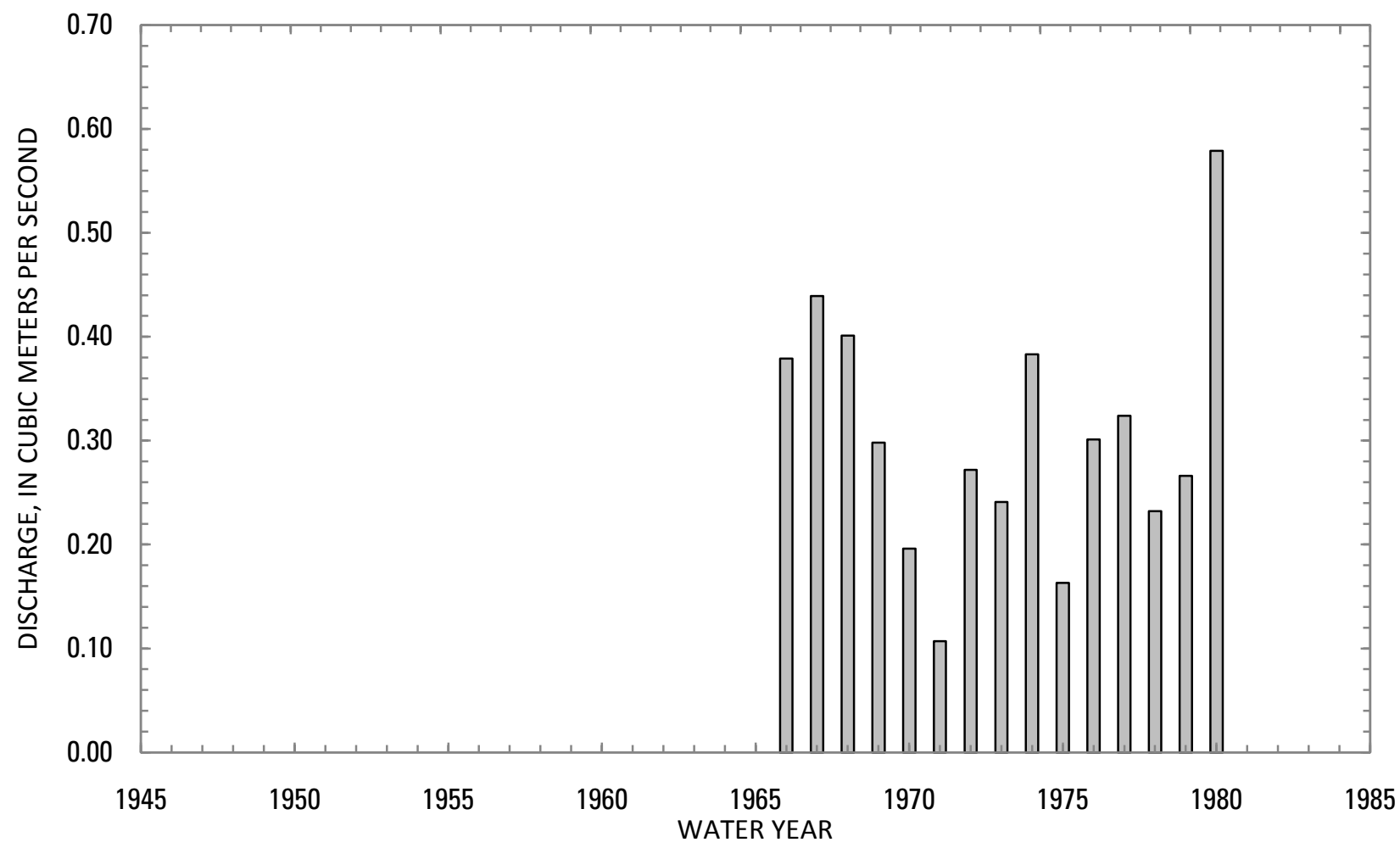




\section{1-9.R00-4W CHAKARI RIVER AT BAND-I-AMIR GHAZI, Continued}

Statistics of monthly and annual mean discharges $\left[\mathrm{m}^{3} / \mathrm{s}\right.$, cubic meters per second]

\begin{tabular}{|c|c|c|c|c|c|c|c|c|}
\hline \multirow[b]{2}{*}{ Month } & \multicolumn{2}{|c|}{ Maximum } & \multicolumn{2}{|c|}{ Minimum } & \multicolumn{4}{|c|}{ Mean } \\
\hline & $\begin{array}{c}\text { Discharge } \\
\left(\mathrm{m}^{3} / \mathrm{s}\right)\end{array}$ & $\begin{array}{c}\text { Water year } \\
\text { of } \\
\text { occurrence }\end{array}$ & $\begin{array}{c}\text { Discharge } \\
\left(\mathrm{m}^{3} / \mathrm{s}\right)\end{array}$ & $\begin{array}{c}\text { Water year } \\
\text { of } \\
\text { occurrence }\end{array}$ & $\begin{array}{c}\text { Discharge } \\
\left(\mathrm{m}^{3} / \mathrm{s}\right)\end{array}$ & $\begin{array}{c}\text { Standard } \\
\text { deviation } \\
\left(\mathrm{m}^{3} / \mathrm{s}\right)\end{array}$ & $\begin{array}{c}\text { Coefficient } \\
\text { of } \\
\text { variation }\end{array}$ & $\begin{array}{c}\text { Percentage } \\
\text { of annual } \\
\text { discharge }\end{array}$ \\
\hline October & 0.68 & 1968 & 0.05 & 1972 & 0.33 & 0.17 & 0.53 & 8.80 \\
\hline November & 1.06 & 1968 & 0.10 & 1971 & 0.34 & 0.24 & 0.73 & 9.06 \\
\hline December & 0.94 & 1968 & 0.02 & 1978 & 0.15 & 0.22 & 1.53 & 3.95 \\
\hline January & 0.25 & 1967 & 0.01 & 1978 & 0.05 & 0.06 & 1.33 & 1.22 \\
\hline February & 0.38 & 1967 & 0.01 & 1978 & 0.10 & 0.11 & 1.17 & 2.57 \\
\hline March & 0.62 & 1980 & 0.01 & 1977 & 0.18 & 0.21 & 1.20 & 4.80 \\
\hline April & 1.71 & 1980 & 0.03 & 1975 & 0.44 & 0.40 & 0.89 & 12.0 \\
\hline May & 0.96 & 1967 & 0.05 & 1975 & 0.55 & 0.26 & 0.47 & 14.9 \\
\hline June & 0.93 & 1974 & 0.04 & 1971 & 0.52 & 0.29 & 0.56 & 14.0 \\
\hline July & 0.97 & 1980 & 0.03 & 1971 & 0.39 & 0.23 & 0.58 & 10.7 \\
\hline August & 0.94 & 1980 & 0.05 & 1971 & 0.34 & 0.22 & 0.65 & 9.14 \\
\hline September & 0.86 & 1979 & 0.06 & 1971 & 0.33 & 0.20 & 0.59 & 8.99 \\
\hline Annual & 0.58 & 1980 & 0.11 & 1971 & 0.31 & 0.12 & 0.39 & 100 \\
\hline
\end{tabular}


1-9.R00-4W CHAKARI RIVER AT BAND-I-AMIR GHAZI, Continued

Monthly and annual flow duration, in cubic meters per second

\begin{tabular}{|c|c|c|c|c|c|c|c|c|c|c|c|c|c|}
\hline \multirow{2}{*}{$\begin{array}{l}\text { Percentage } \\
\text { of days } \\
\text { discharge } \\
\text { equaled or } \\
\text { exceeded }\end{array}$} & \multicolumn{12}{|c|}{ Month } & \multirow{2}{*}{ Annual } \\
\hline & October & November & December & January & February & March & April & May & June & July & August & September & \\
\hline 95 & 0.06 & 0.07 & 0.02 & 0.01 & 0.01 & 0.01 & 0.02 & 0.07 & 0.04 & 0.03 & 0.05 & 0.05 & 0.02 \\
\hline 90 & 0.08 & 0.10 & 0.02 & 0.02 & 0.02 & 0.02 & 0.03 & 0.13 & 0.07 & 0.12 & 0.07 & 0.07 & 0.02 \\
\hline 85 & 0.12 & 0.12 & 0.02 & 0.02 & 0.02 & 0.02 & 0.09 & 0.25 & 0.23 & 0.14 & 0.11 & 0.10 & 0.03 \\
\hline 80 & 0.19 & 0.13 & 0.02 & 0.02 & 0.02 & 0.02 & 0.13 & 0.25 & 0.24 & 0.21 & 0.13 & 0.20 & 0.04 \\
\hline 75 & 0.20 & 0.15 & 0.03 & 0.02 & 0.02 & 0.03 & 0.14 & 0.34 & 0.31 & 0.23 & 0.20 & 0.21 & 0.06 \\
\hline 70 & 0.21 & 0.16 & 0.03 & 0.02 & 0.03 & 0.03 & 0.17 & 0.41 & 0.35 & 0.24 & 0.22 & 0.22 & 0.08 \\
\hline 65 & 0.24 & 0.22 & 0.03 & 0.02 & 0.03 & 0.03 & 0.25 & 0.48 & 0.38 & 0.26 & 0.23 & 0.24 & 0.13 \\
\hline 60 & 0.25 & 0.23 & 0.04 & 0.03 & 0.04 & 0.04 & 0.31 & 0.49 & 0.41 & 0.31 & 0.25 & 0.25 & 0.17 \\
\hline 55 & 0.29 & 0.25 & 0.04 & 0.03 & 0.04 & 0.04 & 0.40 & 0.50 & 0.45 & 0.33 & 0.30 & 0.26 & 0.22 \\
\hline 50 & 0.30 & 0.26 & 0.05 & 0.03 & 0.05 & 0.05 & 0.44 & 0.51 & 0.47 & 0.34 & 0.32 & 0.31 & 0.25 \\
\hline 45 & 0.31 & 0.31 & 0.05 & 0.03 & 0.05 & 0.10 & 0.46 & 0.52 & 0.49 & 0.36 & 0.33 & 0.32 & 0.27 \\
\hline 40 & 0.32 & 0.33 & 0.06 & 0.03 & 0.06 & 0.15 & 0.48 & 0.57 & 0.51 & 0.38 & 0.35 & 0.34 & 0.30 \\
\hline 35 & 0.34 & 0.36 & 0.07 & 0.04 & 0.07 & 0.19 & 0.49 & 0.67 & 0.56 & 0.41 & 0.38 & 0.36 & 0.34 \\
\hline 30 & 0.36 & 0.39 & 0.09 & 0.04 & 0.10 & 0.20 & 0.51 & 0.71 & 0.72 & 0.44 & 0.41 & 0.38 & 0.40 \\
\hline 25 & 0.39 & 0.42 & 0.12 & 0.05 & 0.14 & 0.26 & 0.54 & 0.75 & 0.80 & 0.48 & 0.44 & 0.40 & 0.44 \\
\hline 20 & 0.44 & 0.48 & 0.15 & 0.05 & 0.18 & 0.37 & 0.58 & 0.82 & 0.87 & 0.53 & 0.47 & 0.44 & 0.48 \\
\hline 15 & 0.48 & 0.51 & 0.23 & 0.06 & 0.23 & 0.40 & 0.71 & 0.88 & 0.93 & 0.60 & 0.50 & 0.50 & 0.53 \\
\hline 10 & 0.51 & 0.67 & 0.31 & 0.07 & 0.30 & 0.53 & 0.79 & 1.01 & 1.00 & 0.79 & 0.52 & 0.54 & 0.75 \\
\hline 5 & 0.86 & 0.90 & 0.82 & 0.20 & 0.37 & 0.62 & 1.58 & 1.13 & 1.07 & 0.96 & 0.69 & 0.80 & 0.95 \\
\hline
\end{tabular}




\section{1-9.R00-4W CHAKARI RIVER AT BAND-I-AMIR GHAZI, Continued}

Probability of occurrence of annual high discharges

[ $\mathrm{m}^{3} / \mathrm{s}$, cubic meters per second; ng, not given]

\begin{tabular}{|c|c|c|c|c|c|c|}
\hline \multirow{2}{*}{$\begin{array}{l}\text { Exceedance } \\
\text { probability }\end{array}$} & \multirow{2}{*}{$\begin{array}{c}\text { Recurrence } \\
\text { interval } \\
\text { (years) }\end{array}$} & \multirow{2}{*}{$\begin{array}{c}\text { Maximum } \\
\text { instantaneous } \\
\text { discharge } \\
\left(\mathrm{m}^{3} / \mathrm{s}\right)\end{array}$} & \multicolumn{4}{|c|}{ Maximum daily mean discharge $\left(\mathrm{m}^{3} / \mathrm{s}\right)$} \\
\hline & & & $\begin{array}{l}\text { 3-day } \\
\text { period }\end{array}$ & $\begin{array}{l}\text { 7-day } \\
\text { period }\end{array}$ & $\begin{array}{l}\text { 15-day } \\
\text { period }\end{array}$ & $\begin{array}{l}\text { 30-day } \\
\text { period }\end{array}$ \\
\hline 0.99 & 1.01 & 0.30 & 0.27 & 0.27 & 0.25 & 0.24 \\
\hline 0.95 & 1.05 & 0.40 & 0.39 & 0.39 & 0.36 & 0.34 \\
\hline 0.90 & 1.11 & 0.50 & 0.47 & 0.47 & 0.44 & 0.42 \\
\hline 0.80 & 1.25 & 0.60 & 0.59 & 0.59 & 0.55 & 0.52 \\
\hline 0.50 & 2 & 1.00 & 0.90 & 0.89 & 0.85 & 0.78 \\
\hline 0.20 & 5 & 1.40 & 1.34 & 1.32 & 1.27 & 1.14 \\
\hline 0.10 & 10 & 1.70 & 1.64 & 1.61 & 1.55 & 1.38 \\
\hline 0.04 & 25 & 2.00 & 2.03 & 1.99 & 1.92 & 1.68 \\
\hline 0.02 & 50 & 2.20 & 2.31 & 2.27 & 2.19 & 1.89 \\
\hline 0.01 & 100 & 2.40 & 2.60 & 2.55 & 2.46 & 2.11 \\
\hline 0.005 & 200 & 2.60 & 2.89 & 2.83 & 2.73 & 2.32 \\
\hline 0.002 & 500 & 2.90 & $\mathrm{ng}$ & $\mathrm{ng}$ & $\mathrm{ng}$ & $\mathrm{ng}$ \\
\hline
\end{tabular}

1-9.R00-4W CHAKARI RIVER AT BAND-I-AMIR GHAZI, Continued

Probability of occurrence of annual low discharges

$\left[\mathrm{m}^{3} / \mathrm{s}\right.$, meters per second]

\begin{tabular}{|c|c|c|c|c|c|c|c|c|c|c|}
\hline \multirow{3}{*}{$\begin{array}{c}\text { Nonexceedance } \\
\text { probability }\end{array}$} & \multirow{3}{*}{$\begin{array}{c}\text { Recurrence } \\
\text { interval } \\
\text { (years) }\end{array}$} & \multicolumn{9}{|c|}{ Minimum daily mean discharge $\left(\mathrm{m}^{3} / \mathrm{s}\right)$} \\
\hline & & \multicolumn{9}{|c|}{ Number of consecutive days } \\
\hline & & 1 & 3 & 7 & 14 & 30 & 60 & 90 & 120 & 183 \\
\hline 0.05 & 20 & 0 & 0 & 0 & 0.01 & 0.01 & 0.01 & 0.01 & 0.02 & 0.05 \\
\hline 0.10 & 10 & 0.01 & 0.01 & 0.01 & 0.01 & 0.01 & 0.01 & 0.01 & 0.02 & 0.07 \\
\hline 0.20 & 5 & 0.01 & 0.01 & 0.01 & 0.01 & 0.01 & 0.02 & 0.02 & 0.03 & 0.09 \\
\hline 0.50 & 2 & 0.01 & 0.01 & 0.01 & 0.02 & 0.02 & 0.03 & 0.03 & 0.06 & 0.15 \\
\hline
\end{tabular}




\section{1-9.R00-4W CHAKARI RIVER AT BAND-I-AMIR GHAZI, Continued}

\section{Probability of occurrence of seasonal low discharges}

[ $\mathrm{m}^{3} / \mathrm{s}$, meters per second]

\begin{tabular}{|c|c|c|c|c|c|c|c|c|c|}
\hline \multirow{3}{*}{$\begin{array}{c}\text { Nonexceedance } \\
\text { probability }\end{array}$} & \multirow{3}{*}{$\begin{array}{c}\text { Recurrence } \\
\text { interval } \\
\text { (years) }\end{array}$} & \multicolumn{8}{|c|}{ Minimum daily mean discharge $\left(\mathrm{m}^{3} / \mathrm{s}\right)$} \\
\hline & & \multicolumn{8}{|c|}{ Number of consecutive days } \\
\hline & & 1 & 7 & 14 & 30 & 1 & 7 & 14 & 30 \\
\hline & & \multicolumn{4}{|c|}{ December-January-February } & \multicolumn{4}{|c|}{ March-April-May } \\
\hline 0.05 & 20 & 0.01 & 0.01 & 0.01 & 0.01 & 0.01 & 0.01 & 0.01 & 0.01 \\
\hline 0.10 & 10 & 0.01 & 0.01 & 0.01 & 0.01 & 0.01 & 0.01 & 0.01 & 0.01 \\
\hline 0.20 & 5 & 0.01 & 0.01 & 0.01 & 0.01 & 0.02 & 0.02 & 0.02 & 0.02 \\
\hline \multirow[t]{2}{*}{0.50} & 2 & 0.02 & 0.02 & 0.02 & 0.02 & 0.05 & 0.05 & 0.05 & 0.07 \\
\hline & & \multicolumn{4}{|c|}{ June-July-August } & \multicolumn{4}{|c|}{ September-October-November } \\
\hline 0.05 & 20 & 0.01 & 0.01 & 0.02 & 0.04 & 0 & 0 & 0.04 & 0.06 \\
\hline 0.10 & 10 & 0.01 & 0.02 & 0.03 & 0.07 & 0.01 & 0.01 & 0.06 & 0.08 \\
\hline 0.20 & 5 & 0.03 & 0.04 & 0.06 & 0.13 & 0.03 & 0.04 & 0.08 & 0.12 \\
\hline 0.50 & 2 & 0.11 & 0.13 & 0.18 & 0.27 & 0.11 & 0.12 & 0.16 & 0.22 \\
\hline
\end{tabular}




\section{1-9.R00-4W CHAKARI RIVER AT BAND-I-AMIR GHAZI, Continued}

\begin{tabular}{|c|c|c|c|c|c|}
\hline \multicolumn{6}{|c|}{$\begin{array}{c}\text { Annual peak discharges } \\
\text { [m³/s, meters per second] }\end{array}$} \\
\hline \multicolumn{3}{|c|}{$\begin{array}{c}\text { Annual peak discharge, } \\
\text { by year }\end{array}$} & \multicolumn{3}{|c|}{$\begin{array}{c}\text { Annual peak discharge, } \\
\text { from highest to lowest }\end{array}$} \\
\hline $\begin{array}{l}\text { Water } \\
\text { year }\end{array}$ & Date & $\begin{array}{c}\text { Peak } \\
\text { discharge } \\
\left(\mathrm{m}^{3} / \mathrm{s}\right)\end{array}$ & $\begin{array}{l}\text { Water } \\
\text { year }\end{array}$ & Date & $\begin{array}{c}\text { Peak } \\
\text { discharge } \\
\left(\mathrm{m}^{3} / \mathrm{s}\right)\end{array}$ \\
\hline 1966 & May 1,1966 & 1.00 & 1980 & April 17, 1980 & 1.92 \\
\hline 1967 & May 23, 1967 & 1.26 & 1968 & $\begin{array}{c}\text { December } 9,1967 \\
\text { September } 13,\end{array}$ & 1.67 \\
\hline 1968 & December 9, 1967 & 1.67 & 1979 & 1979 & 1.43 \\
\hline 1969 & March 13, 1968 & 0.55 & 1972 & June 18, 1972 & 1.36 \\
\hline 1970 & July 17,1970 & 0.96 & 1967 & May 23, 1967 & 1.26 \\
\hline 1971 & May 1,1971 & 0.55 & 1978 & June 9, 1978 & 1.19 \\
\hline 1972 & June 18,1972 & 1.36 & 1974 & June 8, 1974 & 1.06 \\
\hline 1973 & April 21, 1973 & 0.55 & 1966 & May 1,1966 & 1.00 \\
\hline 1974 & June 8, 1974 & 1.06 & 1970 & July 17,1970 & 0.96 \\
\hline 1975 & June 8, 1975 & 0.39 & 1976 & May 17,1976 & 0.82 \\
\hline 1976 & May 17,1976 & 0.82 & 1977 & August 9, 1977 & 0.82 \\
\hline 1977 & August 9, 1977 & 0.82 & 1969 & March 13, 1968 & 0.55 \\
\hline 1978 & $\begin{array}{l}\text { June } 9,1978 \\
\text { September } 13 \text {, }\end{array}$ & 1.19 & 1971 & May 1,1971 & 0.55 \\
\hline 1979 & 1979 & 1.43 & 1973 & April 21, 1973 & 0.55 \\
\hline 1980 & April 17, 1980 & 1.92 & 1975 & June 8,1975 & 0.39 \\
\hline
\end{tabular}


1-9.R00-4W CHAKARI RIVER AT BAND-I-AMIR GHAZI, Continued

Monthly and annual mean discharges, in cubic meters per second

[Data may not be rounded in accordance with U.S. Geological Survey publication standards; --, no data]

\begin{tabular}{|c|c|c|c|c|c|c|c|c|c|c|c|c|c|}
\hline \multirow{2}{*}{$\begin{array}{l}\text { Water } \\
\text { year }\end{array}$} & \multicolumn{12}{|c|}{ Monthly mean discharge } & \multirow{2}{*}{$\begin{array}{c}\text { Annual } \\
\text { discharge }\end{array}$} \\
\hline & October & November & December & January & February & March & April & May & June & July & August & September & \\
\hline 1965 & -- & -- & -- & -- & -- & -- & -- & -- & 0.76 & 0.57 & 0.49 & 0.42 & -- \\
\hline 1966 & 0.44 & 0.43 & 0.19 & 0.07 & 0.31 & 0.59 & 0.74 & 0.58 & 0.41 & 0.29 & 0.29 & 0.22 & 0.38 \\
\hline 1967 & 0.35 & 0.33 & 0.05 & 0.25 & 0.38 & 0.40 & 0.33 & 0.96 & 0.90 & 0.54 & 0.32 & 0.46 & 0.44 \\
\hline 1968 & 0.68 & 1.06 & 0.94 & 0.05 & 0.05 & 0.03 & 0.34 & 0.48 & 0.26 & 0.25 & 0.36 & 0.32 & 0.40 \\
\hline 1969 & 0.23 & 0.19 & 0.12 & 0.03 & 0.14 & 0.38 & 0.42 & 0.47 & 0.40 & 0.43 & 0.44 & 0.30 & 0.30 \\
\hline 1970 & 0.29 & 0.39 & 0.07 & 0.03 & 0.15 & 0.19 & 0.37 & 0.31 & 0.22 & 0.15 & 0.11 & 0.08 & 0.20 \\
\hline 1971 & 0.07 & 0.10 & 0.04 & 0.02 & 0.04 & 0.03 & 0.43 & 0.39 & 0.04 & 0.03 & 0.05 & 0.06 & 0.11 \\
\hline 1972 & 0.05 & 0.15 & 0.07 & 0.04 & 0.06 & 0.10 & 0.43 & 0.85 & 0.86 & 0.27 & 0.20 & 0.20 & 0.27 \\
\hline 1973 & 0.20 & 0.10 & 0.05 & 0.05 & 0.03 & 0.03 & 0.28 & 0.47 & 0.50 & 0.49 & 0.34 & 0.34 & 0.24 \\
\hline 1974 & 0.28 & 0.25 & 0.09 & 0.03 & 0.09 & 0.18 & 0.56 & 0.84 & 0.93 & 0.72 & 0.34 & 0.28 & 0.38 \\
\hline 1975 & 0.25 & 0.30 & 0.13 & 0.01 & 0.02 & 0.02 & 0.03 & 0.05 & 0.26 & 0.30 & 0.30 & 0.29 & 0.16 \\
\hline 1976 & 0.43 & 0.22 & 0.06 & 0.02 & 0.02 & 0.07 & 0.46 & 0.77 & 0.76 & 0.24 & 0.26 & 0.31 & 0.30 \\
\hline 1977 & 0.24 & 0.52 & 0.15 & 0.02 & 0.01 & 0.01 & 0.36 & 0.52 & 0.41 & 0.37 & 0.66 & 0.62 & 0.32 \\
\hline 1978 & 0.34 & 0.20 & 0.02 & 0.01 & 0.01 & 0.01 & 0.08 & 0.45 & 0.89 & 0.28 & 0.23 & 0.26 & 0.23 \\
\hline 1979 & 0.45 & 0.56 & 0.16 & 0.01 & 0.02 & 0.02 & 0.11 & 0.24 & 0.26 & 0.42 & 0.09 & 0.86 & 0.27 \\
\hline 1980 & 0.59 & 0.23 & 0.07 & 0.04 & 0.11 & 0.62 & 1.71 & 0.88 & 0.43 & 0.97 & 0.94 & 0.35 & 0.58 \\
\hline
\end{tabular}




\section{1-11.1L0-4W OARGHA RIVER BELOW OARGHA RESERVOIR}

\section{(U.S. Geological Survey identification number: 343300069020000$)$}

LOCATION: Lat $34^{\circ} 33^{\prime} \mathrm{N}$., long 6902'E.

DRAINAGE AREA: $115 \mathrm{~km}^{2}$.

ELEVATION: 1,950 meters above mean sea level.

PERIOD OF RECORD: October 1, 1964 to September 30, 1980.

GAGE: Water-stage recorder.

Annual mean discharge

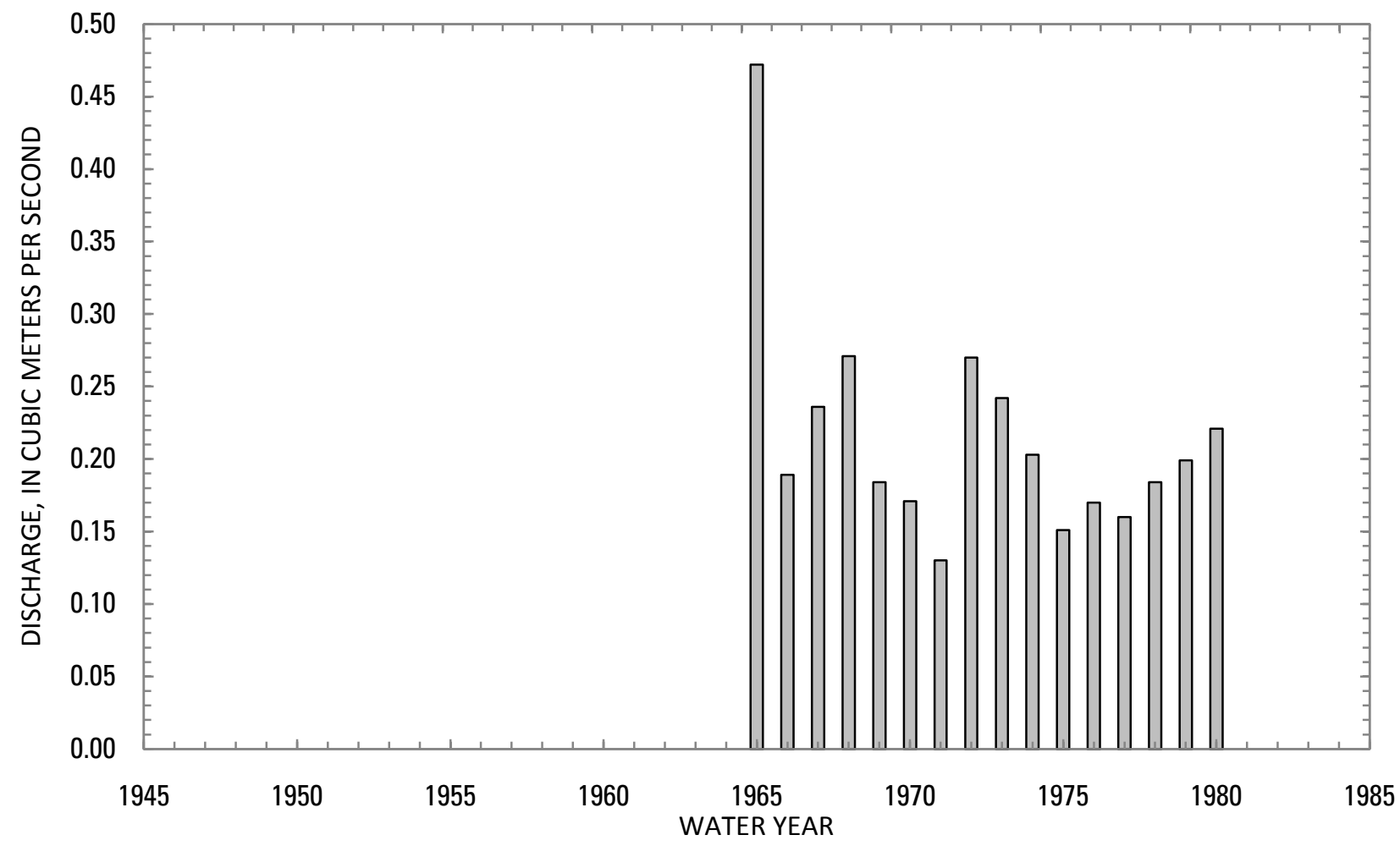




\section{1-11.1L0-4W OARGHA RIVER BELOW OARGHA RESERVOIR, Continued}

Statistics of monthly and annual mean discharges $\left[\mathrm{m}^{3} / \mathrm{s}\right.$, cubic meters per second]

\begin{tabular}{|c|c|c|c|c|c|c|c|c|}
\hline \multirow[b]{2}{*}{ Month } & \multicolumn{2}{|c|}{ Maximum } & \multicolumn{2}{|c|}{ Minimum } & \multicolumn{4}{|c|}{ Mean } \\
\hline & $\begin{array}{c}\text { Discharge } \\
\left(\mathrm{m}^{3} / \mathrm{s}\right)\end{array}$ & $\begin{array}{c}\text { Water year } \\
\text { of } \\
\text { occurrence }\end{array}$ & $\begin{array}{c}\text { Discharge } \\
\left(\mathrm{m}^{3} / \mathrm{s}\right)\end{array}$ & $\begin{array}{c}\text { Water year } \\
\text { of } \\
\text { occurrence }\end{array}$ & $\begin{array}{c}\text { Discharge } \\
\left(\mathrm{m}^{3} / \mathrm{s}\right)\end{array}$ & $\begin{array}{c}\text { Standard } \\
\text { deviation } \\
\left(\mathrm{m}^{3} / \mathrm{s}\right)\end{array}$ & $\begin{array}{c}\text { Coefficient } \\
\text { of } \\
\text { variation }\end{array}$ & $\begin{array}{c}\text { Percentage } \\
\text { of annual } \\
\text { discharge }\end{array}$ \\
\hline October & 0.60 & 1974 & 0.07 & 1972 & 0.24 & 0.13 & 0.55 & 9.26 \\
\hline November & 0.37 & 1974 & 0.06 & 1970 & 0.15 & 0.09 & 0.62 & 5.84 \\
\hline December & 0.33 & 1965 & 0.03 & 1976 & 0.09 & 0.07 & 0.78 & 3.55 \\
\hline January & 0.28 & 1965 & 0.02 & 1976 & 0.08 & 0.06 & 0.76 & 3.08 \\
\hline February & 0.31 & 1965 & 0.02 & 1976 & 0.09 & 0.07 & 0.79 & 3.51 \\
\hline March & 0.31 & 1965 & 0.03 & 1976 & 0.10 & 0.07 & 0.73 & 3.76 \\
\hline April & 0.42 & 1965 & 0.05 & 1976 & 0.13 & 0.09 & 0.67 & 5.21 \\
\hline May & 0.88 & 1972 & 0.12 & 1977 & 0.32 & 0.23 & 0.71 & 12.5 \\
\hline June & 0.97 & 1965 & 0.20 & 1975 & 0.38 & 0.23 & 0.60 & 14.8 \\
\hline July & 0.54 & 1973 & 0.13 & 1966 & 0.33 & 0.11 & 0.34 & 12.9 \\
\hline August & 0.62 & 1965 & 0.14 & 1969 & 0.34 & 0.12 & 0.34 & 13.3 \\
\hline September & 0.68 & 1965 & 0.10 & 1971 & 0.32 & 0.15 & 0.46 & 12.3 \\
\hline Annual & 0.47 & 1965 & 0.13 & 1971 & 0.22 & 0.08 & 0.37 & 100 \\
\hline
\end{tabular}




\section{1-11.1L0-4W OARGHA RIVER BELOW OARGHA RESERVOIR, Continued}

Monthly and annual flow duration, in cubic meters per second

[ng, not given]

\begin{tabular}{|c|c|c|c|c|c|c|c|c|c|c|c|c|c|}
\hline \multirow{2}{*}{$\begin{array}{l}\text { Percentage } \\
\text { of days } \\
\text { discharge } \\
\text { equaled or } \\
\text { exceeded }\end{array}$} & \multicolumn{12}{|c|}{ Month } & \multirow{2}{*}{ Annua } \\
\hline & October & November & December & January & February & March & April & May & June & July & August & September & \\
\hline 95 & 0.07 & 0.04 & 0.03 & 0.03 & 0.03 & 0.03 & 0.04 & 0.09 & 0.17 & 0.15 & 0.13 & 0.10 & 0.04 \\
\hline 90 & 0.08 & 0.06 & 0.04 & 0.03 & 0.03 & 0.04 & 0.05 & 0.11 & 0.20 & 0.19 & 0.16 & 0.12 & 0.05 \\
\hline 85 & 0.09 & 0.06 & 0.04 & 0.04 & 0.04 & 0.04 & 0.06 & 0.13 & 0.20 & 0.21 & 0.21 & 0.15 & 0.06 \\
\hline 80 & 0.12 & 0.07 & 0.05 & 0.04 & 0.04 & 0.04 & 0.07 & 0.14 & 0.21 & 0.22 & 0.24 & 0.20 & 0.07 \\
\hline 75 & 0.15 & 0.07 & 0.05 & 0.04 & 0.04 & 0.05 & 0.08 & 0.16 & 0.21 & 0.25 & 0.27 & 0.23 & 0.08 \\
\hline 70 & 0.17 & 0.08 & 0.06 & 0.05 & 0.05 & 0.05 & 0.09 & 0.17 & 0.25 & 0.26 & 0.30 & 0.24 & 0.10 \\
\hline 65 & 0.19 & 0.09 & 0.06 & 0.05 & 0.05 & 0.06 & 0.10 & 0.18 & 0.26 & 0.28 & 0.31 & 0.26 & 0.11 \\
\hline 60 & 0.20 & 0.10 & 0.07 & 0.06 & 0.06 & 0.07 & 0.10 & 0.19 & 0.27 & 0.30 & 0.31 & 0.29 & 0.13 \\
\hline 55 & 0.21 & 0.10 & 0.07 & 0.06 & 0.07 & 0.09 & 0.11 & 0.21 & 0.29 & 0.31 & 0.32 & 0.30 & 0.14 \\
\hline 50 & 0.23 & 0.12 & 0.07 & 0.07 & 0.07 & 0.09 & 0.12 & 0.22 & 0.31 & 0.32 & 0.34 & 0.32 & 0.16 \\
\hline 45 & 0.25 & 0.13 & 0.08 & 0.07 & 0.09 & 0.10 & 0.12 & 0.24 & 0.33 & 0.35 & 0.35 & 0.33 & 0.19 \\
\hline 40 & 0.26 & 0.15 & 0.08 & 0.08 & 0.09 & 0.10 & 0.13 & 0.26 & 0.36 & 0.36 & 0.35 & 0.34 & 0.22 \\
\hline 35 & 0.28 & 0.17 & 0.09 & 0.09 & 0.10 & 0.10 & 0.13 & 0.28 & 0.37 & 0.40 & 0.37 & 0.36 & 0.26 \\
\hline 30 & 0.30 & 0.20 & 0.10 & 0.09 & 0.12 & 0.11 & 0.13 & 0.30 & 0.40 & 0.41 & 0.40 & 0.37 & 0.29 \\
\hline 25 & 0.32 & 0.22 & 0.12 & 0.10 & 0.13 & 0.12 & 0.14 & 0.34 & 0.44 & 0.42 & 0.43 & 0.38 & 0.32 \\
\hline 20 & 0.33 & 0.26 & 0.13 & 0.10 & 0.13 & 0.13 & 0.16 & 0.41 & 0.52 & 0.43 & 0.45 & 0.41 & 0.34 \\
\hline 15 & 0.35 & 0.28 & 0.14 & 0.12 & 0.14 & 0.15 & 0.20 & 0.50 & 0.60 & 0.47 & 0.49 & 0.47 & 0.39 \\
\hline 10 & 0.42 & 0.32 & 0.16 & 0.15 & 0.18 & 0.20 & 0.27 & 0.64 & 0.69 & 0.52 & 0.51 & 0.50 & 0.44 \\
\hline 5 & ng & 0.37 & $\mathrm{ng}$ & $\mathrm{ng}$ & 0.28 & 0.27 & 0.39 & 1.05 & 0.93 & 0.58 & 0.59 & 0.65 & 0.56 \\
\hline
\end{tabular}




\section{1-11.1L0-4W OARGHA RIVER BELOW OARGHA RESERVOIR, Continued}

Probability of occurrence of annual high discharges

$\left[\mathrm{m}^{3} / \mathrm{s}\right.$, cubic meters per second; ng, not given]

\begin{tabular}{|c|c|c|c|c|c|c|}
\hline \multirow{2}{*}{$\begin{array}{c}\text { Exceedance } \\
\text { probability }\end{array}$} & \multirow{2}{*}{$\begin{array}{l}\text { Recurrence } \\
\text { interval } \\
\text { (years) }\end{array}$} & \multirow{2}{*}{$\begin{array}{l}\text { Maximum } \\
\text { instantaneous } \\
\text { discharge } \\
\left(\mathrm{m}^{3} / \mathrm{s}\right)\end{array}$} & \multicolumn{4}{|c|}{ Maximum daily mean discharge $\left(\mathrm{m}^{3} / \mathrm{s}\right)$} \\
\hline & & & $\begin{array}{l}\text { 3-day } \\
\text { period }\end{array}$ & $\begin{array}{l}\text { 7-day } \\
\text { period }\end{array}$ & $\begin{array}{l}\text { 15-day } \\
\text { period }\end{array}$ & $\begin{array}{l}\text { 30-day } \\
\text { period }\end{array}$ \\
\hline 0.99 & 1.01 & 0.40 & 0.35 & 0.32 & 0.29 & 0.25 \\
\hline 0.95 & 1.05 & 0.40 & 0.37 & 0.34 & 0.32 & 0.28 \\
\hline 0.90 & 1.11 & 0.40 & 0.38 & 0.36 & 0.34 & 0.30 \\
\hline 0.80 & 1.25 & 0.50 & 0.42 & 0.40 & 0.37 & 0.33 \\
\hline 0.50 & 2 & 0.70 & 0.54 & 0.53 & 0.49 & 0.43 \\
\hline 0.20 & 5 & 1.30 & 0.87 & 0.82 & 0.72 & 0.62 \\
\hline 0.10 & 10 & 2.10 & 1.22 & 1.11 & 0.92 & 0.79 \\
\hline 0.04 & 25 & 4.00 & 1.87 & 1.62 & 1.26 & 1.05 \\
\hline 0.02 & 50 & 6.50 & 2.59 & 2.14 & 1.58 & 1.28 \\
\hline 0.01 & 100 & 10.6 & 3.56 & 2.82 & 1.97 & 1.56 \\
\hline 0.005 & 200 & 17.0 & 4.87 & 3.70 & 2.45 & 1.89 \\
\hline 0.002 & 500 & 32.0 & ng & $\mathrm{ng}$ & $\mathrm{ng}$ & $\mathrm{ng}$ \\
\hline
\end{tabular}

\section{1-11.1L0-4W OARGHA RIVER BELOW OARGHA RESERVOIR, Continued}

Probability of occurrence of annual low discharges $\left[\mathrm{m}^{3} / \mathrm{s}\right.$, meters per second]

\begin{tabular}{|c|c|c|c|c|c|c|c|c|c|c|}
\hline \multirow{3}{*}{$\begin{array}{c}\text { Nonexceedance } \\
\text { probability }\end{array}$} & \multirow{3}{*}{$\begin{array}{c}\text { Recurrence } \\
\text { interval } \\
\text { (years) }\end{array}$} & \multicolumn{9}{|c|}{ Minimum daily mean discharge $\left(\mathrm{m}^{3} / \mathrm{s}\right)$} \\
\hline & & \multicolumn{9}{|c|}{ Number of consecutive days } \\
\hline & & 1 & 3 & 7 & 14 & 30 & 60 & 90 & 120 & 183 \\
\hline 0.05 & 20 & 0.02 & 0.02 & 0.02 & 0.02 & 0.02 & 0.03 & 0.03 & 0.03 & 0.06 \\
\hline 0.10 & 10 & 0.02 & 0.03 & 0.03 & 0.03 & 0.03 & 0.03 & 0.03 & 0.04 & 0.07 \\
\hline 0.20 & 5 & 0.03 & 0.03 & 0.03 & 0.03 & 0.03 & 0.04 & 0.04 & 0.05 & 0.08 \\
\hline 0.50 & 2 & 0.03 & 0.04 & 0.04 & 0.04 & 0.04 & 0.05 & 0.06 & 0.07 & 0.11 \\
\hline
\end{tabular}




\section{1-11.1L0-4W OARGHA RIVER BELOW OARGHA RESERVOIR, Continued}

Probability of occurrence of seasonal low discharges

$\left[\mathrm{m}^{3} / \mathrm{s}\right.$, meters per second]

\begin{tabular}{|c|c|c|c|c|c|c|c|c|c|}
\hline \multirow{3}{*}{$\begin{array}{c}\text { Nonexceedance } \\
\text { probability }\end{array}$} & \multirow{3}{*}{$\begin{array}{c}\text { Recurrence } \\
\text { interval } \\
\text { (years) }\end{array}$} & \multicolumn{8}{|c|}{ Minimum daily mean discharge $\left(\mathrm{m}^{3} / \mathrm{s}\right)$} \\
\hline & & \multicolumn{8}{|c|}{ Number of consecutive days } \\
\hline & & 1 & 7 & 14 & 30 & 1 & 7 & 14 & 30 \\
\hline & & \multicolumn{4}{|c|}{ December-January-February } & \multicolumn{4}{|c|}{ March-April-May } \\
\hline 0.05 & 20 & 0.02 & 0.02 & 0.02 & 0.02 & 0.03 & 0.03 & 0.03 & 0.03 \\
\hline 0.10 & 10 & 0.02 & 0.02 & 0.03 & 0.03 & 0.03 & 0.03 & 0.03 & 0.04 \\
\hline 0.20 & 5 & 0.03 & 0.03 & 0.03 & 0.03 & 0.03 & 0.04 & 0.04 & 0.05 \\
\hline \multirow[t]{2}{*}{0.50} & 2 & 0.04 & 0.04 & 0.04 & 0.05 & 0.05 & 0.06 & 0.06 & 0.07 \\
\hline & & \multicolumn{4}{|c|}{ June-July-August } & \multicolumn{4}{|c|}{ September-October-November } \\
\hline 0.05 & 20 & 0.08 & 0.08 & 0.09 & 0.13 & 0.03 & 0.03 & 0.04 & 0.06 \\
\hline 0.10 & 10 & 0.10 & 0.10 & 0.11 & 0.15 & 0.03 & 0.03 & 0.05 & 0.07 \\
\hline 0.20 & 5 & 0.13 & 0.13 & 0.14 & 0.18 & 0.04 & 0.04 & 0.06 & 0.08 \\
\hline 0.50 & 2 & 0.19 & 0.21 & 0.22 & 0.25 & 0.06 & 0.07 & 0.08 & 0.11 \\
\hline
\end{tabular}




\section{1-11.1L0-4W OARGHA RIVER BELOW OARGHA RESERVOIR, Continued}

\begin{tabular}{|c|c|c|c|c|c|}
\hline \multicolumn{6}{|c|}{$\begin{array}{l}\text { Annual peak discharges } \\
\text { [m³/s, meters per second] }\end{array}$} \\
\hline \multicolumn{3}{|c|}{$\begin{array}{c}\text { Annual peak discharge, } \\
\text { by year }\end{array}$} & \multicolumn{3}{|c|}{$\begin{array}{l}\text { Annual peak discharge, } \\
\text { from highest to lowest }\end{array}$} \\
\hline $\begin{array}{l}\text { Water } \\
\text { year }\end{array}$ & Date & $\begin{array}{c}\text { Peak } \\
\text { discharge } \\
\left(\mathrm{m}^{3} / \mathrm{s}\right)\end{array}$ & $\begin{array}{l}\text { Water } \\
\text { year }\end{array}$ & Date & $\begin{array}{c}\text { Peak } \\
\text { discharge } \\
\left(\mathrm{m}^{3} / \mathrm{s}\right)\end{array}$ \\
\hline 1965 & May 11,1965 & 1.27 & 1972 & May 14,1972 & 5.90 \\
\hline 1966 & August 22, 1966 & 0.44 & 1978 & July 6, 1978 & 3.06 \\
\hline 1967 & June 18, 1967 & 0.58 & 1965 & May 11,1965 & 1.27 \\
\hline 1968 & May 2, 1968 & 1.20 & 1968 & May 2, 1968 & 1.20 \\
\hline 1969 & June 1, 1969 & 0.93 & 1969 & June 1, 1969 & 0.93 \\
\hline 1970 & June 20, 1970 & 0.63 & 1973 & July 18,1973 & 0.70 \\
\hline 1971 & June 7, 1971 & 0.50 & 1976 & May 18, 1976 & 0.65 \\
\hline 1972 & May 14, 1972 & 5.90 & 1970 & June 20, 1970 & 0.63 \\
\hline 1973 & July 18,1973 & 0.70 & 1974 & October 1, 1973 & 0.60 \\
\hline 1974 & October 1, 1973 & 0.60 & 1967 & June 18, 1967 & 0.58 \\
\hline 1975 & August 21, 1975 & 0.55 & 1979 & July 11, 1979 & 0.56 \\
\hline 1976 & May 18, 1976 & 0.65 & 1975 & August 21, 1975 & 0.55 \\
\hline 1977 & October 18, 1976 & 0.45 & 1971 & June 7, 1971 & 0.50 \\
\hline 1978 & July 6, 1978 & 3.06 & 1980 & $\begin{array}{c}\text { July } 28,1980 \\
\text { October } 18,\end{array}$ & 0.48 \\
\hline 1979 & July 11, 1979 & 0.56 & 1977 & 1976 & 0.45 \\
\hline 1980 & July 28, 1980 & 0.48 & 1966 & August 22, 1966 & 0.44 \\
\hline
\end{tabular}




\section{1-11.1L0-4W OARGHA RIVER BELOW OARGHA RESERVOIR, Continued}

Monthly and annual mean discharges, in cubic meters per second

[Data may not be rounded in accordance with U.S. Geological Survey publication standards]

\begin{tabular}{|c|c|c|c|c|c|c|c|c|c|c|c|c|c|}
\hline \multirow{2}{*}{$\begin{array}{l}\text { Water } \\
\text { year }\end{array}$} & \multicolumn{12}{|c|}{ Monthly mean discharge } & \multirow{2}{*}{$\begin{array}{c}\text { Annual } \\
\text { discharge }\end{array}$} \\
\hline & October & November & December & January & February & March & April & May & June & July & August & September & \\
\hline 1965 & 0.15 & 0.33 & 0.33 & 0.28 & 0.31 & 0.31 & 0.42 & 0.75 & 0.97 & 0.53 & 0.62 & 0.68 & 0.47 \\
\hline 1966 & 0.37 & 0.14 & 0.12 & 0.09 & 0.12 & 0.12 & 0.13 & 0.17 & 0.28 & 0.13 & 0.33 & 0.27 & 0.19 \\
\hline 1967 & 0.25 & 0.27 & 0.10 & 0.07 & 0.09 & 0.09 & 0.07 & 0.28 & 0.39 & 0.43 & 0.45 & 0.34 & 0.24 \\
\hline 1968 & 0.27 & 0.09 & 0.09 & 0.10 & 0.12 & 0.10 & 0.23 & 0.65 & 0.61 & 0.44 & 0.31 & 0.24 & 0.27 \\
\hline 1969 & 0.13 & 0.11 & 0.13 & 0.14 & 0.18 & 0.19 & 0.22 & 0.27 & 0.35 & 0.23 & 0.14 & 0.11 & 0.18 \\
\hline 1970 & 0.08 & 0.06 & 0.05 & 0.07 & 0.12 & 0.11 & 0.11 & 0.21 & 0.35 & 0.33 & 0.30 & 0.26 & 0.17 \\
\hline 1971 & 0.21 & 0.08 & 0.06 & 0.04 & 0.06 & 0.07 & 0.11 & 0.22 & 0.20 & 0.26 & 0.14 & 0.10 & 0.13 \\
\hline 1972 & 0.07 & 0.08 & 0.07 & 0.08 & 0.03 & 0.04 & 0.10 & 0.88 & 0.83 & 0.38 & 0.45 & 0.23 & 0.27 \\
\hline 1973 & 0.16 & 0.21 & 0.04 & 0.04 & 0.06 & 0.12 & 0.11 & 0.22 & 0.44 & 0.54 & 0.42 & 0.53 & 0.24 \\
\hline 1974 & 0.60 & 0.37 & 0.08 & 0.03 & 0.06 & 0.05 & 0.11 & 0.21 & 0.20 & 0.21 & 0.24 & 0.28 & 0.20 \\
\hline 1975 & 0.19 & 0.07 & 0.06 & 0.06 & 0.04 & 0.04 & 0.06 & 0.14 & 0.20 & 0.27 & 0.37 & 0.32 & 0.15 \\
\hline 1976 & 0.19 & 0.09 & 0.03 & 0.02 & 0.02 & 0.03 & 0.05 & 0.35 & 0.23 & 0.36 & 0.34 & 0.33 & 0.17 \\
\hline 1977 & 0.31 & 0.10 & 0.04 & 0.06 & 0.04 & 0.08 & 0.11 & 0.12 & 0.22 & 0.29 & 0.30 & 0.24 & 0.16 \\
\hline 1978 & 0.22 & 0.13 & 0.07 & 0.07 & 0.09 & 0.10 & 0.10 & 0.24 & 0.24 & 0.27 & 0.34 & 0.34 & 0.18 \\
\hline 1979 & 0.28 & 0.14 & 0.07 & 0.09 & 0.08 & 0.06 & 0.11 & 0.14 & 0.26 & 0.31 & 0.39 & 0.46 & 0.20 \\
\hline 1980 & 0.36 & 0.15 & 0.12 & 0.05 & 0.04 & 0.04 & 0.14 & 0.33 & 0.35 & 0.36 & 0.36 & 0.34 & 0.22 \\
\hline
\end{tabular}




\section{1-11.1L0-5W OARGHA RIVER ABOVE OARGHA RESERVOIR}

\section{(U.S. Geological Survey identification number: 343400069010000 )}

LOCATION: Lat $34^{\circ} 34^{\prime} \mathrm{N} .$, long $69^{\circ} 01^{\prime} \mathrm{E}$.

DRAINAGE AREA: $70 \mathrm{~km}^{2}$.

ELEVATION: 2,035 meters above mean sea level.

PERIOD OF RECORD: April 16, 1963 to September 30, 1966 and March 18, 1970 to September 30, 1980.

GAGE: Water-stage recorder.

Annual mean discharge

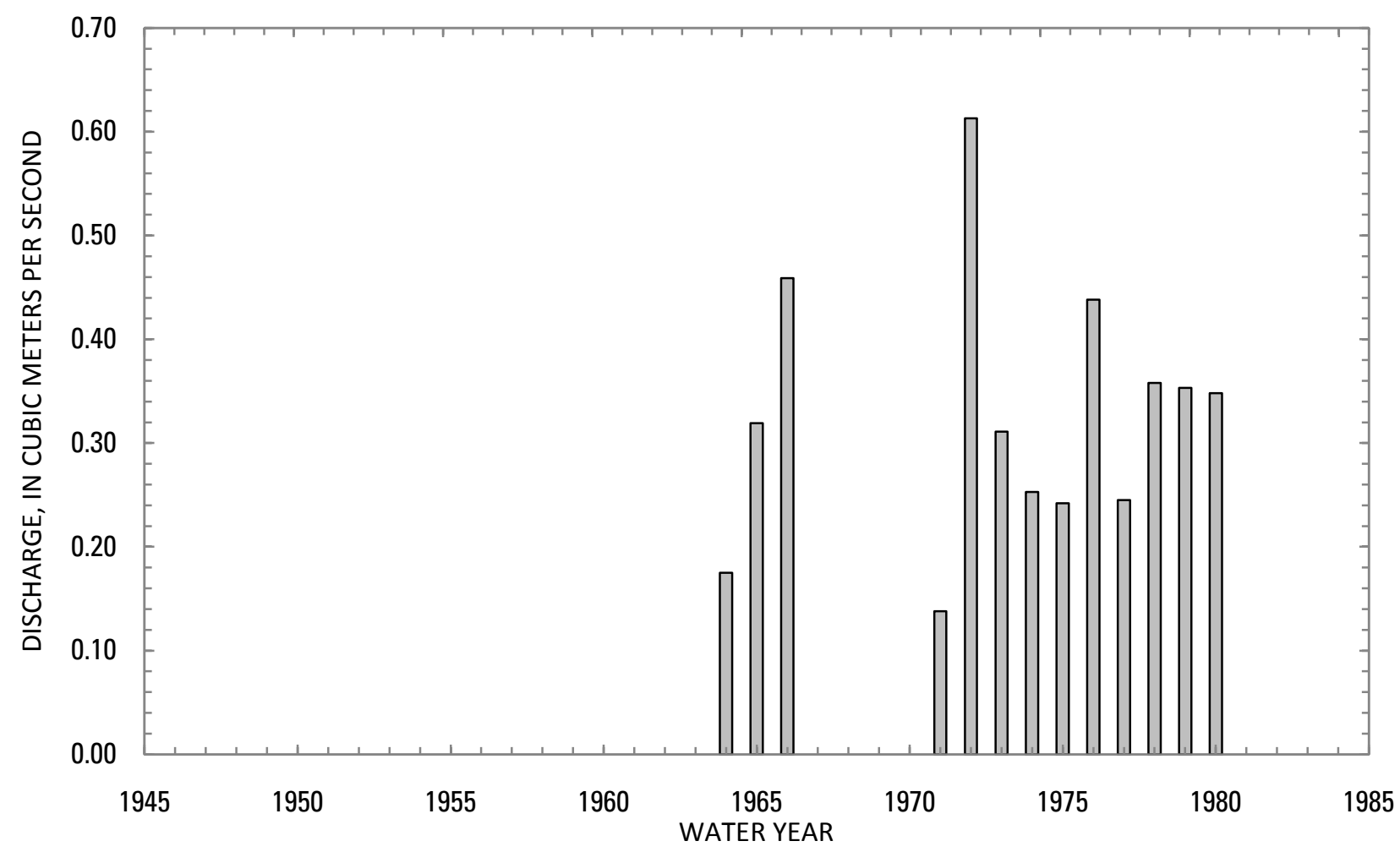




\section{1-11.1L0-5W QARGHA RIVER ABOVE OARGHA RESERVOIR, Continued}

Statistics of monthly and annual mean discharges

[ $\mathrm{m}^{3} / \mathrm{s}$, cubic meters per second; ng, not given]

\begin{tabular}{|c|c|c|c|c|c|c|c|c|}
\hline \multirow[b]{2}{*}{ Month } & \multicolumn{2}{|c|}{ Maximum } & \multicolumn{2}{|c|}{ Minimum } & \multicolumn{4}{|c|}{ Mean } \\
\hline & $\begin{array}{c}\text { Discharge } \\
\left(\mathrm{m}^{3} / \mathrm{s}\right)\end{array}$ & $\begin{array}{c}\text { Water year } \\
\text { of } \\
\text { occurrence }\end{array}$ & $\begin{array}{c}\text { Discharge } \\
\left(\mathrm{m}^{3} / \mathrm{s}\right)\end{array}$ & $\begin{array}{c}\text { Water year } \\
\text { of } \\
\text { occurrence }\end{array}$ & $\begin{array}{c}\text { Discharge } \\
\left(\mathrm{m}^{3} / \mathrm{s}\right)\end{array}$ & $\begin{array}{c}\text { Standard } \\
\text { deviation } \\
\left(\mathrm{m}^{3} / \mathrm{s}\right)\end{array}$ & $\begin{array}{c}\text { Coefficient } \\
\text { of } \\
\text { variation }\end{array}$ & $\begin{array}{c}\text { Percentage } \\
\text { of annual } \\
\text { discharge }\end{array}$ \\
\hline October & 0.06 & 1973 & 0 & ng & 0 & 0.02 & 3.61 & 0.12 \\
\hline November & 0.11 & 1973 & 0 & ng & 0.01 & 0.03 & 3.61 & 0.21 \\
\hline December & 0.12 & 1973 & 0 & ng & 0.01 & 0.04 & 2.62 & 0.35 \\
\hline January & 0.32 & 1980 & 0 & ng & 0.04 & 0.09 & 2.22 & 1.03 \\
\hline February & 0.29 & 1977 & 0 & ng & 0.09 & 0.11 & 1.22 & 2.28 \\
\hline March & 1.23 & 1976 & 0.05 & 1964 & 0.62 & 0.36 & 0.58 & 16.0 \\
\hline April & 3.35 & 1980 & 0.86 & 1964 & 1.93 & 0.82 & 0.42 & 49.7 \\
\hline May & 1.82 & 1979 & 0 & 1977 & 0.87 & 0.56 & 0.65 & 22.4 \\
\hline June & 1.18 & 1972 & 0 & ng & 0.20 & 0.34 & 1.67 & 5.21 \\
\hline July & 0.48 & 1965 & 0 & ng & 0.10 & 0.18 & 1.83 & 2.50 \\
\hline August & 0.16 & 1978 & 0 & ng & 0.01 & 0.04 & 3.87 & 0.27 \\
\hline September & 0.00 & 1963 & 0 & ng & 0 & 0 & ng & 0 \\
\hline Annual & 0.61 & 1972 & 0.14 & 1971 & 0.33 & 0.13 & 0.39 & 100 \\
\hline
\end{tabular}




\section{1-11.1L0-5W OARGHA RIVER ABOVE OARGHA RESERVOIR, Continued}

Monthly and annual flow duration, in cubic meters per second

[ng, not given]

\begin{tabular}{|c|c|c|c|c|c|c|c|c|c|c|c|c|c|}
\hline \multirow{2}{*}{$\begin{array}{l}\text { Percentage } \\
\text { of days } \\
\text { discharge } \\
\text { equaled or } \\
\text { exceeded }\end{array}$} & \multicolumn{12}{|c|}{ Month } & \multirow{2}{*}{ Annua } \\
\hline & October & November & December & January & February & March & April & May & June & July & August & September & \\
\hline 95 & 0 & 0 & 0 & 0 & 0 & 0.01 & 0.55 & 0 & 0 & 0 & 0 & 0 & 0 \\
\hline 90 & 0 & 0 & 0 & 0 & 0 & 0.02 & 0.73 & 0 & 0 & 0 & 0.01 & 0 & 0 \\
\hline 85 & 0 & 0.01 & 0 & 0 & 0 & 0.03 & 0.81 & 0.01 & 0 & 0 & 0.02 & 0 & 0 \\
\hline 80 & 0 & 0.02 & 0 & 0 & 0 & 0.04 & 0.91 & 0.02 & 0 & 0 & 0.02 & 0 & 0 \\
\hline 75 & 0 & 0.02 & 0 & 0 & 0 & 0.06 & 1.04 & 0.08 & 0 & 0 & 0.03 & 0 & 0 \\
\hline 70 & 0 & 0.03 & 0 & 0 & 0 & 0.09 & 1.20 & 0.23 & 0 & 0 & 0.03 & 0 & 0 \\
\hline 65 & 0 & 0.03 & 0 & 0 & 0 & 0.13 & 1.38 & 0.40 & 0 & 0 & 0.04 & 0 & 0 \\
\hline 60 & 0 & 0.04 & 0 & 0 & 0.01 & 0.21 & 1.49 & 0.57 & 0 & 0 & 0.04 & 0 & 0 \\
\hline 55 & 0 & 0.04 & 0 & 0 & 0.02 & 0.33 & 1.60 & 0.64 & 0 & 0 & 0.05 & 0 & 0 \\
\hline 50 & 0 & 0.05 & 0 & 0 & 0.02 & 0.47 & 1.73 & 0.77 & 0 & 0 & 0.05 & 0 & 0 \\
\hline 45 & 0 & 0.05 & 0 & 0 & 0.04 & 0.56 & 1.87 & 0.85 & 0 & 0 & 0.06 & 0 & 0 \\
\hline 40 & 0 & 0.06 & 0 & 0 & 0.05 & 0.67 & 2.02 & 0.93 & 0.02 & 0 & 0.06 & 0 & 0 \\
\hline 35 & 0 & 0.06 & 0 & 0 & 0.07 & 0.77 & 2.17 & 1.02 & 0.07 & 0 & 0.07 & 0 & 0.02 \\
\hline 30 & 0 & 0.07 & 0 & 0.01 & 0.10 & 0.87 & 2.36 & 1.11 & 0.17 & 0 & 0.07 & 0 & 0.08 \\
\hline 25 & 0 & 0.07 & 0 & 0.02 & 0.12 & 0.99 & 2.58 & 1.29 & 0.30 & 0 & 0.08 & 0 & 0.17 \\
\hline 20 & 0 & 0.08 & 0 & 0.06 & 0.15 & 1.14 & 2.87 & 1.53 & 0.42 & 0 & 0.08 & 0 & 0.48 \\
\hline 15 & 0 & 0.08 & 0 & 0.08 & 0.21 & 1.28 & 3.21 & 1.75 & 0.54 & 0.10 & 0.09 & 0 & 0.84 \\
\hline 10 & 0 & 0.09 & 0.05 & 0.10 & 0.28 & 1.45 & 3.69 & 1.93 & 0.77 & 0.42 & 0.09 & 0 & 1.20 \\
\hline 5 & 0.02 & 0.10 & 0.06 & 0.11 & 0.43 & 1.78 & 4.19 & 2.49 & 1.00 & 0.56 & 0.10 & 0 & 1.90 \\
\hline
\end{tabular}




\section{1-11.1L0-5W QARGHA RIVER ABOVE OARGHA RESERVOIR, Continued}

Probability of occurrence of annual high discharges

[ $\mathrm{m}^{3} / \mathrm{s}$, cubic meters per second; ng, not given]

\begin{tabular}{|c|c|c|c|c|c|c|}
\hline \multirow{2}{*}{$\begin{array}{c}\text { Exceedance } \\
\text { probability }\end{array}$} & \multirow{2}{*}{$\begin{array}{c}\text { Recurrence } \\
\text { interval } \\
\text { (years) }\end{array}$} & \multirow{2}{*}{$\begin{array}{l}\text { Maximum } \\
\text { instantaneous } \\
\text { discharge } \\
\left(\mathrm{m}^{3} / \mathrm{s}\right)\end{array}$} & \multicolumn{4}{|c|}{ Maximum daily mean discharge $\left(\mathrm{m}^{3} / \mathrm{s}\right)$} \\
\hline & & & $\begin{array}{l}\text { 3-day } \\
\text { period }\end{array}$ & $\begin{array}{l}\text { 7-day } \\
\text { period }\end{array}$ & $\begin{array}{l}\text { 15-day } \\
\text { period }\end{array}$ & $\begin{array}{l}\text { 30-day } \\
\text { period }\end{array}$ \\
\hline 0.99 & 1.01 & 1.70 & 1.51 & 1.31 & 1.03 & 0.90 \\
\hline 0.95 & 1.05 & 2.30 & 1.94 & 1.65 & 1.33 & 1.13 \\
\hline 0.90 & 1.11 & 2.70 & 2.20 & 1.87 & 1.52 & 1.27 \\
\hline 0.80 & 1.25 & 3.20 & 2.54 & 2.17 & 1.79 & 1.48 \\
\hline 0.50 & 2 & 4.20 & 3.32 & 2.88 & 2.47 & 2.00 \\
\hline 0.20 & 5 & 5.30 & 4.26 & 3.83 & 3.43 & 2.73 \\
\hline 0.10 & 10 & 5.90 & 4.81 & 4.44 & 4.08 & 3.23 \\
\hline 0.04 & 25 & 6.60 & 5.45 & 5.20 & 4.93 & 3.89 \\
\hline 0.02 & 50 & 7.00 & 5.89 & 5.76 & 5.57 & 4.39 \\
\hline 0.01 & 100 & 7.30 & '6.30 & 6.32 & 6.23 & 4.90 \\
\hline 0.005 & 200 & 7.60 & '6.69 & ${ }^{1} 6.87$ & 6.90 & 5.44 \\
\hline 0.002 & 500 & 8.00 & $\mathrm{ng}$ & $\mathrm{ng}$ & $\mathrm{ng}$ & $\mathrm{ng}$ \\
\hline
\end{tabular}

'Data does not fit log-Pearson Type III curve, use with caution.

\section{1-11.1L0-5W QARGHA RIVER ABOVE QARGHA RESERVOIR, Continued}

Probability of occurrence of annual low discharges

[ $\mathrm{m}^{3} / \mathrm{s}$, meters per second; ng, not given]

\begin{tabular}{|c|c|c|c|c|c|c|c|c|c|c|}
\hline \multirow{3}{*}{$\begin{array}{c}\text { Nonexceedance } \\
\text { probability }\end{array}$} & \multirow{3}{*}{$\begin{array}{c}\text { Recurrence } \\
\text { interval } \\
\text { (years) }\end{array}$} & \multicolumn{9}{|c|}{ Minimum daily mean discharge $\left(\mathrm{m}^{3} / \mathrm{s}\right)$} \\
\hline & & \multicolumn{9}{|c|}{ Number of consecutive days } \\
\hline & & 1 & 3 & 7 & 14 & 30 & 60 & 90 & 120 & 183 \\
\hline 0.05 & 20 & ng & $\mathrm{ng}$ & ng & ng & ng & ng & $\mathrm{ng}$ & ng & ng \\
\hline 0.10 & 10 & ng & $\mathrm{ng}$ & ng & $\mathrm{ng}$ & ng & ng & ng & ng & ng \\
\hline 0.20 & 5 & ng & ng & $\mathrm{ng}$ & ng & ng & ng & ng & ng & ng \\
\hline 0.50 & 2 & ng & ng & $\mathrm{ng}$ & ng & ng & ng & ng & ng & ng \\
\hline
\end{tabular}




\section{1-11.1L0-5W QARGHA RIVER ABOVE QARGHA RESERVOIR, Continued}

Probability of occurrence of seasonal low discharges [ $\mathrm{m}^{3} / \mathrm{s}$, meters per second; $\mathrm{ng}$, not given]

\begin{tabular}{|c|c|c|c|c|c|c|c|c|c|}
\hline \multirow{3}{*}{$\begin{array}{c}\text { Nonexceedance } \\
\text { probability }\end{array}$} & \multirow{3}{*}{$\begin{array}{c}\text { Recurrence } \\
\text { interval } \\
\text { (years) }\end{array}$} & \multicolumn{8}{|c|}{ Minimum daily mean discharge $\left(\mathrm{m}^{3} / \mathrm{s}\right)$} \\
\hline & & \multicolumn{8}{|c|}{ Number of consecutive days } \\
\hline & & 1 & 7 & 14 & 30 & 1 & 7 & 14 & 30 \\
\hline & & \multicolumn{4}{|c|}{$\begin{array}{c}\text { December-January- } \\
\text { February } \\
\end{array}$} & \multicolumn{4}{|c|}{ March-April-May } \\
\hline 0.05 & 20 & ng & ng & ng & ng & 0 & 0 & 0 & 0 \\
\hline 0.10 & 10 & ng & ng & ng & ng & 0 & 0 & 0 & 0.01 \\
\hline 0.20 & 5 & ng & ng & ng & ng & 0 & 0 & 0 & 0.04 \\
\hline \multirow[t]{2}{*}{0.50} & 2 & $\mathrm{ng}$ & ng & ng & ng & 0 & 0.01 & 0.03 & 0.35 \\
\hline & & \multicolumn{4}{|c|}{ June-July-August } & \multicolumn{4}{|c|}{ September-October-November } \\
\hline 0.05 & 20 & ng & ng & ng & $\mathrm{ng}$ & ng & ng & $\mathrm{ng}$ & ng \\
\hline 0.10 & 10 & $\mathrm{ng}$ & ng & $\mathrm{ng}$ & ng & ng & ng & ng & $\mathrm{ng}$ \\
\hline 0.20 & 5 & $\mathrm{ng}$ & ng & $\mathrm{ng}$ & ng & $\mathrm{ng}$ & ng & ng & $\mathrm{ng}$ \\
\hline 0.50 & 2 & $\mathrm{ng}$ & ng & $\mathrm{ng}$ & ng & ng & $\mathrm{ng}$ & $\mathrm{ng}$ & $\mathrm{ng}$ \\
\hline
\end{tabular}




\section{1-11.1L0-5W QARGHA RIVER ABOVE OARGHA RESERVOIR, Continued}

\begin{tabular}{|c|c|c|c|c|c|}
\hline \multicolumn{6}{|c|}{$\begin{array}{l}\text { Annual peak discharges } \\
\text { [m³/s, meters per second] }\end{array}$} \\
\hline \multicolumn{3}{|c|}{$\begin{array}{c}\text { Annual peak discharge, } \\
\text { by year }\end{array}$} & \multicolumn{3}{|c|}{$\begin{array}{l}\text { Annual peak discharge, } \\
\text { from highest to lowest }\end{array}$} \\
\hline $\begin{array}{l}\text { Water } \\
\text { year }\end{array}$ & Date & $\begin{array}{c}\text { Peak } \\
\text { discharge } \\
\left(\mathrm{m}^{3} / \mathrm{s}\right)\end{array}$ & $\begin{array}{l}\text { Water } \\
\text { year }\end{array}$ & Date & $\begin{array}{c}\text { Peak } \\
\text { discharge } \\
\left(\mathrm{m}^{3} / \mathrm{s}\right)\end{array}$ \\
\hline 1963 & May 12,1963 & 3.34 & 1966 & April 24, 1966 & 6.15 \\
\hline 1964 & May 8, 1964 & 4.31 & 1980 & April 5, 1980 & 6.11 \\
\hline 1965 & April 21, 1965 & 3.30 & 1974 & March 21, 1974 & 5.60 \\
\hline 1966 & April 24, 1966 & 6.15 & 1972 & April 7, 1972 & 5.25 \\
\hline 1970 & May 1,1970 & 4.80 & 1978 & July 6, 1978 & 5.00 \\
\hline 1971 & April 16, 1971 & 2.50 & 1970 & May 1,1970 & 4.80 \\
\hline 1972 & April 7, 1972 & 5.25 & 1964 & May 8, 1964 & 4.31 \\
\hline 1973 & April 2, 1973 & 2.08 & 1979 & May 19, 1979 & 4.10 \\
\hline 1974 & March 21, 1974 & 5.60 & 1976 & April 22, 1976 & 4.00 \\
\hline 1975 & April 4, 1975 & 3.50 & 1977 & April 15, 1977 & 3.55 \\
\hline 1976 & April 22, 1976 & 4.00 & 1975 & April 4, 1975 & 3.50 \\
\hline 1977 & April 15, 1977 & 3.55 & 1963 & May 12,1963 & 3.34 \\
\hline 1978 & July 6, 1978 & 5.00 & 1965 & April 21, 1965 & 3.30 \\
\hline 1979 & May 19, 1979 & 4.10 & 1971 & April 16, 1971 & 2.50 \\
\hline 1980 & April 5, 1980 & 6.11 & 1973 & April 2, 1973 & 2.08 \\
\hline
\end{tabular}




\section{1-11.1L0-5W QARGHA RIVER ABOVE QARGHA RESERVOIR, Continued}

Monthly and annual mean discharges, in cubic meters per second

[Data may not be rounded in accordance with U.S. Geological Survey publication standards; --, no data]

\begin{tabular}{|c|c|c|c|c|c|c|c|c|c|c|c|c|c|}
\hline \multirow{2}{*}{$\begin{array}{l}\text { Water } \\
\text { year }\end{array}$} & \multicolumn{12}{|c|}{ Monthly mean discharge } & \multirow{2}{*}{$\begin{array}{c}\text { Annual } \\
\text { discharg }\end{array}$} \\
\hline & October & November & December & January & February & March & April & May & June & July & August & September & \\
\hline 1963 & -- & -- & -- & -- & -- & -- & -- & 1.43 & 0.21 & 0 & 0 & 0 & -- \\
\hline 1964 & 0 & 0 & 0 & 0 & 0 & 0.05 & 0.86 & 1.07 & 0.12 & 0 & 0 & 0 & 0.18 \\
\hline 1965 & 0 & 0 & 0 & 0 & 0.01 & 0.28 & 1.39 & 0.96 & 0.71 & 0.48 & 0 & 0 & 0.32 \\
\hline 1966 & 0 & 0 & 0 & 0.01 & 0.17 & 1.03 & 2.47 & 1.31 & 0.36 & 0.18 & 0 & 0 & 0.46 \\
\hline 1970 & -- & -- & -- & -- & -- & -- & 1.31 & 0.63 & 0 & 0 & 0 & 0 & -- \\
\hline 1971 & 0 & 0 & 0 & 0 & 0.01 & 0.45 & 1.19 & 0.01 & 0 & 0 & 0 & 0 & 0.14 \\
\hline 1972 & 0 & 0 & 0 & 0 & 0 & 0.96 & 3.15 & 1.74 & 1.18 & 0.35 & 0 & 0 & 0.61 \\
\hline 1973 & 0.06 & 0.11 & 0.12 & 0 & 0.05 & 1.05 & 0.92 & 1.03 & 0.37 & 0 & 0 & 0 & 0.31 \\
\hline 1974 & 0 & 0 & 0 & 0 & 0.01 & 0.79 & 1.53 & 0.64 & 0.06 & 0 & 0 & 0 & 0.25 \\
\hline 1975 & 0 & 0 & 0 & 0 & 0 & 0.29 & 1.69 & 0.89 & 0.03 & 0 & 0 & 0 & 0.24 \\
\hline 1976 & 0 & 0 & 0.05 & 0.10 & 0.27 & 1.23 & 2.95 & 0.69 & 0 & 0 & 0 & 0 & 0.44 \\
\hline 1977 & 0 & 0 & 0 & 0.03 & 0.29 & 0.53 & 2.12 & 0 & 0 & 0 & 0 & 0 & 0.25 \\
\hline 1978 & 0 & 0 & 0 & 0.05 & 0.07 & 0.64 & 2.30 & 0.62 & 0 & 0.45 & 0.16 & 0 & 0.36 \\
\hline 1979 & 0 & 0 & 0 & 0.01 & 0.21 & 0.45 & 1.75 & 1.82 & 0 & 0 & 0 & 0 & 0.35 \\
\hline 1980 & 0 & 0 & 0.00 & 0.32 & 0.06 & 0.32 & 3.35 & 0.17 & 0 & 0 & 0 & 0 & 0.35 \\
\hline
\end{tabular}




\section{1-11.L00-1A PAGHMAN RIVER AT PUL-I-SOKHTA}

\section{(U.S. Geological Survey identification number: 343000069080000 )}

LOCATION: Lat $34^{\circ} 30^{\prime} \mathrm{N}$., long $69^{\circ} 08^{\prime} \mathrm{E}$.

DRAINAGE AREA: $500 \mathrm{~km}^{2}$.

ELEVATION: 1,805 meters above mean sea level.

PERIOD OF RECORD: March 1, 1963 to September 30, 1980.

GAGE: Water-stage recorder.

Annual mean discharge

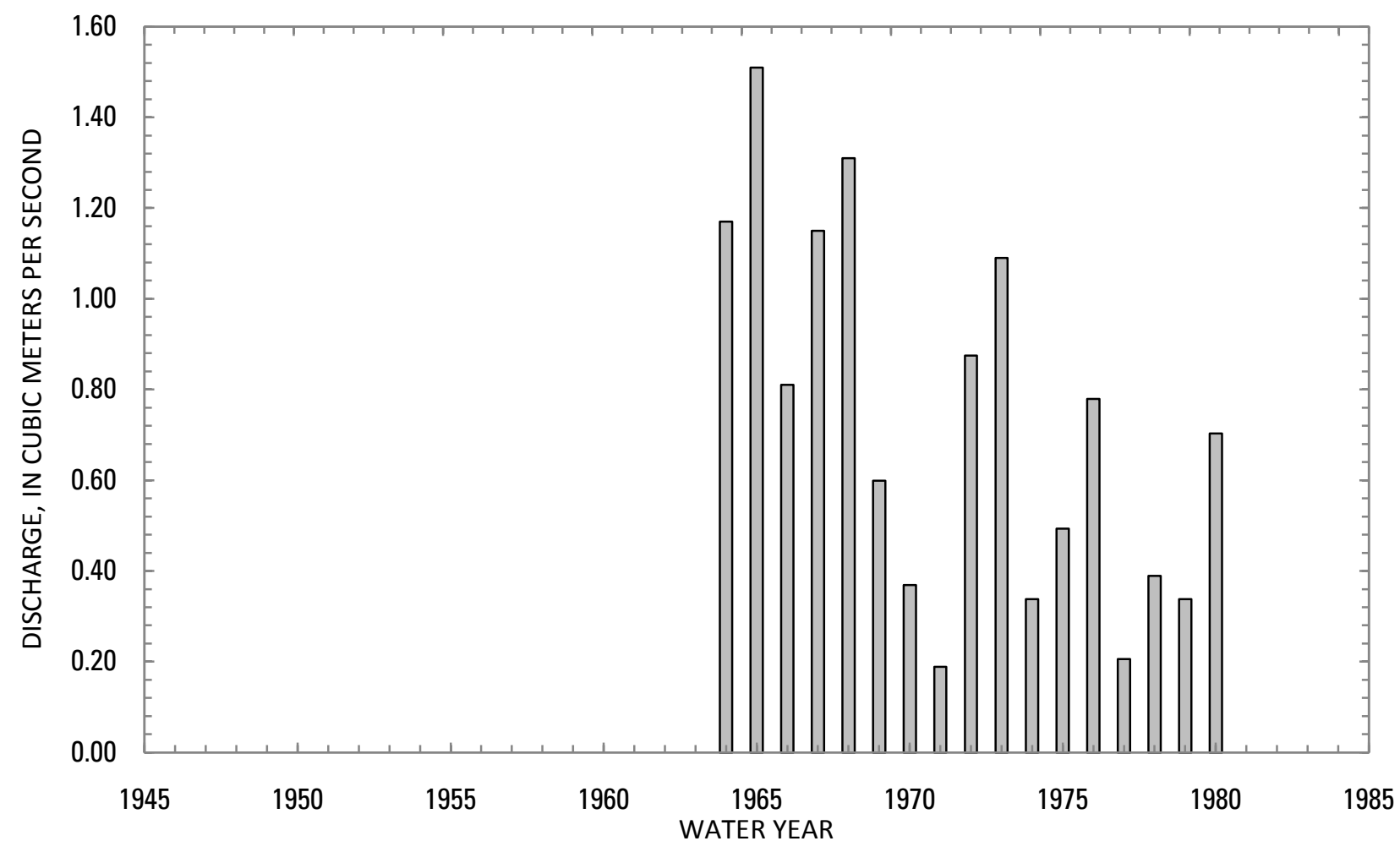




\section{1-11.L00-1A PAGHMAN RIVER AT PUL-I-SOKHTA, Continued}

Statistics of monthly and annual mean discharges [ $\mathrm{m}^{3} / \mathrm{s}$, cubic meters per second]

\begin{tabular}{|c|c|c|c|c|c|c|c|c|}
\hline \multirow[b]{2}{*}{ Month } & \multicolumn{2}{|c|}{ Maximum } & \multicolumn{2}{|c|}{ Minimum } & \multicolumn{4}{|c|}{ Mean } \\
\hline & $\begin{array}{c}\text { Discharge } \\
\left(\mathrm{m}^{3} / \mathrm{s}\right)\end{array}$ & $\begin{array}{c}\text { Water year } \\
\text { of } \\
\text { occurrence }\end{array}$ & $\begin{array}{c}\text { Discharge } \\
\left(\mathrm{m}^{3} / \mathrm{s}\right)\end{array}$ & $\begin{array}{c}\text { Water year } \\
\text { of } \\
\text { occurrence }\end{array}$ & $\begin{array}{c}\text { Discharge } \\
\left(\mathrm{m}^{3} / \mathrm{s}\right)\end{array}$ & $\begin{array}{c}\text { Standard } \\
\text { deviation } \\
\left(\mathrm{m}^{3} / \mathrm{s}\right)\end{array}$ & $\begin{array}{c}\text { Coefficient } \\
\text { of } \\
\text { variation }\end{array}$ & $\begin{array}{c}\text { Percentage } \\
\text { of annual } \\
\text { discharge }\end{array}$ \\
\hline October & 0.32 & 1969 & 0.00 & 1973 & 0.09 & 0.09 & 1.03 & 1.06 \\
\hline November & 0.72 & 1970 & 0.01 & 1972 & 0.22 & 0.24 & 1.06 & 2.59 \\
\hline December & 0.88 & 1968 & 0.04 & 1972 & 0.37 & 0.30 & 0.82 & 4.23 \\
\hline January & 1.16 & 1966 & 0.07 & 1972 & 0.44 & 0.30 & 0.69 & 5.14 \\
\hline February & 1.20 & 1966 & 0.07 & 1972 & 0.48 & 0.28 & 0.59 & 5.50 \\
\hline March & 2.77 & 1968 & 0.38 & 1977 & 1.22 & 0.71 & 0.58 & 14.1 \\
\hline April & 8.88 & 1964 & 0.21 & 1970 & 3.64 & 2.66 & 0.73 & 42.0 \\
\hline May & 6.55 & 1965 & 0.03 & 1978 & 1.68 & 2.03 & 1.21 & 19.4 \\
\hline June & 1.12 & 1965 & 0.01 & 1966 & 0.18 & 0.28 & 1.59 & 2.06 \\
\hline July & 0.79 & 1978 & 0.01 & 1966 & 0.17 & 0.22 & 1.32 & 1.94 \\
\hline August & 0.34 & 1975 & 0.01 & 1977 & 0.10 & 0.10 & 1.02 & 1.14 \\
\hline September & 0.32 & 1968 & 0 & 1966 & 0.08 & 0.09 & 1.11 & 0.89 \\
\hline Annual & 1.51 & 1965 & 0.19 & 1971 & 0.72 & 0.41 & 0.56 & 100 \\
\hline
\end{tabular}


1-11.L00-1A PAGHMAN RIVER AT PUL-I-SOKHTA, Continued

Monthly and annual flow duration, in cubic meters per second

[ng, not given]

\begin{tabular}{|c|c|c|c|c|c|c|c|c|c|c|c|c|c|}
\hline \multirow{2}{*}{$\begin{array}{l}\text { Percentage } \\
\text { of days } \\
\text { discharge } \\
\text { equaled or } \\
\text { exceeded }\end{array}$} & \multicolumn{12}{|c|}{ Month } & \multirow{2}{*}{ Annua } \\
\hline & October & November & December & January & February & March & April & May & June & July & August & September & \\
\hline 95 & 0 & 0.01 & 0.02 & 0.07 & 0.08 & 0.22 & 0.10 & 0.02 & 0.01 & 0.01 & 0.01 & 0 & 0.01 \\
\hline 90 & 0.01 & 0.01 & 0.03 & 0.13 & 0.20 & 0.36 & 0.22 & 0.03 & 0.01 & 0.01 & 0.01 & 0 & 0.02 \\
\hline 85 & 0.01 & 0.01 & 0.05 & 0.18 & 0.22 & 0.44 & 0.56 & 0.04 & 0.02 & 0.02 & 0.02 & 0.01 & 0.02 \\
\hline 80 & 0.01 & 0.02 & 0.08 & 0.20 & 0.22 & 0.50 & 0.89 & 0.05 & 0.02 & 0.02 & 0.02 & 0.01 & 0.03 \\
\hline 75 & 0.02 & 0.02 & 0.09 & 0.22 & 0.23 & 0.57 & 1.18 & 0.06 & 0.03 & 0.02 & 0.02 & 0.02 & 0.04 \\
\hline 70 & 0.02 & 0.02 & 0.13 & 0.23 & 0.27 & 0.63 & 1.41 & 0.09 & 0.03 & 0.03 & 0.02 & 0.02 & 0.06 \\
\hline 65 & 0.02 & 0.03 & 0.17 & 0.26 & 0.29 & 0.68 & 1.62 & 0.12 & 0.04 & 0.03 & 0.03 & 0.02 & 0.08 \\
\hline 60 & 0.02 & 0.04 & 0.19 & 0.29 & 0.36 & 0.74 & 1.85 & 0.14 & 0.05 & 0.04 & 0.03 & 0.03 & 0.11 \\
\hline 55 & 0.03 & 0.06 & 0.22 & 0.31 & 0.38 & 0.81 & 2.10 & 0.16 & 0.06 & 0.05 & 0.04 & 0.04 & 0.14 \\
\hline 50 & 0.04 & 0.14 & 0.25 & 0.33 & 0.41 & 0.96 & 2.50 & 0.27 & 0.07 & 0.05 & 0.05 & 0.05 & 0.18 \\
\hline 45 & 0.08 & 0.17 & 0.31 & 0.37 & 0.43 & 1.03 & 2.82 & 0.36 & 0.09 & 0.06 & 0.07 & 0.07 & 0.23 \\
\hline 40 & 0.11 & 0.20 & 0.49 & 0.50 & 0.48 & 1.11 & 3.13 & 0.49 & 0.11 & 0.08 & 0.08 & 0.08 & 0.28 \\
\hline 35 & 0.13 & 0.24 & 0.57 & 0.56 & 0.55 & 1.24 & 3.55 & 0.78 & 0.12 & 0.11 & 0.09 & 0.09 & 0.36 \\
\hline 30 & 0.15 & 0.30 & 0.60 & 0.62 & 0.60 & 1.37 & 4.09 & 1.22 & 0.13 & 0.13 & 0.11 & 0.10 & 0.47 \\
\hline 25 & 0.17 & 0.36 & 0.63 & 0.67 & 0.64 & 1.49 & 5.06 & 1.72 & 0.16 & 0.14 & 0.13 & 0.12 & 0.60 \\
\hline 20 & 0.18 & 0.42 & 0.66 & 0.72 & 0.72 & 1.79 & 6.24 & 2.48 & 0.19 & 0.16 & 0.15 & 0.14 & 0.74 \\
\hline 15 & 0.20 & 0.50 & 0.71 & 0.77 & 0.77 & 2.15 & 7.47 & 3.65 & 0.26 & 0.21 & 0.17 & 0.15 & 0.97 \\
\hline 10 & 0.25 & 0.66 & 0.77 & 0.91 & 0.83 & 2.59 & 8.84 & 4.76 & 0.46 & 0.44 & 0.21 & 0.19 & 1.56 \\
\hline 5 & 0.32 & 0.80 & 0.91 & $\mathrm{ng}$ & 1.15 & 3.53 & 11.0 & 8.34 & 1.05 & 0.55 & 0.27 & 0.31 & 3.40 \\
\hline
\end{tabular}




\section{1-11.L00-1A PAGHMAN RIVER AT PUL-I-SOKHTA, Continued}

Probability of occurrence of annual high discharges

[ $\mathrm{m}^{3} / \mathrm{s}$, cubic meters per second; ng, not given]

\begin{tabular}{|c|c|c|c|c|c|c|}
\hline \multirow{2}{*}{$\begin{array}{l}\text { Exceedance } \\
\text { probability }\end{array}$} & \multirow{2}{*}{$\begin{array}{c}\text { Recurrence } \\
\text { interval } \\
\text { (years) }\end{array}$} & \multirow{2}{*}{$\begin{array}{c}\text { Maximum } \\
\text { instantaneous } \\
\text { discharge } \\
\left(\mathrm{m}^{3} / \mathrm{s}\right)\end{array}$} & \multicolumn{4}{|c|}{ Maximum daily mean discharge $\left(\mathrm{m}^{3} / \mathrm{s}\right)$} \\
\hline & & & $\begin{array}{l}\text { 3-day } \\
\text { period }\end{array}$ & $\begin{array}{l}\text { 7-day } \\
\text { period }\end{array}$ & $\begin{array}{l}\text { 15-day } \\
\text { period }\end{array}$ & $\begin{array}{l}\text { 30-day } \\
\text { period }\end{array}$ \\
\hline 0.99 & 1.01 & 1.80 & 0.89 & 0.63 & 0.45 & 0.39 \\
\hline 0.95 & 1.05 & 3.40 & 1.70 & 1.25 & 0.97 & 0.80 \\
\hline 0.90 & 1.11 & 4.70 & 2.37 & 1.79 & 1.41 & 1.14 \\
\hline 0.80 & 1.25 & 7.20 & 3.50 & 2.71 & 2.20 & 1.73 \\
\hline 0.50 & 2 & 16.0 & 7.13 & 5.81 & 4.78 & 3.65 \\
\hline 0.20 & 5 & 35.6 & 13.9 & 11.9 & 9.57 & 7.18 \\
\hline 0.10 & 10 & 54.3 & 19.4 & 16.9 & 13.3 & 9.95 \\
\hline 0.04 & 25 & 85.0 & 27.2 & 24.4 & 18.6 & 13.8 \\
\hline 0.02 & 50 & 114 & 33.7 & 30.7 & 22.7 & 16.9 \\
\hline 0.01 & 100 & 148 & 40.6 & 37.6 & 27.0 & 20.1 \\
\hline 0.005 & 200 & 188 & 48.1 & 45.0 & 31.4 & 23.5 \\
\hline 0.002 & 500 & 251 & $\mathrm{ng}$ & $\mathrm{ng}$ & $\mathrm{ng}$ & $\mathrm{ng}$ \\
\hline
\end{tabular}

\section{1-11.L00-1A PAGHMAN RIVER AT PUL-I-SOKHTA, Continued}

Probability of occurrence of annual low discharges

$\left[\mathrm{m}^{3} / \mathrm{s}\right.$, meters per second]

\begin{tabular}{|c|c|c|c|c|c|c|c|c|c|c|}
\hline \multirow{3}{*}{$\begin{array}{c}\text { Nonexceedance } \\
\text { probability }\end{array}$} & \multirow{3}{*}{$\begin{array}{c}\text { Recurrence } \\
\text { interval } \\
\text { (years) }\end{array}$} & \multicolumn{9}{|c|}{ Minimum daily mean discharge $\left(\mathrm{m}^{3} / \mathrm{s}\right)$} \\
\hline & & \multicolumn{9}{|c|}{ Number of consecutive days } \\
\hline & & 1 & 3 & 7 & 14 & 30 & 60 & 90 & 120 & 183 \\
\hline 0.05 & 20 & 0 & 0 & 0 & 0 & 0 & 0 & 0 & 0.01 & 0.01 \\
\hline 0.10 & 10 & 0 & 0 & 0 & 0 & 0 & 0 & 0 & 0.01 & 0.02 \\
\hline 0.20 & 5 & 0 & 0 & 0.01 & 0.01 & 0.01 & 0.01 & 0.01 & 0.01 & 0.03 \\
\hline 0.50 & 2 & 0.01 & 0.01 & 0.02 & 0.02 & 0.02 & 0.03 & 0.04 & 0.04 & 0.08 \\
\hline
\end{tabular}




\section{1-11.L00-1A PAGHMAN RIVER AT PUL-I-SOKHTA, Continued}

Probability of occurrence of seasonal low discharges

[m³/s, meters per second]

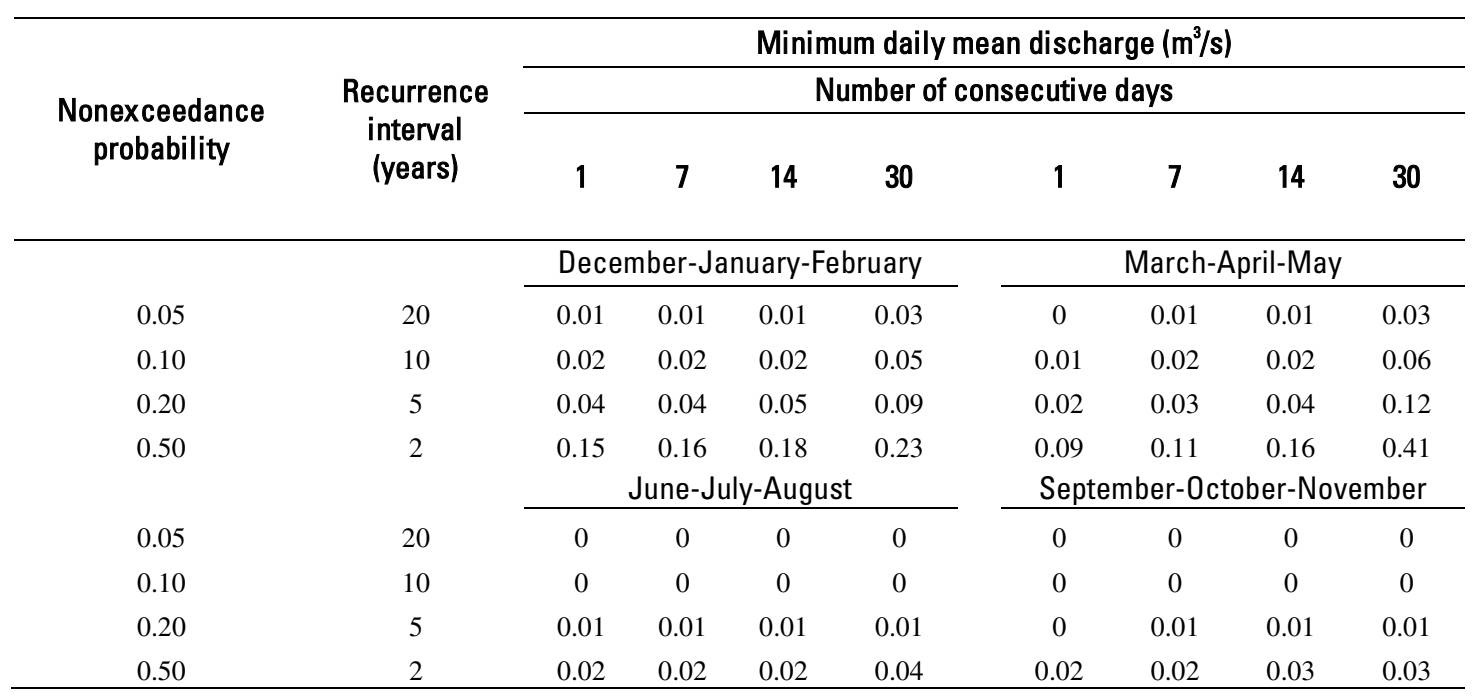




\section{1-11.L00-1A PAGHMAN RIVER AT PUL-I-SOKHTA, Continued}

Annual peak discharges

$\left[\mathrm{m}^{3} / \mathrm{s}\right.$, meters per second]

\begin{tabular}{|c|c|c|c|c|c|}
\hline \multicolumn{3}{|c|}{$\begin{array}{c}\text { Annual peak discharge, } \\
\text { by year }\end{array}$} & \multicolumn{3}{|c|}{$\begin{array}{l}\text { Annual peak discharge, } \\
\text { from highest to lowest }\end{array}$} \\
\hline $\begin{array}{l}\text { Water } \\
\text { year }\end{array}$ & Date & $\begin{array}{c}\text { Peak } \\
\text { discharge } \\
\left(\mathrm{m}^{3} / \mathrm{s}\right)\end{array}$ & $\begin{array}{l}\text { Water } \\
\text { year }\end{array}$ & Date & $\begin{array}{c}\text { Peak } \\
\text { discharge } \\
\left(\mathrm{m}^{3} / \mathrm{s}\right)\end{array}$ \\
\hline 1963 & May 12,1963 & 17.8 & 1975 & August 13,1975 & 80.0 \\
\hline 1964 & April 10, 1964 & 15.5 & 1978 & August 17, 1978 & 70.5 \\
\hline 1965 & May 7, 1965 & 16.0 & 1968 & April 30, 1968 & 48.8 \\
\hline 1966 & April 27, 1966 & 5.56 & 1979 & March 29, 1979 & 39.2 \\
\hline 1967 & April 27, 1967 & 34.8 & 1967 & April 27, 1967 & 34.8 \\
\hline 1968 & April 30, 1968 & 48.8 & 1980 & April 11, 1980 & 23.7 \\
\hline 1969 & April 19, 1969 & 4.65 & 1972 & May 6, 1972 & 19.5 \\
\hline 1970 & July 7, 1970 & 4.00 & 1963 & May 12,1963 & 17.8 \\
\hline 1971 & April 12, 1971 & 3.42 & 1973 & August 2, 1973 & 17.1 \\
\hline 1972 & May 6, 1972 & 19.5 & 1965 & May 7, 1965 & 16.0 \\
\hline 1973 & August 2, 1973 & 17.1 & 1964 & April 10, 1964 & 15.5 \\
\hline 1974 & April 7, 1974 & 10.5 & 1976 & April 27, 1976 & 13.1 \\
\hline 1975 & August 13,1975 & 80.0 & 1974 & April 7, 1974 & 10.5 \\
\hline 1976 & April 27, 1976 & 13.1 & 1977 & April 10, 1977 & 7.40 \\
\hline 1977 & April 10, 1977 & 7.40 & 1966 & April 27, 1966 & 5.56 \\
\hline 1978 & August 17, 1978 & 70.5 & 1969 & April 19, 1969 & 4.65 \\
\hline 1979 & March 29, 1979 & 39.2 & 1970 & July 7, 1970 & 4.00 \\
\hline 1980 & April 11, 1980 & 23.7 & 1971 & April 12, 1971 & 3.42 \\
\hline
\end{tabular}


1-11.L00-1A PAGHMAN RIVER AT PUL-I-SOKHTA, Continued

Monthly and annual mean discharges, in cubic meters per second

[Data may not be rounded in accordance with U.S. Geological Survey publication standards; --, no data]

\begin{tabular}{|c|c|c|c|c|c|c|c|c|c|c|c|c|c|}
\hline \multirow{2}{*}{$\begin{array}{l}\text { Water } \\
\text { year }\end{array}$} & \multicolumn{12}{|c|}{ Monthly mean discharge } & \multirow{2}{*}{$\begin{array}{c}\text { Annual } \\
\text { discharge }\end{array}$} \\
\hline & October & November & December & January & February & March & April & May & June & July & August & September & \\
\hline 1963 & -- & -- & -- & -- & -- & 0.95 & 1.40 & 3.14 & 0.19 & 0.14 & 0.15 & 0.15 & -- \\
\hline 1964 & 0.15 & 0.17 & 0.19 & 0.24 & 0.25 & 2.42 & 8.88 & 1.34 & 0.13 & 0.05 & 0.10 & 0.19 & 1.17 \\
\hline 1965 & 0.18 & 0.31 & 0.58 & 0.53 & 0.58 & 0.55 & 6.73 & 6.55 & 1.12 & 0.57 & 0.20 & 0.14 & 1.51 \\
\hline 1966 & 0.21 & 0.66 & 0.81 & 1.16 & 1.20 & 1.41 & 3.41 & 0.90 & 0.01 & 0.01 & 0.02 & 0.00 & 0.81 \\
\hline 1967 & 0.07 & 0.47 & 0.67 & 0.61 & 0.61 & 0.72 & 4.68 & 4.66 & 0.35 & 0.47 & 0.32 & 0.16 & 1.15 \\
\hline 1968 & 0.21 & 0.47 & 0.88 & 0.81 & 0.63 & 2.77 & 3.91 & 4.69 & 0.67 & 0.18 & 0.16 & 0.32 & 1.31 \\
\hline 1969 & 0.32 & 0.43 & 0.61 & 0.87 & 0.86 & 1.27 & 2.22 & 0.42 & 0.04 & 0.05 & 0.07 & 0.09 & 0.60 \\
\hline 1970 & 0.12 & 0.72 & 0.64 & 0.69 & 0.75 & 0.85 & 0.21 & 0.07 & 0.06 & 0.29 & 0.03 & 0.04 & 0.37 \\
\hline 1971 & 0.07 & 0.04 & 0.14 & 0.26 & 0.41 & 0.58 & 0.67 & 0.06 & 0.02 & 0.02 & 0.02 & 0.02 & 0.19 \\
\hline 1972 & 0.02 & 0.01 & 0.04 & 0.07 & 0.07 & 1.16 & 5.01 & 3.94 & 0.13 & 0.05 & 0.02 & 0.00 & 0.88 \\
\hline 1973 & 0.00 & 0.06 & 0.34 & 0.38 & 0.58 & 2.49 & 8.28 & 0.67 & 0.02 & 0.13 & 0.14 & 0.09 & 1.09 \\
\hline 1974 & 0.12 & 0.19 & 0.68 & 0.62 & 0.34 & 0.64 & 0.93 & 0.12 & 0.12 & 0.11 & 0.10 & 0.08 & 0.34 \\
\hline 1975 & 0.05 & 0.05 & 0.23 & 0.28 & 0.23 & 0.98 & 3.34 & 0.27 & 0.11 & 0.04 & 0.34 & 0.05 & 0.49 \\
\hline 1976 & 0.02 & 0.04 & 0.09 & 0.17 & 0.20 & 1.16 & 5.46 & 2.10 & 0.09 & 0.03 & 0.03 & 0.01 & 0.78 \\
\hline 1977 & 0.01 & 0.04 & 0.19 & 0.28 & 0.32 & 0.38 & 1.19 & 0.03 & 0.02 & 0.01 & 0.01 & 0.01 & 0.21 \\
\hline 1978 & 0.01 & 0.15 & 0.08 & 0.22 & 0.31 & 1.90 & 1.07 & 0.03 & 0.02 & 0.79 & 0.07 & 0.01 & 0.39 \\
\hline 1979 & 0.01 & 0.01 & 0.04 & 0.20 & 0.32 & 0.87 & 2.08 & 0.35 & 0.09 & 0.08 & 0.01 & 0.01 & 0.34 \\
\hline 1980 & 0.01 & 0.01 & 0.04 & 0.18 & 0.43 & 0.87 & 6.00 & 0.89 & 0.04 & 0.02 & 0.02 & 0.03 & 0.70 \\
\hline
\end{tabular}




\section{2-4.000-5A URGUN RIVER AT PIRKOTI}

\section{(U.S. Geological Survey identification number: 325500069150000)}

LOCATION: Lat $32^{\circ} 55^{\prime} \mathrm{N}$., long $69^{\circ} 15^{\prime} \mathrm{E}$.

DRAINAGE AREA: $780 \mathrm{~km}^{2}$.

ELEVATION: 2,108 meters above mean sea level.

PERIOD OF RECORD: August 13, 1970 to September 30, 1978.

GAGE: Water-stage recorder.

Annual mean discharge

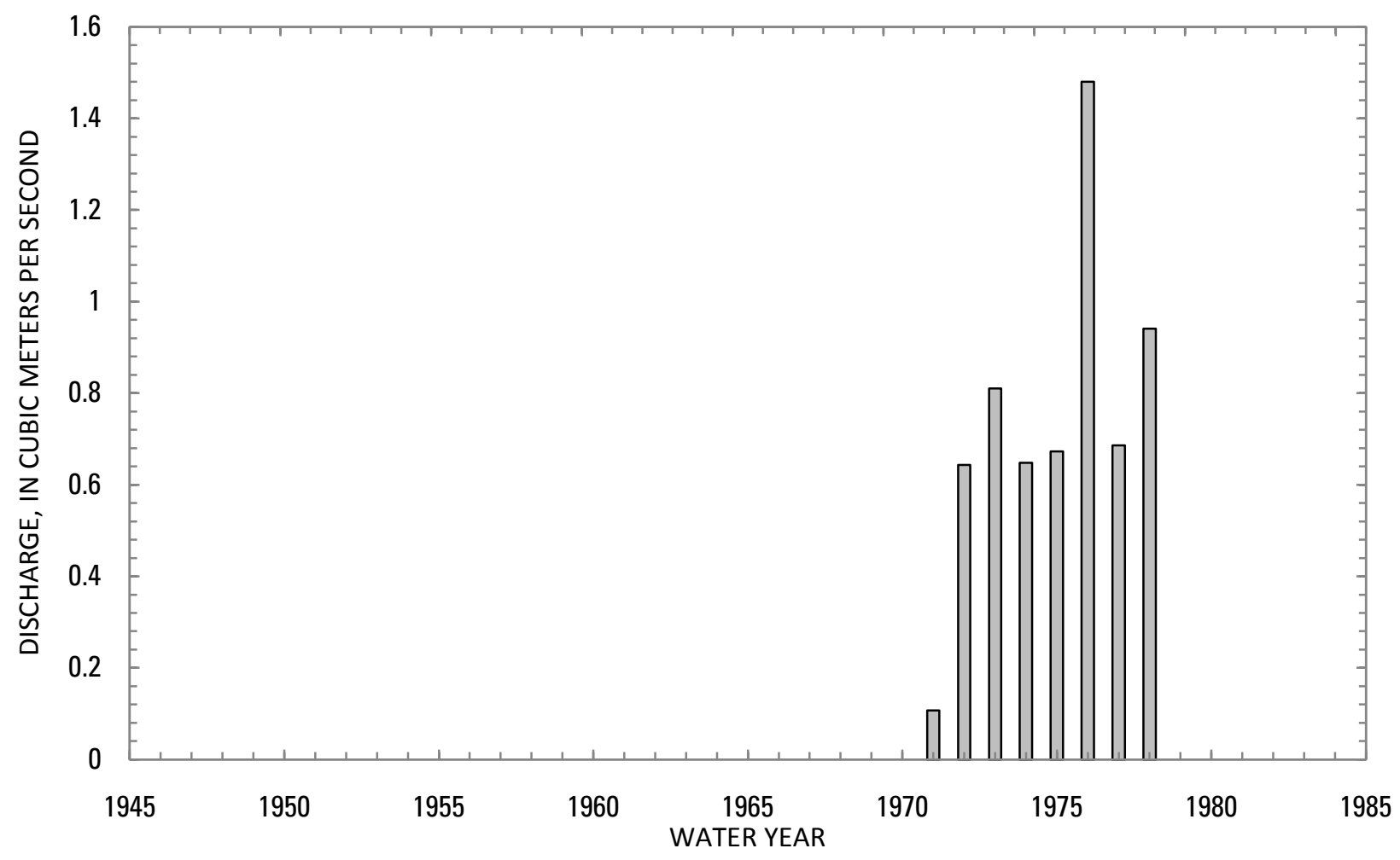




\section{2-4.000-5A URGUN RIVER AT PIRKOTI, Continued}

Statistics of monthly and annual mean discharges [ $\mathrm{m}^{3} / \mathrm{s}$, cubic meters per second]

\begin{tabular}{|c|c|c|c|c|c|c|c|c|}
\hline \multirow[b]{2}{*}{ Month } & \multicolumn{2}{|c|}{ Maximum } & \multicolumn{2}{|c|}{ Minimum } & \multicolumn{4}{|c|}{ Mean } \\
\hline & $\begin{array}{c}\text { Discharge } \\
\left(\mathrm{m}^{3} / \mathrm{s}\right)\end{array}$ & $\begin{array}{l}\text { Water year } \\
\text { of } \\
\text { occurrence }\end{array}$ & $\begin{array}{c}\text { Discharge } \\
\left(\mathrm{m}^{3} / \mathrm{s}\right)\end{array}$ & $\begin{array}{c}\text { Water year } \\
\text { of } \\
\text { occurrence }\end{array}$ & $\begin{array}{c}\text { Discharge } \\
\left(\mathrm{m}^{3} / \mathrm{s}\right)\end{array}$ & $\begin{array}{c}\text { Standard } \\
\text { deviation } \\
\left(\mathrm{m}^{3} / \mathrm{s}\right)\end{array}$ & $\begin{array}{c}\text { Coefficient } \\
\text { of } \\
\text { variation }\end{array}$ & $\begin{array}{c}\text { Percentage } \\
\text { of annual } \\
\text { discharge }\end{array}$ \\
\hline October & 0.30 & 1977 & 0 & 1972 & 0.10 & 0.11 & 1.13 & 1.08 \\
\hline November & 0.49 & 1978 & 0 & 1972 & 0.20 & 0.17 & 0.86 & 2.22 \\
\hline December & 0.50 & 1977 & 0.01 & 1972 & 0.28 & 0.18 & 0.64 & 3.12 \\
\hline January & 0.81 & 1977 & 0.01 & 1972 & 0.32 & 0.24 & 0.74 & 3.55 \\
\hline February & 2.18 & 1973 & 0.24 & 1971 & 0.90 & 0.57 & 0.63 & 10.0 \\
\hline March & 4.27 & 1976 & 0.25 & 1971 & 2.31 & 1.49 & 0.64 & 25.6 \\
\hline April & 7.64 & 1976 & 0.06 & 1971 & 2.11 & 2.41 & 1.14 & 23.4 \\
\hline May & 1.76 & 1972 & 0.04 & 1978 & 0.61 & 0.67 & 1.10 & 6.78 \\
\hline June & 0.74 & 1972 & 0.03 & 1971 & 0.23 & 0.30 & 1.28 & 2.60 \\
\hline July & 6.12 & 1978 & 0.10 & 1974 & 1.27 & 2.01 & 1.59 & 14.03 \\
\hline August & 1.14 & 1977 & 0.01 & 1971 & 0.46 & 0.47 & 1.02 & 5.07 \\
\hline September & 0.50 & 1970 & 0 & 1971 & 0.22 & 0.21 & 0.93 & 2.49 \\
\hline Annual & 1.48 & 1976 & 0.11 & 1971 & 0.75 & 0.38 & 0.51 & 100 \\
\hline
\end{tabular}




\section{2-4.000-5A URGUN RIVER AT PIRKOTI, Continued}

\section{Monthly and annual flow duration, in cubic meters per second}

[ng, not given]

\begin{tabular}{|c|c|c|c|c|c|c|c|c|c|c|c|c|c|}
\hline \multirow{2}{*}{$\begin{array}{l}\text { Percentage } \\
\text { of days } \\
\text { discharge } \\
\text { equaled or } \\
\text { exceeded }\end{array}$} & \multicolumn{12}{|c|}{ Month } & \multirow{2}{*}{ Annua } \\
\hline & October & November & December & January & February & March & April & May & June & July & August & September & \\
\hline 95 & 0 & 0 & 0.01 & 0.01 & 0.18 & 0.25 & 0.05 & 0.02 & 0.01 & 0.02 & 0 & 0 & 0.01 \\
\hline 90 & 0 & 0 & 0.02 & 0.02 & 0.21 & 0.28 & 0.06 & 0.04 & 0.02 & 0.04 & 0 & 0 & 0.02 \\
\hline 85 & 0.01 & 0.02 & 0.05 & 0.15 & 0.22 & 0.47 & 0.19 & 0.05 & 0.02 & 0.06 & 0.01 & 0.01 & 0.03 \\
\hline 80 & 0.01 & 0.03 & 0.10 & 0.18 & 0.23 & 0.66 & 0.31 & 0.06 & 0.02 & 0.07 & 0.01 & 0.02 & 0.04 \\
\hline 75 & 0.02 & 0.04 & 0.13 & 0.20 & 0.24 & 0.85 & 0.38 & 0.07 & 0.03 & 0.08 & 0.02 & 0.02 & 0.06 \\
\hline 70 & 0.03 & 0.05 & 0.14 & 0.21 & 0.25 & 1.02 & 0.50 & 0.08 & 0.04 & 0.10 & 0.02 & 0.03 & 0.08 \\
\hline 65 & 0.03 & 0.06 & 0.21 & 0.22 & 0.31 & 1.15 & 0.60 & 0.14 & 0.04 & 0.12 & 0.02 & 0.05 & 0.11 \\
\hline 60 & 0.04 & 0.06 & 0.23 & 0.24 & 0.37 & 1.40 & 0.74 & 0.17 & 0.05 & 0.14 & 0.03 & 0.07 & 0.17 \\
\hline 55 & 0.04 & 0.08 & 0.28 & 0.25 & 0.46 & 1.50 & 0.90 & 0.23 & 0.05 & 0.20 & 0.07 & 0.08 & 0.20 \\
\hline 50 & 0.04 & 0.10 & 0.30 & 0.26 & 0.51 & 1.83 & 1.10 & 0.31 & 0.05 & 0.24 & 0.09 & 0.10 & 0.24 \\
\hline 45 & 0.05 & 0.14 & 0.32 & 0.31 & 0.56 & 1.99 & 1.34 & 0.35 & 0.06 & 0.31 & 0.14 & 0.11 & 0.29 \\
\hline 40 & 0.07 & 0.18 & 0.34 & 0.34 & 0.71 & 2.41 & 1.50 & 0.39 & 0.08 & 0.39 & 0.21 & 0.12 & 0.34 \\
\hline 35 & 0.10 & 0.20 & 0.40 & 0.36 & 0.81 & 2.77 & 1.70 & 0.46 & 0.10 & 0.48 & 0.31 & 0.17 & 0.42 \\
\hline 30 & 0.14 & 0.21 & 0.42 & 0.38 & 0.99 & 2.98 & 1.97 & 0.55 & 0.13 & 0.54 & 0.41 & 0.21 & 0.49 \\
\hline 25 & 0.16 & 0.25 & 0.43 & 0.40 & 1.08 & 3.19 & 2.41 & 0.89 & 0.19 & 0.65 & 0.49 & 0.27 & 0.56 \\
\hline 20 & 0.20 & 0.28 & 0.50 & 0.44 & 1.15 & 3.90 & 3.74 & 1.21 & 0.26 & 0.95 & 0.55 & 0.36 & 0.85 \\
\hline 15 & 0.22 & 0.31 & 0.52 & 0.48 & 1.22 & 4.30 & 4.95 & 1.66 & 0.31 & 1.53 & 0.66 & 0.38 & 1.18 \\
\hline 10 & 0.31 & 0.44 & 0.54 & 0.62 & 1.48 & 5.34 & 6.19 & 1.89 & 0.50 & 2.39 & 0.85 & 0.41 & 1.87 \\
\hline 5 & ng & 0.51 & 0.56 & 0.00 & 3.31 & 5.74 & 9.00 & 2.15 & 1.13 & 7.95 & 1.51 & 0.75 & 3.73 \\
\hline
\end{tabular}




\section{2-4.000-5A URGUN RIVER AT PIRKOTI, Continued}

Probability of occurrence of annual high discharges

[m $\mathrm{m}^{3} / \mathrm{s}$, cubic meters per second; ng, not given]

\begin{tabular}{|c|c|c|c|c|c|c|}
\hline \multirow{2}{*}{$\begin{array}{c}\text { Exceedance } \\
\text { probability }\end{array}$} & \multirow{2}{*}{$\begin{array}{l}\text { Recurrence } \\
\text { interval } \\
\text { (years) }\end{array}$} & \multirow{2}{*}{$\begin{array}{c}\text { Maximum } \\
\text { instantaneous } \\
\text { discharge }^{1} \\
\left(\mathrm{~m}^{3} / \mathrm{s}\right)\end{array}$} & \multicolumn{4}{|c|}{ Maximum daily mean discharge $\left(\mathrm{m}^{3} / \mathrm{s}\right)$} \\
\hline & & & $\begin{array}{l}\text { 3-day } \\
\text { period }\end{array}$ & $\begin{array}{l}\text { 7-day } \\
\text { period }\end{array}$ & $\begin{array}{l}\text { 15-day } \\
\text { period }\end{array}$ & $\begin{array}{l}\text { 30-day } \\
\text { period }\end{array}$ \\
\hline 0.99 & 1.01 & 6.40 & 0.82 & 0.39 & 0.17 & 0.09 \\
\hline 0.95 & 1.05 & 11.8 & 1.81 & 1.13 & 0.64 & 0.37 \\
\hline 0.90 & 1.11 & 15.8 & 2.65 & 1.84 & 1.16 & 0.72 \\
\hline 0.80 & 1.25 & 21.7 & 4.05 & 3.06 & 2.13 & 1.41 \\
\hline 0.50 & 2 & 36.2 & 8.20 & 6.58 & 5.05 & 3.65 \\
\hline 0.20 & 5 & 53.7 & 14.5 & 11.0 & 8.44 & 6.44 \\
\hline 0.10 & 10 & 63.1 & 18.7 & 13.2 & 9.88 & 7.68 \\
\hline 0.04 & 25 & 73.0 & 23.5 & 15.2 & 11.0 & 8.64 \\
\hline 0.02 & 50 & 78.9 & 26.9 & 16.2 & 11.5 & 9.07 \\
\hline 0.01 & 100 & 84.0 & 29.9 & 17.0 & 11.8 & 9.34 \\
\hline 0.005 & 200 & 88.2 & 32.7 & 17.6 & 12.0 & 9.50 \\
\hline 0.002 & 500 & 92.9 & ng & $\mathrm{ng}$ & $\mathrm{ng}$ & ng \\
\hline
\end{tabular}

'Less than 10 years of data used.

\section{2-4.000-5A URGUN RIVER AT PIRKOTI, Continued}

Probability of occurrence of annual low discharges $\left[\mathrm{m}^{3} / \mathrm{s}\right.$, meters per second]

\begin{tabular}{|c|c|c|c|c|c|c|c|c|c|c|}
\hline \multirow{3}{*}{$\begin{array}{c}\text { Nonexceedance } \\
\text { probability }\end{array}$} & \multirow{3}{*}{$\begin{array}{c}\text { Recurrence } \\
\text { interval } \\
\text { (years) }\end{array}$} & \multicolumn{9}{|c|}{ Minimum daily mean discharge $\left(\mathrm{m}^{3} / \mathrm{s}\right)$} \\
\hline & & \multicolumn{9}{|c|}{ Number of consecutive days } \\
\hline & & 1 & 3 & 7 & 14 & 30 & 60 & 90 & 120 & 183 \\
\hline 0.05 & 20 & 0 & 0 & 0 & 0 & 0 & 0 & 0 & 0 & 0 \\
\hline 0.10 & 10 & 0 & 0 & 0 & 0 & 0 & 0 & 0 & 0 & 0.01 \\
\hline 0.20 & 5 & 0.01 & 0.01 & 0.01 & 0.01 & 0.01 & 0.02 & 0.02 & 0.01 & 0.03 \\
\hline 0.50 & 2 & 0.01 & 0.01 & 0.02 & 0.02 & 0.03 & 0.11 & 0.11 & 0.13 & 0.16 \\
\hline
\end{tabular}




\section{2-4.000-5A URGUN RIVER AT PIRKOTI, Continued}

Probability of occurrence of seasonal low discharges

[ $\mathrm{m}^{3} / \mathrm{s}$, meters per second]

\begin{tabular}{|c|c|c|c|c|c|c|c|c|c|}
\hline \multirow{3}{*}{$\begin{array}{c}\text { Nonexceedance } \\
\text { probability }\end{array}$} & \multirow{3}{*}{$\begin{array}{c}\text { Recurrence } \\
\text { interval } \\
\text { (years) }\end{array}$} & \multicolumn{8}{|c|}{ Minimum daily mean discharge $\left(\mathrm{m}^{3} / \mathrm{s}\right)$} \\
\hline & & \multicolumn{8}{|c|}{ Number of consecutive days } \\
\hline & & 1 & 7 & 14 & 30 & 1 & 7 & 14 & 30 \\
\hline & & \multicolumn{4}{|c|}{ December-January-February } & \multicolumn{4}{|c|}{ March-April-May } \\
\hline 0.05 & 20 & 0 & 0 & 0 & 0.01 & 0.01 & 0.01 & 0.01 & 0.02 \\
\hline 0.10 & 10 & 0 & 0 & 0.01 & 0.03 & 0.02 & 0.02 & 0.03 & 0.04 \\
\hline 0.20 & 5 & 0.05 & 0.06 & 0.04 & 0.07 & 0.03 & 0.04 & 0.05 & 0.08 \\
\hline \multirow[t]{2}{*}{0.50} & 2 & 0.15 & 0.20 & 0.20 & 0.23 & 0.09 & 0.13 & 0.18 & 0.25 \\
\hline & & \multicolumn{4}{|c|}{ June-July-August } & \multicolumn{4}{|c|}{ September-0ctober-November } \\
\hline 0.05 & 20 & 0 & 0 & 0 & 0.01 & 0 & 0 & 0 & 0 \\
\hline 0.10 & 10 & 0 & 0 & 0 & 0.01 & 0 & 0 & 0 & 0 \\
\hline 0.20 & 5 & 0.01 & 0.01 & 0.01 & 0.02 & 0.01 & 0.01 & 0.01 & 0.01 \\
\hline 0.50 & 2 & 0.01 & 0.02 & 0.02 & 0.06 & 0.02 & 0.02 & 0.02 & 0.04 \\
\hline
\end{tabular}

\section{2-4.000-5A URGUN RIVER AT PIRKOTI, Continued}

Annual peak discharges

[ $\mathrm{m}^{3} / \mathrm{s}$, meters per second]

\begin{tabular}{|c|c|c|c|c|c|}
\hline \multicolumn{3}{|c|}{$\begin{array}{c}\text { Annual peak discharge, } \\
\text { by year }\end{array}$} & \multicolumn{3}{|c|}{$\begin{array}{l}\text { Annual peak discharge, } \\
\text { from highest to lowest }\end{array}$} \\
\hline $\begin{array}{l}\text { Water } \\
\text { year }\end{array}$ & Date & $\begin{array}{c}\text { Peak } \\
\text { discharge } \\
\left(\mathrm{m}^{3} / \mathrm{s}\right)\end{array}$ & $\begin{array}{l}\text { Water } \\
\text { year }\end{array}$ & Date & $\begin{array}{c}\text { Peak } \\
\text { discharge } \\
\left(\mathrm{m}^{3} / \mathrm{s}\right)\end{array}$ \\
\hline 1970 & August 22, 1970 & 82.0 & 1970 & August 22, 1970 & 82.0 \\
\hline 1971 & August 4, 1971 & 26.5 & 1975 & July 12,1975 & 45.1 \\
\hline 1972 & May 4, 1972 & 38.0 & 1974 & April 27, 1974 & 42.5 \\
\hline 1973 & August 5, 1973 & 32.6 & 1972 & May 4, 1972 & 38.0 \\
\hline 1974 & April 27, 1974 & 42.5 & 1973 & August 5, 1973 & 32.6 \\
\hline 1975 & July 12,1975 & 45.1 & 1977 & May 27, 1977 & 32.6 \\
\hline 1976 & April 23, 1976 & 10.0 & 1978 & July 5, 1978 & 30.0 \\
\hline 1977 & May 27, 1977 & 32.6 & 1971 & August 4, 1971 & 26.5 \\
\hline 1978 & July 5, 1978 & 30.0 & 1976 & April 23, 1976 & 10.0 \\
\hline
\end{tabular}




\section{2-4.000-5A URGUN RIVER AT PIRKOTI, Continued}

Monthly and annual mean discharges, in cubic meters per second

[Data may not be rounded in accordance with U.S. Geological Survey publication standards; --, no data]

\begin{tabular}{|c|c|c|c|c|c|c|c|c|c|c|c|c|c|}
\hline \multirow{2}{*}{$\begin{array}{l}\text { Water } \\
\text { year }\end{array}$} & \multicolumn{12}{|c|}{ Monthly mean discharge } & \multirow{2}{*}{$\begin{array}{c}\text { Annual } \\
\text { discharge }\end{array}$} \\
\hline & October & November & December & January & February & March & April & May & June & July & August & September & \\
\hline 1970 & -- & -- & -- & -- & -- & -- & -- & -- & -- & -- & -- & 0.50 & -- \\
\hline 1971 & 0.03 & 0.04 & 0.08 & 0.19 & 0.24 & 0.25 & 0.06 & 0.05 & 0.03 & 0.30 & 0.01 & 0 & 0.11 \\
\hline 1972 & 0 & 0 & 0.01 & 0.01 & 0.92 & 1.11 & 2.73 & 1.76 & 0.74 & 0.30 & 0.11 & 0.09 & 0.64 \\
\hline 1973 & 0.10 & 0.15 & 0.25 & 0.22 & 2.18 & 3.27 & 1.02 & 0.39 & 0.06 & 1.16 & 0.62 & 0.39 & 0.81 \\
\hline 1974 & 0.24 & 0.37 & 0.45 & 0.40 & 0.81 & 3.37 & 1.65 & 0.31 & 0.05 & 0.10 & 0.01 & 0.01 & 0.65 \\
\hline 1975 & 0.04 & 0.08 & 0.21 & 0.27 & 0.70 & 3.65 & 2.40 & 0.40 & 0.07 & 0.15 & 0.07 & 0.05 & 0.67 \\
\hline 1976 & 0.07 & 0.18 & 0.30 & 0.42 & 0.80 & 4.27 & 7.64 & 1.59 & 0.21 & 1.14 & 0.68 & 0.45 & 1.48 \\
\hline 1977 & 0.30 & 0.30 & 0.50 & 0.81 & 1.04 & 1.22 & 0.61 & 0.36 & 0.69 & 0.86 & 1.14 & 0.43 & 0.69 \\
\hline 1978 & 0.01 & 0.49 & 0.45 & 0.23 & 0.56 & 1.34 & 0.79 & 0.04 & 0.04 & 6.12 & 1.01 & 0.11 & 0.94 \\
\hline
\end{tabular}




\section{2-4.4L0-2T DAHANE LEGAD RIVER NEAR URGUN}

\section{(U.S. Geological Survey identification number: 325800069090000)}

LOCATION: Lat $32^{\circ} 58^{\prime} \mathrm{N}$., long $69^{\circ} 09^{\prime} \mathrm{E}$.

DRAINAGE AREA: $185 \mathrm{~km}^{2}$.

ELEVATION: 2,215 meters above mean sea level.

PERIOD OF RECORD: April 9, 1972 to September 30, 1978.

GAGE: Water-stage recorder.

Annual mean discharge

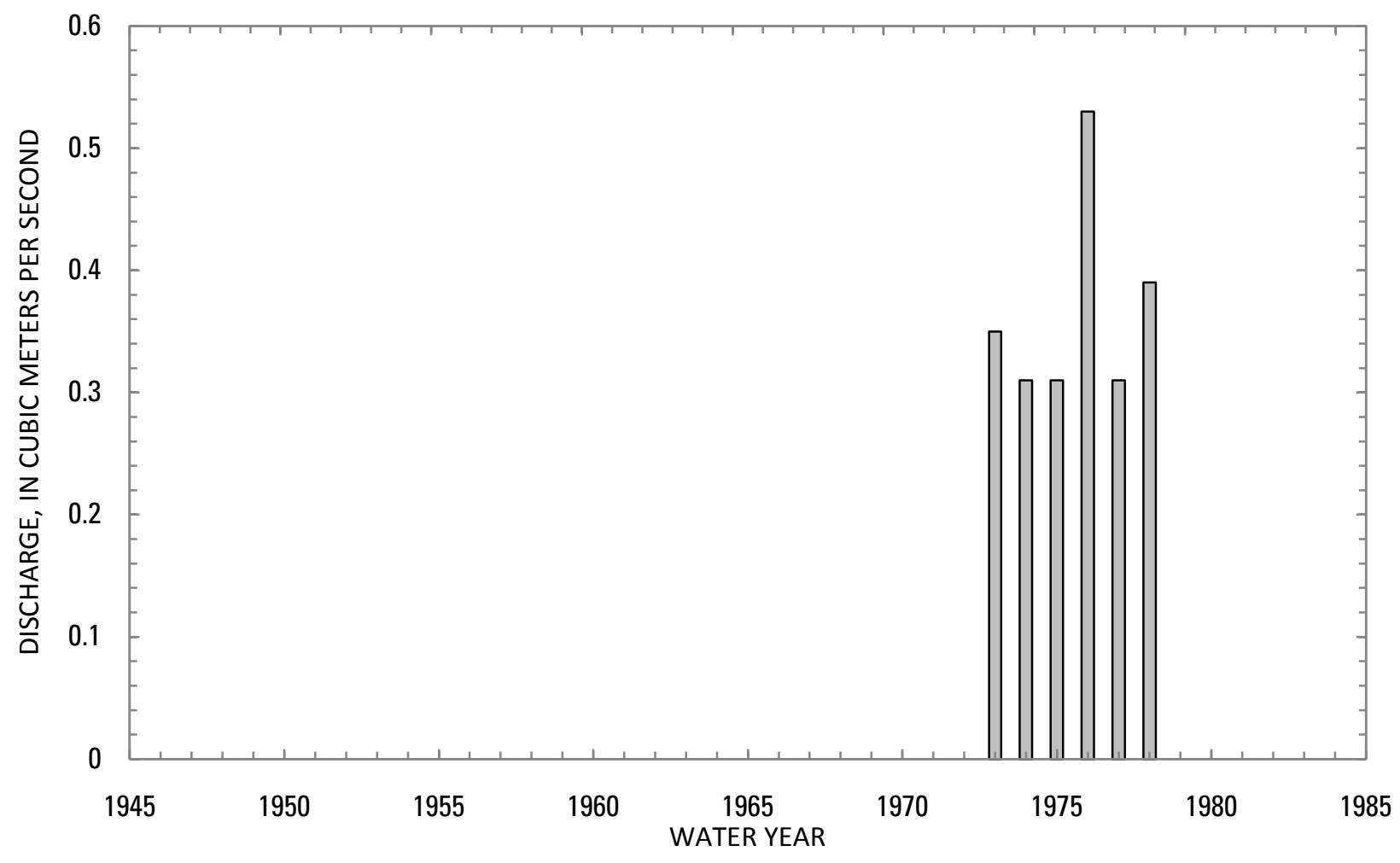




\section{2-4.4L0-2T DAHANE LEGAD RIVER NEAR URGUN, Continued}

Statistics of monthly and annual mean discharges $\left[\mathrm{m}^{3} / \mathrm{s}\right.$, cubic meters per second]

\begin{tabular}{|c|c|c|c|c|c|c|c|c|}
\hline \multirow[b]{2}{*}{ Month } & \multicolumn{2}{|c|}{ Maximum } & \multicolumn{2}{|c|}{ Minimum } & \multicolumn{4}{|c|}{ Mean } \\
\hline & $\begin{array}{c}\text { Discharge } \\
\left(\mathrm{m}^{3} / \mathrm{s}\right)\end{array}$ & $\begin{array}{c}\text { Water year } \\
\text { of } \\
\text { occurrence }\end{array}$ & $\begin{array}{c}\text { Discharge } \\
\left(\mathrm{m}^{3} / \mathrm{s}\right)\end{array}$ & $\begin{array}{c}\text { Water year } \\
\text { of } \\
\text { occurrence }\end{array}$ & $\begin{array}{c}\text { Discharge } \\
\left(\mathrm{m}^{3} / \mathrm{s}\right)\end{array}$ & $\begin{array}{c}\text { Standard } \\
\text { deviation } \\
\left(\mathrm{m}^{3} / \mathrm{s}\right)\end{array}$ & $\begin{array}{c}\text { Coefficient } \\
\text { of } \\
\text { variation }\end{array}$ & $\begin{array}{c}\text { Percentage } \\
\text { of annual } \\
\text { discharge }\end{array}$ \\
\hline October & 0.12 & 1977 & 0.01 & 1974 & 0.03 & 0.04 & 1.52 & 0.66 \\
\hline November & 0.16 & 1978 & 0.01 & 1975 & 0.06 & 0.06 & 0.93 & 1.43 \\
\hline December & 0.17 & 1978 & 0.03 & 1973 & 0.08 & 0.06 & 0.73 & 1.89 \\
\hline January & 0.20 & 1977 & 0.03 & 1976 & 0.09 & 0.06 & 0.70 & 1.95 \\
\hline February & 1.25 & 1973 & 0.12 & 1976 & 0.45 & 0.41 & 0.90 & 10.3 \\
\hline March & 1.68 & 1973 & 0.60 & 1977 & 1.33 & 0.51 & 0.39 & 30.4 \\
\hline April & 2.88 & 1976 & 0.34 & 1978 & 1.09 & 0.95 & 0.88 & 24.8 \\
\hline May & 0.77 & 1972 & 0.03 & 1978 & 0.34 & 0.27 & 0.81 & 7.65 \\
\hline June & 0.42 & 1977 & 0.01 & 1974 & 0.13 & 0.15 & 1.15 & 3.02 \\
\hline July & 2.36 & 1978 & 0.01 & 1974 & 0.53 & 0.83 & 1.55 & 12.2 \\
\hline August & 0.46 & 1977 & 0.01 & 1974 & 0.18 & 0.18 & 1.05 & 4.01 \\
\hline September & 0.23 & 1977 & 0.01 & 1974 & 0.07 & 0.09 & 1.23 & 1.67 \\
\hline Annual & 0.53 & 1976 & 0.31 & 1975 & 0.37 & 0.09 & 0.24 & 100 \\
\hline
\end{tabular}


2-4.4L0-2T DAHANE LEGAD RIVER NEAR URGUN, Continued

Monthly and annual flow duration, in cubic meters per second

[ng, not given]

\begin{tabular}{|c|c|c|c|c|c|c|c|c|c|c|c|c|c|}
\hline \multirow{2}{*}{$\begin{array}{l}\text { Percentage } \\
\text { of days } \\
\text { discharge } \\
\text { equaled or } \\
\text { exceeded }\end{array}$} & \multicolumn{12}{|c|}{ Month } & \multirow{2}{*}{ Annua } \\
\hline & October & November & December & January & February & March & April & May & June & July & August & September & \\
\hline 95 & 0.01 & 0 & 0.01 & 0.01 & 0.03 & 0.35 & 0.12 & 0.02 & 0.01 & 0.01 & 0.01 & 0.01 & 0.01 \\
\hline 90 & 0.01 & 0.01 & 0.02 & 0.01 & 0.05 & 0.46 & 0.17 & 0.02 & 0.01 & 0.02 & 0.01 & 0.01 & 0.01 \\
\hline 85 & 0.01 & 0.01 & 0.02 & 0.02 & 0.05 & 0.51 & 0.27 & 0.04 & 0.01 & 0.02 & 0.01 & 0.01 & 0.02 \\
\hline 80 & 0.01 & 0.01 & 0.02 & 0.02 & 0.06 & 0.56 & 0.32 & 0.05 & 0.01 & 0.02 & 0.01 & 0.01 & 0.02 \\
\hline 75 & 0.01 & 0.01 & 0.03 & 0.02 & 0.08 & 0.68 & 0.39 & 0.07 & 0.02 & 0.03 & 0.02 & 0.01 & 0.02 \\
\hline 70 & 0.01 & 0.02 & 0.04 & 0.03 & 0.11 & 0.77 & 0.43 & 0.09 & 0.02 & 0.03 & 0.02 & 0.02 & 0.02 \\
\hline 65 & 0.01 & 0.02 & 0.05 & 0.04 & 0.15 & 0.83 & 0.51 & 0.13 & 0.02 & 0.05 & 0.02 & 0.02 & 0.03 \\
\hline 60 & 0.02 & 0.02 & 0.06 & 0.04 & 0.18 & 0.95 & 0.54 & 0.15 & 0.02 & 0.06 & 0.02 & 0.02 & 0.05 \\
\hline 55 & 0.02 & 0.02 & 0.06 & 0.05 & 0.20 & 1.00 & 0.58 & 0.17 & 0.03 & 0.08 & 0.03 & 0.02 & 0.06 \\
\hline 50 & 0.02 & 0.02 & 0.07 & 0.06 & 0.22 & 1.06 & 0.66 & 0.21 & 0.05 & 0.09 & 0.03 & 0.02 & 0.08 \\
\hline 45 & 0.02 & 0.03 & 0.07 & 0.06 & 0.23 & 1.17 & 0.73 & 0.25 & 0.06 & 0.10 & 0.05 & 0.02 & 0.11 \\
\hline 40 & 0.02 & 0.03 & 0.09 & 0.07 & 0.27 & 1.26 & 0.82 & 0.30 & 0.07 & 0.11 & 0.06 & 0.02 & 0.15 \\
\hline 35 & 0.02 & 0.05 & 0.10 & 0.10 & 0.30 & 1.41 & 0.92 & 0.34 & 0.09 & 0.13 & 0.08 & 0.03 & 0.17 \\
\hline 30 & 0.02 & 0.06 & 0.14 & 0.13 & 0.39 & 1.57 & 1.11 & 0.40 & 0.11 & 0.16 & 0.10 & 0.04 & 0.19 \\
\hline 25 & 0.02 & 0.07 & 0.14 & 0.15 & 0.47 & 1.86 & 1.57 & 0.48 & 0.12 & 0.20 & 0.11 & 0.05 & 0.27 \\
\hline 20 & 0.03 & 0.09 & 0.15 & 0.16 & 0.56 & 1.96 & 1.84 & 0.63 & 0.16 & 0.29 & 0.15 & 0.05 & 0.43 \\
\hline 15 & 0.08 & 0.10 & 0.15 & 0.20 & 0.66 & 2.26 & 2.38 & 0.78 & 0.22 & 0.53 & 0.17 & 0.16 & 0.64 \\
\hline 10 & ng & 0.13 & 0.16 & 0.21 & 0.84 & 2.45 & 3.01 & 0.90 & 0.26 & 1.12 & 0.21 & 0.17 & 1.04 \\
\hline 5 & ng & 0.16 & 0.20 & 0.28 & 1.85 & 2.99 & 3.36 & 1.04 & 0.41 & 3.98 & 0.48 & 0.18 & 1.93 \\
\hline
\end{tabular}




\section{2-4.4L0-2T DAHANE LEGAD RIVER NEAR URGUN, Continued}

Probability of occurrence of annual high discharges

[m $\mathrm{m}^{3} / \mathrm{s}$, cubic meters per second; ng, not given]

\begin{tabular}{|c|c|c|c|c|c|c|}
\hline \multirow{2}{*}{$\begin{array}{c}\text { Exceedance } \\
\text { probability }\end{array}$} & \multirow{2}{*}{$\begin{array}{c}\text { Recurrence } \\
\text { interval } \\
\text { (years) }\end{array}$} & \multirow{2}{*}{$\begin{array}{c}\text { Maximum } \\
\text { instantaneous } \\
\text { discharge } \\
\left(\mathrm{m}^{3} / \mathrm{s}\right)\end{array}$} & \multicolumn{4}{|c|}{ Maximum daily mean discharge $\left(\mathrm{m}^{3} / \mathrm{s}\right)$} \\
\hline & & & $\begin{array}{l}\text { 3-day } \\
\text { period }\end{array}$ & $\begin{array}{l}\text { 7-day } \\
\text { period }\end{array}$ & $\begin{array}{l}\text { 15-day } \\
\text { period }\end{array}$ & $\begin{array}{l}\text { 30-day } \\
\text { period }\end{array}$ \\
\hline 0.99 & 1.01 & 10.6 & ${ }^{2} 2.01$ & 2.18 & 0.97 & 0.45 \\
\hline 0.95 & 1.05 & 14.2 & 2.51 & 2.36 & 1.38 & 0.82 \\
\hline 0.90 & 1.11 & 16.5 & 2.85 & 2.50 & 1.64 & 1.06 \\
\hline 0.80 & 1.25 & 19.9 & 3.34 & 2.72 & 1.99 & 1.40 \\
\hline 0.50 & 2 & 28.3 & 4.62 & 3.40 & 2.76 & 2.10 \\
\hline 0.20 & 5 & 40.0 & 6.59 & 4.65 & 3.64 & 2.72 \\
\hline 0.10 & 10 & 48.0 & 8.04 & 5.69 & 4.13 & 2.97 \\
\hline 0.04 & 25 & 58.2 & 10.0 & 7.28 & 4.65 & 3.16 \\
\hline 0.02 & 50 & 65.8 & 11.6 & 8.69 & 4.99 & 3.25 \\
\hline 0.01 & 100 & 73.5 & 13.3 & 10.3 & 5.29 & 3.31 \\
\hline 0.005 & 200 & 81.3 & 15.1 & 12.2 & 5.55 & 3.35 \\
\hline 0.002 & 500 & 91.9 & ng & ng & ng & ng \\
\hline
\end{tabular}

\section{2-4.4L0-2T DAHANE LEGAD RIVER NEAR URGUN, Continued}

Probability of occurrence of annual low discharges [ $\mathrm{m}^{3} / \mathrm{s}$, meters per second]

\begin{tabular}{|c|c|c|c|c|c|c|c|c|c|c|}
\hline \multirow{3}{*}{$\begin{array}{c}\text { Nonexceedance } \\
\text { probability }\end{array}$} & \multirow{3}{*}{$\begin{array}{c}\text { Recurrence } \\
\text { interval } \\
\text { (years) }\end{array}$} & \multicolumn{9}{|c|}{ Minimum daily mean discharge $\left(\mathrm{m}^{3} / \mathrm{s}\right)$} \\
\hline & & \multicolumn{9}{|c|}{ Number of consecutive days } \\
\hline & & 1 & 3 & 7 & 14 & 30 & 60 & 90 & 120 & 183 \\
\hline 0.05 & 20 & 0 & 0 & 0 & 0 & 0.01 & 0.01 & 0.01 & 0.01 & 0.01 \\
\hline 0.10 & 10 & 0 & 0 & 0 & 0 & 0.01 & 0.01 & 0.01 & 0.01 & 0.01 \\
\hline 0.20 & 5 & 0 & 0 & 0 & 0.01 & 0.01 & 0.01 & 0.01 & 0.02 & 0.02 \\
\hline 0.50 & 2 & 0.01 & 0.01 & 0.01 & 0.01 & 0.01 & 0.01 & 0.03 & 0.04 & 0.05 \\
\hline
\end{tabular}




\section{2-4.4L0-2T DAHANE LEGAD RIVER NEAR URGUN, Continued}

Probability of occurrence of seasonal low discharges

$\left[\mathrm{m}^{3} / \mathrm{s}\right.$, meters per second]

\begin{tabular}{|c|c|c|c|c|c|c|c|c|c|}
\hline \multirow{3}{*}{$\begin{array}{c}\text { Nonexceedance } \\
\text { probability }\end{array}$} & \multirow{3}{*}{$\begin{array}{c}\text { Recurrence } \\
\text { interval } \\
\text { (years) }\end{array}$} & \multicolumn{8}{|c|}{ Minimum daily mean discharge $\left(\mathrm{m}^{3} / \mathrm{s}\right)$} \\
\hline & & \multicolumn{8}{|c|}{ Number of consecutive days } \\
\hline & & 1 & 7 & 14 & 30 & 1 & 7 & 14 & 30 \\
\hline & & \multicolumn{4}{|c|}{ December-January-February } & \multicolumn{4}{|c|}{ March-April-May } \\
\hline 0.05 & 20 & 0.01 & 0.01 & 0.01 & 0.02 & 0.01 & 0.01 & 0.01 & 0.02 \\
\hline 0.10 & 10 & 0.01 & 0.01 & 0.01 & 0.02 & 0.01 & 0.01 & 0.02 & 0.03 \\
\hline 0.20 & 5 & 0.01 & 0.01 & 0.01 & 0.02 & 0.01 & 0.02 & 0.03 & 0.05 \\
\hline \multirow[t]{2}{*}{0.50} & 2 & 0.01 & 0.01 & 0.02 & 0.03 & 0.02 & 0.07 & 0.10 & 0.15 \\
\hline & & \multicolumn{4}{|c|}{ June-July-August } & \multicolumn{4}{|c|}{ September-0ctober-November } \\
\hline 0.05 & 20 & 0.01 & 0.01 & 0.01 & 0.01 & 0 & 0 & 0 & 0.01 \\
\hline 0.10 & 10 & 0.01 & 0.01 & 0.01 & 0.01 & 0 & 0 & 0.01 & 0.01 \\
\hline 0.20 & 5 & 0.01 & 0.01 & 0.01 & 0.01 & 0.01 & 0 & 0.01 & 0.01 \\
\hline 0.50 & 2 & 0.01 & 0.01 & 0.01 & 0.02 & 0.01 & 0.01 & 0.01 & 0.01 \\
\hline
\end{tabular}

\section{2-4.4L0-2T DAHANE LEGAD RIVER NEAR URGUN, Continued}

Annual peak discharges

$\left[\mathrm{m}^{3} / \mathrm{s}\right.$, meters per second]

\begin{tabular}{|c|c|c|c|c|c|}
\hline \multicolumn{3}{|c|}{$\begin{array}{c}\text { Annual peak discharge, } \\
\text { by year }\end{array}$} & \multicolumn{3}{|c|}{$\begin{array}{l}\text { Annual peak discharge, } \\
\text { from highest to lowest }\end{array}$} \\
\hline $\begin{array}{l}\text { Water } \\
\text { year }\end{array}$ & Date & $\begin{array}{c}\text { Peak } \\
\text { discharge } \\
\left(\mathrm{m}^{3} / \mathrm{s}\right)\end{array}$ & $\begin{array}{l}\text { Water } \\
\text { year }\end{array}$ & Date & $\begin{array}{c}\text { Peak } \\
\text { discharge } \\
\left(\mathrm{m}^{3} / \mathrm{s}\right)\end{array}$ \\
\hline 1973 & September 19, 1973 & 49.0 & 1973 & September 19, 1973 & 49.0 \\
\hline 1974 & April 27, 1974 & 19.8 & 1976 & July 14, 1976 & 37.0 \\
\hline 1975 & August 12,1975 & 35.0 & 1975 & August 12, 1975 & 35.0 \\
\hline 1976 & July 14,1976 & 37.0 & 1977 & May 25, 1977 & 24.4 \\
\hline 1977 & May 25, 1977 & 24.4 & 1974 & April 27, 1974 & 19.8 \\
\hline 1978 & July 5, 1978 & 16.4 & 1978 & July 5,1978 & 16.4 \\
\hline
\end{tabular}


2-4.4L0-2T DAHANE LEGAD RIVER NEAR URGUN, Continued

Monthly and annual mean discharges, in cubic meters per second $[--$, no data]

\begin{tabular}{|c|c|c|c|c|c|c|c|c|c|c|c|c|c|}
\hline \multirow{2}{*}{$\begin{array}{l}\text { Water } \\
\text { year }\end{array}$} & \multicolumn{12}{|c|}{ Monthly mean discharge } & \multirow{2}{*}{$\begin{array}{c}\text { Annual } \\
\text { discharge }\end{array}$} \\
\hline & October & November & December & January & February & March & April & May & June & July & August & September & \\
\hline 1972 & -- & -- & -- & -- & -- & -- & -- & 0.77 & 0.23 & 0.08 & 0.03 & 0.02 & -- \\
\hline 1973 & 0.01 & 0.05 & 0.03 & 0.10 & 1.25 & 1.68 & 0.49 & 0.15 & 0.02 & 0.29 & 0.11 & 0.04 & 0.35 \\
\hline 1974 & 0.01 & 0.03 & 0.05 & 0.06 & 0.48 & 1.63 & 1.24 & 0.20 & 0.01 & 0.01 & 0.01 & 0.01 & 0.31 \\
\hline 1975 & 0.01 & 0.01 & 0.03 & 0.05 & 0.27 & 1.68 & 1.11 & 0.33 & 0.08 & 0.07 & 0.04 & 0.01 & 0.31 \\
\hline 1976 & 0.01 & 0.03 & 0.06 & 0.03 & 0.12 & 1.68 & 2.88 & 0.65 & 0.16 & 0.44 & 0.18 & 0.17 & 0.53 \\
\hline 1977 & 0.12 & 0.11 & 0.15 & 0.20 & 0.24 & 0.60 & 0.45 & 0.22 & 0.42 & 0.49 & 0.46 & 0.23 & 0.31 \\
\hline 1978 & 0.01 & 0.16 & 0.17 & 0.07 & 0.36 & 0.75 & 0.34 & 0.03 & 0.01 & 2.36 & 0.40 & 0.04 & 0.39 \\
\hline
\end{tabular}




\section{8-0.000-1M HARI RUD RIVER AT TIR PUL}

\section{(U.S. Geological Survey identification number: 343600061160000 )}

LOCATION: Lat $34^{\circ} 36^{\prime}$ N., long $61^{\circ} 16^{\prime} \mathrm{E}$.

DRAINAGE AREA: $31,760 \mathrm{~km}^{2}$.

ELEVATION: 760 meters above mean sea level.

PERIOD OF RECORD: March 11, 1969 to September 30, 1978.

GAGE: Water-stage recorder.

Annual mean discharge

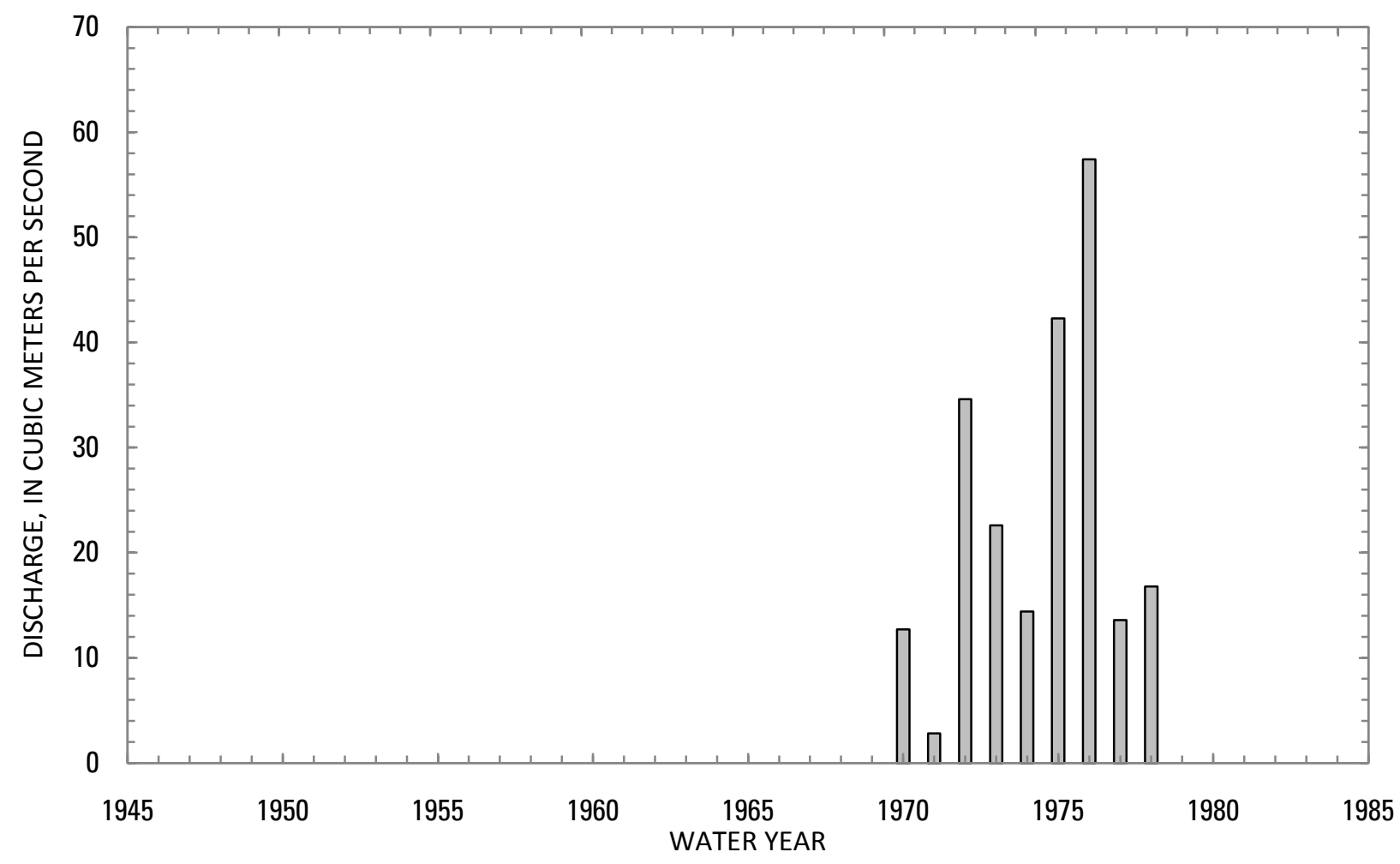




\section{8-0.000-1M HARI RUD RIVER AT TIR PUL, Continued}

Statistics of monthly and annual mean discharges $\left[\mathrm{m}^{3} / \mathrm{s}\right.$, cubic meters per second]

\begin{tabular}{|c|c|c|c|c|c|c|c|c|}
\hline \multirow[b]{2}{*}{ Month } & \multicolumn{2}{|c|}{ Maximum } & \multicolumn{2}{|c|}{ Minimum } & \multicolumn{4}{|c|}{ Mean } \\
\hline & $\begin{array}{c}\text { Discharge } \\
\left(\mathrm{m}^{3} / \mathrm{s}\right)\end{array}$ & $\begin{array}{c}\text { Water year } \\
\text { of } \\
\text { occurrence }\end{array}$ & $\begin{array}{c}\text { Discharge } \\
\left(\mathrm{m}^{3} / \mathrm{s}\right)\end{array}$ & $\begin{array}{c}\text { Water year } \\
\text { of } \\
\text { occurrence }\end{array}$ & $\begin{array}{c}\text { Discharge } \\
\left(\mathrm{m}^{3} / \mathrm{s}\right)\end{array}$ & $\begin{array}{c}\text { Standard } \\
\text { deviation } \\
\left(\mathrm{m}^{3} / \mathrm{s}\right)\end{array}$ & $\begin{array}{c}\text { Coefficient } \\
\text { of } \\
\text { variation }\end{array}$ & $\begin{array}{c}\text { Percentage } \\
\text { of annual } \\
\text { discharge }\end{array}$ \\
\hline October & 0.54 & 1970 & 0.04 & 1974 & 0.16 & 0.15 & 0.94 & 0.05 \\
\hline November & 9.76 & 1977 & 0.04 & 1974 & 2.74 & 3.95 & 1.44 & 0.79 \\
\hline December & 12.8 & 1977 & 0.22 & 1974 & 4.66 & 5.06 & 1.09 & 1.34 \\
\hline January & 20.2 & 1978 & 0.80 & 1971 & 12.8 & 6.42 & 0.50 & 3.67 \\
\hline February & 35.5 & 1976 & 1.30 & 1971 & 22.4 & 10.1 & 0.45 & 6.44 \\
\hline March & 94.0 & 1972 & 3.39 & 1971 & 47.2 & 24.2 & 0.51 & 13.6 \\
\hline April & 374 & 1969 & 16.9 & 1971 & 125 & 112 & 0.89 & 35.8 \\
\hline May & 302 & 1969 & 1.03 & 1970 & 108 & 114 & 1.05 & 31.2 \\
\hline June & 95.3 & 1969 & 0.06 & 1970 & 23.9 & 32.7 & 1.37 & 6.87 \\
\hline July & 5.64 & 1969 & 0.03 & 1974 & 0.88 & 1.79 & 2.04 & 0.25 \\
\hline August & 0.44 & 1969 & 0.01 & 1973 & 0.09 & 0.13 & 1.36 & 0.03 \\
\hline September & 0.23 & 1969 & 0.01 & 1970 & 0.08 & 0.07 & 0.85 & 0.02 \\
\hline Annual & 57.4 & 1976 & 2.83 & 1971 & 24.1 & 17.3 & 0.72 & 100 \\
\hline
\end{tabular}


8-0.000-1M HARI RUD RIVER AT TIR PUL, Continued

Monthly and annual flow duration, in cubic meters per second

[ng, not given]

\begin{tabular}{|c|c|c|c|c|c|c|c|c|c|c|c|c|c|}
\hline \multirow{2}{*}{$\begin{array}{l}\text { Percentage } \\
\text { of days } \\
\text { discharge } \\
\text { equaled or } \\
\text { exceeded }\end{array}$} & \multicolumn{12}{|c|}{ Month } & \multirow{2}{*}{ Annual } \\
\hline & October & November & December & January & February & March & April & May & June & July & August & September & \\
\hline 95 & 0.02 & 0.03 & 0.10 & 0.81 & 1.25 & 1.98 & 7.83 & 0.27 & 0.05 & 0.02 & 0 & 0 & 0.02 \\
\hline 90 & 0.04 & 0.06 & 0.17 & 0.94 & 1.71 & 11.7 & 19.4 & 0.61 & 0.06 & 0.03 & 0.01 & 0 & 0.04 \\
\hline 85 & 0.05 & 0.09 & 0.25 & 1.92 & 13.5 & 25.6 & 30.9 & 2.72 & 0.09 & 0.03 & 0.02 & 0.01 & 0.05 \\
\hline 80 & 0.07 & 0.11 & 0.38 & 2.86 & 15.7 & 29.1 & 36.3 & 5.71 & 0.12 & 0.04 & 0.02 & 0.02 & 0.08 \\
\hline 75 & 0.07 & 0.13 & 0.45 & 4.98 & 16.9 & 32.3 & 40.2 & 10.0 & 0.24 & 0.04 & 0.03 & 0.03 & 0.10 \\
\hline 70 & 0.08 & 0.14 & 0.50 & 6.06 & 18.1 & 33.9 & 44.7 & 16.7 & 0.37 & 0.05 & 0.03 & 0.03 & 0.11 \\
\hline 65 & 0.08 & 0.16 & 0.54 & 6.97 & 19.2 & 35.6 & 50.8 & 23.4 & 0.51 & 0.07 & 0.03 & 0.04 & 0.17 \\
\hline 60 & 0.09 & 0.18 & 0.71 & 7.96 & 19.9 & 37.2 & 60.3 & 30.6 & 0.69 & 0.08 & 0.04 & 0.04 & 0.28 \\
\hline 55 & 0.09 & 0.20 & 1.42 & 10.1 & 20.6 & 38.9 & 72.8 & 37.0 & 1.27 & 0.09 & 0.04 & 0.06 & 0.51 \\
\hline 50 & 0.10 & 0.28 & 1.89 & 12.6 & 21.3 & 40.6 & 84.2 & 66.2 & 3.22 & 0.11 & 0.05 & 0.08 & 1.24 \\
\hline 45 & 0.10 & 0.31 & 2.21 & 14.1 & 22.1 & 42.7 & 93.6 & 89.3 & 5.93 & 0.12 & 0.07 & 0.08 & 3.32 \\
\hline 40 & 0.11 & 0.33 & 3.15 & 15.5 & 23.8 & 44.7 & 105 & 117 & 9.21 & 0.14 & 0.08 & 0.09 & 8.34 \\
\hline 35 & 0.11 & 0.41 & 3.99 & 16.9 & 25.5 & 46.8 & 117 & 134 & 14.4 & 0.17 & 0.08 & 0.09 & 13.5 \\
\hline 30 & 0.12 & 0.97 & 5.36 & 18.2 & 27.3 & 48.9 & 130 & 156 & 21.8 & 0.21 & 0.09 & 0.10 & 18.9 \\
\hline 25 & 0.14 & 1.87 & 7.59 & 19.4 & 30.0 & 52.7 & 149 & 178 & 34.0 & 0.26 & 0.10 & 0.10 & 24.4 \\
\hline 20 & 0.15 & 3.78 & 9.30 & 20.6 & 32.9 & 59.4 & 174 & 207 & 47.2 & 0.41 & 0.11 & 0.11 & 34.1 \\
\hline 15 & 0.19 & 9.40 & 10.7 & 22.0 & 35.2 & 68.6 & 206 & 239 & 61.1 & 0.69 & 0.14 & 0.14 & 43.4 \\
\hline 10 & 0.24 & 12.1 & 12.3 & 23.5 & 38.5 & 83.5 & 282 & 283 & 83.3 & 1.33 & 0.35 & 0.20 & 66.9 \\
\hline 5 & 0.29 & 14.0 & 16.0 & 27.2 & 43.8 & 116 & 486 & 346 & 107 & 5.89 & $\mathrm{ng}$ & 0.22 & 125 \\
\hline
\end{tabular}




\section{8-0.000-1M HARI RUD RIVER AT TIR PUL, Continued}

Probability of occurrence of annual high discharges [m $\mathrm{m}^{3} / \mathrm{s}$, cubic meters per second; ng, not given]

\begin{tabular}{|c|c|c|c|c|c|c|}
\hline \multirow{2}{*}{$\begin{array}{c}\text { Exceedance } \\
\text { probability }\end{array}$} & \multirow{2}{*}{$\begin{array}{c}\text { Recurrence } \\
\text { interval } \\
\text { (years) }\end{array}$} & \multirow{2}{*}{$\begin{array}{l}\text { Maximum } \\
\text { instantaneous } \\
\text { discharge } \\
\left(\mathrm{m}^{3} / \mathrm{s}\right)\end{array}$} & \multicolumn{4}{|c|}{ Maximum daily mean discharge $\left(\mathrm{m}^{3} / \mathrm{s}\right)$} \\
\hline & & & $\begin{array}{l}\text { 3-day } \\
\text { period }\end{array}$ & $\begin{array}{l}\text { 7-day } \\
\text { period }\end{array}$ & $\begin{array}{l}\text { 15-day } \\
\text { period }\end{array}$ & $\begin{array}{l}\text { 30-day } \\
\text { period }\end{array}$ \\
\hline 0.99 & 1.01 & 21.4 & 20.2 & 19.3 & 13.4 & 8.58 \\
\hline 0.95 & 1.05 & 40.6 & 35.1 & 31.9 & 25.1 & 18.4 \\
\hline 0.90 & 1.11 & 57.3 & 47.5 & 42.3 & 34.8 & 27.0 \\
\hline 0.80 & 1.25 & 87.4 & 68.7 & 60.2 & 51.6 & 42.3 \\
\hline 0.50 & 2 & 199 & 141 & 122 & 108 & 95.0 \\
\hline 0.20 & 5 & 458 & 296 & 259 & 223 & 199 \\
\hline 0.10 & 10 & 714 & 438 & 391 & 325 & 286 \\
\hline 0.04 & 25 & 1,150 & 671 & 615 & 481 & 412 \\
\hline 0.02 & 50 & 1,570 & 886 & 831 & 619 & 517 \\
\hline 0.01 & 100 & 2,080 & 1,140 & 1,100 & 775 & 629 \\
\hline 0.005 & 200 & 2,700 & 1,440 & 1,420 & 950 & 748 \\
\hline 0.002 & 500 & 3,710 & ng & ng & ng & ng \\
\hline
\end{tabular}

8-0.000-1M HARI RUD RIVER AT TIR PUL, Continued

Probability of occurrence of annual low discharges $\left[\mathrm{m}^{3} / \mathrm{s}\right.$, meters per second]

\begin{tabular}{|c|c|c|c|c|c|c|c|c|c|c|}
\hline \multirow{3}{*}{$\begin{array}{c}\text { Nonexceedance } \\
\text { probability }\end{array}$} & \multirow{3}{*}{$\begin{array}{c}\text { Recurrence } \\
\text { interval } \\
\text { (years) }\end{array}$} & \multicolumn{9}{|c|}{ Minimum daily mean discharge $\left(\mathrm{m}^{3} / \mathrm{s}\right)$} \\
\hline & & \multicolumn{9}{|c|}{ Number of consecutive days } \\
\hline & & 1 & 3 & 7 & 14 & 30 & 60 & 90 & 120 & 183 \\
\hline 0.05 & 20 & 0 & 0 & 0 & 0 & 0 & 0.01 & 0.02 & 0.02 & 0.03 \\
\hline 0.10 & 10 & 0 & 0 & 0 & 0 & 0 & 0.01 & 0.02 & 0.03 & 0.05 \\
\hline 0.20 & 5 & 0 & 0 & 0 & 0 & 0.01 & 0.02 & 0.03 & 0.03 & 0.10 \\
\hline 0.50 & 2 & 0.02 & 0.02 & 0.02 & 0.02 & 0.03 & 0.04 & 0.05 & 0.07 & 0.41 \\
\hline
\end{tabular}




\section{8-0.000-1M HARI RUD RIVER AT TIR PUL, Continued}

Probability of occurrence of seasonal low discharges [ $\mathrm{m}^{3} / \mathrm{s}$, meters per second]

\begin{tabular}{|c|c|c|c|c|c|c|c|c|c|}
\hline \multirow{3}{*}{$\begin{array}{c}\text { Nonexceedance } \\
\text { probability }\end{array}$} & \multirow{3}{*}{$\begin{array}{c}\text { Recurrence } \\
\text { interval } \\
\text { (years) }\end{array}$} & \multicolumn{8}{|c|}{ Minimum daily mean discharge $\left(\mathrm{m}^{3} / \mathrm{s}\right)$} \\
\hline & & \multicolumn{8}{|c|}{ Number of consecutive days } \\
\hline & & 1 & 7 & 14 & 30 & 1 & 7 & 14 & 30 \\
\hline & & \multicolumn{4}{|c|}{ December-January-February } & \multicolumn{4}{|c|}{ March-April-May } \\
\hline 0.05 & 20 & 0.04 & 0.04 & 0.05 & 0.19 & 0.05 & 0.09 & 0.18 & 0.93 \\
\hline 0.10 & 10 & 0.06 & 0.08 & 0.10 & 0.34 & 0.16 & 0.26 & 0.52 & 2.33 \\
\hline 0.20 & 5 & 0.12 & 0.16 & 0.20 & 0.67 & 0.57 & 0.90 & 1.74 & 6.16 \\
\hline \multirow[t]{2}{*}{0.50} & 2 & 0.45 & 0.71 & 0.85 & 2.32 & 4.59 & 6.95 & 11.7 & 26.5 \\
\hline & & \multicolumn{4}{|c|}{ June-July-August } & \multicolumn{4}{|c|}{ September-0ctober-November } \\
\hline 0.05 & 20 & 0 & 0 & 0 & 0 & 0 & 0 & 0 & 0.01 \\
\hline 0.10 & 10 & 0 & 0 & 0 & 0 & 0 & 0 & 0 & 0.01 \\
\hline 0.20 & 5 & 0 & 0.01 & 0.01 & 0.02 & 0 & 0 & 0 & 0.02 \\
\hline 0.50 & 2 & 0.02 & 0.03 & 0.03 & 0.04 & 0.02 & 0.02 & 0.03 & 0.05 \\
\hline
\end{tabular}

\section{8-0.000-1M HARI RUD RIVER AT TIR PUL, Continued}

Annual peak discharges

[ $\mathrm{m}^{3} / \mathrm{s}$, meters per second]

\begin{tabular}{|c|c|c|c|c|c|}
\hline \multicolumn{3}{|c|}{$\begin{array}{c}\text { Annual peak discharge, } \\
\text { by year }\end{array}$} & \multicolumn{3}{|c|}{$\begin{array}{l}\text { Annual peak discharge, } \\
\text { from highest to lowest }\end{array}$} \\
\hline $\begin{array}{l}\text { Water } \\
\text { year }\end{array}$ & Date & $\begin{array}{c}\text { Peak } \\
\text { discharge } \\
\left(\mathrm{m}^{3} / \mathrm{s}\right)\end{array}$ & $\begin{array}{l}\text { Water } \\
\text { year }\end{array}$ & Date & $\begin{array}{c}\text { Peak } \\
\text { discharge } \\
\left(\mathrm{m}^{3} / \mathrm{s}\right)\end{array}$ \\
\hline 1969 & April 15, 1969 & 776 & 1969 & April 15, 1969 & 776 \\
\hline 1970 & April 1, 1970 & 118 & 1976 & April 24, 1976 & 734 \\
\hline 1971 & April 16, 1971 & 60.0 & 1975 & April 27, 1975 & 480 \\
\hline 1972 & April 27, 1972 & 353 & 1972 & April 27, 1972 & 353 \\
\hline 1973 & May 6, 1973 & 177 & 1973 & May 6, 1973 & 177 \\
\hline 1974 & April 4, 1974 & 140 & 1974 & April 4, 1974 & 140 \\
\hline 1975 & April 27, 1975 & 480 & 1978 & April 19, 1978 & 132 \\
\hline 1976 & April 24, 1976 & 734 & 1970 & April 1, 1970 & 118 \\
\hline 1977 & April 10, 1977 & 47.6 & 1971 & April 16, 1971 & 60.0 \\
\hline 1978 & April 19, 1978 & 132 & 1977 & April 10, 1977 & 47.6 \\
\hline
\end{tabular}




\section{8-0.000-1M HARI RUD RIVER AT TIR PUL, Continued}

Monthly and annual mean discharges, in cubic meters per second $[--$, no data]

\begin{tabular}{|c|c|c|c|c|c|c|c|c|c|c|c|c|c|}
\hline \multirow{2}{*}{$\begin{array}{l}\text { Water } \\
\text { year }\end{array}$} & \multicolumn{12}{|c|}{ Monthly mean discharge } & \multirow{2}{*}{$\begin{array}{c}\text { Annual } \\
\text { discharge }\end{array}$} \\
\hline & October & November & December & January & February & March & April & May & June & July & August & September & \\
\hline 1969 & -- & -- & -- & -- & -- & -- & 374 & 302 & 95.3 & 5.64 & 0.44 & 0.23 & -- \\
\hline 1970 & 0.54 & 8.96 & 2.18 & 19.2 & 34.2 & 47.7 & 40.7 & 1.03 & 0.06 & 0.04 & 0.02 & 0.01 & 12.7 \\
\hline 1971 & 0.18 & 0.31 & 0.46 & 0.80 & 1.30 & 3.39 & 16.9 & 7.51 & 2.91 & 0.20 & 0.05 & 0.07 & 2.83 \\
\hline 1972 & 0.09 & 1.34 & 1.26 & 7.74 & 21.5 & 94.0 & 149 & 120 & 20.2 & 0.12 & 0.09 & 0.09 & 34.6 \\
\hline 1973 & 0.11 & 0.18 & 1.70 & 14.3 & 19.6 & 54.3 & 81.2 & 96.1 & 3.38 & 0.06 & 0.01 & 0.01 & 22.6 \\
\hline 1974 & 0.04 & 0.04 & 0.22 & 11.2 & 27.4 & 45.0 & 78.4 & 12.8 & 0.08 & 0.03 & 0.03 & 0.07 & 14.4 \\
\hline 1975 & 0.08 & 0.09 & 2.68 & 8.41 & 18.3 & 51.0 & 183 & 194 & 48.8 & 0.22 & 0.02 & 0.02 & 42.3 \\
\hline 1976 & 0.08 & 0.19 & 8.55 & 14.2 & 35.5 & 60.2 & 229 & 281 & 58.3 & 2.17 & 0.09 & 0.08 & 57.4 \\
\hline 1977 & 0.13 & 9.76 & 12.8 & 19.0 & 23.1 & 30.4 & 38.8 & 22.5 & 7.51 & 0.19 & 0.11 & 0.11 & 13.6 \\
\hline 1978 & 0.21 & 3.84 & 12.1 & 20.2 & 20.8 & 38.9 & 55.2 & 47.6 & 2.47 & 0.13 & 0.06 & 0.09 & 16.8 \\
\hline
\end{tabular}




\section{8-0.000-2M HARI RUD RIVER AT PUL-I-HASHIMI}

\section{(U.S. Geological Survey identification number: 342000061560000)}

LOCATION: Lat $34^{\circ} 20^{\prime} \mathrm{N}$., long $61^{\circ} 56^{\prime} \mathrm{E}$.

DRAINAGE AREA: $27,260 \mathrm{~km}^{2}$.

ELEVATION: 850 meters above mean sea level.

PERIOD OF RECORD: December 14, 1972 to September 30, 1978.

GAGE: Water-stage recorder. Staff gage at same site and datum prior to 1977 water year.

Annual mean discharge

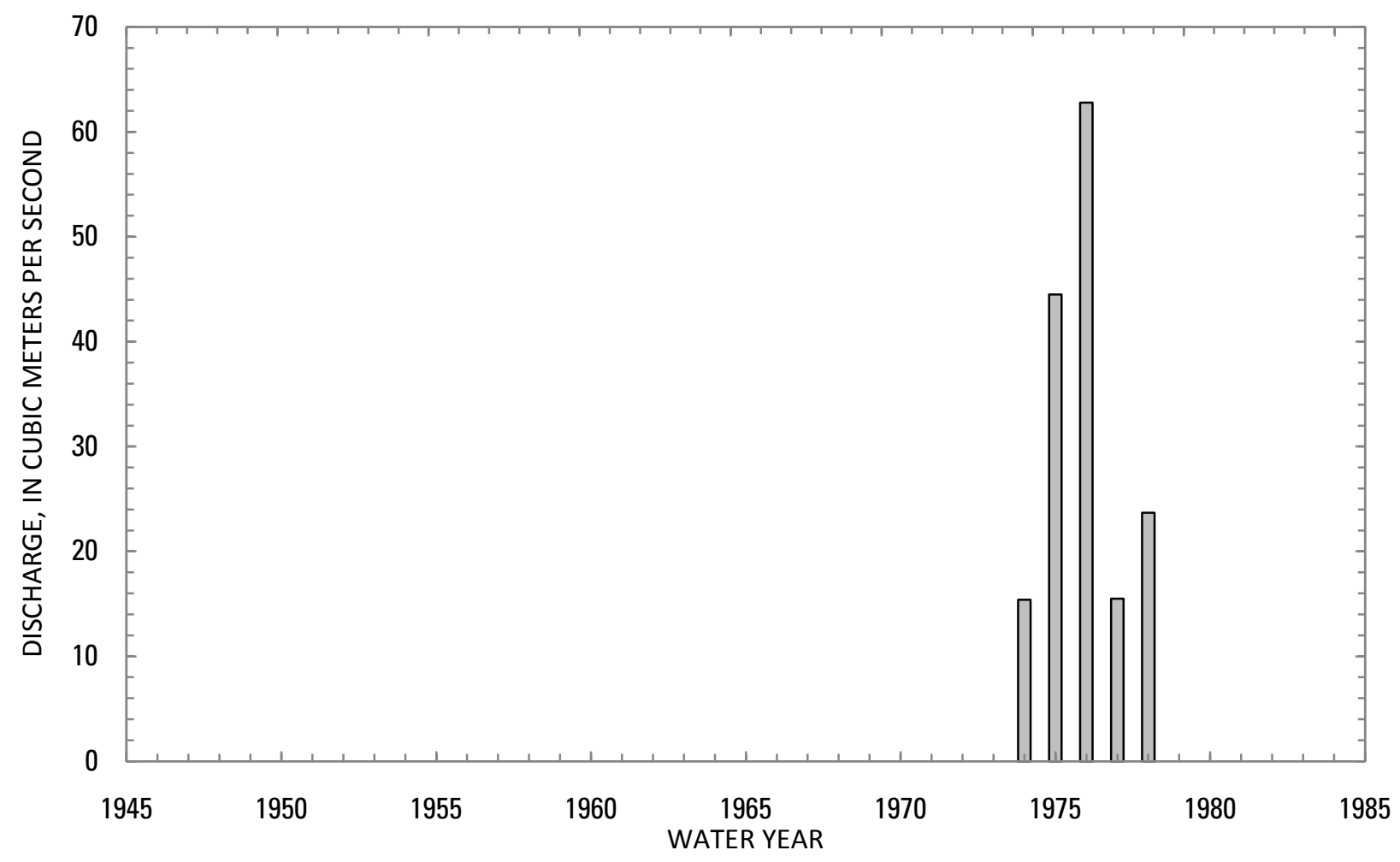




\section{8-0.000-2M HARI RUD RIVER AT PUL-I-HASHIMI, Continued}

Statistics of monthly and annual mean discharges $\left[\mathrm{m}^{3} / \mathrm{s}\right.$, cubic meters per second]

\begin{tabular}{|c|c|c|c|c|c|c|c|c|}
\hline \multirow[b]{2}{*}{ Month } & \multicolumn{2}{|c|}{ Maximum } & \multicolumn{2}{|c|}{ Minimum } & \multicolumn{4}{|c|}{ Mean } \\
\hline & $\begin{array}{c}\text { Discharge } \\
\left(\mathrm{m}^{3} / \mathrm{s}\right)\end{array}$ & $\begin{array}{c}\text { Water year } \\
\text { of } \\
\text { occurrence }\end{array}$ & $\begin{array}{c}\text { Discharge } \\
\left(\mathrm{m}^{3} / \mathrm{s}\right)\end{array}$ & $\begin{array}{c}\text { Water year } \\
\text { of } \\
\text { occurrence }\end{array}$ & $\begin{array}{c}\text { Discharge } \\
\left(\mathrm{m}^{3} / \mathrm{s}\right)\end{array}$ & $\begin{array}{c}\text { Standard } \\
\text { deviation } \\
\left(\mathrm{m}^{3} / \mathrm{s}\right)\end{array}$ & $\begin{array}{c}\text { Coefficient } \\
\text { of } \\
\text { variation }\end{array}$ & $\begin{array}{c}\text { Percentage } \\
\text { of annual } \\
\text { discharge }\end{array}$ \\
\hline October & 2.28 & 1978 & 1.05 & 1975 & 1.68 & 0.46 & 0.27 & 0.44 \\
\hline November & 12.2 & 1977 & 1.38 & 1975 & 4.96 & 4.58 & 0.92 & 1.30 \\
\hline December & 13.8 & 1978 & 3.71 & 1974 & 9.40 & 4.59 & 0.49 & 2.46 \\
\hline January & 20.4 & 1978 & 6.20 & 1975 & 13.0 & 5.28 & 0.41 & 3.40 \\
\hline February & 28.6 & 1976 & 9.16 & 1975 & 20.3 & 6.69 & 0.33 & 5.30 \\
\hline March & 68.3 & 1973 & 27.1 & 1977 & 48.3 & 14.5 & 0.30 & 12.6 \\
\hline April & 247 & 1976 & 57.0 & 1977 & 130 & 77.5 & 0.59 & 34.2 \\
\hline May & 315 & 1976 & 21.5 & 1974 & 127 & 115 & 0.91 & 33.4 \\
\hline June & 72.5 & 1976 & 0.74 & 1974 & 23.0 & 29.8 & 1.29 & 6.03 \\
\hline July & 3.01 & 1976 & 0.67 & 1974 & 1.52 & 0.85 & 0.56 & 0.40 \\
\hline August & 1.50 & 1977 & 0.71 & 1974 & 1.00 & 0.28 & 0.28 & 0.26 \\
\hline September & 1.28 & 1978 & 0.86 & 1974 & 1.11 & 0.17 & 0.15 & 0.29 \\
\hline Annual & 62.8 & 1976 & 15.4 & 1974 & 32.4 & 20.7 & 0.64 & 100 \\
\hline
\end{tabular}


8-0.000-2M HARI RUD RIVER AT PUL-I-HASHIMI, Continued

Monthly and annual flow duration, in cubic meters per second

\begin{tabular}{|c|c|c|c|c|c|c|c|c|c|c|c|c|c|}
\hline \multirow{2}{*}{$\begin{array}{l}\text { Percentage } \\
\text { of days } \\
\text { discharge } \\
\text { equaled or } \\
\text { exceeded }\end{array}$} & \multicolumn{12}{|c|}{ Month } & \multirow{2}{*}{ Annual } \\
\hline & October & November & December & January & February & March & April & May & June & July & August & September & \\
\hline 95 & 0.86 & 1.27 & 2.37 & 4.17 & 7.99 & 21.8 & 31.9 & 5.35 & 0.71 & 0.68 & 0.67 & 0.79 & 0.75 \\
\hline 90 & 1.14 & 1.33 & 3.00 & 4.98 & 9.10 & 24.8 & 46.6 & 16.2 & 0.75 & 0.69 & 0.75 & 0.81 & 0.88 \\
\hline 85 & 1.18 & 1.39 & 3.44 & 5.25 & 12.0 & 28.3 & 51.2 & 21.9 & 0.89 & 0.71 & 0.76 & 0.85 & 1.05 \\
\hline 80 & 1.32 & 1.49 & 3.88 & 5.64 & 13.0 & 30.4 & 57.4 & 24.9 & 1.00 & 0.86 & 0.78 & 0.87 & 1.20 \\
\hline 75 & 1.41 & 1.57 & 4.38 & 6.22 & 13.7 & 31.6 & 62.5 & 30.3 & 1.12 & 0.90 & 0.79 & 0.95 & 1.41 \\
\hline 70 & 1.42 & 1.66 & 6.34 & 6.49 & 15.5 & 33.6 & 68.0 & 37.9 & 1.53 & 0.92 & 0.80 & 1.00 & 1.63 \\
\hline 65 & 1.44 & 2.10 & 7.57 & 7.54 & 16.6 & 35.6 & 77.9 & 44.7 & 1.91 & 0.95 & 0.81 & 1.08 & 1.89 \\
\hline 60 & 1.46 & 2.18 & 8.61 & 11.1 & 17.2 & 37.5 & 83.5 & 52.0 & 2.13 & 1.00 & 0.87 & 1.11 & 2.28 \\
\hline 55 & 1.56 & 2.25 & 9.39 & 11.7 & 17.7 & 39.3 & 89.1 & 61.5 & 2.43 & 1.12 & 0.93 & 1.12 & 3.60 \\
\hline 50 & 1.62 & 2.48 & 9.77 & 12.3 & 18.2 & 41.3 & 100 & 87.9 & 3.56 & 1.15 & 0.95 & 1.13 & 6.97 \\
\hline 45 & 1.65 & 2.62 & 10.1 & 14.1 & 19.0 & 43.4 & 107 & 112 & 6.25 & 1.18 & 0.99 & 1.15 & 10.6 \\
\hline 40 & 1.71 & 2.75 & 10.4 & 15.3 & 20.0 & 46.5 & 118 & 135 & 9.47 & 1.46 & 1.01 & 1.19 & 13.6 \\
\hline 35 & 1.77 & 2.87 & 11.0 & 15.8 & 20.9 & 50.8 & 128 & 152 & 13.3 & 1.73 & 1.08 & 1.20 & 17.1 \\
\hline 30 & 1.86 & 3.22 & 11.6 & 16.3 & 21.7 & 56.3 & 136 & 175 & 18.4 & 1.79 & 1.09 & 1.21 & 20.9 \\
\hline 25 & 1.99 & 9.09 & 12.0 & 17.1 & 22.7 & 59.0 & 144 & 203 & 24.8 & 1.85 & 1.10 & 1.23 & 27.9 \\
\hline 20 & 2.05 & 11.5 & 12.6 & 18.9 & 25.8 & 63.2 & 161 & 235 & 33.2 & 1.90 & 1.12 & 1.26 & 37.5 \\
\hline 15 & 2.16 & 12.0 & 13.4 & 20.4 & 29.1 & 66.7 & 182 & 271 & 55.4 & 1.94 & 1.19 & 1.32 & 54.5 \\
\hline 10 & 2.23 & 12.8 & 14.0 & 21.1 & 31.0 & 78.3 & 233 & 319 & 87.2 & 1.99 & 1.56 & 1.47 & 85.1 \\
\hline 5 & 2.80 & 15.5 & 22.6 & 21.8 & 41.2 & 94.3 & 396 & 358 & 119 & 3.87 & 1.60 & 1.50 & 159 \\
\hline
\end{tabular}




\section{8-0.000-2M HARI RUD RIVER AT PUL-I-HASHIMI, Continued}

Probability of occurrence of annual high discharges

[m $\mathrm{m}^{3} / \mathrm{s}$, cubic meters per second; ng, not given]

\begin{tabular}{|c|c|c|c|c|c|c|}
\hline \multirow{2}{*}{$\begin{array}{l}\text { Exceedance } \\
\text { probability }\end{array}$} & \multirow{2}{*}{$\begin{array}{c}\text { Recurrence } \\
\text { interval } \\
\text { (years) }\end{array}$} & \multirow{2}{*}{$\begin{array}{c}\text { Maximum } \\
\text { instantaneous } \\
\text { discharge }^{1} \\
\left(\mathrm{~m}^{3} / \mathrm{s}\right)\end{array}$} & \multicolumn{4}{|c|}{ Maximum daily mean discharge $\left(\mathrm{m}^{3} / \mathrm{s}\right)$} \\
\hline & & & $\begin{array}{c}\text { 3-day } \\
\text { period }\end{array}$ & $\begin{array}{l}\text { 7-day } \\
\text { period }\end{array}$ & $\begin{array}{l}\text { 15-day } \\
\text { period }\end{array}$ & $\begin{array}{l}\text { 30-day } \\
\text { period }\end{array}$ \\
\hline 0.99 & 1.01 & 35.9 & ${ }^{2} 28.1$ & 29.4 & 28.9 & 27.7 \\
\hline 0.95 & 1.05 & 56.1 & 47.4 & 46.5 & 45.4 & 42.8 \\
\hline 0.90 & 1.11 & 72.4 & 63.4 & 60.5 & 58.4 & 54.7 \\
\hline 0.80 & 1.25 & 100 & 91.4 & 84.6 & 80.2 & 74.4 \\
\hline 0.50 & 2 & 197 & 191 & 170 & 152 & 139 \\
\hline 0.20 & 5 & 413 & 420 & 366 & 303 & 274 \\
\hline 0.10 & 10 & 628 & 648 & 564 & 442 & 398 \\
\hline 0.04 & 25 & 1,000 & 1,050 & 915 & 672 & 602 \\
\hline 0.02 & 50 & 1,370 & 1,440 & 1,270 & 887 & 793 \\
\hline 0.01 & 100 & 1,840 & 1,920 & 1,710 & 1,150 & 1,020 \\
\hline 0.005 & 200 & 2,410 & 2,520 & 2,280 & 1,460 & 1,300 \\
\hline 0.002 & 500 & 3,390 & ng & ng & ng & ng \\
\hline
\end{tabular}

\section{8-0.000-2M HARI RUD RIVER AT PUL-I-HASHIMI, Continued}

Probability of occurrence of annual low discharges $\left[\mathrm{m}^{3} / \mathrm{s}\right.$, meters per second]

\begin{tabular}{|c|c|c|c|c|c|c|c|c|c|c|}
\hline \multirow{3}{*}{$\begin{array}{c}\text { Nonexceedance } \\
\text { probability }\end{array}$} & \multirow{3}{*}{$\begin{array}{c}\text { Recurrence } \\
\text { interval } \\
\text { (years) }\end{array}$} & \multicolumn{9}{|c|}{ Minimum daily mean discharge $\left(\mathrm{m}^{3} / \mathrm{s}\right)$} \\
\hline & & \multicolumn{9}{|c|}{ Number of consecutive days } \\
\hline & & 1 & 3 & 7 & 14 & 30 & 60 & 90 & 120 & 183 \\
\hline 0.05 & 20 & 0.61 & 0.60 & 0.60 & 0.60 & 0.61 & 0.61 & 0.63 & 0.66 & 0.67 \\
\hline 0.10 & 10 & 0.67 & 0.67 & 0.67 & 0.67 & 0.68 & 0.68 & 0.73 & 0.79 & 0.90 \\
\hline 0.20 & 5 & 0.75 & 0.75 & 0.76 & 0.77 & 0.77 & 0.79 & 0.86 & 0.96 & 1.26 \\
\hline 0.50 & 2 & 0.92 & 0.93 & 0.95 & 0.95 & 0.96 & 1.00 & 1.12 & 1.29 & 2.33 \\
\hline
\end{tabular}




\section{8-0.000-2M HARI RUD RIVER AT PUL-I-HASHIMI, Continued}

Probability of occurrence of seasonal low discharges $\left[\mathrm{m}^{3} / \mathrm{s}\right.$, meters per second]

\begin{tabular}{|c|c|c|c|c|c|c|c|c|c|}
\hline \multirow{3}{*}{$\begin{array}{c}\text { Nonexceedance } \\
\text { probability }\end{array}$} & \multirow{3}{*}{$\begin{array}{c}\text { Recurrence } \\
\text { interval } \\
\text { (years) }\end{array}$} & \multicolumn{8}{|c|}{ Minimum daily mean discharge $\left(\mathrm{m}^{3} / \mathrm{s}\right)$} \\
\hline & & \multicolumn{8}{|c|}{ Number of consecutive days } \\
\hline & & 1 & 7 & 14 & 30 & 1 & 7 & 14 & 30 \\
\hline & & \multicolumn{4}{|c|}{ December-January-February } & \multicolumn{4}{|c|}{ March-April-May } \\
\hline 0.05 & 20 & 1.23 & 1.35 & 1.67 & 2.85 & 0.98 & 1.30 & 4.54 & 18.0 \\
\hline 0.10 & 10 & 1.77 & 1.98 & 2.36 & 3.79 & 2.40 & 3.16 & 7.87 & 21.5 \\
\hline 0.20 & 5 & 2.66 & 3.04 & 3.49 & 5.22 & 5.81 & 7.57 & 13.8 & 26.6 \\
\hline \multirow[t]{2}{*}{0.50} & 2 & 5.40 & 6.30 & 6.78 & 8.86 & 18.0 & 23.2 & 29.3 & 39.2 \\
\hline & & \multicolumn{4}{|c|}{ June-July-August } & \multicolumn{4}{|c|}{ September-October-November } \\
\hline 0.05 & 20 & 0.62 & 0.62 & 0.62 & 0.63 & 0.60 & 0.68 & 0.70 & 0.80 \\
\hline 0.10 & 10 & 0.67 & 0.68 & 0.67 & 0.68 & 0.67 & 0.74 & 0.76 & 0.86 \\
\hline 0.20 & 5 & 0.73 & 0.75 & 0.75 & 0.75 & 0.77 & 0.82 & 0.84 & 0.94 \\
\hline 0.50 & 2 & 0.88 & 0.91 & 0.92 & 0.93 & 0.95 & 0.98 & 0.99 & 1.09 \\
\hline
\end{tabular}

8-0.000-2M HARI RUD RIVER AT PUL-I-HASHIMI, Continued

Annual peak discharges

$\left[\mathrm{m}^{3} / \mathrm{s}\right.$, meters per second]

\begin{tabular}{|c|c|c|c|c|c|}
\hline \multicolumn{3}{|c|}{$\begin{array}{c}\text { Annual peak discharge, } \\
\text { by year }\end{array}$} & \multicolumn{3}{|c|}{$\begin{array}{l}\text { Annual peak discharge, } \\
\text { from highest to lowest }\end{array}$} \\
\hline $\begin{array}{l}\text { Water } \\
\text { year }\end{array}$ & Date & $\begin{array}{c}\text { Peak } \\
\text { discharge } \\
\left(\mathrm{m}^{3} / \mathrm{s}\right)\end{array}$ & $\begin{array}{l}\text { Water } \\
\text { year }\end{array}$ & Date & $\begin{array}{c}\text { Peak } \\
\text { discharge } \\
\left(\mathrm{m}^{3} / \mathrm{s}\right)\end{array}$ \\
\hline 1973 & May 1, 1973 & 190 & 1976 & April 23, 1976 & 680 \\
\hline 1974 & April 4, 1974 & 111 & 1975 & April 26, 1975 & 448 \\
\hline 1975 & April 26, 1975 & 448 & 1973 & May 1,1973 & 190 \\
\hline 1976 & April 23, 1976 & 680 & 1978 & April 19, 1978 & 169 \\
\hline 1977 & April 15, 1977 & 71.3 & 1974 & April 4, 1974 & 111 \\
\hline 1978 & April 19, 1978 & 169 & 1977 & April 15, 1977 & 71.3 \\
\hline
\end{tabular}


8-0.000-2M HARI RUD RIVER AT PUL-I-HASHIMI, Continued

Monthly and annual mean discharges, in cubic meters per second $[--$, no data]

\begin{tabular}{|c|c|c|c|c|c|c|c|c|c|c|c|c|c|}
\hline \multirow{2}{*}{$\begin{array}{c}\text { Water } \\
\text { year }\end{array}$} & \multicolumn{12}{|c|}{ Monthly mean discharge } & \multirow{2}{*}{$\begin{array}{c}\text { Annual } \\
\text { discharge }\end{array}$} \\
\hline & October & November & December & January & February & March & April & May & June & July & August & September & \\
\hline 1973 & -- & -- & -- & 15.2 & 19.4 & 68.3 & 119 & 101 & 7.80 & 1.08 & 1.03 & 1.13 & -- \\
\hline 1974 & 1.46 & 1.86 & 3.71 & 8.19 & 21.4 & 46.5 & 78.4 & 21.5 & 0.74 & 0.67 & 0.71 & 0.86 & 15.4 \\
\hline 1975 & 1.05 & 1.38 & 5.50 & 6.20 & 9.16 & 38.8 & 204 & 216 & 47.5 & 1.64 & 0.80 & 0.97 & 44.5 \\
\hline 1976 & 1.84 & 2.56 & 10.6 & 11.9 & 28.6 & 58.0 & 247 & 315 & 72.5 & 3.01 & 1.09 & 1.27 & 62.8 \\
\hline 1977 & 1.76 & 12.2 & 13.4 & 16.0 & 17.9 & 27.1 & 57.0 & 30.3 & 6.09 & 1.84 & 1.50 & 1.18 & 15.5 \\
\hline 1978 & 2.28 & 6.80 & 13.8 & 20.4 & 25.1 & 50.9 & 77.5 & 80.7 & 3.53 & 0.92 & 0.88 & 1.28 & 23.7 \\
\hline
\end{tabular}




\section{8-0.000-3S HARI RUD RIVER AT PUL-I-PASHTOON}

\section{(U.S. Geological Survey identification number: 341700062130000)}

LOCATION: Lat $34^{\circ} 17^{\prime} \mathrm{N}$., long $62^{\circ} 13^{\prime} \mathrm{E}$.

DRAINAGE AREA: $26,130 \mathrm{~km}^{2}$.

ELEVATION: 940 meters above mean sea level.

PERIOD OF RECORD: April 1, 1963 to January 31, 1977.

GAGE: Water-stage recorder. Staff gage at same site and datum prior to 1969 water year.

Annual mean discharge

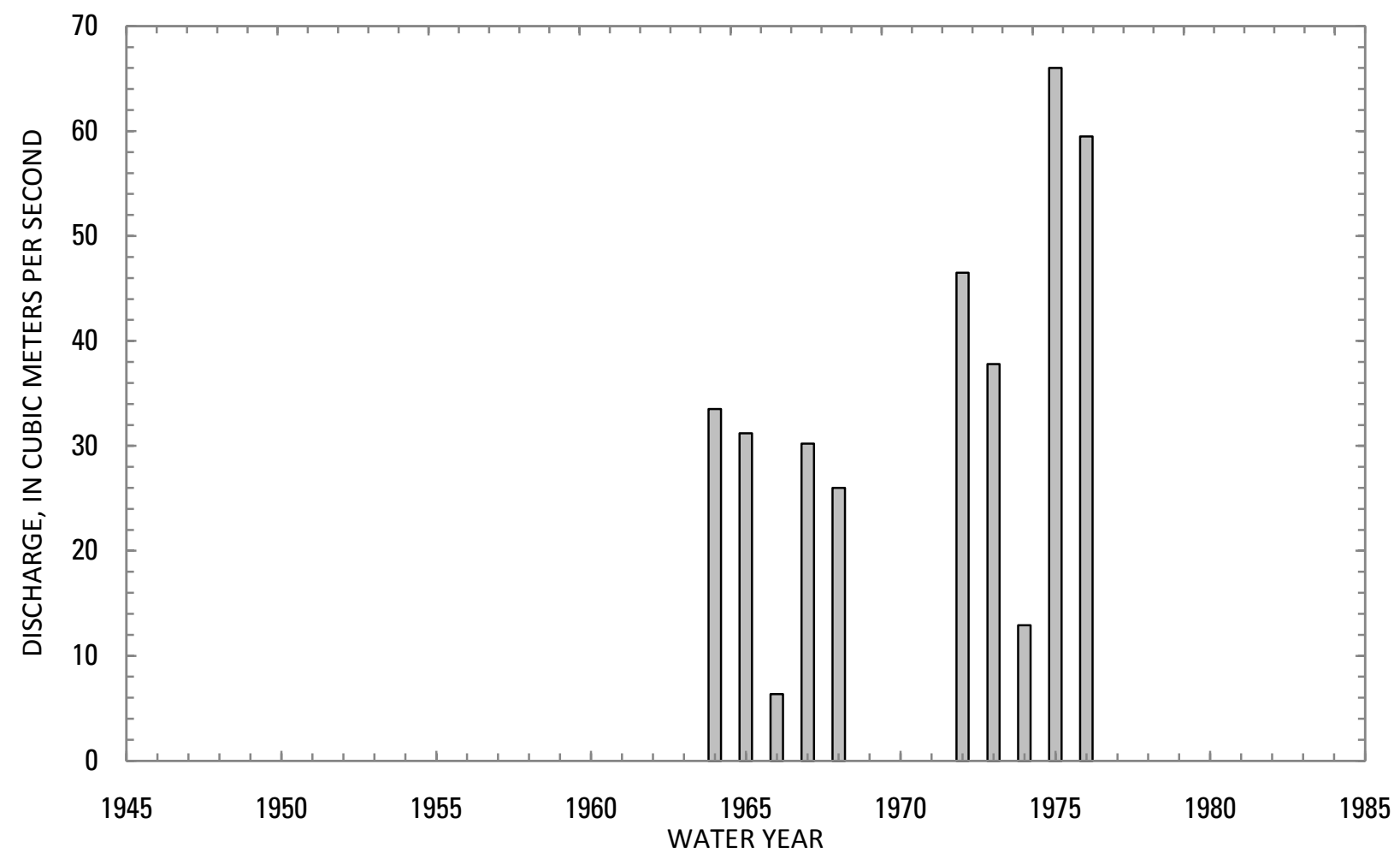




\section{8-0.000-3S HARI RUD RIVER AT PUL-I-PASHTOON, Continued}

Statistics of monthly and annual mean discharges $\left[\mathrm{m}^{3} / \mathrm{s}\right.$, cubic meters per second]

\begin{tabular}{|c|c|c|c|c|c|c|c|c|}
\hline \multirow[b]{2}{*}{ Month } & \multicolumn{2}{|c|}{ Maximum } & \multicolumn{2}{|c|}{ Minimum } & \multicolumn{4}{|c|}{ Mean } \\
\hline & $\begin{array}{c}\text { Discharge } \\
\left(\mathrm{m}^{3} / \mathrm{s}\right)\end{array}$ & $\begin{array}{c}\text { Water year } \\
\text { of } \\
\text { occurrence }\end{array}$ & $\begin{array}{c}\text { Discharge } \\
\left(\mathrm{m}^{3} / \mathrm{s}\right)\end{array}$ & $\begin{array}{c}\text { Water year } \\
\text { of } \\
\text { occurrence }\end{array}$ & $\begin{array}{c}\text { Discharge } \\
\left(\mathrm{m}^{3} / \mathrm{s}\right)\end{array}$ & $\begin{array}{c}\text { Standard } \\
\text { deviation } \\
\left(\mathrm{m}^{3} / \mathrm{s}\right)\end{array}$ & $\begin{array}{c}\text { Coefficient } \\
\text { of } \\
\text { variation }\end{array}$ & $\begin{array}{c}\text { Percentage } \\
\text { of annual } \\
\text { discharge }\end{array}$ \\
\hline October & 1.03 & 1967 & 0.35 & 1975 & 0.57 & 0.20 & 0.35 & 0.14 \\
\hline November & 9.59 & 1977 & 0.35 & 1975 & 1.83 & 2.68 & 1.47 & 0.45 \\
\hline December & 8.65 & 1977 & 0.05 & 1965 & 2.29 & 3.15 & 1.37 & 0.56 \\
\hline January & 12.3 & 1964 & 0.89 & 1967 & 5.69 & 3.62 & 0.64 & 1.40 \\
\hline February & 29.6 & 1964 & 1.37 & 1975 & 12.9 & 9.03 & 0.70 & 3.16 \\
\hline March & 128 & 1972 & 10.5 & 1967 & 50.7 & 34.2 & 0.67 & 12.5 \\
\hline April & 266 & 1975 & 30.0 & 1963 & 133 & 82.4 & 0.62 & 32.6 \\
\hline May & 398 & 1975 & 13.9 & 1974 & 168 & 112 & 0.67 & 41.3 \\
\hline June & 79.5 & 1975 & 0.21 & 1974 & 31.6 & 30.3 & 0.96 & 7.75 \\
\hline July & 1.40 & 1976 & 0.02 & 1968 & 0.42 & 0.40 & 0.95 & 0.10 \\
\hline August & 0.52 & 1967 & 0.04 & 1968 & 0.28 & 0.17 & 0.62 & 0.07 \\
\hline September & 0.78 & 1964 & 0.08 & 1968 & 0.34 & 0.18 & 0.53 & 0.08 \\
\hline Annual & 66.0 & 1975 & 6.35 & 1966 & 35.0 & 18.7 & 0.53 & 100 \\
\hline
\end{tabular}


8-0.000-3S HARI RUD RIVER AT PUL-I-PASHTOON, Continued

Monthly and annual flow duration, in cubic meters per second

[ng, not given]

\begin{tabular}{|c|c|c|c|c|c|c|c|c|c|c|c|c|c|}
\hline \multirow{2}{*}{$\begin{array}{l}\text { Percentage } \\
\text { of days } \\
\text { discharge } \\
\text { equaled or } \\
\text { exceeded }\end{array}$} & \multicolumn{12}{|c|}{ Month } & \multirow{2}{*}{ Annua } \\
\hline & October & November & December & January & February & March & April & May & June & July & August & September & \\
\hline 95 & 0.24 & 0.24 & 0.04 & 0.41 & 1.13 & 3.48 & 4.84 & 1.53 & 0.23 & 0.03 & 0.03 & 0.07 & 0.06 \\
\hline 90 & 0.32 & 0.33 & 0.06 & 0.67 & 1.40 & 5.42 & 13.3 & 12.1 & 0.27 & 0.03 & 0.04 & 0.10 & 0.18 \\
\hline 85 & 0.33 & 0.36 & 0.08 & 0.80 & 1.90 & 6.33 & 30.4 & 32.8 & 0.38 & 0.04 & 0.06 & 0.14 & 0.28 \\
\hline 80 & 0.38 & 0.39 & 0.30 & 0.89 & 3.61 & 9.34 & 44.4 & 49.2 & 0.52 & 0.08 & 0.10 & 0.22 & 0.35 \\
\hline 75 & 0.40 & 0.43 & 0.36 & 0.99 & 4.62 & 12.0 & 58.8 & 67.0 & 0.73 & 0.14 & 0.12 & 0.23 & 0.41 \\
\hline 70 & 0.42 & 0.48 & 0.42 & 1.16 & 6.55 & 15.6 & 68.7 & 86.2 & 0.89 & 0.16 & 0.17 & 0.28 & 0.47 \\
\hline 65 & 0.43 & 0.53 & 0.47 & 1.39 & 8.04 & 21.9 & 76.0 & 112 & 1.00 & 0.18 & 0.19 & 0.30 & 0.52 \\
\hline 60 & 0.45 & 0.60 & 0.52 & 1.91 & 8.87 & 25.5 & 83.5 & 131 & 1.20 & 0.20 & 0.22 & 0.30 & 0.63 \\
\hline 55 & 0.47 & 0.67 & 0.56 & 2.52 & 9.62 & 28.7 & 94.4 & 147 & 2.15 & 0.24 & 0.25 & 0.31 & 0.83 \\
\hline 50 & 0.49 & 0.73 & 0.71 & 3.08 & 10.3 & 32.8 & 105 & 159 & 5.59 & 0.28 & 0.28 & 0.32 & 1.02 \\
\hline 45 & 0.50 & 0.79 & 0.91 & 3.82 & 11.0 & 37.9 & 119 & 171 & 9.77 & 0.32 & 0.31 & 0.33 & 1.46 \\
\hline 40 & 0.52 & 0.89 & 0.98 & 5.12 & 12.1 & 45.7 & 133 & 182 & 14.6 & 0.36 & 0.33 & 0.34 & 3.47 \\
\hline 35 & 0.53 & 1.00 & 1.06 & 6.49 & 14.1 & 51.7 & 145 & 196 & 21.0 & 0.42 & 0.40 & 0.39 & 6.92 \\
\hline 30 & 0.56 & 1.12 & 2.09 & 7.56 & 18.5 & 61.5 & 158 & 216 & 31.3 & 0.49 & 0.42 & 0.41 & 10.7 \\
\hline 25 & 0.62 & 1.30 & 2.69 & 8.74 & 20.4 & 68.6 & 170 & 235 & 46.6 & 0.57 & 0.44 & 0.42 & 21.0 \\
\hline 20 & 0.69 & 1.89 & 3.86 & 9.91 & 21.5 & 75.9 & 191 & 270 & 62.7 & 0.65 & 0.46 & 0.44 & 40.5 \\
\hline 15 & 0.76 & 3.45 & 7.30 & 11.1 & 22.5 & 85.8 & 218 & 310 & 84.4 & 0.74 & 0.49 & 0.47 & 77.5 \\
\hline 10 & 0.98 & 6.21 & 8.23 & 12.2 & 27.4 & 111 & 269 & 344 & 103 & 0.85 & 0.53 & 0.52 & 131 \\
\hline 5 & 1.31 & 8.68 & 9.33 & 17.8 & 33.6 & 184 & 397 & 378 & 139 & 1.06 & 0.58 & $\mathrm{ng}$ & 209 \\
\hline
\end{tabular}




\section{8-0.000-3S HARI RUD RIVER AT PUL-I-PASHTOON, Continued}

Probability of occurrence of annual high discharges

[ $\mathrm{m}^{3} / \mathrm{s}$, cubic meters per second; ng, not given]

\begin{tabular}{|c|c|c|c|c|c|c|}
\hline \multirow{2}{*}{$\begin{array}{c}\text { Exceedance } \\
\text { probability }\end{array}$} & \multirow{2}{*}{$\begin{array}{c}\text { Recurrence } \\
\text { interval } \\
\text { (years) }\end{array}$} & \multirow{2}{*}{$\begin{array}{l}\text { Maximum } \\
\text { instantaneous } \\
\text { discharge } \\
\left(\mathrm{m}^{3} / \mathrm{s}\right)\end{array}$} & \multicolumn{4}{|c|}{ Maximum daily mean discharge $\left(\mathrm{m}^{3} / \mathrm{s}\right)$} \\
\hline & & & $\begin{array}{l}\text { 3-day } \\
\text { period }\end{array}$ & $\begin{array}{l}\text { 7-day } \\
\text { period }\end{array}$ & $\begin{array}{l}\text { 15-day } \\
\text { period }\end{array}$ & $\begin{array}{l}\text { 30-day } \\
\text { period }\end{array}$ \\
\hline 0.99 & 1.01 & 84.3 & 66.5 & 49.9 & 31.2 & 23.7 \\
\hline 0.95 & 1.05 & 132 & 110 & 88.6 & 64.5 & 51.3 \\
\hline 0.90 & 1.11 & 165 & 141 & 117 & 90.6 & 73.6 \\
\hline 0.80 & 1.25 & 215 & 187 & 160 & 131 & 109 \\
\hline 0.50 & 2 & 341 & 308 & 272 & 234 & 202 \\
\hline 0.20 & 5 & 518 & 476 & 423 & 360 & 319 \\
\hline 0.10 & 10 & 632 & 584 & 514 & 427 & 384 \\
\hline 0.04 & 25 & 772 & 714 & 619 & 495 & 449 \\
\hline 0.02 & 50 & 873 & 805 & 690 & 534 & 488 \\
\hline 0.01 & 100 & 970 & 892 & 754 & 567 & 520 \\
\hline 0.005 & 200 & 1,060 & 975 & 813 & 593 & 546 \\
\hline 0.002 & 500 & 1,190 & $\mathrm{ng}$ & ng & ng & ng \\
\hline
\end{tabular}

\section{8-0.000-3S HARI RUD RIVER AT PUL-I-PASHTOON, Continued}

Probability of occurrence of annual low discharges $\left[\mathrm{m}^{3} / \mathrm{s}\right.$, meters per second]

\begin{tabular}{|c|c|c|c|c|c|c|c|c|c|c|}
\hline \multirow{3}{*}{$\begin{array}{c}\text { Nonexceedance } \\
\text { probability }\end{array}$} & \multirow{3}{*}{$\begin{array}{c}\text { Recurrence } \\
\text { interval } \\
\text { (years) }\end{array}$} & \multicolumn{9}{|c|}{ Minimum daily mean discharge $\left(\mathrm{m}^{3} / \mathrm{s}\right)$} \\
\hline & & \multicolumn{9}{|c|}{ Number of consecutive days } \\
\hline & & 1 & 3 & 7 & 14 & 30 & 60 & 90 & 120 & 183 \\
\hline 0.05 & 20 & 0.01 & 0.01 & 0.01 & 0.01 & 0.02 & 0.04 & 0.10 & 0.15 & 0.30 \\
\hline 0.10 & 10 & 0.01 & 0.01 & 0.01 & 0.02 & 0.03 & 0.07 & 0.13 & 0.19 & 0.31 \\
\hline 0.20 & 5 & 0.02 & 0.02 & 0.02 & 0.03 & 0.05 & 0.11 & 0.17 & 0.23 & 0.35 \\
\hline 0.50 & 2 & 0.06 & 0.06 & 0.07 & 0.09 & 0.13 & 0.22 & 0.26 & 0.32 & 0.47 \\
\hline
\end{tabular}




\section{8-0.000-3S HARI RUD RIVER AT PUL-I-PASHTOON, Continued}

Probability of occurrence of seasonal low discharges $\left[\mathrm{m}^{3} / \mathrm{s}\right.$, meters per second]

\begin{tabular}{|c|c|c|c|c|c|c|c|c|c|}
\hline \multirow{3}{*}{$\begin{array}{c}\text { Nonexceedance } \\
\text { probability }\end{array}$} & \multirow{3}{*}{$\begin{array}{c}\text { Recurrence } \\
\text { interval } \\
\text { (years) }\end{array}$} & \multicolumn{8}{|c|}{ Minimum daily mean discharge $\left(\mathrm{m}^{3} / \mathrm{s}\right)$} \\
\hline & & \multicolumn{8}{|c|}{ Number of consecutive days } \\
\hline & & 1 & 7 & 14 & 30 & 1 & 7 & 14 & 30 \\
\hline & & \multicolumn{4}{|c|}{ December-January-February } & \multicolumn{4}{|c|}{ March-April-May } \\
\hline 0.05 & 20 & 0.01 & 0.01 & 0.01 & 0.03 & 0.28 & 0.38 & 0.92 & 6.90 \\
\hline 0.10 & 10 & 0.02 & 0.03 & 0.03 & 0.06 & 0.59 & 0.84 & 1.68 & 10.2 \\
\hline 0.20 & 5 & 0.05 & 0.07 & 0.09 & 0.15 & 1.34 & 2.06 & 3.38 & 16.0 \\
\hline \multirow[t]{2}{*}{0.50} & 2 & 0.33 & 0.44 & 0.51 & 0.70 & 5.53 & 8.66 & 11.8 & 35.3 \\
\hline & & \multicolumn{4}{|c|}{ June-July-August } & \multicolumn{4}{|c|}{ September-October-November } \\
\hline 0.05 & 20 & 0 & 0.01 & 0.02 & 0.02 & 0.01 & 0.08 & 0.10 & 0.14 \\
\hline 0.10 & 10 & 0 & 0.02 & 0.03 & 0.04 & 0.03 & 0.10 & 0.14 & 0.17 \\
\hline 0.20 & 5 & 0.03 & 0.04 & 0.05 & 0.07 & 0.06 & 0.14 & 0.18 & 0.22 \\
\hline 0.50 & 2 & 0.13 & 0.14 & 0.15 & 0.20 & 0.19 & 0.24 & 0.29 & 0.33 \\
\hline
\end{tabular}

\section{8-0.000-3S HARI RUD RIVER AT PUL-I-PASHTOON, Continued}

\section{Annual peak discharges}

$\left[\mathrm{m}^{3} / \mathrm{s}\right.$, meters per second]

\begin{tabular}{|c|c|c|c|c|c|}
\hline \multicolumn{3}{|c|}{$\begin{array}{c}\text { Annual peak discharge, } \\
\text { by year }\end{array}$} & \multicolumn{3}{|c|}{$\begin{array}{l}\text { Annual peak discharge, } \\
\text { from highest to lowest }\end{array}$} \\
\hline $\begin{array}{l}\text { Water } \\
\text { year }\end{array}$ & Date & $\begin{array}{c}\text { Peak } \\
\text { discharge } \\
\left(\mathrm{m}^{3} / \mathrm{s}\right)\end{array}$ & $\begin{array}{l}\text { Water } \\
\text { year }\end{array}$ & Date & $\begin{array}{c}\text { Peak } \\
\text { discharge } \\
\left(\mathrm{m}^{3} / \mathrm{s}\right)\end{array}$ \\
\hline 1963 & May 8, 1963 & 320 & 1975 & May 17,1975 & 652 \\
\hline 1964 & April 26, 1964 & 275 & 1976 & April 23, 1976 & 650 \\
\hline 1965 & May 7, 1965 & 259 & 1967 & April 27, 1967 & 560 \\
\hline 1966 & April 27, 1966 & 135 & 1972 & May 8, 1972 & 410 \\
\hline 1967 & April 27, 1967 & 560 & 1968 & May 1,1968 & 340 \\
\hline 1968 & May 1, 1968 & 340 & 1973 & April 25, 1973 & 333 \\
\hline 1972 & May 8, 1972 & 410 & 1963 & May 8, 1963 & 320 \\
\hline 1973 & April 25, 1973 & 333 & 1964 & April 26, 1964 & 275 \\
\hline 1974 & April 4, 1974 & 150 & 1965 & May 7, 1965 & 259 \\
\hline 1975 & May 17,1975 & 652 & 1974 & April 4, 1974 & 150 \\
\hline 1976 & April 23, 1976 & 650 & 1966 & April 27, 1966 & 135 \\
\hline
\end{tabular}


8-0.000-3S HARI RUD RIVER AT PUL-I-PASHTOON, Continued

Monthly and annual mean discharges, in cubic meters per second $[--$, no data]

\begin{tabular}{|c|c|c|c|c|c|c|c|c|c|c|c|c|c|}
\hline \multirow{2}{*}{$\begin{array}{l}\text { Water } \\
\text { year }\end{array}$} & \multicolumn{12}{|c|}{ Monthly mean discharge } & \multirow{2}{*}{$\begin{array}{c}\text { Annual } \\
\text { discharge }\end{array}$} \\
\hline & October & November & December & January & February & March & April & May & June & July & August & September & \\
\hline 1963 & -- & -- & -- & -- & -- & -- & 30.0 & 130 & 33.1 & 0.19 & 0.30 & 0.23 & -- \\
\hline 1964 & 0.43 & 2.24 & 0.07 & 12.3 & 29.6 & 64.8 & 167 & 118 & 7.11 & 0.65 & 0.51 & 0.78 & 33.5 \\
\hline 1965 & 0.44 & 0.46 & 0.05 & 6.59 & 14.6 & 48.1 & 81.1 & 182 & 37.9 & 0.28 & 0.30 & 0.29 & 31.2 \\
\hline 1966 & 0.46 & 0.66 & 1.00 & 3.29 & 5.68 & 12.0 & 36.7 & 15.3 & 0.76 & 0.35 & 0.14 & 0.31 & 6.35 \\
\hline 1967 & 1.03 & 0.96 & 0.45 & 0.89 & 1.85 & 10.5 & 143 & 180 & 21.2 & 0.75 & 0.52 & 0.42 & 30.2 \\
\hline 1968 & 0.43 & 0.53 & 0.93 & 4.16 & 8.30 & 35.2 & 49.9 & 145 & 66.7 & 0.02 & 0.04 & 0.08 & 26.0 \\
\hline 1972 & 0.56 & 2.62 & 2.05 & 5.63 & 22.4 & 128 & 200 & 181 & 14.9 & 0.04 & 0.04 & 0.19 & 46.5 \\
\hline 1973 & 0.56 & 1.33 & 2.88 & 6.68 & 14.3 & 79.3 & 165 & 173 & 8.13 & 0.23 & 0.24 & 0.40 & 37.8 \\
\hline 1974 & 0.69 & 0.44 & 0.52 & 3.54 & 12.1 & 43.1 & 81.0 & 13.9 & 0.21 & 0.20 & 0.17 & 0.26 & 12.9 \\
\hline 1975 & 0.35 & 0.35 & 0.43 & 0.94 & 1.37 & 39.7 & 266 & 398 & 79.5 & 0.50 & 0.35 & 0.35 & 66.0 \\
\hline 1976 & 0.80 & 0.95 & 8.19 & 8.58 & 18.5 & 46.6 & 239 & 311 & 77.5 & 1.40 & 0.47 & 0.45 & 59.5 \\
\hline 1977 & 0.53 & 9.59 & 8.65 & 10.0 & -- & -- & -- & -- & -- & -- & -- & -- & -- \\
\hline
\end{tabular}




\section{8-0.000-4M HARI RUD RIVER AT ROBAT-I-AKHOND}

\section{(U.S. Geological Survey identification number: 341600062560000 )}

LOCATION: Lat $34^{\circ} 16^{\prime} \mathrm{N}$., long 62 $56^{\circ} \mathrm{E}$.

DRAINAGE AREA: $21,630 \mathrm{~km}^{2}$.

ELEVATION: 1,170 meters above mean sea level.

PERIOD OF RECORD: April 18, 1966 to September 30, 1978.

GAGE: Water-stage recorder. Staff gage at same site and datum prior to 1969 water year.

Annual mean discharge

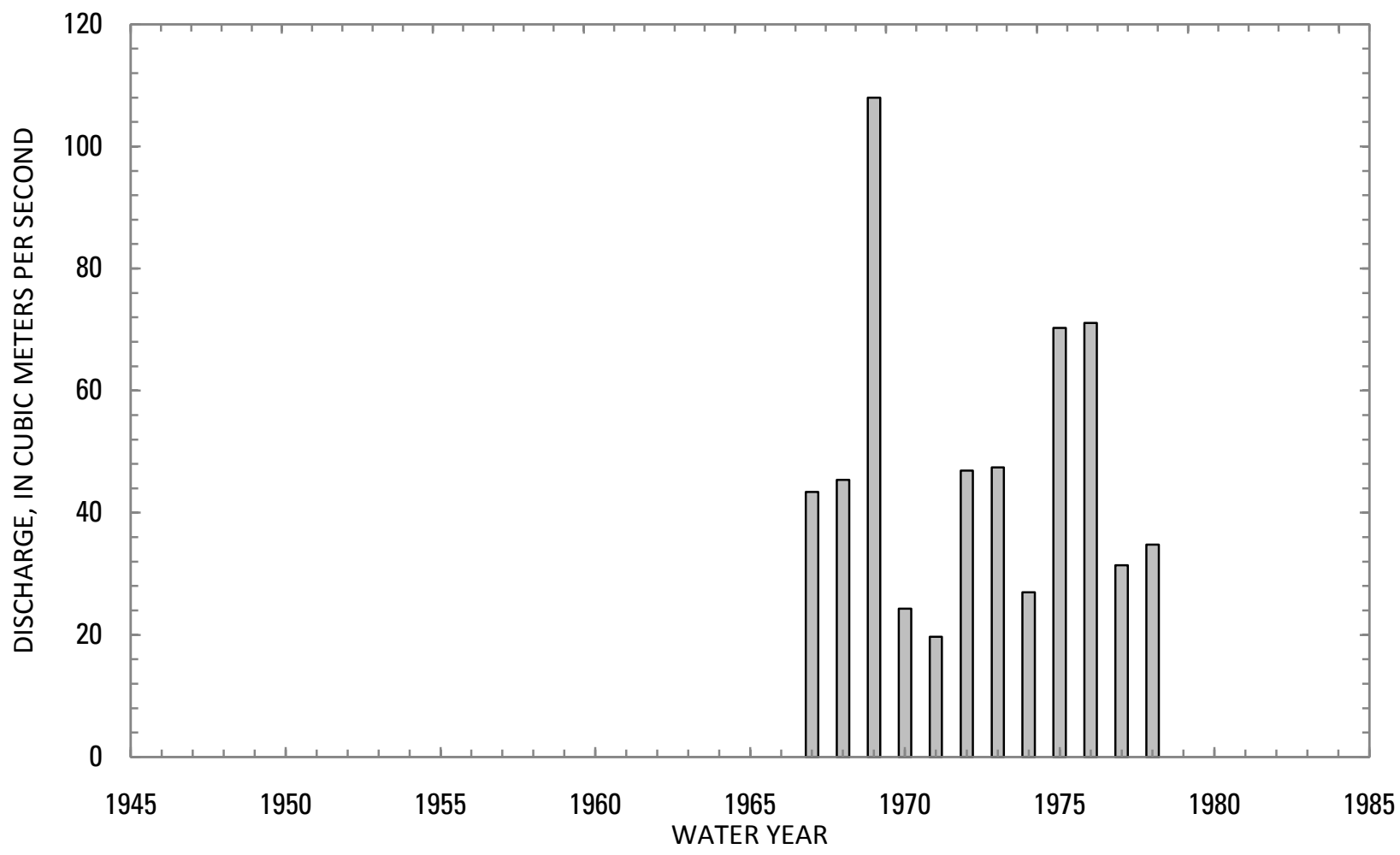




\section{8-0.000-4M HARI RUD RIVER AT ROBAT-I-AKHOND, Continued}

Statistics of monthly and annual mean discharges $\left[\mathrm{m}^{3} / \mathrm{s}\right.$, cubic meters per second]

\begin{tabular}{|c|c|c|c|c|c|c|c|c|}
\hline \multirow[b]{2}{*}{ Month } & \multicolumn{2}{|c|}{ Maximum } & \multicolumn{2}{|c|}{ Minimum } & \multicolumn{4}{|c|}{ Mean } \\
\hline & $\begin{array}{c}\text { Discharge } \\
\left(\mathrm{m}^{3} / \mathrm{s}\right)\end{array}$ & $\begin{array}{c}\text { Water year } \\
\text { of } \\
\text { occurrence }\end{array}$ & $\begin{array}{c}\text { Discharge } \\
\left(\mathrm{m}^{3} / \mathrm{s}\right)\end{array}$ & $\begin{array}{c}\text { Water year } \\
\text { of } \\
\text { occurrence }\end{array}$ & $\begin{array}{c}\text { Discharge } \\
\left(\mathrm{m}^{3} / \mathrm{s}\right)\end{array}$ & $\begin{array}{c}\text { Standard } \\
\text { deviation } \\
\left(\mathrm{m}^{3} / \mathrm{s}\right)\end{array}$ & $\begin{array}{c}\text { Coefficient } \\
\text { of } \\
\text { variation }\end{array}$ & $\begin{array}{c}\text { Percentage } \\
\text { of annual } \\
\text { discharge }\end{array}$ \\
\hline October & 14.2 & 1970 & 3.23 & 1972 & 6.50 & 3.79 & 0.58 & 1.17 \\
\hline November & 28.5 & 1970 & 3.97 & 1972 & 10.1 & 7.52 & 0.75 & 1.82 \\
\hline December & 25.5 & 1969 & 3.84 & 1972 & 12.2 & 7.05 & 0.58 & 2.19 \\
\hline January & 21.9 & 1969 & 5.54 & 1967 & 12.6 & 5.89 & 0.47 & 2.27 \\
\hline February & 25.2 & 1969 & 8.63 & 1967 & 15.9 & 4.98 & 0.31 & 2.86 \\
\hline March & 219 & 1969 & 20.0 & 1967 & 57.0 & 52.1 & 0.91 & 10.3 \\
\hline April & 419 & 1969 & 94.4 & 1970 & 183 & 92.9 & 0.51 & 32.9 \\
\hline May & 377 & 1969 & 48.4 & 1970 & 182 & 118 & 0.65 & 32.8 \\
\hline June & 139 & 1969 & 8.22 & 1970 & 54.9 & 43.5 & 0.79 & 9.88 \\
\hline July & 27.4 & 1969 & 1.41 & 1970 & 10.9 & 7.57 & 0.69 & 1.96 \\
\hline August & 11.6 & 1969 & 1.63 & 1970 & 5.27 & 2.42 & 0.46 & 0.95 \\
\hline September & 11.3 & 1969 & 3.04 & 1971 & 5.11 & 2.42 & 0.47 & 0.92 \\
\hline Annual & 108 & 1969 & 19.7 & 1971 & 47.5 & 25.1 & 0.53 & 100 \\
\hline
\end{tabular}




\section{8-0.000-4M HARI RUD RIVER AT ROBAT-I-AKHOND, Continued}

Monthly and annual flow duration, in cubic meters per second

[ng, not given]

\begin{tabular}{|c|c|c|c|c|c|c|c|c|c|c|c|c|c|}
\hline \multirow{2}{*}{$\begin{array}{c}\text { Percentage } \\
\text { of days } \\
\text { discharge } \\
\text { equaled or } \\
\text { exceeded }\end{array}$} & \multicolumn{12}{|c|}{ Month } & \multirow{2}{*}{ Annua } \\
\hline & October & November & December & January & February & March & April & May & June & July & August & September & \\
\hline 95 & 3.17 & 3.93 & 4.10 & 5.24 & 8.70 & 14.5 & 61.9 & 36.6 & 5.28 & 1.58 & 2.09 & 2.56 & 3.48 \\
\hline 90 & 3.41 & 4.27 & 5.04 & 6.03 & 9.62 & 16.9 & 69.1 & 47.6 & 7.22 & 3.27 & 2.81 & 2.93 & 4.09 \\
\hline 85 & 3.77 & 4.47 & 5.41 & 6.27 & 10.3 & 18.6 & 82.0 & 57.9 & 9.16 & 4.20 & 3.17 & 3.20 & 4.55 \\
\hline 80 & 3.90 & 4.66 & 5.77 & 6.83 & 10.6 & 20.3 & 92.2 & 67.2 & 10.0 & 4.60 & 3.45 & 3.51 & 5.11 \\
\hline 75 & 4.00 & 4.85 & 6.07 & 7.57 & 11.2 & 22.0 & 102 & 77.1 & 11.7 & 4.88 & 3.68 & 3.70 & 5.68 \\
\hline 70 & 4.11 & 5.03 & 6.35 & 8.19 & 11.6 & 24.7 & 109 & 89.3 & 14.7 & 5.20 & 3.89 & 3.80 & 6.61 \\
\hline 65 & 4.28 & 5.30 & 6.62 & 8.85 & 12.2 & 26.6 & 115 & 101 & 18.1 & 5.55 & 4.12 & 3.88 & 7.72 \\
\hline 60 & 4.43 & 5.71 & 7.15 & 9.29 & 13.1 & 29.0 & 122 & 117 & 20.6 & 6.58 & 4.31 & 3.96 & 9.09 \\
\hline 55 & 4.59 & 6.32 & 10.4 & 9.68 & 14.1 & 33.3 & 130 & 132 & 26.0 & 7.50 & 4.45 & 4.12 & 10.8 \\
\hline 50 & 4.81 & 7.46 & 11.5 & 10.9 & 15.0 & 36.7 & 136 & 154 & 33.9 & 8.08 & 4.61 & 4.31 & 12.4 \\
\hline 45 & 5.13 & 8.15 & 11.9 & 12.4 & 16.3 & 41.5 & 145 & 174 & 40.6 & 8.71 & 4.93 & 4.57 & 14.8 \\
\hline 40 & 5.71 & 8.55 & 12.2 & 13.6 & 17.1 & 47.5 & 165 & 199 & 49.7 & 9.47 & 5.32 & 4.79 & 17.5 \\
\hline 35 & 6.31 & 10.3 & 14.0 & 14.9 & 17.5 & 51.6 & 187 & 221 & 59.8 & 10.9 & 5.62 & 4.94 & 20.1 \\
\hline 30 & 6.73 & 11.3 & 17.3 & 16.6 & 17.9 & 55.9 & 208 & 241 & 70.1 & 12.4 & 6.15 & 5.16 & 24.9 \\
\hline 25 & 7.25 & 12.0 & 18.0 & 17.4 & 18.7 & 61.6 & 239 & 264 & 80.1 & 13.7 & 6.52 & 5.49 & 37.7 \\
\hline 20 & 8.99 & 13.0 & 18.6 & 18.4 & 19.9 & 68.6 & 269 & 294 & 98.9 & 16.0 & 6.81 & 6.45 & 62.3 \\
\hline 15 & 11.9 & 18.1 & 19.1 & 19.4 & 21.4 & 80.5 & 305 & 312 & 116 & 20.1 & 7.44 & 7.29 & 99.4 \\
\hline 10 & 13.8 & 21.5 & 21.1 & 19.9 & 24.0 & 118 & 355 & 349 & 143 & 24.3 & 8.21 & 9.37 & 145 \\
\hline 5 & 14.8 & 26.7 & 22.7 & 20.7 & 27.1 & 205 & 456 & 418 & 174 & 30.6 & 10.8 & ng & 245 \\
\hline
\end{tabular}




\section{8-0.000-4M HARI RUD RIVER AT ROBAT-I-AKHOND, Continued}

Probability of occurrence of annual high discharges

[m $\mathrm{m}^{3} / \mathrm{s}$, cubic meters per second; ng, not given]

\begin{tabular}{|c|c|c|c|c|c|c|}
\hline \multirow{2}{*}{$\begin{array}{l}\text { Exceedance } \\
\text { probability }\end{array}$} & \multirow{2}{*}{$\begin{array}{c}\text { Recurrence } \\
\text { interval } \\
\text { (years) }\end{array}$} & \multirow{2}{*}{$\begin{array}{l}\text { Maximum } \\
\text { instantaneous } \\
\text { discharge } \\
\left(\mathrm{m}^{3} / \mathrm{s}\right)\end{array}$} & \multicolumn{4}{|c|}{ Maximum daily mean discharge $\left(\mathrm{m}^{3} / \mathrm{s}\right)$} \\
\hline & & & $\begin{array}{l}\text { 3-day } \\
\text { period }\end{array}$ & $\begin{array}{l}\text { 7-day } \\
\text { period }\end{array}$ & $\begin{array}{l}\text { 15-day } \\
\text { period }\end{array}$ & $\begin{array}{l}\text { 30-day } \\
\text { period }\end{array}$ \\
\hline 0.99 & 1.01 & 74.1 & 67.6 & 67.1 & 65.7 & 57.2 \\
\hline 0.95 & 1.05 & 107 & 102 & 98.6 & 93.8 & 83.9 \\
\hline 0.90 & 1.11 & 131 & 127 & 121 & 114 & 103 \\
\hline 0.80 & 1.25 & 167 & 165 & 156 & 143 & 131 \\
\hline 0.50 & 2 & 275 & 274 & 254 & 225 & 206 \\
\hline 0.20 & 5 & 461 & 454 & 415 & 354 & 323 \\
\hline 0.10 & 10 & 611 & 591 & 538 & 450 & 406 \\
\hline 0.04 & 25 & 830 & 783 & 711 & 582 & 518 \\
\hline 0.02 & 50 & 1,020 & 938 & 852 & 687 & 605 \\
\hline 0.01 & 100 & 1,220 & 1,100 & 1,000 & 799 & 695 \\
\hline 0.005 & 200 & 1,450 & 1,280 & 1,170 & 918 & 789 \\
\hline 0.002 & 500 & 1,790 & $\mathrm{ng}$ & $\mathrm{ng}$ & $\mathrm{ng}$ & ng \\
\hline
\end{tabular}

8-0.000-4M HARI RUD RIVER AT ROBAT-I-AKHOND, Continued

Probability of occurrence of annual low discharges

$\left[\mathrm{m}^{3} / \mathrm{s}\right.$, meters per second]

\begin{tabular}{|c|c|c|c|c|c|c|c|c|c|c|}
\hline \multirow{3}{*}{$\begin{array}{c}\text { Nonexceedance } \\
\text { probability }\end{array}$} & \multirow{3}{*}{$\begin{array}{c}\text { Recurrence } \\
\text { interval } \\
\text { (years) }\end{array}$} & \multicolumn{9}{|c|}{ Minimum daily mean discharge $\left(\mathrm{m}^{3} / \mathrm{s}\right)$} \\
\hline & & \multicolumn{9}{|c|}{ Number of consecutive days } \\
\hline & & 1 & 3 & 7 & 14 & 30 & 60 & 90 & 120 & 183 \\
\hline 0.05 & 20 & 0.97 & 0.97 & 1.09 & 1.33 & 1.63 & 1.83 & 2.26 & 2.56 & 3.25 \\
\hline 0.10 & 10 & 1.40 & 1.40 & 1.54 & 1.80 & 2.09 & 2.30 & 2.69 & 2.98 & 3.87 \\
\hline 0.20 & 5 & 2.09 & 2.10 & 2.24 & 2.52 & 2.76 & 2.99 & 3.33 & 3.62 & 4.79 \\
\hline 0.50 & 2 & 3.90 & 3.92 & 4.04 & 4.30 & 4.42 & 4.71 & 5.03 & 5.47 & 7.32 \\
\hline
\end{tabular}




\section{8-0.000-4M HARI RUD RIVER AT ROBAT-I-AKHOND, Continued}

Probability of occurrence of seasonal low discharges

$\left[\mathrm{m}^{3} / \mathrm{s}\right.$, meters per second]

\begin{tabular}{|c|c|c|c|c|c|c|c|c|c|}
\hline \multirow{3}{*}{$\begin{array}{c}\text { Nonexceedance } \\
\text { probability }\end{array}$} & \multirow{3}{*}{$\begin{array}{c}\text { Recurrence } \\
\text { interval } \\
\text { (years) }\end{array}$} & \multicolumn{8}{|c|}{ Minimum daily mean discharge $\left(\mathrm{m}^{3} / \mathrm{s}\right)$} \\
\hline & & \multicolumn{8}{|c|}{ Number of consecutive days } \\
\hline & & 1 & 7 & 14 & 30 & 1 & 7 & 14 & 30 \\
\hline & & \multicolumn{4}{|c|}{ December-January-February } & \multicolumn{4}{|c|}{ March-April-May } \\
\hline 0.05 & 20 & 2.80 & 3.00 & 3.22 & 3.75 & 10.3 & 11.4 & 13.2 & 23.3 \\
\hline 0.10 & 10 & 3.61 & 3.90 & 4.14 & 4.69 & 11.8 & 12.8 & 14.4 & 24.8 \\
\hline 0.20 & 5 & 4.84 & 5.27 & 5.55 & 6.11 & 13.9 & 15.0 & 16.5 & 27.7 \\
\hline \multirow[t]{2}{*}{0.50} & 2 & 8.22 & 9.00 & 9.34 & 9.90 & 18.9 & 21.4 & 23.8 & 38.8 \\
\hline & & \multicolumn{4}{|c|}{ June-July-August } & \multicolumn{4}{|c|}{ September-October-November } \\
\hline 0.05 & 20 & 1.09 & 1.25 & 1.49 & 1.82 & 1.95 & 2.28 & 2.56 & 2.70 \\
\hline 0.10 & 10 & 1.57 & 1.76 & 2.05 & 2.38 & 2.21 & 2.55 & 2.79 & 2.93 \\
\hline 0.20 & 5 & 2.30 & 2.54 & 2.86 & 3.19 & 2.60 & 2.95 & 3.16 & 3.29 \\
\hline 0.50 & 2 & 4.10 & 4.37 & 4.71 & 5.08 & 3.72 & 4.08 & 4.22 & 4.40 \\
\hline
\end{tabular}

8-0.000-4M HARI RUD RIVER AT ROBAT-I-AKHOND, Continued

Annual peak discharges

$\left[\mathrm{m}^{3} / \mathrm{s}\right.$, meters per second]

\begin{tabular}{|c|c|c|c|c|c|}
\hline \multicolumn{3}{|c|}{$\begin{array}{c}\text { Annual peak discharge, } \\
\text { by year }\end{array}$} & \multicolumn{3}{|c|}{$\begin{array}{l}\text { Annual peak discharge, } \\
\text { from highest to lowest }\end{array}$} \\
\hline $\begin{array}{l}\text { Water } \\
\text { year }\end{array}$ & Date & $\begin{array}{c}\text { Peak } \\
\text { discharge } \\
\left(\mathrm{m}^{3} / \mathrm{s}\right)\end{array}$ & $\begin{array}{l}\text { Water } \\
\text { year }\end{array}$ & Date & $\begin{array}{c}\text { Peak } \\
\text { discharge } \\
\left(\mathrm{m}^{3} / \mathrm{s}\right)\end{array}$ \\
\hline 1966 & April 26, 1966 & 167 & 1969 & April 16, 1969 & 740 \\
\hline 1967 & April 26, 1967 & 355 & 1975 & May 17, 1975 & 592 \\
\hline 1968 & May 1,1968 & 425 & 1976 & April 24, 1976 & 570 \\
\hline 1969 & April 16, 1969 & 740 & 1968 & May 1, 1968 & 425 \\
\hline 1970 & April 12, 1970 & 122 & 1967 & April 26, 1967 & 355 \\
\hline 1971 & April 13, 1971 & 174 & 1973 & May 1, 1973 & 305 \\
\hline 1972 & May 7, 1972 & 261 & 1978 & April 18, 1978 & 292 \\
\hline 1973 & May 1, 1973 & 305 & 1972 & May 7, 1972 & 261 \\
\hline 1974 & April 8, 1974 & 143 & 1971 & April 13, 1971 & 174 \\
\hline 1975 & May 17, 1975 & 592 & 1966 & April 26, 1966 & 167 \\
\hline 1976 & April 24, 1976 & 570 & 1974 & April 8, 1974 & 143 \\
\hline 1977 & April 30, 1977 & 141 & 1977 & April 30, 1977 & 141 \\
\hline 1978 & April 18, 1978 & 292 & 1970 & April 12, 1970 & 122 \\
\hline
\end{tabular}




\section{8-0.000-4M HARI RUD RIVER AT ROBAT-I-AKHOND, Continued}

Monthly and annual mean discharges, in cubic meters per second $[--$, no data]

\begin{tabular}{|c|c|c|c|c|c|c|c|c|c|c|c|c|c|}
\hline \multirow{2}{*}{$\begin{array}{l}\text { Water } \\
\text { year }\end{array}$} & \multicolumn{12}{|c|}{ Monthly mean discharge } & \multirow{2}{*}{$\begin{array}{c}\text { Annual } \\
\text { discharge }\end{array}$} \\
\hline & October & November & December & January & February & March & April & May & June & July & August & September & \\
\hline 1966 & -- & -- & -- & -- & -- & -- & -- & 75.8 & 13.3 & 3.86 & 3.35 & 3.44 & -- \\
\hline 1967 & 4.02 & 4.89 & 5.59 & 5.54 & 8.63 & 20.0 & 147 & 224 & 78.0 & 12.6 & 5.00 & 4.75 & 43.4 \\
\hline 1968 & 6.32 & 8.30 & 12.0 & 13.8 & 16.5 & 44.7 & 159 & 189 & 77.7 & 8.40 & 4.76 & 5.12 & 45.4 \\
\hline 1969 & 6.40 & 10.6 & 25.5 & 21.9 & 25.2 & 219 & 419 & 377 & 139 & 27.4 & 11.6 & 11.3 & 108 \\
\hline 1970 & 14.2 & 28.5 & 21.2 & 20.3 & 18.1 & 32.3 & 94.4 & 48.4 & 8.22 & 1.41 & 1.63 & 3.08 & 24.3 \\
\hline 1971 & 4.00 & 4.79 & 6.06 & 6.28 & 10.8 & 28.3 & 99.0 & 50.0 & 12.0 & 7.41 & 4.75 & 3.04 & 19.7 \\
\hline 1972 & 3.23 & 3.97 & 3.84 & 7.21 & 12.4 & 55.6 & 178 & 194 & 79.8 & 14.5 & 6.66 & 4.06 & 46.9 \\
\hline 1973 & 4.45 & 7.89 & 11.2 & 9.48 & 14.5 & 52.2 & 189 & 207 & 54.2 & 9.84 & 3.73 & 3.85 & 47.4 \\
\hline 1974 & 4.28 & 4.28 & 6.01 & 8.55 & 11.5 & 50.5 & 127 & 78.5 & 13.6 & 8.21 & 6.22 & 4.80 & 27.0 \\
\hline 1975 & 4.71 & 5.59 & 6.57 & 8.33 & 12.5 & 48.3 & 275 & 338 & 108 & 22.2 & 5.65 & 6.64 & 70.3 \\
\hline 1976 & 8.35 & 12.4 & 17.7 & 18.1 & 20.7 & 37.8 & 248 & 366 & 90.2 & 16.3 & 7.27 & 8.58 & 71.1 \\
\hline 1977 & 13.9 & 21.1 & 18.2 & 19.0 & 18.8 & 45.8 & 108 & 96.4 & 22.3 & 4.66 & 4.00 & 4.08 & 31.4 \\
\hline 1978 & 4.19 & 8.79 & 12.3 & 12.6 & 21.0 & 49.3 & 152 & 128 & 17.3 & 4.80 & 3.92 & 3.67 & 34.8 \\
\hline
\end{tabular}




\section{8-0.000-5M HARI RUD RIVER AT TAGAW GHAZA}

\section{(U.S. Geological Survey identification number: 342100063390000)}

LOCATION: Lat $34^{\circ} 21^{\prime} \mathrm{N}$., long 63³9'E.

DRAINAGE AREA: $11,920 \mathrm{~km}^{2}$.

ELEVATION: 1,460 meters above mean sea level.

PERIOD OF RECORD: October 1, 1961 to September 30, 1978.

GAGE: Water-stage recorder. Staff gage at same site and datum prior to 1963 water year.

Annual mean discharge

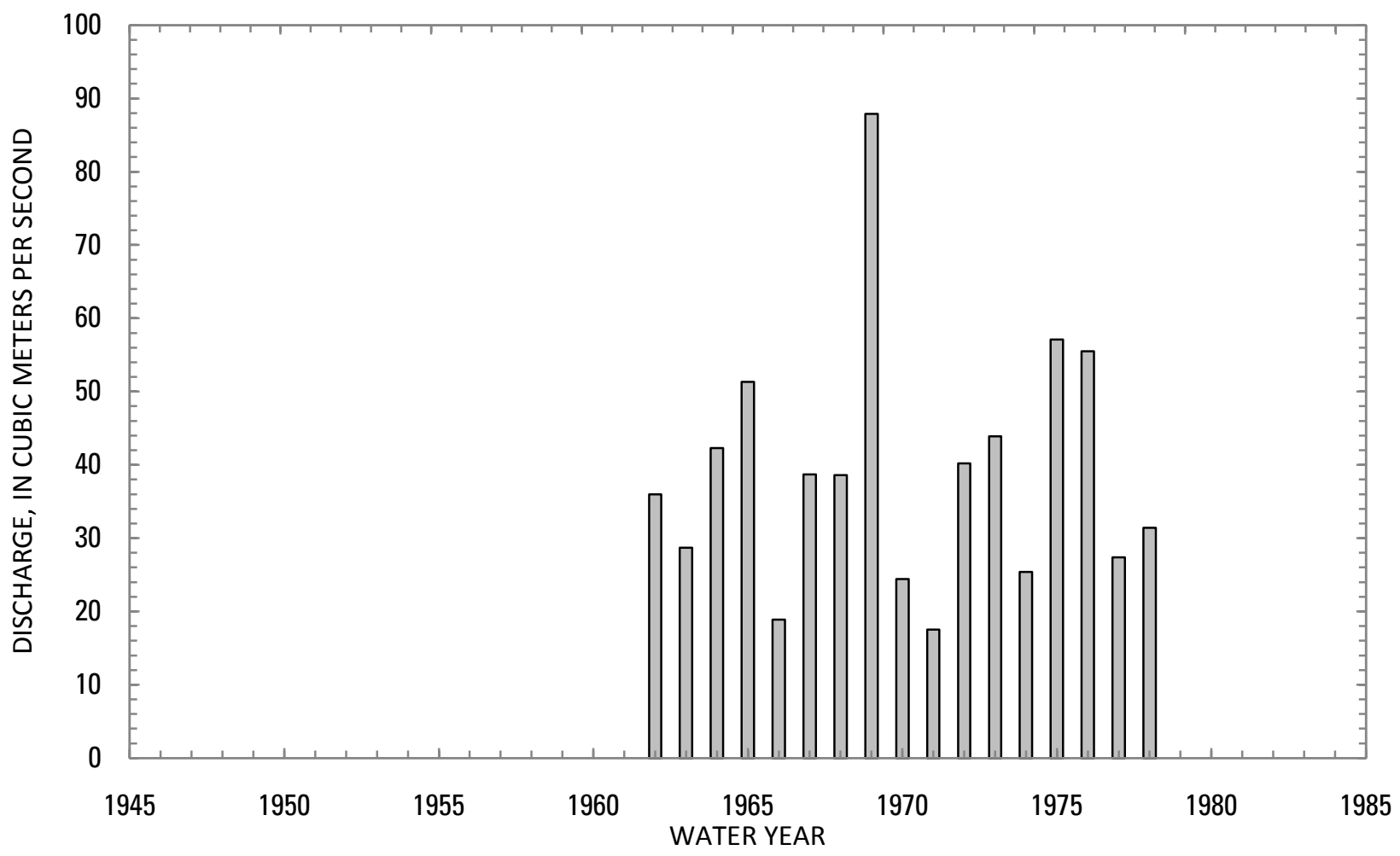




\section{8-0.000-5M HARI RUD RIVER AT TAGAW GHAZA, Continued}

Statistics of monthly and annual mean discharges $\left[\mathrm{m}^{3} / \mathrm{s}\right.$, cubic meters per second]

\begin{tabular}{|c|c|c|c|c|c|c|c|c|}
\hline \multirow[b]{2}{*}{ Month } & \multicolumn{2}{|c|}{ Maximum } & \multicolumn{2}{|c|}{ Minimum } & \multicolumn{4}{|c|}{ Mean } \\
\hline & $\begin{array}{c}\text { Discharge } \\
\left(\mathrm{m}^{3} / \mathrm{s}\right)\end{array}$ & $\begin{array}{c}\text { Water year } \\
\text { of } \\
\text { occurrence }\end{array}$ & $\begin{array}{c}\text { Discharge } \\
\left(\mathrm{m}^{3} / \mathrm{s}\right)\end{array}$ & $\begin{array}{c}\text { Water year } \\
\text { of } \\
\text { occurrence }\end{array}$ & $\begin{array}{c}\text { Discharge } \\
\left(\mathrm{m}^{3} / \mathrm{s}\right)\end{array}$ & $\begin{array}{c}\text { Standard } \\
\text { deviation } \\
\left(\mathrm{m}^{3} / \mathrm{s}\right)\end{array}$ & $\begin{array}{c}\text { Coefficient } \\
\text { of } \\
\text { variation }\end{array}$ & $\begin{array}{c}\text { Percentage } \\
\text { of annual } \\
\text { discharge }\end{array}$ \\
\hline October & 12.9 & 1970 & 5.34 & 1972 & 7.40 & 1.95 & 0.26 & 1.58 \\
\hline November & 20.8 & 1970 & 5.49 & 1975 & 8.23 & 3.69 & 0.45 & 1.76 \\
\hline December & 14.6 & 1970 & 5.12 & 1974 & 7.76 & 2.44 & 0.31 & 1.66 \\
\hline January & 15.5 & 1969 & 5.18 & 1975 & 8.13 & 3.09 & 0.38 & 1.74 \\
\hline February & 14.8 & 1973 & 6.20 & 1972 & 9.59 & 2.79 & 0.29 & 2.05 \\
\hline March & 132 & 1969 & 10.8 & 1966 & 33.6 & 27.5 & 0.82 & 7.17 \\
\hline April & 352 & 1969 & 43.8 & 1966 & 138 & 68.8 & 0.50 & 29.4 \\
\hline May & 331 & 1969 & 56.0 & 1971 & 170 & 83.5 & 0.49 & 36.4 \\
\hline June & 124 & 1969 & 10.9 & 1971 & 56.7 & 33.3 & 0.59 & 12.1 \\
\hline July & 31.1 & 1969 & 4.26 & 1971 & 14.2 & 7.38 & 0.52 & 3.02 \\
\hline August & 14.3 & 1969 & 3.17 & 1971 & 7.54 & 2.97 & 0.39 & 1.61 \\
\hline September & 13.7 & 1969 & 4.47 & 1971 & 7.34 & 2.32 & 0.32 & 1.57 \\
\hline Annual & 87.9 & 1969 & 17.5 & 1971 & 39.1 & 17.2 & 0.44 & 100 \\
\hline
\end{tabular}


8-0.000-5M HARI RUD RIVER AT TAGAW GHAZA, Continued

Monthly and annual flow duration, in cubic meters per second

[ng, not given]

\begin{tabular}{|c|c|c|c|c|c|c|c|c|c|c|c|c|c|}
\hline \multirow{2}{*}{$\begin{array}{l}\text { Percentage } \\
\text { of days } \\
\text { discharge } \\
\text { equaled or } \\
\text { exceeded }\end{array}$} & \multicolumn{12}{|c|}{ Month } & \multirow{2}{*}{ Annua } \\
\hline & October & November & December & January & February & March & April & May & June & July & August & September & \\
\hline 95 & 5.55 & 5.20 & 4.74 & 4.64 & 5.37 & 8.73 & 27.2 & 55.2 & 10.5 & 4.91 & 3.66 & 4.95 & 5.10 \\
\hline 90 & 5.62 & 5.39 & 5.20 & 5.01 & 5.94 & 10.2 & 43.4 & 69.2 & 13.7 & 5.80 & 4.39 & 5.32 & 5.59 \\
\hline 85 & 5.68 & 5.58 & 5.44 & 5.25 & 6.34 & 11.8 & 53.3 & 76.3 & 17.0 & 6.58 & 4.78 & 5.50 & 5.93 \\
\hline 80 & 5.86 & 5.77 & 5.73 & 5.48 & 6.86 & 13.3 & 68.4 & 83.7 & 20.2 & 7.30 & 5.25 & 5.83 & 6.27 \\
\hline 75 & 6.13 & 5.93 & 5.94 & 5.78 & 7.11 & 14.6 & 76.5 & 91.8 & 24.3 & 7.80 & 5.45 & 6.01 & 6.66 \\
\hline 70 & 6.30 & 6.10 & 6.16 & 6.02 & 7.40 & 15.7 & 81.9 & 103 & 27.5 & 8.51 & 5.59 & 6.14 & 7.04 \\
\hline 65 & 6.64 & 6.36 & 6.36 & 6.25 & 7.74 & 16.8 & 88.0 & 115 & 31.0 & 9.33 & 5.85 & 6.22 & 7.47 \\
\hline 60 & 6.73 & 6.60 & 6.53 & 6.47 & 8.10 & 17.8 & 94.8 & 129 & 34.3 & 10.1 & 6.20 & 6.30 & 8.12 \\
\hline 55 & 6.82 & 6.82 & 6.80 & 6.73 & 8.51 & 19.3 & 104 & 140 & 37.5 & 11.0 & 6.50 & 6.42 & 8.81 \\
\hline 50 & 6.91 & 7.17 & 7.27 & 6.99 & 8.87 & 21.2 & 112 & 157 & 42.6 & 12.0 & 6.80 & 6.55 & 9.74 \\
\hline 45 & 6.99 & 7.45 & 7.66 & 7.28 & 9.19 & 22.8 & 120 & 169 & 47.8 & 12.9 & 7.13 & 6.71 & 10.9 \\
\hline 40 & 7.08 & 7.65 & 8.06 & 7.79 & 9.42 & 26.5 & 128 & 183 & 52.9 & 13.8 & 7.48 & 6.87 & 12.5 \\
\hline 35 & 7.15 & 7.98 & 8.36 & 8.21 & 9.69 & 30.4 & 138 & 200 & 58.7 & 14.8 & 8.10 & 7.04 & 15.2 \\
\hline 30 & 7.23 & 8.58 & 8.64 & 8.61 & 10.6 & 33.9 & 156 & 216 & 66.9 & 16.2 & 8.55 & 7.59 & 19.9 \\
\hline 25 & 7.64 & 9.26 & 8.92 & 9.06 & 11.4 & 37.4 & 179 & 234 & 76.5 & 17.8 & 9.13 & 8.59 & 30.6 \\
\hline 20 & 9.30 & 9.57 & 9.32 & 10.1 & 12.1 & 44.8 & 204 & 247 & 88.6 & 20.1 & 10.1 & 8.90 & 51.1 \\
\hline 15 & 9.54 & 9.79 & 10.0 & 10.9 & 13.1 & 54.9 & 241 & 263 & 103 & 22.6 & 10.9 & 10.1 & 82.9 \\
\hline 10 & 9.71 & 10.4 & 10.9 & 12.9 & 14.8 & 65.8 & 271 & 286 & 128 & 26.7 & 12.0 & 10.5 & 123 \\
\hline 5 & 12.7 & 16.6 & 14.1 & 14.6 & 16.9 & 93.4 & 325 & 337 & 150 & 31.8 & 13.5 & $\mathrm{ng}$ & 199 \\
\hline
\end{tabular}




\section{8-0.000-5M HARI RUD RIVER AT TAGAW GHAZA, Continued}

Probability of occurrence of annual high discharges

[ $\mathrm{m}^{3} / \mathrm{s}$, cubic meters per second; $\mathrm{ng}$, not given]

\begin{tabular}{|c|c|c|c|c|c|c|}
\hline \multirow{2}{*}{$\begin{array}{c}\text { Exceedance } \\
\text { probability }\end{array}$} & \multirow{2}{*}{$\begin{array}{l}\text { Recurrence } \\
\text { interval } \\
\text { (years) }\end{array}$} & \multirow{2}{*}{$\begin{array}{c}\text { Maximum } \\
\text { instantaneous } \\
\text { discharge } \\
\left(\mathrm{m}^{3} / \mathrm{s}\right)\end{array}$} & \multicolumn{4}{|c|}{ Maximum daily mean discharge $\left(\mathrm{m}^{3} / \mathrm{s}\right)$} \\
\hline & & & $\begin{array}{l}\text { 3-day } \\
\text { period }\end{array}$ & $\begin{array}{l}\text { 7-day } \\
\text { period }\end{array}$ & $\begin{array}{l}\text { 15-day } \\
\text { period }\end{array}$ & $\begin{array}{l}\text { 30-day } \\
\text { period }\end{array}$ \\
\hline 0.99 & 1.01 & 64.8 & 64.1 & 62.2 & 58.0 & 56.0 \\
\hline 0.95 & 1.05 & 99.2 & 95.7 & 91.9 & 85.3 & 81.4 \\
\hline 0.90 & 1.11 & 124 & 118 & 112 & 104 & 98.6 \\
\hline 0.80 & 1.25 & 162 & 151 & 143 & 131 & 123 \\
\hline 0.50 & 2 & 269 & 239 & 222 & 200 & 185 \\
\hline 0.20 & 5 & 441 & 372 & 339 & 297 & 269 \\
\hline 0.10 & 10 & 569 & 464 & 419 & 362 & 323 \\
\hline 0.04 & 25 & 744 & 586 & 522 & 442 & 390 \\
\hline 0.02 & 50 & 883 & 679 & 599 & 502 & 439 \\
\hline 0.01 & 100 & 1,030 & 773 & 677 & 560 & 486 \\
\hline 0.005 & 200 & 1,180 & 869 & 756 & 618 & 533 \\
\hline 0.002 & 500 & 1,400 & ng & $\mathrm{ng}$ & $\mathrm{ng}$ & $\mathrm{ng}$ \\
\hline
\end{tabular}

\section{8-0.000-5M HARI RUD RIVER AT TAGAW GHAZA, Continued}

Probability of occurrence of annual low discharges $\left[\mathrm{m}^{3} / \mathrm{s}\right.$, meters per second]

\begin{tabular}{|c|c|c|c|c|c|c|c|c|c|c|}
\hline \multirow{3}{*}{$\begin{array}{c}\text { Nonexceedance } \\
\text { probability }\end{array}$} & \multirow{3}{*}{$\begin{array}{c}\text { Recurrence } \\
\text { interval } \\
\text { (years) }\end{array}$} & \multicolumn{9}{|c|}{ Minimum daily mean discharge $\left(\mathrm{m}^{3} / \mathrm{s}\right)$} \\
\hline & & \multicolumn{9}{|c|}{ Number of consecutive days } \\
\hline & & 1 & 3 & 7 & 14 & 30 & 60 & 90 & 120 & 183 \\
\hline 0.05 & 20 & 2.95 & 3.00 & 3.14 & 3.34 & 3.63 & 4.00 & 4.35 & 4.66 & 4.98 \\
\hline 0.10 & 10 & 3.38 & 3.43 & 3.57 & 3.78 & 4.04 & 4.38 & 4.70 & 4.97 & 5.31 \\
\hline 0.20 & 5 & 3.96 & 4.02 & 4.17 & 4.38 & 4.62 & 4.94 & 5.21 & 5.44 & 5.80 \\
\hline 0.50 & 2 & 5.28 & 5.40 & 5.57 & 5.83 & 6.04 & 6.33 & 6.55 & 6.74 & 7.13 \\
\hline
\end{tabular}




\section{8-0.000-5M HARI RUD RIVER AT TAGAW GHAZA, Continued}

Probability of occurrence of seasonal low discharges [ $\mathrm{m}^{3} / \mathrm{s}$, meters per second]

\begin{tabular}{|c|c|c|c|c|c|c|c|c|c|}
\hline \multirow{3}{*}{$\begin{array}{c}\text { Nonexceedance } \\
\text { probability }\end{array}$} & \multirow{3}{*}{$\begin{array}{c}\text { Recurrence } \\
\text { interval } \\
\text { (years) }\end{array}$} & \multicolumn{8}{|c|}{ Minimum daily mean discharge $\left(\mathrm{m}^{3} / \mathrm{s}\right)$} \\
\hline & & \multicolumn{8}{|c|}{ Number of consecutive days } \\
\hline & & 1 & 7 & 14 & 30 & 1 & 7 & 14 & 30 \\
\hline & & \multicolumn{4}{|c|}{ December-January-February } & \multicolumn{4}{|c|}{ March-April-May } \\
\hline 0.05 & 20 & 3.52 & 3.99 & 4.35 & 4.58 & 6.53 & 7.36 & 8.64 & 13.0 \\
\hline 0.10 & 10 & 3.86 & 4.35 & 4.70 & 4.93 & 7.19 & 8.19 & 9.38 & 14.3 \\
\hline 0.20 & 5 & 4.34 & 4.85 & 5.20 & 5.43 & 8.20 & 9.48 & 10.6 & 16.6 \\
\hline \multirow[t]{2}{*}{0.50} & 2 & 5.44 & 6.02 & 6.43 & 6.73 & 11.0 & 13.3 & 14.9 & 24.4 \\
\hline & & \multicolumn{4}{|c|}{ June-July-August } & \multicolumn{4}{|c|}{ September-October-November } \\
\hline 0.05 & 20 & 3.21 & 3.33 & 3.43 & 3.65 & 4.04 & 4.25 & 4.43 & 4.74 \\
\hline 0.10 & 10 & 3.71 & 3.87 & 3.96 & 4.22 & 4.36 & 4.55 & 4.71 & 4.99 \\
\hline 0.20 & 5 & 4.42 & 4.61 & 4.71 & 5.03 & 4.82 & 4.99 & 5.13 & 5.38 \\
\hline 0.50 & 2 & 6.19 & 6.43 & 6.53 & 7.00 & 6.07 & 6.20 & 6.29 & 6.47 \\
\hline
\end{tabular}


8-0.000-5M HARI RUD RIVER AT TAGAW GHAZA, Continued

Annual peak discharges

$\left[\mathrm{m}^{3} / \mathrm{s}\right.$, meters per second]

\begin{tabular}{|c|c|c|c|c|c|}
\hline \multicolumn{3}{|c|}{$\begin{array}{c}\text { Annual peak discharge, } \\
\text { by year }\end{array}$} & \multicolumn{3}{|c|}{$\begin{array}{l}\text { Annual peak discharge, } \\
\text { from highest to lowest }\end{array}$} \\
\hline $\begin{array}{l}\text { Water } \\
\text { year }\end{array}$ & Date & $\begin{array}{c}\text { Peak } \\
\text { discharge } \\
\left(\mathrm{m}^{3} / \mathrm{s}\right)\end{array}$ & $\begin{array}{l}\text { Water } \\
\text { year }\end{array}$ & Date & $\begin{array}{c}\text { Peak } \\
\text { discharge } \\
\left(\mathrm{m}^{3} / \mathrm{s}\right)\end{array}$ \\
\hline 1962 & April 26, 1962 & 297 & 1969 & May 10, 1969 & 723 \\
\hline 1963 & May 5,1963 & 221 & 1975 & May 17, 1975 & 670 \\
\hline 1964 & May 2, 1964 & 300 & 1976 & May 13, 1976 & 500 \\
\hline 1965 & May 22, 1965 & 350 & 1967 & April 27, 1967 & 414 \\
\hline 1966 & May 8, 1966 & 132 & 1968 & April 30, 1968 & 351 \\
\hline 1967 & April 27, 1967 & 414 & 1965 & May 22, 1965 & 350 \\
\hline 1968 & April 30, 1968 & 351 & 1973 & May 3, 1973 & 340 \\
\hline 1969 & May 10, 1969 & 723 & 1964 & May 2, 1964 & 300 \\
\hline 1970 & April 27, 1970 & 124 & 1962 & April 26, 1962 & 297 \\
\hline 1971 & May 2, 1971 & 97.2 & 1978 & April 17, 1978 & 282 \\
\hline 1972 & May 9, 1972 & 244 & 1972 & May 9, 1972 & 244 \\
\hline 1973 & May 3, 1973 & 340 & 1963 & May 5, 1963 & 221 \\
\hline 1974 & April 22, 1974 & 134 & 1977 & April 19, 1977 & 146 \\
\hline 1975 & May 17, 1975 & 670 & 1974 & April 22, 1974 & 134 \\
\hline 1976 & May 13, 1976 & 500 & 1966 & May 8, 1966 & 132 \\
\hline 1977 & April 19, 1977 & 146 & 1970 & April 27, 1970 & 124 \\
\hline 1978 & April 17, 1978 & 282 & 1971 & May 2, 1971 & 97.2 \\
\hline
\end{tabular}




\section{8-0.000-5M HARI RUD RIVER AT TAGAW GHAZA, Continued}

\section{Monthly and annual mean discharges, in cubic meters per second}

\begin{tabular}{|c|c|c|c|c|c|c|c|c|c|c|c|c|c|}
\hline \multirow{2}{*}{$\begin{array}{l}\text { Water } \\
\text { year }\end{array}$} & \multicolumn{12}{|c|}{ Monthly mean discharge } & \multirow{2}{*}{$\begin{array}{c}\text { Annual } \\
\text { discharge }\end{array}$} \\
\hline & October & November & December & January & February & March & April & May & June & July & August & September & \\
\hline 1962 & 5.60 & 6.04 & 5.83 & 6.00 & 10.9 & 37.3 & 157 & 141 & 39.3 & 10.5 & 6.05 & 5.68 & 36.0 \\
\hline 1963 & 7.08 & 6.99 & 5.98 & 6.07 & 7.77 & 15.3 & 62.0 & 141 & 63.0 & 13.4 & 7.60 & 7.14 & 28.7 \\
\hline 1964 & 7.01 & 10.9 & 8.41 & 6.28 & 9.69 & 41.7 & 160 & 186 & 47.8 & 14.6 & 8.14 & 6.85 & 42.3 \\
\hline 1965 & 6.70 & 6.50 & 6.50 & 7.89 & 10.6 & 45.7 & 133 & 256 & 99.4 & 22.7 & 9.17 & 8.72 & 51.3 \\
\hline 1966 & 9.51 & 9.65 & 8.41 & 8.15 & 9.78 & 10.8 & 43.8 & 84.7 & 23.3 & 7.12 & 5.48 & 5.71 & 18.9 \\
\hline 1967 & 6.32 & 5.93 & 5.24 & 5.46 & 6.47 & 15.0 & 117 & 196 & 75.6 & 15.8 & 7.45 & 6.18 & 38.7 \\
\hline 1968 & 5.88 & 5.66 & 8.30 & 7.70 & 8.36 & 25.1 & 127 & 174 & 75.4 & 12.4 & 6.31 & 6.59 & 38.6 \\
\hline 1969 & 6.95 & 7.93 & 10.3 & 15.5 & 14.3 & 132 & 352 & 331 & 124 & 31.1 & 14.3 & 13.7 & 87.9 \\
\hline 1970 & 12.9 & 20.8 & 14.6 & 13.2 & 13.8 & 22.7 & 90.4 & 69.5 & 19.1 & 6.39 & 4.34 & 5.82 & 24.4 \\
\hline 1971 & 7.33 & 7.56 & 6.88 & 6.65 & 7.23 & 20.2 & 75.6 & 56.0 & 10.9 & 4.26 & 3.17 & 4.47 & 17.5 \\
\hline 1972 & 5.34 & 5.67 & 5.86 & 5.66 & 6.20 & 23.6 & 123 & 195 & 75.1 & 18.3 & 9.09 & 8.53 & 40.2 \\
\hline 1973 & 7.11 & 7.09 & 7.84 & 12.2 & 14.8 & 42.5 & 174 & 187 & 47.8 & 12.8 & 6.87 & 6.20 & 43.9 \\
\hline 1974 & 5.79 & 5.51 & 5.12 & 5.18 & 6.57 & 36.4 & 108 & 87.0 & 24.5 & 8.29 & 5.54 & 5.76 & 25.4 \\
\hline 1975 & 6.07 & 5.49 & 5.28 & 5.18 & 6.48 & 27.2 & 192 & 285 & 102 & 24.4 & 12.4 & 10.5 & 57.1 \\
\hline 1976 & 9.35 & 9.60 & 10.1 & 10.7 & 11.5 & 18.6 & 178 & 294 & 79.6 & 21.8 & 11.4 & 10.2 & 55.5 \\
\hline 1977 & 9.78 & 10.4 & 9.62 & 9.63 & 9.58 & 34.9 & 110 & 86.3 & 26.8 & 8.14 & 6.17 & 6.58 & 27.4 \\
\hline 1978 & 7.08 & 8.16 & 7.71 & 6.80 & 9.05 & 21.8 & 140 & 126 & 31.0 & 8.56 & 4.70 & 6.11 & 31.4 \\
\hline
\end{tabular}




\section{8-0.000-7M HARI RUD RIVER AT CHEKHCHERAN}

\section{(U.S. Geological Survey identification number: 343100065150000 )}

LOCATION: Lat $34^{\circ} 31^{\prime} \mathrm{N}$., long $65^{\circ} 15^{\prime} \mathrm{E}$.

DRAINAGE AREA: $6,090 \mathrm{~km}^{2}$.

ELEVATION: 2,250 meters above mean sea level.

PERIOD OF RECORD: October 1, 1961 to September 30, 1978.

GAGE: Water-stage recorder.

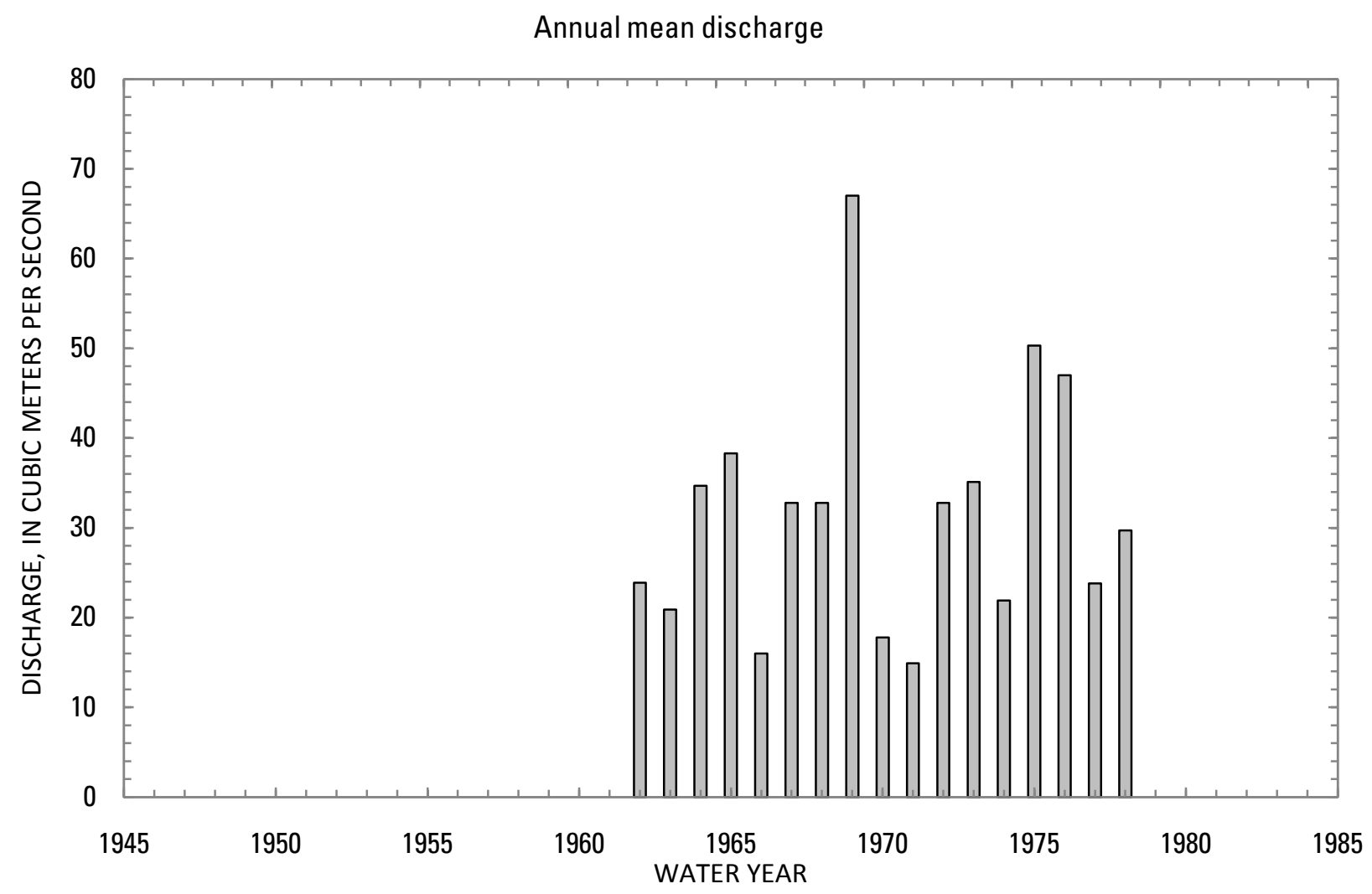




\section{8-0.000-7M HARI RUD RIVER AT CHEKHCHERAN, Continued}

Statistics of monthly and annual mean discharges $\left[\mathrm{m}^{3} / \mathrm{s}\right.$, cubic meters per second]

\begin{tabular}{|c|c|c|c|c|c|c|c|c|}
\hline \multirow[b]{2}{*}{ Month } & \multicolumn{2}{|c|}{ Maximum } & \multicolumn{2}{|c|}{ Minimum } & \multicolumn{4}{|c|}{ Mean } \\
\hline & $\begin{array}{c}\text { Discharge } \\
\left(\mathrm{m}^{3} / \mathrm{s}\right)\end{array}$ & $\begin{array}{c}\text { Water year } \\
\text { of } \\
\text { occurrence }\end{array}$ & $\begin{array}{c}\text { Discharge } \\
\left(\mathrm{m}^{3} / \mathrm{s}\right)\end{array}$ & $\begin{array}{c}\text { Water year } \\
\text { of } \\
\text { occurrence }\end{array}$ & $\begin{array}{c}\text { Discharge } \\
\left(\mathrm{m}^{3} / \mathrm{s}\right)\end{array}$ & $\begin{array}{c}\text { Standard } \\
\text { deviation } \\
\left(\mathrm{m}^{3} / \mathrm{s}\right)\end{array}$ & $\begin{array}{c}\text { Coefficient } \\
\text { of } \\
\text { variation }\end{array}$ & $\begin{array}{c}\text { Percentage } \\
\text { of annual } \\
\text { discharge }\end{array}$ \\
\hline October & 7.42 & 1970 & 3.47 & 1972 & 5.48 & 1.10 & 0.20 & 1.44 \\
\hline November & 10.5 & 1970 & 3.33 & 1971 & 5.53 & 1.67 & 0.30 & 1.45 \\
\hline December & 6.66 & 1970 & 2.42 & 1965 & 4.96 & 1.10 & 0.22 & 1.30 \\
\hline January & 6.60 & 1976 & 3.03 & 1965 & 4.75 & 1.02 & 0.21 & 1.25 \\
\hline February & 8.30 & 1969 & 4.36 & 1974 & 5.78 & 1.11 & 0.19 & 1.52 \\
\hline March & 74.4 & 1969 & 5.75 & 1966 & 19.4 & 16.4 & 0.85 & 5.10 \\
\hline April & 255 & 1969 & 38.6 & 1966 & 110 & 50.1 & 0.45 & 29.0 \\
\hline May & 309 & 1969 & 55.3 & 1971 & 153 & 80.4 & 0.52 & 40.3 \\
\hline June & 104 & 1975 & 11.2 & 1971 & 48.0 & 28.1 & 0.59 & 12.6 \\
\hline July & 23.2 & 1969 & 3.06 & 1971 & 11.3 & 5.72 & 0.51 & 2.97 \\
\hline August & 11.1 & 1969 & 3.00 & 1971 & 5.80 & 2.12 & 0.37 & 1.53 \\
\hline September & 8.74 & 1969 & 3.00 & 1971 & 5.79 & 1.59 & 0.27 & 1.52 \\
\hline Annual & 67.0 & 1969 & 14.9 & 1971 & 31.8 & 13.6 & 0.43 & 100 \\
\hline
\end{tabular}


8-0.000-7M HARI RUD RIVER AT CHEKHCHERAN, Continued

Monthly and annual flow duration, in cubic meters per second

\begin{tabular}{|c|c|c|c|c|c|c|c|c|c|c|c|c|c|}
\hline \multirow{2}{*}{$\begin{array}{l}\text { Percentage } \\
\text { of days } \\
\text { discharge } \\
\text { equaled or } \\
\text { exceeded }\end{array}$} & \multicolumn{12}{|c|}{ Month } & \multirow{2}{*}{ Annual } \\
\hline & October & November & December & January & February & March & April & May & June & July & August & September & \\
\hline 95 & 3.83 & 3.24 & 2.50 & 2.97 & 3.95 & 5.12 & 15.0 & 53.1 & 9.65 & 3.21 & 3.12 & 3.08 & 3.45 \\
\hline 90 & 3.90 & 3.85 & 3.50 & 3.33 & 4.03 & 5.35 & 28.5 & 67.4 & 11.5 & 4.90 & 3.61 & 3.86 & 3.99 \\
\hline 85 & 4.36 & 4.02 & 3.67 & 3.48 & 4.38 & 5.77 & 36.9 & 72.1 & 14.0 & 5.56 & 3.75 & 3.99 & 4.35 \\
\hline 80 & 4.61 & 4.31 & 4.05 & 3.73 & 4.44 & 6.66 & 47.0 & 75.8 & 16.6 & 5.88 & 3.85 & 4.31 & 4.69 \\
\hline 75 & 4.67 & 4.42 & 4.14 & 3.93 & 4.67 & 7.32 & 53.8 & 81.7 & 19.6 & 6.46 & 3.97 & 4.87 & 4.96 \\
\hline 70 & 4.81 & 4.54 & 4.37 & 4.21 & 5.01 & 7.96 & 58.8 & 87.7 & 22.3 & 7.22 & 4.40 & 4.93 & 5.22 \\
\hline 65 & 4.98 & 4.70 & 4.48 & 4.29 & 5.07 & 8.63 & 66.9 & 94.4 & 25.2 & 7.87 & 4.68 & 4.99 & 5.48 \\
\hline 60 & 5.08 & 4.93 & 4.66 & 4.40 & 5.13 & 9.31 & 75.8 & 103 & 28.6 & 8.46 & 4.85 & 5.23 & 5.81 \\
\hline 55 & 5.24 & 5.08 & 4.73 & 4.59 & 5.39 & 10.4 & 82.0 & 114 & 32.1 & 8.88 & 5.01 & 5.38 & 6.15 \\
\hline 50 & 5.39 & 5.20 & 4.84 & 4.82 & 5.50 & 11.6 & 89.4 & 129 & 36.3 & 9.26 & 5.22 & 5.62 & 6.48 \\
\hline 45 & 5.66 & 5.43 & 5.11 & 4.90 & 5.87 & 12.8 & 98.0 & 147 & 40.2 & 9.75 & 5.46 & 5.71 & 7.13 \\
\hline 40 & 5.76 & 5.65 & 5.28 & 4.97 & 5.97 & 14.7 & 106 & 160 & 43.7 & 10.5 & 5.83 & 6.03 & 7.91 \\
\hline 35 & 6.00 & 5.82 & 5.49 & 5.23 & 6.03 & 15.7 & 114 & 173 & 47.6 & 11.5 & 6.52 & 6.41 & 9.47 \\
\hline 30 & 6.33 & 5.96 & 5.64 & 5.48 & 6.10 & 17.2 & 125 & 192 & 53.5 & 12.6 & 6.78 & 6.60 & 12.9 \\
\hline 25 & 6.38 & 6.10 & 5.75 & 5.65 & 6.33 & 19.9 & 147 & 210 & 63.5 & 14.1 & 7.11 & 6.96 & 21.1 \\
\hline 20 & 6.44 & 6.47 & 6.07 & 5.97 & 6.67 & 23.2 & 172 & 229 & 74.1 & 15.7 & 7.43 & 7.32 & 38.3 \\
\hline 15 & 6.50 & 6.85 & 6.28 & 6.05 & 7.33 & 31.8 & 203 & 251 & 89.0 & 18.6 & 7.91 & 7.89 & 68.6 \\
\hline 10 & 7.13 & 7.26 & 6.55 & 6.14 & 7.96 & 40.4 & 230 & 273 & 109 & 21.9 & 8.73 & 8.23 & 100 \\
\hline 5 & 7.30 & 8.33 & 7.04 & 6.46 & 9.38 & 66.3 & 270 & 320 & 133 & 26.3 & 10.4 & 8.54 & 169 \\
\hline
\end{tabular}




\section{8-0.000-7M HARI RUD RIVER AT CHEKHCHERAN, Continued}

Probability of occurrence of annual high discharges

[m $\mathrm{m}^{3} / \mathrm{s}$, cubic meters per second; ng, not given]

\begin{tabular}{|c|c|c|c|c|c|c|}
\hline \multirow{2}{*}{$\begin{array}{c}\text { Exceedance } \\
\text { probability }\end{array}$} & \multirow{2}{*}{$\begin{array}{c}\text { Recurrence } \\
\text { interval } \\
\text { (years) }\end{array}$} & \multirow{2}{*}{$\begin{array}{l}\text { Maximum } \\
\text { instantaneous } \\
\text { discharge } \\
\left(\mathrm{m}^{3} / \mathrm{s}\right)\end{array}$} & \multicolumn{4}{|c|}{ Maximum daily mean discharge $\left(\mathrm{m}^{3} / \mathrm{s}\right)$} \\
\hline & & & $\begin{array}{l}\text { 3-day } \\
\text { period }\end{array}$ & $\begin{array}{l}\text { 7-day } \\
\text { period }\end{array}$ & $\begin{array}{l}\text { 15-day } \\
\text { period }\end{array}$ & $\begin{array}{l}\text { 30-day } \\
\text { period }\end{array}$ \\
\hline 0.99 & 1.01 & 60.4 & 60.0 & 57.4 & 52.9 & 49.8 \\
\hline 0.95 & 1.05 & 86.8 & 85.3 & 81.3 & 75.5 & 70.7 \\
\hline 0.90 & 1.11 & 106 & 103 & 98.1 & 91.1 & 85.0 \\
\hline 0.80 & 1.25 & 136 & 130 & 123 & 114 & 106 \\
\hline 0.50 & 2 & 224 & 206 & 191 & 174 & 159 \\
\hline 0.20 & 5 & 381 & 329 & 298 & 264 & 236 \\
\hline 0.10 & 10 & 508 & 423 & 376 & 327 & 288 \\
\hline 0.04 & 25 & 697 & 554 & 484 & 409 & 356 \\
\hline 0.02 & 50 & 860 & 662 & 569 & 473 & 407 \\
\hline 0.01 & 100 & 1,040 & 777 & 659 & 538 & 458 \\
\hline 0.005 & 200 & 1,250 & 902 & 754 & 605 & 510 \\
\hline 0.002 & 500 & 1,550 & ng & $\mathrm{ng}$ & $\mathrm{ng}$ & $\mathrm{ng}$ \\
\hline
\end{tabular}

\section{8-0.000-7M HARI RUD RIVER AT CHEKHCHERAN, Continued}

Probability of occurrence of annual low discharges

$\left[\mathrm{m}^{3} / \mathrm{s}\right.$, meters per second]

\begin{tabular}{|c|c|c|c|c|c|c|c|c|c|c|}
\hline \multirow{3}{*}{$\begin{array}{c}\text { Nonexceedance } \\
\text { probability }\end{array}$} & \multirow{3}{*}{$\begin{array}{c}\text { Recurrence } \\
\text { interval } \\
\text { (years) }\end{array}$} & \multicolumn{9}{|c|}{ Minimum daily mean discharge $\left(\mathrm{m}^{3} / \mathrm{s}\right)$} \\
\hline & & \multicolumn{9}{|c|}{ Number of consecutive days } \\
\hline & & 1 & 3 & 7 & 14 & 30 & 60 & 90 & 120 & 183 \\
\hline 0.05 & 20 & 2.40 & 2.46 & 2.51 & 2.53 & 2.61 & 2.73 & 3.08 & 3.27 & 3.67 \\
\hline 0.10 & 10 & 2.62 & 2.69 & 2.77 & 2.80 & 2.92 & 3.10 & 3.41 & 3.59 & 3.94 \\
\hline 0.20 & 5 & 2.91 & 3.00 & 3.11 & 3.17 & 3.31 & 3.56 & 3.83 & 4.00 & 4.31 \\
\hline 0.50 & 2 & 3.58 & 3.71 & 3.87 & 3.97 & 4.16 & 4.51 & 4.68 & 4.86 & 5.12 \\
\hline
\end{tabular}




\section{8-0.000-7M HARI RUD RIVER AT CHEKHCHERAN, Continued}

Probability of occurrence of seasonal low discharges [ $\mathrm{m}^{3} / \mathrm{s}$, meters per second]

\begin{tabular}{|c|c|c|c|c|c|c|c|c|c|}
\hline \multirow{3}{*}{$\begin{array}{c}\text { Nonexceedance } \\
\text { probability }\end{array}$} & \multirow{3}{*}{$\begin{array}{c}\text { Recurrence } \\
\text { interval } \\
\text { (years) }\end{array}$} & \multicolumn{8}{|c|}{ Minimum daily mean discharge $\left(\mathrm{m}^{3} / \mathrm{s}\right)$} \\
\hline & & \multicolumn{8}{|c|}{ Number of consecutive days } \\
\hline & & 1 & 7 & 14 & 30 & 1 & 7 & 14 & 30 \\
\hline & & \multicolumn{4}{|c|}{ December-January-February } & \multicolumn{4}{|c|}{ March-April-May } \\
\hline 0.05 & 20 & 2.48 & 2.65 & 2.70 & 2.79 & 3.77 & 4.24 & 4.51 & 5.97 \\
\hline 0.10 & 10 & 2.74 & 2.93 & 3.01 & 3.14 & 4.24 & 4.74 & 5.15 & 6.92 \\
\hline 0.20 & 5 & 3.06 & 3.30 & 3.40 & 3.58 & 4.92 & 5.47 & 6.11 & 8.50 \\
\hline \multirow[t]{2}{*}{0.50} & 2 & 3.76 & 4.07 & 4.19 & 4.44 & 6.76 & 7.38 & 8.78 & 13.7 \\
\hline & & \multicolumn{4}{|c|}{ June-July-August } & \multicolumn{4}{|c|}{ September-October-November } \\
\hline 0.05 & 20 & 2.94 & 2.99 & 3.04 & 3.13 & 2.73 & 2.81 & 2.96 & 3.15 \\
\hline 0.10 & 10 & 3.23 & 3.31 & 3.37 & 3.50 & 3.06 & 3.14 & 3.31 & 3.54 \\
\hline 0.20 & 5 & 3.67 & 3.77 & 3.84 & 4.02 & 3.50 & 3.59 & 3.78 & 4.03 \\
\hline 0.50 & 2 & 4.77 & 4.93 & 5.02 & 5.34 & 4.44 & 4.59 & 4.80 & 5.08 \\
\hline
\end{tabular}


8-0.000-7M HARI RUD RIVER AT CHEKHCHERAN, Continued

Annual peak discharges

[ $\mathrm{m}^{3} / \mathrm{s}$, meters per second]

\begin{tabular}{|c|c|c|c|c|c|}
\hline \multicolumn{3}{|c|}{$\begin{array}{c}\text { Annual peak discharge, } \\
\text { by year }\end{array}$} & \multicolumn{3}{|c|}{$\begin{array}{l}\text { Annual peak discharge, } \\
\text { from highest to lowest }\end{array}$} \\
\hline $\begin{array}{l}\text { Water } \\
\text { year }\end{array}$ & Date & $\begin{array}{c}\text { Peak } \\
\text { discharge } \\
\left(\mathrm{m}^{3} / \mathrm{s}\right)\end{array}$ & $\begin{array}{l}\text { Water } \\
\text { year }\end{array}$ & Date & $\begin{array}{c}\text { Peak } \\
\text { discharge } \\
\left(\mathrm{m}^{3} / \mathrm{s}\right)\end{array}$ \\
\hline 1962 & April 21, 1962 & 158 & 1969 & May 5, 1969 & 693 \\
\hline 1963 & May 5,1963 & 158 & 1975 & May 16,1975 & 610 \\
\hline 1964 & May 2, 1964 & 277 & 1976 & May 12, 1976 & 438 \\
\hline 1965 & May 4, 1965 & 285 & 1968 & April 29, 1968 & 348 \\
\hline 1966 & May 6, 1966 & 109 & 1967 & April 26, 1967 & 334 \\
\hline 1967 & April 26, 1967 & 334 & 1965 & May 4, 1965 & 285 \\
\hline 1968 & April 29, 1968 & 348 & 1978 & April 16, 1978 & 280 \\
\hline 1969 & May 5, 1969 & 693 & 1964 & May 2, 1964 & 277 \\
\hline 1970 & April 25, 1970 & 118 & 1973 & May 1, 1973 & 275 \\
\hline 1971 & April 30, 1971 & 97.6 & 1972 & April 27, 1972 & 235 \\
\hline 1972 & April 27, 1972 & 235 & 1962 & April 21, 1962 & 158 \\
\hline 1973 & May 1, 1973 & 275 & 1963 & May 5, 1963 & 158 \\
\hline 1974 & April 16, 1974 & 106 & 1977 & April 19, 1977 & 129 \\
\hline 1975 & May 16, 1975 & 610 & 1970 & April 25, 1970 & 118 \\
\hline 1976 & May 12, 1976 & 438 & 1966 & May 6, 1966 & 109 \\
\hline 1977 & April 19, 1977 & 129 & 1974 & April 16, 1974 & 106 \\
\hline 1978 & April 16, 1978 & 280 & 1971 & April 30, 1971 & 97.6 \\
\hline
\end{tabular}


8-0.000-7M HARI RUD RIVER AT CHEKHCHERAN, Continued

Monthly and annual mean discharges, in cubic meters per second

\begin{tabular}{|c|c|c|c|c|c|c|c|c|c|c|c|c|c|}
\hline \multirow{2}{*}{$\begin{array}{c}\text { Water } \\
\text { year }\end{array}$} & \multicolumn{12}{|c|}{ Monthly mean discharge } & \multirow{2}{*}{$\begin{array}{c}\text { Annual } \\
\text { discharge }\end{array}$} \\
\hline & October & November & December & January & February & March & April & May & June & July & August & September & \\
\hline 1962 & 4.81 & 4.62 & 4.68 & 3.77 & 4.79 & 23.6 & 94.4 & 93.8 & 30.1 & 10.1 & 5.83 & 6.12 & 23.9 \\
\hline 1963 & 6.42 & 5.37 & 4.22 & 3.80 & 6.13 & 12.9 & 48.8 & 96.6 & 40.4 & 11.2 & 6.98 & 7.56 & 20.9 \\
\hline 1964 & 6.09 & 6.45 & 6.03 & 4.50 & 7.50 & 25.3 & 126 & 170 & 41.8 & 11.4 & 5.30 & 5.72 & 34.7 \\
\hline 1965 & 5.17 & 4.21 & 2.42 & 3.03 & 5.83 & 20.4 & 89.0 & 216 & 73.0 & 22.0 & 8.61 & 7.89 & 38.3 \\
\hline 1966 & 7.12 & 7.02 & 5.96 & 6.00 & 5.39 & 5.75 & 38.6 & 80.9 & 21.5 & 5.90 & 3.69 & 3.88 & 16.0 \\
\hline 1967 & 4.13 & 3.96 & 3.99 & 4.23 & 4.83 & 8.46 & 94.3 & 174 & 71.9 & 12.3 & 4.75 & 5.47 & 32.8 \\
\hline 1968 & 5.72 & 5.61 & 4.69 & 4.24 & 4.84 & 8.62 & 112 & 153 & 67.2 & 11.8 & 8.02 & 7.48 & 32.8 \\
\hline 1969 & 6.46 & 6.37 & 6.39 & 5.64 & 8.30 & 74.4 & 255 & 309 & 87.0 & 23.2 & 11.1 & 8.74 & 67.0 \\
\hline 1970 & 7.42 & 10.5 & 6.66 & 6.00 & 6.40 & 9.73 & 68.4 & 66.6 & 17.5 & 6.61 & 3.90 & 4.06 & 17.8 \\
\hline 1971 & 4.17 & 3.33 & 4.00 & 5.00 & 6.00 & 16.0 & 64.8 & 55.3 & 11.2 & 3.06 & 3.00 & 3.00 & 14.9 \\
\hline 1972 & 3.47 & 5.13 & 5.40 & 4.63 & 5.94 & 14.1 & 109 & 166 & 59.5 & 10.2 & 5.09 & 5.17 & 32.8 \\
\hline 1973 & 4.93 & 4.50 & 4.60 & 4.48 & 4.43 & 12.7 & 141 & 181 & 41.8 & 9.92 & 5.02 & 4.90 & 35.1 \\
\hline 1974 & 4.59 & 4.14 & 4.47 & 4.55 & 4.36 & 32.9 & 93.7 & 80.7 & 18.6 & 5.51 & 3.91 & 4.87 & 21.9 \\
\hline 1975 & 4.77 & 4.62 & 3.95 & 3.24 & 4.38 & 10.4 & 154 & 279 & 104 & 19.2 & 7.26 & 6.90 & 50.3 \\
\hline 1976 & 5.75 & 5.43 & 5.20 & 6.60 & 6.40 & 6.91 & 135 & 283 & 79.3 & 15.0 & 7.12 & 6.82 & 47.0 \\
\hline 1977 & 6.41 & 6.97 & 5.70 & 5.84 & 6.63 & 31.5 & 101 & 79.2 & 23.9 & 6.74 & 5.12 & 5.58 & 23.8 \\
\hline 1978 & 5.72 & 5.82 & 5.97 & 5.25 & 6.18 & 15.8 & 149 & 120 & 27.3 & 7.76 & 3.90 & 4.27 & 29.7 \\
\hline
\end{tabular}




\section{8-0.000-9M HARI RUD RIVER AT DAULATYAR}

\section{(U.S. Geological Survey identification number: 343300065460000$)$}

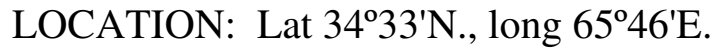

DRAINAGE AREA: $2,840 \mathrm{~km}^{2}$.

ELEVATION: 2,440 meters above mean sea level.

PERIOD OF RECORD: August 6, 1969 to September 30, 1978.

GAGE: Water-stage recorder.

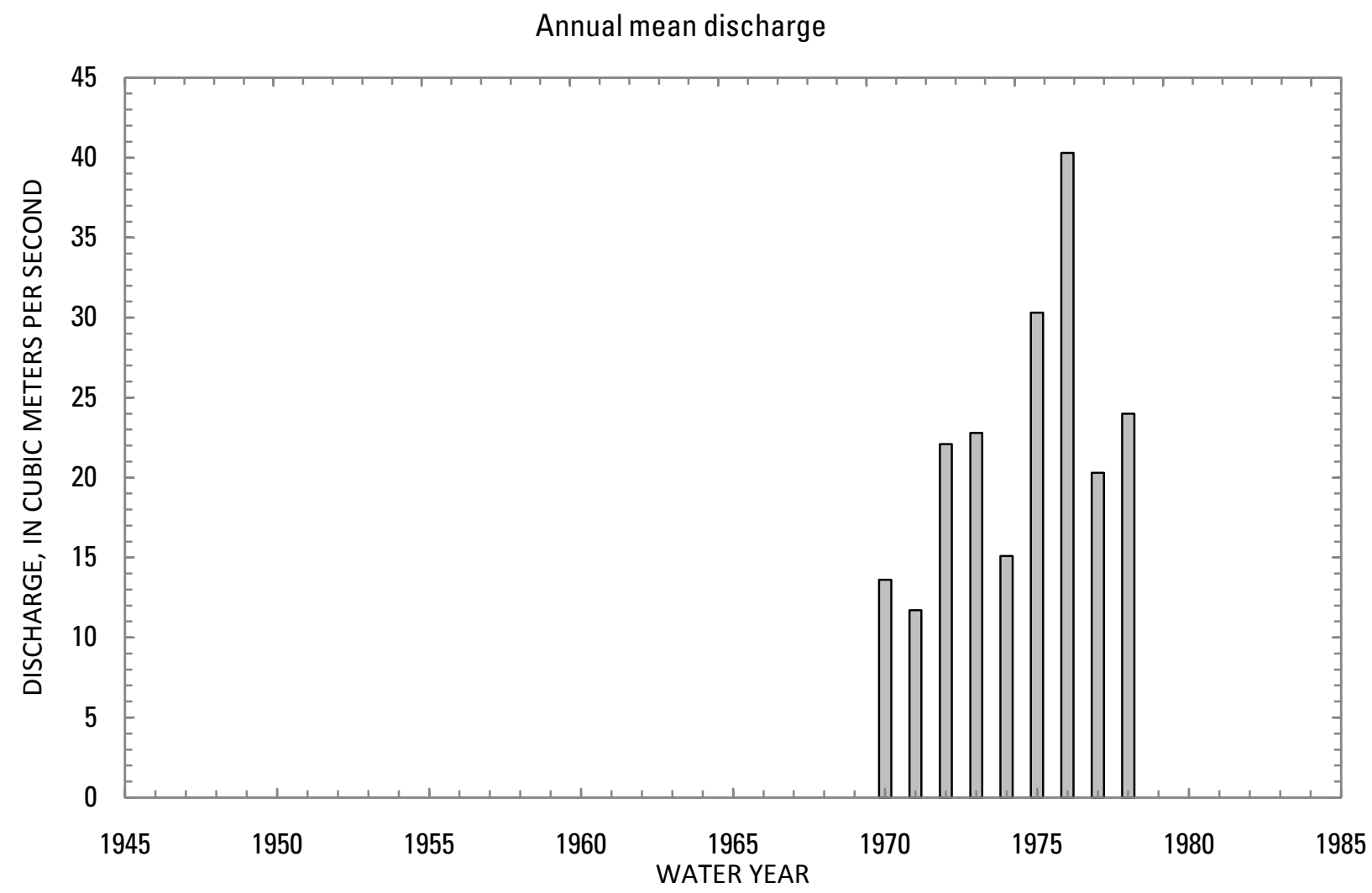




\section{8-0.000-9M HARI RUD RIVER AT DAULATYAR, Continued}

Statistics of monthly and annual mean discharges $\left[\mathrm{m}^{3} / \mathrm{s}\right.$, cubic meters per second]

\begin{tabular}{|c|c|c|c|c|c|c|c|c|}
\hline \multirow[b]{2}{*}{ Month } & \multicolumn{2}{|c|}{ Maximum } & \multicolumn{2}{|c|}{ Minimum } & \multicolumn{4}{|c|}{ Mean } \\
\hline & $\begin{array}{c}\text { Discharge } \\
\left(\mathrm{m}^{3} / \mathrm{s}\right)\end{array}$ & $\begin{array}{c}\text { Water year } \\
\text { of } \\
\text { occurrence }\end{array}$ & $\begin{array}{c}\text { Discharge } \\
\left(\mathrm{m}^{3} / \mathrm{s}\right)\end{array}$ & $\begin{array}{c}\text { Water year } \\
\text { of } \\
\text { occurrence }\end{array}$ & $\begin{array}{c}\text { Discharge } \\
\left(\mathrm{m}^{3} / \mathrm{s}\right)\end{array}$ & $\begin{array}{c}\text { Standard } \\
\text { deviation } \\
\left(\mathrm{m}^{3} / \mathrm{s}\right)\end{array}$ & $\begin{array}{c}\text { Coefficient } \\
\text { of } \\
\text { variation }\end{array}$ & $\begin{array}{c}\text { Percentage } \\
\text { of annual } \\
\text { discharge }\end{array}$ \\
\hline October & 5.73 & 1970 & 2.29 & 1972 & 3.75 & 0.97 & 0.26 & 1.41 \\
\hline November & 7.29 & 1970 & 2.57 & 1972 & 3.66 & 1.42 & 0.39 & 1.37 \\
\hline December & 5.46 & 1970 & 2.71 & 1973 & 3.38 & 0.86 & 0.25 & 1.27 \\
\hline January & 4.96 & 1970 & 2.40 & 1975 & 3.46 & 0.86 & 0.25 & 1.30 \\
\hline February & 5.39 & 1977 & 2.96 & 1975 & 3.86 & 0.82 & 0.21 & 1.45 \\
\hline March & 27.3 & 1977 & 5.07 & 1976 & 11.7 & 7.86 & 0.67 & 4.38 \\
\hline April & 126 & 1978 & 58.2 & 1970 & 81.5 & 25.1 & 0.31 & 30.6 \\
\hline May & 257 & 1976 & 36.3 & 1971 & 108 & 72.4 & 0.67 & 40.5 \\
\hline June & 65.6 & 1976 & 7.53 & 1971 & 30.9 & 22.1 & 0.71 & 11.6 \\
\hline July & 15.4 & 1975 & 2.90 & 1971 & 7.50 & 3.79 & 0.51 & 2.82 \\
\hline August & 6.48 & 1976 & 2.20 & 1971 & 4.29 & 1.33 & 0.31 & 1.61 \\
\hline September & 6.59 & 1969 & 2.42 & 1971 & 4.36 & 1.26 & 0.29 & 1.64 \\
\hline Annual & 40.3 & 1976 & 11.7 & 1971 & 22.2 & 8.91 & 0.40 & 100 \\
\hline
\end{tabular}


8-0.000-9M HARI RUD RIVER AT DAULATYAR, Continued

Monthly and annual flow duration, in cubic meters per second

[ng, not given]

\begin{tabular}{|c|c|c|c|c|c|c|c|c|c|c|c|c|c|}
\hline \multirow{2}{*}{$\begin{array}{l}\text { Percentage } \\
\text { of days } \\
\text { discharge } \\
\text { equaled or } \\
\text { exceeded }\end{array}$} & \multicolumn{12}{|c|}{ Month } & \multirow{2}{*}{ Annua } \\
\hline & October & November & December & January & February & March & April & May & June & July & August & September & \\
\hline 95 & 2.27 & 2.05 & 2.44 & 2.35 & 2.91 & 3.37 & 19.0 & 27.9 & 6.73 & 2.84 & 2.30 & 2.40 & 2.52 \\
\hline 90 & 2.47 & 2.54 & 2.50 & 2.52 & 3.00 & 3.56 & 27.0 & 36.9 & 7.68 & 3.50 & 2.50 & 2.86 & 2.95 \\
\hline 85 & 3.06 & 2.89 & 2.63 & 2.93 & 3.04 & 3.85 & 32.3 & 45.5 & 8.53 & 3.95 & 3.23 & 3.27 & 3.08 \\
\hline 80 & 3.10 & 2.98 & 2.69 & 2.95 & 3.07 & 4.35 & 39.1 & 50.4 & 9.35 & 4.28 & 3.34 & 3.36 & 3.21 \\
\hline 75 & 3.14 & 3.06 & 2.76 & 2.97 & 3.12 & 4.62 & 45.3 & 53.4 & 11.0 & 4.50 & 3.39 & 3.46 & 3.34 \\
\hline 70 & 3.22 & 3.13 & 2.90 & 2.99 & 3.17 & 4.86 & 52.3 & 57.8 & 12.8 & 4.74 & 3.45 & 3.52 & 3.48 \\
\hline 65 & 3.28 & 3.21 & 3.02 & 3.01 & 3.33 & 5.23 & 55.6 & 62.1 & 14.1 & 5.07 & 3.50 & 3.59 & 3.73 \\
\hline 60 & 3.32 & 3.27 & 3.10 & 3.18 & 3.41 & 5.82 & 60.2 & 66.2 & 16.3 & 5.57 & 3.70 & 3.75 & 3.98 \\
\hline 55 & 3.37 & 3.31 & 3.15 & 3.22 & 3.50 & 6.16 & 65.1 & 73.3 & 17.9 & 5.92 & 3.89 & 4.12 & 4.28 \\
\hline 50 & 3.66 & 3.35 & 3.19 & 3.26 & 3.88 & 6.55 & 68.9 & 78.8 & 20.8 & 6.18 & 4.08 & 4.25 & 4.65 \\
\hline 45 & 3.78 & 3.39 & 3.27 & 3.37 & 3.97 & 7.43 & 73.1 & 87.9 & 24.2 & 6.79 & 4.37 & 4.45 & 5.07 \\
\hline 40 & 3.90 & 3.43 & 3.34 & 3.39 & 4.00 & 8.28 & 78.0 & 102 & 28.3 & 7.08 & 4.49 & 4.57 & 5.59 \\
\hline 35 & 4.00 & 3.48 & 3.41 & 3.41 & 4.03 & 8.95 & 84.5 & 114 & 31.3 & 7.69 & 4.61 & 4.85 & 6.50 \\
\hline 30 & 4.05 & 3.53 & 3.46 & 3.43 & 4.06 & 12.3 & 93.3 & 130 & 34.3 & 8.07 & 4.95 & 4.99 & 8.06 \\
\hline 25 & 4.10 & 3.59 & 3.51 & 3.48 & 4.26 & 14.6 & 103 & 146 & 39.0 & 8.37 & 5.09 & 5.15 & 14.2 \\
\hline 20 & 4.29 & 3.88 & 3.78 & 4.25 & 4.54 & 16.2 & 109 & 160 & 44.2 & 9.83 & 5.59 & 5.56 & 26.1 \\
\hline 15 & 4.47 & 4.11 & 4.22 & 4.59 & 4.66 & 18.5 & 129 & 178 & 55.2 & 10.9 & 5.87 & 5.86 & 47.5 \\
\hline 10 & 5.38 & 5.92 & 5.17 & 4.87 & 4.99 & 26.9 & 146 & 229 & 68.5 & 14.0 & 6.04 & 5.98 & 68.1 \\
\hline 5 & 5.70 & 7.14 & $\mathrm{ng}$ & 5.27 & 5.87 & 46.3 & 189 & 286 & 91.8 & 18.1 & 6.81 & 6.44 & 112 \\
\hline
\end{tabular}




\section{8-0.000-9M HARI RUD RIVER AT DAULATYAR, Continued}

Probability of occurrence of annual high discharges

[m $\mathrm{m}^{3} / \mathrm{s}$, cubic meters per second; ng, not given]

\begin{tabular}{|c|c|c|c|c|c|c|}
\hline \multirow{2}{*}{$\begin{array}{c}\text { Exceedance } \\
\text { probability }\end{array}$} & \multirow{2}{*}{$\begin{array}{l}\text { Recurrence } \\
\text { interval } \\
\text { (years) }\end{array}$} & \multirow{2}{*}{$\begin{array}{l}\text { Maximum } \\
\text { instantaneous } \\
\text { discharge } \\
\left(\mathrm{m}^{3} / \mathrm{s}\right)\end{array}$} & \multicolumn{4}{|c|}{ Maximum daily mean discharge $\left(\mathrm{m}^{3} / \mathrm{s}\right)$} \\
\hline & & & $\begin{array}{l}\text { 3-day } \\
\text { period }\end{array}$ & $\begin{array}{l}\text { 7-day } \\
\text { period }\end{array}$ & $\begin{array}{l}\text { 15-day } \\
\text { period }\end{array}$ & $\begin{array}{l}\text { 30-day } \\
\text { period }\end{array}$ \\
\hline 0.99 & 1.01 & 49.7 & 44.7 & 43.3 & 42.1 & 39.4 \\
\hline 0.95 & 1.05 & 66.6 & 61.5 & 58.9 & 56.3 & 52.3 \\
\hline 0.90 & 1.11 & 79.2 & 73.8 & 70.2 & 66.4 & 61.4 \\
\hline 0.80 & 1.25 & 99.2 & 93.1 & 87.8 & 81.9 & 75.2 \\
\hline 0.50 & 2 & 161 & 151 & 139 & 126 & 114 \\
\hline 0.20 & 5 & 279 & 256 & 232 & 203 & 179 \\
\hline 0.10 & 10 & 384 & 345 & 308 & 264 & 230 \\
\hline 0.04 & 25 & 551 & 483 & 423 & 355 & 304 \\
\hline 0.02 & 50 & 705 & 604 & 523 & 433 & 366 \\
\hline 0.01 & 100 & 888 & 744 & 638 & 520 & 435 \\
\hline 0.005 & 200 & 1,100 & 905 & 768 & 618 & 511 \\
\hline 0.002 & 500 & 1,450 & $\mathrm{ng}$ & $\mathrm{ng}$ & $\mathrm{ng}$ & ng \\
\hline
\end{tabular}

\section{8-0.000-9M HARI RUD RIVER AT DAULATYAR, Continued}

Probability of occurrence of annual low discharges [ $\mathrm{m}^{3} / \mathrm{s}$, meters per second]

\begin{tabular}{|c|c|c|c|c|c|c|c|c|c|c|}
\hline \multirow{3}{*}{$\begin{array}{c}\text { Nonexceedance } \\
\text { probability }\end{array}$} & \multirow{3}{*}{$\begin{array}{c}\text { Recurrence } \\
\text { interval } \\
\text { (years) }\end{array}$} & \multicolumn{9}{|c|}{ Minimum daily mean discharge $\left(\mathrm{m}^{3} / \mathrm{s}\right)$} \\
\hline & & \multicolumn{9}{|c|}{ Number of consecutive days } \\
\hline & & 1 & 3 & 7 & 14 & 30 & 60 & 90 & 120 & 183 \\
\hline 0.05 & 20 & 1.42 & 1.50 & 1.60 & 1.78 & 2.04 & 2.19 & 2.29 & 2.35 & 2.62 \\
\hline 0.10 & 10 & 1.59 & 1.68 & 1.78 & 1.94 & 2.20 & 2.33 & 2.43 & 2.50 & 2.74 \\
\hline 0.20 & 5 & 1.81 & 1.90 & 2.01 & 2.15 & 2.41 & 2.52 & 2.61 & 2.68 & 2.91 \\
\hline 0.50 & 2 & 2.28 & 2.39 & 2.51 & 2.60 & 2.81 & 2.91 & 2.99 & 3.08 & 3.30 \\
\hline
\end{tabular}




\section{8-0.000-9M HARI RUD RIVER AT DAULATYAR, Continued}

Probability of occurrence of seasonal low discharges $\left[\mathrm{m}^{3} / \mathrm{s}\right.$, meters per second]

\begin{tabular}{|c|c|c|c|c|c|c|c|c|c|}
\hline \multirow{3}{*}{$\begin{array}{c}\text { Nonexceedance } \\
\text { probability }\end{array}$} & \multirow{3}{*}{$\begin{array}{c}\text { Recurrence } \\
\text { interval } \\
\text { (years) }\end{array}$} & \multicolumn{8}{|c|}{ Minimum daily mean discharge $\left(\mathrm{m}^{3} / \mathrm{s}\right)$} \\
\hline & & \multicolumn{8}{|c|}{ Number of consecutive days } \\
\hline & & 1 & 7 & 14 & 30 & 1 & 7 & 14 & 30 \\
\hline & & \multicolumn{4}{|c|}{ December-January-February } & \multicolumn{4}{|c|}{ March-April-May } \\
\hline 0.05 & 20 & 1.77 & 1.97 & 2.16 & 2.41 & 2.79 & 2.95 & 3.33 & 4.36 \\
\hline 0.10 & 10 & 1.89 & 2.10 & 2.26 & 2.50 & 3.08 & 3.19 & 3.49 & 4.89 \\
\hline 0.20 & 5 & 2.07 & 2.29 & 2.41 & 2.64 & 3.49 & 3.57 & 3.79 & 5.76 \\
\hline \multirow[t]{2}{*}{0.50} & 2 & 2.53 & 2.76 & 2.82 & 3.03 & 4.57 & 4.68 & 4.86 & 8.64 \\
\hline & & \multicolumn{4}{|c|}{ June-July-August } & \multicolumn{4}{|c|}{ September-0ctober-November } \\
\hline 0.05 & 20 & 1.80 & 1.96 & 2.09 & 2.28 & 1.49 & 1.71 & 1.88 & 2.16 \\
\hline 0.10 & 10 & 2.20 & 2.34 & 2.46 & 2.65 & 1.73 & 1.94 & 2.09 & 2.34 \\
\hline 0.20 & 5 & 2.72 & 2.85 & 2.94 & 3.13 & 2.06 & 2.25 & 2.38 & 2.60 \\
\hline 0.50 & 2 & 3.82 & 3.90 & 3.97 & 4.19 & 2.82 & 2.97 & 3.07 & 3.24 \\
\hline
\end{tabular}

\section{8-0.000-9M HARI RUD RIVER AT DAULATYAR, Continued}

Annual peak discharges

[ $\mathrm{m}^{3} / \mathrm{s}$, meters per second]

\begin{tabular}{|c|c|c|c|c|c|}
\hline \multicolumn{3}{|c|}{$\begin{array}{c}\text { Annual peak discharge, } \\
\text { by year }\end{array}$} & \multicolumn{3}{|c|}{$\begin{array}{l}\text { Annual peak discharge, } \\
\text { from highest to lowest }\end{array}$} \\
\hline $\begin{array}{l}\text { Water } \\
\text { year }\end{array}$ & Date & $\begin{array}{c}\text { Peak } \\
\text { discharge } \\
\left(\mathrm{m}^{3} / \mathrm{s}\right)\end{array}$ & $\begin{array}{l}\text { Water } \\
\text { year }\end{array}$ & Date & $\begin{array}{c}\text { Peak } \\
\text { discharge } \\
\left(\mathrm{m}^{3} / \mathrm{s}\right)\end{array}$ \\
\hline 1970 & April 25, 1970 & 107 & 1975 & May 14, 1975 & 446 \\
\hline 1971 & April 22, 1971 & 92.0 & 1976 & April 24, 1976 & 350 \\
\hline 1972 & April 28, 1972 & 157 & 1978 & April 15, 1978 & 299 \\
\hline 1973 & May 1,1973 & 170 & 1973 & May 1, 1973 & 170 \\
\hline 1974 & April 12, 1974 & 81.8 & 1972 & April 28, 1972 & 157 \\
\hline 1975 & May 14, 1975 & 446 & 1977 & April 18, 1977 & 111 \\
\hline 1976 & April 24, 1976 & 350 & 1970 & April 25, 1970 & 107 \\
\hline 1977 & April 18, 1977 & 111 & 1971 & April 22, 1971 & 92.0 \\
\hline 1978 & April 15, 1978 & 299 & 1974 & April 12, 1974 & 81.8 \\
\hline
\end{tabular}




\section{8-0.000-9M HARI RUD RIVER AT DAULATYAR, Continued}

Monthly and annual mean discharges, in cubic meters per second $[--$, no data]

\begin{tabular}{|c|c|c|c|c|c|c|c|c|c|c|c|c|c|}
\hline \multirow{2}{*}{$\begin{array}{c}\text { Water } \\
\text { year }\end{array}$} & \multicolumn{12}{|c|}{ Monthly mean discharge } & \multirow{2}{*}{$\begin{array}{c}\text { Annual } \\
\text { discharge }\end{array}$} \\
\hline & October & November & December & January & February & March & April & May & June & July & August & September & \\
\hline 1969 & -- & -- & -- & -- & -- & -- & -- & -- & -- & -- & -- & 6.59 & -- \\
\hline 1970 & 5.73 & 7.29 & 5.46 & 4.96 & 4.71 & 7.28 & 58.2 & 46.6 & 11.6 & 4.85 & 3.35 & 3.35 & 13.6 \\
\hline 1971 & 3.19 & 2.71 & 2.82 & 3.40 & 4.00 & 11.1 & 62.1 & 36.3 & 7.53 & 2.90 & 2.20 & 2.42 & 11.7 \\
\hline 1972 & 2.29 & 2.57 & 3.12 & 3.02 & 4.00 & 7.23 & 67.5 & 110 & 46.9 & 8.58 & 4.62 & 4.81 & 22.1 \\
\hline 1973 & 4.16 & 3.59 & 2.71 & 3.00 & 3.00 & 8.91 & 88.0 & 113 & 29.0 & 8.47 & 4.65 & 4.23 & 22.8 \\
\hline 1974 & 4.01 & 3.39 & 3.35 & 3.20 & 3.10 & 22.7 & 62.0 & 53.3 & 13.8 & 5.09 & 3.44 & 3.46 & 15.1 \\
\hline 1975 & 3.27 & 3.20 & 2.86 & 2.40 & 2.96 & 5.90 & 64.2 & 187 & 62.6 & 15.4 & 5.89 & 5.25 & 30.3 \\
\hline 1976 & 3.68 & 3.08 & 2.98 & 3.02 & 3.48 & 5.07 & 115 & 257 & 65.6 & 10.7 & 6.48 & 5.75 & 40.3 \\
\hline 1977 & 4.28 & 3.86 & 3.90 & 4.80 & 5.39 & 27.3 & 90.9 & 69.8 & 20.3 & 5.37 & 3.60 & 3.42 & 20.3 \\
\hline 1978 & 3.17 & 3.26 & 3.19 & 3.35 & 4.05 & 9.53 & 126 & 98.8 & 20.9 & 6.15 & 4.39 & 4.31 & 24.0 \\
\hline
\end{tabular}




\section{8-1.R00-9T SENJAB RIVER AT KHUSH RABAT}

\section{(U.S. Geological Survey identification number: 344100062070000)}

LOCATION: Lat $34^{\circ} 41^{\prime} \mathrm{N}$., long $62^{\circ} 07^{\prime} \mathrm{E}$.

DRAINAGE AREA: $65 \mathrm{~km}^{2}$.

ELEVATION: 1,320 meters above mean sea level.

PERIOD OF RECORD: May 24, 1969 to September 30, 1978.

GAGE: Water-stage recorder. Staff gage at same site and datum prior to 1970 water year.

Annual mean discharge

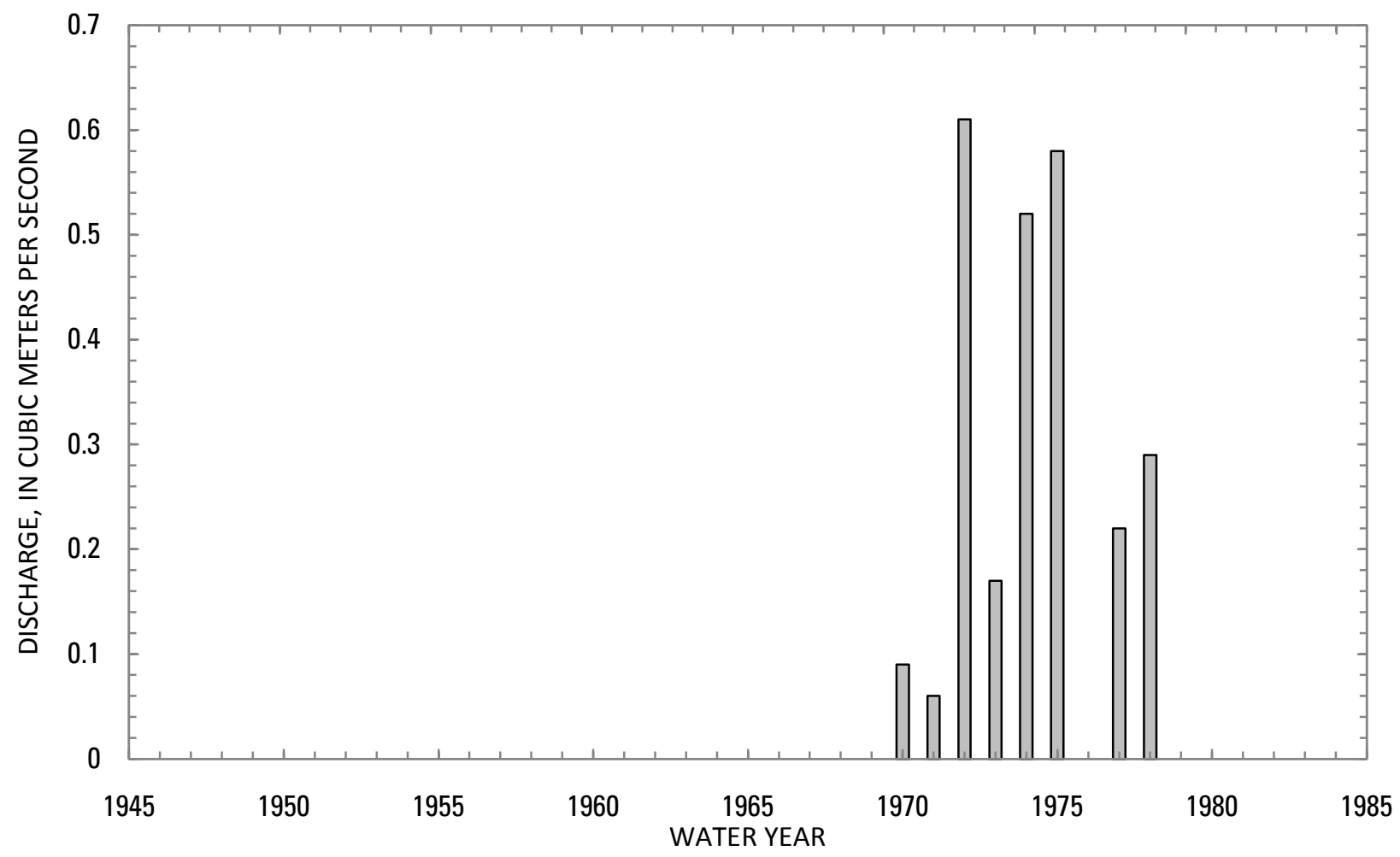




\section{8-1.R00-9T SENJAB RIVER AT KHUSH RABAT, Continued}

Statistics of monthly and annual mean discharges $\left[\mathrm{m}^{3} / \mathrm{s}\right.$, cubic meters per second]

\begin{tabular}{|c|c|c|c|c|c|c|c|c|}
\hline \multirow[b]{2}{*}{ Month } & \multicolumn{2}{|c|}{ Maximum } & \multicolumn{2}{|c|}{ Minimum } & \multicolumn{4}{|c|}{ Mean } \\
\hline & $\begin{array}{c}\text { Discharge } \\
\left(\mathrm{m}^{3} / \mathrm{s}\right)\end{array}$ & $\begin{array}{c}\text { Water year } \\
\text { of } \\
\text { occurrence }\end{array}$ & $\begin{array}{c}\text { Discharge } \\
\left(\mathrm{m}^{3} / \mathrm{s}\right)\end{array}$ & $\begin{array}{c}\text { Water year } \\
\text { of } \\
\text { occurrence }\end{array}$ & $\begin{array}{c}\text { Discharge } \\
\left(\mathrm{m}^{3} / \mathrm{s}\right)\end{array}$ & $\begin{array}{c}\text { Standard } \\
\text { deviation } \\
\left(\mathrm{m}^{3} / \mathrm{s}\right)\end{array}$ & $\begin{array}{c}\text { Coefficient } \\
\text { of } \\
\text { variation }\end{array}$ & $\begin{array}{c}\text { Percentage } \\
\text { of annual } \\
\text { discharge }\end{array}$ \\
\hline October & 0.06 & 1975 & 0 & 1972 & 0.01 & 0.02 & 1.57 & 0.33 \\
\hline November & 0.37 & 1972 & 0 & 1974 & 0.13 & 0.13 & 1.06 & 3.18 \\
\hline December & 0.99 & 1978 & 0.01 & 1974 & 0.22 & 0.33 & 1.47 & 5.68 \\
\hline January & 1.33 & 1972 & 0.06 & 1971 & 0.59 & 0.50 & 0.85 & 14.9 \\
\hline February & 1.62 & 1972 & 0.06 & 1971 & 0.61 & 0.62 & 1.02 & 15.4 \\
\hline March & 2.62 & 1972 & 0.20 & 1971 & 1.12 & 0.81 & 0.72 & 28.5 \\
\hline April & 2.44 & 1975 & 0.24 & 1973 & 1.05 & 0.95 & 0.90 & 26.7 \\
\hline May & 0.27 & 1976 & 0.04 & 1973 & 0.13 & 0.08 & 0.60 & 3.38 \\
\hline June & 0.13 & 1969 & 0 & 1971 & 0.05 & 0.05 & 1.09 & 1.20 \\
\hline July & 0.08 & 1972 & 0 & 1971 & 0.02 & 0.03 & 1.31 & 0.54 \\
\hline August & 0.03 & 1972 & 0 & 1969 & 0.01 & 0.01 & 1.68 & 0.19 \\
\hline September & 0.02 & 1976 & 0 & 1969 & 0 & 0.01 & 1.74 & 0.12 \\
\hline Annual & 0.61 & 1972 & 0.06 & 1971 & 0.32 & 0.22 & 0.69 & 100 \\
\hline
\end{tabular}


8-1.R00-9T SENJAB RIVER AT KHUSH RABAT, Continued

Monthly and annual flow duration, in cubic meters per second

[ng, not given]

\begin{tabular}{|c|c|c|c|c|c|c|c|c|c|c|c|c|c|}
\hline \multirow{2}{*}{$\begin{array}{l}\text { Percentage } \\
\text { of days } \\
\text { discharge } \\
\text { equaled or } \\
\text { exceeded }\end{array}$} & \multicolumn{12}{|c|}{ Month } & \multirow{2}{*}{ Annua } \\
\hline & October & November & December & January & February & March & April & May & June & July & August & September & \\
\hline 95 & 0 & 0 & 0 & 0.01 & 0.03 & 0.02 & 0.10 & 0.02 & 0 & 0 & 0 & 0 & 0 \\
\hline 90 & 0 & 0 & 0 & 0.02 & 0.05 & 0.03 & 0.14 & 0.03 & 0 & 0 & 0 & 0 & 0 \\
\hline 85 & 0 & 0 & 0.01 & 0.02 & 0.06 & 0.08 & 0.17 & 0.04 & 0 & 0 & 0 & 0 & 0 \\
\hline 80 & 0 & 0.01 & 0.02 & 0.02 & 0.08 & 0.25 & 0.20 & 0.05 & 0.01 & 0 & 0 & 0 & 0 \\
\hline 75 & 0 & 0.01 & 0.02 & 0.03 & 0.08 & 0.36 & 0.23 & 0.06 & 0.01 & 0 & 0 & 0 & 0 \\
\hline 70 & 0 & 0.01 & 0.02 & 0.08 & 0.09 & 0.44 & 0.27 & 0.07 & 0.01 & 0 & 0 & 0 & 0.01 \\
\hline 65 & 0 & 0.02 & 0.03 & 0.09 & 0.10 & 0.50 & 0.29 & 0.09 & 0.02 & 0 & 0 & 0 & 0.02 \\
\hline 60 & 0 & 0.02 & 0.03 & 0.10 & 0.14 & 0.57 & 0.31 & 0.10 & 0.02 & 0.01 & 0 & 0 & 0.02 \\
\hline 55 & 0 & 0.02 & 0.04 & 0.13 & 0.21 & 0.70 & 0.40 & 0.10 & 0.02 & 0.01 & 0 & 0 & 0.03 \\
\hline 50 & 0 & 0.03 & 0.04 & 0.20 & 0.26 & 0.77 & 0.47 & 0.11 & 0.03 & 0.02 & 0 & 0 & 0.04 \\
\hline 45 & 0.01 & 0.04 & 0.05 & 0.26 & 0.33 & 0.86 & 0.56 & 0.11 & 0.03 & 0.02 & 0 & 0 & 0.05 \\
\hline 40 & 0.01 & 0.04 & 0.06 & 0.34 & 0.51 & 1.00 & 0.72 & 0.13 & 0.04 & 0.02 & 0 & 0 & 0.07 \\
\hline 35 & 0.01 & 0.05 & 0.07 & 0.47 & 0.64 & 1.12 & 0.79 & 0.14 & 0.05 & 0.02 & 0 & 0 & 0.09 \\
\hline 30 & 0.02 & 0.05 & 0.08 & 0.57 & 0.78 & 1.23 & 0.89 & 0.15 & 0.07 & 0.03 & 0.01 & 0 & 0.12 \\
\hline 25 & 0.02 & 0.07 & 0.10 & 0.72 & 0.99 & 1.37 & 1.06 & 0.18 & 0.09 & 0.03 & 0.02 & 0.01 & 0.21 \\
\hline 20 & 0.03 & 0.10 & 0.15 & 0.89 & 1.20 & 1.53 & 1.37 & 0.2 & 0.11 & 0.05 & 0.02 & 0.02 & 0.30 \\
\hline 15 & 0.04 & 0.20 & 0.30 & 1.07 & 1.36 & 1.83 & 2.17 & 0.23 & 0.12 & 0.06 & 0.03 & 0.02 & 0.57 \\
\hline 10 & ng & 0.28 & 0.37 & 1.45 & 1.60 & 2.47 & 2.97 & 0.27 & 0.13 & 0.08 & 0.03 & 0.02 & 0.92 \\
\hline 5 & ng & 0.52 & 0.87 & 2.55 & 1.98 & 3.49 & 4.06 & 0.37 & 0.16 & $\mathrm{ng}$ & 0.04 & 0.03 & 1.5 \\
\hline
\end{tabular}




\section{8-1.R00-9T SENJAB RIVER AT KHUSH RABAT, Continued}

Probability of occurrence of annual high discharges

[ $\mathrm{m}^{3} / \mathrm{s}$, cubic meters per second; ng, not given]

\begin{tabular}{|c|c|c|c|c|c|c|}
\hline \multirow{2}{*}{$\begin{array}{c}\text { Exceedance } \\
\text { probability }\end{array}$} & \multirow{2}{*}{$\begin{array}{c}\text { Recurrence } \\
\text { interval } \\
\text { (years) }\end{array}$} & \multirow{2}{*}{$\begin{array}{c}\text { Maximum } \\
\text { instantaneous } \\
\text { discharge } \\
\left(\mathrm{m}^{3} / \mathrm{s}\right)\end{array}$} & \multicolumn{4}{|c|}{ Maximum daily mean discharge $\left(\mathrm{m}^{3} / \mathrm{s}\right)$} \\
\hline & & & $\begin{array}{l}\text { 3-day } \\
\text { period }\end{array}$ & $\begin{array}{l}\text { 7-day } \\
\text { period }\end{array}$ & $\begin{array}{l}\text { 15-day } \\
\text { period }\end{array}$ & $\begin{array}{l}\text { 30-day } \\
\text { period }\end{array}$ \\
\hline 0.99 & 1.01 & 4.40 & 0.72 & 0.49 & 0.29 & 0.21 \\
\hline 0.95 & 1.05 & 6.50 & 1.19 & 0.78 & 0.49 & 0.37 \\
\hline 0.90 & 1.11 & 8.10 & 1.54 & 0.99 & 0.66 & 0.50 \\
\hline 0.80 & 1.25 & 10.7 & 2.12 & 1.35 & 0.93 & 0.71 \\
\hline 0.50 & 2 & 18.6 & 3.85 & 2.43 & 1.79 & 1.34 \\
\hline 0.20 & 5 & 33.7 & 6.90 & 4.47 & 3.42 & 2.48 \\
\hline 0.10 & 10 & 46.7 & 9.31 & 6.18 & 4.78 & 3.37 \\
\hline 0.04 & 25 & 67.0 & 12.8 & 8.79 & 6.84 & 4.64 \\
\hline 0.02 & 50 & 85.1 & 15.6 & 11.1 & 8.60 & 5.68 \\
\hline 0.01 & 100 & 106 & 18.7 & 13.6 & 10.6 & 6.78 \\
\hline 0.005 & 200 & 130 & 22.0 & 16.5 & 12.7 & 7.96 \\
\hline 0.002 & 500 & 168 & $\mathrm{ng}$ & $\mathrm{ng}$ & ng & $\mathrm{ng}$ \\
\hline
\end{tabular}

'Less than 10 years of data used.

\section{8-1.R00-9T SENJAB RIVER AT KHUSH RABAT, Continued}

Probability of occurrence of annual low discharges

[ $\mathrm{m}^{3} / \mathrm{s}$, meters per second; $\mathrm{ng}$, not given]

\begin{tabular}{|c|c|c|c|c|c|c|c|c|c|c|}
\hline \multirow{3}{*}{$\begin{array}{c}\text { Nonexceedance } \\
\text { probability }\end{array}$} & \multirow{3}{*}{$\begin{array}{c}\text { Recurrence } \\
\text { interval } \\
\text { (years) }\end{array}$} & \multicolumn{9}{|c|}{ Minimum daily mean discharge $\left(\mathrm{m}^{3} / \mathrm{s}\right)$} \\
\hline & & \multicolumn{9}{|c|}{ Number of consecutive days } \\
\hline & & 1 & 3 & 7 & 14 & 30 & 60 & 90 & 120 & 183 \\
\hline 0.05 & 20 & $\mathrm{ng}$ & ng & $\mathrm{ng}$ & $\mathrm{ng}$ & ng & 0 & 0 & 0 & 0 \\
\hline 0.10 & 10 & ng & ng & $\mathrm{ng}$ & ng & ng & 0 & 0 & 0 & 0 \\
\hline 0.20 & 5 & ng & ng & $\mathrm{ng}$ & $\mathrm{ng}$ & ng & 0 & 0 & 0 & 0 \\
\hline 0.50 & 2 & ng & ng & $\mathrm{ng}$ & $\mathrm{ng}$ & ng & 0 & 0 & 0 & 0.01 \\
\hline
\end{tabular}




\section{8-1.R00-9T SENJAB RIVER AT KHUSH RABAT, Continued}

Probability of occurrence of seasonal low discharges

[ $\mathrm{m}^{3} / \mathrm{s}$, meters per second; $\mathrm{ng}$, not given]

\begin{tabular}{|c|c|c|c|c|c|c|c|c|c|}
\hline \multirow{3}{*}{$\begin{array}{c}\text { Nonexceedance } \\
\text { probability }\end{array}$} & \multirow{3}{*}{$\begin{array}{c}\text { Recurrence } \\
\text { interval } \\
\text { (years) }\end{array}$} & \multicolumn{8}{|c|}{ Minimum daily mean discharge $\left(\mathrm{m}^{3} / \mathrm{s}\right)$} \\
\hline & & \multicolumn{8}{|c|}{ Number of consecutive days } \\
\hline & & 1 & 7 & 14 & 30 & 1 & 7 & 14 & 30 \\
\hline & & \multicolumn{4}{|c|}{ December-January-February } & \multicolumn{4}{|c|}{ March-April-May } \\
\hline 0.05 & 20 & 0 & 0 & 0 & 0.01 & 0 & 0 & 0.01 & 0.03 \\
\hline 0.10 & 10 & 0 & 0 & 0 & 0.01 & 0 & 0.01 & 0.01 & 0.04 \\
\hline 0.20 & 5 & 0 & 0 & 0.01 & 0.01 & 0.01 & 0.01 & 0.02 & 0.06 \\
\hline \multirow[t]{2}{*}{0.50} & 2 & 0 & 0.02 & 0.02 & 0.04 & 0.04 & 0.05 & 0.06 & 0.11 \\
\hline & & \multicolumn{4}{|c|}{ June-July-August } & \multicolumn{4}{|c|}{ September-October-November } \\
\hline 0.05 & 20 & 0 & 0 & 0 & 0 & ng & ng & $\mathrm{ng}$ & ng \\
\hline 0.10 & 10 & 0 & 0 & 0 & 0 & $\mathrm{ng}$ & ng & $\mathrm{ng}$ & $\mathrm{ng}$ \\
\hline 0.20 & 5 & 0 & 0 & 0 & 0 & $\mathrm{ng}$ & ng & $\mathrm{ng}$ & $\mathrm{ng}$ \\
\hline 0.50 & 2 & 0 & 0 & 0 & 0 & $\mathrm{ng}$ & $\mathrm{ng}$ & $\mathrm{ng}$ & ng \\
\hline
\end{tabular}

\section{8-1.R00-9T SENJAB RIVER AT KHUSH RABAT, Continued}

Annual peak discharges

[ $\mathrm{m}^{3} / \mathrm{s}$, meters per second]

\begin{tabular}{|c|c|c|c|c|c|}
\hline \multicolumn{3}{|c|}{$\begin{array}{c}\text { Annual peak discharge, } \\
\text { by year }\end{array}$} & \multicolumn{3}{|c|}{$\begin{array}{l}\text { Annual peak discharge, } \\
\text { from highest to lowest }\end{array}$} \\
\hline $\begin{array}{l}\text { Water } \\
\text { year }\end{array}$ & Date & $\begin{array}{c}\text { Peak } \\
\text { discharge } \\
\left(\mathrm{m}^{3} / \mathrm{s}\right)\end{array}$ & $\begin{array}{c}\text { Water } \\
\text { year }\end{array}$ & Date & $\begin{array}{c}\text { Peak } \\
\text { discharge } \\
\left(\mathrm{m}^{3} / \mathrm{s}\right)\end{array}$ \\
\hline 1970 & April 13, 1970 & 7.38 & 1976 & April 22, 1976 & 54.0 \\
\hline 1971 & March 30, 1971 & 17.0 & 1972 & April 14, 1972 & 48.0 \\
\hline 1972 & April 14, 1972 & 48.0 & 1978 & December 24, 1977 & 27.4 \\
\hline 1973 & February 23, 1973 & 18.1 & 1973 & February 23, 1973 & 18.1 \\
\hline 1974 & April 1, 1974 & 14.6 & 1975 & March 20, 1975 & 17.5 \\
\hline 1975 & March 20, 1975 & 17.5 & 1971 & March 30, 1971 & 17.0 \\
\hline 1976 & April 22, 1976 & 54.0 & 1974 & April 1, 1974 & 14.6 \\
\hline 1977 & April 22, 1977 & 8.40 & 1977 & April 22, 1977 & 8.40 \\
\hline 1978 & December 24, 1977 & 27.4 & 1970 & April 13, 1970 & 7.38 \\
\hline
\end{tabular}


8-1.R00-9T SENJAB RIVER AT KHUSH RABAT, Continued

Monthly and annual mean discharges, in cubic meters per second $[-$, no data]

\begin{tabular}{|c|c|c|c|c|c|c|c|c|c|c|c|c|c|}
\hline \multirow{2}{*}{$\begin{array}{l}\text { Water } \\
\text { year }\end{array}$} & \multicolumn{12}{|c|}{ Monthly mean discharge } & \multirow{2}{*}{$\begin{array}{c}\text { Annual } \\
\text { discharge }\end{array}$} \\
\hline & October & November & December & January & February & March & April & May & June & July & August & September & \\
\hline 1969 & -- & -- & -- & -- & -- & -- & -- & -- & 0.13 & 0.03 & 0 & 0 & -- \\
\hline 1970 & 0.01 & 0.12 & 0.03 & 0.15 & 0.09 & 0.25 & 0.41 & 0.05 & 0.01 & 0 & 0 & 0 & 0.09 \\
\hline 1971 & 0 & 0.02 & 0.02 & 0.06 & 0.06 & 0.20 & 0.28 & 0.09 & 0 & 0 & 0 & 0 & 0.06 \\
\hline 1972 & 0 & 0.37 & 0.05 & 1.33 & 1.62 & 2.62 & 0.92 & 0.20 & 0.12 & 0.08 & 0.03 & 0.01 & 0.61 \\
\hline 1973 & 0 & 0.01 & 0.05 & 0.36 & 0.38 & 1.01 & 0.24 & 0.04 & 0 & 0 & 0 & 0 & 0.17 \\
\hline 1974 & 0 & 0 & 0.01 & 1.19 & 0.56 & 1.94 & 2.23 & 0.17 & 0.04 & 0.02 & 0.02 & 0.02 & 0.52 \\
\hline 1975 & 0.06 & 0.04 & 0.38 & 0.91 & 1.54 & 1.44 & 2.44 & 0.17 & 0.01 & 0 & 0 & 0 & 0.58 \\
\hline 1976 & 0.01 & 0.18 & 0.41 & 0.27 & -- & 0.62 & 2.17 & 0.27 & 0.11 & 0.06 & 0.02 & 0.02 & -- \\
\hline 1977 & 0.03 & 0.10 & 0.07 & 0.91 & 0.37 & 0.63 & 0.46 & 0.06 & 0.02 & 0.01 & 0 & 0 & 0.22 \\
\hline 1978 & 0.01 & 0.30 & 0.99 & 0.09 & 0.25 & 1.37 & 0.28 & 0.16 & 0.03 & 0.01 & 0 & 0 & 0.29 \\
\hline
\end{tabular}




\section{8-2.R00-3A KARUKH RIVER NEAR HERAT}

\section{(U.S. Geological Survey identification number: 342600062280000$)$}

LOCATION: Lat $34^{\circ} 26^{\prime} \mathrm{N}$., long $62^{\circ} 28^{\prime} \mathrm{E}$.

DRAINAGE AREA: $1,390 \mathrm{~km}^{2}$.

ELEVATION: 1,145 meters above mean sea level.

PERIOD OF RECORD: December 11, 1973 to September 30, 1977.

GAGE: Water-stage recorder.

Annual mean discharge

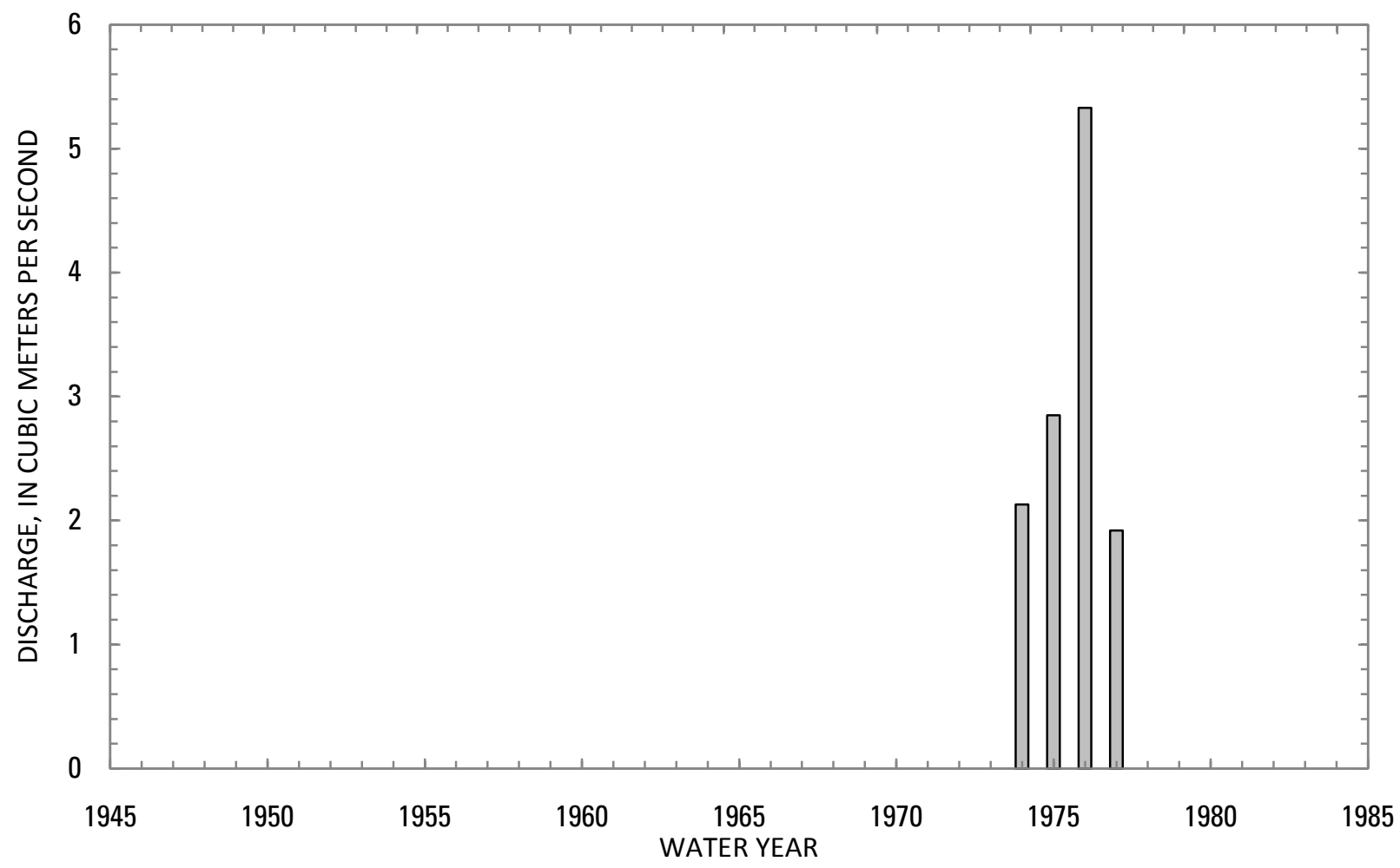




\section{8-2.R00-3A KARUKH RIVER NEAR HERAT, Continued}

Statistics of monthly and annual mean discharges $\left[\mathrm{m}^{3} / \mathrm{s}\right.$, cubic meters per second]

\begin{tabular}{|c|c|c|c|c|c|c|c|c|}
\hline \multirow[b]{2}{*}{ Month } & \multicolumn{2}{|c|}{ Maximum } & \multicolumn{2}{|c|}{ Minimum } & \multicolumn{4}{|c|}{ Mean } \\
\hline & $\begin{array}{c}\text { Discharge } \\
\left(\mathrm{m}^{3} / \mathrm{s}\right)\end{array}$ & $\begin{array}{c}\text { Water year } \\
\text { of } \\
\text { occurrence }\end{array}$ & $\begin{array}{c}\text { Discharge } \\
\left(\mathrm{m}^{3} / \mathrm{s}\right)\end{array}$ & $\begin{array}{c}\text { Water year } \\
\text { of } \\
\text { occurrence }\end{array}$ & $\begin{array}{c}\text { Discharge } \\
\left(\mathrm{m}^{3} / \mathrm{s}\right)\end{array}$ & $\begin{array}{c}\text { Standard } \\
\text { deviation } \\
\left(\mathrm{m}^{3} / \mathrm{s}\right)\end{array}$ & $\begin{array}{c}\text { Coefficient } \\
\text { of } \\
\text { variation }\end{array}$ & $\begin{array}{c}\text { Percentage } \\
\text { of annual } \\
\text { discharge }\end{array}$ \\
\hline October & 1.30 & 1974 & 0.99 & 1975 & 1.16 & 0.14 & 0.12 & 3.38 \\
\hline November & 1.53 & 1977 & 1.19 & 1975 & 1.36 & 0.15 & 0.11 & 3.96 \\
\hline December & 2.48 & 1977 & 1.31 & 1975 & 1.70 & 0.53 & 0.31 & 4.97 \\
\hline January & 2.31 & 1973 & 0.78 & 1975 & 1.83 & 0.67 & 0.36 & 5.35 \\
\hline February & 5.63 & 1976 & 2.13 & 1975 & 3.40 & 1.32 & 0.39 & 9.94 \\
\hline March & 17.8 & 1976 & 4.37 & 1977 & 8.40 & 5.39 & 0.64 & 24.5 \\
\hline April & 17.5 & 1976 & 3.31 & 1973 & 9.45 & 6.63 & 0.70 & 27.6 \\
\hline May & 13.7 & 1976 & 0.96 & 1973 & 4.33 & 5.39 & 1.24 & 12.7 \\
\hline June & 0.90 & 1976 & 0.40 & 1974 & 0.59 & 0.21 & 0.35 & 1.73 \\
\hline July & 1.00 & 1976 & 0.46 & 1977 & 0.62 & 0.22 & 0.37 & 1.80 \\
\hline August & 0.95 & 1976 & 0.41 & 1973 & 0.60 & 0.21 & 0.34 & 1.75 \\
\hline September & 1.02 & 1973 & 0.61 & 1975 & 0.79 & 0.16 & 0.21 & 2.32 \\
\hline Annual & 5.33 & 1976 & 1.92 & 1977 & 3.06 & 1.57 & 0.51 & 100 \\
\hline
\end{tabular}


8-2.R00-3A KARUKH RIVER NEAR HERAT, Continued

Monthly and annual flow duration, in cubic meters per second

[ng, not given]

\begin{tabular}{|c|c|c|c|c|c|c|c|c|c|c|c|c|c|}
\hline \multirow{2}{*}{$\begin{array}{l}\text { Percentage } \\
\text { of days } \\
\text { discharge } \\
\text { equaled or } \\
\text { exceeded }\end{array}$} & \multicolumn{12}{|c|}{ Month } & \multirow{2}{*}{ Annua } \\
\hline & October & November & December & January & February & March & April & May & June & July & August & September & \\
\hline 95 & 0.96 & 1.10 & 0.85 & 0.55 & 1.29 & 3.34 & 2.02 & 0.52 & 0.37 & 0.44 & 0.40 & 0.51 & 0.45 \\
\hline 90 & 0.96 & 1.10 & 0.99 & 0.73 & 1.78 & 3.40 & 2.99 & 0.55 & 0.39 & 0.47 & 0.40 & 0.52 & 0.49 \\
\hline 85 & 0.97 & 1.11 & 1.31 & 0.75 & 2.29 & 3.46 & 3.13 & 0.76 & 0.40 & 0.48 & 0.41 & 0.59 & 0.54 \\
\hline 80 & 1.00 & 1.23 & 1.33 & 1.36 & 2.38 & 3.57 & 3.97 & 0.83 & 0.41 & 0.49 & 0.45 & 0.61 & 0.63 \\
\hline 75 & 1.01 & 1.24 & 1.35 & 1.41 & 2.42 & 4.00 & 4.17 & 0.93 & 0.41 & 0.49 & 0.51 & 0.67 & 0.74 \\
\hline 70 & 1.10 & 1.25 & 1.37 & 1.52 & 2.45 & 4.67 & 4.61 & 0.97 & 0.42 & 0.50 & 0.52 & 0.70 & 0.82 \\
\hline 65 & 1.10 & 1.30 & 1.39 & 1.56 & 2.49 & 4.92 & 4.86 & 1.01 & 0.44 & 0.50 & 0.52 & 0.70 & 0.96 \\
\hline 60 & 1.11 & 1.31 & 1.41 & 1.60 & 2.54 & 6.03 & 5.03 & 1.06 & 0.59 & 0.50 & 0.52 & 0.71 & 1.06 \\
\hline 55 & 1.15 & 1.32 & 1.43 & 1.69 & 2.61 & 6.24 & 5.20 & 1.16 & 0.60 & 0.50 & 0.53 & 0.71 & 1.19 \\
\hline 50 & 1.20 & 1.40 & 1.46 & 1.83 & 2.70 & 6.49 & 6.13 & 1.99 & 0.60 & 0.51 & 0.55 & 0.76 & 1.29 \\
\hline 45 & 1.21 & 1.41 & 1.51 & 1.96 & 2.82 & 6.91 & 8.07 & 2.11 & 0.61 & 0.51 & 0.56 & 0.79 & 1.37 \\
\hline 40 & 1.23 & 1.41 & 1.72 & 2.02 & 2.89 & 7.25 & 9.11 & 2.22 & 0.61 & 0.56 & 0.60 & 0.80 & 1.45 \\
\hline 35 & 1.29 & 1.42 & 1.80 & 2.08 & 2.95 & 7.93 & 10.1 & 2.45 & 0.62 & 0.61 & 0.60 & 0.81 & 1.89 \\
\hline 30 & 1.29 & 1.42 & 1.85 & 2.15 & 3.02 & 8.32 & 11.6 & 3.07 & 0.64 & 0.62 & 0.61 & 0.91 & 2.23 \\
\hline 25 & 1.30 & 1.42 & 1.89 & 2.46 & 3.69 & 8.79 & 13.6 & 4.01 & 0.71 & 0.62 & 0.61 & 0.92 & 2.91 \\
\hline 20 & 1.30 & 1.43 & 1.93 & 2.51 & 4.06 & 9.31 & 14.9 & 7.50 & 0.73 & 0.63 & 0.78 & 0.93 & 3.57 \\
\hline 15 & 1.31 & 1.52 & 1.97 & 2.55 & 6.45 & 10.3 & 17.5 & 9.78 & 0.81 & $\mathrm{ng}$ & ng & 1.01 & 4.92 \\
\hline 10 & $\mathrm{ng}$ & 1.62 & 2.01 & 2.65 & 6.84 & 14.8 & 19.1 & 14.7 & 0.83 & $\mathrm{ng}$ & $\mathrm{ng}$ & 1.08 & 7.74 \\
\hline 5 & $\mathrm{ng}$ & $\mathrm{ng}$ & 3.13 & 2.94 & 7.58 & $\mathrm{ng}$ & 20 & 18.4 & 1.05 & ng & $\mathrm{ng}$ & $\mathrm{ng}$ & 14.3 \\
\hline
\end{tabular}




\section{8-2.R00-3A KARUKH RIVER NEAR HERAT, Continued}

Probability of occurrence of annual high discharges

[ $\mathrm{m}^{3} / \mathrm{s}$, cubic meters per second; $\mathrm{ng}$, not given]

\begin{tabular}{|c|c|c|c|c|c|c|}
\hline \multirow{2}{*}{$\begin{array}{c}\text { Exceedance } \\
\text { probability }\end{array}$} & \multirow{2}{*}{$\begin{array}{l}\text { Recurrence } \\
\text { interval } \\
\text { (years) }\end{array}$} & \multirow{2}{*}{$\begin{array}{c}\text { Maximum } \\
\text { instantaneous } \\
\text { discharge }^{1} \\
\left(\mathrm{~m}^{3} / \mathrm{s}\right)\end{array}$} & \multicolumn{4}{|c|}{ Maximum daily mean discharge $\left(\mathrm{m}^{3} / \mathrm{s}\right)$} \\
\hline & & & $\begin{array}{l}\text { 3-day } \\
\text { period }\end{array}$ & $\begin{array}{l}\text { 7-day } \\
\text { period }\end{array}$ & $\begin{array}{l}\text { 15-day } \\
\text { period }\end{array}$ & $\begin{array}{l}\text { 30-day } \\
\text { period }\end{array}$ \\
\hline 0.99 & 1.01 & 9.80 & 5.85 & 3.56 & 2.79 & 2.47 \\
\hline 0.95 & 1.05 & 10.3 & 7.10 & 5.15 & 4.34 & 3.90 \\
\hline 0.90 & 1.11 & 10.9 & 8.11 & 6.34 & 5.50 & 4.95 \\
\hline 0.80 & 1.25 & 12.4 & 9.84 & 8.24 & 7.35 & 6.57 \\
\hline 0.50 & 2 & 20.0 & 15.9 & 14.0 & 12.9 & 11.1 \\
\hline 0.20 & 5 & 49.7 & 30.2 & 25.0 & 22.9 & 18.3 \\
\hline 0.10 & 10 & 98.0 & 45.1 & 34.4 & 31.0 & 23.6 \\
\hline 0.04 & 25 & 239 & 73.3 & 49.1 & 43.0 & 30.8 \\
\hline 0.02 & 50 & 468 & 103 & 62.2 & 53.3 & 36.4 \\
\hline 0.01 & 100 & 916 & 144 & 77.4 & 64.6 & 42.2 \\
\hline 0.005 & 200 & 1,790 & 199 & 94.9 & 77.2 & 48.2 \\
\hline 0.002 & 500 & 4,390 & $\mathrm{ng}$ & $\mathrm{ng}$ & ng & $\mathrm{ng}$ \\
\hline
\end{tabular}

'Less than 10 years of data used.

\section{8-2.R00-3A KARUKH RIVER NEAR HERAT, Continued}

Probability of occurrence of annual low discharges

[ $\mathrm{m}^{3} / \mathrm{s}$, meters per second; $\mathrm{ng}$, not given]

\begin{tabular}{|c|c|c|c|c|c|c|c|c|c|c|}
\hline \multirow{3}{*}{$\begin{array}{c}\text { Nonexceedance } \\
\text { probability }\end{array}$} & \multirow{3}{*}{$\begin{array}{c}\text { Recurrence } \\
\text { interval } \\
\text { (years) }\end{array}$} & \multicolumn{9}{|c|}{ Minimum daily mean discharge $\left(\mathrm{m}^{3} / \mathrm{s}\right)$} \\
\hline & & \multicolumn{9}{|c|}{ Number of consecutive days } \\
\hline & & 1 & 3 & 7 & 14 & 30 & 60 & 90 & 120 & 183 \\
\hline 0.05 & 20 & ng & ng & ng & ng & ng & ng & ng & ng & ng \\
\hline 0.10 & 10 & ng & ng & $\mathrm{ng}$ & ng & ng & ng & ng & ng & ng \\
\hline 0.20 & 5 & ng & ng & $\mathrm{ng}$ & ng & ng & $\mathrm{ng}$ & ng & ng & $\mathrm{ng}$ \\
\hline 0.50 & 2 & $\mathrm{ng}$ & ng & $\mathrm{ng}$ & ng & ng & $\mathrm{ng}$ & ng & ng & ng \\
\hline
\end{tabular}




\section{8-2.R00-3A KARUKH RIVER NEAR HERAT, Continued}

Probability of occurrence of seasonal low discharges [m $\mathrm{m}^{3} / \mathrm{s}$, meters per second; $\mathrm{ng}$, not given]

\begin{tabular}{|c|c|c|c|c|c|c|c|c|c|}
\hline \multirow{3}{*}{$\begin{array}{c}\text { Nonexceedance } \\
\text { probability }\end{array}$} & \multirow{3}{*}{$\begin{array}{c}\text { Recurrence } \\
\text { interval } \\
\text { (years) }\end{array}$} & \multicolumn{8}{|c|}{ Minimum daily mean discharge $\left(\mathrm{m}^{3} / \mathrm{s}\right)$} \\
\hline & & \multicolumn{8}{|c|}{ Number of consecutive days } \\
\hline & & 1 & 7 & 14 & 30 & 1 & 7 & 14 & 30 \\
\hline & & \multicolumn{4}{|c|}{ December-January-February } & \multicolumn{4}{|c|}{ March-April-May } \\
\hline 0.05 & 20 & 0.33 & 0.33 & 0.39 & 0.51 & 0.32 & 0.45 & 0.49 & 0.62 \\
\hline 0.10 & 10 & 0.48 & 0.49 & 0.54 & 0.67 & 0.37 & 0.47 & 0.53 & 0.74 \\
\hline 0.20 & 5 & 0.70 & 0.73 & 0.77 & 0.90 & 0.44 & 0.53 & 0.62 & 0.98 \\
\hline \multirow{2}{*}{0.50} & 2 & 1.22 & 1.28 & 1.31 & 1.44 & 0.69 & 0.81 & 1.07 & 2.03 \\
\hline & & \multicolumn{4}{|c|}{ June-July-August } & \multicolumn{4}{|c|}{ September-0ctober-November } \\
\hline 0.05 & 20 & 0.31 & 0.32 & 0.33 & 0.35 & ng & ng & ng & ng \\
\hline 0.10 & 10 & 0.33 & 0.34 & 0.34 & 0.36 & ng & $\mathrm{ng}$ & ng & ng \\
\hline 0.20 & 5 & 0.35 & 0.36 & 0.37 & 0.38 & ng & $\mathrm{ng}$ & ng & ng \\
\hline 0.50 & 2 & 0.42 & 0.43 & 0.44 & 0.45 & ng & $\mathrm{ng}$ & ng & ng \\
\hline
\end{tabular}

\section{8-2.R00-3A KARUKH RIVER NEAR HERAT, Continued}

\section{Annual peak discharges}

[ $\mathrm{m}^{3} / \mathrm{s}$, meters per second]

\begin{tabular}{|c|c|c|c|c|c|}
\hline \multicolumn{3}{|c|}{$\begin{array}{c}\text { Annual peak discharge, } \\
\text { by year }\end{array}$} & \multicolumn{3}{|c|}{$\begin{array}{c}\text { Annual peak discharge, } \\
\text { from highest to lowest }\end{array}$} \\
\hline $\begin{array}{l}\text { Water } \\
\text { year }\end{array}$ & Date & $\begin{array}{c}\text { Peak } \\
\text { discharge } \\
\left(\mathrm{m}^{3} / \mathrm{s}\right)\end{array}$ & $\begin{array}{l}\text { Water } \\
\text { year }\end{array}$ & Date & $\begin{array}{c}\text { Peak } \\
\text { discharge } \\
\left(\mathrm{m}^{3} / \mathrm{s}\right)\end{array}$ \\
\hline 1973 & January 16, 1973 & 21.4 & 1976 & April 22, 1976 & 150 \\
\hline 1974 & April 1, 1974 & 13.0 & 1975 & $\begin{array}{l}\text { April 18, } 1975 \\
\text { January } 16,\end{array}$ & 22.5 \\
\hline 1975 & April 18, 1975 & 22.5 & 1973 & 1973 & 21.4 \\
\hline 1976 & April 22, 1976 & 150 & 1977 & March 28, 1977 & 15.0 \\
\hline 1977 & March 28, 1977 & 15.0 & 1974 & April 1, 1974 & 13.0 \\
\hline
\end{tabular}




\section{8-2.R00-3A KARUKH RIVER NEAR HERAT, Continued}

Monthly and annual mean discharges, in cubic meters per second $[-$, no data]

\begin{tabular}{|c|c|c|c|c|c|c|c|c|c|c|c|c|c|}
\hline \multirow{2}{*}{$\begin{array}{l}\text { Water } \\
\text { year }\end{array}$} & \multicolumn{12}{|c|}{ Monthly mean discharge } & \multirow{2}{*}{$\begin{array}{c}\text { Annual } \\
\text { discharge }\end{array}$} \\
\hline & October & November & December & January & February & March & April & May & June & July & August & September & \\
\hline 1973 & -- & -- & -- & 2.31 & 3.18 & 7.54 & 3.31 & 0.96 & 0.60 & 0.50 & 0.41 & 1.02 & -- \\
\hline 1974 & 1.30 & 1.40 & 1.45 & 2.23 & 3.24 & 6.57 & 6.08 & 1.15 & 0.40 & 0.50 & 0.60 & 0.72 & 2.13 \\
\hline 1975 & 0.99 & 1.19 & 1.31 & 0.78 & 2.13 & 5.71 & 15.7 & 4.14 & 0.66 & 0.62 & 0.51 & 0.61 & 2.85 \\
\hline 1976 & 1.23 & 1.30 & 1.57 & 1.54 & 5.63 & 17.8 & 17.5 & 13.7 & 0.90 & 1.00 & 0.95 & 0.90 & 5.33 \\
\hline 1977 & 1.10 & 1.53 & 2.48 & 2.29 & 2.83 & 4.37 & 4.64 & 1.72 & 0.41 & 0.46 & 0.53 & 0.73 & 1.92 \\
\hline
\end{tabular}




\section{8-3.L00-1A KOWGAN RIVER AT LANGAR}

\section{(U.S. Geological Survey identification number: 341300063000000 )}

LOCATION: Lat $34^{\circ} 13^{\prime} \mathrm{N}$., long $63^{\circ} 00^{\prime} \mathrm{E}$.

DRAINAGE AREA: $7,490 \mathrm{~km}^{2}$.

ELEVATION: 1,230 meters above mean sea level.

PERIOD OF RECORD: April 25, 1962 to September 30, 1978.

GAGE: Water-stage recorder. Staff gage at same site and datum prior to 1965 water year.

Annual mean discharge

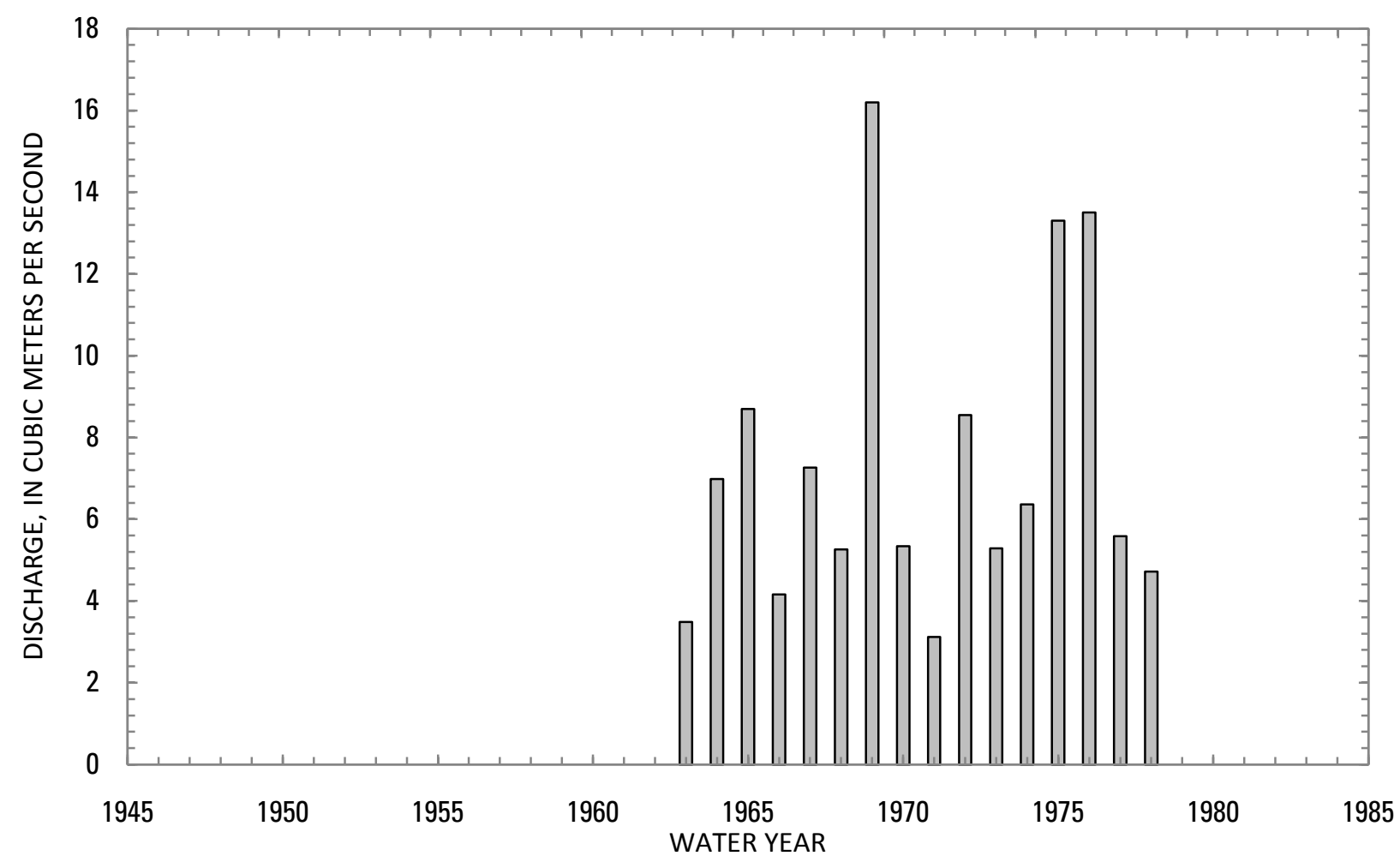




\section{8-3.L00-1A KOWGAN RIVER AT LANGAR, Continued}

Statistics of monthly and annual mean discharges [ $\mathrm{m}^{3} / \mathrm{s}$, cubic meters per second]

\begin{tabular}{|c|c|c|c|c|c|c|c|c|}
\hline \multirow[b]{2}{*}{ Month } & \multicolumn{2}{|c|}{ Maximum } & \multicolumn{2}{|c|}{ Minimum } & \multicolumn{4}{|c|}{ Mean } \\
\hline & $\begin{array}{c}\text { Discharge } \\
\left(\mathrm{m}^{3} / \mathrm{s}\right)\end{array}$ & $\begin{array}{c}\text { Water year } \\
\text { of } \\
\text { occurrence }\end{array}$ & $\begin{array}{c}\text { Discharge } \\
\left(\mathrm{m}^{3} / \mathrm{s}\right)\end{array}$ & $\begin{array}{c}\text { Water year } \\
\text { of } \\
\text { occurrence }\end{array}$ & $\begin{array}{c}\text { Discharge } \\
\left(\mathrm{m}^{3} / \mathrm{s}\right)\end{array}$ & $\begin{array}{c}\text { Standard } \\
\text { deviation } \\
\left(\mathrm{m}^{3} / \mathrm{s}\right)\end{array}$ & $\begin{array}{c}\text { Coefficient } \\
\text { of } \\
\text { variation }\end{array}$ & $\begin{array}{c}\text { Percentage } \\
\text { of annual } \\
\text { discharge }\end{array}$ \\
\hline October & 5.68 & 1977 & 1.41 & 1964 & 3.03 & 1.38 & 0.46 & 3.51 \\
\hline November & 8.72 & 1970 & 2.09 & 1972 & 4.40 & 2.02 & 0.46 & 5.10 \\
\hline December & 8.42 & 1969 & 2.25 & 1964 & 4.42 & 1.75 & 0.40 & 5.12 \\
\hline January & 7.38 & 1969 & 1.81 & 1964 & 4.43 & 1.64 & 0.37 & 5.14 \\
\hline February & 11.1 & 1969 & 2.99 & 1972 & 5.94 & 2.16 & 0.36 & 6.89 \\
\hline March & 43.3 & 1969 & 4.27 & 1963 & 13.7 & 9.05 & 0.66 & 15.9 \\
\hline April & 66.0 & 1969 & 5.51 & 1963 & 25.1 & 18.9 & 0.75 & 29.2 \\
\hline May & 42.2 & 1975 & 1.86 & 1971 & 15.4 & 13.0 & 0.84 & 17.9 \\
\hline June & 11.0 & 1975 & 0.77 & 1963 & 3.91 & 3.31 & 0.85 & 4.53 \\
\hline July & 5.10 & 1975 & 0.19 & 1963 & 1.84 & 1.54 & 0.83 & 2.14 \\
\hline August & 4.50 & 1974 & 0.29 & 1963 & 1.74 & 1.35 & 0.77 & 2.02 \\
\hline September & 4.79 & 1969 & 0.86 & 1966 & 2.29 & 1.25 & 0.54 & 2.66 \\
\hline Annual & 16.2 & 1969 & 3.12 & 1971 & 7.36 & 3.84 & 0.52 & 100 \\
\hline
\end{tabular}


8-3.L00-1A KOWGAN RIVER AT LANGAR, Continued

Monthly and annual flow duration, in cubic meters per second

\begin{tabular}{|c|c|c|c|c|c|c|c|c|c|c|c|c|c|}
\hline \multirow{2}{*}{$\begin{array}{l}\text { Percentage } \\
\text { of days } \\
\text { discharge } \\
\text { equaled or } \\
\text { exceeded }\end{array}$} & \multicolumn{12}{|c|}{ Month } & \multirow{2}{*}{ Annual } \\
\hline & October & November & December & January & February & March & April & May & June & July & August & September & \\
\hline 95 & 1.39 & 1.94 & 2.31 & 1.67 & 2.84 & 4.22 & 5.07 & 2.12 & 0.29 & 0.19 & 0.22 & 0.62 & 0.60 \\
\hline 90 & 1.52 & 2.49 & 2.62 & 2.20 & 3.19 & 4.70 & 7.12 & 2.66 & 0.57 & 0.23 & 0.29 & 0.92 & 1.11 \\
\hline 85 & 1.66 & 2.64 & 2.81 & 2.59 & 3.61 & 5.34 & 8.16 & 3.27 & 0.77 & 0.33 & 0.40 & 1.15 & 1.43 \\
\hline 80 & 1.78 & 2.72 & 2.98 & 2.93 & 3.86 & 5.79 & 8.72 & 3.82 & 0.93 & 0.45 & 0.65 & 1.25 & 1.76 \\
\hline 75 & 1.96 & 2.81 & 3.10 & 3.35 & 4.17 & 6.47 & 9.51 & 4.40 & 1.18 & 0.65 & 0.82 & 1.34 & 2.13 \\
\hline 70 & 2.17 & 2.94 & 3.23 & 3.60 & 4.48 & 7.26 & 11.0 & 5.27 & 1.37 & 0.78 & 0.90 & 1.47 & 2.51 \\
\hline 65 & 2.27 & 3.06 & 3.36 & 3.73 & 4.67 & 7.75 & 12.7 & 6.30 & 1.55 & 0.91 & 0.97 & 1.61 & 2.91 \\
\hline 60 & 2.39 & 3.28 & 3.50 & 3.88 & 4.93 & 8.61 & 14.2 & 7.38 & 1.73 & 1.04 & 1.08 & 1.73 & 3.30 \\
\hline 55 & 2.49 & 3.46 & 3.63 & 4.09 & 5.22 & 9.14 & 15.6 & 8.39 & 1.95 & 1.17 & 1.18 & 1.85 & 3.69 \\
\hline 50 & 2.58 & 3.59 & 3.80 & 4.29 & 5.39 & 9.82 & 17.0 & 9.75 & 2.19 & 1.30 & 1.31 & 1.95 & 4.04 \\
\hline 45 & 2.70 & 3.73 & 4.05 & 4.48 & 5.56 & 10.8 & 18.8 & 10.8 & 2.60 & 1.44 & 1.43 & 2.07 & 4.40 \\
\hline 40 & 2.95 & 4.11 & 4.40 & 4.68 & 5.85 & 11.7 & 21.8 & 13.2 & 3.06 & 1.64 & 1.55 & 2.20 & 4.78 \\
\hline 35 & 3.24 & 4.32 & 4.81 & 4.88 & 6.31 & 12.7 & 26.3 & 15.6 & 3.62 & 2.00 & 1.69 & 2.37 & 5.40 \\
\hline 30 & 3.43 & 4.59 & 5.09 & 5.08 & 6.74 & 14.6 & 29.3 & 18.5 & 4.58 & 2.30 & 1.85 & 2.55 & 6.02 \\
\hline 25 & 3.89 & 4.95 & 5.50 & 5.40 & 7.20 & 16.4 & 32.5 & 21.9 & 5.55 & 2.64 & 2.24 & 2.98 & 7.11 \\
\hline 20 & 4.36 & 5.57 & 5.86 & 5.88 & 7.66 & 18.2 & 37.0 & 26.6 & 6.81 & 3.55 & 3.43 & 3.56 & 8.61 \\
\hline 15 & 5.05 & 6.13 & 6.42 & 6.25 & 8.20 & 21.7 & 42.5 & 33.3 & 8.22 & 4.01 & 3.70 & 4.16 & 11.3 \\
\hline 10 & 5.25 & 6.97 & 6.71 & 6.56 & 9.42 & 26.7 & 52.8 & 37.9 & 10.0 & 4.40 & 4.00 & 4.62 & 16.6 \\
\hline 5 & 5.65 & 8.19 & 7.21 & 7.09 & 10.8 & 41.7 & 70.8 & 47.1 & 12.2 & 4.90 & 4.41 & 4.94 & 29.6 \\
\hline
\end{tabular}




\section{8-3.L00-1A KOWGAN RIVER AT LANGAR, Continued}

Probability of occurrence of annual high discharges

[ $\mathrm{m}^{3} / \mathrm{s}$, cubic meters per second; $\mathrm{ng}$, not given]

\begin{tabular}{|c|c|c|c|c|c|c|}
\hline \multirow{2}{*}{$\begin{array}{c}\text { Exceedance } \\
\text { probability }\end{array}$} & \multirow{2}{*}{$\begin{array}{c}\text { Recurrence } \\
\text { interval } \\
\text { (years) }\end{array}$} & \multirow{2}{*}{$\begin{array}{c}\text { Maximum } \\
\text { instantaneous } \\
\text { discharge } \\
\left(\mathrm{m}^{3} / \mathrm{s}\right)\end{array}$} & \multicolumn{4}{|c|}{ Maximum daily mean discharge $\left(\mathrm{m}^{3} / \mathrm{s}\right)$} \\
\hline & & & $\begin{array}{l}\text { 3-day } \\
\text { period }\end{array}$ & $\begin{array}{l}\text { 7-day } \\
\text { period }\end{array}$ & $\begin{array}{l}\text { 15-day } \\
\text { period }\end{array}$ & $\begin{array}{l}\text { 30-day } \\
\text { period }\end{array}$ \\
\hline 0.99 & 1.01 & 18.1 & 11.5 & 8.82 & 7.54 & 5.91 \\
\hline 0.95 & 1.05 & 25.6 & 15.2 & 12.1 & 10.5 & 8.54 \\
\hline 0.90 & 1.11 & 31.4 & 18.2 & 14.6 & 12.7 & 10.5 \\
\hline 0.80 & 1.25 & 40.9 & 23.0 & 18.7 & 16.2 & 13.7 \\
\hline 0.50 & 2 & 71.4 & 39.0 & 32.0 & 27.4 & 23.4 \\
\hline 0.20 & 5 & 134 & 73.7 & 59.7 & 49.6 & 41.9 \\
\hline 0.10 & 10 & 192 & 108 & 85.7 & 69.6 & 57.8 \\
\hline 0.04 & 25 & 289 & 167 & 129 & 102 & 82.8 \\
\hline 0.02 & 50 & 381 & 225 & 172 & 132 & 105 \\
\hline 0.01 & 100 & 493 & 300 & 224 & 169 & 131 \\
\hline 0.005 & 200 & 629 & 394 & 288 & 212 & 161 \\
\hline 0.002 & 500 & 853 & ng & $\mathrm{ng}$ & $\mathrm{ng}$ & $\mathrm{ng}$ \\
\hline
\end{tabular}

8-3.LO0-1A KOWGAN RIVER AT LANGAR, Continued

Probability of occurrence of annual low discharges $\left[\mathrm{m}^{3} / \mathrm{s}\right.$, meters per second]

\begin{tabular}{|c|c|c|c|c|c|c|c|c|c|c|}
\hline \multirow{3}{*}{$\begin{array}{c}\text { Nonexceedance } \\
\text { probability }\end{array}$} & \multirow{3}{*}{$\begin{array}{c}\text { Recurrence } \\
\text { interval } \\
\text { (years) }\end{array}$} & \multicolumn{9}{|c|}{ Minimum daily mean discharge $\left(\mathrm{m}^{3} / \mathrm{s}\right)$} \\
\hline & & \multicolumn{9}{|c|}{ Number of consecutive days } \\
\hline & & 1 & 3 & 7 & 14 & 30 & 60 & 90 & 120 & 183 \\
\hline 0.05 & 20 & 0 & 0 & 0.13 & 0.19 & 0.23 & 0.28 & 0.35 & 0.54 & 1.02 \\
\hline 0.10 & 10 & 0.16 & 0.18 & 0.21 & 0.30 & 0.35 & 0.41 & 0.50 & 0.70 & 1.22 \\
\hline 0.20 & 5 & 0.33 & 0.35 & 0.38 & 0.48 & 0.55 & 0.64 & 0.75 & 0.96 & 1.52 \\
\hline 0.50 & 2 & 0.87 & 0.91 & 0.98 & 1.10 & 1.21 & 1.34 & 1.50 & 1.72 & 2.34 \\
\hline
\end{tabular}




\section{8-3.L00-1A KOWGAN RIVER AT LANGAR, Continued}

Probability of occurrence of seasonal low discharges $\left[\mathrm{m}^{3} / \mathrm{s}\right.$, meters per second]

\begin{tabular}{|c|c|c|c|c|c|c|c|c|c|}
\hline \multirow{3}{*}{$\begin{array}{c}\text { Nonexceedance } \\
\text { probability }\end{array}$} & \multirow{3}{*}{$\begin{array}{c}\text { Recurrence } \\
\text { interval } \\
\text { (years) }\end{array}$} & \multicolumn{8}{|c|}{ Minimum daily mean discharge $\left(\mathrm{m}^{3} / \mathrm{s}\right)$} \\
\hline & & \multicolumn{8}{|c|}{ Number of consecutive days } \\
\hline & & 1 & 7 & 14 & 30 & 1 & 7 & 14 & 30 \\
\hline & & \multicolumn{4}{|c|}{ December-January-February } & \multicolumn{4}{|c|}{ March-April-May } \\
\hline 0.05 & 20 & 1.18 & 1.28 & 1.36 & 1.71 & 1.04 & 1.38 & 1.65 & 2.37 \\
\hline 0.10 & 10 & 1.51 & 1.65 & 1.75 & 2.08 & 1.32 & 1.74 & 2.07 & 3.08 \\
\hline 0.20 & 5 & 1.99 & 2.19 & 2.31 & 2.60 & 1.79 & 2.33 & 2.76 & 4.23 \\
\hline \multirow[t]{2}{*}{0.50} & 2 & 3.16 & 3.47 & 3.64 & 3.82 & 3.37 & 4.23 & 4.97 & 7.74 \\
\hline & & \multicolumn{4}{|c|}{ June-July-August } & \multicolumn{4}{|c|}{ September-October-November } \\
\hline 0.05 & 20 & 0 & 0.11 & 0.18 & 0.22 & 0.30 & 0.52 & 0.61 & 0.87 \\
\hline 0.10 & 10 & 0.11 & 0.19 & 0.28 & 0.33 & 0.43 & 0.66 & 0.77 & 1.04 \\
\hline 0.20 & 5 & 0.25 & 0.33 & 0.44 & 0.51 & 0.67 & 0.89 & 1.02 & 1.30 \\
\hline 0.50 & 2 & 0.78 & 0.88 & 1.01 & 1.14 & 1.40 & 1.56 & 1.72 & 2.00 \\
\hline
\end{tabular}




\section{8-3.L00-1A KOWGAN RIVER AT LANGAR, Continued}

\begin{tabular}{|c|c|c|c|c|c|}
\hline \multicolumn{6}{|c|}{$\begin{array}{l}\text { Annual peak discharges } \\
\text { [m³/s, meters per second] }\end{array}$} \\
\hline \multicolumn{3}{|c|}{$\begin{array}{c}\text { Annual peak discharge, } \\
\text { by year }\end{array}$} & \multicolumn{3}{|c|}{$\begin{array}{l}\text { Annual peak discharge, } \\
\text { from highest to lowest }\end{array}$} \\
\hline $\begin{array}{l}\text { Water } \\
\text { year }\end{array}$ & Date & $\begin{array}{c}\text { Peak } \\
\text { discharge } \\
\left(\mathrm{m}^{3} / \mathrm{s}\right)\end{array}$ & $\begin{array}{l}\text { Water } \\
\text { year }\end{array}$ & Date & $\begin{array}{c}\text { Peak } \\
\text { discharge } \\
\left(\mathrm{m}^{3} / \mathrm{s}\right)\end{array}$ \\
\hline 1962 & April 25, 1962 & 22.3 & 1969 & April 13, 1969 & 290 \\
\hline 1963 & May 7, 1963 & 32.4 & 1975 & April 25, 1975 & 240 \\
\hline 1964 & April 23, 1964 & 198 & 1964 & April 23, 1964 & 198 \\
\hline 1965 & April 22, 1965 & 47.1 & 1968 & May 19, 1968 & 148 \\
\hline 1966 & April 26, 1966 & 67.0 & 1976 & April 25, 1976 & 103 \\
\hline 1967 & April 27, 1967 & 78.4 & 1972 & March 26, 1972 & 86.0 \\
\hline 1968 & May 19, 1968 & 148 & 1967 & April 27, 1967 & 78.4 \\
\hline 1969 & April 13, 1969 & 290 & 1977 & May 26, 1977 & 71.1 \\
\hline 1970 & November 6, 1969 & 43.4 & 1971 & April 13, 1971 & 70.4 \\
\hline 1971 & April 13, 1971 & 70.4 & 1966 & April 26, 1966 & 67.0 \\
\hline 1972 & March 26, 1972 & 86.0 & 1973 & March 25, 1973 & 60.5 \\
\hline 1973 & March 25, 1973 & 60.5 & 1965 & April 22, 1965 & 47.1 \\
\hline 1974 & April 3, 1974 & 39.5 & 1978 & November 18, 1977 & 46.6 \\
\hline 1975 & April 25, 1975 & 240 & 1970 & November 6, 1969 & 43.4 \\
\hline 1976 & April 25, 1976 & 103 & 1974 & April 3, 1974 & 39.5 \\
\hline 1977 & May 26, 1977 & 71.1 & 1963 & May 7, 1963 & 32.4 \\
\hline 1978 & November 18, 1977 & 46.6 & 1962 & April 25, 1962 & 22.3 \\
\hline
\end{tabular}




\section{8-3.L00-1A KOWGAN RIVER AT LANGAR, Continued}

Monthly and annual mean discharges, in cubic meters per second $[--$, no data]

\begin{tabular}{|c|c|c|c|c|c|c|c|c|c|c|c|c|c|}
\hline \multirow{2}{*}{$\begin{array}{l}\text { Water } \\
\text { year }\end{array}$} & \multicolumn{12}{|c|}{ Monthly mean discharge } & \multirow{2}{*}{$\begin{array}{c}\text { Annual } \\
\text { discharge }\end{array}$} \\
\hline & October & November & December & January & February & March & April & May & June & July & August & September & \\
\hline 1962 & -- & -- & -- & 4.49 & 5.65 & 7.13 & 10.4 & 5.85 & 1.12 & 0.87 & 0.93 & 1.89 & -- \\
\hline 1963 & 2.69 & 3.59 & 3.80 & 3.72 & 3.83 & 4.27 & 5.51 & 12.2 & 0.77 & 0.19 & 0.29 & 0.96 & 3.49 \\
\hline 1964 & 1.41 & 3.21 & 2.25 & 1.81 & 4.19 & 15.2 & 36.2 & 13.0 & 2.62 & 1.34 & 1.28 & 1.57 & 6.98 \\
\hline 1965 & 2.01 & 2.99 & 3.07 & 4.78 & 9.24 & 17.9 & 26.3 & 25.3 & 6.51 & 1.91 & 1.63 & 2.93 & 8.70 \\
\hline 1966 & 3.97 & 4.40 & 4.89 & 4.90 & 5.47 & 5.64 & 12.6 & 5.51 & 1.08 & 0.41 & 0.42 & 0.86 & 4.16 \\
\hline 1967 & 1.97 & 2.85 & 3.10 & 2.11 & 3.82 & 7.69 & 26.9 & 26.6 & 6.65 & 2.00 & 1.46 & 1.86 & 7.26 \\
\hline 1968 & 3.46 & 4.51 & 4.95 & 5.02 & 4.90 & 11.2 & 8.12 & 11.2 & 4.68 & 1.18 & 1.51 & 2.38 & 5.26 \\
\hline 1969 & 3.42 & 5.40 & 8.42 & 7.38 & 11.1 & 43.3 & 66.0 & 30.0 & 7.37 & 4.01 & 3.78 & 4.79 & 16.2 \\
\hline 1970 & 5.47 & 8.72 & 5.70 & 6.46 & 6.83 & 11.1 & 11.0 & 3.76 & 1.36 & 0.95 & 1.00 & 1.80 & 5.34 \\
\hline 1971 & 2.66 & 3.63 & 3.89 & 3.61 & 4.11 & 5.80 & 9.07 & 1.86 & 0.85 & 0.60 & 0.60 & 0.98 & 3.12 \\
\hline 1972 & 1.56 & 2.09 & 2.83 & 2.13 & 2.99 & 22.5 & 37.0 & 18.0 & 6.87 & 2.74 & 1.74 & 2.23 & 8.55 \\
\hline 1973 & 2.54 & 3.18 & 3.38 & 3.86 & 6.78 & 13.9 & 16.8 & 6.90 & 2.26 & 1.11 & 1.22 & 1.76 & 5.29 \\
\hline 1974 & 1.97 & 2.63 & 2.78 & 3.19 & 3.83 & 15.4 & 24.4 & 8.74 & 2.41 & 3.44 & 4.50 & 2.99 & 6.36 \\
\hline 1975 & 2.02 & 3.00 & 3.36 & 4.35 & 6.34 & 16.1 & 57.8 & 42.2 & 11.0 & 5.10 & 3.72 & 4.33 & 13.3 \\
\hline 1976 & 5.18 & 6.14 & 6.85 & 6.26 & 7.15 & 13.8 & 54.1 & 40.9 & 8.85 & 4.39 & 3.85 & 4.57 & 13.5 \\
\hline 1977 & 5.68 & 8.68 & 6.79 & 6.75 & 7.58 & 9.84 & 12.4 & 4.66 & 1.18 & 0.62 & 1.19 & 1.88 & 5.58 \\
\hline 1978 & 2.42 & 5.38 & 4.60 & 4.51 & 7.22 & 11.7 & 12.8 & 5.21 & 0.89 & 0.50 & 0.49 & 1.17 & 4.72 \\
\hline
\end{tabular}




\section{8-3.L00-6A KOWGAN RIVER AT TANGI AZU}

\section{(U.S. Geological Survey identification number: 340800064120000)}

LOCATION: Lat $34^{\circ} 08^{\prime} \mathrm{N}$., long $64^{\circ} 12^{\prime} \mathrm{E}$.

DRAINAGE AREA: $2,030 \mathrm{~km}^{2}$.

ELEVATION: 2,200 meters above mean sea level.

PERIOD OF RECORD: October 16, 1962 to September 30, 1978.

GAGE: Water-stage recorder.

Annual mean discharge

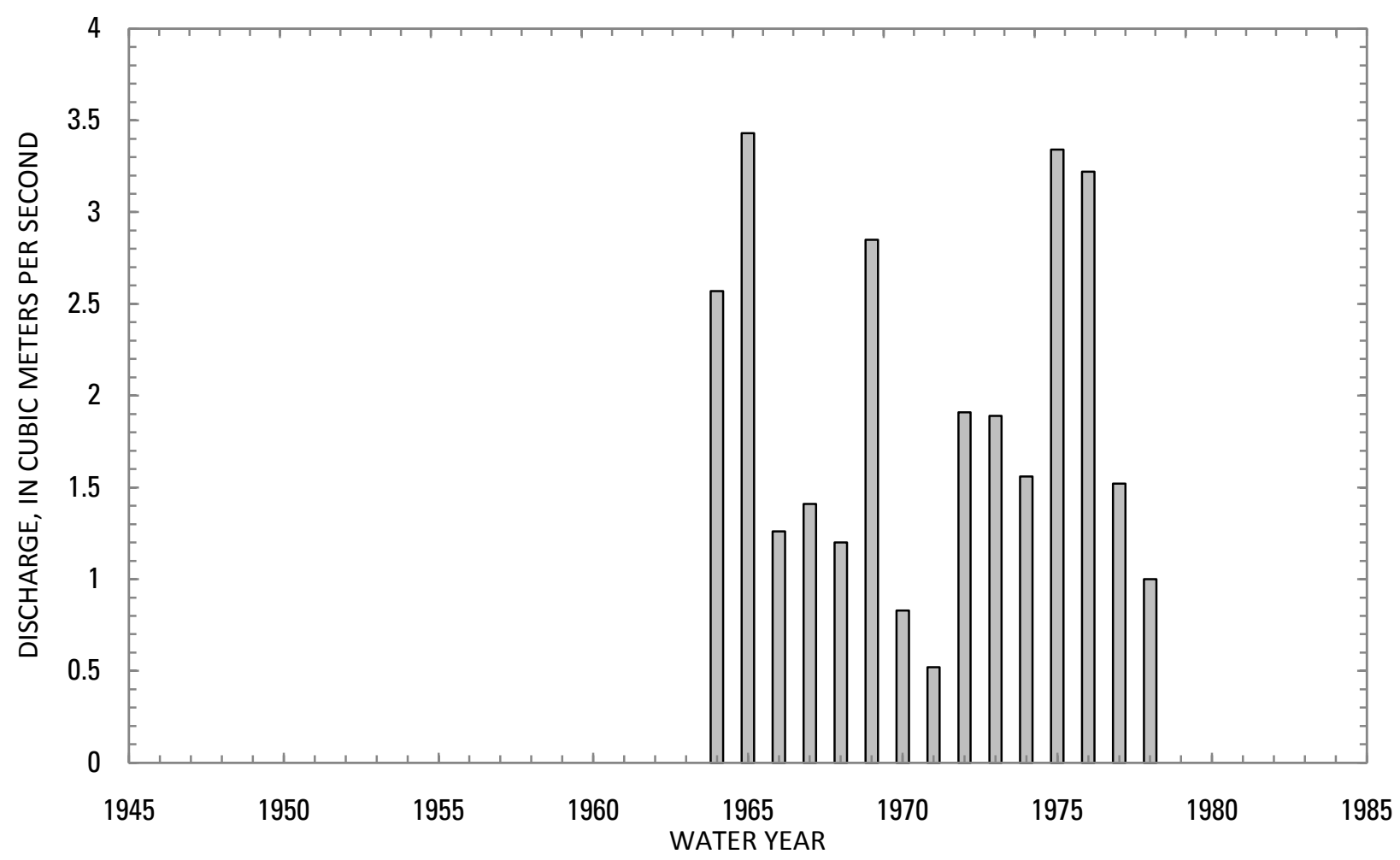




\section{8-3.L00-6A KOWGAN RIVER AT TANGI AZU, Continued}

Statistics of monthly and annual mean discharges [ $\mathrm{m}^{3} / \mathrm{s}$, cubic meters per second]

\begin{tabular}{|c|c|c|c|c|c|c|c|c|}
\hline \multirow[b]{2}{*}{ Month } & \multicolumn{2}{|c|}{ Maximum } & \multicolumn{2}{|c|}{ Minimum } & \multicolumn{4}{|c|}{ Mean } \\
\hline & $\begin{array}{c}\text { Discharge } \\
\left(\mathrm{m}^{3} / \mathrm{s}\right)\end{array}$ & $\begin{array}{c}\text { Water year } \\
\text { of } \\
\text { occurrence }\end{array}$ & $\begin{array}{c}\text { Discharge } \\
\left(\mathrm{m}^{3} / \mathrm{s}\right)\end{array}$ & $\begin{array}{c}\text { Water year } \\
\text { of } \\
\text { occurrence }\end{array}$ & $\begin{array}{c}\text { Discharge } \\
\left(\mathrm{m}^{3} / \mathrm{s}\right)\end{array}$ & $\begin{array}{c}\text { Standard } \\
\text { deviation } \\
\left(\mathrm{m}^{3} / \mathrm{s}\right)\end{array}$ & $\begin{array}{c}\text { Coefficient } \\
\text { of } \\
\text { variation }\end{array}$ & $\begin{array}{c}\text { Percentage } \\
\text { of annual } \\
\text { discharge }\end{array}$ \\
\hline October & 1.62 & 1966 & 0.44 & 1971 & 0.79 & 0.36 & 0.46 & 3.54 \\
\hline November & 1.49 & 1966 & 0.38 & 1970 & 0.84 & 0.36 & 0.43 & 3.76 \\
\hline December & 1.60 & 1970 & 0.32 & 1971 & 0.86 & 0.34 & 0.39 & 3.86 \\
\hline January & 1.47 & 1965 & 0.24 & 1971 & 0.81 & 0.35 & 0.43 & 3.64 \\
\hline February & 2.29 & 1965 & 0.29 & 1971 & 0.90 & 0.54 & 0.60 & 4.04 \\
\hline March & 8.36 & 1969 & 0.61 & 1967 & 3.15 & 2.29 & 0.73 & 14.2 \\
\hline April & 17.7 & 1975 & 1.03 & 1971 & 6.99 & 5.12 & 0.73 & 31.5 \\
\hline May & 9.84 & 1975 & 0.38 & 1971 & 4.54 & 3.18 & 0.70 & 20.4 \\
\hline June & 3.78 & 1975 & 0.36 & 1971 & 1.39 & 1.08 & 0.78 & 6.24 \\
\hline July & 1.38 & 1975 & 0.34 & 1978 & 0.64 & 0.29 & 0.44 & 2.88 \\
\hline August & 1.22 & 1975 & 0.30 & 1978 & 0.62 & 0.25 & 0.41 & 2.78 \\
\hline September & 1.58 & 1975 & 0.38 & 1978 & 0.71 & 0.35 & 0.50 & 3.20 \\
\hline Annual & 3.43 & 1965 & 0.52 & 1971 & 1.90 & 0.96 & 0.50 & 100 \\
\hline
\end{tabular}


8-3.L00-6A KOWGAN RIVER AT TANGI AZU, Continued

Monthly and annual flow duration, in cubic meters per second

[ng, not given]

\begin{tabular}{|c|c|c|c|c|c|c|c|c|c|c|c|c|c|}
\hline \multirow{2}{*}{$\begin{array}{l}\text { Percentage } \\
\text { of days } \\
\text { discharge } \\
\text { equaled or } \\
\text { exceeded }\end{array}$} & \multicolumn{12}{|c|}{ Month } & \multirow{2}{*}{ Annua } \\
\hline & October & November & December & January & February & March & April & May & June & July & August & September & \\
\hline 95 & 0.45 & 0.37 & 0.34 & 0.25 & 0.27 & 0.46 & 0.66 & 0.39 & 0.38 & 0.34 & 0.33 & 0.39 & 0.36 \\
\hline 90 & 0.50 & 0.47 & 0.47 & 0.34 & 0.33 & 0.61 & 1.34 & 0.88 & 0.41 & 0.37 & 0.38 & 0.41 & 0.41 \\
\hline 85 & 0.51 & 0.50 & 0.53 & 0.42 & 0.36 & 0.84 & 1.53 & 1.16 & 0.48 & 0.38 & 0.38 & 0.45 & 0.46 \\
\hline 80 & 0.52 & 0.53 & 0.61 & 0.49 & 0.39 & 1.00 & 1.80 & 1.45 & 0.52 & 0.39 & 0.42 & 0.46 & 0.52 \\
\hline 75 & 0.53 & 0.61 & 0.62 & 0.53 & 0.45 & 1.13 & 1.94 & 1.70 & 0.55 & 0.45 & 0.45 & 0.51 & 0.56 \\
\hline 70 & 0.57 & 0.64 & 0.63 & 0.57 & 0.49 & 1.30 & 2.30 & 2.01 & 0.57 & 0.46 & 0.46 & 0.53 & 0.61 \\
\hline 65 & 0.60 & 0.67 & 0.64 & 0.60 & 0.54 & 1.45 & 3.24 & 2.36 & 0.61 & 0.47 & 0.47 & 0.54 & 0.65 \\
\hline 60 & 0.61 & 0.68 & 0.68 & 0.62 & 0.64 & 1.59 & 4.12 & 2.76 & 0.65 & 0.47 & 0.49 & 0.55 & 0.69 \\
\hline 55 & 0.62 & 0.70 & 0.70 & 0.68 & 0.74 & 1.74 & 4.75 & 3.12 & 0.75 & 0.52 & 0.52 & 0.56 & 0.73 \\
\hline 50 & 0.63 & 0.72 & 0.73 & 0.73 & 0.82 & 1.84 & 5.49 & 3.64 & 0.86 & 0.54 & 0.53 & 0.62 & 0.81 \\
\hline 45 & 0.64 & 0.73 & 0.78 & 0.78 & 0.88 & 1.94 & 6.92 & 4.10 & 0.95 & 0.56 & 0.60 & 0.63 & 0.92 \\
\hline 40 & 0.69 & 0.75 & 0.85 & 0.84 & 0.94 & 2.09 & 7.65 & 4.77 & 1.03 & 0.68 & 0.62 & 0.64 & 1.04 \\
\hline 35 & 0.71 & 0.89 & 0.97 & 0.90 & 1.03 & 2.45 & 8.73 & 5.43 & 1.08 & 0.73 & 0.70 & 0.71 & 1.18 \\
\hline 30 & 0.84 & 0.97 & 1.07 & 1.01 & 1.16 & 3.07 & 9.62 & 6.37 & 1.35 & 0.76 & 0.71 & 0.76 & 1.35 \\
\hline 25 & 0.93 & 1.19 & 1.16 & 1.12 & 1.26 & 4.15 & 10.4 & 7.02 & 1.69 & 0.78 & 0.76 & 0.77 & 1.53 \\
\hline 20 & 1.23 & 1.29 & 1.22 & 1.19 & 1.33 & 5.05 & 11.4 & 7.66 & 2.09 & 0.87 & 0.80 & 0.78 & 1.82 \\
\hline 15 & 1.31 & 1.37 & 1.25 & 1.25 & 1.42 & 6.66 & 12.7 & 8.48 & 2.44 & 0.96 & 0.85 & 1.06 & 2.76 \\
\hline 10 & 1.41 & 1.45 & 1.41 & 1.34 & 1.57 & 8.43 & 14.5 & 9.30 & 3.19 & 1.00 & 0.99 & 1.48 & 5.09 \\
\hline 5 & $\mathrm{ng}$ & 1.55 & 1.58 & 1.53 & 2.14 & 10.1 & 19.4 & 11.4 & 5.03 & 1.25 & 1.20 & $\mathrm{ng}$ & 8.74 \\
\hline
\end{tabular}




\section{8-3.L00-6A KOWGAN RIVER AT TANGI AZU, Continued}

Probability of occurrence of annual high discharges

[m $\mathrm{m}^{3} / \mathrm{s}$, cubic meters per second; ng, not given]

\begin{tabular}{|c|c|c|c|c|c|c|}
\hline \multirow{2}{*}{$\begin{array}{c}\text { Exceedance } \\
\text { probability }\end{array}$} & \multirow{2}{*}{$\begin{array}{l}\text { Recurrence } \\
\text { interval } \\
\text { (years) }\end{array}$} & \multirow{2}{*}{$\begin{array}{l}\text { Maximum } \\
\text { instantaneous } \\
\text { discharge } \\
\left(\mathrm{m}^{3} / \mathrm{s}\right)\end{array}$} & \multicolumn{4}{|c|}{ Maximum daily mean discharge $\left(\mathrm{m}^{3} / \mathrm{s}\right)$} \\
\hline & & & $\begin{array}{l}\text { 3-day } \\
\text { period }\end{array}$ & $\begin{array}{l}\text { 7-day } \\
\text { period }\end{array}$ & $\begin{array}{l}\text { 15-day } \\
\text { period }\end{array}$ & $\begin{array}{l}\text { 30-day } \\
\text { period }\end{array}$ \\
\hline 0.99 & 1.01 & ng & 1.30 & 1.03 & 0.80 & 0.72 \\
\hline 0.95 & 1.05 & ng & 2.51 & 2.05 & 1.68 & 1.52 \\
\hline 0.90 & 1.11 & 4.40 & 3.48 & 2.90 & 2.42 & 2.19 \\
\hline 0.80 & 1.25 & 6.70 & 5.04 & 4.30 & 3.69 & 3.32 \\
\hline 0.50 & 2 & 13.2 & 9.56 & 8.52 & 7.61 & 6.78 \\
\hline 0.20 & 5 & 22.2 & 16.6 & 15.5 & 14.2 & 12.6 \\
\hline 0.10 & 10 & 27.6 & 21.5 & 20.5 & 19.0 & 16.7 \\
\hline 0.04 & 25 & 33.3 & 27.6 & 27.0 & 25.2 & 22.0 \\
\hline 0.02 & 50 & 37.0 & 32.1 & 31.9 & 29.8 & 25.9 \\
\hline 0.01 & 100 & 40.1 & '36.4 & 36.7 & 34.3 & 29.8 \\
\hline 0.005 & 200 & 42.7 & ${ }^{1} 40.6$ & 41.5 & 38.8 & 33.6 \\
\hline 0.002 & 500 & 45.7 & $\mathrm{ng}$ & $\mathrm{ng}$ & $\mathrm{ng}$ & $\mathrm{ng}$ \\
\hline
\end{tabular}

'Data does not fit log-Pearson Type III curve, use with caution.

\section{8-3.L00-6A KOWGAN RIVER AT TANGI AZU, Continued}

Probability of occurrence of annual low discharges

$\left[\mathrm{m}^{3} / \mathrm{s}\right.$, meters per second]

\begin{tabular}{|c|c|c|c|c|c|c|c|c|c|c|}
\hline \multirow{3}{*}{$\begin{array}{c}\text { Nonexceedance } \\
\text { probability }\end{array}$} & \multirow{3}{*}{$\begin{array}{c}\text { Recurrence } \\
\text { interval } \\
\text { (years) }\end{array}$} & \multicolumn{9}{|c|}{ Minimum daily mean discharge $\left(\mathrm{m}^{3} / \mathrm{s}\right)$} \\
\hline & & \multicolumn{9}{|c|}{ Number of consecutive days } \\
\hline & & 1 & 3 & 7 & 14 & 30 & 60 & 90 & 120 & 183 \\
\hline 0.05 & 20 & 0.04 & 0.07 & 0.10 & 0.19 & 0.25 & 0.28 & 0.31 & 0.33 & 0.38 \\
\hline 0.10 & 10 & 0.06 & 0.11 & 0.15 & 0.23 & 0.28 & 0.32 & 0.34 & 0.37 & 0.42 \\
\hline 0.20 & 5 & 0.11 & 0.17 & 0.22 & 0.28 & 0.33 & 0.37 & 0.39 & 0.42 & 0.48 \\
\hline 0.50 & 2 & 0.29 & 0.34 & 0.41 & 0.42 & 0.46 & 0.51 & 0.54 & 0.57 & 0.65 \\
\hline
\end{tabular}




\section{8-3.L00-6A KOWGAN RIVER AT TANGI AZU, Continued}

Probability of occurrence of seasonal low discharges [m $\mathrm{m}^{3} / \mathrm{s}$, meters per second]

\begin{tabular}{|c|c|c|c|c|c|c|c|c|c|}
\hline \multirow{3}{*}{$\begin{array}{c}\text { Nonexceedance } \\
\text { probability }\end{array}$} & \multirow{3}{*}{$\begin{array}{c}\text { Recurrence } \\
\text { interval } \\
\text { (years) }\end{array}$} & \multicolumn{8}{|c|}{ Minimum daily mean discharge $\left(\mathrm{m}^{3} / \mathrm{s}\right)$} \\
\hline & & \multicolumn{8}{|c|}{ Number of consecutive days } \\
\hline & & 1 & 7 & 14 & 30 & 1 & 7 & 14 & 30 \\
\hline & & \multicolumn{4}{|c|}{ December-January-February } & \multicolumn{4}{|c|}{ March-April-May } \\
\hline 0.05 & 20 & 0.06 & 0.11 & 0.20 & 0.25 & 0.32 & 0.34 & 0.37 & 0.47 \\
\hline 0.10 & 10 & 0.10 & 0.17 & 0.25 & 0.31 & 0.39 & 0.43 & 0.48 & 0.66 \\
\hline 0.20 & 5 & 0.18 & 0.28 & 0.34 & 0.39 & 0.50 & 0.57 & 0.67 & 0.99 \\
\hline \multirow[t]{2}{*}{0.50} & 2 & 0.44 & 0.55 & 0.57 & 0.61 & 0.81 & 0.97 & 1.24 & 2.06 \\
\hline & & \multicolumn{4}{|c|}{ June-July-August } & \multicolumn{4}{|c|}{ September-October-November } \\
\hline 0.05 & 20 & 0.29 & 0.29 & 0.29 & 0.29 & 0.10 & 0.29 & 0.32 & 0.36 \\
\hline 0.10 & 10 & 0.31 & 0.32 & 0.32 & 0.33 & 0.17 & 0.32 & 0.35 & 0.39 \\
\hline 0.20 & 5 & 0.34 & 0.35 & 0.37 & 0.38 & 0.30 & 0.38 & 0.40 & 0.44 \\
\hline 0.50 & 2 & 0.44 & 0.47 & 0.49 & 0.52 & 0.62 & 0.54 & 0.56 & 0.59 \\
\hline
\end{tabular}




\section{8-3.L00-6A KOWGAN RIVER AT TANGI AZU, Continued}

\begin{tabular}{|c|c|c|c|c|c|}
\hline \multicolumn{6}{|c|}{$\begin{array}{l}\text { Annual peak discharges } \\
\text { [m³/s, meters per second] }\end{array}$} \\
\hline \multicolumn{3}{|c|}{$\begin{array}{c}\text { Annual peak discharge, } \\
\text { by year }\end{array}$} & \multicolumn{3}{|c|}{$\begin{array}{l}\text { Annual peak discharge, } \\
\text { from highest to lowest }\end{array}$} \\
\hline $\begin{array}{l}\text { Water } \\
\text { year }\end{array}$ & Date & $\begin{array}{c}\text { Peak } \\
\text { discharge } \\
\left(\mathrm{m}^{3} / \mathrm{s}\right)\end{array}$ & $\begin{array}{l}\text { Water } \\
\text { year }\end{array}$ & Date & $\begin{array}{c}\text { Peak } \\
\text { discharge } \\
\left(\mathrm{m}^{3} / \mathrm{s}\right)\end{array}$ \\
\hline 1963 & May 11, 1963 & 5.36 & 1968 & June 8,1968 & 33.0 \\
\hline 1964 & April 23, 1964 & 19.3 & 1976 & April 24, 1976 & 27.0 \\
\hline 1965 & April 22, 1965 & 15.7 & 1969 & April 16, 1969 & 24.0 \\
\hline 1966 & April 25, 1966 & 8.86 & 1975 & April 8, 1975 & 24.0 \\
\hline 1967 & April 27, 1967 & 14.8 & 1964 & April 23, 1964 & 19.3 \\
\hline 1968 & June 8, 1968 & 33.0 & 1965 & April 22, 1965 & 15.7 \\
\hline 1969 & April 16, 1969 & 24.0 & 1967 & April 27, 1967 & 14.8 \\
\hline 1970 & April 26, 1970 & 1.90 & 1972 & March 23, 1972 & 13.0 \\
\hline 1971 & April 8, 1971 & 10.9 & 1973 & April 14, 1973 & 12.9 \\
\hline 1972 & March 23, 1972 & 13.0 & 1974 & March 25, 1974 & 11.4 \\
\hline 1973 & April 14, 1973 & 12.9 & 1971 & April 8, 1971 & 10.9 \\
\hline 1974 & March 25, 1974 & 11.4 & 1966 & April 25, 1966 & 8.86 \\
\hline 1975 & April 8, 1975 & 24.0 & 1977 & April 11, 1977 & 6.28 \\
\hline 1976 & April 24, 1976 & 27.0 & 1963 & May 11, 1963 & 5.36 \\
\hline 1977 & April 11, 1977 & 6.28 & 1978 & April 17, 1978 & 5.32 \\
\hline 1978 & April 17, 1978 & 5.32 & 1970 & April 26, 1970 & 1.90 \\
\hline
\end{tabular}


8-3.L00-6A KOWGAN RIVER AT TANGI AZU, Continued

Monthly and annual mean discharges, in cubic meters per second $[--$, no data]

\begin{tabular}{|c|c|c|c|c|c|c|c|c|c|c|c|c|c|}
\hline \multirow{2}{*}{$\begin{array}{l}\text { Water } \\
\text { year }\end{array}$} & \multicolumn{12}{|c|}{ Monthly mean discharge } & \multirow{2}{*}{$\begin{array}{c}\text { Annual } \\
\text { discharge }\end{array}$} \\
\hline & October & November & December & January & February & March & April & May & June & July & August & September & \\
\hline 1963 & -- & 1.00 & 0.85 & 0.76 & 0.82 & 1.83 & 1.66 & 2.98 & 0.73 & 0.73 & 0.70 & 0.73 & -- \\
\hline 1964 & 0.53 & 0.61 & 0.65 & 0.61 & 0.87 & 5.61 & 11.6 & 6.34 & 1.61 & 0.94 & 0.75 & 0.76 & 2.57 \\
\hline 1965 & 0.80 & 0.89 & 1.07 & 1.47 & 2.29 & 7.30 & 11.9 & 9.68 & 2.26 & 0.93 & 1.05 & 1.47 & 3.43 \\
\hline 1966 & 1.62 & 1.49 & 1.14 & 1.12 & 1.15 & 1.53 & 2.76 & 2.13 & 0.58 & 0.54 & 0.51 & 0.53 & 1.26 \\
\hline 1967 & 0.60 & 0.65 & 0.55 & 0.39 & 0.35 & 0.61 & 4.85 & 5.47 & 1.37 & 0.72 & 0.63 & 0.73 & 1.41 \\
\hline 1968 & 1.01 & 1.30 & 1.17 & 0.88 & 0.73 & 1.66 & 2.17 & 2.40 & 1.74 & 0.38 & 0.41 & 0.48 & 1.20 \\
\hline 1969 & 0.61 & 0.70 & 0.65 & 0.74 & 1.04 & 8.36 & 11.3 & 6.92 & 1.59 & 0.86 & 0.75 & 0.54 & 2.85 \\
\hline 1970 & 0.59 & 0.38 & 1.60 & 1.20 & 1.29 & 1.28 & 1.09 & 0.88 & 0.47 & 0.36 & 0.37 & 0.43 & 0.83 \\
\hline 1971 & 0.44 & 0.41 & 0.32 & 0.24 & 0.29 & 1.22 & 1.03 & 0.38 & 0.36 & 0.41 & 0.50 & 0.58 & 0.52 \\
\hline 1972 & 0.66 & 0.67 & 0.64 & 0.69 & 0.48 & 3.80 & 7.67 & 5.29 & 1.33 & 0.45 & 0.56 & 0.65 & 1.91 \\
\hline 1973 & 0.65 & 0.70 & 0.67 & 0.68 & 0.58 & 4.43 & 9.22 & 3.74 & 0.70 & 0.47 & 0.40 & 0.42 & 1.89 \\
\hline 1974 & 0.54 & 0.72 & 0.90 & 0.66 & 0.35 & 3.75 & 6.79 & 2.83 & 0.56 & 0.46 & 0.48 & 0.60 & 1.56 \\
\hline 1975 & 0.61 & 0.51 & 0.53 & 0.48 & 0.51 & 2.02 & 17.7 & 9.84 & 3.78 & 1.38 & 1.22 & 1.58 & 3.34 \\
\hline 1976 & 1.36 & 1.38 & 1.27 & 1.34 & 1.40 & 2.00 & 13.9 & 9.75 & 3.74 & 0.80 & 0.79 & 0.98 & 3.22 \\
\hline 1977 & 1.29 & 1.32 & 1.09 & 1.12 & 1.55 & 3.57 & 4.32 & 1.80 & 0.79 & 0.49 & 0.47 & 0.50 & 1.52 \\
\hline 1978 & 0.52 & 0.65 & 0.63 & 0.55 & 0.65 & 1.42 & 3.80 & 2.23 & 0.56 & 0.34 & 0.30 & 0.38 & 1.00 \\
\hline
\end{tabular}




\section{8-11.L00-1A LAL RIVER AT SHINYA}

\section{(U.S. Geological Survey identification number: 343000065400000 )}

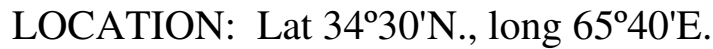

DRAINAGE AREA: $1,685 \mathrm{~km}^{2}$.

ELEVATION: 2,420 meters above mean sea level.

PERIOD OF RECORD: October 27, 1976 to September 30, 1978.

GAGE: Water-stage recorder.

Annual mean discharge

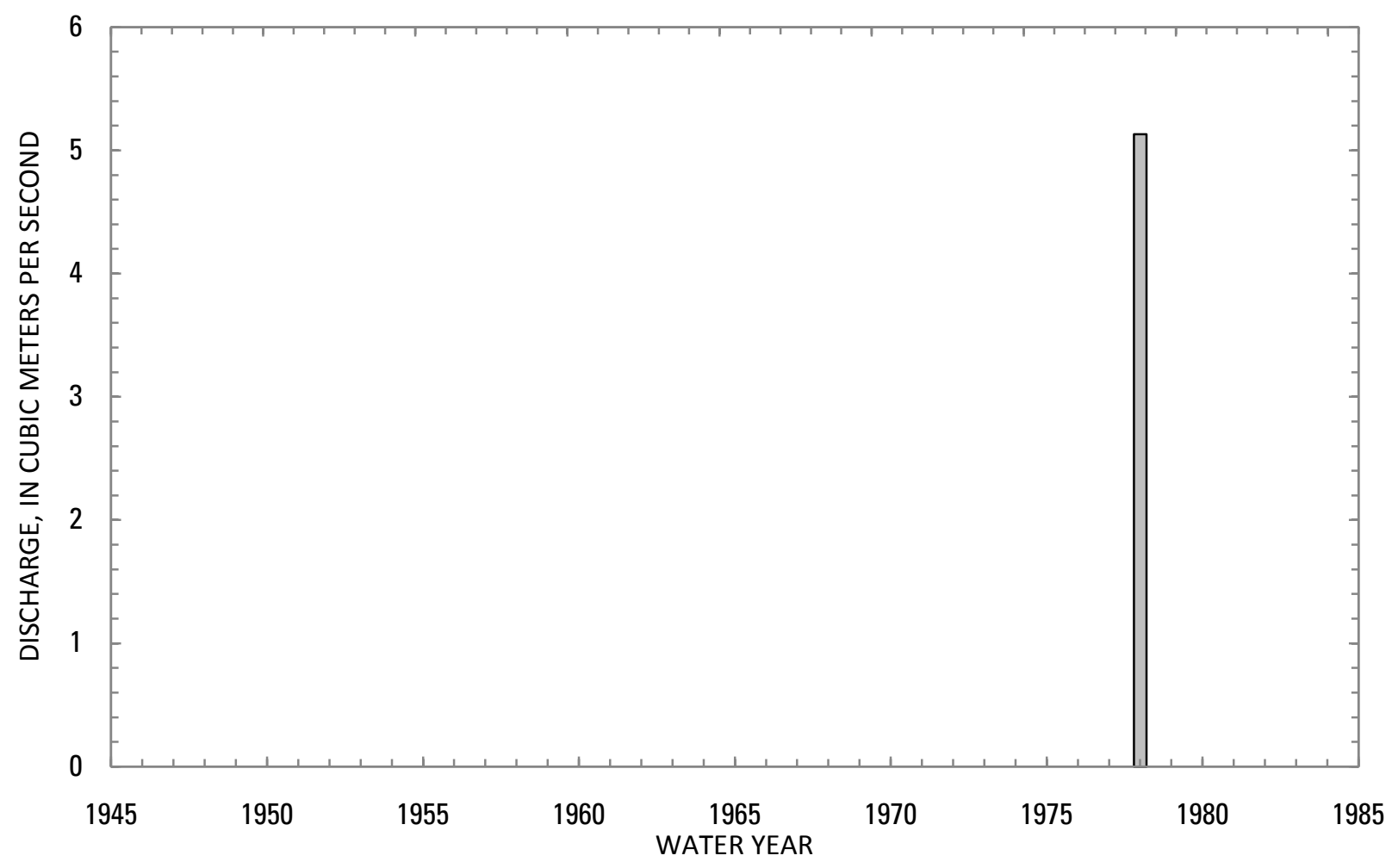


8-11.L00-1A LAL RIVER AT SHINYA, Continued

Statistics of monthly and annual mean discharges

[ $\mathrm{m}^{3} / \mathrm{s}$, cubic meters per second; ng, not given]

\begin{tabular}{|c|c|c|c|c|c|c|c|c|}
\hline \multirow[b]{2}{*}{ Month } & \multicolumn{2}{|c|}{ Maximum } & \multicolumn{2}{|c|}{ Minimum } & \multicolumn{4}{|c|}{ Mean } \\
\hline & $\begin{array}{c}\text { Discharge } \\
\left(\mathrm{m}^{3} / \mathrm{s}\right)\end{array}$ & $\begin{array}{c}\text { Water year } \\
\text { of } \\
\text { occurrence }\end{array}$ & $\begin{array}{c}\text { Discharge } \\
\left(\mathrm{m}^{3} / \mathrm{s}\right)\end{array}$ & $\begin{array}{c}\text { Water year } \\
\text { of } \\
\text { occurrence }\end{array}$ & $\begin{array}{c}\text { Discharge } \\
\left(\mathrm{m}^{3} / \mathrm{s}\right)\end{array}$ & $\begin{array}{c}\text { Standard } \\
\text { deviation } \\
\left(\mathrm{m}^{3} / \mathrm{s}\right)\end{array}$ & $\begin{array}{c}\text { Coefficient } \\
\text { of } \\
\text { variation }\end{array}$ & $\begin{array}{c}\text { Percentage } \\
\text { of annual } \\
\text { discharge }\end{array}$ \\
\hline October & 0.95 & 1978 & 0.95 & 1978 & 0.95 & ng & ng & 1.73 \\
\hline November & 1.06 & 1978 & 0.95 & 1977 & 1.01 & 0.07 & 0.07 & 1.84 \\
\hline December & 1.32 & 1978 & 0.81 & 1977 & 1.07 & 0.36 & 0.34 & 1.95 \\
\hline January & 1.66 & 1978 & 0.80 & 1977 & 1.23 & 0.61 & 0.50 & 2.24 \\
\hline February & 1.26 & 1978 & 0.76 & 1977 & 1.01 & 0.36 & 0.35 & 1.84 \\
\hline March & 3.53 & 1977 & 2.19 & 1978 & 2.86 & 0.95 & 0.33 & 5.22 \\
\hline April & 22.7 & 1978 & 11.6 & 1977 & 17.2 & 7.85 & 0.46 & 31.3 \\
\hline May & 25.6 & 1978 & 17.5 & 1977 & 21.6 & 5.73 & 0.27 & 39.3 \\
\hline June & 5.87 & 1977 & 4.19 & 1978 & 5.03 & 1.19 & 0.24 & 9.17 \\
\hline July & 1.38 & 1977 & 0.38 & 1978 & 0.88 & 0.71 & 0.81 & 1.60 \\
\hline August & 2.29 & 1977 & 0.08 & 1978 & 1.18 & 1.57 & 1.32 & 2.16 \\
\hline September & 1.82 & 1977 & 0.08 & 1978 & 0.95 & 1.23 & 1.30 & 1.73 \\
\hline Annual & 5.13 & 1978 & 5.13 & 1978 & 5.13 & ng & ng & 100 \\
\hline
\end{tabular}


8-11.L00-1A LAL RIVER AT SHINYA, Continued

Monthly and annual flow duration, in cubic meters per second

[ng, not given]

\begin{tabular}{|c|c|c|c|c|c|c|c|c|c|c|c|c|c|}
\hline \multirow{2}{*}{$\begin{array}{l}\text { Percentage } \\
\text { of days } \\
\text { discharge } \\
\text { equaled or } \\
\text { exceeded }\end{array}$} & \multicolumn{12}{|c|}{ Month } & \multirow{2}{*}{ Annua } \\
\hline & October & November & December & January & February & March & April & May & June & July & August & September & \\
\hline 95 & 0.70 & 0.69 & 0.68 & 0.76 & 0.70 & 0.87 & 5.76 & 13.9 & 1.12 & 0.18 & 0.05 & 0.05 & 0.06 \\
\hline 90 & 0.71 & 0.70 & 0.69 & 0.76 & 0.71 & 1.01 & 5.95 & 14.2 & 1.32 & 0.21 & 0.06 & 0.06 & 0.08 \\
\hline 85 & 0.75 & 0.75 & 0.74 & 0.77 & 0.71 & 1.04 & 7.55 & 14.8 & 1.55 & 0.22 & 0.06 & 0.06 & 0.11 \\
\hline 80 & 0.76 & 0.76 & 0.76 & 0.79 & 0.75 & 1.07 & 9.46 & 15.1 & 1.69 & 0.31 & 0.06 & 0.08 & 0.31 \\
\hline 75 & 0.76 & 0.86 & 0.86 & 0.80 & 0.75 & 1.09 & 11.2 & 15.9 & 1.77 & 0.33 & 0.07 & 0.08 & 0.64 \\
\hline 70 & 0.76 & 0.87 & 0.87 & 0.81 & 0.76 & 1.47 & 12.1 & 16.2 & 1.91 & 0.42 & 0.08 & 0.09 & 0.77 \\
\hline 65 & 0.77 & 0.87 & 0.89 & 0.83 & 0.76 & 1.55 & 12.5 & 16.8 & 2.26 & 0.45 & 0.09 & 0.09 & 0.86 \\
\hline 60 & 0.87 & 0.87 & 0.89 & 0.84 & 0.80 & 1.97 & 12.8 & 17.9 & 2.55 & 0.56 & 0.11 & 0.10 & 1.13 \\
\hline 55 & 0.88 & 0.88 & 0.90 & 0.85 & 0.89 & 2.44 & 13.2 & 18.7 & 3.02 & 0.69 & 0.13 & 0.11 & 1.22 \\
\hline 50 & 0.88 & 0.88 & 1.09 & 0.86 & 0.91 & 2.55 & 14.3 & 19.3 & 3.56 & 0.78 & 0.15 & 0.12 & 1.30 \\
\hline 45 & 0.88 & 0.89 & 1.10 & 1.55 & 1.09 & 2.79 & 14.7 & 20.4 & 4.56 & 0.88 & 1.86 & 1.53 & 1.39 \\
\hline 40 & 0.89 & 1.11 & 1.23 & 1.58 & 1.10 & 3.56 & 15.2 & 21.1 & 5.39 & 1.09 & 1.94 & 1.62 & 1.48 \\
\hline 35 & 1.09 & 1.12 & 1.24 & 1.59 & 1.10 & 3.92 & 17.6 & 22.5 & 6.19 & 1.16 & 2.06 & 1.69 & 1.56 \\
\hline 30 & 1.10 & 1.22 & 1.34 & 1.60 & 1.11 & 4.63 & 24.7 & 23.4 & 6.91 & 1.35 & 2.22 & 1.75 & 1.71 \\
\hline 25 & 1.21 & 1.22 & 1.35 & 1.61 & $\mathrm{ng}$ & $\mathrm{ng}$ & 26.1 & 26.1 & 7.50 & 1.46 & 2.42 & 1.80 & 2.52 \\
\hline 20 & 1.22 & 1.32 & 1.36 & 1.67 & ng & ng & 27.0 & 29.4 & 8.50 & 1.53 & ng & 1.86 & 4.58 \\
\hline 15 & 1.22 & 1.33 & $\mathrm{ng}$ & ng & $\mathrm{ng}$ & $\mathrm{ng}$ & 29.2 & 32.2 & 9.24 & 1.67 & $\mathrm{ng}$ & ng & 11.4 \\
\hline 10 & 1.23 & 1.34 & $\mathrm{ng}$ & ng & $\mathrm{ng}$ & $\mathrm{ng}$ & 31.8 & 33.3 & 10.4 & ng & ng & ng & 23.4 \\
\hline 5 & $\mathrm{ng}$ & 1.35 & $\mathrm{ng}$ & ng & $\mathrm{ng}$ & $\mathrm{ng}$ & 33.7 & 35.9 & 12.3 & ng & $\mathrm{ng}$ & $\mathrm{ng}$ & 30.00 \\
\hline
\end{tabular}




\section{8-11.L00-1A LAL RIVER AT SHINYA, Continued}

Probability of occurrence of annual high discharges

[m $\mathrm{m}^{3} / \mathrm{s}$, cubic meters per second; ng, not given]

\begin{tabular}{|c|c|c|c|c|c|c|}
\hline \multirow{2}{*}{$\begin{array}{c}\text { Exceedance } \\
\text { probability }\end{array}$} & \multirow{2}{*}{$\begin{array}{c}\text { Recurrence } \\
\text { interval } \\
\text { (years) }\end{array}$} & \multirow{2}{*}{$\begin{array}{l}\text { Maximum } \\
\text { instantaneous } \\
\text { discharge } \\
\left(\mathrm{m}^{3} / \mathrm{s}\right)\end{array}$} & \multicolumn{4}{|c|}{ Maximum daily mean discharge $\left(\mathrm{m}^{3} / \mathrm{s}\right)$} \\
\hline & & & $\begin{array}{l}\text { 3-day } \\
\text { period }\end{array}$ & $\begin{array}{l}\text { 7-day } \\
\text { period }\end{array}$ & $\begin{array}{l}\text { 15-day } \\
\text { period }\end{array}$ & $\begin{array}{l}\text { 30-day } \\
\text { period }\end{array}$ \\
\hline 0.99 & 1.01 & ng & ng & ng & ng & ng \\
\hline 0.95 & 1.05 & $\mathrm{ng}$ & $\mathrm{ng}$ & ng & ng & ng \\
\hline 0.90 & 1.11 & $\mathrm{ng}$ & $\mathrm{ng}$ & ng & ng & ng \\
\hline 0.80 & 1.25 & $\mathrm{ng}$ & $\mathrm{ng}$ & ng & ng & ng \\
\hline 0.50 & 2 & $\mathrm{ng}$ & $\mathrm{ng}$ & $\mathrm{ng}$ & $\mathrm{ng}$ & ng \\
\hline 0.20 & 5 & $\mathrm{ng}$ & $\mathrm{ng}$ & ng & $\mathrm{ng}$ & ng \\
\hline 0.10 & 10 & $\mathrm{ng}$ & $\mathrm{ng}$ & ng & $\mathrm{ng}$ & ng \\
\hline 0.04 & 25 & ng & $\mathrm{ng}$ & ng & ng & ng \\
\hline 0.02 & 50 & ng & ng & ng & ng & ng \\
\hline 0.01 & 100 & ng & ng & ng & ng & ng \\
\hline 0.005 & 200 & ng & ng & ng & ng & ng \\
\hline 0.002 & 500 & ng & ng & ng & ng & ng \\
\hline
\end{tabular}

8-11.L00-1A LAL RIVER AT SHINYA, Continued

Probability of occurrence of annual low discharges

[ $\mathrm{m}^{3} / \mathrm{s}$, meters per second; $\mathrm{ng}$, not given]

\begin{tabular}{|c|c|c|c|c|c|c|c|c|c|c|}
\hline \multirow{3}{*}{$\begin{array}{c}\text { Nonexceedance } \\
\text { probability }\end{array}$} & \multirow{3}{*}{$\begin{array}{c}\text { Recurrence } \\
\text { interval } \\
\text { (years) }\end{array}$} & \multicolumn{9}{|c|}{ Minimum daily mean discharge $\left(\mathrm{m}^{3} / \mathrm{s}\right)$} \\
\hline & & \multicolumn{9}{|c|}{ Number of consecutive days } \\
\hline & & 1 & 3 & 7 & 14 & 30 & 60 & 90 & 120 & 183 \\
\hline 0.05 & 20 & ng & ng & $\mathrm{ng}$ & ng & ng & ng & ng & ng & ng \\
\hline 0.10 & 10 & ng & ng & $\mathrm{ng}$ & ng & ng & ng & ng & ng & ng \\
\hline 0.20 & 5 & ng & ng & $\mathrm{ng}$ & $\mathrm{ng}$ & ng & ng & ng & ng & ng \\
\hline 0.50 & 2 & ng & ng & $\mathrm{ng}$ & $\mathrm{ng}$ & ng & ng & ng & ng & ng \\
\hline
\end{tabular}




\section{8-11.L00-1A LAL RIVER AT SHINYA, Continued}

Probability of occurrence of seasonal low discharges [ $\mathrm{m}^{3} / \mathrm{s}$, meters per second; $\mathrm{ng}$, not given]

\begin{tabular}{|c|c|c|c|c|c|c|c|c|c|}
\hline \multirow{3}{*}{$\begin{array}{c}\text { Nonexceedance } \\
\text { probability }\end{array}$} & \multirow{3}{*}{$\begin{array}{c}\text { Recurrence } \\
\text { interval } \\
\text { (years) }\end{array}$} & \multicolumn{8}{|c|}{ Minimum daily mean discharge $\left(\mathrm{m}^{3} / \mathrm{s}\right)$} \\
\hline & & \multicolumn{8}{|c|}{ Number of consecutive days } \\
\hline & & 1 & 7 & 14 & 30 & 1 & 7 & 14 & 30 \\
\hline & & \multicolumn{4}{|c|}{ December-January-February } & \multicolumn{4}{|c|}{ March-April-May } \\
\hline 0.10 & 10 & ng & $\mathrm{ng}$ & ng & ng & ng & $\mathrm{ng}$ & ng & ng \\
\hline 0.20 & 5 & $\mathrm{ng}$ & ng & ng & ng & ng & ng & ng & ng \\
\hline 0.50 & 2 & ng & $\mathrm{ng}$ & ng & ng & ng & ng & $\mathrm{ng}$ & ng \\
\hline 0.20 & 5 & ng & ng & $\mathrm{ng}$ & $\mathrm{ng}$ & $\mathrm{ng}$ & $\mathrm{ng}$ & $\mathrm{ng}$ & $\mathrm{ng}$ \\
\hline 0.50 & 2 & $\mathrm{ng}$ & $\mathrm{ng}$ & $\mathrm{ng}$ & $\mathrm{ng}$ & $\mathrm{ng}$ & $\mathrm{ng}$ & $\mathrm{ng}$ & $\mathrm{ng}$ \\
\hline
\end{tabular}

\section{8-11.L00-1A LAL RIVER AT SHINYA, Continued}

Annual peak discharges

[ $\mathrm{m}^{3} / \mathrm{s}$, meters per second]

\begin{tabular}{|c|c|c|c|c|c|}
\hline \multicolumn{3}{|c|}{$\begin{array}{c}\text { Annual peak discharge, } \\
\text { by year }\end{array}$} & \multicolumn{3}{|c|}{$\begin{array}{l}\text { Annual peak discharge, } \\
\text { from highest to lowest }\end{array}$} \\
\hline $\begin{array}{l}\text { Water } \\
\text { year }\end{array}$ & Date & $\begin{array}{c}\text { Peak } \\
\text { discharge } \\
\left(\mathrm{m}^{3} / \mathrm{s}\right)\end{array}$ & $\begin{array}{c}\text { Water } \\
\text { year }\end{array}$ & Date & $\begin{array}{c}\text { Peak } \\
\text { discharge } \\
\left(\mathrm{m}^{3} / \mathrm{s}\right)\end{array}$ \\
\hline 1977 & May 22, 1977 & 23.3 & 1978 & May 5, 1978 & 38.5 \\
\hline 1978 & May 5, 1978 & 38.5 & 1977 & May 22, 1977 & 23.3 \\
\hline
\end{tabular}


8-11.L00-1A LAL RIVER AT SHINYA, Continued

Monthly and annual mean discharges, in cubic meters per second

$[--$, no data]

\begin{tabular}{|c|c|c|c|c|c|c|c|c|c|c|c|c|c|}
\hline \multirow{2}{*}{$\begin{array}{l}\text { Water } \\
\text { year }\end{array}$} & \multicolumn{12}{|c|}{ Monthly mean discharge } & \multirow{2}{*}{$\begin{array}{c}\text { Annual } \\
\text { discharge }\end{array}$} \\
\hline & October & November & December & January & February & March & April & May & June & July & August & September & \\
\hline 1977 & -- & 0.95 & 0.81 & 0.80 & 0.76 & 3.53 & 11.6 & 17.5 & 5.87 & 1.38 & 2.29 & 1.82 & -- \\
\hline 1978 & 0.95 & 1.06 & 1.32 & 1.66 & 1.26 & 2.19 & 22.7 & 25.6 & 4.19 & 0.38 & 0.08 & 0.08 & 5.13 \\
\hline
\end{tabular}




\section{9-0.000-1M MURGHAB RIVER AT BALA MURGHAB}

\section{(U.S. Geological Survey identification number: 353500063190000)}

LOCATION: Lat $35^{\circ} 35^{\prime}$ N., long 63º' $19^{\prime} \mathrm{E}$.

DRAINAGE AREA: $20,525 \mathrm{~km}^{2}$.

ELEVATION: 468 meters above mean sea level.

PERIOD OF RECORD: April 1, 1969 to September 30, 1978.

GAGE: Water-stage recorder. Staff gage prior to 1970 water year.

Annual mean discharge

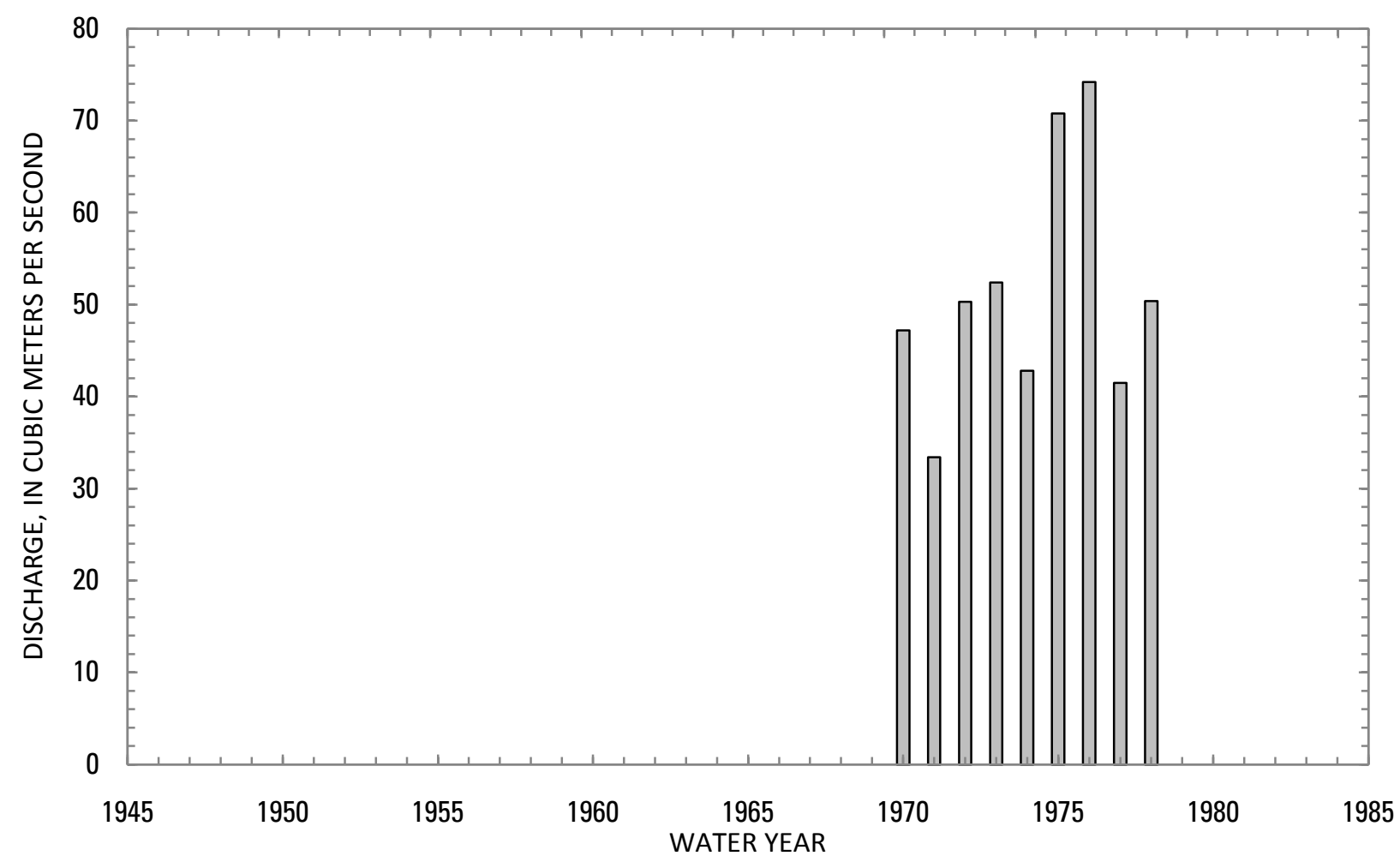




\section{9-0.000-1M MURGHAB RIVER AT BALA MURGHAB, Continued}

Statistics of monthly and annual mean discharges [ $\mathrm{m}^{3} / \mathrm{s}$, cubic meters per second]

\begin{tabular}{|c|c|c|c|c|c|c|c|c|}
\hline \multirow[b]{2}{*}{ Month } & \multicolumn{2}{|c|}{ Maximum } & \multicolumn{2}{|c|}{ Minimum } & \multicolumn{4}{|c|}{ Mean } \\
\hline & $\begin{array}{c}\text { Discharge } \\
\left(\mathrm{m}^{3} / \mathrm{s}\right)\end{array}$ & $\begin{array}{l}\text { Water year } \\
\text { of } \\
\text { occurrence }\end{array}$ & $\begin{array}{c}\text { Discharge } \\
\left(\mathrm{m}^{3} / \mathrm{s}\right)\end{array}$ & $\begin{array}{c}\text { Water year } \\
\text { of } \\
\text { occurrence }\end{array}$ & $\begin{array}{c}\text { Discharge } \\
\left(\mathrm{m}^{3} / \mathrm{s}\right)\end{array}$ & $\begin{array}{c}\text { Standard } \\
\text { deviation } \\
\left(\mathrm{m}^{3} / \mathrm{s}\right)\end{array}$ & $\begin{array}{c}\text { Coefficient } \\
\text { of } \\
\text { variation }\end{array}$ & $\begin{array}{c}\text { Percentage } \\
\text { of annual } \\
\text { discharge }\end{array}$ \\
\hline October & 50.0 & 1977 & 29.2 & 1972 & 38.3 & 8.21 & 0.21 & 5.74 \\
\hline November & 52.5 & 1970 & 29.6 & 1972 & 38.1 & 8.41 & 0.22 & 5.71 \\
\hline December & 44.5 & 1970 & 27.1 & 1972 & 36.0 & 6.78 & 0.19 & 5.40 \\
\hline January & 41.7 & 1970 & 26.4 & 1972 & 33.3 & 5.56 & 0.17 & 4.99 \\
\hline February & 44.8 & 1976 & 25.4 & 1972 & 33.9 & 5.91 & 0.17 & 5.09 \\
\hline March & 58.9 & 1973 & 33.3 & 1971 & 48.4 & 8.04 & 0.17 & 7.26 \\
\hline April & 281 & 1969 & 51.5 & 1971 & 113 & 67.9 & 0.60 & 17.0 \\
\hline May & 228 & 1969 & 46.4 & 1971 & 116 & 63.5 & 0.55 & 17.4 \\
\hline June & 167 & 1969 & 34.6 & 1971 & 80 & 42.5 & 0.53 & 12.0 \\
\hline July & 93.5 & 1969 & 30.0 & 1971 & 50.7 & 21.3 & 0.42 & 7.61 \\
\hline August & 64.3 & 1969 & 26.8 & 1971 & 40.3 & 12.9 & 0.32 & 6.05 \\
\hline September & 54.1 & 1969 & 27.9 & 1971 & 38.5 & 9.4 & 0.24 & 5.77 \\
\hline Annual & 74.2 & 1976 & 33.4 & 1971 & 51.4 & 13.3 & 0.26 & 100 \\
\hline
\end{tabular}




\section{9-0.000-1M MURGHAB RIVER AT BALA MURGHAB, Continued}

Monthly and annual flow duration, in cubic meters per second

[ng, not given]

\begin{tabular}{|c|c|c|c|c|c|c|c|c|c|c|c|c|c|}
\hline \multirow{2}{*}{$\begin{array}{c}\text { Percentage } \\
\text { of days } \\
\text { discharge } \\
\text { equaled or } \\
\text { exceeded }\end{array}$} & \multicolumn{12}{|c|}{ Month } & \multirow{2}{*}{ Annua } \\
\hline & October & November & December & January & February & March & April & May & June & July & August & September & \\
\hline 95 & 29.4 & 29.3 & 27.0 & 26.5 & 25.2 & 30.2 & 46.6 & 46.6 & 34.6 & 28.4 & 27.1 & 27.9 & 28.0 \\
\hline 90 & 29.7 & 29.7 & 28.7 & 26.7 & 26.6 & 31.3 & 53.9 & 49.4 & 36.5 & 29.9 & 27.7 & 29.4 & 29.3 \\
\hline 85 & 30.8 & 30.0 & 29.3 & 27.6 & 28.2 & 35.1 & 57.4 & 51.0 & 40.1 & 31.3 & 28.3 & 30.5 & 30.6 \\
\hline 80 & 31.1 & 30.2 & 29.7 & 28.2 & 28.6 & 38.0 & 61.8 & 60.5 & 44.1 & 33.8 & 29.1 & 30.9 & 31.6 \\
\hline 75 & 31.9 & 31.3 & 30.5 & 28.4 & 29.0 & 38.6 & 67.0 & 65.2 & 46.7 & 35.0 & 31.9 & 32.0 & 32.5 \\
\hline 70 & 32.9 & 31.6 & 31.0 & 28.6 & 29.4 & 39.2 & 72.7 & 70.1 & 49.7 & 36.6 & 33.1 & 32.5 & 33.5 \\
\hline 65 & 33.9 & 32.6 & 31.4 & 29.4 & 29.9 & 39.8 & 76.8 & 73.0 & 52.7 & 37.5 & 33.9 & 32.9 & 34.5 \\
\hline 60 & 34.2 & 34.0 & 31.9 & 30.4 & 30.5 & 40.4 & 79.7 & 85.2 & 56.1 & 38.7 & 34.8 & 34.2 & 36.1 \\
\hline 55 & 34.5 & 34.5 & 32.5 & 31.4 & 31.9 & 41.6 & 83.2 & 93.7 & 59.7 & 40.8 & 35.3 & 34.7 & 37.7 \\
\hline 50 & 34.9 & 35.1 & 33.7 & 32.4 & 33.3 & 42.9 & 87.7 & 97.9 & 65.9 & 42.9 & 35.8 & 35.3 & 39.8 \\
\hline 45 & 35.8 & 36.3 & 34.5 & 33.0 & 33.8 & 46.1 & 93.0 & 104 & 71.6 & 44.6 & 36.2 & 35.8 & 41.8 \\
\hline 40 & 36.1 & 37.9 & 37.3 & 34.8 & 34.2 & 47.2 & 98.5 & 111 & 80.2 & 46.7 & 37.1 & 36.2 & 44.3 \\
\hline 35 & 37.3 & 40.8 & 39.4 & 35.2 & 36.2 & 48.3 & 104 & 124 & 87.4 & 51.0 & 38.2 & 36.6 & 46.9 \\
\hline 30 & 46.4 & 43.6 & 41.8 & 36.7 & 37.1 & 51.8 & 115 & 147 & 94.3 & 58.6 & 49.1 & 47.8 & 49.9 \\
\hline 25 & 47.1 & 45.0 & 43.3 & 38.2 & 37.5 & 53.8 & 125 & 176 & 105 & 64.7 & 51.8 & 48.9 & 54.1 \\
\hline 20 & 48.5 & 45.6 & 43.8 & 39.3 & 37.8 & 56.2 & 138 & 184 & 118 & 69.8 & 54.9 & 50.4 & 61.2 \\
\hline 15 & 49.2 & 47.6 & 44.3 & 40.9 & 40.0 & 60.3 & 172 & 193 & 133 & 76.6 & 57.4 & 51.2 & 73.7 \\
\hline 10 & ng & 49.4 & 45.1 & 41.4 & 43.9 & 68.1 & 217 & 202 & 143 & 85.3 & 58.6 & 51.8 & 92.2 \\
\hline 5 & ng & 53.2 & 46 & ng & 46.5 & 83.2 & 258 & 219 & 171 & 94.6 & 65.1 & 53.1 & 121 \\
\hline
\end{tabular}




\section{9-0.000-1M MURGHAB RIVER AT BALA MURGHAB, Continued}

Probability of occurrence of annual high discharges

[m $\mathrm{m}^{3} / \mathrm{s}$, cubic meters per second; ng, not given]

\begin{tabular}{|c|c|c|c|c|c|c|}
\hline \multirow{2}{*}{$\begin{array}{c}\text { Exceedance } \\
\text { probability }\end{array}$} & \multirow{2}{*}{$\begin{array}{c}\text { Recurrence } \\
\text { interval } \\
\text { (years) }\end{array}$} & \multirow{2}{*}{$\begin{array}{l}\text { Maximum } \\
\text { instantaneous } \\
\text { discharge } \\
\left(\mathrm{m}^{3} / \mathrm{s}\right)\end{array}$} & \multicolumn{4}{|c|}{ Maximum daily mean discharge $\left(\mathrm{m}^{3} / \mathrm{s}\right)$} \\
\hline & & & $\begin{array}{l}\text { 3-day } \\
\text { period }\end{array}$ & $\begin{array}{l}\text { 7-day } \\
\text { period }\end{array}$ & $\begin{array}{l}\text { 15-day } \\
\text { period }\end{array}$ & $\begin{array}{l}\text { 30-day } \\
\text { period }\end{array}$ \\
\hline 0.99 & 1.01 & 40.7 & ${ }^{1} 45.7$ & 46.1 & 41.8 & 37.5 \\
\hline 0.95 & 1.05 & 64.9 & 59.0 & 56.9 & 53.1 & 48.9 \\
\hline 0.90 & 1.11 & 83.5 & 68.4 & 64.6 & 60.9 & 56.8 \\
\hline 0.80 & 1.25 & 114 & 82.3 & 76.3 & 72.6 & 68.5 \\
\hline 0.50 & 2 & 208 & 121 & 110 & 105 & 101 \\
\hline 0.20 & 5 & 387 & 184 & 167 & 158 & 152 \\
\hline 0.10 & 10 & 539 & 234 & 214 & 199 & 192 \\
\hline 0.04 & 25 & 769 & 304 & 283 & 258 & 247 \\
\hline 0.02 & 50 & 971 & 363 & 343 & 307 & 293 \\
\hline 0.01 & 100 & 1200 & 428 & 411 & 361 & 342 \\
\hline 0.005 & 200 & 1460 & 499 & 488 & 421 & 396 \\
\hline 0.002 & 500 & 1850 & $\mathrm{ng}$ & $\mathrm{ng}$ & $\mathrm{ng}$ & ng \\
\hline
\end{tabular}

'Data does not fit log-Pearson Type III curve, use with caution.

\section{9-0.000-1M MURGHAB RIVER AT BALA MURGHAB, Continued}

Probability of occurrence of annual low discharges [ $\mathrm{m}^{3} / \mathrm{s}$, meters per second]

\begin{tabular}{|c|c|c|c|c|c|c|c|c|c|c|}
\hline \multirow{3}{*}{$\begin{array}{c}\text { Nonexceedance } \\
\text { probability }\end{array}$} & \multirow{3}{*}{$\begin{array}{c}\text { Recurrence } \\
\text { interval } \\
\text { (years) }\end{array}$} & \multicolumn{9}{|c|}{ Minimum daily mean discharge $\left(\mathrm{m}^{3} / \mathrm{s}\right)$} \\
\hline & & \multicolumn{9}{|c|}{ Number of consecutive days } \\
\hline & & 1 & 3 & 7 & 14 & 30 & 60 & 90 & 120 & 183 \\
\hline 0.05 & 20 & 22.6 & 23.0 & 23.6 & 23.9 & 24.7 & 25.2 & 25.5 & 26.1 & 26.6 \\
\hline 0.10 & 10 & 23.9 & 24.2 & 24.7 & 25.0 & 25.7 & 26.3 & 26.7 & 27.3 & 28.0 \\
\hline 0.20 & 5 & 25.6 & 25.8 & 26.2 & 26.4 & 27.1 & 27.8 & 28.4 & 29.0 & 30.0 \\
\hline 0.50 & 2 & 29.4 & 29.5 & 29.7 & 29.9 & 30.5 & 31.4 & 32.2 & 33.0 & 34.6 \\
\hline
\end{tabular}




\section{9-0.000-1M MURGHAB RIVER AT BALA MURGHAB, Continued}

Probability of occurrence of seasonal low discharges [ $\mathrm{m}^{3} / \mathrm{s}$, meters per second]

\begin{tabular}{|c|c|c|c|c|c|c|c|c|c|}
\hline \multirow{3}{*}{$\begin{array}{c}\text { Nonexceedance } \\
\text { probability }\end{array}$} & \multirow{3}{*}{$\begin{array}{c}\text { Recurrence } \\
\text { interval } \\
\text { (years) }\end{array}$} & \multicolumn{8}{|c|}{ Minimum daily mean discharge $\left(\mathrm{m}^{3} / \mathrm{s}\right)$} \\
\hline & & \multicolumn{8}{|c|}{ Number of consecutive days } \\
\hline & & 1 & 7 & 14 & 30 & 1 & 7 & 14 & 30 \\
\hline & & \multicolumn{4}{|c|}{ December-January-February } & \multicolumn{4}{|c|}{ March-April-May } \\
\hline 0.05 & 20 & 22.6 & 23.6 & 23.9 & 24.8 & 24.9 & 27.3 & 30.5 & 34.1 \\
\hline 0.10 & 10 & 24.2 & 25.0 & 25.3 & 26.1 & 27.1 & 29.2 & 32.0 & 37.4 \\
\hline 0.20 & 5 & 26.2 & 26.9 & 27.1 & 27.9 & 29.9 & 31.7 & 34.1 & 41.4 \\
\hline \multirow[t]{2}{*}{0.50} & 2 & 30.4 & 30.8 & 31.0 & 31.6 & 35.7 & 37.1 & 38.6 & 48.5 \\
\hline & & \multicolumn{4}{|c|}{ June-July-August } & \multicolumn{4}{|c|}{ September-0 ctober-November } \\
\hline 0.05 & 20 & 24.3 & 24.4 & 24.6 & 25.0 & 25.1 & 25.2 & 25.5 & 26.1 \\
\hline 0.10 & 10 & 26.0 & 26.2 & 26.4 & 26.9 & 26.6 & 26.7 & 27.0 & 27.6 \\
\hline 0.20 & 5 & 28.5 & 28.7 & 29.1 & 29.8 & 28.7 & 28.9 & 29.1 & 29.8 \\
\hline 0.50 & 2 & 35.2 & 35.4 & 36.0 & 37.3 & 33.7 & 34.0 & 34.3 & 35.1 \\
\hline
\end{tabular}

\section{9-0.000-1M MURGHAB RIVER AT BALA MURGHAB, Continued}

\section{Annual peak discharges}

$\left[\mathrm{m}^{3} / \mathrm{s}\right.$, meters per second]

\begin{tabular}{|c|c|c|c|c|c|}
\hline \multicolumn{3}{|c|}{$\begin{array}{c}\text { Annual peak discharge, } \\
\text { by year }\end{array}$} & \multicolumn{3}{|c|}{$\begin{array}{l}\text { Annual peak discharge, } \\
\text { from highest to lowest }\end{array}$} \\
\hline $\begin{array}{l}\text { Water } \\
\text { year }\end{array}$ & Date & $\begin{array}{c}\text { Peak } \\
\text { discharge } \\
\left(\mathrm{m}^{3} / \mathrm{s}\right)\end{array}$ & $\begin{array}{l}\text { Water } \\
\text { year }\end{array}$ & Date & $\begin{array}{c}\text { Peak } \\
\text { discharge } \\
\left(\mathrm{m}^{3} / \mathrm{s}\right)\end{array}$ \\
\hline 1969 & April 15, 1969 & 765 & 1969 & April 15, 1969 & 765 \\
\hline 1970 & March 25, 1970 & 101 & 1975 & May 9, 1975 & 359 \\
\hline 1971 & April 18, 1971 & 69.8 & 1972 & April 26, 1972 & 330 \\
\hline 1972 & April 26, 1972 & 330 & 1977 & April 9, 1977 & 278 \\
\hline 1973 & March 26, 1973 & 250 & 1976 & April 24, 1976 & 270 \\
\hline 1974 & April 13, 1974 & 103 & 1973 & March 26, 1973 & 250 \\
\hline 1975 & May 9, 1975 & 359 & 1978 & December 25, 1977 & 139 \\
\hline 1976 & April 24, 1976 & 270 & 1974 & April 13, 1974 & 103 \\
\hline 1977 & April 9, 1977 & 278 & 1970 & March 25, 1970 & 101 \\
\hline 1978 & December 25, 1977 & 139 & 1971 & April 18, 1971 & 69.8 \\
\hline
\end{tabular}




\section{9-0.000-1M MURGHAB RIVER AT BALA MURGHAB, Continued}

Monthly and annual mean discharges, in cubic meters per second $[--$, no data]

\begin{tabular}{|c|c|c|c|c|c|c|c|c|c|c|c|c|c|}
\hline \multirow{2}{*}{$\begin{array}{l}\text { Water } \\
\text { year }\end{array}$} & \multicolumn{12}{|c|}{ Monthly mean discharge } & \multirow{2}{*}{$\begin{array}{c}\text { Annual } \\
\text { discharge }\end{array}$} \\
\hline & October & November & December & January & February & March & April & May & June & July & August & September & \\
\hline 1969 & -- & -- & -- & -- & -- & -- & 281 & 228 & 167 & 93.5 & 64.3 & 54.1 & -- \\
\hline 1970 & 49.7 & 52.5 & 44.5 & 41.7 & 38.0 & 44.9 & 71.4 & 69.9 & 49.5 & 38.2 & 33.1 & 32.0 & 47.2 \\
\hline 1971 & 32.3 & 30.4 & 29.9 & 29.0 & 28.9 & 33.3 & 51.5 & 46.4 & 34.6 & 30.0 & 26.8 & 27.9 & 33.4 \\
\hline 1972 & 29.2 & 29.6 & 27.1 & 26.4 & 25.4 & 52.5 & 96.0 & 118 & 80.8 & 46.6 & 36.7 & 34.3 & 50.3 \\
\hline 1973 & 34.6 & 33.3 & 31.9 & 29.6 & 33.0 & 58.9 & 106 & 107 & 73.5 & 46.7 & 37.6 & 36.2 & 52.4 \\
\hline 1974 & 36.1 & 34.9 & 33.5 & 32.3 & 30.6 & 43.1 & 81.2 & 70.2 & 49.6 & 36.3 & 32.8 & 32.5 & 42.8 \\
\hline 1975 & 31.2 & 30.6 & 30.4 & 27.7 & 30.4 & 51.2 & 160 & 191 & 120 & 72.1 & 53.9 & 49.1 & 70.8 \\
\hline 1976 & 46.7 & 44.6 & 42.0 & 39.9 & 44.8 & 56.7 & 138 & 182 & 118 & 72.1 & 55.6 & 51.3 & 74.2 \\
\hline 1977 & 50.0 & 47.8 & 44.2 & 37.3 & 36.4 & 42.9 & 57.6 & 50.9 & 41.3 & 30.4 & 27.9 & 31.0 & 41.5 \\
\hline 1978 & 34.5 & 38.8 & 40.6 & 35.6 & 37.9 & 52.2 & 90.1 & 96.9 & 65.9 & 41.4 & 34.6 & 36.2 & 50.4 \\
\hline
\end{tabular}




\section{9-0.000-5M MURGHAB RIVER AT OALA-I-NIAZKHAN}

\section{(U.S. Geological Survey identification number: 350200064010000)}

LOCATION: Lat $35^{\circ} 02^{\prime} \mathrm{N}$., long $64^{\circ} 01^{\prime} \mathrm{E}$.

DRAINAGE AREA: $13,805 \mathrm{~km}^{2}$.

ELEVATION: 800 meters above mean sea level.

PERIOD OF RECORD: January 8, 1966 to September 30, 1978.

GAGE: Water-stage recorder.

Annual mean discharge

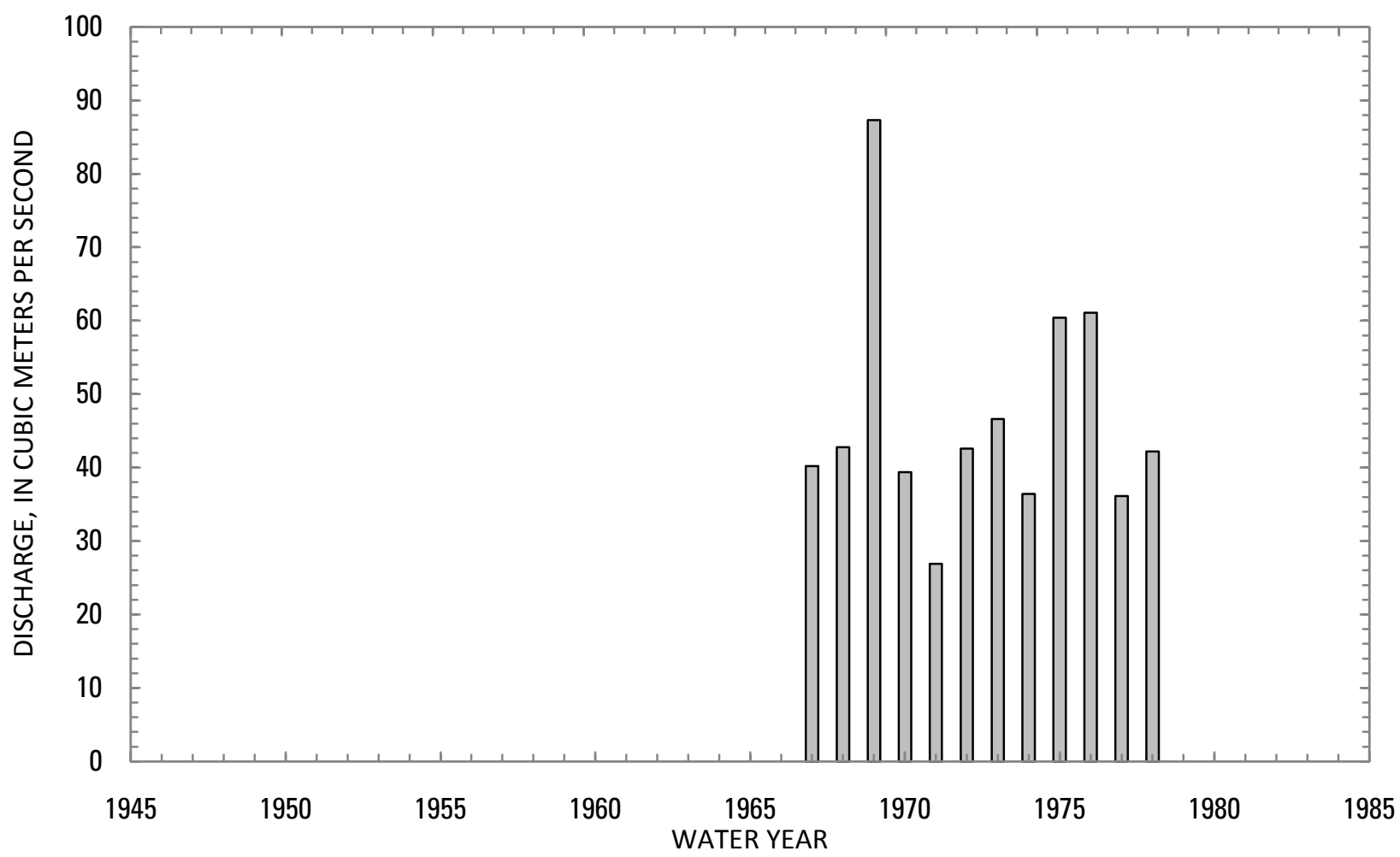




\section{9-0.000-5M MURGHAB RIVER AT QALA-I-NIAZKHAN, Continued}

Statistics of monthly and annual mean discharges $\left[\mathrm{m}^{3} / \mathrm{s}\right.$, cubic meters per second]

\begin{tabular}{|c|c|c|c|c|c|c|c|c|}
\hline \multirow[b]{2}{*}{ Month } & \multicolumn{2}{|c|}{ Maximum } & \multicolumn{2}{|c|}{ Minimum } & \multicolumn{4}{|c|}{ Mean } \\
\hline & $\begin{array}{c}\text { Discharge } \\
\left(\mathrm{m}^{3} / \mathrm{s}\right)\end{array}$ & $\begin{array}{c}\text { Water year } \\
\text { of } \\
\text { occurrence }\end{array}$ & $\begin{array}{c}\text { Discharge } \\
\left(\mathrm{m}^{3} / \mathrm{s}\right)\end{array}$ & $\begin{array}{c}\text { Water year } \\
\text { of } \\
\text { occurrence }\end{array}$ & $\begin{array}{c}\text { Discharge } \\
\left(\mathrm{m}^{3} / \mathrm{s}\right)\end{array}$ & $\begin{array}{c}\text { Standard } \\
\text { deviation } \\
\left(\mathrm{m}^{3} / \mathrm{s}\right)\end{array}$ & $\begin{array}{c}\text { Coefficient } \\
\text { of } \\
\text { variation }\end{array}$ & $\begin{array}{c}\text { Percentage } \\
\text { of annual } \\
\text { discharge }\end{array}$ \\
\hline October & 44.3 & 1976 & 19.9 & 1972 & 32.7 & 7.17 & 0.22 & 5.94 \\
\hline November & 43.2 & 1970 & 22.3 & 1972 & 31.5 & 6.31 & 0.20 & 5.73 \\
\hline December & 37.0 & 1976 & 22.2 & 1972 & 29.5 & 5.23 & 0.18 & 5.36 \\
\hline January & 35.5 & 1976 & 22.4 & 1971 & 28.7 & 4.13 & 0.14 & 5.21 \\
\hline February & 39.3 & 1976 & 20.3 & 1972 & 28.4 & 4.97 & 0.18 & 5.16 \\
\hline March & 113 & 1969 & 26.6 & 1971 & 41.2 & 22.3 & 0.54 & 7.49 \\
\hline April & 214 & 1969 & 42.0 & 1966 & 80.3 & 46.4 & 0.58 & 14.6 \\
\hline May & 202 & 1969 & 36.8 & 1971 & 92.0 & 50.6 & 0.55 & 16.7 \\
\hline June & 146 & 1969 & 28.5 & 1971 & 67.5 & 34.1 & 0.50 & 12.3 \\
\hline July & 89.0 & 1969 & 24.9 & 1971 & 45.9 & 17.4 & 0.38 & 8.35 \\
\hline August & 59.2 & 1969 & 22.9 & 1971 & 37.6 & 10.6 & 0.28 & 6.84 \\
\hline September & 48.2 & 1975 & 21.1 & 1971 & 34.7 & 8.31 & 0.24 & 6.31 \\
\hline Annual & 87.3 & 1969 & 26.9 & 1971 & 46.8 & 16.0 & 0.34 & 100 \\
\hline
\end{tabular}


9-0.000-5M MURGHAB RIVER AT QALA-I-NIAZKHAN, Continued

Monthly and annual flow duration, in cubic meters per second

\begin{tabular}{|c|c|c|c|c|c|c|c|c|c|c|c|c|c|}
\hline \multirow{2}{*}{$\begin{array}{l}\text { Percentage } \\
\text { of days } \\
\text { discharge } \\
\text { equaled or } \\
\text { exceeded }\end{array}$} & \multicolumn{12}{|c|}{ Month } & \multirow{2}{*}{ Annual } \\
\hline & October & November & December & January & February & March & April & May & June & July & August & September & \\
\hline 95 & 20.3 & 22.5 & 22.1 & 22.1 & 20.4 & 23.5 & 33.4 & 37.5 & 28.3 & 25.5 & 23.2 & 21.5 & 22.5 \\
\hline 90 & 25.0 & 23.5 & 22.8 & 22.5 & 22.2 & 25.9 & 37.3 & 42.5 & 35.9 & 29.9 & 28.2 & 26.8 & 24.5 \\
\hline 85 & 25.6 & 24.3 & 23.5 & 24.0 & 22.9 & 27.5 & 41.4 & 46.9 & 38.0 & 31.9 & 28.9 & 27.6 & 26.2 \\
\hline 80 & 26.2 & 25.0 & 24.1 & 24.4 & 23.3 & 28.3 & 44.2 & 51.3 & 39.9 & 33.2 & 29.4 & 28.3 & 27.7 \\
\hline 75 & 27.3 & 26.0 & 24.9 & 25.4 & 25.6 & 28.9 & 46.2 & 55.5 & 42.1 & 34.0 & 29.9 & 29.8 & 29.0 \\
\hline 70 & 29.2 & 28.1 & 26.3 & 26.4 & 26.3 & 29.6 & 48.3 & 60.2 & 44.9 & 35.1 & 30.4 & 30.1 & 30.0 \\
\hline 65 & 29.6 & 29.3 & 27.0 & 27.0 & 26.8 & 30.8 & 51.0 & 63.3 & 47.6 & 36.5 & 31.0 & 30.4 & 31.0 \\
\hline 60 & 30.6 & 30.0 & 27.5 & 27.6 & 27.2 & 31.9 & 55.0 & 68.3 & 49.6 & 38.0 & 32.3 & 30.8 & 32.1 \\
\hline 55 & 31.1 & 30.6 & 28.2 & 28.4 & 27.9 & 33.0 & 57.6 & 73.9 & 52.1 & 39.1 & 34.4 & 31.6 & 33.7 \\
\hline 50 & 31.8 & 31.3 & 28.6 & 28.9 & 28.7 & 34.1 & 62.0 & 77.8 & 56.1 & 41.3 & 36.1 & 34.2 & 35.2 \\
\hline 45 & 32.9 & 31.7 & 29.2 & 29.3 & 29.0 & 35.1 & 68.0 & 81.7 & 60.3 & 42.9 & 36.6 & 34.7 & 36.7 \\
\hline 40 & 33.7 & 32.0 & 32.0 & 30.0 & 29.4 & 36.2 & 73.4 & 87.0 & 63.3 & 44.5 & 37.3 & 35.5 & 38.6 \\
\hline 35 & 34.9 & 32.3 & 32.4 & 30.3 & 29.8 & 37.1 & 79.3 & 90.7 & 68.8 & 46.6 & 38.0 & 36.2 & 40.9 \\
\hline 30 & 35.5 & 33.8 & 32.8 & 30.6 & 30.4 & 38.0 & 89.1 & 95.9 & 77.9 & 48.7 & 39.5 & 36.7 & 44.5 \\
\hline 25 & 37.5 & 35.2 & 33.7 & 31.1 & 30.8 & 39.4 & 96.0 & 109 & 86.8 & 54.6 & 40.7 & 37.5 & 48.7 \\
\hline 20 & 40.8 & 36.5 & 35.0 & 31.8 & 31.4 & 41.2 & 101 & 140 & 93.9 & 58.4 & 49.2 & 45.6 & 56.4 \\
\hline 15 & 41.6 & 38.2 & 36.2 & 32.8 & 32.7 & 45.6 & 117 & 155 & 109 & 63.8 & 51.6 & 46.4 & 66.7 \\
\hline 10 & 43.1 & 40.7 & 36.9 & 33.8 & 34.6 & 60.0 & 157 & 173 & 121 & 72.2 & 54.6 & 47.6 & 84.6 \\
\hline 5 & 44.7 & 42.3 & 37.3 & 35.3 & 38.0 & 108 & 180 & 193 & 142 & 81.6 & 56.8 & 49.0 & 117 \\
\hline
\end{tabular}




\section{9-0.000-5M MURGHAB RIVER AT QALA-I-NIAZKHAN, Continued}

Probability of occurrence of annual high discharges

[ $\mathrm{m}^{3} / \mathrm{s}$, cubic meters per second; $\mathrm{ng}$, statistic not given]

\begin{tabular}{|c|c|c|c|c|c|c|}
\hline \multirow{2}{*}{$\begin{array}{c}\text { Exceedance } \\
\text { probability }\end{array}$} & \multirow{2}{*}{$\begin{array}{c}\text { Recurrence } \\
\text { interval } \\
\text { (years) }\end{array}$} & \multirow{2}{*}{$\begin{array}{c}\text { Maximum } \\
\text { instantaneous } \\
\text { discharge } \\
\left(\mathrm{m}^{3} / \mathrm{s}\right)\end{array}$} & \multicolumn{4}{|c|}{ Maximum daily mean discharge $\left(\mathrm{m}^{3} / \mathrm{s}\right)$} \\
\hline & & & $\begin{array}{l}\text { 3-day } \\
\text { period }\end{array}$ & $\begin{array}{l}\text { 7-day } \\
\text { period }\end{array}$ & $\begin{array}{l}\text { 15-day } \\
\text { period }\end{array}$ & $\begin{array}{l}\text { 30-day } \\
\text { period }\end{array}$ \\
\hline 0.99 & 1.01 & 31.7 & 42.3 & 39.8 & 37.9 & 33.5 \\
\hline 0.95 & 1.05 & 48.8 & 52.1 & 50.0 & 47.2 & 43.2 \\
\hline 0.90 & 1.11 & 61.4 & 59.4 & 57.3 & 53.9 & 50.0 \\
\hline 0.80 & 1.25 & 80.9 & 70.7 & 68.5 & 64.2 & 60.3 \\
\hline 0.50 & 2 & 137 & 105 & 101 & 94.2 & 88.8 \\
\hline 0.20 & 5 & 231 & 169 & 158 & 148 & 136 \\
\hline 0.10 & 10 & 304 & 224 & 205 & 192 & 173 \\
\hline 0.04 & 25 & 406 & 311 & 277 & 259 & 227 \\
\hline 0.02 & 50 & 489 & 390 & 340 & 319 & 272 \\
\hline 0.01 & 100 & 578 & 483 & 411 & 388 & 321 \\
\hline 0.005 & 200 & 673 & 593 & 493 & 467 & 376 \\
\hline 0.002 & 500 & 810 & ng & $\mathrm{ng}$ & $\mathrm{ng}$ & ng \\
\hline
\end{tabular}

\section{9-0.000-5M MURGHAB RIVER AT QALA-I-NIAZKHAN, Continued}

Probability of occurrence of annual low discharges $\left[\mathrm{m}^{3} / \mathrm{s}\right.$, meters per second]

\begin{tabular}{|c|c|c|c|c|c|c|c|c|c|c|}
\hline \multirow{3}{*}{$\begin{array}{c}\text { Nonexceedance } \\
\text { probability }\end{array}$} & \multirow{3}{*}{$\begin{array}{c}\text { Recurrence } \\
\text { interval } \\
\text { (years) }\end{array}$} & \multicolumn{9}{|c|}{ Minimum daily mean discharge $\left(\mathrm{m}^{3} / \mathrm{s}\right)$} \\
\hline & & \multicolumn{9}{|c|}{ Number of consecutive days } \\
\hline & & 1 & 3 & 7 & 14 & 30 & 60 & 90 & 120 & 183 \\
\hline 0.05 & 20 & 19.3 & 19.5 & 19.6 & 19.9 & 20.3 & 20.8 & 21.2 & 21.4 & 21.9 \\
\hline 0.10 & 10 & 20.7 & 20.8 & 21.0 & 21.3 & 21.7 & 22.3 & 22.6 & 23.0 & 23.6 \\
\hline 0.20 & 5 & 22.4 & 22.5 & 22.8 & 23.1 & 23.5 & 24.1 & 24.5 & 24.9 & 25.8 \\
\hline 0.50 & 2 & 25.7 & 25.9 & 26.3 & 26.7 & 27.1 & 27.8 & 28.3 & 28.8 & 30.2 \\
\hline
\end{tabular}




\section{9-0.000-5M MURGHAB RIVER AT QALA-I-NIAZKHAN, Continued}

Probability of occurrence of seasonal low discharges

$\left[\mathrm{m}^{3} / \mathrm{s}\right.$, meters per second]

\begin{tabular}{|c|c|c|c|c|c|c|c|c|c|}
\hline \multirow{3}{*}{$\begin{array}{c}\text { Nonexceedance } \\
\text { probability }\end{array}$} & \multirow{3}{*}{$\begin{array}{c}\text { Recurrence } \\
\text { interval } \\
\text { (years) }\end{array}$} & \multicolumn{8}{|c|}{ Minimum daily mean discharge $\left(\mathrm{m}^{3} / \mathrm{s}\right)$} \\
\hline & & \multicolumn{8}{|c|}{ Number of consecutive days } \\
\hline & & 1 & 7 & 14 & 30 & 1 & 7 & 14 & 30 \\
\hline & & \multicolumn{4}{|c|}{ December-January-February } & \multicolumn{4}{|c|}{ March-April-May } \\
\hline 0.05 & 20 & 19.8 & 20.1 & 20.3 & 20.6 & 20.9 & 21.7 & 24.7 & 28.2 \\
\hline 0.10 & 10 & 21.0 & 21.3 & 21.6 & 22.0 & 22.4 & 22.7 & 25.0 & 28.5 \\
\hline 0.20 & 5 & 22.6 & 22.9 & 23.3 & 23.7 & 24.3 & 24.2 & 25.7 & 29.1 \\
\hline \multirow[t]{2}{*}{0.50} & 2 & 25.7 & 26.3 & 26.7 & 27.2 & 28.0 & 28.5 & 29.0 & 33.2 \\
\hline & & \multicolumn{4}{|c|}{ June-July-August } & \multicolumn{4}{|c|}{ September-October-November } \\
\hline 0.05 & 20 & 23.3 & 23.3 & 23.4 & 23.9 & 20.1 & 20.2 & 20.4 & 20.9 \\
\hline 0.10 & 10 & 25.2 & 25.2 & 25.4 & 25.9 & 22.0 & 22.3 & 22.5 & 23.0 \\
\hline 0.20 & 5 & 27.7 & 27.9 & 28.1 & 28.7 & 24.3 & 24.8 & 25.0 & 25.6 \\
\hline 0.50 & 2 & 33.9 & 34.3 & 34.7 & 35.6 & 29.0 & 29.7 & 30.1 & 30.8 \\
\hline
\end{tabular}

9-0.000-5M MURGHAB RIVER AT QALA-I-NIAZKHAN, Continued

Annual peak discharges

$\left[\mathrm{m}^{3} / \mathrm{s}\right.$, meters per second]

\begin{tabular}{|c|c|c|c|c|c|}
\hline \multicolumn{3}{|c|}{$\begin{array}{c}\text { Annual peak discharge, } \\
\text { by year }\end{array}$} & \multicolumn{3}{|c|}{$\begin{array}{l}\text { Annual peak discharge, } \\
\text { from highest to lowest }\end{array}$} \\
\hline $\begin{array}{l}\text { Water } \\
\text { year }\end{array}$ & Date & $\begin{array}{c}\text { Peak } \\
\text { discharge } \\
\left(\mathrm{m}^{3} / \mathrm{s}\right)\end{array}$ & $\begin{array}{l}\text { Water } \\
\text { year }\end{array}$ & Date & $\begin{array}{c}\text { Peak } \\
\text { discharge } \\
\left(\mathrm{m}^{3} / \mathrm{s}\right)\end{array}$ \\
\hline 1966 & April 27, 1966 & 57.1 & 1969 & April 11, 1969 & 381 \\
\hline 1967 & April 24, 1967 & 295 & 1967 & April 24, 1967 & 295 \\
\hline 1968 & April 25, 1968 & 170 & 1975 & May 8, 1975 & 229 \\
\hline 1969 & April 11, 1969 & 381 & 1973 & March 25, 1973 & 215 \\
\hline 1970 & April 27, 1970 & 74.0 & 1976 & April 25, 1976 & 184 \\
\hline 1971 & April 16, 1971 & 56.6 & 1968 & April 25, 1968 & 170 \\
\hline 1972 & March 19, 1972 & 150 & 1972 & March 19, 1972 & 150 \\
\hline 1973 & March 25, 1973 & 215 & 1977 & April 9, 1977 & 131 \\
\hline 1974 & May 3, 1974 & 74.0 & 1978 & May 6, 1978 & 96.7 \\
\hline 1975 & May 8, 1975 & 229 & 1970 & April 27, 1970 & 74.0 \\
\hline 1976 & April 25, 1976 & 184 & 1974 & May 3, 1974 & 74.0 \\
\hline 1977 & April 9, 1977 & 131 & 1966 & April 27, 1966 & 57.1 \\
\hline 1978 & May 6, 1978 & 96.7 & 1971 & April 16, 1971 & 56.6 \\
\hline
\end{tabular}




\section{9-0.000-5M MURGHAB RIVER AT QALA-I-NIAZKHAN, Continued}

Monthly and annual mean discharges, in cubic meters per second $[--$, no data]

\begin{tabular}{|c|c|c|c|c|c|c|c|c|c|c|c|c|c|}
\hline \multirow{2}{*}{$\begin{array}{l}\text { Water } \\
\text { year }\end{array}$} & \multicolumn{12}{|c|}{ Monthly mean discharge } & \multirow{2}{*}{$\begin{array}{c}\text { Annual } \\
\text { discharge }\end{array}$} \\
\hline & October & November & December & January & February & March & April & May & June & July & August & September & \\
\hline 1966 & -- & -- & -- & -- & 29.8 & 29.4 & 42.0 & 51.0 & 40.4 & 33.7 & 31.1 & 30.8 & -- \\
\hline 1967 & 31.0 & 29.0 & 27.4 & 28.7 & 26.6 & 28.4 & 62.2 & 72.7 & 55.2 & 45.2 & 39.3 & 36.1 & 40.2 \\
\hline 1968 & 34.0 & 31.7 & 26.9 & 26.8 & 25.8 & 34.7 & 55.4 & 85.6 & 75.7 & 44.4 & 36.7 & 36.3 & 42.8 \\
\hline 1969 & 35.5 & 34.0 & 36.3 & 34.8 & 33.4 & 113 & 214 & 202 & 146 & 89.0 & 59.2 & 47.3 & 87.3 \\
\hline 1970 & 40.5 & 43.2 & 34.9 & 31.1 & 28.1 & 34.4 & 63.2 & 62.3 & 43.7 & 35.1 & 29.2 & 27.3 & 39.4 \\
\hline 1971 & 25.6 & 24.8 & 23.7 & 22.4 & 22.5 & 26.6 & 42.6 & 36.8 & 28.5 & 24.9 & 22.9 & 21.1 & 26.9 \\
\hline 1972 & 19.9 & 22.3 & 22.2 & 23.0 & 20.3 & 41.1 & 79.1 & 98.5 & 78.8 & 42.7 & 32.6 & 30.3 & 42.6 \\
\hline 1973 & 31.3 & 31.7 & 32.1 & 30.0 & 31.0 & 48.1 & 91.6 & 86.9 & 57.6 & 45.4 & 38.7 & 34.6 & 46.6 \\
\hline 1974 & 32.4 & 29.9 & 27.3 & 27.6 & 29.8 & 37.4 & 55.5 & 58.4 & 45.4 & 35.9 & 29.6 & 27.9 & 36.4 \\
\hline 1975 & 26.2 & 24.3 & 23.0 & 24.6 & 23.2 & 31.7 & 123 & 172 & 113 & 64.0 & 50.9 & 48.2 & 60.4 \\
\hline 1976 & 44.3 & 37.9 & 37.0 & 35.5 & 39.3 & 39.1 & 94.8 & 143 & 98.5 & 64.7 & 52.5 & 46.5 & 61.1 \\
\hline 1977 & 42.1 & 39.3 & 33.6 & 30.3 & 30.5 & 35.5 & 49.6 & 44.6 & 38.3 & 30.3 & 29.2 & 29.9 & 36.1 \\
\hline 1978 & 29.4 & 30.0 & 29.5 & 29.2 & 28.3 & 36.3 & 71.0 & 82.0 & 56.4 & 41.7 & 37.0 & 34.7 & 42.2 \\
\hline
\end{tabular}




\section{9-1.000-1A GULRAN RIVER AT CHAR TAKHTA}

\section{(U.S. Geological Survey identification number: 352300062020000 )}

LOCATION: Lat $35^{\circ} 23^{\prime} \mathrm{N}$., long $62^{\circ} 02^{\prime} \mathrm{E}$.

DRAINAGE AREA: $2,895 \mathrm{~km}^{2}$.

ELEVATION: 568 meters above mean sea level.

PERIOD OF RECORD: June 20, 1977 to September 30, 1978.

GAGE: Water-stage recorder.

Annual mean discharge

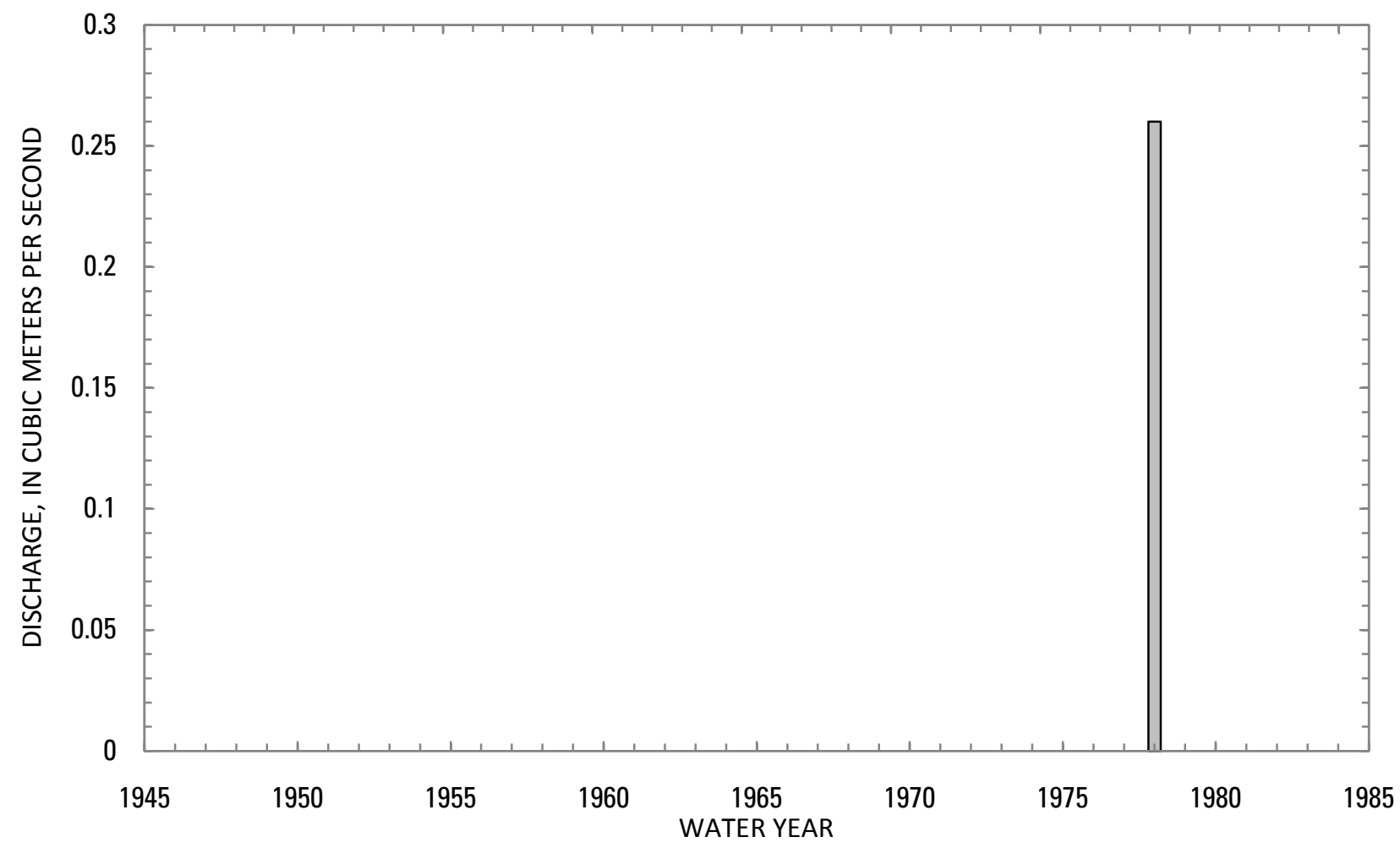




\section{9-1.000-1A GULRAN RIVER AT CHAR TAKHTA, Continued}

Statistics of monthly and annual mean discharges

[ $\mathrm{m}^{3} / \mathrm{s}$, cubic meters per second; ng, not given]

\begin{tabular}{|c|c|c|c|c|c|c|c|c|}
\hline \multirow[b]{2}{*}{ Month } & \multicolumn{2}{|c|}{ Maximum } & \multicolumn{2}{|c|}{ Minimum } & \multicolumn{4}{|c|}{ Mean } \\
\hline & $\begin{array}{c}\text { Discharge } \\
\left(\mathrm{m}^{3} / \mathrm{s}\right)\end{array}$ & $\begin{array}{c}\text { Water year } \\
\text { of } \\
\text { occurrence }\end{array}$ & $\begin{array}{c}\text { Discharge } \\
\left(\mathrm{m}^{3} / \mathrm{s}\right)\end{array}$ & $\begin{array}{c}\text { Water year } \\
\text { of } \\
\text { occurrence }\end{array}$ & $\begin{array}{c}\text { Discharge } \\
\left(\mathrm{m}^{3} / \mathrm{s}\right)\end{array}$ & $\begin{array}{c}\text { Standard } \\
\text { deviation } \\
\left(\mathrm{m}^{3} / \mathrm{s}\right)\end{array}$ & $\begin{array}{c}\text { Coefficient } \\
\text { of } \\
\text { variation }\end{array}$ & $\begin{array}{c}\text { Percentage } \\
\text { of annual } \\
\text { discharge }\end{array}$ \\
\hline October & 0.13 & 1978 & 0.13 & 1978 & 0.12 & ng & ng & 3.95 \\
\hline November & 0.57 & 1978 & 0.57 & 1978 & 0.57 & ng & ng & 18.0 \\
\hline December & 0.53 & 1978 & 0.53 & 1978 & 0.53 & ng & ng & 16.8 \\
\hline January & 0.03 & 1978 & 0.03 & 1978 & 0.03 & ng & ng & 0.98 \\
\hline February & 0.37 & 1978 & 0.37 & 1978 & 0.37 & ng & ng & 11.7 \\
\hline March & 0.78 & 1978 & 0.78 & 1978 & 0.78 & ng & ng & 24.8 \\
\hline April & 0.48 & 1978 & 0.48 & 1978 & 0.48 & ng & ng & 15.3 \\
\hline May & 0.06 & 1978 & 0.06 & 1978 & 0.06 & ng & ng & 1.99 \\
\hline June & 0.05 & 1978 & 0.05 & 1978 & 0.05 & ng & ng & 1.61 \\
\hline July & 0.04 & 1978 & 0.04 & 1977 & 0.04 & 0 & 0.05 & 1.25 \\
\hline August & 0.06 & 1977 & 0.04 & 1978 & 0.05 & 0.02 & 0.33 & 1.64 \\
\hline September & 0.09 & 1977 & 0.04 & 1978 & 0.07 & 0.04 & 0.55 & 2.12 \\
\hline Annual & 0.26 & 1978 & 0.26 & 1978 & 0.26 & ng & ng & 100 \\
\hline
\end{tabular}


9-1.000-1A GULRAN RIVER AT CHAR TAKHTA, Continued

Monthly and annual flow duration, in cubic meters per second

[ng, not given]

\begin{tabular}{|c|c|c|c|c|c|c|c|c|c|c|c|c|c|}
\hline \multirow{2}{*}{$\begin{array}{c}\text { Percentage } \\
\text { of days } \\
\text { discharge } \\
\text { equaled or } \\
\text { exceeded }\end{array}$} & \multicolumn{12}{|c|}{ Month } & \multirow{2}{*}{ Annua } \\
\hline & October & November & December & January & February & March & April & May & June & July & August & September & \\
\hline 95 & 0.11 & 0.20 & 0.09 & 0.03 & 0.03 & 0.50 & 0.11 & 0.04 & ng & 0.03 & 0.04 & 0.04 & 0.03 \\
\hline 90 & 0.12 & 0.21 & 0.10 & 0.03 & 0.03 & 0.50 & 0.11 & 0.04 & ng & 0.04 & 0.04 & 0.04 & 0.04 \\
\hline 85 & 0.12 & 0.25 & 0.10 & 0.03 & 0.03 & 0.51 & 0.11 & 0.04 & ng & ng & 0.04 & 0.04 & 0.04 \\
\hline 80 & 0.12 & 0.25 & 0.10 & 0.03 & 0.03 & 0.51 & 0.12 & 0.05 & $\mathrm{ng}$ & ng & 0.04 & 0.04 & 0.04 \\
\hline 75 & 0.12 & 0.25 & 0.10 & 0.03 & 0.03 & 0.51 & 0.12 & 0.05 & ng & ng & 0.05 & 0.05 & 0.04 \\
\hline 70 & 0.12 & 0.25 & 0.11 & 0.03 & 0.04 & 0.51 & 0.15 & 0.05 & ng & ng & 0.05 & 0.05 & 0.05 \\
\hline 65 & 0.12 & 0.26 & 0.11 & 0.03 & 0.04 & 0.52 & 0.19 & 0.05 & ng & ng & 0.05 & 0.05 & 0.05 \\
\hline 60 & 0.12 & 0.26 & 0.11 & 0.03 & 0.04 & 0.52 & 0.22 & 0.06 & ng & ng & 0.05 & 0.05 & 0.05 \\
\hline 55 & 0.13 & 0.26 & 0.11 & 0.03 & 0.04 & 0.6 & 0.31 & 0.06 & ng & ng & 0.05 & 0.05 & 0.06 \\
\hline 50 & 0.13 & 0.26 & 0.14 & 0.04 & 0.04 & 0.61 & 0.33 & 0.07 & ng & ng & 0.05 & 0.07 & 0.07 \\
\hline 45 & 0.13 & 0.31 & 0.14 & 0.04 & 0.05 & 0.71 & 0.42 & 0.07 & ng & ng & ng & 0.08 & 0.09 \\
\hline 40 & 0.13 & 0.33 & 0.15 & 0.04 & 0.05 & 0.72 & 0.51 & 0.07 & ng & ng & ng & 0.09 & 0.11 \\
\hline 35 & 0.13 & 0.41 & 0.15 & 0.04 & 0.06 & 0.81 & 0.58 & ng & ng & ng & ng & 0.09 & 0.12 \\
\hline 30 & 0.13 & 0.49 & 0.15 & 0.04 & 0.11 & 0.82 & 0.60 & ng & ng & ng & ng & 0.10 & 0.15 \\
\hline 25 & ng & 0.51 & 0.16 & 0.04 & 0.23 & 0.98 & 0.68 & ng & ng & ng & ng & 0.10 & 0.20 \\
\hline 20 & $\mathrm{ng}$ & 0.53 & 0.16 & 0.04 & 0.95 & 0.99 & 0.73 & $\mathrm{ng}$ & ng & ng & ng & ng & 0.31 \\
\hline 15 & ng & 1.10 & 0.30 & 0.04 & 1.04 & 1.00 & 0.78 & $\mathrm{ng}$ & $\mathrm{ng}$ & ng & $\mathrm{ng}$ & $\mathrm{ng}$ & 0.54 \\
\hline 10 & ng & 1.51 & 1.08 & 0.04 & ng & 1.01 & 1.13 & ng & ng & ng & ng & ng & 0.73 \\
\hline 5 & $\mathrm{ng}$ & $\mathrm{ng}$ & $\mathrm{ng}$ & $\mathrm{ng}$ & $\mathrm{ng}$ & $\mathrm{ng}$ & $\mathrm{ng}$ & $\mathrm{ng}$ & $\mathrm{ng}$ & $\mathrm{ng}$ & $\mathrm{ng}$ & $\mathrm{ng}$ & 1.11 \\
\hline
\end{tabular}




\section{9-1.000-1A GULRAN RIVER AT CHAR TAKHTA, Continued}

Probability of occurrence of annual high discharges

[m $\mathrm{m}^{3} / \mathrm{s}$, cubic meters per second; ng, not given]

\begin{tabular}{|c|c|c|c|c|c|c|}
\hline \multirow{2}{*}{$\begin{array}{l}\text { Exceedance } \\
\text { probability }\end{array}$} & \multirow{2}{*}{$\begin{array}{c}\text { Recurrence } \\
\text { interval } \\
\text { (years) }\end{array}$} & \multirow{2}{*}{$\begin{array}{l}\text { Maximum } \\
\text { instantaneous } \\
\text { discharge } \\
\left(\mathrm{m}^{3} / \mathrm{s}\right)\end{array}$} & \multicolumn{4}{|c|}{ Maximum daily mean discharge $\left(\mathrm{m}^{3} / \mathrm{s}\right)$} \\
\hline & & & $\begin{array}{l}\text { 3-day } \\
\text { period }\end{array}$ & $\begin{array}{l}\text { 7-day } \\
\text { period }\end{array}$ & $\begin{array}{l}\text { 15-day } \\
\text { period }\end{array}$ & $\begin{array}{l}\text { 30-day } \\
\text { period }\end{array}$ \\
\hline 0.99 & 1.01 & ng & ng & ng & $\mathrm{ng}$ & ng \\
\hline 0.95 & 1.05 & ng & ng & ng & $\mathrm{ng}$ & ng \\
\hline 0.90 & 1.11 & ng & ng & ng & $\mathrm{ng}$ & ng \\
\hline 0.80 & 1.25 & ng & ng & ng & $\mathrm{ng}$ & $\mathrm{ng}$ \\
\hline 0.50 & 2 & ng & $\mathrm{ng}$ & ng & ng & ng \\
\hline 0.20 & 5 & ng & $\mathrm{ng}$ & ng & ng & ng \\
\hline 0.10 & 10 & ng & $\mathrm{ng}$ & ng & $\mathrm{ng}$ & $\mathrm{ng}$ \\
\hline 0.04 & 25 & ng & $\mathrm{ng}$ & ng & $\mathrm{ng}$ & ng \\
\hline 0.02 & 50 & ng & $\mathrm{ng}$ & ng & $\mathrm{ng}$ & $\mathrm{ng}$ \\
\hline 0.01 & 100 & ng & $\mathrm{ng}$ & $\mathrm{ng}$ & $\mathrm{ng}$ & $\mathrm{ng}$ \\
\hline 0.005 & 200 & ng & ng & ng & ng & $\mathrm{ng}$ \\
\hline 0.002 & 500 & $\mathrm{ng}$ & ng & $\mathrm{ng}$ & $\mathrm{ng}$ & $\mathrm{ng}$ \\
\hline
\end{tabular}

\section{9-1.000-1A GULRAN RIVER AT CHAR TAKHTA, Continued}

Probability of occurrence of annual low discharges [ $\mathrm{m}^{3} / \mathrm{s}$, meters per second]

\begin{tabular}{|c|c|c|c|c|c|c|c|c|c|c|}
\hline \multirow{3}{*}{$\begin{array}{c}\text { Nonexceedance } \\
\text { probability }\end{array}$} & \multirow{3}{*}{$\begin{array}{c}\text { Recurrence } \\
\text { interval } \\
\text { (years) }\end{array}$} & \multicolumn{9}{|c|}{ Minimum daily mean discharge $\left(\mathrm{m}^{3} / \mathrm{s}\right)$} \\
\hline & & \multicolumn{9}{|c|}{ Number of consecutive days } \\
\hline & & 1 & 3 & 7 & 14 & 30 & 60 & 90 & 120 & 183 \\
\hline 0.05 & 20 & ng & ng & ng & ng & ng & ng & ng & ng & $\mathrm{ng}$ \\
\hline 0.10 & 10 & ng & $\mathrm{ng}$ & ng & ng & ng & ng & ng & ng & ng \\
\hline 0.20 & 5 & $\mathrm{ng}$ & ng & ng & ng & ng & ng & ng & ng & ng \\
\hline 0.50 & 2 & $\mathrm{ng}$ & ng & $\mathrm{ng}$ & $\mathrm{ng}$ & ng & ng & ng & ng & ng \\
\hline
\end{tabular}




\section{9-1.000-1A GULRAN RIVER AT CHAR TAKHTA, Continued}

Probability of occurrence of seasonal low discharges [ $\mathrm{m}^{3} / \mathrm{s}$, meters per second; $\mathrm{ng}$, not given]

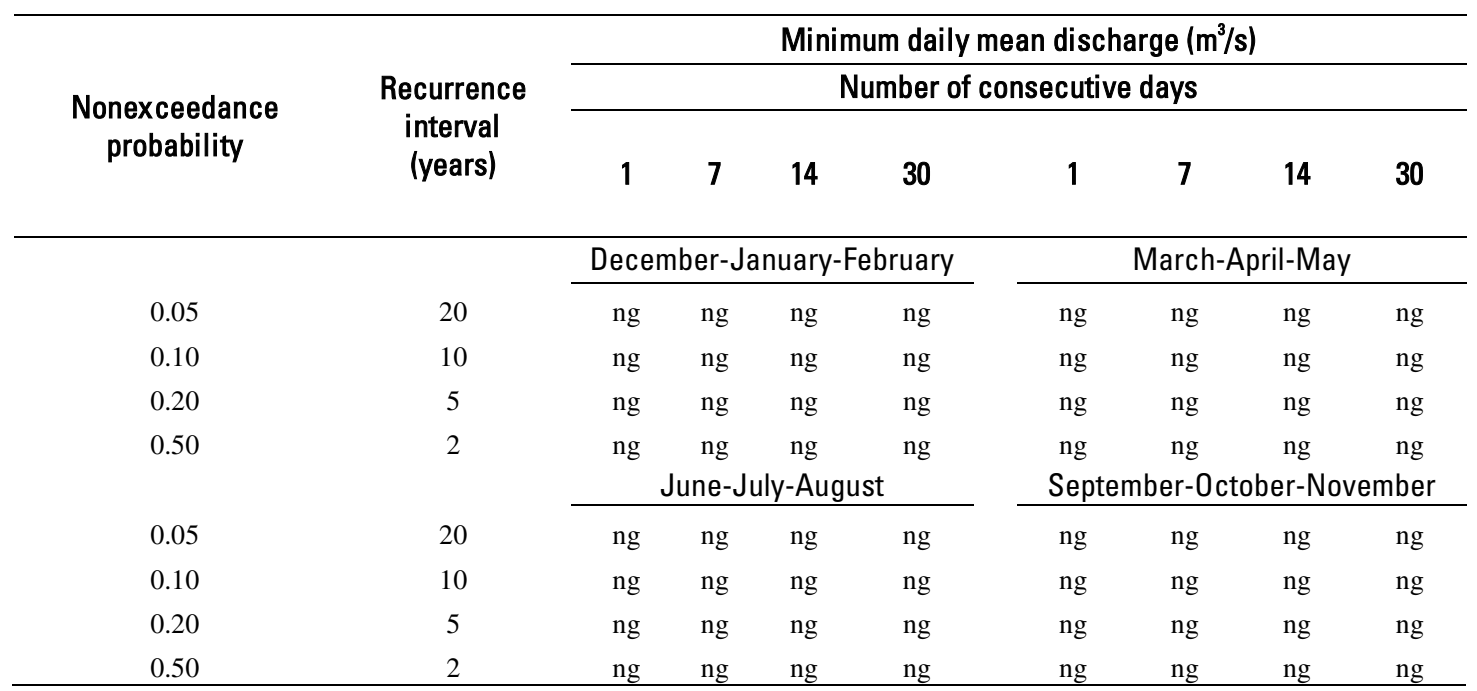

\section{9-1.000-1A GULRAN RIVER AT CHAR TAKHTA, Continued}

\section{Annual peak discharges}

$\left[\mathrm{m}^{3} / \mathrm{s}\right.$, meters per second]

\begin{tabular}{|c|c|c|c|c|c|}
\hline \multicolumn{3}{|c|}{$\begin{array}{c}\text { Annual peak discharge, } \\
\text { by year }\end{array}$} & \multicolumn{3}{|c|}{$\begin{array}{l}\text { Annual peak discharge, } \\
\text { from highest to lowest }\end{array}$} \\
\hline $\begin{array}{l}\text { Water } \\
\text { year }\end{array}$ & Date & $\begin{array}{c}\text { Peak } \\
\text { discharge } \\
\left(\mathrm{m}^{3} / \mathrm{s}\right)\end{array}$ & $\begin{array}{l}\text { Water } \\
\text { year }\end{array}$ & Date & $\begin{array}{c}\text { Peak } \\
\text { discharge } \\
\left(\mathrm{m}^{3} / \mathrm{s}\right)\end{array}$ \\
\hline 1978 & November 18, 1977 & 23.4 & 1978 & November 18, 1977 & 23.4 \\
\hline
\end{tabular}


9-1.000-1A GULRAN RIVER AT CHAR TAKHTA, Continued

Monthly and annual mean discharges, in cubic meters per second [--, no data]

\begin{tabular}{|c|c|c|c|c|c|c|c|c|c|c|c|c|c|}
\hline \multirow{2}{*}{$\begin{array}{c}\text { Water } \\
\text { year }\end{array}$} & \multicolumn{12}{|c|}{ Monthly mean discharge } & \multirow{2}{*}{$\begin{array}{l}\text { Annual } \\
\text { discharge }\end{array}$} \\
\hline & October & November & December & January & February & March & April & May & June & July & August & September & \\
\hline 1977 & -- & -- & -- & -- & -- & -- & -- & -- & -- & 0.04 & 0.06 & 0.09 & -- \\
\hline 1978 & 0.13 & 0.57 & 0.53 & 0.03 & 0.37 & 0.78 & 0.48 & 0.06 & 0.05 & 0.04 & 0.04 & 0.04 & 0.26 \\
\hline
\end{tabular}




\section{9-2.000-1A KUSHK RIVER AT CHIL DUKHTARAN}

\section{(U.S. Geological Survey identification number: 351300062170000)}

LOCATION: Lat $35^{\circ} 13^{\prime} \mathrm{N} .$, long $62^{\circ} 17^{\prime} \mathrm{E}$.

DRAINAGE AREA: $2,240 \mathrm{~km}^{2}$.

ELEVATION: 768 meters above mean sea level.

PERIOD OF RECORD: July 6, 1970 to March 29, 1975 and June 14, 1977 to September 30, 1978.

GAGE: Water-stage recorder.

Annual mean discharge

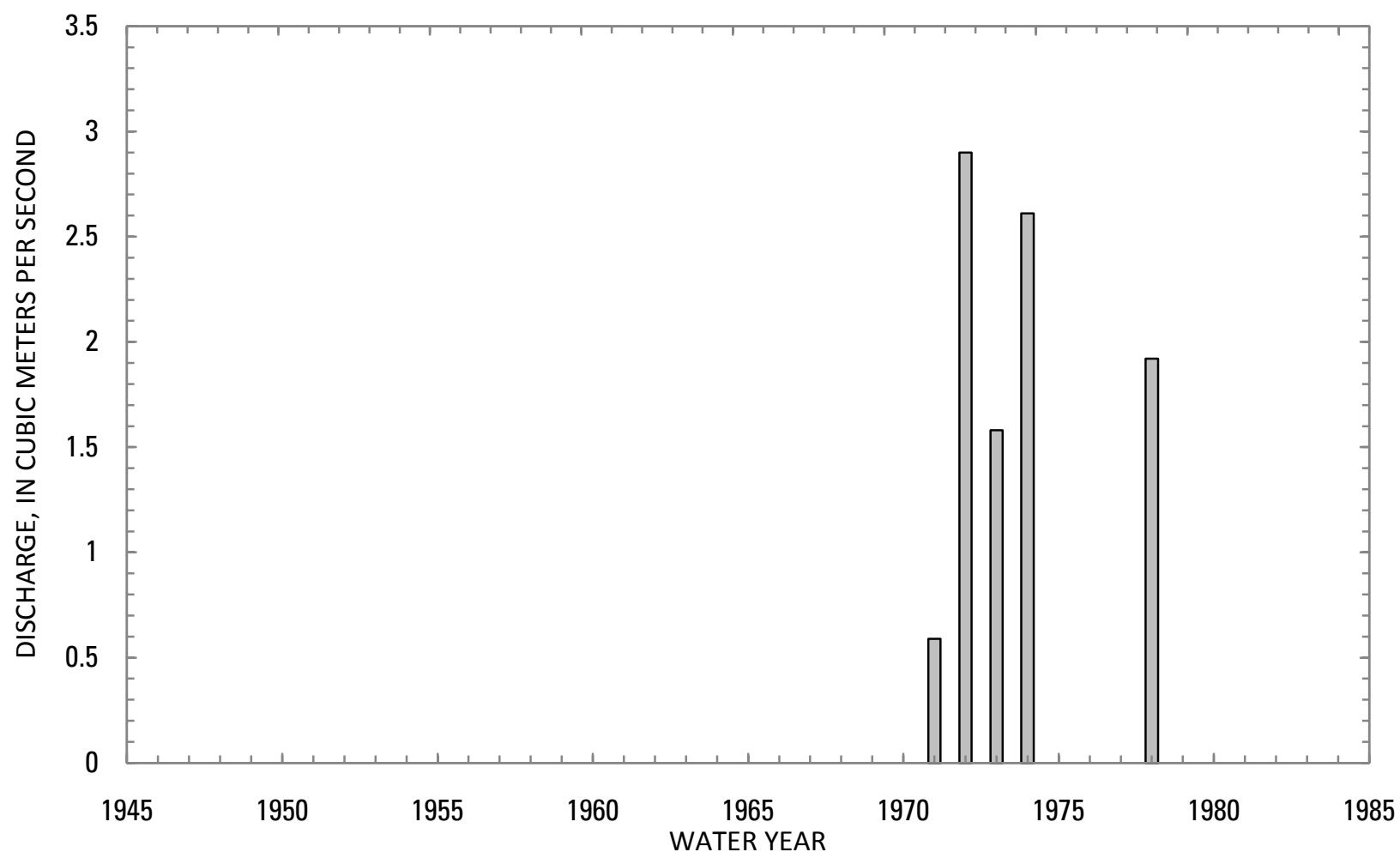




\section{9-2.000-1A KUSHK RIVER AT CHIL DUKHTARAN, Continued}

Statistics of monthly and annual mean discharges $\left[\mathrm{m}^{3} / \mathrm{s}\right.$, cubic meters per second]

\begin{tabular}{|c|c|c|c|c|c|c|c|c|}
\hline \multirow[b]{2}{*}{ Month } & \multicolumn{2}{|c|}{ Maximum } & \multicolumn{2}{|c|}{ Minimum } & \multicolumn{4}{|c|}{ Mean } \\
\hline & $\begin{array}{c}\text { Discharge } \\
\left(\mathrm{m}^{3} / \mathrm{s}\right)\end{array}$ & $\begin{array}{c}\text { Water year } \\
\text { of } \\
\text { occurrence }\end{array}$ & $\begin{array}{c}\text { Discharge } \\
\left(\mathrm{m}^{3} / \mathrm{s}\right)\end{array}$ & $\begin{array}{c}\text { Water year } \\
\text { of } \\
\text { occurrence }\end{array}$ & $\begin{array}{c}\text { Discharge } \\
\left(\mathrm{m}^{3} / \mathrm{s}\right)\end{array}$ & $\begin{array}{c}\text { Standard } \\
\text { deviation } \\
\left(\mathrm{m}^{3} / \mathrm{s}\right)\end{array}$ & $\begin{array}{c}\text { Coefficient } \\
\text { of } \\
\text { variation }\end{array}$ & $\begin{array}{c}\text { Percentage } \\
\text { of annual } \\
\text { discharge }\end{array}$ \\
\hline October & 0.35 & 1973 & 0.09 & 1974 & 0.18 & 0.10 & 0.55 & 0.77 \\
\hline November & 1.07 & 1972 & 0.17 & 1974 & 0.58 & 0.40 & 0.69 & 2.48 \\
\hline December & 1.85 & 1978 & 0.57 & 1972 & 1.02 & 0.52 & 0.52 & 4.37 \\
\hline January & 2.03 & 1974 & 0.65 & 1971 & 1.41 & 0.48 & 0.34 & 6.08 \\
\hline February & 3.68 & 1975 & 1.57 & 1978 & 2.70 & 0.96 & 0.35 & 11.6 \\
\hline March & 10.1 & 1972 & 1.11 & 1971 & 7.06 & 3.60 & 0.51 & 30.3 \\
\hline April & 13.6 & 1974 & 2.31 & 1971 & 7.45 & 4.22 & 0.57 & 32.0 \\
\hline May & 7.18 & 1972 & 0.16 & 1971 & 2.06 & 2.98 & 1.45 & 8.84 \\
\hline June & 1.65 & 1972 & 0.04 & 1974 & 0.42 & 0.69 & 1.64 & 1.81 \\
\hline July & 0.48 & 1972 & 0.04 & 1974 & 0.15 & 0.17 & 1.13 & 0.65 \\
\hline August & 0.38 & 1972 & 0.02 & 1977 & 0.12 & 0.15 & 1.18 & 0.53 \\
\hline September & 0.26 & 1978 & 0.05 & 1973 & 0.12 & 0.07 & 0.59 & 0.54 \\
\hline Annual & 2.90 & 1972 & 0.59 & 1971 & 1.92 & 0.91 & 0.47 & 100 \\
\hline
\end{tabular}


9-2.000-1A KUSHK RIVER AT CHIL DUKHTARAN, Continued

Monthly and annual flow duration, in cubic meters per second

[ng, not given]

\begin{tabular}{|c|c|c|c|c|c|c|c|c|c|c|c|c|c|}
\hline \multirow{2}{*}{$\begin{array}{l}\text { Percentage } \\
\text { of days } \\
\text { discharge } \\
\text { equaled or } \\
\text { exceeded }\end{array}$} & \multicolumn{12}{|c|}{ Month } & \multirow{2}{*}{ Annua } \\
\hline & October & November & December & January & February & March & April & May & June & July & August & September & \\
\hline 95 & 0.08 & 0.10 & 0.30 & 0.54 & 0.75 & 0.14 & 0.50 & 0.04 & 0.04 & 0.02 & 0.01 & 0.03 & 0.04 \\
\hline 90 & 0.09 & 0.13 & 0.37 & 0.60 & 0.99 & 0.81 & 1.14 & 0.07 & 0.05 & 0.03 & 0.02 & 0.04 & 0.05 \\
\hline 85 & 0.09 & 0.16 & 0.40 & 0.64 & 1.03 & 1.38 & 1.67 & 0.09 & 0.05 & 0.04 & 0.02 & 0.05 & 0.06 \\
\hline 80 & 0.10 & 0.17 & 0.43 & 0.73 & 1.07 & 3.15 & 2.35 & 0.10 & 0.05 & 0.04 & 0.02 & 0.05 & 0.09 \\
\hline 75 & 0.10 & 0.24 & 0.47 & 0.77 & 1.34 & 3.44 & 2.97 & 0.12 & 0.06 & 0.05 & 0.03 & 0.06 & 0.12 \\
\hline 70 & 0.10 & 0.27 & 0.51 & 0.92 & 1.54 & 3.72 & 4.19 & 0.14 & 0.06 & 0.05 & 0.03 & 0.07 & 0.16 \\
\hline 65 & 0.12 & 0.30 & 0.56 & 0.98 & 1.69 & 4.22 & 4.87 & 0.2 & 0.07 & 0.05 & 0.04 & 0.07 & 0.23 \\
\hline 60 & 0.13 & 0.34 & 0.63 & 1.03 & 1.83 & 5.08 & 5.39 & 0.22 & 0.07 & 0.06 & 0.04 & 0.08 & 0.28 \\
\hline 55 & 0.15 & 0.36 & 0.68 & 1.06 & 2.01 & 6.07 & 5.92 & 0.23 & 0.08 & 0.06 & 0.05 & 0.08 & 0.37 \\
\hline 50 & 0.16 & 0.38 & 0.72 & 1.10 & 2.09 & 6.71 & 6.46 & 0.32 & 0.11 & 0.07 & 0.05 & 0.09 & 0.44 \\
\hline 45 & 0.17 & 0.41 & 0.76 & 1.15 & 2.17 & 7.35 & 7.22 & 0.45 & 0.13 & 0.11 & 0.06 & 0.11 & 0.55 \\
\hline 40 & 0.19 & 0.51 & 0.80 & 1.20 & 2.51 & 8.18 & 7.86 & 0.57 & 0.14 & 0.13 & 0.07 & 0.12 & 0.75 \\
\hline 35 & 0.20 & 0.54 & 0.88 & 1.26 & 2.77 & 9.01 & 8.45 & 0.84 & 0.15 & 0.14 & 0.09 & 0.14 & 0.92 \\
\hline 30 & 0.21 & 0.56 & 0.98 & 1.35 & 2.98 & 9.64 & 9.48 & 1.31 & 0.29 & 0.14 & 0.12 & 0.15 & 1.16 \\
\hline 25 & 0.21 & 0.60 & 1.13 & 1.55 & 3.21 & 10.3 & 10.8 & 3.58 & 0.33 & 0.22 & 0.27 & 0.19 & 1.65 \\
\hline 20 & 0.22 & 0.66 & 1.29 & 1.88 & 3.92 & 10.9 & 12.1 & 5.16 & 0.37 & 0.25 & 0.29 & 0.24 & 2.56 \\
\hline 15 & 0.24 & 0.76 & 1.43 & 2.02 & 4.42 & 11.9 & 13.2 & 6.62 & 0.75 & 0.32 & 0.30 & 0.26 & 4.22 \\
\hline 10 & 0.39 & 0.92 & 1.63 & 2.63 & 4.95 & 13.2 & 15.4 & 7.22 & 0.98 & 0.36 & 0.35 & 0.27 & 6.73 \\
\hline 5 & 0.47 & 1.38 & 2.12 & 3.16 & 6.07 & 15.5 & 17.6 & 8.44 & 2.82 & 0.47 & ng & 0.28 & 10.1 \\
\hline
\end{tabular}




\section{9-2.000-1A KUSHK RIVER AT CHIL DUKHTARAN, Continued}

Probability of occurrence of annual high discharges

[m $\mathrm{m}^{3} / \mathrm{s}$, cubic meters per second; ng, not given]

\begin{tabular}{|c|c|c|c|c|c|c|}
\hline \multirow{2}{*}{$\begin{array}{c}\text { Exceedance } \\
\text { probability }\end{array}$} & \multirow{2}{*}{$\begin{array}{c}\text { Recurrence } \\
\text { interval } \\
\text { (years) }\end{array}$} & \multirow{2}{*}{$\begin{array}{c}\text { Maximum } \\
\text { instantaneous } \\
\text { discharge } \\
\left(\mathrm{m}^{3} / \mathrm{s}\right)\end{array}$} & \multicolumn{4}{|c|}{ Maximum daily mean discharge $\left(\mathrm{m}^{3} / \mathrm{s}\right)$} \\
\hline & & & $\begin{array}{l}\text { 3-day } \\
\text { period }\end{array}$ & $\begin{array}{l}\text { 7-day } \\
\text { period }\end{array}$ & $\begin{array}{l}\text { 15-day } \\
\text { period }\end{array}$ & $\begin{array}{l}\text { 30-day } \\
\text { period }\end{array}$ \\
\hline 0.99 & 1.01 & 7.90 & 5.75 & 3.17 & 1.55 & 0.96 \\
\hline 0.95 & 1.05 & 14.0 & 8.20 & 5.69 & 3.54 & 2.45 \\
\hline 0.90 & 1.11 & 18.8 & 9.71 & 7.36 & 5.07 & 3.69 \\
\hline 0.80 & 1.25 & 26.5 & 11.7 & 9.56 & 7.31 & 5.60 \\
\hline 0.50 & 2 & 49.1 & 15.9 & 13.7 & 12.1 & 9.96 \\
\hline 0.20 & 5 & 86.7 & 20.3 & 16.9 & 16.0 & 13.8 \\
\hline 0.10 & 10 & 114 & 22.5 & 17.9 & 17.3 & 15.1 \\
\hline 0.04 & 25 & 152 & 24.8 & 18.6 & 18.1 & 16.0 \\
\hline 0.02 & 50 & 181 & 26.1 & 18.9 & 18.4 & 16.4 \\
\hline 0.01 & 100 & 211 & 27.3 & 19.1 & 18.6 & 16.6 \\
\hline 0.005 & 200 & 241 & 28.2 & 19.2 & 18.7 & 16.7 \\
\hline 0.002 & 500 & 283 & ng & ng & $\mathrm{ng}$ & ng \\
\hline
\end{tabular}

\section{9-2.000-1A KUSHK RIVER AT CHIL DUKHTARAN, Continued}

Probability of occurrence of annual low discharges $\left[\mathrm{m}^{3} / \mathrm{s}\right.$, meters per second]

\begin{tabular}{|c|c|c|c|c|c|c|c|c|c|c|}
\hline \multirow{3}{*}{$\begin{array}{c}\text { Nonexceedance } \\
\text { probability }\end{array}$} & \multirow{3}{*}{$\begin{array}{c}\text { Recurrence } \\
\text { interval } \\
\text { (years) }\end{array}$} & \multicolumn{9}{|c|}{ Minimum daily mean discharge $\left(\mathrm{m}^{3} / \mathrm{s}\right)$} \\
\hline & & \multicolumn{9}{|c|}{ Number of consecutive days } \\
\hline & & 1 & 3 & 7 & 14 & 30 & 60 & 90 & 120 & 183 \\
\hline 0.05 & 20 & 0.01 & 0.01 & 0.01 & 0.01 & 0.02 & 0.03 & 0.03 & 0.03 & 0.04 \\
\hline 0.10 & 10 & 0.01 & 0.01 & 0.01 & 0.01 & 0.02 & 0.03 & 0.03 & 0.04 & 0.05 \\
\hline 0.20 & 5 & 0.01 & 0.01 & 0.02 & 0.02 & 0.03 & 0.03 & 0.04 & 0.05 & 0.06 \\
\hline 0.50 & 2 & 0.02 & 0.02 & 0.02 & 0.03 & 0.04 & 0.06 & 0.08 & 0.09 & 0.11 \\
\hline
\end{tabular}




\section{9-2.000-1A KUSHK RIVER AT CHIL DUKHTARAN, Continued}

Probability of occurrence of seasonal low discharges [ $\mathrm{m}^{3} / \mathrm{s}$, meters per second]

\begin{tabular}{|c|c|c|c|c|c|c|c|c|c|}
\hline \multirow{3}{*}{$\begin{array}{c}\text { Nonexceedance } \\
\text { probability }\end{array}$} & \multirow{3}{*}{$\begin{array}{c}\text { Recurrence } \\
\text { interval } \\
\text { (years) }\end{array}$} & \multicolumn{8}{|c|}{ Minimum daily mean discharge $\left(\mathrm{m}^{3} / \mathrm{s}\right)$} \\
\hline & & \multicolumn{8}{|c|}{ Number of consecutive days } \\
\hline & & 1 & 7 & 14 & 30 & 1 & 7 & 14 & 30 \\
\hline & & \multicolumn{4}{|c|}{ December-January-February } & \multicolumn{4}{|c|}{ March-April-May } \\
\hline 0.05 & 20 & 0.01 & 0.29 & 0.34 & 0.45 & 0.01 & 0.02 & 0.04 & 0.09 \\
\hline 0.10 & 10 & 0.03 & 0.30 & 0.36 & 0.51 & 0.02 & 0.02 & 0.05 & 0.13 \\
\hline 0.20 & 5 & 0.08 & 0.31 & 0.40 & 0.59 & 0.02 & 0.03 & 0.06 & 0.21 \\
\hline \multirow[t]{2}{*}{0.50} & 2 & 0.31 & 0.38 & 0.52 & 0.80 & 0.07 & 0.10 & 0.15 & 0.65 \\
\hline & & \multicolumn{4}{|c|}{ June-July-August } & \multicolumn{4}{|c|}{ September-0ctober-November } \\
\hline 0.05 & 20 & 0.01 & 0.01 & 0.01 & 0.02 & 0.02 & 0.03 & 0.04 & 0.04 \\
\hline 0.10 & 10 & 0.01 & 0.01 & 0.01 & 0.02 & 0.02 & 0.03 & 0.04 & 0.05 \\
\hline 0.20 & 5 & 0.01 & 0.02 & 0.02 & 0.03 & 0.02 & 0.03 & 0.04 & 0.06 \\
\hline 0.50 & 2 & 0.04 & 0.04 & 0.05 & 0.06 & 0.03 & 0.04 & 0.05 & 0.09 \\
\hline
\end{tabular}

\section{9-2.000-1A KUSHK RIVER AT CHIL DUKHTARAN, Continued}

\section{Annual peak discharges}

$\left[\mathrm{m}^{3} / \mathrm{s}\right.$, meters per second]

\begin{tabular}{|c|c|c|c|c|c|}
\hline \multicolumn{3}{|c|}{$\begin{array}{c}\text { Annual peak discharge, } \\
\text { by year }\end{array}$} & \multicolumn{3}{|c|}{$\begin{array}{c}\text { Annual peak discharge, } \\
\text { from highest to lowest }\end{array}$} \\
\hline $\begin{array}{l}\text { Water } \\
\text { year }\end{array}$ & Date & $\begin{array}{c}\text { Peak } \\
\text { discharge } \\
\left(\mathrm{m}^{3} / \mathrm{s}\right)\end{array}$ & $\begin{array}{l}\text { Water } \\
\text { year }\end{array}$ & Date & $\begin{array}{c}\text { Peak } \\
\text { discharge } \\
\left(\mathrm{m}^{3} / \mathrm{s}\right)\end{array}$ \\
\hline 1971 & April 9, 1971 & 17.8 & 1978 & December 24, 1977 & 116 \\
\hline 1972 & March 18, 1972 & 58.0 & 1973 & March 24, 1973 & 61.0 \\
\hline 1973 & March 24, 1973 & 61.0 & 1972 & March 18, 1972 & 58.0 \\
\hline 1974 & April 12, 1974 & 32.8 & 1974 & April 12, 1974 & 32.8 \\
\hline 1978 & December 24, 1977 & 116 & 1971 & April 9, 1971 & 17.8 \\
\hline
\end{tabular}


9-2.000-1A KUSHK RIVER AT CHIL DUKHTARAN, Continued

Monthly and annual mean discharges, in cubic meters per second $[--$, no data]

\begin{tabular}{|c|c|c|c|c|c|c|c|c|c|c|c|c|c|}
\hline \multirow{2}{*}{$\begin{array}{l}\text { Water } \\
\text { year }\end{array}$} & \multicolumn{12}{|c|}{ Monthly mean discharge } & \multirow{2}{*}{$\begin{array}{c}\text { Annual } \\
\text { discharge }\end{array}$} \\
\hline & October & November & December & January & February & March & April & May & June & July & August & September & \\
\hline 1970 & -- & -- & -- & -- & -- & -- & -- & -- & -- & -- & 0.06 & 0.08 & -- \\
\hline 1971 & 0.18 & 0.33 & 0.59 & 0.65 & 1.57 & 1.11 & 2.31 & 0.16 & 0.10 & 0.06 & 0.06 & 0.11 & 0.59 \\
\hline 1972 & 0.15 & 1.07 & 0.57 & 1.62 & 2.55 & 10.1 & 8.90 & 7.18 & 1.65 & 0.48 & 0.38 & 0.18 & 2.90 \\
\hline 1973 & 0.35 & 0.56 & 1.21 & 1.69 & 3.28 & 6.33 & 5.22 & 0.33 & 0.06 & 0.05 & 0.04 & 0.05 & 1.58 \\
\hline 1974 & 0.09 & 0.17 & 0.60 & 2.03 & 3.53 & 9.16 & 13.6 & 2.17 & 0.04 & 0.04 & 0.04 & 0.06 & 2.61 \\
\hline 1975 & 0.09 & 0.27 & 1.29 & 1.36 & 3.68 & -- & -- & -- & -- & -- & -- & -- & -- \\
\hline 1977 & -- & -- & -- & -- & -- & -- & -- & -- & -- & 0.10 & 0.02 & 0.13 & -- \\
\hline 1978 & 0.21 & 1.07 & 1.85 & 1.14 & 1.57 & 8.60 & 7.21 & 0.44 & 0.26 & 0.18 & 0.28 & 0.26 & 1.92 \\
\hline
\end{tabular}




\section{9-3.000-1A KASHAN RIVER AT BABULAI}

\section{(U.S. Geological Survey identification number: 352000062550000 )}

LOCATION: Lat $35^{\circ} 20^{\prime} \mathrm{N}$., long $62^{\circ} 55^{\prime} \mathrm{E}$.

DRAINAGE AREA: $5,265 \mathrm{~km}^{2}$.

ELEVATION: 557 meters above mean sea level.

PERIOD OF RECORD: December 22, 1973 to September 30, 1978.

GAGE: Water-stage recorder.

Annual mean discharge

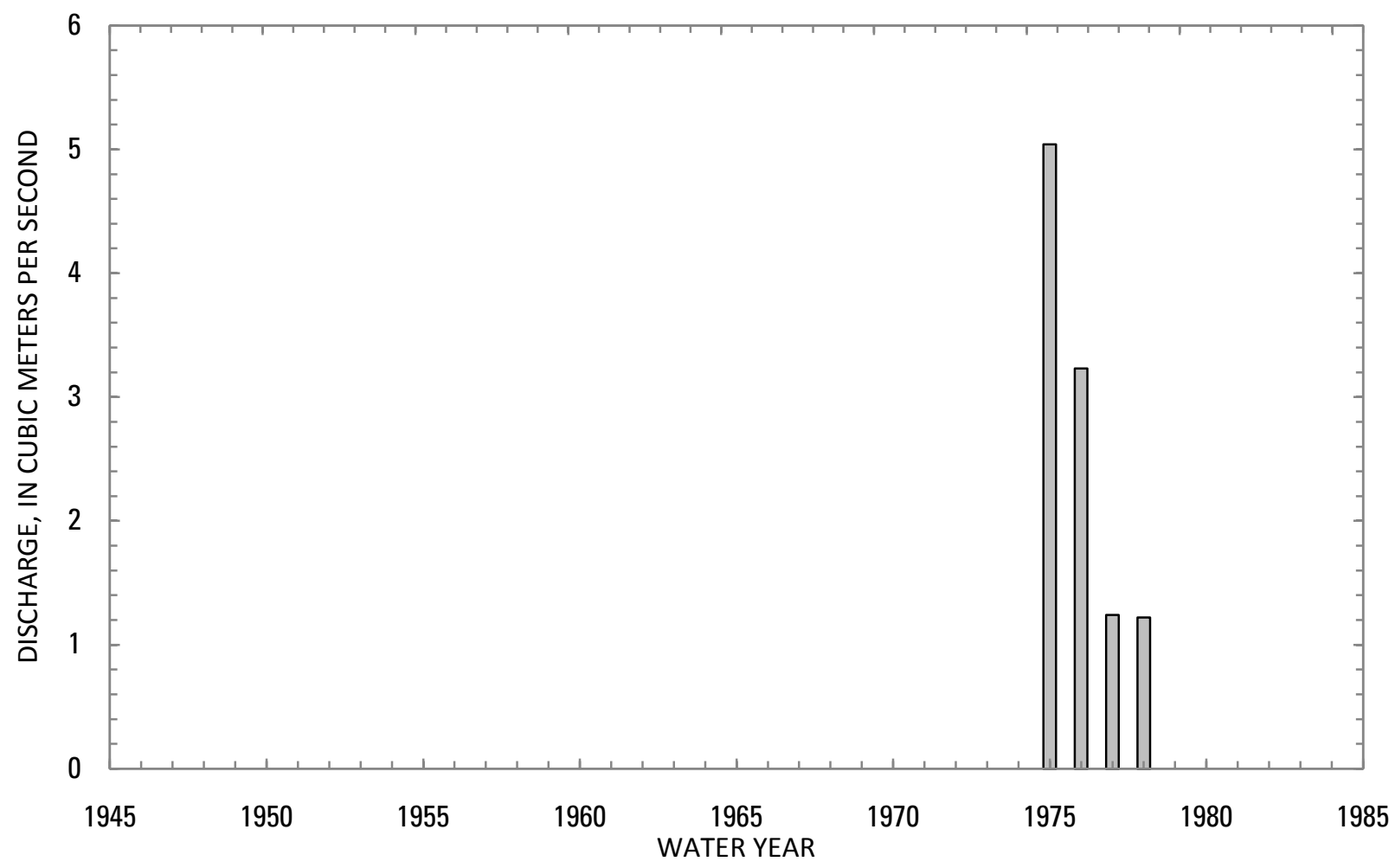




\section{9-3.000-1A KASHAN RIVER AT BABULAI, Continued}

Statistics of monthly and annual mean discharges [ $\mathrm{m}^{3} / \mathrm{s}$, cubic meters per second]

\begin{tabular}{|c|c|c|c|c|c|c|c|c|}
\hline \multirow[b]{2}{*}{ Month } & \multicolumn{2}{|c|}{ Maximum } & \multicolumn{2}{|c|}{ Minimum } & \multicolumn{4}{|c|}{ Mean } \\
\hline & $\begin{array}{c}\text { Discharge } \\
\left(\mathrm{m}^{3} / \mathrm{s}\right)\end{array}$ & $\begin{array}{c}\text { Water year } \\
\text { of } \\
\text { occurrence }\end{array}$ & $\begin{array}{c}\text { Discharge } \\
\left(\mathrm{m}^{3} / \mathrm{s}\right)\end{array}$ & $\begin{array}{c}\text { Water year } \\
\text { of } \\
\text { occurrence }\end{array}$ & $\begin{array}{c}\text { Discharge } \\
\left(\mathrm{m}^{3} / \mathrm{s}\right)\end{array}$ & $\begin{array}{c}\text { Standard } \\
\text { deviation } \\
\left(\mathrm{m}^{3} / \mathrm{s}\right)\end{array}$ & $\begin{array}{c}\text { Coefficient } \\
\text { of } \\
\text { variation }\end{array}$ & $\begin{array}{c}\text { Percentage } \\
\text { of annual } \\
\text { discharge }\end{array}$ \\
\hline October & 0.77 & 1977 & 0.14 & 1978 & 0.45 & 0.30 & 0.66 & 1.61 \\
\hline November & 1.06 & 1978 & 0.87 & 1975 & 0.99 & 0.09 & 0.09 & 3.50 \\
\hline December & 4.38 & 1978 & 1.14 & 1976 & 2.52 & 1.59 & 0.63 & 8.93 \\
\hline January & 2.29 & 1975 & 0.77 & 1974 & 1.38 & 0.56 & 0.40 & 4.88 \\
\hline February & 4.82 & 1975 & 0.84 & 1974 & 2.75 & 1.90 & 0.69 & 9.72 \\
\hline March & 14.8 & 1975 & 1.37 & 1977 & 5.87 & 5.58 & 0.95 & 20.8 \\
\hline April & 26.9 & 1975 & 1.88 & 1978 & 10.6 & 10.6 & 1.00 & 37.5 \\
\hline May & 6.99 & 1975 & 0.09 & 1978 & 3.39 & 3.22 & 0.95 & 12.0 \\
\hline June & 0.37 & 1975 & 0.01 & 1978 & 0.17 & 0.18 & 1.03 & 0.62 \\
\hline July & 0.20 & 1976 & 0 & 1978 & 0.06 & 0.09 & 1.46 & 0.21 \\
\hline August & 0.04 & 1976 & 0 & 1975 & 0.01 & 0.02 & 1.82 & 0.04 \\
\hline September & 0.35 & 1976 & 0 & 1974 & 0.07 & 0.16 & 2.22 & 0.25 \\
\hline Annual & 5.04 & 1975 & 1.22 & 1978 & 2.68 & 1.83 & 0.68 & 100 \\
\hline
\end{tabular}


9-3.000-1A KASHAN RIVER AT BABULAl, Continued

Monthly and annual flow duration, in cubic meters per second

[ng, not given]

\begin{tabular}{|c|c|c|c|c|c|c|c|c|c|c|c|c|c|}
\hline \multirow{2}{*}{$\begin{array}{l}\text { Percentage } \\
\text { of days } \\
\text { discharge } \\
\text { equaled or } \\
\text { exceeded }\end{array}$} & \multicolumn{12}{|c|}{ Month } & \multirow{2}{*}{ Annua } \\
\hline & October & November & December & January & February & March & April & May & June & July & August & September & \\
\hline 95 & 0 & 0.58 & 0.80 & 0.54 & 0.69 & 0.91 & 0.41 & 0.02 & 0 & 0 & 0 & 0 & 0 \\
\hline 90 & 0 & 0.70 & 0.85 & 0.57 & 0.74 & 1.04 & 0.70 & 0.05 & 0 & 0 & 0 & 0 & 0 \\
\hline 85 & 0.01 & 0.73 & 0.89 & 0.61 & 0.85 & 1.21 & 0.88 & 0.09 & 0.01 & 0 & 0 & 0 & 0 \\
\hline 80 & 0.04 & 0.75 & 1.02 & 0.70 & 0.94 & 1.33 & 1.19 & 0.12 & 0.01 & 0 & 0 & 0 & 0.01 \\
\hline 75 & 0.10 & 0.82 & 1.09 & 0.74 & 1.02 & 1.44 & 1.44 & 0.15 & 0.02 & 0 & 0 & 0 & 0.02 \\
\hline 70 & 0.12 & 0.85 & 1.15 & 0.83 & 1.07 & 1.52 & 1.98 & 0.20 & 0.02 & 0 & 0 & 0 & 0.19 \\
\hline 65 & 0.29 & 0.87 & 1.19 & 0.96 & 1.11 & 1.61 & 2.31 & 0.22 & 0.03 & 0 & 0 & 0 & 0.27 \\
\hline 60 & 0.31 & 0.90 & 1.22 & 1.04 & 1.32 & 1.78 & 2.57 & 0.24 & 0.03 & 0 & 0 & 0 & 0.42 \\
\hline 55 & 0.48 & 0.94 & 1.26 & 1.23 & 1.44 & 2.28 & 3.43 & 0.26 & 0.04 & 0.01 & 0 & 0 & 0.66 \\
\hline 50 & 0.52 & 0.99 & 1.30 & 1.27 & 1.49 & 2.58 & 4.52 & 0.31 & 0.05 & 0.01 & 0 & 0 & 0.80 \\
\hline 45 & 0.55 & 1.04 & 1.33 & 1.29 & 1.54 & 2.78 & 5.77 & 0.62 & 0.06 & 0.01 & 0 & 0 & 0.95 \\
\hline 40 & 0.59 & 1.06 & 1.36 & 1.32 & 1.64 & 2.97 & 7.56 & 1.15 & 0.23 & 0.02 & 0 & 0 & 1.12 \\
\hline 35 & 0.64 & 1.07 & 1.40 & 1.35 & 1.79 & 4.21 & 9.01 & 1.96 & 0.25 & 0.02 & 0.01 & 0 & 1.26 \\
\hline 30 & 0.67 & 1.09 & 1.43 & 1.41 & 2.01 & 5.38 & 11.5 & 2.56 & 0.26 & 0.02 & 0.01 & 0 & 1.39 \\
\hline 25 & 0.71 & 1.10 & 1.49 & 1.46 & 3.00 & 5.93 & 13.6 & 3.20 & 0.27 & 0.08 & 0.01 & 0 & 1.52 \\
\hline 20 & 0.74 & 1.13 & 1.56 & 1.52 & 3.81 & 6.81 & 15.8 & 4.79 & 0.29 & ng & 0.02 & 0.02 & 1.99 \\
\hline 15 & 0.78 & 1.18 & 1.63 & 1.57 & 4.63 & 8.14 & 18.1 & 6.02 & 0.31 & ng & 0.02 & 0.25 & 3.28 \\
\hline 10 & 0.85 & 1.24 & 1.92 & 1.77 & 6.12 & 12.9 & 30.2 & 7.47 & 0.46 & ng & 0.02 & 0.27 & 5.88 \\
\hline 5 & 0.97 & 1.33 & 8.32 & 2.81 & 11.0 & 18.3 & 40.8 & 14.6 & 0.61 & ng & ng & $\mathrm{ng}$ & 12.3 \\
\hline
\end{tabular}




\section{9-3.000-1A KASHAN RIVER AT BABULAI, Continued}

Probability of occurrence of annual high discharges [ $\mathrm{m}^{3} / \mathrm{s}$, cubic meters per second; $\mathrm{ng}$, not given]

\begin{tabular}{|c|c|c|c|c|c|c|}
\hline \multirow{2}{*}{$\begin{array}{c}\text { Exceedance } \\
\text { probability }\end{array}$} & \multirow{2}{*}{$\begin{array}{c}\text { Recurrence } \\
\text { interval } \\
\text { (years) }\end{array}$} & \multirow{2}{*}{$\begin{array}{l}\text { Maximum } \\
\text { instantaneous } \\
\text { discharge } \\
\left(\mathrm{m}^{3} / \mathrm{s}\right)\end{array}$} & \multicolumn{4}{|c|}{ Maximum daily mean discharge $\left(\mathrm{m}^{3} / \mathrm{s}\right)$} \\
\hline & & & $\begin{array}{l}\text { 3-day } \\
\text { period }\end{array}$ & $\begin{array}{l}\text { 7-day } \\
\text { period }\end{array}$ & $\begin{array}{l}\text { 15-day } \\
\text { period }\end{array}$ & $\begin{array}{l}\text { 30-day } \\
\text { period }\end{array}$ \\
\hline 0.99 & 1.01 & ng & 25.6 & 9.21 & 3.17 & 1.35 \\
\hline 0.95 & 1.05 & ng & 27.1 & 10.6 & 4.36 & 2.22 \\
\hline 0.90 & 1.11 & ng & 28.5 & 11.8 & 5.35 & 2.96 \\
\hline 0.80 & 1.25 & ng & 31.2 & 14.0 & 7.13 & 4.30 \\
\hline 0.50 & 2 & 82.9 & 40.9 & 22.7 & 14.0 & 9.45 \\
\hline 0.20 & 5 & 111 & 62.8 & 46.2 & 32.7 & 23.0 \\
\hline 0.10 & 10 & 119 & 84.5 & 74.2 & 54.9 & 38.2 \\
\hline 0.04 & 25 & 125 & ${ }^{2} 123$ & 134 & 101 & 67.8 \\
\hline 0.02 & 50 & 127 & ${ }^{2} 162$ & 205 & 156 & 100 \\
\hline 0.01 & 100 & 128 & ${ }^{2} 212$ & 310 & 234 & 144 \\
\hline 0.005 & 200 & 129 & ${ }^{2} 277$ & 467 & 348 & 202 \\
\hline 0.002 & 500 & 129 & $\mathrm{ng}$ & $\mathrm{ng}$ & $\mathrm{ng}$ & $\mathrm{ng}$ \\
\hline
\end{tabular}

\section{9-3.000-1A KASHAN RIVER AT BABULAI, Continued}

Probability of occurrence of annual low discharges

[ $\mathrm{m}^{3} / \mathrm{s}$, meters per second; ng, not given]

\begin{tabular}{|c|c|c|c|c|c|c|c|c|c|c|}
\hline \multirow{3}{*}{$\begin{array}{c}\text { Nonexceedance } \\
\text { probability }\end{array}$} & \multirow{3}{*}{$\begin{array}{c}\text { Recurrence } \\
\text { interval } \\
\text { (years) }\end{array}$} & \multicolumn{9}{|c|}{ Minimum daily mean discharge $\left(\mathrm{m}^{3} / \mathrm{s}\right)$} \\
\hline & & \multicolumn{9}{|c|}{ Number of consecutive days } \\
\hline & & 1 & 3 & 7 & 14 & 30 & 60 & 90 & 120 & 183 \\
\hline 0.05 & 20 & ng & ng & $\mathrm{ng}$ & ng & ng & ng & $\mathrm{ng}$ & ng & $\mathrm{ng}$ \\
\hline 0.10 & 10 & ng & ng & $\mathrm{ng}$ & ng & ng & ng & ng & ng & ng \\
\hline 0.20 & 5 & ng & ng & $\mathrm{ng}$ & ng & ng & ng & ng & ng & ng \\
\hline 0.50 & 2 & ng & ng & ng & ng & ng & ng & ng & ng & $\mathrm{ng}$ \\
\hline
\end{tabular}




\section{9-3.000-1A KASHAN RIVER AT BABULAI, Continued}

Probability of occurrence of seasonal low discharges [ $\mathrm{m}^{3} / \mathrm{s}$, meters per second; $\mathrm{ng}$, not given]

\begin{tabular}{|c|c|c|c|c|c|c|c|c|c|}
\hline \multirow{3}{*}{$\begin{array}{c}\text { Nonexceedance } \\
\text { probability }\end{array}$} & \multirow{3}{*}{$\begin{array}{c}\text { Recurrence } \\
\text { interval } \\
\text { (years) }\end{array}$} & \multicolumn{8}{|c|}{ Minimum daily mean discharge $\left(\mathrm{m}^{3} / \mathrm{s}\right)$} \\
\hline & & \multicolumn{8}{|c|}{ Number of consecutive days } \\
\hline & & 1 & 7 & 14 & 30 & 1 & 7 & 14 & 30 \\
\hline & & \multicolumn{4}{|c|}{ December-January-February } & \multicolumn{4}{|c|}{ March-April-May } \\
\hline 0.05 & 20 & 0.38 & 0.46 & 0.50 & 0.73 & 0.01 & 0.01 & 0 & 0.03 \\
\hline 0.10 & 10 & 0.44 & 0.54 & 0.59 & 0.80 & 0.01 & 0.01 & 0.01 & 0.06 \\
\hline 0.20 & 5 & 0.52 & 0.64 & 0.72 & 0.91 & 0.04 & 0.04 & 0.04 & 0.13 \\
\hline \multirow[t]{2}{*}{0.50} & 2 & 0.73 & 0.90 & 1.00 & 1.18 & 0.19 & 0.23 & 0.31 & 0.63 \\
\hline & & \multicolumn{4}{|c|}{ June-July-August } & \multicolumn{4}{|c|}{ September-0ctober-November } \\
\hline 0.05 & 20 & ng & ng & ng & ng & ng & ng & ng & $\mathrm{ng}$ \\
\hline 0.10 & 10 & ng & ng & ng & ng & ng & $\mathrm{ng}$ & $\mathrm{ng}$ & ng \\
\hline 0.20 & 5 & $\mathrm{ng}$ & ng & $\mathrm{ng}$ & ng & ng & $\mathrm{ng}$ & $\mathrm{ng}$ & $\mathrm{ng}$ \\
\hline 0.50 & 2 & $\mathrm{ng}$ & ng & $\mathrm{ng}$ & ng & ng & $\mathrm{ng}$ & ng & $\mathrm{ng}$ \\
\hline
\end{tabular}

\section{9-3.000-1A KASHAN RIVER AT BABULAI, Continued}

Annual peak discharges

[ $\mathrm{m}^{3} / \mathrm{s}$, meters per second]

\begin{tabular}{|c|c|c|c|c|c|}
\hline \multicolumn{3}{|c|}{$\begin{array}{c}\text { Annual peak discharge, } \\
\text { by year }\end{array}$} & \multicolumn{3}{|c|}{$\begin{array}{l}\text { Annual peak discharge, } \\
\text { from highest to lowest }\end{array}$} \\
\hline $\begin{array}{l}\text { Water } \\
\text { year }\end{array}$ & Date & $\begin{array}{c}\text { Peak } \\
\text { discharge } \\
\left(\mathrm{m}^{3} / \mathrm{s}\right)\end{array}$ & $\begin{array}{l}\text { Water } \\
\text { year }\end{array}$ & Date & $\begin{array}{c}\text { Peak } \\
\text { discharge } \\
\left(\mathrm{m}^{3} / \mathrm{s}\right)\end{array}$ \\
\hline 1974 & April 7, 1974 & 26.0 & 1975 & April 3, 1975 & 106 \\
\hline 1975 & April 3, 1975 & 106 & 1977 & April 9, 1977 & 102 \\
\hline 1976 & April 28, 1976 & 75.1 & 1978 & December 24, 1977 & 80.4 \\
\hline 1977 & April 9, 1977 & 102 & 1976 & April 28, 1976 & 75.1 \\
\hline 1978 & December 24,1977 & 80.4 & 1974 & April 7, 1974 & 26.0 \\
\hline
\end{tabular}


9-3.000-1A KASHAN RIVER AT BABULAI, Continued

Monthly and annual mean discharges, in cubic meters per second $[--$, no data]

\begin{tabular}{|c|c|c|c|c|c|c|c|c|c|c|c|c|c|}
\hline \multirow{2}{*}{$\begin{array}{l}\text { Water } \\
\text { year }\end{array}$} & \multicolumn{12}{|c|}{ Monthly mean discharge } & \multirow{2}{*}{$\begin{array}{c}\text { Annual } \\
\text { discharge }\end{array}$} \\
\hline & October & November & December & January & February & March & April & May & June & July & August & September & \\
\hline 1974 & -- & -- & -- & 0.77 & 0.84 & 1.60 & 4.46 & 0.24 & 0.04 & 0.01 & 0.01 & 0 & -- \\
\hline 1975 & 0.27 & 0.87 & 3.31 & 2.29 & 4.82 & 14.8 & 26.9 & 6.99 & 0.37 & 0.08 & 0 & 0 & 5.04 \\
\hline 1976 & 0.64 & 0.96 & 1.14 & 1.20 & 4.76 & 7.58 & 15.6 & 6.14 & 0.36 & 0.20 & 0.04 & 0.35 & 3.23 \\
\hline 1977 & 0.77 & 1.06 & 1.26 & 1.35 & 1.48 & 1.37 & 4.05 & 3.48 & 0.09 & 0 & 0 & 0 & 1.24 \\
\hline 1978 & 0.14 & 1.06 & 4.38 & 1.28 & 1.83 & 4.01 & 1.88 & 0.09 & 0.01 & 0 & 0 & 0 & 1.22 \\
\hline
\end{tabular}




\section{9-4.R00-8A CHICHAKTU RIVER AT CHICHAKTU}

\section{(U.S. Geological Survey identification number: 354400064050000 )}

LOCATION: Lat $35^{\circ} 44^{\prime} \mathrm{N}$., long $64^{\circ} 05^{\prime} \mathrm{E}$.

DRAINAGE AREA: $415 \mathrm{~km}^{2}$.

ELEVATION: 1,023 meters above mean sea level.

PERIOD OF RECORD: May 1, 1970 to September 30, 1978

GAGE: Water-stage recorder.

Annual mean discharge

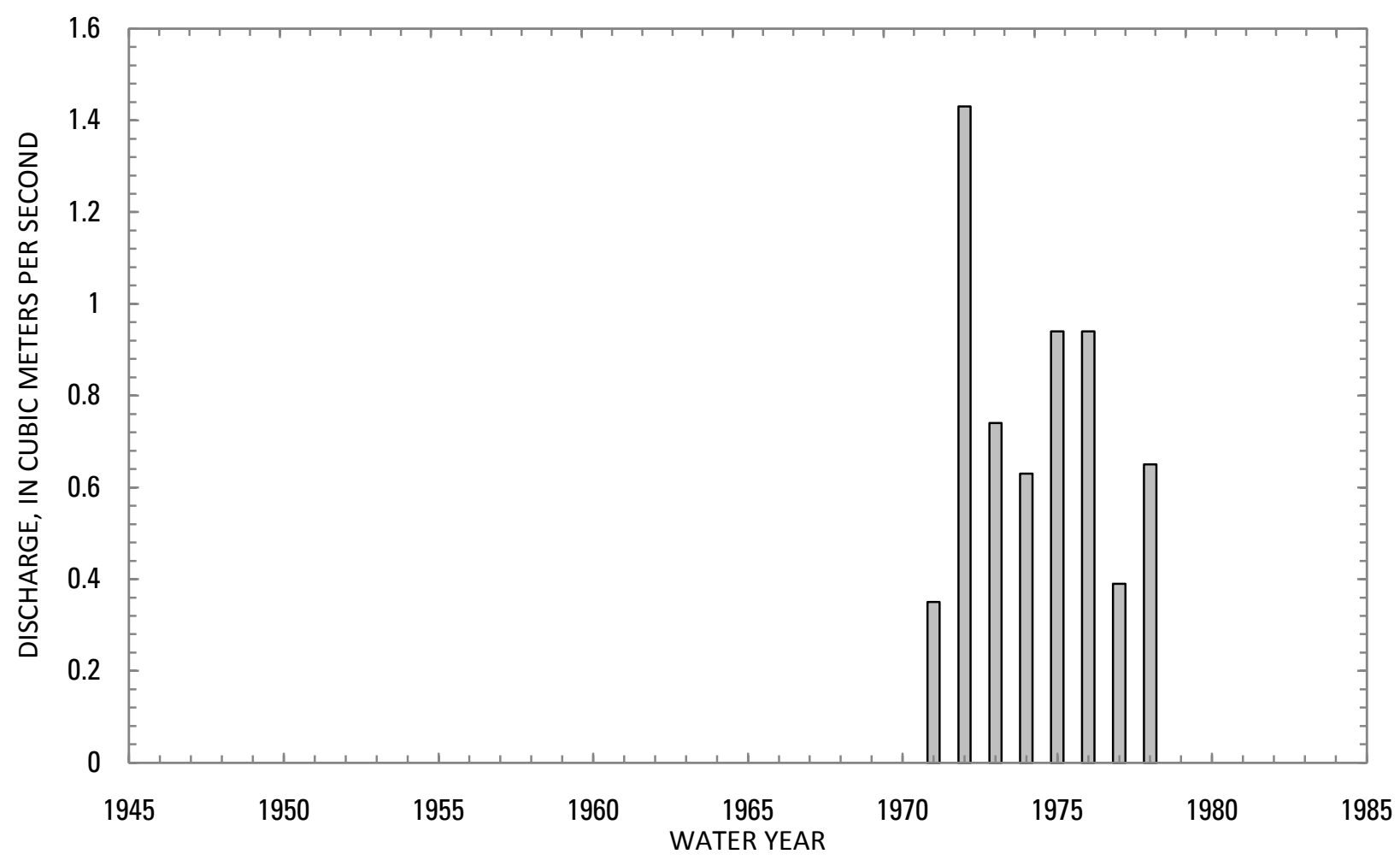




\section{9-4.R00-8A CHICHAKTU RIVER AT CHICHAKTU, Continued}

Statistics of monthly and annual mean discharges $\left[\mathrm{m}^{3} / \mathrm{s}\right.$, cubic meters per second]

\begin{tabular}{|c|c|c|c|c|c|c|c|c|}
\hline \multirow[b]{2}{*}{ Month } & \multicolumn{2}{|c|}{ Maximum } & \multicolumn{2}{|c|}{ Minimum } & \multicolumn{4}{|c|}{ Mean } \\
\hline & $\begin{array}{c}\text { Discharge } \\
\left(\mathrm{m}^{3} / \mathrm{s}\right)\end{array}$ & $\begin{array}{c}\text { Water year } \\
\text { of } \\
\text { occurrence }\end{array}$ & $\begin{array}{c}\text { Discharge } \\
\left(\mathrm{m}^{3} / \mathrm{s}\right)\end{array}$ & $\begin{array}{c}\text { Water year } \\
\text { of } \\
\text { occurrence }\end{array}$ & $\begin{array}{c}\text { Discharge } \\
\left(\mathrm{m}^{3} / \mathrm{s}\right)\end{array}$ & $\begin{array}{c}\text { Standard } \\
\text { deviation } \\
\left(\mathrm{m}^{3} / \mathrm{s}\right)\end{array}$ & $\begin{array}{c}\text { Coefficient } \\
\text { of } \\
\text { variation }\end{array}$ & $\begin{array}{c}\text { Percentage } \\
\text { of annual } \\
\text { discharge }\end{array}$ \\
\hline October & 0.57 & 1975 & 0.29 & 1978 & 0.44 & 0.10 & 0.24 & 4.94 \\
\hline November & 0.61 & 1975 & 0.26 & 1978 & 0.45 & 0.14 & 0.31 & 5.09 \\
\hline December & 0.90 & 1978 & 0.35 & 1972 & 0.54 & 0.19 & 0.35 & 6.14 \\
\hline January & 0.76 & 1973 & 0.26 & 1978 & 0.52 & 0.17 & 0.33 & 5.82 \\
\hline February & 1.24 & 1975 & 0.53 & 1974 & 0.71 & 0.23 & 0.33 & 8.01 \\
\hline March & 5.19 & 1972 & 0.51 & 1977 & 1.64 & 1.62 & 0.99 & 18.5 \\
\hline April & 5.10 & 1972 & 0.45 & 1977 & 1.96 & 1.62 & 0.82 & 22.1 \\
\hline May & 3.58 & 1976 & 0.18 & 1971 & 1.05 & 1.10 & 1.05 & 11.8 \\
\hline June & 1.42 & 1972 & 0.15 & 1977 & 0.58 & 0.43 & 0.73 & 6.56 \\
\hline July & 0.60 & 1976 & 0.12 & 1977 & 0.35 & 0.18 & 0.52 & 3.94 \\
\hline August & 0.57 & 1975 & 0.14 & 1970 & 0.32 & 0.15 & 0.46 & 3.61 \\
\hline September & 0.49 & 1973 & 0.21 & 1971 & 0.31 & 0.11 & 0.35 & 3.54 \\
\hline Annual & 1.43 & 1972 & 0.35 & 1971 & 0.76 & 0.35 & 0.46 & 100 \\
\hline
\end{tabular}


9-4.R00-8A CHICHAKTU RIVER AT CHICHAKTU, Continued

Monthly and annual flow duration, in cubic meters per second

[ng, not given]

\begin{tabular}{|c|c|c|c|c|c|c|c|c|c|c|c|c|c|}
\hline \multirow{2}{*}{$\begin{array}{l}\text { Percentage } \\
\text { of days } \\
\text { discharge } \\
\text { equaled or } \\
\text { exceeded }\end{array}$} & \multicolumn{12}{|c|}{ Month } & \multirow{2}{*}{ Annua } \\
\hline & October & November & December & January & February & March & April & May & June & July & August & September & \\
\hline 95 & 0.19 & 0.26 & 0.31 & 0.25 & 0.25 & 0.38 & 0.21 & 0.08 & 0.05 & 0.03 & 0.03 & 0.14 & 0.15 \\
\hline 90 & 0.26 & 0.28 & 0.35 & 0.31 & 0.33 & 0.52 & 0.31 & 0.15 & 0.13 & 0.08 & 0.09 & 0.17 & 0.21 \\
\hline 85 & 0.29 & 0.29 & 0.36 & 0.39 & 0.46 & 0.55 & 0.44 & 0.20 & 0.17 & 0.11 & 0.14 & 0.20 & 0.26 \\
\hline 80 & 0.32 & 0.29 & 0.37 & 0.40 & 0.48 & 0.58 & 0.51 & 0.23 & 0.20 & 0.16 & 0.16 & 0.21 & 0.28 \\
\hline 75 & 0.33 & 0.31 & 0.38 & 0.40 & 0.53 & 0.60 & 0.59 & 0.27 & 0.23 & 0.19 & 0.19 & 0.24 & 0.31 \\
\hline 70 & 0.34 & 0.35 & 0.39 & 0.41 & 0.56 & 0.65 & 0.69 & 0.3 & 0.27 & 0.22 & 0.21 & 0.26 & 0.34 \\
\hline 65 & 0.35 & 0.36 & 0.41 & 0.43 & 0.57 & 0.70 & 0.86 & 0.32 & 0.31 & 0.26 & 0.24 & 0.28 & 0.38 \\
\hline 60 & 0.37 & 0.37 & 0.43 & 0.47 & 0.58 & 0.72 & 0.93 & 0.35 & 0.36 & 0.29 & 0.26 & 0.29 & 0.42 \\
\hline 55 & 0.39 & 0.42 & 0.46 & 0.48 & 0.59 & 0.75 & 1.00 & 0.41 & 0.40 & 0.30 & 0.28 & 0.29 & 0.45 \\
\hline 50 & 0.41 & 0.44 & 0.48 & 0.49 & 0.60 & 0.77 & 1.12 & 0.61 & 0.55 & 0.31 & 0.30 & 0.30 & 0.49 \\
\hline 45 & 0.45 & 0.49 & 0.49 & 0.50 & 0.61 & 0.81 & 1.23 & 0.76 & 0.62 & 0.37 & 0.31 & 0.31 & 0.52 \\
\hline 40 & 0.49 & 0.50 & 0.50 & 0.51 & 0.64 & 0.86 & 1.31 & 0.89 & 0.68 & 0.40 & 0.36 & 0.31 & 0.56 \\
\hline 35 & 0.51 & 0.53 & 0.52 & 0.58 & 0.66 & 0.94 & 1.38 & 0.99 & 0.75 & 0.45 & 0.38 & 0.33 & 0.60 \\
\hline 30 & 0.52 & 0.57 & 0.53 & 0.59 & 0.69 & 1.08 & 1.71 & 1.10 & 0.80 & 0.50 & 0.40 & 0.38 & 0.65 \\
\hline 25 & 0.53 & 0.58 & 0.59 & 0.61 & 0.71 & 1.24 & 2.24 & 1.22 & 0.84 & 0.52 & 0.43 & 0.40 & 0.71 \\
\hline 20 & 0.57 & 0.59 & 0.63 & 0.70 & 0.74 & 1.47 & 2.83 & 1.64 & 0.89 & 0.55 & 0.50 & 0.43 & 0.77 \\
\hline 15 & 0.61 & 0.60 & 0.66 & 0.73 & 0.8 & 1.98 & 3.75 & 1.81 & 1.00 & 0.59 & 0.53 & 0.48 & 0.96 \\
\hline 10 & 0.63 & 0.69 & 0.69 & 0.77 & 1.10 & 3.01 & 4.53 & 1.98 & 1.29 & 0.64 & 0.58 & 0.51 & 1.26 \\
\hline 5 & 0.67 & 0.71 & 0.82 & 0.82 & 1.42 & 6.89 & 6.79 & 2.9 & 1.51 & $\mathrm{ng}$ & ng & 0.54 & 1.96 \\
\hline
\end{tabular}




\section{9-4.R00-8A CHICHAKTU RIVER AT CHICHAKTU, Continued}

Probability of occurrence of annual high discharges

[ $\mathrm{m}^{3} / \mathrm{s}$, cubic meters per second; ng, not given]

\begin{tabular}{|c|c|c|c|c|c|c|}
\hline \multirow{2}{*}{$\begin{array}{c}\text { Exceedance } \\
\text { probability }\end{array}$} & \multirow{2}{*}{$\begin{array}{c}\text { Recurrence } \\
\text { interval } \\
\text { (years) }\end{array}$} & \multirow{2}{*}{$\begin{array}{l}\text { Maximum } \\
\text { instantaneous } \\
\text { discharge } \\
\left(\mathrm{m}^{3} / \mathrm{s}\right)\end{array}$} & \multicolumn{4}{|c|}{ Maximum daily mean discharge $\left(\mathrm{m}^{3} / \mathrm{s}\right)$} \\
\hline & & & $\begin{array}{l}\text { 3-day } \\
\text { period }\end{array}$ & $\begin{array}{l}\text { 7-day } \\
\text { period }\end{array}$ & $\begin{array}{l}\text { 15-day } \\
\text { period }\end{array}$ & $\begin{array}{l}\text { 30-day } \\
\text { period }\end{array}$ \\
\hline 0.99 & 1.01 & 0.50 & 0.47 & 0.35 & ${ }^{2} 0.26$ & 0.27 \\
\hline 0.95 & 1.05 & 1.40 & 0.91 & 0.66 & 0.51 & 0.50 \\
\hline 0.90 & 1.11 & 2.50 & 1.29 & 0.93 & 0.73 & 0.69 \\
\hline 0.80 & 1.25 & 4.60 & 1.98 & 1.42 & 1.13 & 1.02 \\
\hline 0.50 & 2 & 13.2 & 4.54 & 3.27 & 2.53 & 2.09 \\
\hline 0.20 & 5 & 32.3 & 10.6 & 7.75 & 5.61 & 4.22 \\
\hline 0.10 & 10 & 48.7 & 16.6 & 12.3 & 8.47 & 6.04 \\
\hline 0.04 & 25 & 72.2 & 26.8 & 20.3 & 13.1 & 8.82 \\
\hline 0.02 & 50 & 91.1 & 36.7 & 28.2 & 17.3 & 11.2 \\
\hline 0.01 & 100 & 111 & 48.7 & 38.0 & 22.1 & 13.9 \\
\hline 0.005 & 200 & 131 & 63.2 & 50.1 & 27.8 & 16.9 \\
\hline 0.002 & 500 & 157 & $\mathrm{ng}$ & ng & $\mathrm{ng}$ & ng \\
\hline
\end{tabular}

\section{9-4.R00-8A CHICHAKTU RIVER AT CHICHAKTU, Continued}

Probability of occurrence of annual low discharges [ $\mathrm{m}^{3} / \mathrm{s}$, meters per second]

\begin{tabular}{|c|c|c|c|c|c|c|c|c|c|c|}
\hline \multirow{3}{*}{$\begin{array}{c}\text { Nonexceedance } \\
\text { probability }\end{array}$} & \multirow{3}{*}{$\begin{array}{c}\text { Recurrence } \\
\text { interval } \\
\text { (years) }\end{array}$} & \multicolumn{9}{|c|}{ Minimum daily mean discharge $\left(\mathrm{m}^{3} / \mathrm{s}\right)$} \\
\hline & & \multicolumn{9}{|c|}{ Number of consecutive days } \\
\hline & & 1 & 3 & 7 & 14 & 30 & 60 & 90 & 120 & 183 \\
\hline 0.05 & 20 & 0 & 0 & 0.04 & 0.08 & 0.10 & 0.11 & 0.13 & 0.14 & 0.18 \\
\hline 0.10 & 10 & 0 & 0 & 0.06 & 0.09 & 0.12 & 0.13 & 0.16 & 0.17 & 0.22 \\
\hline 0.20 & 5 & 0 & 0.02 & 0.07 & 0.11 & 0.14 & 0.17 & 0.20 & 0.22 & 0.27 \\
\hline 0.50 & 2 & 0.06 & 0.09 & 0.14 & 0.16 & 0.20 & 0.25 & 0.30 & 0.33 & 0.38 \\
\hline
\end{tabular}




\section{9-4.R00-8A CHICHAKTU RIVER AT CHICHAKTU, Continued}

Probability of occurrence of seasonal low discharges [ $\mathrm{m}^{3} / \mathrm{s}$, meters per second]

\begin{tabular}{|c|c|c|c|c|c|c|c|c|c|}
\hline \multirow{3}{*}{$\begin{array}{c}\text { Nonexceedance } \\
\text { probability }\end{array}$} & \multirow{3}{*}{$\begin{array}{c}\text { Recurrence } \\
\text { interval } \\
\text { (years) }\end{array}$} & \multicolumn{8}{|c|}{ Minimum daily mean discharge $\left(\mathrm{m}^{3} / \mathrm{s}\right)$} \\
\hline & & \multicolumn{8}{|c|}{ Number of consecutive days } \\
\hline & & 1 & 7 & 14 & 30 & 1 & 7 & 14 & 30 \\
\hline & & \multicolumn{4}{|c|}{ December-January-February } & \multicolumn{4}{|c|}{ March-April-May } \\
\hline 0.05 & 20 & 0.15 & 0.19 & 0.20 & 0.22 & 0.01 & 0.10 & 0.11 & 0.15 \\
\hline 0.10 & 10 & 0.19 & 0.23 & 0.24 & 0.26 & 0.02 & 0.13 & 0.15 & 0.20 \\
\hline 0.20 & 5 & 0.24 & 0.29 & 0.30 & 0.32 & 0.04 & 0.19 & 0.21 & 0.29 \\
\hline \multirow[t]{2}{*}{0.50} & 2 & 0.36 & 0.39 & 0.41 & 0.42 & 0.16 & 0.35 & 0.40 & 0.55 \\
\hline & & \multicolumn{4}{|c|}{ June-July-August } & \multicolumn{4}{|c|}{ September-October-November } \\
\hline 0.05 & 20 & 0 & 0.05 & 0.08 & 0.09 & 0.01 & 0.10 & 0.13 & 0.18 \\
\hline 0.10 & 10 & 0 & 0.06 & 0.09 & 0.12 & 0.02 & 0.12 & 0.14 & 0.19 \\
\hline 0.20 & 5 & 0 & 0.09 & 0.12 & 0.15 & 0.05 & 0.14 & 0.16 & 0.21 \\
\hline 0.50 & 2 & 0.07 & 0.18 & 0.20 & 0.24 & 0.14 & 0.21 & 0.23 & 0.28 \\
\hline
\end{tabular}

\section{9-4.R00-8A CHICHAKTU RIVER AT CHICHAKTU, Continued}

Annual peak discharges

[ $\mathrm{m}^{3} / \mathrm{s}$, meters per second]

\begin{tabular}{|c|c|c|c|c|c|}
\hline \multicolumn{3}{|c|}{$\begin{array}{c}\text { Annual peak discharge, } \\
\text { by year }\end{array}$} & \multicolumn{3}{|c|}{$\begin{array}{l}\text { Annual peak discharge, } \\
\text { from highest to lowest }\end{array}$} \\
\hline $\begin{array}{l}\text { Water } \\
\text { year }\end{array}$ & Date & $\begin{array}{c}\text { Peak } \\
\text { discharge } \\
\left(\mathrm{m}^{3} / \mathrm{s}\right)\end{array}$ & $\begin{array}{l}\text { Water } \\
\text { year }\end{array}$ & Date & $\begin{array}{c}\text { Peak } \\
\text { discharge } \\
\left(\mathrm{m}^{3} / \mathrm{s}\right)\end{array}$ \\
\hline 1971 & April 8, 1971 & 2.82 & 1977 & April 9, 1977 & 56.5 \\
\hline 1972 & March 27, 1972 & 20.0 & 1978 & December 24, 1977 & 26.4 \\
\hline 1973 & June 6, 1973 & 1.74 & 1976 & May 12, 1976 & 25.0 \\
\hline 1974 & March 31, 1974 & 8.86 & 1972 & March 27, 1972 & 20.0 \\
\hline 1975 & April 7, 1975 & 11.3 & 1975 & April 7, 1975 & 11.3 \\
\hline 1976 & May 12, 1976 & 25.0 & 1974 & March 31, 1974 & 8.86 \\
\hline 1977 & April 9, 1977 & 56.5 & 1971 & April 8, 1971 & 2.82 \\
\hline 1978 & December 24, 1977 & 26.4 & 1973 & June 6, 1973 & 1.74 \\
\hline
\end{tabular}




\section{9-4.R00-8A CHICHAKTU RIVER AT CHICHAKTU, Continued}

Monthly and annual mean discharges, in cubic meters per second $[--$, no data]

\begin{tabular}{|c|c|c|c|c|c|c|c|c|c|c|c|c|c|}
\hline \multirow{2}{*}{$\begin{array}{c}\text { Water } \\
\text { year }\end{array}$} & \multicolumn{12}{|c|}{ Monthly mean discharge } & \multirow{2}{*}{$\begin{array}{c}\text { Annual } \\
\text { discharge }\end{array}$} \\
\hline & October & November & December & January & February & March & April & May & June & July & August & September & \\
\hline 1970 & -- & -- & -- & -- & -- & -- & -- & 0.22 & 0.28 & 0.16 & 0.14 & 0.24 & -- \\
\hline 1971 & 0.33 & 0.34 & 0.37 & 0.46 & 0.61 & 0.61 & 0.55 & 0.18 & 0.15 & 0.25 & 0.18 & 0.21 & 0.35 \\
\hline 1972 & 0.42 & 0.38 & 0.35 & 0.53 & 0.58 & 5.19 & 5.10 & 1.90 & 1.42 & 0.40 & 0.41 & 0.42 & 1.43 \\
\hline 1973 & 0.52 & 0.59 & 0.52 & 0.76 & 0.70 & 0.80 & 1.35 & 1.13 & 1.02 & 0.54 & 0.45 & 0.49 & 0.74 \\
\hline 1974 & 0.50 & 0.54 & 0.48 & 0.53 & 0.53 & 0.89 & 1.55 & 0.99 & 0.48 & 0.23 & 0.32 & 0.47 & 0.63 \\
\hline 1975 & 0.57 & 0.61 & 0.72 & 0.75 & 1.24 & 1.45 & 3.08 & 0.82 & 0.68 & 0.56 & 0.57 & 0.25 & 0.94 \\
\hline 1976 & 0.36 & 0.33 & 0.42 & 0.42 & 0.57 & 0.80 & 2.89 & 3.58 & 0.73 & 0.60 & 0.35 & 0.22 & 0.94 \\
\hline 1977 & 0.53 & 0.56 & 0.60 & 0.43 & 0.62 & 0.51 & 0.45 & 0.26 & 0.15 & 0.12 & 0.16 & 0.27 & 0.39 \\
\hline 1978 & 0.29 & 0.26 & 0.90 & 0.26 & 0.85 & 2.86 & 0.74 & 0.34 & 0.35 & 0.31 & 0.31 & 0.28 & 0.65 \\
\hline
\end{tabular}




\section{9-5.L00-1A BUM RIVER AT LUKA-I-SURKH}

\section{(U.S. Geological Survey identification number: 351400063280000$)$}

LOCATION: Lat $35^{\circ} 14^{\prime} \mathrm{N}$., long $63^{\circ} 28^{\prime} \mathrm{E}$.

DRAINAGE AREA: $1,225 \mathrm{~km}^{2}$.

ELEVATION: 594 meters above mean sea level.

PERIOD OF RECORD: April 3, 1969 to September 30, 1978.

GAGE: Water-stage recorder.

Annual mean discharge

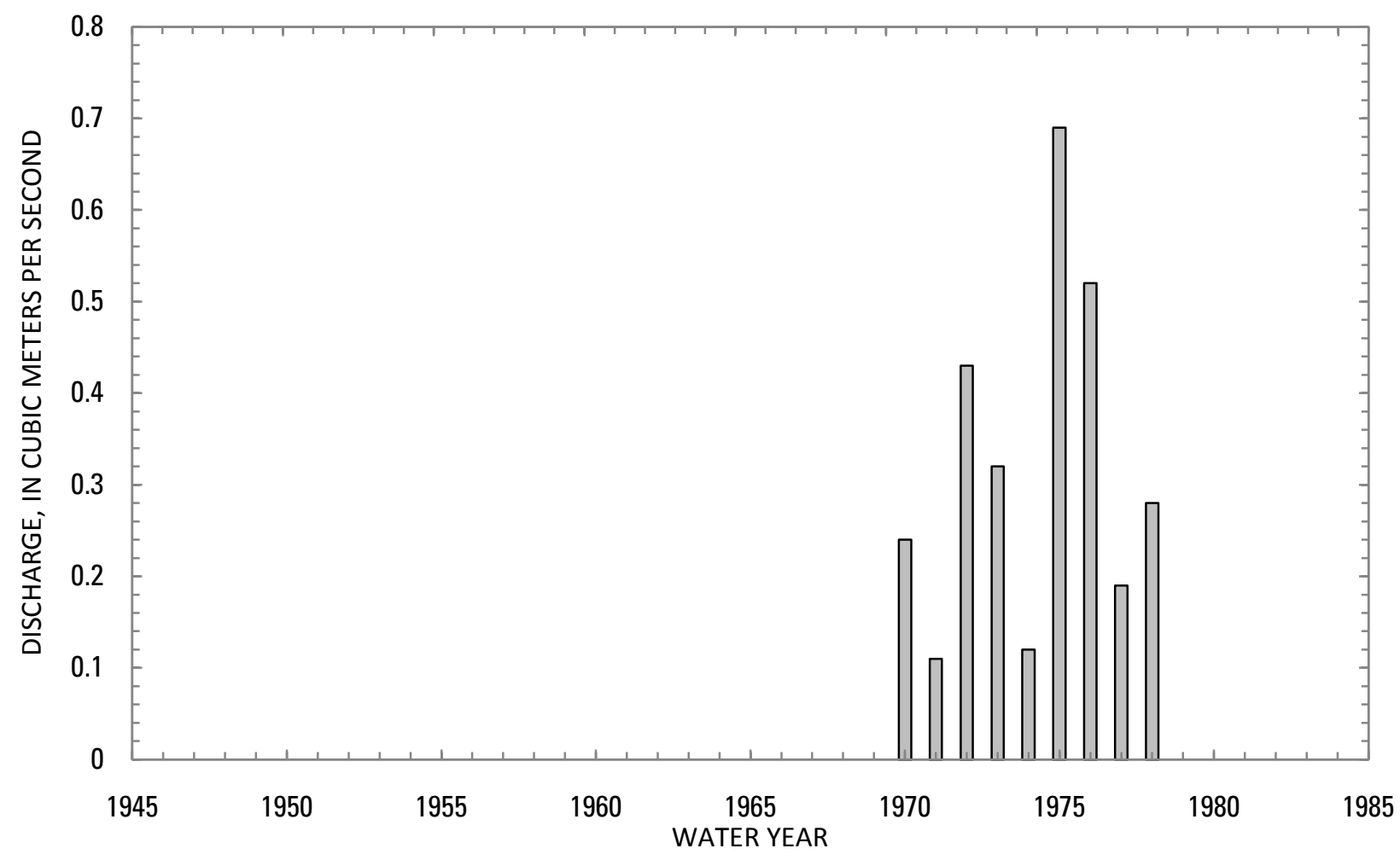




\section{9-5.L00-1A BUM RIVER AT LUKA-I-SURKH, Continued}

Statistics of monthly and annual mean discharges $\left[\mathrm{m}^{3} / \mathrm{s}\right.$, cubic meters per second]

\begin{tabular}{|c|c|c|c|c|c|c|c|c|}
\hline \multirow[b]{2}{*}{ Month } & \multicolumn{2}{|c|}{ Maximum } & \multicolumn{2}{|c|}{ Minimum } & \multicolumn{4}{|c|}{ Mean } \\
\hline & $\begin{array}{c}\text { Discharge } \\
\left(\mathrm{m}^{3} / \mathrm{s}\right)\end{array}$ & $\begin{array}{c}\text { Water year } \\
\text { of } \\
\text { occurrence }\end{array}$ & $\begin{array}{c}\text { Discharge } \\
\left(\mathrm{m}^{3} / \mathrm{s}\right)\end{array}$ & $\begin{array}{c}\text { Water year } \\
\text { of } \\
\text { occurrence }\end{array}$ & $\begin{array}{c}\text { Discharge } \\
\left(\mathrm{m}^{3} / \mathrm{s}\right)\end{array}$ & $\begin{array}{c}\text { Standard } \\
\text { deviation } \\
\left(\mathrm{m}^{3} / \mathrm{s}\right)\end{array}$ & $\begin{array}{c}\text { Coefficient } \\
\text { of } \\
\text { variation }\end{array}$ & $\begin{array}{c}\text { Percentage } \\
\text { of annual } \\
\text { discharge }\end{array}$ \\
\hline October & 0.38 & 1970 & 0.07 & 1973 & 0.16 & 0.09 & 0.59 & 3.89 \\
\hline November & 0.54 & 1970 & 0.08 & 1975 & 0.20 & 0.14 & 0.70 & 4.95 \\
\hline December & 0.53 & 1978 & 0.12 & 1974 & 0.22 & 0.13 & 0.59 & 5.49 \\
\hline January & 0.38 & 1973 & 0.11 & 1976 & 0.21 & 0.11 & 0.51 & 5.07 \\
\hline February & 0.38 & 1975 & 0.11 & 1971 & 0.28 & 0.10 & 0.37 & 6.87 \\
\hline March & 1.44 & 1972 & 0.18 & 1974 & 0.65 & 0.42 & 0.64 & 16.0 \\
\hline April & 3.48 & 1975 & 0.13 & 1970 & 1.16 & 1.09 & 0.94 & 28.3 \\
\hline May & 2.66 & 1969 & 0.02 & 1971 & 0.88 & 1.02 & 1.15 & 21.6 \\
\hline June & 0.47 & 1975 & 0.01 & 1971 & 0.15 & 0.16 & 1.07 & 3.74 \\
\hline July & 0.13 & 1976 & 0.01 & 1971 & 0.04 & 0.04 & 1.02 & 0.98 \\
\hline August & 0.09 & 1969 & 0.01 & 1972 & 0.03 & 0.03 & 0.95 & 0.72 \\
\hline September & 0.27 & 1969 & 0.01 & 1974 & 0.10 & 0.08 & 0.79 & 2.49 \\
\hline Annual & 0.69 & 1975 & 0.11 & 1971 & 0.32 & 0.19 & 0.60 & 100 \\
\hline
\end{tabular}


9-5.L00-1A BUM RIVER AT LUKA-I-SURKH, Continued

Monthly and annual flow duration, in cubic meters per second

\begin{tabular}{|c|c|c|c|c|c|c|c|c|c|c|c|c|c|}
\hline \multirow{2}{*}{$\begin{array}{l}\text { Percentage } \\
\text { of days } \\
\text { discharge } \\
\text { equaled or } \\
\text { exceeded }\end{array}$} & \multicolumn{12}{|c|}{ Month } & \multirow{2}{*}{ Annual } \\
\hline & October & November & December & January & February & March & April & May & June & July & August & September & \\
\hline 95 & 0.05 & 0.07 & 0.09 & 0.07 & 0.08 & 0.09 & 0.06 & 0.01 & 0.01 & 0 & 0.01 & 0.01 & 0.01 \\
\hline 90 & 0.06 & 0.09 & 0.10 & 0.09 & 0.10 & 0.15 & 0.10 & 0.02 & 0.01 & 0.01 & 0.01 & 0.01 & 0.02 \\
\hline 85 & 0.07 & 0.09 & 0.11 & 0.10 & 0.12 & 0.18 & 0.18 & 0.02 & 0.01 & 0.01 & 0.01 & 0.02 & 0.02 \\
\hline 80 & 0.08 & 0.10 & 0.13 & 0.12 & 0.15 & 0.23 & 0.24 & 0.02 & 0.02 & 0.01 & 0.01 & 0.02 & 0.02 \\
\hline 75 & 0.10 & 0.11 & 0.14 & 0.13 & 0.18 & 0.24 & 0.32 & 0.03 & 0.02 & 0.01 & 0.01 & 0.02 & 0.04 \\
\hline 70 & 0.10 & 0.13 & 0.15 & 0.14 & 0.19 & 0.27 & 0.38 & 0.05 & 0.02 & 0.01 & 0.01 & 0.03 & 0.07 \\
\hline 65 & 0.12 & 0.14 & 0.16 & 0.15 & 0.23 & 0.31 & 0.44 & 0.06 & 0.02 & 0.02 & 0.02 & 0.04 & 0.09 \\
\hline 60 & 0.13 & 0.15 & 0.16 & 0.15 & 0.24 & 0.34 & 0.57 & 0.09 & 0.02 & 0.02 & 0.02 & 0.06 & 0.11 \\
\hline 55 & 0.14 & 0.16 & 0.17 & 0.16 & 0.26 & 0.36 & 0.70 & 0.15 & 0.03 & 0.02 & 0.02 & 0.07 & 0.14 \\
\hline 50 & 0.15 & 0.17 & 0.19 & 0.17 & 0.27 & 0.39 & 0.81 & 0.25 & 0.05 & 0.02 & 0.02 & 0.10 & 0.15 \\
\hline 45 & 0.16 & 0.19 & 0.20 & 0.18 & 0.32 & 0.42 & 0.90 & 0.38 & 0.07 & 0.02 & 0.02 & 0.11 & 0.17 \\
\hline 40 & 0.16 & 0.20 & 0.22 & 0.21 & 0.33 & 0.48 & 1.00 & 0.58 & 0.13 & 0.02 & 0.02 & 0.13 & 0.19 \\
\hline 35 & 0.19 & 0.20 & 0.23 & 0.24 & 0.34 & 0.57 & 1.10 & 0.73 & 0.18 & 0.02 & 0.03 & 0.14 & 0.22 \\
\hline 30 & 0.20 & 0.21 & 0.24 & 0.25 & 0.35 & 0.63 & 1.31 & 1.13 & 0.21 & 0.04 & 0.03 & 0.15 & 0.26 \\
\hline 25 & 0.20 & 0.24 & 0.25 & 0.27 & 0.36 & 0.72 & 1.62 & 1.38 & 0.24 & 0.06 & 0.04 & 0.16 & 0.31 \\
\hline 20 & 0.21 & 0.26 & 0.26 & 0.30 & 0.38 & 0.94 & 2.12 & 1.61 & 0.28 & 0.08 & 0.05 & 0.17 & 0.36 \\
\hline 15 & 0.25 & 0.32 & 0.32 & 0.35 & 0.40 & 1.18 & 2.38 & 1.98 & 0.35 & 0.10 & 0.07 & 0.20 & 0.44 \\
\hline 10 & 0.32 & 0.40 & 0.36 & 0.38 & 0.43 & 1.49 & 2.76 & 2.70 & 0.42 & 0.14 & 0.08 & 0.24 & 0.70 \\
\hline 5 & 0.38 & 0.42 & 0.39 & 0.43 & 0.52 & 2.17 & 3.88 & 3.09 & 0.66 & 0.20 & 0.12 & 0.27 & 1.42 \\
\hline
\end{tabular}




\section{9-5.L00-1A BUM RIVER AT LUKA-I-SURKH, Continued}

Probability of occurrence of annual high discharges

[ $\mathrm{m}^{3} / \mathrm{s}$, cubic meters per second; $\mathrm{ng}$, not given]

\begin{tabular}{|c|c|c|c|c|c|c|}
\hline \multirow{2}{*}{$\begin{array}{c}\text { Exceedance } \\
\text { probability }\end{array}$} & \multirow{2}{*}{$\begin{array}{l}\text { Recurrence } \\
\text { interval } \\
\text { (years) }\end{array}$} & \multirow{2}{*}{$\begin{array}{l}\text { Maximum } \\
\text { instantaneous } \\
\text { discharge } \\
\left(\mathrm{m}^{3} / \mathrm{s}\right)\end{array}$} & \multicolumn{4}{|c|}{ Maximum daily mean discharge $\left(\mathrm{m}^{3} / \mathrm{s}\right)$} \\
\hline & & & $\begin{array}{l}\text { 3-day } \\
\text { period }\end{array}$ & $\begin{array}{l}\text { 7-day } \\
\text { period }\end{array}$ & $\begin{array}{l}\text { 15-day } \\
\text { period }\end{array}$ & $\begin{array}{l}\text { 30-day } \\
\text { period }\end{array}$ \\
\hline 0.99 & 1.01 & 0.80 & 0.31 & 0.27 & 0.26 & 0.18 \\
\hline 0.95 & 1.05 & 1.80 & 0.63 & 0.49 & 0.40 & 0.29 \\
\hline 0.90 & 1.11 & 2.80 & 0.90 & 0.67 & 0.52 & 0.37 \\
\hline 0.80 & 1.25 & 4.50 & 1.35 & 0.95 & 0.70 & 0.52 \\
\hline 0.50 & 2 & 11.2 & 2.72 & 1.81 & 1.30 & 1.00 \\
\hline 0.20 & 5 & 27.4 & 4.98 & 3.29 & 2.49 & 2.04 \\
\hline 0.10 & 10 & 43.2 & 6.61 & 4.41 & 3.54 & 3.01 \\
\hline 0.04 & 25 & 69.6 & 8.72 & 5.96 & 5.20 & 4.66 \\
\hline 0.02 & 50 & 94.4 & 10.3 & 7.18 & 6.71 & 6.22 \\
\hline 0.01 & 100 & 124 & 11.8 & ${ }^{1} 8.44$ & 8.47 & 8.13 \\
\hline 0.005 & 200 & 158 & 13.4 & ${ }^{1} 9.76$ & 10.5 & 10.4 \\
\hline 0.002 & 500 & 213 & $\mathrm{ng}$ & $\mathrm{ng}$ & $\mathrm{ng}$ & $\mathrm{ng}$ \\
\hline
\end{tabular}

'Data does not fit log-Pearson Type III curve, use with caution.

\section{9-5.L00-1A BUM RIVER AT LUKA-I-SURKH, Continued}

Probability of occurrence of annual low discharges $\left[\mathrm{m}^{3} / \mathrm{s}\right.$, meters per second]

\begin{tabular}{|c|c|c|c|c|c|c|c|c|c|c|}
\hline \multirow{3}{*}{$\begin{array}{c}\text { Nonexceedance } \\
\text { probability }\end{array}$} & \multirow{3}{*}{$\begin{array}{c}\text { Recurrence } \\
\text { interval } \\
\text { (years) }\end{array}$} & \multicolumn{9}{|c|}{ Minimum daily mean discharge $\left(\mathrm{m}^{3} / \mathrm{s}\right)$} \\
\hline & & \multicolumn{9}{|c|}{ Number of consecutive days } \\
\hline & & 1 & 3 & 7 & 14 & 30 & 60 & 90 & 120 & 183 \\
\hline 0.05 & 20 & 0 & 0 & 0 & 0 & 0 & 0.01 & 0.01 & 0.01 & 0.02 \\
\hline 0.10 & 10 & 0 & 0 & 0 & 0 & 0 & 0.01 & 0.01 & 0.01 & 0.03 \\
\hline 0.20 & 5 & 0.01 & 0.01 & 0.01 & 0.01 & 0.01 & 0.01 & 0.01 & 0.01 & 0.04 \\
\hline 0.50 & 2 & 0.01 & 0.01 & 0.01 & 0.01 & 0.01 & 0.02 & 0.02 & 0.03 & 0.07 \\
\hline
\end{tabular}




\section{9-5.L00-1A BUM RIVER AT LUKA-I-SURKH, Continued}

Probability of occurrence of seasonal low discharges [ $\mathrm{m}^{3} / \mathrm{s}$, meters per second]

\begin{tabular}{|c|c|c|c|c|c|c|c|c|c|}
\hline \multirow{3}{*}{$\begin{array}{c}\text { Nonexceedance } \\
\text { probability }\end{array}$} & \multirow{3}{*}{$\begin{array}{c}\text { Recurrence } \\
\text { interval } \\
\text { (years) }\end{array}$} & \multicolumn{8}{|c|}{ Minimum daily mean discharge $\left(\mathrm{m}^{3} / \mathrm{s}\right)$} \\
\hline & & \multicolumn{8}{|c|}{ Number of consecutive days } \\
\hline & & 1 & 7 & 14 & 30 & 1 & 7 & 14 & 30 \\
\hline & & \multicolumn{4}{|c|}{ December-January-February } & \multicolumn{4}{|c|}{ March-April-May } \\
\hline 0.05 & 20 & 0.01 & 0.05 & 0.06 & 0.08 & 0 & 0.01 & 0 & 0.01 \\
\hline 0.10 & 10 & 0.01 & 0.05 & 0.07 & 0.09 & 0.01 & 0.01 & 0.01 & 0.01 \\
\hline 0.20 & 5 & 0.02 & 0.06 & 0.08 & 0.10 & 0.01 & 0.02 & 0.02 & 0.03 \\
\hline \multirow[t]{2}{*}{0.50} & 2 & 0.04 & 0.09 & 0.11 & 0.13 & 0.03 & 0.05 & 0.07 & 0.15 \\
\hline & & \multicolumn{4}{|c|}{ June-July-August } & \multicolumn{4}{|c|}{ September-0ctober-November } \\
\hline 0.05 & 20 & 0 & 0 & 0 & 0 & 0.01 & 0.01 & 0.01 & 0.01 \\
\hline 0.10 & 10 & 0 & 0 & 0 & 0 & 0.01 & 0.01 & 0.01 & 0.02 \\
\hline 0.20 & 5 & 0.01 & 0.01 & 0.01 & 0.01 & 0.01 & 0.01 & 0.02 & 0.03 \\
\hline 0.50 & 2 & 0.01 & 0.01 & 0.01 & 0.01 & 0.02 & 0.04 & 0.05 & 0.07 \\
\hline
\end{tabular}

\section{9-5.L00-1A BUM RIVER AT LUKA-I-SURKH, Continued}

\section{Annual peak discharges}

[ $\mathrm{m}^{3} / \mathrm{s}$, meters per second]

\begin{tabular}{|c|c|c|c|c|c|}
\hline \multicolumn{3}{|c|}{$\begin{array}{c}\text { Annual peak discharge, } \\
\text { by year }\end{array}$} & \multicolumn{3}{|c|}{$\begin{array}{l}\text { Annual peak discharge, } \\
\text { from highest to lowest }\end{array}$} \\
\hline $\begin{array}{l}\text { Water } \\
\text { year }\end{array}$ & Date & $\begin{array}{c}\text { Peak } \\
\text { discharge } \\
\left(\mathrm{m}^{3} / \mathrm{s}\right)\end{array}$ & $\begin{array}{l}\text { Water } \\
\text { year }\end{array}$ & Date & $\begin{array}{c}\text { Peak } \\
\text { discharge } \\
\left(\mathrm{m}^{3} / \mathrm{s}\right)\end{array}$ \\
\hline 1969 & April 14, 1969 & 39.4 & 1972 & May 7, 1972 & 40.0 \\
\hline 1970 & March 25, 1970 & 9.00 & 1969 & April 14, 1969 & 39.4 \\
\hline 1971 & April 4, 1971 & 2.24 & 1975 & May 8,1975 & 39.0 \\
\hline 1972 & May 7, 1972 & 40.0 & 1977 & April 9, 1977 & 18.8 \\
\hline 1973 & March 26, 1973 & 4.91 & 1978 & December 27, 1977 & 9.45 \\
\hline 1974 & April 8, 1974 & 2.77 & 1970 & March 25, 1970 & 9.00 \\
\hline 1975 & May 8, 1975 & 39.0 & 1976 & April 27, 1976 & 8.98 \\
\hline 1976 & April 27, 1976 & 8.98 & 1973 & March 26, 1973 & 4.91 \\
\hline 1977 & April 9, 1977 & 18.8 & 1974 & April 8, 1974 & 2.77 \\
\hline 1978 & December 27, 1977 & 9.45 & 1971 & April 4, 1971 & 2.24 \\
\hline
\end{tabular}


9-5.L00-1A BUM RIVER AT LUKA-I-SURKH, Continued

Monthly and annual mean discharges, in cubic meters per second $[--$, no data]

\begin{tabular}{|c|c|c|c|c|c|c|c|c|c|c|c|c|c|}
\hline \multirow{2}{*}{$\begin{array}{l}\text { Water } \\
\text { year }\end{array}$} & \multicolumn{12}{|c|}{ Monthly mean discharge } & \multirow{2}{*}{$\begin{array}{c}\text { Annual } \\
\text { discharge }\end{array}$} \\
\hline & October & November & December & January & February & March & April & May & June & July & August & September & \\
\hline 1969 & -- & -- & -- & -- & -- & -- & -- & 2.66 & 0.32 & 0.07 & 0.09 & 0.27 & -- \\
\hline 1970 & 0.38 & 0.54 & 0.35 & 0.38 & 0.32 & 0.48 & 0.13 & 0.04 & 0.03 & 0.01 & 0.03 & 0.14 & 0.24 \\
\hline 1971 & 0.17 & 0.16 & 0.14 & 0.13 & 0.11 & 0.19 & 0.34 & 0.02 & 0.01 & 0.01 & 0.02 & 0.04 & 0.11 \\
\hline 1972 & 0.08 & 0.13 & 0.13 & 0.20 & 0.37 & 1.44 & 1.33 & 1.38 & 0.02 & 0.01 & 0.01 & 0.02 & 0.43 \\
\hline 1973 & 0.07 & 0.10 & 0.20 & 0.38 & 0.30 & 0.88 & 1.24 & 0.23 & 0.15 & 0.07 & 0.06 & 0.16 & 0.32 \\
\hline 1974 & 0.17 & 0.13 & 0.12 & 0.12 & 0.17 & 0.18 & 0.46 & 0.06 & 0.01 & 0.01 & 0.01 & 0.01 & 0.12 \\
\hline 1975 & 0.07 & 0.08 & 0.15 & 0.14 & 0.38 & 0.82 & 3.48 & 2.46 & 0.47 & 0.07 & 0.01 & 0.09 & 0.69 \\
\hline 1976 & 0.20 & 0.18 & 0.20 & 0.11 & 0.36 & 0.98 & 2.20 & 1.38 & 0.32 & 0.13 & 0.01 & 0.15 & 0.52 \\
\hline 1977 & 0.17 & 0.21 & 0.20 & 0.22 & 0.35 & 0.34 & 0.31 & 0.32 & 0.15 & 0.01 & 0.01 & 0.05 & 0.19 \\
\hline 1978 & 0.14 & 0.31 & 0.53 & 0.18 & 0.17 & 0.59 & 0.92 & 0.29 & 0.05 & 0.02 & 0.04 & 0.08 & 0.28 \\
\hline
\end{tabular}




\section{0-0.000-3M SHIRIN TAGAB RIVER AT PATA BABA}

\section{(U.S. Geological Survey identification number: 363500064520000 )}

LOCATION: Lat $36^{\circ} 35^{\prime} \mathrm{N}$., long 64'52'E.

DRAINAGE AREA: $11,775 \mathrm{~km}^{2}$.

ELEVATION: 371 meters above mean sea level.

PERIOD OF RECORD: May 23, 1964 to September 30, 1978.

GAGE: Water-stage recorder. Staff gage at same site and datum prior to 1970 water year.

Annual mean discharge

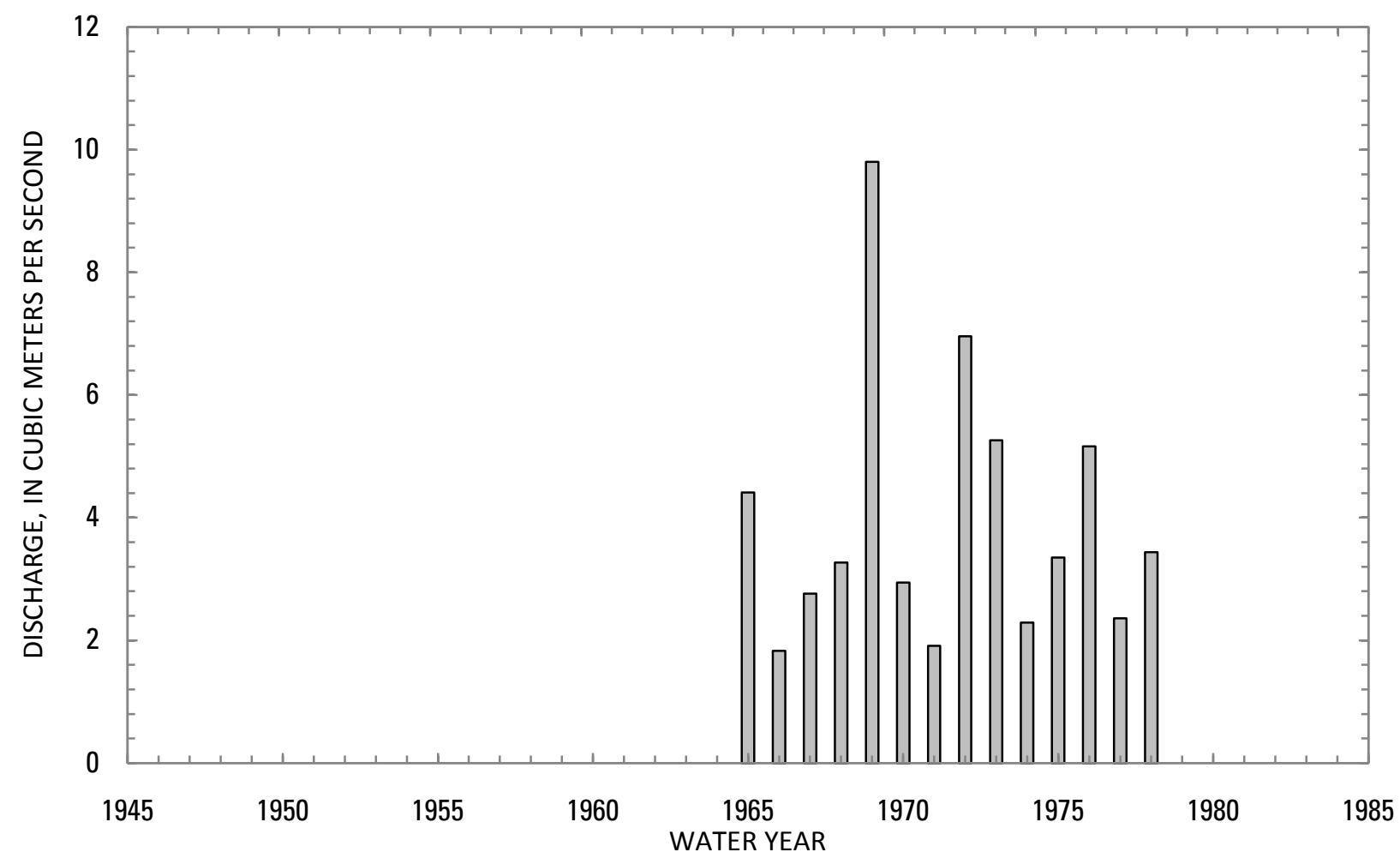


10-0.000-3M SHIRIN TAGAB RIVER AT PATA BABA, Continued

Statistics of monthly and annual mean discharges

$\left[\mathrm{m}^{3} / \mathrm{s}\right.$, cubic meters per second]

\begin{tabular}{|c|c|c|c|c|c|c|c|c|}
\hline \multirow[b]{2}{*}{ Month } & \multicolumn{2}{|c|}{ Maximum } & \multicolumn{2}{|c|}{ Minimum } & \multicolumn{4}{|c|}{ Mean } \\
\hline & $\begin{array}{c}\text { Discharge } \\
\left(\mathrm{m}^{3} / \mathrm{s}\right)\end{array}$ & $\begin{array}{l}\text { Water year } \\
\text { of } \\
\text { occurrence }\end{array}$ & $\begin{array}{c}\text { Discharge } \\
\left(\mathrm{m}^{3} / \mathrm{s}\right)\end{array}$ & $\begin{array}{c}\text { Water year } \\
\text { of } \\
\text { occurrence }\end{array}$ & $\begin{array}{c}\text { Discharge } \\
\left(\mathrm{m}^{3} / \mathrm{s}\right)\end{array}$ & $\begin{array}{c}\text { Standard } \\
\text { deviation } \\
\left(\mathrm{m}^{3} / \mathrm{s}\right)\end{array}$ & $\begin{array}{c}\text { Coefficient } \\
\text { of } \\
\text { variation }\end{array}$ & $\begin{array}{c}\text { Percentage } \\
\text { of annual } \\
\text { discharge }\end{array}$ \\
\hline October & 3.42 & 1970 & 0.82 & 1976 & 1.87 & 0.70 & 0.38 & 3.90 \\
\hline November & 5.91 & 1970 & 1.26 & 1975 & 2.76 & 1.21 & 0.44 & 5.76 \\
\hline December & 10.2 & 1969 & 1.16 & 1972 & 3.72 & 2.41 & 0.65 & 7.78 \\
\hline January & 8.09 & 1973 & 1.10 & 1972 & 3.45 & 1.88 & 0.54 & 7.22 \\
\hline February & 9.16 & 1973 & 2.59 & 1972 & 4.12 & 1.86 & 0.45 & 8.60 \\
\hline March & 22.4 & 1972 & 2.57 & 1977 & 7.74 & 6.51 & 0.84 & 16.2 \\
\hline April & 42.4 & 1969 & 1.98 & 1966 & 11.9 & 11.1 & 0.94 & 24.8 \\
\hline May & 21.4 & 1969 & 0.53 & 1971 & 7.59 & 7.50 & 0.99 & 15.9 \\
\hline June & 8.03 & 1972 & 0.59 & 1974 & 1.77 & 1.95 & 1.10 & 3.70 \\
\hline July & 2.48 & 1972 & 0.47 & 1977 & 1.04 & 0.63 & 0.61 & 2.16 \\
\hline August & 2.03 & 1969 & 0.37 & 1977 & 0.97 & 0.50 & 0.51 & 2.03 \\
\hline September & 2.10 & 1969 & 0.42 & 1975 & 0.96 & 0.48 & 0.50 & 2.02 \\
\hline Annual & 9.80 & 1969 & 1.83 & 1966 & 3.98 & 2.22 & 0.56 & 100 \\
\hline
\end{tabular}


10-0.000-3M SHIRIN TAGAB RIVER AT PATA BABA, Continued

Monthly and annual flow duration, in cubic meters per second

\begin{tabular}{|c|c|c|c|c|c|c|c|c|c|c|c|c|c|}
\hline \multirow{2}{*}{$\begin{array}{l}\text { Percentage } \\
\text { of days } \\
\text { discharge } \\
\text { equaled or } \\
\text { exceeded }\end{array}$} & \multicolumn{12}{|c|}{ Month } & \multirow{2}{*}{ Annual } \\
\hline & October & November & December & January & February & March & April & May & June & July & August & September & \\
\hline 95 & 0.71 & 1.10 & 1.20 & 1.11 & 2.04 & 2.14 & 1.05 & 0.46 & 0.40 & 0.31 & 0.30 & 0.35 & 0.42 \\
\hline 90 & 0.77 & 1.26 & 1.37 & 1.41 & 2.25 & 2.57 & 1.50 & 0.59 & 0.47 & 0.36 & 0.33 & 0.39 & 0.57 \\
\hline 85 & 0.87 & 1.38 & 1.68 & 1.60 & 2.48 & 2.76 & 1.71 & 0.62 & 0.57 & 0.41 & 0.37 & 0.45 & 0.71 \\
\hline 80 & 0.97 & 1.56 & 1.84 & 1.80 & 2.71 & 2.95 & 2.06 & 0.66 & 0.66 & 0.47 & 0.48 & 0.52 & 0.84 \\
\hline 75 & 1.09 & 1.83 & 2.04 & 1.98 & 2.92 & 3.11 & 2.40 & 0.69 & 0.71 & 0.50 & 0.53 & 0.56 & 1.02 \\
\hline 70 & 1.23 & 2.05 & 2.34 & 2.22 & 3.11 & 3.28 & 2.69 & 0.90 & 0.78 & 0.54 & 0.62 & 0.60 & 1.19 \\
\hline 65 & 1.34 & 2.33 & 2.58 & 2.41 & 3.24 & 3.45 & 3.06 & 1.18 & 0.85 & 0.61 & 0.67 & 0.69 & 1.41 \\
\hline 60 & 1.49 & 2.53 & 2.70 & 2.55 & 3.34 & 3.63 & 3.51 & 1.39 & 0.92 & 0.67 & 0.71 & 0.74 & 1.65 \\
\hline 55 & 1.68 & 2.60 & 2.83 & 2.70 & 3.42 & 3.80 & 4.32 & 1.69 & 1.00 & 0.72 & 0.74 & 0.77 & 1.90 \\
\hline 50 & 1.80 & 2.67 & 2.95 & 2.88 & 3.50 & 4.10 & 5.22 & 2.68 & 1.07 & 0.78 & 0.80 & 0.81 & 2.15 \\
\hline 45 & 1.95 & 2.75 & 3.08 & 3.13 & 3.58 & 4.41 & 6.19 & 3.59 & 1.16 & 0.87 & 0.90 & 0.87 & 2.41 \\
\hline 40 & 2.09 & 2.83 & 3.24 & 3.38 & 3.68 & 4.82 & 7.70 & 4.84 & 1.27 & 1.04 & 1.05 & 0.97 & 2.68 \\
\hline 35 & 2.18 & 2.96 & 3.45 & 3.56 & 3.78 & 5.63 & 9.66 & 6.54 & 1.39 & 1.13 & 1.12 & 1.05 & 2.98 \\
\hline 30 & 2.28 & 3.11 & 3.75 & 3.77 & 3.88 & 6.74 & 11.4 & 9.19 & 1.54 & 1.31 & 1.19 & 1.13 & 3.30 \\
\hline 25 & 2.39 & 3.28 & 4.04 & 4.06 & 4.23 & 8.27 & 12.6 & 11.2 & 1.74 & 1.53 & 1.34 & 1.24 & 3.68 \\
\hline 20 & 2.55 & 3.43 & 4.29 & 4.52 & 4.76 & 9.37 & 14.5 & 13.5 & 2.02 & 1.69 & 1.45 & 1.42 & 4.18 \\
\hline 15 & 2.71 & 3.63 & 4.73 & 5.22 & 5.45 & 11.2 & 19.5 & 16.2 & 2.41 & 1.84 & 1.65 & 1.62 & 5.31 \\
\hline 10 & 3.04 & 3.96 & 5.39 & 6.31 & 7.02 & 15.2 & 30.6 & 20.8 & 3.16 & 2.12 & 1.93 & 1.89 & 7.70 \\
\hline 5 & 3.80 & 5.68 & 6.56 & 8.16 & 8.64 & 24.6 & 53.3 & 27.7 & 4.98 & 2.39 & 2.13 & 2.09 & 13.4 \\
\hline
\end{tabular}




\section{0-0.000-3M SHIRIN TAGAB RIVER AT PATA BABA, Continued}

Probability of occurrence of annual high discharges

[m $\mathrm{m}^{3} / \mathrm{s}$, cubic meters per second; ng, not given]

\begin{tabular}{|c|c|c|c|c|c|c|}
\hline \multirow{2}{*}{$\begin{array}{l}\text { Exceedance } \\
\text { probability }\end{array}$} & \multirow{2}{*}{$\begin{array}{l}\text { Recurrence } \\
\text { interval } \\
\text { (years) }\end{array}$} & \multirow{2}{*}{$\begin{array}{l}\text { Maximum } \\
\text { instantaneous } \\
\text { discharge } \\
\left(\mathrm{m}^{3} / \mathrm{s}\right)\end{array}$} & \multicolumn{4}{|c|}{ Maximum daily mean discharge $\left(\mathrm{m}^{3} / \mathrm{s}\right)$} \\
\hline & & & $\begin{array}{l}\text { 3-day } \\
\text { period }\end{array}$ & $\begin{array}{l}\text { 7-day } \\
\text { period }\end{array}$ & $\begin{array}{l}\text { 15-day } \\
\text { period }\end{array}$ & $\begin{array}{l}\text { 30-day } \\
\text { period }\end{array}$ \\
\hline 0.99 & 1.01 & $\mathrm{ng}$ & 2.13 & 2.06 & ${ }^{1} 1.88$ & 2.02 \\
\hline 0.95 & 1.05 & $\mathrm{ng}$ & 6.63 & 5.12 & 3.99 & 3.49 \\
\hline 0.90 & 1.11 & 21.9 & 11.1 & 7.86 & 5.79 & 4.65 \\
\hline 0.80 & 1.25 & 40.8 & 18.9 & 12.5 & 8.83 & 6.56 \\
\hline 0.50 & 2 & 94.5 & 41.4 & 26.4 & 18.3 & 12.5 \\
\hline 0.20 & 5 & 149 & 68.6 & 46.4 & 34.1 & 23.4 \\
\hline 0.10 & 10 & 168 & 81.4 & 58.3 & 45.5 & 32.3 \\
\hline 0.04 & 25 & 180 & 92.4 & 71.2 & 60.2 & 45.2 \\
\hline 0.02 & 50 & 185 & 97.8 & 79.3 & 71.1 & 56.1 \\
\hline 0.01 & 100 & 187 & 102 & 86.2 & 81.7 & 68.0 \\
\hline 0.005 & 200 & 188 & 104 & ${ }^{1} 92.1$ & 92.2 & 80.9 \\
\hline 0.002 & 500 & 189 & ng & $\mathrm{ng}$ & $\mathrm{ng}$ & $\mathrm{ng}$ \\
\hline
\end{tabular}

'Data does not fit log-Pearson Type III curve, use with caution.

10-0.000-3M SHIRIN TAGAB RIVER AT PATA BABA, Continued

Probability of occurrence of annual low discharges

[m $\mathrm{m}^{3} / \mathrm{s}$, meters per second]

\begin{tabular}{|c|c|c|c|c|c|c|c|c|c|c|}
\hline \multirow{3}{*}{$\begin{array}{c}\text { Nonexceedance } \\
\text { probability }\end{array}$} & \multirow{3}{*}{$\begin{array}{c}\text { Recurrence } \\
\text { interval } \\
\text { (years) }\end{array}$} & \multicolumn{9}{|c|}{ Minimum daily mean discharge $\left(\mathrm{m}^{3} / \mathrm{s}\right)$} \\
\hline & & \multicolumn{9}{|c|}{ Number of consecutive days } \\
\hline & & 1 & 3 & 7 & 14 & 30 & 60 & 90 & 120 & 183 \\
\hline 0.05 & 20 & 0.16 & 0.18 & 0.20 & 0.26 & 0.32 & 0.37 & 0.40 & 0.44 & 0.59 \\
\hline 0.10 & 10 & 0.18 & 0.19 & 0.22 & 0.27 & 0.35 & 0.41 & 0.45 & 0.49 & 0.67 \\
\hline 0.20 & 5 & 0.20 & 0.22 & 0.25 & 0.31 & 0.40 & 0.47 & 0.52 & 0.58 & 0.80 \\
\hline 0.50 & 2 & 0.30 & 0.32 & 0.36 & 0.44 & 0.57 & 0.68 & 0.75 & 0.83 & 1.17 \\
\hline
\end{tabular}




\section{0-0.000-3M SHIRIN TAGAB RIVER AT PATA BABA, Continued}

Probability of occurrence of seasonal low discharges

$\left[\mathrm{m}^{3} / \mathrm{s}\right.$, meters per second]

\begin{tabular}{|c|c|c|c|c|c|c|c|c|c|}
\hline \multirow{3}{*}{$\begin{array}{c}\text { Nonexceedance } \\
\text { probability }\end{array}$} & \multirow{3}{*}{$\begin{array}{c}\text { Recurrence } \\
\text { interval } \\
\text { (years) }\end{array}$} & \multicolumn{8}{|c|}{ Minimum daily mean discharge $\left(\mathrm{m}^{3} / \mathrm{s}\right)$} \\
\hline & & \multicolumn{8}{|c|}{ Number of consecutive days } \\
\hline & & 1 & 7 & 14 & 30 & 1 & 7 & 14 & 30 \\
\hline & & \multicolumn{4}{|c|}{ December-January-February } & \multicolumn{4}{|c|}{ March-April-May } \\
\hline 0.05 & 20 & 0.86 & 0.94 & 1.02 & 1.13 & 0.27 & 0.35 & 0.36 & 0.42 \\
\hline 0.10 & 10 & 1.04 & 1.16 & 1.25 & 1.37 & 0.32 & 0.42 & 0.48 & 0.6 \\
\hline 0.20 & 5 & 1.28 & 1.46 & 1.57 & 1.71 & 0.41 & 0.57 & 0.68 & 0.92 \\
\hline \multirow[t]{2}{*}{0.50} & 2 & 1.88 & 2.16 & 2.31 & 2.6 & 0.79 & 1.17 & 1.49 & 2.26 \\
\hline & & \multicolumn{4}{|c|}{ June-July-August } & \multicolumn{4}{|c|}{ September-October-November } \\
\hline 0.05 & 20 & 0.15 & 0.2 & 0.26 & 0.36 & 0.19 & 0.27 & 0.34 & 0.41 \\
\hline 0.10 & 10 & 0.18 & 0.22 & 0.29 & 0.41 & 0.23 & 0.31 & 0.38 & 0.46 \\
\hline 0.20 & 5 & 0.22 & 0.27 & 0.34 & 0.49 & 0.29 & 0.37 & 0.45 & 0.55 \\
\hline 0.50 & 2 & 0.36 & 0.43 & 0.52 & 0.73 & 0.49 & 0.57 & 0.66 & 0.79 \\
\hline
\end{tabular}


10-0.000-3M SHIRIN TAGAB RIVER AT PATA BABA, Continued

\begin{tabular}{|c|c|c|c|c|c|}
\hline \multicolumn{6}{|c|}{$\begin{array}{c}\text { Annual peak discharges } \\
{\left[\mathrm{m}^{3} / \mathrm{s} \text {, meters per second] }\right.}\end{array}$} \\
\hline \multicolumn{3}{|c|}{$\begin{array}{c}\text { Annual peak discharge, } \\
\text { by year }\end{array}$} & \multicolumn{3}{|c|}{$\begin{array}{l}\text { Annual peak discharge, } \\
\text { from highest to lowest }\end{array}$} \\
\hline $\begin{array}{l}\text { Water } \\
\text { year }\end{array}$ & Date & $\begin{array}{c}\text { Peak } \\
\text { discharge } \\
\left(\mathrm{m}^{3} / \mathrm{s}\right)\end{array}$ & $\begin{array}{l}\text { Water } \\
\text { year }\end{array}$ & Date & $\begin{array}{c}\text { Peak } \\
\text { discharge } \\
\left(\mathrm{m}^{3} / \mathrm{s}\right)\end{array}$ \\
\hline 1965 & April 23, 1965 & 134 & 1977 & April 10, 1977 & 195 \\
\hline 1965 & December 5, 1965 & 5.45 & 1976 & April 9, 1976 & 160 \\
\hline 1967 & May 10, 1967 & 50.0 & 1969 & April 14, 1969 & 140 \\
\hline 1968 & April 26, 1968 & 87.0 & 1972 & March 19, 1972 & 135 \\
\hline 1969 & April 14, 1969 & 140 & 1965 & April 23, 1965 & 134 \\
\hline 1970 & March 27, 1970 & 80.0 & 1974 & April 7, 1974 & 100 \\
\hline 1971 & April 16, 1971 & 27.0 & 1968 & April 26, 1968 & 87.0 \\
\hline 1972 & March 19, 1972 & 135 & 1970 & March 27, 1970 & 80.0 \\
\hline 1973 & March 26, 1973 & 72.0 & 1973 & March 26, 1973 & 72.0 \\
\hline 1974 & April 7, 1974 & 100 & 1978 & December 25, 1977 & 61.1 \\
\hline 1975 & May 4, 1975 & 60.0 & 1975 & May 4, 1975 & 60.0 \\
\hline 1976 & April 9, 1976 & 160 & 1967 & May 10, 1967 & 50.0 \\
\hline 1977 & $\begin{array}{l}\text { April 10, } 1977 \\
\text { December 25, }\end{array}$ & 195 & 1971 & April 16, 1971 & 27.0 \\
\hline 1978 & 1977 & 61.1 & 1965 & December 5, 1965 & 5.45 \\
\hline
\end{tabular}




\section{0-0.000-3M SHIRIN TAGAB RIVER AT PATA BABA, Continued}

Monthly and annual mean discharges, in cubic meters per second $[--$, no data]

\begin{tabular}{|c|c|c|c|c|c|c|c|c|c|c|c|c|c|}
\hline \multirow{2}{*}{$\begin{array}{l}\text { Water } \\
\text { year }\end{array}$} & \multicolumn{12}{|c|}{ Monthly mean discharge } & \multirow{2}{*}{$\begin{array}{c}\text { Annual } \\
\text { discharge }\end{array}$} \\
\hline & October & November & December & January & February & March & April & May & June & July & August & September & \\
\hline 1964 & -- & -- & -- & -- & -- & -- & -- & -- & 1.21 & 1.26 & 1.13 & 1.09 & -- \\
\hline 1965 & 2.17 & 3.24 & 3.79 & 4.30 & 6.16 & 8.19 & 15.2 & 5.95 & 1.60 & 0.86 & 0.91 & 0.77 & 4.41 \\
\hline 1966 & 2.37 & 3.07 & 3.25 & 1.65 & 2.73 & 3.37 & 1.98 & 0.81 & 0.91 & 0.51 & 0.57 & 0.74 & 1.83 \\
\hline 1967 & 1.69 & 2.12 & 1.42 & 2.24 & 3.13 & 3.58 & 5.01 & 10.6 & 0.75 & 0.61 & 1.01 & 0.95 & 2.76 \\
\hline 1968 & 2.22 & 3.28 & 3.23 & 3.20 & 3.44 & 3.39 & 8.83 & 8.64 & 1.08 & 0.81 & 0.61 & 0.51 & 3.27 \\
\hline 1969 & 1.69 & 3.03 & 10.2 & 6.22 & 5.98 & 16.4 & 42.4 & 21.4 & 4.10 & 2.11 & 2.03 & 2.10 & 9.80 \\
\hline 1970 & 3.42 & 5.91 & 4.78 & 3.69 & 3.66 & 7.71 & 2.66 & 0.61 & 0.72 & 0.53 & 0.56 & 1.01 & 2.94 \\
\hline 1971 & 2.36 & 2.94 & 2.96 & 2.69 & 3.60 & 3.47 & 2.07 & 0.53 & 0.60 & 0.51 & 0.71 & 0.62 & 1.91 \\
\hline 1972 & 0.94 & 1.38 & 1.16 & 1.10 & 2.59 & 22.4 & 21.9 & 19.1 & 8.03 & 2.48 & 1.41 & 0.91 & 6.96 \\
\hline 1973 & 2.14 & 2.97 & 4.56 & 8.09 & 9.16 & 18.2 & 11.3 & 1.55 & 1.05 & 1.36 & 1.28 & 1.58 & 5.26 \\
\hline 1974 & 1.59 & 1.46 & 2.94 & 3.42 & 3.01 & 2.69 & 8.07 & 2.25 & 0.59 & 0.50 & 0.60 & 0.46 & 2.29 \\
\hline 1975 & 0.88 & 1.26 & 2.05 & 2.20 & 2.96 & 3.95 & 12.0 & 12.0 & 1.19 & 0.81 & 0.52 & 0.42 & 3.35 \\
\hline 1976 & 0.82 & 1.58 & 1.86 & 1.89 & 2.64 & 4.31 & 23.3 & 18.1 & 2.43 & 1.67 & 1.86 & 1.52 & 5.16 \\
\hline 1977 & 2.22 & 2.67 & 2.82 & 3.28 & 3.57 & 2.57 & 5.97 & 2.98 & 1.05 & 0.47 & 0.37 & 0.54 & 2.36 \\
\hline 1978 & 1.64 & 3.68 & 7.09 & 4.37 & 4.99 & 8.08 & 5.13 & 1.80 & 1.25 & 1.03 & 1.03 & 1.24 & 3.44 \\
\hline
\end{tabular}




\section{0-0.000-4M SHIRIN TAGAB RIVER AT DAULATABAD}

\section{(U.S. Geological Survey identification number: 362700064530000)}

LOCATION: Lat $36^{\circ} 27^{\prime} \mathrm{N}$., long 6453'E.

DRAINAGE AREA: $4,645 \mathrm{~km}^{2}$.

ELEVATION: 391 meters above mean sea level.

PERIOD OF RECORD: January 16, 1969 to May 31, 1970 and June 1, 1973 to September 30, 1978.

GAGE: Staff gage from January 16, 1969 to May 31, 1970. Water-stage recorder at same site and datum June 1, 1973 to September 30, 1978.

Annual mean discharge

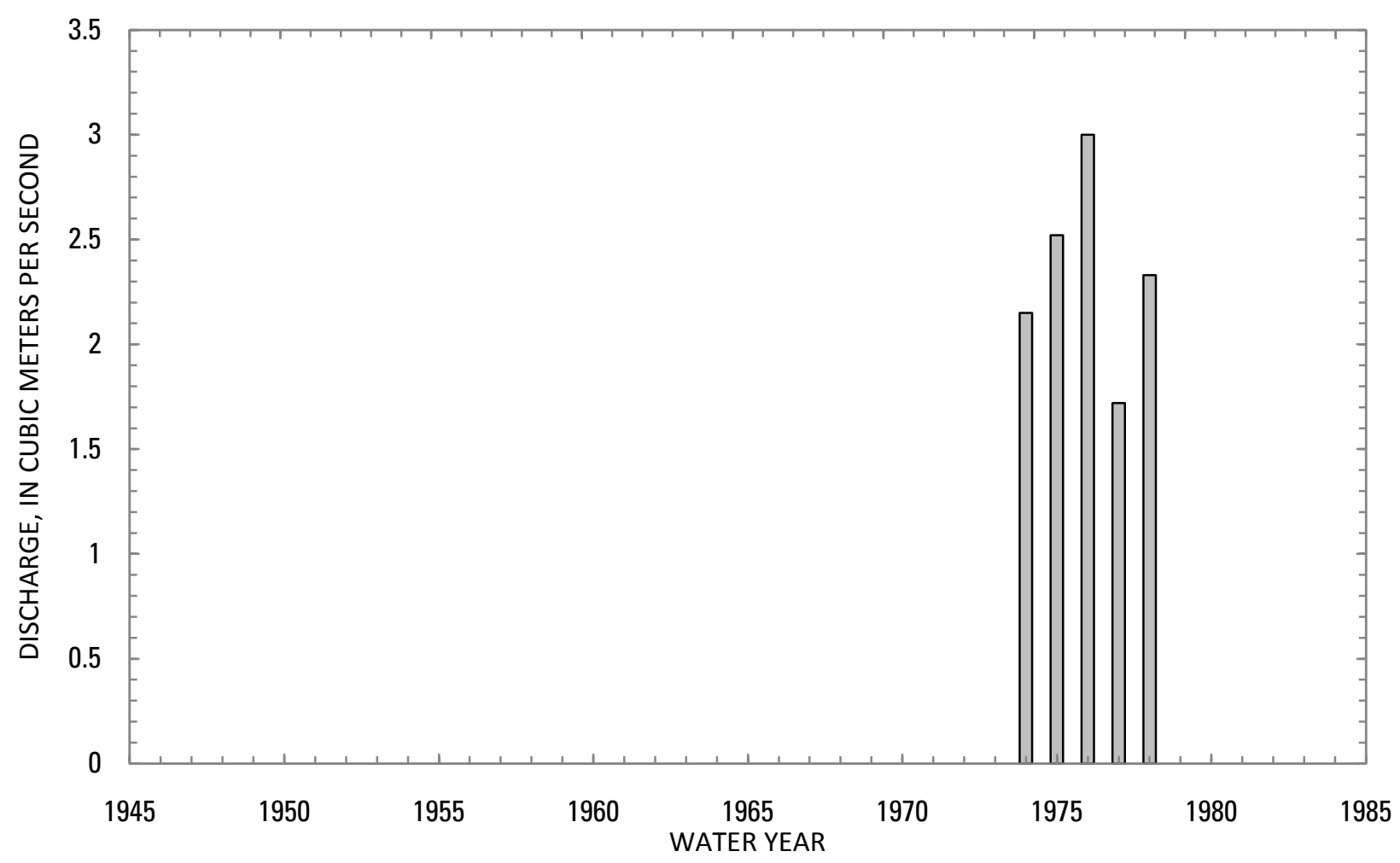


10-0.000-4M SHIRIN TAGAB RIVER AT DAULATABAD, Continued

Statistics of monthly and annual mean discharges

$\left[\mathrm{m}^{3} / \mathrm{s}\right.$, cubic meters per second]

\begin{tabular}{|c|c|c|c|c|c|c|c|c|}
\hline \multirow[b]{2}{*}{ Month } & \multicolumn{2}{|c|}{ Maximum } & \multicolumn{2}{|c|}{ Minimum } & \multicolumn{4}{|c|}{ Mean } \\
\hline & $\begin{array}{c}\text { Discharge } \\
\left(\mathrm{m}^{3} / \mathrm{s}\right)\end{array}$ & $\begin{array}{l}\text { Water year } \\
\text { of } \\
\text { occurrence }\end{array}$ & $\begin{array}{c}\text { Discharge } \\
\left(\mathrm{m}^{3} / \mathrm{s}\right)\end{array}$ & $\begin{array}{c}\text { Water year } \\
\text { of } \\
\text { occurrence }\end{array}$ & $\begin{array}{c}\text { Discharge } \\
\left(\mathrm{m}^{3} / \mathrm{s}\right)\end{array}$ & $\begin{array}{c}\text { Standard } \\
\text { deviation } \\
\left(\mathrm{m}^{3} / \mathrm{s}\right)\end{array}$ & $\begin{array}{c}\text { Coefficient } \\
\text { of } \\
\text { variation }\end{array}$ & $\begin{array}{c}\text { Percentage } \\
\text { of annual } \\
\text { discharge }\end{array}$ \\
\hline October & 4.97 & 1970 & 0.83 & 1978 & 1.77 & 1.59 & 0.89 & 5.51 \\
\hline November & 7.50 & 1970 & 1.31 & 1975 & 2.87 & 2.30 & 0.80 & 8.91 \\
\hline December & 4.87 & 1970 & 1.86 & 1975 & 3.19 & 1.28 & 0.40 & 9.93 \\
\hline January & 3.58 & 1974 & 1.83 & 1975 & 2.70 & 0.75 & 0.28 & 8.40 \\
\hline February & 4.55 & 1978 & 2.77 & 1969 & 3.54 & 0.63 & 0.18 & 11.0 \\
\hline March & 6.74 & 1978 & 2.15 & 1977 & 4.74 & 1.72 & 0.36 & 14.8 \\
\hline April & 13.5 & 1969 & 1.41 & 1970 & 5.92 & 4.73 & 0.80 & 18.4 \\
\hline May & 7.35 & 1975 & 0.54 & 1970 & 3.25 & 2.85 & 0.88 & 10.1 \\
\hline June & 1.69 & 1969 & 0.87 & 1974 & 1.18 & 0.29 & 0.25 & 3.65 \\
\hline July & 1.62 & 1969 & 0.63 & 1977 & 1.04 & 0.37 & 0.35 & 3.25 \\
\hline August & 1.89 & 1969 & 0.67 & 1978 & 1.05 & 0.46 & 0.44 & 3.26 \\
\hline September & 2.33 & 1969 & 0.54 & 1975 & 0.91 & 0.65 & 0.71 & 2.84 \\
\hline Annual & 3.00 & 1976 & 1.72 & 1977 & 2.34 & 0.47 & 0.20 & 100 \\
\hline
\end{tabular}


10-0.000-4M SHIRIN TAGAB RIVER AT DAULATABAD, Continued

Monthly and annual flow duration, in cubic meters per second

\begin{tabular}{|c|c|c|c|c|c|c|c|c|c|c|c|c|c|}
\hline \multirow{2}{*}{$\begin{array}{l}\text { Percentage } \\
\text { of days } \\
\text { discharge } \\
\text { equaled or } \\
\text { exceeded }\end{array}$} & \multicolumn{12}{|c|}{ Month } & \multirow{2}{*}{ Annual } \\
\hline & October & November & December & January & February & March & April & May & June & July & August & September & \\
\hline 95 & 0.51 & 1.00 & 1.56 & 1.42 & 2.27 & 1.12 & 0.57 & 0.31 & 0.33 & 0.25 & 0.22 & 0.25 & 0.32 \\
\hline 90 & 0.61 & 1.25 & 1.75 & 1.54 & 2.47 & 1.92 & 0.78 & 0.35 & 0.36 & 0.35 & 0.25 & 0.28 & 0.42 \\
\hline 85 & 0.73 & 1.43 & 2.03 & 1.64 & 2.55 & 2.54 & 0.99 & 0.39 & 0.46 & 0.38 & 0.31 & 0.33 & 0.54 \\
\hline 80 & 0.81 & 1.64 & 2.17 & 1.75 & 2.64 & 2.68 & 1.18 & 0.45 & 0.62 & 0.41 & 0.41 & 0.41 & 0.67 \\
\hline 75 & 0.87 & 1.73 & 2.28 & 1.93 & 2.72 & 3.03 & 1.30 & 0.51 & 0.70 & 0.45 & 0.44 & 0.47 & 0.84 \\
\hline 70 & 0.92 & 1.83 & 2.32 & 2.03 & 2.87 & 3.37 & 1.43 & 0.57 & 0.89 & 0.51 & 0.49 & 0.50 & 1.00 \\
\hline 65 & 0.97 & 1.93 & 2.35 & 2.20 & 2.99 & 3.55 & 1.58 & 0.65 & 1.03 & 0.60 & 0.58 & 0.52 & 1.12 \\
\hline 60 & 1.02 & 2.01 & 2.39 & 2.29 & 3.27 & 3.73 & 1.92 & 0.79 & 1.09 & 0.67 & 0.74 & 0.56 & 1.25 \\
\hline 55 & 1.07 & 2.08 & 2.52 & 2.43 & 3.40 & 3.91 & 2.40 & 1.01 & 1.15 & 0.81 & 0.81 & 0.61 & 1.40 \\
\hline 50 & 1.19 & 2.14 & 2.65 & 2.54 & 3.51 & 4.09 & 2.82 & 1.24 & 1.22 & 0.95 & 0.88 & 0.65 & 1.59 \\
\hline 45 & 1.31 & 2.20 & 2.78 & 2.65 & 3.63 & 4.30 & 3.61 & 1.70 & 1.30 & 1.08 & 0.98 & 0.72 & 1.83 \\
\hline 40 & 1.38 & 2.27 & 3.11 & 2.78 & 3.70 & 4.53 & 4.03 & 2.15 & 1.34 & 1.15 & 1.05 & 0.79 & 2.07 \\
\hline 35 & 1.44 & 2.34 & 3.25 & 2.91 & 3.80 & 4.83 & 4.61 & 2.85 & 1.38 & 1.20 & 1.13 & 0.84 & 2.29 \\
\hline 30 & 1.50 & 2.44 & 3.40 & 3.07 & 3.91 & 5.20 & 5.54 & 3.62 & 1.45 & 1.25 & 1.33 & 0.93 & 2.52 \\
\hline 25 & 1.79 & 2.58 & 3.56 & 3.26 & 4.01 & 5.52 & 6.88 & 4.84 & 1.55 & 1.53 & 1.54 & 1.03 & 2.76 \\
\hline 20 & 1.95 & 2.80 & 4.00 & 3.46 & 4.14 & 5.93 & 7.96 & 5.72 & 1.65 & 1.75 & 1.68 & 1.19 & 3.31 \\
\hline 15 & 3.13 & 6.35 & 4.37 & 3.75 & 4.31 & 6.76 & 8.78 & 6.49 & 1.71 & 1.94 & 1.88 & 1.50 & 3.84 \\
\hline 10 & 4.47 & 7.36 & 5.07 & 4.01 & 4.49 & 7.80 & 12.4 & 7.7 & 1.81 & 2.03 & 2.03 & 2.44 & 4.56 \\
\hline 5 & 5.97 & 8.22 & 5.60 & 4.66 & 5.61 & 9.67 & 18.5 & 9.34 & 1.99 & 2.19 & 2.78 & 2.77 & 6.41 \\
\hline
\end{tabular}


10-0.000-4M SHIRIN TAGAB RIVER AT DAULATABAD, Continued

Probability of occurrence of annual high discharges

[ $\mathrm{m}^{3} / \mathrm{s}$, cubic meters per second; ng, not given]

\begin{tabular}{|c|c|c|c|c|c|c|}
\hline \multirow{2}{*}{$\begin{array}{l}\text { Exceedance } \\
\text { probability }\end{array}$} & \multirow{2}{*}{$\begin{array}{c}\text { Recurrence } \\
\text { interval } \\
\text { (years) }\end{array}$} & \multirow{2}{*}{$\begin{array}{c}\text { Maximum } \\
\text { instantaneous } \\
\text { discharge } \\
\left(\mathrm{m}^{3} / \mathrm{s}\right)\end{array}$} & \multicolumn{4}{|c|}{ Maximum daily mean discharge $\left(\mathrm{m}^{3} / \mathrm{s}\right)$} \\
\hline & & & $\begin{array}{l}\text { 3-day } \\
\text { period }\end{array}$ & $\begin{array}{l}\text { 7-day } \\
\text { period }\end{array}$ & $\begin{array}{l}\text { 15-day } \\
\text { period }\end{array}$ & $\begin{array}{l}\text { 30-day } \\
\text { period }\end{array}$ \\
\hline 0.99 & 1.01 & 20.9 & 10.5 & 6.23 & 3.78 & 1.73 \\
\hline 0.95 & 1.05 & 28.5 & 12.5 & 7.46 & 4.87 & 2.69 \\
\hline 0.90 & 1.11 & 33.8 & 13.8 & 8.31 & 5.60 & 3.36 \\
\hline 0.80 & 1.25 & 41.9 & 15.9 & 9.60 & 6.66 & 4.37 \\
\hline 0.50 & 2 & 64.9 & 21.4 & 13.2 & 9.42 & 6.99 \\
\hline 0.20 & 5 & 104 & 30.3 & 19.2 & 13.6 & 10.8 \\
\hline 0.10 & 10 & 135 & 37.2 & 23.9 & 16.6 & 13.3 \\
\hline 0.04 & 25 & 179 & 46.9 & 30.7 & 20.6 & 16.4 \\
\hline 0.02 & 50 & 217 & 55.0 & 36.6 & 23.8 & 18.8 \\
\hline 0.01 & 100 & 258 & 63.9 & 43.1 & 27.1 & 21.1 \\
\hline 0.005 & 200 & 304 & 73.6 & 50.3 & 30.6 & 23.3 \\
\hline 0.002 & 500 & 372 & ng & ng & ng & ng \\
\hline
\end{tabular}

'Less than 10 years of data used.

\section{0-0.000-4M SHIRIN TAGAB RIVER AT DAULATABAD, Continued}

Probability of occurrence of annual low discharges

$\left[\mathrm{m}^{3} / \mathrm{s}\right.$, meters per second]

\begin{tabular}{|c|c|c|c|c|c|c|c|c|c|c|}
\hline \multirow{3}{*}{$\begin{array}{c}\text { Nonexceedance } \\
\text { probability }\end{array}$} & \multirow{3}{*}{$\begin{array}{c}\text { Recurrence } \\
\text { interval } \\
\text { (years) }\end{array}$} & \multicolumn{9}{|c|}{ Minimum daily mean discharge $\left(\mathrm{m}^{3} / \mathrm{s}\right)$} \\
\hline & & \multicolumn{9}{|c|}{ Number of consecutive days } \\
\hline & & 1 & 3 & 7 & 14 & 30 & 60 & 90 & 120 & 183 \\
\hline 0.05 & 20 & 0.14 & 0.16 & 0.20 & 0.28 & 0.30 & 0.48 & 0.53 & 0.55 & 0.68 \\
\hline 0.10 & 10 & 0.17 & 0.19 & 0.22 & 0.30 & 0.33 & 0.51 & 0.57 & 0.59 & 0.72 \\
\hline 0.20 & 5 & 0.21 & 0.22 & 0.25 & 0.34 & 0.37 & 0.57 & 0.63 & 0.66 & 0.79 \\
\hline 0.50 & 2 & 0.29 & 0.30 & 0.31 & 0.46 & 0.53 & 0.75 & 0.83 & 0.88 & 1.09 \\
\hline
\end{tabular}


10-0.000-4M SHIRIN TAGAB RIVER AT DAULATABAD, Continued

Probability of occurrence of seasonal low discharges

$\left[\mathrm{m}^{3} / \mathrm{s}\right.$, meters per second]

\begin{tabular}{|c|c|c|c|c|c|c|c|c|c|}
\hline \multirow{3}{*}{$\begin{array}{c}\text { Nonexceedance } \\
\text { probability }\end{array}$} & \multirow{3}{*}{$\begin{array}{c}\text { Recurrence } \\
\text { interval } \\
\text { (years) }\end{array}$} & \multicolumn{8}{|c|}{ Minimum daily mean discharge $\left(\mathrm{m}^{3} / \mathrm{s}\right)$} \\
\hline & & \multicolumn{8}{|c|}{ Number of consecutive days } \\
\hline & & 1 & 7 & 14 & 30 & 1 & 7 & 14 & 30 \\
\hline & & \multicolumn{4}{|c|}{ December-January-February } & \multicolumn{4}{|c|}{ March-April-May } \\
\hline 0.05 & 20 & 0.91 & 1.10 & 1.19 & 1.36 & 0.20 & 0.20 & 0.21 & 0.24 \\
\hline 0.10 & 10 & 1.03 & 1.24 & 1.35 & 1.56 & 0.24 & 0.25 & 0.28 & 0.36 \\
\hline 0.20 & 5 & 1.19 & 1.44 & 1.55 & 1.83 & 0.29 & 0.34 & 0.41 & 0.60 \\
\hline \multirow[t]{2}{*}{0.50} & 2 & 1.57 & 1.88 & 2.04 & 2.43 & 0.51 & 0.68 & 0.95 & 1.54 \\
\hline & & \multicolumn{4}{|c|}{ June-July-August } & \multicolumn{4}{|c|}{ September-October-November } \\
\hline 0.05 & 20 & 0.14 & 0.21 & 0.31 & 0.52 & 0.18 & 0.25 & 0.32 & 0.42 \\
\hline 0.10 & 10 & 0.16 & 0.23 & 0.34 & 0.58 & 0.21 & 0.29 & 0.34 & 0.44 \\
\hline 0.20 & 5 & 0.20 & 0.26 & 0.39 & 0.67 & 0.26 & 0.34 & 0.39 & 0.50 \\
\hline 0.50 & 2 & 0.27 & 0.32 & 0.52 & 0.87 & 0.37 & 0.50 & 0.57 & 0.70 \\
\hline
\end{tabular}

10-0.000-4M SHIRIN TAGAB RIVER AT DAULATABAD, Continued

Annual peak discharges

$\left[\mathrm{m}^{3} / \mathrm{s}\right.$, meters per second]

\begin{tabular}{|c|c|c|c|c|c|}
\hline \multicolumn{3}{|c|}{$\begin{array}{c}\text { Annual peak discharge, } \\
\text { by year }\end{array}$} & \multicolumn{3}{|c|}{$\begin{array}{l}\text { Annual peak discharge, } \\
\text { from highest to lowest }\end{array}$} \\
\hline $\begin{array}{l}\text { Water } \\
\text { year }\end{array}$ & Date & $\begin{array}{c}\text { Peak } \\
\text { discharge } \\
\left(\mathrm{m}^{3} / \mathrm{s}\right)\end{array}$ & $\begin{array}{l}\text { Water } \\
\text { year }\end{array}$ & Date & $\begin{array}{c}\text { Peak } \\
\text { discharge } \\
\left(\mathrm{m}^{3} / \mathrm{s}\right)\end{array}$ \\
\hline 1969 & April 14, 1969 & 80.0 & 1977 & April 10, 1977 & 141 \\
\hline 1970 & March 26, 1970 & 41.0 & 1976 & April 9, 1976 & 120 \\
\hline 1974 & April 7, 1974 & 63.0 & 1969 & April 14, 1969 & 80.0 \\
\hline 1975 & May 4,1975 & 49.0 & 1974 & April 7, 1974 & 63.0 \\
\hline 1976 & April 9, 1976 & 120 & 1975 & May 4,1975 & 49.0 \\
\hline 1977 & April 10, 1977 & 141 & 1970 & $\begin{array}{c}\text { March 26, } 1970 \\
\text { December 25, }\end{array}$ & 41.0 \\
\hline 1978 & December 25, 1977 & 33.4 & 1978 & 1977 & 33.4 \\
\hline
\end{tabular}


10-0.000-4M SHIRIN TAGAB RIVER AT DAULATABAD, Continued

Monthly and annual mean discharges, in cubic meters per second $[--$, no data]

\begin{tabular}{|c|c|c|c|c|c|c|c|c|c|c|c|c|c|}
\hline \multirow{2}{*}{$\begin{array}{l}\text { Water } \\
\text { year }\end{array}$} & \multicolumn{12}{|c|}{ Monthly mean discharge } & \multirow{2}{*}{$\begin{array}{c}\text { Annual } \\
\text { discharge }\end{array}$} \\
\hline & October & November & December & January & February & March & April & May & June & July & August & September & \\
\hline 1969 & -- & -- & -- & -- & 2.77 & 6.50 & 13.5 & 6.68 & 1.69 & 1.62 & 1.89 & 2.33 & -- \\
\hline 1970 & 4.97 & 7.50 & 4.87 & 3.18 & 3.60 & 5.57 & 1.41 & 0.54 & -- & -- & -- & -- & -- \\
\hline 1973 & -- & -- & -- & -- & -- & -- & -- & -- & 1.11 & 1.32 & 1.27 & 1.05 & -- \\
\hline 1974 & 1.36 & 1.94 & 3.16 & 3.58 & 4.04 & 2.98 & 4.24 & 1.57 & 0.87 & 0.90 & 0.80 & 0.55 & 2.15 \\
\hline 1975 & 0.87 & 1.31 & 1.86 & 1.83 & 3.63 & 4.38 & 5.78 & 7.35 & 1.25 & 0.88 & 0.69 & 0.54 & 2.52 \\
\hline 1976 & 1.14 & 1.90 & 2.37 & 2.20 & 3.23 & 4.87 & 11.4 & 4.28 & 1.38 & 1.27 & 1.31 & 0.76 & 3.00 \\
\hline 1977 & 1.48 & 2.12 & 2.26 & 2.08 & 2.93 & 2.15 & 3.25 & 1.51 & 1.04 & 0.63 & 0.70 & 0.61 & 1.72 \\
\hline 1978 & 0.83 & 2.43 & 4.64 & 3.34 & 4.55 & 6.74 & 1.87 & 0.83 & 0.88 & 0.68 & 0.67 & 0.56 & 2.33 \\
\hline
\end{tabular}




\section{0-0.000-6M SHIRIN TAGAB RIVER AT KHISHT PUL}

\section{(U.S. Geological Survey identification number: 355700064540000)}

LOCATION: Lat 355'N., long 6454'E.

DRAINAGE AREA: $3,280 \mathrm{~km}^{2}$.

ELEVATION: 769 meters above mean sea level.

PERIOD OF RECORD: January 1, 1966 to September 30, 1978.

GAGE: Water-stage recorder. Staff gage at same site and datum prior to 1969 water year.

Annual mean discharge

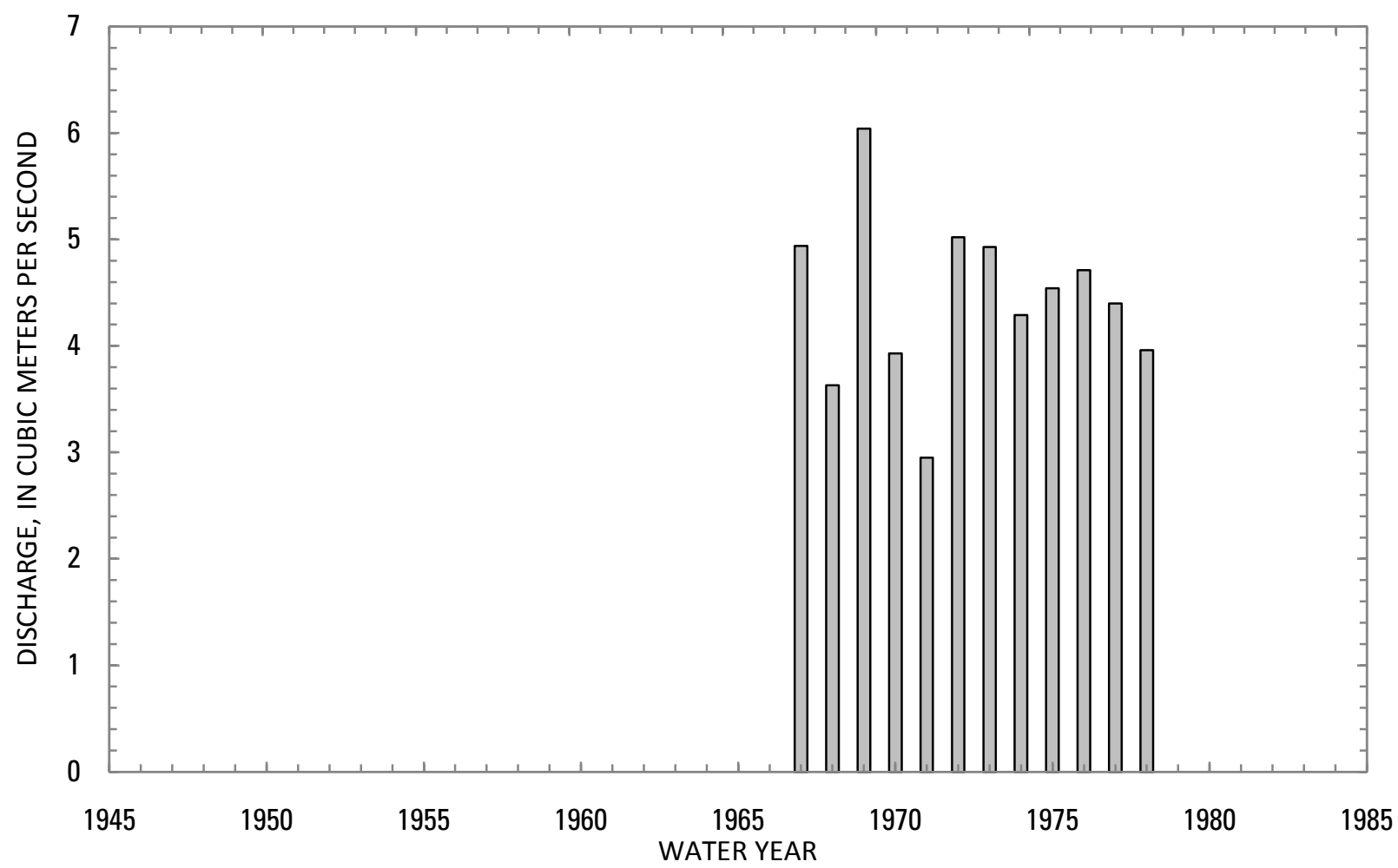




\section{0-0.000-6M SHIRIN TAGAB RIVER AT KHISHT PUL, Continued}

Statistics of monthly and annual mean discharges $\left[\mathrm{m}^{3} / \mathrm{s}\right.$, cubic meters per second]

\begin{tabular}{|c|c|c|c|c|c|c|c|c|}
\hline \multirow[b]{2}{*}{ Month } & \multicolumn{2}{|c|}{ Maximum } & \multicolumn{2}{|c|}{ Minimum } & \multicolumn{4}{|c|}{ Mean } \\
\hline & $\begin{array}{c}\text { Discharge } \\
\left(\mathrm{m}^{3} / \mathrm{s}\right)\end{array}$ & $\begin{array}{c}\text { Water year } \\
\text { of } \\
\text { occurrence }\end{array}$ & $\begin{array}{c}\text { Discharge } \\
\left(\mathrm{m}^{3} / \mathrm{s}\right)\end{array}$ & $\begin{array}{c}\text { Water year } \\
\text { of } \\
\text { occurrence }\end{array}$ & $\begin{array}{c}\text { Discharge } \\
\left(\mathrm{m}^{3} / \mathrm{s}\right)\end{array}$ & $\begin{array}{c}\text { Standard } \\
\text { deviation } \\
\left(\mathrm{m}^{3} / \mathrm{s}\right)\end{array}$ & $\begin{array}{c}\text { Coefficient } \\
\text { of } \\
\text { variation }\end{array}$ & $\begin{array}{c}\text { Percentage } \\
\text { of annual } \\
\text { discharge }\end{array}$ \\
\hline October & 6.18 & 1970 & 2.72 & 1972 & 3.96 & 0.99 & 0.25 & 7.55 \\
\hline November & 6.46 & 1970 & 3.06 & 1972 & 4.52 & 0.97 & 0.21 & 8.63 \\
\hline December & 6.16 & 1969 & 2.23 & 1972 & 4.89 & 1.14 & 0.23 & 9.34 \\
\hline January & 6.13 & 1973 & 3.43 & 1966 & 4.78 & 0.88 & 0.18 & 9.13 \\
\hline February & 7.11 & 1973 & 3.89 & 1968 & 5.23 & 0.94 & 0.18 & 9.99 \\
\hline March & 12.5 & 1972 & 4.98 & 1976 & 6.85 & 2.59 & 0.38 & 13.1 \\
\hline April & 16.8 & 1969 & 4.55 & 1971 & 8.49 & 3.57 & 0.42 & 16.2 \\
\hline May & 11.1 & 1972 & 1.48 & 1970 & 5.30 & 3.11 & 0.59 & 10.1 \\
\hline June & 5.94 & 1972 & 0.92 & 1966 & 2.24 & 1.30 & 0.58 & 4.27 \\
\hline July & 3.00 & 1969 & 0.60 & 1971 & 1.88 & 0.68 & 0.36 & 3.60 \\
\hline August & 3.50 & 1969 & 0.29 & 1971 & 1.72 & 0.83 & 0.48 & 3.29 \\
\hline September & 4.22 & 1969 & 1.08 & 1971 & 2.53 & 0.83 & 0.33 & 4.83 \\
\hline Annual & 6.04 & 1969 & 2.95 & 1971 & 4.45 & 0.79 & 0.18 & 100 \\
\hline
\end{tabular}


10-0.000-6M SHIRIN TAGAB RIVER AT KHISHT PUL, Continued

Monthly and annual flow duration, in cubic meters per second

[ng, not given]

\begin{tabular}{|c|c|c|c|c|c|c|c|c|c|c|c|c|c|}
\hline \multirow{2}{*}{$\begin{array}{l}\text { Percentage } \\
\text { of days } \\
\text { discharge } \\
\text { equaled or } \\
\text { exceeded }\end{array}$} & \multicolumn{12}{|c|}{ Month } & \multirow{2}{*}{ Annua } \\
\hline & October & November & December & January & February & March & April & May & June & July & August & September & \\
\hline 95 & 2.59 & 3.14 & 2.28 & 3.50 & 3.93 & 4.23 & 3.71 & 0.77 & 0.69 & 0.59 & 0.30 & 1.16 & 1.12 \\
\hline 90 & 2.72 & 3.28 & 3.63 & 3.68 & 4.00 & 4.43 & 4.07 & 1.18 & 1.03 & 0.86 & 0.76 & 1.33 & 1.53 \\
\hline 85 & 2.83 & 3.54 & 3.85 & 3.74 & 4.11 & 4.57 & 4.42 & 1.72 & 1.12 & 1.09 & 0.87 & 1.52 & 1.87 \\
\hline 80 & 2.94 & 3.73 & 4.16 & 3.95 & 4.26 & 4.71 & 4.75 & 1.95 & 1.20 & 1.23 & 1.01 & 1.69 & 2.14 \\
\hline 75 & 3.28 & 3.87 & 4.29 & 4.03 & 4.37 & 4.84 & 4.97 & 2.25 & 1.29 & 1.40 & 1.08 & 1.82 & 2.41 \\
\hline 70 & 3.42 & 4.10 & 4.42 & 4.11 & 4.50 & 4.96 & 5.20 & 2.59 & 1.39 & 1.55 & 1.17 & 1.93 & 2.71 \\
\hline 65 & 3.47 & 4.16 & 4.55 & 4.27 & 4.56 & 5.09 & 5.39 & 2.82 & 1.50 & 1.64 & 1.29 & 2.09 & 3.03 \\
\hline 60 & 3.51 & 4.23 & 4.68 & 4.37 & 4.61 & 5.21 & 5.58 & 3.07 & 1.66 & 1.74 & 1.45 & 2.21 & 3.47 \\
\hline 55 & 3.68 & 4.33 & 4.79 & 4.47 & 4.91 & 5.53 & 5.78 & 3.33 & 1.90 & 1.96 & 1.58 & 2.39 & 3.81 \\
\hline 50 & 3.74 & 4.46 & 4.88 & 4.57 & 5.02 & 5.73 & 6.01 & 3.60 & 2.04 & 2.04 & 1.70 & 2.57 & 4.08 \\
\hline 45 & 3.79 & 4.55 & 4.96 & 4.84 & 5.11 & 5.83 & 6.26 & 4.02 & 2.17 & 2.11 & 1.81 & 2.71 & 4.36 \\
\hline 40 & 4.09 & 4.67 & 5.05 & 4.94 & 5.32 & 5.92 & 6.52 & 4.55 & 2.28 & 2.17 & 1.94 & 2.83 & 4.60 \\
\hline 35 & 4.26 & 4.81 & 5.22 & 5.09 & 5.58 & 6.02 & 6.88 & 4.91 & 2.39 & 2.22 & 2.08 & 2.99 & 4.82 \\
\hline 30 & 4.36 & 4.87 & 5.41 & 5.25 & 5.76 & 6.12 & 7.27 & 5.39 & 2.53 & 2.28 & 2.15 & 3.13 & 5.05 \\
\hline 25 & 4.44 & 4.93 & 5.55 & 5.38 & 5.95 & 6.29 & 8.52 & 6.01 & 2.67 & 2.35 & 2.22 & 3.23 & 5.28 \\
\hline 20 & 4.56 & 5.19 & 5.68 & 5.55 & 6.13 & 6.56 & 9.54 & 6.87 & 2.82 & 2.44 & 2.28 & 3.33 & 5.66 \\
\hline 15 & 4.85 & 5.78 & 5.81 & 5.77 & 6.25 & 6.97 & 11.0 & 7.72 & 2.97 & 2.53 & 2.52 & 3.51 & 6.10 \\
\hline 10 & 5.53 & 6.06 & 6.26 & 6.10 & 6.56 & 8.23 & 13.5 & 8.50 & 3.16 & 2.82 & 3.10 & 3.71 & 6.65 \\
\hline 5 & 6.08 & $\mathrm{ng}$ & 6.63 & 6.41 & 6.95 & 19.0 & 23.8 & 15.0 & 4.24 & 3.09 & 3.37 & 4.09 & 7.92 \\
\hline
\end{tabular}




\section{0-0.000-6M SHIRIN TAGAB RIVER AT KHISHT PUL, Continued}

Probability of occurrence of annual high discharges

[m $\mathrm{m}^{3} / \mathrm{s}$, cubic meters per second; ng, not given]

\begin{tabular}{|c|c|c|c|c|c|c|}
\hline \multirow{2}{*}{$\begin{array}{l}\text { Exceedance } \\
\text { probability }\end{array}$} & \multirow{2}{*}{$\begin{array}{c}\text { Recurrence } \\
\text { interval } \\
\text { (years) }\end{array}$} & \multirow{2}{*}{$\begin{array}{l}\text { Maximum } \\
\text { instantaneous } \\
\text { discharge } \\
\left(\mathrm{m}^{3} / \mathrm{s}\right)\end{array}$} & \multicolumn{4}{|c|}{ Maximum daily mean discharge $\left(\mathrm{m}^{3} / \mathrm{s}\right)$} \\
\hline & & & $\begin{array}{l}\text { 3-day } \\
\text { period }\end{array}$ & $\begin{array}{l}\text { 7-day } \\
\text { period }\end{array}$ & $\begin{array}{l}\text { 15-day } \\
\text { period }\end{array}$ & $\begin{array}{l}\text { 30-day } \\
\text { period }\end{array}$ \\
\hline 0.99 & 1.01 & 15.2 & 5.16 & 4.18 & 3.64 & 3.59 \\
\hline 0.95 & 1.05 & 26.2 & 8.78 & 6.27 & 5.26 & 4.91 \\
\hline 0.90 & 1.11 & 34.2 & 11.4 & 7.75 & 6.38 & 5.78 \\
\hline 0.80 & 1.25 & 46.0 & 15.1 & 9.98 & 8.03 & 7.02 \\
\hline 0.50 & 2 & 75.4 & 24.5 & 15.9 & 12.3 & 10.1 \\
\hline 0.20 & 5 & 113 & 36.3 & 25.0 & 18.7 & 14.3 \\
\hline 0.10 & 10 & 135 & 43.1 & 31.4 & 23.1 & 17.0 \\
\hline 0.04 & 25 & 160 & 50.7 & 39.9 & 28.9 & 20.5 \\
\hline 0.02 & 50 & 176 & 55.6 & 46.4 & 33.3 & 23.1 \\
\hline 0.01 & 100 & 190 & 60.0 & 53.1 & 37.7 & 25.6 \\
\hline 0.005 & 200 & 203 & 63.9 & 59.9 & 42.3 & 28.1 \\
\hline 0.002 & 500 & 218 & $\mathrm{ng}$ & $\mathrm{ng}$ & $\mathrm{ng}$ & $\mathrm{ng}$ \\
\hline
\end{tabular}

\section{0-0.000-6M SHIRIN TAGAB RIVER AT KHISHT PUL, Continued}

Probability of occurrence of annual low discharges

$\left[\mathrm{m}^{3} / \mathrm{s}\right.$, meters per second]

\begin{tabular}{|c|c|c|c|c|c|c|c|c|c|c|}
\hline \multirow{3}{*}{$\begin{array}{c}\text { Nonexceedance } \\
\text { probability }\end{array}$} & \multirow{3}{*}{$\begin{array}{c}\text { Recurrence } \\
\text { interval } \\
\text { (years) }\end{array}$} & \multicolumn{9}{|c|}{ Minimum daily mean discharge $\left(\mathrm{m}^{3} / \mathrm{s}\right)$} \\
\hline & & \multicolumn{9}{|c|}{ Number of consecutive days } \\
\hline & & 1 & 3 & 7 & 14 & 30 & 60 & 90 & 120 & 183 \\
\hline 0.05 & 20 & 0 & 0 & 0.23 & 0.27 & 0.35 & 0.48 & 0.70 & 0.79 & 1.41 \\
\hline 0.10 & 10 & 0.21 & 0.26 & 0.35 & 0.40 & 0.51 & 0.68 & 0.87 & 0.98 & 1.63 \\
\hline 0.20 & 5 & 0.50 & 0.56 & 0.56 & 0.62 & 0.77 & 0.98 & 1.13 & 1.24 & 1.92 \\
\hline 0.50 & 2 & 1.04 & 1.09 & 1.12 & 1.20 & 1.41 & 1.67 & 1.72 & 1.86 & 2.55 \\
\hline
\end{tabular}




\section{0-0.000-6M SHIRIN TAGAB RIVER AT KHISHT PUL, Continued}

\section{Probability of occurrence of seasonal low discharges}

$\left[\mathrm{m}^{3} / \mathrm{s}\right.$, meters per second]

\begin{tabular}{|c|c|c|c|c|c|c|c|c|c|}
\hline \multirow{3}{*}{$\begin{array}{c}\text { Nonexceedance } \\
\text { probability }\end{array}$} & \multirow{3}{*}{$\begin{array}{c}\text { Recurrence } \\
\text { interval } \\
\text { (years) }\end{array}$} & \multicolumn{8}{|c|}{ Minimum daily mean discharge $\left(\mathrm{m}^{3} / \mathrm{s}\right)$} \\
\hline & & \multicolumn{8}{|c|}{ Number of consecutive days } \\
\hline & & 1 & 7 & 14 & 30 & 1 & 7 & 14 & 30 \\
\hline & & \multicolumn{4}{|c|}{ December-January-February } & \multicolumn{4}{|c|}{ March-April-May } \\
\hline 0.05 & 20 & 2.23 & 2.44 & 2.47 & 2.64 & 0.46 & 0.6 & 0.71 & 1.34 \\
\hline 0.10 & 10 & 2.69 & 2.94 & 3.00 & 3.14 & 0.66 & 0.84 & 0.99 & 1.73 \\
\hline 0.20 & 5 & 3.23 & 3.53 & 3.63 & 3.73 & 0.98 & 1.23 & 1.43 & 2.32 \\
\hline \multirow[t]{2}{*}{0.50} & 2 & 4.08 & 4.46 & 4.63 & 4.74 & 1.94 & 2.34 & 2.68 & 3.87 \\
\hline & & \multicolumn{4}{|c|}{ June-July-August } & \multicolumn{4}{|c|}{ September-October-November } \\
\hline 0.05 & 20 & 0 & 0.24 & 0.30 & 0.39 & 0.43 & 0.59 & 0.78 & 1.23 \\
\hline 0.10 & 10 & 0.25 & 0.38 & 0.45 & 0.58 & 0.63 & 0.84 & 1.04 & 1.49 \\
\hline 0.20 & 5 & 0.54 & 0.60 & 0.69 & 0.87 & 0.95 & 1.21 & 1.40 & 1.84 \\
\hline 0.50 & 2 & 1.09 & 1.17 & 1.27 & 1.53 & 1.79 & 2.08 & 2.21 & 2.58 \\
\hline
\end{tabular}

10-0.000-6M SHIRIN TAGAB RIVER AT KHISHT PUL, Continued

Annual peak discharges

$\left[\mathrm{m}^{3} / \mathrm{s}\right.$, meters per second]

\begin{tabular}{|c|c|c|c|c|c|}
\hline \multicolumn{3}{|c|}{$\begin{array}{c}\text { Annual peak discharge, } \\
\text { by year }\end{array}$} & \multicolumn{3}{|c|}{$\begin{array}{l}\text { Annual peak discharge, } \\
\text { from highest to lowest }\end{array}$} \\
\hline $\begin{array}{l}\text { Water } \\
\text { year }\end{array}$ & Date & $\begin{array}{c}\text { Peak } \\
\text { discharge } \\
\left(\mathrm{m}^{3} / \mathrm{s}\right)\end{array}$ & $\begin{array}{l}\text { Water } \\
\text { year }\end{array}$ & Date & $\begin{array}{c}\text { Peak } \\
\text { discharge } \\
\left(\mathrm{m}^{3} / \mathrm{s}\right)\end{array}$ \\
\hline 1967 & May 9, 1967 & 57.9 & 1977 & April 10, 1977 & 150 \\
\hline 1968 & April 25, 1968 & 100 & 1976 & April 8, 1976 & 140 \\
\hline 1969 & April 14, 1969 & 103 & 1969 & April 14, 1969 & 103 \\
\hline 1970 & March 25, 1970 & 50.0 & 1968 & April 25, 1968 & 100 \\
\hline 1971 & April 14, 1971 & 21.3 & 1972 & May 15,1972 & 95.0 \\
\hline 1972 & May 15, 1972 & 95.0 & 1974 & April 7, 1974 & 78.7 \\
\hline 1973 & March 26, 1973 & 60.6 & 1973 & March 26, 1973 & 60.6 \\
\hline 1974 & April 7, 1974 & 78.7 & 1975 & May 3, 1975 & 60.0 \\
\hline 1975 & May 3, 1975 & 60.0 & 1967 & May 9, 1967 & 57.9 \\
\hline 1976 & April 8, 1976 & 140 & 1970 & March 25, 1970 & 50.0 \\
\hline 1977 & April 10, 1977 & 150 & 1978 & April 26, 1978 & 43.4 \\
\hline 1978 & April 26, 1978 & 43.4 & 1971 & April 14, 1971 & 21.3 \\
\hline
\end{tabular}


10-0.000-6M SHIRIN TAGAB RIVER AT KHISHT PUL, Continued

Monthly and annual mean discharges, in cubic meters per second

[Data may not be rounded in accordance with U.S. Geological Survey publication standards; --, no data]

\begin{tabular}{|c|c|c|c|c|c|c|c|c|c|c|c|c|c|}
\hline \multirow{2}{*}{$\begin{array}{l}\text { Water } \\
\text { year }\end{array}$} & \multicolumn{12}{|c|}{ Monthly mean discharge } & \multirow{2}{*}{$\begin{array}{c}\text { Annual } \\
\text { discharge }\end{array}$} \\
\hline & October & November & December & January & February & March & April & May & June & July & August & September & \\
\hline 1966 & -- & -- & -- & 3.43 & 4.19 & 5.31 & 5.55 & 2.64 & 0.92 & 1.00 & 1.10 & 2.58 & -- \\
\hline 1967 & 5.24 & 5.99 & 5.93 & 5.79 & 6.46 & 6.18 & 9.42 & 8.36 & 1.87 & 1.57 & 1.07 & 1.55 & 4.94 \\
\hline 1968 & 3.26 & 4.48 & 4.47 & 3.94 & 3.89 & 5.31 & 6.41 & 5.51 & 1.60 & 1.54 & 1.13 & 2.06 & 3.63 \\
\hline 1969 & 3.63 & 3.78 & 6.16 & 5.86 & 5.94 & 8.05 & 16.8 & 8.82 & 2.87 & 3.00 & 3.50 & 4.22 & 6.04 \\
\hline 1970 & 6.18 & 6.46 & 5.82 & 5.19 & 5.10 & 6.23 & 4.79 & 1.48 & 1.16 & 1.25 & 1.14 & 2.45 & 3.93 \\
\hline 1971 & 4.21 & 4.55 & 3.85 & 3.86 & 4.69 & 5.13 & 4.55 & 1.49 & 1.22 & 0.60 & 0.29 & 1.08 & 2.95 \\
\hline 1972 & 2.72 & 3.06 & 2.23 & 3.94 & 4.21 & 12.5 & 8.75 & 11.1 & 5.94 & 2.09 & 1.59 & 2.01 & 5.02 \\
\hline 1973 & 2.73 & 3.49 & 5.82 & 6.13 & 7.11 & 12.3 & 9.96 & 2.25 & 2.30 & 2.22 & 1.99 & 3.06 & 4.93 \\
\hline 1974 & 3.57 & 4.20 & 4.76 & 5.55 & 5.73 & 6.20 & 8.64 & 4.16 & 2.03 & 2.37 & 1.81 & 2.63 & 4.29 \\
\hline 1975 & 3.53 & 4.00 & 4.30 & 4.30 & 5.03 & 5.28 & 10.8 & 8.41 & 1.27 & 2.03 & 2.39 & 3.25 & 4.54 \\
\hline 1976 & 4.19 & 4.57 & 4.63 & 4.66 & 4.60 & 4.98 & 12.9 & 6.37 & 2.31 & 2.22 & 2.25 & 2.91 & 4.71 \\
\hline 1977 & 4.19 & 4.78 & 4.83 & 5.03 & 5.67 & 6.17 & 6.17 & 4.48 & 3.02 & 2.72 & 2.60 & 3.20 & 4.40 \\
\hline 1978 & 4.04 & 4.92 & 5.93 & 4.51 & 5.43 & 5.40 & 5.66 & 3.87 & 2.56 & 1.89 & 1.56 & 1.90 & 3.96 \\
\hline
\end{tabular}




\section{0-1.1L0-7A OAISAR RIVER AT OAISAR}

\section{(U.S. Geological Survey identification number: 354200064180000 )}

LOCATION: Lat $35^{\circ} 42^{\prime} \mathrm{N}$., long $64^{\circ} 18^{\prime} \mathrm{E}$.

DRAINAGE AREA: $425 \mathrm{~km}^{2}$.

ELEVATION: 1,312 meters above mean sea level.

PERIOD OF RECORD: March 30, 1969 to September 30, 1978.

GAGE: Water-stage recorder.

Annual mean discharge

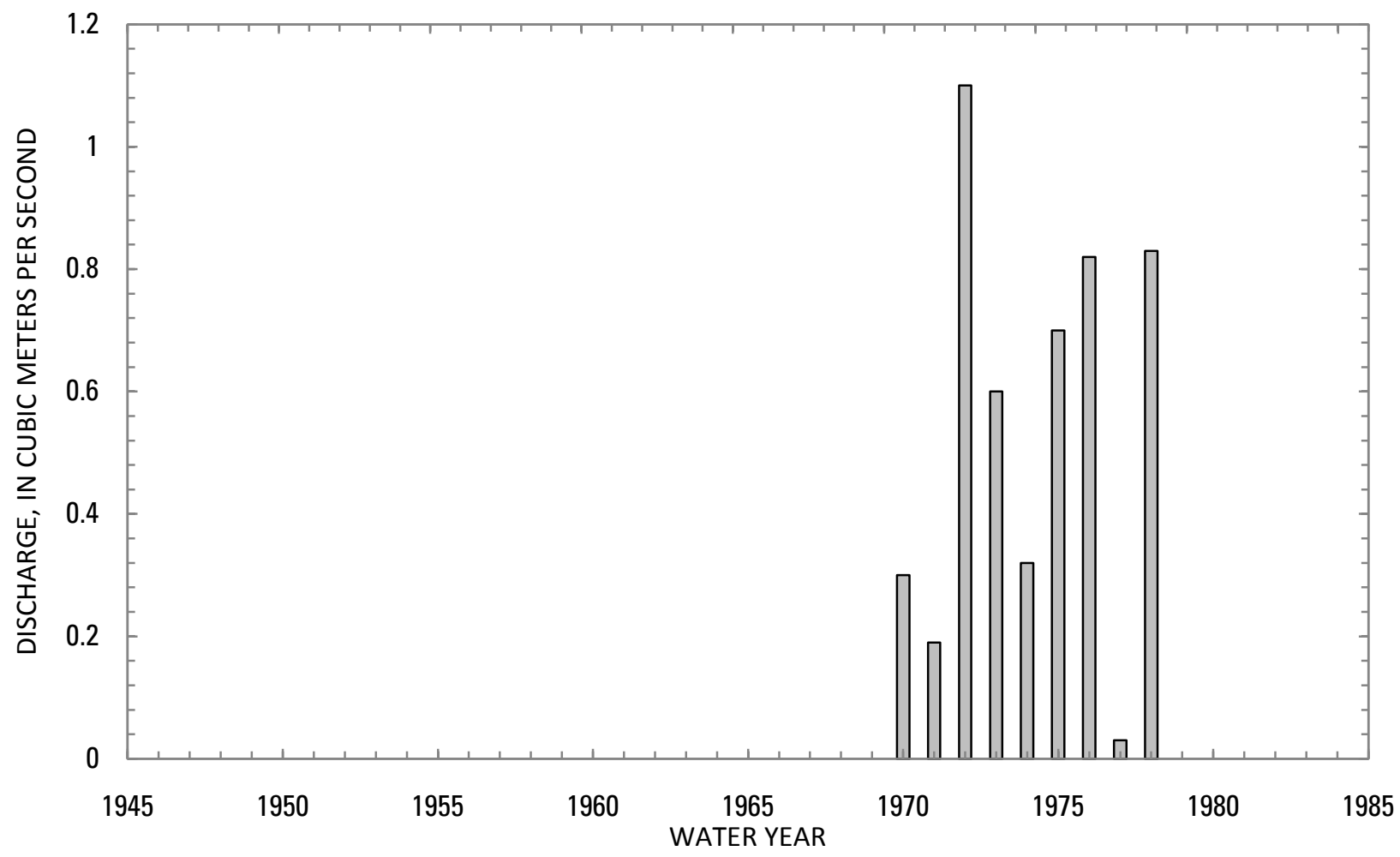




\section{0-1.1L0-7A OAISAR RIVER AT OAISAR, Continued}

Statistics of monthly and annual mean discharges $\left[\mathrm{m}^{3} / \mathrm{s}\right.$, cubic meters per second]

\begin{tabular}{|c|c|c|c|c|c|c|c|c|}
\hline \multirow[b]{2}{*}{ Month } & \multicolumn{2}{|c|}{ Maximum } & \multicolumn{2}{|c|}{ Minimum } & \multicolumn{4}{|c|}{ Mean } \\
\hline & $\begin{array}{c}\text { Discharge } \\
\left(\mathrm{m}^{3} / \mathrm{s}\right)\end{array}$ & $\begin{array}{l}\text { Water year } \\
\text { of } \\
\text { occurrence }\end{array}$ & $\begin{array}{c}\text { Discharge } \\
\left(\mathrm{m}^{3} / \mathrm{s}\right)\end{array}$ & $\begin{array}{c}\text { Water year } \\
\text { of } \\
\text { occurrence }\end{array}$ & $\begin{array}{c}\text { Discharge } \\
\left(\mathrm{m}^{3} / \mathrm{s}\right)\end{array}$ & $\begin{array}{c}\text { Standard } \\
\text { deviation } \\
\left(\mathrm{m}^{3} / \mathrm{s}\right)\end{array}$ & $\begin{array}{c}\text { Coefficient } \\
\text { of } \\
\text { variation }\end{array}$ & $\begin{array}{c}\text { Percentage } \\
\text { of annual } \\
\text { discharge }\end{array}$ \\
\hline October & 0.01 & 1975 & 0 & 1970 & 0 & 0 & 1.66 & 0.02 \\
\hline November & 0.13 & 1978 & 0 & 1972 & 0.02 & 0.04 & 1.89 & 0.31 \\
\hline December & 0.59 & 1978 & 0 & 1970 & 0.08 & 0.19 & 2.40 & 1.10 \\
\hline January & 0.03 & 1974 & 0 & 1970 & 0.01 & 0.01 & 1.29 & 0.10 \\
\hline February & 0.87 & 1975 & 0 & 1970 & 0.13 & 0.29 & 2.20 & 1.77 \\
\hline March & 4.44 & 1972 & 0.02 & 1971 & 1.30 & 1.51 & 1.16 & 17.8 \\
\hline April & 7.30 & 1969 & 0.33 & 1977 & 3.79 & 2.06 & 0.55 & 51.7 \\
\hline May & 5.57 & 1969 & 0.02 & 1977 & 1.90 & 2.03 & 1.07 & 25.9 \\
\hline June & 0.51 & 1972 & 0 & 1970 & 0.08 & 0.16 & 1.89 & 1.13 \\
\hline July & 0.02 & 1975 & 0 & 1969 & 0 & 0.01 & 1.89 & 0.05 \\
\hline August & 0.01 & 1976 & 0 & 1969 & 0 & 0 & 1.69 & 0.02 \\
\hline September & 0.02 & 1978 & 0 & 1970 & 0 & 0.01 & 1.66 & 0.06 \\
\hline Annual & 1.10 & 1972 & 0.03 & 1977 & 0.54 & 0.35 & 0.65 & 100 \\
\hline
\end{tabular}


10-1.1L0-7A QAISAR RIVER AT QAISAR, Continued

Monthly and annual flow duration, in cubic meters per second

\begin{tabular}{|c|c|c|c|c|c|c|c|c|c|c|c|c|c|}
\hline \multirow{2}{*}{$\begin{array}{l}\text { Percentage } \\
\text { of days } \\
\text { discharge } \\
\text { equaled or } \\
\text { exceeded }\end{array}$} & \multicolumn{12}{|c|}{ Month } & \multirow{2}{*}{ Annual } \\
\hline & October & November & December & January & February & March & April & May & June & July & August & September & \\
\hline 95 & 0 & 0 & 0 & 0 & 0 & 0 & 0.05 & 0 & 0 & 0 & 0 & 0 & 0 \\
\hline 90 & 0 & 0 & 0 & 0 & 0 & 0 & 0.27 & 0 & 0 & 0 & 0 & 0 & 0 \\
\hline 85 & 0 & 0 & 0 & 0 & 0 & 0 & 0.50 & 0.01 & 0 & 0 & 0 & 0 & 0 \\
\hline 80 & 0 & 0 & 0 & 0 & 0 & 0 & 1.38 & 0.02 & 0 & 0 & 0 & 0 & 0 \\
\hline 75 & 0 & 0 & 0 & 0 & 0 & 0.01 & 1.68 & 0.04 & 0 & 0 & 0 & 0 & 0 \\
\hline 70 & 0 & 0 & 0 & 0 & 0 & 0.02 & 1.87 & 0.05 & 0 & 0 & 0 & 0 & 0 \\
\hline 65 & 0 & 0 & 0 & 0 & 0 & 0.02 & 2.07 & 0.11 & 0 & 0 & 0 & 0 & 0 \\
\hline 60 & 0 & 0 & 0 & 0 & 0 & 0.06 & 2.33 & 0.18 & 0 & 0 & 0 & 0 & 0 \\
\hline 55 & 0 & 0 & 0 & 0 & 0 & 0.19 & 2.60 & 0.23 & 0 & 0 & 0 & 0 & 0 \\
\hline 50 & 0 & 0 & 0 & 0 & 0 & 0.35 & 3.02 & 0.41 & 0 & 0 & 0 & 0 & 0 \\
\hline 45 & 0 & 0 & 0 & 0 & 0 & 0.38 & 3.83 & 0.76 & 0 & 0 & 0 & 0 & 0 \\
\hline 40 & 0 & 0 & 0 & 0 & 0 & 0.41 & 4.36 & 1.23 & 0 & 0 & 0 & 0 & 0 \\
\hline 35 & 0 & 0 & 0 & 0 & 0 & 0.50 & 4.80 & 2.05 & 0.01 & 0 & 0 & 0 & 0.01 \\
\hline 30 & 0 & 0 & 0.01 & 0 & 0.01 & 0.93 & 5.24 & 2.39 & 0.02 & 0 & 0 & 0 & 0.02 \\
\hline 25 & 0 & 0 & 0.01 & 0 & 0.02 & 1.22 & 5.67 & 3.27 & 0.02 & 0 & 0 & 0 & 0.04 \\
\hline 20 & 0 & 0 & 0.02 & 0.01 & 0.04 & 1.65 & 6.09 & 3.97 & 0.04 & 0 & 0 & 0 & 0.14 \\
\hline 15 & 0 & 0.01 & 0.03 & 0.02 & 0.06 & 2.19 & 6.89 & 5.02 & 0.10 & 0 & 0 & 0.01 & 0.51 \\
\hline 10 & 0 & 0.04 & 0.05 & 0.04 & 0.45 & 3.99 & 7.83 & 6.02 & 0.29 & 0.02 & 0 & 0.02 & 1.96 \\
\hline 5 & 0.02 & 0.06 & 0.07 & 0.05 & 1 & 6.22 & 9.52 & 7.07 & 0.43 & 0.03 & 0.01 & 0.05 & 4.20 \\
\hline
\end{tabular}




\section{0-1.1L0-7A OAISAR RIVER AT OAISAR, Continued}

Probability of occurrence of annual high discharges

[m $\mathrm{m}^{3} / \mathrm{s}$, cubic meters per second; ng, not given]

\begin{tabular}{|c|c|c|c|c|c|c|}
\hline \multirow{2}{*}{$\begin{array}{l}\text { Exceedance } \\
\text { probability }\end{array}$} & \multirow{2}{*}{$\begin{array}{c}\text { Recurrence } \\
\text { interval } \\
\text { (years) }\end{array}$} & \multirow{2}{*}{$\begin{array}{l}\text { Maximum } \\
\text { instantaneous } \\
\text { discharge } \\
\left(\mathrm{m}^{3} / \mathrm{s}\right)\end{array}$} & \multicolumn{4}{|c|}{ Maximum daily mean discharge $\left(\mathrm{m}^{3} / \mathrm{s}\right)$} \\
\hline & & & $\begin{array}{l}\text { 3-day } \\
\text { period }\end{array}$ & $\begin{array}{l}\text { 7-day } \\
\text { period }\end{array}$ & $\begin{array}{l}\text { 15-day } \\
\text { period }\end{array}$ & $\begin{array}{l}\text { 30-day } \\
\text { period }\end{array}$ \\
\hline 0.99 & 1.01 & 3.20 & 1.10 & 0.49 & 0.25 & 0.13 \\
\hline 0.95 & 1.05 & 4.40 & 1.99 & 1.22 & 0.80 & 0.51 \\
\hline 0.90 & 1.11 & 5.20 & 2.68 & 1.85 & 1.34 & 0.93 \\
\hline 0.80 & 1.25 & 6.50 & 3.78 & 2.92 & 2.30 & 1.73 \\
\hline 0.50 & 2 & 10.0 & 6.93 & 5.98 & 4.96 & 4.10 \\
\hline 0.20 & 5 & 15.7 & 12.0 & 10.2 & 7.98 & 6.71 \\
\hline 0.10 & 10 & 20.0 & 15.5 & 12.5 & 9.31 & 7.74 \\
\hline 0.04 & 25 & 26.0 & 20.2 & 15.0 & 10.4 & 8.48 \\
\hline 0.02 & 50 & 31.0 & 23.6 & 16.5 & 10.9 & 8.77 \\
\hline 0.01 & 100 & 36.4 & 27.1 & 17.7 & 11.2 & 8.95 \\
\hline 0.005 & 200 & 42.2 & 30.6 & 18.7 & 11.4 & 9.05 \\
\hline 0.002 & 500 & 50.6 & $\mathrm{ng}$ & $\mathrm{ng}$ & $\mathrm{ng}$ & $\mathrm{ng}$ \\
\hline
\end{tabular}

10-1.1L0-7A QAISAR RIVER AT QAISAR, Continued

Probability of occurrence of annual low discharges

[ $\mathrm{m}^{3} / \mathrm{s}$, meters per second; $\mathrm{ng}$, not given]

\begin{tabular}{|c|c|c|c|c|c|c|c|c|c|c|}
\hline \multirow{3}{*}{$\begin{array}{c}\text { Nonexceedance } \\
\text { probability }\end{array}$} & \multirow{3}{*}{$\begin{array}{c}\text { Recurrence } \\
\text { interval } \\
\text { (years) }\end{array}$} & \multicolumn{9}{|c|}{ Minimum daily mean discharge $\left(\mathrm{m}^{3} / \mathrm{s}\right)$} \\
\hline & & \multicolumn{9}{|c|}{ Number of consecutive days } \\
\hline & & 1 & 3 & 7 & 14 & 30 & 60 & 90 & 120 & 183 \\
\hline 0.05 & 20 & ng & $\mathrm{ng}$ & $\mathrm{ng}$ & $\mathrm{ng}$ & ng & ng & 0 & 0 & 0 \\
\hline 0.10 & 10 & ng & $\mathrm{ng}$ & $\mathrm{ng}$ & $\mathrm{ng}$ & ng & ng & 0 & 0 & 0 \\
\hline 0.20 & 5 & ng & $\mathrm{ng}$ & $\mathrm{ng}$ & $\mathrm{ng}$ & ng & ng & 0 & 0 & 0 \\
\hline 0.50 & 2 & ng & $\mathrm{ng}$ & $\mathrm{ng}$ & ng & ng & ng & 0 & 0 & 0 \\
\hline
\end{tabular}




\section{0-1.1L0-7A OAISAR RIVER AT OAISAR, Continued}

Probability of occurrence of seasonal low discharges [m³/s, meters per second; ng, not given]

\begin{tabular}{|c|c|c|c|c|c|c|c|c|c|}
\hline \multirow{3}{*}{$\begin{array}{c}\text { Nonexceedance } \\
\text { probability }\end{array}$} & \multirow{3}{*}{$\begin{array}{c}\text { Recurrence } \\
\text { interval } \\
\text { (years) }\end{array}$} & \multicolumn{8}{|c|}{ Minimum daily mean discharge $\left(\mathrm{m}^{3} / \mathrm{s}\right)$} \\
\hline & & \multicolumn{8}{|c|}{ Number of consecutive days } \\
\hline & & 1 & 7 & 14 & 30 & 1 & 7 & 14 & 30 \\
\hline & & \multicolumn{4}{|c|}{$\begin{array}{c}\text { December-January- } \\
\text { February } \\
\end{array}$} & \multicolumn{4}{|c|}{ March-April-May } \\
\hline 0.05 & 20 & 0 & 0 & 0 & 0 & 0 & 0 & 0 & 0.01 \\
\hline 0.10 & 10 & 0 & 0 & 0 & 0 & 0 & 0 & 0 & 0.02 \\
\hline 0.20 & 5 & 0 & 0 & 0 & 0 & 0 & 0 & 0 & 0.05 \\
\hline \multirow{2}{*}{0.50} & 2 & 0 & 0 & 0 & 0 & 0 & 0.01 & 0.02 & 0.28 \\
\hline & & \multicolumn{4}{|c|}{ June-July-August } & \multicolumn{4}{|c|}{ September-0ctober-November } \\
\hline 0.05 & 20 & ng & ng & ng & ng & ng & $\mathrm{ng}$ & $\mathrm{ng}$ & $\mathrm{ng}$ \\
\hline 0.10 & 10 & ng & ng & ng & $\mathrm{ng}$ & ng & $\mathrm{ng}$ & $\mathrm{ng}$ & ng \\
\hline 0.20 & 5 & $\mathrm{ng}$ & ng & ng & $\mathrm{ng}$ & $\mathrm{ng}$ & $\mathrm{ng}$ & $\mathrm{ng}$ & ng \\
\hline 0.50 & 2 & $\mathrm{ng}$ & $\mathrm{ng}$ & ng & ng & $\mathrm{ng}$ & ng & $\mathrm{ng}$ & $\mathrm{ng}$ \\
\hline
\end{tabular}

10-1.1L0-7A OAISAR RIVER AT QAISAR, Continued

\begin{tabular}{|c|c|c|c|c|c|}
\hline \multicolumn{6}{|c|}{$\begin{array}{l}\text { Annual peak discharges } \\
\text { [m³/s, meters per second] }\end{array}$} \\
\hline \multicolumn{3}{|c|}{$\begin{array}{c}\text { Annual peak discharge, } \\
\text { by year }\end{array}$} & \multicolumn{3}{|c|}{$\begin{array}{l}\text { Annual peak discharge, } \\
\text { from highest to lowest }\end{array}$} \\
\hline $\begin{array}{l}\text { Water } \\
\text { year }\end{array}$ & Date & $\begin{array}{c}\text { Peak } \\
\text { discharge } \\
\left(\mathrm{m}^{3} / \mathrm{s}\right)\end{array}$ & $\begin{array}{l}\text { Water } \\
\text { year }\end{array}$ & Date & $\begin{array}{c}\text { Peak } \\
\text { discharge } \\
\left(\mathrm{m}^{3} / \mathrm{s}\right)\end{array}$ \\
\hline 1969 & April 14, 1969 & 11.2 & 1976 & May 11, 1976 & 25.0 \\
\hline 1970 & March 25, 1970 & 10.5 & 1972 & March 27, 1972 & 20.0 \\
\hline 1971 & April 14, 1971 & 9.08 & 1978 & April 12, 1978 & 12.0 \\
\hline 1972 & March 27, 1972 & 20.0 & 1969 & April 14, 1969 & 11.2 \\
\hline 1973 & April 7, 1973 & 7.00 & 1970 & March 25, 1970 & 10.5 \\
\hline 1974 & May 2, 1974 & 7.40 & 1971 & April 14, 1971 & 9.08 \\
\hline 1975 & April 19, 1975 & 8.58 & 1975 & April 19, 1975 & 8.58 \\
\hline 1976 & May 11, 1976 & 25.0 & 1974 & May 2, 1974 & 7.40 \\
\hline 1977 & April 9, 1977 & 4.00 & 1973 & April 7, 1973 & 7.00 \\
\hline 1978 & April 12, 1978 & 12.0 & 1977 & April 9, 1977 & 4.00 \\
\hline
\end{tabular}




\section{0-1.1L0-7A QAISAR RIVER AT QAISAR, Continued}

Monthly and annual mean discharges, in cubic meters per second $[--$, no data]

\begin{tabular}{|c|c|c|c|c|c|c|c|c|c|c|c|c|c|}
\hline \multirow{2}{*}{$\begin{array}{l}\text { Water } \\
\text { year }\end{array}$} & \multicolumn{12}{|c|}{ Monthly mean discharge } & \multirow{2}{*}{$\begin{array}{c}\text { Annual } \\
\text { discharge }\end{array}$} \\
\hline & October & November & December & January & February & March & April & May & June & July & August & September & \\
\hline 1969 & -- & -- & -- & -- & -- & -- & 7.30 & 5.57 & 0.07 & 0 & 0 & 0 & -- \\
\hline 1970 & 0 & 0.05 & 0 & 0 & 0 & 0.79 & 2.71 & 0.03 & 0 & 0.01 & 0 & 0 & 0.30 \\
\hline 1971 & 0 & 0.02 & 0.01 & 0.01 & 0.01 & 0.02 & 1.74 & 0.51 & 0 & 0 & 0 & 0.01 & 0.19 \\
\hline 1972 & 0 & 0 & 0.01 & 0 & 0 & 4.44 & 5.75 & 2.53 & 0.51 & 0 & 0 & 0 & 1.10 \\
\hline 1973 & 0 & 0 & 0 & 0 & 0.04 & 1.16 & 4.37 & 1.61 & 0 & 0 & 0 & 0 & 0.60 \\
\hline 1974 & 0 & 0 & 0.01 & 0.03 & 0.01 & 0.67 & 2.22 & 0.81 & 0.10 & 0 & 0 & 0.01 & 0.32 \\
\hline 1975 & 0.01 & 0 & 0.10 & 0.01 & 0.87 & 1.11 & 4.31 & 1.97 & 0.01 & 0.02 & 0 & 0 & 0.70 \\
\hline 1976 & 0 & 0 & 0 & 0.01 & 0.02 & 0.31 & 4.03 & 5.29 & 0.14 & 0.01 & 0.01 & 0.01 & 0.82 \\
\hline 1977 & 0.01 & 0 & 0.01 & 0 & 0.01 & 0.05 & 0.33 & 0.02 & 0 & 0 & 0 & 0 & 0.03 \\
\hline 1978 & 0 & 0.13 & 0.59 & 0.01 & 0.21 & 3.15 & 5.12 & 0.66 & 0 & 0 & 0.01 & 0.02 & 0.83 \\
\hline
\end{tabular}




\section{0-1.L00-1T MAIMANA RIVER NEAR PATA BABA}

\section{(U.S. Geological Survey identification number: 363200064530000 )}

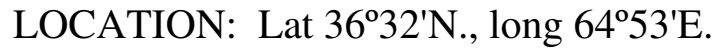

DRAINAGE AREA: $6,685 \mathrm{~km}^{2}$.

ELEVATION: 381 meters above mean sea level.

PERIOD OF RECORD: November 11, 1967 to September 30, 1972

GAGE: Water-stage recorder. Staff gage prior to 1970 water year.

Annual mean discharge

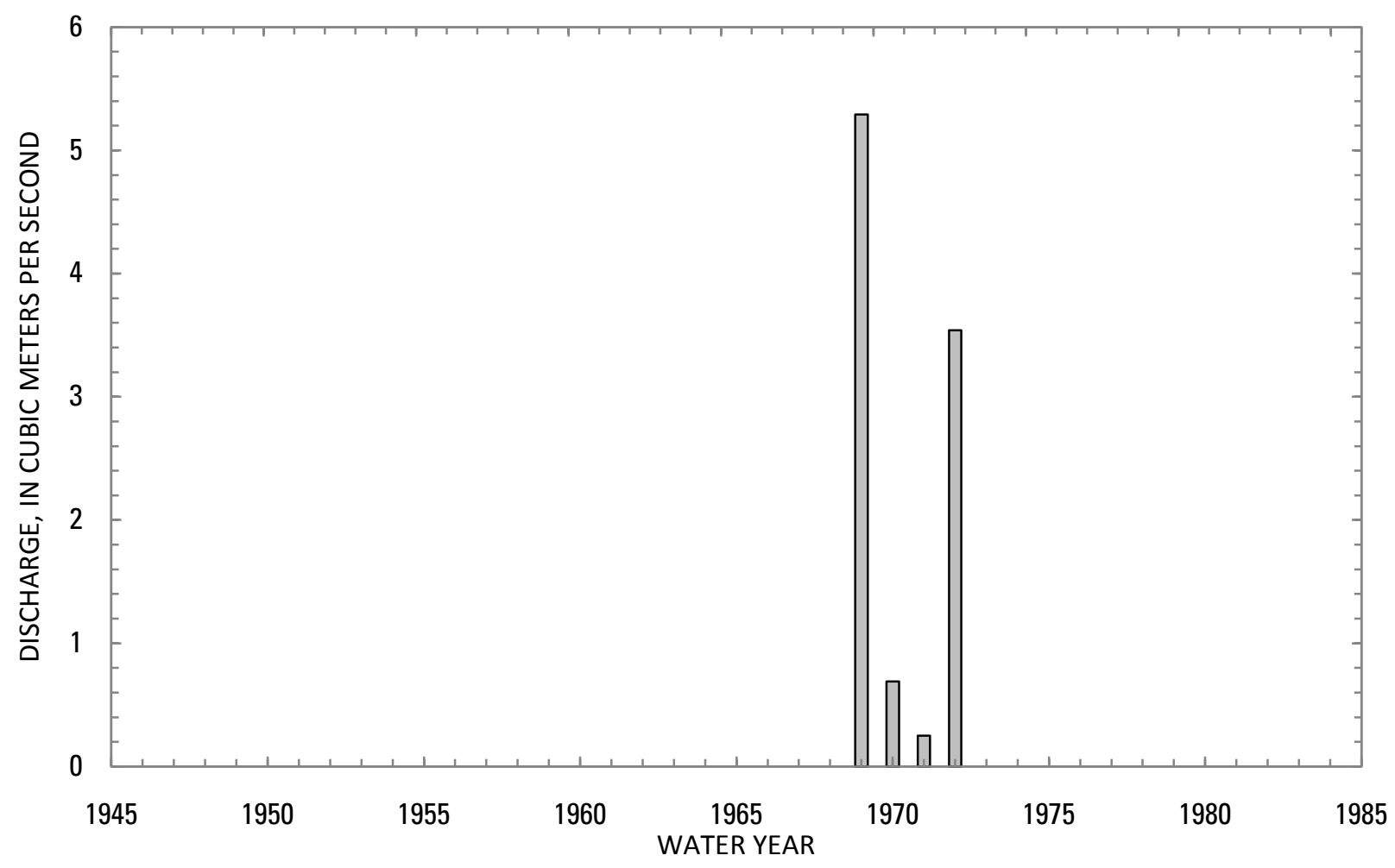




\section{0-1.L00-1T MAIMANA RIVER NEAR PATA BABA, Continued}

Statistics of monthly and annual mean discharges $\left[\mathrm{m}^{3} / \mathrm{s}\right.$, cubic meters per second]

\begin{tabular}{|c|c|c|c|c|c|c|c|c|}
\hline \multirow[b]{2}{*}{ Month } & \multicolumn{2}{|c|}{ Maximum } & \multicolumn{2}{|c|}{ Minimum } & \multicolumn{4}{|c|}{ Mean } \\
\hline & $\begin{array}{c}\text { Discharge } \\
\left(\mathrm{m}^{3} / \mathrm{s}\right)\end{array}$ & $\begin{array}{c}\text { Water year } \\
\text { of } \\
\text { occurrence }\end{array}$ & $\begin{array}{c}\text { Discharge } \\
\left(\mathrm{m}^{3} / \mathrm{s}\right)\end{array}$ & $\begin{array}{c}\text { Water year } \\
\text { of } \\
\text { occurrence }\end{array}$ & $\begin{array}{c}\text { Discharge } \\
\left(\mathrm{m}^{3} / \mathrm{s}\right)\end{array}$ & $\begin{array}{c}\text { Standard } \\
\text { deviation } \\
\left(\mathrm{m}^{3} / \mathrm{s}\right)\end{array}$ & $\begin{array}{c}\text { Coefficient } \\
\text { of } \\
\text { variation }\end{array}$ & $\begin{array}{c}\text { Percentage } \\
\text { of annual } \\
\text { discharge }\end{array}$ \\
\hline October & 0.71 & 1970 & 0.06 & 1972 & 0.32 & 0.28 & 0.87 & 1.26 \\
\hline November & 1.30 & 1970 & 0.23 & 1972 & 0.51 & 0.52 & 1.02 & 2.01 \\
\hline December & 2.33 & 1969 & 0.16 & 1968 & 0.80 & 0.91 & 1.15 & 3.12 \\
\hline January & 1.50 & 1969 & 0.13 & 1968 & 0.68 & 0.52 & 0.77 & 2.66 \\
\hline February & 5.38 & 1972 & 0.30 & 1968 & 2.08 & 2.34 & 1.12 & 8.13 \\
\hline March & 9.74 & 1972 & 0.53 & 1971 & 4.29 & 4.31 & 1.00 & 16.8 \\
\hline April & 28.0 & 1969 & 0.62 & 1971 & 9.05 & 11.5 & 1.27 & 35.4 \\
\hline May & 16.9 & 1969 & 0.01 & 1971 & 6.27 & 7.24 & 1.15 & 24.6 \\
\hline June & 3.45 & 1972 & 0 & 1971 & 1.07 & 1.51 & 1.41 & 4.19 \\
\hline July & 0.47 & 1972 & 0 & 1970 & 0.13 & 0.20 & 1.56 & 0.50 \\
\hline August & 0.45 & 1972 & 0 & 1970 & 0.13 & 0.20 & 1.50 & 0.52 \\
\hline September & 0.48 & 1969 & 0 & 1971 & 0.21 & 0.23 & 1.11 & 0.81 \\
\hline Annual & 5.29 & 1969 & 0.25 & 1971 & 2.44 & 2.39 & 0.98 & 100 \\
\hline
\end{tabular}


10-1.L00-1T MAIMANA RIVER NEAR PATA BABA, Continued

Monthly and annual flow duration, in cubic meters per second

[ng, not given]

\begin{tabular}{|c|c|c|c|c|c|c|c|c|c|c|c|c|c|}
\hline \multirow{2}{*}{$\begin{array}{l}\text { Percentage } \\
\text { of days } \\
\text { discharge } \\
\text { equaled or } \\
\text { exceeded }\end{array}$} & \multicolumn{12}{|c|}{ Month } & \multirow{2}{*}{ Annua } \\
\hline & October & November & December & January & February & March & April & May & June & July & August & September & \\
\hline 95 & 0.01 & 0.19 & 0.01 & 0 & 0.18 & 0.10 & 0.07 & 0 & 0 & 0 & 0 & 0 & 0 \\
\hline 90 & 0.03 & 0.21 & 0.05 & 0 & 0.21 & 0.14 & 0.13 & 0.01 & 0 & 0 & 0 & 0 & 0 \\
\hline 85 & 0.08 & 0.22 & 0.10 & 0.02 & 0.29 & 0.17 & 0.20 & 0.01 & 0 & 0 & 0 & 0 & 0 \\
\hline 80 & 0.11 & 0.23 & 0.13 & 0.10 & 0.39 & 0.24 & 0.22 & 0.02 & 0 & 0 & 0 & 0 & 0.02 \\
\hline 75 & 0.13 & 0.23 & 0.16 & 0.22 & 0.43 & 0.36 & 0.31 & 0.02 & 0 & 0 & 0 & 0 & 0.10 \\
\hline 70 & 0.17 & 0.24 & 0.19 & 0.25 & 0.46 & 0.42 & 0.39 & 0.05 & 0.03 & 0 & 0 & 0 & 0.18 \\
\hline 65 & 0.21 & 0.25 & 0.22 & 0.30 & 0.49 & 0.50 & 0.51 & 0.09 & 0.04 & 0 & 0 & 0.01 & 0.22 \\
\hline 60 & 0.22 & 0.25 & 0.25 & 0.35 & 0.53 & 0.64 & 0.75 & 0.45 & 0.06 & 0 & 0 & 0.02 & 0.27 \\
\hline 55 & 0.23 & 0.26 & 0.28 & 0.37 & 0.56 & 0.73 & 1.04 & 1.29 & 0.11 & 0 & 0 & 0.10 & 0.32 \\
\hline 50 & 0.24 & 0.26 & 0.34 & 0.39 & 0.60 & 0.85 & 1.71 & 1.90 & 0.15 & 0.02 & 0 & 0.13 & 0.37 \\
\hline 45 & 0.25 & 0.27 & 0.39 & 0.44 & 0.67 & 1.37 & 2.76 & 3.70 & 0.19 & 0.02 & 0 & 0.17 & 0.43 \\
\hline 40 & 0.29 & 0.31 & 0.44 & 0.52 & 0.76 & 2.27 & 5.88 & 4.97 & 0.23 & 0.03 & 0.01 & 0.24 & 0.48 \\
\hline 35 & 0.31 & 0.33 & 0.50 & 0.58 & 0.95 & 4.00 & 7.20 & 6.06 & 0.45 & 0.04 & 0.14 & 0.34 & 0.57 \\
\hline 30 & 0.33 & 0.35 & 0.63 & 0.65 & 2.59 & 5.07 & 9.05 & 7.53 & 0.66 & 0.12 & 0.26 & 0.41 & 0.71 \\
\hline 25 & 0.37 & 0.37 & 0.70 & 0.71 & 3.71 & 6.12 & 10.6 & 9.64 & 1.01 & 0.29 & 0.30 & 0.43 & 1.00 \\
\hline 20 & 0.54 & 1.04 & 0.84 & 0.85 & 4.36 & 7.53 & 13.2 & 12.5 & 1.20 & 0.41 & ng & 0.45 & 1.60 \\
\hline 15 & 0.63 & 1.14 & 1.12 & 1.04 & 5.47 & 8.97 & 18.9 & 14.1 & 1.94 & 0.46 & ng & 0.48 & 4.13 \\
\hline 10 & 0.67 & 1.39 & 1.63 & 1.21 & 6.00 & 12.0 & 30.1 & 15.7 & 3.29 & 0.49 & ng & 0.51 & 6.64 \\
\hline 5 & 1.03 & 1.56 & 3.80 & 1.48 & 6.53 & 16.2 & 48.7 & 28.0 & 5.50 & $\mathrm{ng}$ & ng & 0.65 & 12.9 \\
\hline
\end{tabular}




\section{0-1.L00-1T MAIMANA RIVER NEAR PATA BABA, Continued}

Probability of occurrence of annual high discharges

[m $\mathrm{m}^{3} / \mathrm{s}$, cubic meters per second; ng, not given]

\begin{tabular}{|c|c|c|c|c|c|c|}
\hline \multirow{2}{*}{$\begin{array}{l}\text { Exceedance } \\
\text { probability }\end{array}$} & \multirow{2}{*}{$\begin{array}{l}\text { Recurrence } \\
\text { interval } \\
\text { (years) }\end{array}$} & \multirow{2}{*}{$\begin{array}{l}\text { Maximum } \\
\text { instantaneous } \\
\text { discharge } \\
\left(\mathrm{m}^{3} / \mathrm{s}\right)\end{array}$} & \multicolumn{4}{|c|}{ Maximum daily mean discharge $\left(\mathrm{m}^{3} / \mathrm{s}\right)$} \\
\hline & & & $\begin{array}{l}\text { 3-day } \\
\text { period }\end{array}$ & $\begin{array}{l}\text { 7-day } \\
\text { period }\end{array}$ & $\begin{array}{l}\text { 15-day } \\
\text { period }\end{array}$ & $\begin{array}{l}\text { 30-day } \\
\text { period }\end{array}$ \\
\hline 0.99 & 1.01 & ng & 0.77 & 0.38 & 0.13 & 0.08 \\
\hline 0.95 & 1.05 & ng & 2.62 & 1.20 & 0.50 & 0.34 \\
\hline 0.90 & 1.11 & ng & 4.64 & 2.12 & 1.01 & 0.71 \\
\hline 0.80 & 1.25 & ng & 8.65 & 4.09 & 2.23 & 1.60 \\
\hline 0.50 & 2 & 40.1 & 23.1 & 13.1 & 9.01 & 6.70 \\
\hline 0.20 & 5 & 60.8 & 48.2 & 37.3 & 31.1 & 23.4 \\
\hline 0.10 & 10 & 70.7 & 64.8 & 61.5 & 55.9 & 42.0 \\
\hline 0.04 & 25 & 79.4 & ${ }^{2} 83.6$ & 101 & 100 & 74.6 \\
\hline 0.02 & 50 & 84.0 & ${ }^{2} 95.6$ & ${ }^{2} 137$ & 142 & 105 \\
\hline 0.01 & 100 & 87.3 & ${ }^{2} 106$ & ${ }^{2} 179$ & 193 & 141 \\
\hline 0.005 & 200 & 89.8 & ${ }^{2} 115$ & ${ }^{2} 225$ & 251 & 182 \\
\hline 0.002 & 500 & 92.1 & $\mathrm{ng}$ & $\mathrm{ng}$ & ng & ng \\
\hline
\end{tabular}

\section{0-1.L00-1T MAIMANA RIVER NEAR PATA BABA, Continued}

Probability of occurrence of annual low discharges

[ $\mathrm{m}^{3} / \mathrm{s}$, meters per second; ng, not given]

\begin{tabular}{|c|c|c|c|c|c|c|c|c|c|c|}
\hline \multirow{3}{*}{$\begin{array}{c}\text { Nonexceedance } \\
\text { probability }\end{array}$} & \multirow{3}{*}{$\begin{array}{c}\text { Recurrence } \\
\text { interval } \\
\text { (years) }\end{array}$} & \multicolumn{9}{|c|}{ Minimum daily mean discharge $\left(\mathrm{m}^{3} / \mathrm{s}\right)$} \\
\hline & & \multicolumn{9}{|c|}{ Number of consecutive days } \\
\hline & & 1 & 3 & 7 & 14 & 30 & 60 & 90 & 120 & 183 \\
\hline 0.05 & 20 & ng & $\mathrm{ng}$ & ng & ng & ng & ng & $\mathrm{ng}$ & ng & ng \\
\hline 0.10 & 10 & ng & $\mathrm{ng}$ & ng & ng & ng & ng & $\mathrm{ng}$ & ng & ng \\
\hline 0.20 & 5 & ng & $\mathrm{ng}$ & ng & ng & ng & ng & $\mathrm{ng}$ & ng & ng \\
\hline 0.50 & 2 & ng & ng & $\mathrm{ng}$ & ng & ng & ng & ng & ng & ng \\
\hline
\end{tabular}




\section{0-1.L00-1T MAIMANA RIVER NEAR PATA BABA, Continued}

Probability of occurrence of seasonal low discharges

[m $\mathrm{m}^{3} / \mathrm{s}$, meters per second; ng, not given]

\begin{tabular}{|c|c|c|c|c|c|c|c|c|c|}
\hline \multirow{3}{*}{$\begin{array}{c}\text { Nonexceedance } \\
\text { probability }\end{array}$} & \multirow{3}{*}{$\begin{array}{c}\text { Recurrence } \\
\text { interval } \\
\text { (years) }\end{array}$} & \multicolumn{8}{|c|}{ Minimum daily mean discharge $\left(\mathrm{m}^{3} / \mathrm{s}\right)$} \\
\hline & & \multicolumn{8}{|c|}{ Number of consecutive days } \\
\hline & & 1 & 7 & 14 & 30 & 1 & 7 & 14 & 30 \\
\hline & & \multicolumn{4}{|c|}{ December-January-February } & \multicolumn{4}{|c|}{ March-April-May } \\
\hline 0.05 & 20 & 0 & 0 & 0 & 0 & 0 & 0 & 0 & 0 \\
\hline 0.10 & 10 & 0 & 0 & 0 & 0.01 & 0 & 0 & 0 & 0.01 \\
\hline 0.20 & 5 & 0 & 0 & 0.01 & 0.03 & 0 & 0 & 0.02 & 0.03 \\
\hline \multirow[t]{2}{*}{0.50} & 2 & 0.07 & 0 & 0.13 & 0.27 & 0.02 & 0.14 & 0.20 & 0.50 \\
\hline & & \multicolumn{4}{|c|}{ June-July-August } & \multicolumn{4}{|c|}{ September-October-November } \\
\hline 0.05 & 20 & $\mathrm{ng}$ & ng & ng & ng & ng & ng & ng & ng \\
\hline 0.10 & 10 & ng & ng & ng & ng & ng & ng & ng & ng \\
\hline 0.20 & 5 & ng & ng & ng & ng & ng & ng & ng & $\mathrm{ng}$ \\
\hline 0.50 & 2 & ng & ng & $\mathrm{ng}$ & ng & ng & ng & ng & $\mathrm{ng}$ \\
\hline
\end{tabular}

\section{0-1.L00-1T MAIMANA RIVER NEAR PATA BABA, Continued}

Annual peak discharges

[ $\mathrm{m}^{3} / \mathrm{s}$, meters per second]

\begin{tabular}{|c|c|c|c|c|c|}
\hline \multicolumn{3}{|c|}{$\begin{array}{c}\text { Annual peak discharge, } \\
\text { by year }\end{array}$} & \multicolumn{3}{|c|}{$\begin{array}{l}\text { Annual peak discharge, } \\
\text { from highest to lowest }\end{array}$} \\
\hline $\begin{array}{l}\text { Water } \\
\text { year }\end{array}$ & Date & $\begin{array}{c}\text { Peak } \\
\text { discharge } \\
\left(\mathrm{m}^{3} / \mathrm{s}\right)\end{array}$ & $\begin{array}{l}\text { Water } \\
\text { year }\end{array}$ & Date & $\begin{array}{c}\text { Peak } \\
\text { discharge } \\
\left(\mathrm{m}^{3} / \mathrm{s}\right)\end{array}$ \\
\hline 1968 & April 27, 1968 & 31.0 & 1969 & April 14, 1969 & 60.0 \\
\hline 1969 & April 14, 1969 & 60.0 & 1972 & March 19, 1972 & 60.0 \\
\hline 1970 & April 15, 1970 & 38.8 & 1970 & April 15, 1970 & 38.8 \\
\hline 1971 & April 16, 1971 & 12.0 & 1968 & April 27, 1968 & 31.0 \\
\hline 1972 & March 19, 1972 & 60.0 & 1971 & April 16, 1971 & 12.0 \\
\hline
\end{tabular}


10-1.L00-1T MAIMANA RIVER NEAR PATA BABA, Continued

Monthly and annual mean discharges, in cubic meters per second $[--$, no data]

\begin{tabular}{|c|c|c|c|c|c|c|c|c|c|c|c|c|c|}
\hline \multirow{2}{*}{$\begin{array}{l}\text { Water } \\
\text { year }\end{array}$} & \multicolumn{12}{|c|}{ Monthly mean discharge } & \multirow{2}{*}{$\begin{array}{c}\text { Annual } \\
\text { discharge }\end{array}$} \\
\hline & October & November & December & January & February & March & April & May & June & July & August & September & \\
\hline 1968 & -- & -- & 0.16 & 0.13 & 0.30 & 0.73 & 3.51 & 4.30 & 0.16 & 0.04 & 0 & 0.08 & -- \\
\hline 1969 & 0.25 & 0.24 & 2.33 & 1.50 & 3.73 & 8.08 & 28.0 & 16.9 & 1.71 & 0.13 & 0.22 & 0.48 & 5.29 \\
\hline 1970 & 0.71 & 1.30 & 0.95 & 0.82 & 0.48 & 2.40 & 1.50 & 0.04 & 0.03 & 0 & 0 & 0.04 & 0.69 \\
\hline 1971 & 0.27 & 0.28 & 0.37 & 0.39 & 0.50 & 0.53 & 0.62 & 0.01 & 0 & 0 & 0 & 0 & 0.25 \\
\hline 1972 & 0.06 & 0.23 & 0.18 & 0.58 & 5.38 & 9.74 & 11.6 & 10.1 & 3.45 & 0.47 & 0.45 & 0.43 & 3.54 \\
\hline
\end{tabular}




\section{1-0.000-4M SARE PUL RIVER AT ASIABAD}

\section{(U.S. Geological Survey identification number: 361200065570000)}

LOCATION: Lat $36^{\circ} 12^{\prime} \mathrm{N}$., long $65^{\circ} 57^{\prime} \mathrm{E}$.

DRAINAGE AREA: $6,950 \mathrm{~km}^{2}$.

ELEVATION: 642 meters above mean sea level.

PERIOD OF RECORD: October 1, 1964 to September 30, 1978.

GAGE: Water-stage recorder. Staff gage at same site and datum prior to 1970 water year.

Annual mean discharge

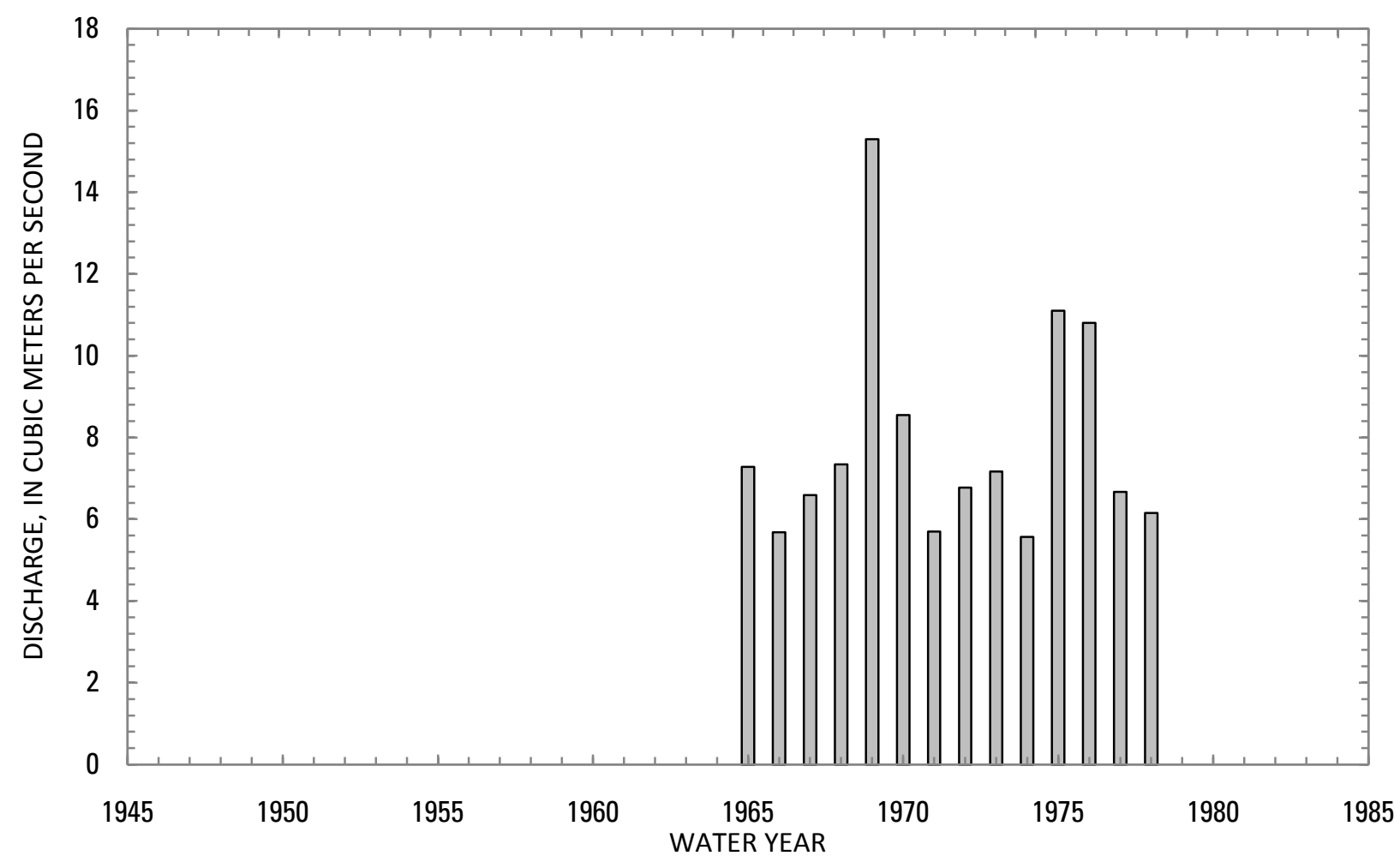




\section{1-0.000-4M SARE PUL RIVER AT ASIABAD, Continued}

Statistics of monthly and annual mean discharges $\left[\mathrm{m}^{3} / \mathrm{s}\right.$, cubic meters per second]

\begin{tabular}{|c|c|c|c|c|c|c|c|c|}
\hline \multirow[b]{2}{*}{ Month } & \multicolumn{2}{|c|}{ Maximum } & \multicolumn{2}{|c|}{ Minimum } & \multicolumn{4}{|c|}{ Mean } \\
\hline & $\begin{array}{c}\text { Discharge } \\
\left(\mathrm{m}^{3} / \mathrm{s}\right)\end{array}$ & $\begin{array}{c}\text { Water year } \\
\text { of } \\
\text { occurrence }\end{array}$ & $\begin{array}{c}\text { Discharge } \\
\left(\mathrm{m}^{3} / \mathrm{s}\right)\end{array}$ & $\begin{array}{c}\text { Water year } \\
\text { of } \\
\text { occurrence }\end{array}$ & $\begin{array}{c}\text { Discharge } \\
\left(\mathrm{m}^{3} / \mathrm{s}\right)\end{array}$ & $\begin{array}{c}\text { Standard } \\
\text { deviation } \\
\left(\mathrm{m}^{3} / \mathrm{s}\right)\end{array}$ & $\begin{array}{c}\text { Coefficient } \\
\text { of } \\
\text { variation }\end{array}$ & $\begin{array}{c}\text { Percentage } \\
\text { of annual } \\
\text { discharge }\end{array}$ \\
\hline October & 13.1 & 1970 & 4.74 & 1972 & 7.21 & 2.23 & 0.31 & 7.60 \\
\hline November & 11.7 & 1970 & 5.38 & 1973 & 7.70 & 1.75 & 0.23 & 8.12 \\
\hline December & 10.3 & 1976 & 5.11 & 1973 & 7.36 & 1.75 & 0.24 & 7.76 \\
\hline January & 9.41 & 1976 & 5.73 & 1973 & 7.01 & 1.29 & 0.18 & 7.39 \\
\hline February & 9.81 & 1976 & 5.80 & 1968 & 7.59 & 1.26 & 0.17 & 8.00 \\
\hline March & 15.7 & 1969 & 6.29 & 1967 & 9.31 & 2.53 & 0.27 & 9.81 \\
\hline April & 31.1 & 1969 & 6.36 & 1966 & 12.2 & 7.06 & 0.58 & 12.9 \\
\hline May & 29.9 & 1969 & 3.77 & 1971 & 12.5 & 8.19 & 0.66 & 13.2 \\
\hline June & 23.6 & 1969 & 3.14 & 1977 & 7.91 & 5.62 & 0.71 & 8.34 \\
\hline July & 15.6 & 1969 & 2.27 & 1977 & 5.52 & 3.53 & 0.64 & 5.82 \\
\hline August & 12.1 & 1969 & 2.19 & 1977 & 4.90 & 2.71 & 0.55 & 5.16 \\
\hline September & 12.4 & 1969 & 3.27 & 1966 & 5.65 & 2.56 & 0.45 & 5.95 \\
\hline Annual & 15.3 & 1969 & 5.57 & 1974 & 7.90 & 2.74 & 0.35 & 100 \\
\hline
\end{tabular}


11-0.000-4M SARE PUL RIVER AT ASIABAD, Continued

Monthly and annual flow duration, in cubic meters per second

\begin{tabular}{|c|c|c|c|c|c|c|c|c|c|c|c|c|c|}
\hline \multirow{2}{*}{$\begin{array}{l}\text { Percentage } \\
\text { of days } \\
\text { discharge } \\
\text { equaled or } \\
\text { exceeded }\end{array}$} & \multicolumn{12}{|c|}{ Month } & \multirow{2}{*}{ Annual } \\
\hline & October & November & December & January & February & March & April & May & June & July & August & September & \\
\hline 95 & 4.25 & 5.23 & 4.73 & 5.23 & 5.68 & 6.06 & 5.28 & 3.42 & 2.51 & 2.11 & 1.93 & 2.56 & 2.80 \\
\hline 90 & 4.82 & 5.69 & 5.26 & 5.54 & 5.88 & 6.36 & 5.64 & 4.22 & 2.88 & 2.43 & 2.28 & 3.01 & 3.63 \\
\hline 85 & 5.30 & 5.94 & 5.51 & 5.70 & 6.08 & 6.63 & 5.91 & 4.93 & 3.38 & 2.59 & 2.47 & 3.32 & 4.21 \\
\hline 80 & 5.49 & 6.13 & 5.69 & 5.80 & 6.23 & 6.87 & 6.15 & 5.22 & 3.63 & 2.92 & 2.72 & 3.51 & 4.79 \\
\hline 75 & 5.68 & 6.40 & 5.96 & 5.92 & 6.36 & 7.09 & 6.40 & 5.53 & 3.89 & 3.23 & 2.97 & 3.81 & 5.24 \\
\hline 70 & 5.87 & 6.59 & 6.24 & 6.05 & 6.57 & 7.30 & 6.73 & 6.22 & 4.23 & 3.55 & 3.18 & 3.97 & 5.60 \\
\hline 65 & 6.01 & 6.78 & 6.47 & 6.12 & 6.77 & 7.51 & 7.09 & 6.76 & 4.63 & 3.79 & 3.40 & 4.19 & 5.95 \\
\hline 60 & 6.17 & 7.04 & 6.68 & 6.20 & 6.93 & 7.73 & 7.67 & 7.54 & 5.02 & 4.00 & 3.66 & 4.41 & 6.26 \\
\hline 55 & 6.39 & 7.37 & 6.88 & 6.29 & 7.10 & 7.95 & 8.24 & 8.17 & 5.62 & 4.21 & 3.80 & 4.69 & 6.56 \\
\hline 50 & 6.77 & 7.52 & 7.13 & 6.46 & 7.25 & 8.17 & 8.79 & 8.84 & 6.05 & 4.42 & 3.91 & 4.94 & 6.86 \\
\hline 45 & 7.11 & 7.70 & 7.35 & 6.82 & 7.37 & 8.39 & 9.33 & 9.79 & 6.80 & 4.79 & 4.20 & 5.13 & 7.21 \\
\hline 40 & 7.40 & 7.86 & 7.55 & 7.13 & 7.57 & 8.69 & 9.98 & 10.7 & 7.48 & 5.08 & 4.73 & 5.82 & 7.56 \\
\hline 35 & 7.66 & 7.98 & 7.71 & 7.45 & 8.02 & 9.05 & 10.8 & 11.9 & 8.13 & 5.35 & 5.05 & 6.10 & 7.94 \\
\hline 30 & 7.97 & 8.09 & 7.87 & 7.68 & 8.54 & 9.70 & 12.1 & 13.0 & 9.19 & 5.64 & 5.33 & 6.45 & 8.39 \\
\hline 25 & 8.32 & 8.45 & 8.21 & 7.85 & 8.84 & 10.1 & 13.5 & 14.6 & 9.95 & 6.33 & 5.92 & 7.03 & 8.83 \\
\hline 20 & 8.63 & 8.78 & 8.76 & 8.45 & 9.06 & 10.4 & 14.6 & 17.8 & 10.9 & 7.34 & 6.65 & 7.45 & 9.60 \\
\hline 15 & 9.11 & 10.1 & 9.67 & 9.01 & 9.27 & 10.9 & 16.3 & 21.9 & 12.7 & 8.25 & 8.33 & 8.42 & 10.5 \\
\hline 10 & 10.1 & 10.5 & 10.4 & 9.24 & 9.64 & 12.8 & 22.0 & 25.3 & 15.9 & 10.6 & 9.06 & 8.97 & 12.1 \\
\hline 5 & 12.7 & 11.1 & 10.8 & 9.44 & 10.2 & 16.1 & 30.4 & 30.9 & 20.7 & 14.6 & 11.7 & 12.0 & 15.8 \\
\hline
\end{tabular}




\section{1-0.000-4M SARE PUL RIVER AT ASIABAD, Continued}

Probability of occurrence of annual high discharges

[ $\mathrm{m}^{3} / \mathrm{s}$, cubic meters per second; ng, not given]

\begin{tabular}{|c|c|c|c|c|c|c|}
\hline \multirow{2}{*}{$\begin{array}{l}\text { Exceedance } \\
\text { probability }\end{array}$} & \multirow{2}{*}{$\begin{array}{c}\text { Recurrence } \\
\text { interval } \\
\text { (years) }\end{array}$} & \multirow{2}{*}{$\begin{array}{l}\text { Maximum } \\
\text { instantaneous } \\
\text { discharge } \\
\left(\mathrm{m}^{3} / \mathrm{s}\right)\end{array}$} & \multicolumn{4}{|c|}{ Maximum daily mean discharge $\left(\mathrm{m}^{3} / \mathrm{s}\right)$} \\
\hline & & & $\begin{array}{l}\text { 3-day } \\
\text { period }\end{array}$ & $\begin{array}{l}\text { 7-day } \\
\text { period }\end{array}$ & $\begin{array}{l}\text { 15-day } \\
\text { period }\end{array}$ & $\begin{array}{l}\text { 30-day } \\
\text { period }\end{array}$ \\
\hline 0.99 & 1.01 & 5.00 & ${ }^{1} 5.20$ & ${ }^{1} 5.08$ & ${ }^{1} 5.36$ & 5.73 \\
\hline 0.95 & 1.05 & 11.3 & 8.28 & 7.33 & 7.04 & 6.98 \\
\hline 0.90 & 1.11 & 17.0 & 10.6 & 8.99 & 8.25 & 7.89 \\
\hline 0.80 & 1.25 & 27.6 & 14.5 & 11.6 & 10.1 & 9.29 \\
\hline 0.50 & 2 & 64.9 & 26.2 & 19.3 & 15.7 & 13.4 \\
\hline 0.20 & 5 & 141 & 47.8 & 33.3 & 25.9 & 20.7 \\
\hline 0.10 & 10 & 205 & 65.8 & 44.7 & 34.5 & 26.8 \\
\hline 0.04 & 25 & 300 & 92.8 & 62.0 & 47.7 & 36.2 \\
\hline 0.02 & 50 & 378 & 116 & 77.0 & 59.4 & 44.5 \\
\hline 0.01 & 100 & 462 & 142 & 93.9 & 73.0 & 54.0 \\
\hline 0.005 & 200 & 552 & 171 & 113 & 88.6 & 65.1 \\
\hline 0.002 & 500 & 679 & $\mathrm{ng}$ & $\mathrm{ng}$ & $\mathrm{ng}$ & $\mathrm{ng}$ \\
\hline
\end{tabular}

'Data does not fit log-Pearson Type III curve, use with caution.

11-0.000-4M SARE PUL RIVER AT ASIABAD, Continued

Probability of occurrence of annual low discharges

$\left[\mathrm{m}^{3} / \mathrm{s}\right.$, meters per second]

\begin{tabular}{|c|c|c|c|c|c|c|c|c|c|c|}
\hline \multirow{3}{*}{$\begin{array}{c}\text { Nonexceedance } \\
\text { probability }\end{array}$} & \multirow{3}{*}{$\begin{array}{l}\text { Recurrence } \\
\text { interval } \\
\text { (years) }\end{array}$} & \multicolumn{9}{|c|}{ Minimum daily mean discharge $\left(\mathrm{m}^{3} / \mathrm{s}\right)$} \\
\hline & & \multicolumn{9}{|c|}{ Number of consecutive days } \\
\hline & & 1 & 3 & 7 & 14 & 30 & 60 & 90 & 120 & 183 \\
\hline 0.05 & 20 & 1.34 & 1.52 & 1.60 & 2.05 & 2.15 & 2.23 & 2.38 & 2.57 & 3.26 \\
\hline 0.10 & 10 & 1.54 & 1.70 & 1.79 & 2.31 & 2.43 & 2.55 & 2.72 & 2.93 & 3.62 \\
\hline 0.20 & 5 & 1.86 & 2.00 & 2.10 & 2.70 & 2.85 & 3.01 & 3.22 & 3.46 & 4.14 \\
\hline 0.50 & 2 & 2.81 & 2.91 & 3.03 & 3.81 & 4.01 & 4.27 & 4.55 & 4.84 & 5.50 \\
\hline
\end{tabular}




\section{1-0.000-4M SARE PUL RIVER AT ASIABAD, Continued}

Probability of occurrence of seasonal low discharges [ $\mathrm{m}^{3} / \mathrm{s}$, meters per second]

\begin{tabular}{|c|c|c|c|c|c|c|c|c|c|}
\hline \multirow{3}{*}{$\begin{array}{c}\text { Nonexceedance } \\
\text { probability }\end{array}$} & \multirow{3}{*}{$\begin{array}{c}\text { Recurrence } \\
\text { interval } \\
\text { (years) }\end{array}$} & \multicolumn{8}{|c|}{ Minimum daily mean discharge $\left(\mathrm{m}^{3} / \mathrm{s}\right)$} \\
\hline & & \multicolumn{8}{|c|}{ Number of consecutive days } \\
\hline & & 1 & 7 & 14 & 30 & 1 & 7 & 14 & 30 \\
\hline & & \multicolumn{4}{|c|}{ December-January-February } & \multicolumn{4}{|c|}{ March-April-May } \\
\hline 0.05 & 20 & 3.77 & 4.03 & 4.50 & 4.97 & 2.34 & 2.79 & 3.44 & 3.98 \\
\hline 0.10 & 10 & 4.16 & 4.40 & 4.81 & 5.22 & 2.80 & 3.22 & 3.90 & 4.51 \\
\hline 0.20 & 5 & 4.68 & 4.90 & 5.23 & 5.57 & 3.45 & 3.85 & 4.56 & 5.26 \\
\hline \multirow[t]{2}{*}{0.50} & 2 & 5.86 & 6.04 & 6.24 & 6.46 & 4.99 & 5.50 & 6.22 & 7.15 \\
\hline & & \multicolumn{4}{|c|}{ June-July-August } & \multicolumn{4}{|c|}{ September-October-November } \\
\hline 0.05 & 20 & 1.40 & 1.71 & 2.14 & 2.24 & 1.68 & 2.12 & 2.59 & 3.02 \\
\hline 0.10 & 10 & 1.55 & 1.85 & 2.34 & 2.46 & 1.94 & 2.37 & 2.90 & 3.33 \\
\hline 0.20 & 5 & 1.82 & 2.09 & 2.66 & 2.82 & 2.33 & 2.75 & 3.36 & 3.80 \\
\hline 0.50 & 2 & 2.69 & 2.92 & 3.69 & 3.91 & 3.49 & 3.89 & 4.64 & 5.10 \\
\hline
\end{tabular}

\section{1-0.000-4M SARE PUL RIVER AT ASIABAD, Continued}

Annual peak discharges

[ $\mathrm{m}^{3} / \mathrm{s}$, meters per second]

\begin{tabular}{|c|c|c|c|c|c|}
\hline \multicolumn{3}{|c|}{$\begin{array}{c}\text { Annual peak discharge, } \\
\text { by year }\end{array}$} & \multicolumn{3}{|c|}{$\begin{array}{l}\text { Annual peak discharge, } \\
\text { from highest to lowest }\end{array}$} \\
\hline $\begin{array}{l}\text { Water } \\
\text { year }\end{array}$ & Date & $\begin{array}{c}\text { Peak } \\
\text { discharge } \\
\left(\mathrm{m}^{3} / \mathrm{s}\right)\end{array}$ & $\begin{array}{l}\text { Water } \\
\text { year }\end{array}$ & Date & $\begin{array}{c}\text { Peak } \\
\text { discharge } \\
\left(\mathrm{m}^{3} / \mathrm{s}\right)\end{array}$ \\
\hline 1965 & May 6, 1965 & 24.5 & 1976 & April 28, 1976 & 220 \\
\hline 1966 & March 22, 1966 & 15.0 & 1977 & May 29, 1977 & 195 \\
\hline 1967 & May 1,1967 & 97.2 & 1972 & March 27, 1972 & 170 \\
\hline 1968 & November 27, 1967 & 58.0 & 1975 & April 22, 1975 & 150 \\
\hline 1969 & March 29, 1969 & 95.0 & 1967 & May 1,1967 & 97.2 \\
\hline 1970 & March 8, 1970 & 30.0 & 1978 & April 26, 1978 & 97.0 \\
\hline 1971 & April 15, 1971 & 23.9 & 1969 & March 29, 1969 & 95.0 \\
\hline 1972 & March 27, 1972 & 170 & 1973 & $\begin{array}{c}\text { March 25, } 1973 \\
\text { November 27, }\end{array}$ & 60.4 \\
\hline 1973 & March 25, 1973 & 60.4 & 1968 & 1967 & 58.0 \\
\hline 1974 & May 3, 1974 & 11.8 & 1970 & March 8, 1970 & 30.0 \\
\hline 1975 & April 22, 1975 & 150 & 1965 & May 6, 1965 & 24.5 \\
\hline 1976 & April 28, 1976 & 220 & 1971 & April 15, 1971 & 23.9 \\
\hline 1977 & May 29, 1977 & 195 & 1966 & March 22, 1966 & 15.0 \\
\hline 1978 & April 26, 1978 & 97.0 & 1974 & May 3, 1974 & 11.8 \\
\hline
\end{tabular}




\section{1-0.000-4M SARE PUL RIVER AT ASIABAD, Continued}

Monthly and annual mean discharges, in cubic meters per second

[Data may not be rounded in accordance with U.S. Geological Survey publication standards]

\begin{tabular}{|c|c|c|c|c|c|c|c|c|c|c|c|c|c|}
\hline \multirow{2}{*}{$\begin{array}{l}\text { Water } \\
\text { year }\end{array}$} & \multicolumn{12}{|c|}{ Monthly mean discharge } & \multirow{2}{*}{$\begin{array}{l}\text { Annual } \\
\text { discharge }\end{array}$} \\
\hline & October & November & December & January & February & March & April & May & June & July & August & September & \\
\hline 1965 & 6.96 & 7.62 & 7.56 & 7.43 & 8.08 & 9.37 & 8.87 & 9.96 & 6.67 & 4.71 & 4.35 & 5.88 & 7.28 \\
\hline 1966 & 7.82 & 7.39 & 6.64 & 5.93 & 7.73 & 9.31 & 6.36 & 4.70 & 3.33 & 2.95 & 2.81 & 3.27 & 5.68 \\
\hline 1967 & 5.20 & 6.05 & 5.57 & 5.90 & 6.00 & 6.29 & 7.52 & 15.4 & 7.49 & 4.97 & 4.20 & 4.48 & 6.59 \\
\hline 1968 & 5.76 & 7.09 & 5.57 & 5.83 & 5.80 & 7.25 & 9.76 & 12.5 & 9.79 & 6.38 & 5.84 & 6.58 & 7.34 \\
\hline 1969 & 7.98 & 8.39 & 9.75 & 7.81 & 8.55 & 15.7 & 31.1 & 29.9 & 23.6 & 15.6 & 12.1 & 12.4 & 15.3 \\
\hline 1970 & 13.1 & 11.7 & 10.2 & 9.31 & 9.55 & 11.3 & 11.0 & 7.27 & 4.72 & 4.63 & 4.43 & 5.41 & 8.55 \\
\hline 1971 & 7.53 & 7.90 & 7.97 & 7.33 & 7.30 & 6.83 & 7.16 & 3.77 & 3.16 & 2.90 & 3.05 & 3.59 & 5.70 \\
\hline 1972 & 4.74 & 5.51 & 5.58 & 6.19 & 7.13 & 11.1 & 7.91 & 14.3 & 7.60 & 3.89 & 3.38 & 3.91 & 6.77 \\
\hline 1973 & 5.41 & 5.38 & 5.11 & 5.73 & 6.75 & 11.1 & 14.5 & 10.6 & 7.31 & 4.45 & 4.14 & 5.63 & 7.17 \\
\hline 1974 & 6.23 & 6.53 & 6.36 & 6.05 & 6.65 & 7.06 & 6.67 & 6.58 & 4.21 & 3.45 & 3.21 & 3.94 & 5.57 \\
\hline 1975 & 6.02 & 7.05 & 6.72 & 5.97 & 7.55 & 8.93 & 20.6 & 28.6 & 15.1 & 9.96 & 8.15 & 8.37 & 11.1 \\
\hline 1976 & 9.67 & 10.3 & 10.3 & 9.41 & 9.81 & 10.7 & 19.3 & 17.5 & 10.1 & 7.38 & 7.60 & 8.17 & 10.8 \\
\hline 1977 & 8.80 & 8.76 & 7.73 & 8.26 & 8.88 & 7.80 & 12.4 & 6.39 & 3.14 & 2.27 & 2.19 & 3.61 & 6.67 \\
\hline 1978 & 5.74 & 8.17 & 8.01 & 6.96 & 6.44 & 7.56 & 8.14 & 7.45 & 4.51 & 3.81 & 3.15 & 3.83 & 6.15 \\
\hline
\end{tabular}




\section{1-1.R00-1A SHORAB RIVER NEAR SARE PUL}

\section{(U.S. Geological Survey identification number: $\mathbf{3 6 1 1 0 0 0 6 6 0 2 0 0 0 0 )}$}

LOCATION: Lat $36^{\circ} 11^{\prime} \mathrm{N}$., long $66^{\circ} 02^{\prime} \mathrm{E}$.

DRAINAGE AREA: $3,700 \mathrm{~km}^{2}$.

ELEVATION: 703 meters above mean sea level.

PERIOD OF RECORD: November 24, 1969 to September 30, 1978.

GAGE: Water-stage recorder.

Annual mean discharge

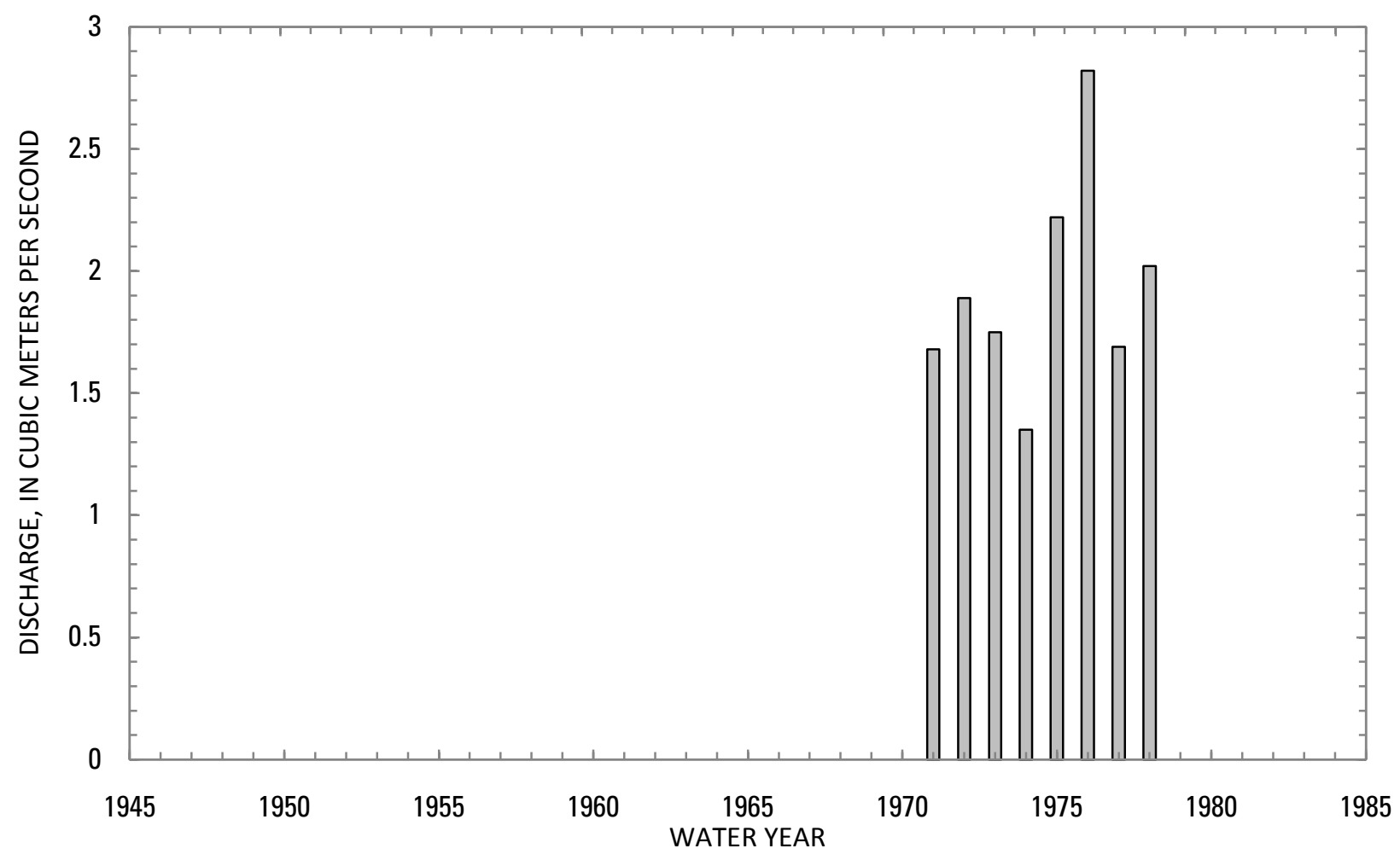




\section{1-1.R00-1A SHORAB RIVER NEAR SARE PUL, Continued}

Statistics of monthly and annual mean discharges [m $\mathrm{m}^{3} / \mathrm{s}$, cubic meters per second]

\begin{tabular}{|c|c|c|c|c|c|c|c|c|}
\hline \multirow[b]{2}{*}{ Month } & \multicolumn{2}{|c|}{ Maximum } & \multicolumn{2}{|c|}{ Minimum } & \multicolumn{4}{|c|}{ Mean } \\
\hline & $\begin{array}{c}\text { Discharge } \\
\left(\mathrm{m}^{3} / \mathrm{s}\right)\end{array}$ & $\begin{array}{c}\text { Water year } \\
\text { of } \\
\text { occurrence }\end{array}$ & $\begin{array}{c}\text { Discharge } \\
\left(\mathrm{m}^{3} / \mathrm{s}\right)\end{array}$ & $\begin{array}{c}\text { Water year } \\
\text { of } \\
\text { occurrence }\end{array}$ & $\begin{array}{c}\text { Discharge } \\
\left(\mathrm{m}^{3} / \mathrm{s}\right)\end{array}$ & $\begin{array}{c}\text { Standard } \\
\text { deviation } \\
\left(\mathrm{m}^{3} / \mathrm{s}\right)\end{array}$ & $\begin{array}{c}\text { Coefficient } \\
\text { of } \\
\text { variation }\end{array}$ & $\begin{array}{c}\text { Percentage } \\
\text { of annual } \\
\text { discharge }\end{array}$ \\
\hline October & 3.11 & 1976 & 1.77 & 1975 & 2.28 & 0.53 & 0.23 & 9.87 \\
\hline November & 4.05 & 1978 & 2.24 & 1973 & 2.81 & 0.74 & 0.26 & 12.1 \\
\hline December & 3.31 & 1976 & 2.08 & 1972 & 2.60 & 0.49 & 0.19 & 11.3 \\
\hline January & 3.04 & 1977 & 1.67 & 1973 & 2.31 & 0.38 & 0.17 & 9.99 \\
\hline February & 3.74 & 1971 & 2.01 & 1973 & 2.70 & 0.54 & 0.20 & 11.7 \\
\hline March & 4.28 & 1970 & 2.37 & 1974 & 3.30 & 0.71 & 0.22 & 14.3 \\
\hline April & 6.54 & 1976 & 1.40 & 1974 & 3.39 & 1.51 & 0.45 & 14.6 \\
\hline May & 6.11 & 1975 & 0.56 & 1971 & 2.30 & 2.21 & 0.96 & 9.94 \\
\hline June & 0.95 & 1972 & 0.08 & 1974 & 0.26 & 0.27 & 1.05 & 1.11 \\
\hline July & 0.39 & 1973 & 0.01 & 1972 & 0.10 & 0.13 & 1.21 & 0.45 \\
\hline August & 0.58 & 1973 & 0.03 & 1977 & 0.25 & 0.18 & 0.72 & 1.09 \\
\hline September & 1.46 & 1975 & 0.31 & 1974 & 0.82 & 0.43 & 0.53 & 3.56 \\
\hline Annual & 2.82 & 1976 & 1.35 & 1974 & 1.93 & 0.44 & 0.23 & 100 \\
\hline
\end{tabular}


11-1.R00-1A SHORAB RIVER NEAR SARE PUL, Continued

Monthly and annual flow duration, in cubic meters per second

\begin{tabular}{|c|c|c|c|c|c|c|c|c|c|c|c|c|c|}
\hline \multirow{2}{*}{$\begin{array}{l}\text { Percentage } \\
\text { of days } \\
\text { discharge } \\
\text { equaled or } \\
\text { exceeded }\end{array}$} & \multicolumn{12}{|c|}{ Month } & \multirow{2}{*}{ Annual } \\
\hline & October & November & December & January & February & March & April & May & June & July & August & September & \\
\hline 95 & 1.31 & 2.11 & 1.85 & 1.70 & 1.85 & 1.96 & 0.72 & 0.03 & 0.01 & 0.01 & 0.01 & 0.02 & 0.02 \\
\hline 90 & 1.51 & 2.22 & 2.03 & 1.89 & 2.01 & 2.06 & 1.25 & 0.05 & 0.02 & 0.01 & 0.02 & 0.05 & 0.03 \\
\hline 85 & 1.75 & 2.25 & 2.12 & 1.94 & 2.08 & 2.14 & 1.54 & 0.14 & 0.02 & 0.02 & 0.02 & 0.14 & 0.04 \\
\hline 80 & 1.87 & 2.28 & 2.18 & 1.99 & 2.13 & 2.26 & 1.77 & 0.24 & 0.02 & 0.02 & 0.02 & 0.18 & 0.16 \\
\hline 75 & 1.92 & 2.30 & 2.22 & 2.03 & 2.19 & 2.46 & 1.98 & 0.36 & 0.03 & 0.02 & 0.03 & 0.27 & 0.39 \\
\hline 70 & 1.96 & 2.33 & 2.26 & 2.08 & 2.25 & 2.62 & 2.12 & 0.45 & 0.03 & 0.02 & 0.03 & 0.37 & 0.65 \\
\hline 65 & 1.99 & 2.35 & 2.29 & 2.13 & 2.30 & 2.72 & 2.31 & 0.60 & 0.03 & 0.02 & 0.04 & 0.49 & 1.14 \\
\hline 60 & 2.02 & 2.37 & 2.32 & 2.17 & 2.35 & 2.86 & 2.57 & 0.82 & 0.04 & 0.03 & 0.04 & 0.60 & 1.61 \\
\hline 55 & 2.05 & 2.39 & 2.35 & 2.21 & 2.42 & 3.01 & 2.81 & 1.08 & 0.04 & 0.03 & 0.07 & 0.74 & 1.93 \\
\hline 50 & 2.11 & 2.41 & 2.41 & 2.26 & 2.49 & 3.19 & 2.97 & 1.32 & 0.08 & 0.03 & 0.13 & 0.82 & 2.05 \\
\hline 45 & 2.19 & 2.54 & 2.52 & 2.31 & 2.62 & 3.28 & 3.16 & 1.56 & 0.11 & 0.03 & 0.16 & 0.92 & 2.17 \\
\hline 40 & 2.30 & 2.63 & 2.60 & 2.38 & 2.80 & 3.35 & 3.42 & 1.8 & 0.13 & 0.04 & 0.19 & 1.03 & 2.29 \\
\hline 35 & 2.42 & 2.73 & 2.69 & 2.44 & 2.90 & 3.46 & 3.56 & 2.33 & 0.15 & 0.04 & 0.27 & 1.20 & 2.42 \\
\hline 30 & 2.52 & 2.83 & 2.79 & 2.50 & 3.00 & 3.62 & 3.70 & 2.72 & 0.17 & 0.05 & 0.32 & 1.27 & 2.61 \\
\hline 25 & 2.60 & 3.23 & 2.90 & 2.57 & 3.18 & 3.77 & 3.91 & 3.46 & 0.24 & 0.13 & 0.43 & 1.34 & 2.80 \\
\hline 20 & 2.71 & 3.41 & 3.11 & 2.65 & 3.33 & 3.89 & 4.31 & 4.15 & 0.30 & 0.20 & 0.52 & 1.42 & 2.98 \\
\hline 15 & 2.91 & 3.81 & 3.25 & 2.75 & 3.44 & 4.25 & 4.99 & 4.89 & 0.42 & 0.29 & 0.61 & 1.50 & 3.30 \\
\hline 10 & 3.16 & 3.93 & 3.39 & 2.96 & 3.70 & 4.68 & 5.64 & 5.72 & 0.64 & 0.37 & 0.70 & 1.59 & 3.68 \\
\hline 5 & 3.73 & 4.16 & 3.61 & 3.35 & 3.90 & 5.83 & 6.40 & 7.05 & 1.50 & 0.47 & 0.92 & 1.76 & 4.53 \\
\hline
\end{tabular}




\section{1-1.R00-1A SHORAB RIVER NEAR SARE PUL, Continued}

Probability of occurrence of annual high discharges

[m $\mathrm{m}^{3} / \mathrm{s}$, cubic meters per second; ng, not given]

\begin{tabular}{|c|c|c|c|c|c|c|}
\hline \multirow{2}{*}{$\begin{array}{c}\text { Exceedance } \\
\text { probability }\end{array}$} & \multirow{2}{*}{$\begin{array}{l}\text { Recurrence } \\
\text { interval } \\
\text { (years) }\end{array}$} & \multirow{2}{*}{$\begin{array}{c}\text { Maximum } \\
\text { instantaneous } \\
\text { discharge }^{1} \\
\left(\mathrm{~m}^{3} / \mathrm{s}\right)\end{array}$} & \multicolumn{4}{|c|}{ Maximum daily mean discharge $\left(\mathrm{m}^{3} / \mathrm{s}\right)$} \\
\hline & & & $\begin{array}{l}\text { 3-day } \\
\text { period }\end{array}$ & $\begin{array}{l}\text { 7-day } \\
\text { period }\end{array}$ & $\begin{array}{l}\text { 15-day } \\
\text { period }\end{array}$ & $\begin{array}{l}\text { 30-day } \\
\text { period }\end{array}$ \\
\hline 0.99 & 1.01 & 3.60 & 2.10 & 2.02 & 2.01 & ${ }^{2} 2.02$ \\
\hline 0.95 & 1.05 & 5.50 & 2.67 & 2.57 & 2.52 & 2.47 \\
\hline 0.90 & 1.11 & 7.00 & 3.12 & 2.98 & 2.87 & 2.77 \\
\hline 0.80 & 1.25 & 9.50 & 3.89 & 3.64 & 3.39 & 3.21 \\
\hline 0.50 & 2 & 17.9 & 6.54 & 5.64 & 4.77 & 4.34 \\
\hline 0.20 & 5 & 35.3 & 12.7 & 9.51 & 6.94 & 6.06 \\
\hline 0.10 & 10 & 51.5 & 19.1 & 12.9 & 8.57 & 7.31 \\
\hline 0.04 & 25 & 78.4 & 31.1 & 18.5 & 10.9 & 9.02 \\
\hline 0.02 & 50 & 104 & 43.7 & 23.6 & 12.7 & 10.4 \\
\hline 0.01 & 100 & 134 & 60.5 & 29.8 & 14.7 & 11.8 \\
\hline 0.005 & 200 & 171 & 82.7 & 37.2 & 16.9 & 13.4 \\
\hline 0.002 & 500 & 231 & $\mathrm{ng}$ & $\mathrm{ng}$ & $\mathrm{ng}$ & $\mathrm{ng}$ \\
\hline
\end{tabular}

11-1.R00-1A SHORAB RIVER NEAR SARE PUL, Continued

Probability of occurrence of annual low discharges

$\left[\mathrm{m}^{3} / \mathrm{s}\right.$, meters per second]

\begin{tabular}{|c|c|c|c|c|c|c|c|c|c|c|}
\hline \multirow{3}{*}{$\begin{array}{c}\text { Nonexceedance } \\
\text { probability }\end{array}$} & \multirow{3}{*}{$\begin{array}{c}\text { Recurrence } \\
\text { interval } \\
\text { (years) }\end{array}$} & \multicolumn{9}{|c|}{ Minimum daily mean discharge $\left(\mathrm{m}^{3} / \mathrm{s}\right)$} \\
\hline & & \multicolumn{9}{|c|}{ Number of consecutive days } \\
\hline & & 1 & 3 & 7 & 14 & 30 & 60 & 90 & 120 & 183 \\
\hline 0.05 & 20 & 0.01 & 0.01 & 0.01 & 0.01 & 0.01 & 0.01 & 0.03 & 0.13 & 0.44 \\
\hline 0.10 & 10 & 0.01 & 0.01 & 0.01 & 0.01 & 0.01 & 0.02 & 0.04 & 0.15 & 0.49 \\
\hline 0.20 & 5 & 0.01 & 0.01 & 0.01 & 0.01 & 0.02 & 0.02 & 0.06 & 0.18 & 0.58 \\
\hline 0.50 & 2 & 0.01 & 0.01 & 0.01 & 0.03 & 0.03 & 0.05 & 0.14 & 0.26 & 0.79 \\
\hline
\end{tabular}




\section{1-1.R00-1A SHORAB RIVER NEAR SARE PUL, Continued}

Probability of occurrence of seasonal low discharges

[ $\mathrm{m}^{3} / \mathrm{s}$, meters per second]

\begin{tabular}{|c|c|c|c|c|c|c|c|c|c|}
\hline \multirow{3}{*}{$\begin{array}{c}\text { Nonexceedance } \\
\text { probability }\end{array}$} & \multirow{3}{*}{$\begin{array}{c}\text { Recurrence } \\
\text { interval } \\
\text { (years) }\end{array}$} & \multicolumn{8}{|c|}{ Minimum daily mean discharge $\left(\mathrm{m}^{3} / \mathrm{s}\right)$} \\
\hline & & \multicolumn{8}{|c|}{ Number of consecutive days } \\
\hline & & 1 & 7 & 14 & 30 & 1 & 7 & 14 & 30 \\
\hline & & \multicolumn{4}{|c|}{ December-January-February } & \multicolumn{4}{|c|}{ March-April-May } \\
\hline 0.05 & 20 & 0.72 & 0.88 & 0.98 & 1.59 & 0.01 & 0.02 & 0.04 & 0.30 \\
\hline 0.10 & 10 & 0.98 & 1.15 & 1.25 & 1.74 & 0.01 & 0.03 & 0.07 & 0.42 \\
\hline 0.20 & 5 & 1.33 & 1.49 & 1.59 & 1.92 & 0.02 & 0.06 & 0.15 & 0.62 \\
\hline \multirow[t]{2}{*}{0.50} & 2 & 1.90 & 2.03 & 2.10 & 2.19 & 0.12 & 0.25 & 0.58 & 1.26 \\
\hline & & \multicolumn{4}{|c|}{ June-July-August } & \multicolumn{4}{|c|}{ September-0ctober-November } \\
\hline 0.05 & 20 & 0.01 & 0.01 & 0.01 & 0.01 & 0 & 0.02 & 0.05 & 0.29 \\
\hline 0.10 & 10 & 0.01 & 0.01 & 0.01 & 0.01 & 0.01 & 0.03 & 0.08 & 0.37 \\
\hline 0.20 & 5 & 0.01 & 0.01 & 0.01 & 0.02 & 0.01 & 0.05 & 0.14 & 0.49 \\
\hline 0.50 & 2 & 0.01 & 0.01 & 0.02 & 0.03 & 0.06 & 0.19 & 0.40 & 0.80 \\
\hline
\end{tabular}

\section{1-1.R00-1A SHORAB RIVER NEAR SARE PUL, Continued}

\section{Annual peak discharges}

[ $\mathrm{m}^{3} / \mathrm{s}$, meters per second]

\begin{tabular}{|c|c|c|c|c|c|}
\hline \multicolumn{3}{|c|}{$\begin{array}{c}\text { Annual peak discharge, } \\
\text { by year }\end{array}$} & \multicolumn{3}{|c|}{$\begin{array}{l}\text { Annual peak discharge, } \\
\text { from highest to lowest }\end{array}$} \\
\hline $\begin{array}{l}\text { Water } \\
\text { year }\end{array}$ & Date & $\begin{array}{c}\text { Peak } \\
\text { discharge } \\
\left(\mathrm{m}^{3} / \mathrm{s}\right)\end{array}$ & $\begin{array}{l}\text { Water } \\
\text { year }\end{array}$ & Date & $\begin{array}{c}\text { Peak } \\
\text { discharge } \\
\left(\mathrm{m}^{3} / \mathrm{s}\right)\end{array}$ \\
\hline 1970 & April 13, 1970 & 16.2 & 1976 & April 28, 1976 & 66.0 \\
\hline 1971 & April 15, 1971 & 10.5 & 1975 & May 4, 1975 & 53.0 \\
\hline 1972 & May 16, 1972 & 23.0 & 1972 & May 16, 1972 & 23.0 \\
\hline 1973 & April 14, 1973 & 16.2 & 1977 & May 29, 1977 & 16.4 \\
\hline 1974 & April 1, 1974 & 5.1 & 1970 & April 13, 1970 & 16.2 \\
\hline 1975 & May 4, 1975 & 53.0 & 1973 & April 14, 1973 & 16.2 \\
\hline 1976 & April 28, 1976 & 66.0 & 1978 & April 26, 1978 & 14.0 \\
\hline 1977 & May 29, 1977 & 16.4 & 1971 & April 15, 1971 & 10.5 \\
\hline 1978 & April 26, 1978 & 14.0 & 1974 & April 1, 1974 & 5.1 \\
\hline
\end{tabular}


11-1.R00-1A SHORAB RIVER NEAR SARE PUL, Continued

Monthly and annual mean discharges, in cubic meters per second

[Data may not be rounded in accordance with U.S. Geological Survey publication standards; --, no data]

\begin{tabular}{|c|c|c|c|c|c|c|c|c|c|c|c|c|c|}
\hline \multirow{2}{*}{$\begin{array}{l}\text { Water } \\
\text { year }\end{array}$} & \multicolumn{12}{|c|}{ Monthly mean discharge } & \multirow{2}{*}{$\begin{array}{c}\text { Annual } \\
\text { discharge }\end{array}$} \\
\hline & October & November & December & January & February & March & April & May & June & July & August & September & \\
\hline 1970 & -- & -- & 3.15 & 2.38 & 2.51 & 4.28 & 2.99 & 0.80 & 0.15 & 0.16 & 0.13 & 0.94 & -- \\
\hline 1971 & 2.25 & 2.34 & 2.29 & 2.42 & 3.74 & 2.85 & 2.58 & 0.56 & 0.12 & 0.04 & 0.38 & 0.72 & 1.68 \\
\hline 1972 & 1.84 & 2.30 & 2.08 & 2.37 & 2.71 & 3.81 & 3.50 & 2.66 & 0.95 & 0.01 & 0.08 & 0.45 & 1.89 \\
\hline 1973 & 1.97 & 2.24 & 2.14 & 1.67 & 2.01 & 3.18 & 3.97 & 1.15 & 0.30 & 0.39 & 0.58 & 1.44 & 1.75 \\
\hline 1974 & 2.03 & 2.33 & 2.25 & 1.97 & 2.18 & 2.37 & 1.40 & 0.96 & 0.08 & 0.20 & 0.16 & 0.31 & 1.35 \\
\hline 1975 & 1.77 & 2.45 & 2.43 & 2.06 & 2.53 & 2.71 & 4.52 & 6.11 & 0.20 & 0.02 & 0.41 & 1.46 & 2.22 \\
\hline 1976 & 3.11 & 3.89 & 3.31 & 2.44 & 2.86 & 4.14 & 6.54 & 5.95 & 0.12 & 0.03 & 0.33 & 1.08 & 2.82 \\
\hline 1977 & 2.20 & 2.86 & 2.55 & 3.04 & 3.32 & 2.58 & 2.14 & 0.76 & 0.29 & 0.02 & 0.03 & 0.57 & 1.69 \\
\hline 1978 & 3.08 & 4.05 & 3.24 & 2.43 & 2.43 & 3.76 & 2.83 & 1.74 & 0.09 & 0.05 & 0.17 & 0.42 & 2.02 \\
\hline
\end{tabular}




\section{2-0.000-1M BALKH RIVER AT RABAT-I-BALA}

\section{(U.S. Geological Survey identification number: 363500066580000 )}

LOCATION: Lat $36^{\circ} 35^{\prime} \mathrm{N}$., long $66^{\circ} 58^{\prime} \mathrm{E}$.

DRAINAGE AREA: $18,035 \mathrm{~km}^{2}$.

ELEVATION: 432 meters above mean sea level.

PERIOD OF RECORD: April 1, 1964 to September 30, 1978.

GAGE: Water-stage recorder. Staff gage at same site and datum prior to 1968 water year.

Annual mean discharge

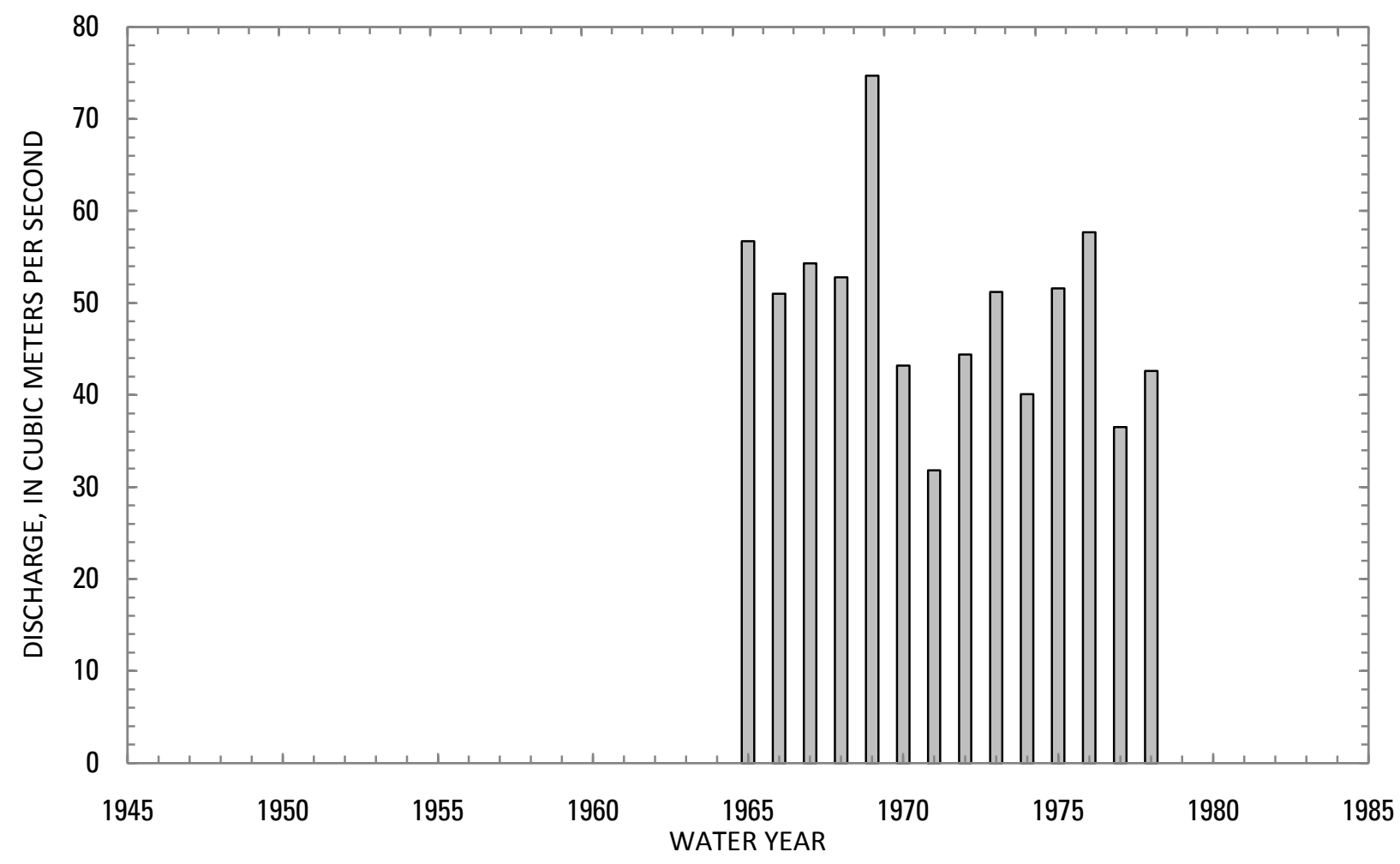




\section{2-0.000-1M BALKH RIVER AT RABAT-I-BALA, Continued}

Statistics of monthly and annual mean discharges [ $\mathrm{m}^{3} / \mathrm{s}$, cubic meters per second]

\begin{tabular}{|c|c|c|c|c|c|c|c|c|}
\hline \multirow[b]{2}{*}{ Month } & \multicolumn{2}{|c|}{ Maximum } & \multicolumn{2}{|c|}{ Minimum } & \multicolumn{4}{|c|}{ Mean } \\
\hline & $\begin{array}{c}\text { Discharge } \\
\left(\mathrm{m}^{3} / \mathrm{s}\right)\end{array}$ & $\begin{array}{c}\text { Water year } \\
\text { of } \\
\text { occurrence }\end{array}$ & $\begin{array}{l}\text { Discharge } \\
\left(\mathrm{m}^{3} / \mathrm{s}\right)\end{array}$ & $\begin{array}{c}\text { Water year } \\
\text { of } \\
\text { occurrence }\end{array}$ & $\begin{array}{c}\text { Discharge } \\
\left(\mathrm{m}^{3} / \mathrm{s}\right)\end{array}$ & $\begin{array}{c}\text { Standard } \\
\text { deviation } \\
\left(\mathrm{m}^{3} / \mathrm{s}\right)\end{array}$ & $\begin{array}{c}\text { Coefficient } \\
\text { of } \\
\text { variation }\end{array}$ & $\begin{array}{c}\text { Percentage } \\
\text { of annual } \\
\text { discharge }\end{array}$ \\
\hline October & 51.1 & 1970 & 22.7 & 1978 & 36.6 & 7.91 & 0.22 & 6.17 \\
\hline November & 53.5 & 1970 & 26.0 & 1978 & 36.9 & 7.66 & 0.21 & 6.23 \\
\hline December & 47.3 & 1966 & 27.1 & 1978 & 35.8 & 6.47 & 0.18 & 6.04 \\
\hline January & 47.3 & 1966 & 25.5 & 1973 & 34.5 & 5.91 & 0.17 & 5.83 \\
\hline February & 48.2 & 1966 & 27.9 & 1975 & 34.8 & 5.47 & 0.16 & 5.86 \\
\hline March & 55.9 & 1966 & 28.9 & 1971 & 39.6 & 7.53 & 0.19 & 6.67 \\
\hline April & 104 & 1969 & 42.1 & 1971 & 59.4 & 15.3 & 0.26 & 10.02 \\
\hline May & 150 & 1976 & 52.4 & 1971 & 101 & 31.1 & 0.31 & 17.07 \\
\hline June & 169 & 1969 & 29.9 & 1971 & 95.3 & 39.5 & 0.41 & 16.07 \\
\hline July & 104 & 1969 & 19.8 & 1971 & 47.7 & 20.1 & 0.42 & 8.05 \\
\hline August & 65.0 & 1969 & 19.8 & 1971 & 35.8 & 10.7 & 0.30 & 6.04 \\
\hline September & 55.4 & 1969 & 20.8 & 1971 & 35.3 & 8.15 & 0.23 & 5.95 \\
\hline Annual & 74.7 & 1969 & 31.8 & 1971 & 49.2 & 10.7 & 0.22 & 100 \\
\hline
\end{tabular}


12-0.000-1M BALKH RIVER AT RABAT-I-BALA, Continued

Monthly and annual flow duration, in cubic meters per second

[ng, not given]

\begin{tabular}{|c|c|c|c|c|c|c|c|c|c|c|c|c|c|}
\hline \multirow{2}{*}{$\begin{array}{l}\text { Percentage } \\
\text { of days } \\
\text { discharge } \\
\text { equaled or } \\
\text { exceeded }\end{array}$} & \multicolumn{12}{|c|}{ Month } & \multirow{2}{*}{ Annua } \\
\hline & October & November & December & January & February & March & April & May & June & July & August & September & \\
\hline 95 & 23.1 & 24.2 & 26.7 & 26.3 & 27.5 & 28.7 & 35.6 & 46.7 & 31.4 & 21.4 & 20.2 & 22.0 & 24.4 \\
\hline 90 & 24.0 & 27.0 & 27.4 & 27.3 & 28.1 & 30.9 & 37.9 & 54.9 & 37.0 & 25.3 & 23.0 & 24.8 & 28.0 \\
\hline 85 & 29.0 & 29.2 & 28.3 & 27.9 & 28.7 & 32.2 & 40.3 & 59.9 & 44.2 & 28.8 & 24.6 & 28.2 & 29.5 \\
\hline 80 & 30.9 & 30.2 & 28.9 & 29.4 & 29.8 & 33.3 & 42.9 & 64.4 & 51.8 & 31.4 & 28.2 & 29.9 & 31.1 \\
\hline 75 & 32.0 & 31.5 & 29.6 & 30.1 & 30.3 & 33.8 & 44.7 & 69.9 & 58.8 & 33.5 & 28.9 & 30.7 & 32.8 \\
\hline 70 & 33.0 & 32.4 & 32.0 & 31.9 & 30.7 & 34.5 & 45.7 & 75.1 & 63.9 & 35.5 & 31.2 & 31.5 & 34.0 \\
\hline 65 & 33.7 & 33.2 & 33.0 & 32.5 & 31.8 & 35.3 & 46.7 & 78.4 & 70.0 & 37.7 & 32.4 & 32.3 & 35.2 \\
\hline 60 & 34.3 & 34.3 & 34.5 & 33.0 & 32.4 & 36.2 & 48.0 & 81.2 & 76.7 & 39.9 & 33.2 & 33.0 & 36.3 \\
\hline 55 & 35.0 & 36.0 & 35.4 & 33.6 & 33.3 & 37.0 & 49.8 & 85.2 & 82.3 & 42.1 & 33.8 & 33.8 & 37.4 \\
\hline 50 & 37.2 & 36.8 & 35.9 & 34.4 & 34.1 & 37.7 & 51.7 & 94.3 & 87.7 & 44.0 & 34.6 & 35.5 & 38.5 \\
\hline 45 & 39.6 & 38.8 & 36.6 & 35.0 & 35.6 & 38.1 & 53.7 & 102 & 94.6 & 45.8 & 35.6 & 36.4 & 39.6 \\
\hline 40 & 39.9 & 39.6 & 37.3 & 35.5 & 36.3 & 38.5 & 55.5 & 106 & 104 & 47.7 & 37.3 & 37.1 & 41.6 \\
\hline 35 & 40.2 & 40.1 & 37.8 & 36.5 & 36.8 & 39.5 & 57.4 & 116 & 114 & 49.7 & 38.1 & 37.7 & 43.7 \\
\hline 30 & 40.6 & 40.7 & 39.0 & 36.9 & 37.3 & 41.1 & 60.1 & 124 & 119 & 52.1 & 38.8 & 38.4 & 46.3 \\
\hline 25 & 41.1 & 41.3 & 40.1 & 37.5 & 38.0 & 42.3 & 63.7 & 131 & 129 & 55.1 & 40.0 & 39.1 & 50.3 \\
\hline 20 & 41.6 & 41.9 & 41.5 & 38.4 & 38.8 & 45.8 & 69.6 & 138 & 141 & 58.0 & 42.3 & 39.8 & 56.1 \\
\hline 15 & 44.2 & 44.8 & 43.4 & 39.5 & 39.7 & 49.7 & 78.2 & 144 & 148 & 65.4 & 43.5 & 41.6 & 67.5 \\
\hline 10 & 45.7 & 46.7 & 46.5 & 43.5 & 40.8 & 53.1 & 90.9 & 153 & 156 & 76.2 & 45.1 & 43.4 & 87.3 \\
\hline 5 & 50.8 & 51.3 & 47.1 & $\mathrm{ng}$ & 47.3 & 57.8 & 113 & 169 & 178 & 90.8 & 60.5 & 55.2 & 125 \\
\hline
\end{tabular}




\section{2-0.000-1M BALKH RIVER AT RABAT-I-BALA, Continued}

Probability of occurrence of annual high discharges

[ $\mathrm{m}^{3} / \mathrm{s}$, cubic meters per second; ng, not given]

\begin{tabular}{|c|c|c|c|c|c|c|}
\hline \multirow{2}{*}{$\begin{array}{l}\text { Exceedance } \\
\text { probability }\end{array}$} & \multirow{2}{*}{$\begin{array}{c}\text { Recurrence } \\
\text { interval } \\
\text { (years) }\end{array}$} & \multirow{2}{*}{$\begin{array}{c}\text { Maximum } \\
\text { instantaneous } \\
\text { discharge } \\
\left(\mathrm{m}^{3} / \mathrm{s}\right)\end{array}$} & \multicolumn{4}{|c|}{ Maximum daily mean discharge $\left(\mathrm{m}^{3} / \mathrm{s}\right)$} \\
\hline & & & $\begin{array}{l}\text { 3-day } \\
\text { period }\end{array}$ & $\begin{array}{l}\text { 7-day } \\
\text { period }\end{array}$ & $\begin{array}{l}\text { 15-day } \\
\text { period }\end{array}$ & $\begin{array}{l}\text { 30-day } \\
\text { period }\end{array}$ \\
\hline 0.99 & 1.01 & 39.1 & 46.4 & 44.3 & 43.1 & 38.9 \\
\hline 0.95 & 1.05 & 68.8 & 66.9 & 65.7 & 62.1 & 55.7 \\
\hline 0.90 & 1.11 & 91.6 & 80.5 & 79.3 & 74.1 & 66.4 \\
\hline 0.80 & 1.25 & 128 & 99.7 & 97.6 & 90.3 & 81.0 \\
\hline 0.50 & 2 & 231 & 147 & 137 & 126 & 114 \\
\hline 0.20 & 5 & 397 & 208 & 180 & 165 & 152 \\
\hline 0.10 & 10 & 516 & 247 & 201 & 186 & 173 \\
\hline 0.04 & 25 & 673 & 293 & 223 & 209 & 197 \\
\hline 0.02 & 50 & 791 & 326 & 237 & 223 & 212 \\
\hline 0.01 & 100 & 911 & 357 & 248 & 235 & 226 \\
\hline 0.005 & 200 & 1,030 & 387 & 258 & 246 & 238 \\
\hline 0.002 & 500 & 1,190 & $\mathrm{ng}$ & $\mathrm{ng}$ & $\mathrm{ng}$ & $\mathrm{ng}$ \\
\hline
\end{tabular}

\section{2-0.000-1M BALKH RIVER AT RABAT-I-BALA, Continued}

Probability of occurrence of annual low discharges

$\left[\mathrm{m}^{3} / \mathrm{s}\right.$, meters per second]

\begin{tabular}{|c|c|c|c|c|c|c|c|c|c|c|}
\hline \multirow{3}{*}{$\begin{array}{c}\text { Nonexceedance } \\
\text { probability }\end{array}$} & \multirow{3}{*}{$\begin{array}{c}\text { Recurrence } \\
\text { interval } \\
\text { (years) }\end{array}$} & \multicolumn{9}{|c|}{ Minimum daily mean discharge $\left(\mathrm{m}^{3} / \mathrm{s}\right)$} \\
\hline & & \multicolumn{9}{|c|}{ Number of consecutive days } \\
\hline & & 1 & 3 & 7 & 14 & 30 & 60 & 90 & 120 & 183 \\
\hline 0.05 & 20 & 18.5 & 18.6 & 19.2 & 19.6 & 20.6 & 21.1 & 21.6 & 22.3 & 23.6 \\
\hline 0.10 & 10 & 20.9 & 21.1 & 21.5 & 21.9 & 22.9 & 23.6 & 24.2 & 24.9 & 26.0 \\
\hline 0.20 & 5 & 23.9 & 24.1 & 24.4 & 24.7 & 25.7 & 26.7 & 27.4 & 28.1 & 29.1 \\
\hline 0.50 & 2 & 29.8 & 29.9 & 30.1 & 30.4 & 31.2 & 32.5 & 33.5 & 34.1 & 35.0 \\
\hline
\end{tabular}




\section{2-0.000-1M BALKH RIVER AT RABAT-I-BALA, Continued}

Probability of occurrence of seasonal low discharges $\left[\mathrm{m}^{3} / \mathrm{s}\right.$, meters per second]

\begin{tabular}{|c|c|c|c|c|c|c|c|c|c|}
\hline \multirow{3}{*}{$\begin{array}{c}\text { Nonexceedance } \\
\text { probability }\end{array}$} & \multirow{3}{*}{$\begin{array}{c}\text { Recurrence } \\
\text { interval } \\
\text { (years) }\end{array}$} & \multicolumn{8}{|c|}{ Minimum daily mean discharge $\left(\mathrm{m}^{3} / \mathrm{s}\right)$} \\
\hline & & \multicolumn{8}{|c|}{ Number of consecutive days } \\
\hline & & 1 & 7 & 14 & 30 & 1 & 7 & 14 & 30 \\
\hline & & \multicolumn{4}{|c|}{ December-January-February } & \multicolumn{4}{|c|}{ March-April-May } \\
\hline 0.05 & 20 & 23.1 & 23.5 & 24.1 & 25.2 & 27.7 & 28.5 & 28.9 & 30.6 \\
\hline 0.10 & 10 & 24.5 & 24.9 & 25.3 & 26.4 & 28.5 & 29.3 & 29.9 & 31.7 \\
\hline 0.20 & 5 & 26.4 & 26.7 & 27.0 & 28.1 & 29.7 & 30.7 & 31.4 & 33.3 \\
\hline \multirow[t]{2}{*}{0.50} & 2 & 30.7 & 30.8 & 31.1 & 32.0 & 32.9 & 34.3 & 35.4 & 37.7 \\
\hline & & \multicolumn{4}{|c|}{ June-July-August } & \multicolumn{4}{|c|}{ September-October-November } \\
\hline 0.05 & 20 & 19.3 & 20.0 & 20.4 & 21.4 & 20.2 & 20.6 & 21.0 & 22.0 \\
\hline 0.10 & 10 & 21.9 & 22.5 & 22.8 & 23.7 & 22.9 & 23.3 & 23.7 & 24.7 \\
\hline 0.20 & 5 & 25.3 & 25.8 & 26.1 & 26.9 & 26.4 & 26.7 & 27.1 & 28.1 \\
\hline 0.50 & 2 & 32.7 & 33.1 & 33.5 & 34.2 & 33.3 & 33.6 & 33.9 & 34.8 \\
\hline
\end{tabular}




\section{2-0.000-1M BALKH RIVER AT RABAT-I-BALA, Continued}

\begin{tabular}{|c|c|c|c|c|c|}
\hline \multicolumn{6}{|c|}{$\begin{array}{l}\text { Annual peak discharges } \\
\text { [m³/s, meters per second] }\end{array}$} \\
\hline \multicolumn{3}{|c|}{$\begin{array}{c}\text { Annual peak discharge, } \\
\text { by year }\end{array}$} & \multicolumn{3}{|c|}{$\begin{array}{l}\text { Annual peak discharge, } \\
\text { from highest to lowest }\end{array}$} \\
\hline $\begin{array}{l}\text { Water } \\
\text { year }\end{array}$ & Date & $\begin{array}{c}\text { Peak } \\
\text { discharge } \\
\left(\mathrm{m}^{3} / \mathrm{s}\right)\end{array}$ & $\begin{array}{l}\text { Water } \\
\text { year }\end{array}$ & Date & $\begin{array}{c}\text { Peak } \\
\text { discharge } \\
\left(\mathrm{m}^{3} / \mathrm{s}\right)\end{array}$ \\
\hline 1964 & May 20, 1964 & 152 & 1977 & May 29, 1977 & 531 \\
\hline 1965 & June 4, 1965 & 152 & 1972 & June 2, 1972 & 507 \\
\hline 1966 & April 16, 1966 & 117 & 1976 & April 28, 1976 & 490 \\
\hline 1967 & May 9, 1967 & 157 & 1973 & April 25, 1973 & 441 \\
\hline 1968 & May 20, 1968 & 168 & 1975 & May 4, 1975 & 345 \\
\hline 1969 & April 20, 1969 & 309 & 1969 & April 20, 1969 & 309 \\
\hline 1970 & May 23, 1970 & 79.9 & 1978 & April 27, 1978 & 309 \\
\hline 1971 & May 21,1971 & 64.5 & 1974 & May 26, 1974 & 235 \\
\hline 1972 & June 2, 1972 & 507 & 1968 & May 20, 1968 & 168 \\
\hline 1973 & April 25, 1973 & 441 & 1967 & May 9, 1967 & 157 \\
\hline 1974 & May 26, 1974 & 235 & 1964 & May 20, 1964 & 152 \\
\hline 1975 & May 4, 1975 & 345 & 1965 & June 4, 1965 & 152 \\
\hline 1976 & April 28, 1976 & 490 & 1966 & April 16, 1966 & 117 \\
\hline 1977 & May 29, 1977 & 531 & 1970 & May 23, 1970 & 79.9 \\
\hline 1978 & April 27, 1978 & 309 & 1971 & May 21, 1971 & 64.5 \\
\hline
\end{tabular}


12-0.000-1M BALKH RIVER AT RABAT-I-BALA, Continued

Monthly and annual mean discharges, in cubic meters per second

[Data may not be rounded in accordance with U.S. Geological Survey publication standards; --, no data]

\begin{tabular}{|c|c|c|c|c|c|c|c|c|c|c|c|c|c|}
\hline \multirow{2}{*}{$\begin{array}{l}\text { Water } \\
\text { year }\end{array}$} & \multicolumn{12}{|c|}{ Monthly mean discharge } & \multirow{2}{*}{$\begin{array}{c}\text { Annual } \\
\text { discharge }\end{array}$} \\
\hline & October & November & December & January & February & March & April & May & June & July & August & September & \\
\hline 1964 & -- & -- & -- & -- & -- & -- & 66.1 & 128 & 97.2 & 52.3 & 36.8 & 37.5 & -- \\
\hline 1965 & 40.2 & 39.5 & 37.0 & 36.6 & 37.8 & 42.8 & 52.8 & 110 & 131 & 66.2 & 42.6 & 42.5 & 56.7 \\
\hline 1966 & 45.0 & 45.9 & 47.3 & 47.3 & 48.2 & 55.9 & 61.8 & 73.7 & 67.6 & 44.0 & 37.6 & 37.3 & 51.0 \\
\hline 1967 & 42.1 & 41.7 & 40.9 & 37.8 & 37.6 & 40.0 & 61.7 & 104 & 112 & 54.2 & 40.4 & 39.3 & 54.3 \\
\hline 1968 & 42.2 & 41.6 & 39.0 & 35.7 & 33.5 & 37.0 & 54.9 & 88.7 & 120 & 56.1 & 45.2 & 40.1 & 52.8 \\
\hline 1969 & 39.8 & 40.5 & 40.3 & 36.0 & 39.1 & 55.3 & 104 & 147 & 169 & 104 & 65.0 & 55.4 & 74.7 \\
\hline 1970 & 51.1 & 53.5 & 45.3 & 43.1 & 35.5 & 40.7 & 50.2 & 64.6 & 46.5 & 29.8 & 26.7 & 31.1 & 43.2 \\
\hline 1971 & 34.9 & 35.8 & 34.7 & 31.9 & 30.3 & 28.9 & 42.1 & 52.4 & 29.9 & 19.8 & 19.8 & 20.8 & 31.8 \\
\hline 1972 & 23.7 & 26.3 & 27.4 & 29.0 & 29.6 & 35.5 & 45.6 & 92.5 & 121 & 41.4 & 31.0 & 30.0 & 44.4 \\
\hline 1973 & 30.0 & 29.6 & 28.3 & 25.5 & 28.3 & 37.7 & 68.9 & 142 & 108 & 44.9 & 34.7 & 35.3 & 51.2 \\
\hline 1974 & 33.4 & 32.9 & 34.5 & 33.4 & 32.0 & 35.8 & 44.7 & 79.0 & 55.2 & 34.1 & 32.7 & 32.7 & 40.1 \\
\hline 1975 & 34.6 & 33.7 & 32.6 & 29.9 & 27.9 & 34.9 & 55.5 & 113 & 124 & 54.8 & 38.4 & 39.4 & 51.6 \\
\hline 1976 & 40.0 & 38.5 & 37.5 & 36.8 & 36.7 & 38.4 & 72.3 & 150 & 125 & 50.6 & 34.1 & 31.9 & 57.7 \\
\hline 1977 & 32.7 & 31.7 & 29.1 & 33.1 & 38.5 & 36.2 & 49.2 & 66.5 & 48.0 & 25.4 & 23.1 & 24.4 & 36.5 \\
\hline 1978 & 22.7 & 26.0 & 27.1 & 27.5 & 31.7 & 34.9 & 61.0 & 107 & 74.4 & 38.1 & 29.1 & 31.3 & 42.6 \\
\hline
\end{tabular}




\section{2-0.000-9M BALKH RIVER NEAR NAYAK}

\section{(U.S. Geological Survey identification number: 344500067000000)}

LOCATION: Lat $34^{\circ} 45^{\prime} \mathrm{N}$., long $67^{\circ} 00^{\prime} \mathrm{E}$.

DRAINAGE AREA: $1,460 \mathrm{~km}^{2}$.

ELEVATION: 2,580 meters above mean sea level.

PERIOD OF RECORD: January 15, 1969 to September 30, 1978.

GAGE: Water-stage recorder.

Annual mean discharge

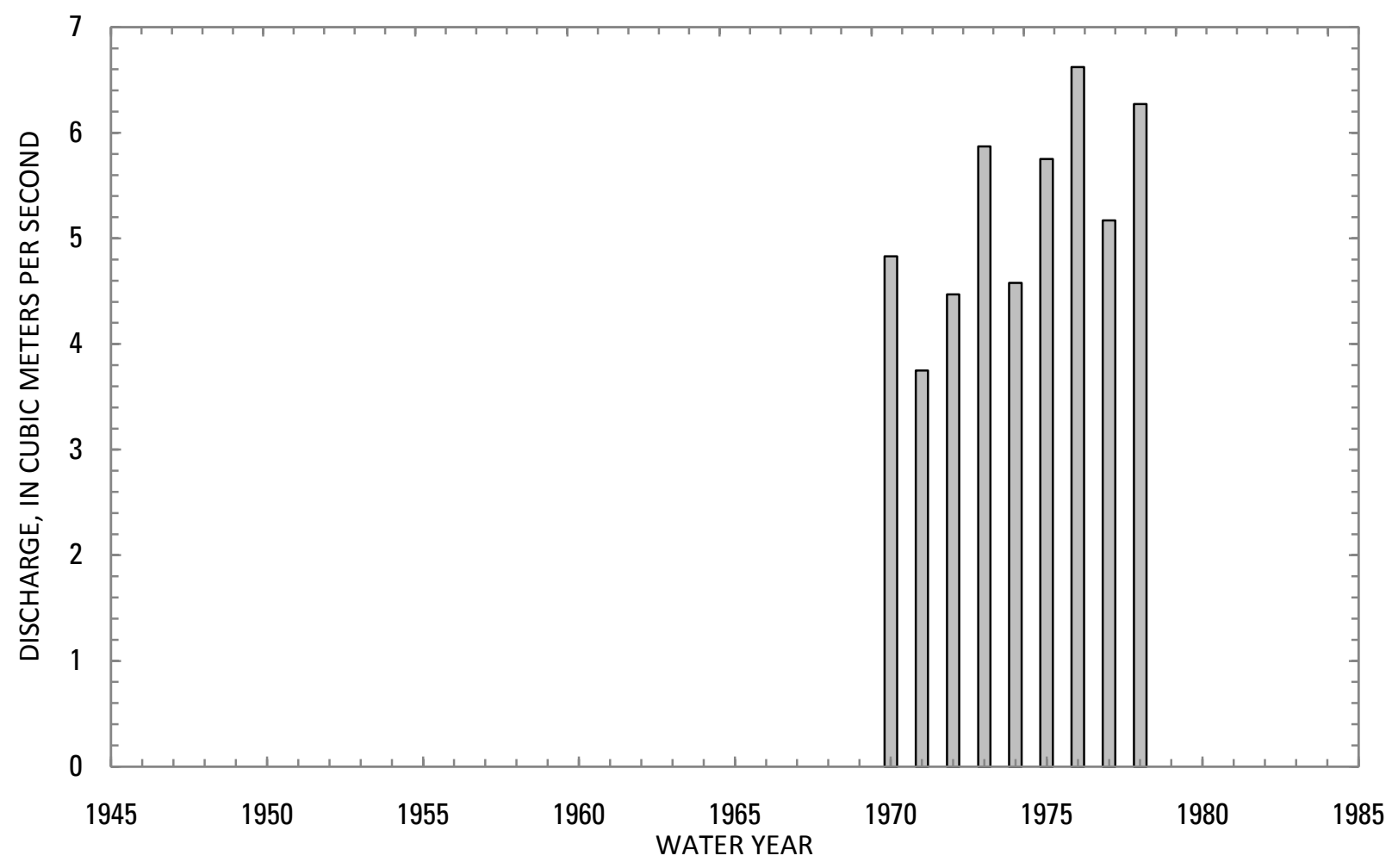




\section{2-0.000-9M BALKH RIVER NEAR NAYAK, Continued}

Statistics of monthly and annual mean discharges $\left[\mathrm{m}^{3} / \mathrm{s}\right.$, cubic meters per second]

\begin{tabular}{|c|c|c|c|c|c|c|c|c|}
\hline \multirow[b]{2}{*}{ Month } & \multicolumn{2}{|c|}{ Maximum } & \multicolumn{2}{|c|}{ Minimum } & \multicolumn{4}{|c|}{ Mean } \\
\hline & $\begin{array}{c}\text { Discharge } \\
\left(\mathrm{m}^{3} / \mathrm{s}\right)\end{array}$ & $\begin{array}{c}\text { Water year } \\
\text { of } \\
\text { occurrence }\end{array}$ & $\begin{array}{c}\text { Discharge } \\
\left(\mathrm{m}^{3} / \mathrm{s}\right)\end{array}$ & $\begin{array}{c}\text { Water year } \\
\text { of } \\
\text { occurrence }\end{array}$ & $\begin{array}{c}\text { Discharge } \\
\left(\mathrm{m}^{3} / \mathrm{s}\right)\end{array}$ & $\begin{array}{c}\text { Standard } \\
\text { deviation } \\
\left(\mathrm{m}^{3} / \mathrm{s}\right)\end{array}$ & $\begin{array}{c}\text { Coefficient } \\
\text { of } \\
\text { variation }\end{array}$ & $\begin{array}{c}\text { Percentage } \\
\text { of annual } \\
\text { discharge }\end{array}$ \\
\hline October & 5.77 & 1970 & 2.39 & 1972 & 3.81 & 0.89 & 0.23 & 5.84 \\
\hline November & 5.50 & 1970 & 2.43 & 1972 & 3.68 & 0.86 & 0.23 & 5.63 \\
\hline December & 4.54 & 1970 & 2.44 & 1972 & 3.55 & 0.66 & 0.19 & 5.44 \\
\hline January & 4.26 & 1970 & 2.27 & 1972 & 3.55 & 0.71 & 0.20 & 5.44 \\
\hline February & 4.82 & 1978 & 1.87 & 1972 & 3.47 & 0.99 & 0.29 & 5.31 \\
\hline March & 5.22 & 1978 & 2.17 & 1972 & 3.87 & 0.95 & 0.24 & 5.93 \\
\hline April & 9.40 & 1978 & 2.93 & 1972 & 6.41 & 1.69 & 0.26 & 9.83 \\
\hline May & 19.5 & 1976 & 6.83 & 1971 & 13.6 & 4.61 & 0.34 & 20.9 \\
\hline June & 20.6 & 1969 & 3.44 & 1971 & 10.7 & 5.50 & 0.52 & 16.4 \\
\hline July & 9.99 & 1969 & 2.96 & 1971 & 4.95 & 2.11 & 0.43 & 7.59 \\
\hline August & 6.56 & 1969 & 2.73 & 1971 & 3.78 & 1.10 & 0.29 & 5.79 \\
\hline September & 6.12 & 1969 & 2.47 & 1971 & 3.91 & 0.92 & 0.23 & 6.00 \\
\hline Annual & 6.62 & 1976 & 3.75 & 1971 & 5.26 & 0.94 & 0.18 & 100 \\
\hline
\end{tabular}


12-0.000-9M BALKH RIVER NEAR NAYAK, Continued

Monthly and annual flow duration, in cubic meters per second

[ng, not given]

\begin{tabular}{|c|c|c|c|c|c|c|c|c|c|c|c|c|c|}
\hline \multirow{2}{*}{$\begin{array}{l}\text { Percentage } \\
\text { of days } \\
\text { discharge } \\
\text { equaled or } \\
\text { exceeded }\end{array}$} & \multicolumn{12}{|c|}{ Month } & \multirow{2}{*}{ Annua } \\
\hline & October & November & December & January & February & March & April & May & June & July & August & September & \\
\hline 95 & 2.36 & 2.40 & 2.43 & 2.17 & 1.92 & 2.13 & 2.62 & 6.61 & 3.28 & 2.96 & 2.72 & 2.61 & 2.46 \\
\hline 90 & 2.53 & 2.60 & 2.68 & 2.29 & 1.96 & 2.37 & 3.67 & 7.26 & 3.84 & 3.03 & 2.74 & 2.67 & 2.79 \\
\hline 85 & 3.35 & 3.00 & 2.81 & 2.57 & 2.03 & 2.70 & 4.24 & 7.63 & 4.09 & 3.09 & 2.78 & 3.33 & 2.99 \\
\hline 80 & 3.39 & 3.18 & 3.01 & 2.99 & 2.22 & 3.19 & 4.52 & 8.07 & 4.60 & 3.28 & 2.82 & 3.38 & 3.27 \\
\hline 75 & 3.52 & 3.24 & 3.12 & 3.13 & 2.79 & 3.32 & 4.82 & 8.86 & 5.26 & 3.43 & 2.95 & 3.56 & 3.39 \\
\hline 70 & 3.55 & 3.38 & 3.17 & 3.16 & 3.00 & 3.57 & 5.03 & 9.35 & 6.02 & 3.51 & 3.00 & 3.62 & 3.50 \\
\hline 65 & 3.58 & 3.41 & 3.36 & 3.38 & 3.21 & 3.66 & 5.21 & 9.89 & 6.80 & 3.58 & 3.14 & 3.67 & 3.60 \\
\hline 60 & 3.60 & 3.45 & 3.39 & 3.53 & 3.40 & 3.77 & 5.40 & 10.6 & 7.58 & 3.70 & 3.30 & 3.75 & 3.71 \\
\hline 55 & 3.63 & 3.48 & 3.41 & 3.58 & 3.68 & 3.88 & 5.71 & 11.1 & 8.51 & 3.91 & 3.54 & 3.81 & 3.83 \\
\hline 50 & 3.66 & 3.52 & 3.43 & 3.72 & 3.84 & 3.97 & 6.15 & 12.1 & 9.30 & 4.46 & 3.73 & 3.84 & 3.94 \\
\hline 45 & 3.69 & 3.55 & 3.60 & 3.78 & 3.89 & 4.01 & 6.30 & 13.0 & 10.0 & 4.63 & 3.78 & 3.86 & 4.06 \\
\hline 40 & 3.72 & 3.72 & 3.78 & 3.92 & 3.93 & 4.05 & 6.47 & 14.5 & 11.7 & 4.83 & 3.83 & 3.89 & 4.18 \\
\hline 35 & 3.86 & 3.80 & 3.94 & 3.96 & 3.98 & 4.09 & 6.77 & 15.7 & 12.8 & 5.17 & 3.90 & 3.92 & 4.30 \\
\hline 30 & 3.91 & 3.89 & 4.00 & 4.11 & 4.02 & 4.32 & 7.20 & 17.5 & 14.2 & 5.45 & 4.09 & 4.10 & 4.59 \\
\hline 25 & 4.08 & 4.11 & 4.06 & 4.15 & 4.14 & 4.44 & 7.44 & 18.6 & 15.2 & 5.65 & 4.16 & 4.16 & 5.10 \\
\hline 20 & 4.20 & 4.20 & 4.21 & ng & 4.27 & 4.63 & 7.90 & 19.6 & 16.0 & 6.33 & 4.23 & 4.22 & 5.86 \\
\hline 15 & 4.27 & 4.31 & 4.26 & ng & 4.41 & 4.86 & 8.45 & 20.4 & 17.6 & 6.94 & 4.28 & 4.30 & 7.26 \\
\hline 10 & 5.60 & 4.74 & 4.42 & ng & 4.55 & 5.30 & 10.3 & 21.5 & 19.8 & 8.32 & 4.58 & 4.52 & 9.64 \\
\hline 5 & 5.74 & 5.70 & 4.52 & ng & 4.75 & 5.49 & 11.7 & 24.6 & 22.9 & 9.29 & 6.45 & 5.98 & 14.4 \\
\hline
\end{tabular}




\section{2-0.000-9M BALKH RIVER NEAR NAYAK, Continued}

Probability of occurrence of annual high discharges

[ $\mathrm{m}^{3} / \mathrm{s}$, cubic meters per second; $\mathrm{ng}$, not given]

\begin{tabular}{|c|c|c|c|c|c|c|}
\hline \multirow{2}{*}{$\begin{array}{c}\text { Exceedance } \\
\text { probability }\end{array}$} & \multirow{2}{*}{$\begin{array}{c}\text { Recurrence } \\
\text { interval } \\
\text { (years) }\end{array}$} & \multirow{2}{*}{$\begin{array}{l}\text { Maximum } \\
\text { instantaneous } \\
\text { discharge } \\
\left(\mathrm{m}^{3} / \mathrm{s}\right)\end{array}$} & \multicolumn{4}{|c|}{ Maximum daily mean discharge $\left(\mathrm{m}^{3} / \mathrm{s}\right)$} \\
\hline & & & $\begin{array}{l}\text { 3-day } \\
\text { period }\end{array}$ & $\begin{array}{l}\text { 7-day } \\
\text { period }\end{array}$ & $\begin{array}{l}\text { 15-day } \\
\text { period }\end{array}$ & $\begin{array}{l}\text { 30-day } \\
\text { period }\end{array}$ \\
\hline 0.99 & 1.01 & 5.00 & 5.23 & 5.07 & 4.81 & 4.52 \\
\hline 0.95 & 1.05 & 8.10 & 7.64 & 7.24 & 6.89 & 6.47 \\
\hline 0.90 & 1.11 & 10.3 & 9.21 & 8.67 & 8.26 & 7.75 \\
\hline 0.80 & 1.25 & 13.4 & 11.4 & 10.7 & 10.2 & 9.55 \\
\hline 0.50 & 2 & 21.1 & 16.6 & 15.6 & 14.9 & 13.9 \\
\hline 0.20 & 5 & 30.9 & 23.1 & 21.9 & 20.9 & 19.5 \\
\hline 0.10 & 10 & 36.6 & 26.9 & 25.8 & 24.7 & 22.9 \\
\hline 0.04 & 25 & 43.1 & 31.3 & 30.6 & 29.2 & 27.0 \\
\hline 0.02 & 50 & 47.4 & 34.3 & 33.9 & 32.3 & 29.8 \\
\hline 0.01 & 100 & 51.3 & 37.1 & 37.0 & 35.3 & 32.5 \\
\hline 0.005 & 200 & 54.8 & ${ }^{1} 39.6$ & 40.1 & 38.2 & 35.1 \\
\hline 0.002 & 500 & 59.0 & $\mathrm{ng}$ & $\mathrm{ng}$ & ng & $\mathrm{ng}$ \\
\hline
\end{tabular}

'Data does not fit log-Pearson Type III curve, use with caution.

\section{2-0.000-9M BALKH RIVER NEAR NAYAK, Continued}

Probability of occurrence of annual low discharges $\left[\mathrm{m}^{3} / \mathrm{s}\right.$, meters per second]

\begin{tabular}{|c|c|c|c|c|c|c|c|c|c|c|}
\hline \multirow{3}{*}{$\begin{array}{c}\text { Nonexceedance } \\
\text { probability }\end{array}$} & \multirow{3}{*}{$\begin{array}{l}\text { Recurrence } \\
\text { interval } \\
\text { (years) }\end{array}$} & \multicolumn{9}{|c|}{ Minimum daily mean discharge $\left(\mathrm{m}^{3} / \mathrm{s}\right)$} \\
\hline & & \multicolumn{9}{|c|}{ Number of consecutive days } \\
\hline & & 1 & 3 & 7 & 14 & 30 & 60 & 90 & 120 & 183 \\
\hline 0.05 & 20 & 1.40 & 1.40 & 1.60 & 1.77 & 1.97 & 2.12 & 2.18 & 2.29 & 2.37 \\
\hline 0.10 & 10 & 1.66 & 1.68 & 1.86 & 2.01 & 2.23 & 2.39 & 2.47 & 2.57 & 2.65 \\
\hline 0.20 & 5 & 2.01 & 2.05 & 2.19 & 2.32 & 2.55 & 2.72 & 2.82 & 2.90 & 2.99 \\
\hline 0.50 & 2 & 2.69 & 2.79 & 2.86 & 2.95 & 3.17 & 3.32 & 3.44 & 3.50 & 3.62 \\
\hline
\end{tabular}




\section{2-0.000-9M BALKH RIVER NEAR NAYAK, Continued}

Probability of occurrence of seasonal low discharges $\left[\mathrm{m}^{3} / \mathrm{s}\right.$, meters per second]

\begin{tabular}{|c|c|c|c|c|c|c|c|c|c|}
\hline \multirow{3}{*}{$\begin{array}{c}\text { Nonexceedance } \\
\text { probability }\end{array}$} & \multirow{3}{*}{$\begin{array}{c}\text { Recurrence } \\
\text { interval } \\
\text { (years) }\end{array}$} & \multicolumn{8}{|c|}{ Minimum daily mean discharge $\left(\mathrm{m}^{3} / \mathrm{s}\right)$} \\
\hline & & \multicolumn{8}{|c|}{ Number of consecutive days } \\
\hline & & 1 & 7 & 14 & 30 & 1 & 7 & 14 & 30 \\
\hline & & \multicolumn{4}{|c|}{ December-January-February } & \multicolumn{4}{|c|}{ March-April-May } \\
\hline 0.05 & 20 & 1.42 & 1.64 & 1.81 & 2.00 & 1.80 & 1.93 & 1.98 & 2.24 \\
\hline 0.10 & 10 & 1.76 & 1.98 & 2.14 & 2.33 & 2.08 & 2.25 & 2.32 & 2.59 \\
\hline 0.20 & 5 & 2.20 & 2.42 & 2.56 & 2.73 & 2.46 & 2.67 & 2.75 & 3.03 \\
\hline \multirow[t]{2}{*}{0.50} & 2 & 3.04 & 3.23 & 3.33 & 3.45 & 3.25 & 3.50 & 3.61 & 3.89 \\
\hline & & \multicolumn{4}{|c|}{ June-July-August } & \multicolumn{4}{|c|}{ September-October-November } \\
\hline 0.05 & 20 & 2.42 & 2.51 & 2.54 & 2.62 & 2.13 & 2.25 & 2.36 & 2.45 \\
\hline 0.10 & 10 & 2.53 & 2.60 & 2.63 & 2.72 & 2.37 & 2.48 & 2.57 & 2.64 \\
\hline 0.20 & 5 & 2.70 & 2.75 & 2.79 & 2.89 & 2.68 & 2.78 & 2.85 & 2.90 \\
\hline 0.50 & 2 & 3.19 & 3.23 & 3.27 & 3.41 & 3.29 & 3.39 & 3.43 & 3.49 \\
\hline
\end{tabular}

\section{2-0.000-9M BALKH RIVER NEAR NAYAK, Continued}

\section{Annual peak discharges}

$\left[\mathrm{m}^{3} / \mathrm{s}\right.$, meters per second]

\begin{tabular}{|c|c|c|c|c|c|}
\hline \multicolumn{3}{|c|}{$\begin{array}{c}\text { Annual peak discharge, } \\
\text { by year }\end{array}$} & \multicolumn{3}{|c|}{$\begin{array}{l}\text { Annual peak discharge, } \\
\text { from highest to lowest }\end{array}$} \\
\hline $\begin{array}{l}\text { Water } \\
\text { year }\end{array}$ & Date & $\begin{array}{c}\text { Peak } \\
\text { discharge } \\
\left(\mathrm{m}^{3} / \mathrm{s}\right)\end{array}$ & $\begin{array}{l}\text { Water } \\
\text { year }\end{array}$ & Date & $\begin{array}{c}\text { Peak } \\
\text { discharge } \\
\left(\mathrm{m}^{3} / \mathrm{s}\right)\end{array}$ \\
\hline 1969 & May 31, 1969 & 29.0 & 1975 & May 15, 1975 & 41.0 \\
\hline 1970 & April 13, 1970 & 10.3 & 1976 & June 3, 1976 & 29.7 \\
\hline 1971 & May 9, 1971 & 8.12 & 1969 & May 31, 1969 & 29.0 \\
\hline 1972 & May 31, 1972 & 26.4 & 1972 & May 31, 1972 & 26.4 \\
\hline 1973 & May 16, 1973 & 21.8 & 1978 & May 24, 1978 & 22.8 \\
\hline 1974 & May 25, 1974 & 19.0 & 1973 & May 16, 1973 & 21.8 \\
\hline 1975 & May 15, 1975 & 41.0 & 1974 & May 25, 1974 & 19.0 \\
\hline 1976 & June 3, 1976 & 29.7 & 1977 & May 26, 1977 & 14.2 \\
\hline 1977 & May 26, 1977 & 14.2 & 1970 & April 13, 1970 & 10.3 \\
\hline 1978 & May 24, 1978 & 22.8 & 1971 & May 9, 1971 & 8.12 \\
\hline
\end{tabular}




\section{2-0.000-9M BALKH RIVER NEAR NAYAK, Continued}

Monthly and annual mean discharges, in cubic meters per second

[Data may not be rounded in accordance with U.S. Geological Survey publication standards; --, no data]

\begin{tabular}{|c|c|c|c|c|c|c|c|c|c|c|c|c|c|}
\hline \multirow{2}{*}{$\begin{array}{l}\text { Water } \\
\text { year }\end{array}$} & \multicolumn{12}{|c|}{ Monthly mean discharge } & \multirow{2}{*}{$\begin{array}{c}\text { Annual } \\
\text { discharge }\end{array}$} \\
\hline & October & November & December & January & February & March & April & May & June & July & August & September & \\
\hline 1969 & -- & -- & -- & -- & 1.99 & 2.70 & 6.58 & 16.4 & 20.6 & 9.99 & 6.56 & 6.12 & -- \\
\hline 1970 & 5.77 & 5.50 & 4.54 & 4.26 & 4.00 & 4.01 & 7.22 & 7.96 & 4.93 & 3.42 & 3.00 & 3.38 & 4.83 \\
\hline 1971 & 3.40 & 3.26 & 3.26 & 3.52 & 3.67 & 4.12 & 5.31 & 6.83 & 3.44 & 2.96 & 2.73 & 2.47 & 3.75 \\
\hline 1972 & 2.39 & 2.43 & 2.44 & 2.27 & 1.87 & 2.17 & 2.93 & 12.0 & 12.0 & 5.12 & 3.98 & 4.01 & 4.47 \\
\hline 1973 & 3.74 & 3.50 & 3.35 & 3.15 & 3.15 & 3.69 & 7.71 & 17.0 & 11.5 & 5.36 & 4.24 & 3.93 & 5.87 \\
\hline 1974 & 3.70 & 3.37 & 3.30 & 3.65 & 3.86 & 4.46 & 6.35 & 10.2 & 5.10 & 3.67 & 3.37 & 3.80 & 4.58 \\
\hline 1975 & 3.49 & 3.10 & 2.97 & 2.73 & 2.80 & 3.65 & 5.45 & 16.1 & 15.3 & 5.87 & 3.82 & 3.64 & 5.75 \\
\hline 1976 & 3.74 & 3.98 & 3.94 & 4.11 & 4.21 & 3.66 & 6.28 & 19.5 & 15.6 & 6.12 & 3.98 & 4.27 & 6.62 \\
\hline 1977 & 4.27 & 4.28 & 4.22 & 4.08 & 4.32 & 5.06 & 6.92 & 10.9 & 7.65 & 3.22 & 3.18 & 4.00 & 5.17 \\
\hline 1978 & 3.83 & 3.68 & 3.92 & 4.18 & 4.82 & 5.22 & 9.40 & 19.2 & 10.6 & 3.81 & 2.96 & 3.53 & 6.27 \\
\hline
\end{tabular}




\section{2-0.000-10M BALKH RIVER BELOW BAND-I-AMIR}

\section{(U.S. Geological Survey identification number: 344900067100000)}

LOCATION: Lat $34^{\circ} 49^{\prime} \mathrm{N}$., long $67^{\circ} 10^{\prime} \mathrm{E}$.

DRAINAGE AREA: $445 \mathrm{~km}^{2}$.

ELEVATION: 2,882 meters above mean sea level.

PERIOD OF RECORD: October 1, 1969 to March 20, 1976.

GAGE: Staff gage.

Annual mean discharge

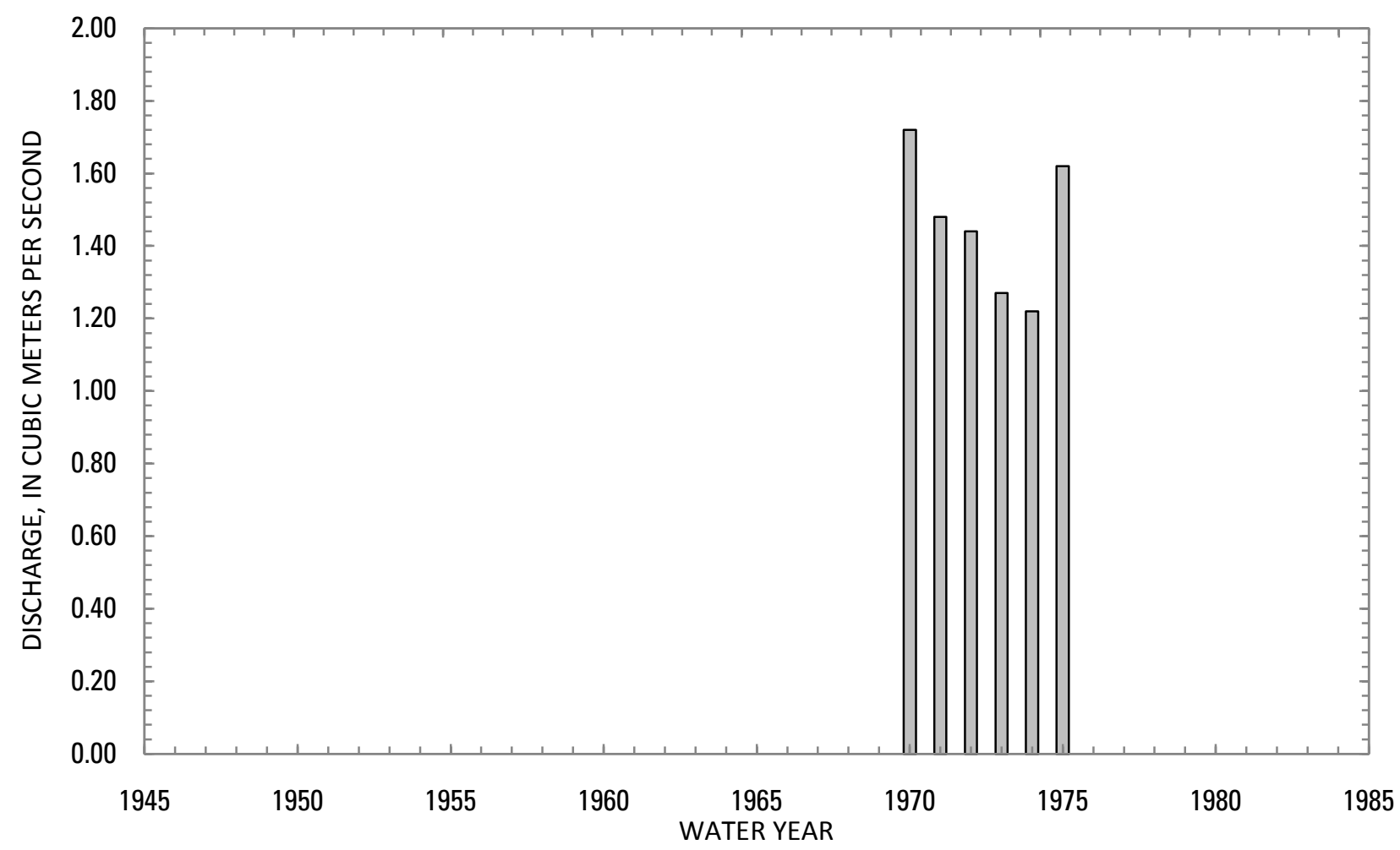




\section{2-0.000-10M BALKH RIVER BELOW BAND-I-AMIR, Continued}

Statistics of monthly and annual mean discharges [m $\mathrm{m}^{3} / \mathrm{s}$, cubic meters per second]

\begin{tabular}{|c|c|c|c|c|c|c|c|c|}
\hline \multirow[b]{2}{*}{ Month } & \multicolumn{2}{|c|}{ Maximum } & \multicolumn{2}{|c|}{ Minimum } & \multicolumn{4}{|c|}{ Mean } \\
\hline & $\begin{array}{c}\text { Discharge } \\
\left(\mathrm{m}^{3} / \mathrm{s}\right)\end{array}$ & $\begin{array}{c}\text { Water year } \\
\text { of } \\
\text { occurrence }\end{array}$ & $\begin{array}{c}\text { Discharge } \\
\left(\mathrm{m}^{3} / \mathrm{s}\right)\end{array}$ & $\begin{array}{c}\text { Water year } \\
\text { of } \\
\text { occurrence }\end{array}$ & $\begin{array}{c}\text { Discharge } \\
\left(\mathrm{m}^{3} / \mathrm{s}\right)\end{array}$ & $\begin{array}{c}\text { Standard } \\
\text { deviation } \\
\left(\mathrm{m}^{3} / \mathrm{s}\right)\end{array}$ & $\begin{array}{c}\text { Coefficient } \\
\text { of } \\
\text { variation }\end{array}$ & $\begin{array}{c}\text { Percentage } \\
\text { of annual } \\
\text { discharge }\end{array}$ \\
\hline October & 1.95 & 1970 & 1.08 & 1976 & 1.24 & 0.31 & 0.25 & 7.15 \\
\hline November & 1.83 & 1970 & 0.83 & 1975 & 1.27 & 0.33 & 0.26 & 7.29 \\
\hline December & 1.61 & 1970 & 0.64 & 1975 & 1.16 & 0.30 & 0.26 & 6.70 \\
\hline January & 1.75 & 1970 & 0.51 & 1975 & 1.18 & 0.41 & 0.35 & 6.78 \\
\hline February & 1.85 & 1970 & 0.65 & 1975 & 1.30 & 0.41 & 0.32 & 7.50 \\
\hline March & 2.18 & 1970 & 0.76 & 1975 & 1.47 & 0.48 & 0.33 & 8.48 \\
\hline April & 3.02 & 1971 & 1.17 & 1974 & 2.17 & 0.74 & 0.34 & 12.5 \\
\hline May & 6.83 & 1975 & 1.26 & 1974 & 2.71 & 2.05 & 0.76 & 15.6 \\
\hline June & 1.91 & 1975 & 1.07 & 1971 & 1.52 & 0.31 & 0.20 & 8.76 \\
\hline July & 1.29 & 1974 & 0.93 & 1971 & 1.17 & 0.13 & 0.11 & 6.73 \\
\hline August & 1.25 & 1975 & 0.93 & 1971 & 1.06 & 0.12 & 0.11 & 6.13 \\
\hline September & 1.24 & 1975 & 0.87 & 1973 & 1.11 & 0.13 & 0.11 & 6.38 \\
\hline Annual & 1.72 & 1970 & 1.22 & 1974 & 1.46 & 0.19 & 0.13 & 100 \\
\hline
\end{tabular}




\section{2-0.000-10M BALKH RIVER BELOW BAND-I-AMIR, Continued}

Monthly and annual flow duration, in cubic meters per second [ng, not given]

\begin{tabular}{|c|c|c|c|c|c|c|c|c|c|c|c|c|c|}
\hline \multirow{2}{*}{$\begin{array}{c}\text { Percentage } \\
\text { of days } \\
\text { discharge } \\
\text { equaled or } \\
\text { exceeded }\end{array}$} & \multicolumn{12}{|c|}{ Month } & \multirow{2}{*}{ Annua } \\
\hline & October & November & December & January & February & March & April & May & June & July & August & September & \\
\hline 95 & 0.97 & 0.78 & 0.60 & 0.51 & 0.64 & 0.71 & 1.16 & 1.28 & 0.87 & 0.93 & 0.92 & 0.88 & 0.74 \\
\hline 90 & 1.01 & 0.90 & 0.70 & 0.55 & 0.65 & 0.73 & 1.18 & 1.31 & 1.17 & 0.94 & 0.93 & 0.91 & 0.89 \\
\hline 85 & 1.05 & 0.97 & 1.01 & 0.87 & 0.95 & 0.88 & 1.20 & 1.35 & 1.26 & 0.94 & 0.93 & 0.91 & 0.95 \\
\hline 80 & 1.07 & 1.07 & 1.07 & 0.93 & 1.04 & 1.18 & 1.38 & 1.47 & 1.28 & 1.09 & 0.93 & 0.94 & 1.04 \\
\hline 75 & 1.08 & 1.09 & 1.09 & 0.95 & 1.08 & 1.20 & 1.47 & 1.57 & 1.30 & 1.10 & 0.94 & 1.02 & 1.08 \\
\hline 70 & 1.09 & 1.11 & 1.09 & 0.97 & 1.10 & 1.28 & 1.59 & 1.75 & 1.32 & 1.13 & 1.00 & 1.02 & 1.12 \\
\hline 65 & 1.09 & 1.15 & 1.10 & 1.05 & 1.12 & 1.31 & 1.69 & 1.93 & 1.34 & 1.14 & 1.01 & 1.06 & 1.16 \\
\hline 60 & 1.10 & 1.17 & 1.11 & 1.08 & 1.14 & 1.33 & 1.74 & 1.99 & 1.42 & 1.18 & 1.01 & 1.09 & 1.19 \\
\hline 55 & 1.13 & 1.18 & 1.11 & 1.10 & 1.19 & 1.35 & 1.78 & 2.02 & 1.44 & 1.18 & 1.01 & 1.10 & 1.23 \\
\hline 50 & 1.15 & 1.19 & 1.12 & 1.11 & 1.24 & 1.44 & 1.81 & 2.06 & 1.47 & 1.19 & 1.02 & 1.10 & 1.27 \\
\hline 45 & 1.17 & 1.20 & 1.14 & 1.14 & 1.27 & 1.59 & 1.94 & 2.10 & 1.49 & 1.20 & 1.09 & 1.11 & 1.32 \\
\hline 40 & 1.18 & 1.21 & 1.16 & 1.42 & 1.50 & 1.62 & 2.08 & 2.17 & 1.51 & 1.21 & 1.09 & 1.14 & 1.36 \\
\hline 35 & 1.19 & 1.27 & 1.19 & 1.45 & 1.60 & 1.65 & 2.54 & 2.24 & 1.60 & 1.25 & 1.10 & 1.18 & 1.46 \\
\hline 30 & 1.21 & 1.34 & 1.35 & 1.47 & 1.62 & 1.68 & 2.69 & 2.38 & 1.64 & 1.26 & 1.11 & 1.19 & 1.56 \\
\hline 25 & 1.26 & 1.49 & 1.40 & 1.49 & 1.65 & 1.70 & 2.80 & 2.46 & 1.68 & 1.26 & 1.18 & 1.20 & 1.64 \\
\hline 20 & 1.28 & 1.57 & 1.42 & 1.51 & 1.67 & 1.78 & 2.99 & 2.55 & 1.75 & 1.26 & 1.19 & 1.25 & 1.74 \\
\hline 15 & 1.34 & 1.60 & 1.44 & 1.53 & 1.69 & 2.10 & 3.13 & 4.23 & 1.84 & 1.27 & 1.23 & 1.32 & 1.85 \\
\hline 10 & ng & 1.70 & 1.53 & $\mathrm{ng}$ & ng & 2.14 & 3.35 & 5.82 & 2.01 & 1.34 & 1.27 & 1.34 & 2.04 \\
\hline 5 & ng & ng & ng & ng & ng & 2.27 & 3.75 & 8.39 & 2.18 & 1.35 & ng & 1.41 & 2.59 \\
\hline
\end{tabular}




\section{2-0.000-10M BALKH RIVER BELOW BAND-I-AMIR, Continued}

Probability of occurrence of annual high discharges

[ $\mathrm{m}^{3} / \mathrm{s}$, cubic meters per second; ng, not given]

\begin{tabular}{|c|c|c|c|c|c|c|}
\hline \multirow{2}{*}{$\begin{array}{c}\text { Exceedance } \\
\text { probability }\end{array}$} & \multirow{2}{*}{$\begin{array}{c}\text { Recurrence } \\
\text { interval } \\
\text { (years) }\end{array}$} & \multirow{2}{*}{$\begin{array}{l}\text { Maximum } \\
\text { instantaneous } \\
\text { discharge } \\
\left(\mathrm{m}^{3} / \mathrm{s}\right)\end{array}$} & \multicolumn{4}{|c|}{ Maximum daily mean discharge $\left(\mathrm{m}^{3} / \mathrm{s}\right)$} \\
\hline & & & $\begin{array}{l}\text { 3-day } \\
\text { period }\end{array}$ & $\begin{array}{l}\text { 7-day } \\
\text { period }\end{array}$ & $\begin{array}{l}\text { 15-day } \\
\text { period }\end{array}$ & $\begin{array}{l}\text { 30-day } \\
\text { period }\end{array}$ \\
\hline 0.99 & 1.01 & ng & ${ }^{1} 1.31$ & ${ }^{1} 1.32$ & 1.34 & 1.31 \\
\hline 0.95 & 1.05 & ng & 1.49 & 1.49 & 1.49 & 1.46 \\
\hline 0.90 & 1.11 & ng & 1.65 & 1.63 & 1.62 & 1.58 \\
\hline 0.80 & 1.25 & ng & 1.92 & 1.88 & 1.85 & 1.79 \\
\hline 0.50 & 2 & ng & 2.87 & 2.78 & 2.66 & 2.51 \\
\hline 0.20 & 5 & ng & 5.08 & 4.91 & 4.58 & 4.07 \\
\hline 0.10 & 10 & ng & 7.37 & 7.15 & 6.56 & 5.59 \\
\hline 0.04 & 25 & ng & 11.6 & 11.4 & 10.3 & 8.25 \\
\hline 0.02 & 50 & ng & 16.1 & 15.9 & 14.2 & 10.9 \\
\hline 0.01 & 100 & ng & 22.2 & 22.0 & 19.5 & 14.4 \\
\hline 0.005 & 200 & ng & 30.2 & 30.2 & 26.6 & 18.8 \\
\hline 0.002 & 500 & ng & $\mathrm{ng}$ & $\mathrm{ng}$ & $\mathrm{ng}$ & $\mathrm{ng}$ \\
\hline
\end{tabular}

'Data does not fit log-Pearson Type III curve, use with caution.

\section{2-0.000-10M BALKH RIVER BELOW BAND-I-AMIR, Continued}

Probability of occurrence of annual low discharges [ $\mathrm{m}^{3} / \mathrm{s}$, meters per second]

\begin{tabular}{|c|c|c|c|c|c|c|c|c|c|c|}
\hline \multirow{3}{*}{$\begin{array}{c}\text { Nonexceedance } \\
\text { probability }\end{array}$} & \multirow{3}{*}{$\begin{array}{c}\text { Recurrence } \\
\text { interval } \\
\text { (years) }\end{array}$} & \multicolumn{9}{|c|}{ Minimum daily mean discharge $\left(\mathrm{m}^{3} / \mathrm{s}\right)$} \\
\hline & & \multicolumn{9}{|c|}{ Number of consecutive days } \\
\hline & & 1 & 3 & 7 & 14 & 30 & 60 & 90 & 120 & 183 \\
\hline 0.05 & 20 & 0.41 & 0.41 & 0.42 & 0.44 & 0.45 & 0.51 & 0.55 & 0.59 & 0.72 \\
\hline 0.10 & 10 & 0.49 & 0.49 & 0.51 & 0.53 & 0.56 & 0.61 & 0.65 & 0.70 & 0.81 \\
\hline 0.20 & 5 & 0.59 & 0.60 & 0.62 & 0.65 & 0.68 & 0.73 & 0.78 & 0.83 & 0.92 \\
\hline 0.50 & 2 & 0.80 & 0.82 & 0.84 & 0.87 & 0.91 & 0.95 & 0.99 & 1.04 & 1.08 \\
\hline
\end{tabular}




\section{2-0.000-10M BALKH RIVER BELOW BAND-I-AMIR, Continued}

\section{Probability of occurrence of seasonal low discharges}

[ $\mathrm{m}^{3} / \mathrm{s}$, meters per second]

\begin{tabular}{|c|c|c|c|c|c|c|c|c|c|}
\hline \multirow{3}{*}{$\begin{array}{c}\text { Nonexceedance } \\
\text { probability }\end{array}$} & \multirow{3}{*}{$\begin{array}{c}\text { Recurrence } \\
\text { interval } \\
\text { (years) }\end{array}$} & \multicolumn{8}{|c|}{ Minimum daily mean discharge $\left(\mathrm{m}^{3} / \mathrm{s}\right)$} \\
\hline & & \multicolumn{8}{|c|}{ Number of consecutive days } \\
\hline & & 1 & 7 & 14 & 30 & 1 & 7 & 14 & 30 \\
\hline & & \multicolumn{4}{|c|}{ December-January-February } & \multicolumn{4}{|c|}{ March-April-May } \\
\hline 0.05 & 20 & 0.46 & 0.47 & 0.49 & 0.50 & 0.68 & 0.68 & 0.67 & 0.71 \\
\hline 0.10 & 10 & 0.59 & 0.59 & 0.61 & 0.62 & 0.81 & 0.82 & 0.82 & 0.86 \\
\hline 0.20 & 5 & 0.75 & 0.76 & 0.77 & 0.79 & 0.97 & 1.00 & 1.00 & 1.04 \\
\hline \multirow[t]{2}{*}{0.50} & 2 & 1.08 & 1.09 & 1.10 & 1.13 & 1.28 & 1.32 & 1.35 & 1.41 \\
\hline & & \multicolumn{4}{|c|}{ June-July-August } & \multicolumn{4}{|c|}{ September-October-November } \\
\hline 0.05 & 20 & 0.74 & 0.79 & 0.83 & 0.87 & 0.70 & 0.72 & 0.73 & 0.79 \\
\hline 0.10 & 10 & 0.79 & 0.84 & 0.86 & 0.91 & 0.74 & 0.76 & 0.79 & 0.84 \\
\hline 0.20 & 5 & 0.85 & 0.89 & 0.91 & 0.96 & 0.79 & 0.82 & 0.85 & 0.90 \\
\hline 0.50 & 2 & 0.96 & 0.99 & 1.00 & 1.04 & 0.90 & 0.92 & 0.97 & 1.01 \\
\hline
\end{tabular}

\section{2-0.000-10M BALKH RIVER BELOW BAND-I-AMIR, Continued}

Annual peak discharges

[ $\mathrm{m}^{3} / \mathrm{s}$, meters per second]

\begin{tabular}{|c|c|c|c|c|c|}
\hline \multicolumn{3}{|c|}{$\begin{array}{c}\text { Annual peak discharge, } \\
\text { by year }\end{array}$} & \multicolumn{3}{|c|}{$\begin{array}{c}\text { Annual peak discharge, } \\
\text { from highest to lowest }\end{array}$} \\
\hline $\begin{array}{l}\text { Water } \\
\text { year }\end{array}$ & Date & $\begin{array}{c}\text { Peak } \\
\text { discharge } \\
\left(\mathrm{m}^{3} / \mathrm{s}\right)\end{array}$ & $\begin{array}{l}\text { Water } \\
\text { year }\end{array}$ & Date & $\begin{array}{c}\text { Peak } \\
\text { discharge } \\
\left(\mathrm{m}^{3} / \mathrm{s}\right)\end{array}$ \\
\hline 1970 & April 12, 1970 & 4.00 & 1975 & May 21, 1975 & 9.89 \\
\hline 1971 & April 18, 1971 & 3.43 & 1970 & April 12, 1970 & 4.00 \\
\hline 1972 & May 20, 1972 & 2.63 & 1971 & April 18, 1971 & 3.43 \\
\hline 1973 & May 16,1973 & 2.10 & 1972 & May 20, 1972 & 2.63 \\
\hline 1974 & June 7, 1974 & 1.67 & 1973 & May 16, 1973 & 2.10 \\
\hline 1975 & May 21,1975 & 9.89 & 1974 & June 7, 1974 & 1.67 \\
\hline
\end{tabular}




\section{2-0.000-10M BALKH RIVER BELOW BAND-I-AMIR, Continued}

Monthly and annual mean discharges, in cubic meters per second

[Data may not be rounded in accordance with U.S. Geological Survey publication standards; --, no data]

\begin{tabular}{|c|c|c|c|c|c|c|c|c|c|c|c|c|c|}
\hline \multirow{2}{*}{$\begin{array}{l}\text { Water } \\
\text { year }\end{array}$} & \multicolumn{12}{|c|}{ Monthly mean discharge } & \multirow{2}{*}{$\begin{array}{c}\text { Annual } \\
\text { discharge }\end{array}$} \\
\hline & October & November & December & January & February & March & April & May & June & July & August & September & \\
\hline 1970 & 1.95 & 1.83 & 1.61 & 1.75 & 1.85 & 2.18 & 2.93 & 1.81 & 1.29 & 1.22 & 1.12 & 1.12 & 1.72 \\
\hline 1971 & 1.11 & 1.27 & 1.41 & 1.49 & 1.67 & 1.71 & 3.02 & 2.01 & 1.07 & 0.93 & 0.93 & 1.19 & 1.48 \\
\hline 1972 & 1.10 & 1.11 & 1.15 & 1.44 & 1.56 & 1.64 & 1.82 & 2.36 & 1.78 & 1.24 & 0.96 & 1.09 & 1.44 \\
\hline 1973 & 1.19 & 1.20 & 1.10 & 1.10 & 1.13 & 1.25 & 1.65 & 2.00 & 1.55 & 1.16 & 1.03 & 0.87 & 1.27 \\
\hline 1974 & 1.16 & 1.54 & 1.13 & 0.92 & 1.13 & 1.31 & 1.17 & 1.26 & 1.54 & 1.29 & 1.10 & 1.14 & 1.22 \\
\hline 1975 & 1.11 & 0.83 & 0.64 & 0.51 & 0.65 & 0.76 & 2.43 & 6.83 & 1.91 & 1.18 & 1.25 & 1.24 & 1.62 \\
\hline 1976 & 1.08 & 1.09 & 1.11 & 1.04 & 1.14 & -- & -- & -- & -- & -- & -- & -- & -- \\
\hline
\end{tabular}




\section{2-1.R00-1A SUF RIVER NEAR KISHANDEH}

\section{(U.S. Geological Survey identification number: 360800066570000 )}

LOCATION: Lat $36^{\circ} 08^{\prime} \mathrm{N}$., long 66 $56^{\circ} \mathrm{E}$.

DRAINAGE AREA: $3,070 \mathrm{~km}^{2}$.

ELEVATION: 753 meters above mean sea level.

PERIOD OF RECORD: January 9, 1969 to September 30, 1978.

GAGE: Water-stage recorder.

Annual mean discharge

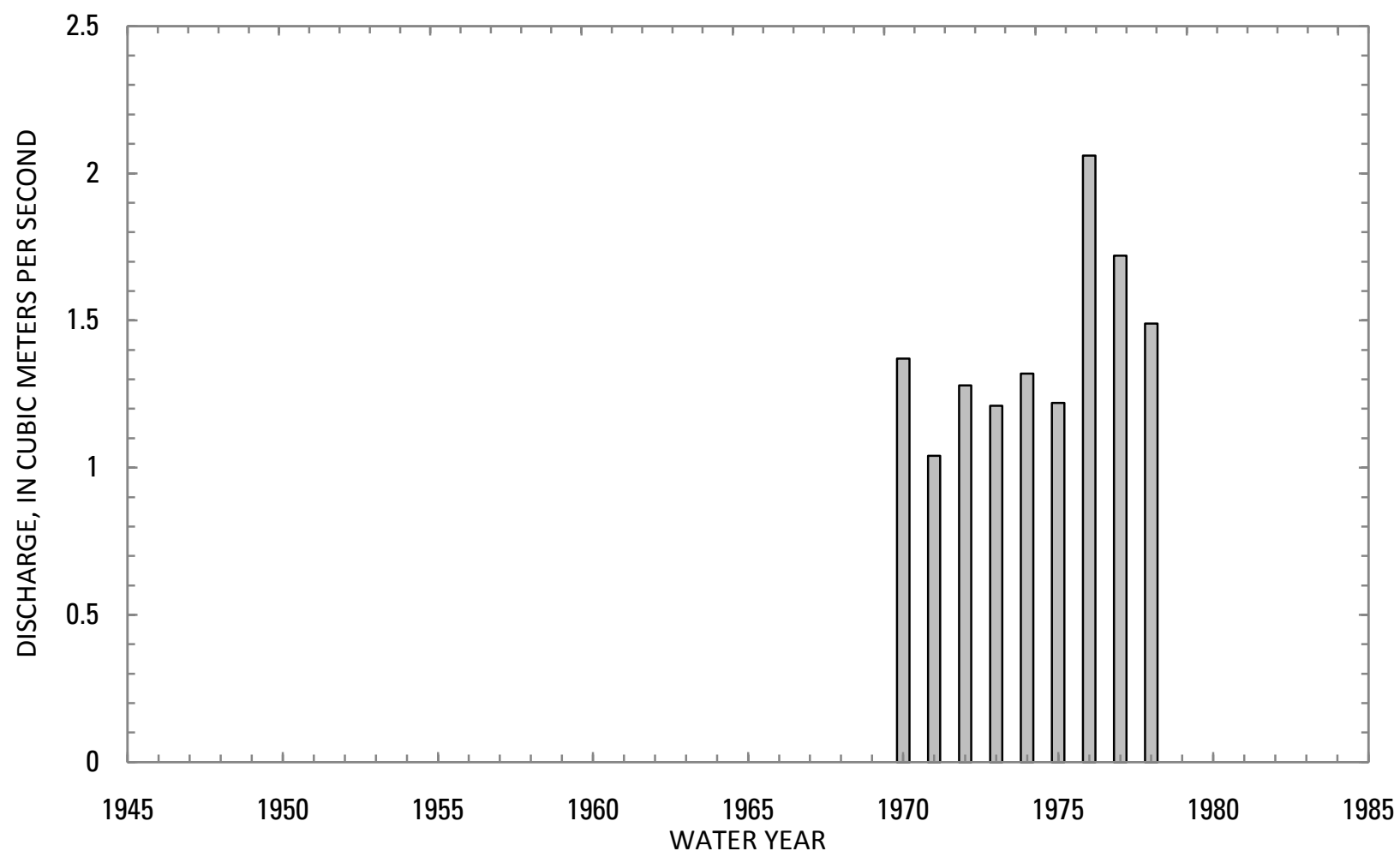




\section{2-1.R00-1A SUF RIVER NEAR KISHANDEH, Continued}

Statistics of monthly and annual mean discharges

[ $\mathrm{m}^{3} / \mathrm{s}$, cubic meters per second; ng, not given]

\begin{tabular}{|c|c|c|c|c|c|c|c|c|}
\hline \multirow[b]{2}{*}{ Month } & \multicolumn{2}{|c|}{ Maximum } & \multicolumn{2}{|c|}{ Minimum } & \multicolumn{4}{|c|}{ Mean } \\
\hline & $\begin{array}{c}\text { Discharge } \\
\left(\mathrm{m}^{3} / \mathrm{s}\right)\end{array}$ & $\begin{array}{c}\text { Water year } \\
\text { of } \\
\text { occurrence }\end{array}$ & $\begin{array}{c}\text { Discharge } \\
\left(\mathrm{m}^{3} / \mathrm{s}\right)\end{array}$ & $\begin{array}{c}\text { Water year } \\
\text { of } \\
\text { occurrence }\end{array}$ & $\begin{array}{c}\text { Discharge } \\
\left(\mathrm{m}^{3} / \mathrm{s}\right)\end{array}$ & $\begin{array}{c}\text { Standard } \\
\text { deviation } \\
\left(\mathrm{m}^{3} / \mathrm{s}\right)\end{array}$ & $\begin{array}{c}\text { Coefficient } \\
\text { of } \\
\text { variation }\end{array}$ & $\begin{array}{c}\text { Percentage } \\
\text { of annual } \\
\text { discharge }\end{array}$ \\
\hline October & 2.12 & 1970 & 0.40 & 1972 & 1.38 & 0.52 & 0.38 & 7.74 \\
\hline November & 2.67 & 1977 & 0.67 & 1972 & 1.71 & 0.53 & 0.31 & 9.60 \\
\hline December & 3.38 & 1977 & 0.96 & 1972 & 1.97 & 0.72 & 0.36 & 11.0 \\
\hline January & 3.15 & 1977 & 1.13 & 1972 & 2.06 & 0.58 & 0.28 & 11.5 \\
\hline February & 2.89 & 1969 & 1.27 & 1972 & 2.02 & 0.52 & 0.26 & 11.3 \\
\hline March & 2.98 & 1969 & 1.67 & 1973 & 2.05 & 0.44 & 0.22 & 11.5 \\
\hline April & 4.53 & 1976 & 1.18 & 1974 & 2.22 & 1.03 & 0.46 & 12.5 \\
\hline May & 7.70 & 1976 & 0.09 & 1971 & 2.39 & 2.34 & 0.98 & 13.4 \\
\hline June & 2.64 & 1972 & 0 & 1975 & 0.52 & 0.82 & 1.57 & 2.93 \\
\hline July & 1.11 & 1969 & 0 & ng & 0.16 & 0.34 & 2.19 & 0.88 \\
\hline August & 1.57 & 1969 & 0.02 & 1971 & 0.37 & 0.46 & 1.25 & 2.05 \\
\hline September & 2.04 & 1969 & 0.12 & 1971 & 1.00 & 0.53 & 0.53 & 5.60 \\
\hline Annual & 2.06 & 1976 & 1.04 & 1971 & 1.41 & 0.31 & 0.22 & 100 \\
\hline
\end{tabular}


12-1.R00-1A SUF RIVER NEAR KISHANDEH, Continued

Monthly and annual flow duration, in cubic meters per second

[ng, not given]

\begin{tabular}{|c|c|c|c|c|c|c|c|c|c|c|c|c|c|}
\hline \multirow{2}{*}{$\begin{array}{l}\text { Percentage } \\
\text { of days } \\
\text { discharge } \\
\text { equaled or } \\
\text { exceeded }\end{array}$} & \multicolumn{12}{|c|}{ Month } & \multirow{2}{*}{ Annua } \\
\hline & October & November & December & January & February & March & April & May & June & July & August & September & \\
\hline 95 & 0.37 & 0.66 & 0.98 & 1.14 & 1.15 & 1.30 & 0.78 & 0 & 0 & 0 & 0 & 0.04 & 0 \\
\hline 90 & 0.47 & 0.75 & 1.05 & 1.16 & 1.27 & 1.40 & 0.97 & 0 & 0 & 0 & 0 & 0.30 & 0 \\
\hline 85 & 1.00 & 1.27 & 1.48 & 1.61 & 1.40 & 1.56 & 1.13 & 0 & 0 & 0 & 0 & 0.47 & 0 \\
\hline 80 & 1.05 & 1.39 & 1.62 & 1.73 & 1.53 & 1.69 & 1.24 & 0 & 0 & 0 & 0 & 0.58 & 0.09 \\
\hline 75 & 1.08 & 1.44 & 1.71 & 1.75 & 1.60 & 1.73 & 1.31 & 0.01 & 0 & 0 & 0 & 0.62 & 0.39 \\
\hline 70 & 1.14 & 1.55 & 1.75 & 1.77 & 1.71 & 1.76 & 1.37 & 0.45 & 0 & 0 & 0 & 0.67 & 0.64 \\
\hline 65 & 1.23 & 1.66 & 1.77 & 1.85 & 1.76 & 1.80 & 1.50 & 0.73 & 0 & 0 & 0 & 0.72 & 0.92 \\
\hline 60 & 1.37 & 1.70 & 1.78 & 1.92 & 1.93 & 1.86 & 1.65 & 0.89 & 0 & 0 & 0.01 & 0.78 & 1.08 \\
\hline 55 & 1.41 & 1.72 & 1.80 & 1.93 & 2.07 & 1.90 & 1.76 & 1.06 & 0.01 & 0 & 0.02 & 0.86 & 1.25 \\
\hline 50 & 1.43 & 1.74 & 1.81 & 1.95 & 2.10 & 1.93 & 1.87 & 1.23 & 0.02 & 0 & 0.18 & 0.93 & 1.41 \\
\hline 45 & 1.45 & 1.77 & 1.83 & 2.05 & 2.18 & 1.96 & 1.97 & 1.42 & 0.04 & 0 & 0.25 & 0.98 & 1.54 \\
\hline 40 & 1.47 & 1.79 & 1.85 & 2.09 & 2.22 & 2.00 & 2.03 & 1.61 & 0.11 & 0 & 0.28 & 1.05 & 1.64 \\
\hline 35 & 1.49 & 1.82 & 1.88 & 2.15 & 2.26 & 2.05 & 2.09 & 1.79 & 0.18 & 0.01 & 0.39 & 1.12 & 1.74 \\
\hline 30 & 1.57 & 1.86 & 1.91 & 2.23 & 2.31 & 2.12 & 2.16 & 2.21 & 0.39 & 0.04 & 0.47 & 1.22 & 1.84 \\
\hline 25 & 1.68 & 1.92 & 1.95 & 2.31 & 2.38 & 2.25 & 2.25 & 2.92 & 0.72 & 0.09 & 0.59 & 1.33 & 1.96 \\
\hline 20 & 1.96 & 2.02 & 2.45 & 2.55 & 2.41 & 2.39 & 2.39 & 3.74 & 0.90 & 0.22 & 0.66 & 1.48 & 2.10 \\
\hline 15 & 2.07 & 2.22 & 3.06 & 2.73 & 2.52 & 2.80 & 2.64 & 5.18 & 1.01 & 0.30 & 0.71 & 1.80 & 2.25 \\
\hline 10 & 2.12 & 2.36 & 3.18 & 3.03 & 2.77 & 2.97 & 3.23 & 6.66 & 1.23 & 0.71 & 1.16 & 1.93 & 2.42 \\
\hline 5 & 2.16 & $\mathrm{ng}$ & $\mathrm{ng}$ & $\mathrm{ng}$ & ng & 3.12 & 4.75 & 9.09 & 1.61 & $\mathrm{ng}$ & 1.58 & 2.04 & 3.15 \\
\hline
\end{tabular}




\section{2-1.R00-1A SUF RIVER NEAR KISHANDEH, Continued}

Probability of occurrence of annual high discharges

[ $\mathrm{m}^{3} / \mathrm{s}$, cubic meters per second; ng, not given]

\begin{tabular}{|c|c|c|c|c|c|c|}
\hline \multirow{2}{*}{$\begin{array}{l}\text { Exceedance } \\
\text { probability }\end{array}$} & \multirow{2}{*}{$\begin{array}{c}\text { Recurrence } \\
\text { interval } \\
\text { (years) }\end{array}$} & \multirow{2}{*}{$\begin{array}{c}\text { Maximum } \\
\text { instantaneous } \\
\text { discharge } \\
\left(\mathrm{m}^{3} / \mathrm{s}\right)\end{array}$} & \multicolumn{4}{|c|}{ Maximum daily mean discharge $\left(\mathrm{m}^{3} / \mathrm{s}\right)$} \\
\hline & & & $\begin{array}{l}\text { 3-day } \\
\text { period }\end{array}$ & $\begin{array}{l}\text { 7-day } \\
\text { period }\end{array}$ & $\begin{array}{l}\text { 15-day } \\
\text { period }\end{array}$ & $\begin{array}{l}\text { 30-day } \\
\text { period }\end{array}$ \\
\hline 0.99 & 1.01 & 1.40 & ${ }^{1} 1.24$ & ${ }^{1} 1.45$ & 1.78 & 1.57 \\
\hline 0.95 & 1.05 & 3.30 & ${ }^{1} 1.95$ & ${ }^{1} 1.92$ & 1.99 & 1.79 \\
\hline 0.90 & 1.11 & 5.00 & 2.49 & 2.26 & 2.16 & 1.97 \\
\hline 0.80 & 1.25 & 8.00 & 3.37 & 2.80 & 2.45 & 2.24 \\
\hline 0.50 & 2 & 18.0 & 6.18 & 4.43 & 3.40 & 3.07 \\
\hline 0.20 & 5 & 36.4 & 11.6 & 7.48 & 5.37 & 4.63 \\
\hline 0.10 & 10 & 50.5 & 16.4 & 10.1 & 7.23 & 5.99 \\
\hline 0.04 & 25 & 69.5 & 23.8 & 14.2 & 10.4 & 8.14 \\
\hline 0.02 & 50 & 84.1 & 30.4 & 18.0 & 13.5 & 10.1 \\
\hline 0.01 & 100 & 98.8 & 38.0 & 22.3 & 17.4 & 12.4 \\
\hline 0.005 & 200 & 114 & 46.9 & 27.4 & 22.2 & 15.2 \\
\hline 0.002 & 500 & 133 & $\mathrm{ng}$ & $\mathrm{ng}$ & $\mathrm{ng}$ & $\mathrm{ng}$ \\
\hline
\end{tabular}

'Data does not fit log-Pearson Type III curve, use with caution.

\section{2-1.R00-1A SUF RIVER NEAR KISHANDEH, Continued}

Probability of occurrence of annual low discharges [ $\mathrm{m}^{3} / \mathrm{s}$, meters per second; $\mathrm{ng}$, not given]

\begin{tabular}{|c|c|c|c|c|c|c|c|c|c|c|}
\hline \multirow{3}{*}{$\begin{array}{c}\text { Nonexceedance } \\
\text { probability }\end{array}$} & \multirow{3}{*}{$\begin{array}{c}\text { Recurrence } \\
\text { interval } \\
\text { (years) }\end{array}$} & \multicolumn{9}{|c|}{ Minimum daily mean discharge $\left(\mathrm{m}^{3} / \mathrm{s}\right)$} \\
\hline & & \multicolumn{9}{|c|}{ Number of consecutive days } \\
\hline & & 1 & 3 & 7 & 14 & 30 & 60 & 90 & 120 & 183 \\
\hline 0.05 & 20 & ng & ng & ng & 0 & 0 & 0 & 0.03 & 0.03 & 0.14 \\
\hline 0.10 & 10 & ng & ng & $\mathrm{ng}$ & 0 & 0 & 0 & 0.04 & 0.06 & 0.22 \\
\hline 0.20 & 5 & $\mathrm{ng}$ & ng & $\mathrm{ng}$ & 0 & 0 & 0.01 & 0.06 & 0.12 & 0.36 \\
\hline 0.50 & 2 & ng & ng & ng & 0 & 0 & 0.02 & 0.13 & 0.34 & 0.72 \\
\hline
\end{tabular}




\section{2-1.R00-1A SUF RIVER NEAR KISHANDEH, Continued}

Probability of occurrence of seasonal low discharges [ $\mathrm{m}^{3} / \mathrm{s}$, meters per second; $\mathrm{ng}$, not given]

\begin{tabular}{|c|c|c|c|c|c|c|c|c|c|}
\hline \multirow{3}{*}{$\begin{array}{c}\text { Nonexceedance } \\
\text { probability }\end{array}$} & \multirow{3}{*}{$\begin{array}{c}\text { Recurrence } \\
\text { interval } \\
\text { (years) }\end{array}$} & \multicolumn{8}{|c|}{ Minimum daily mean discharge $\left(\mathrm{m}^{3} / \mathrm{s}\right)$} \\
\hline & & \multicolumn{8}{|c|}{ Number of consecutive days } \\
\hline & & 1 & 7 & 14 & 30 & 1 & 7 & 14 & 30 \\
\hline & & \multicolumn{4}{|c|}{ December-January-February } & \multicolumn{4}{|c|}{ March-April-May } \\
\hline 0.05 & 20 & 0.87 & 0.90 & 0.94 & 1.01 & 0 & 0 & 0 & 0.10 \\
\hline 0.10 & 10 & 1.00 & 1.05 & 1.08 & 1.15 & 0 & 0 & 0 & 0.21 \\
\hline 0.20 & 5 & 1.16 & 1.23 & 1.27 & 1.33 & 0 & 0 & 0 & 0.44 \\
\hline \multirow[t]{2}{*}{0.50} & 2 & 1.51 & 1.61 & 1.64 & 1.72 & 0 & 0 & 0.36 & 1.21 \\
\hline & & \multicolumn{4}{|c|}{ June-July-August } & \multicolumn{4}{|c|}{ September-October-November } \\
\hline 0.05 & 20 & ng & ng & 0 & 0 & 0 & 0 & 0.05 & 0.16 \\
\hline 0.10 & 10 & ng & ng & 0 & 0 & 0 & 0 & 0.12 & 0.27 \\
\hline 0.20 & 5 & ng & ng & 0 & 0 & 0.10 & 0.36 & 0.26 & 0.46 \\
\hline 0.50 & 2 & ng & $\mathrm{ng}$ & 0 & 0 & 0.46 & 0.53 & 0.75 & 0.97 \\
\hline
\end{tabular}

\section{2-1.R00-1A SUF RIVER NEAR KISHANDEH, Continued}

\section{Annual peak discharges}

[ $\mathrm{m}^{3} / \mathrm{s}$, meters per second]

\begin{tabular}{|c|c|c|c|c|c|}
\hline \multicolumn{3}{|c|}{$\begin{array}{c}\text { Annual peak discharge, } \\
\text { by year }\end{array}$} & \multicolumn{3}{|c|}{$\begin{array}{l}\text { Annual peak discharge, } \\
\text { from highest to lowest }\end{array}$} \\
\hline $\begin{array}{l}\text { Water } \\
\text { year }\end{array}$ & Date & $\begin{array}{c}\text { Peak } \\
\text { discharge } \\
\left(\mathrm{m}^{3} / \mathrm{s}\right)\end{array}$ & $\begin{array}{l}\text { Water } \\
\text { year }\end{array}$ & Date & $\begin{array}{c}\text { Peak } \\
\text { discharge } \\
\left(\mathrm{m}^{3} / \mathrm{s}\right)\end{array}$ \\
\hline 1969 & April 20, 1969 & 10.0 & 1976 & April 19, 1976 & 66.8 \\
\hline 1970 & April 16, 1970 & 5.92 & 1977 & April 15, 1977 & 40.4 \\
\hline 1971 & April 10, 1971 & 3.02 & 1972 & May 13, 1972 & 25.6 \\
\hline 1972 & May 13, 1972 & 25.6 & 1973 & April 24, 1973 & 24.3 \\
\hline 1973 & April 24, 1973 & 24.3 & 1975 & May 3, 1975 & 24.3 \\
\hline 1974 & March 28, 1974 & 14.5 & 1978 & May 26, 1978 & 16.0 \\
\hline 1975 & May 3, 1975 & 24.3 & 1974 & March 28, 1974 & 14.5 \\
\hline 1976 & April 19, 1976 & 66.8 & 1969 & April 20, 1969 & 10.0 \\
\hline 1977 & April 15, 1977 & 40.4 & 1970 & April 16, 1970 & 5.92 \\
\hline 1978 & May 26, 1978 & 16.0 & 1971 & April 10, 1971 & 3.02 \\
\hline
\end{tabular}


12-1.R00-1A SUF RIVER NEAR KISHANDEH, Continued

Monthly and annual mean discharges, in cubic meters per second

[Data may not be rounded in accordance with U.S. Geological Survey publication standards; --, no data]

\begin{tabular}{|c|c|c|c|c|c|c|c|c|c|c|c|c|c|}
\hline \multirow{2}{*}{$\begin{array}{l}\text { Water } \\
\text { year }\end{array}$} & \multicolumn{12}{|c|}{ Monthly mean discharge } & \multirow{2}{*}{$\begin{array}{c}\text { Annual } \\
\text { discharge }\end{array}$} \\
\hline & October & November & December & January & February & March & April & May & June & July & August & September & \\
\hline 1969 & -- & -- & -- & -- & 2.89 & 2.98 & 3.35 & 3.80 & 0.87 & 1.11 & 1.57 & 2.04 & -- \\
\hline 1970 & 2.12 & 2.13 & 1.89 & 1.94 & 1.72 & 2.70 & 2.25 & 0.55 & 0.10 & 0.15 & 0.18 & 0.69 & 1.37 \\
\hline 1971 & 1.43 & 1.72 & 1.82 & 2.07 & 2.29 & 1.77 & 1.23 & 0.09 & 0.03 & 0.03 & 0.02 & 0.12 & 1.04 \\
\hline 1972 & 0.40 & 0.67 & 0.96 & 1.13 & 1.27 & 1.76 & 2.02 & 3.69 & 2.64 & 0 & 0.10 & 0.73 & 1.28 \\
\hline 1973 & 1.39 & 1.73 & 1.74 & 1.95 & 1.36 & 1.67 & 1.73 & 1.41 & 0.02 & 0.04 & 0.64 & 0.84 & 1.21 \\
\hline 1974 & 1.08 & 1.59 & 2.85 & 2.67 & 2.32 & 2.14 & 1.18 & 0.73 & 0.11 & 0 & 0.18 & 1.01 & 1.32 \\
\hline 1975 & 1.38 & 1.69 & 1.74 & 1.70 & 1.60 & 1.76 & 2.01 & 1.58 & 0 & 0.19 & 0.35 & 0.70 & 1.22 \\
\hline 1976 & 1.08 & 1.45 & 1.83 & 2.19 & 2.06 & 2.02 & 4.53 & 7.70 & 0.16 & 0.04 & 0.26 & 1.40 & 2.06 \\
\hline 1977 & 2.06 & 2.67 & 3.38 & 3.15 & 2.49 & 1.81 & 2.41 & 0.71 & 0.93 & 0 & 0.08 & 1.03 & 1.72 \\
\hline 1978 & 1.50 & 1.78 & 1.54 & 1.75 & 2.17 & 1.92 & 1.53 & 3.64 & 0.36 & 0 & 0.28 & 1.45 & 1.49 \\
\hline
\end{tabular}




\section{3-0.000-1M KHULM RIVER AT TANGI TASHQURGHAN}

\section{(U.S. Geological Survey identification number: 364000067420000)}

LOCATION: Lat $36^{\circ} 40^{\prime} \mathrm{N}$., long $67^{\circ} 42^{\prime} \mathrm{E}$.

DRAINAGE AREA: $8,220 \mathrm{~km}^{2}$.

ELEVATION: 515 meters above mean sea level.

PERIOD OF RECORD: February 17, 1969 to September 30, 1978.

GAGE: Water-stage recorder. Staff gage at same site and datum prior to 1970 water year.

Annual mean discharge

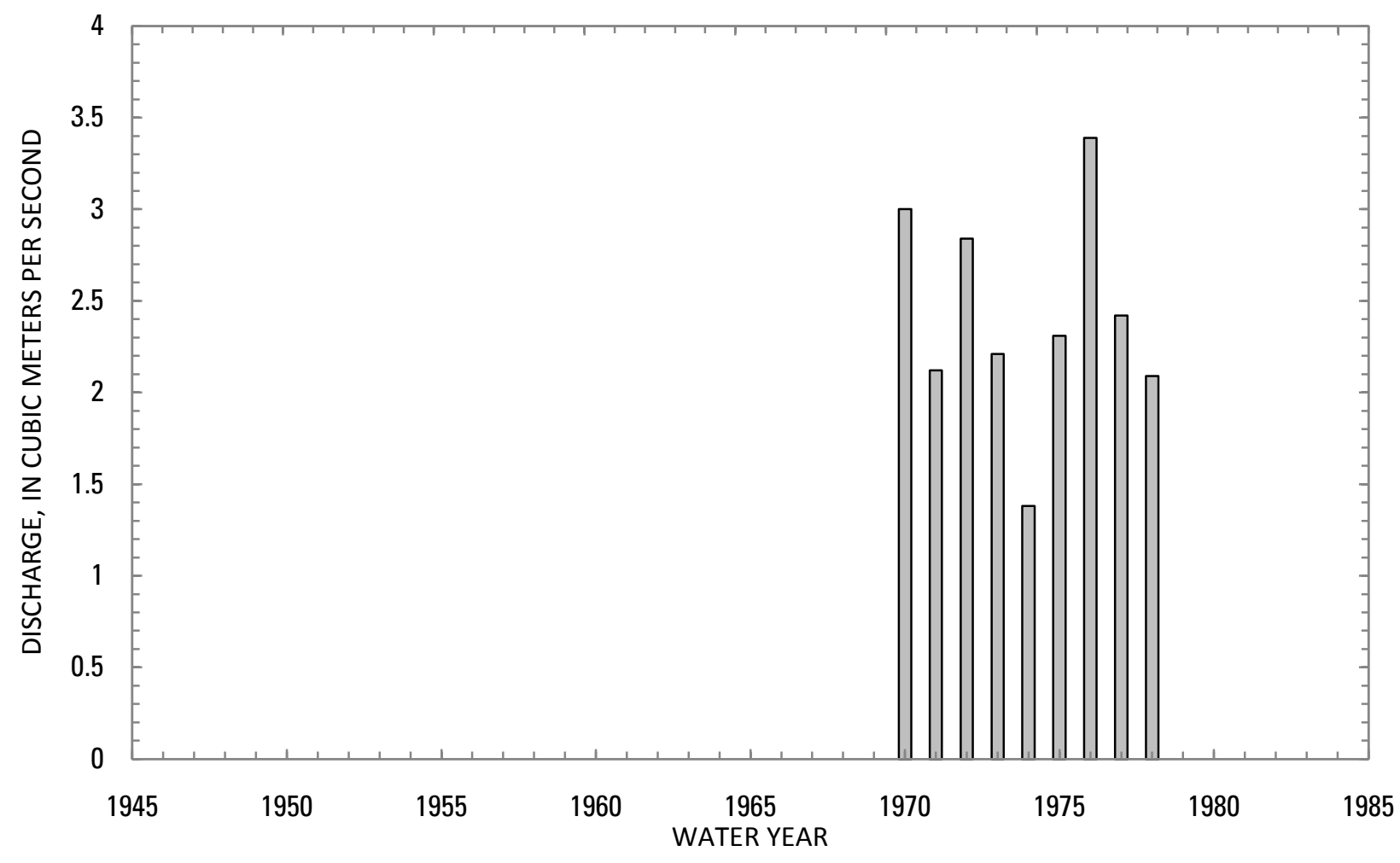




\section{3-0.000-1M KHULM RIVER AT TANGI TASHQURGHAN, Continued}

Statistics of monthly and annual mean discharges

$\left[\mathrm{m}^{3} / \mathrm{s}\right.$, cubic meters per second]

\begin{tabular}{|c|c|c|c|c|c|c|c|c|}
\hline \multirow[b]{2}{*}{ Month } & \multicolumn{2}{|c|}{ Maximum } & \multicolumn{2}{|c|}{ Minimum } & \multicolumn{4}{|c|}{ Mean } \\
\hline & $\begin{array}{c}\text { Discharge } \\
\left(\mathrm{m}^{3} / \mathrm{s}\right)\end{array}$ & $\begin{array}{c}\text { Water year } \\
\text { of } \\
\text { occurrence }\end{array}$ & $\begin{array}{c}\text { Discharge } \\
\left(\mathrm{m}^{3} / \mathrm{s}\right)\end{array}$ & $\begin{array}{c}\text { Water year } \\
\text { of } \\
\text { occurrence }\end{array}$ & $\begin{array}{c}\text { Discharge } \\
\left(\mathrm{m}^{3} / \mathrm{s}\right)\end{array}$ & $\begin{array}{c}\text { Standard } \\
\text { deviation } \\
\left(\mathrm{m}^{3} / \mathrm{s}\right)\end{array}$ & $\begin{array}{c}\text { Coefficient } \\
\text { of } \\
\text { variation }\end{array}$ & $\begin{array}{c}\text { Percentage } \\
\text { of annual } \\
\text { discharge }\end{array}$ \\
\hline October & 2.26 & 1978 & 1.21 & 1974 & 1.71 & 0.35 & 0.21 & 5.50 \\
\hline November & 4.75 & 1978 & 1.61 & 1974 & 2.59 & 1.01 & 0.39 & 8.35 \\
\hline December & 4.56 & 1970 & 1.70 & 1974 & 2.97 & 0.99 & 0.33 & 9.57 \\
\hline January & 4.05 & 1970 & 1.44 & 1974 & 2.81 & 0.75 & 0.27 & 9.04 \\
\hline February & 3.55 & 1970 & 1.44 & 1974 & 2.64 & 0.66 & 0.25 & 8.51 \\
\hline March & 6.12 & 1969 & 2.31 & 1973 & 3.57 & 1.30 & 0.36 & 11.5 \\
\hline April & 11.7 & 1976 & 1.09 & 1978 & 3.96 & 3.13 & 0.79 & 12.8 \\
\hline May & 11.3 & 1969 & 1.13 & 1971 & 4.66 & 3.45 & 0.74 & 15.0 \\
\hline June & 4.90 & 1969 & 0.94 & 1974 & 2.10 & 1.18 & 0.56 & 6.77 \\
\hline July & 2.61 & 1969 & 0.82 & 1977 & 1.36 & 0.52 & 0.38 & 4.37 \\
\hline August & 2.33 & 1969 & 0.70 & 1978 & 1.22 & 0.43 & 0.35 & 3.94 \\
\hline September & 2.52 & 1969 & 0.76 & 1978 & 1.45 & 0.46 & 0.32 & 4.68 \\
\hline Annual & 3.39 & 1976 & 1.38 & 1974 & 2.42 & 0.59 & 0.24 & 100 \\
\hline
\end{tabular}


13-0.000-1M KHULM RIVER AT TANGI TASHQURGHAN, Continued

Monthly and annual flow duration, in cubic meters per second

[ng, not given]

\begin{tabular}{|c|c|c|c|c|c|c|c|c|c|c|c|c|c|}
\hline \multirow{2}{*}{$\begin{array}{l}\text { Percentage } \\
\text { of days } \\
\text { discharge } \\
\text { equaled or } \\
\text { exceeded }\end{array}$} & \multicolumn{12}{|c|}{ Month } & \multirow{2}{*}{ Annua } \\
\hline & October & November & December & January & February & March & April & May & June & July & August & September & \\
\hline 95 & 0.96 & 1.21 & 1.39 & 1.33 & 1.31 & 1.47 & 0.89 & 0.62 & 0.82 & 0.76 & 0.58 & 0.76 & 0.81 \\
\hline 90 & 1.07 & 1.42 & 1.55 & 1.68 & 1.78 & 1.76 & 1.07 & 0.93 & 0.94 & 0.82 & 0.69 & 0.87 & 0.95 \\
\hline 85 & 1.16 & 1.53 & 1.77 & 2.09 & 1.89 & 1.96 & 1.22 & 1.10 & 1.01 & 0.86 & 0.74 & 0.95 & 1.06 \\
\hline 80 & 1.30 & 1.59 & 2.17 & 2.21 & 2.00 & 2.23 & 1.34 & 1.22 & 1.06 & 0.89 & 0.78 & 0.99 & 1.15 \\
\hline 75 & 1.33 & 1.66 & 2.35 & 2.32 & 2.16 & 2.46 & 1.48 & 1.31 & 1.18 & 0.94 & 0.83 & 1.12 & 1.25 \\
\hline 70 & 1.39 & 1.90 & 2.44 & 2.39 & 2.30 & 2.61 & 1.65 & 1.39 & 1.25 & 0.97 & 0.92 & 1.22 & 1.35 \\
\hline 65 & 1.45 & 2.07 & 2.51 & 2.48 & 2.37 & 2.73 & 1.86 & 1.48 & 1.31 & 1.04 & 1.00 & 1.26 & 1.45 \\
\hline 60 & 1.50 & 2.20 & 2.59 & 2.59 & 2.45 & 2.88 & 2.09 & 1.58 & 1.35 & 1.09 & 1.04 & 1.29 & 1.57 \\
\hline 55 & 1.54 & 2.33 & 2.67 & 2.68 & 2.51 & 3.03 & 2.33 & 1.71 & 1.40 & 1.14 & 1.08 & 1.32 & 1.70 \\
\hline 50 & 1.57 & 2.42 & 2.99 & 2.76 & 2.56 & 3.19 & 2.57 & 2.02 & 1.47 & 1.20 & 1.12 & 1.35 & 1.84 \\
\hline 45 & 1.61 & 2.52 & 3.10 & 2.81 & 2.66 & 3.46 & 2.91 & 2.51 & 1.55 & 1.24 & 1.16 & 1.40 & 2.06 \\
\hline 40 & 1.74 & 2.62 & 3.20 & 2.85 & 2.76 & 3.66 & 3.21 & 2.98 & 1.62 & 1.31 & 1.21 & 1.44 & 2.26 \\
\hline 35 & 1.84 & 2.73 & 3.41 & 2.92 & 2.90 & 3.98 & 3.46 & 3.60 & 1.69 & 1.39 & 1.27 & 1.48 & 2.45 \\
\hline 30 & 1.92 & 2.83 & 3.52 & 3.14 & 3.10 & 4.13 & 3.70 & 4.34 & 1.86 & 1.46 & 1.35 & 1.55 & 2.64 \\
\hline 25 & 2.02 & 2.99 & 3.64 & 3.24 & 3.24 & 4.27 & 4.00 & 5.05 & 2.00 & 1.51 & 1.45 & 1.67 & 2.90 \\
\hline 20 & 2.15 & 3.30 & 3.96 & 3.53 & 3.37 & 4.43 & 4.32 & 6.42 & 2.39 & 1.64 & 1.56 & 1.75 & 3.19 \\
\hline 15 & 2.33 & 3.81 & 4.34 & 3.93 & 3.49 & 4.63 & 4.90 & 7.85 & 3.63 & 1.84 & 1.68 & 1.91 & 3.63 \\
\hline 10 & 2.47 & 4.19 & 4.52 & 4.02 & 3.56 & 5.28 & 6.26 & 10.8 & 4.06 & 2.14 & 1.86 & 2.12 & 4.17 \\
\hline 5 & 2.78 & 4.46 & 4.70 & 4.28 & 3.62 & 6.35 & 10.4 & 18.4 & 5.60 & $\mathrm{ng}$ & 2.44 & 2.59 & 4.77 \\
\hline
\end{tabular}




\section{3-0.000-1M KHULM RIVER AT TANGI TASHQURGHAN, Continued}

Probability of occurrence of annual high discharges

[m $\mathrm{m}^{3} / \mathrm{s}$, cubic meters per second; ng, not given]

\begin{tabular}{|c|c|c|c|c|c|c|}
\hline \multirow{2}{*}{$\begin{array}{c}\text { Exceedance } \\
\text { probability }\end{array}$} & \multirow{2}{*}{$\begin{array}{c}\text { Recurrence } \\
\text { interval } \\
\text { (years) }\end{array}$} & \multirow{2}{*}{$\begin{array}{c}\text { Maximum } \\
\text { instantaneous } \\
\text { discharge } \\
\left(\mathrm{m}^{3} / \mathrm{s}\right)\end{array}$} & \multicolumn{4}{|c|}{ Maximum daily mean discharge $\left(\mathrm{m}^{3} / \mathrm{s}\right)$} \\
\hline & & & $\begin{array}{l}\text { 3-day } \\
\text { period }\end{array}$ & $\begin{array}{l}\text { 7-day } \\
\text { period }\end{array}$ & $\begin{array}{l}\text { 15-day } \\
\text { period }\end{array}$ & $\begin{array}{l}\text { 30-day } \\
\text { period }\end{array}$ \\
\hline 0.99 & 1.01 & 0.90 & ${ }^{1} 1.63$ & ${ }^{1} 2.00$ & 2.09 & 2.00 \\
\hline 0.95 & 1.05 & 3.20 & ${ }^{1} 2.81$ & 2.83 & 2.70 & 2.52 \\
\hline 0.90 & 1.11 & 5.90 & 3.77 & 3.47 & 3.16 & 2.89 \\
\hline 0.80 & 1.25 & 11.4 & 5.40 & 4.55 & 3.92 & 3.45 \\
\hline 0.50 & 2 & 33.6 & 10.8 & 8.18 & 6.31 & 5.06 \\
\hline 0.20 & 5 & 78.1 & 21.7 & 16.1 & 11.2 & 7.88 \\
\hline 0.10 & 10 & 111 & 31.4 & 23.9 & 15.7 & 10.2 \\
\hline 0.04 & 25 & 153 & 46.7 & 37.5 & 23.3 & 13.6 \\
\hline 0.02 & 50 & 182 & 60.5 & 51.1 & 30.5 & 16.6 \\
\hline 0.01 & 100 & 209 & 76.3 & 68.2 & 39.5 & 20.0 \\
\hline 0.005 & 200 & 234 & 94.6 & 89.7 & 50.5 & 23.8 \\
\hline 0.002 & 500 & 264 & $\mathrm{ng}$ & $\mathrm{ng}$ & $\mathrm{ng}$ & $\mathrm{ng}$ \\
\hline
\end{tabular}

'Data does not fit log-Pearson Type III curve, use with caution.

\section{3-0.000-1M KHULM RIVER AT TANGI TASHQURGHAN, Continued}

Probability of occurrence of annual low discharges [ $\mathrm{m}^{3} / \mathrm{s}$, meters per second]

\begin{tabular}{|c|c|c|c|c|c|c|c|c|c|c|}
\hline \multirow{3}{*}{$\begin{array}{c}\text { Nonexceedance } \\
\text { probability }\end{array}$} & \multirow{3}{*}{$\begin{array}{c}\text { Recurrence } \\
\text { interval } \\
\text { (years) }\end{array}$} & \multicolumn{9}{|c|}{ Minimum daily mean discharge $\left(\mathrm{m}^{3} / \mathrm{s}\right)$} \\
\hline & & \multicolumn{9}{|c|}{ Number of consecutive days } \\
\hline & & 1 & 3 & 7 & 14 & 30 & 60 & 90 & 120 & 183 \\
\hline 0.05 & 20 & 0.06 & 0.09 & 0.11 & 0.60 & 0.77 & 0.95 & 1.05 & 1.09 & 1.12 \\
\hline 0.10 & 10 & 0.11 & 0.15 & 0.22 & 0.65 & 0.79 & 0.96 & 1.05 & 1.10 & 1.18 \\
\hline 0.20 & 5 & 0.19 & 0.27 & 0.40 & 0.73 & 0.84 & 0.99 & 1.07 & 1.13 & 1.27 \\
\hline 0.50 & 2 & 0.48 & 0.61 & 0.81 & 0.91 & 0.99 & 1.11 & 1.17 & 1.24 & 1.54 \\
\hline
\end{tabular}




\section{3-0.000-1M KHULM RIVER AT TANGI TASHQURGHAN, Continued}

Probability of occurrence of seasonal low discharges

$\left[\mathrm{m}^{3} / \mathrm{s}\right.$, meters per second]

\begin{tabular}{|c|c|c|c|c|c|c|c|c|c|}
\hline \multirow{3}{*}{$\begin{array}{c}\text { Nonexceedance } \\
\text { probability }\end{array}$} & \multirow{3}{*}{$\begin{array}{c}\text { Recurrence } \\
\text { interval } \\
\text { (years) }\end{array}$} & \multicolumn{8}{|c|}{ Minimum daily mean discharge $\left(\mathrm{m}^{3} / \mathrm{s}\right)$} \\
\hline & & \multicolumn{8}{|c|}{ Number of consecutive days } \\
\hline & & 1 & 7 & 14 & 30 & 1 & 7 & 14 & 30 \\
\hline & & \multicolumn{4}{|c|}{ December-January-February } & \multicolumn{4}{|c|}{ March-April-May } \\
\hline 0.05 & 20 & 0.92 & 1.00 & 1.13 & 1.43 & 0.12 & 0.51 & 0.57 & 0.87 \\
\hline 0.10 & 10 & 1.03 & 1.15 & 1.30 & 1.60 & 0.22 & 0.60 & 0.74 & 1.07 \\
\hline 0.20 & 5 & 1.19 & 1.36 & 1.53 & 1.82 & 0.40 & 0.74 & 1.00 & 1.36 \\
\hline \multirow[t]{2}{*}{0.50} & 2 & 1.60 & 1.86 & 2.05 & 2.29 & 0.93 & 1.13 & 1.69 & 2.19 \\
\hline & & \multicolumn{4}{|c|}{ June-July-August } & \multicolumn{4}{|c|}{ September-October-November } \\
\hline 0.05 & 20 & 0.12 & 0.12 & 0.63 & 0.71 & 0.43 & 0.76 & 0.98 & 1.05 \\
\hline 0.10 & 10 & 0.21 & 0.23 & 0.66 & 0.74 & 0.53 & 0.81 & 1.02 & 1.1 \\
\hline 0.20 & 5 & 0.36 & 0.42 & 0.71 & 0.79 & 0.67 & 0.86 & 1.07 & 1.17 \\
\hline 0.50 & 2 & 0.72 & 0.86 & 0.86 & 0.97 & 0.92 & 0.98 & 1.19 & 1.35 \\
\hline
\end{tabular}

13-0.000-1M KHULM RIVER AT TANGI TASHQURGHAN, Continued

Annual peak discharges

$\left[\mathrm{m}^{3} / \mathrm{s}\right.$, meters per second]

\begin{tabular}{|c|c|c|c|c|c|}
\hline \multicolumn{3}{|c|}{$\begin{array}{c}\text { Annual peak discharge, } \\
\text { by year }\end{array}$} & \multicolumn{3}{|c|}{$\begin{array}{l}\text { Annual peak discharge, } \\
\text { from highest to lowest }\end{array}$} \\
\hline $\begin{array}{l}\text { Water } \\
\text { year }\end{array}$ & Date & $\begin{array}{c}\text { Peak } \\
\text { discharge } \\
\left(\mathrm{m}^{3} / \mathrm{s}\right)\end{array}$ & $\begin{array}{l}\text { Water } \\
\text { year }\end{array}$ & Date & $\begin{array}{c}\text { Peak } \\
\text { discharge } \\
\left(\mathrm{m}^{3} / \mathrm{s}\right)\end{array}$ \\
\hline 1969 & May 11, 1969 & 50.4 & 1976 & May 25, 1976 & 85.2 \\
\hline 1970 & May 10, 1970 & 7.54 & 1973 & May 16, 1973 & 80.0 \\
\hline 1971 & April 14, 1971 & 4.60 & 1978 & November 8, 1977 & 72.8 \\
\hline 1972 & May 16, 1972 & 30.0 & 1977 & May 29, 1977 & 57.0 \\
\hline 1973 & May 16, 1973 & 80.0 & 1969 & May 11, 1969 & 50.4 \\
\hline 1974 & May 10, 1974 & 4.80 & 1975 & May 16, 1975 & 49.2 \\
\hline 1975 & May 16, 1975 & 49.2 & 1972 & May 16,1972 & 30.0 \\
\hline 1976 & May 25, 1976 & 85.2 & 1970 & May 10,1970 & 7.54 \\
\hline 1977 & May 29, 1977 & 57.0 & 1974 & May 10, 1974 & 4.80 \\
\hline 1978 & November 8, 1977 & 72.8 & 1971 & April 14, 1971 & 4.60 \\
\hline
\end{tabular}


13-0.000-1M KHULM RIVER AT TANGI TASHQURGHAN, Continued

Monthly and annual mean discharges, in cubic meters per second

[Data may not be rounded in accordance with U.S. Geological Survey publication standards; --, no data]

\begin{tabular}{|c|c|c|c|c|c|c|c|c|c|c|c|c|c|}
\hline \multirow{2}{*}{$\begin{array}{l}\text { Water } \\
\text { year }\end{array}$} & \multicolumn{12}{|c|}{ Monthly mean discharge } & \multirow{2}{*}{$\begin{array}{c}\text { Annual } \\
\text { discharge }\end{array}$} \\
\hline & October & November & December & January & February & March & April & May & June & July & August & September & \\
\hline 1969 & -- & -- & -- & -- & -- & 6.12 & 6.67 & 11.3 & 4.90 & 2.61 & 2.33 & 2.52 & -- \\
\hline 1970 & 2.25 & 3.78 & 4.56 & 4.05 & 3.55 & 4.16 & 3.30 & 3.44 & 2.72 & 1.79 & 1.05 & 1.39 & 3.00 \\
\hline 1971 & 1.49 & 2.52 & 3.44 & 2.77 & 2.98 & 3.11 & 2.64 & 1.13 & 1.24 & 1.34 & 1.15 & 1.68 & 2.12 \\
\hline 1972 & 1.84 & 1.99 & 2.12 & 2.39 & 2.26 & 4.20 & 3.82 & 9.22 & 2.25 & 1.38 & 1.04 & 1.48 & 2.84 \\
\hline 1973 & 1.57 & 2.26 & 2.50 & 2.86 & 2.64 & 2.31 & 3.62 & 3.35 & 1.38 & 1.26 & 1.32 & 1.51 & 2.21 \\
\hline 1974 & 1.21 & 1.61 & 1.70 & 1.44 & 1.44 & 2.40 & 1.31 & 1.30 & 0.94 & 1.00 & 1.07 & 1.13 & 1.38 \\
\hline 1975 & 1.53 & 2.26 & 3.18 & 2.77 & 2.26 & 2.66 & 2.75 & 4.95 & 1.78 & 1.28 & 1.03 & 1.24 & 2.31 \\
\hline 1976 & 1.51 & 2.23 & 3.04 & 2.42 & 2.83 & 5.17 & 11.7 & 6.57 & 1.54 & 1.17 & 1.29 & 1.24 & 3.39 \\
\hline 1977 & 1.70 & 1.92 & 2.00 & 3.64 & 3.45 & 3.03 & 2.69 & 4.07 & 2.95 & 0.82 & 1.24 & 1.59 & 2.42 \\
\hline 1978 & 2.26 & 4.75 & 4.20 & 2.91 & 2.37 & 2.52 & 1.09 & 1.28 & 1.31 & 0.93 & 0.70 & 0.76 & 2.09 \\
\hline
\end{tabular}




\section{3-0.000-2M KHULM RIVER AT SAYAD}

\section{(U.S. Geological Survey identification number: 363500067470000)}

LOCATION: Lat $36^{\circ} 35^{\prime} \mathrm{N}$., long $67^{\circ} 47^{\prime} \mathrm{E}$.

DRAINAGE AREA: $8,170 \mathrm{~km}^{2}$.

ELEVATION: 669 meters above mean sea level.

PERIOD OF RECORD: May 13, 1964 to September 30, 1978.

GAGE: Water-stage recorder. Staff gage at same site and datum prior to 1970 water year.

Annual mean discharge

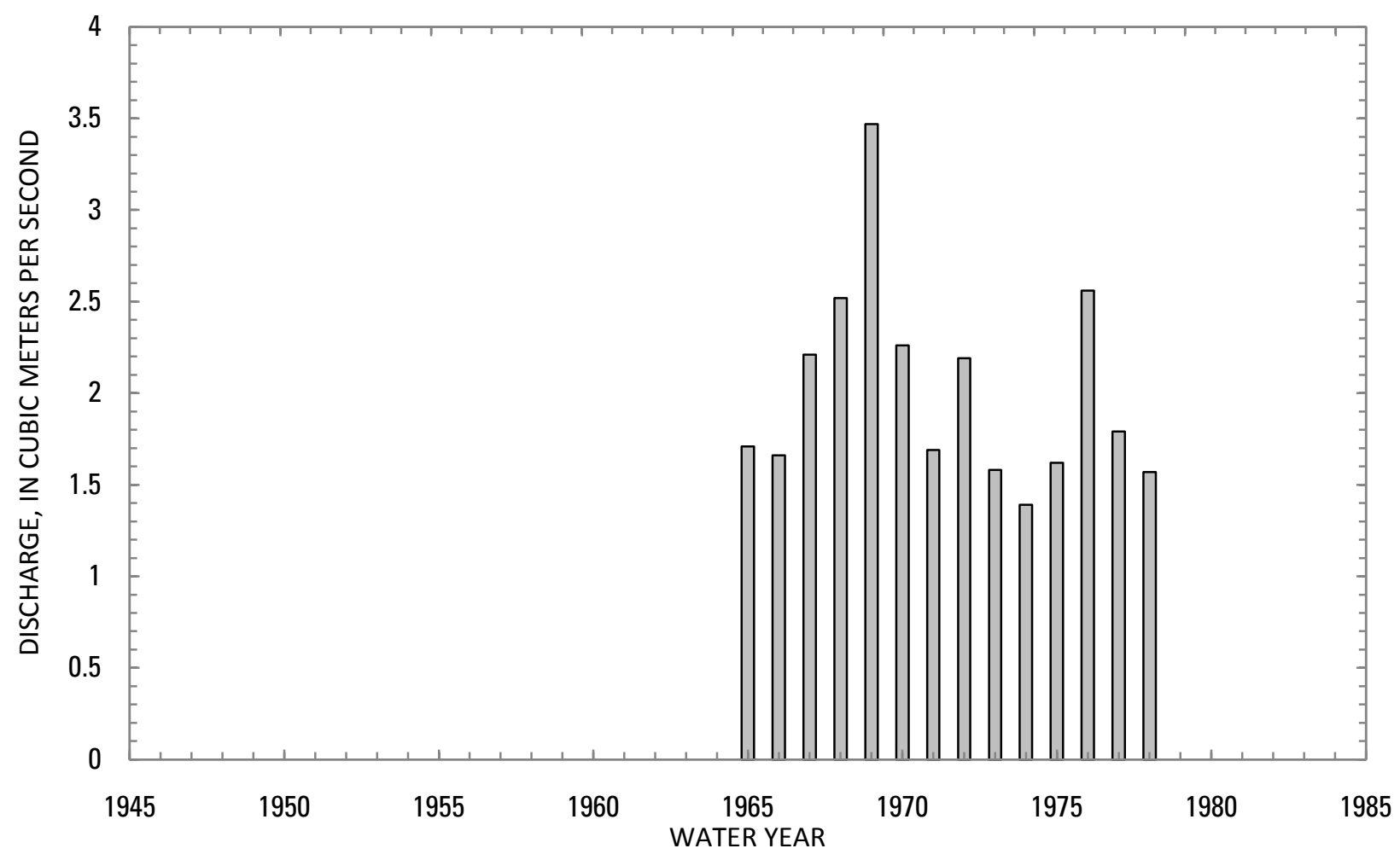




\section{3-0.000-2M KHULM RIVER AT SAYAD, Continued}

Statistics of monthly and annual mean discharges $\left[\mathrm{m}^{3} / \mathrm{s}\right.$, cubic meters per second]

\begin{tabular}{|c|c|c|c|c|c|c|c|c|}
\hline \multirow[b]{2}{*}{ Month } & \multicolumn{2}{|c|}{ Maximum } & \multicolumn{2}{|c|}{ Minimum } & \multicolumn{4}{|c|}{ Mean } \\
\hline & $\begin{array}{c}\text { Discharge } \\
\left(\mathrm{m}^{3} / \mathrm{s}\right)\end{array}$ & $\begin{array}{c}\text { Water year } \\
\text { of } \\
\text { occurrence }\end{array}$ & $\begin{array}{c}\text { Discharge } \\
\left(\mathrm{m}^{3} / \mathrm{s}\right)\end{array}$ & $\begin{array}{c}\text { Water year } \\
\text { of } \\
\text { occurrence }\end{array}$ & $\begin{array}{c}\text { Discharge } \\
\left(\mathrm{m}^{3} / \mathrm{s}\right)\end{array}$ & $\begin{array}{c}\text { Standard } \\
\text { deviation } \\
\left(\mathrm{m}^{3} / \mathrm{s}\right)\end{array}$ & $\begin{array}{c}\text { Coefficient } \\
\text { of } \\
\text { variation }\end{array}$ & $\begin{array}{c}\text { Percentage } \\
\text { of annual } \\
\text { discharge }\end{array}$ \\
\hline October & 2.41 & 1968 & 0.80 & 1965 & 1.44 & 0.44 & 0.30 & 5.98 \\
\hline November & 3.38 & 1970 & 0.91 & 1965 & 1.92 & 0.77 & 0.40 & 7.98 \\
\hline December & 3.25 & 1970 & 0.75 & 1965 & 2.12 & 0.67 & 0.32 & 8.80 \\
\hline January & 3.06 & 1969 & 1.21 & 1965 & 2.06 & 0.48 & 0.23 & 8.53 \\
\hline February & 3.33 & 1969 & 1.63 & 1973 & 2.13 & 0.43 & 0.20 & 8.83 \\
\hline March & 5.52 & 1969 & 1.73 & 1977 & 2.66 & 0.98 & 0.37 & 11.0 \\
\hline April & 10.1 & 1976 & 1.07 & 1978 & 2.91 & 2.31 & 0.79 & 12.1 \\
\hline May & 9.73 & 1969 & 0.99 & 1978 & 3.90 & 2.69 & 0.69 & 16.2 \\
\hline June & 3.25 & 1968 & 0.64 & 1976 & 1.45 & 0.78 & 0.53 & 6.02 \\
\hline July & 1.82 & 1969 & 0.71 & 1975 & 1.15 & 0.35 & 0.30 & 4.79 \\
\hline August & 1.84 & 1967 & 0.84 & 1976 & 1.10 & 0.30 & 0.28 & 4.58 \\
\hline September & 1.99 & 1967 & 0.84 & 1978 & 1.25 & 0.36 & 0.29 & 5.18 \\
\hline Annual & 3.47 & 1969 & 1.39 & 1974 & 2.02 & 0.56 & 0.28 & 100 \\
\hline
\end{tabular}


13-0.000-2M KHULM RIVER AT SAYAD, Continued

Monthly and annual flow duration, in cubic meters per second

\begin{tabular}{|c|c|c|c|c|c|c|c|c|c|c|c|c|c|}
\hline \multirow{2}{*}{$\begin{array}{l}\text { Percentage } \\
\text { of days } \\
\text { discharge } \\
\text { equaled or } \\
\text { exceeded }\end{array}$} & \multicolumn{12}{|c|}{ Month } & \multirow{2}{*}{ Annual } \\
\hline & October & November & December & January & February & March & April & May & June & July & August & September & \\
\hline 95 & 0.72 & 0.85 & 0.78 & 1.27 & 1.36 & 1.29 & 0.92 & 0.87 & 0.56 & 0.69 & 0.79 & 0.72 & 0.78 \\
\hline 90 & 0.85 & 0.93 & 1.28 & 1.38 & 1.46 & 1.55 & 1.06 & 0.95 & 0.71 & 0.74 & 0.82 & 0.81 & 0.87 \\
\hline 85 & 0.89 & 1.01 & 1.52 & 1.57 & 1.57 & 1.70 & 1.16 & 0.99 & 0.79 & 0.79 & 0.84 & 0.89 & 0.94 \\
\hline 80 & 0.95 & 1.14 & 1.60 & 1.64 & 1.65 & 1.89 & 1.24 & 1.03 & 0.86 & 0.83 & 0.86 & 0.92 & 1.00 \\
\hline 75 & 0.99 & 1.28 & 1.67 & 1.71 & 1.78 & 1.98 & 1.33 & 1.10 & 0.89 & 0.87 & 0.87 & 0.95 & 1.07 \\
\hline 70 & 1.03 & 1.52 & 1.74 & 1.77 & 1.87 & 2.06 & 1.44 & 1.18 & 0.93 & 0.89 & 0.89 & 0.98 & 1.15 \\
\hline 65 & 1.09 & 1.59 & 1.82 & 1.83 & 1.95 & 2.22 & 1.55 & 1.32 & 0.97 & 0.91 & 0.92 & 1.00 & 1.26 \\
\hline 60 & 1.14 & 1.65 & 1.89 & 1.94 & 2.05 & 2.31 & 1.67 & 1.45 & 1.00 & 0.94 & 0.94 & 1.02 & 1.39 \\
\hline 55 & 1.21 & 1.70 & 1.99 & 2.02 & 2.10 & 2.38 & 1.81 & 1.60 & 1.04 & 0.96 & 0.96 & 1.05 & 1.51 \\
\hline 50 & 1.30 & 1.76 & 2.09 & 2.08 & 2.15 & 2.46 & 1.97 & 1.80 & 1.08 & 1.00 & 0.98 & 1.08 & 1.64 \\
\hline 45 & 1.39 & 1.83 & 2.17 & 2.11 & 2.19 & 2.55 & 2.12 & 2.06 & 1.12 & 1.07 & 1.00 & 1.15 & 1.75 \\
\hline 40 & 1.48 & 1.91 & 2.26 & 2.15 & 2.24 & 2.65 & 2.29 & 2.36 & 1.16 & 1.14 & 1.02 & 1.20 & 1.87 \\
\hline 35 & 1.59 & 2.00 & 2.34 & 2.20 & 2.29 & 2.76 & 2.45 & 2.84 & 1.25 & 1.19 & 1.04 & 1.31 & 2.00 \\
\hline 30 & 1.69 & 2.10 & 2.45 & 2.25 & 2.33 & 2.86 & 2.65 & 3.43 & 1.36 & 1.27 & 1.08 & 1.41 & 2.14 \\
\hline 25 & 1.78 & 2.31 & 2.64 & 2.32 & 2.37 & 3.04 & 2.87 & 3.83 & 1.49 & 1.36 & 1.13 & 1.48 & 2.30 \\
\hline 20 & 1.89 & 2.60 & 2.81 & 2.43 & 2.44 & 3.22 & 3.11 & 4.69 & 1.66 & 1.54 & 1.20 & 1.62 & 2.45 \\
\hline 15 & 1.99 & 2.84 & 2.94 & 2.57 & 2.55 & 3.41 & 3.46 & 6.14 & 1.82 & 1.64 & 1.43 & 1.81 & 2.71 \\
\hline 10 & 2.15 & 3.12 & 3.09 & 2.77 & 2.71 & 3.73 & 4.29 & 9.14 & 2.30 & 1.79 & 1.73 & 2.03 & 3.08 \\
\hline 5 & 2.60 & 3.42 & 3.27 & 3.12 & 2.98 & 4.20 & 6.46 & 16.5 & 4.38 & 2.03 & 1.95 & 2.16 & 3.81 \\
\hline
\end{tabular}




\section{3-0.000-2M KHULM RIVER AT SAYAD, Continued}

Probability of occurrence of annual high discharges [ $\mathrm{m}^{3} / \mathrm{s}$, cubic meters per second; ng, not given]

\begin{tabular}{|c|c|c|c|c|c|c|}
\hline \multirow{2}{*}{$\begin{array}{c}\text { Exceedance } \\
\text { probability }\end{array}$} & \multirow{2}{*}{$\begin{array}{c}\text { Recurrence } \\
\text { interval } \\
\text { (years) }\end{array}$} & \multirow{2}{*}{$\begin{array}{c}\text { Maximum } \\
\text { instantaneous } \\
\text { discharge } \\
\left(\mathrm{m}^{3} / \mathrm{s}\right)\end{array}$} & \multicolumn{4}{|c|}{ Maximum daily mean discharge $\left(\mathrm{m}^{3} / \mathrm{s}\right)$} \\
\hline & & & $\begin{array}{l}\text { 3-day } \\
\text { period }\end{array}$ & $\begin{array}{l}\text { 7-day } \\
\text { period }\end{array}$ & $\begin{array}{l}\text { 15-day } \\
\text { period }\end{array}$ & $\begin{array}{l}\text { 30-day } \\
\text { period }\end{array}$ \\
\hline 0.99 & 1.01 & 1.00 & ${ }^{1} 1.30$ & ${ }^{1} 1.51$ & ${ }^{1} 1.63$ & 1.70 \\
\hline 0.95 & 1.05 & 2.90 & 2.50 & 2.40 & 2.27 & 2.15 \\
\hline 0.90 & 1.11 & 4.80 & 3.49 & 3.10 & 2.74 & 2.47 \\
\hline 0.80 & 1.25 & 8.50 & 5.19 & 4.25 & 3.48 & 2.97 \\
\hline 0.50 & 2 & 22.6 & 10.7 & 7.93 & 5.72 & 4.38 \\
\hline 0.20 & 5 & 52.0 & 21.1 & 15.3 & 9.88 & 6.86 \\
\hline 0.10 & 10 & 76.1 & 29.6 & 21.7 & 13.4 & 8.88 \\
\hline 0.04 & 25 & 110 & 41.8 & 31.9 & 18.9 & 11.9 \\
\hline 0.02 & 50 & 137 & 51.9 & 41.2 & 23.9 & 14.6 \\
\hline 0.01 & 100 & 164 & 62.8 & 51.9 & 29.5 & 17.6 \\
\hline 0.005 & 200 & 192 & 74.5 & 64.4 & 36.1 & 21.0 \\
\hline 0.002 & 500 & 228 & $\mathrm{ng}$ & ng & ng & ng \\
\hline
\end{tabular}

'Data does not fit log-Pearson Type III curve, use with caution.

\section{3-0.000-2M KHULM RIVER AT SAYAD, Continued}

Probability of occurrence of annual low discharges

$\left[\mathrm{m}^{3} / \mathrm{s}\right.$, meters per second]

\begin{tabular}{|c|c|c|c|c|c|c|c|c|c|c|}
\hline \multirow{3}{*}{$\begin{array}{c}\text { Nonexceedance } \\
\text { probability }\end{array}$} & \multirow{3}{*}{$\begin{array}{c}\text { Recurrence } \\
\text { interval } \\
\text { (years) }\end{array}$} & \multicolumn{9}{|c|}{ Minimum daily mean discharge $\left(\mathrm{m}^{3} / \mathrm{s}\right)$} \\
\hline & & \multicolumn{9}{|c|}{ Number of consecutive days } \\
\hline & & 1 & 3 & 7 & 14 & 30 & 60 & 90 & 120 & 183 \\
\hline 0.05 & 20 & 0.38 & 0.41 & 0.42 & 0.47 & 0.56 & 0.64 & 0.70 & 0.77 & 0.92 \\
\hline 0.10 & 10 & 0.45 & 0.47 & 0.49 & 0.54 & 0.62 & 0.70 & 0.76 & 0.83 & 0.97 \\
\hline 0.20 & 5 & 0.53 & 0.56 & 0.58 & 0.64 & 0.70 & 0.79 & 0.85 & 0.91 & 1.05 \\
\hline 0.50 & 2 & 0.71 & 0.74 & 0.77 & 0.85 & 0.91 & 1.01 & 1.06 & 1.11 & 1.27 \\
\hline
\end{tabular}




\section{3-0.000-2M KHULM RIVER AT SAYAD, Continued}

\section{Probability of occurrence of seasonal low discharges}

[ $\mathrm{m}^{3} / \mathrm{s}$, meters per second]

\begin{tabular}{|c|c|c|c|c|c|c|c|c|c|}
\hline \multirow{3}{*}{$\begin{array}{c}\text { Nonexceedance } \\
\text { probability }\end{array}$} & \multirow{3}{*}{$\begin{array}{c}\text { Recurrence } \\
\text { interval } \\
\text { (years) }\end{array}$} & \multicolumn{8}{|c|}{ Minimum daily mean discharge $\left(\mathrm{m}^{3} / \mathrm{s}\right)$} \\
\hline & & \multicolumn{8}{|c|}{ Number of consecutive days } \\
\hline & & 1 & 7 & 14 & 30 & 1 & 7 & 14 & 30 \\
\hline & & \multicolumn{4}{|c|}{ December-January-February } & \multicolumn{4}{|c|}{ March-April-May } \\
\hline 0.05 & 20 & 0.77 & 0.83 & 0.87 & 0.95 & 0.72 & 0.8 & 0.84 & 0.93 \\
\hline 0.10 & 10 & 0.91 & 0.99 & 1.03 & 1.14 & 0.74 & 0.83 & 0.91 & 1.04 \\
\hline 0.20 & 5 & 1.08 & 1.19 & 1.25 & 1.38 & 0.78 & 0.88 & 1.02 & 1.21 \\
\hline \multirow[t]{2}{*}{0.50} & 2 & 1.44 & 1.59 & 1.68 & 1.85 & 0.91 & 1.06 & 1.33 & 1.69 \\
\hline & & \multicolumn{4}{|c|}{ June-July-August } & \multicolumn{4}{|c|}{ September-October-November } \\
\hline 0.05 & 20 & 0.41 & 0.46 & 0.50 & 0.59 & 0.57 & 0.61 & 0.68 & 0.79 \\
\hline 0.10 & 10 & 0.48 & 0.53 & 0.58 & 0.65 & 0.61 & 0.65 & 0.74 & 0.84 \\
\hline 0.20 & 5 & 0.57 & 0.63 & 0.68 & 0.74 & 0.66 & 0.72 & 0.82 & 0.92 \\
\hline 0.50 & 2 & 0.76 & 0.83 & 0.89 & 0.95 & 0.81 & 0.89 & 1.03 & 1.12 \\
\hline
\end{tabular}

\section{3-0.000-2M KHULM RIVER AT SAYAD, Continued}

Annual peak discharges

[ $\mathrm{m}^{3} / \mathrm{s}$, meters per second]

\begin{tabular}{|c|c|c|c|c|c|}
\hline \multicolumn{3}{|c|}{$\begin{array}{c}\text { Annual peak discharge, } \\
\text { by year }\end{array}$} & \multicolumn{3}{|c|}{$\begin{array}{l}\text { Annual peak discharge, } \\
\text { from highest to lowest }\end{array}$} \\
\hline $\begin{array}{l}\text { Water } \\
\text { year }\end{array}$ & Date & $\begin{array}{c}\text { Peak } \\
\text { discharge } \\
\left(\mathrm{m}^{3} / \mathrm{s}\right)\end{array}$ & $\begin{array}{l}\text { Water } \\
\text { year }\end{array}$ & Date & $\begin{array}{c}\text { Peak } \\
\text { discharge } \\
\left(\mathrm{m}^{3} / \mathrm{s}\right)\end{array}$ \\
\hline 1965 & May 7, 1965 & 24.4 & 1976 & May 25, 1976 & 80.0 \\
\hline 1966 & April 14, 1966 & 4.08 & 1973 & May 16, 1973 & 75.0 \\
\hline 1967 & May 24, 1967 & 17.0 & 1978 & November 8, 1977 & 70.0 \\
\hline 1968 & May 20, 1968 & 23.6 & 1969 & May 11, 1969 & 45.0 \\
\hline 1969 & May 11, 1969 & 45.0 & 1977 & April 14, 1977 & 36.5 \\
\hline 1970 & April 7, 1970 & 10.2 & 1972 & May 16, 1972 & 25.0 \\
\hline 1971 & March 13, 1971 & 3.87 & 1975 & May 17, 1975 & 25.0 \\
\hline 1972 & May 16, 1972 & 25.0 & 1965 & May 7, 1965 & 24.4 \\
\hline 1973 & May 16, 1973 & 75.0 & 1968 & May 20, 1968 & 23.6 \\
\hline 1974 & March 15, 1974 & 3.20 & 1967 & May 24, 1967 & 17.0 \\
\hline 1975 & May 17,1975 & 25.0 & 1970 & April 7, 1970 & 10.2 \\
\hline 1976 & May 25, 1976 & 80.0 & 1966 & April 14, 1966 & 4.08 \\
\hline 1977 & April 14, 1977 & 36.5 & 1971 & March 13, 1971 & 3.87 \\
\hline 1978 & November 8, 1977 & 70.0 & 1974 & March 15, 1974 & 3.20 \\
\hline
\end{tabular}




\section{3-0.000-2M KHULM RIVER AT SAYAD, Continued}

Monthly and annual mean discharges, in cubic meters per second

[Data may not be rounded in accordance with U.S. Geological Survey publication standards; --, no data]

\begin{tabular}{|c|c|c|c|c|c|c|c|c|c|c|c|c|c|}
\hline \multirow{2}{*}{$\begin{array}{l}\text { Water } \\
\text { year }\end{array}$} & \multicolumn{12}{|c|}{ Monthly mean discharge } & \multirow{2}{*}{$\begin{array}{c}\text { Annual } \\
\text { discharge }\end{array}$} \\
\hline & October & November & December & January & February & March & April & May & June & July & August & September & \\
\hline 1964 & -- & -- & -- & -- & -- & -- & -- & -- & 0.94 & 0.90 & 0.97 & 0.96 & -- \\
\hline 1965 & 0.80 & 0.91 & 0.75 & 1.21 & 2.05 & 2.25 & 2.27 & 5.31 & 1.39 & 1.29 & 0.91 & 1.35 & 1.71 \\
\hline 1966 & 1.31 & 1.95 & 2.22 & 2.11 & 2.25 & 2.45 & 1.99 & 1.51 & 1.15 & 1.05 & 0.90 & 1.10 & 1.66 \\
\hline 1967 & 1.75 & 1.98 & 2.20 & 2.31 & 2.23 & 1.86 & 1.72 & 5.40 & 1.52 & 1.64 & 1.84 & 1.99 & 2.21 \\
\hline 1968 & 2.41 & 3.00 & 2.85 & 2.13 & 1.85 & 2.31 & 2.64 & 5.15 & 3.25 & 1.51 & 1.39 & 1.75 & 2.52 \\
\hline 1969 & 1.60 & 1.63 & 2.86 & 3.06 & 3.33 & 5.52 & 5.37 & 9.73 & 3.05 & 1.82 & 1.68 & 1.87 & 3.47 \\
\hline 1970 & 2.07 & 3.38 & 3.25 & 2.81 & 2.40 & 3.50 & 2.56 & 1.71 & 1.31 & 1.53 & 1.21 & 1.44 & 2.26 \\
\hline 1971 & 1.42 & 2.02 & 2.72 & 2.18 & 2.24 & 2.31 & 1.87 & 1.05 & 1.24 & 1.23 & 1.03 & 1.01 & 1.69 \\
\hline 1972 & 0.93 & 1.22 & 1.52 & 1.82 & 2.12 & 3.14 & 3.10 & 7.50 & 1.58 & 1.05 & 1.07 & 1.20 & 2.19 \\
\hline 1973 & 1.14 & 1.41 & 1.53 & 1.61 & 1.63 & 2.29 & 2.76 & 2.27 & 1.20 & 1.20 & 0.96 & 0.97 & 1.58 \\
\hline 1974 & 1.43 & 1.43 & 1.70 & 1.71 & 1.85 & 2.42 & 1.22 & 1.19 & 0.93 & 0.95 & 0.90 & 0.97 & 1.39 \\
\hline 1975 & 1.15 & 1.48 & 1.95 & 1.95 & 1.78 & 2.15 & 2.18 & 3.52 & 0.72 & 0.71 & 0.84 & 0.97 & 1.62 \\
\hline 1976 & 1.09 & 1.60 & 1.93 & 1.62 & 1.94 & 3.30 & 10.10 & 5.90 & 0.64 & 0.72 & 0.84 & 1.10 & 2.56 \\
\hline 1977 & 1.40 & 1.62 & 1.70 & 2.20 & 2.46 & 1.73 & 1.94 & 3.40 & 2.01 & 0.80 & 1.03 & 1.22 & 1.79 \\
\hline 1978 & 1.67 & 3.31 & 2.50 & 2.06 & 1.66 & 2.01 & 1.07 & 0.99 & 0.85 & 0.90 & 0.99 & 0.84 & 1.57 \\
\hline
\end{tabular}




\section{4-0.000-1M KUNDUZ RIVER AT KULUKH TEPA}

\section{(U.S. Geological Survey identification number: 365900068180000)}

LOCATION: Lat $36^{\circ} 59^{\prime} \mathrm{N}$., long $68^{\circ} 18^{\prime} \mathrm{E}$.

DRAINAGE AREA: $37,100 \mathrm{~km}^{2}$.

ELEVATION: 320 meters above mean sea level.

PERIOD OF RECORD: October 1, 1965 to September 30, 1978.

GAGE: Water-stage recorder. Staff gage at same site and datum prior to 1969 water year.

Annual mean discharge

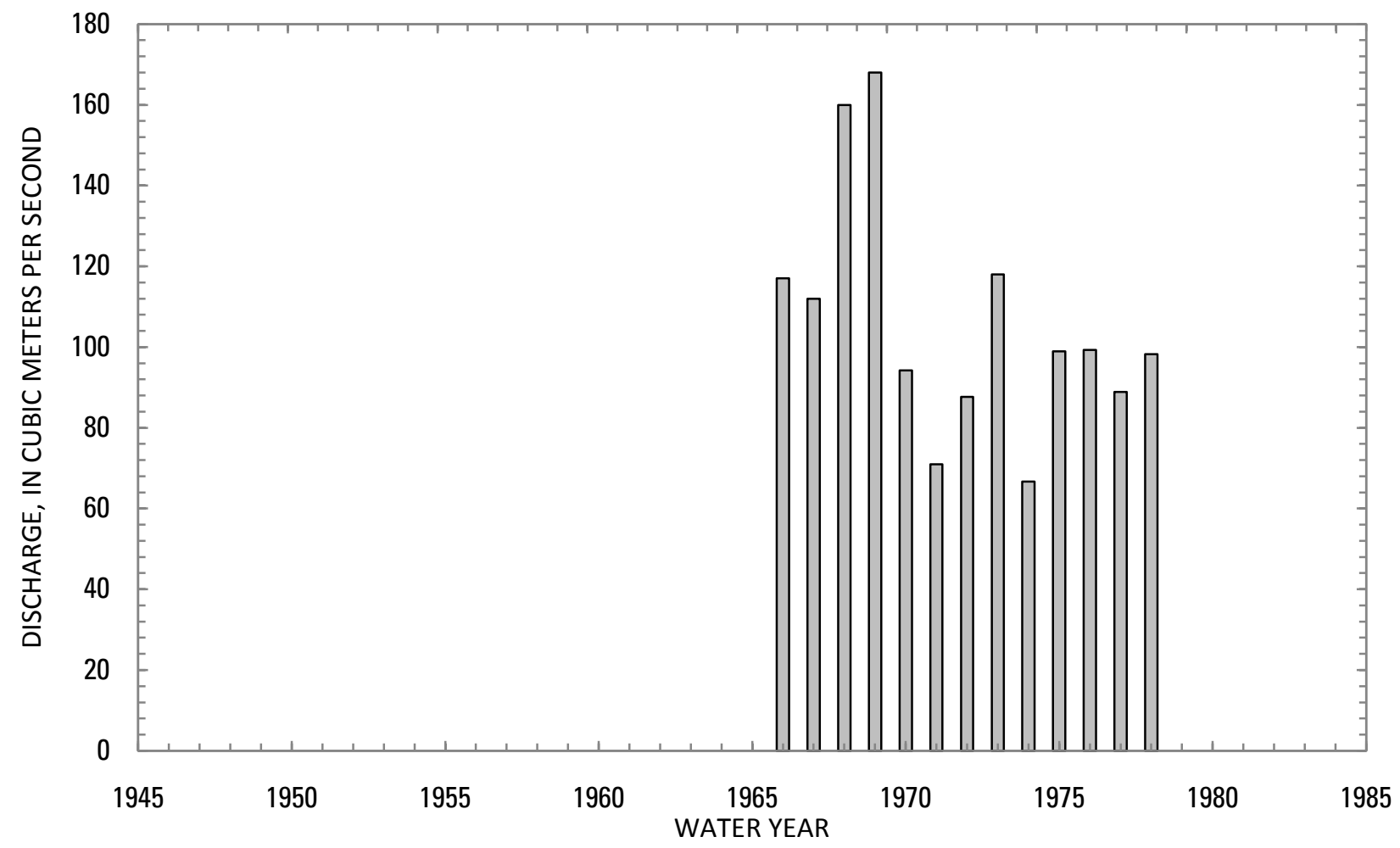




\section{4-0.000-1M KUNDUZ RIVER AT KULUKH TEPA, Continued}

Statistics of monthly and annual mean discharges [ $\mathrm{m}^{3} / \mathrm{s}$, cubic meters per second]

\begin{tabular}{|c|c|c|c|c|c|c|c|c|}
\hline \multirow[b]{2}{*}{ Month } & \multicolumn{2}{|c|}{ Maximum } & \multicolumn{2}{|c|}{ Minimum } & \multicolumn{4}{|c|}{ Mean } \\
\hline & $\begin{array}{c}\text { Discharge } \\
\left(\mathrm{m}^{3} / \mathrm{s}\right)\end{array}$ & $\begin{array}{c}\text { Water year } \\
\text { of } \\
\text { occurrence }\end{array}$ & $\begin{array}{c}\text { Discharge } \\
\left(\mathrm{m}^{3} / \mathrm{s}\right)\end{array}$ & $\begin{array}{c}\text { Water year } \\
\text { of } \\
\text { occurrence }\end{array}$ & $\begin{array}{c}\text { Discharge } \\
\left(\mathrm{m}^{3} / \mathrm{s}\right)\end{array}$ & $\begin{array}{c}\text { Standard } \\
\text { deviation } \\
\left(\mathrm{m}^{3} / \mathrm{s}\right)\end{array}$ & $\begin{array}{c}\text { Coefficient } \\
\text { of } \\
\text { variation }\end{array}$ & $\begin{array}{c}\text { Percentage } \\
\text { of annual } \\
\text { discharge }\end{array}$ \\
\hline October & 101 & 1970 & 40.9 & 1975 & 71.0 & 16.6 & 0.23 & 5.57 \\
\hline November & 103 & 1970 & 61.4 & 1973 & 84.1 & 11.4 & 0.14 & 6.60 \\
\hline December & 95.1 & 1969 & 66.3 & 1973 & 81.8 & 9.05 & 0.11 & 6.42 \\
\hline January & 80.7 & 1969 & 58.3 & 1975 & 69.9 & 8.36 & 0.12 & 5.49 \\
\hline February & 74.1 & 1970 & 53.8 & 1975 & 65.8 & 5.88 & 0.09 & 5.17 \\
\hline March & 96.3 & 1969 & 55.2 & 1975 & 74.0 & 10.8 & 0.15 & 5.81 \\
\hline April & 179 & 1969 & 30.8 & 1977 & 76.9 & 39.5 & 0.51 & 6.04 \\
\hline May & 236 & 1973 & 28.1 & 1974 & 141 & 58.5 & 0.41 & 11.1 \\
\hline June & 618 & 1968 & 148 & 1971 & 320 & 132 & 0.41 & 25.1 \\
\hline July & 408 & 1968 & 12.0 & 1971 & 204 & 112 & 0.55 & 16.0 \\
\hline August & 154 & 1969 & 5.47 & 1974 & 54.7 & 46.4 & 0.85 & 4.29 \\
\hline September & 60.4 & 1969 & 4.44 & 1974 & 30.8 & 14.6 & 0.47 & 2.41 \\
\hline Annual & 168 & 1969 & 66.7 & 1974 & 106 & 29.9 & 0.28 & 100 \\
\hline
\end{tabular}




\section{4-0.000-1M KUNDUZ RIVER AT KULUKH TEPA, Continued}

Monthly and annual flow duration, in cubic meters per second

\begin{tabular}{|c|c|c|c|c|c|c|c|c|c|c|c|c|c|}
\hline \multirow{2}{*}{$\begin{array}{l}\text { Percentage } \\
\text { of days } \\
\text { discharge } \\
\text { equaled or } \\
\text { exceeded }\end{array}$} & \multicolumn{12}{|c|}{ Month } & \multirow{2}{*}{ Annual } \\
\hline & October & November & December & January & February & March & April & May & June & July & August & September & \\
\hline 95 & 34.6 & 60.0 & 62.1 & 52.3 & 51.7 & 51.2 & 19.5 & 15.0 & 86.6 & 11.3 & 3.88 & 5.04 & 17.6 \\
\hline 90 & 41.3 & 64.3 & 66.1 & 56.1 & 56.1 & 55.6 & 25.7 & 25.9 & 125 & 39.8 & 7.50 & 11.7 & 28.1 \\
\hline 85 & 47.6 & 71.4 & 69.0 & 58.4 & 58.2 & 58.5 & 32.9 & 33.0 & 163 & 64.9 & 11.5 & 16.1 & 38.4 \\
\hline 80 & 51.6 & 75.3 & 71.8 & 60.8 & 59.8 & 61.0 & 38.5 & 49.4 & 189 & 82.2 & 15.2 & 17.6 & 49.0 \\
\hline 75 & 55.3 & 76.9 & 74.5 & 63.1 & 61.6 & 63.2 & 43.6 & 63.5 & 207 & 97.8 & 19.9 & 19.5 & 56.0 \\
\hline 70 & 58.9 & 79.4 & 76.5 & 64.6 & 63.1 & 65.2 & 48.6 & 77.6 & 220 & 113 & 22.9 & 21.6 & 61.3 \\
\hline 65 & 61.9 & 81.6 & 78.2 & 65.8 & 64.1 & 67.0 & 54.0 & 89.2 & 236 & 126 & 28.0 & 23.8 & 64.8 \\
\hline 60 & 64.0 & 83.0 & 81.4 & 67.6 & 64.9 & 68.7 & 59.4 & 103 & 255 & 144 & 31.8 & 25.4 & 68.3 \\
\hline 55 & 66.1 & 84.2 & 83.4 & 69.3 & 65.7 & 70.3 & 64.9 & 117 & 275 & 162 & 35.2 & 27.0 & 71.9 \\
\hline 50 & 69.9 & 85.0 & 84.3 & 70.5 & 66.4 & 71.8 & 69.5 & 132 & 292 & 180 & 38.9 & 28.5 & 75.6 \\
\hline 45 & 72.9 & 85.9 & 85.2 & 71.6 & 67.2 & 73.8 & 74.2 & 148 & 309 & 197 & 42.1 & 30.5 & 79.4 \\
\hline 40 & 75.0 & 86.8 & 86.1 & 73.1 & 67.9 & 75.9 & 79.2 & 166 & 327 & 217 & 45.8 & 32.4 & 83.2 \\
\hline 35 & 77.2 & 88.0 & 87.1 & 74.7 & 68.8 & 78.8 & 84.2 & 184 & 354 & 241 & 50.7 & 34.1 & 88.2 \\
\hline 30 & 80.8 & 89.3 & 88.5 & 76.2 & 70.0 & 82.4 & 90.9 & 197 & 388 & 269 & 62.8 & 35.9 & 94.2 \\
\hline 25 & 85.1 & 91.0 & 90.0 & 78.1 & 71.0 & 85.6 & 97.9 & 211 & 414 & 297 & 71.3 & 37.7 & 100 \\
\hline 20 & 90.2 & 92.9 & 91.2 & 79.5 & 72.1 & 88.2 & 106 & 229 & 446 & 328 & 83.9 & 41.1 & 122 \\
\hline 15 & 96.3 & 95.3 & 92.4 & 81.2 & 73.2 & 91.3 & 115 & 247 & 485 & 368 & 101 & 47.9 & 176 \\
\hline 10 & 104 & 98.3 & 94.4 & 83.4 & 75.0 & 95.2 & 129 & 266 & 551 & 410 & 134 & 56.0 & 238 \\
\hline 5 & 115 & 109 & 98.4 & 85.8 & 77.3 & 102 & 168 & 301 & 664 & 471 & 182 & 66.9 & 339 \\
\hline
\end{tabular}




\section{4-0.000-1M KUNDUZ RIVER AT KULUKH TEPA, Continued}

Probability of occurrence of annual high discharges

[ $\mathrm{m}^{3} / \mathrm{s}$, cubic meters per second; $\mathrm{ng}$, not given]

\begin{tabular}{|c|c|c|c|c|c|c|}
\hline \multirow{2}{*}{$\begin{array}{c}\text { Exceedance } \\
\text { probability }\end{array}$} & \multirow{2}{*}{$\begin{array}{c}\text { Recurrence } \\
\text { interval } \\
\text { (years) }\end{array}$} & \multirow{2}{*}{$\begin{array}{c}\text { Maximum } \\
\text { instantaneous } \\
\text { discharge } \\
\left(\mathrm{m}^{3} / \mathrm{s}\right)\end{array}$} & \multicolumn{4}{|c|}{ Maximum daily mean discharge $\left(\mathrm{m}^{3} / \mathrm{s}\right)$} \\
\hline & & & $\begin{array}{l}\text { 3-day } \\
\text { period }\end{array}$ & $\begin{array}{l}\text { 7-day } \\
\text { period }\end{array}$ & $\begin{array}{l}\text { 15-day } \\
\text { period }\end{array}$ & $\begin{array}{l}\text { 30-day } \\
\text { period }\end{array}$ \\
\hline 0.99 & 1.01 & 252 & 221 & 216 & 188 & 166 \\
\hline 0.95 & 1.05 & 306 & 273 & 262 & 227 & 199 \\
\hline 0.90 & 1.11 & 340 & 307 & 292 & 252 & 221 \\
\hline 0.80 & 1.25 & 388 & 353 & 333 & 288 & 252 \\
\hline 0.50 & 2 & 505 & 464 & 432 & 378 & 329 \\
\hline 0.20 & 5 & 665 & 614 & 568 & 508 & 440 \\
\hline 0.10 & 10 & 773 & 713 & 659 & 599 & 517 \\
\hline 0.04 & 25 & 911 & 837 & 775 & 718 & 619 \\
\hline 0.02 & 50 & 1,020 & 929 & 863 & 812 & 698 \\
\hline 0.01 & 100 & 1,120 & 1,020 & 951 & 908 & 779 \\
\hline 0.005 & 200 & 1,230 & 1,110 & 1,040 & 1,010 & 865 \\
\hline 0.002 & 500 & 1,380 & ng & ng & ng & ng \\
\hline
\end{tabular}

14-0.000-1M KUNDUZ RIVER AT KULUKH TEPA, Continued

Probability of occurrence of annual low discharges

$\left[\mathrm{m}^{3} / \mathrm{s}\right.$, meters per second]

\begin{tabular}{|c|c|c|c|c|c|c|c|c|c|c|}
\hline \multirow{3}{*}{$\begin{array}{c}\text { Nonexceedance } \\
\text { probability }\end{array}$} & \multirow{3}{*}{$\begin{array}{c}\text { Recurrence } \\
\text { interval } \\
\text { (years) }\end{array}$} & \multicolumn{9}{|c|}{ Minimum daily mean discharge $\left(\mathrm{m}^{3} / \mathrm{s}\right)$} \\
\hline & & \multicolumn{9}{|c|}{ Number of consecutive days } \\
\hline & & 1 & 3 & 7 & 14 & 30 & 60 & 90 & 120 & 183 \\
\hline 0.05 & 20 & 3.11 & 3.12 & 3.12 & 3.38 & 3.73 & 6.27 & 14.7 & 24.4 & 39.6 \\
\hline 0.10 & 10 & 4.92 & 4.97 & 5.02 & 5.40 & 6.09 & 9.79 & 19.9 & 30.4 & 44.5 \\
\hline 0.20 & 5 & 8.14 & 8.28 & 8.44 & 9.00 & 10.3 & 15.8 & 27.6 & 38.4 & 50.5 \\
\hline 0.50 & 2 & 18.3 & 18.8 & 19.3 & 20.3 & 23.4 & 32.7 & 45.8 & 55.3 & 62.5 \\
\hline
\end{tabular}




\section{4-0.000-1M KUNDUZ RIVER AT KULUKH TEPA, Continued}

Probability of occurrence of seasonal low discharges $\left[\mathrm{m}^{3} / \mathrm{s}\right.$, meters per second]

\begin{tabular}{|c|c|c|c|c|c|c|c|c|c|}
\hline \multirow{3}{*}{$\begin{array}{c}\text { Nonexceedance } \\
\text { probability }\end{array}$} & \multirow{3}{*}{$\begin{array}{c}\text { Recurrence } \\
\text { interval } \\
\text { (years) }\end{array}$} & \multicolumn{8}{|c|}{ Minimum daily mean discharge $\left(\mathrm{m}^{3} / \mathrm{s}\right)$} \\
\hline & & \multicolumn{8}{|c|}{ Number of consecutive days } \\
\hline & & 1 & 7 & 14 & 30 & 1 & 7 & 14 & 30 \\
\hline & & \multicolumn{4}{|c|}{ December-January-February } & \multicolumn{4}{|c|}{ March-April-May } \\
\hline 0.05 & 20 & 40.4 & 46.6 & 50.0 & 52.6 & 5.84 & 7.21 & 8.77 & 16.9 \\
\hline 0.10 & 10 & 43.8 & 49.3 & 52.5 & 55.2 & 8.81 & 11.3 & 14.1 & 23.6 \\
\hline 0.20 & 5 & 48.0 & 52.8 & 55.7 & 58.3 & 13.9 & 18.3 & 22.9 & 33.5 \\
\hline \multirow[t]{2}{*}{0.50} & 2 & 56.2 & 59.4 & 61.9 & 64.2 & 29.3 & 37.7 & 45.6 & 56.4 \\
\hline & & \multicolumn{4}{|c|}{ June-July-August } & \multicolumn{4}{|c|}{ September-October-November } \\
\hline 0.05 & 20 & 3.38 & 3.39 & 3.68 & 5.12 & 4.16 & 4.23 & 4.67 & 7.04 \\
\hline 0.10 & 10 & 5.60 & 5.66 & 6.13 & 8.51 & 6.53 & 6.75 & 7.62 & 10.9 \\
\hline 0.20 & 5 & 9.72 & 9.98 & 10.8 & 15.1 & 10.5 & 11.0 & 12.6 & 17.1 \\
\hline 0.50 & 2 & 23.4 & 25.3 & 28.1 & 39.2 & 21.6 & 22.8 & 25.6 & 31.8 \\
\hline
\end{tabular}

\section{4-0.000-1M KUNDUZ RIVER AT KULUKH TEPA, Continued}

Annual peak discharges

$\left[\mathrm{m}^{3} / \mathrm{s}\right.$, meters per second]

\begin{tabular}{|c|c|c|c|c|c|}
\hline \multicolumn{3}{|c|}{$\begin{array}{c}\text { Annual peak discharge, } \\
\text { by year }\end{array}$} & \multicolumn{3}{|c|}{$\begin{array}{l}\text { Annual peak discharge, } \\
\text { from highest to lowest }\end{array}$} \\
\hline $\begin{array}{l}\text { Water } \\
\text { year }\end{array}$ & Date & $\begin{array}{c}\text { Peak } \\
\text { discharge } \\
\left(\mathrm{m}^{3} / \mathrm{s}\right)\end{array}$ & $\begin{array}{l}\text { Water } \\
\text { year }\end{array}$ & Date & $\begin{array}{c}\text { Peak } \\
\text { discharge } \\
\left(\mathrm{m}^{3} / \mathrm{s}\right)\end{array}$ \\
\hline 1966 & June 21, 1966 & 739 & 1968 & June 13, 1968 & 830 \\
\hline 1967 & June 13, 1967 & 588 & 1977 & May 29, 1977 & 800 \\
\hline 1968 & June 13, 1968 & 830 & 1966 & June 21, 1966 & 739 \\
\hline 1969 & June 22, 1969 & 640 & 1969 & June 22, 1969 & 640 \\
\hline 1970 & June 2, 1970 & 348 & 1967 & June 13, 1967 & 588 \\
\hline 1971 & May 30, 1971 & 340 & 1973 & June 11, 1973 & 531 \\
\hline 1972 & June 25, 1972 & 503 & 1972 & June 25, 1972 & 503 \\
\hline 1973 & June 11,1973 & 531 & 1975 & June 19, 1975 & 450 \\
\hline 1974 & June 18, 1974 & 337 & 1976 & June 6, 1976 & 450 \\
\hline 1975 & June 19, 1975 & 450 & 1978 & June 9, 1978 & 390 \\
\hline 1976 & June 6, 1976 & 450 & 1970 & June 2, 1970 & 348 \\
\hline 1977 & May 29, 1977 & 800 & 1971 & May 30, 1971 & 340 \\
\hline 1978 & June 9, 1978 & 390 & 1974 & June 18, 1974 & 337 \\
\hline
\end{tabular}




\section{4-0.000-1M KUNDUZ RIVER AT KULUKH TEPA, Continued}

Monthly and annual mean discharges, in cubic meters per second

[Data may not be rounded in accordance with U.S. Geological Survey publication standards]

\begin{tabular}{|c|c|c|c|c|c|c|c|c|c|c|c|c|c|}
\hline \multirow{2}{*}{$\begin{array}{l}\text { Water } \\
\text { year }\end{array}$} & \multicolumn{12}{|c|}{ Monthly mean discharge } & \multirow{2}{*}{$\begin{array}{c}\text { Annual } \\
\text { discharge }\end{array}$} \\
\hline & October & November & December & January & February & March & April & May & June & July & August & September & \\
\hline 1966 & 69.8 & 87.0 & 86.2 & 71.9 & 61.8 & 83.8 & 95.5 & 157 & 428 & 157 & 64.8 & 37.0 & 117 \\
\hline 1967 & 86.5 & 93.8 & 80.0 & 71.1 & 68.7 & 66.5 & 60.6 & 112 & 334 & 258 & 78.4 & 32.5 & 112 \\
\hline 1968 & 70.1 & 83.2 & 87.1 & 79.6 & 67.7 & 62.2 & 85.7 & 174 & 618 & 408 & 142 & 46.2 & 160 \\
\hline 1969 & 78.7 & 88.7 & 95.1 & 80.7 & 70.9 & 96.3 & 179 & 234 & 484 & 390 & 154 & 60.4 & 168 \\
\hline 1970 & 101 & 103 & 91.1 & 80.3 & 74.1 & 76.3 & 63.2 & 150 & 198 & 107 & 37.4 & 47.8 & 94.2 \\
\hline 1971 & 75.1 & 80.6 & 88.7 & 75.6 & 67.8 & 69.0 & 62.9 & 138 & 148 & 12.0 & 10.8 & 23.8 & 71.0 \\
\hline 1972 & 52.8 & 77.5 & 67.3 & 60.5 & 55.9 & 66.0 & 68.1 & 90.0 & 285 & 166 & 44.2 & 19.8 & 87.7 \\
\hline 1973 & 51.0 & 61.4 & 66.3 & 59.0 & 65.2 & 84.7 & 130 & 236 & 364 & 230 & 39.7 & 31.6 & 118 \\
\hline 1974 & 87.1 & 76.7 & 81.8 & 61.4 & 63.4 & 73.7 & 38.9 & 28.1 & 181 & 99.9 & 5.47 & 4.44 & 66.7 \\
\hline 1975 & 40.9 & 69.8 & 73.1 & 58.3 & 53.8 & 55.2 & 66.6 & 150 & 304 & 236 & 52.2 & 24.6 & 98.9 \\
\hline 1976 & 79.5 & 98.7 & 75.2 & 64.1 & 66.4 & 73.2 & 55.4 & 137 & 211 & 261 & 42.7 & 25.9 & 99.3 \\
\hline 1977 & 60.6 & 83.6 & 82.5 & 70.9 & 68.6 & 73.4 & 30.8 & 68.6 & 314 & 182 & 13.0 & 18.2 & 88.9 \\
\hline 1978 & 69.2 & 88.8 & 89.0 & 75.6 & 71.4 & 81.0 & 63.1 & 161 & 286 & 140 & 26.2 & 27.5 & 98.2 \\
\hline
\end{tabular}




\section{4-0.000-2M KUNDUZ RIVER AT CHAR DARA}

\section{(U.S. Geological Survey identification number: $\mathbf{3 6 4 2 0 0 0 6 8 5 0 0 0 0 0 )}$}

LOCATION: Lat $36^{\circ} 42^{\prime} \mathrm{N}$., long $68^{\circ} 50^{\prime} \mathrm{E}$.

DRAINAGE AREA: $24,820 \mathrm{~km}^{2}$.

ELEVATION: 401 meters above mean sea level.

PERIOD OF RECORD: August 5, 1964 to September 30, 1978.

GAGE: Water-stage recorder.

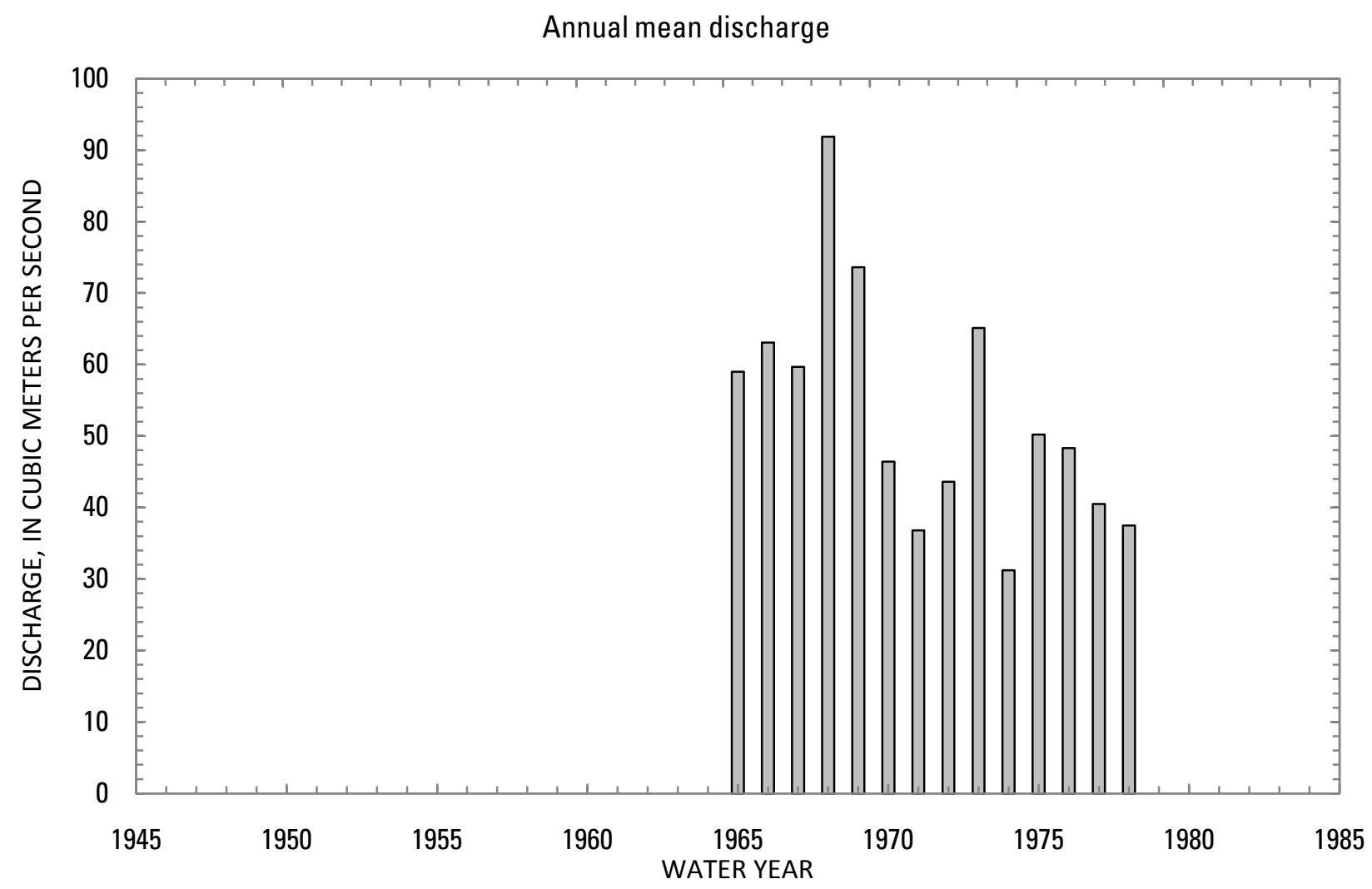




\section{4-0.000-2M KUNDUZ RIVER AT CHAR DARA, Continued}

Statistics of monthly and annual mean discharges $\left[\mathrm{m}^{3} / \mathrm{s}\right.$, cubic meters per second]

\begin{tabular}{|c|c|c|c|c|c|c|c|c|}
\hline \multirow[b]{2}{*}{ Month } & \multicolumn{2}{|c|}{ Maximum } & \multicolumn{2}{|c|}{ Minimum } & \multicolumn{4}{|c|}{ Mean } \\
\hline & $\begin{array}{c}\text { Discharge } \\
\left(\mathrm{m}^{3} / \mathrm{s}\right)\end{array}$ & $\begin{array}{c}\text { Water year } \\
\text { of } \\
\text { occurrence }\end{array}$ & $\begin{array}{c}\text { Discharge } \\
\left(\mathrm{m}^{3} / \mathrm{s}\right)\end{array}$ & $\begin{array}{c}\text { Water year } \\
\text { of } \\
\text { occurrence }\end{array}$ & $\begin{array}{c}\text { Discharge } \\
\left(\mathrm{m}^{3} / \mathrm{s}\right)\end{array}$ & $\begin{array}{c}\text { Standard } \\
\text { deviation } \\
\left(\mathrm{m}^{3} / \mathrm{s}\right)\end{array}$ & $\begin{array}{c}\text { Coefficient } \\
\text { of } \\
\text { variation }\end{array}$ & $\begin{array}{c}\text { Percentage } \\
\text { of annual } \\
\text { discharge }\end{array}$ \\
\hline October & 41.7 & 1969 & 10.0 & 1972 & 27.2 & 8.89 & 0.33 & 4.25 \\
\hline November & 49.2 & 1970 & 19.7 & 1972 & 32.6 & 7.94 & 0.24 & 5.10 \\
\hline December & 44.9 & 1969 & 21.0 & 1972 & 35.0 & 6.00 & 0.17 & 5.46 \\
\hline January & 40.8 & 1969 & 21.0 & 1972 & 32.4 & 4.81 & 0.15 & 5.06 \\
\hline February & 40.4 & 1969 & 21.0 & 1972 & 31.4 & 4.48 & 0.14 & 4.90 \\
\hline March & 42.7 & 1966 & 24.6 & 1972 & 33.8 & 4.63 & 0.14 & 5.28 \\
\hline April & 77.8 & 1973 & 16.8 & 1974 & 37.9 & 16.9 & 0.45 & 5.92 \\
\hline May & 159 & 1973 & 45.1 & 1974 & 91.6 & 33.5 & 0.37 & 14.3 \\
\hline June & 406 & 1968 & 72.2 & 1971 & 186 & 88.4 & 0.48 & 29.0 \\
\hline July & 203 & 1968 & 13.1 & 1971 & 93.1 & 57.0 & 0.61 & 14.6 \\
\hline August & 66.3 & 1968 & 2.16 & 1974 & 23.6 & 20.1 & 0.85 & 3.69 \\
\hline September & 32.7 & 1968 & 2.59 & 1974 & 16.1 & 8.76 & 0.54 & 2.51 \\
\hline Annual & 91.9 & 1968 & 31.2 & 1974 & 53.4 & 16.6 & 0.31 & 100 \\
\hline
\end{tabular}


14-0.000-2M KUNDUZ RIVER AT CHAR DARA, Continued

Monthly and annual flow duration, in cubic meters per second

\begin{tabular}{|c|c|c|c|c|c|c|c|c|c|c|c|c|c|}
\hline \multirow{2}{*}{$\begin{array}{l}\text { Percentage } \\
\text { of days } \\
\text { discharge } \\
\text { equaled or } \\
\text { exceeded }\end{array}$} & \multicolumn{12}{|c|}{ Month } & \multirow{2}{*}{ Annual } \\
\hline & October & November & December & January & February & March & April & May & June & July & August & September & \\
\hline 95 & 10.3 & 19.2 & 21.4 & 21.4 & 21.3 & 23.9 & 9.80 & 11.0 & 57.7 & 9.26 & 1.89 & 3.05 & 7.80 \\
\hline 90 & 15.3 & 21.5 & 29.0 & 27.3 & 26.4 & 25.2 & 15.3 & 18.3 & 78.3 & 15.9 & 2.83 & 4.09 & 13.5 \\
\hline 85 & 17.5 & 23.6 & 30.6 & 27.9 & 27.4 & 27.3 & 19.2 & 30.9 & 89.0 & 24.5 & 3.49 & 6.82 & 19.1 \\
\hline 80 & 18.8 & 26.5 & 31.7 & 28.6 & 28.0 & 28.7 & 22.0 & 35.1 & 96.9 & 32.4 & 4.83 & 8.05 & 22.6 \\
\hline 75 & 20.6 & 28.4 & 32.2 & 29.6 & 29.2 & 29.9 & 23.9 & 38.8 & 106 & 38.7 & 5.93 & 10.1 & 25.4 \\
\hline 70 & 21.8 & 29.7 & 32.6 & 30.1 & 29.9 & 30.7 & 25.3 & 44.2 & 121 & 46.6 & 7.66 & 11.6 & 27.5 \\
\hline 65 & 23.5 & 30.6 & 33.1 & 30.8 & 30.3 & 31.5 & 27.5 & 53.4 & 140 & 55.7 & 10.1 & 12.4 & 29.5 \\
\hline 60 & 25.1 & 31.1 & 33.6 & 31.7 & 30.6 & 32.2 & 29.4 & 66.8 & 150 & 64.6 & 11.8 & 13.2 & 31.1 \\
\hline 55 & 26.8 & 31.7 & 34.1 & 32.3 & 31.0 & 32.6 & 31.0 & 78.6 & 158 & 73.2 & 14.1 & 13.9 & 32.4 \\
\hline 50 & 27.6 & 32.4 & 34.6 & 32.8 & 31.5 & 33.0 & 32.7 & 86.2 & 172 & 79.4 & 18.2 & 14.8 & 33.6 \\
\hline 45 & 29.1 & 33.2 & 35.0 & 33.2 & 32.0 & 33.7 & 34.6 & 94.4 & 185 & 86.8 & 20.5 & 16.2 & 34.8 \\
\hline 40 & 29.8 & 34.2 & 36.2 & 33.6 & 32.3 & 34.6 & 36.9 & 103 & 201 & 96.5 & 23.0 & 17.6 & 36.1 \\
\hline 35 & 30.4 & 35.2 & 36.8 & 34.0 & 32.5 & 35.5 & 39.5 & 111 & 223 & 106 & 26.2 & 18.4 & 38.5 \\
\hline 30 & 31.4 & 36.1 & 37.9 & 34.6 & 33.1 & 36.6 & 44.7 & 121 & 234 & 119 & 29.5 & 19.2 & 41.8 \\
\hline 25 & 32.8 & 37.5 & 38.4 & 35.3 & 34.1 & 38.0 & 48.4 & 133 & 245 & 135 & 34.2 & 20.6 & 47.0 \\
\hline 20 & 35.7 & 39.0 & 39.4 & 36.0 & 35.1 & 38.8 & 52.6 & 146 & 259 & 151 & 39.2 & 22.9 & 61.7 \\
\hline 15 & 38.3 & 40.1 & 41.3 & 36.9 & 36.0 & 40.0 & 58.0 & 159 & 275 & 172 & 45.7 & 27.7 & 90.2 \\
\hline 10 & 39.5 & 42.7 & 43.8 & 38.9 & 37.5 & 42.8 & 65.1 & 175 & 296 & 197 & 54.8 & 30.8 & 133 \\
\hline 5 & 42.1 & 48.1 & 45.6 & 40.8 & 39.0 & 45.6 & 81.2 & 199 & 346 & 223 & 70.8 & 33.2 & 191 \\
\hline
\end{tabular}




\section{4-0.000-2M KUNDUZ RIVER AT CHAR DARA, Continued}

Probability of occurrence of annual high discharges

[m $\mathrm{m}^{3} / \mathrm{s}$, cubic meters per second; ng, not given]

\begin{tabular}{|c|c|c|c|c|c|c|}
\hline \multirow{2}{*}{$\begin{array}{c}\text { Exceedance } \\
\text { probability }\end{array}$} & \multirow{2}{*}{$\begin{array}{c}\text { Recurrence } \\
\text { interval } \\
\text { (years) }\end{array}$} & \multirow{2}{*}{$\begin{array}{l}\text { Maximum } \\
\text { instantaneous } \\
\text { discharge } \\
\left(\mathrm{m}^{3} / \mathrm{s}\right)\end{array}$} & \multicolumn{4}{|c|}{ Maximum daily mean discharge $\left(\mathrm{m}^{3} / \mathrm{s}\right)$} \\
\hline & & & $\begin{array}{l}\text { 3-day } \\
\text { period }\end{array}$ & $\begin{array}{l}\text { 7-day } \\
\text { period }\end{array}$ & $\begin{array}{l}\text { 15-day } \\
\text { period }\end{array}$ & $\begin{array}{l}\text { 30-day } \\
\text { period }\end{array}$ \\
\hline 0.99 & 1.01 & 113 & 105 & 95.7 & 80.6 & 74.7 \\
\hline 0.95 & 1.05 & 149 & 136 & 124 & 107 & 97.3 \\
\hline 0.90 & 1.11 & 172 & 156 & 142 & 124 & 112 \\
\hline 0.80 & 1.25 & 202 & 184 & 168 & 148 & 133 \\
\hline 0.50 & 2 & 271 & 249 & 230 & 209 & 185 \\
\hline 0.20 & 5 & 354 & 333 & 316 & 295 & 258 \\
\hline 0.10 & 10 & 403 & 387 & 372 & 354 & 307 \\
\hline 0.04 & 25 & 459 & 451 & 444 & 428 & 370 \\
\hline 0.02 & 50 & 498 & 498 & 497 & 485 & 418 \\
\hline 0.01 & 100 & 535 & ${ }^{1} 543$ & 550 & 542 & 466 \\
\hline 0.005 & 200 & 569 & ${ }^{1} 587$ & 603 & 600 & 515 \\
\hline 0.002 & 500 & 612 & ng & $\mathrm{ng}$ & $\mathrm{ng}$ & $\mathrm{ng}$ \\
\hline
\end{tabular}

'Data does not fit log-Pearson Type III curve, use with caution.

\section{4-0.000-2M KUNDUZ RIVER AT CHAR DARA, Continued}

Probability of occurrence of annual low discharges $\left[\mathrm{m}^{3} / \mathrm{s}\right.$, meters per second]

\begin{tabular}{|c|c|c|c|c|c|c|c|c|c|c|}
\hline \multirow{3}{*}{$\begin{array}{c}\text { Nonexceedance } \\
\text { probability }\end{array}$} & \multirow{3}{*}{$\begin{array}{c}\text { Recurrence } \\
\text { interval } \\
\text { (years) }\end{array}$} & \multicolumn{9}{|c|}{ Minimum daily mean discharge $\left(\mathrm{m}^{3} / \mathrm{s}\right)$} \\
\hline & & \multicolumn{9}{|c|}{ Number of consecutive days } \\
\hline & & 1 & 3 & 7 & 14 & 30 & 60 & 90 & 120 & 183 \\
\hline 0.05 & 20 & 1.68 & 1.7 & 1.75 & 1.84 & 2.17 & 2.92 & 6.02 & 8.37 & 13.9 \\
\hline 0.10 & 10 & 2.78 & 2.81 & 2.88 & 3.01 & 3.48 & 4.61 & 8.25 & 11.1 & 16.7 \\
\hline 0.20 & 5 & 4.78 & 4.84 & 4.93 & 5.14 & 5.81 & 7.56 & 11.7 & 14.9 & 20.4 \\
\hline 0.50 & 2 & 11.1 & 11.4 & 11.6 & 12.0 & 13.1 & 16.3 & 20.3 & 23.8 & 27.7 \\
\hline
\end{tabular}


14-0.000-2M KUNDUZ RIVER AT CHAR DARA, Continued

Probability of occurrence of seasonal low discharges $\left[\mathrm{m}^{3} / \mathrm{s}\right.$, meters per second]

\begin{tabular}{|c|c|c|c|c|c|c|c|c|c|}
\hline \multirow{3}{*}{$\begin{array}{c}\text { Nonexceedance } \\
\text { probability }\end{array}$} & \multirow{3}{*}{$\begin{array}{c}\text { Recurrence } \\
\text { interval } \\
\text { (years) }\end{array}$} & \multicolumn{8}{|c|}{ Minimum daily mean discharge $\left(\mathrm{m}^{3} / \mathrm{s}\right)$} \\
\hline & & \multicolumn{8}{|c|}{ Number of consecutive days } \\
\hline & & 1 & 7 & 14 & 30 & 1 & 7 & 14 & 30 \\
\hline & & \multicolumn{4}{|c|}{ December-January-February } & \multicolumn{4}{|c|}{ March-April-May } \\
\hline 0.05 & 20 & 22.5 & 22.7 & 22.9 & 23.2 & 5.68 & 6.77 & 7.49 & 10.5 \\
\hline 0.10 & 10 & 23.8 & 24.1 & 24.4 & 25.0 & 7.50 & 9.06 & 10.2 & 13.8 \\
\hline 0.20 & 5 & 25.4 & 25.8 & 26.3 & 27.1 & 10.2 & 12.4 & 14.3 & 18.5 \\
\hline \multirow[t]{2}{*}{0.50} & 2 & 28.4 & 29.1 & 29.8 & 31.0 & 17.3 & 20.6 & 23.5 & 28.2 \\
\hline & & \multicolumn{4}{|c|}{ June-July-August } & \multicolumn{4}{|c|}{ September-October-November } \\
\hline 0.05 & 20 & 1.34 & 1.42 & 1.49 & 1.98 & 1.98 & 2.06 & 2.16 & 3.76 \\
\hline 0.10 & 10 & 2.28 & 2.40 & 2.50 & 3.29 & 3.30 & 3.49 & 3.68 & 5.53 \\
\hline 0.20 & 5 & 4.13 & 4.30 & 4.50 & 5.87 & 5.66 & 6.08 & 6.39 & 8.36 \\
\hline 0.50 & 2 & 11.0 & 11.4 & 12.1 & 16.0 & 12.7 & 13.6 & 14.2 & 15.8 \\
\hline
\end{tabular}

14-0.000-2M KUNDUZ RIVER AT CHAR DARA, Continued

Annual peak discharges

$\left[\mathrm{m}^{3} / \mathrm{s}\right.$, meters per second]

\begin{tabular}{|c|c|c|c|c|c|}
\hline \multicolumn{3}{|c|}{$\begin{array}{c}\text { Annual peak discharge, } \\
\text { by year }\end{array}$} & \multicolumn{3}{|c|}{$\begin{array}{l}\text { Annual peak discharge, } \\
\text { from highest to lowest }\end{array}$} \\
\hline $\begin{array}{l}\text { Water } \\
\text { year }\end{array}$ & Date & $\begin{array}{c}\text { Peak } \\
\text { discharge } \\
\left(\mathrm{m}^{3} / \mathrm{s}\right)\end{array}$ & $\begin{array}{l}\text { Water } \\
\text { year }\end{array}$ & Date & $\begin{array}{c}\text { Peak } \\
\text { discharge } \\
\left(\mathrm{m}^{3} / \mathrm{s}\right)\end{array}$ \\
\hline 1965 & June 17,1965 & 290 & 1968 & June 12,1968 & 500 \\
\hline 1966 & June 20, 1966 & 340 & 1966 & June 20, 1966 & 340 \\
\hline 1967 & June 12, 1967 & 297 & 1973 & June 11,1973 & 340 \\
\hline 1968 & June 12, 1968 & 500 & 1969 & June 21, 1969 & 331 \\
\hline 1969 & June 21, 1969 & 331 & 1976 & May 25, 1976 & 308 \\
\hline 1970 & May 20, 1970 & 190 & 1967 & June 12, 1967 & 297 \\
\hline 1971 & May 22, 1971 & 200 & 1965 & June 17,1965 & 290 \\
\hline 1972 & June 18, 1972 & 275 & 1972 & June 18, 1972 & 275 \\
\hline 1973 & June 11, 1973 & 340 & 1977 & May 29, 1977 & 272 \\
\hline 1974 & June 19, 1974 & 146 & 1975 & June 19,1975 & 269 \\
\hline 1975 & June 19, 1975 & 269 & 1971 & May 22, 1971 & 200 \\
\hline 1976 & May 25, 1976 & 308 & 1970 & May 20, 1970 & 190 \\
\hline 1977 & May 29, 1977 & 272 & 1978 & May 25, 1978 & 156 \\
\hline 1978 & May 25, 1978 & 156 & 1974 & June 19, 1974 & 146 \\
\hline
\end{tabular}




\section{4-0.000-2M KUNDUZ RIVER AT CHAR DARA, Continued}

Monthly and annual mean discharges, in cubic meters per second $[--$, no data]

\begin{tabular}{|c|c|c|c|c|c|c|c|c|c|c|c|c|c|}
\hline \multirow{2}{*}{$\begin{array}{l}\text { Water } \\
\text { year }\end{array}$} & \multicolumn{12}{|c|}{ Monthly mean discharge } & \multirow{2}{*}{$\begin{array}{c}\text { Annual } \\
\text { discharge }\end{array}$} \\
\hline & October & November & December & January & February & March & April & May & June & July & August & September & \\
\hline 1964 & -- & -- & -- & -- & -- & -- & -- & -- & -- & -- & -- & 15.4 & -- \\
\hline 1965 & 18.6 & 22.5 & 29.8 & 28.6 & 28.7 & 29.1 & 37.7 & 57.9 & 211 & 172 & 48.1 & 23.2 & 59.0 \\
\hline 1966 & 29.5 & 31.9 & 37.0 & 34.5 & 34.4 & 42.7 & 58.3 & 116 & 240 & 83.3 & 31.5 & 18.9 & 63.1 \\
\hline 1967 & 32.5 & 33.7 & 33.9 & 32.9 & 36.0 & 34.3 & 30.3 & 80.5 & 213 & 135 & 37.4 & 15.7 & 59.7 \\
\hline 1968 & 34.7 & 41.2 & 40.2 & 34.5 & 29.8 & 27.8 & 47.6 & 140 & 406 & 203 & 66.3 & 32.7 & 91.9 \\
\hline 1969 & 41.7 & 40.6 & 44.9 & 40.8 & 40.4 & 39.9 & 48.3 & 107 & 237 & 161 & 49.0 & 31.8 & 73.6 \\
\hline 1970 & 40.0 & 49.2 & 43.3 & 38.8 & 33.0 & 37.1 & 34.9 & 86.3 & 96.2 & 51.2 & 24.7 & 21.1 & 46.4 \\
\hline 1971 & 30.8 & 34.1 & 35.5 & 33.5 & 31.7 & 33.1 & 41.2 & 108 & 72.2 & 13.1 & 3.86 & 5.41 & 36.8 \\
\hline 1972 & 10.0 & 19.7 & 21.0 & 21.0 & 21.0 & 24.6 & 20.5 & 51.4 & 215 & 92.9 & 14.6 & 12.7 & 43.6 \\
\hline 1973 & 24.4 & 29.3 & 31.0 & 28.5 & 28.0 & 35.4 & 77.8 & 159 & 242 & 88.4 & 17.4 & 20.4 & 65.1 \\
\hline 1974 & 29.6 & 31.6 & 32.7 & 31.3 & 29.2 & 33.8 & 16.8 & 45.1 & 90.9 & 29.1 & 2.16 & 2.59 & 31.2 \\
\hline 1975 & 15.5 & 22.6 & 32.0 & 28.7 & 29.4 & 34.9 & 32.5 & 93.8 & 188 & 101 & 14.6 & 8.77 & 50.2 \\
\hline 1976 & 25.4 & 33.9 & 36.7 & 33.4 & 34.3 & 34.0 & 43.5 & 99.0 & 125 & 88.6 & 12.8 & 12.3 & 48.3 \\
\hline 1977 & 25.8 & 30.2 & 33.4 & 32.6 & 31.9 & 31.9 & 17.0 & 54.6 & 165 & 48.7 & 5.87 & 10.8 & 40.5 \\
\hline 1978 & 22.1 & 36.4 & 38.2 & 34.4 & 31.8 & 34.8 & 24.4 & 83.6 & 96.2 & 36.5 & 2.53 & 9.46 & 37.5 \\
\hline
\end{tabular}




\section{4-0.000-3M KUNDUZ RIVER AT GERDAB}

\section{(U.S. Geological Survey identification number: 362200068520000 )}

LOCATION: Lat $36^{\circ} 22^{\prime} \mathrm{N}$., long $68^{\circ} 52^{\prime} \mathrm{E}$.

DRAINAGE AREA: $22,930 \mathrm{~km}^{2}$.

ELEVATION: 464 meters above mean sea level.

PERIOD OF RECORD: April 21, 1964 to September 30, 1978.

GAGE: Staff gage. Water-stage recorder at same site and datum during the 1978 water year.

Annual mean discharge

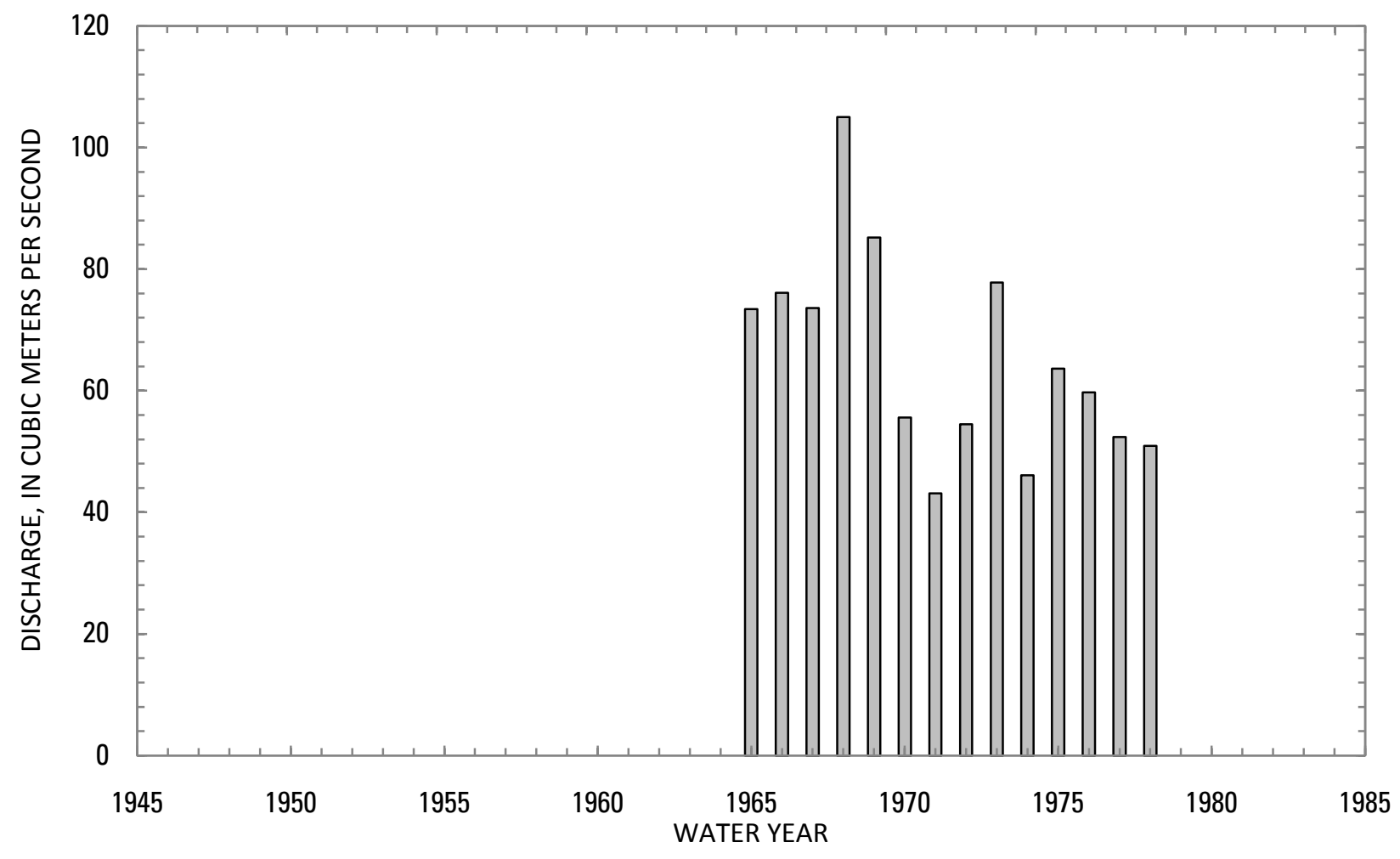




\section{4-0.000-3M KUNDUZ RIVER AT GERDAB, Continued}

Statistics of monthly and annual mean discharges $\left[\mathrm{m}^{3} / \mathrm{s}\right.$, cubic meters per second]

\begin{tabular}{|c|c|c|c|c|c|c|c|c|}
\hline \multirow[b]{2}{*}{ Month } & \multicolumn{2}{|c|}{ Maximum } & \multicolumn{2}{|c|}{ Minimum } & \multicolumn{4}{|c|}{ Mean } \\
\hline & $\begin{array}{c}\text { Discharge } \\
\left(\mathrm{m}^{3} / \mathrm{s}\right)\end{array}$ & $\begin{array}{c}\text { Water year } \\
\text { of } \\
\text { occurrence }\end{array}$ & $\begin{array}{c}\text { Discharge } \\
\left(\mathrm{m}^{3} / \mathrm{s}\right)\end{array}$ & $\begin{array}{c}\text { Water year } \\
\text { of } \\
\text { occurrence }\end{array}$ & $\begin{array}{l}\text { Discharge } \\
\left(\mathrm{m}^{3} / \mathrm{s}\right)\end{array}$ & $\begin{array}{c}\text { Standard } \\
\text { deviation } \\
\left(\mathrm{m}^{3} / \mathrm{s}\right)\end{array}$ & $\begin{array}{c}\text { Coefficient } \\
\text { of } \\
\text { variation }\end{array}$ & $\begin{array}{c}\text { Percentage } \\
\text { of annual } \\
\text { discharge }\end{array}$ \\
\hline October & 51.5 & 1969 & 23.3 & 1972 & 38.8 & 8.17 & 0.21 & 4.95 \\
\hline November & 56.8 & 1970 & 29.4 & 1972 & 41.7 & 7.49 & 0.18 & 5.33 \\
\hline December & 51.4 & 1969 & 28.4 & 1972 & 42.3 & 5.53 & 0.13 & 5.4 \\
\hline January & 44.8 & 1969 & 28.3 & 1972 & 39.1 & 4.58 & 0.12 & 4.99 \\
\hline February & 44.7 & 1974 & 29 & 1972 & 37.4 & 3.97 & 0.11 & 4.77 \\
\hline March & 47.3 & 1970 & 31.1 & 1972 & 39 & 4.95 & 0.13 & 4.97 \\
\hline April & 93.6 & 1973 & 27.1 & 1972 & 51.2 & 18.2 & 0.36 & 6.53 \\
\hline May & 184 & 1973 & 63.5 & 1974 & 112 & 34.3 & 0.31 & 14.3 \\
\hline June & 400 & 1968 & 77.5 & 1971 & 204 & 82.5 & 0.4 & 26 \\
\hline July & 239 & 1968 & 18.9 & 1971 & 112 & 60 & 0.54 & 14.3 \\
\hline August & 92.4 & 1968 & 10.6 & 1971 & 37.5 & 22.8 & 0.61 & 4.79 \\
\hline September & 48.6 & 1968 & 13.5 & 1971 & 28.9 & 10.6 & 0.37 & 3.69 \\
\hline Annual & 105 & 1968 & 43.1 & 1971 & 65.5 & 17.2 & 0.26 & 100 \\
\hline
\end{tabular}




\section{4-0.000-3M KUNDUZ RIVER AT GERDAB, Continued}

Monthly and annual flow duration, in cubic meters per second

[Data may not be rounded in accordance with U.S. Geological Survey publication standards]

\begin{tabular}{|c|c|c|c|c|c|c|c|c|c|c|c|c|c|}
\hline \multirow{2}{*}{$\begin{array}{c}\text { Percentage } \\
\text { of days } \\
\text { discharge } \\
\text { equaled or } \\
\text { exceeded }\end{array}$} & \multicolumn{12}{|c|}{ Month } & \multirow{2}{*}{ Annua } \\
\hline & October & November & December & January & February & March & April & May & June & July & August & September & \\
\hline 95 & 23.4 & 30.2 & 28.7 & 28.8 & 29.4 & 29.6 & 22.8 & 26.6 & 67.7 & 19.9 & 11 & 12.3 & 19.7 \\
\hline 90 & 29.2 & 31.1 & 36.4 & 33.7 & 32.1 & 31.4 & 28 & 37 & 88.7 & 29.4 & 14.4 & 15 & 26.5 \\
\hline 85 & 30.4 & 33.5 & 38.1 & 34.5 & 33 & 32.1 & 30.6 & 47.1 & 102 & 37.8 & 15.3 & 17.3 & 29.8 \\
\hline 80 & 32.8 & 34.8 & 38.5 & 35.1 & 33.6 & 33.1 & 32.5 & 53.7 & 113 & 45.2 & 16.6 & 19.5 & 32.2 \\
\hline 75 & 33.4 & 36.2 & 38.9 & 35.9 & 34.3 & 34 & 34.2 & 58 & 123 & 55.2 & 18.1 & 20.8 & 34.3 \\
\hline 70 & 34 & 38.4 & 39.5 & 36.4 & 35 & 34.8 & 36.7 & 66.5 & 148 & 66 & 20.1 & 22.1 & 36 \\
\hline 65 & 34.6 & 39.6 & 40.5 & 36.8 & 35.6 & 35.7 & 38.1 & 76 & 163 & 74.8 & 23.6 & 23.9 & 37.4 \\
\hline 60 & 35.4 & 40.1 & 41.7 & 37.4 & 35.9 & 36.7 & 39.5 & 86 & 174 & 80.4 & 26.9 & 25.3 & 38.9 \\
\hline 55 & 36.4 & 40.5 & 42.3 & 39.3 & 36.3 & 37.7 & 41 & 95.2 & 185 & 88.1 & 28.8 & 26.1 & 40.4 \\
\hline 50 & 37.3 & 40.9 & 42.8 & 40.1 & 37.1 & 38.9 & 42.6 & 105 & 198 & 95.4 & 30.7 & 27.1 & 41.8 \\
\hline 45 & 38.9 & 41.5 & 43.3 & 40.9 & 37.8 & 39.6 & 45.1 & 113 & 212 & 104 & 33.2 & 28.3 & 43.3 \\
\hline 40 & 40.3 & 42.2 & 43.9 & 41.5 & 38.7 & 40.2 & 50.2 & 123 & 228 & 114 & 36.1 & 29.8 & 44.7 \\
\hline 35 & 41.8 & 43.3 & 44.7 & 42 & 39.1 & 40.7 & 54.8 & 131 & 244 & 130 & 41.2 & 31.5 & 47.5 \\
\hline 30 & 44.3 & 46 & 45.6 & 42.5 & 39.7 & 41.2 & 58.1 & 141 & 257 & 143 & 45.1 & 33.2 & 50.3 \\
\hline 25 & 46.8 & 47.2 & 46.4 & 43 & 40.4 & 41.9 & 62.7 & 156 & 266 & 157 & 48.4 & 36.5 & 57.1 \\
\hline 20 & 48.1 & 48.3 & 46.9 & 43.4 & 41.4 & 43 & 67.4 & 171 & 275 & 174 & 53.6 & 42.2 & 78.9 \\
\hline 15 & 48.9 & 49.5 & 47.5 & 43.8 & 42.5 & 45.3 & 73.4 & 188 & 293 & 195 & 62.5 & 43.6 & 110 \\
\hline 10 & 50.2 & 51.8 & 48.5 & 44.3 & 43.6 & 48.2 & 85.3 & 202 & 320 & 216 & 72 & 45.3 & 154 \\
\hline 5 & 51.6 & 55.2 & 51.2 & 45.5 & 44.2 & 53.7 & 106 & 230 & 372 & 250 & 88.3 & 47.7 & 217 \\
\hline
\end{tabular}




\section{4-0.000-3M KUNDUZ RIVER AT GERDAB, Continued}

Probability of occurrence of annual high discharges [m3/s, cubic meters per second; ng, not given]

\begin{tabular}{|c|c|c|c|c|c|c|}
\hline \multirow{2}{*}{$\begin{array}{c}\text { Exceedance } \\
\text { probability }\end{array}$} & \multirow{2}{*}{$\begin{array}{c}\text { Recurrence } \\
\text { interval } \\
\text { (years) }\end{array}$} & \multirow{2}{*}{$\begin{array}{c}\text { Maximum } \\
\text { instantaneous } \\
\text { discharge } \\
\left(\mathrm{m}^{3} / \mathrm{s}\right)\end{array}$} & \multicolumn{4}{|c|}{ Maximum daily mean discharge $\left(\mathrm{m}^{3} / \mathrm{s}\right)$} \\
\hline & & & $\begin{array}{l}\text { 3-day } \\
\text { period }\end{array}$ & $\begin{array}{l}\text { 7-day } \\
\text { period }\end{array}$ & $\begin{array}{l}\text { 15-day } \\
\text { period }\end{array}$ & $\begin{array}{l}\text { 30-day } \\
\text { period }\end{array}$ \\
\hline 0.99 & 1.01 & 181 & 159 & 134 & 113 & 101 \\
\hline 0.95 & 1.05 & 209 & 186 & 162 & 139 & 125 \\
\hline 0.90 & 1.11 & 226 & 203 & 180 & 157 & 140 \\
\hline 0.80 & 1.25 & 249 & 226 & 204 & 180 & 161 \\
\hline 0.50 & 2 & 301 & 280 & 261 & 237 & 211 \\
\hline 0.20 & 5 & 369 & 349 & 336 & 313 & 279 \\
\hline 0.10 & 10 & 413 & 394 & 383 & 363 & 323 \\
\hline 0.04 & 25 & 466 & 449 & 442 & 426 & 379 \\
\hline 0.02 & 50 & 504 & 489 & 485 & 473 & 420 \\
\hline 0.01 & 100 & 542 & 529 & 528 & 519 & 462 \\
\hline 0.005 & 200 & 580 & ${ }^{1} 569$ & 571 & 566 & 503 \\
\hline 0.002 & 500 & 631 & $\mathrm{ng}$ & ng & $\mathrm{ng}$ & ng \\
\hline
\end{tabular}

'Data does not fit log-Pearson Type III curve, use with caution.

\section{4-0.000-3M KUNDUZ RIVER AT GERDAB, Continued}

Probability of occurrence of annual low discharges $\left[\mathrm{m}^{3} / \mathrm{s}\right.$, meters per second]

\begin{tabular}{|c|c|c|c|c|c|c|c|c|c|c|}
\hline \multirow{3}{*}{$\begin{array}{c}\text { Nonexceedance } \\
\text { probability }\end{array}$} & \multirow{3}{*}{$\begin{array}{c}\text { Recurrence } \\
\text { interval } \\
\text { (years) }\end{array}$} & \multicolumn{9}{|c|}{ Minimum daily mean discharge $\left(\mathrm{m}^{3} / \mathrm{s}\right)$} \\
\hline & & \multicolumn{9}{|c|}{ Number of consecutive days } \\
\hline & & 1 & 3 & 7 & 14 & 30 & 60 & 90 & 120 & 183 \\
\hline 0.05 & 20 & 7.95 & 8.3 & 8.59 & 9.47 & 10.8 & 12.6 & 16.1 & 18.9 & 23.4 \\
\hline 0.10 & 10 & 10.2 & 10.6 & 10.9 & 11.7 & 13.2 & 15.6 & 19.3 & 22.3 & 26.6 \\
\hline 0.20 & 5 & 13.4 & 13.9 & 14.2 & 15 & 16.7 & 19.7 & 23.6 & 26.7 & 30.5 \\
\hline 0.50 & 2 & 21.3 & 21.8 & 22.3 & 23 & 24.9 & 28.6 & 32.2 & 35 & 37.5 \\
\hline
\end{tabular}




\section{4-0.000-3M KUNDUZ RIVER AT GERDAB, Continued}

\section{Probability of occurrence of seasonal low discharges}

[ $\mathrm{m}^{3} / \mathrm{s}$, meters per second]

\begin{tabular}{|c|c|c|c|c|c|c|c|c|c|}
\hline \multirow{3}{*}{$\begin{array}{c}\text { Nonexceedance } \\
\text { probability }\end{array}$} & \multirow{3}{*}{$\begin{array}{c}\text { Recurrence } \\
\text { interval } \\
\text { (years) }\end{array}$} & \multicolumn{8}{|c|}{ Minimum daily mean discharge $\left(\mathrm{m}^{3} / \mathrm{s}\right)$} \\
\hline & & \multicolumn{8}{|c|}{ Number of consecutive days } \\
\hline & & 1 & 7 & 14 & 30 & 1 & 7 & 14 & 30 \\
\hline & & \multicolumn{4}{|c|}{ December-January-February } & \multicolumn{4}{|c|}{ March-April-May } \\
\hline 0.05 & 20 & 26.6 & 28.4 & 29.1 & 29.8 & 13 & 16.1 & 17.8 & 23.8 \\
\hline 0.10 & 10 & 28.1 & 29.5 & 30.3 & 31.4 & 16.2 & 19.4 & 21.2 & 26.5 \\
\hline 0.20 & 5 & 30 & 31 & 31.9 & 33.3 & 20.4 & 23.5 & 25.3 & 29.8 \\
\hline \multirow[t]{2}{*}{0.50} & 2 & 33.7 & 34.2 & 35 & 36.8 & 28.4 & 30.9 & 32.7 & 36 \\
\hline & & \multicolumn{4}{|c|}{ June-July-August } & \multicolumn{4}{|c|}{ September-October-November } \\
\hline 0.05 & 20 & 8.24 & 10.1 & 10.4 & 11.6 & 8.49 & 9.18 & 10.2 & 14.3 \\
\hline 0.10 & 10 & 10.5 & 12.3 & 12.7 & 14.4 & 10.7 & 11.4 & 12.5 & 16.7 \\
\hline 0.20 & 5 & 13.9 & 15.6 & 16.3 & 18.7 & 13.9 & 14.6 & 15.8 & 19.9 \\
\hline 0.50 & 2 & 23.1 & 24.8 & 26.1 & 31.1 & 22.1 & 22.9 & 24.2 & 27.7 \\
\hline
\end{tabular}




\section{4-0.000-3M KUNDUZ RIVER AT GERDAB, Continued}

\begin{tabular}{|c|c|c|c|c|c|}
\hline \multicolumn{6}{|c|}{$\begin{array}{c}\text { Annual peak discharges } \\
{\left[\mathrm{m}^{3} / \mathrm{s} \text {, meters per second] }\right.}\end{array}$} \\
\hline \multicolumn{3}{|c|}{$\begin{array}{c}\text { Annual peak discharge, } \\
\text { by year }\end{array}$} & \multicolumn{3}{|c|}{$\begin{array}{l}\text { Annual peak discharge, } \\
\text { from highest to lowest }\end{array}$} \\
\hline $\begin{array}{l}\text { Water } \\
\text { year }\end{array}$ & Date & $\begin{array}{c}\text { Peak } \\
\text { discharge } \\
\left(\mathrm{m}^{3} / \mathrm{s}\right)\end{array}$ & $\begin{array}{l}\text { Water } \\
\text { year }\end{array}$ & Date & $\begin{array}{c}\text { Peak } \\
\text { discharge } \\
\left(\mathrm{m}^{3} / \mathrm{s}\right)\end{array}$ \\
\hline 1964 & June 29, 1964 & 275 & 1968 & June 12, 1968 & 498 \\
\hline 1965 & June 23, 1965 & 317 & 1969 & June 21, 1969 & 376 \\
\hline 1966 & June 19, 1966 & 358 & 1973 & June 15,1973 & 376 \\
\hline 1967 & June 8, 1967 & 274 & 1966 & June 19, 1966 & 358 \\
\hline 1968 & June 12, 1968 & 498 & 1977 & May 28, 1977 & 350 \\
\hline 1969 & June 21, 1969 & 376 & 1976 & June 5, 1976 & 328 \\
\hline 1970 & May 19, 1970 & 224 & 1965 & June 23, 1965 & 317 \\
\hline 1971 & May 21, 1971 & 252 & 1972 & June 15, 1972 & 314 \\
\hline 1972 & June 15, 1972 & 314 & 1975 & June 19, 1975 & 303 \\
\hline 1973 & June 15,1973 & 376 & 1964 & June 29, 1964 & 275 \\
\hline 1974 & June 19, 1974 & 220 & 1967 & June 8, 1967 & 274 \\
\hline 1975 & June 19, 1975 & 303 & 1971 & May 21, 1971 & 252 \\
\hline 1976 & June 5, 1976 & 328 & 1970 & May 19, 1970 & 224 \\
\hline 1977 & May 28, 1977 & 350 & 1974 & June 19, 1974 & 220 \\
\hline 1978 & June 6, 1978 & 210 & 1978 & June 6, 1978 & 210 \\
\hline
\end{tabular}




\section{4-0.000-3M KUNDUZ RIVER AT GERDAB, Continued}

Monthly and annual mean discharges, in cubic meters per second $[--$, no data]

\begin{tabular}{|c|c|c|c|c|c|c|c|c|c|c|c|c|c|}
\hline \multirow{2}{*}{$\begin{array}{l}\text { Water } \\
\text { year }\end{array}$} & \multicolumn{12}{|c|}{ Monthly mean discharge } & \multirow{2}{*}{$\begin{array}{c}\text { Annual } \\
\text { discharge }\end{array}$} \\
\hline & October & November & December & January & February & March & April & May & June & July & August & September & \\
\hline 1964 & -- & -- & -- & -- & -- & -- & -- & 74.5 & 196 & 138 & 40.3 & 22.1 & -- \\
\hline 1965 & 32.0 & 32.7 & 38.5 & 35.8 & 37.1 & 36.9 & 50.6 & 85.5 & 239 & 199 & 60.5 & 32.4 & 73.4 \\
\hline 1966 & 39.2 & 41.1 & 43.2 & 39.0 & 36.1 & 46.8 & 72.6 & 134 & 263 & 105 & 49.2 & 43.4 & 76.1 \\
\hline 1967 & 49.4 & 48.9 & 46.3 & 43.9 & 43.8 & 41.2 & 43.4 & 105 & 232 & 146 & 53.4 & 28.8 & 73.6 \\
\hline 1968 & 44.6 & 42.1 & 47.3 & 41.9 & 35.8 & 34.2 & 66.5 & 164 & 400 & 239 & 92.4 & 48.6 & 105 \\
\hline 1969 & 51.5 & 50.3 & 51.4 & 44.8 & 41.0 & 41.9 & 60.4 & 133 & 267 & 168 & 66.3 & 44.5 & 85.2 \\
\hline 1970 & 49.6 & 56.8 & 46.5 & 42.2 & 38.6 & 47.3 & 49.1 & 108 & 110 & 58.0 & 30.5 & 29.8 & 55.6 \\
\hline 1971 & 35.5 & 37.7 & 38.5 & 36.7 & 34.6 & 37.2 & 48.1 & 128 & 77.5 & 18.9 & 10.6 & 13.5 & 43.1 \\
\hline 1972 & 23.3 & 29.4 & 28.4 & 28.3 & 29.0 & 31.1 & 27.1 & 68.9 & 238 & 110 & 22.4 & 18.9 & 54.5 \\
\hline 1973 & 30.4 & 33.7 & 39.2 & 34.4 & 36.1 & 40.1 & 93.6 & 184 & 263 & 108 & 32.7 & 38.0 & 77.8 \\
\hline 1974 & 43.0 & 40.4 & 44.5 & 43.4 & 44.7 & 44.5 & 27.6 & 63.5 & 116 & 52.3 & 17.5 & 16.5 & 46.1 \\
\hline 1975 & 34.3 & 38.1 & 39.0 & 40.6 & 34.0 & 33.9 & 51.1 & 119 & 204 & 115 & 29.4 & 24.8 & 63.6 \\
\hline 1976 & 39.7 & 44.2 & 42.0 & 36.2 & 38.0 & 38.0 & 55.0 & 116 & 152 & 107 & 24.5 & 23.7 & 59.7 \\
\hline 1977 & 37.2 & 40.8 & 43.0 & 37.7 & 36.8 & 34.2 & 32.7 & 81.9 & 183 & 61.5 & 16.4 & 24.9 & 52.4 \\
\hline 1978 & 33.0 & 48.1 & 45.0 & 42.7 & 37.5 & 38.4 & 38.3 & 117 & 120 & 49.6 & 16.9 & 23.7 & 50.9 \\
\hline
\end{tabular}




\section{4-0.000-4M KUNDUZ RIVER AT BAGHLAN}

\section{(U.S. Geological Survey identification number: $\mathbf{3 6 0 6 0 0 0 6 8 4 0 0 0 0 0 )}$}

LOCATION: Lat $36^{\circ} 06^{\prime} \mathrm{N}$., long $68^{\circ} 40^{\prime} \mathrm{E}$.

DRAINAGE AREA: $19,740 \mathrm{~km}^{2}$.

ELEVATION: 562 meters above mean sea level.

PERIOD OF RECORD: April 7, 1968 to September 30, 1978.

GAGE: Water-stage recorder.

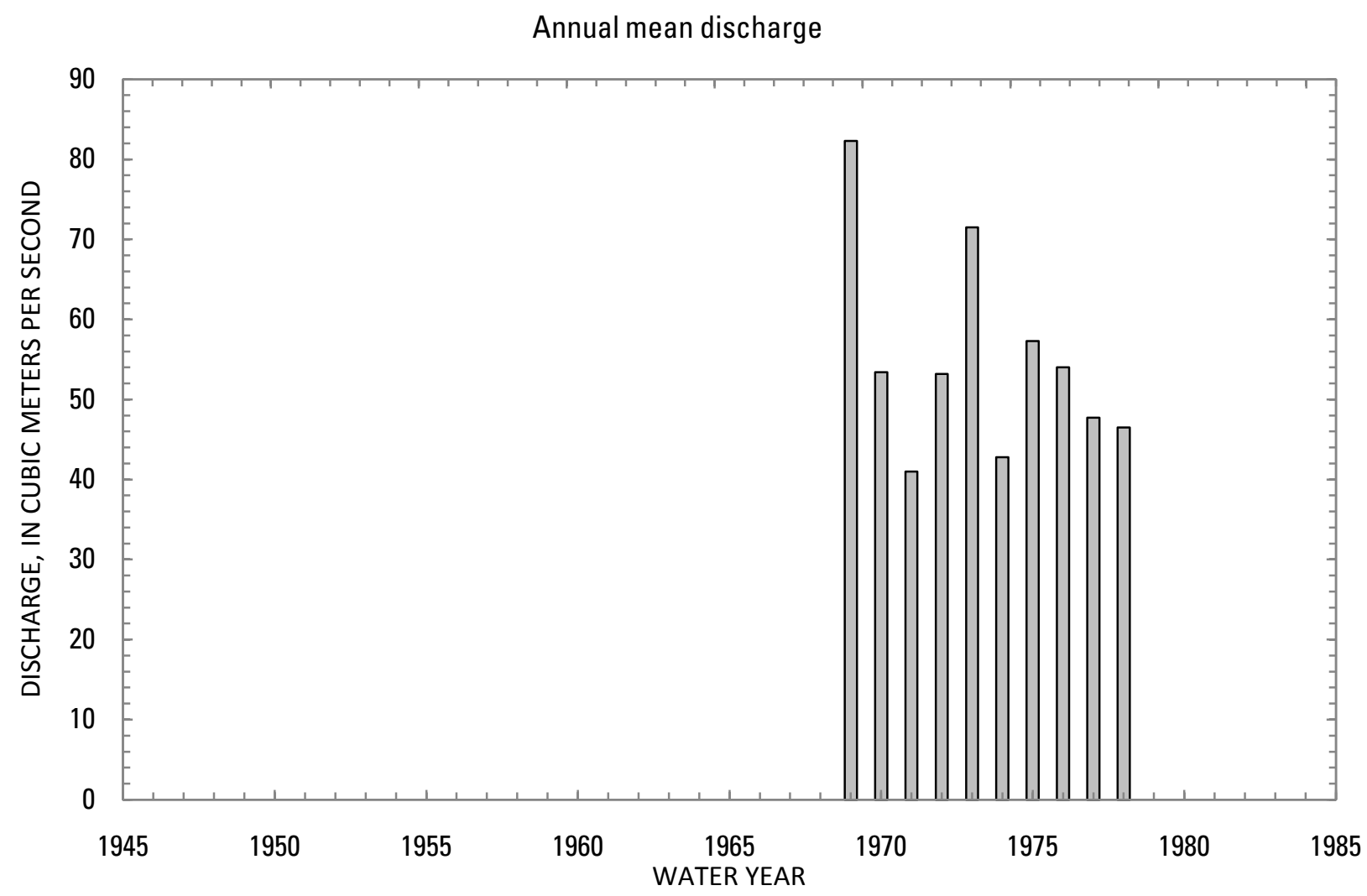




\section{4-0.000-4M KUNDUZ RIVER AT BAGHLAN, Continued}

Statistics of monthly and annual mean discharges [m $\mathrm{m}^{3} / \mathrm{s}$, cubic meters per second]

\begin{tabular}{|c|c|c|c|c|c|c|c|c|}
\hline \multirow[b]{2}{*}{ Month } & \multicolumn{2}{|c|}{ Maximum } & \multicolumn{2}{|c|}{ Minimum } & \multicolumn{4}{|c|}{ Mean } \\
\hline & $\begin{array}{c}\text { Discharge } \\
\left(\mathrm{m}^{3} / \mathrm{s}\right)\end{array}$ & $\begin{array}{c}\text { Water year } \\
\text { of } \\
\text { occurrence }\end{array}$ & $\begin{array}{c}\text { Discharge } \\
\left(\mathrm{m}^{3} / \mathrm{s}\right)\end{array}$ & $\begin{array}{c}\text { Water year } \\
\text { of } \\
\text { occurrence }\end{array}$ & $\begin{array}{c}\text { Discharge } \\
\left(\mathrm{m}^{3} / \mathrm{s}\right)\end{array}$ & $\begin{array}{c}\text { Standard } \\
\text { deviation } \\
\left(\mathrm{m}^{3} / \mathrm{s}\right)\end{array}$ & $\begin{array}{c}\text { Coefficient } \\
\text { of } \\
\text { variation }\end{array}$ & $\begin{array}{c}\text { Percentage } \\
\text { of annual } \\
\text { discharge }\end{array}$ \\
\hline October & 43.8 & 1969 & 21.0 & 1972 & 32.5 & 7.15 & 0.22 & 4.60 \\
\hline November & 48.5 & 1970 & 27.0 & 1972 & 35.2 & 6.73 & 0.19 & 4.99 \\
\hline December & 45.1 & 1969 & 25.9 & 1972 & 34.8 & 5.31 & 0.15 & 4.94 \\
\hline January & 38.4 & 1970 & 26.0 & 1972 & 32.0 & 3.49 & 0.11 & 4.54 \\
\hline February & 35.4 & 1970 & 24.8 & 1978 & 29.8 & 3.40 & 0.11 & 4.23 \\
\hline March & 37.7 & 1970 & 26.4 & 1972 & 32.3 & 3.77 & 0.12 & 4.58 \\
\hline April & 82.2 & 1973 & 22.0 & 1972 & 43.4 & 17.4 & 0.40 & 6.15 \\
\hline May & 169 & 1973 & 58.9 & 1972 & 111 & 35.0 & 0.32 & 15.7 \\
\hline June & 375 & 1968 & 79.8 & 1971 & 184 & 85.6 & 0.46 & 26.1 \\
\hline July & 248 & 1968 & 25.1 & 1971 & 102 & 64.7 & 0.64 & 14.4 \\
\hline August & 97.1 & 1968 & 15.0 & 1971 & 39.7 & 25.4 & 0.64 & 5.63 \\
\hline September & 48.9 & 1968 & 15.8 & 1971 & 29.1 & 10.6 & 0.37 & 4.13 \\
\hline Annual & 82.3 & 1969 & 41.0 & 1971 & 55.0 & 12.9 & 0.23 & 100 \\
\hline
\end{tabular}


14-0.000-4M KUNDUZ RIVER AT BAGHLAN, Continued

Monthly and annual flow duration, in cubic meters per second

\begin{tabular}{|c|c|c|c|c|c|c|c|c|c|c|c|c|c|}
\hline \multirow{2}{*}{$\begin{array}{l}\text { Percentage } \\
\text { of days } \\
\text { discharge } \\
\text { equaled or } \\
\text { exceeded }\end{array}$} & \multicolumn{12}{|c|}{ Month } & \multirow{2}{*}{ Annual } \\
\hline & October & November & December & January & February & March & April & May & June & July & August & September & \\
\hline 95 & 20.8 & 27.5 & 25.9 & 25.8 & 24.4 & 24.2 & 18.9 & 27.7 & 56.7 & 24.8 & 14.9 & 15.1 & 20.8 \\
\hline 90 & 24.2 & 27.9 & 28.6 & 27.7 & 25.0 & 25.7 & 21.6 & 34.5 & 85.9 & 28.3 & 18.6 & 19.3 & 23.9 \\
\hline 85 & 27.4 & 28.7 & 31.0 & 29.0 & 25.9 & 26.6 & 25.0 & 43.6 & 96.5 & 34.0 & 19.7 & 20.4 & 26.0 \\
\hline 80 & 27.9 & 29.8 & 32.0 & 29.5 & 26.5 & 28.0 & 27.3 & 52.1 & 106 & 44.9 & 20.9 & 20.9 & 27.5 \\
\hline 75 & 28.4 & 30.2 & 32.3 & 30.5 & 26.9 & 28.7 & 29.0 & 56.1 & 114 & 48.6 & 22.0 & 21.3 & 29.1 \\
\hline 70 & 28.8 & 30.7 & 32.5 & 30.9 & 27.9 & 29.2 & 31.1 & 67.6 & 122 & 55.7 & 23.1 & 22.3 & 30.0 \\
\hline 65 & 29.2 & 31.2 & 32.8 & 31.2 & 28.3 & 29.7 & 32.4 & 80.9 & 133 & 62.0 & 24.7 & 23.3 & 30.8 \\
\hline 60 & 29.5 & 31.8 & 33.1 & 31.4 & 28.9 & 30.2 & 33.7 & 90.2 & 146 & 68.3 & 26.7 & 24.1 & 31.7 \\
\hline 55 & 29.8 & 33.0 & 33.3 & 31.6 & 29.2 & 30.8 & 35.4 & 101 & 157 & 74.9 & 29.0 & 24.7 & 32.7 \\
\hline 50 & 30.2 & 33.7 & 33.6 & 31.8 & 29.9 & 31.4 & 37.0 & 112 & 169 & 81.0 & 31.2 & 25.5 & 34.1 \\
\hline 45 & 31.2 & 34.4 & 34.0 & 31.9 & 30.2 & 32.1 & 38.7 & 120 & 179 & 88.6 & 33.1 & 26.4 & 35.4 \\
\hline 40 & 32.0 & 35.5 & 34.4 & 32.2 & 30.6 & 33.5 & 40.6 & 127 & 189 & 96.9 & 35.3 & 28.8 & 37.4 \\
\hline 35 & 32.8 & 36.7 & 34.9 & 32.5 & 31.0 & 34.3 & 44.0 & 136 & 198 & 107 & 38.4 & 30.5 & 39.8 \\
\hline 30 & 35.2 & 38.2 & 35.7 & 32.9 & 32.0 & 34.9 & 48.7 & 143 & 224 & 122 & 41.4 & 33.1 & 43.7 \\
\hline 25 & 37.7 & 39.3 & 37.1 & 33.4 & 32.7 & 35.7 & 50.7 & 150 & 242 & 136 & 44.2 & 34.7 & 49.1 \\
\hline 20 & 39.4 & 41.1 & 39.8 & 34.8 & 33.5 & 36.5 & 57.3 & 161 & 260 & 151 & 53.4 & 35.8 & 65.7 \\
\hline 15 & 41.9 & 44.2 & 41.7 & 36.0 & 34.0 & 37.3 & 63.5 & 172 & 277 & 168 & 65.9 & 44.1 & 95.2 \\
\hline 10 & 44.1 & 45.4 & 42.9 & 37.9 & 35.0 & 39.0 & 77.5 & 191 & 307 & 198 & 83.9 & 45.9 & 128 \\
\hline 5 & 45.2 & 48.9 & 45.2 & 39.1 & 35.3 & 40.7 & 90.3 & 224 & 411 & 243 & 94.8 & 49.1 & 177 \\
\hline
\end{tabular}




\section{4-0.000-4M KUNDUZ RIVER AT BAGHLAN, Continued}

Probability of occurrence of annual high discharges

[m $\mathrm{m}^{3} / \mathrm{s}$, cubic meters per second; ng, not given]

\begin{tabular}{|c|c|c|c|c|c|c|}
\hline \multirow{2}{*}{$\begin{array}{c}\text { Exceedance } \\
\text { probability }\end{array}$} & \multirow{2}{*}{$\begin{array}{c}\text { Recurrence } \\
\text { interval } \\
\text { (years) }\end{array}$} & \multirow{2}{*}{$\begin{array}{l}\text { Maximum } \\
\text { instantaneous } \\
\text { discharge } \\
\left(\mathrm{m}^{3} / \mathrm{s}\right)\end{array}$} & \multicolumn{4}{|c|}{ Maximum daily mean discharge $\left(\mathrm{m}^{3} / \mathrm{s}\right)$} \\
\hline & & & $\begin{array}{l}\text { 3-day } \\
\text { period }\end{array}$ & $\begin{array}{l}\text { 7-day } \\
\text { period }\end{array}$ & $\begin{array}{l}\text { 15-day } \\
\text { period }\end{array}$ & $\begin{array}{l}\text { 30-day } \\
\text { period }\end{array}$ \\
\hline 0.99 & 1.01 & 166 & 143 & 122 & 106 & 97.0 \\
\hline 0.95 & 1.05 & 192 & 167 & 145 & 127 & 115 \\
\hline 0.90 & 1.11 & 208 & 181 & 158 & 140 & 127 \\
\hline 0.80 & 1.25 & 231 & 200 & 177 & 158 & 142 \\
\hline 0.50 & 2 & 286 & 242 & 219 & 199 & 178 \\
\hline 0.20 & 5 & 362 & 292 & 274 & 251 & 225 \\
\hline 0.10 & 10 & 412 & 322 & 308 & 285 & 255 \\
\hline 0.04 & 25 & 477 & 358 & 349 & 327 & 291 \\
\hline 0.02 & 50 & 525 & 383 & 379 & 357 & 318 \\
\hline 0.01 & 100 & 574 & '406 & 409 & 387 & 345 \\
\hline 0.005 & 200 & 625 & '429 & 438 & 417 & 371 \\
\hline 0.002 & 500 & 694 & $\mathrm{ng}$ & $\mathrm{ng}$ & $\mathrm{ng}$ & ng \\
\hline
\end{tabular}

'Data does not fit log-Pearson Type III curve, use with caution.

\section{4-0.000-4M KUNDUZ RIVER AT BAGHLAN, Continued}

Probability of occurrence of annual low discharges

$\left[\mathrm{m}^{3} / \mathrm{s}\right.$, meters per second]

\begin{tabular}{|c|c|c|c|c|c|c|c|c|c|c|}
\hline \multirow{3}{*}{$\begin{array}{c}\text { Nonexceedance } \\
\text { probability }\end{array}$} & \multirow{3}{*}{$\begin{array}{c}\text { Recurrence } \\
\text { interval } \\
\text { (years) }\end{array}$} & \multicolumn{9}{|c|}{ Minimum daily mean discharge $\left(\mathrm{m}^{3} / \mathrm{s}\right)$} \\
\hline & & \multicolumn{9}{|c|}{ Number of consecutive days } \\
\hline & & 1 & 3 & 7 & 14 & 30 & 60 & 90 & 120 & 183 \\
\hline 0.05 & 20 & 13.3 & 13.4 & 13.7 & 14.0 & 14.7 & 16.2 & 18.2 & 19.7 & 22.5 \\
\hline 0.10 & 10 & 14.6 & 14.8 & 15.1 & 15.6 & 16.6 & 18.5 & 20.3 & 22.0 & 24.2 \\
\hline 0.20 & 5 & 16.5 & 16.7 & 17.1 & 17.7 & 19.1 & 21.3 & 23.0 & 24.7 & 26.3 \\
\hline 0.50 & 2 & 21.1 & 21.3 & 21.8 & 22.6 & 24.4 & 26.6 & 28.1 & 29.6 & 30.6 \\
\hline
\end{tabular}




\section{4-0.000-4M KUNDUZ RIVER AT BAGHLAN, Continued}

Probability of occurrence of seasonal low discharges [ $\mathrm{m}^{3} / \mathrm{s}$, meters per second]

\begin{tabular}{|c|c|c|c|c|c|c|c|c|c|}
\hline \multirow{3}{*}{$\begin{array}{c}\text { Nonexceedance } \\
\text { probability }\end{array}$} & \multirow{3}{*}{$\begin{array}{c}\text { Recurrence } \\
\text { interval } \\
\text { (years) }\end{array}$} & \multicolumn{8}{|c|}{ Minimum daily mean discharge $\left(\mathrm{m}^{3} / \mathrm{s}\right)$} \\
\hline & & \multicolumn{8}{|c|}{ Number of consecutive days } \\
\hline & & 1 & 7 & 14 & 30 & 1 & 7 & 14 & 30 \\
\hline & & \multicolumn{4}{|c|}{ December-January-February } & \multicolumn{4}{|c|}{ March-April-May } \\
\hline 0.05 & 20 & 21.8 & 22.6 & 23.1 & 24.1 & 16.1 & 17.1 & 18.3 & 22.2 \\
\hline 0.10 & 10 & 22.9 & 23.6 & 24.3 & 25.2 & 18.1 & 19.1 & 20.2 & 23.9 \\
\hline 0.20 & 5 & 24.3 & 24.9 & 25.7 & 26.7 & 20.6 & 21.6 & 22.7 & 26.1 \\
\hline \multirow[t]{2}{*}{0.50} & 2 & 27.3 & 27.8 & 28.6 & 29.5 & 25.2 & 26.2 & 27.2 & 30.2 \\
\hline & & \multicolumn{4}{|c|}{ June-July-August } & \multicolumn{4}{|c|}{ September-0ctober-November } \\
\hline 0.05 & 20 & 14.2 & 14.4 & 14.6 & 15.2 & 13.9 & 14.1 & 14.9 & 16.4 \\
\hline 0.10 & 10 & 15.6 & 15.9 & 16.3 & 17.4 & 15.7 & 15.9 & 16.8 & 18.3 \\
\hline 0.20 & 5 & 17.9 & 18.3 & 19.0 & 21.0 & 18.2 & 18.5 & 19.5 & 21.0 \\
\hline 0.50 & 2 & 24.9 & 25.6 & 27.3 & 31.8 & 24.3 & 24.7 & 25.9 & 27.3 \\
\hline
\end{tabular}

\section{4-0.000-4M KUNDUZ RIVER AT BAGHLAN, Continued}

Annual peak discharges

[m $\mathrm{m}^{3} / \mathrm{s}$, meters per second]

\begin{tabular}{|c|c|c|c|c|c|}
\hline \multicolumn{3}{|c|}{$\begin{array}{c}\text { Annual peak discharge, } \\
\text { by year }\end{array}$} & \multicolumn{3}{|c|}{$\begin{array}{l}\text { Annual peak discharge, } \\
\text { from highest to lowest }\end{array}$} \\
\hline $\begin{array}{l}\text { Water } \\
\text { year }\end{array}$ & Date & $\begin{array}{c}\text { Peak } \\
\text { discharge } \\
\left(\mathrm{m}^{3} / \mathrm{s}\right)\end{array}$ & $\begin{array}{l}\text { Water } \\
\text { year }\end{array}$ & Date & $\begin{array}{c}\text { Peak } \\
\text { discharge } \\
\left(\mathrm{m}^{3} / \mathrm{s}\right)\end{array}$ \\
\hline 1968 & June 25,1968 & 492 & 1968 & June 25,1968 & 492 \\
\hline 1969 & June 17, 1969 & 337 & 1972 & June 22, 1972 & 338 \\
\hline 1970 & May 22, 1970 & 222 & 1969 & June 17, 1969 & 337 \\
\hline 1971 & May 27, 1971 & 235 & 1973 & June 9, 1973 & 331 \\
\hline 1972 & June 22, 1972 & 338 & 1977 & May 29, 1977 & 327 \\
\hline 1973 & June 9, 1973 & 331 & 1976 & May 24, 1976 & 320 \\
\hline 1974 & June 18, 1974 & 200 & 1975 & June 16,1975 & 286 \\
\hline 1975 & June 16, 1975 & 286 & 1971 & May 27, 1971 & 235 \\
\hline 1976 & May 24, 1976 & 320 & 1970 & May 22, 1970 & 222 \\
\hline 1977 & May 29, 1977 & 327 & 1978 & June 5, 1978 & 214 \\
\hline 1978 & June 5, 1978 & 214 & 1974 & June 18,1974 & 200 \\
\hline
\end{tabular}




\section{4-0.000-4M KUNDUZ RIVER AT BAGHLAN, Continued}

Monthly and annual mean discharges, in cubic meters per second $[--$, no data]

\begin{tabular}{|c|c|c|c|c|c|c|c|c|c|c|c|c|c|}
\hline \multirow{2}{*}{$\begin{array}{l}\text { Water } \\
\text { year }\end{array}$} & \multicolumn{12}{|c|}{ Monthly mean discharge } & \multirow{2}{*}{$\begin{array}{l}\text { Annual } \\
\text { discharge }\end{array}$} \\
\hline & October & November & December & January & February & March & April & May & June & July & August & September & \\
\hline 1968 & -- & -- & -- & -- & -- & -- & -- & 156 & 375 & 248 & 97.1 & 48.9 & -- \\
\hline 1969 & 43.8 & 43.6 & 45.1 & 36.8 & 33.7 & 35.0 & 59.9 & 122 & 261 & 180 & 78.4 & 47.3 & 82.3 \\
\hline 1970 & 43.8 & 48.5 & 41.8 & 38.4 & 35.4 & 37.7 & 46.0 & 106 & 112 & 61.0 & 37.3 & 31.9 & 53.4 \\
\hline 1971 & 30.6 & 32.0 & 33.3 & 30.5 & 28.3 & 32.5 & 45.5 & 123 & 79.8 & 25.1 & 15.0 & 15.8 & 41.0 \\
\hline 1972 & 21.0 & 27.0 & 25.9 & 26.0 & 25.4 & 26.4 & 22.0 & 58.9 & 217 & 128 & 37.9 & 23.8 & 53.2 \\
\hline 1973 & 29.2 & 30.7 & 34.3 & 32.1 & 30.6 & 34.6 & 82.2 & 169 & 242 & 98.0 & 40.3 & 34.9 & 71.5 \\
\hline 1974 & 37.3 & 37.6 & 34.7 & 32.7 & 32.3 & 36.7 & 26.7 & 63.8 & 111 & 53.6 & 25.3 & 22.4 & 42.8 \\
\hline 1975 & 28.3 & 29.8 & 32.8 & 32.0 & 29.2 & 29.3 & 42.0 & 114 & 184 & 104 & 35.3 & 25.8 & 57.3 \\
\hline 1976 & 30.3 & 30.2 & 31.5 & 30.5 & 30.3 & 32.0 & 42.2 & 116 & 148 & 104 & 28.8 & 23.9 & 54.0 \\
\hline 1977 & 31.3 & 36.6 & 35.5 & 31.5 & 28.0 & 30.9 & 31.8 & 71.3 & 170 & 61.0 & 20.7 & 23.9 & 47.7 \\
\hline 1978 & 29.1 & 35.9 & 33.4 & 29.9 & 24.8 & 27.7 & 35.6 & 116 & 125 & 56.2 & 21.0 & 21.8 & 46.5 \\
\hline
\end{tabular}




\section{4-0.000-5S KUNDUZ RIVER AT PUL-I-KHUMRI}

\section{(U.S. Geological Survey identification number: 355600068430000 )}

LOCATION: Lat $35^{\circ} 56^{\prime} \mathrm{N}$., long 68²43'E.

DRAINAGE AREA: $17,405 \mathrm{~km}^{2}$.

ELEVATION: 639 meters above mean sea level.

PERIOD OF RECORD: Monthly mean values April 1, 1950 to September 30 1959. Daily mean values October 1, 1959 to March 26, 1968.

GAGE: Water-stage recorder. Head works gage prior to January 1966 at hydropower station $2 \mathrm{~km}$ downstream.

Annual mean discharge

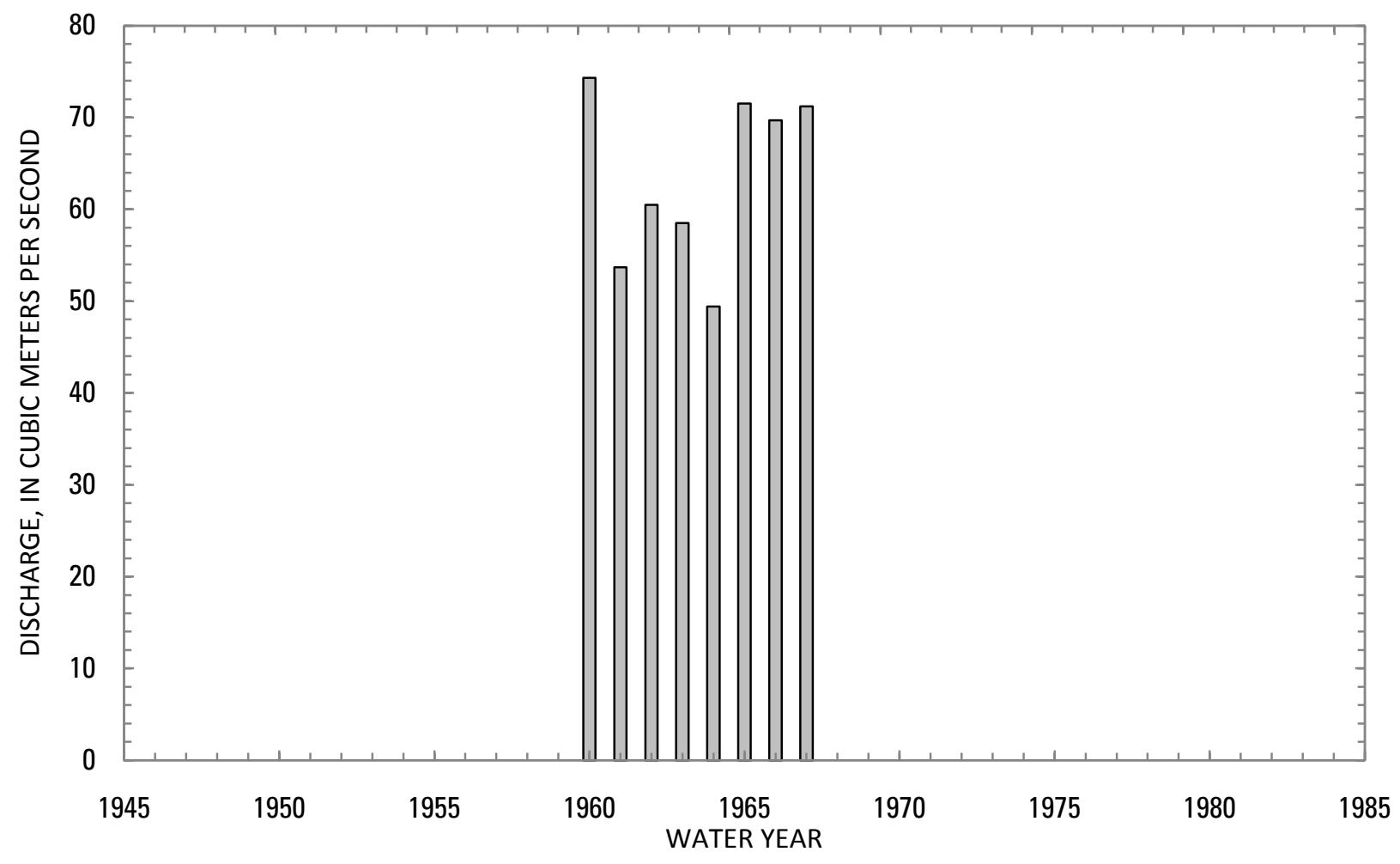




\section{4-0.000-5S KUNDUZ RIVER AT PUL-I-KHUMRI, Continued}

Statistics of monthly and annual mean discharges $\left[\mathrm{m}^{3} / \mathrm{s}\right.$, cubic meters per second]

\begin{tabular}{|c|c|c|c|c|c|c|c|c|}
\hline \multirow[b]{2}{*}{ Month } & \multicolumn{2}{|c|}{ Maximum } & \multicolumn{2}{|c|}{ Minimum } & \multicolumn{4}{|c|}{ Mean } \\
\hline & $\begin{array}{c}\text { Discharge } \\
\left(\mathrm{m}^{3} / \mathrm{s}\right)\end{array}$ & $\begin{array}{c}\text { Water year } \\
\text { of } \\
\text { occurrence }\end{array}$ & $\begin{array}{c}\text { Discharge } \\
\left(\mathrm{m}^{3} / \mathrm{s}\right)\end{array}$ & $\begin{array}{c}\text { Water year } \\
\text { of } \\
\text { occurrence }\end{array}$ & $\begin{array}{c}\text { Discharge } \\
\left(\mathrm{m}^{3} / \mathrm{s}\right)\end{array}$ & $\begin{array}{c}\text { Standard } \\
\text { deviation } \\
\left(\mathrm{m}^{3} / \mathrm{s}\right)\end{array}$ & $\begin{array}{c}\text { Coefficient } \\
\text { of } \\
\text { variation }\end{array}$ & $\begin{array}{c}\text { Percentage } \\
\text { of annual } \\
\text { discharge }\end{array}$ \\
\hline October & 46.5 & 1968 & 26.6 & 1962 & 34.6 & 6.70 & 0.19 & 4.50 \\
\hline November & 43.6 & 1968 & 25.3 & 1965 & 31.9 & 6.23 & 0.20 & 4.15 \\
\hline December & 39.9 & 1968 & 22.6 & 1964 & 29.4 & 6.17 & 0.21 & 3.83 \\
\hline January & 36.6 & 1966 & 20.0 & 1964 & 27.5 & 6.05 & 0.22 & 3.58 \\
\hline February & 35.5 & 1966 & 20.6 & 1964 & 26.7 & 4.70 & 0.18 & 3.48 \\
\hline March & 34.7 & 1966 & 20.3 & 1964 & 26.0 & 4.27 & 0.16 & 3.38 \\
\hline April & 53.9 & 1966 & 26.5 & 1961 & 36.9 & 9.55 & 0.26 & 4.80 \\
\hline May & 139 & 1966 & 67.5 & 1964 & 95.1 & 21.9 & 0.23 & 12.4 \\
\hline June & 261 & 1963 & 153 & 1964 & 224 & 36.9 & 0.16 & 29.2 \\
\hline July & 229 & 1960 & 97.2 & 1966 & 143 & 53.7 & 0.38 & 18.6 \\
\hline August & 81.8 & 1960 & 40.2 & 1963 & 56.7 & 15.3 & 0.27 & 7.38 \\
\hline September & 44.2 & 1966 & 28.8 & 1963 & 36.5 & 6.37 & 0.17 & 4.74 \\
\hline Annual & 74.3 & 1960 & 49.4 & 1964 & 63.6 & 9.31 & 0.15 & 100 \\
\hline
\end{tabular}


14-0.000-5S KUNDUZ RIVER AT PUL-I-KHUMRI, Continued

Monthly and annual flow duration, in cubic meters per second

\begin{tabular}{|c|c|c|c|c|c|c|c|c|c|c|c|c|c|}
\hline \multirow{2}{*}{$\begin{array}{l}\text { Percentage } \\
\text { of days } \\
\text { discharge } \\
\text { equaled or } \\
\text { exceeded }\end{array}$} & \multicolumn{12}{|c|}{ Month } & \multirow{2}{*}{ Annual } \\
\hline & October & November & December & January & February & March & April & May & June & July & August & September & \\
\hline 95 & 22.3 & 23.4 & 21.2 & 20.2 & 20.2 & 20.0 & 22.4 & 41.7 & 111 & 65.8 & 31.1 & 26.8 & 21.4 \\
\hline 90 & 25.4 & 24.4 & 22.9 & 20.4 & 20.4 & 20.2 & 24.0 & 53.6 & 141 & 74.7 & 32.7 & 27.5 & 23.1 \\
\hline 85 & 27.8 & 25.2 & 23.5 & 21.1 & 22.2 & 21.5 & 24.9 & 60.0 & 153 & 80.4 & 36.3 & 28.2 & 24.1 \\
\hline 80 & 29.1 & 25.9 & 24.2 & 22.4 & 22.8 & 21.8 & 25.6 & 66.0 & 163 & 84.9 & 38.4 & 28.6 & 25.1 \\
\hline 75 & 30.2 & 27.0 & 24.6 & 23.1 & 23.1 & 22.7 & 26.3 & 69.8 & 171 & 90.4 & 42.4 & 29.5 & 26.3 \\
\hline 70 & 31.0 & 27.5 & 24.9 & 23.5 & 23.4 & 23.1 & 26.9 & 72.6 & 182 & 96.1 & 45.4 & 30.6 & 27.7 \\
\hline 65 & 31.6 & 28.4 & 25.3 & 23.7 & 23.6 & 23.7 & 27.5 & 75.1 & 198 & 103 & 48.2 & 31.6 & 29.3 \\
\hline 60 & 32.1 & 29.2 & 25.9 & 24.2 & 24.1 & 24.0 & 28.7 & 78.1 & 211 & 113 & 50.6 & 33.0 & 31.0 \\
\hline 55 & 32.6 & 30.1 & 26.5 & 24.7 & 24.6 & 24.5 & 30.5 & 81.7 & 220 & 122 & 52.2 & 34.5 & 32.7 \\
\hline 50 & 33.2 & 31.2 & 27.4 & 25.2 & 25.2 & 25.0 & 32.1 & 86.4 & 232 & 130 & 54.0 & 36.8 & 34.5 \\
\hline 45 & 34.5 & 31.8 & 28.4 & 26.0 & 26.8 & 25.6 & 33.5 & 90.3 & 242 & 136 & 56.0 & 37.6 & 36.3 \\
\hline 40 & 35.0 & 33.0 & 29.8 & 27.5 & 27.6 & 26.1 & 35.1 & 95.1 & 252 & 141 & 58.5 & 40.0 & 39.5 \\
\hline 35 & 36.4 & 34.4 & 31.9 & 29.6 & 29.0 & 27.0 & 36.7 & 101 & 261 & 148 & 61.2 & 40.9 & 43.7 \\
\hline 30 & 37.8 & 34.7 & 34.5 & 33.6 & 29.7 & 27.7 & 40.2 & 107 & 269 & 156 & 63.3 & 42.0 & 53.6 \\
\hline 25 & 40.2 & 35.1 & 35.0 & 34.4 & 30.4 & 28.5 & 44.2 & 115 & 277 & 178 & 65.2 & 42.9 & 68.3 \\
\hline 20 & 42.5 & 38.2 & 35.7 & 34.9 & 31.0 & 29.3 & 48.3 & 126 & 285 & 198 & 69.9 & 43.5 & 84.8 \\
\hline 15 & 43.8 & 39.6 & 36.9 & 35.5 & 31.8 & 31.8 & 53.0 & 138 & 295 & 223 & 76.1 & 44.4 & 116 \\
\hline 10 & 44.9 & 42.4 & 38.6 & 36.2 & 32.7 & 34.2 & 59.6 & 152 & 306 & 246 & 80.9 & 45.7 & 159 \\
\hline 5 & 46.2 & 43.8 & 40.5 & 37.1 & 35.5 & 34.9 & 67.5 & 183 & 322 & 283 & 95.8 & 47.1 & 236 \\
\hline
\end{tabular}




\section{4-0.000-5S KUNDUZ RIVER AT PUL-I-KHUMRI, Continued}

Probability of occurrence of annual high discharges

[ $\mathrm{m}^{3} / \mathrm{s}$, cubic meters per second; $\mathrm{ng}$, not given]

\begin{tabular}{|c|c|c|c|c|c|c|}
\hline \multirow{2}{*}{$\begin{array}{c}\text { Exceedance } \\
\text { probability }\end{array}$} & \multirow{2}{*}{$\begin{array}{c}\text { Recurrence } \\
\text { interval } \\
\text { (years) }\end{array}$} & \multirow{2}{*}{$\begin{array}{c}\text { Maximum } \\
\text { instantaneous } \\
\text { discharge } \\
\left(\mathrm{m}^{3} / \mathrm{s}\right)\end{array}$} & \multicolumn{4}{|c|}{ Maximum daily mean discharge $\left(\mathrm{m}^{3} / \mathrm{s}\right)$} \\
\hline & & & $\begin{array}{l}\text { 3-day } \\
\text { period }\end{array}$ & $\begin{array}{l}\text { 7-day } \\
\text { period }\end{array}$ & $\begin{array}{l}\text { 15-day } \\
\text { period }\end{array}$ & $\begin{array}{l}\text { 30-day } \\
\text { period }\end{array}$ \\
\hline 0.99 & 1.01 & 235 & 233 & 229 & 191 & 158 \\
\hline 0.95 & 1.05 & 265 & 257 & 247 & 213 & 180 \\
\hline 0.90 & 1.11 & 280 & 269 & 256 & 225 & 192 \\
\hline 0.80 & 1.25 & 299 & 284 & 268 & 241 & 207 \\
\hline 0.50 & 2 & 334 & 311 & 292 & 271 & 238 \\
\hline 0.20 & 5 & 365 & 335 & 317 & 303 & 270 \\
\hline 0.10 & 10 & 380 & 346 & 330 & 320 & 287 \\
\hline 0.04 & 25 & 394 & 358 & 345 & 338 & 306 \\
\hline 0.02 & 50 & 403 & 364 & 355 & 350 & 318 \\
\hline 0.01 & 100 & 410 & 370 & 363 & 361 & 329 \\
\hline 0.005 & 200 & 416 & 375 & 372 & 371 & 340 \\
\hline 0.002 & 500 & 423 & ng & ng & ng & ng \\
\hline
\end{tabular}

\section{4-0.000-5S KUNDUZ RIVER AT PUL-I-KHUMRI, Continued}

Probability of occurrence of annual low discharges $\left[\mathrm{m}^{3} / \mathrm{s}\right.$, meters per second]

\begin{tabular}{|c|c|c|c|c|c|c|c|c|c|c|}
\hline \multirow{3}{*}{$\begin{array}{c}\text { Nonexceedance } \\
\text { probability }\end{array}$} & \multirow{3}{*}{$\begin{array}{c}\text { Recurrence } \\
\text { interval } \\
\text { (years) }\end{array}$} & \multicolumn{9}{|c|}{ Minimum daily mean discharge $\left(\mathrm{m}^{3} / \mathrm{s}\right)$} \\
\hline & & \multicolumn{9}{|c|}{ Number of consecutive days } \\
\hline & & 1 & 3 & 7 & 14 & 30 & 60 & 90 & 120 & 183 \\
\hline 0.05 & 20 & 18.8 & 18.9 & 19.4 & 19.5 & 19.6 & 19.8 & 19.7 & 20.1 & 22.0 \\
\hline 0.10 & 10 & 19.2 & 19.6 & 20.1 & 20.3 & 20.3 & 20.6 & 20.6 & 21.1 & 23.0 \\
\hline 0.20 & 5 & 19.7 & 20.4 & 21.0 & 21.4 & 21.4 & 21.7 & 21.9 & 22.5 & 24.4 \\
\hline 0.50 & 2 & 21.1 & 22.2 & 22.8 & 23.6 & 24.1 & 24.6 & 25.2 & 25.9 & 27.7 \\
\hline
\end{tabular}




\section{4-0.000-5S KUNDUZ RIVER AT PUL-I-KHUMRI, Continued}

Probability of occurrence of seasonal low discharges $\left[\mathrm{m}^{3} / \mathrm{s}\right.$, meters per second]

\begin{tabular}{|c|c|c|c|c|c|c|c|c|c|}
\hline \multirow{3}{*}{$\begin{array}{c}\text { Nonexceedance } \\
\text { probability }\end{array}$} & \multirow{3}{*}{$\begin{array}{c}\text { Recurrence } \\
\text { interval } \\
\text { (years) }\end{array}$} & \multicolumn{8}{|c|}{ Minimum daily mean discharge $\left(\mathrm{m}^{3} / \mathrm{s}\right)$} \\
\hline & & \multicolumn{8}{|c|}{ Number of consecutive days } \\
\hline & & 1 & 7 & 14 & 30 & 1 & 7 & 14 & 30 \\
\hline & & \multicolumn{4}{|c|}{ December-January-February } & \multicolumn{4}{|c|}{ March-April-May } \\
\hline 0.05 & 20 & 18.4 & 19.1 & 19.4 & 19.6 & 19.1 & 19.8 & 19.9 & 20.6 \\
\hline 0.10 & 10 & 19.1 & 20.0 & 20.4 & 20.6 & 19.4 & 20.3 & 20.5 & 21.2 \\
\hline 0.20 & 5 & 20.2 & 21.3 & 21.8 & 22.1 & 19.9 & 21.0 & 21.5 & 22.3 \\
\hline \multirow[t]{2}{*}{0.50} & 2 & 22.7 & 24.1 & 24.8 & 25.5 & 21.7 & 23.1 & 23.9 & 24.9 \\
\hline & & \multicolumn{4}{|c|}{ June-July-August } & \multicolumn{4}{|c|}{ September-October-November } \\
\hline 0.05 & 20 & 25.2 & 27.4 & 28.8 & 35.7 & 17.3 & 19.2 & 20.4 & 21.7 \\
\hline 0.10 & 10 & 27.4 & 30.1 & 31.8 & 38.9 & 18.9 & 20.8 & 22.0 & 23.3 \\
\hline 0.20 & 5 & 30.6 & 33.6 & 35.7 & 43.2 & 21.1 & 23.0 & 24.2 & 25.4 \\
\hline 0.50 & 2 & 37.8 & 41.6 & 44.6 & 53.5 & 26.5 & 28.2 & 29.3 & 30.3 \\
\hline
\end{tabular}




\section{4-0.000-5S KUNDUZ RIVER AT PUL-I-KHUMRI, Continued}

\begin{tabular}{|c|c|c|c|c|c|}
\hline \multicolumn{6}{|c|}{$\begin{array}{c}\text { Annual peak discharges } \\
\text { [m³/s, meters per second] }\end{array}$} \\
\hline \multicolumn{3}{|c|}{$\begin{array}{c}\text { Annual peak discharge, } \\
\text { by year }\end{array}$} & \multicolumn{3}{|c|}{$\begin{array}{l}\text { Annual peak discharge, } \\
\text { from highest to lowest }^{2}\end{array}$} \\
\hline $\begin{array}{l}\text { Water } \\
\text { year }\end{array}$ & Date & $\begin{array}{c}\text { Peak } \\
\text { discharge } \\
\left(\mathrm{m}^{3} / \mathrm{s}\right)\end{array}$ & $\begin{array}{l}\text { Water } \\
\text { year }\end{array}$ & Date & $\begin{array}{c}\text { Peak } \\
\text { discharge } \\
\left(\mathrm{m}^{3} / \mathrm{s}\right)\end{array}$ \\
\hline 1950 & June 15,1950 & $403^{1}$ & 1960 & July 3, 1960 & 382 \\
\hline 1951 & May 29, 1951 & $280^{1}$ & 1965 & June 22, 1965 & 368 \\
\hline 1952 & June 22, 1952 & $305^{1}$ & 1966 & June 18, 1966 & 358 \\
\hline 1953 & June 14,1953 & $373^{1}$ & 1962 & June 13, 1962 & 338 \\
\hline 1954 & June 4, 1954 & $406^{1}$ & 1963 & June 8, 1963 & 320 \\
\hline 1955 & June 12, 1955 & $727^{1}$ & 1961 & June 3, 1961 & 319 \\
\hline 1956 & July 27, 1956 & $208^{1}$ & 1967 & June 14, 1967 & 300 \\
\hline 1957 & June 12, 1957 & $416^{1}$ & 1964 & June 28, 1964 & 264 \\
\hline 1958 & June 18,1958 & $408^{1}$ & & & \\
\hline 1959 & June 4, 1959 & $358^{1}$ & & & \\
\hline 1960 & July 3, 1960 & 382 & & & \\
\hline 1961 & June 3, 1961 & 319 & & & \\
\hline 1962 & June 13, 1962 & 338 & & & \\
\hline 1963 & June 8, 1963 & 320 & & & \\
\hline 1964 & June 28, 1964 & 264 & & & \\
\hline 1965 & June 22, 1965 & 368 & & & \\
\hline 1966 & June 18,1966 & 358 & & & \\
\hline 1967 & June 14,1967 & 300 & & & \\
\hline
\end{tabular}

${ }^{1}$ Published as a maximum daily discharge.

${ }^{2}$ Only instantaneous annual peaks shown. 


\section{4-0.000-5S KUNDUZ RIVER AT PUL-I-KHUMRI, Continued}

Monthly and annual mean discharges, in cubic meters per second $[--$, no data]

\begin{tabular}{|c|c|c|c|c|c|c|c|c|c|c|c|c|c|}
\hline \multirow{2}{*}{$\begin{array}{c}\text { Water } \\
\text { year }\end{array}$} & \multicolumn{12}{|c|}{ Monthly mean discharge } & \multirow{2}{*}{$\begin{array}{c}\text { Annual } \\
\text { discharge }\end{array}$} \\
\hline & October & November & December & January & February & March & April & May & June & July & August & September & \\
\hline 1960 & 33.1 & 31.1 & 27.2 & 25.1 & 26.0 & 24.4 & 35.5 & 95.4 & 241 & 229 & 81.8 & 41.1 & 74.3 \\
\hline 1961 & 36.6 & 31.3 & 29.2 & 26.4 & 24.8 & 25.5 & 26.5 & 82.6 & 191 & 99.3 & 41.1 & 30.0 & 53.7 \\
\hline 1962 & 26.6 & 26.8 & 24.0 & 23.3 & 24.0 & 27.5 & 48.0 & 110 & 216 & 115 & 49.3 & 34.5 & 60.5 \\
\hline 1963 & 32.3 & 28.8 & 26.2 & 25.2 & 24.7 & 24.7 & 29.3 & 83.1 & 261 & 97.8 & 40.2 & 28.8 & 58.5 \\
\hline 1964 & 30.7 & 26.1 & 22.6 & 20.0 & 20.6 & 20.3 & 29.3 & 67.5 & 153 & 127 & 45.7 & 29.8 & 49.4 \\
\hline 1965 & 27.2 & 25.3 & 24.3 & 22.2 & 23.2 & 23.0 & 36.8 & 83.9 & 261 & 217 & 71.7 & 40.8 & 71.5 \\
\hline 1966 & 34.9 & 35.0 & 34.5 & 36.6 & 35.5 & 34.7 & 53.9 & 139 & 232 & 97.2 & 57.9 & 44.2 & 69.7 \\
\hline 1967 & 43.1 & 39.0 & 36.6 & 34.0 & 30.5 & 27.9 & 35.8 & 99.5 & 240 & 159 & 65.7 & 42.4 & 71.2 \\
\hline 1968 & 46.5 & 43.6 & 39.9 & 34.8 & 31.2 & -- & -- & -- & -- & -- & -- & -- & -- \\
\hline
\end{tabular}




\section{4-0.000-6M KUNDUZ RIVER AT PUL-I-KONDA SANG}

(U.S. Geological Survey identification number: $\mathbf{3 5 3 6 0 0 0 6 8 3 6 0 0 0 0 )}$

LOCATION: Lat $35^{\circ} 36^{\prime} \mathrm{N}$. , long $68^{\circ} 36^{\prime} \mathrm{E}$.

DRAINAGE AREA: $12,610 \mathrm{~km}^{2}$.

ELEVATION: 899 meters above mean sea level.

PERIOD OF RECORD: October 1, 1967 to September 30, 1978.

GAGE: Water-stage recorder.

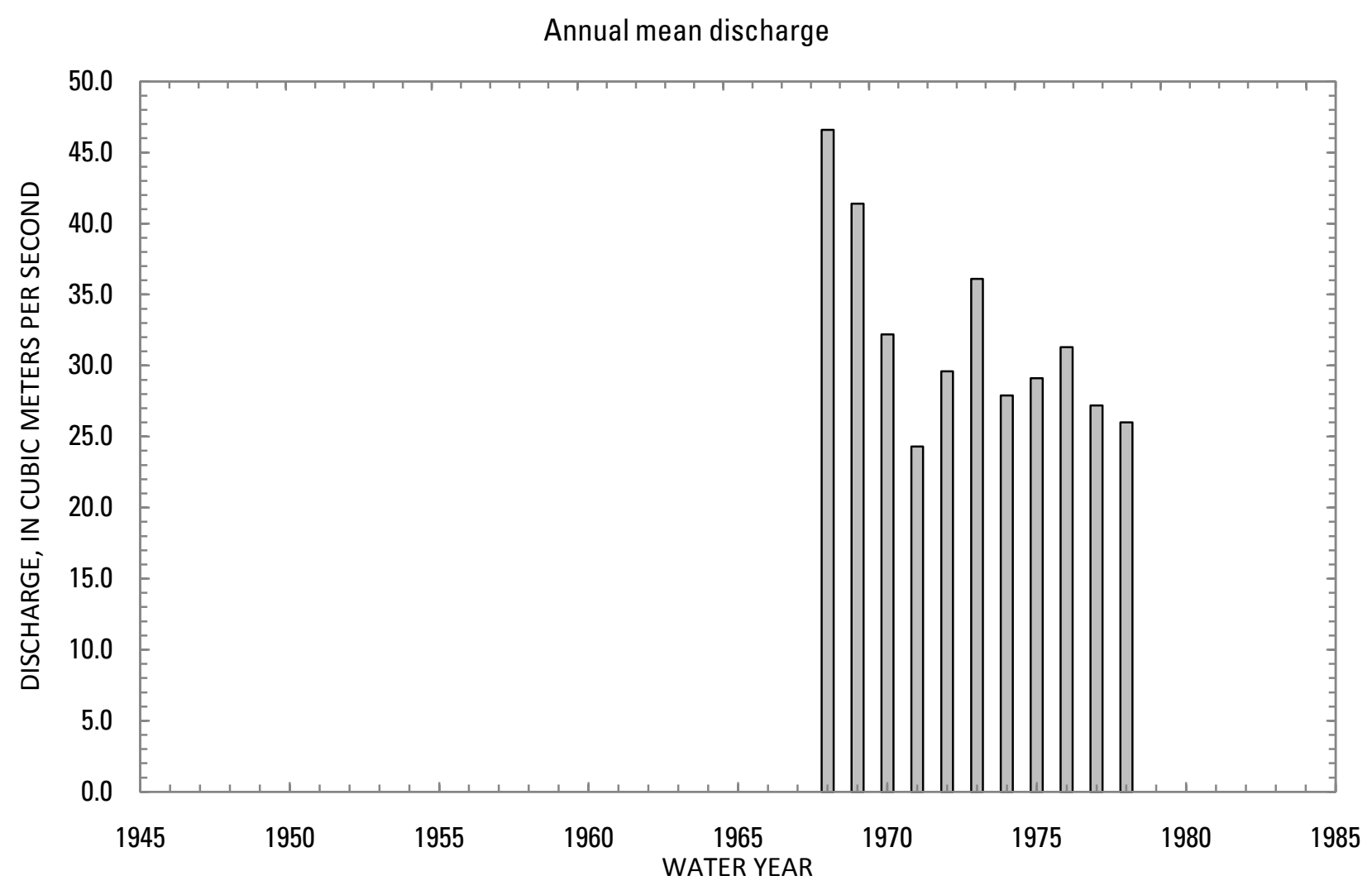


14-0.000-6M KUNDUZ RIVER AT PUL-I-KONDA SANG, Continued

Statistics of monthly and annual mean discharges

$\left[\mathrm{m}^{3} / \mathrm{s}\right.$, cubic meters per second]

\begin{tabular}{|c|c|c|c|c|c|c|c|c|}
\hline \multirow[b]{2}{*}{ Month } & \multicolumn{2}{|c|}{ Maximum } & \multicolumn{2}{|c|}{ Minimum } & \multicolumn{4}{|c|}{ Mean } \\
\hline & $\begin{array}{c}\text { Discharge } \\
\left(\mathrm{m}^{3} / \mathrm{s}\right)\end{array}$ & $\begin{array}{l}\text { Water year } \\
\text { of } \\
\text { occurrence }\end{array}$ & $\begin{array}{c}\text { Discharge } \\
\left(\mathrm{m}^{3} / \mathrm{s}\right)\end{array}$ & $\begin{array}{c}\text { Water year } \\
\text { of } \\
\text { occurrence }\end{array}$ & $\begin{array}{c}\text { Discharge } \\
\left(\mathrm{m}^{3} / \mathrm{s}\right)\end{array}$ & $\begin{array}{c}\text { Standard } \\
\text { deviation } \\
\left(\mathrm{m}^{3} / \mathrm{s}\right)\end{array}$ & $\begin{array}{c}\text { Coefficient } \\
\text { of } \\
\text { variation }\end{array}$ & $\begin{array}{c}\text { Percentage } \\
\text { of annual } \\
\text { discharge }\end{array}$ \\
\hline October & 29.6 & 1970 & 14.2 & 1978 & 22.2 & 4.64 & 0.21 & 5.78 \\
\hline November & 28.6 & 1970 & 14.5 & 1978 & 21.3 & 4.20 & 0.20 & 5.56 \\
\hline December & 25.9 & 1970 & 11.5 & 1978 & 19.4 & 4.00 & 0.21 & 5.06 \\
\hline January & 23.2 & 1970 & 13.8 & 1978 & 18.1 & 2.94 & 0.16 & 4.73 \\
\hline February & 23.0 & 1970 & 14.0 & 1976 & 17.6 & 2.87 & 0.16 & 4.58 \\
\hline March & 22.8 & 1974 & 14.8 & 1978 & 18.1 & 2.71 & 0.15 & 4.71 \\
\hline April & 29.3 & 1968 & 16.7 & 1971 & 21.3 & 4.52 & 0.21 & 5.55 \\
\hline May & 64.3 & 1971 & 35.0 & 1972 & 51.4 & 11.7 & 0.23 & 13.4 \\
\hline June & 166 & 1968 & 41.8 & 1971 & 92.6 & 33.4 & 0.36 & 24.2 \\
\hline July & 98.9 & 1968 & 17.8 & 1971 & 52.8 & 23.6 & 0.45 & 13.8 \\
\hline August & 42.9 & 1968 & 16.2 & 1977 & 26.6 & 8.56 & 0.32 & 6.92 \\
\hline September & 33.3 & 1968 & 15.1 & 1977 & 22.2 & 6.06 & 0.27 & 5.78 \\
\hline Annual & 46.6 & 1968 & 26.0 & 1978 & 32.0 & 6.84 & 0.21 & 100 \\
\hline
\end{tabular}


14-0.000-6M KUNDUZ RIVER AT PUL-I-KONDA SANG, Continued

Monthly and annual flow duration, in cubic meters per second

\begin{tabular}{|c|c|c|c|c|c|c|c|c|c|c|c|c|c|}
\hline \multirow{2}{*}{$\begin{array}{l}\text { Percentage } \\
\text { of days } \\
\text { discharge } \\
\text { equaled or } \\
\text { exceeded }\end{array}$} & \multicolumn{12}{|c|}{ Month } & \multirow{2}{*}{ Annual } \\
\hline & October & November & December & January & February & March & April & May & June & July & August & September & \\
\hline 95 & 14.3 & 13.0 & 11.5 & 13.8 & 13.8 & 14.4 & 14.1 & 16.0 & 40.8 & 17.6 & 16.0 & 15.0 & 14.5 \\
\hline 90 & 16.7 & 16.7 & 13.8 & 14.4 & 14.0 & 14.8 & 14.8 & 20.0 & 52.7 & 20.5 & 16.6 & 15.2 & 15.2 \\
\hline 85 & 17.7 & 17.2 & 15.9 & 15.2 & 14.5 & 15.2 & 15.2 & 22.4 & 59.2 & 25.0 & 17.3 & 16.0 & 16.0 \\
\hline 80 & 18.2 & 18.1 & 16.6 & 15.7 & 14.9 & 15.4 & 16.0 & 26.8 & 65.6 & 28.9 & 18.7 & 17.0 & 16.6 \\
\hline 75 & 19.1 & 19.1 & 17.0 & 15.8 & 15.3 & 15.9 & 16.5 & 30.9 & 70.2 & 31.5 & 21.1 & 17.3 & 17.3 \\
\hline 70 & 19.3 & 19.5 & 17.3 & 16.1 & 15.8 & 16.1 & 17.0 & 34.6 & 73.7 & 34.4 & 21.9 & 18.1 & 18.1 \\
\hline 65 & 19.6 & 19.7 & 18.0 & 16.7 & 16.0 & 16.6 & 17.4 & 38.9 & 76.7 & 37.6 & 22.5 & 18.5 & 19.0 \\
\hline 60 & 19.8 & 19.9 & 18.3 & 16.9 & 16.6 & 16.8 & 18.2 & 40.7 & 80.2 & 40.9 & 23.0 & 19.3 & 19.9 \\
\hline 55 & 20.4 & 20.1 & 19.1 & 17.2 & 16.8 & 17.0 & 19.4 & 43.2 & 84.0 & 43.9 & 23.9 & 19.9 & 20.7 \\
\hline 50 & 22.8 & 20.4 & 19.3 & 17.6 & 17.0 & 17.3 & 20.0 & 47.0 & 87.9 & 46.5 & 24.9 & 20.4 & 21.7 \\
\hline 45 & 23.2 & 20.8 & 19.7 & 18.2 & 17.2 & 18.1 & 20.5 & 49.1 & 91.6 & 50.3 & 25.7 & 23.0 & 22.8 \\
\hline 40 & 23.6 & 22.3 & 20.1 & 18.7 & 17.4 & 18.8 & 21.1 & 54.0 & 95.1 & 55.3 & 26.3 & 23.6 & 24.0 \\
\hline 35 & 24.5 & 22.8 & 20.5 & 19.0 & 18.2 & 19.2 & 22.0 & 58.4 & 101 & 59.8 & 27.3 & 24.1 & 25.2 \\
\hline 30 & 24.7 & 23.4 & 21.5 & 19.6 & 19.1 & 19.6 & 23.8 & 62.7 & 106 & 63.8 & 28.1 & 24.5 & 27.3 \\
\hline 25 & 25.2 & 24.2 & 22.1 & 20.2 & 19.8 & 20.4 & 24.7 & 69.0 & 110 & 67.4 & 30.4 & 25.0 & 30.8 \\
\hline 20 & 25.8 & 25.5 & 22.9 & 21.0 & 20.7 & 20.8 & 25.7 & 76.0 & 119 & 73.1 & 33.0 & 25.5 & 38.2 \\
\hline 15 & 26.8 & 27.2 & 24.6 & 22.0 & 21.9 & 21.1 & 28.5 & 80.9 & 127 & 83.0 & 35.2 & 31.2 & 52.0 \\
\hline 10 & 28.5 & 28.1 & 25.3 & 22.7 & 22.2 & 22.0 & 30.2 & 88.5 & 144 & 91.9 & 39.7 & 32.0 & 70.6 \\
\hline 5 & 29.4 & 29.1 & 25.9 & 22.9 & 22.6 & 22.4 & 33.0 & 101 & 172 & 109 & 47.4 & 33.6 & 93.0 \\
\hline
\end{tabular}




\section{4-0.000-6M KUNDUZ RIVER AT PUL-I-KONDA SANG, Continued}

Probability of occurrence of annual high discharges

[ $\mathrm{m}^{3} / \mathrm{s}$, cubic meters per second; $\mathrm{ng}$, not given]

\begin{tabular}{|c|c|c|c|c|c|c|}
\hline \multirow{2}{*}{$\begin{array}{c}\text { Exceedance } \\
\text { probability }\end{array}$} & \multirow{2}{*}{$\begin{array}{c}\text { Recurrence } \\
\text { interval } \\
\text { (years) }\end{array}$} & \multirow{2}{*}{$\begin{array}{l}\text { Maximum } \\
\text { instantaneous } \\
\text { discharge } \\
\left(\mathrm{m}^{3} / \mathrm{s}\right)\end{array}$} & \multicolumn{4}{|c|}{ Maximum daily mean discharge $\left(\mathrm{m}^{3} / \mathrm{s}\right)$} \\
\hline & & & $\begin{array}{l}\text { 3-day } \\
\text { period }\end{array}$ & $\begin{array}{l}\text { 7-day } \\
\text { period }\end{array}$ & $\begin{array}{l}\text { 15-day } \\
\text { period }\end{array}$ & $\begin{array}{l}\text { 30-day } \\
\text { period }\end{array}$ \\
\hline 0.99 & 1.01 & 97.0 & 81.3 & 76.4 & 73.5 & 66.8 \\
\hline 0.95 & 1.05 & 104 & 86.9 & 81.6 & 77.6 & 70.5 \\
\hline 0.90 & 1.11 & 109 & 91.1 & 85.5 & 80.9 & 73.4 \\
\hline 0.80 & 1.25 & 117 & 97.5 & 91.6 & 86.2 & 78.1 \\
\hline 0.50 & 2 & 138 & 116 & 109 & 102 & 92.1 \\
\hline 0.20 & 5 & 170 & 145 & 138 & 130 & 117 \\
\hline 0.10 & 10 & 194 & 168 & 160 & 152 & 136 \\
\hline 0.04 & 25 & 227 & 200 & 192 & 184 & 164 \\
\hline 0.02 & 50 & 254 & 226 & 218 & 211 & 188 \\
\hline 0.01 & 100 & 282 & 255 & 247 & 241 & 214 \\
\hline 0.005 & 200 & 313 & 286 & 279 & 274 & 243 \\
\hline 0.002 & 500 & 357 & $\mathrm{ng}$ & $\mathrm{ng}$ & $\mathrm{ng}$ & ng \\
\hline
\end{tabular}

\section{4-0.000-6M KUNDUZ RIVER AT PUL-I-KONDA SANG, Continued}

Probability of occurrence of annual low discharges $\left[\mathrm{m}^{3} / \mathrm{s}\right.$, meters per second]

\begin{tabular}{|c|c|c|c|c|c|c|c|c|c|c|}
\hline \multirow{3}{*}{$\begin{array}{c}\text { Nonexceedance } \\
\text { probability }\end{array}$} & \multirow{3}{*}{$\begin{array}{c}\text { Recurrence } \\
\text { interval } \\
\text { (years) }\end{array}$} & \multicolumn{9}{|c|}{ Minimum daily mean discharge $\left(\mathrm{m}^{3} / \mathrm{s}\right)$} \\
\hline & & \multicolumn{9}{|c|}{ Number of consecutive days } \\
\hline & & 1 & 3 & 7 & 14 & 30 & 60 & 90 & 120 & 183 \\
\hline 0.05 & 20 & 9.39 & 10.4 & 10.7 & 11.4 & 11.8 & 12.5 & 13.1 & 13.6 & 14.2 \\
\hline 0.10 & 10 & 10.1 & 10.9 & 11.2 & 12.0 & 12.7 & 13.4 & 13.9 & 14.4 & 15.1 \\
\hline 0.20 & 5 & 11.0 & 11.6 & 12.0 & 12.8 & 13.8 & 14.5 & 15.0 & 15.5 & 16.3 \\
\hline 0.50 & 2 & 13.0 & 13.3 & 13.9 & 14.8 & 16.1 & 16.9 & 17.3 & 17.7 & 19.0 \\
\hline
\end{tabular}




\section{4-0.000-6M KUNDUZ RIVER AT PUL-I-KONDA SANG, Continued}

Probability of occurrence of seasonal low discharges

$\left[\mathrm{m}^{3} / \mathrm{s}\right.$, meters per second]

\begin{tabular}{|c|c|c|c|c|c|c|c|c|c|}
\hline \multirow{3}{*}{$\begin{array}{c}\text { Nonexceedance } \\
\text { probability }\end{array}$} & \multirow{3}{*}{$\begin{array}{c}\text { Recurrence } \\
\text { interval } \\
\text { (years) }\end{array}$} & \multicolumn{8}{|c|}{ Minimum daily mean discharge $\left(\mathrm{m}^{3} / \mathrm{s}\right)$} \\
\hline & & \multicolumn{8}{|c|}{ Number of consecutive days } \\
\hline & & 1 & 7 & 14 & 30 & 1 & 7 & 14 & 30 \\
\hline & & \multicolumn{4}{|c|}{ December-January-February } & \multicolumn{4}{|c|}{ March-April-May } \\
\hline 0.05 & 20 & 10.9 & 11.3 & 11.6 & 12.0 & 9.80 & 11.6 & 13.0 & 13.9 \\
\hline 0.10 & 10 & 11.7 & 12.1 & 12.4 & 13.0 & 10.9 & 12.4 & 13.6 & 14.5 \\
\hline 0.20 & 5 & 12.8 & 13.1 & 13.5 & 14.2 & 12.2 & 13.5 & 14.5 & 15.3 \\
\hline \multirow[t]{2}{*}{0.50} & 2 & 15.1 & 15.5 & 15.9 & 16.7 & 14.7 & 15.7 & 16.3 & 17.1 \\
\hline & & \multicolumn{4}{|c|}{ June-July-August } & \multicolumn{4}{|c|}{ September-October-November } \\
\hline 0.05 & 20 & 12.9 & 13.8 & 14.5 & 15.7 & 12.2 & 12.4 & 12.6 & 14.4 \\
\hline 0.10 & 10 & 14.5 & 15.3 & 15.9 & 17.2 & 13.4 & 13.8 & 14.0 & 15.4 \\
\hline 0.20 & 5 & 16.7 & 17.3 & 18.0 & 19.3 & 15.0 & 15.5 & 15.7 & 16.8 \\
\hline 0.50 & 2 & 21.6 & 21.9 & 22.7 & 24.7 & 18.3 & 18.9 & 19.4 & 20.0 \\
\hline
\end{tabular}

14-0.000-6M KUNDUZ RIVER AT PUL-I-KONDA SANG, Continued

Annual peak discharges

$\left[\mathrm{m}^{3} / \mathrm{s}\right.$, meters per second]

\begin{tabular}{|c|c|c|c|c|c|}
\hline \multicolumn{3}{|c|}{$\begin{array}{c}\text { Annual peak discharge, } \\
\text { by year }\end{array}$} & \multicolumn{3}{|c|}{$\begin{array}{l}\text { Annual peak discharge, } \\
\text { from highest to lowest }\end{array}$} \\
\hline $\begin{array}{l}\text { Water } \\
\text { year }\end{array}$ & Date & $\begin{array}{c}\text { Peak } \\
\text { discharge } \\
\left(\mathrm{m}^{3} / \mathrm{s}\right)\end{array}$ & $\begin{array}{l}\text { Water } \\
\text { year }\end{array}$ & Date & $\begin{array}{c}\text { Peak } \\
\text { discharge } \\
\left(\mathrm{m}^{3} / \mathrm{s}\right)\end{array}$ \\
\hline 1968 & June 10, 1968 & 236 & 1968 & June 10, 1968 & 236 \\
\hline 1969 & June 17, 1969 & 150 & 1973 & June 4, 1973 & 180 \\
\hline 1970 & May 22, 1970 & 133 & 1972 & June 22, 1972 & 154 \\
\hline 1971 & May 20, 1971 & 126 & 1976 & June 3, 1976 & 154 \\
\hline 1972 & June 22, 1972 & 154 & 1969 & June 17, 1969 & 150 \\
\hline 1973 & June 4, 1973 & 180 & 1975 & June 17,1975 & 142 \\
\hline 1974 & June 4, 1974 & 114 & 1970 & May 22, 1970 & 133 \\
\hline 1975 & June 17, 1975 & 142 & 1971 & May 20, 1971 & 126 \\
\hline 1976 & June 3, 1976 & 154 & 1974 & June 4, 1974 & 114 \\
\hline 1977 & June 23, 1977 & 113 & 1977 & June 23, 1977 & 113 \\
\hline 1978 & June 7, 1978 & 106 & 1978 & June 7, 1978 & 106 \\
\hline
\end{tabular}




\section{4-0.000-6M KUNDUZ RIVER AT PUL-I-KONDA SANG, Continued}

Monthly and annual mean discharges, in cubic meters per second

[Data may not be rounded in accordance with U.S. Geological Survey publication standards]

\begin{tabular}{|c|c|c|c|c|c|c|c|c|c|c|c|c|c|}
\hline \multirow{2}{*}{$\begin{array}{l}\text { Water } \\
\text { year }\end{array}$} & \multicolumn{12}{|c|}{ Monthly mean discharge } & \multirow{2}{*}{$\begin{array}{c}\text { Annual } \\
\text { discharge }\end{array}$} \\
\hline & October & November & December & January & February & March & April & May & June & July & August & September & \\
\hline 1968 & 25.8 & 24.0 & 22.0 & 19.9 & 16.8 & 17.0 & 29.3 & 63.1 & 166 & 98.9 & 42.9 & 33.3 & 46.6 \\
\hline 1969 & 27.5 & 27.5 & 25.0 & 22.2 & 21.5 & 20.5 & 29.2 & 54.3 & 114 & 82.1 & 40.4 & 31.7 & 41.4 \\
\hline 1970 & 29.6 & 28.6 & 25.9 & 23.2 & 23.0 & 21.6 & 23.4 & 63.1 & 60.9 & 34.8 & 26.6 & 24.5 & 32.2 \\
\hline 1971 & 24.1 & 20.8 & 19.3 & 16.8 & 19.0 & 16.8 & 16.7 & 64.3 & 41.8 & 17.8 & 16.8 & 16.8 & 24.3 \\
\hline 1972 & 17.5 & 16.9 & 16.3 & 15.1 & 15.9 & 17.5 & 17.2 & 35.0 & 95.9 & 62.7 & 26.5 & 19.2 & 29.6 \\
\hline 1973 & 19.9 & 18.8 & 17.5 & 16.5 & 16.7 & 20.0 & 21.9 & 63.3 & 125 & 59.1 & 29.8 & 24.3 & 36.1 \\
\hline 1974 & 23.2 & 20.5 & 18.2 & 18.8 & 16.9 & 22.8 & 21.5 & 41.1 & 74.8 & 39.0 & 20.3 & 17.8 & 27.9 \\
\hline 1975 & 18.8 & 19.7 & 18.6 & 16.7 & 15.7 & 15.1 & 18.2 & 35.6 & 91.9 & 54.7 & 23.4 & 20.7 & 29.1 \\
\hline 1976 & 19.2 & 20.5 & 18.5 & 16.4 & 14.0 & 16.0 & 18.5 & 53.3 & 87.9 & 61.3 & 25.8 & 23.9 & 31.3 \\
\hline 1977 & 24.0 & 22.6 & 20.5 & 19.9 & 19.3 & 16.6 & 17.1 & 39.6 & 82.7 & 32.6 & 16.2 & 15.1 & 27.2 \\
\hline 1978 & 14.2 & 14.5 & 11.5 & 13.8 & 14.2 & 14.8 & 20.9 & 52.1 & 77.9 & 37.8 & 23.3 & 16.6 & 26.0 \\
\hline
\end{tabular}




\section{4-0.000-8M KUNDUZ RIVER AT DASHT-I-SAFED}

\section{(U.S. Geological Survey identification number: 351800067550000)}

LOCATION: Lat $35^{\circ} 18^{\prime} \mathrm{N}$., long $67^{\circ} 55^{\prime} \mathrm{E}$.

DRAINAGE AREA: $3,795 \mathrm{~km}^{2}$.

ELEVATION: 1,588 meters above mean sea level.

PERIOD OF RECORD: October 12, 1967 to May 5, 1975 and August 13, 1977 to September 30, 1978.

GAGE: Water-stage recorder. Site damaged by floods on May 5, 1975 and re-established $10 \mathrm{~km}$ downstream (Kunduz River at Doab) on August 13, 1977.

Annual mean discharge

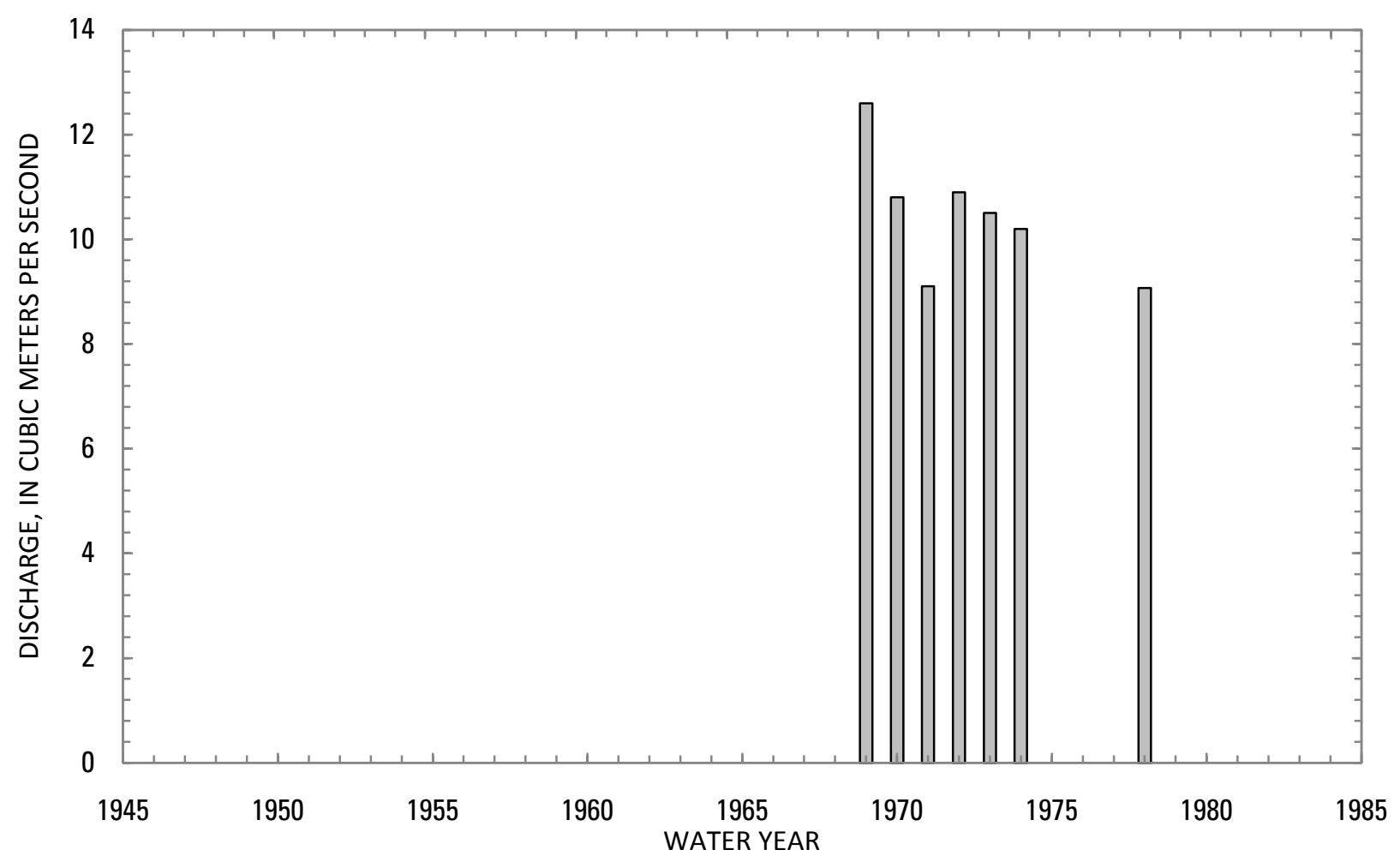




\section{4-0.000-8M KUNDUZ RIVER AT DASHT-I-SAFED, Continued}

Statistics of monthly and annual mean discharges $\left[\mathrm{m}^{3} / \mathrm{s}\right.$, cubic meters per second]

\begin{tabular}{|c|c|c|c|c|c|c|c|c|}
\hline \multirow[b]{2}{*}{ Month } & \multicolumn{2}{|c|}{ Maximum } & \multicolumn{2}{|c|}{ Minimum } & \multicolumn{4}{|c|}{ Mean } \\
\hline & $\begin{array}{c}\text { Discharge } \\
\left(\mathrm{m}^{3} / \mathrm{s}\right)\end{array}$ & $\begin{array}{c}\text { Water year } \\
\text { of } \\
\text { occurrence }\end{array}$ & $\begin{array}{c}\text { Discharge } \\
\left(\mathrm{m}^{3} / \mathrm{s}\right)\end{array}$ & $\begin{array}{c}\text { Water year } \\
\text { of } \\
\text { occurrence }\end{array}$ & $\begin{array}{c}\text { Discharge } \\
\left(\mathrm{m}^{3} / \mathrm{s}\right)\end{array}$ & $\begin{array}{c}\text { Standard } \\
\text { deviation } \\
\left(\mathrm{m}^{3} / \mathrm{s}\right)\end{array}$ & $\begin{array}{c}\text { Coefficient } \\
\text { of } \\
\text { variation }\end{array}$ & $\begin{array}{c}\text { Percentage } \\
\text { of annual } \\
\text { discharge }\end{array}$ \\
\hline October & 13.0 & 1970 & 8.78 & 1971 & 10.6 & 1.67 & 0.16 & 8.33 \\
\hline November & 12.8 & 1970 & 7.91 & 1971 & 9.96 & 1.38 & 0.14 & 7.85 \\
\hline December & 13.5 & 1970 & 7.64 & 1971 & 10.5 & 1.81 & 0.17 & 8.31 \\
\hline January & 13.7 & 1969 & 9.37 & 1972 & 11.0 & 1.48 & 0.13 & 8.65 \\
\hline February & 12.9 & 1969 & 8.22 & 1978 & 10.2 & 1.46 & 0.14 & 8.02 \\
\hline March & 12.5 & 1969 & 7.07 & 1978 & 9.51 & 1.54 & 0.16 & 7.49 \\
\hline April & 12.4 & 1969 & 7.64 & 1978 & 9.62 & 1.43 & 0.15 & 7.58 \\
\hline May & 14.2 & 1972 & 8.13 & 1974 & 10.4 & 1.93 & 0.19 & 8.21 \\
\hline June & 21.7 & 1972 & 7.32 & 1971 & 13.4 & 5.52 & 0.41 & 10.5 \\
\hline July & 13.8 & 1968 & 8.31 & 1970 & 11.2 & 1.90 & 0.17 & 8.80 \\
\hline August & 12.7 & 1968 & 8.82 & 1970 & 10.5 & 1.22 & 0.12 & 8.28 \\
\hline September & 12.8 & 1969 & 8.49 & 1972 & 10.1 & 1.66 & 0.16 & 7.96 \\
\hline Annual & 12.6 & 1969 & 9.07 & 1978 & 10.5 & 1.21 & 0.12 & 100 \\
\hline
\end{tabular}


14-0.000-8M KUNDUZ RIVER AT DASHT-I-SAFED, Continued

Monthly and annual flow duration, in cubic meters per second

[ng, not given]

\begin{tabular}{|c|c|c|c|c|c|c|c|c|c|c|c|c|c|}
\hline \multirow{2}{*}{$\begin{array}{l}\text { Percentage } \\
\text { of days } \\
\text { discharge } \\
\text { equaled or } \\
\text { exceeded }\end{array}$} & \multicolumn{12}{|c|}{ Month } & \multirow{2}{*}{ Annua } \\
\hline & October & November & December & January & February & March & April & May & June & July & August & September & \\
\hline 95 & 8.35 & 7.61 & 7.26 & 9.22 & 7.94 & 7.01 & 7.16 & 7.92 & 7.19 & 8.08 & 8.41 & 7.67 & 7.56 \\
\hline 90 & 8.73 & 7.69 & 7.74 & 9.26 & 8.29 & 7.14 & 7.62 & 8.13 & 7.37 & 8.52 & 8.89 & 8.07 & 7.95 \\
\hline 85 & 8.80 & 8.41 & 8.86 & 9.30 & 8.40 & 7.55 & 8.11 & 8.34 & 8.05 & 8.88 & 9.05 & 8.35 & 8.32 \\
\hline 80 & 8.87 & 8.77 & 8.98 & 9.34 & 8.49 & 8.46 & 8.32 & 8.55 & 8.57 & 9.29 & 9.13 & 8.47 & 8.68 \\
\hline 75 & 9.25 & 8.92 & 9.61 & 10.2 & 8.57 & 8.84 & 8.52 & 8.91 & 9.00 & 9.44 & 9.21 & 8.90 & 9.03 \\
\hline 70 & 9.30 & 9.10 & 9.73 & 10.3 & 9.67 & 9.06 & 8.92 & 9.23 & 9.65 & 9.58 & 9.29 & 9.14 & 9.28 \\
\hline 65 & 9.35 & 9.64 & 9.90 & 10.4 & 10.1 & 9.20 & 9.17 & 9.39 & 9.88 & 10.2 & 9.75 & 9.19 & 9.45 \\
\hline 60 & 10.1 & 9.70 & 10.2 & 10.6 & 10.2 & 9.32 & 9.39 & 9.50 & 10.1 & 10.9 & 10.1 & 9.24 & 9.61 \\
\hline 55 & 10.1 & 9.75 & 10.3 & 10.6 & 10.2 & 9.48 & 9.54 & 9.61 & 10.5 & 11.0 & 10.4 & 9.29 & 9.78 \\
\hline 50 & 10.2 & 9.99 & 10.7 & 10.7 & 10.4 & 9.59 & 9.64 & 9.72 & 10.9 & 11.3 & 10.7 & 9.70 & 10.1 \\
\hline 45 & 10.6 & 10.1 & 10.9 & 10.7 & 10.5 & 9.74 & 9.74 & 9.84 & 11.2 & 11.6 & 10.9 & 9.82 & 10.4 \\
\hline 40 & 11.0 & 10.2 & 11.0 & 10.7 & 10.6 & 9.92 & 9.89 & 9.97 & 12.2 & 11.7 & 11.0 & 10.2 & 10.7 \\
\hline 35 & 11.2 & 10.4 & 11.1 & 11.0 & 10.7 & 9.97 & 10.1 & 10.1 & 12.6 & 12.0 & 11.1 & 11.0 & 10.9 \\
\hline 30 & 11.9 & 10.6 & 11.4 & 11.5 & 10.8 & 10.0 & 10.4 & 10.5 & 14.8 & 12.1 & 11.2 & 11.5 & 11.1 \\
\hline 25 & 12.3 & 10.7 & 11.6 & 12.0 & 11.1 & 10.1 & 10.5 & 10.8 & 15.5 & 12.9 & 11.6 & 11.6 & 11.6 \\
\hline 20 & 12.5 & 11.0 & 12.1 & 12.3 & 11.2 & 10.2 & 10.7 & 11.1 & 17.1 & 13.4 & 11.9 & 11.9 & 12.1 \\
\hline 15 & 12.6 & 11.1 & 12.5 & 13.0 & 11.5 & 10.6 & 10.8 & 11.7 & 19.4 & 13.7 & 12.1 & 12.1 & 12.4 \\
\hline 10 & 12.9 & 12.1 & 13.0 & 13.4 & 12.6 & 12.2 & 11.3 & 12.6 & 21.6 & ng & 12.4 & 12.6 & 12.9 \\
\hline 5 & 13.1 & 13.0 & 13.5 & $\mathrm{ng}$ & 12.8 & $\mathrm{ng}$ & 11.9 & 13.4 & 26.7 & $\mathrm{ng}$ & $\mathrm{ng}$ & $\mathrm{ng}$ & 13.5 \\
\hline
\end{tabular}




\section{4-0.000-8M KUNDUZ RIVER AT DASHT-I-SAFED, Continued}

Probability of occurrence of annual high discharges

[m $\mathrm{m}^{3} / \mathrm{s}$, cubic meters per second; ng, not given]

\begin{tabular}{|c|c|c|c|c|c|c|}
\hline \multirow{2}{*}{$\begin{array}{c}\text { Exceedance } \\
\text { probability }\end{array}$} & \multirow{2}{*}{$\begin{array}{c}\text { Recurrence } \\
\text { interval } \\
\text { (years) }\end{array}$} & \multirow{2}{*}{$\begin{array}{c}\text { Maximum } \\
\text { instantaneous } \\
\text { discharge } \\
\left(\mathrm{m}^{3} / \mathrm{s}\right)\end{array}$} & \multicolumn{4}{|c|}{ Maximum daily mean discharge $\left(\mathrm{m}^{3} / \mathrm{s}\right)$} \\
\hline & & & $\begin{array}{l}\text { 3-day } \\
\text { period }\end{array}$ & $\begin{array}{l}\text { 7-day } \\
\text { period }\end{array}$ & $\begin{array}{l}\text { 15-day } \\
\text { period }\end{array}$ & $\begin{array}{l}\text { 30-day } \\
\text { period }\end{array}$ \\
\hline 0.99 & 1.01 & 10.6 & 10.6 & 10.5 & 10.3 & 9.90 \\
\hline 0.95 & 1.05 & 12.0 & 10.8 & 10.7 & 10.5 & 10.1 \\
\hline 0.90 & 1.11 & 13.0 & 10.9 & 10.9 & 10.7 & 10.4 \\
\hline 0.80 & 1.25 & 14.6 & 11.4 & 11.4 & 11.2 & 10.9 \\
\hline 0.50 & 2 & 19.6 & 13.8 & 13.6 & 13.4 & 12.8 \\
\hline 0.20 & 5 & 29.0 & 20.8 & 19.5 & 18.8 & 17.1 \\
\hline 0.10 & 10 & 37.1 & 28.7 & 25.6 & 24.3 & 21.0 \\
\hline 0.04 & 25 & 49.8 & 44.2 & 36.8 & 34.2 & 27.5 \\
\hline 0.02 & 50 & 61.5 & 61.4 & 48.5 & 44.3 & 33.7 \\
\hline 0.01 & 100 & 75.2 & 85.5 & 63.8 & 57.3 & 41.2 \\
\hline 0.005 & 200 & 91.5 & 119 & 84.1 & 74.1 & 50.3 \\
\hline 0.002 & 500 & 118 & $\mathrm{ng}$ & $\mathrm{ng}$ & ng & ng \\
\hline
\end{tabular}

'Less than 10 years of data used.

\section{4-0.000-8M KUNDUZ RIVER AT DASHT-I-SAFED, Continued}

Probability of occurrence of annual low discharges

$\left[\mathrm{m}^{3} / \mathrm{s}\right.$, meters per second]

\begin{tabular}{|c|c|c|c|c|c|c|c|c|c|c|}
\hline \multirow{3}{*}{$\begin{array}{c}\text { Nonexceedance } \\
\text { probability }\end{array}$} & \multirow{3}{*}{$\begin{array}{c}\text { Recurrence } \\
\text { interval } \\
\text { (years) }\end{array}$} & \multicolumn{9}{|c|}{ Minimum daily mean discharge $\left(\mathrm{m}^{3} / \mathrm{s}\right)$} \\
\hline & & \multicolumn{9}{|c|}{ Number of consecutive days } \\
\hline & & 1 & 3 & 7 & 14 & 30 & 60 & 90 & 120 & 183 \\
\hline 0.05 & 20 & 6.43 & 6.44 & 6.42 & 6.55 & 6.76 & 7.23 & 7.58 & 7.94 & 8.02 \\
\hline 0.10 & 10 & 6.68 & 6.71 & 6.71 & 6.84 & 7.07 & 7.52 & 7.94 & 8.33 & 8.45 \\
\hline 0.20 & 5 & 7.02 & 7.06 & 7.10 & 7.23 & 7.48 & 7.91 & 8.41 & 8.83 & 9.02 \\
\hline 0.50 & 2 & 7.81 & 7.87 & 7.97 & 8.11 & 8.40 & 8.80 & 9.44 & 9.90 & 10.2 \\
\hline
\end{tabular}




\section{4-0.000-8M KUNDUZ RIVER AT DASHT-I-SAFED, Continued}

Probability of occurrence of seasonal low discharges [ $\mathrm{m}^{3} / \mathrm{s}$, meters per second]

\begin{tabular}{|c|c|c|c|c|c|c|c|c|c|}
\hline \multirow{3}{*}{$\begin{array}{c}\text { Nonexceedance } \\
\text { probability }\end{array}$} & \multirow{3}{*}{$\begin{array}{c}\text { Recurrence } \\
\text { interval } \\
\text { (years) }\end{array}$} & \multicolumn{8}{|c|}{ Minimum daily mean discharge $\left(\mathrm{m}^{3} / \mathrm{s}\right)$} \\
\hline & & \multicolumn{8}{|c|}{ Number of consecutive days } \\
\hline & & 1 & 7 & 14 & 30 & 1 & 7 & 14 & 30 \\
\hline & & \multicolumn{4}{|c|}{ December-January-February } & \multicolumn{4}{|c|}{ March-April-May } \\
\hline 0.05 & 20 & 6.69 & 6.73 & 7.04 & 7.47 & 5.81 & 6.14 & 6.36 & 6.69 \\
\hline 0.10 & 10 & 7.15 & 7.20 & 7.47 & 7.90 & 6.19 & 6.50 & 6.71 & 7.06 \\
\hline 0.20 & 5 & 7.73 & 7.80 & 8.03 & 8.46 & 6.70 & 6.98 & 7.18 & 7.56 \\
\hline \multirow[t]{2}{*}{0.50} & 2 & 8.91 & 9.01 & 9.20 & 9.62 & 7.84 & 8.07 & 8.26 & 8.69 \\
\hline & & \multicolumn{4}{|c|}{ June-July-August } & \multicolumn{4}{|c|}{ September-0ctober-November } \\
\hline 0.05 & 20 & 6.57 & 6.89 & 6.97 & 7.12 & 6.68 & 6.81 & 7.04 & 7.76 \\
\hline 0.10 & 10 & 6.98 & 7.29 & 7.41 & 7.65 & 7.03 & 7.14 & 7.34 & 8.01 \\
\hline 0.20 & 5 & 7.54 & 7.84 & 8.00 & 8.32 & 7.51 & 7.60 & 7.77 & 8.38 \\
\hline 0.50 & 2 & 8.86 & 9.10 & 9.31 & 9.75 & 8.65 & 8.70 & 8.84 & 9.33 \\
\hline
\end{tabular}

\section{4-0.000-8M KUNDUZ RIVER AT DASHT-I-SAFED, Continued}

Annual peak discharges

$\left[\mathrm{m}^{3} / \mathrm{s}\right.$, meters per second]

\begin{tabular}{|c|c|c|c|c|c|}
\hline \multicolumn{3}{|c|}{$\begin{array}{c}\text { Annual peak discharge, } \\
\text { by year }\end{array}$} & \multicolumn{3}{|c|}{$\begin{array}{l}\text { Annual peak discharge, } \\
\text { from highest to lowest }\end{array}$} \\
\hline $\begin{array}{l}\text { Water } \\
\text { year }\end{array}$ & Date & $\begin{array}{c}\text { Peak } \\
\text { discharge } \\
\left(\mathrm{m}^{3} / \mathrm{s}\right)\end{array}$ & $\begin{array}{l}\text { Water } \\
\text { year }\end{array}$ & Date & $\begin{array}{c}\text { Peak } \\
\text { discharge } \\
\left(\mathrm{m}^{3} / \mathrm{s}\right)\end{array}$ \\
\hline 1968 & June 11, 1968 & 28.0 & 1972 & June 2, 1972 & 47.0 \\
\hline 1969 & June 7, 1969 & 18.0 & 1968 & June 11, 1968 & 28.0 \\
\hline 1970 & April 27, 1970 & 16.5 & 1978 & April 26, 1978 & 27.5 \\
\hline 1971 & April 4, 1971 & 18.2 & 1971 & April 4, 1971 & 18.2 \\
\hline 1972 & June 2, 1972 & 47.0 & 1969 & June 7, 1969 & 18.0 \\
\hline 1973 & January 5, 1973 & 12.9 & 1970 & April 27, 1970 & 16.5 \\
\hline 1974 & July 23, 1974 & 14.9 & 1974 & July 23, 1974 & 14.9 \\
\hline 1978 & April 26, 1978 & 27.5 & 1973 & January 5, 1973 & 12.9 \\
\hline
\end{tabular}


14-0.000-8M KUNDUZ RIVER AT DASHT-I-SAFED, Continued

Monthly and annual mean discharges, in cubic meters per second $[-$, no data]

\begin{tabular}{|c|c|c|c|c|c|c|c|c|c|c|c|c|c|}
\hline \multirow{2}{*}{$\begin{array}{l}\text { Water } \\
\text { year }\end{array}$} & \multicolumn{12}{|c|}{ Monthly mean discharge } & \multirow{2}{*}{$\begin{array}{c}\text { Annual } \\
\text { discharge }\end{array}$} \\
\hline & October & November & December & January & February & March & April & May & June & July & August & September & \\
\hline 1968 & -- & 8.95 & 9.86 & 10.4 & 10.5 & 10.00 & 10.4 & 10.6 & 20.9 & 13.8 & 12.7 & 11.8 & -- \\
\hline 1969 & 12.2 & 10.1 & 12.2 & 13.7 & 12.9 & 12.5 & 12.4 & 11.8 & 15.9 & 13.4 & 11.3 & 12.8 & 12.6 \\
\hline 1970 & 13.0 & 12.8 & 13.5 & 12.6 & 11.1 & 10.1 & 10.2 & 10.7 & 9.72 & 8.31 & 8.82 & 8.66 & 10.8 \\
\hline 1971 & 8.78 & 7.91 & 7.64 & 9.85 & 10.5 & 10.0 & 10.1 & 8.54 & 7.32 & 9.95 & 9.57 & 9.06 & 9.10 \\
\hline 1972 & 9.04 & 9.58 & 10.2 & 9.37 & 8.52 & 8.97 & 8.29 & 14.2 & 21.7 & 11.3 & 10.7 & 8.49 & 10.9 \\
\hline 1973 & 10.2 & 10.8 & 11.4 & 11.9 & 8.99 & 7.79 & 9.52 & 9.58 & 11.00 & 11.5 & 11.2 & 12.0 & 10.5 \\
\hline 1974 & 12.1 & 10.5 & 11.4 & 10.8 & 10.9 & 9.50 & 8.34 & 8.13 & 8.74 & 11.7 & 10.0 & 9.99 & 10.2 \\
\hline 1975 & 10.4 & 9.86 & 10.2 & 10.7 & 10.0 & 9.67 & 9.72 & -- & -- & -- & -- & -- & -- \\
\hline 1977 & -- & -- & -- & -- & -- & -- & -- & -- & -- & -- & -- & 8.62 & -- \\
\hline 1978 & 8.85 & 9.13 & 8.47 & 9.46 & 8.22 & 7.07 & 7.64 & 9.84 & 11.5 & 9.35 & 9.80 & 9.47 & 9.07 \\
\hline
\end{tabular}




\section{4-1.1L0-1A BANGI RIVER AT PUL-I-BANGI}

\section{(U.S. Geological Survey identification number: 364200069150000)}

LOCATION: Lat $36^{\circ} 42^{\prime} \mathrm{N}$., long $69^{\circ} 15^{\prime} \mathrm{E}$.

DRAINAGE AREA: $4,200 \mathrm{~km}^{2}$.

ELEVATION: 610 meters above mean sea level.

PERIOD OF RECORD: August 19, 1964 to September 30, 1978.

GAGE: Water-stage recorder.

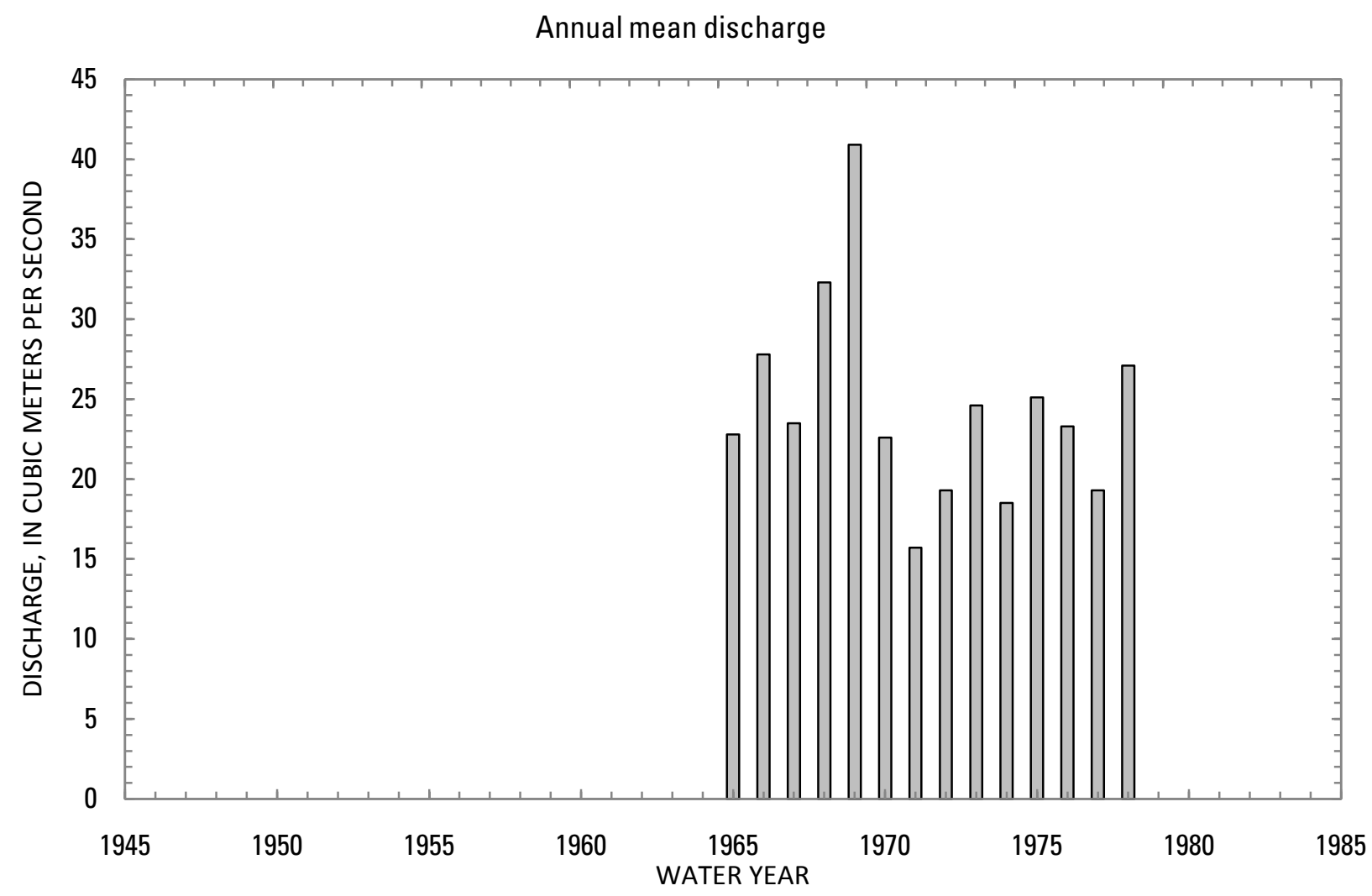




\section{4-1.1L0-1A BANGI RIVER AT PUL-I-BANGI, Continued}

Statistics of monthly and annual mean discharges $\left[\mathrm{m}^{3} / \mathrm{s}\right.$, cubic meters per second]

\begin{tabular}{|c|c|c|c|c|c|c|c|c|}
\hline \multirow[b]{2}{*}{ Month } & \multicolumn{2}{|c|}{ Maximum } & \multicolumn{2}{|c|}{ Minimum } & \multicolumn{4}{|c|}{ Mean } \\
\hline & $\begin{array}{c}\text { Discharge } \\
\left(\mathrm{m}^{3} / \mathrm{s}\right)\end{array}$ & $\begin{array}{c}\text { Water year } \\
\text { of } \\
\text { occurrence }\end{array}$ & $\begin{array}{c}\text { Discharge } \\
\left(\mathrm{m}^{3} / \mathrm{s}\right)\end{array}$ & $\begin{array}{c}\text { Water year } \\
\text { of } \\
\text { occurrence }\end{array}$ & $\begin{array}{c}\text { Discharge } \\
\left(\mathrm{m}^{3} / \mathrm{s}\right)\end{array}$ & $\begin{array}{c}\text { Standard } \\
\text { deviation } \\
\left(\mathrm{m}^{3} / \mathrm{s}\right)\end{array}$ & $\begin{array}{c}\text { Coefficient } \\
\text { of } \\
\text { variation }\end{array}$ & $\begin{array}{c}\text { Percentage } \\
\text { of annual } \\
\text { discharge }\end{array}$ \\
\hline October & 20.9 & 1970 & 9.01 & 1972 & 13.2 & 3.12 & 0.24 & 4.51 \\
\hline November & 17.3 & 1970 & 9.20 & 1975 & 12.9 & 2.42 & 0.19 & 4.41 \\
\hline December & 17.6 & 1969 & 9.52 & 1972 & 12.8 & 2.26 & 0.18 & 4.36 \\
\hline January & 17.1 & 1969 & 8.98 & 1972 & 12.2 & 2.08 & 0.17 & 4.15 \\
\hline February & 17.0 & 1969 & 7.76 & 1975 & 11.7 & 2.51 & 0.21 & 3.99 \\
\hline March & 21.5 & 1969 & 7.55 & 1975 & 12.7 & 3.70 & 0.29 & 4.33 \\
\hline April & 43.4 & 1969 & 8.93 & 1977 & 21.2 & 8.61 & 0.41 & 7.24 \\
\hline May & 57.0 & 1969 & 27.6 & 1972 & 39.9 & 10.1 & 0.25 & 13.6 \\
\hline June & 113 & 1966 & 34.9 & 1971 & 72.1 & 25.1 & 0.35 & 24.6 \\
\hline July & 107 & 1969 & 12.7 & 1971 & 50.0 & 25.5 & 0.51 & 17.1 \\
\hline August & 51.3 & 1969 & 9.02 & 1971 & 21.5 & 11.0 & 0.51 & 7.32 \\
\hline September & 23.6 & 1969 & 7.51 & 1971 & 13.0 & 3.79 & 0.29 & 4.43 \\
\hline Annual & 40.9 & 1969 & 15.7 & 1971 & 24.5 & 6.35 & 0.26 & 100 \\
\hline
\end{tabular}


14-1.1L0-1A BANGI RIVER AT PUL-I-BANGI, Continued

Monthly and annual flow duration, in cubic meters per second

\begin{tabular}{|c|c|c|c|c|c|c|c|c|c|c|c|c|c|}
\hline \multirow{2}{*}{$\begin{array}{l}\text { Percentage } \\
\text { of days } \\
\text { discharge } \\
\text { equaled or } \\
\text { exceeded }\end{array}$} & \multicolumn{12}{|c|}{ Month } & \multirow{2}{*}{ Annual } \\
\hline & October & November & December & January & February & March & April & May & June & July & August & September & \\
\hline 95 & 9.05 & 9.11 & 9.59 & 9.20 & 8.05 & 7.61 & 8.53 & 13.0 & 30.2 & 13.1 & 9.76 & 7.94 & 8.75 \\
\hline 90 & 9.41 & 9.63 & 10.4 & 9.50 & 8.55 & 8.04 & 10.0 & 16.5 & 35.0 & 18.3 & 10.9 & 8.42 & 9.61 \\
\hline 85 & 10.0 & 10.7 & 10.7 & 9.98 & 8.80 & 8.89 & 11.1 & 19.8 & 38.2 & 23.1 & 12.3 & 8.84 & 10.4 \\
\hline 80 & 10.4 & 11.1 & 10.9 & 10.3 & 9.06 & 9.50 & 12.7 & 24.4 & 41.2 & 26.0 & 13.1 & 9.65 & 11.1 \\
\hline 75 & 10.7 & 11.5 & 11.3 & 10.5 & 9.40 & 9.92 & 13.4 & 27.2 & 45.5 & 28.8 & 13.7 & 11.0 & 11.7 \\
\hline 70 & 11.5 & 11.7 & 11.6 & 10.9 & 10.3 & 10.2 & 13.9 & 29.1 & 49.6 & 31.3 & 14.6 & 11.7 & 12.2 \\
\hline 65 & 12.1 & 11.9 & 11.8 & 11.4 & 10.8 & 10.5 & 14.4 & 30.7 & 51.6 & 33.6 & 15.5 & 12.0 & 12.6 \\
\hline 60 & 12.5 & 12.1 & 11.9 & 11.7 & 11.2 & 10.9 & 15.3 & 32.6 & 54.1 & 36.7 & 16.3 & 12.2 & 13.1 \\
\hline 55 & 12.7 & 12.3 & 12.1 & 11.9 & 11.5 & 11.5 & 16.4 & 34.9 & 58.8 & 39.2 & 17.0 & 12.6 & 13.5 \\
\hline 50 & 12.9 & 12.6 & 12.3 & 12.1 & 11.7 & 11.9 & 17.5 & 37.1 & 63.5 & 42.7 & 17.8 & 12.9 & 14.2 \\
\hline 45 & 13.2 & 12.7 & 12.5 & 12.4 & 11.8 & 12.5 & 18.5 & 39.5 & 67.1 & 47.9 & 18.7 & 13.2 & 15.0 \\
\hline 40 & 13.8 & 12.8 & 12.8 & 12.7 & 12.3 & 12.8 & 20.5 & 41.6 & 71.1 & 52.0 & 19.6 & 13.4 & 16.4 \\
\hline 35 & 14.1 & 13.6 & 13.0 & 12.9 & 12.7 & 13.3 & 22.0 & 44.2 & 76.0 & 56.0 & 20.7 & 13.7 & 18.2 \\
\hline 30 & 14.5 & 14.0 & 13.3 & 13.0 & 13.0 & 13.8 & 23.3 & 47.8 & 81.4 & 59.5 & 22.0 & 14.0 & 20.9 \\
\hline 25 & 15.1 & 14.2 & 13.8 & 13.2 & 13.3 & 14.2 & 24.5 & 51.2 & 86.7 & 63.2 & 24.5 & 14.5 & 26.6 \\
\hline 20 & 15.4 & 15.1 & 14.1 & 13.8 & 13.7 & 15.1 & 27.8 & 54.6 & 93.7 & 70.6 & 27.9 & 15.0 & 33.2 \\
\hline 15 & 15.6 & 15.9 & 15.4 & 14.0 & 14.2 & 16.8 & 30.1 & 59.5 & 108 & 75.5 & 31.7 & 15.5 & 42.5 \\
\hline 10 & 16.3 & 17.1 & 16.9 & 14.5 & 15.0 & 19.5 & 34.8 & 64.0 & 123 & 93.8 & 37.1 & 16.1 & 55.7 \\
\hline 5 & 20.1 & 17.4 & 17.6 & 16.7 & 17.1 & 21.2 & 45.9 & 74.2 & 154 & 117 & 49.6 & 22.4 & 73.7 \\
\hline
\end{tabular}




\section{4-1.1L0-1A BANGI RIVER AT PUL-I-BANGI, Continued}

Probability of occurrence of annual high discharges

[m $\mathrm{m}^{3} / \mathrm{s}$, cubic meters per second; ng, not given]

\begin{tabular}{|c|c|c|c|c|c|c|}
\hline \multirow{2}{*}{$\begin{array}{c}\text { Exceedance } \\
\text { probability }\end{array}$} & \multirow{2}{*}{$\begin{array}{c}\text { Recurrence } \\
\text { interval } \\
\text { (years) }\end{array}$} & \multirow{2}{*}{$\begin{array}{c}\text { Maximum } \\
\text { instantaneous } \\
\text { discharge } \\
\left(\mathrm{m}^{3} / \mathrm{s}\right)\end{array}$} & \multicolumn{4}{|c|}{ Maximum daily mean discharge $\left(\mathrm{m}^{3} / \mathrm{s}\right)$} \\
\hline & & & $\begin{array}{l}\text { 3-day } \\
\text { period }\end{array}$ & $\begin{array}{l}\text { 7-day } \\
\text { period }\end{array}$ & $\begin{array}{l}\text { 15-day } \\
\text { period }\end{array}$ & $\begin{array}{l}\text { 30-day } \\
\text { period }\end{array}$ \\
\hline 0.99 & 1.01 & 40.6 & 51.9 & 48.1 & 42.1 & 37.6 \\
\hline 0.95 & 1.05 & 67.9 & 62.8 & 57.8 & 51.0 & 45.4 \\
\hline 0.90 & 1.11 & 88.1 & 70.2 & 64.5 & 56.9 & 50.4 \\
\hline 0.80 & 1.25 & 120 & 81.3 & 74.3 & 65.2 & 57.3 \\
\hline 0.50 & 2 & 207 & 111 & 100 & 86.1 & 74.0 \\
\hline 0.20 & 5 & 343 & 160 & 142 & 116 & 96.8 \\
\hline 0.10 & 10 & 439 & 197 & 173 & 138 & 112 \\
\hline 0.04 & 25 & 564 & 250 & 217 & 165 & 131 \\
\hline 0.02 & 50 & 658 & 294 & 253 & 187 & 146 \\
\hline 0.01 & 100 & 754 & 343 & 293 & 210 & 161 \\
\hline 0.005 & 200 & 850 & 396 & 335 & 233 & 176 \\
\hline 0.002 & 500 & 978 & ng & $\mathrm{ng}$ & $\mathrm{ng}$ & $\mathrm{ng}$ \\
\hline
\end{tabular}

14-1.1L0-1A BANGI RIVER AT PUL-I-BANGI, Continued

Probability of occurrence of annual low discharges $\left[\mathrm{m}^{3} / \mathrm{s}\right.$, meters per second]

\begin{tabular}{|c|c|c|c|c|c|c|c|c|c|c|}
\hline \multirow{3}{*}{$\begin{array}{c}\text { Nonexceedance } \\
\text { probability }\end{array}$} & \multirow{3}{*}{$\begin{array}{c}\text { Recurrence } \\
\text { interval } \\
\text { (years) }\end{array}$} & \multicolumn{9}{|c|}{ Minimum daily mean discharge $\left(\mathrm{m}^{3} / \mathrm{s}\right)$} \\
\hline & & \multicolumn{9}{|c|}{ Number of consecutive days } \\
\hline & & 1 & 3 & 7 & 14 & 30 & 60 & 90 & 120 & 183 \\
\hline 0.05 & 20 & 5.68 & 5.88 & 5.94 & 6.17 & 6.94 & 7.58 & 8.18 & 8.71 & 9.09 \\
\hline 0.10 & 10 & 6.37 & 6.57 & 6.65 & 6.84 & 7.54 & 8.17 & 8.74 & 9.21 & 9.71 \\
\hline 0.20 & 5 & 7.26 & 7.44 & 7.56 & 7.73 & 8.35 & 8.95 & 9.48 & 9.88 & 10.5 \\
\hline 0.50 & 2 & 9.05 & 9.19 & 9.46 & 9.67 & 10.1 & 10.6 & 11.1 & 11.4 & 12.2 \\
\hline
\end{tabular}




\section{4-1.1L0-1A BANGI RIVER AT PUL-I-BANGI, Continued}

Probability of occurrence of seasonal low discharges [m³/s, meters per second]

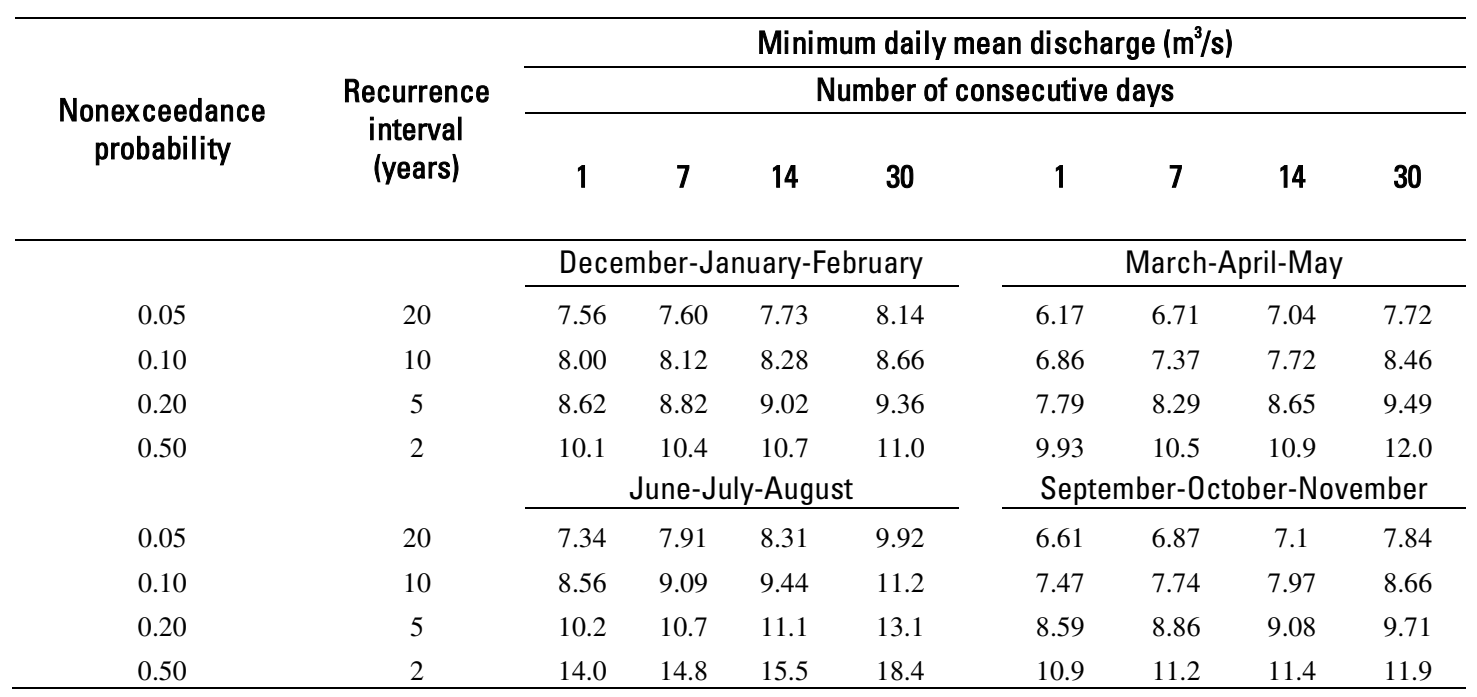




\section{4-1.1L0-1A BANGI RIVER AT PUL-I-BANGI, Continued}

\begin{tabular}{|c|c|c|c|c|c|}
\hline \multicolumn{6}{|c|}{$\begin{array}{c}\text { Annual peak discharges } \\
\text { [m } \mathrm{m}^{3} / \mathrm{s} \text {, meters per second] }\end{array}$} \\
\hline \multicolumn{3}{|c|}{$\begin{array}{c}\text { Annual peak discharge, } \\
\text { by year }\end{array}$} & \multicolumn{3}{|c|}{$\begin{array}{l}\text { Annual peak discharge, } \\
\text { from highest to lowest }\end{array}$} \\
\hline $\begin{array}{l}\text { Water } \\
\text { year }\end{array}$ & Date & $\begin{array}{c}\text { Peak } \\
\text { discharge } \\
\left(\mathrm{m}^{3} / \mathrm{s}\right)\end{array}$ & $\begin{array}{l}\text { Water } \\
\text { year }\end{array}$ & Date & $\begin{array}{c}\text { Peak } \\
\text { discharge } \\
\left(\mathrm{m}^{3} / \mathrm{s}\right)\end{array}$ \\
\hline 1965 & June 15,1965 & 91.0 & 1969 & May 22, 1969 & 398 \\
\hline 1966 & June 15, 1966 & 390 & 1966 & June 15, 1966 & 390 \\
\hline 1967 & May 23, 1967 & 377 & 1972 & May 12, 1972 & 382 \\
\hline 1968 & June 10, 1968 & 215 & 1967 & May 23, 1967 & 377 \\
\hline 1969 & May 22, 1969 & 398 & 1976 & April 27, 1976 & 370 \\
\hline 1970 & June 2, 1970 & 94.0 & 1977 & May 29, 1977 & 300 \\
\hline 1971 & May 27, 1971 & 152 & 1978 & April 27, 1978 & 230 \\
\hline 1972 & May 12, 1972 & 382 & 1968 & June 10, 1968 & 215 \\
\hline 1973 & June 25, 1973 & 82.0 & 1971 & May 27, 1971 & 152 \\
\hline 1974 & June 19, 1974 & 88.0 & 1975 & May 16, 1975 & 148 \\
\hline 1975 & May 16, 1975 & 148 & 1970 & June 2, 1970 & 94.0 \\
\hline 1976 & April 27, 1976 & 370 & 1965 & June 15,1965 & 91.0 \\
\hline 1977 & May 29, 1977 & 300 & 1974 & June 19, 1974 & 88.0 \\
\hline 1978 & April 27, 1978 & 230 & 1973 & June 25, 1973 & 82.0 \\
\hline
\end{tabular}




\section{4-1.1L0-1A BANGI RIVER AT PUL-I-BANGI, Continued}

Monthly and annual mean discharges, in cubic meters per second

[Data may not be rounded in accordance with U.S. Geological Survey publication standards; --, no data]

\begin{tabular}{|c|c|c|c|c|c|c|c|c|c|c|c|c|c|}
\hline \multirow{2}{*}{$\begin{array}{l}\text { Water } \\
\text { year }\end{array}$} & \multicolumn{12}{|c|}{ Monthly mean discharge } & \multirow{2}{*}{$\begin{array}{c}\text { Annual } \\
\text { discharge }\end{array}$} \\
\hline & October & November & December & January & February & March & April & May & June & July & August & September & \\
\hline 1964 & -- & -- & -- & -- & -- & -- & -- & -- & -- & -- & -- & 11.4 & -- \\
\hline 1965 & 13.0 & 12.0 & 11.9 & 10.5 & 9.31 & 10.5 & 17.1 & 30.8 & 72.8 & 48.1 & 21.0 & 15.7 & 22.8 \\
\hline 1966 & 13.2 & 13.8 & 13.3 & 12.0 & 13.3 & 17.0 & 27.7 & 46.9 & 113 & 31.1 & 18.5 & 13.7 & 27.8 \\
\hline 1967 & 15.7 & 15.2 & 13.3 & 13.0 & 11.4 & 10.0 & 12.9 & 31.4 & 63.4 & 59.8 & 21.3 & 13.5 & 23.5 \\
\hline 1968 & 14.5 & 14.1 & 13.5 & 13.0 & 11.3 & 11.6 & 17.3 & 40.3 & 113 & 86.6 & 36.1 & 16.0 & 32.3 \\
\hline 1969 & 15.3 & 17.0 & 17.6 & 17.1 & 17.0 & 21.5 & 43.4 & 57.0 & 101 & 107 & 51.3 & 23.6 & 40.9 \\
\hline 1970 & 20.9 & 17.3 & 16.1 & 14.1 & 14.4 & 14.7 & 20.6 & 41.4 & 51.8 & 31.1 & 16.0 & 12.5 & 22.6 \\
\hline 1971 & 12.7 & 12.4 & 11.3 & 11.3 & 12.4 & 12.7 & 14.4 & 37.5 & 34.9 & 12.7 & 9.02 & 7.51 & 15.7 \\
\hline 1972 & 9.01 & 9.71 & 9.52 & 8.98 & 8.65 & 11.5 & 14.6 & 27.6 & 55.2 & 38.8 & 25.6 & 12.5 & 19.3 \\
\hline 1973 & 10.2 & 10.9 & 11.7 & 12.6 & 13.4 & 14.1 & 20.0 & 37.2 & 66.7 & 61.0 & 23.9 & 12.7 & 24.6 \\
\hline 1974 & 12.3 & 11.6 & 12.0 & 11.8 & 11.4 & 15.8 & 23.3 & 28.1 & 44.1 & 30.2 & 12.9 & 8.82 & 18.5 \\
\hline 1975 & 10.3 & 9.20 & 10.8 & 10.2 & 7.76 & 7.55 & 21.9 & 46.3 & 69.7 & 69.9 & 22.3 & 13.6 & 25.1 \\
\hline 1976 & 13.0 & 12.1 & 11.1 & 12.1 & 11.6 & 10.3 & 26.5 & 50.5 & 49.8 & 54.5 & 14.3 & 12.9 & 23.3 \\
\hline 1977 & 15.5 & 13.6 & 11.4 & 9.82 & 8.90 & 8.33 & 8.93 & 28.0 & 79.4 & 26.0 & 12.7 & 8.63 & 19.3 \\
\hline 1978 & 9.56 & 12.2 & 15.4 & 14.0 & 13.0 & 12.3 & 28.4 & 55.0 & 94.5 & 43.0 & 15.6 & 11.8 & 27.1 \\
\hline
\end{tabular}




\section{4-1.R00-2A TALOQAN RIVER AT PUL-I-CHUGHA}

\section{(U.S. Geological Survey identification number: 364400069120000)}

LOCATION: Lat $36^{\circ} 44^{\prime} \mathrm{N} .$, long $69^{\circ} 12^{\prime} \mathrm{E}$.

DRAINAGE AREA: $9,760 \mathrm{~km}^{2}$.

ELEVATION: 558 meters above mean sea level.

PERIOD OF RECORD: April 19, 1960 to September 30, 1960 and August 2, 1964 to September 30, 1978.

GAGE: Water-stage recorder.

Annual mean discharge

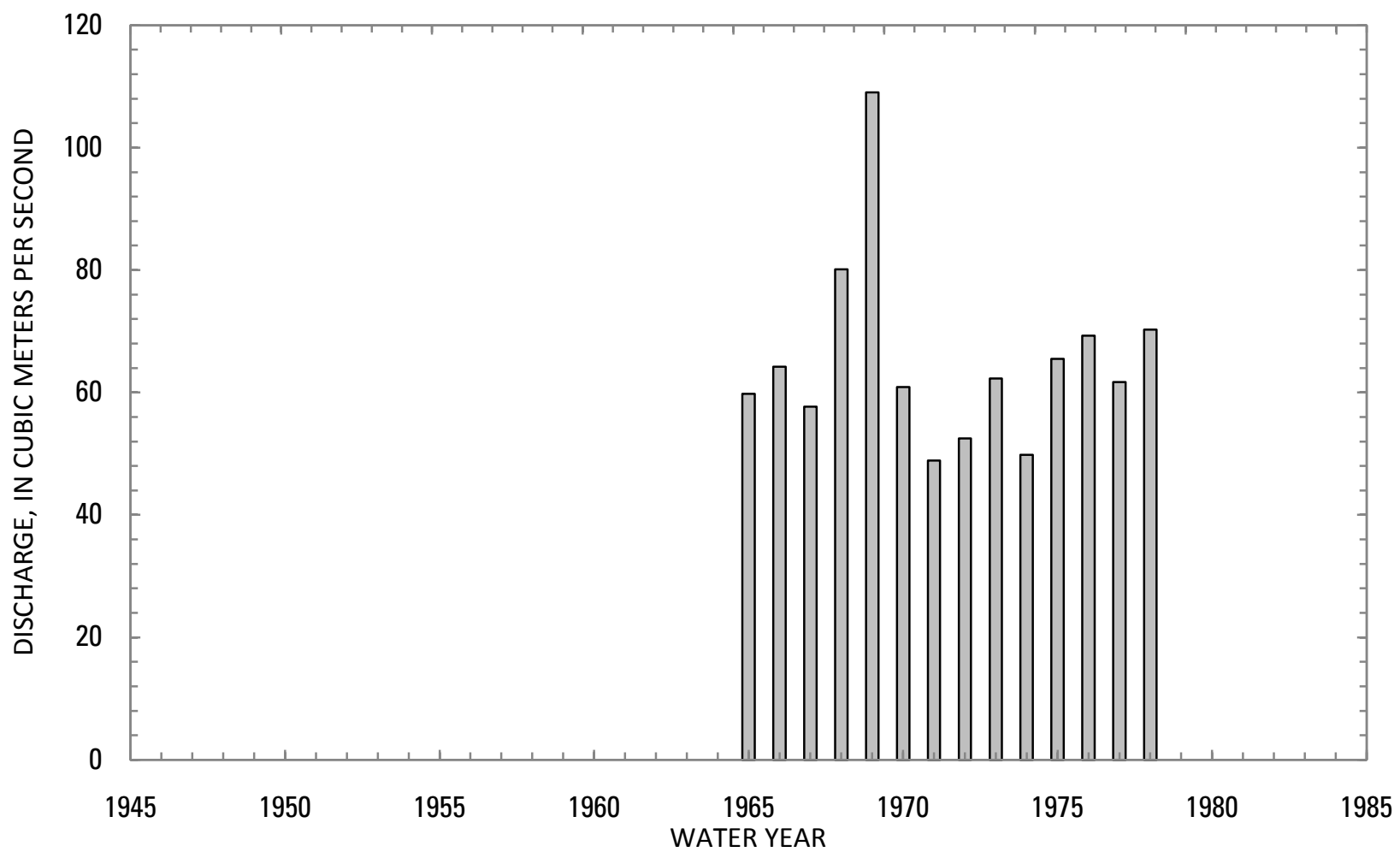




\section{4-1.R00-2A TALOQAN RIVER AT PUL-I-CHUGHA, Continued}

Statistics of monthly and annual mean discharges $\left[\mathrm{m}^{3} / \mathrm{s}\right.$, cubic meters per second]

\begin{tabular}{|c|c|c|c|c|c|c|c|c|}
\hline \multirow[b]{2}{*}{ Month } & \multicolumn{2}{|c|}{ Maximum } & \multicolumn{2}{|c|}{ Minimum } & \multicolumn{4}{|c|}{ Mean } \\
\hline & $\begin{array}{c}\text { Discharge } \\
\left(\mathrm{m}^{3} / \mathrm{s}\right)\end{array}$ & $\begin{array}{c}\text { Water year } \\
\text { of } \\
\text { occurrence }\end{array}$ & $\begin{array}{c}\text { Discharge } \\
\left(\mathrm{m}^{3} / \mathrm{s}\right)\end{array}$ & $\begin{array}{c}\text { Water year } \\
\text { of } \\
\text { occurrence }\end{array}$ & $\begin{array}{c}\text { Discharge } \\
\left(\mathrm{m}^{3} / \mathrm{s}\right)\end{array}$ & $\begin{array}{c}\text { Standard } \\
\text { deviation } \\
\left(\mathrm{m}^{3} / \mathrm{s}\right)\end{array}$ & $\begin{array}{c}\text { Coefficient } \\
\text { of } \\
\text { variation }\end{array}$ & $\begin{array}{c}\text { Percentage } \\
\text { of annual } \\
\text { discharge }\end{array}$ \\
\hline October & 47.0 & 1970 & 29.3 & 1966 & 36.2 & 5.05 & 0.14 & 4.71 \\
\hline November & 42.9 & 1970 & 31.0 & 1973 & 36.3 & 3.26 & 0.09 & 4.72 \\
\hline December & 42.1 & 1969 & 29.4 & 1972 & 34.6 & 3.88 & 0.11 & 4.51 \\
\hline January & 35.8 & 1977 & 23.0 & 1966 & 29.5 & 3.54 & 0.12 & 3.84 \\
\hline February & 35.2 & 1976 & 22.4 & 1965 & 27.8 & 3.81 & 0.14 & 3.62 \\
\hline March & 50.2 & 1969 & 20.3 & 1967 & 31.5 & 7.44 & 0.24 & 4.11 \\
\hline April & 144 & 1969 & 24.9 & 1977 & 52.5 & 28.9 & 0.55 & 6.84 \\
\hline May & 178 & 1969 & 51.9 & 1974 & 88.7 & 30.6 & 0.35 & 11.6 \\
\hline June & 285 & 1969 & 90.0 & 1960 & 179 & 57.0 & 0.32 & 23.4 \\
\hline July & 267 & 1969 & 53.0 & 1971 & 146 & 56.2 & 0.38 & 19.0 \\
\hline August & 133 & 1969 & 46.3 & 1974 & 69.4 & 22.1 & 0.32 & 9.04 \\
\hline September & 55.2 & 1969 & 26.1 & 1977 & 35.8 & 7.34 & 0.20 & 4.66 \\
\hline Annual & 109 & 1969 & 48.9 & 1971 & 65.1 & 15.1 & 0.23 & 100 \\
\hline
\end{tabular}


14-1.R00-2A TALOQAN RIVER AT PUL-I-CHUGHA, Continued

Monthly and annual flow duration, in cubic meters per second

\begin{tabular}{|c|c|c|c|c|c|c|c|c|c|c|c|c|c|}
\hline \multirow{2}{*}{$\begin{array}{l}\text { Percentage } \\
\text { of days } \\
\text { discharge } \\
\text { equaled or } \\
\text { exceeded }\end{array}$} & \multicolumn{12}{|c|}{ Month } & \multirow{2}{*}{ Annual } \\
\hline & October & November & December & January & February & March & April & May & June & July & August & September & \\
\hline 95 & 28.3 & 30.7 & 29.4 & 22.0 & 21.7 & 20.3 & 23.4 & 37.1 & 78.2 & 55.1 & 39.9 & 25.5 & 24.0 \\
\hline 90 & 30.2 & 31.0 & 29.8 & 24.5 & 22.6 & 21.6 & 26.2 & 44.4 & 85.7 & 73.2 & 41.7 & 27.7 & 26.4 \\
\hline 85 & 31.1 & 31.4 & 30.3 & 25.0 & 23.8 & 22.7 & 29.9 & 48.1 & 97.8 & 83.4 & 45.1 & 28.9 & 28.5 \\
\hline 80 & 31.4 & 32.3 & 30.9 & 25.6 & 24.1 & 24.1 & 31.3 & 51.0 & 112 & 93.0 & 48.0 & 29.9 & 29.6 \\
\hline 75 & 31.7 & 32.5 & 31.1 & 26.3 & 24.4 & 25.2 & 32.9 & 53.3 & 123 & 99.4 & 50.3 & 30.7 & 30.7 \\
\hline 70 & 32.7 & 33.2 & 31.4 & 26.8 & 24.9 & 26.4 & 34.7 & 56.0 & 133 & 105 & 52.6 & 31.3 & 31.8 \\
\hline 65 & 33.0 & 34.5 & 32.4 & 27.6 & 25.8 & 27.4 & 36.2 & 59.7 & 140 & 110 & 56.4 & 31.9 & 33.1 \\
\hline 60 & 33.7 & 35.6 & 32.7 & 28.7 & 26.4 & 28.6 & 37.5 & 66.4 & 151 & 117 & 59.3 & 32.5 & 34.4 \\
\hline 55 & 34.6 & 36.3 & 32.9 & 29.4 & 26.9 & 29.5 & 39.4 & 71.1 & 158 & 123 & 61.3 & 32.9 & 36.1 \\
\hline 50 & 36.1 & 36.7 & 33.9 & 30.1 & 27.8 & 30.3 & 42.3 & 75.2 & 167 & 131 & 63.2 & 33.4 & 37.9 \\
\hline 45 & 37.1 & 37.3 & 34.3 & 30.5 & 28.2 & 31.0 & 44.4 & 81.8 & 176 & 140 & 65.1 & 34.2 & 40.1 \\
\hline 40 & 37.9 & 38.1 & 35.5 & 30.7 & 28.6 & 31.7 & 46.5 & 89.2 & 187 & 146 & 67.7 & 36.0 & 42.7 \\
\hline 35 & 38.4 & 38.4 & 36.4 & 30.8 & 29.3 & 32.5 & 48.7 & 96.0 & 201 & 155 & 71.3 & 36.9 & 48.2 \\
\hline 30 & 39.3 & 38.6 & 36.9 & 31.0 & 29.8 & 34.4 & 51.3 & 102 & 212 & 165 & 75.1 & 37.5 & 56.6 \\
\hline 25 & 40.1 & 38.9 & 38.0 & 31.6 & 31.2 & 36.0 & 54.7 & 113 & 227 & 178 & 78.8 & 38.1 & 70.6 \\
\hline 20 & 40.6 & 39.8 & 39.2 & 32.8 & 31.5 & 37.4 & 61.3 & 125 & 244 & 192 & 84.5 & 40.6 & 91.3 \\
\hline 15 & 42.0 & 40.2 & 40.2 & 34.1 & 32.6 & 39.1 & 71.1 & 138 & 269 & 211 & 91.1 & 45.0 & 118 \\
\hline 10 & 45.1 & 40.5 & 40.9 & 34.8 & 33.9 & 41.1 & 84.6 & 155 & 288 & 250 & 102 & 49.4 & 154 \\
\hline 5 & 46.1 & 42.7 & 41.8 & 36.9 & 34.8 & 44.7 & 126 & 181 & 324 & 304 & 131 & 53.6 & 203 \\
\hline
\end{tabular}




\section{4-1.R00-2A TALOQAN RIVER AT PUL-I-CHUGHA, Continued}

Probability of occurrence of annual high discharges

[m $\mathrm{m}^{3} / \mathrm{s}$, cubic meters per second; ng, not given]

\begin{tabular}{|c|c|c|c|c|c|c|}
\hline \multirow{2}{*}{$\begin{array}{l}\text { Exceedance } \\
\text { probability }\end{array}$} & \multirow{2}{*}{$\begin{array}{c}\text { Recurrence } \\
\text { interval } \\
\text { (years) }\end{array}$} & \multirow{2}{*}{$\begin{array}{c}\text { Maximum } \\
\text { instantaneous } \\
\text { discharge } \\
\left(\mathrm{m}^{3} / \mathrm{s}\right)\end{array}$} & \multicolumn{4}{|c|}{ Maximum daily mean discharge $\left(\mathrm{m}^{3} / \mathrm{s}\right)$} \\
\hline & & & $\begin{array}{l}\text { 3-day } \\
\text { period }\end{array}$ & $\begin{array}{l}\text { 7-day } \\
\text { period }\end{array}$ & $\begin{array}{l}\text { 15-day } \\
\text { period }\end{array}$ & $\begin{array}{l}\text { 30-day } \\
\text { period }\end{array}$ \\
\hline 0.99 & 1.01 & 150 & 156 & 140 & 117 & 112 \\
\hline 0.95 & 1.05 & 176 & 178 & 163 & 141 & 133 \\
\hline 0.90 & 1.11 & 193 & 193 & 177 & 156 & 145 \\
\hline 0.80 & 1.25 & 220 & 213 & 197 & 176 & 162 \\
\hline 0.50 & 2 & 292 & 262 & 245 & 223 & 201 \\
\hline 0.20 & 5 & 409 & 333 & 310 & 281 & 250 \\
\hline 0.10 & 10 & 498 & 382 & 353 & 318 & 281 \\
\hline 0.04 & 25 & 625 & 446 & 407 & 362 & 319 \\
\hline 0.02 & 50 & 731 & 495 & 447 & 394 & 346 \\
\hline 0.01 & 100 & 847 & 546 & 488 & 425 & 372 \\
\hline 0.005 & 200 & 975 & 598 & 529 & 456 & 399 \\
\hline 0.002 & 500 & 1,160 & $\mathrm{ng}$ & $\mathrm{ng}$ & $\mathrm{ng}$ & $\mathrm{ng}$ \\
\hline
\end{tabular}

\section{4-1.R00-2A TALOQAN RIVER AT PUL-I-CHUGHA, Continued}

Probability of occurrence of annual low discharges

$\left[\mathrm{m}^{3} / \mathrm{s}\right.$, meters per second]

\begin{tabular}{|c|c|c|c|c|c|c|c|c|c|c|}
\hline \multirow{3}{*}{$\begin{array}{c}\text { Nonexceedance } \\
\text { probability }\end{array}$} & \multirow{3}{*}{$\begin{array}{c}\text { Recurrence } \\
\text { interval } \\
\text { (years) }\end{array}$} & \multicolumn{9}{|c|}{ Minimum daily mean discharge $\left(\mathrm{m}^{3} / \mathrm{s}\right)$} \\
\hline & & \multicolumn{9}{|c|}{ Number of consecutive days } \\
\hline & & 1 & 3 & 7 & 14 & 30 & 60 & 90 & 120 & 183 \\
\hline 0.05 & 20 & 16.7 & 16.8 & 17.8 & 19.1 & 20.3 & 22.5 & 23.9 & 25.7 & 28.7 \\
\hline 0.10 & 10 & 17.8 & 18.0 & 18.8 & 20.0 & 21.5 & 23.7 & 25.2 & 26.8 & 29.4 \\
\hline 0.20 & 5 & 19.1 & 19.4 & 20.0 & 21.2 & 22.9 & 25.2 & 26.7 & 28.2 & 30.3 \\
\hline 0.50 & 2 & 21.7 & 22.1 & 22.5 & 23.9 & 25.6 & 27.8 & 29.3 & 30.7 & 32.3 \\
\hline
\end{tabular}




\section{4-1.R00-2A TALOQAN RIVER AT PUL-I-CHUGHA, Continued}

Probability of occurrence of seasonal low discharges

[ $\mathrm{m}^{3} / \mathrm{s}$, meters per second]

\begin{tabular}{|c|c|c|c|c|c|c|c|c|c|}
\hline \multirow{3}{*}{$\begin{array}{c}\text { Nonexceedance } \\
\text { probability }\end{array}$} & \multirow{3}{*}{$\begin{array}{c}\text { Recurrence } \\
\text { interval } \\
\text { (years) }\end{array}$} & \multicolumn{8}{|c|}{ Minimum daily mean discharge $\left(\mathrm{m}^{3} / \mathrm{s}\right)$} \\
\hline & & \multicolumn{8}{|c|}{ Number of consecutive days } \\
\hline & & 1 & 7 & 14 & 30 & 1 & 7 & 14 & 30 \\
\hline 0.05 & 20 & 19.3 & 19.8 & 20.1 & 21.3 & 16.6 & 17.8 & 19.4 & 21.2 \\
\hline 0.10 & 10 & 20.2 & 20.8 & 21.3 & 22.4 & 18 & 19.4 & 20.8 & 22.8 \\
\hline 0.20 & 5 & 21.3 & 22.1 & 22.7 & 23.8 & 19.9 & 21.4 & 22.7 & 25.0 \\
\hline 0.50 & 2 & 23.9 & 24.7 & 25.4 & 26.6 & 23.8 & 25.9 & 27.1 & 30.0 \\
\hline 0.20 & 5 & 35.7 & 39.2 & 43.1 & 52.3 & 25.1 & 26.1 & 26.6 & 28.4 \\
\hline 0.50 & 2 & 42.8 & 46.9 & 51.3 & 62.7 & 29.3 & 30.0 & 30.5 & 32.0 \\
\hline
\end{tabular}




\section{4-1.R00-2A TALOQAN RIVER AT PUL-I-CHUGHA, Continued}

\begin{tabular}{|c|c|c|c|c|c|}
\hline \multicolumn{6}{|c|}{$\begin{array}{l}\text { Annual peak discharges } \\
\text { [m³/s, meters per second] }\end{array}$} \\
\hline \multicolumn{3}{|c|}{$\begin{array}{c}\text { Annual peak discharge, } \\
\text { by year }\end{array}$} & \multicolumn{3}{|c|}{$\begin{array}{l}\text { Annual peak discharge, } \\
\text { from highest to lowest }\end{array}$} \\
\hline $\begin{array}{l}\text { Water } \\
\text { year }\end{array}$ & Date & $\begin{array}{c}\text { Peak } \\
\text { discharge } \\
\left(\mathrm{m}^{3} / \mathrm{s}\right)\end{array}$ & $\begin{array}{l}\text { Water } \\
\text { year }\end{array}$ & Date & $\begin{array}{c}\text { Peak } \\
\text { discharge } \\
\left(\mathrm{m}^{3} / \mathrm{s}\right)\end{array}$ \\
\hline 1960 & May 17, 1960 & 180 & 1969 & April 20, 1969 & 640 \\
\hline 1965 & June 15, 1965 & 240 & 1977 & May 29, 1977 & 540 \\
\hline 1966 & June 20, 1966 & 447 & 1966 & June 20, 1966 & 447 \\
\hline 1967 & June 12, 1967 & 244 & 1976 & April 27, 1976 & 382 \\
\hline 1968 & July 10, 1968 & 377 & 1968 & July 10, 1968 & 377 \\
\hline 1969 & April 20, 1969 & 640 & 1975 & June 18, 1975 & 330 \\
\hline 1970 & June 30, 1970 & 300 & 1978 & June 7, 1978 & 305 \\
\hline 1971 & June 1, 1971 & 216 & 1970 & June 30, 1970 & 300 \\
\hline 1972 & June 13, 1972 & 199 & 1974 & June 19, 1974 & 249 \\
\hline 1973 & June 13, 1973 & 220 & 1967 & June 12, 1967 & 244 \\
\hline 1974 & June 19, 1974 & 249 & 1965 & June 15,1965 & 240 \\
\hline 1975 & June 18, 1975 & 330 & 1973 & June 13, 1973 & 220 \\
\hline 1976 & April 27, 1976 & 382 & 1971 & June 1, 1971 & 216 \\
\hline 1977 & May 29, 1977 & 540 & 1972 & June 13, 1972 & 199 \\
\hline 1978 & June 7, 1978 & 305 & 1960 & May 17,1960 & 180 \\
\hline
\end{tabular}


14-1.R00-2A TALOQAN RIVER AT PUL-I-CHUGHA, Continued

Monthly and annual mean discharges, in cubic meters per second $[--$, no data]

\begin{tabular}{|c|c|c|c|c|c|c|c|c|c|c|c|c|c|}
\hline \multirow{2}{*}{$\begin{array}{l}\text { Water } \\
\text { year }\end{array}$} & \multicolumn{12}{|c|}{ Monthly mean discharge } & \multirow{2}{*}{$\begin{array}{c}\text { Annual } \\
\text { discharge }\end{array}$} \\
\hline & October & November & December & January & February & March & April & May & June & July & August & September & \\
\hline 1960 & -- & -- & -- & -- & -- & -- & -- & 60.2 & 90.0 & 117 & 59.5 & 31.9 & -- \\
\hline 1964 & -- & -- & -- & -- & -- & -- & -- & -- & -- & -- & 64.0 & 32.7 & -- \\
\hline 1965 & 31.4 & 32.0 & 30.8 & 25.3 & 22.4 & 26.4 & 41.3 & 68.8 & 193 & 144 & 62.9 & 38.3 & 59.8 \\
\hline 1966 & 29.3 & 34.1 & 32.1 & 23.0 & 24.4 & 32.9 & 45.0 & 97.5 & 237 & 109 & 67.0 & 38.2 & 64.2 \\
\hline 1967 & 39.1 & 39.0 & 32.4 & 25.6 & 24.8 & 20.3 & 33.2 & 64.1 & 166 & 154 & 62.6 & 29.9 & 57.7 \\
\hline 1968 & 31.2 & 37.8 & 32.0 & 30.2 & 25.3 & 30.4 & 47.6 & 83.6 & 257 & 239 & 106 & 40.3 & 80.1 \\
\hline 1969 & 43.0 & 38.5 & 42.1 & 33.9 & 29.2 & 50.2 & 144 & 178 & 285 & 267 & 133 & 55.2 & 109 \\
\hline 1970 & 47.0 & 42.9 & 38.2 & 31.3 & 30.2 & 29.6 & 41.9 & 93.1 & 161 & 99.8 & 66.3 & 47.9 & 60.9 \\
\hline 1971 & 41.0 & 38.6 & 37.2 & 30.8 & 29.6 & 31.6 & 36.8 & 84.8 & 119 & 53.0 & 51.6 & 32.5 & 48.9 \\
\hline 1972 & 36.7 & 33.1 & 29.4 & 26.7 & 23.8 & 26.7 & 40.3 & 69.2 & 148 & 98.6 & 65.0 & 32.4 & 52.5 \\
\hline 1973 & 31.0 & 31.0 & 31.0 & 31.0 & 32.6 & 40.7 & 50.4 & 88.8 & 162 & 144 & 70.4 & 32.1 & 62.3 \\
\hline 1974 & 35.6 & 33.5 & 36.7 & 29.6 & 24.8 & 37.5 & 47.5 & 51.9 & 111 & 113 & 46.3 & 28.4 & 49.8 \\
\hline 1975 & 37.1 & 35.4 & 33.9 & 32.6 & 28.2 & 27.4 & 45.9 & 88 & 172 & 164 & 84.9 & 33.9 & 65.5 \\
\hline 1976 & 36.9 & 38.1 & 32.5 & 27.7 & 35.2 & 33.7 & 74.7 & 101 & 137 & 210 & 65.6 & 37.7 & 69.3 \\
\hline 1977 & 33.0 & 37.5 & 40.6 & 35.8 & 32.0 & 25.1 & 24.9 & 77.8 & 213 & 146 & 48.0 & 26.1 & 61.7 \\
\hline 1978 & 34.5 & 36.2 & 36.0 & 29.0 & 26.9 & 29.0 & 62.1 & 123 & 240 & 133 & 57.7 & 35.3 & 70.3 \\
\hline
\end{tabular}




\section{4-1.R00-5A FARKHAR RIVER NEAR TALOQAN}

\section{(U.S. Geological Survey identification number: 363800069430000 )}

LOCATION: Lat $36^{\circ} 38^{\prime} \mathrm{N}$., long 69² $43^{\prime} \mathrm{E}$.

DRAINAGE AREA: $4,110 \mathrm{~km}^{2}$.

ELEVATION: 991 meters above mean sea level.

PERIOD OF RECORD: November 22, 1966 to September 30, 1978.

GAGE: Water-stage recorder.

Annual mean discharge

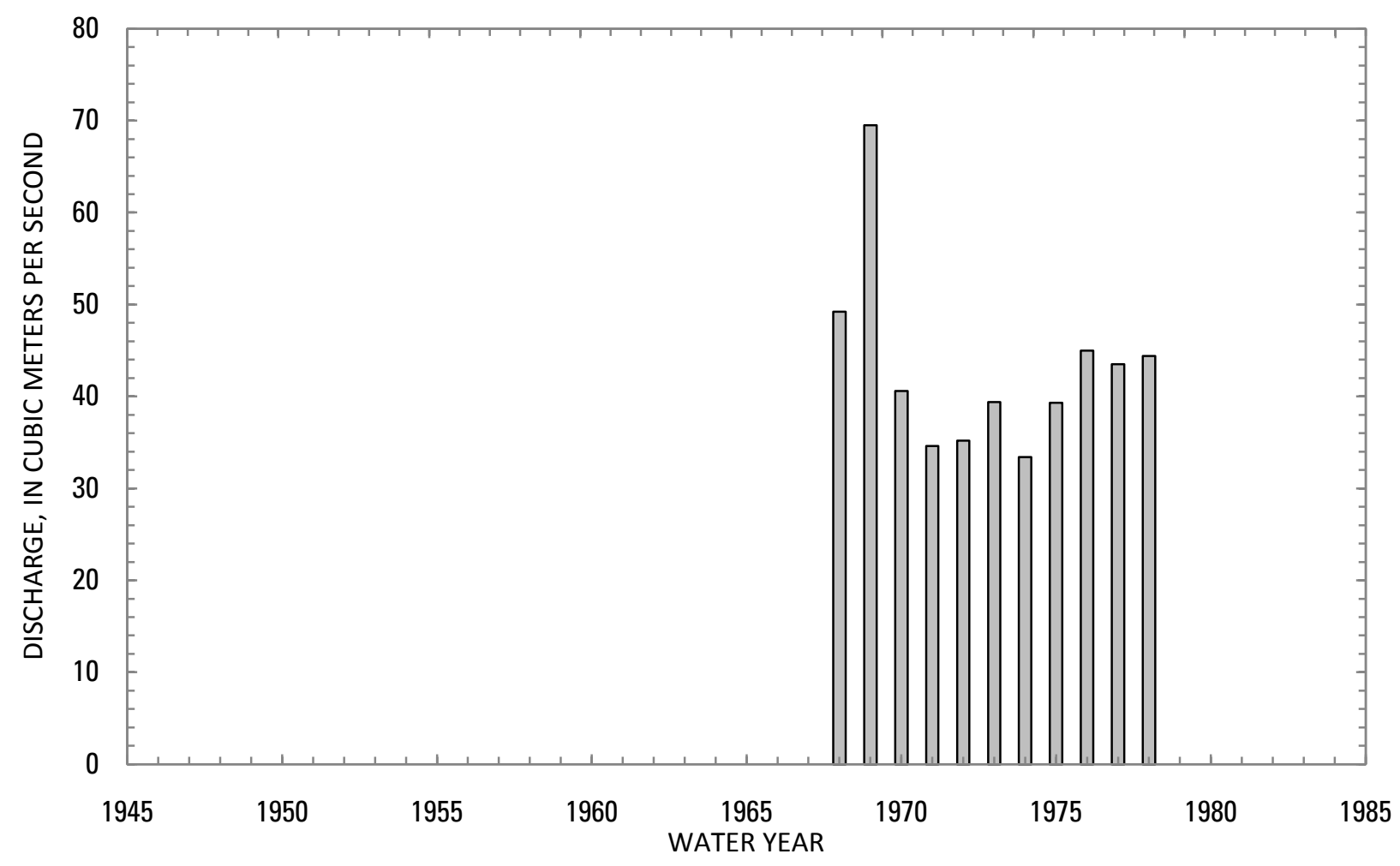




\section{4-1.R00-5A FARKHAR RIVER NEAR TALOQAN, Continued}

Statistics of monthly and annual mean discharges $\left[\mathrm{m}^{3} / \mathrm{s}\right.$, cubic meters per second]

\begin{tabular}{|c|c|c|c|c|c|c|c|c|}
\hline \multirow[b]{2}{*}{ Month } & \multicolumn{2}{|c|}{ Maximum } & \multicolumn{2}{|c|}{ Minimum } & \multicolumn{4}{|c|}{ Mean } \\
\hline & $\begin{array}{c}\text { Discharge } \\
\left(\mathrm{m}^{3} / \mathrm{s}\right)\end{array}$ & $\begin{array}{c}\text { Water year } \\
\text { of } \\
\text { occurrence }\end{array}$ & $\begin{array}{c}\text { Discharge } \\
\left(\mathrm{m}^{3} / \mathrm{s}\right)\end{array}$ & $\begin{array}{c}\text { Water year } \\
\text { of } \\
\text { occurrence }\end{array}$ & $\begin{array}{c}\text { Discharge } \\
\left(\mathrm{m}^{3} / \mathrm{s}\right)\end{array}$ & $\begin{array}{c}\text { Standard } \\
\text { deviation } \\
\left(\mathrm{m}^{3} / \mathrm{s}\right)\end{array}$ & $\begin{array}{c}\text { Coefficient } \\
\text { of } \\
\text { variation }\end{array}$ & $\begin{array}{c}\text { Percentage } \\
\text { of annual } \\
\text { discharge }\end{array}$ \\
\hline October & 24.5 & 1970 & 15.9 & 1972 & 20.1 & 2.74 & 0.14 & 3.95 \\
\hline November & 18.2 & 1970 & 12.6 & 1975 & 15.7 & 1.74 & 0.11 & 3.08 \\
\hline December & 18.4 & 1969 & 11.6 & 1972 & 13.4 & 1.88 & 0.14 & 2.63 \\
\hline January & 14.7 & 1969 & 10.2 & 1971 & 11.7 & 1.56 & 0.13 & 2.30 \\
\hline February & 14.1 & 1973 & 8.83 & 1971 & 11.1 & 1.67 & 0.15 & 2.18 \\
\hline March & 20.4 & 1969 & 9.49 & 1967 & 12.8 & 3.32 & 0.26 & 2.51 \\
\hline April & 82.0 & 1969 & 14.0 & 1977 & 29.0 & 17.9 & 0.62 & 5.70 \\
\hline May & 123 & 1969 & 35.4 & 1974 & 59.8 & 22.1 & 0.37 & 11.8 \\
\hline June & 184 & 1969 & 85.0 & 1974 & 124 & 30.5 & 0.25 & 24.4 \\
\hline July & 182 & 1969 & 56.1 & 1971 & 113 & 38.8 & 0.34 & 22.3 \\
\hline August & 110 & 1969 & 47.2 & 1974 & 65.1 & 17.9 & 0.28 & 12.8 \\
\hline September & 48.7 & 1970 & 24.0 & 1973 & 32.5 & 7.36 & 0.23 & 6.39 \\
\hline Annual & 69.5 & 1969 & 33.4 & 1974 & 43.1 & 10.0 & 0.23 & 100 \\
\hline
\end{tabular}


14-1.R00-5A FARKHAR RIVER NEAR TALOQAN, Continued

Monthly and annual flow duration, in cubic meters per second

\begin{tabular}{|c|c|c|c|c|c|c|c|c|c|c|c|c|c|}
\hline \multirow{2}{*}{$\begin{array}{l}\text { Percentage } \\
\text { of days } \\
\text { discharge } \\
\text { equaled or } \\
\text { exceeded }\end{array}$} & \multicolumn{12}{|c|}{ Month } & \multirow{2}{*}{ Annual } \\
\hline & October & November & December & January & February & March & April & May & June & July & August & September & \\
\hline 95 & 14.9 & 11.9 & 11.4 & 9.70 & 8.99 & 9.01 & 11.0 & 19.5 & 64.4 & 55.0 & 37.7 & 21.3 & 9.83 \\
\hline 90 & 15.6 & 12.7 & 11.6 & 10.0 & 9.11 & 9.34 & 12.0 & 23.7 & 71.5 & 64.6 & 41.9 & 23.0 & 10.8 \\
\hline 85 & 17.0 & 13.2 & 11.8 & 10.1 & 9.53 & 9.59 & 12.7 & 28.5 & 77.2 & 69.0 & 44.7 & 23.9 & 11.6 \\
\hline 80 & 17.4 & 13.8 & 12.0 & 10.5 & 9.66 & 9.82 & 13.6 & 31.1 & 84.7 & 73.3 & 47.2 & 25.0 & 12.4 \\
\hline 75 & 17.8 & 14.2 & 12.0 & 10.6 & 9.93 & 9.99 & 14.4 & 33.7 & 90.1 & 79.7 & 48.6 & 26.2 & 13.1 \\
\hline 70 & 18.1 & 14.6 & 12.1 & 10.7 & 9.99 & 10.2 & 15.4 & 36.2 & 95.4 & 84.2 & 50.5 & 26.9 & 13.9 \\
\hline 65 & 18.3 & 15.1 & 12.4 & 11.0 & 10.0 & 10.4 & 17.6 & 38.9 & 101 & 88.0 & 53.1 & 27.8 & 15.2 \\
\hline 60 & 18.6 & 15.4 & 12.5 & 11.1 & 10.1 & 10.7 & 19.6 & 42.3 & 106 & 91.3 & 55.7 & 28.9 & 16.7 \\
\hline 55 & 19.0 & 15.7 & 12.7 & 11.2 & 10.4 & 11.1 & 20.6 & 46.7 & 111 & 94.1 & 58.3 & 30.0 & 18.4 \\
\hline 50 & 19.6 & 16.1 & 12.9 & 11.3 & 10.6 & 11.8 & 22.1 & 50.9 & 117 & 99.6 & 60.9 & 30.7 & 20.6 \\
\hline 45 & 20.1 & 16.2 & 13.0 & 11.5 & 10.7 & 12.0 & 23.8 & 55.3 & 122 & 104 & 64.8 & 31.7 & 24.5 \\
\hline 40 & 20.5 & 16.6 & 13.1 & 11.6 & 11.0 & 12.2 & 25.3 & 60.7 & 127 & 109 & 68.3 & 32.9 & 29.4 \\
\hline 35 & 21.0 & 16.7 & 13.4 & 11.9 & 11.9 & 12.4 & 27.1 & 66.4 & 134 & 116 & 70.8 & 34.1 & 36.4 \\
\hline 30 & 22.0 & 16.8 & 13.7 & 12.6 & 12.1 & 13.0 & 29.5 & 71.3 & 140 & 126 & 73.6 & 35.2 & 45.7 \\
\hline 25 & 23.0 & 17.1 & 14.2 & 13.0 & 12.3 & 13.7 & 34.0 & 75.9 & 149 & 141 & 75.5 & 36.2 & 59.2 \\
\hline 20 & 23.7 & 17.4 & 15.0 & 13.1 & 12.9 & 14.8 & 37.7 & 81.8 & 159 & 156 & 77.5 & 38.0 & 72.1 \\
\hline 15 & 24.4 & 17.8 & 15.5 & 13.7 & 13.3 & 16.3 & 41.3 & 92.3 & 174 & 170 & 82.1 & 39.9 & 86.8 \\
\hline 10 & 25.2 & 18.0 & 16.1 & 14.3 & 13.7 & 18.2 & 47.2 & 109 & 190 & 194 & 94.5 & 45.2 & 109 \\
\hline 5 & 26.9 & 19.0 & 18.1 & 14.9 & 14.1 & 20.3 & 71.9 & 137 & 221 & 212 & 114 & 53.7 & 146 \\
\hline
\end{tabular}




\section{4-1.R00-5A FARKHAR RIVER NEAR TALOQAN, Continued}

Probability of occurrence of annual high discharges

[m $\mathrm{m}^{3} / \mathrm{s}$, cubic meters per second; ng, not given]

\begin{tabular}{|c|c|c|c|c|c|c|}
\hline \multirow{2}{*}{$\begin{array}{l}\text { Exceedance } \\
\text { probability }\end{array}$} & \multirow{2}{*}{$\begin{array}{l}\text { Recurrence } \\
\text { interval } \\
\text { (years) }\end{array}$} & \multirow{2}{*}{$\begin{array}{c}\text { Maximum } \\
\text { instantaneous } \\
\text { discharge } \\
\left(\mathrm{m}^{3} / \mathrm{s}\right)\end{array}$} & \multicolumn{4}{|c|}{ Maximum daily mean discharge $\left(\mathrm{m}^{3} / \mathrm{s}\right)$} \\
\hline & & & $\begin{array}{l}\text { 3-day } \\
\text { period }\end{array}$ & $\begin{array}{l}\text { 7-day } \\
\text { period }\end{array}$ & $\begin{array}{l}\text { 15-day } \\
\text { period }\end{array}$ & $\begin{array}{l}\text { 30-day } \\
\text { period }\end{array}$ \\
\hline 0.99 & 1.01 & 104 & 102 & 92.0 & 84.1 & 80.2 \\
\hline 0.95 & 1.05 & 128 & 118 & 109 & 98.7 & 93.3 \\
\hline 0.90 & 1.11 & 143 & 129 & 119 & 108 & 102 \\
\hline 0.80 & 1.25 & 166 & 144 & 134 & 121 & 113 \\
\hline 0.50 & 2 & 223 & 181 & 170 & 153 & 140 \\
\hline 0.20 & 5 & 305 & 233 & 220 & 197 & 177 \\
\hline 0.10 & 10 & 363 & 268 & 253 & 227 & 201 \\
\hline 0.04 & 25 & 440 & 314 & 297 & 265 & 231 \\
\hline 0.02 & 50 & 499 & 349 & 330 & 295 & 254 \\
\hline 0.01 & 100 & 561 & 384 & 363 & 325 & 277 \\
\hline 0.005 & 200 & 625 & 421 & 398 & 355 & 300 \\
\hline 0.002 & 500 & 715 & ng & $\mathrm{ng}$ & $\mathrm{ng}$ & $\mathrm{ng}$ \\
\hline
\end{tabular}

14-1.R00-5A FARKHAR RIVER NEAR TALOQAN, Continued

Probability of occurrence of annual low discharges $\left[\mathrm{m}^{3} / \mathrm{s}\right.$, meters per second]

\begin{tabular}{|c|c|c|c|c|c|c|c|c|c|c|}
\hline \multirow{3}{*}{$\begin{array}{c}\text { Nonexceedance } \\
\text { probability }\end{array}$} & \multirow{3}{*}{$\begin{array}{c}\text { Recurrence } \\
\text { interval } \\
\text { (years) }\end{array}$} & \multicolumn{9}{|c|}{ Minimum daily mean discharge $\left(\mathrm{m}^{3} / \mathrm{s}\right)$} \\
\hline & & \multicolumn{9}{|c|}{ Number of consecutive days } \\
\hline & & 1 & 3 & 7 & 14 & 30 & 60 & 90 & 120 & 183 \\
\hline 0.05 & 20 & 8.17 & 8.24 & 8.48 & 8.62 & 8.83 & 9.02 & 9.51 & 10.2 & 12.1 \\
\hline 0.10 & 10 & 8.45 & 8.54 & 8.76 & 8.91 & 9.12 & 9.37 & 9.83 & 10.5 & 12.5 \\
\hline 0.20 & 5 & 8.85 & 8.95 & 9.15 & 9.31 & 9.53 & 9.84 & 10.3 & 10.9 & 13.1 \\
\hline 0.50 & 2 & 9.81 & 9.92 & 10.1 & 10.3 & 10.5 & 10.9 & 11.4 & 11.9 & 14.2 \\
\hline
\end{tabular}




\section{4-1.R00-5A FARKHAR RIVER NEAR TALOQAN, Continued}

Probability of occurrence of seasonal low discharges [m $\mathrm{m}^{3} / \mathrm{s}$, meters per second]

\begin{tabular}{|c|c|c|c|c|c|c|c|c|c|}
\hline \multirow{3}{*}{$\begin{array}{c}\text { Nonexceedance } \\
\text { probability }\end{array}$} & \multirow{3}{*}{$\begin{array}{c}\text { Recurrence } \\
\text { interval } \\
\text { (years) }\end{array}$} & \multicolumn{8}{|c|}{ Minimum daily mean discharge $\left(\mathrm{m}^{3} / \mathrm{s}\right)$} \\
\hline & & \multicolumn{8}{|c|}{ Number of consecutive days } \\
\hline & & 1 & 7 & 14 & 30 & 1 & 7 & 14 & 30 \\
\hline & & \multicolumn{4}{|c|}{ December-January-February } & \multicolumn{4}{|c|}{ March-April-May } \\
\hline 0.05 & 20 & 8.23 & 8.53 & 8.72 & 8.97 & 7.88 & 8.25 & 8.4 & 8.96 \\
\hline 0.10 & 10 & 8.52 & 8.77 & 8.95 & 9.21 & 8.31 & 8.69 & 8.86 & 9.46 \\
\hline 0.20 & 5 & 8.92 & 9.12 & 9.29 & 9.57 & 8.87 & 9.29 & 9.49 & 10.2 \\
\hline \multirow[t]{2}{*}{0.50} & 2 & 9.88 & 10.0 & 10.2 & 10.5 & 10.1 & 10.6 & 11.0 & 12.0 \\
\hline & & \multicolumn{4}{|c|}{ June-July-August } & \multicolumn{4}{|c|}{ September-October-November } \\
\hline 0.05 & 20 & 28.7 & 31.6 & 37.4 & 46.1 & 10.5 & 11.4 & 11.8 & 12.3 \\
\hline 0.10 & 10 & 30.7 & 33.8 & 39.1 & 47.9 & 11.3 & 12.1 & 12.5 & 13.1 \\
\hline 0.20 & 5 & 33.6 & 37.0 & 41.8 & 50.9 & 12.4 & 12.9 & 13.3 & 14.1 \\
\hline 0.50 & 2 & 41.0 & 44.8 & 49.6 & 59.8 & 14.2 & 14.5 & 14.9 & 15.8 \\
\hline
\end{tabular}

\section{4-1.R00-5A FARKHAR RIVER NEAR TALOQAN, Continued}

Annual peak discharges

[m $\mathrm{m}^{3} / \mathrm{s}$, meters per second]

\begin{tabular}{|c|c|c|c|c|c|}
\hline \multicolumn{3}{|c|}{$\begin{array}{c}\text { Annual peak discharge, } \\
\text { by year }\end{array}$} & \multicolumn{3}{|c|}{$\begin{array}{l}\text { Annual peak discharge, } \\
\text { from highest to lowest }\end{array}$} \\
\hline $\begin{array}{l}\text { Water } \\
\text { year }\end{array}$ & Date & $\begin{array}{c}\text { Peak } \\
\text { discharge } \\
\left(\mathrm{m}^{3} / \mathrm{s}\right)\end{array}$ & $\begin{array}{l}\text { Water } \\
\text { year }\end{array}$ & Date & $\begin{array}{c}\text { Peak } \\
\text { discharge } \\
\left(\mathrm{m}^{3} / \mathrm{s}\right)\end{array}$ \\
\hline 1967 & June 11, 1967 & 150 & 1969 & April 19, 1969 & 400 \\
\hline 1968 & July 9, 1968 & 276 & 1976 & May 24, 1976 & 343 \\
\hline 1969 & April 19, 1969 & 400 & 1978 & April 22, 1978 & 330 \\
\hline 1970 & June 2, 1970 & 167 & 1977 & June 23, 1977 & 320 \\
\hline 1971 & May 28, 1971 & 191 & 1968 & July 9, 1968 & 276 \\
\hline 1972 & April 15, 1972 & 189 & 1974 & June 18,1974 & 202 \\
\hline 1973 & June 12, 1973 & 135 & 1971 & May 28, 1971 & 191 \\
\hline 1974 & June 18,1974 & 202 & 1972 & April 15, 1972 & 189 \\
\hline 1975 & June 16,1975 & 181 & 1975 & June 16, 1975 & 181 \\
\hline 1976 & May 24, 1976 & 343 & 1970 & June 2, 1970 & 167 \\
\hline 1977 & June 23, 1977 & 320 & 1967 & June 11, 1967 & 150 \\
\hline 1978 & April 22, 1978 & 330 & 1973 & June 12, 1973 & 135 \\
\hline
\end{tabular}


14-1.R00-5A FARKHAR RIVER NEAR TALOQAN, Continued

Monthly and annual mean discharges, in cubic meters per second $[--$, no data]

\begin{tabular}{|c|c|c|c|c|c|c|c|c|c|c|c|c|c|}
\hline \multirow{2}{*}{$\begin{array}{l}\text { Water } \\
\text { year }\end{array}$} & \multicolumn{12}{|c|}{ Monthly mean discharge } & \multirow{2}{*}{$\begin{array}{c}\text { Annual } \\
\text { discharge }\end{array}$} \\
\hline & October & November & December & January & February & March & April & May & June & July & August & September & \\
\hline 1967 & -- & -- & 11.8 & 10.3 & 9.63 & 9.49 & 15.4 & 44.5 & 110 & 102 & 56.6 & 32.9 & -- \\
\hline 1968 & 21.8 & 16.6 & 12.9 & 11.1 & 9.99 & 12.2 & 24.9 & 43.7 & 154 & 162 & 86.5 & 32.8 & 49.2 \\
\hline 1969 & 21.7 & 17.2 & 18.4 & 14.7 & 11.5 & 20.4 & 82.0 & 123 & 184 & 182 & 110 & 43.7 & 69.5 \\
\hline 1970 & 24.5 & 18.2 & 13.3 & 10.4 & 10.4 & 10.8 & 21.6 & 59.4 & 112 & 83.1 & 73.2 & 48.7 & 40.6 \\
\hline 1971 & 22.3 & 15.8 & 12.8 & 10.2 & 8.83 & 10.1 & 21.5 & 70.6 & 109 & 56.1 & 49.7 & 26.6 & 34.6 \\
\hline 1972 & 15.9 & 13.6 & 11.6 & 10.7 & 10.2 & 13.0 & 24.3 & 52.5 & 107 & 78.6 & 56.4 & 28.0 & 35.2 \\
\hline 1973 & 18.2 & 16.8 & 15.5 & 14.4 & 14.1 & 16.2 & 28.1 & 64.0 & 105 & 92.8 & 61.7 & 24.0 & 39.4 \\
\hline 1974 & 17.6 & 13.9 & 12.2 & 12.0 & 13.1 & 16.4 & 24.5 & 35.4 & 85.0 & 95.3 & 47.2 & 26.3 & 33.4 \\
\hline 1975 & 16.7 & 12.6 & 12.8 & 10.8 & 9.98 & 10.2 & 22.0 & 54.4 & 113 & 104 & 68.6 & 34.2 & 39.3 \\
\hline 1976 & 22.5 & 17.0 & 14.0 & 13.0 & 12.1 & 12.0 & 34.0 & 54.1 & 96.6 & 164 & 63.7 & 35.6 & 45.0 \\
\hline 1977 & 20.5 & 14.9 & 13.0 & 12.0 & 10.1 & 9.82 & 14.0 & 54.8 & 152 & 136 & 54.5 & 27.9 & 43.5 \\
\hline 1978 & 19.3 & 16.0 & 12.5 & 11.0 & 13.2 & 12.5 & 36.0 & 61.6 & 161 & 106 & 52.9 & 29.7 & 44.4 \\
\hline
\end{tabular}




\section{4-5.R00-1A ANDARAB RIVER AT DOSHI}

\section{(U.S. Geological Survey identification number: 353600068410000)}

LOCATION: Lat $35^{\circ} 36^{\prime} \mathrm{N}$., long 68' $41^{\prime} \mathrm{E}$.

DRAINAGE AREA: $3,705 \mathrm{~km}^{2}$.

ELEVATION: 860 meters above mean sea level.

PERIOD OF RECORD: October 1, 1964 to September 30, 1978.

GAGE: Water-stage recorder.

Annual mean discharge

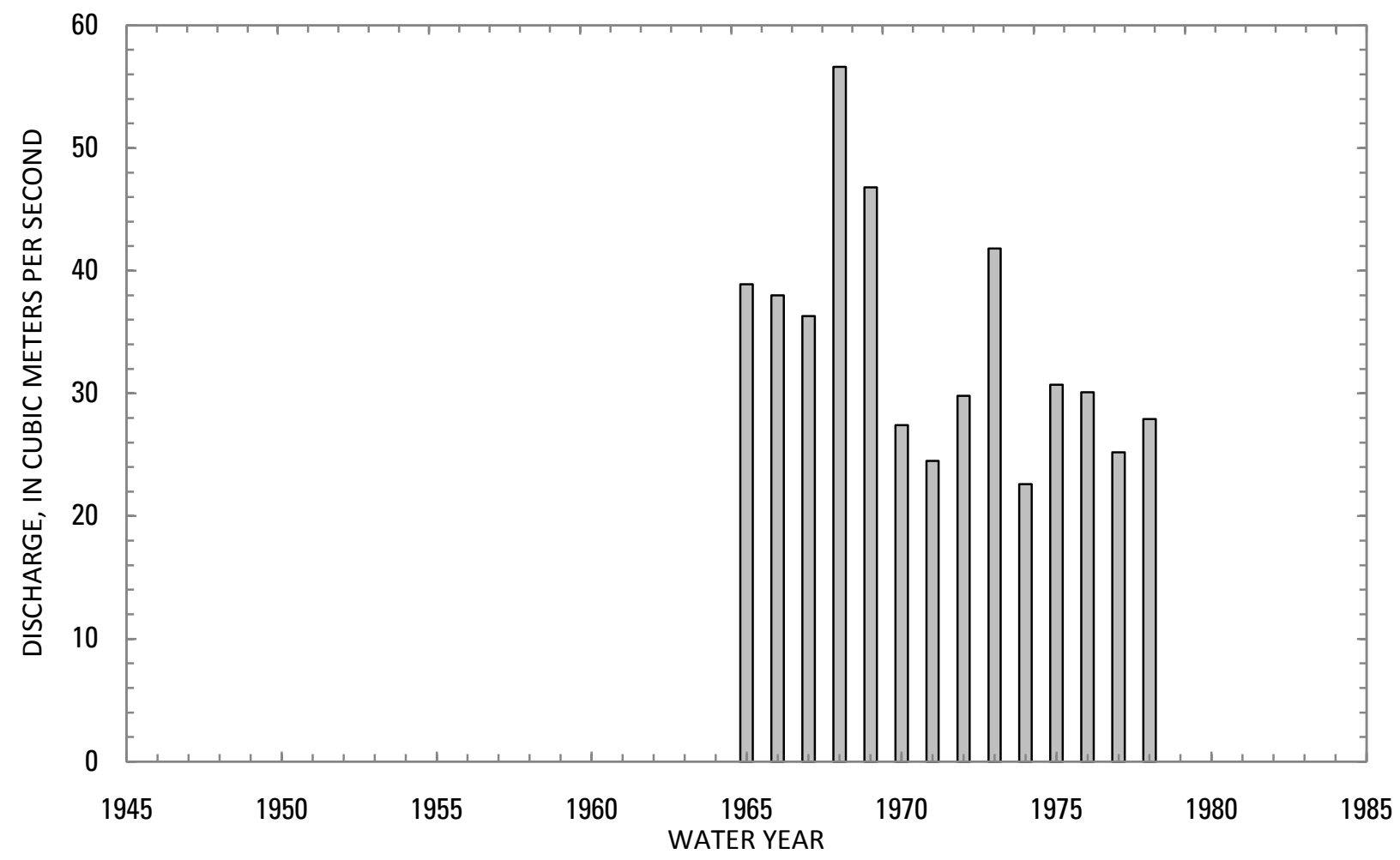




\section{4-5.R00-1A ANDARAB RIVER AT DOSHI, Continued}

Statistics of monthly and annual mean discharges [ $\mathrm{m}^{3} / \mathrm{s}$, cubic meters per second]

\begin{tabular}{|c|c|c|c|c|c|c|c|c|}
\hline \multirow[b]{2}{*}{ Month } & \multicolumn{2}{|c|}{ Maximum } & \multicolumn{2}{|c|}{ Minimum } & \multicolumn{4}{|c|}{ Mean } \\
\hline & $\begin{array}{c}\text { Discharge } \\
\left(\mathrm{m}^{3} / \mathrm{s}\right)\end{array}$ & $\begin{array}{l}\text { Water year } \\
\text { of } \\
\text { occurrence }\end{array}$ & $\begin{array}{c}\text { Discharge } \\
\left(\mathrm{m}^{3} / \mathrm{s}\right)\end{array}$ & $\begin{array}{c}\text { Water year } \\
\text { of } \\
\text { occurrence }\end{array}$ & $\begin{array}{c}\text { Discharge } \\
\left(\mathrm{m}^{3} / \mathrm{s}\right)\end{array}$ & $\begin{array}{c}\text { Standard } \\
\text { deviation } \\
\left(\mathrm{m}^{3} / \mathrm{s}\right)\end{array}$ & $\begin{array}{c}\text { Coefficient } \\
\text { of } \\
\text { variation }\end{array}$ & $\begin{array}{c}\text { Percentage } \\
\text { of annual } \\
\text { discharge }\end{array}$ \\
\hline October & 22.5 & 1969 & 11.7 & 1972 & 16.5 & 3.31 & 0.20 & 4.05 \\
\hline November & 18.6 & 1978 & 10.3 & 1975 & 14.0 & 2.51 & 0.18 & 3.44 \\
\hline December & 15.0 & 1973 & 9.40 & 1972 & 12.2 & 1.98 & 0.16 & 3.00 \\
\hline January & 14.0 & 1973 & 8.49 & 1975 & 11.2 & 1.75 & 0.16 & 2.75 \\
\hline February & 13.6 & 1973 & 8.13 & 1975 & 10.9 & 1.70 & 0.16 & 2.66 \\
\hline March & 19.3 & 1966 & 8.12 & 1975 & 12.2 & 3.23 & 0.26 & 3.00 \\
\hline April & 61.2 & 1973 & 10.1 & 1972 & 24.1 & 12.9 & 0.54 & 5.91 \\
\hline May & 111 & 1973 & 37.9 & 1972 & 67.2 & 21.0 & 0.31 & 16.5 \\
\hline June & 224 & 1968 & 57.7 & 1971 & 117 & 48.1 & 0.41 & 28.7 \\
\hline July & 169 & 1968 & 28.3 & 1971 & 73.6 & 39.1 & 0.53 & 18.1 \\
\hline August & 58.0 & 1968 & 17.8 & 1971 & 31.1 & 12.6 & 0.41 & 7.62 \\
\hline September & 27.5 & 1969 & 10.9 & 1974 & 17.4 & 4.58 & 0.26 & 4.28 \\
\hline Annual & 56.6 & 1968 & 22.6 & 1974 & 34.0 & 9.62 & 0.28 & 100 \\
\hline
\end{tabular}


14-5.R00-1A ANDARAB RIVER AT DOSHI, Continued

Monthly and annual flow duration, in cubic meters per second

[ng, not given]

\begin{tabular}{|c|c|c|c|c|c|c|c|c|c|c|c|c|c|}
\hline \multirow{2}{*}{$\begin{array}{l}\text { Percentage } \\
\text { of days } \\
\text { discharge } \\
\text { equaled or } \\
\text { exceeded }\end{array}$} & \multicolumn{12}{|c|}{ Month } & \multirow{2}{*}{ Annual } \\
\hline & October & November & December & January & February & March & April & May & June & July & August & September & \\
\hline 95 & 11.4 & 10.2 & 9.10 & 8.48 & 8.10 & 8.03 & 9.44 & 16.2 & 44.5 & 25.6 & 15.7 & 11.3 & 9.03 \\
\hline 90 & 12.3 & 11.0 & 9.53 & 8.73 & 8.59 & 8.26 & 10.1 & 26.6 & 51.5 & 31.1 & 17.6 & 12.3 & 10.0 \\
\hline 85 & 13.1 & 11.4 & 10.0 & 9.01 & 9.05 & 8.64 & 10.8 & 30.5 & 58.8 & 35.3 & 18.9 & 13.0 & 10.6 \\
\hline 80 & 14.0 & 11.6 & 10.2 & 9.41 & 9.35 & 8.99 & 11.7 & 34.4 & 70.1 & 41.4 & 19.7 & 13.3 & 11.2 \\
\hline 75 & 14.4 & 11.8 & 10.6 & 10.0 & 9.66 & 9.45 & 12.8 & 37.8 & 75.2 & 44.3 & 20.5 & 14.0 & 12.0 \\
\hline 70 & 14.9 & 12.3 & 10.9 & 10.1 & 9.77 & 9.82 & 13.9 & 44.2 & 80.8 & 46.9 & 21.3 & 14.3 & 12.7 \\
\hline 65 & 15.1 & 13.1 & 11.1 & 10.3 & 10.0 & 10.2 & 14.7 & 49.4 & 85.0 & 50.1 & 22.3 & 15.1 & 13.3 \\
\hline 60 & 15.4 & 13.3 & 11.6 & 10.7 & 10.2 & 10.5 & 15.5 & 53.9 & 88.9 & 52.7 & 23.4 & 15.5 & 13.9 \\
\hline 55 & 15.8 & 13.5 & 11.8 & 11.0 & 10.3 & 10.9 & 16.3 & 59.1 & 97.1 & 55.1 & 25.2 & 16.2 & 14.8 \\
\hline 50 & 16.0 & 13.9 & 12.0 & 11.1 & 10.6 & 11.3 & 17.8 & 63.9 & 103 & 57.8 & 26.5 & 16.9 & 16.0 \\
\hline 45 & 16.4 & 14.1 & 12.5 & 11.3 & 11.0 & 12.1 & 19.6 & 69.4 & 118 & 60.9 & 28.4 & 17.9 & 17.6 \\
\hline 40 & 16.8 & 14.3 & 12.7 & 11.7 & 11.2 & 12.7 & 23.1 & 72.3 & 129 & 66.0 & 30.6 & 18.2 & 19.6 \\
\hline 35 & 17.0 & 14.6 & 13.1 & 12.0 & 11.3 & 13.2 & 24.5 & 75.2 & 143 & 72.8 & 32.3 & 18.5 & 22.6 \\
\hline 30 & 17.5 & 15.0 & 13.6 & 12.5 & 11.7 & 13.7 & 26.0 & 80.8 & 151 & 83.5 & 34.1 & 18.9 & 28.3 \\
\hline 25 & 18.1 & 15.6 & 14.1 & 12.7 & 12.1 & 14.4 & 29.6 & 87.6 & 157 & 91.2 & 36.5 & 19.4 & 36.8 \\
\hline 20 & 19.1 & 16.2 & 14.3 & 13.0 & 12.6 & 15.0 & 32.8 & 97.2 & 164 & 112 & 40.0 & 20.3 & 48.8 \\
\hline 15 & 20.5 & 17.1 & 14.6 & 13.2 & 13.1 & 15.9 & 39.9 & 108 & 174 & 124 & 43.0 & 22.3 & 66.1 \\
\hline 10 & 22.0 & 18.2 & 15.0 & 13.8 & 14.0 & 17.5 & 47.1 & 118 & 187 & 138 & 48.3 & 24.8 & 84.3 \\
\hline 5 & 23.7 & 19.6 & 15.8 & 14.2 & 14.2 & 19.9 & 59.1 & 140 & 206 & 159 & 66.1 & 27.4 & 129 \\
\hline
\end{tabular}




\section{4-5.R00-1A ANDARAB RIVER AT DOSHI, Continued}

Probability of occurrence of annual high discharges

[ $\mathrm{m}^{3} / \mathrm{s}$, cubic meters per second; ng, not given]

\begin{tabular}{|c|c|c|c|c|c|c|}
\hline \multirow{2}{*}{$\begin{array}{c}\text { Exceedance } \\
\text { probability }\end{array}$} & \multirow{2}{*}{$\begin{array}{c}\text { Recurrence } \\
\text { interval } \\
\text { (years) }\end{array}$} & \multirow{2}{*}{$\begin{array}{l}\text { Maximum } \\
\text { instantaneous } \\
\text { discharge } \\
\left(\mathrm{m}^{3} / \mathrm{s}\right)\end{array}$} & \multicolumn{4}{|c|}{ Maximum daily mean discharge $\left(\mathrm{m}^{3} / \mathrm{s}\right)$} \\
\hline & & & $\begin{array}{l}\text { 3-day } \\
\text { period }\end{array}$ & $\begin{array}{l}\text { 7-day } \\
\text { period }\end{array}$ & $\begin{array}{l}\text { 15-day } \\
\text { period }\end{array}$ & $\begin{array}{l}\text { 30-day } \\
\text { period }\end{array}$ \\
\hline 0.99 & 1.01 & 128 & 79.1 & 66.5 & 58.0 & 52.6 \\
\hline 0.95 & 1.05 & 134 & 96.0 & 82.9 & 74.0 & 67.0 \\
\hline 0.90 & 1.11 & 139 & 107 & 93.6 & 84.4 & 76.2 \\
\hline 0.80 & 1.25 & 147 & 122 & 109 & 98.8 & 89.1 \\
\hline 0.50 & 2 & 173 & 159 & 146 & 134 & 120 \\
\hline 0.20 & 5 & 222 & 210 & 199 & 181 & 163 \\
\hline 0.10 & 10 & 262 & 245 & 236 & 212 & 191 \\
\hline 0.04 & 25 & 323 & 289 & 283 & 251 & 227 \\
\hline 0.02 & 50 & 375 & 323 & 320 & 280 & 253 \\
\hline 0.01 & 100 & 435 & 357 & 357 & 309 & 280 \\
\hline 0.005 & 200 & 502 & '393 & 395 & 338 & 307 \\
\hline 0.002 & 500 & 606 & $\mathrm{ng}$ & ng & ng & ng \\
\hline
\end{tabular}

'Data does not fit log-Pearson Type III curve, use with caution.

14-5.R00-1A ANDARAB RIVER AT DOSHI, Continued

Probability of occurrence of annual low discharges

$\left[\mathrm{m}^{3} / \mathrm{s}\right.$, meters per second]

\begin{tabular}{|c|c|c|c|c|c|c|c|c|c|c|}
\hline \multirow{3}{*}{$\begin{array}{c}\text { Nonexceedance } \\
\text { probability }\end{array}$} & \multirow{3}{*}{$\begin{array}{c}\text { Recurrence } \\
\text { interval } \\
\text { (years) }\end{array}$} & \multicolumn{9}{|c|}{ Minimum daily mean discharge $\left(\mathrm{m}^{3} / \mathrm{s}\right)$} \\
\hline & & \multicolumn{9}{|c|}{ Number of consecutive days } \\
\hline & & 1 & 3 & 7 & 14 & 30 & 60 & 90 & 120 & 183 \\
\hline 0.05 & 20 & 7.09 & 7.29 & 7.49 & 7.69 & 8.01 & 8.18 & 8.42 & 8.72 & 9.64 \\
\hline 0.10 & 10 & 7.46 & 7.62 & 7.79 & 8.00 & 8.35 & 8.59 & 8.86 & 9.23 & 10.3 \\
\hline 0.20 & 5 & 7.93 & 8.04 & 8.17 & 8.40 & 8.77 & 9.13 & 9.44 & 9.89 & 11.1 \\
\hline 0.50 & 2 & 8.86 & 8.91 & 9.02 & 9.26 & 9.64 & 10.3 & 10.7 & 11.2 & 12.7 \\
\hline
\end{tabular}


14-5.R00-1A ANDARAB RIVER AT DOSHI, Continued

Probability of occurrence of seasonal low discharges $\left[\mathrm{m}^{3} / \mathrm{s}\right.$, meters per second]

\begin{tabular}{|c|c|c|c|c|c|c|c|c|c|}
\hline \multirow{3}{*}{$\begin{array}{c}\text { Nonexceedance } \\
\text { probability }\end{array}$} & \multirow{3}{*}{$\begin{array}{c}\text { Recurrence } \\
\text { interval } \\
\text { (years) }\end{array}$} & \multicolumn{8}{|c|}{ Minimum daily mean discharge $\left(\mathrm{m}^{3} / \mathrm{s}\right)$} \\
\hline & & \multicolumn{8}{|c|}{ Number of consecutive days } \\
\hline & & 1 & 7 & 14 & 30 & 1 & 7 & 14 & 30 \\
\hline & & \multicolumn{4}{|c|}{ December-January-February } & \multicolumn{4}{|c|}{ March-April-May } \\
\hline 0.05 & 20 & 7.87 & 7.83 & 8.00 & 8.27 & 6.75 & 7.16 & 7.53 & 7.96 \\
\hline 0.10 & 10 & 8.13 & 8.16 & 8.34 & 8.65 & 7.30 & 7.68 & 8.03 & 8.56 \\
\hline 0.20 & 5 & 8.50 & 8.61 & 8.80 & 9.15 & 8.05 & 8.41 & 8.74 & 9.41 \\
\hline \multirow[t]{2}{*}{0.50} & 2 & 9.39 & 9.64 & 9.85 & 10.2 & 9.83 & 10.2 & 10.5 & 11.5 \\
\hline & & \multicolumn{4}{|c|}{ June-July-August } & \multicolumn{4}{|c|}{ September-October-November } \\
\hline 0.05 & 20 & 11.0 & 12.5 & 14.3 & 16.8 & 9.16 & 9.50 & 9.82 & 10.2 \\
\hline 0.10 & 10 & 12.5 & 14.0 & 15.7 & 18.5 & 9.79 & 10.1 & 10.4 & 10.9 \\
\hline 0.20 & 5 & 14.6 & 16.0 & 17.6 & 20.9 & 10.6 & 10.9 & 11.2 & 11.8 \\
\hline 0.50 & 2 & 19.4 & 20.7 & 22.6 & 27.6 & 12.2 & 12.5 & 12.9 & 13.7 \\
\hline
\end{tabular}

14-5.R00-1A ANDARAB RIVER AT DOSHI, Continued

Annual peak discharges

$\left[\mathrm{m}^{3} / \mathrm{s}\right.$, meters per second]

\begin{tabular}{|c|c|c|c|c|c|}
\hline \multicolumn{3}{|c|}{$\begin{array}{c}\text { Annual peak discharge, } \\
\text { by year }\end{array}$} & \multicolumn{3}{|c|}{$\begin{array}{l}\text { Annual peak discharge, } \\
\text { from highest to lowest }\end{array}$} \\
\hline $\begin{array}{l}\text { Water } \\
\text { year }\end{array}$ & Date & $\begin{array}{c}\text { Peak } \\
\text { discharge } \\
\left(\mathrm{m}^{3} / \mathrm{s}\right)\end{array}$ & $\begin{array}{l}\text { Water } \\
\text { year }\end{array}$ & Date & $\begin{array}{c}\text { Peak } \\
\text { discharge } \\
\left(\mathrm{m}^{3} / \mathrm{s}\right)\end{array}$ \\
\hline 1965 & June 17,1965 & 190 & 1968 & June 17,1968 & 381 \\
\hline 1966 & June 18, 1966 & 220 & 1966 & June 18, 1966 & 220 \\
\hline 1967 & May 24, 1967 & 190 & 1969 & June 19, 1969 & 220 \\
\hline 1968 & June 17, 1968 & 381 & 1965 & June 17,1965 & 190 \\
\hline 1969 & June 19, 1969 & 220 & 1967 & May 24, 1967 & 190 \\
\hline 1970 & April 12, 1970 & 160 & 1973 & June 8, 1973 & 190 \\
\hline 1971 & May 20, 1971 & 150 & 1972 & June 21, 1972 & 180 \\
\hline 1972 & June 21, 1972 & 180 & 1976 & June 4, 1976 & 180 \\
\hline 1973 & June 8, 1973 & 190 & 1975 & May 15,1975 & 170 \\
\hline 1974 & May 9, 1974 & 161 & 1974 & May 9, 1974 & 161 \\
\hline 1975 & May 15,1975 & 170 & 1970 & April 12, 1970 & 160 \\
\hline 1976 & June 4, 1976 & 180 & 1977 & May 28, 1977 & 160 \\
\hline 1977 & May 28, 1977 & 160 & 1971 & May 20, 1971 & 150 \\
\hline 1978 & May 22, 1978 & 115 & 1978 & May 22, 1978 & 115 \\
\hline
\end{tabular}




\section{4-5.R00-1A ANDARAB RIVER AT DOSHI, Continued}

Monthly and annual mean discharges, in cubic meters per second

[Data may not be rounded in accordance with U.S. Geological Survey publication standards]

\begin{tabular}{|c|c|c|c|c|c|c|c|c|c|c|c|c|c|}
\hline \multirow{2}{*}{$\begin{array}{l}\text { Water } \\
\text { year }\end{array}$} & \multicolumn{12}{|c|}{ Monthly mean discharge } & \multirow{2}{*}{$\begin{array}{c}\text { Annual } \\
\text { discharge }\end{array}$} \\
\hline & October & November & December & January & February & March & April & May & June & July & August & September & \\
\hline 1965 & 15.0 & 11.8 & 12.6 & 12.4 & 12.9 & 13.2 & 19.1 & 53.0 & 144 & 126 & 31.0 & 14.0 & 38.9 \\
\hline 1966 & 16.9 & 16.0 & 14.7 & 12.9 & 12.1 & 19.3 & 34.6 & 61.5 & 162 & 54.4 & 33.1 & 18.7 & 38.0 \\
\hline 1967 & 17.3 & 14.9 & 11.5 & 11.5 & 11.4 & 11.9 & 17.0 & 59.1 & 144 & 94.5 & 26.6 & 15.2 & 36.3 \\
\hline 1968 & 17.1 & 14.3 & 13.1 & 11.2 & 9.64 & 9.67 & 27.6 & 99.5 & 224 & 169 & 58.0 & 24.7 & 56.6 \\
\hline 1969 & 22.5 & 17.9 & 10.5 & 13.0 & 13.4 & 15.8 & 33.7 & 78.6 & 157 & 112 & 57.3 & 27.5 & 46.8 \\
\hline 1970 & 21.9 & 16.5 & 14.4 & 13.4 & 11.2 & 11.6 & 22.6 & 58.1 & 60.3 & 48.7 & 34.4 & 14.0 & 27.4 \\
\hline 1971 & 14.7 & 12.0 & 11.5 & 10.5 & 10.9 & 14.8 & 25.7 & 76.5 & 57.7 & 28.3 & 17.8 & 13.1 & 24.5 \\
\hline 1972 & 11.7 & 11.3 & 9.40 & 9.14 & 9.23 & 9.89 & 10.1 & 37.9 & 138 & 73.0 & 23.8 & 14.3 & 29.8 \\
\hline 1973 & 15.0 & 13.7 & 15.0 & 14.0 & 13.6 & 15.9 & 61.2 & 111 & 130 & 58.2 & 31.8 & 20.8 & 41.8 \\
\hline 1974 & 14.4 & 13.8 & 12.9 & 10.7 & 10.5 & 10.7 & 15.0 & 43.5 & 62.8 & 47.5 & 18.0 & 10.9 & 22.6 \\
\hline 1975 & 11.9 & 10.3 & 9.60 & 8.49 & 8.13 & 8.12 & 18.3 & 73.8 & 101 & 68.0 & 31.2 & 18.3 & 30.7 \\
\hline 1976 & 16.0 & 13.3 & 10.8 & 9.14 & 9.74 & 9.67 & 21.9 & 71.8 & 81.2 & 66.9 & 30.9 & 18.5 & 30.1 \\
\hline 1977 & 15.8 & 12.0 & 10.2 & 10.6 & 10.2 & 8.60 & 12.5 & 42.4 & 97.7 & 43.2 & 20.5 & 18.5 & 25.2 \\
\hline 1978 & 20.9 & 18.6 & 14.7 & 9.65 & 8.89 & 12.1 & 17.9 & 74.5 & 80.3 & 40.2 & 20.5 & 15.7 & 27.9 \\
\hline
\end{tabular}




\section{4-9.5R0-1T FOLADI RIVER AT BAMYAN}

\section{(U.S. Geological Survey identification number: 344900067490000)}

LOCATION: Lat $34^{\circ} 49^{\prime} \mathrm{N}$., long $67^{\circ} 49^{\prime} \mathrm{E}$.

DRAINAGE AREA: $320 \mathrm{~km}^{2}$.

ELEVATION: 2,507 meters above mean sea level.

PERIOD OF RECORD: December 4, 1969 to May 21, 1977.

GAGE: Staff gage.

Annual mean discharge

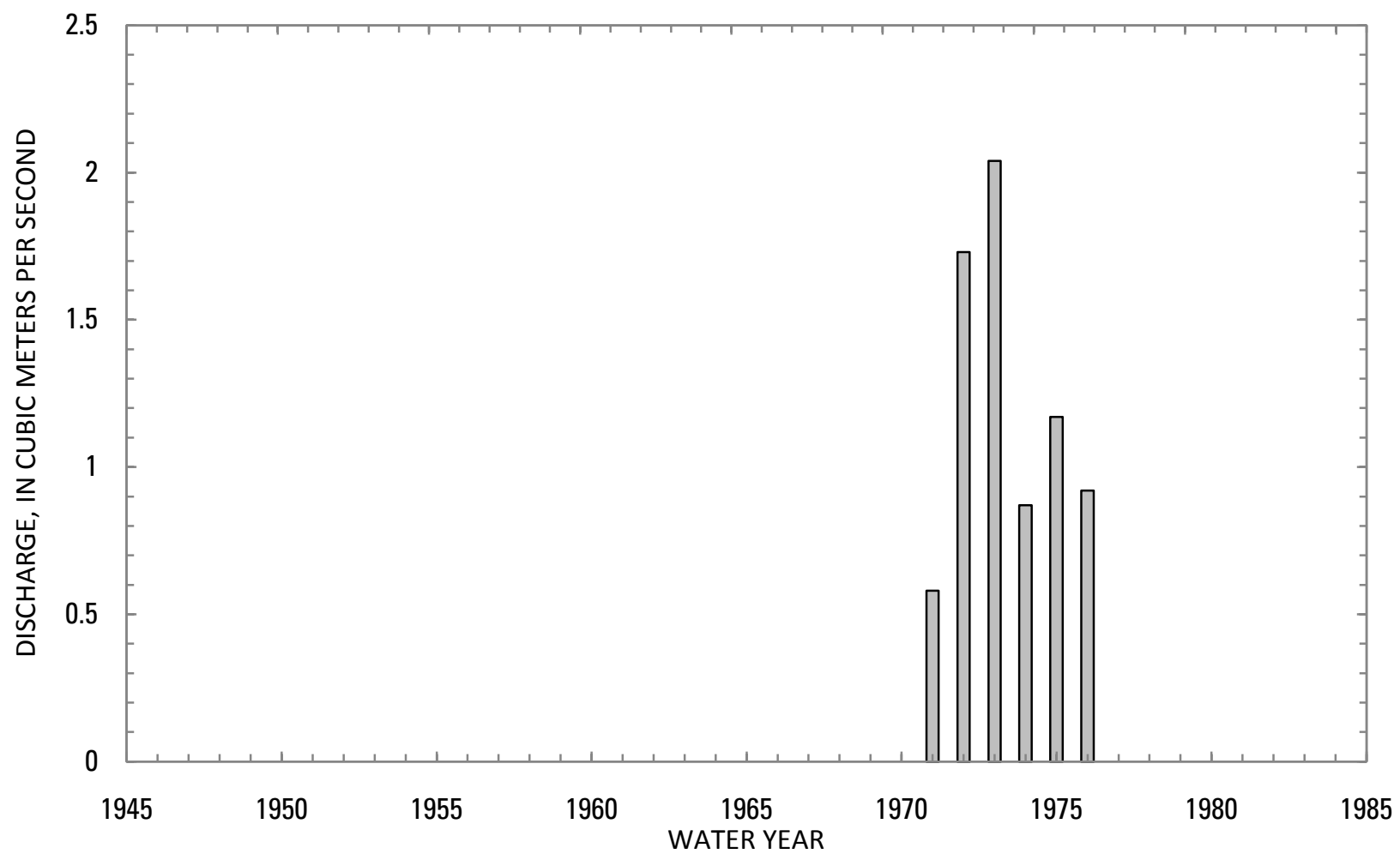




\section{4-9.5R0-1T FOLADI RIVER AT BAMYAN, Continued}

Statistics of monthly and annual mean discharges [ $\mathrm{m}^{3} / \mathrm{s}$, cubic meters per second]

\begin{tabular}{|c|c|c|c|c|c|c|c|c|}
\hline \multirow[b]{2}{*}{ Month } & \multicolumn{2}{|c|}{ Maximum } & \multicolumn{2}{|c|}{ Minimum } & \multicolumn{4}{|c|}{ Mean } \\
\hline & $\begin{array}{c}\text { Discharge } \\
\left(\mathrm{m}^{3} / \mathrm{s}\right)\end{array}$ & $\begin{array}{c}\text { Water year } \\
\text { of } \\
\text { occurrence }\end{array}$ & $\begin{array}{c}\text { Discharge } \\
\left(\mathrm{m}^{3} / \mathrm{s}\right)\end{array}$ & $\begin{array}{c}\text { Water year } \\
\text { of } \\
\text { occurrence }\end{array}$ & $\begin{array}{c}\text { Discharge } \\
\left(\mathrm{m}^{3} / \mathrm{s}\right)\end{array}$ & $\begin{array}{c}\text { Standard } \\
\text { deviation } \\
\left(\mathrm{m}^{3} / \mathrm{s}\right)\end{array}$ & $\begin{array}{c}\text { Coefficient } \\
\text { of } \\
\text { variation }\end{array}$ & $\begin{array}{c}\text { Percentage } \\
\text { of annual } \\
\text { discharge }\end{array}$ \\
\hline October & 1.22 & 1973 & 0.56 & 1972 & 0.94 & 0.24 & 0.26 & 6.45 \\
\hline November & 1.20 & 1973 & 0.29 & 1976 & 0.69 & 0.33 & 0.47 & 4.78 \\
\hline December & 1.25 & 1977 & 0.39 & 1972 & 0.84 & 0.37 & 0.44 & 5.78 \\
\hline January & 1.20 & 1970 & 0.55 & 1974 & 0.82 & 0.24 & 0.29 & 5.65 \\
\hline February & 1.04 & 1970 & 0.40 & 1975 & 0.72 & 0.21 & 0.29 & 4.94 \\
\hline March & 0.91 & 1973 & 0.28 & 1971 & 0.57 & 0.22 & 0.38 & 3.95 \\
\hline April & 1.62 & 1973 & 0.07 & 1971 & 0.47 & 0.52 & 1.10 & 3.25 \\
\hline May & 4.26 & 1973 & 0.99 & 1970 & 1.87 & 1.21 & 0.65 & 12.9 \\
\hline June & 10.6 & 1972 & 0.60 & 1971 & 4.17 & 3.30 & 0.79 & 28.8 \\
\hline July & 2.64 & 1975 & 0.09 & 1971 & 1.64 & 0.95 & 0.58 & 11.3 \\
\hline August & 2.15 & 1973 & 0.08 & 1971 & 0.84 & 0.67 & 0.80 & 5.75 \\
\hline September & 1.58 & 1973 & 0.65 & 1974 & 0.94 & 0.35 & 0.37 & 6.50 \\
\hline Annual & 2.04 & 1973 & 0.58 & 1971 & 1.22 & 0.56 & 0.46 & 100 \\
\hline
\end{tabular}


14-9.5R0-1T FOLADI RIVER AT BAMYAN, Continued

Monthly and annual flow duration, in cubic meters per second

[ng, not given]

\begin{tabular}{|c|c|c|c|c|c|c|c|c|c|c|c|c|c|}
\hline \multirow{2}{*}{$\begin{array}{l}\text { Percentage } \\
\text { of days } \\
\text { discharge } \\
\text { equaled or } \\
\text { exceeded }\end{array}$} & \multicolumn{12}{|c|}{ Month } & \multirow{2}{*}{ Annua } \\
\hline & October & November & December & January & February & March & April & May & June & July & August & September & \\
\hline 95 & 0.49 & 0.20 & 0.16 & 0.51 & 0.39 & 0.24 & 0.03 & 0.04 & 0.44 & 0.09 & 0.08 & 0.52 & 0.09 \\
\hline 90 & 0.50 & 0.28 & 0.23 & 0.52 & 0.42 & 0.26 & 0.06 & 0.06 & 0.61 & 0.10 & 0.09 & 0.57 & 0.22 \\
\hline 85 & 0.60 & 0.32 & 0.27 & 0.53 & 0.47 & 0.31 & 0.08 & 0.16 & 0.89 & 0.22 & 0.41 & 0.62 & 0.32 \\
\hline 80 & 0.71 & 0.37 & 0.44 & 0.57 & 0.49 & 0.34 & 0.09 & 0.26 & 1.27 & 0.46 & 0.46 & 0.66 & 0.45 \\
\hline 75 & 0.72 & 0.40 & 0.48 & 0.59 & 0.54 & 0.40 & 0.11 & 0.49 & 1.68 & 0.82 & 0.50 & 0.70 & 0.50 \\
\hline 70 & 0.73 & 0.47 & 0.68 & 0.64 & 0.57 & 0.43 & 0.15 & 0.56 & 2.01 & 0.90 & 0.52 & 0.73 & 0.55 \\
\hline 65 & 0.75 & 0.50 & 0.73 & 0.67 & 0.60 & 0.48 & 0.20 & 0.92 & 2.28 & 0.95 & 0.54 & 0.75 & 0.60 \\
\hline 60 & 0.76 & 0.58 & 0.77 & 0.70 & 0.64 & 0.51 & 0.22 & 1.33 & 2.52 & 1.00 & 0.59 & 0.78 & 0.64 \\
\hline 55 & 0.90 & 0.68 & 0.83 & 0.75 & 0.67 & 0.54 & 0.24 & 1.57 & 2.74 & 1.17 & 0.61 & 0.80 & 0.70 \\
\hline 50 & 0.97 & 0.72 & 0.92 & 0.79 & 0.73 & 0.57 & 0.27 & 1.72 & 2.94 & 1.36 & 0.63 & 0.83 & 0.76 \\
\hline 45 & 0.99 & 0.73 & 0.96 & 0.81 & 0.74 & 0.60 & 0.31 & 1.83 & 3.57 & 1.46 & 0.65 & 0.87 & 0.82 \\
\hline 40 & 1.03 & 0.75 & 0.99 & 0.86 & 0.76 & 0.62 & 0.40 & 1.94 & 4.13 & 1.78 & 0.73 & 0.91 & 0.89 \\
\hline 35 & 1.19 & 0.77 & 1.04 & 0.91 & 0.79 & 0.65 & 0.45 & 2.10 & 4.57 & 1.98 & 0.82 & 0.96 & 0.97 \\
\hline 30 & 1.20 & 0.81 & 1.13 & 0.98 & 0.84 & 0.69 & 0.55 & 2.32 & 5.37 & 2.37 & 0.93 & 1.01 & 1.09 \\
\hline 25 & 1.22 & 0.90 & 1.18 & 1.01 & 0.88 & 0.75 & 0.71 & 2.66 & 6.13 & 2.45 & 1.17 & 1.18 & 1.23 \\
\hline 20 & 1.23 & 1.14 & 1.21 & 1.02 & 0.92 & 0.81 & 0.81 & 3.22 & 6.77 & 2.54 & 1.25 & 1.38 & 1.43 \\
\hline 15 & 1.25 & 1.21 & 1.23 & 1.04 & 0.96 & 0.84 & 0.93 & 3.68 & 7.71 & 2.64 & 1.32 & 1.46 & 1.99 \\
\hline 10 & 1.28 & 1.24 & 1.26 & 1.22 & 0.99 & 0.88 & 1.32 & 4.34 & 9.73 & 2.99 & 2.11 & 1.56 & 2.52 \\
\hline 5 & 1.33 & 1.28 & 1.51 & 1.33 & 1.18 & 0.96 & 1.7 & 5.39 & 11.1 & 4.14 & ng & 1.71 & 4.14 \\
\hline
\end{tabular}




\section{4-9.5R0-1T FOLADI RIVER AT BAMYAN, Continued}

Probability of occurrence of annual high discharges

[m $\mathrm{m}^{3} / \mathrm{s}$, cubic meters per second; ng, not given]

\begin{tabular}{|c|c|c|c|c|c|c|}
\hline \multirow{2}{*}{$\begin{array}{c}\text { Exceedance } \\
\text { probability }\end{array}$} & \multirow{2}{*}{$\begin{array}{l}\text { Recurrence } \\
\text { interval } \\
\text { (years) }\end{array}$} & \multirow{2}{*}{$\begin{array}{c}\text { Maximum } \\
\text { instantaneous } \\
\text { discharge } \\
\left(\mathrm{m}^{3} / \mathrm{s}\right)\end{array}$} & \multicolumn{4}{|c|}{ Maximum daily mean discharge $\left(\mathrm{m}^{3} / \mathrm{s}\right)$} \\
\hline & & & $\begin{array}{l}\text { 3-day } \\
\text { period }\end{array}$ & $\begin{array}{l}\text { 7-day } \\
\text { period }\end{array}$ & $\begin{array}{l}\text { 15-day } \\
\text { period }\end{array}$ & $\begin{array}{l}\text { 30-day } \\
\text { period }\end{array}$ \\
\hline 0.99 & 1.01 & 2.30 & 2.14 & 2.02 & 1.48 & 1.13 \\
\hline 0.95 & 1.05 & 3.30 & 2.74 & 2.54 & 2.02 & 1.58 \\
\hline 0.90 & 1.11 & 3.90 & 3.17 & 2.91 & 2.40 & 1.90 \\
\hline 0.80 & 1.25 & 4.90 & 3.82 & 3.50 & 3.00 & 2.42 \\
\hline 0.50 & 2 & 7.10 & 5.69 & 5.21 & 4.74 & 3.98 \\
\hline 0.20 & 5 & 10.1 & 8.92 & 8.31 & 7.78 & 6.90 \\
\hline 0.10 & 10 & 11.9 & 11.5 & 10.9 & 10.3 & 9.40 \\
\hline 0.04 & 25 & 14.1 & 15.4 & 15.0 & 13.9 & 13.3 \\
\hline 0.02 & 50 & 15.7 & 18.8 & 18.5 & 17.1 & 16.8 \\
\hline 0.01 & 100 & 17.1 & ${ }^{2} 22.6$ & 22.7 & ${ }^{2} 20.7$ & 20.8 \\
\hline 0.005 & 200 & 18.5 & ${ }^{2} 26.9$ & 27.5 & ${ }^{2} 24.7$ & 25.5 \\
\hline 0.002 & 500 & 20.3 & ng & ng & ng & ng \\
\hline
\end{tabular}

\section{4-9.5R0-1T FOLADI RIVER AT BAMYAN, Continued}

Probability of occurrence of annual low discharges $\left[\mathrm{m}^{3} / \mathrm{s}\right.$, meters per second]

\begin{tabular}{|c|c|c|c|c|c|c|c|c|c|c|}
\hline \multirow{3}{*}{$\begin{array}{c}\text { Nonexceedance } \\
\text { probability }\end{array}$} & \multirow{3}{*}{$\begin{array}{c}\text { Recurrence } \\
\text { interval } \\
\text { (years) }\end{array}$} & \multicolumn{9}{|c|}{ Minimum daily mean discharge $\left(\mathrm{m}^{3} / \mathrm{s}\right)$} \\
\hline & & \multicolumn{9}{|c|}{ Number of consecutive days } \\
\hline & & 1 & 3 & 7 & 14 & 30 & 60 & 90 & 120 & 183 \\
\hline 0.05 & 20 & 0 & 0 & 0 & 0.01 & 0.03 & 0.09 & 0.18 & 0.31 & 0.32 \\
\hline 0.10 & 10 & 0 & 0 & 0 & 0.02 & 0.05 & 0.15 & 0.24 & 0.37 & 0.39 \\
\hline 0.20 & 5 & 0 & 0 & 0 & 0.03 & 0.08 & 0.24 & 0.34 & 0.45 & 0.50 \\
\hline 0.50 & 2 & 0.04 & 0.05 & 0.07 & 0.12 & 0.19 & 0.49 & 0.57 & 0.63 & 0.72 \\
\hline
\end{tabular}




\section{4-9.5R0-1T FOLADI RIVER AT BAMYAN, Continued}

Probability of occurrence of seasonal low discharges [m $\mathrm{m}^{3} / \mathrm{s}$, meters per second]

\begin{tabular}{|c|c|c|c|c|c|c|c|c|c|}
\hline \multirow{3}{*}{$\begin{array}{c}\text { Nonexceedance } \\
\text { probability }\end{array}$} & \multirow{3}{*}{$\begin{array}{c}\text { Recurrence } \\
\text { interval } \\
\text { (years) }\end{array}$} & \multicolumn{8}{|c|}{ Minimum daily mean discharge $\left(\mathrm{m}^{3} / \mathrm{s}\right)$} \\
\hline & & \multicolumn{8}{|c|}{ Number of consecutive days } \\
\hline & & 1 & 7 & 14 & 30 & 1 & 7 & 14 & 30 \\
\hline & & \multicolumn{4}{|c|}{ December-January-February } & \multicolumn{4}{|c|}{ March-April-May } \\
\hline 0.05 & 20 & 0.08 & 0.11 & 0.14 & 0.33 & 0 & 0 & 0.01 & 0.03 \\
\hline 0.10 & 10 & 0.11 & 0.15 & 0.18 & 0.36 & 0 & 0 & 0.02 & 0.05 \\
\hline 0.20 & 5 & 0.16 & 0.21 & 0.24 & 0.40 & 0 & 0 & 0.03 & 0.08 \\
\hline \multirow[t]{2}{*}{0.50} & 2 & 0.33 & 0.39 & 0.42 & 0.52 & 0.04 & 0.07 & 0.13 & 0.20 \\
\hline & & \multicolumn{4}{|c|}{ June-July-August } & \multicolumn{4}{|c|}{ September-0ctober-November } \\
\hline 0.05 & 20 & 0.08 & 0.08 & 0.08 & 0.08 & 0.06 & 0.12 & 0.18 & 0.25 \\
\hline 0.10 & 10 & 0.11 & 0.13 & 0.13 & 0.14 & 0.10 & 0.17 & 0.23 & 0.32 \\
\hline 0.20 & 5 & 0.18 & 0.22 & 0.24 & 0.27 & 0.17 & 0.25 & 0.31 & 0.41 \\
\hline 0.50 & 2 & 0.43 & 0.54 & 0.59 & 0.69 & 0.38 & 0.45 & 0.49 & 0.61 \\
\hline
\end{tabular}

\section{4-9.5R0-1T FOLADI RIVER AT BAMYAN, Continued}

Annual peak discharges

[ $\mathrm{m}^{3} / \mathrm{s}$, meters per second]

\begin{tabular}{|c|c|c|c|c|c|}
\hline \multicolumn{3}{|c|}{$\begin{array}{c}\text { Annual peak discharge, } \\
\text { by year }\end{array}$} & \multicolumn{3}{|c|}{$\begin{array}{l}\text { Annual peak discharge, } \\
\text { from highest to lowest }\end{array}$} \\
\hline $\begin{array}{l}\text { Water } \\
\text { year }\end{array}$ & Date & $\begin{array}{c}\text { Peak } \\
\text { discharge } \\
\left(\mathrm{m}^{3} / \mathrm{s}\right)\end{array}$ & $\begin{array}{l}\text { Water } \\
\text { year }\end{array}$ & Date & $\begin{array}{c}\text { Peak } \\
\text { discharge } \\
\left(\mathrm{m}^{3} / \mathrm{s}\right)\end{array}$ \\
\hline 1970 & June 28,1970 & 9.29 & 1972 & June 22, 1972 & 13.1 \\
\hline 1971 & May 19, 1971 & 3.42 & 1970 & June 28,1970 & 9.29 \\
\hline 1972 & June 22, 1972 & 13.1 & 1973 & May 7, 1973 & 8.00 \\
\hline 1973 & May 7, 1973 & 8.00 & 1975 & June 20, 1975 & 7.50 \\
\hline 1974 & June 6, 1974 & 5.00 & 1976 & May 24, 1976 & 6.39 \\
\hline 1975 & June 20, 1975 & 7.50 & 1974 & June 6, 1974 & 5.00 \\
\hline 1976 & May 24, 1976 & 6.39 & 1971 & May 19, 1971 & 3.42 \\
\hline
\end{tabular}


14-9.5R0-1T FOLADI RIVER AT BAMYAN, Continued

Monthly and annual mean discharges, in cubic meters per second $[--$, no data]

\begin{tabular}{|c|c|c|c|c|c|c|c|c|c|c|c|c|c|}
\hline \multirow{2}{*}{$\begin{array}{l}\text { Water } \\
\text { year }\end{array}$} & \multicolumn{12}{|c|}{ Monthly mean discharge } & \multirow{2}{*}{$\begin{array}{c}\text { Annual } \\
\text { discharge }\end{array}$} \\
\hline & October & November & December & January & February & March & April & May & June & July & August & September & \\
\hline 1970 & -- & -- & -- & 1.20 & 1.04 & 0.33 & 0.21 & 0.99 & 2.26 & 2.05 & 1.20 & 1.00 & -- \\
\hline 1971 & 1.00 & 0.69 & 0.63 & 0.80 & 0.53 & 0.28 & 0.07 & 1.52 & 0.60 & 0.09 & 0.08 & 0.69 & 0.58 \\
\hline 1972 & 0.56 & 0.32 & 0.39 & 0.71 & 0.77 & 0.76 & 0.80 & 2.69 & 10.6 & 1.83 & 0.66 & 0.79 & 1.73 \\
\hline 1973 & 1.22 & 1.20 & 1.21 & 0.99 & 0.93 & 0.91 & 1.62 & 4.26 & 5.74 & 2.59 & 2.15 & 1.58 & 2.04 \\
\hline 1974 & 1.16 & 0.66 & 0.87 & 0.55 & 0.69 & 0.58 & 0.23 & 1.03 & 2.81 & 0.69 & 0.48 & 0.65 & 0.87 \\
\hline 1975 & 0.74 & 0.73 & 1.11 & 0.60 & 0.40 & 0.49 & 0.10 & 1.01 & 4.71 & 2.64 & 0.76 & 0.68 & 1.17 \\
\hline 1976 & 0.78 & 0.29 & 0.42 & 0.66 & 0.61 & 0.51 & 0.30 & 1.58 & 2.50 & 1.60 & 0.53 & 1.22 & 0.92 \\
\hline 1977 & 1.10 & 0.96 & 1.25 & 1.06 & 0.78 & 0.74 & 0.44 & -- & -- & -- & -- & -- & -- \\
\hline
\end{tabular}




\section{4-9.R00-1A BAMYAN RIVER AT DOAB}

\section{(U.S. Geological Survey identification number: 351600067590000$)$}

LOCATION: Lat $35^{\circ} 16^{\prime} \mathrm{N}$., long $67^{\circ} 59^{\prime} \mathrm{E}$.

DRAINAGE AREA: $5,005 \mathrm{~km}^{2}$.

ELEVATION: 1,468 meters above mean sea level.

PERIOD OF RECORD: October 1, 1967 to September 30, 1978.

GAGE: Water-stage recorder.

Annual mean discharge

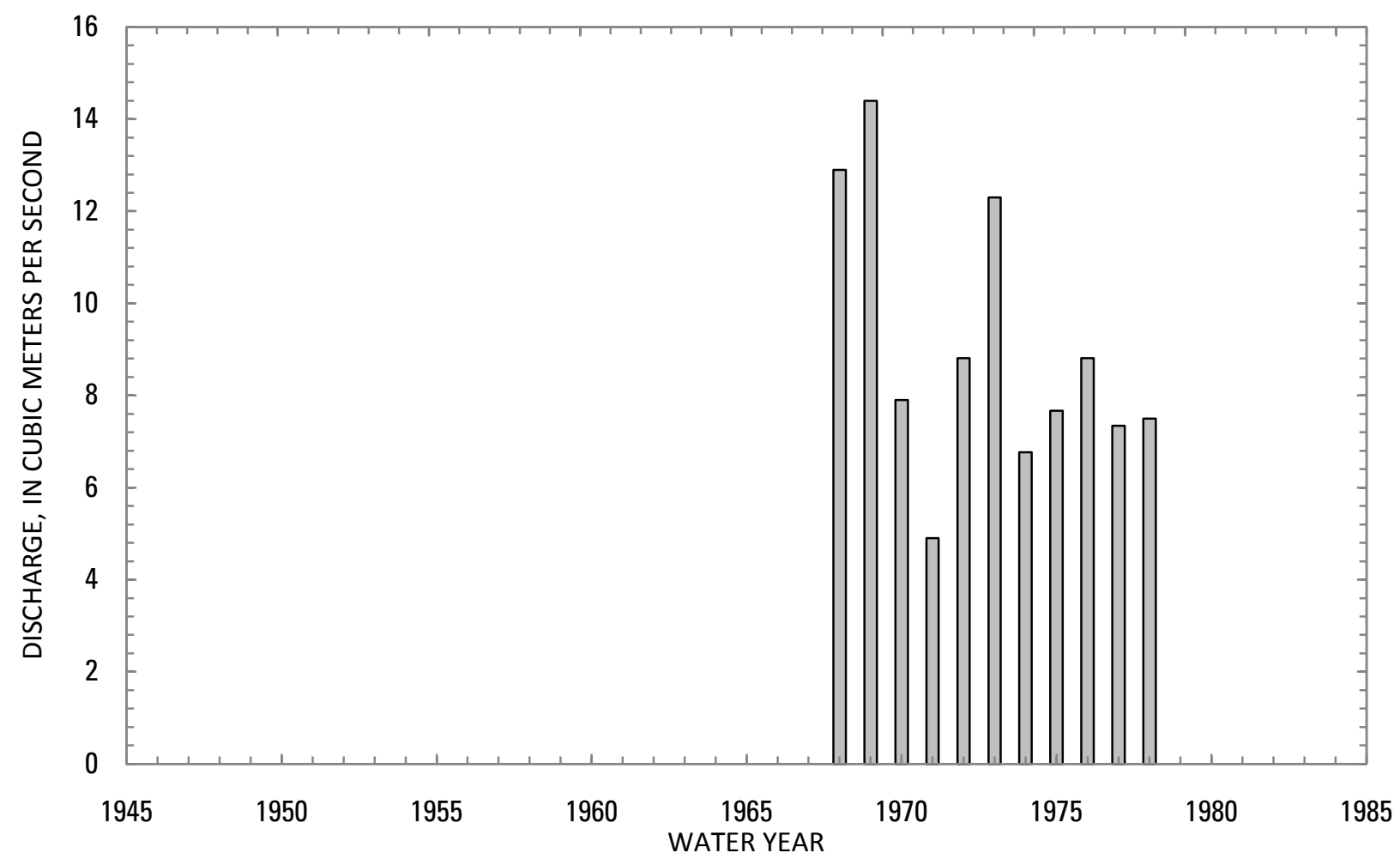




\section{4-9.R00-1A BAMYAN RIVER AT DOAB, Continued}

Statistics of monthly and annual mean discharges $\left[\mathrm{m}^{3} / \mathrm{s}\right.$, cubic meters per second]

\begin{tabular}{|c|c|c|c|c|c|c|c|c|}
\hline \multirow[b]{2}{*}{ Month } & \multicolumn{2}{|c|}{ Maximum } & \multicolumn{2}{|c|}{ Minimum } & \multicolumn{4}{|c|}{ Mean } \\
\hline & $\begin{array}{c}\text { Discharge } \\
\left(\mathrm{m}^{3} / \mathrm{s}\right)\end{array}$ & $\begin{array}{c}\text { Water year } \\
\text { of } \\
\text { occurrence }\end{array}$ & $\begin{array}{c}\text { Discharge } \\
\left(\mathrm{m}^{3} / \mathrm{s}\right)\end{array}$ & $\begin{array}{c}\text { Water year } \\
\text { of } \\
\text { occurrence }\end{array}$ & $\begin{array}{c}\text { Discharge } \\
\left(\mathrm{m}^{3} / \mathrm{s}\right)\end{array}$ & $\begin{array}{c}\text { Standard } \\
\text { deviation } \\
\left(\mathrm{m}^{3} / \mathrm{s}\right)\end{array}$ & $\begin{array}{c}\text { Coefficient } \\
\text { of } \\
\text { variation }\end{array}$ & $\begin{array}{c}\text { Percentage } \\
\text { of annual } \\
\text { discharge }\end{array}$ \\
\hline October & 8.55 & 1970 & 3.10 & 1972 & 5.99 & 1.69 & 0.28 & 5.53 \\
\hline November & 8.82 & 1970 & 3.26 & 1972 & 5.70 & 1.64 & 0.29 & 5.27 \\
\hline December & 7.92 & 1970 & 2.98 & 1972 & 4.85 & 1.33 & 0.27 & 4.48 \\
\hline January & 6.69 & 1970 & 2.70 & 1978 & 4.43 & 1.16 & 0.26 & 4.10 \\
\hline February & 5.85 & 1969 & 2.46 & 1978 & 4.15 & 1.15 & 0.28 & 3.83 \\
\hline March & 5.32 & 1968 & 2.82 & 1975 & 4.18 & 0.85 & 0.20 & 3.86 \\
\hline April & 8.25 & 1968 & 3.06 & 1972 & 4.90 & 1.67 & 0.34 & 4.52 \\
\hline May & 26.6 & 1973 & 10.4 & 1975 & 14.9 & 4.83 & 0.32 & 13.8 \\
\hline June & 56.1 & 1969 & 9.35 & 1971 & 30.7 & 14.6 & 0.47 & 28.4 \\
\hline July & 31.5 & 1969 & 2.66 & 1971 & 15.5 & 8.79 & 0.57 & 14.3 \\
\hline August & 13.3 & 1969 & 2.65 & 1971 & 6.85 & 3.18 & 0.46 & 6.33 \\
\hline September & 10.5 & 1969 & 1.97 & 1971 & 6.06 & 2.61 & 0.43 & 5.60 \\
\hline Annual & 14.4 & 1969 & 4.90 & 1971 & 9.03 & 2.92 & 0.32 & 100 \\
\hline
\end{tabular}


14-9.R00-1A BAMYAN RIVER AT DOAB, Continued

Monthly and annual flow duration, in cubic meters per second

\begin{tabular}{|c|c|c|c|c|c|c|c|c|c|c|c|c|c|}
\hline \multirow{2}{*}{$\begin{array}{l}\text { Percentage } \\
\text { of days } \\
\text { discharge } \\
\text { equaled or } \\
\text { exceeded }\end{array}$} & \multicolumn{12}{|c|}{ Month } & \multirow{2}{*}{ Annual } \\
\hline & October & November & December & January & February & March & April & May & June & July & August & September & \\
\hline 95 & 3.15 & 3.23 & 3.07 & 2.75 & 2.45 & 2.74 & 2.68 & 3.88 & 10.2 & 2.80 & 2.75 & 1.62 & 2.77 \\
\hline 90 & 3.98 & 3.61 & 3.22 & 3.06 & 2.55 & 2.96 & 2.85 & 4.56 & 13.6 & 3.81 & 3.78 & 3.52 & 3.17 \\
\hline 85 & 4.51 & 3.89 & 3.45 & 3.23 & 2.69 & 3.01 & 2.98 & 5.66 & 16.0 & 5.27 & 4.08 & 4.01 & 3.52 \\
\hline 80 & 4.64 & 4.37 & 3.64 & 3.51 & 2.98 & 3.20 & 3.06 & 6.22 & 17.9 & 6.42 & 4.22 & 4.20 & 3.91 \\
\hline 75 & 4.86 & 4.52 & 3.83 & 3.58 & 3.16 & 3.38 & 3.24 & 6.87 & 20.1 & 7.05 & 4.36 & 4.38 & 4.15 \\
\hline 70 & 4.98 & 4.78 & 4.06 & 3.65 & 3.34 & 3.48 & 3.58 & 7.73 & 21.8 & 8.53 & 4.77 & 4.57 & 4.38 \\
\hline 65 & 5.10 & 4.90 & 4.30 & 3.81 & 3.66 & 3.77 & 3.82 & 8.79 & 23.2 & 9.61 & 4.96 & 4.91 & 4.60 \\
\hline 60 & 5.23 & 5.02 & 4.44 & 3.97 & 3.89 & 3.97 & 4.01 & 10.0 & 24.3 & 10.9 & 5.13 & 5.05 & 4.81 \\
\hline 55 & 5.43 & 5.11 & 4.50 & 4.11 & 4.26 & 4.03 & 4.15 & 11.6 & 25.8 & 11.8 & 5.46 & 5.19 & 5.02 \\
\hline 50 & 5.59 & 5.21 & 4.57 & 4.24 & 4.32 & 4.22 & 4.35 & 13.5 & 27.6 & 13.0 & 5.88 & 5.41 & 5.33 \\
\hline 45 & 5.79 & 5.44 & 4.90 & 4.41 & 4.39 & 4.34 & 4.56 & 15.1 & 29.2 & 15.1 & 6.40 & 5.70 & 5.66 \\
\hline 40 & 6.26 & 5.97 & 5.02 & 4.58 & 4.48 & 4.53 & 4.80 & 16.4 & 31.8 & 16.4 & 7.15 & 6.05 & 6.17 \\
\hline 35 & 6.51 & 6.30 & 5.11 & 4.80 & 4.61 & 4.64 & 5.02 & 17.5 & 34.7 & 18.2 & 7.58 & 6.62 & 6.81 \\
\hline 30 & 6.99 & 6.67 & 5.19 & 5.01 & 4.88 & 4.76 & 5.44 & 18.7 & 37.3 & 19.3 & 8.02 & 7.05 & 7.59 \\
\hline 25 & 7.41 & 6.88 & 5.53 & 5.27 & 5.03 & 4.95 & 6.07 & 20.2 & 40.9 & 20.9 & 8.54 & 7.39 & 8.49 \\
\hline 20 & 8.02 & 7.19 & 5.74 & 5.49 & 5.29 & 5.04 & 6.57 & 22.2 & 46.2 & 24.2 & 9.23 & 7.85 & 10.4 \\
\hline 15 & 8.26 & 7.64 & 6.09 & 5.86 & 5.43 & 5.11 & 7.44 & 25.0 & 51.7 & 27.1 & 9.69 & 8.83 & 15.1 \\
\hline 10 & 8.46 & 8.13 & 6.52 & 6.26 & 5.67 & 5.31 & 8.09 & 28.5 & 54.6 & 31.3 & 11.1 & 9.94 & 21.2 \\
\hline 5 & 8.76 & 9.09 & 7.92 & 6.70 & 5.91 & 6.00 & 8.71 & 34.2 & 58.1 & 36.5 & 14.2 & 11.7 & 30.7 \\
\hline
\end{tabular}




\section{4-9.R00-1A BAMYAN RIVER AT DOAB, Continued}

Probability of occurrence of annual high discharges [ $\mathrm{m}^{3} / \mathrm{s}$, cubic meters per second; $\mathrm{ng}$, not given]

\begin{tabular}{|c|c|c|c|c|c|c|}
\hline \multirow{2}{*}{$\begin{array}{c}\text { Exceedance } \\
\text { probability }\end{array}$} & \multirow{2}{*}{$\begin{array}{c}\text { Recurrence } \\
\text { interval } \\
\text { (years) }\end{array}$} & \multirow{2}{*}{$\begin{array}{l}\text { Maximum } \\
\text { instantaneous } \\
\text { discharge } \\
\left(\mathrm{m}^{3} / \mathrm{s}\right)\end{array}$} & \multicolumn{4}{|c|}{ Maximum daily mean discharge $\left(\mathrm{m}^{3} / \mathrm{s}\right)$} \\
\hline & & & $\begin{array}{l}\text { 3-day } \\
\text { period }\end{array}$ & $\begin{array}{l}\text { 7-day } \\
\text { period }\end{array}$ & $\begin{array}{l}\text { 15-day } \\
\text { period }\end{array}$ & $\begin{array}{l}\text { 30-day } \\
\text { period }\end{array}$ \\
\hline 0.99 & 1.01 & 25.3 & 15.3 & 13.7 & 12.3 & 11.2 \\
\hline 0.95 & 1.05 & 28.6 & 20.3 & 18.2 & 16.4 & 14.9 \\
\hline 0.90 & 1.11 & 30.9 & 23.5 & 21.2 & 19.2 & 17.4 \\
\hline 0.80 & 1.25 & 34.5 & 27.9 & 25.4 & 23.1 & 20.9 \\
\hline 0.50 & 2 & 44.8 & 38.1 & 35.4 & 32.8 & 29.8 \\
\hline 0.20 & 5 & 62.3 & 51.0 & 48.9 & 46.5 & 42.5 \\
\hline 0.10 & 10 & 76.3 & 59.0 & 57.6 & 55.7 & 51.1 \\
\hline 0.04 & 25 & 96.9 & 68.6 & 68.3 & 67.5 & 62.2 \\
\hline 0.02 & 50 & 115 & ${ }^{1} 75.4$ & ${ }^{1} 76.1$ & 76.3 & 70.6 \\
\hline 0.01 & 100 & 134 & '81.9 & ${ }^{1} 83.8$ & 85.2 & 79.2 \\
\hline 0.005 & 200 & 157 & ${ }^{1} 88.2$ & ${ }^{1} 91.5$ & 94.3 & 87.9 \\
\hline 0.002 & 500 & 191 & $\mathrm{ng}$ & $\mathrm{ng}$ & $\mathrm{ng}$ & $\mathrm{ng}$ \\
\hline
\end{tabular}

'Data does not fit log-Pearson Type III curve, use with caution.

\section{4-9.R00-1A BAMYAN RIVER AT DOAB, Continued}

Probability of occurrence of annual low discharges $\left[\mathrm{m}^{3} / \mathrm{s}\right.$, meters per second]

\begin{tabular}{|c|c|c|c|c|c|c|c|c|c|c|}
\hline \multirow{3}{*}{$\begin{array}{c}\text { Nonexceedance } \\
\text { probability }\end{array}$} & \multirow{3}{*}{$\begin{array}{c}\text { Recurrence } \\
\text { interval } \\
\text { (years) }\end{array}$} & \multicolumn{9}{|c|}{ Minimum daily mean discharge $\left(\mathrm{m}^{3} / \mathrm{s}\right)$} \\
\hline & & \multicolumn{9}{|c|}{ Number of consecutive days } \\
\hline & & 1 & 3 & 7 & 14 & 30 & 60 & 90 & 120 & 183 \\
\hline 0.05 & 20 & 0.98 & 1.08 & 1.27 & 1.52 & 1.96 & 2.17 & 2.36 & 2.57 & 2.84 \\
\hline 0.10 & 10 & 1.20 & 1.33 & 1.52 & 1.79 & 2.17 & 2.47 & 2.66 & 2.87 & 3.19 \\
\hline 0.20 & 5 & 1.52 & 1.67 & 1.87 & 2.15 & 2.47 & 2.87 & 3.07 & 3.28 & 3.65 \\
\hline 0.50 & 2 & 2.26 & 2.45 & 2.64 & 2.94 & 3.16 & 3.73 & 3.96 & 4.17 & 4.67 \\
\hline
\end{tabular}




\section{4-9.R00-1A BAMYAN RIVER AT DOAB, Continued}

Probability of occurrence of seasonal low discharges [ $\mathrm{m}^{3} / \mathrm{s}$, meters per second]

\begin{tabular}{|c|c|c|c|c|c|c|c|c|c|}
\hline \multirow{3}{*}{$\begin{array}{c}\text { Nonexceedance } \\
\text { probability }\end{array}$} & \multirow{3}{*}{$\begin{array}{c}\text { Recurrence } \\
\text { interval } \\
\text { (years) }\end{array}$} & \multicolumn{8}{|c|}{ Minimum daily mean discharge $\left(\mathrm{m}^{3} / \mathrm{s}\right)$} \\
\hline & & \multicolumn{8}{|c|}{ Number of consecutive days } \\
\hline & & 1 & 7 & 14 & 30 & 1 & 7 & 14 & 30 \\
\hline & & \multicolumn{4}{|c|}{ December-January-February } & \multicolumn{4}{|c|}{ March-April-May } \\
\hline 0.05 & 20 & 1.38 & 1.60 & 2.06 & 2.29 & 1.22 & 1.66 & 2.23 & 2.54 \\
\hline 0.10 & 10 & 1.66 & 1.95 & 2.36 & 2.58 & 1.57 & 1.95 & 2.44 & 2.77 \\
\hline 0.20 & 5 & 2.05 & 2.42 & 2.76 & 2.97 & 2.05 & 2.35 & 2.73 & 3.07 \\
\hline \multirow[t]{2}{*}{0.50} & 2 & 2.93 & 3.42 & 3.61 & 3.83 & 3.08 & 3.20 & 3.38 & 3.75 \\
\hline & & \multicolumn{4}{|c|}{ June-July-August } & \multicolumn{4}{|c|}{ September-0ctober-November } \\
\hline 0.05 & 20 & 1.77 & 2.09 & 2.33 & 2.84 & 1.58 & 1.71 & 1.77 & 2.29 \\
\hline 0.10 & 10 & 2.36 & 2.67 & 2.90 & 3.36 & 2.13 & 2.34 & 2.41 & 2.89 \\
\hline 0.20 & 5 & 3.22 & 3.50 & 3.71 & 4.12 & 2.90 & 3.22 & 3.30 & 3.71 \\
\hline 0.50 & 2 & 5.28 & 5.47 & 5.64 & 6.11 & 4.56 & 5.03 & 5.17 & 5.40 \\
\hline
\end{tabular}

\section{4-9.R00-1A BAMYAN RIVER AT DOAB, Continued}

Annual peak discharges

[m $\mathrm{m}^{3} / \mathrm{s}$, meters per second]

\begin{tabular}{|c|c|c|c|c|c|}
\hline \multicolumn{3}{|c|}{$\begin{array}{c}\text { Annual peak discharge, } \\
\text { by year }\end{array}$} & \multicolumn{3}{|c|}{$\begin{array}{l}\text { Annual peak discharge, } \\
\text { from highest to lowest }\end{array}$} \\
\hline $\begin{array}{l}\text { Water } \\
\text { year }\end{array}$ & Date & $\begin{array}{c}\text { Peak } \\
\text { discharge } \\
\left(\mathrm{m}^{3} / \mathrm{s}\right)\end{array}$ & $\begin{array}{l}\text { Water } \\
\text { year }\end{array}$ & Date & $\begin{array}{c}\text { Peak } \\
\text { discharge } \\
\left(\mathrm{m}^{3} / \mathrm{s}\right)\end{array}$ \\
\hline 1968 & June 15,1968 & 61.5 & 1969 & May 26, 1969 & 101 \\
\hline 1969 & May 26, 1969 & 101 & 1973 & June 9, 1973 & 61.8 \\
\hline 1970 & June 3, 1970 & 30.3 & 1968 & June 15,1968 & 61.5 \\
\hline 1971 & May 20, 1971 & 32.0 & 1972 & June 2, 1972 & 61.4 \\
\hline 1972 & June 2, 1972 & 61.4 & 1978 & June 7, 1978 & 45.0 \\
\hline 1973 & June 9, 1973 & 61.8 & 1975 & May 16, 1975 & 44.6 \\
\hline 1974 & June 6, 1974 & 33.8 & 1977 & May 28, 1977 & 40.5 \\
\hline 1975 & May 16, 1975 & 44.6 & 1976 & June 2, 1976 & 40.0 \\
\hline 1976 & June 2, 1976 & 40.0 & 1974 & June 6, 1974 & 33.8 \\
\hline 1977 & May 28, 1977 & 40.5 & 1971 & May 20, 1971 & 32.0 \\
\hline 1978 & June 7, 1978 & 45.0 & 1970 & June 3, 1970 & 30.3 \\
\hline
\end{tabular}


14-9.R00-1A BAMYAN RIVER AT DOAB, Continued

Monthly and annual mean discharges, in cubic meters per second

\begin{tabular}{|c|c|c|c|c|c|c|c|c|c|c|c|c|c|}
\hline \multirow{2}{*}{$\begin{array}{l}\text { Water } \\
\text { year }\end{array}$} & \multicolumn{12}{|c|}{ Monthly mean discharge } & \multirow{2}{*}{$\begin{array}{c}\text { Annual } \\
\text { discharge }\end{array}$} \\
\hline & October & November & December & January & February & March & April & May & June & July & August & September & \\
\hline 1968 & 7.22 & 6.19 & 4.57 & 4.34 & 4.97 & 5.32 & 8.25 & 14.4 & 51.0 & 27.5 & 11.3 & 10.3 & 12.9 \\
\hline 1969 & 7.44 & 7.22 & 5.19 & 5.55 & 5.85 & 5.19 & 6.02 & 18.5 & 56.1 & 31.5 & 13.3 & 10.5 & 14.4 \\
\hline 1970 & 8.55 & 8.82 & 7.92 & 6.69 & 5.41 & 4.80 & 5.81 & 11.4 & 16.4 & 8.37 & 5.51 & 5.08 & 7.90 \\
\hline 1971 & 5.14 & 4.79 & 4.31 & 3.64 & 3.32 & 3.81 & 3.98 & 13.0 & 9.35 & 2.66 & 2.65 & 1.97 & 4.90 \\
\hline 1972 & 3.10 & 3.26 & 2.98 & 3.55 & 4.05 & 3.32 & 3.06 & 11.1 & 36.8 & 19.8 & 8.02 & 6.89 & 8.81 \\
\hline 1973 & 8.08 & 7.44 & 5.89 & 5.72 & 5.18 & 4.23 & 5.90 & 26.6 & 43.7 & 18.6 & 8.06 & 7.62 & 12.3 \\
\hline 1974 & 6.29 & 5.90 & 4.21 & 3.60 & 2.56 & 4.32 & 3.17 & 13.7 & 21.5 & 7.82 & 4.03 & 4.00 & 6.77 \\
\hline 1975 & 4.83 & 3.80 & 3.51 & 3.86 & 3.21 & 2.82 & 3.25 & 10.4 & 25.4 & 18.2 & 6.95 & 5.69 & 7.67 \\
\hline 1976 & 5.19 & 5.12 & 5.02 & 4.59 & 4.41 & 4.47 & 6.23 & 18.9 & 25.1 & 16.1 & 4.85 & 5.55 & 8.81 \\
\hline 1977 & 5.66 & 5.28 & 5.62 & 4.54 & 4.24 & 4.67 & 4.67 & 11.0 & 23.9 & 9.47 & 4.57 & 4.40 & 7.34 \\
\hline 1978 & 4.34 & 4.93 & 4.15 & 2.70 & 2.46 & 3.01 & 3.52 & 15.2 & 28.3 & 10.5 & 6.11 & 4.62 & 7.50 \\
\hline
\end{tabular}




\section{4-9.R00-6T BAMYAN RIVER AT AHANGARAN}

\section{(U.S. Geological Survey identification number: 344900067550000)}

LOCATION: Lat $34^{\circ} 49^{\prime} \mathrm{N}$., long $67^{\circ} 55^{\prime} \mathrm{E}$.

DRAINAGE AREA: $1,660 \mathrm{~km}^{2}$.

ELEVATION: 2,450 meters above mean sea level.

PERIOD OF RECORD: April 14, 1975 to May 21, 1976.

GAGE: Staff gage.

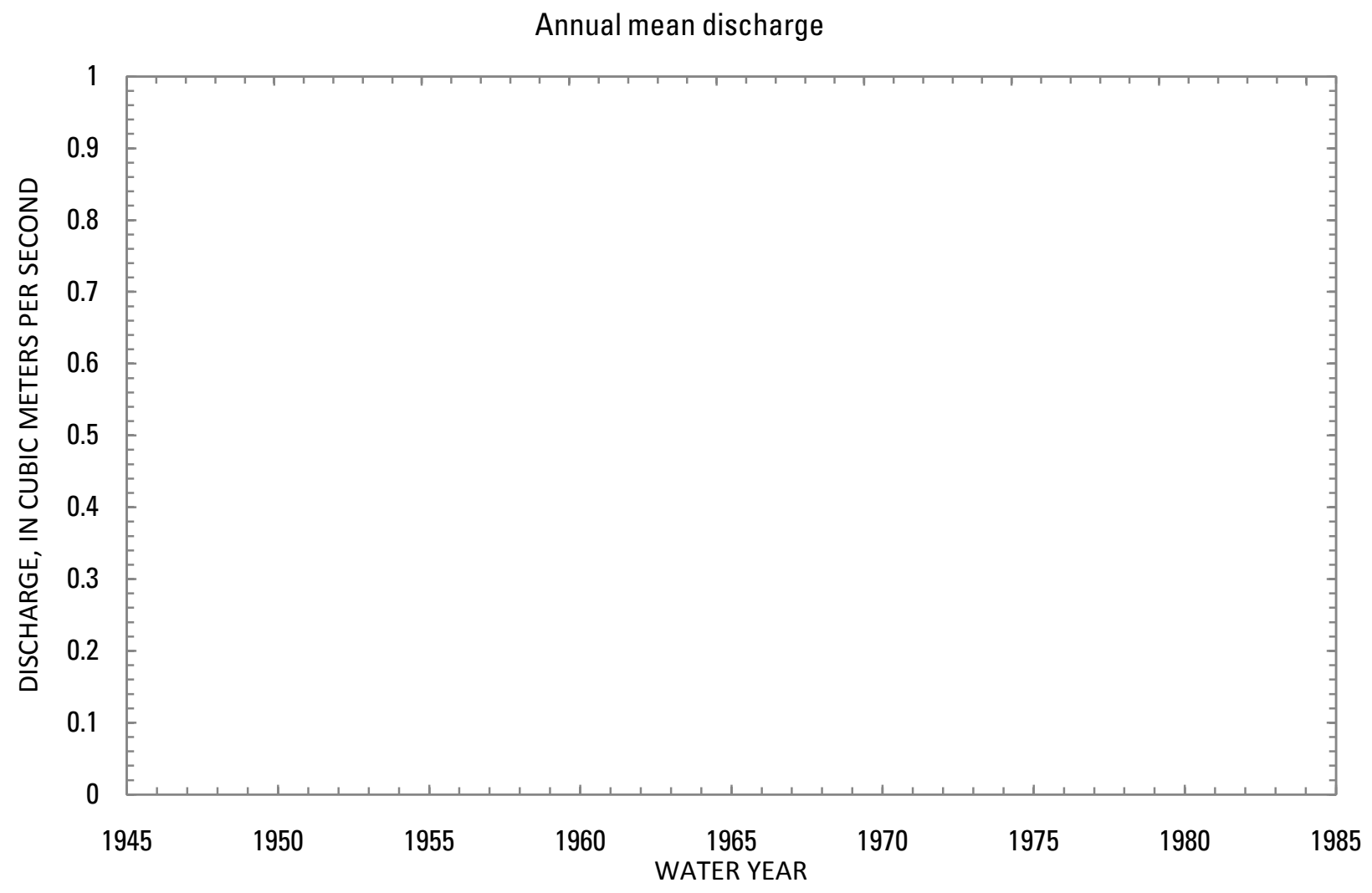




\section{4-9.R00-6T BAMYAN RIVER AT AHANGARAN, Continued}

Statistics of monthly and annual mean discharges

[ $\mathrm{m}^{3} / \mathrm{s}$, cubic meters per second; ng, not given]

\begin{tabular}{|c|c|c|c|c|c|c|c|c|}
\hline \multirow[b]{2}{*}{ Month } & \multicolumn{2}{|c|}{ Maximum } & \multicolumn{2}{|c|}{ Minimum } & \multicolumn{4}{|c|}{ Mean } \\
\hline & $\begin{array}{c}\text { Discharge } \\
\left(\mathrm{m}^{3} / \mathrm{s}\right)\end{array}$ & $\begin{array}{c}\text { Water year } \\
\text { of } \\
\text { occurrence }\end{array}$ & $\begin{array}{c}\text { Discharge } \\
\left(\mathrm{m}^{3} / \mathrm{s}\right)\end{array}$ & $\begin{array}{c}\text { Water year } \\
\text { of } \\
\text { occurrence }\end{array}$ & $\begin{array}{c}\text { Discharge } \\
\left(\mathrm{m}^{3} / \mathrm{s}\right)\end{array}$ & $\begin{array}{c}\text { Standard } \\
\text { deviation } \\
\left(\mathrm{m}^{3} / \mathrm{s}\right)\end{array}$ & $\begin{array}{c}\text { Coefficient } \\
\text { of } \\
\text { variation }\end{array}$ & $\begin{array}{c}\text { Percentage } \\
\text { of annual } \\
\text { discharge }\end{array}$ \\
\hline October & 2.97 & 1976 & 2.97 & 1976 & 2.97 & ng & ng & 5.49 \\
\hline November & 2.59 & 1976 & 2.59 & 1976 & 2.59 & ng & ng & 4.79 \\
\hline December & 2.53 & 1976 & 2.53 & 1976 & 2.53 & ng & ng & 4.68 \\
\hline January & 2.29 & 1976 & 2.29 & 1976 & 2.29 & ng & ng & 4.23 \\
\hline February & 2.32 & 1976 & 2.32 & 1976 & 2.32 & ng & ng & 4.29 \\
\hline March & 2.24 & 1976 & 2.24 & 1976 & 2.24 & ng & ng & 4.14 \\
\hline April & 3.24 & 1976 & 3.24 & 1976 & 3.23 & ng & ng & 5.98 \\
\hline May & 6.77 & 1975 & 6.77 & 1975 & 6.77 & ng & ng & 12.5 \\
\hline June & 12.8 & 1975 & 12.8 & 1975 & 12.8 & ng & ng & 23.7 \\
\hline July & 7.75 & 1975 & 7.75 & 1975 & 7.75 & ng & ng & 14.3 \\
\hline August & 4.47 & 1975 & 4.47 & 1975 & 4.47 & ng & ng & 8.26 \\
\hline September & 4.12 & 1975 & 4.12 & 1975 & 4.12 & ng & ng & 7.62 \\
\hline Annual & ng & ng & ng & ng & ng & ng & ng & 100 \\
\hline
\end{tabular}




\section{4-9.R00-6T BAMYAN RIVER AT AHANGARAN, Continued}

Monthly and annual flow duration, in cubic meters per second

[ng, not given]

\begin{tabular}{|c|c|c|c|c|c|c|c|c|c|c|c|c|c|}
\hline \multirow{2}{*}{$\begin{array}{l}\text { Percentage } \\
\text { of days } \\
\text { discharge } \\
\text { equaled or } \\
\text { exceeded }\end{array}$} & \multicolumn{12}{|c|}{ Month } & \multirow{2}{*}{ Annua } \\
\hline & October & November & December & January & February & March & April & May & June & July & August & September & \\
\hline 95 & 2.70 & 1.20 & 2.05 & 1.57 & 2.04 & 2.03 & 1.75 & 1.69 & 8.63 & 6.32 & 3.90 & 3.42 & ng \\
\hline 90 & 2.70 & 2.40 & 2.37 & 2.02 & 2.21 & 2.03 & 1.91 & 1.73 & 9.68 & 6.35 & 4.12 & 3.43 & ng \\
\hline 85 & 2.71 & ng & 2.38 & 2.04 & 2.21 & 2.04 & 1.95 & 1.78 & 10.1 & 6.37 & 4.14 & 3.45 & ng \\
\hline 80 & 2.71 & ng & 2.38 & 2.21 & 2.21 & 2.04 & 1.99 & 2.09 & 10.5 & 6.40 & 4.32 & 3.67 & ng \\
\hline 75 & 2.71 & ng & 2.39 & 2.22 & 2.21 & 2.04 & 2.05 & 2.41 & 11.4 & 6.43 & 4.33 & 3.89 & ng \\
\hline 70 & 2.87 & ng & 2.39 & 2.23 & 2.22 & 2.05 & 2.10 & 2.48 & 11.9 & 6.75 & 4.33 & 3.91 & ng \\
\hline 65 & 2.88 & ng & 2.40 & 2.23 & 2.22 & 2.05 & 2.19 & 2.55 & 12.0 & 6.81 & 4.34 & 3.92 & ng \\
\hline 60 & 2.88 & ng & 2.53 & 2.24 & 2.22 & 2.20 & 2.38 & 3.51 & 12.1 & 7.25 & 4.34 & 4.12 & ng \\
\hline 55 & $\mathrm{ng}$ & $\mathrm{ng}$ & 2.53 & 2.25 & $\mathrm{ng}$ & 2.20 & 2.41 & 4.08 & 13.0 & 7.29 & 4.35 & 4.14 & ng \\
\hline 50 & ng & ng & 2.54 & 2.37 & ng & 2.21 & 2.45 & 4.62 & 13.1 & 7.32 & 4.35 & 4.15 & ng \\
\hline 45 & ng & ng & 2.55 & 2.38 & ng & 2.21 & 2.86 & 4.90 & 13.1 & 7.36 & 4.57 & 4.35 & ng \\
\hline 40 & ng & ng & 2.55 & 2.39 & ng & 2.21 & 3.10 & 7.84 & 13.2 & 7.57 & 4.58 & 4.36 & ng \\
\hline 35 & ng & ng & 2.69 & 2.40 & ng & 2.22 & 3.38 & 10.0 & 13.6 & 7.85 & 4.59 & 4.37 & ng \\
\hline 30 & ng & ng & 2.70 & 2.41 & ng & 2.22 & 3.88 & 10.3 & 13.7 & 8.20 & 4.60 & 4.38 & ng \\
\hline 25 & ng & ng & 2.70 & $\mathrm{ng}$ & ng & ng & 4.16 & 10.6 & 14.5 & 8.50 & 4.61 & ng & ng \\
\hline 20 & ng & ng & 2.71 & ng & ng & ng & 4.34 & 11.3 & 15.2 & 9.30 & 4.81 & ng & ng \\
\hline 15 & ng & ng & 2.72 & $\mathrm{ng}$ & ng & ng & 4.98 & 13.5 & 15.7 & 10.1 & 4.83 & ng & $\mathrm{ng}$ \\
\hline 10 & ng & ng & 2.73 & ng & ng & ng & 5.12 & 14.2 & 16.0 & 10.2 & 4.85 & ng & ng \\
\hline 5 & $\mathrm{ng}$ & $\mathrm{ng}$ & $\mathrm{ng}$ & $\mathrm{ng}$ & ng & $\mathrm{ng}$ & $\mathrm{ng}$ & $\mathrm{ng}$ & $\mathrm{ng}$ & $\mathrm{ng}$ & $\mathrm{ng}$ & ng & $\mathrm{ng}$ \\
\hline
\end{tabular}




\section{4-9.R00-6T BAMYAN RIVER AT AHANGARAN, Continued}

Probability of occurrence of annual high discharges

[ $\mathrm{m}^{3} / \mathrm{s}$, cubic meters per second; $\mathrm{ng}$, not given]

\begin{tabular}{|c|c|c|c|c|c|c|}
\hline \multirow{2}{*}{$\begin{array}{l}\text { Exceedance } \\
\text { probability }\end{array}$} & \multirow{2}{*}{$\begin{array}{c}\text { Recurrence } \\
\text { interval } \\
\text { (years) }\end{array}$} & \multirow{2}{*}{$\begin{array}{l}\text { Maximum } \\
\text { instantaneous } \\
\text { discharge } \\
\left(\mathrm{m}^{3} / \mathrm{s}\right)\end{array}$} & \multicolumn{4}{|c|}{ Maximum daily mean discharge $\left(\mathrm{m}^{3} / \mathrm{s}\right)$} \\
\hline & & & $\begin{array}{l}\text { 3-day } \\
\text { period }\end{array}$ & $\begin{array}{l}\text { 7-day } \\
\text { period }\end{array}$ & $\begin{array}{l}\text { 15-day } \\
\text { period }\end{array}$ & $\begin{array}{l}\text { 30-day } \\
\text { period }\end{array}$ \\
\hline 0.99 & 1.01 & ng & ng & ng & $\mathrm{ng}$ & ng \\
\hline 0.95 & 1.05 & ng & ng & ng & $\mathrm{ng}$ & ng \\
\hline 0.90 & 1.11 & ng & ng & ng & $\mathrm{ng}$ & ng \\
\hline 0.80 & 1.25 & ng & ng & ng & $\mathrm{ng}$ & $\mathrm{ng}$ \\
\hline 0.50 & 2 & ng & $\mathrm{ng}$ & ng & ng & ng \\
\hline 0.20 & 5 & ng & $\mathrm{ng}$ & ng & ng & ng \\
\hline 0.10 & 10 & ng & $\mathrm{ng}$ & ng & $\mathrm{ng}$ & $\mathrm{ng}$ \\
\hline 0.04 & 25 & ng & $\mathrm{ng}$ & ng & $\mathrm{ng}$ & ng \\
\hline 0.02 & 50 & ng & $\mathrm{ng}$ & ng & $\mathrm{ng}$ & $\mathrm{ng}$ \\
\hline 0.01 & 100 & ng & $\mathrm{ng}$ & $\mathrm{ng}$ & $\mathrm{ng}$ & $\mathrm{ng}$ \\
\hline 0.005 & 200 & ng & ng & ng & ng & $\mathrm{ng}$ \\
\hline 0.002 & 500 & $\mathrm{ng}$ & ng & $\mathrm{ng}$ & $\mathrm{ng}$ & $\mathrm{ng}$ \\
\hline
\end{tabular}

\section{4-9.R00-6T BAMYAN RIVER AT AHANGARAN, Continued}

Probability of occurrence of annual low discharges

[m $\mathrm{m}^{3} / \mathrm{s}$, meters per second; ng, not given]

\begin{tabular}{|c|c|c|c|c|c|c|c|c|c|c|}
\hline \multirow{3}{*}{$\begin{array}{c}\text { Nonexceedance } \\
\text { probability }\end{array}$} & \multirow{3}{*}{$\begin{array}{c}\text { Recurrence } \\
\text { interval } \\
\text { (years) }\end{array}$} & \multicolumn{9}{|c|}{ Minimum daily mean discharge $\left(\mathrm{m}^{3} / \mathrm{s}\right)$} \\
\hline & & \multicolumn{9}{|c|}{ Number of consecutive days } \\
\hline & & 1 & 3 & 7 & 14 & 30 & 60 & 90 & 120 & 183 \\
\hline 0.05 & 20 & ng & ng & $\mathrm{ng}$ & ng & ng & ng & ng & ng & ng \\
\hline 0.10 & 10 & ng & ng & $\mathrm{ng}$ & ng & ng & ng & ng & ng & ng \\
\hline 0.20 & 5 & ng & ng & $\mathrm{ng}$ & $\mathrm{ng}$ & ng & ng & ng & ng & ng \\
\hline 0.50 & 2 & ng & ng & $\mathrm{ng}$ & $\mathrm{ng}$ & ng & ng & ng & ng & ng \\
\hline
\end{tabular}




\section{4-9.R00-6T BAMYAN RIVER AT AHANGARAN, Continued}

Probability of occurrence of seasonal low discharges [ $\mathrm{m}^{3} / \mathrm{s}$, meters per second; $\mathrm{ng}$, not given]

\begin{tabular}{|c|c|c|c|c|c|c|c|c|c|}
\hline \multirow{3}{*}{$\begin{array}{c}\text { Nonexceedance } \\
\text { probability }\end{array}$} & \multirow{3}{*}{$\begin{array}{c}\text { Recurrence } \\
\text { interval } \\
\text { (years) }\end{array}$} & \multicolumn{8}{|c|}{ Minimum daily mean discharge $\left(\mathrm{m}^{3} / \mathrm{s}\right)$} \\
\hline & & \multicolumn{8}{|c|}{ Number of consecutive days } \\
\hline & & 1 & 7 & 14 & 30 & 1 & 7 & 14 & 30 \\
\hline & & \multicolumn{4}{|c|}{ December-January-February } & \multicolumn{4}{|c|}{ March-April-May } \\
\hline 0.05 & 20 & ng & ng & ng & ng & ng & ng & ng & $\mathrm{ng}$ \\
\hline 0.10 & 10 & ng & ng & ng & ng & ng & ng & ng & $\mathrm{ng}$ \\
\hline 0.20 & 5 & ng & ng & ng & ng & ng & ng & ng & $\mathrm{ng}$ \\
\hline \multirow[t]{2}{*}{0.50} & 2 & ng & ng & ng & ng & ng & ng & ng & $\mathrm{ng}$ \\
\hline & & \multicolumn{4}{|c|}{ June-July-August } & \multicolumn{4}{|c|}{ September-October-November } \\
\hline 0.05 & 20 & ng & ng & ng & $\mathrm{ng}$ & ng & ng & ng & ng \\
\hline 0.10 & 10 & ng & ng & ng & ng & ng & ng & ng & ng \\
\hline 0.20 & 5 & ng & ng & ng & ng & ng & ng & $\mathrm{ng}$ & $\mathrm{ng}$ \\
\hline 0.50 & 2 & $\mathrm{ng}$ & $\mathrm{ng}$ & $\mathrm{ng}$ & $\mathrm{ng}$ & $\mathrm{ng}$ & $\mathrm{ng}$ & $\mathrm{ng}$ & $\mathrm{ng}$ \\
\hline
\end{tabular}

\section{4-9.R00-6T BAMYAN RIVER AT AHANGARAN, Continued}

Annual peak discharges

[ $\mathrm{m}^{3} / \mathrm{s}$, meters per second]

\begin{tabular}{|c|c|c|c|c|c|}
\hline \multicolumn{3}{|c|}{$\begin{array}{c}\text { Annual peak discharge, } \\
\text { by year }\end{array}$} & \multicolumn{3}{|c|}{$\begin{array}{l}\text { Annual peak discharge, } \\
\text { from highest to lowest }\end{array}$} \\
\hline $\begin{array}{l}\text { Water } \\
\text { year }\end{array}$ & Date & $\begin{array}{c}\text { Peak } \\
\text { discharge } \\
\left(\mathrm{m}^{3} / \mathrm{s}\right)\end{array}$ & $\begin{array}{l}\text { Water } \\
\text { year }\end{array}$ & Date & $\begin{array}{c}\text { Peak } \\
\text { discharge } \\
\left(\mathrm{m}^{3} / \mathrm{s}\right)\end{array}$ \\
\hline 1975 & June 20, 1975 & 23.1 & 1975 & June 20,1975 & 23.1 \\
\hline
\end{tabular}


14-9.R00-6T BAMYAN RIVER AT AHANGARAN, Continued

Monthly and annual mean discharges, in cubic meters per second

[Data may not be rounded in accordance with U.S. Geological Survey publication standards; --, no data]

\begin{tabular}{|c|c|c|c|c|c|c|c|c|c|c|c|c|c|}
\hline \multirow{2}{*}{$\begin{array}{l}\text { Water } \\
\text { year }\end{array}$} & \multicolumn{12}{|c|}{ Monthly mean discharge } & \multirow{2}{*}{$\begin{array}{c}\text { Annual } \\
\text { discharge }\end{array}$} \\
\hline & October & November & December & January & February & March & April & May & June & July & August & September & \\
\hline 1975 & -- & -- & -- & -- & -- & -- & -- & 6.77 & 12.8 & 7.75 & 4.47 & 4.12 & -- \\
\hline 1976 & 2.97 & 2.59 & 2.53 & 2.29 & 2.32 & 2.24 & 3.24 & -- & -- & -- & -- & -- & -- \\
\hline
\end{tabular}




\section{4-9.R00-8A BAMYAN RIVER AT BAMYAN}

\section{(U.S. Geological Survey identification number: 345000067490000)}

LOCATION: Lat $34^{\circ} 50^{\prime} \mathrm{N}$., long 67049'E.

DRAINAGE AREA: $945 \mathrm{~km}^{2}$.

ELEVATION: 2,506 meters above mean sea level.

PERIOD OF RECORD: October 1, 1969 to May 21, 1977.

GAGE: Staff gage.

Annual mean discharge

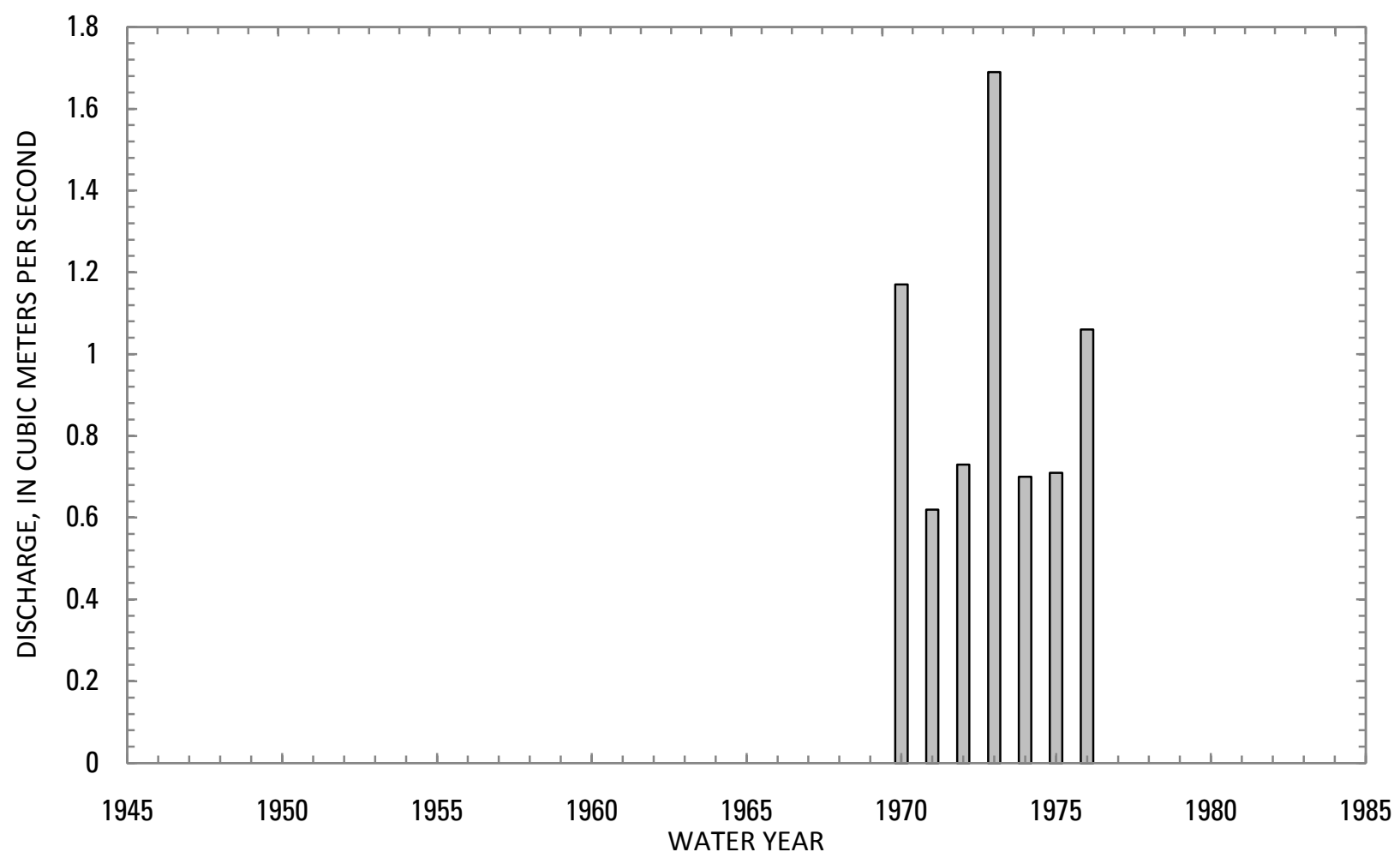




\section{4-9.R00-8A BAMYAN RIVER AT BAMYAN, Continued}

Statistics of monthly and annual mean discharges

$\left[\mathrm{m}^{3} / \mathrm{s}\right.$, cubic meters per second]

\begin{tabular}{|c|c|c|c|c|c|c|c|c|}
\hline \multirow[b]{2}{*}{ Month } & \multicolumn{2}{|c|}{ Maximum } & \multicolumn{2}{|c|}{ Minimum } & \multicolumn{4}{|c|}{ Mean } \\
\hline & $\begin{array}{c}\text { Discharge } \\
\left(\mathrm{m}^{3} / \mathrm{s}\right)\end{array}$ & $\begin{array}{c}\text { Water year } \\
\text { of } \\
\text { occurrence }\end{array}$ & $\begin{array}{c}\text { Discharge } \\
\left(\mathrm{m}^{3} / \mathrm{s}\right)\end{array}$ & $\begin{array}{c}\text { Water year } \\
\text { of } \\
\text { occurrence }\end{array}$ & $\begin{array}{c}\text { Discharge } \\
\left(\mathrm{m}^{3} / \mathrm{s}\right)\end{array}$ & $\begin{array}{c}\text { Standard } \\
\text { deviation } \\
\left(\mathrm{m}^{3} / \mathrm{s}\right)\end{array}$ & $\begin{array}{c}\text { Coefficient } \\
\text { of } \\
\text { variation }\end{array}$ & $\begin{array}{c}\text { Percentage } \\
\text { of annual } \\
\text { discharge }\end{array}$ \\
\hline October & 1.97 & 1970 & 0.78 & 1977 & 1.07 & 0.39 & 0.36 & 9.39 \\
\hline November & 1.96 & 1970 & 0.59 & 1971 & 1.05 & 0.44 & 0.42 & 9.29 \\
\hline December & 1.54 & 1970 & 0.36 & 1971 & 0.76 & 0.45 & 0.58 & 6.74 \\
\hline January & 1.43 & 1973 & 0.23 & 1975 & 0.67 & 0.41 & 0.61 & 5.92 \\
\hline February & 0.93 & 1976 & 0.26 & 1975 & 0.63 & 0.25 & 0.41 & 5.51 \\
\hline March & 1.46 & 1971 & 0.46 & 1972 & 0.98 & 0.40 & 0.41 & 8.65 \\
\hline April & 2.33 & 1973 & 0.41 & 1972 & 1.07 & 0.70 & 0.66 & 9.46 \\
\hline May & 3.01 & 1973 & 0.63 & 1972 & 1.38 & 0.81 & 0.58 & 12.2 \\
\hline June & 4.15 & 1973 & 0.52 & 1974 & 1.33 & 1.27 & 0.96 & 11.7 \\
\hline July & 2.09 & 1973 & 0.38 & 1975 & 0.86 & 0.59 & 0.69 & 7.59 \\
\hline August & 1.10 & 1973 & 0.19 & 1971 & 0.71 & 0.29 & 0.41 & 6.25 \\
\hline September & 1.19 & 1973 & 0.68 & 1971 & 0.83 & 0.17 & 0.21 & 7.33 \\
\hline Annual & 1.69 & 1973 & 0.62 & 1971 & 0.96 & 0.39 & 0.40 & 100 \\
\hline
\end{tabular}


14-9.R00-8A BAMYAN RIVER AT BAMYAN, Continued

Monthly and annual flow duration, in cubic meters per second

\begin{tabular}{|c|c|c|c|c|c|c|c|c|c|c|c|c|c|}
\hline \multirow{2}{*}{$\begin{array}{l}\text { Percentage } \\
\text { of days } \\
\text { discharge } \\
\text { equaled or } \\
\text { exceeded }\end{array}$} & \multicolumn{12}{|c|}{ Month } & \multirow{2}{*}{ Annual } \\
\hline & October & November & December & January & February & March & April & May & June & July & August & September & \\
\hline 95 & 0.68 & 0.44 & 0.32 & 0.21 & 0.21 & 0.38 & 0.19 & 0.35 & 0.42 & 0.19 & 0.24 & 0.55 & 0.29 \\
\hline 90 & 0.73 & 0.53 & 0.36 & 0.28 & 0.30 & 0.42 & 0.30 & 0.48 & 0.45 & 0.25 & 0.30 & 0.63 & 0.36 \\
\hline 85 & 0.75 & 0.61 & 0.37 & 0.29 & 0.31 & 0.43 & 0.33 & 0.54 & 0.47 & 0.30 & 0.42 & 0.64 & 0.41 \\
\hline 80 & 0.79 & 0.64 & 0.37 & 0.30 & 0.37 & 0.46 & 0.39 & 0.59 & 0.48 & 0.38 & 0.49 & 0.68 & 0.46 \\
\hline 75 & 0.81 & 0.70 & 0.39 & 0.34 & 0.41 & 0.49 & 0.44 & 0.63 & 0.57 & 0.46 & 0.56 & 0.68 & 0.52 \\
\hline 70 & 0.87 & 0.75 & 0.41 & 0.37 & 0.42 & 0.53 & 0.58 & 0.69 & 0.61 & 0.53 & 0.63 & 0.69 & 0.58 \\
\hline 65 & 0.89 & 0.84 & 0.42 & 0.42 & 0.43 & 0.58 & 0.63 & 0.76 & 0.65 & 0.61 & 0.64 & 0.75 & 0.64 \\
\hline 60 & 0.91 & 0.89 & 0.48 & 0.49 & 0.56 & 0.69 & 0.66 & 0.85 & 0.70 & 0.67 & 0.67 & 0.77 & 0.71 \\
\hline 55 & 0.93 & 0.97 & 0.54 & 0.55 & 0.61 & 0.82 & 0.69 & 0.95 & 0.76 & 0.71 & 0.73 & 0.80 & 0.76 \\
\hline 50 & 0.95 & 1.00 & 0.61 & 0.57 & 0.62 & 0.88 & 0.72 & 1.03 & 0.80 & 0.73 & 0.77 & 0.81 & 0.82 \\
\hline 45 & 1.04 & 1.03 & 0.67 & 0.60 & 0.67 & 0.94 & 0.77 & 1.12 & 0.87 & 0.76 & 0.79 & 0.86 & 0.86 \\
\hline 40 & 1.09 & 1.10 & 0.74 & 0.63 & 0.69 & 0.97 & 0.88 & 1.27 & 0.94 & 0.79 & 0.81 & 0.88 & 0.90 \\
\hline 35 & 1.12 & 1.14 & 0.82 & 0.73 & 0.76 & 1.02 & 1.01 & 1.53 & 1.01 & 0.82 & 0.82 & 0.90 & 0.98 \\
\hline 30 & 1.13 & 1.29 & 0.96 & 0.83 & 0.79 & 1.09 & 1.12 & 1.67 & 1.12 & 0.96 & 0.84 & 0.91 & 1.05 \\
\hline 25 & 1.15 & 1.35 & 1.12 & 0.98 & 0.81 & 1.17 & 1.41 & 2.02 & 1.27 & 1.09 & 0.89 & 0.92 & 1.10 \\
\hline 20 & 1.16 & 1.40 & 1.32 & 1.03 & 0.85 & 1.51 & 1.76 & 2.31 & 1.82 & 1.16 & 0.92 & 0.94 & 1.21 \\
\hline 15 & 1.32 & 1.45 & 1.39 & 1.10 & 0.90 & 1.60 & 2.15 & 2.67 & 2.42 & 1.37 & 1.02 & 1.04 & 1.41 \\
\hline 10 & 1.88 & 1.87 & 1.44 & 1.25 & 0.96 & 1.99 & 2.49 & 2.90 & 3.72 & 1.79 & 1.10 & 1.18 & 1.79 \\
\hline 5 & 2.01 & 1.99 & 1.65 & 1.54 & 1.14 & 2.31 & 2.83 & 3.19 & 4.52 & 2.15 & 1.14 & 1.21 & 2.33 \\
\hline
\end{tabular}




\section{4-9.R00-8A BAMYAN RIVER AT BAMYAN, Continued}

Probability of occurrence of annual high discharges

[ $\mathrm{m}^{3} / \mathrm{s}$, cubic meters per second; $\mathrm{ng}$, not given]

\begin{tabular}{|c|c|c|c|c|c|c|}
\hline \multirow{2}{*}{$\begin{array}{c}\text { Exceedance } \\
\text { probability }\end{array}$} & \multirow{2}{*}{$\begin{array}{l}\text { Recurrence } \\
\text { interval } \\
\text { (years) }\end{array}$} & \multirow{2}{*}{$\begin{array}{c}\text { Maximum } \\
\text { instantaneous } \\
\text { discharge }^{1} \\
\left(\mathrm{~m}^{3} / \mathrm{s}\right)\end{array}$} & \multicolumn{4}{|c|}{ Maximum daily mean discharge $\left(\mathrm{m}^{3} / \mathrm{s}\right)$} \\
\hline & & & $\begin{array}{l}\text { 3-day } \\
\text { period }\end{array}$ & $\begin{array}{l}\text { 7-day } \\
\text { period }\end{array}$ & $\begin{array}{l}\text { 15-day } \\
\text { period }\end{array}$ & $\begin{array}{l}\text { 30-day } \\
\text { period }\end{array}$ \\
\hline 0.99 & 1.01 & 1.20 & 1.17 & ${ }^{2} 1.03$ & ${ }^{2} 1.01$ & 1.07 \\
\hline 0.95 & 1.05 & 1.60 & 1.56 & 1.33 & 1.22 & 1.16 \\
\hline 0.90 & 1.11 & 1.90 & 1.82 & 1.53 & 1.36 & 1.24 \\
\hline 0.80 & 1.25 & 2.30 & 2.18 & 1.82 & 1.57 & 1.36 \\
\hline 0.50 & 2 & 3.40 & 3.08 & 2.58 & 2.16 & 1.76 \\
\hline 0.20 & 5 & 5.10 & 4.32 & 3.71 & 3.16 & 2.56 \\
\hline 0.10 & 10 & 6.30 & 5.13 & 4.52 & 3.95 & 3.26 \\
\hline 0.04 & 25 & 7.90 & 6.17 & 5.60 & 5.10 & 4.41 \\
\hline 0.02 & 50 & 9.10 & 6.93 & 6.45 & 6.08 & 5.48 \\
\hline 0.01 & 100 & 10.4 & 7.69 & 7.33 & 7.18 & 6.77 \\
\hline 0.005 & 200 & 11.8 & 8.46 & ${ }^{2} 8.26$ & 8.40 & 8.33 \\
\hline 0.002 & 500 & 13.7 & $\mathrm{ng}$ & $\mathrm{ng}$ & $\mathrm{ng}$ & $\mathrm{ng}$ \\
\hline
\end{tabular}

\section{4-9.R00-8A BAMYAN RIVER AT BAMYAN, Continued}

Probability of occurrence of annual low discharges

$\left[\mathrm{m}^{3} / \mathrm{s}\right.$, meters per second]

\begin{tabular}{|c|c|c|c|c|c|c|c|c|c|c|}
\hline \multirow{3}{*}{$\begin{array}{c}\text { Nonexceedance } \\
\text { probability }\end{array}$} & \multirow{3}{*}{$\begin{array}{c}\text { Recurrence } \\
\text { interval } \\
\text { (years) }\end{array}$} & \multicolumn{9}{|c|}{ Minimum daily mean discharge $\left(\mathrm{m}^{3} / \mathrm{s}\right)$} \\
\hline & & \multicolumn{9}{|c|}{ Number of consecutive days } \\
\hline & & 1 & 3 & 7 & 14 & 30 & 60 & 90 & 120 & 183 \\
\hline 0.05 & 20 & 0 & 0 & 0 & 0 & 0.10 & 0.21 & 0.26 & 0.30 & 0.49 \\
\hline 0.10 & 10 & 0 & 0 & 0 & 0 & 0.14 & 0.24 & 0.29 & 0.33 & 0.52 \\
\hline 0.20 & 5 & 0.12 & 0.12 & 0.12 & 0.16 & 0.19 & 0.28 & 0.33 & 0.38 & 0.56 \\
\hline 0.50 & 2 & 0.18 & 0.19 & 0.21 & 0.24 & 0.30 & 0.37 & 0.44 & 0.49 & 0.65 \\
\hline
\end{tabular}




\section{4-9.R00-8A BAMYAN RIVER AT BAMYAN, Continued}

Probability of occurrence of seasonal low discharges [ $\mathrm{m}^{3} / \mathrm{s}$, meters per second]

\begin{tabular}{|c|c|c|c|c|c|c|c|c|c|}
\hline \multirow{3}{*}{$\begin{array}{c}\text { Nonexceedance } \\
\text { probability }\end{array}$} & \multirow{3}{*}{$\begin{array}{c}\text { Recurrence } \\
\text { interval } \\
\text { (years) }\end{array}$} & \multicolumn{8}{|c|}{ Minimum daily mean discharge $\left(\mathrm{m}^{3} / \mathrm{s}\right)$} \\
\hline & & \multicolumn{8}{|c|}{ Number of consecutive days } \\
\hline & & 1 & 7 & 14 & 30 & 1 & 7 & 14 & 30 \\
\hline & & \multicolumn{4}{|c|}{ December-January-February } & \multicolumn{4}{|c|}{ March-April-May } \\
\hline 0.05 & 20 & 0.14 & 0.14 & 0.16 & 0.18 & 0.14 & 0.14 & 0.17 & 0.34 \\
\hline 0.10 & 10 & 0.19 & 0.19 & 0.21 & 0.23 & 0.17 & 0.18 & 0.22 & 0.38 \\
\hline 0.20 & 5 & 0.25 & 0.27 & 0.28 & 0.31 & 0.22 & 0.25 & 0.28 & 0.43 \\
\hline \multirow{2}{*}{0.50} & 2 & 0.41 & 0.45 & 0.47 & 0.51 & 0.34 & 0.41 & 0.45 & 0.56 \\
\hline & & \multicolumn{4}{|c|}{ June-July-August } & \multicolumn{4}{|c|}{ September-0 ctober-November } \\
\hline 0.05 & 20 & 0 & 0 & 0 & 0.11 & 0.32 & 0.39 & 0.44 & 0.59 \\
\hline 0.10 & 10 & 0 & 0 & 0 & 0.17 & 0.35 & 0.41 & 0.47 & 0.62 \\
\hline 0.20 & 5 & 0.14 & 0.19 & 0.23 & 0.27 & 0.38 & 0.45 & 0.5 & 0.65 \\
\hline 0.50 & 2 & 0.36 & 0.42 & 0.47 & 0.57 & 0.45 & 0.53 & 0.59 & 0.71 \\
\hline
\end{tabular}

\section{4-9.R00-8A BAMYAN RIVER AT BAMYAN, Continued}

Annual peak discharges

$\left[\mathrm{m}^{3} / \mathrm{s}\right.$, meters per second]

\begin{tabular}{|c|c|c|c|c|c|}
\hline \multicolumn{3}{|c|}{$\begin{array}{c}\text { Annual peak discharge, } \\
\text { by year }\end{array}$} & \multicolumn{3}{|c|}{$\begin{array}{l}\text { Annual peak discharge, } \\
\text { from highest to lowest }\end{array}$} \\
\hline $\begin{array}{l}\text { Water } \\
\text { year }\end{array}$ & Date & $\begin{array}{c}\text { Peak } \\
\text { discharge } \\
\left(\mathrm{m}^{3} / \mathrm{s}\right)\end{array}$ & $\begin{array}{l}\text { Water } \\
\text { year }\end{array}$ & Date & $\begin{array}{c}\text { Peak } \\
\text { discharge } \\
\left(\mathrm{m}^{3} / \mathrm{s}\right)\end{array}$ \\
\hline 1970 & April 5, 1970 & 2.90 & 1976 & April 22, 1976 & 6.14 \\
\hline 1971 & March 20, 1971 & 2.04 & 1973 & June 14,1973 & 5.30 \\
\hline 1972 & June 24, 1972 & 2.04 & 1975 & May 16, 1975 & 5.18 \\
\hline 1973 & June 14, 1973 & 5.30 & 1970 & April 5, 1970 & 2.90 \\
\hline 1974 & March 20, 1974 & 2.90 & 1974 & March 20, 1974 & 2.90 \\
\hline 1975 & May 16,1975 & 5.18 & 1971 & March 20, 1971 & 2.04 \\
\hline 1976 & April 22, 1976 & 6.14 & 1972 & June 24,1972 & 2.04 \\
\hline
\end{tabular}




\section{4-9.R00-8A BAMYAN RIVER AT BAMYAN, Continued}

Monthly and annual mean discharges, in cubic meters per second $[--$, no data]

\begin{tabular}{|c|c|c|c|c|c|c|c|c|c|c|c|c|c|}
\hline \multirow{2}{*}{$\begin{array}{l}\text { Water } \\
\text { year }\end{array}$} & \multicolumn{12}{|c|}{ Monthly mean discharge } & \multirow{2}{*}{$\begin{array}{c}\text { Annual } \\
\text { discharge }\end{array}$} \\
\hline & October & November & December & January & February & March & April & May & June & July & August & September & \\
\hline 1970 & 1.97 & 1.96 & 1.54 & 1.06 & 0.85 & 1.06 & 1.07 & 1.34 & 0.74 & 0.76 & 0.80 & 0.89 & 1.17 \\
\hline 1971 & 0.83 & 0.59 & 0.36 & 0.38 & 0.49 & 1.46 & 0.59 & 0.67 & 0.68 & 0.47 & 0.19 & 0.68 & 0.62 \\
\hline 1972 & 0.81 & 0.83 & 0.56 & 0.54 & 0.62 & 0.46 & 0.41 & 0.63 & 1.07 & 1.13 & 0.89 & 0.86 & 0.73 \\
\hline 1973 & 1.09 & 1.27 & 1.06 & 1.43 & 0.88 & 0.72 & 2.33 & 3.01 & 4.15 & 2.09 & 1.10 & 1.19 & 1.69 \\
\hline 1974 & 1.11 & 0.75 & 0.37 & 0.29 & 0.33 & 1.39 & 0.57 & 1.04 & 0.52 & 0.66 & 0.63 & 0.71 & 0.70 \\
\hline 1975 & 1.00 & 0.92 & 0.37 & 0.23 & 0.26 & 0.48 & 1.13 & 1.34 & 1.14 & 0.38 & 0.54 & 0.75 & 0.71 \\
\hline 1976 & 0.94 & 1.32 & 1.20 & 0.79 & 0.93 & 0.93 & 1.92 & 1.67 & 0.97 & 0.55 & 0.81 & 0.76 & 1.06 \\
\hline 1977 & 0.78 & 0.81 & 0.66 & 0.65 & 0.66 & 1.36 & 0.58 & -- & -- & -- & -- & -- & -- \\
\hline
\end{tabular}




\section{5-0.000-1M KOKCHA RIVER AT KHOJAGHAR}

\section{(U.S. Geological Survey identification number: 370500069280000)}

LOCATION: Lat $37^{\circ} 05^{\prime} \mathrm{N}$., long $69^{\circ} 28^{\prime} \mathrm{E}$.

DRAINAGE AREA: $20,645 \mathrm{~km}^{2}$.

ELEVATION: 446 meters above mean sea level.

PERIOD OF RECORD: April 26, 1964 to September 30, 1978.

GAGE: Staff gage. Water-stage recorder at same site and datum during water years 1970-73 and 1978.

Annual mean discharge

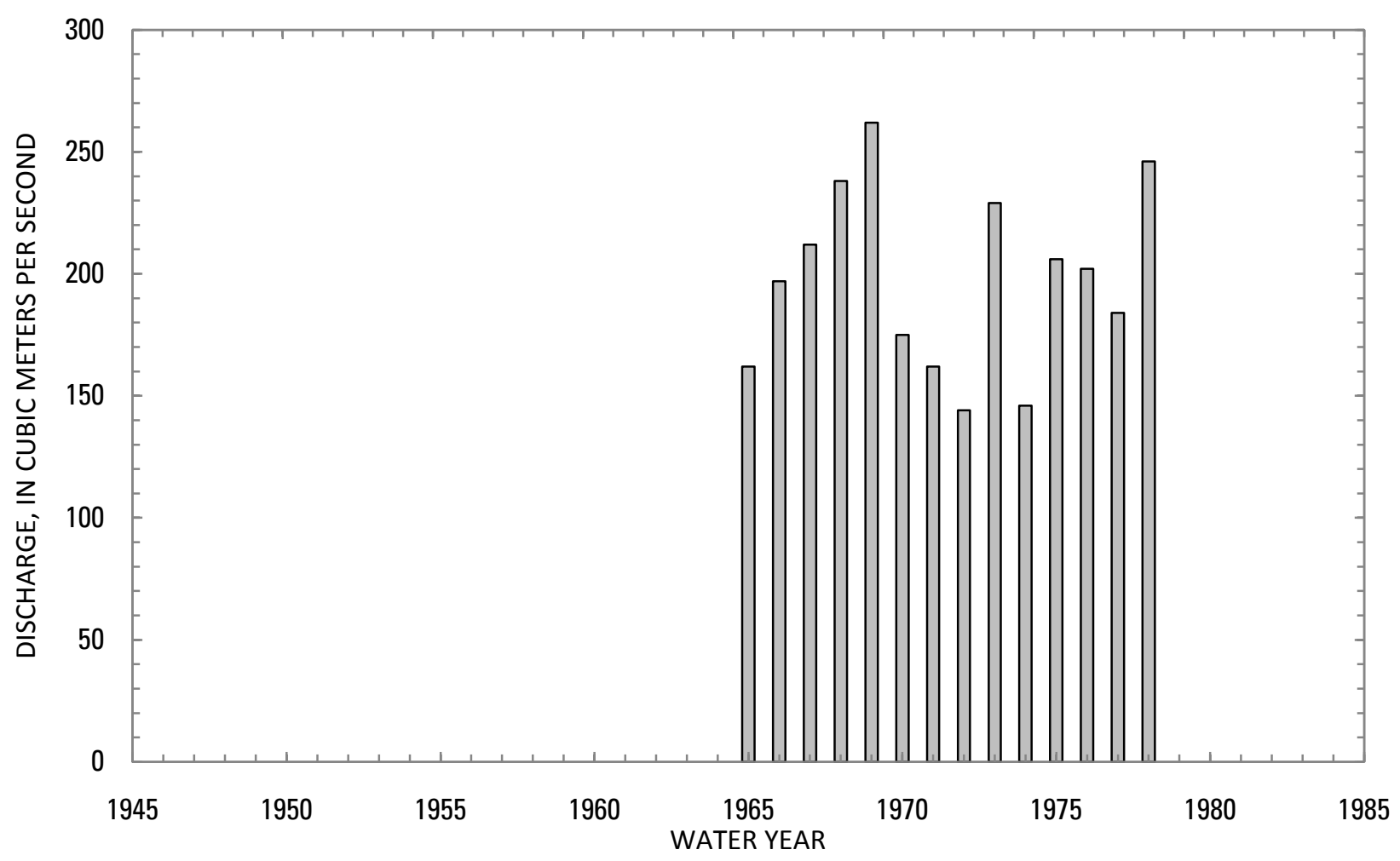




\section{5-0.000-1M KOKCHA RIVER AT KHOJAGHAR, Continued}

Statistics of monthly and annual mean discharges [ $\mathrm{m}^{3} / \mathrm{s}$, cubic meters per second]

\begin{tabular}{|c|c|c|c|c|c|c|c|c|}
\hline \multirow[b]{2}{*}{ Month } & \multicolumn{2}{|c|}{ Maximum } & \multicolumn{2}{|c|}{ Minimum } & \multicolumn{4}{|c|}{ Mean } \\
\hline & $\begin{array}{c}\text { Discharge } \\
\left(\mathrm{m}^{3} / \mathrm{s}\right)\end{array}$ & $\begin{array}{c}\text { Water year } \\
\text { of } \\
\text { occurrence }\end{array}$ & $\begin{array}{c}\text { Discharge } \\
\left(\mathrm{m}^{3} / \mathrm{s}\right)\end{array}$ & $\begin{array}{c}\text { Water year } \\
\text { of } \\
\text { occurrence }\end{array}$ & $\begin{array}{c}\text { Discharge } \\
\left(\mathrm{m}^{3} / \mathrm{s}\right)\end{array}$ & $\begin{array}{c}\text { Standard } \\
\text { deviation } \\
\left(\mathrm{m}^{3} / \mathrm{s}\right)\end{array}$ & $\begin{array}{c}\text { Coefficient } \\
\text { of } \\
\text { variation }\end{array}$ & $\begin{array}{c}\text { Percentage } \\
\text { of annual } \\
\text { discharge }\end{array}$ \\
\hline October & 142 & 1968 & 82.2 & 1972 & 110 & 19.9 & 0.18 & 4.67 \\
\hline November & 111 & 1970 & 64.8 & 1978 & 85.9 & 16.3 & 0.19 & 3.65 \\
\hline December & 99.8 & 1970 & 53.1 & 1973 & 72.3 & 13.8 & 0.19 & 3.08 \\
\hline January & 92.7 & 1970 & 42.5 & 1973 & 62.6 & 15.2 & 0.24 & 2.66 \\
\hline February & 84.3 & 1970 & 46.5 & 1971 & 63.3 & 14.1 & 0.22 & 2.69 \\
\hline March & 153 & 1969 & 42.7 & 1977 & 80.4 & 24.2 & 0.30 & 3.42 \\
\hline April & 201 & 1978 & 60.7 & 1977 & 133 & 47.4 & 0.36 & 5.67 \\
\hline May & 368 & 1969 & 128 & 1974 & 225 & 70.7 & 0.31 & 9.57 \\
\hline June & 748 & 1978 & 291 & 1974 & 484 & 137 & 0.28 & 20.6 \\
\hline July & 703 & 1969 & 313 & 1970 & 511 & 133 & 0.26 & 21.7 \\
\hline August & 472 & 1967 & 205 & 1972 & 338 & 76.5 & 0.23 & 14.4 \\
\hline September & 240 & 1967 & 130 & 1972 & 186 & 30.9 & 0.17 & 7.90 \\
\hline Annual & 262 & 1969 & 144 & 1972 & 198 & 37.4 & 0.19 & 100 \\
\hline
\end{tabular}


15-0.000-1M KOKCHA RIVER AT KHOJAGHAR, Continued

Monthly and annual flow duration, in cubic meters per second

\begin{tabular}{|c|c|c|c|c|c|c|c|c|c|c|c|c|c|}
\hline \multirow{2}{*}{$\begin{array}{l}\text { Percentage } \\
\text { of days } \\
\text { discharge } \\
\text { equaled or } \\
\text { exceeded }\end{array}$} & \multicolumn{12}{|c|}{ Month } & \multirow{2}{*}{ Annual } \\
\hline & October & November & December & January & February & March & April & May & June & July & August & September & \\
\hline 95 & 73.6 & 59.6 & 50.0 & 40.5 & 42.8 & 41.9 & 58.9 & 97.4 & 205 & 267 & 208 & 120 & 49.0 \\
\hline 90 & 77.3 & 63.7 & 53.8 & 43.0 & 44.4 & 54.0 & 67.8 & 112 & 240 & 296 & 229 & 131 & 56.8 \\
\hline 85 & 81.9 & 66.8 & 56.1 & 44.2 & 46.2 & 56.6 & 72.8 & 125 & 268 & 327 & 242 & 135 & 64.0 \\
\hline 80 & 86.5 & 69.2 & 58.8 & 46.4 & 48.2 & 59.3 & 78.1 & 138 & 295 & 355 & 254 & 139 & 68.4 \\
\hline 75 & 91.3 & 71.8 & 61.4 & 50.5 & 49.5 & 62.0 & 83.4 & 146 & 326 & 380 & 267 & 150 & 72.5 \\
\hline 70 & 94.4 & 73.5 & 63.3 & 51.9 & 50.6 & 64.7 & 89.9 & 156 & 360 & 400 & 279 & 157 & 77.4 \\
\hline 65 & 98.3 & 75.7 & 65.2 & 54.9 & 52.7 & 66.7 & 94.7 & 167 & 384 & 422 & 287 & 164 & 83.8 \\
\hline 60 & 103 & 79.2 & 67.1 & 57.5 & 55.7 & 68.5 & 100 & 176 & 408 & 443 & 296 & 170 & 91.5 \\
\hline 55 & 109 & 82.9 & 69.6 & 60.8 & 58.4 & 70.1 & 106 & 185 & 434 & 462 & 307 & 178 & 101 \\
\hline 50 & 113 & 85.2 & 71.6 & 63.1 & 61.9 & 71.6 & 112 & 198 & 458 & 485 & 321 & 185 & 115 \\
\hline 45 & 115 & 88.0 & 73.7 & 64.7 & 64.3 & 73.0 & 119 & 215 & 479 & 511 & 335 & 190 & 132 \\
\hline 40 & 118 & 90.8 & 76.0 & 66.1 & 66.2 & 74.8 & 127 & 230 & 512 & 547 & 350 & 198 & 153 \\
\hline 35 & 121 & 93.2 & 78.0 & 67.3 & 67.9 & 77.0 & 134 & 248 & 549 & 569 & 364 & 203 & 186 \\
\hline 30 & 124 & 95.4 & 80.3 & 68.6 & 72.6 & 79.6 & 144 & 267 & 584 & 587 & 385 & 209 & 225 \\
\hline 25 & 128 & 98.0 & 82.1 & 70.3 & 75.4 & 83.1 & 160 & 283 & 635 & 608 & 403 & 219 & 267 \\
\hline 20 & 132 & 103 & 84.5 & 73.8 & 77.6 & 90.3 & 179 & 307 & 677 & 652 & 422 & 227 & 318 \\
\hline 15 & 136 & 107 & 88.5 & 78.6 & 80.3 & 99.8 & 196 & 336 & 721 & 717 & 453 & 238 & 388 \\
\hline 10 & 141 & 110 & 91.0 & 86.3 & 86.0 & 124 & 221 & 362 & 764 & 780 & 484 & 252 & 468 \\
\hline 5 & 149 & 115 & 101 & 92.8 & 90.3 & 158 & 288 & 400 & 833 & 844 & 525 & 270 & 594 \\
\hline
\end{tabular}




\section{5-0.000-1M KOKCHA RIVER AT KHOJAGHAR, Continued}

Probability of occurrence of annual high discharges

[ $\mathrm{m}^{3} / \mathrm{s}$, cubic meters per second; ng, not given]

\begin{tabular}{|c|c|c|c|c|c|c|}
\hline \multirow{2}{*}{$\begin{array}{c}\text { Exceedance } \\
\text { probability }\end{array}$} & \multirow{2}{*}{$\begin{array}{c}\text { Recurrence } \\
\text { interval } \\
\text { (years) }\end{array}$} & \multirow{2}{*}{$\begin{array}{l}\text { Maximum } \\
\text { instantaneous } \\
\text { discharge } \\
\left(\mathrm{m}^{3} / \mathrm{s}\right)\end{array}$} & \multicolumn{4}{|c|}{ Maximum daily mean discharge $\left(\mathrm{m}^{3} / \mathrm{s}\right)$} \\
\hline & & & $\begin{array}{l}\text { 3-day } \\
\text { period }\end{array}$ & $\begin{array}{l}\text { 7-day } \\
\text { period }\end{array}$ & $\begin{array}{l}\text { 15-day } \\
\text { period }\end{array}$ & $\begin{array}{l}\text { 30-day } \\
\text { period }\end{array}$ \\
\hline 0.99 & 1.01 & 398 & 361 & 343 & 325 & 287 \\
\hline 0.95 & 1.05 & 483 & 457 & 426 & 400 & 358 \\
\hline 0.90 & 1.11 & 537 & 514 & 476 & 445 & 400 \\
\hline 0.80 & 1.25 & 613 & 588 & 541 & 504 & 457 \\
\hline 0.50 & 2 & 797 & 742 & 682 & 633 & 582 \\
\hline 0.20 & 5 & 1,050 & 909 & 844 & 785 & 728 \\
\hline 0.10 & 10 & 1,220 & 1,000 & 936 & 873 & 813 \\
\hline 0.04 & 25 & 1,430 & 1,100 & 1,040 & 975 & 911 \\
\hline 0.02 & 50 & 1,590 & 1,160 & 1,110 & 1,040 & 977 \\
\hline 0.01 & 100 & 1,760 & 1,220 & 1,170 & 1,110 & 1,040 \\
\hline 0.005 & 200 & 1,920 & 1,270 & 1,230 & 1,170 & 1,100 \\
\hline 0.002 & 500 & 2,150 & $\mathrm{ng}$ & ng & $\mathrm{ng}$ & ng \\
\hline
\end{tabular}

\section{5-0.000-1M KOKCHA RIVER AT KHOJAGHAR, Continued}

Probability of occurrence of annual low discharges $\left[\mathrm{m}^{3} / \mathrm{s}\right.$, meters per second]

\begin{tabular}{|c|c|c|c|c|c|c|c|c|c|c|}
\hline \multirow{3}{*}{$\begin{array}{c}\text { Nonexceedance } \\
\text { probability }\end{array}$} & \multirow{3}{*}{$\begin{array}{c}\text { Recurrence } \\
\text { interval } \\
\text { (years) }\end{array}$} & \multicolumn{9}{|c|}{ Minimum daily mean discharge $\left(\mathrm{m}^{3} / \mathrm{s}\right)$} \\
\hline & & \multicolumn{9}{|c|}{ Number of consecutive days } \\
\hline & & 1 & 3 & 7 & 14 & 30 & 60 & 90 & 120 & 183 \\
\hline 0.05 & 20 & 32.4 & 33.7 & 34.9 & 36.3 & 38.0 & 41.0 & 44.0 & 47.9 & 58.5 \\
\hline 0.10 & 10 & 35.2 & 36.5 & 38.0 & 39.6 & 41.4 & 44.3 & 47.3 & 51.1 & 62.1 \\
\hline 0.20 & 5 & 39.1 & 40.3 & 42.1 & 44.0 & 45.9 & 48.8 & 51.7 & 55.3 & 66.8 \\
\hline 0.50 & 2 & 48.0 & 49.1 & 51.3 & 53.7 & 55.9 & 58.8 & 61.6 & 65.0 & 77.4 \\
\hline
\end{tabular}




\section{5-0.000-1M KOKCHA RIVER AT KHOJAGHAR, Continued}

Probability of occurrence of seasonal low discharges

$\left[\mathrm{m}^{3} / \mathrm{s}\right.$, meters per second]

\begin{tabular}{|c|c|c|c|c|c|c|c|c|c|}
\hline \multirow{3}{*}{$\begin{array}{c}\text { Nonexceedance } \\
\text { probability }\end{array}$} & \multirow{3}{*}{$\begin{array}{c}\text { Recurrence } \\
\text { interval } \\
\text { (years) }\end{array}$} & \multicolumn{8}{|c|}{ Minimum daily mean discharge $\left(\mathrm{m}^{3} / \mathrm{s}\right)$} \\
\hline & & \multicolumn{8}{|c|}{ Number of consecutive days } \\
\hline & & 1 & 7 & 14 & 30 & 1 & 7 & 14 & 30 \\
\hline & & \multicolumn{4}{|c|}{ December-January-February } & \multicolumn{4}{|c|}{ March-April-May } \\
\hline 0.05 & 20 & 33.4 & 36.4 & 37.8 & 39.6 & 38.0 & 42.2 & 44.4 & 51.2 \\
\hline 0.10 & 10 & 36.4 & 39.5 & 41.1 & 42.9 & 42.2 & 46.5 & 48.0 & 55.1 \\
\hline 0.20 & 5 & 40.5 & 43.6 & 45.5 & 47.4 & 47.6 & 52.1 & 53.0 & 60.7 \\
\hline \multirow[t]{2}{*}{0.50} & 2 & 49.8 & 52.9 & 55.4 & 57.6 & 58.3 & 63.8 & 65.1 & 74.7 \\
\hline & & \multicolumn{4}{|c|}{ June-July-August } & \multicolumn{4}{|c|}{ September-October-November } \\
\hline 0.05 & 20 & 144 & 172 & 192 & 215 & 51.8 & 53.0 & 55.3 & 61.4 \\
\hline 0.10 & 10 & 163 & 189 & 210 & 239 & 56.7 & 58.0 & 60.2 & 66.0 \\
\hline 0.20 & 5 & 186 & 210 & 232 & 269 & 62.8 & 64.4 & 66.4 & 71.9 \\
\hline 0.50 & 2 & 228 & 251 & 280 & 331 & 75.4 & 77.4 & 79.3 & 84.7 \\
\hline
\end{tabular}




\section{5-0.000-1M KOKCHA RIVER AT KHOJAGHAR, Continued}

\begin{tabular}{|c|c|c|c|c|c|}
\hline \multicolumn{6}{|c|}{$\begin{array}{l}\text { Annual peak discharges } \\
\text { [m³/s, meters per second] }\end{array}$} \\
\hline \multicolumn{3}{|c|}{$\begin{array}{c}\text { Annual peak discharge, } \\
\text { by year }\end{array}$} & \multicolumn{3}{|c|}{$\begin{array}{l}\text { Annual peak discharge, } \\
\text { from highest to lowest }\end{array}$} \\
\hline $\begin{array}{l}\text { Water } \\
\text { year }\end{array}$ & Date & $\begin{array}{c}\text { Peak } \\
\text { discharge } \\
\left(\mathrm{m}^{3} / \mathrm{s}\right)\end{array}$ & $\begin{array}{l}\text { Water } \\
\text { year }\end{array}$ & Date & $\begin{array}{c}\text { Peak } \\
\text { discharge } \\
\left(\mathrm{m}^{3} / \mathrm{s}\right)\end{array}$ \\
\hline 1964 & June 30, 1964 & 775 & 1969 & May 22, 1969 & 1,550 \\
\hline 1965 & June 21, 1965 & 555 & 1977 & June 23, 1977 & 1,110 \\
\hline 1966 & June 19, 1966 & 805 & 1978 & June 30, 1978 & 1,065 \\
\hline 1967 & June 29, 1967 & 751 & 1973 & June 12, 1973 & 1,008 \\
\hline 1968 & July 8, 1968 & 969 & 1968 & July 8, 1968 & 969 \\
\hline 1969 & May 22, 1969 & 1,550 & 1975 & June 18, 1975 & 889 \\
\hline 1970 & June 30, 1970 & 484 & 1976 & May 25, 1976 & 889 \\
\hline 1971 & June 10, 1971 & 600 & 1966 & June 19, 1966 & 805 \\
\hline 1972 & June 24, 1972 & 680 & 1964 & June 30, 1964 & 775 \\
\hline 1973 & June 12, 1973 & 1,008 & 1967 & June 29, 1967 & 751 \\
\hline 1974 & July 1, 1974 & 520 & 1972 & June 24, 1972 & 680 \\
\hline 1975 & June 18, 1975 & 889 & 1971 & June 10, 1971 & 600 \\
\hline 1976 & May 25, 1976 & 889 & 1965 & June 21, 1965 & 555 \\
\hline 1977 & June 23, 1977 & 1,110 & 1974 & July 1,1974 & 520 \\
\hline 1978 & June 30, 1978 & 1,065 & 1970 & June 30,1970 & 484 \\
\hline
\end{tabular}




\section{5-0.000-1M KOKCHA RIVER AT KHOJAGHAR, Continued}

Monthly and annual mean discharges, in cubic meters per second

[Data may not be rounded in accordance with U.S. Geological Survey publication standards; --, no data]

\begin{tabular}{|c|c|c|c|c|c|c|c|c|c|c|c|c|c|}
\hline \multirow{2}{*}{$\begin{array}{l}\text { Water } \\
\text { year }\end{array}$} & \multicolumn{12}{|c|}{ Monthly mean discharge } & \multirow{2}{*}{$\begin{array}{c}\text { Annual } \\
\text { discharge }\end{array}$} \\
\hline & October & November & December & January & February & March & April & May & June & July & August & September & \\
\hline 1964 & -- & -- & -- & -- & -- & -- & -- & 191 & 445 & 466 & 312 & 188 & -- \\
\hline 1965 & 108 & 74.2 & 68.2 & 67.7 & 83.3 & 80.6 & 113 & 161 & 380 & 412 & 243 & 147 & 162 \\
\hline 1966 & 105 & 95.3 & 80.5 & 70.2 & 78.4 & 84.9 & 108 & 201 & 503 & 476 & 360 & 189 & 197 \\
\hline 1967 & 125 & 90.3 & 78.8 & 68.3 & 66.8 & 68.7 & 102 & 177 & 460 & 581 & 472 & 240 & 212 \\
\hline 1968 & 142 & 108 & 86.0 & 67.1 & 66.5 & 84.0 & 135 & 219 & 648 & 672 & 429 & 191 & 238 \\
\hline 1969 & 126 & 100 & 89.9 & 87.0 & 79.9 & 153 & 194 & 368 & 615 & 703 & 433 & 178 & 262 \\
\hline 1970 & 123 & 111 & 99.8 & 92.7 & 84.3 & 76.9 & 115 & 216 & 364 & 313 & 294 & 209 & 175 \\
\hline 1971 & 94.7 & 67.0 & 55.4 & 43.6 & 46.5 & 63.4 & 99.9 & 210 & 429 & 339 & 332 & 159 & 162 \\
\hline 1972 & 82.2 & 72.2 & 63.1 & 53.1 & 49.0 & 80.3 & 110 & 176 & 373 & 337 & 205 & 130 & 144 \\
\hline 1973 & 84.9 & 72.6 & 53.1 & 42.5 & 50.2 & 85.5 & 191 & 316 & 690 & 592 & 338 & 224 & 229 \\
\hline 1974 & 113 & 84.1 & 72.1 & 64.1 & 57.8 & 74.2 & 73.8 & 128 & 291 & 382 & 250 & 153 & 146 \\
\hline 1975 & 92.4 & 66.1 & 61.8 & 52.3 & 51.9 & 62.3 & 185 & 298 & 531 & 499 & 364 & 195 & 206 \\
\hline 1976 & 126 & 98.7 & 75.6 & 67.7 & 70.3 & 85.5 & 179 & 236 & 328 & 604 & 330 & 218 & 202 \\
\hline 1977 & 133 & 98.0 & 70.6 & 53.8 & 46.5 & 42.7 & 60.7 & 152 & 452 & 631 & 295 & 162 & 184 \\
\hline 1978 & 83.5 & 64.8 & 57.7 & 45.8 & 55.0 & 83.1 & 201 & 326 & 748 & 654 & 418 & 202 & 246 \\
\hline
\end{tabular}




\section{5-0.000-3M KOKCHA RIVER NEAR KESHEM}

\section{(U.S. Geological Survey identification number: $\mathbf{3 6 5 7 0 0 0 7 0 0 3 0 0 0 0 )}$}

LOCATION: Lat $36^{\circ} 57^{\prime} \mathrm{N}$., long $70^{\circ} 03^{\prime} \mathrm{E}$.

DRAINAGE AREA: $16,765 \mathrm{~km}^{2}$.

ELEVATION: 805 meters above mean sea level.

PERIOD OF RECORD: October 1, 1969 to September 30, 1978.

GAGE: Water-stage recorder.

Annual mean discharge

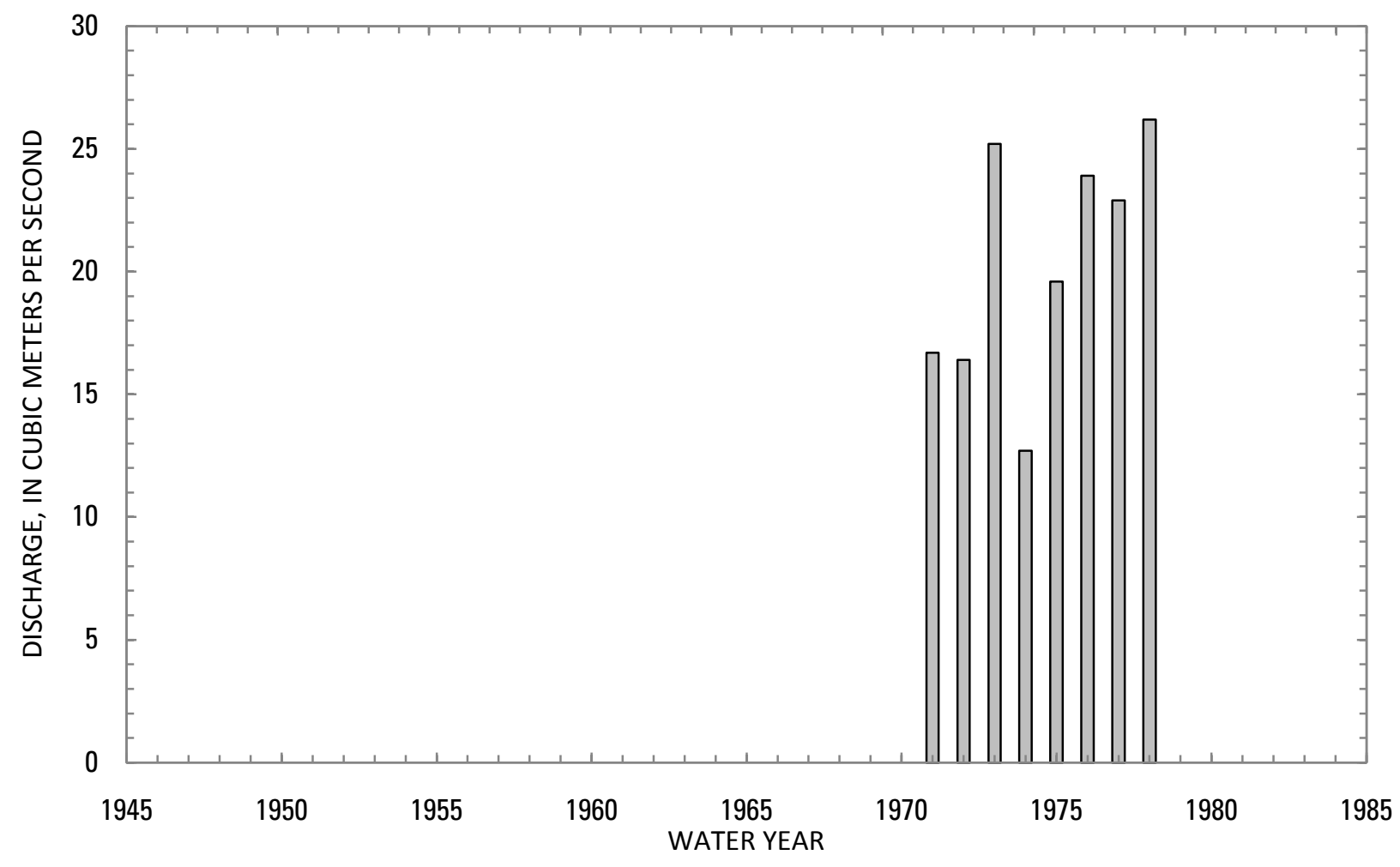




\section{5-0.000-3M KOKCHA RIVER NEAR KESHEM, Continued}

Statistics of monthly and annual mean discharges $\left[\mathrm{m}^{3} / \mathrm{s}\right.$, cubic meters per second]

\begin{tabular}{|c|c|c|c|c|c|c|c|c|}
\hline \multirow[b]{2}{*}{ Month } & \multicolumn{2}{|c|}{ Maximum } & \multicolumn{2}{|c|}{ Minimum } & \multicolumn{4}{|c|}{ Mean } \\
\hline & $\begin{array}{c}\text { Discharge } \\
\left(\mathrm{m}^{3} / \mathrm{s}\right)\end{array}$ & $\begin{array}{c}\text { Water year } \\
\text { of } \\
\text { occurrence }\end{array}$ & $\begin{array}{c}\text { Discharge } \\
\left(\mathrm{m}^{3} / \mathrm{s}\right)\end{array}$ & $\begin{array}{c}\text { Water year } \\
\text { of } \\
\text { occurrence }\end{array}$ & $\begin{array}{c}\text { Discharge } \\
\left(\mathrm{m}^{3} / \mathrm{s}\right)\end{array}$ & $\begin{array}{c}\text { Standard } \\
\text { deviation } \\
\left(\mathrm{m}^{3} / \mathrm{s}\right)\end{array}$ & $\begin{array}{c}\text { Coefficient } \\
\text { of } \\
\text { variation }\end{array}$ & $\begin{array}{c}\text { Percentage } \\
\text { of annual } \\
\text { discharge }\end{array}$ \\
\hline October & 116 & 1970 & 69.5 & 1978 & 92.9 & 15.6 & 0.17 & 4.86 \\
\hline November & 105 & 1970 & 47.7 & 1978 & 71.1 & 16.7 & 0.23 & 3.72 \\
\hline December & 80.7 & 1970 & 41.0 & 1978 & 56.1 & 11.3 & 0.20 & 2.93 \\
\hline January & 73.0 & 1970 & 35.9 & 1978 & 48.4 & 11.4 & 0.24 & 2.53 \\
\hline February & 67.4 & 1970 & 39.0 & 1977 & 48.1 & 9.37 & 0.19 & 2.51 \\
\hline March & 67.5 & 1973 & 39.6 & 1977 & 57.0 & 8.59 & 0.15 & 2.98 \\
\hline April & 153 & 1975 & 49.3 & 1977 & 102 & 38.0 & 0.37 & 5.34 \\
\hline May & 271 & 1978 & 105 & 1977 & 196 & 61.8 & 0.31 & 10.3 \\
\hline June & 666 & 1978 & 262 & 1974 & 402 & 146 & 0.36 & 21.0 \\
\hline July & 579 & 1978 & 285 & 1970 & 414 & 111 & 0.27 & 21.6 \\
\hline August & 374 & 1978 & 171 & 1972 & 274 & 56.2 & 0.21 & 14.3 \\
\hline September & 180 & 1978 & 104 & 1972 & 151 & 25.9 & 0.17 & 7.89 \\
\hline Annual & 210 & 1978 & 122 & 1972 & 160 & 29.3 & 0.18 & 100 \\
\hline
\end{tabular}


15-0.000-3M KOKCHA RIVER NEAR KESHEM, Continued

Monthly and annual flow duration, in cubic meters per second

\begin{tabular}{|c|c|c|c|c|c|c|c|c|c|c|c|c|c|}
\hline \multirow{2}{*}{$\begin{array}{l}\text { Percentage } \\
\text { of days } \\
\text { discharge } \\
\text { equaled or } \\
\text { exceeded }\end{array}$} & \multicolumn{12}{|c|}{ Month } & \multirow{2}{*}{ Annual } \\
\hline & October & November & December & January & February & March & April & May & June & July & August & September & \\
\hline 95 & 60.2 & 47.0 & 40.1 & 34.9 & 36.6 & 37.2 & 45.4 & 67.4 & 164 & 210 & 163 & 98.3 & 40.0 \\
\hline 90 & 72.0 & 50.1 & 42.7 & 35.8 & 38.5 & 41.4 & 52.0 & 83.7 & 189 & 254 & 183 & 105 & 44.7 \\
\hline 85 & 75.2 & 54.9 & 44.7 & 36.7 & 39.9 & 45.3 & 56.9 & 98.1 & 205 & 279 & 206 & 111 & 48.2 \\
\hline 80 & 77.3 & 59.4 & 46.2 & 38.0 & 40.3 & 47.2 & 59.3 & 105 & 224 & 297 & 224 & 114 & 51.6 \\
\hline 75 & 79.0 & 61.2 & 47.9 & 40.5 & 40.7 & 48.8 & 62.4 & 117 & 250 & 324 & 235 & 117 & 56.4 \\
\hline 70 & 81.1 & 62.7 & 49.5 & 42.0 & 41.1 & 50.1 & 68.0 & 125 & 274 & 339 & 244 & 121 & 60.4 \\
\hline 65 & 82.6 & 63.9 & 50.5 & 43.0 & 42.1 & 51.6 & 71.9 & 137 & 297 & 351 & 252 & 129 & 65.4 \\
\hline 60 & 84.0 & 65.3 & 51.5 & 44.0 & 43.7 & 53.4 & 76.5 & 153 & 321 & 363 & 260 & 135 & 71.7 \\
\hline 55 & 87.6 & 66.7 & 52.7 & 45.1 & 44.6 & 54.2 & 79.7 & 170 & 345 & 376 & 266 & 141 & 79.5 \\
\hline 50 & 90.6 & 67.8 & 54.3 & 46.8 & 46.0 & 55.1 & 82.8 & 194 & 368 & 396 & 274 & 147 & 90.7 \\
\hline 45 & 94.9 & 68.8 & 55.4 & 47.6 & 46.7 & 56.0 & 88.2 & 209 & 388 & 409 & 281 & 153 & 105 \\
\hline 40 & 99.6 & 70.1 & 56.9 & 48.2 & 48.0 & 57.1 & 92.9 & 232 & 420 & 421 & 287 & 159 & 121 \\
\hline 35 & 103 & 71.7 & 58.3 & 49.1 & 49.3 & 58.3 & 101 & 251 & 453 & 446 & 294 & 166 & 150 \\
\hline 30 & 105 & 74.1 & 60.2 & 50.3 & 52.3 & 59.7 & 108 & 260 & 486 & 470 & 302 & 172 & 188 \\
\hline 25 & 107 & 77.7 & 61.1 & 52.9 & 54.2 & 61.7 & 118 & 269 & 530 & 485 & 313 & 180 & 229 \\
\hline 20 & 109 & 82.1 & 63.4 & 57.4 & 55.3 & 63.9 & 135 & 280 & 581 & 505 & 326 & 189 & 271 \\
\hline 15 & 111 & 89.0 & 68.3 & 59.0 & 56.5 & 65.7 & 162 & 292 & 627 & 546 & 341 & 197 & 308 \\
\hline 10 & 115 & 96.1 & 76.8 & 71.1 & 65.1 & 69.5 & 179 & 309 & 680 & 613 & 359 & 207 & 367 \\
\hline 5 & 122 & 107 & 81.5 & 73.1 & 68.5 & 88.3 & 218 & 345 & 738 & 700 & 404 & 220 & 474 \\
\hline
\end{tabular}




\section{5-0.000-3M KOKCHA RIVER NEAR KESHEM, Continued}

Probability of occurrence of annual high discharges

[ $\mathrm{m}^{3} / \mathrm{s}$, cubic meters per second; ng, not given]

\begin{tabular}{|c|c|c|c|c|c|c|}
\hline \multirow{2}{*}{$\begin{array}{l}\text { Exceedance } \\
\text { probability }\end{array}$} & \multirow{2}{*}{$\begin{array}{c}\text { Recurrence } \\
\text { interval } \\
\text { (years) }\end{array}$} & \multirow{2}{*}{$\begin{array}{l}\text { Maximum } \\
\text { instantaneous } \\
\text { discharge } \\
\left(\mathrm{m}^{3} / \mathrm{s}\right)\end{array}$} & \multicolumn{4}{|c|}{ Maximum daily mean discharge $\left(\mathrm{m}^{3} / \mathrm{s}\right)$} \\
\hline & & & $\begin{array}{l}\text { 3-day } \\
\text { period }\end{array}$ & $\begin{array}{l}\text { 7-day } \\
\text { period }\end{array}$ & $\begin{array}{l}\text { 15-day } \\
\text { period }\end{array}$ & $\begin{array}{l}\text { 30-day } \\
\text { period }\end{array}$ \\
\hline 0.99 & 1.01 & 392 & 389 & 337 & 307 & 273 \\
\hline 0.95 & 1.05 & 449 & 433 & 384 & 350 & 312 \\
\hline 0.90 & 1.11 & 485 & 462 & 413 & 378 & 338 \\
\hline 0.80 & 1.25 & 532 & 502 & 456 & 417 & 375 \\
\hline 0.50 & 2 & 642 & 601 & 560 & 517 & 467 \\
\hline 0.20 & 5 & 782 & 742 & 706 & 662 & 602 \\
\hline 0.10 & 10 & 871 & 838 & 805 & 763 & 696 \\
\hline 0.04 & 25 & 981 & 963 & 935 & 897 & 821 \\
\hline 0.02 & 50 & 1,060 & 1,060 & 1,030 & 1,000 & 919 \\
\hline 0.01 & 100 & 1,140 & 1,160 & 1,140 & 1,110 & 1,020 \\
\hline 0.005 & 200 & 1,220 & 1,260 & 1,240 & 1,230 & 1,130 \\
\hline 0.002 & 500 & 1,320 & $\mathrm{ng}$ & $\mathrm{ng}$ & $\mathrm{ng}$ & $\mathrm{ng}$ \\
\hline
\end{tabular}

\section{5-0.000-3M KOKCHA RIVER NEAR KESHEM, Continued}

Probability of occurrence of annual low discharges $\left[\mathrm{m}^{3} / \mathrm{s}\right.$, meters per second]

\begin{tabular}{|c|c|c|c|c|c|c|c|c|c|c|}
\hline \multirow{3}{*}{$\begin{array}{c}\text { Nonexceedance } \\
\text { probability }\end{array}$} & \multirow{3}{*}{$\begin{array}{c}\text { Recurrence } \\
\text { interval } \\
\text { (years) }\end{array}$} & \multicolumn{9}{|c|}{ Minimum daily mean discharge $\left(\mathrm{m}^{3} / \mathrm{s}\right)$} \\
\hline & & \multicolumn{9}{|c|}{ Number of consecutive days } \\
\hline & & 1 & 3 & 7 & 14 & 30 & 60 & 90 & 120 & 183 \\
\hline 0.05 & 20 & 31.6 & 31.6 & 32.0 & 32.6 & 34.2 & 35.5 & 37.2 & 40.4 & 50.5 \\
\hline 0.10 & 10 & 33.1 & 33.2 & 33.6 & 34.2 & 35.8 & 37.1 & 38.9 & 41.9 & 52.1 \\
\hline 0.20 & 5 & 35.2 & 35.4 & 35.8 & 36.3 & 37.8 & 39.3 & 41.0 & 43.9 & 54.3 \\
\hline 0.50 & 2 & 40.0 & 40.3 & 40.6 & 41.2 & 42.5 & 44.2 & 45.7 & 48.3 & 59.1 \\
\hline
\end{tabular}




\section{5-0.000-3M KOKCHA RIVER NEAR KESHEM, Continued}

Probability of occurrence of seasonal low discharges [ $\mathrm{m}^{3} / \mathrm{s}$, meters per second]

\begin{tabular}{|c|c|c|c|c|c|c|c|c|c|}
\hline \multirow{3}{*}{$\begin{array}{c}\text { Nonexceedance } \\
\text { probability }\end{array}$} & \multirow{3}{*}{$\begin{array}{c}\text { Recurrence } \\
\text { interval } \\
\text { (years) }\end{array}$} & \multicolumn{8}{|c|}{ Minimum daily mean discharge $\left(\mathrm{m}^{3} / \mathrm{s}\right)$} \\
\hline & & \multicolumn{8}{|c|}{ Number of consecutive days } \\
\hline & & 1 & 7 & 14 & 30 & 1 & 7 & 14 & 30 \\
\hline & & \multicolumn{4}{|c|}{ December-January-February } & \multicolumn{4}{|c|}{ March-April-May } \\
\hline 0.05 & 20 & 31.4 & 32.0 & 32.7 & 34.4 & 34.9 & 36.5 & 37.5 & 41.2 \\
\hline 0.10 & 10 & 33.0 & 33.6 & 34.3 & 35.9 & 37.5 & 39.6 & 40.7 & 45.3 \\
\hline 0.20 & 5 & 35.4 & 36.0 & 36.7 & 38.1 & 40.9 & 43.2 & 44.5 & 50.0 \\
\hline \multirow[t]{2}{*}{0.50} & 2 & 41.5 & 42.1 & 42.7 & 44.0 & 47.5 & 50.0 & 51.4 & 58.0 \\
\hline & & \multicolumn{4}{|c|}{ June-July-August } & \multicolumn{4}{|c|}{ September-October-November } \\
\hline 0.05 & 20 & 103 & 127 & 154 & 176 & 42.4 & 43.7 & 45.1 & 48.6 \\
\hline 0.10 & 10 & 119 & 144 & 167 & 195 & 46.1 & 47.1 & 48.7 & 52.4 \\
\hline 0.20 & 5 & 140 & 165 & 184 & 219 & 50.6 & 51.5 & 53.3 & 57.1 \\
\hline 0.50 & 2 & 182 & 208 & 224 & 267 & 59.4 & 60.4 & 62.1 & 66.6 \\
\hline
\end{tabular}

\section{5-0.000-3M KOKCHA RIVER NEAR KESHEM, Continued}

Annual peak discharges

[ $\mathrm{m}^{3} / \mathrm{s}$, meters per second]

\begin{tabular}{|c|c|c|c|c|c|}
\hline \multicolumn{3}{|c|}{$\begin{array}{c}\text { Annual peak discharge, } \\
\text { by year }\end{array}$} & \multicolumn{3}{|c|}{$\begin{array}{l}\text { Annual peak discharge, } \\
\text { from highest to lowest }\end{array}$} \\
\hline $\begin{array}{l}\text { Water } \\
\text { year }\end{array}$ & Date & $\begin{array}{c}\text { Peak } \\
\text { discharge } \\
\left(\mathrm{m}^{3} / \mathrm{s}\right)\end{array}$ & $\begin{array}{c}\text { Water } \\
\text { year }\end{array}$ & Date & $\begin{array}{c}\text { Peak } \\
\text { discharge } \\
\left(\mathrm{m}^{3} / \mathrm{s}\right)\end{array}$ \\
\hline 1970 & June 30, 1970 & 490 & 1978 & June 29, 1978 & 930 \\
\hline 1971 & June 10,1971 & 550 & 1973 & June 12, 1973 & 800 \\
\hline 1972 & June 23, 1972 & 600 & 1977 & June 24, 1977 & 800 \\
\hline 1973 & June 12, 1973 & 800 & 1975 & June 18,1975 & 659 \\
\hline 1974 & June 17, 1974 & 480 & 1976 & July 8, 1976 & 650 \\
\hline 1975 & June 18, 1975 & 659 & 1972 & June 23, 1972 & 600 \\
\hline 1976 & July 8, 1976 & 650 & 1971 & June 10, 1971 & 550 \\
\hline 1977 & June 24, 1977 & 800 & 1970 & June 30,1970 & 490 \\
\hline 1978 & June 29,1978 & 930 & 1974 & June 17, 1974 & 480 \\
\hline
\end{tabular}


15-0.000-3M KOKCHA RIVER NEAR KESHEM, Continued

Monthly and annual mean discharges, in cubic meters per second

\begin{tabular}{|c|c|c|c|c|c|c|c|c|c|c|c|c|c|}
\hline \multirow{2}{*}{$\begin{array}{c}\text { Water } \\
\text { year }\end{array}$} & \multicolumn{12}{|c|}{ Monthly mean discharge } & \multirow{2}{*}{$\begin{array}{c}\text { Annual } \\
\text { discharge }\end{array}$} \\
\hline & October & November & December & January & February & March & April & May & June & July & August & September & \\
\hline 1970 & 116 & 105 & 80.7 & 73.0 & 67.4 & 63.4 & 84.8 & 199 & 330 & 285 & 264 & 160 & 153 \\
\hline 1971 & 96.1 & 59.8 & 46.0 & 43.0 & 40.5 & 49.6 & 89.8 & 215 & 390 & 307 & 296 & 141 & 148 \\
\hline 1972 & 78.5 & 64.4 & 53.5 & 48.0 & 45.5 & 58.1 & 79.4 & 145 & 318 & 297 & 171 & 104 & 122 \\
\hline 1973 & 80.1 & 67.0 & 50.4 & 38.6 & 43.2 & 67.5 & 132 & 260 & 621 & 527 & 287 & 179 & 197 \\
\hline 1974 & 111 & 72.4 & 61.0 & 58.0 & 55.1 & 56 & 59 & 112 & 262 & 336 & 225 & 122 & 128 \\
\hline 1975 & 87.5 & 64.1 & 54.0 & 49.3 & 46.3 & 55 & 153 & 239 & 417 & 424 & 303 & 160 & 172 \\
\hline 1976 & 104 & 88.0 & 59.5 & 48.8 & 55.2 & 58.7 & 128 & 221 & 266 & 462 & 290 & 170 & 163 \\
\hline 1977 & 93.2 & 71.3 & 58.6 & 40.7 & 39.0 & 39.6 & 49.3 & 105 & 352 & 505 & 254 & 142 & 147 \\
\hline 1978 & 69.5 & 47.7 & 41.0 & 35.9 & 40.6 & 65.5 & 144 & 271 & 666 & 579 & 374 & 180 & 210 \\
\hline
\end{tabular}




\section{5-0.000-6M KOKCHA RIVER NEAR JURM}

\section{(U.S. Geological Survey identification number: 365600070520000 )}

LOCATION: Lat $36^{\circ} 56^{\prime} \mathrm{N}$., long $70^{\circ} 52^{\prime} \mathrm{E}$.

DRAINAGE AREA: $7,670 \mathrm{~km}^{2}$.

ELEVATION: 1,449 meters above mean sea level.

PERIOD OF RECORD: October 1, 1969 to September 30, 1978.

GAGE: Water-stage recorder.

Annual mean discharge

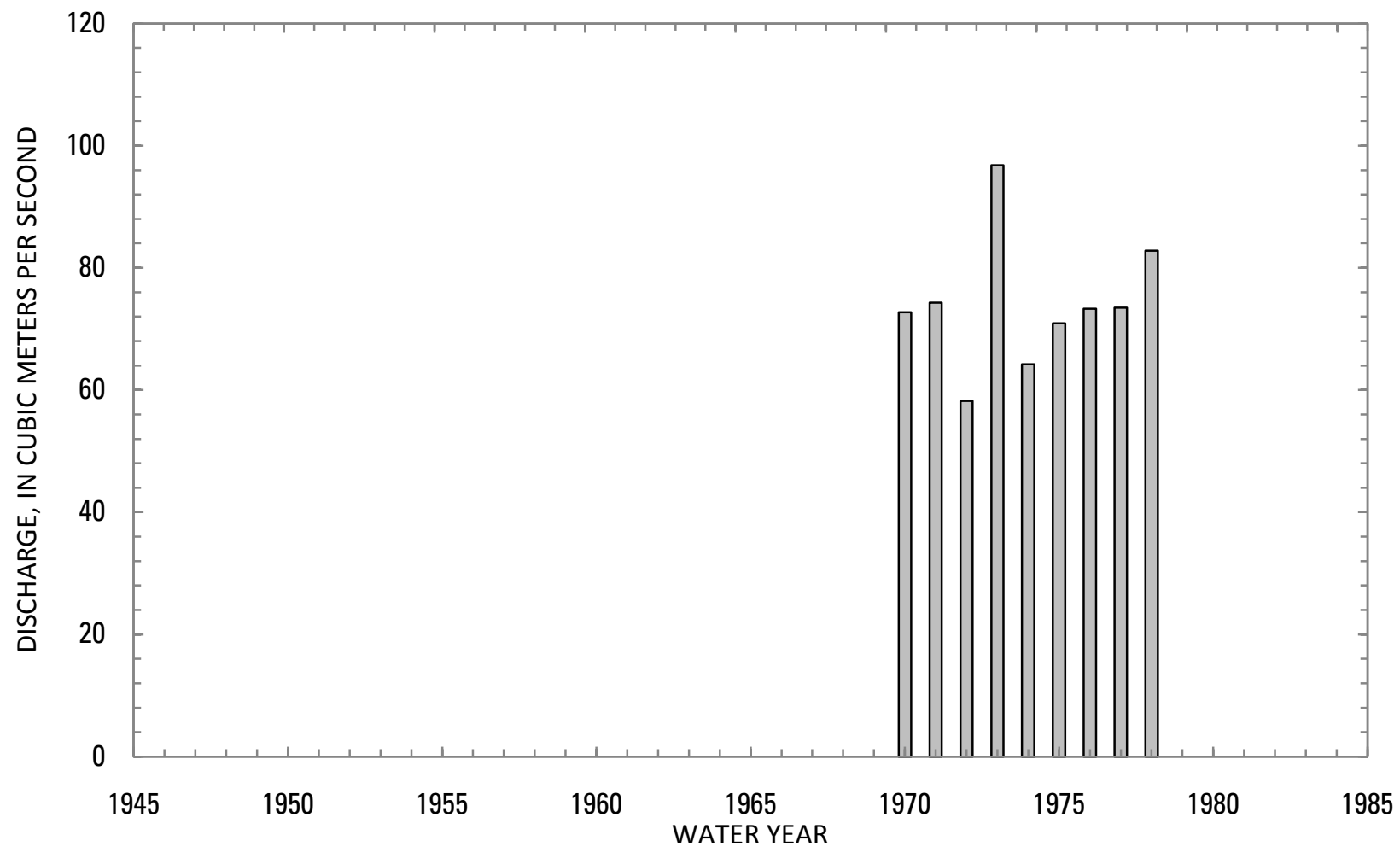




\section{5-0.000-6M KOKCHA RIVER NEAR JURM, Continued}

Statistics of monthly and annual mean discharges $\left[\mathrm{m}^{3} / \mathrm{s}\right.$, cubic meters per second]

\begin{tabular}{|c|c|c|c|c|c|c|c|c|}
\hline \multirow[b]{2}{*}{ Month } & \multicolumn{2}{|c|}{ Maximum } & \multicolumn{2}{|c|}{ Minimum } & \multicolumn{4}{|c|}{ Mean } \\
\hline & $\begin{array}{c}\text { Discharge } \\
\left(\mathrm{m}^{3} / \mathrm{s}\right)\end{array}$ & $\begin{array}{l}\text { Water year } \\
\text { of } \\
\text { occurrence }\end{array}$ & $\begin{array}{c}\text { Discharge } \\
\left(\mathrm{m}^{3} / \mathrm{s}\right)\end{array}$ & $\begin{array}{l}\text { Water year } \\
\text { of } \\
\text { occurrence }\end{array}$ & $\begin{array}{c}\text { Discharge } \\
\left(\mathrm{m}^{3} / \mathrm{s}\right)\end{array}$ & $\begin{array}{c}\text { Standard } \\
\text { deviation } \\
\left(\mathrm{m}^{3} / \mathrm{s}\right)\end{array}$ & $\begin{array}{c}\text { Coefficient } \\
\text { of } \\
\text { variation }\end{array}$ & $\begin{array}{c}\text { Percentage } \\
\text { of annual } \\
\text { discharge }\end{array}$ \\
\hline October & 54.4 & 1970 & 35.3 & 1975 & 46.3 & 6.78 & 0.15 & 5.23 \\
\hline November & 41.4 & 1974 & 29.4 & 1975 & 34.6 & 4.10 & 0.12 & 3.91 \\
\hline December & 32.8 & 1977 & 24.0 & 1975 & 28.2 & 3.05 & 0.11 & 3.19 \\
\hline January & 30.0 & 1970 & 19.1 & 1972 & 24.2 & 3.40 & 0.14 & 2.73 \\
\hline February & 27.9 & 1974 & 17.2 & 1971 & 22.0 & 3.56 & 0.16 & 2.48 \\
\hline March & 23.6 & 1971 & 17.9 & 1978 & 20.5 & 1.76 & 0.09 & 2.31 \\
\hline April & 39.2 & 1971 & 18.2 & 1977 & 26.6 & 6.94 & 0.26 & 3.01 \\
\hline May & 112 & 1971 & 38.0 & 1972 & 63.3 & 25.4 & 0.40 & 7.15 \\
\hline June & 282 & 1973 & 107 & 1976 & 175 & 52.5 & 0.30 & 19.7 \\
\hline July & 302 & 1973 & 150 & 1970 & 207 & 53.9 & 0.26 & 23.3 \\
\hline August & 186 & 1973 & 112 & 1972 & 151 & 23.5 & 0.16 & 17.0 \\
\hline September & 112 & 1970 & 69.5 & 1972 & 88.0 & 12.5 & 0.14 & 9.94 \\
\hline Annual & 96.8 & 1973 & 58.2 & 1972 & 74.1 & 10.9 & 0.15 & 100 \\
\hline
\end{tabular}


15-0.000-6M KOKCHA RIVER NEAR JURM, Continued

Monthly and annual flow duration, in cubic meters per second

\begin{tabular}{|c|c|c|c|c|c|c|c|c|c|c|c|c|c|}
\hline \multirow{2}{*}{$\begin{array}{l}\text { Percentage } \\
\text { of days } \\
\text { discharge } \\
\text { equaled or } \\
\text { exceeded }\end{array}$} & \multicolumn{12}{|c|}{ Month } & \multirow{2}{*}{ Annual } \\
\hline & October & November & December & January & February & March & April & May & June & July & August & September & \\
\hline 95 & 34.2 & 28.1 & 23.2 & 18.5 & 16.0 & 17.5 & 17.4 & 20.9 & 74.8 & 129 & 101 & 55.8 & 18.3 \\
\hline 90 & 34.8 & 29.2 & 23.9 & 20.1 & 17.1 & 17.8 & 18.1 & 24.9 & 82.0 & 136 & 113 & 61.2 & 19.8 \\
\hline 85 & 35.4 & 29.9 & 24.8 & 20.9 & 17.9 & 18.0 & 18.5 & 29.1 & 90.1 & 147 & 118 & 64.7 & 21.0 \\
\hline 80 & 36.6 & 30.4 & 25.5 & 21.4 & 18.9 & 18.2 & 18.9 & 33.7 & 106 & 151 & 122 & 67.8 & 22.6 \\
\hline 75 & 37.9 & 31.3 & 26.0 & 21.9 & 19.4 & 18.9 & 19.3 & 35.9 & 117 & 160 & 131 & 71.9 & 24.4 \\
\hline 70 & 40.5 & 31.6 & 26.5 & 22.0 & 20.1 & 19.3 & 19.6 & 37.1 & 125 & 166 & 135 & 74.5 & 26.5 \\
\hline 65 & 41.9 & 32.0 & 26.8 & 22.6 & 20.5 & 19.9 & 20.0 & 38.8 & 135 & 172 & 140 & 77.6 & 28.6 \\
\hline 60 & 43.3 & 32.7 & 27.0 & 23.0 & 20.9 & 20.0 & 20.5 & 41.8 & 146 & 178 & 144 & 79.1 & 30.9 \\
\hline 55 & 44.8 & 33.2 & 27.1 & 23.8 & 21.3 & 20.1 & 21.6 & 48.3 & 159 & 184 & 148 & 80.8 & 33.7 \\
\hline 50 & 45.6 & 34.0 & 28.0 & 24.0 & 22.2 & 20.2 & 22.5 & 55.0 & 168 & 190 & 151 & 83.0 & 37.4 \\
\hline 45 & 46.9 & 34.7 & 29.1 & 24.3 & 22.6 & 20.3 & 23.5 & 60.9 & 175 & 195 & 155 & 86.4 & 42.6 \\
\hline 40 & 47.8 & 35.3 & 29.3 & 24.9 & 23.0 & 20.5 & 25.9 & 64.7 & 186 & 202 & 158 & 91.2 & 52.1 \\
\hline 35 & 49.0 & 35.6 & 30.0 & 25.5 & 23.3 & 20.8 & 27.6 & 69.2 & 195 & 211 & 162 & 95.2 & 66.6 \\
\hline 30 & 51.0 & 36.9 & 30.2 & 25.9 & 23.6 & 21.2 & 30.0 & 75.6 & 208 & 225 & 166 & 99.2 & 86.0 \\
\hline 25 & 52.4 & 38.3 & 30.4 & 26.8 & 24.3 & 21.4 & 32.3 & 84.2 & 221 & 249 & 171 & 103 & 113 \\
\hline 20 & 53.3 & 39.1 & 31.3 & 27.5 & 25.0 & 22.0 & 35.5 & 95.3 & 233 & 267 & 175 & 108 & 133 \\
\hline 15 & 56.0 & 40.0 & 32.1 & 28.7 & 26.1 & 22.2 & 38.5 & 110 & 249 & 282 & 180 & 113 & 155 \\
\hline 10 & 59.4 & 41.2 & 33.1 & 29.4 & 27.2 & 23.0 & 40.6 & 117 & 287 & 295 & 185 & 117 & 178 \\
\hline 5 & 61.8 & 43.1 & 33.6 & 30.3 & 28.2 & 26.2 & 44.7 & 123 & 319 & 331 & 193 & 130 & 218 \\
\hline
\end{tabular}




\section{5-0.000-6M KOKCHA RIVER NEAR JURM, Continued}

Probability of occurrence of annual high discharges

[m $\mathrm{m}^{3} / \mathrm{s}$, cubic meters per second; ng, not given]

\begin{tabular}{|c|c|c|c|c|c|c|}
\hline \multirow{2}{*}{$\begin{array}{c}\text { Exceedance } \\
\text { probability }\end{array}$} & \multirow{2}{*}{$\begin{array}{c}\text { Recurrence } \\
\text { interval } \\
\text { (years) }\end{array}$} & \multirow{2}{*}{$\begin{array}{c}\text { Maximum } \\
\text { instantaneous } \\
\text { discharge } \\
\left(\mathrm{m}^{3} / \mathrm{s}\right)\end{array}$} & \multicolumn{4}{|c|}{ Maximum daily mean discharge $\left(\mathrm{m}^{3} / \mathrm{s}\right)$} \\
\hline & & & $\begin{array}{l}\text { 3-day } \\
\text { period }\end{array}$ & $\begin{array}{l}\text { 7-day } \\
\text { period }\end{array}$ & $\begin{array}{l}\text { 15-day } \\
\text { period }\end{array}$ & $\begin{array}{l}\text { 30-day } \\
\text { period }\end{array}$ \\
\hline 0.99 & 1.01 & 172 & ${ }^{2} 158$ & 162 & 148 & 140 \\
\hline 0.95 & 1.05 & 203 & 185 & 183 & 171 & 159 \\
\hline 0.90 & 1.11 & 223 & 202 & 197 & 185 & 170 \\
\hline 0.80 & 1.25 & 251 & 225 & 215 & 204 & 186 \\
\hline 0.50 & 2 & 318 & 281 & 262 & 249 & 223 \\
\hline 0.20 & 5 & 409 & 355 & 326 & 306 & 272 \\
\hline 0.10 & 10 & 470 & 403 & 370 & 342 & 304 \\
\hline 0.04 & 25 & 548 & 464 & 426 & 387 & 343 \\
\hline 0.02 & 50 & 607 & 510 & 469 & 420 & 373 \\
\hline 0.01 & 100 & 666 & 555 & 513 & 453 & 402 \\
\hline 0.005 & 200 & 727 & 601 & 559 & 485 & 432 \\
\hline 0.002 & 500 & 810 & $\mathrm{ng}$ & $\mathrm{ng}$ & $\mathrm{ng}$ & $\mathrm{ng}$ \\
\hline
\end{tabular}

\section{5-0.000-6M KOKCHA RIVER NEAR JURM, Continued}

Probability of occurrence of annual low discharges $\left[\mathrm{m}^{3} / \mathrm{s}\right.$, meters per second]

\begin{tabular}{|c|c|c|c|c|c|c|c|c|c|c|}
\hline \multirow{3}{*}{$\begin{array}{c}\text { Nonexceedance } \\
\text { probability }\end{array}$} & \multirow{3}{*}{$\begin{array}{c}\text { Recurrence } \\
\text { interval } \\
\text { (years) }\end{array}$} & \multicolumn{9}{|c|}{ Minimum daily mean discharge $\left(\mathrm{m}^{3} / \mathrm{s}\right)$} \\
\hline & & \multicolumn{9}{|c|}{ Number of consecutive days } \\
\hline & & 1 & 3 & 7 & 14 & 30 & 60 & 90 & 120 & 183 \\
\hline 0.05 & 20 & 14.0 & 14.1 & 14.6 & 15.2 & 16.4 & 17.4 & 18.6 & 19.9 & 24.1 \\
\hline 0.10 & 10 & 14.8 & 14.9 & 15.4 & 15.8 & 16.9 & 18.0 & 19.2 & 20.6 & 25.1 \\
\hline 0.20 & 5 & 15.8 & 15.9 & 16.3 & 16.6 & 17.5 & 18.7 & 20.0 & 21.4 & 26.4 \\
\hline 0.50 & 2 & 17.5 & 17.8 & 18.1 & 18.3 & 18.9 & 20.5 & 21.6 & 23.2 & 28.9 \\
\hline
\end{tabular}


15-0.000-6M KOKCHA RIVER NEAR JURM, Continued

Probability of occurrence of seasonal low discharges $\left[\mathrm{m}^{3} / \mathrm{s}\right.$, meters per second]

\begin{tabular}{|c|c|c|c|c|c|c|c|c|c|}
\hline \multirow{3}{*}{$\begin{array}{c}\text { Nonexceedance } \\
\text { probability }\end{array}$} & \multirow{3}{*}{$\begin{array}{c}\text { Recurrence } \\
\text { interval } \\
\text { (years) }\end{array}$} & \multicolumn{8}{|c|}{ Minimum daily mean discharge $\left(\mathrm{m}^{3} / \mathrm{s}\right)$} \\
\hline & & \multicolumn{8}{|c|}{ Number of consecutive days } \\
\hline & & 1 & 7 & 14 & 30 & 1 & 7 & 14 & 30 \\
\hline & & \multicolumn{4}{|c|}{ December-January-February } & \multicolumn{4}{|c|}{ March-April-May } \\
\hline 0.05 & 20 & 14.1 & 14.6 & 15.3 & 16.7 & 15.0 & 16.6 & 17.1 & 17.4 \\
\hline 0.10 & 10 & 15.4 & 15.8 & 16.4 & 17.6 & 15.7 & 17.1 & 17.5 & 17.8 \\
\hline 0.20 & 5 & 16.9 & 17.3 & 17.8 & 18.8 & 16.6 & 17.6 & 18.1 & 18.3 \\
\hline \multirow[t]{2}{*}{0.50} & 2 & 19.7 & 20.2 & 20.5 & 21.4 & 18.1 & 18.6 & 19 & 19.5 \\
\hline & & \multicolumn{4}{|c|}{ June-July-August } & \multicolumn{4}{|c|}{ September-October-November } \\
\hline 0.05 & 20 & 47.5 & 57.9 & 69.5 & 103 & 26.0 & 26.2 & 26.9 & 28.4 \\
\hline 0.10 & 10 & 57.1 & 67.1 & 77.7 & 110 & 26.9 & 27.2 & 27.8 & 29.3 \\
\hline 0.20 & 5 & 69.6 & 79.1 & 88.6 & 120 & 28.1 & 28.4 & 29.0 & 30.6 \\
\hline 0.50 & 2 & 94.6 & 104 & 112 & 140 & 30.6 & 31.0 & 31.7 & 33.6 \\
\hline
\end{tabular}

\section{5-0.000-6M KOKCHA RIVER NEAR JURM, Continued}

Annual peak discharges

$\left[\mathrm{m}^{3} / \mathrm{s}\right.$, meters per second]

\begin{tabular}{|c|c|c|c|c|c|}
\hline \multicolumn{3}{|c|}{$\begin{array}{c}\text { Annual peak discharge, } \\
\text { by year }\end{array}$} & \multicolumn{3}{|c|}{$\begin{array}{l}\text { Annual peak discharge, } \\
\text { from highest to lowest }\end{array}$} \\
\hline $\begin{array}{l}\text { Water } \\
\text { year }\end{array}$ & Date & $\begin{array}{c}\text { Peak } \\
\text { discharge } \\
\left(\mathrm{m}^{3} / \mathrm{s}\right)\end{array}$ & $\begin{array}{l}\text { Water } \\
\text { year }\end{array}$ & Date & $\begin{array}{c}\text { Peak } \\
\text { discharge } \\
\left(\mathrm{m}^{3} / \mathrm{s}\right)\end{array}$ \\
\hline 1970 & June 29, 1970 & 240 & 1978 & July 8, 1978 & 465 \\
\hline 1971 & June 11, 1971 & 230 & 1973 & June 25, 1973 & 460 \\
\hline 1972 & June 22, 1972 & 310 & 1977 & June 24, 1977 & 446 \\
\hline 1973 & June 25, 1973 & 460 & 1976 & July 24, 1976 & 323 \\
\hline 1974 & July 1, 1974 & 233 & 1972 & June 22, 1972 & 310 \\
\hline 1975 & June 19,1975 & 296 & 1975 & June 19, 1975 & 296 \\
\hline 1976 & July 24, 1976 & 323 & 1970 & June 29, 1970 & 240 \\
\hline 1977 & June 24, 1977 & 446 & 1974 & July 1, 1974 & 233 \\
\hline 1978 & July 8,1978 & 465 & 1971 & June 11, 1971 & 230 \\
\hline
\end{tabular}




\section{5-0.000-6M KOKCHA RIVER NEAR JURM, Continued}

Monthly and annual mean discharges, in cubic meters per second

\begin{tabular}{|c|c|c|c|c|c|c|c|c|c|c|c|c|c|}
\hline \multirow{2}{*}{$\begin{array}{l}\text { Water } \\
\text { year }\end{array}$} & \multicolumn{12}{|c|}{ Monthly mean discharge } & \multirow{2}{*}{$\begin{array}{c}\text { Annual } \\
\text { discharge }\end{array}$} \\
\hline & October & November & December & January & February & March & April & May & June & July & August & September & \\
\hline 1970 & 54.4 & 38.4 & 30.2 & 30.0 & 24.8 & 20.9 & 28.6 & 71.8 & 156 & 150 & 152 & 112 & 72.7 \\
\hline 1971 & 52.5 & 36.0 & 29.1 & 23.5 & 17.2 & 23.6 & 39.2 & 112 & 197 & 152 & 131 & 75.5 & 74.3 \\
\hline 1972 & 37.0 & 30.5 & 24.8 & 19.1 & 17.4 & 19.1 & 19.0 & 38.0 & 146 & 165 & 112 & 69.5 & 58.2 \\
\hline 1973 & 41.8 & 32.5 & 26.9 & 21.2 & 24.3 & 20.4 & 33.2 & 89.6 & 282 & 302 & 186 & 97.5 & 96.8 \\
\hline 1974 & 51.4 & 41.4 & 32.0 & 27.5 & 27.9 & 22.4 & 20.3 & 39.2 & 127 & 172 & 125 & 81.2 & 64.2 \\
\hline 1975 & 35.3 & 29.4 & 24.0 & 22.0 & 20.5 & 20.0 & 29.5 & 55.0 & 178 & 186 & 160 & 87.3 & 70.9 \\
\hline 1976 & 48.9 & 35.1 & 27.6 & 24.4 & 23.2 & 20.9 & 24.9 & 53.0 & 107 & 257 & 163 & 89.4 & 73.3 \\
\hline 1977 & 47.3 & 37.3 & 32.8 & 26.8 & 22.7 & 19.0 & 18.2 & 39.7 & 161 & 230 & 157 & 86.1 & 73.5 \\
\hline 1978 & 47.8 & 30.6 & 26.6 & 23.4 & 19.8 & 17.9 & 26.7 & 71.6 & 217 & 245 & 169 & 93.6 & 82.8 \\
\hline
\end{tabular}




\section{5-1.L00-1A KESHEM RIVER NEAR KESHEM}

\section{(U.S. Geological Survey identification number: 365500070030000 )}

LOCATION: Lat $36^{\circ} 55^{\prime} \mathrm{N}$., long $70^{\circ} 03^{\prime} \mathrm{E}$.

DRAINAGE AREA: $2,145 \mathrm{~km}^{2}$.

ELEVATION: 822 meters above mean sea level.

PERIOD OF RECORD: October 8, 1969 to September 30, 1978.

GAGE: Water-stage recorder.

Annual mean discharge

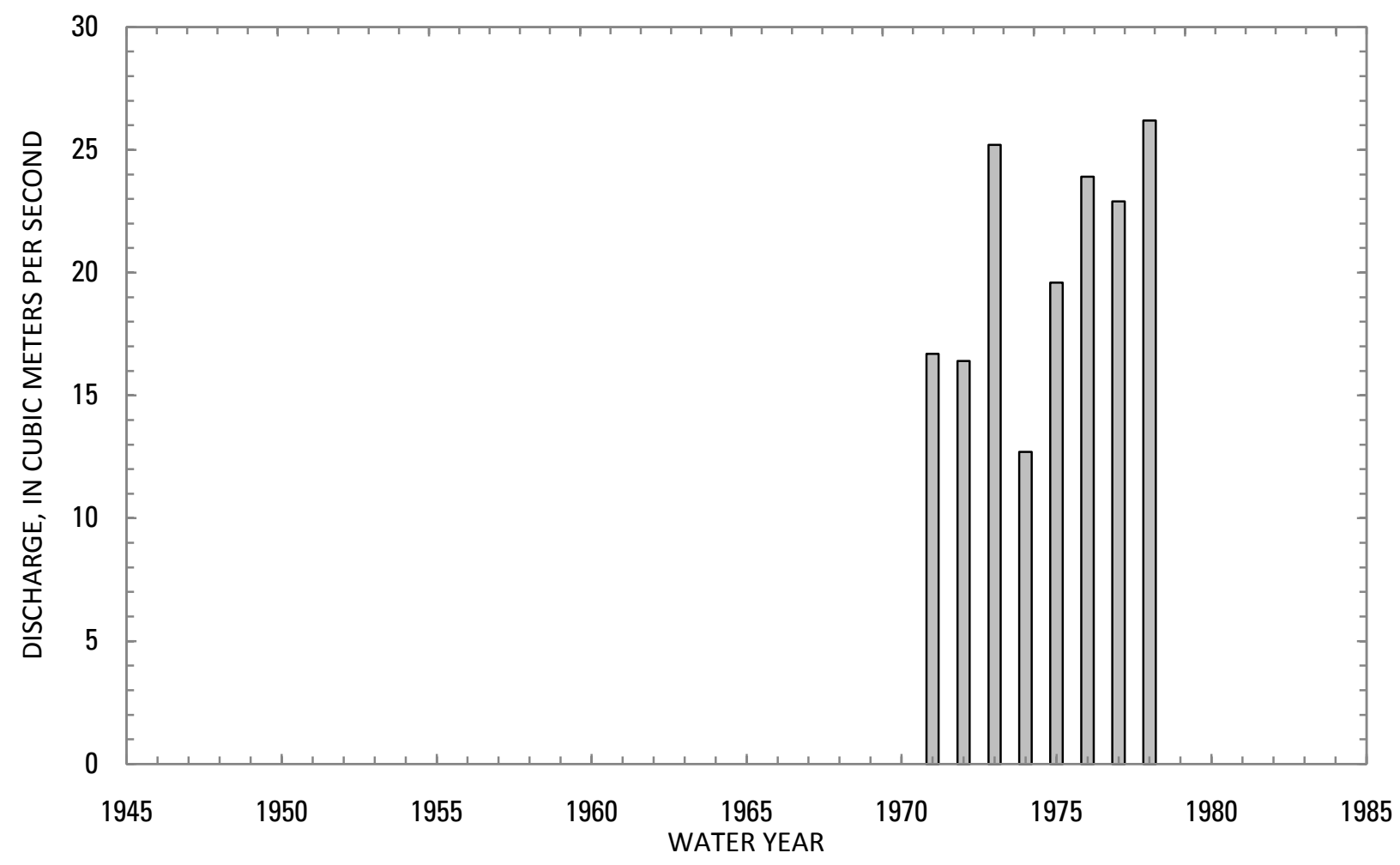




\section{5-1.L00-1A KESHEM RIVER NEAR KESHEM, Continued}

Statistics of monthly and annual mean discharges $\left[\mathrm{m}^{3} / \mathrm{s}\right.$, cubic meters per second]

\begin{tabular}{|c|c|c|c|c|c|c|c|c|}
\hline \multirow[b]{2}{*}{ Month } & \multicolumn{2}{|c|}{ Maximum } & \multicolumn{2}{|c|}{ Minimum } & \multicolumn{4}{|c|}{ Mean } \\
\hline & $\begin{array}{c}\text { Discharge } \\
\left(\mathrm{m}^{3} / \mathrm{s}\right)\end{array}$ & $\begin{array}{c}\text { Water year } \\
\text { of } \\
\text { occurrence }\end{array}$ & $\begin{array}{c}\text { Discharge } \\
\left(\mathrm{m}^{3} / \mathrm{s}\right)\end{array}$ & $\begin{array}{c}\text { Water year } \\
\text { of } \\
\text { occurrence }\end{array}$ & $\begin{array}{c}\text { Discharge } \\
\left(\mathrm{m}^{3} / \mathrm{s}\right)\end{array}$ & $\begin{array}{c}\text { Standard } \\
\text { deviation } \\
\left(\mathrm{m}^{3} / \mathrm{s}\right)\end{array}$ & $\begin{array}{c}\text { Coefficient } \\
\text { of } \\
\text { variation }\end{array}$ & $\begin{array}{c}\text { Percentage } \\
\text { of annual } \\
\text { discharge }\end{array}$ \\
\hline October & 12.2 & 1973 & 9.39 & 1972 & 11.4 & 0.99 & 0.09 & 4.78 \\
\hline November & 10.6 & 1973 & 7.67 & 1974 & 8.93 & 1.05 & 0.12 & 3.75 \\
\hline December & 10.1 & 1978 & 5.56 & 1970 & 7.60 & 1.54 & 0.20 & 3.19 \\
\hline January & 8.25 & 1978 & 5.20 & 1970 & 6.45 & 1.11 & 0.17 & 2.71 \\
\hline February & 8.01 & 1978 & 3.83 & 1972 & 5.84 & 1.48 & 0.25 & 2.46 \\
\hline March & 11.7 & 1973 & 4.59 & 1974 & 7.09 & 2.11 & 0.30 & 2.98 \\
\hline April & 24.4 & 1978 & 4.90 & 1974 & 14.5 & 6.65 & 0.46 & 6.1 \\
\hline May & 48.3 & 1978 & 8.62 & 1974 & 28.6 & 11.1 & 0.39 & 12.0 \\
\hline June & 74.7 & 1978 & 28.8 & 1974 & 50.7 & 16.6 & 0.33 & 21.3 \\
\hline July & 83.8 & 1976 & 27.5 & 1970 & 50.1 & 19.8 & 0.40 & 21.00 \\
\hline August & 41.3 & 1973 & 20.8 & 1974 & 29.5 & 6.12 & 0.21 & 12.4 \\
\hline September & 23.4 & 1973 & 13.0 & 1971 & 17.2 & 3.84 & 0.22 & 7.25 \\
\hline Annual & 26.2 & 1978 & 12.7 & 1974 & 20.5 & 4.85 & 0.24 & 100 \\
\hline
\end{tabular}


15-1.L00-1A KESHEM RIVER NEAR KESHEM, Continued

Monthly and annual flow duration, in cubic meters per second

[ng, not given]

\begin{tabular}{|c|c|c|c|c|c|c|c|c|c|c|c|c|c|}
\hline \multirow{2}{*}{$\begin{array}{l}\text { Percentage } \\
\text { of days } \\
\text { discharge } \\
\text { equaled or } \\
\text { exceeded }\end{array}$} & \multicolumn{12}{|c|}{ Month } & \multirow{2}{*}{ Annua } \\
\hline & October & November & December & January & February & March & April & May & June & July & August & September & \\
\hline 95 & 8.72 & 6.87 & 5.27 & 4.81 & 3.67 & 4.28 & 4.62 & 5.83 & 23.0 & 24.8 & 19.3 & 10.5 & 4.81 \\
\hline 90 & 9.41 & 7.22 & 5.88 & 5.11 & 4.29 & 4.60 & 5.62 & 9.81 & 26.7 & 26.2 & 21.4 & 11.9 & 5.52 \\
\hline 85 & 9.72 & 7.63 & 6.03 & 5.24 & 4.56 & 4.82 & 6.15 & 12.4 & 29.8 & 28.3 & 22.7 & 12.6 & 6.12 \\
\hline 80 & 9.96 & 7.88 & 6.17 & 5.43 & 4.60 & 5.05 & 7.26 & 15.2 & 32.4 & 30.8 & 23.3 & 13.4 & 6.94 \\
\hline 75 & 10.3 & 8.24 & 6.28 & 5.51 & 4.65 & 5.26 & 8.06 & 17.6 & 35.3 & 33.8 & 23.7 & 14.0 & 7.52 \\
\hline 70 & 10.5 & 8.30 & 6.40 & 5.64 & 4.69 & 5.44 & 8.57 & 19.2 & 38.9 & 36.0 & 24.1 & 14.4 & 8.07 \\
\hline 65 & 10.7 & 8.36 & 6.50 & 5.71 & 4.80 & 5.69 & 9.30 & 20.6 & 40.8 & 38.2 & 24.9 & 14.8 & 8.60 \\
\hline 60 & 11.0 & 8.68 & 6.60 & 5.90 & 5.04 & 5.93 & 10.3 & 22.0 & 42.7 & 40.1 & 25.9 & 15.2 & 9.32 \\
\hline 55 & 11.2 & 8.75 & 7.09 & 5.97 & 5.57 & 6.14 & 10.9 & 23.7 & 44.5 & 41.8 & 26.7 & 16.0 & 10.1 \\
\hline 50 & 11.5 & 8.86 & 7.38 & 6.26 & 5.76 & 6.39 & 11.6 & 26.7 & 46.4 & 44.2 & 27.7 & 16.5 & 11.1 \\
\hline 45 & 11.6 & 9.02 & 7.88 & 6.55 & 5.87 & 6.69 & 12.6 & 29.1 & 48.7 & 46.4 & 29.8 & 17.1 & 12.7 \\
\hline 40 & 11.8 & 9.16 & 8.15 & 6.63 & 6.06 & 7.18 & 14.0 & 32.9 & 52.7 & 48.9 & 31.1 & 17.7 & 15.0 \\
\hline 35 & 12.1 & 9.34 & 8.29 & 6.86 & 6.58 & 7.61 & 15.4 & 35.6 & 56.3 & 52.4 & 32.6 & 18.4 & 18.7 \\
\hline 30 & 12.3 & 9.60 & 8.44 & 7.17 & 6.82 & 7.86 & 16.7 & 38.2 & 59.4 & 57.2 & 34.0 & 19.2 & 23.3 \\
\hline 25 & 12.4 & 9.83 & 8.64 & 7.58 & 7.35 & 8.18 & 18.2 & 41.1 & 62.2 & 61.1 & 35.3 & 20.7 & 28.1 \\
\hline 20 & 12.6 & 10.0 & 8.83 & 7.74 & 7.49 & 8.57 & 20.8 & 44.1 & 65.7 & 65.6 & 36.6 & 21.9 & 34.2 \\
\hline 15 & 13.0 & 10.4 & 9.05 & 7.97 & 7.59 & 8.85 & 22.2 & 46.8 & 69.8 & 72.1 & 38.1 & 22.6 & 40.9 \\
\hline 10 & 13.2 & 10.5 & 9.35 & 8.13 & 7.79 & 9.26 & 25.1 & 48.9 & 75.9 & 82.1 & 40.2 & 23.6 & 47.8 \\
\hline 5 & 13.7 & 11.0 & 9.74 & $\mathrm{ng}$ & 8.01 & 10.5 & 31.4 & 51.9 & 97.3 & 99.7 & 42.5 & 25.5 & 61.0 \\
\hline
\end{tabular}




\section{5-1.L00-1A KESHEM RIVER NEAR KESHEM, Continued}

Probability of occurrence of annual high discharges

[ $\mathrm{m}^{3} / \mathrm{s}$, cubic meters per second; $\mathrm{ng}$, not given]

\begin{tabular}{|c|c|c|c|c|c|c|}
\hline \multirow{2}{*}{$\begin{array}{c}\text { Exceedance } \\
\text { probability }\end{array}$} & \multirow{2}{*}{$\begin{array}{c}\text { Recurrence } \\
\text { interval } \\
\text { (years) }\end{array}$} & \multirow{2}{*}{$\begin{array}{c}\text { Maximum } \\
\text { instantaneous } \\
\text { discharge } \\
\left(\mathrm{m}^{3} / \mathrm{s}\right)\end{array}$} & \multicolumn{4}{|c|}{ Maximum daily mean discharge $\left(\mathrm{m}^{3} / \mathrm{s}\right)$} \\
\hline & & & $\begin{array}{l}\text { 3-day } \\
\text { period }\end{array}$ & $\begin{array}{l}\text { 7-day } \\
\text { period }\end{array}$ & $\begin{array}{l}\text { 15-day } \\
\text { period }\end{array}$ & $\begin{array}{l}\text { 30-day } \\
\text { period }\end{array}$ \\
\hline 0.99 & 1.01 & 34.7 & 35.4 & 31.5 & 30.4 & 28.9 \\
\hline 0.95 & 1.05 & 44.3 & 42.7 & 39.1 & 37.5 & 35.4 \\
\hline 0.90 & 1.11 & 51.0 & 47.8 & 44.2 & 42.1 & 39.6 \\
\hline 0.80 & 1.25 & 60.9 & 55.2 & 51.6 & 48.7 & 45.5 \\
\hline 0.50 & 2 & 88.0 & 75.3 & 70.7 & 65.5 & 60.0 \\
\hline 0.20 & 5 & 132 & 108 & 99.7 & 89.9 & 80.4 \\
\hline 0.10 & 10 & 166 & 132 & 121 & 107 & 94.3 \\
\hline 0.04 & 25 & 214 & 167 & 149 & 130 & 112 \\
\hline 0.02 & 50 & 254 & 196 & 172 & 147 & 126 \\
\hline 0.01 & 100 & 297 & 227 & 195 & 166 & 140 \\
\hline 0.005 & 200 & 345 & 261 & 221 & 185 & 155 \\
\hline 0.002 & 500 & 415 & ng & $\mathrm{ng}$ & $\mathrm{ng}$ & ng \\
\hline
\end{tabular}

\section{5-1.L00-1A KESHEM RIVER NEAR KESHEM, Continued}

Probability of occurrence of annual low discharges $\left[\mathrm{m}^{3} / \mathrm{s}\right.$, meters per second]

\begin{tabular}{|c|c|c|c|c|c|c|c|c|c|c|}
\hline \multirow{3}{*}{$\begin{array}{c}\text { Nonexceedance } \\
\text { probability }\end{array}$} & \multirow{3}{*}{$\begin{array}{c}\text { Recurrence } \\
\text { interval } \\
\text { (years) }\end{array}$} & \multicolumn{9}{|c|}{ Minimum daily mean discharge $\left(\mathrm{m}^{3} / \mathrm{s}\right)$} \\
\hline & & \multicolumn{9}{|c|}{ Number of consecutive days } \\
\hline & & 1 & 3 & 7 & 14 & 30 & 60 & 90 & 120 & 183 \\
\hline 0.05 & 20 & 2.85 & 2.90 & 3.10 & 3.43 & 3.54 & 4.08 & 4.48 & 4.86 & 6.30 \\
\hline 0.10 & 10 & 3.16 & 3.24 & 3.45 & 3.76 & 3.88 & 4.36 & 4.77 & 5.17 & 6.60 \\
\hline 0.20 & 5 & 3.60 & 3.70 & 3.93 & 4.22 & 4.35 & 4.75 & 5.17 & 5.60 & 7.00 \\
\hline 0.50 & 2 & 4.63 & 4.77 & 5.05 & 5.28 & 5.46 & 5.70 & 6.11 & 6.57 & 7.87 \\
\hline
\end{tabular}




\section{5-1.L00-1A KESHEM RIVER NEAR KESHEM, Continued}

Probability of occurrence of seasonal low discharges [ $\mathrm{m}^{3} / \mathrm{s}$, meters per second]

\begin{tabular}{|c|c|c|c|c|c|c|c|c|c|}
\hline \multirow{3}{*}{$\begin{array}{c}\text { Nonexceedance } \\
\text { probability }\end{array}$} & \multirow{3}{*}{$\begin{array}{c}\text { Recurrence } \\
\text { interval } \\
\text { (years) }\end{array}$} & \multicolumn{8}{|c|}{ Minimum daily mean discharge $\left(\mathrm{m}^{3} / \mathrm{s}\right)$} \\
\hline & & \multicolumn{8}{|c|}{ Number of consecutive days } \\
\hline & & 1 & 7 & 14 & 30 & 1 & 7 & 14 & 30 \\
\hline & & \multicolumn{4}{|c|}{ December-January-February } & \multicolumn{4}{|c|}{ March-April-May } \\
\hline 0.05 & 20 & 3.00 & 3.28 & 3.54 & 3.77 & 3.25 & 3.64 & 4.00 & 4.60 \\
\hline 0.10 & 10 & 3.36 & 3.64 & 3.86 & 4.06 & 3.57 & 3.94 & 4.28 & 4.91 \\
\hline 0.20 & 5 & 3.84 & 4.13 & 4.30 & 4.46 & 4.03 & 4.37 & 4.67 & 5.36 \\
\hline \multirow[t]{2}{*}{0.50} & 2 & 4.98 & 5.27 & 5.34 & 5.44 & 5.15 & 5.41 & 5.67 & 6.57 \\
\hline & & \multicolumn{4}{|c|}{ June-July-August } & \multicolumn{4}{|c|}{ September-0ctober-November } \\
\hline 0.05 & 20 & 10.2 & 17.1 & 18.4 & 20.8 & 6.07 & 6.38 & 6.59 & 7.31 \\
\hline 0.10 & 10 & 12.6 & 18.2 & 19.6 & 22.3 & 6.45 & 6.76 & 6.99 & 7.63 \\
\hline 0.20 & 5 & 15.7 & 19.6 & 21.2 & 24.2 & 6.96 & 7.24 & 7.50 & 8.04 \\
\hline 0.50 & 2 & 21.4 & 23.3 & 25.0 & 28.6 & 8.01 & 8.24 & 8.51 & 8.92 \\
\hline
\end{tabular}

\section{5-1.L00-1A KESHEM RIVER NEAR KESHEM, Continued}

\section{Annual peak discharges}

[ $\mathrm{m}^{3} / \mathrm{s}$, meters per second]

\begin{tabular}{|c|c|c|c|c|c|}
\hline \multicolumn{3}{|c|}{$\begin{array}{c}\text { Annual peak discharge, } \\
\text { by year }\end{array}$} & \multicolumn{3}{|c|}{$\begin{array}{l}\text { Annual peak discharge, } \\
\text { from highest to lowest }\end{array}$} \\
\hline $\begin{array}{l}\text { Water } \\
\text { year }\end{array}$ & Date & $\begin{array}{c}\text { Peak } \\
\text { discharge } \\
\left(\mathrm{m}^{3} / \mathrm{s}\right)\end{array}$ & $\begin{array}{l}\text { Water } \\
\text { year }\end{array}$ & Date & $\begin{array}{c}\text { Peak } \\
\text { discharge } \\
\left(\mathrm{m}^{3} / \mathrm{s}\right)\end{array}$ \\
\hline 1970 & June 20, 1970 & 54.2 & 1977 & June 24, 1977 & 177 \\
\hline 1971 & June 9, 1971 & 53.6 & 1976 & July 10, 1976 & 150 \\
\hline 1972 & July 2, 1972 & 62.2 & 1978 & April 29, 1978 & 149 \\
\hline 1973 & July 1,1973 & 86.0 & 1975 & June 16, 1975 & 94.4 \\
\hline 1974 & July 1, 1974 & 69.2 & 1973 & July 1, 1973 & 86.0 \\
\hline 1975 & June 16, 1975 & 94.4 & 1974 & July 1, 1974 & 69.2 \\
\hline 1976 & July 10, 1976 & 150 & 1972 & July 2, 1972 & 62.2 \\
\hline 1977 & June 24, 1977 & 177 & 1970 & June 20, 1970 & 54.2 \\
\hline 1978 & April 29, 1978 & 149 & 1971 & June 9, 1971 & 53.6 \\
\hline
\end{tabular}




\section{5-1.L00-1A KESHEM RIVER NEAR KESHEM, Continued}

Monthly and annual mean discharges, in cubic meters per second $[--$, no data]

\begin{tabular}{|c|c|c|c|c|c|c|c|c|c|c|c|c|c|}
\hline \multirow{2}{*}{$\begin{array}{c}\text { Water } \\
\text { year }\end{array}$} & \multicolumn{12}{|c|}{ Monthly mean discharge } & \multirow{2}{*}{$\begin{array}{c}\text { Annual } \\
\text { discharge }\end{array}$} \\
\hline & October & November & December & January & February & March & April & May & June & July & August & September & \\
\hline 1970 & -- & 8.47 & 5.56 & 5.20 & 6.11 & 6.07 & 10.7 & 27.2 & 32.3 & 27.5 & 24.3 & 20.9 & -- \\
\hline 1971 & 11.9 & 8.46 & 6.97 & 6.07 & 4.73 & 6.29 & 13.1 & 29.5 & 40.0 & 32.4 & 27.0 & 13.0 & 16.7 \\
\hline 1972 & 9.39 & 8.27 & 6.11 & 5.57 & 3.83 & 7.07 & 12.3 & 20.2 & 43.0 & 38.4 & 26.3 & 15.6 & 16.4 \\
\hline 1973 & 12.2 & 10.6 & 8.83 & 7.71 & 7.56 & 11.7 & 20.4 & 38.0 & 62.4 & 56.6 & 41.3 & 23.4 & 25.2 \\
\hline 1974 & 11.9 & 7.67 & 6.35 & 5.46 & 4.56 & 4.59 & 4.9 & 8.62 & 28.8 & 35.0 & 20.8 & 13.3 & 12.7 \\
\hline 1975 & 10.3 & 7.76 & 7.14 & 5.61 & 4.88 & 6.08 & 12.9 & 31.1 & 53.6 & 46.0 & 32.5 & 16.7 & 19.6 \\
\hline 1976 & 11.7 & 9.22 & 8.27 & 7.07 & 7.20 & 7.67 & 23.1 & 31.2 & 49.2 & 83.8 & 31.5 & 15.6 & 23.9 \\
\hline 1977 & 11.7 & 10.0 & 9.08 & 7.08 & 5.72 & 5.59 & 8.90 & 23.2 & 72.5 & 77.5 & 27.7 & 14.8 & 22.9 \\
\hline 1978 & 11.9 & 9.92 & 10.1 & 8.25 & 8.01 & 8.77 & 24.4 & 48.3 & 74.7 & 53.3 & 34.5 & 21.9 & 26.2 \\
\hline
\end{tabular}




\section{5-10.R00-1A WARDUJ RIVER AT SHASHPUL \\ (U.S. Geological Survey identification number: $\mathbf{3 7 0 1 0 0 0 7 0 5 0 0 0 0 0 )}$}

LOCATION: Lat $37^{\circ} 01^{\prime} \mathrm{N}$., long $70^{\circ} 50^{\prime} \mathrm{E}$.

DRAINAGE AREA: $4,485 \mathrm{~km}^{2}$.

ELEVATION: 1,407 meters above mean sea level.

PERIOD OF RECORD: October 1, 1969 to September 30, 1978.

GAGE: Water-stage recorder.

Annual mean discharge

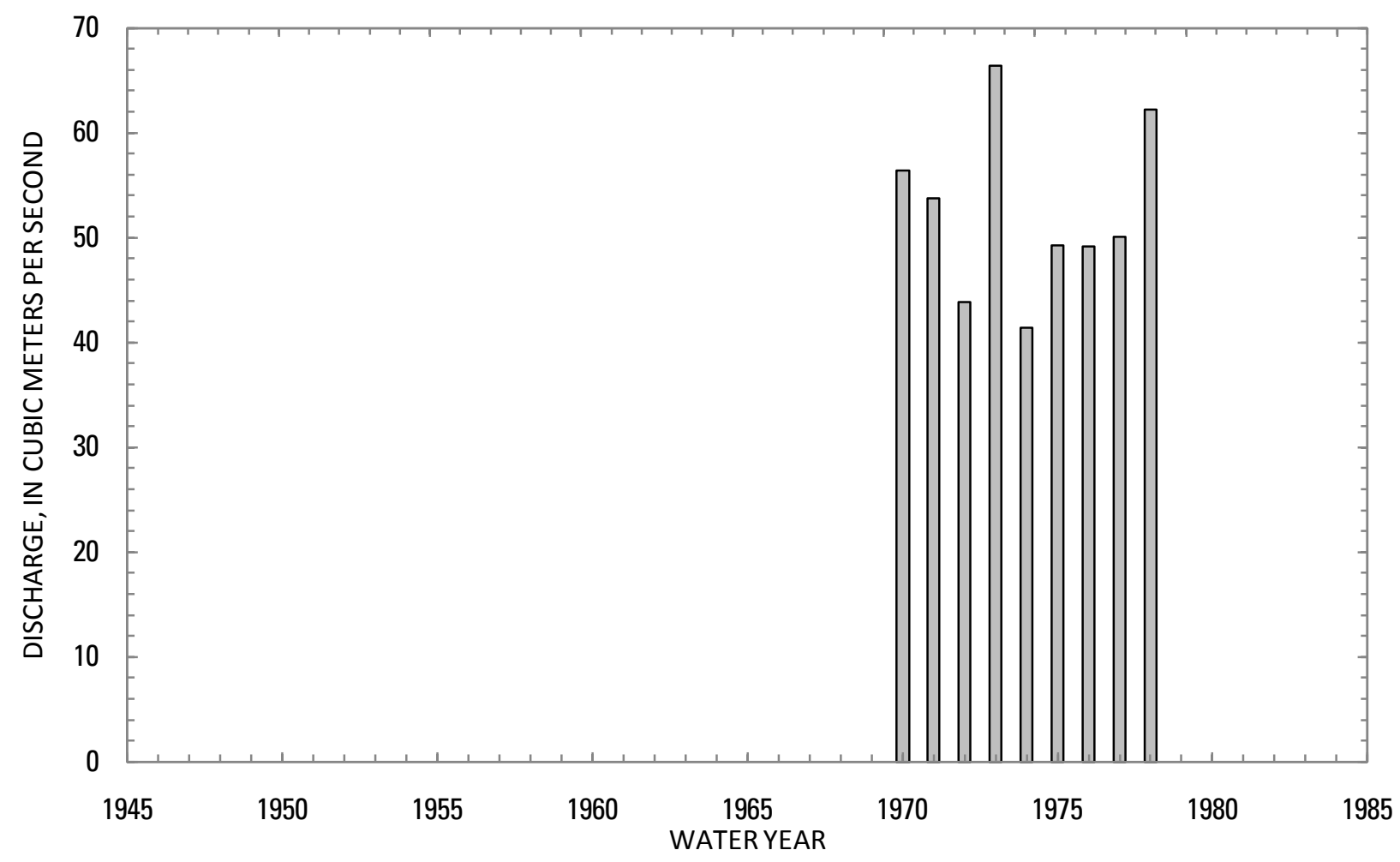




\section{5-10.R00-1A WARDUJ RIVER AT SHASHPUL, Continued}

Statistics of monthly and annual mean discharges $\left[\mathrm{m}^{3} / \mathrm{s}\right.$, cubic meters per second]

\begin{tabular}{|c|c|c|c|c|c|c|c|c|}
\hline \multirow[b]{2}{*}{ Month } & \multicolumn{2}{|c|}{ Maximum } & \multicolumn{2}{|c|}{ Minimum } & \multicolumn{4}{|c|}{ Mean } \\
\hline & $\begin{array}{c}\text { Discharge } \\
\left(\mathrm{m}^{3} / \mathrm{s}\right)\end{array}$ & $\begin{array}{c}\text { Water year } \\
\text { of } \\
\text { occurrence }\end{array}$ & $\begin{array}{c}\text { Discharge } \\
\left(\mathrm{m}^{3} / \mathrm{s}\right)\end{array}$ & $\begin{array}{c}\text { Water year } \\
\text { of } \\
\text { occurrence }\end{array}$ & $\begin{array}{c}\text { Discharge } \\
\left(\mathrm{m}^{3} / \mathrm{s}\right)\end{array}$ & $\begin{array}{c}\text { Standard } \\
\text { deviation } \\
\left(\mathrm{m}^{3} / \mathrm{s}\right)\end{array}$ & $\begin{array}{c}\text { Coefficient } \\
\text { of } \\
\text { variation }\end{array}$ & $\begin{array}{c}\text { Percentage } \\
\text { of annual } \\
\text { discharge }\end{array}$ \\
\hline October & 45.1 & 1970 & 25.8 & 1972 & 31.3 & 5.82 & 0.19 & 4.99 \\
\hline November & 30.5 & 1970 & 19.5 & 1976 & 23.5 & 3.30 & 0.14 & 3.74 \\
\hline December & 23.5 & 1970 & 16.2 & 1976 & 19.1 & 2.17 & 0.11 & 3.04 \\
\hline January & 23.2 & 1970 & 13.5 & 1972 & 15.8 & 2.91 & 0.18 & 2.52 \\
\hline February & 21.0 & 1970 & 11.8 & 1972 & 14.6 & 2.75 & 0.19 & 2.33 \\
\hline March & 16.0 & 1970 & 11.8 & 1975 & 13.6 & 1.34 & 0.10 & 2.17 \\
\hline April & 29.2 & 1978 & 13.8 & 1977 & 20.4 & 4.99 & 0.24 & 3.25 \\
\hline May & 70.6 & 1973 & 23.4 & 1974 & 45.2 & 17.6 & 0.39 & 7.21 \\
\hline June & 203 & 1973 & 84.2 & 1976 & 134 & 39.7 & 0.30 & 21.3 \\
\hline July & 184 & 1973 & 115 & 1970 & 146 & 27.9 & 0.19 & 23.3 \\
\hline August & 126 & 1978 & 77.3 & 1974 & 105 & 15.4 & 0.15 & 16.7 \\
\hline September & 74.1 & 1970 & 44.3 & 1974 & 59.5 & 11.3 & 0.19 & 9.48 \\
\hline Annual & 66.4 & 1973 & 41.4 & 1974 & 52.5 & 8.14 & 0.15 & 100 \\
\hline
\end{tabular}


15-10.R00-1A WARDUJ RIVER AT SHASHPUL, Continued

Monthly and annual flow duration, in cubic meters per second

[ng, not given]

\begin{tabular}{|c|c|c|c|c|c|c|c|c|c|c|c|c|c|}
\hline \multirow{2}{*}{$\begin{array}{l}\text { Percentage } \\
\text { of days } \\
\text { discharge } \\
\text { equaled or } \\
\text { exceeded }\end{array}$} & \multicolumn{12}{|c|}{ Month } & \multirow{2}{*}{ Annua } \\
\hline & October & November & December & January & February & March & April & May & June & July & August & September & \\
\hline 95 & 23.6 & 19.0 & 15.7 & 12.2 & 10.5 & 11.4 & 12.3 & 15.9 & 47.5 & 93.0 & 68.1 & 34.9 & 12.6 \\
\hline 90 & 24.5 & 19.7 & 16.2 & 13.3 & 11.6 & 11.6 & 12.9 & 18.5 & 56.5 & 99.3 & 74.9 & 37.9 & 13.5 \\
\hline 85 & 25.4 & 20.1 & 16.6 & 13.7 & 12.6 & 12.0 & 13.3 & 20.9 & 62.0 & 104 & 80.0 & 40.5 & 14.4 \\
\hline 80 & 26.1 & 20.5 & 17.0 & 14.0 & 13.0 & 12.2 & 13.8 & 24.0 & 69.8 & 108 & 83.3 & 43.3 & 15.4 \\
\hline 75 & 26.5 & 20.9 & 17.4 & 14.2 & 13.3 & 12.4 & 14.3 & 26.4 & 79.4 & 114 & 89.0 & 45.1 & 16.4 \\
\hline 70 & 27.0 & 21.1 & 17.5 & 14.4 & 13.5 & 12.6 & 14.7 & 28.0 & 93.8 & 120 & 93.7 & 47.1 & 17.6 \\
\hline 65 & 27.5 & 21.4 & 17.7 & 14.7 & 13.7 & 12.7 & 15.2 & 29.8 & 104 & 124 & 96.3 & 50.6 & 19.4 \\
\hline 60 & 28.2 & 22.0 & 18.1 & 15.3 & 14.0 & 12.9 & 15.7 & 32.5 & 114 & 128 & 101 & 52.6 & 21.5 \\
\hline 55 & 28.8 & 22.5 & 18.3 & 15.5 & 14.1 & 13.0 & 16.2 & 36.0 & 122 & 132 & 104 & 54.8 & 23.6 \\
\hline 50 & 29.6 & 22.8 & 18.5 & 15.6 & 14.2 & 13.2 & 16.7 & 40.0 & 131 & 137 & 106 & 57.8 & 26.1 \\
\hline 45 & 30.5 & 23.6 & 18.9 & 15.7 & 14.4 & 13.5 & 17.2 & 42.9 & 139 & 142 & 109 & 60.1 & 29.6 \\
\hline 40 & 31.4 & 24.1 & 19.4 & 15.8 & 14.9 & 13.6 & 18.4 & 46.0 & 147 & 145 & 111 & 62.2 & 36.6 \\
\hline 35 & 32.2 & 24.4 & 19.8 & 15.9 & 15.2 & 13.9 & 19.9 & 49.2 & 153 & 151 & 112 & 64.4 & 45.6 \\
\hline 30 & 33.0 & 25.1 & 20.0 & 16.1 & 15.4 & 14.1 & 22.2 & 52.7 & 162 & 160 & 114 & 67.9 & 60.0 \\
\hline 25 & 34.8 & 25.3 & 20.4 & 16.2 & 15.6 & 14.7 & 24.5 & 59.5 & 174 & 170 & 116 & 70.6 & 74.4 \\
\hline 20 & 36.0 & 25.6 & 21.3 & 16.5 & 15.7 & 15.4 & 27.4 & 64.4 & 186 & 183 & 120 & 73.3 & 96.0 \\
\hline 15 & 39.3 & 25.9 & 22.4 & 16.9 & 16.0 & 15.7 & 29.4 & 69.7 & 198 & 190 & 126 & 80.1 & 111 \\
\hline 10 & 41.8 & 26.8 & 23.1 & 21.6 & 20.2 & 16.4 & 35.3 & 80.0 & 218 & 214 & 139 & 84.1 & 132 \\
\hline 5 & 44.7 & 30.2 & $\mathrm{ng}$ & $\mathrm{ng}$ & 21.3 & 17.7 & 38.9 & 97.3 & 252 & 239 & 147 & 95.9 & 163 \\
\hline
\end{tabular}




\section{5-10.R00-1A WARDUJ RIVER AT SHASHPUL, Continued}

Probability of occurrence of annual high discharges

[m $\mathrm{m}^{3} / \mathrm{s}$, cubic meters per second; ng, not given]

\begin{tabular}{|c|c|c|c|c|c|c|}
\hline \multirow{2}{*}{$\begin{array}{c}\text { Exceedance } \\
\text { probability }\end{array}$} & \multirow{2}{*}{$\begin{array}{c}\text { Recurrence } \\
\text { interval } \\
\text { (years) }\end{array}$} & \multirow{2}{*}{$\begin{array}{c}\text { Maximum } \\
\text { instantaneous } \\
\text { discharge } \\
\left(\mathrm{m}^{3} / \mathrm{s}\right)\end{array}$} & \multicolumn{4}{|c|}{ Maximum daily mean discharge $\left(\mathrm{m}^{3} / \mathrm{s}\right)$} \\
\hline & & & $\begin{array}{l}\text { 3-day } \\
\text { period }\end{array}$ & $\begin{array}{l}\text { 7-day } \\
\text { period }\end{array}$ & $\begin{array}{l}\text { 15-day } \\
\text { period }\end{array}$ & $\begin{array}{l}\text { 30-day } \\
\text { period }\end{array}$ \\
\hline 0.99 & 1.01 & 147 & 140 & 124 & 122 & 108 \\
\hline 0.95 & 1.05 & 176 & 155 & 139 & 134 & 121 \\
\hline 0.90 & 1.11 & 194 & 165 & 149 & 142 & 129 \\
\hline 0.80 & 1.25 & 221 & 179 & 162 & 154 & 140 \\
\hline 0.50 & 2 & 286 & 216 & 196 & 182 & 166 \\
\hline 0.20 & 5 & 377 & 269 & 242 & 220 & 199 \\
\hline 0.10 & 10 & 439 & 307 & 273 & 247 & 220 \\
\hline 0.04 & 25 & 521 & 357 & 313 & 280 & 247 \\
\hline 0.02 & 50 & 583 & 396 & 344 & 306 & 266 \\
\hline 0.01 & 100 & 647 & 436 & 375 & 332 & 285 \\
\hline 0.005 & 200 & 713 & 479 & 407 & 359 & 304 \\
\hline 0.002 & 500 & 804 & $\mathrm{ng}$ & ng & ng & $\mathrm{ng}$ \\
\hline
\end{tabular}

15-10.R00-1A WARDUJ RIVER AT SHASHPUL, Continued

Probability of occurrence of annual low discharges

$\left[\mathrm{m}^{3} / \mathrm{s}\right.$, meters per second]

\begin{tabular}{|c|c|c|c|c|c|c|c|c|c|c|}
\hline \multirow{3}{*}{$\begin{array}{c}\text { Nonexceedance } \\
\text { probability }\end{array}$} & \multirow{3}{*}{$\begin{array}{c}\text { Recurrence } \\
\text { interval } \\
\text { (years) }\end{array}$} & \multicolumn{9}{|c|}{ Minimum daily mean discharge $\left(\mathrm{m}^{3} / \mathrm{s}\right)$} \\
\hline & & \multicolumn{9}{|c|}{ Number of consecutive days } \\
\hline & & 1 & 3 & 7 & 14 & 30 & 60 & 90 & 120 & 183 \\
\hline 0.05 & 20 & 7.74 & 8.25 & 9.48 & 10.1 & 10.9 & 11.8 & 12.6 & 13.8 & 17.0 \\
\hline 0.10 & 10 & 8.06 & 8.57 & 9.87 & 10.5 & 11.2 & 12.1 & 12.9 & 14.2 & 17.5 \\
\hline 0.20 & 5 & 8.51 & 9.00 & 10.3 & 11.0 & 11.6 & 12.5 & 13.2 & 14.5 & 18.0 \\
\hline 0.50 & 2 & 9.58 & 9.99 & 11.2 & 12.0 & 12.6 & 13.4 & 13.9 & 15.2 & 19.0 \\
\hline
\end{tabular}




\section{5-10.R00-1A WARDUJ RIVER AT SHASHPUL, Continued}

\section{Probability of occurrence of seasonal low discharges}

[ $\mathrm{m}^{3} / \mathrm{s}$, meters per second]

\begin{tabular}{|c|c|c|c|c|c|c|c|c|c|}
\hline \multirow{3}{*}{$\begin{array}{c}\text { Nonexceedance } \\
\text { probability }\end{array}$} & \multirow{3}{*}{$\begin{array}{l}\text { Recurrence } \\
\text { interval } \\
\text { (years) }\end{array}$} & \multicolumn{8}{|c|}{ Minimum daily mean discharge $\left(\mathrm{m}^{3} / \mathrm{s}\right)$} \\
\hline & & \multicolumn{8}{|c|}{ Number of consecutive days } \\
\hline & & 1 & 7 & 14 & 30 & 1 & 7 & 14 & 30 \\
\hline & & \multicolumn{4}{|c|}{ December-January-February } & \multicolumn{4}{|c|}{ March-April-May } \\
\hline 0.05 & 20 & 7.52 & 9.51 & 10.7 & 11.8 & 8.4 & 9.43 & 10.4 & 11.5 \\
\hline 0.10 & 10 & 8.28 & 10.1 & 11.1 & 12.1 & 9.01 & 10.2 & 10.9 & 11.9 \\
\hline 0.20 & 5 & 9.34 & 10.9 & 11.6 & 12.5 & 9.76 & 11 & 11.4 & 12.4 \\
\hline \multirow[t]{2}{*}{0.50} & 2 & 11.9 & 12.9 & 13.3 & 13.8 & 11.2 & 12.2 & 12.4 & 13.3 \\
\hline & & \multicolumn{4}{|c|}{ June-July-August } & \multicolumn{4}{|c|}{ September-October-November } \\
\hline 0.05 & 20 & 32.3 & 39.9 & 47.5 & 75.6 & 17.9 & 18 & 18.3 & 19.2 \\
\hline 0.10 & 10 & 37.6 & 45.6 & 53.5 & 80.7 & 18.2 & 18.5 & 18.8 & 19.9 \\
\hline 0.20 & 5 & 44.8 & 53.5 & 61.4 & 87.2 & 18.6 & 19.1 & 19.6 & 20.8 \\
\hline 0.50 & 2 & 61.1 & 71.5 & 79.2 & 101 & 19.7 & 20.6 & 21.1 & 22.6 \\
\hline
\end{tabular}

\section{5-10.R00-1A WARDUJ RIVER AT SHASHPUL, Continued}

\section{Annual peak discharges}

[ $\mathrm{m}^{3} / \mathrm{s}$, meters per second]

\begin{tabular}{|c|c|c|c|c|c|}
\hline \multicolumn{3}{|c|}{$\begin{array}{c}\text { Annual peak discharge, } \\
\text { by year }\end{array}$} & \multicolumn{3}{|c|}{$\begin{array}{l}\text { Annual peak discharge, } \\
\text { from highest to lowest }\end{array}$} \\
\hline $\begin{array}{l}\text { Water } \\
\text { year }\end{array}$ & Date & $\begin{array}{c}\text { Peak } \\
\text { discharge } \\
\left(\mathrm{m}^{3} / \mathrm{s}\right)\end{array}$ & $\begin{array}{l}\text { Water } \\
\text { year }\end{array}$ & Date & $\begin{array}{c}\text { Peak } \\
\text { discharge } \\
\left(\mathrm{m}^{3} / \mathrm{s}\right)\end{array}$ \\
\hline 1970 & July 2, 1970 & 226 & 1977 & June 24, 1977 & 495 \\
\hline 1971 & June 10, 1971 & 239 & 1973 & July 1, 1973 & 382 \\
\hline 1972 & July 4, 1972 & 180 & 1978 & July 1, 1978 & 378 \\
\hline 1973 & July 1, 1973 & 382 & 1976 & July 7, 1976 & 311 \\
\hline 1974 & June 30, 1974 & 232 & 1975 & July 14, 1975 & 286 \\
\hline 1975 & July 14, 1975 & 286 & 1971 & June 10, 1971 & 239 \\
\hline 1976 & July 7, 1976 & 311 & 1974 & June 30, 1974 & 232 \\
\hline 1977 & June 24, 1977 & 495 & 1970 & July 2, 1970 & 226 \\
\hline 1978 & July 1,1978 & 378 & 1972 & July 4, 1972 & 180 \\
\hline
\end{tabular}




\section{5-10.R00-1A WARDUJ RIVER AT SHASHPUL, Continued}

Monthly and annual mean discharges, in cubic meters per second

\begin{tabular}{|c|c|c|c|c|c|c|c|c|c|c|c|c|c|}
\hline \multirow{2}{*}{$\begin{array}{c}\text { Water } \\
\text { year }\end{array}$} & \multicolumn{12}{|c|}{ Monthly mean discharge } & \multirow{2}{*}{$\begin{array}{c}\text { Annual } \\
\text { discharge }\end{array}$} \\
\hline & October & November & December & January & February & March & April & May & June & July & August & September & \\
\hline 1970 & 45.1 & 30.5 & 23.5 & 23.2 & 21.0 & 16.0 & 21.7 & 60.0 & 135 & 115 & 110 & 74.1 & 56.4 \\
\hline 1971 & 34.0 & 25.9 & 21.3 & 15.1 & 12.0 & 13.8 & 25.3 & 67.0 & 147 & 120 & 110 & 50.0 & 53.7 \\
\hline 1972 & 25.8 & 20.4 & 17.4 & 13.5 & 11.8 & 13.0 & 16.6 & 28.9 & 96.5 & 119 & 101 & 59.8 & 43.8 \\
\hline 1973 & 32.8 & 24.2 & 18.0 & 13.9 & 13.2 & 12.5 & 23.6 & 70.6 & 203 & 184 & 125 & 72.1 & 66.4 \\
\hline 1974 & 31.1 & 23.0 & 19.0 & 16.4 & 15.2 & 14.2 & 15.9 & 23.4 & 91.2 & 124 & 77.3 & 44.3 & 41.4 \\
\hline 1975 & 27.0 & 21.2 & 18.3 & 15.2 & 14.1 & 11.8 & 17.9 & 36.9 & 132 & 140 & 102 & 53.1 & 49.3 \\
\hline 1976 & 27.9 & 19.5 & 16.2 & 15.8 & 15.8 & 15.2 & 19.3 & 42.9 & 84.2 & 169 & 102 & 60.5 & 49.2 \\
\hline 1977 & 28.5 & 22.8 & 18.7 & 15.4 & 13.7 & 12.7 & 13.8 & 28.1 & 135 & 170 & 91.0 & 48.6 & 50.1 \\
\hline 1978 & 29.5 & 23.6 & 19.2 & 14.1 & 14.9 & 13.5 & 29.2 & 49.2 & 178 & 174 & 126 & 72.7 & 62.2 \\
\hline
\end{tabular}




\section{5-10.R00-2A WARDUJ RIVER NEAR BAHARAK}

\section{(U.S. Geological Survey identification number: 365800070540000 )}

LOCATION: Lat $36^{\circ} 58^{\prime} \mathrm{N}$., long 7054'E.

DRAINAGE AREA: $3,350 \mathrm{~km}^{2}$.

ELEVATION: 1,465 meters above mean sea level.

PERIOD OF RECORD: April 22, 1969 to September 30, 1978.

GAGE: Water-stage recorder.

Annual mean discharge

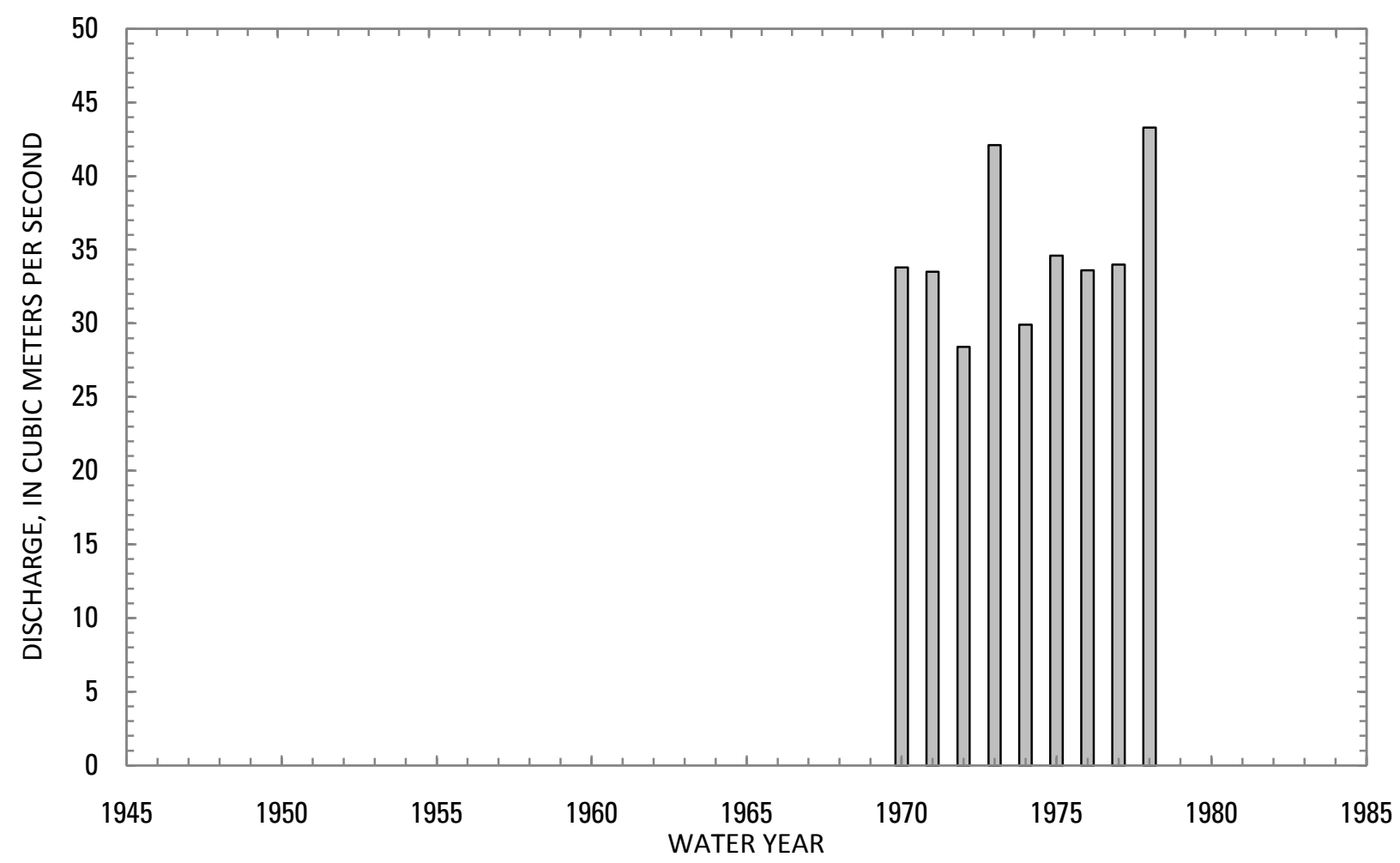




\section{5-10.R00-2A WARDUJ RIVER NEAR BAHARAK, Continued}

Statistics of monthly and annual mean discharges [ $\mathrm{m}^{3} / \mathrm{s}$, cubic meters per second]

\begin{tabular}{|c|c|c|c|c|c|c|c|c|}
\hline \multirow[b]{2}{*}{ Month } & \multicolumn{2}{|c|}{ Maximum } & \multicolumn{2}{|c|}{ Minimum } & \multicolumn{4}{|c|}{ Mean } \\
\hline & $\begin{array}{c}\text { Discharge } \\
\left(\mathrm{m}^{3} / \mathrm{s}\right)\end{array}$ & $\begin{array}{l}\text { Water year } \\
\text { of } \\
\text { occurrence }\end{array}$ & $\begin{array}{c}\text { Discharge } \\
\left(\mathrm{m}^{3} / \mathrm{s}\right)\end{array}$ & $\begin{array}{c}\text { Water year } \\
\text { of } \\
\text { occurrence }\end{array}$ & $\begin{array}{c}\text { Discharge } \\
\left(\mathrm{m}^{3} / \mathrm{s}\right)\end{array}$ & $\begin{array}{c}\text { Standard } \\
\text { deviation } \\
\left(\mathrm{m}^{3} / \mathrm{s}\right)\end{array}$ & $\begin{array}{c}\text { Coefficient } \\
\text { of } \\
\text { variation }\end{array}$ & $\begin{array}{c}\text { Percentage } \\
\text { of annual } \\
\text { discharge }\end{array}$ \\
\hline October & 25.4 & 1970 & 18.8 & 1973 & 21.5 & 2.19 & 0.10 & 5.14 \\
\hline November & 16.1 & 1973 & 14.3 & 1977 & 15.4 & 0.58 & 0.04 & 3.67 \\
\hline December & 13.7 & 1973 & 11.4 & 1977 & 12.8 & 0.69 & 0.05 & 3.05 \\
\hline January & 12.4 & 1970 & 9.86 & 1977 & 11.2 & 0.98 & 0.09 & 2.68 \\
\hline February & 11.4 & 1973 & 8.44 & 1971 & 10.5 & 1.12 & 0.11 & 2.51 \\
\hline March & 11.2 & 1972 & 7.38 & 1971 & 9.55 & 1.14 & 0.12 & 2.28 \\
\hline April & 12.1 & 1978 & 7.35 & 1977 & 10.5 & 1.57 & 0.15 & 2.52 \\
\hline May & 28.5 & 1971 & 10.3 & 1977 & 19.3 & 7.57 & 0.39 & 4.62 \\
\hline June & 119 & 1973 & 43.9 & 1976 & 74.8 & 26.0 & 0.35 & 17.9 \\
\hline July & 128 & 1978 & 73.3 & 1972 & 103 & 22.1 & 0.21 & 24.7 \\
\hline August & 113 & 1969 & 61.0 & 1974 & 84.6 & 15.8 & 0.19 & 20.2 \\
\hline September & 56.3 & 1973 & 36.2 & 1972 & 45.2 & 7.61 & 0.17 & 10.8 \\
\hline Annual & 43.3 & 1978 & 28.4 & 1972 & 34.8 & 4.94 & 0.14 & 100 \\
\hline
\end{tabular}


15-10.R00-2A WARDUJ RIVER NEAR BAHARAK, Continued

Monthly and annual flow duration, in cubic meters per second

[ng, not given]

\begin{tabular}{|c|c|c|c|c|c|c|c|c|c|c|c|c|c|}
\hline \multirow{2}{*}{$\begin{array}{l}\text { Percentage } \\
\text { of days } \\
\text { discharge } \\
\text { equaled or } \\
\text { exceeded }\end{array}$} & \multicolumn{12}{|c|}{ Month } & \multirow{2}{*}{ Annua } \\
\hline & October & November & December & January & February & March & April & May & June & July & August & September & \\
\hline 95 & 16.8 & 13.0 & 11.2 & 9.21 & 8.40 & 7.39 & 7.18 & 8.00 & 26.2 & 58.5 & 52.2 & 26.2 & 8.61 \\
\hline 90 & 17.1 & 13.7 & 11.6 & 9.51 & 9.05 & 8.06 & 7.93 & 8.61 & 29.5 & 63.0 & 59.4 & 28.1 & 9.46 \\
\hline 85 & 17.7 & 13.9 & 11.7 & 9.81 & 9.10 & 8.17 & 8.37 & 9.18 & 31.5 & 67.0 & 66.1 & 30.8 & 10.1 \\
\hline 80 & 18.1 & 14.0 & 12.1 & 9.87 & 9.15 & 8.65 & 8.62 & 9.64 & 35.3 & 71.2 & 69.0 & 32.0 & 10.7 \\
\hline 75 & 18.8 & 14.3 & 12.2 & 10.6 & 9.37 & 8.76 & 8.78 & 10.2 & 40.4 & 77.0 & 71.8 & 33.7 & 11.3 \\
\hline 70 & 19.0 & 14.4 & 12.6 & 10.7 & 9.45 & 9.18 & 9.42 & 11.3 & 45.6 & 84.6 & 74.6 & 35.1 & 11.9 \\
\hline 65 & 19.1 & 14.5 & 12.6 & 10.8 & 10.3 & 9.44 & 9.66 & 12.4 & 52.4 & 87.6 & 76.4 & 37.3 & 12.6 \\
\hline 60 & 20.1 & 15.0 & 12.7 & 11.2 & 10.7 & 9.50 & 9.84 & 13.1 & 59.1 & 93.0 & 78.2 & 39.2 & 13.3 \\
\hline 55 & 20.4 & 15.4 & 12.7 & 11.3 & 10.8 & 9.55 & 10.0 & 14.2 & 64.6 & 97.4 & 80.1 & 40.6 & 14.3 \\
\hline 50 & 20.7 & 15.5 & 13.0 & 11.5 & 11.2 & 9.61 & 10.2 & 15.8 & 71.0 & 102 & 81.7 & 43.2 & 15.8 \\
\hline 45 & 21.4 & 15.5 & 13.1 & 11.6 & 11.2 & 9.84 & 10.3 & 16.9 & 75.8 & 106 & 83.4 & 46.4 & 18.0 \\
\hline 40 & 21.9 & 16.1 & 13.1 & 11.6 & 11.2 & 9.90 & 10.9 & 18.8 & 78.5 & 111 & 85.2 & 47.8 & 21.9 \\
\hline 35 & 22.7 & 16.2 & 13.2 & 11.7 & 11.3 & 10.3 & 11.2 & 20.7 & 82.7 & 115 & 87.6 & 51.3 & 26.8 \\
\hline 30 & 23.0 & 16.3 & 13.5 & 12.1 & 11.3 & 10.4 & 11.5 & 22.3 & 89.9 & 120 & 91.9 & 53.0 & 35.2 \\
\hline 25 & 23.8 & 16.5 & 13.6 & 12.1 & 11.3 & 10.4 & 11.8 & 24.4 & 97.4 & 126 & 96.2 & 54.4 & 51.8 \\
\hline 20 & 24.5 & 16.6 & 13.6 & 12.2 & 11.3 & 10.7 & 12.2 & 26.5 & 107 & 132 & 99.3 & 56.2 & 65.0 \\
\hline 15 & 25.5 & 16.7 & 13.7 & 12.5 & 11.6 & 10.8 & 12.8 & 29.2 & 120 & 139 & 103 & 60.7 & 77.4 \\
\hline 10 & 26.4 & 17.1 & ng & 12.6 & 11.7 & 11.1 & 13.5 & 33.6 & 132 & 147 & 112 & 63.3 & 90.0 \\
\hline 5 & 28.1 & 18.0 & $\mathrm{ng}$ & 12.7 & ng & 11.3 & 14.4 & 44.0 & 156 & 158 & 123 & 68.5 & 113 \\
\hline
\end{tabular}




\section{5-10.R00-2A WARDUJ RIVER NEAR BAHARAK, Continued}

Probability of occurrence of annual high discharges

[ $\mathrm{m}^{3} / \mathrm{s}$, cubic meters per second; $\mathrm{ng}$, not given]

\begin{tabular}{|c|c|c|c|c|c|c|}
\hline \multirow{2}{*}{$\begin{array}{c}\text { Exceedance } \\
\text { probability }\end{array}$} & \multirow{2}{*}{$\begin{array}{c}\text { Recurrence } \\
\text { interval } \\
\text { (years) }\end{array}$} & \multirow{2}{*}{$\begin{array}{l}\text { Maximum } \\
\text { instantaneous } \\
\text { discharge } \\
\left(\mathrm{m}^{3} / \mathrm{s}\right)\end{array}$} & \multicolumn{4}{|c|}{ Maximum daily mean discharge $\left(\mathrm{m}^{3} / \mathrm{s}\right)$} \\
\hline & & & $\begin{array}{l}\text { 3-day } \\
\text { period }\end{array}$ & $\begin{array}{l}\text { 7-day } \\
\text { period }\end{array}$ & $\begin{array}{l}\text { 15-day } \\
\text { period }\end{array}$ & $\begin{array}{l}\text { 30-day } \\
\text { period }\end{array}$ \\
\hline 0.99 & 1.01 & 107 & 80.6 & 74.8 & 70.2 & 65.9 \\
\hline 0.95 & 1.05 & 132 & 98.0 & 90.9 & 83.1 & 76.5 \\
\hline 0.90 & 1.11 & 147 & 108 & 100 & 90.7 & 82.9 \\
\hline 0.80 & 1.25 & 166 & 120 & 111 & 101 & 91.2 \\
\hline 0.50 & 2 & 205 & 145 & 133 & 122 & 110 \\
\hline 0.20 & 5 & 246 & 171 & 154 & 146 & 131 \\
\hline 0.10 & 10 & 269 & 185 & 165 & 159 & 145 \\
\hline 0.04 & 25 & 293 & 198 & 176 & 175 & 160 \\
\hline 0.02 & 50 & 308 & 207 & ${ }^{1} 182$ & 186 & 171 \\
\hline 0.01 & 100 & 322 & 215 & ${ }^{1} 188$ & 196 & 181 \\
\hline 0.005 & 200 & 335 & 222 & ${ }^{1} 192$ & 205 & 191 \\
\hline 0.002 & 500 & 350 & ng & $\mathrm{ng}$ & ng & ng \\
\hline
\end{tabular}

'Data does not fit log-Pearson Type III curve, use with caution.

\section{5-10.R00-2A WARDUJ RIVER NEAR BAHARAK, Continued}

Probability of occurrence of annual low discharges $\left[\mathrm{m}^{3} / \mathrm{s}\right.$, meters per second]

\begin{tabular}{|c|c|c|c|c|c|c|c|c|c|c|}
\hline \multirow{3}{*}{$\begin{array}{c}\text { Nonexceedance } \\
\text { probability }\end{array}$} & \multirow{3}{*}{$\begin{array}{c}\text { Recurrence } \\
\text { interval } \\
\text { (years) }\end{array}$} & \multicolumn{9}{|c|}{ Minimum daily mean discharge $\left(\mathrm{m}^{3} / \mathrm{s}\right)$} \\
\hline & & \multicolumn{9}{|c|}{ Number of consecutive days } \\
\hline & & 1 & 3 & 7 & 14 & 30 & 60 & 90 & 120 & 183 \\
\hline 0.05 & 20 & 6.22 & 6.29 & 6.43 & 6.72 & 6.96 & 7.75 & 8.46 & 9.32 & 12.4 \\
\hline 0.10 & 10 & 6.64 & 6.72 & 6.86 & 7.12 & 7.42 & 8.24 & 8.93 & 9.72 & 12.6 \\
\hline 0.20 & 5 & 7.16 & 7.25 & 7.37 & 7.61 & 7.96 & 8.81 & 9.49 & 10.2 & 12.9 \\
\hline 0.50 & 2 & 8.14 & 8.24 & 8.34 & 8.52 & 8.93 & 9.86 & 10.4 & 11.0 & 13.5 \\
\hline
\end{tabular}




\section{5-10.R00-2A WARDUJ RIVER NEAR BAHARAK, Continued}

Probability of occurrence of seasonal low discharges $\left[\mathrm{m}^{3} / \mathrm{s}\right.$, meters per second]

\begin{tabular}{|c|c|c|c|c|c|c|c|c|c|}
\hline \multirow{3}{*}{$\begin{array}{c}\text { Nonexceedance } \\
\text { probability }\end{array}$} & \multirow{3}{*}{$\begin{array}{c}\text { Recurrence } \\
\text { interval } \\
\text { (years) }\end{array}$} & \multicolumn{8}{|c|}{ Minimum daily mean discharge $\left(\mathrm{m}^{3} / \mathrm{s}\right)$} \\
\hline & & \multicolumn{8}{|c|}{ Number of consecutive days } \\
\hline & & 1 & 7 & 14 & 30 & 1 & 7 & 14 & 30 \\
\hline & & \multicolumn{4}{|c|}{ December-January-February } & \multicolumn{4}{|c|}{ March-April-May } \\
\hline 0.05 & 20 & 7.30 & 7.71 & 8.04 & 8.45 & 6.28 & 6.50 & 6.79 & 7.08 \\
\hline 0.10 & 10 & 7.89 & 8.31 & 8.62 & 8.99 & 6.80 & 7.03 & 7.27 & 7.55 \\
\hline 0.20 & 5 & 8.58 & 9.00 & 9.30 & 9.61 & 7.43 & 7.66 & 7.86 & 8.13 \\
\hline \multirow[t]{2}{*}{0.50} & 2 & 9.77 & 10.2 & 10.4 & 10.7 & 8.60 & 8.83 & 8.96 & 9.23 \\
\hline & & \multicolumn{4}{|c|}{ June-July-August } & \multicolumn{4}{|c|}{ September-October-November } \\
\hline 0.05 & 20 & 16.2 & 20.3 & 24.5 & 43.9 & 11.9 & 12.3 & 12.6 & 14.3 \\
\hline 0.10 & 10 & 18.6 & 23.1 & 27.5 & 48.0 & 12.4 & 12.7 & 13.1 & 14.6 \\
\hline 0.20 & 5 & 21.9 & 27.2 & 31.9 & 53.6 & 12.9 & 13.2 & 13.6 & 14.9 \\
\hline 0.50 & 2 & 29.8 & 38.4 & 43.7 & 66.6 & 13.7 & 14.1 & 14.4 & 15.4 \\
\hline
\end{tabular}

\section{5-10.R00-2A WARDUJ RIVER NEAR BAHARAK, Continued}

Annual peak discharges

[ $\mathrm{m}^{3} / \mathrm{s}$, meters per second]

\begin{tabular}{|c|c|c|c|c|c|}
\hline \multicolumn{3}{|c|}{$\begin{array}{c}\text { Annual peak discharge, } \\
\text { by year }\end{array}$} & \multicolumn{3}{|c|}{$\begin{array}{l}\text { Annual peak discharge, } \\
\text { from highest to lowest }\end{array}$} \\
\hline $\begin{array}{c}\text { Water } \\
\text { year }\end{array}$ & Date & $\begin{array}{c}\text { Peak } \\
\text { discharge } \\
\left(\mathrm{m}^{3} / \mathrm{s}\right)\end{array}$ & $\begin{array}{c}\text { Water } \\
\text { year }\end{array}$ & Date & $\begin{array}{c}\text { Peak } \\
\text { discharge } \\
\left(\mathrm{m}^{3} / \mathrm{s}\right)\end{array}$ \\
\hline 1969 & July 24, 1969 & 215 & 1977 & June 23, 1977 & 288 \\
\hline 1970 & June 27,1970 & 152 & 1973 & June 25, 1973 & 243 \\
\hline 1971 & June 9, 1971 & 152 & 1978 & June 30, 1978 & 231 \\
\hline 1972 & July 3, 1972 & 137 & 1976 & July 7, 1976 & 217 \\
\hline 1973 & June 25, 1973 & 243 & 1969 & July 24, 1969 & 215 \\
\hline 1974 & July 1, 1974 & 209 & 1975 & July 16, 1975 & 215 \\
\hline 1975 & July 16,1975 & 215 & 1974 & July 1, 1974 & 209 \\
\hline 1976 & July 7, 1976 & 217 & 1970 & June 27,1970 & 152 \\
\hline 1977 & June 23, 1977 & 288 & 1971 & June 9, 1971 & 152 \\
\hline 1978 & June 30,1978 & 231 & 1972 & July 3, 1972 & 137 \\
\hline
\end{tabular}


15-10.R00-2A WARDUJ RIVER NEAR BAHARAK, Continued

Monthly and annual mean discharges, in cubic meters per second [--, no data]

\begin{tabular}{|c|c|c|c|c|c|c|c|c|c|c|c|c|c|}
\hline \multirow{2}{*}{$\begin{array}{l}\text { Water } \\
\text { year }\end{array}$} & \multicolumn{12}{|c|}{ Monthly mean discharge } & \multirow{2}{*}{$\begin{array}{c}\text { Annual } \\
\text { discharge }\end{array}$} \\
\hline & October & November & December & January & February & March & April & May & June & July & August & September & \\
\hline 1969 & -- & -- & -- & -- & -- & -- & -- & 10.9 & 60.8 & 123 & 113 & 44.6 & -- \\
\hline 1970 & 25.4 & 15.3 & 13.0 & 12.4 & 11.0 & 10.2 & 11.0 & 28.2 & 71.3 & 74.1 & 79.3 & 52.6 & 33.8 \\
\hline 1971 & 21.8 & 15.5 & 12.5 & 9.90 & 8.44 & 7.38 & 9.02 & 28.5 & 90.6 & 76.4 & 82.2 & 37.7 & 33.5 \\
\hline 1972 & 19.5 & 15.0 & 13.1 & 11.3 & 11.0 & 11.2 & 11.8 & 15.1 & 56.3 & 73.3 & 66.2 & 36.2 & 28.4 \\
\hline 1973 & 18.8 & 16.1 & 13.7 & 12.3 & 11.4 & 9.68 & 11.8 & 26.7 & 119 & 110 & 97.2 & 56.3 & 42.1 \\
\hline 1974 & 23.9 & 15.9 & 13.2 & 11.6 & 11.4 & 9.79 & 9.78 & 10.4 & 50.3 & 102 & 61.0 & 37.9 & 29.9 \\
\hline 1975 & 19.4 & 15.6 & 13.2 & 11.9 & 11.3 & 10.3 & 11.5 & 20.6 & 64.9 & 98.1 & 89.5 & 47.4 & 34.6 \\
\hline 1976 & 21.7 & 14.8 & 12.1 & 11.5 & 11.2 & 10.1 & 10.4 & 16.8 & 43.9 & 126 & 80.9 & 40.7 & 33.6 \\
\hline 1977 & 20.6 & 14.3 & 11.4 & 9.86 & 9.38 & 8.51 & 7.35 & 10.3 & 74.7 & 121 & 76.2 & 42.4 & 34.0 \\
\hline 1978 & 22.4 & 15.8 & 12.8 & 10.3 & 9.4 & 8.84 & 12.1 & 25.7 & 116 & 128 & 100 & 56.1 & 43.3 \\
\hline
\end{tabular}


Prepared by the Pembroke Publishing Service Center.

For more information concerning this report, contact:

Chief

International Water Resources Branch

U.S. Geological Survey

420 National Center

Reston, VA 20192

vrschnei@usgs.gov

or visit our Web site at:

http://water.usgs.gov/international 
